\title{
Final Status Survey Report for Corrective Action Unit 117 - Pluto Disassembly Facility, Building 2201, Nevada National Security Site, Nevada
}

\author{
National Security Technologies, LLC \\ Environmental Restoration Group \\ Nevada National Security Site, Nevada
}

Jeremy Gwin

Douglas Frenette

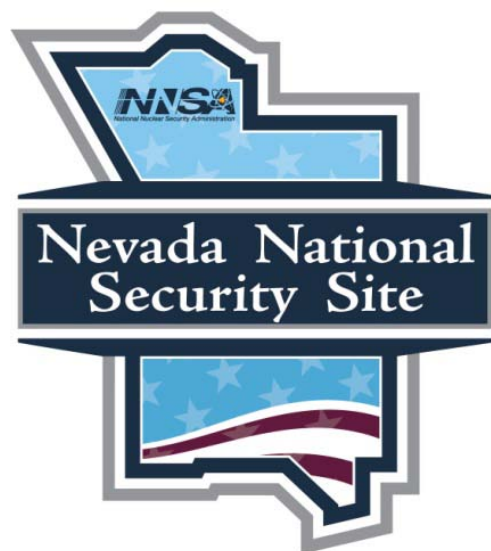

September 2010 


\section{DISCLAIMER}

Reference herein to any specific commercial product, process, or service by trade name, trademark, manufacturer, or otherwise, does not necessarily constitute or imply its endorsement, recommendation, or favoring by the U.S. Government or any agency thereof. 


\section{Final Status Survey Report for Corrective Action Unit 117 - Pluto Disassembly Facility, Building 2201, Nevada National Security Site, Nevada}

\section{Prepared By:}

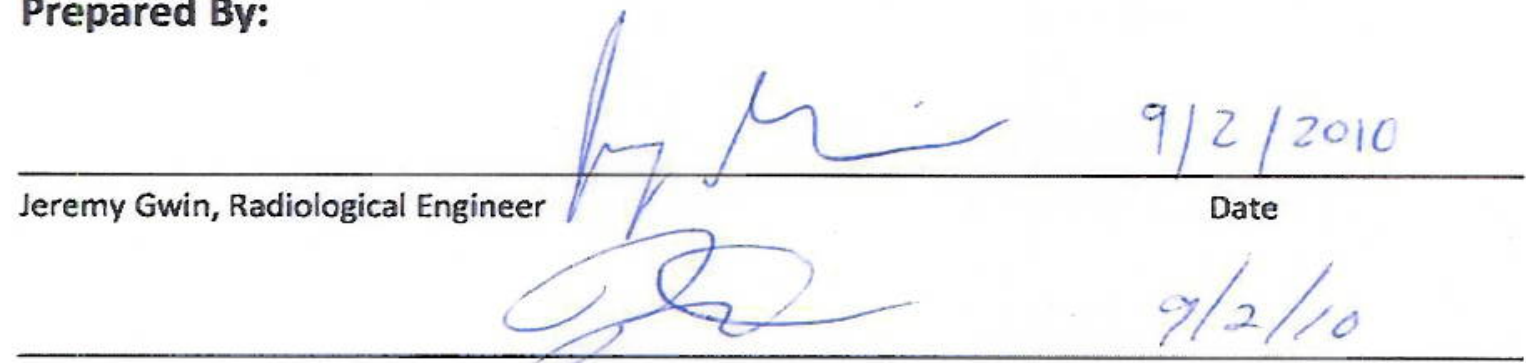

Douglas Frenette, Health Physics Supervisor

Date

Approved By:

Amute Painow

Annette Primrose, Environmental Restoration Manager

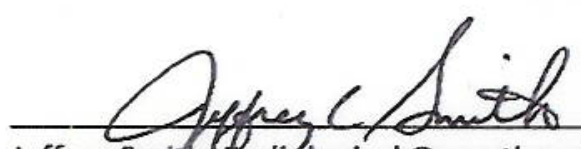

Jeffrey Smjch, Radiblogical Operations Manager

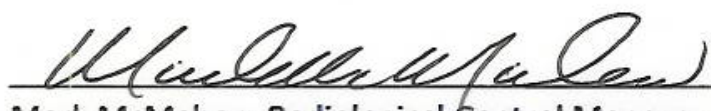

Mark McMahon, Radiological Control Manager
$9 / 2 / 10$

Date

Date

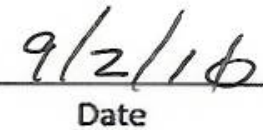




\section{Table of Contents}

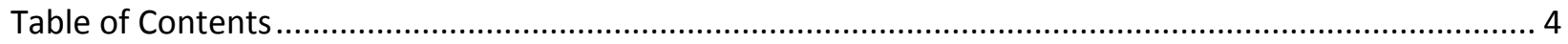

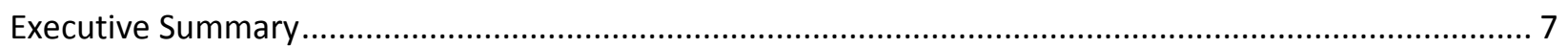

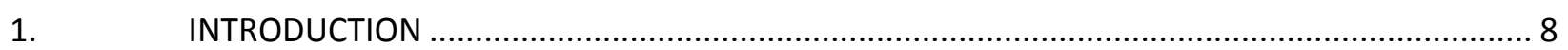

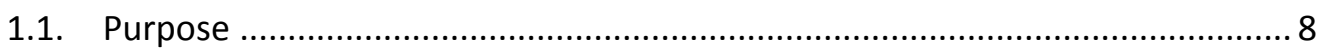

1.2. Authorized Limits for Release ................................................................... 9

1.3. Site Location and History..................................................................... 10

1.4. Multi Agency Radiation Survey and Site Investigation Manual (MARSSIM)

Methodology and Final Status Survey Design .......................................................... 12

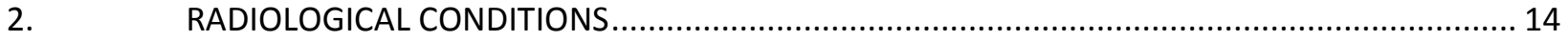

2.1. Historical Site Assessment (HSA) ............................................................... 14

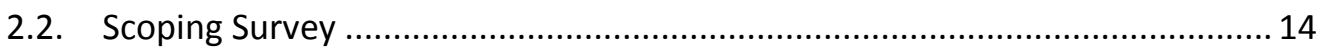

2.3. Characterization Survey ….................................................................... 15

2.4. Remedial Action Support Survey (Decontamination) Before FSS .................... 16

2.5. Remedial Action Support Survey (Decontamination) During FSS .................... 17

2.6. Volumetric Evaluation of Building Materials................................................. 17

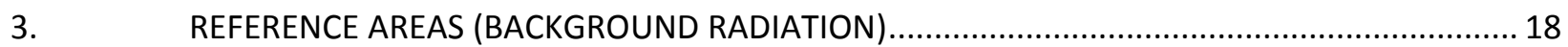

4. PERFORMANCE OF THE FINAL STATUS SURVEYS (Data Collection) ...................................... 20

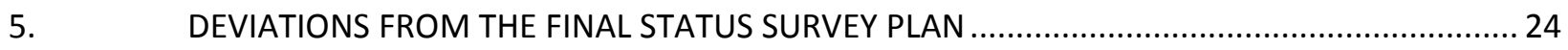

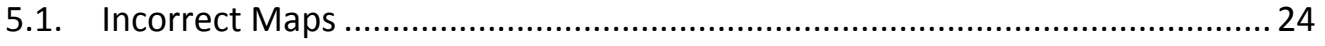

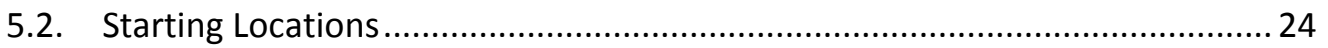

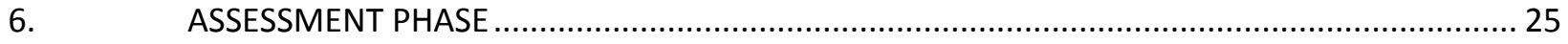

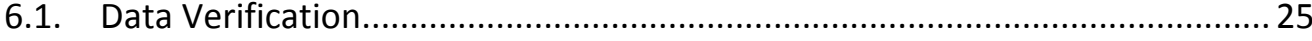

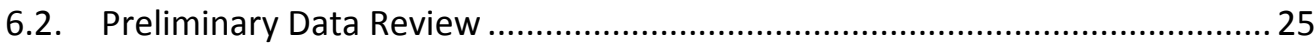

6.3. Investigation of Elevated Readings ……....................................................... 27

6.4. Negative Beta Surface Activity ............................................................. 28

6.5. Graphical Representation of Data ............................................................ 29

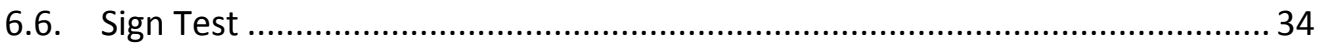

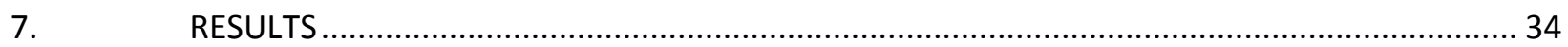

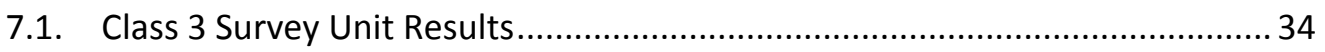




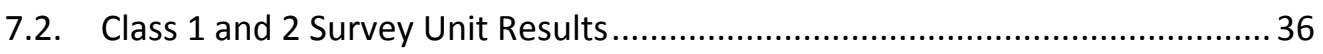

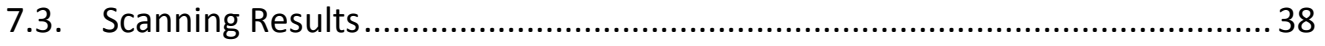

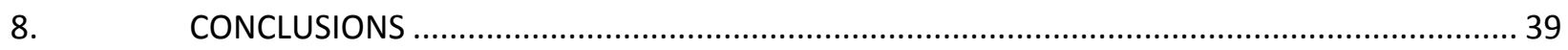

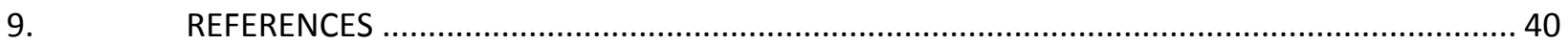

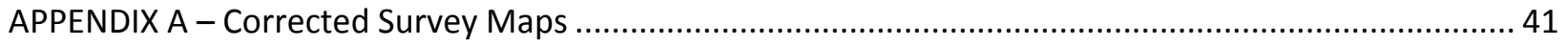

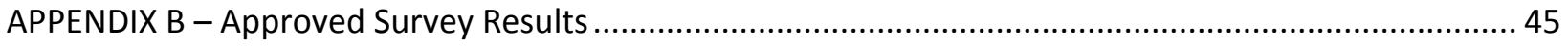

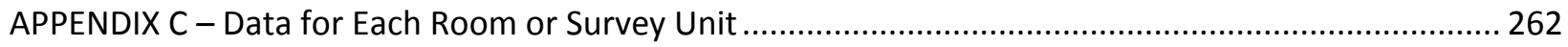

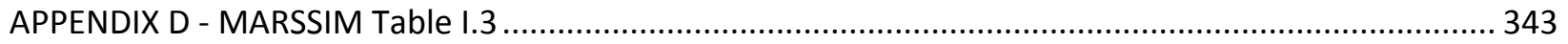

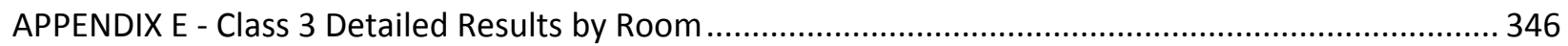

APPENDIX F - Room 102 and Ledges Detailed Results by Survey Unit ................................................... 350

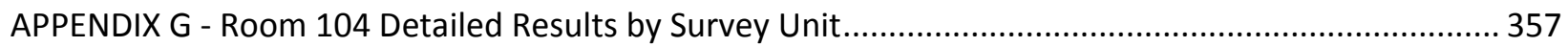

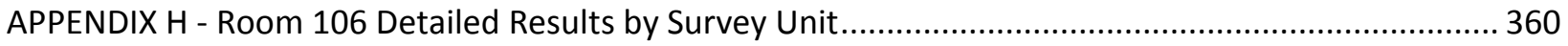

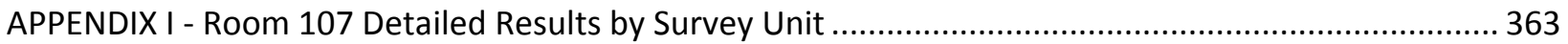

\section{List of Figures}

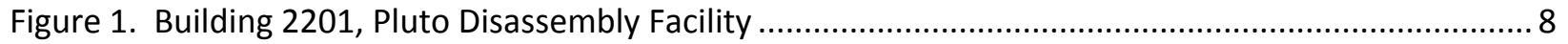

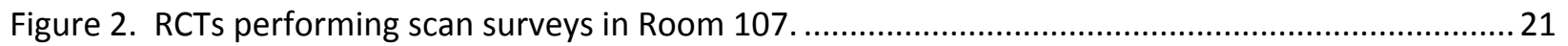

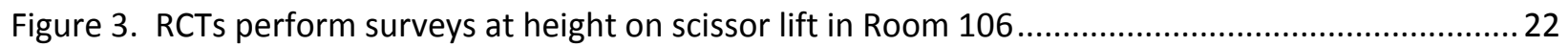

Figure 4. RCTs perform surveys at height on a knuckle boom lift in Room 107 .....................................23

Figure 5. RCTs accessing roof in preparation for surveys in Rooms 201 and 301 ................................24

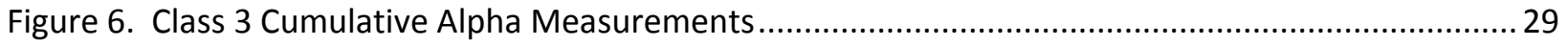

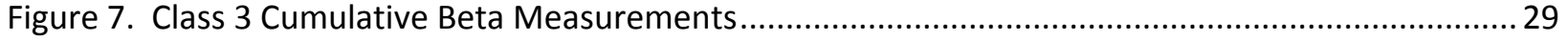

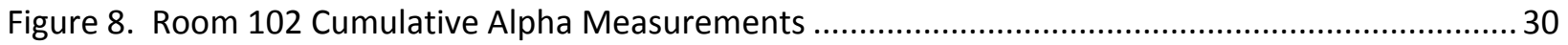

Figure 9. Room 102 Cumulative Beta Measurements ............................................................................ 30

Figure 10. Room 104 Cumulative Alpha Measurements ….................................................................. 31

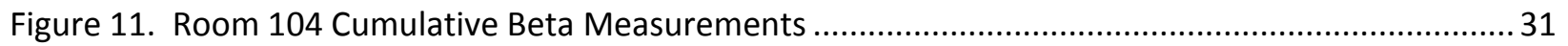

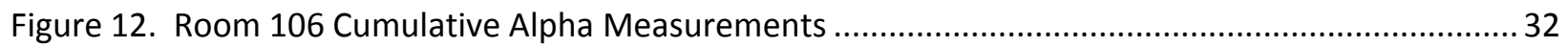

Figure 13. Room 106 Cumulative Beta Measurements ..................................................................... 32

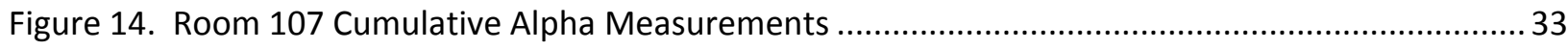

Figure 15. Room 107 Cumulative Beta Measurements …...................................................................... 33 
Final Status Survey Report for

Corrective Action Unit 117-Pluto

Disassembly Facility, Building 2201

\section{List of Tables}

Table 1. Table 4-2 from the NNSS Radiological Control Manual ............................................................. 9

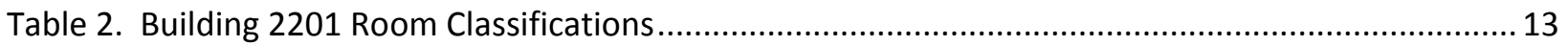

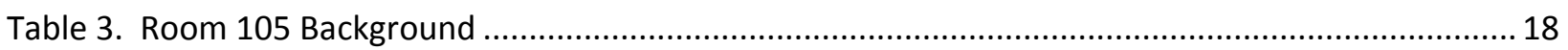

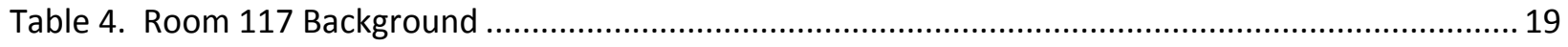

Table 5. Electra 1120 Background for Different Materials .................................................................. 19

Table 6. Electra 1504 Background for Different Materials .................................................................... 20

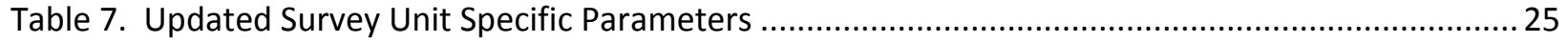

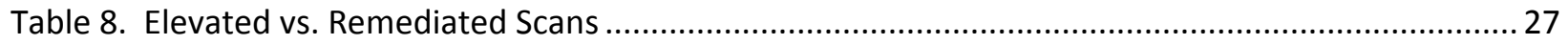

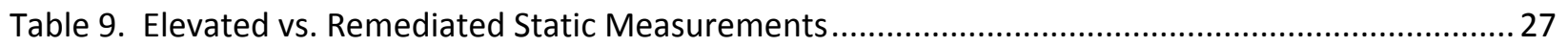

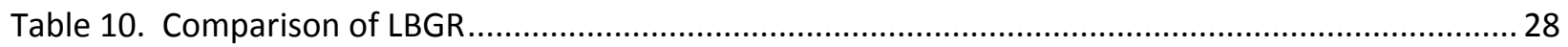

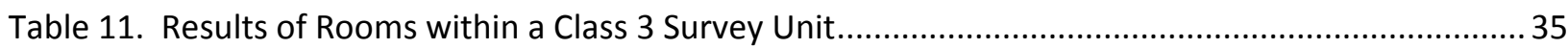

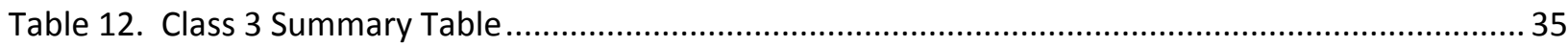

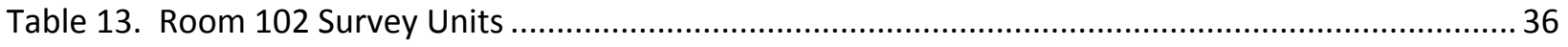

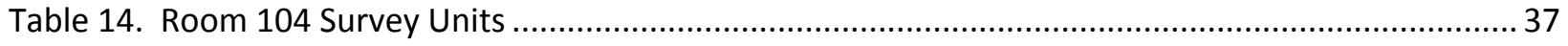

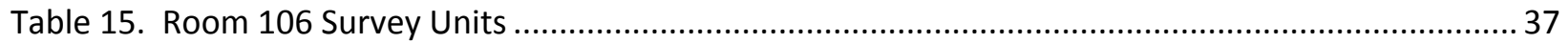

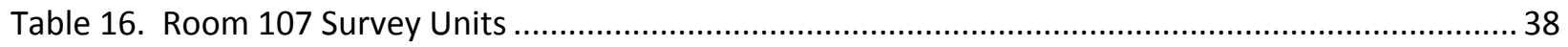

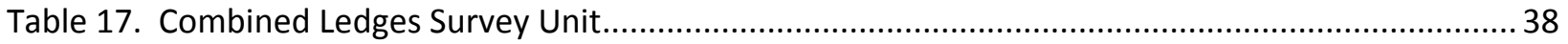

\section{List of Attachments}

ATTACHMENT 1. Survey Plan 10-015, "Final Status Survey Plan for Corrective Action Unit 117 - Pluto Disassembly Facility, Building 2201"

ATTACHMENT 2. Survey Plan 09-022, "Pluto Disassembly Facility - Building Radiological Characterization"

ATTACHMENT 3. Survey Plan 09-031, "Pluto Disassembly Facility - Radiological Characterization (Phase II) and Post Remediation Survey Requirements" 
Final Status Survey Report for

Corrective Action Unit 117-Pluto

Disassembly Facility, Building 2201

\section{Executive Summary}

This document contains the process knowledge, radiological data and subsequent statistical methodology and analysis to support approval for the radiological release of Corrective Action Unit (CAU) 117 - Pluto Disassembly Facility, Building 2201 located in Area 26 of the Nevada National Security Site (NNSS).

Preparations for release of the building began in 2009 and followed the methodology described in the Multi-Agency Radiation Survey and Site Investigation Manual (MARSSIM). MARSSIM is the DOE approved process for release of Real Property (buildings and landmasses) to a set of established criteria or authorized limits. The pre-approved authorized limits for surface contamination values and corresponding assumptions were established by DOE O 5400.5. The release criteria coincide with the acceptance criteria of the U10C landfill permit. The U10C landfill is the proposed location to dispose of the radiologically non-impacted, or "clean," building rubble following demolition. However, other disposition options that include the building and/or waste remaining at the NNSS may be considered providing that the same release limits apply.

The Final Status Survey was designed following MARSSIM guidance by reviewing historical documentation and radiological survey data. Following this review a formal radiological characterization survey was performed in two phases. The characterization revealed multiple areas of residual radioactivity above the release criteria. These locations were remediated (decontaminated) and then the surface activity was verified to be less than the release criteria. Once remediation efforts had been successfully completed, a Final Status Survey Plan (10-015, "Final Status Survey Plan for Corrective Action Unit 117 - Pluto Disassembly Facility, Building 2201") was developed and implemented to complete the final step in the MARSSIM process, the Final Status Survey.

The Final Status Survey Plan consisted of categorizing each individual room into one of three categories: Class 1, Class 2 or Class 3 (a fourth category is a "Non-Impacted Class" which in the case of Building 2201 only pertained to exterior surfaces of the building.) The majority of the rooms were determined to fall in the less restrictive Class 3 category, however, Rooms 102, 104, 106, and 107 were identified as containing Class 1 and 2 areas. Building 2201 was divided into "survey units" and surveyed following the requirements of the Final Status Survey Plan for each particular class.

As each survey unit was completed and documented, the survey results were evaluated. Each sample (static measurement) with units of counts per minute (cpm) was corrected for the appropriate background and converted to a value with units of $\mathrm{dpm} / 100 \mathrm{~cm}^{2}$. With a surface contamination value in the appropriate units, it was compared to the surface contamination limits, or in this case the derived concentration guideline level (DCGLw). The appropriate statistical test (sign test) was then performed. If the survey unit was statistically determined to be below the DCGLw, then the survey unit passed and the null hypothesis (that the survey unit is above limits) was rejected. If the survey unit was equal to or below the critical value in the sign test, the null hypothesis was not rejected. This process was performed for all survey units within Building 2201. A total of thirty-three "Class 1," four "Class 2," and one "Class 3" survey units were developed, surveyed, and evaluated. All survey units successfully passed the statistical test.

Building 2201 meets the release criteria commensurate with the Waste Acceptance Criteria (for radiological purposes) of the U10C landfill permit residing within NNSS boundaries. Based on the thorough statistical sampling and scanning of the building's interior, Building 2201 may be considered radiologically "clean," or free of contamination. 


\section{INTRODUCTION}

This document contains the process knowledge, radiological data and subsequent statistical methodology and analysis to support approval for the radiological release of Corrective Action Unit (CAU) 117 - Pluto Disassembly Facility, Building 2201 (Figure 1) located in Area 26 of the Nevada National Security Site (NNSS), formerly known as the Nevada Test Site (NTS). CAU 117 is located approximately 10 miles northwest of Mercury, Nevada, in the southwest region of Area 26 at the NNSS and comprises one Corrective Action Site (CAS) 26-41-01.

Figure 1. Building 2201, Pluto Disassembly Facility

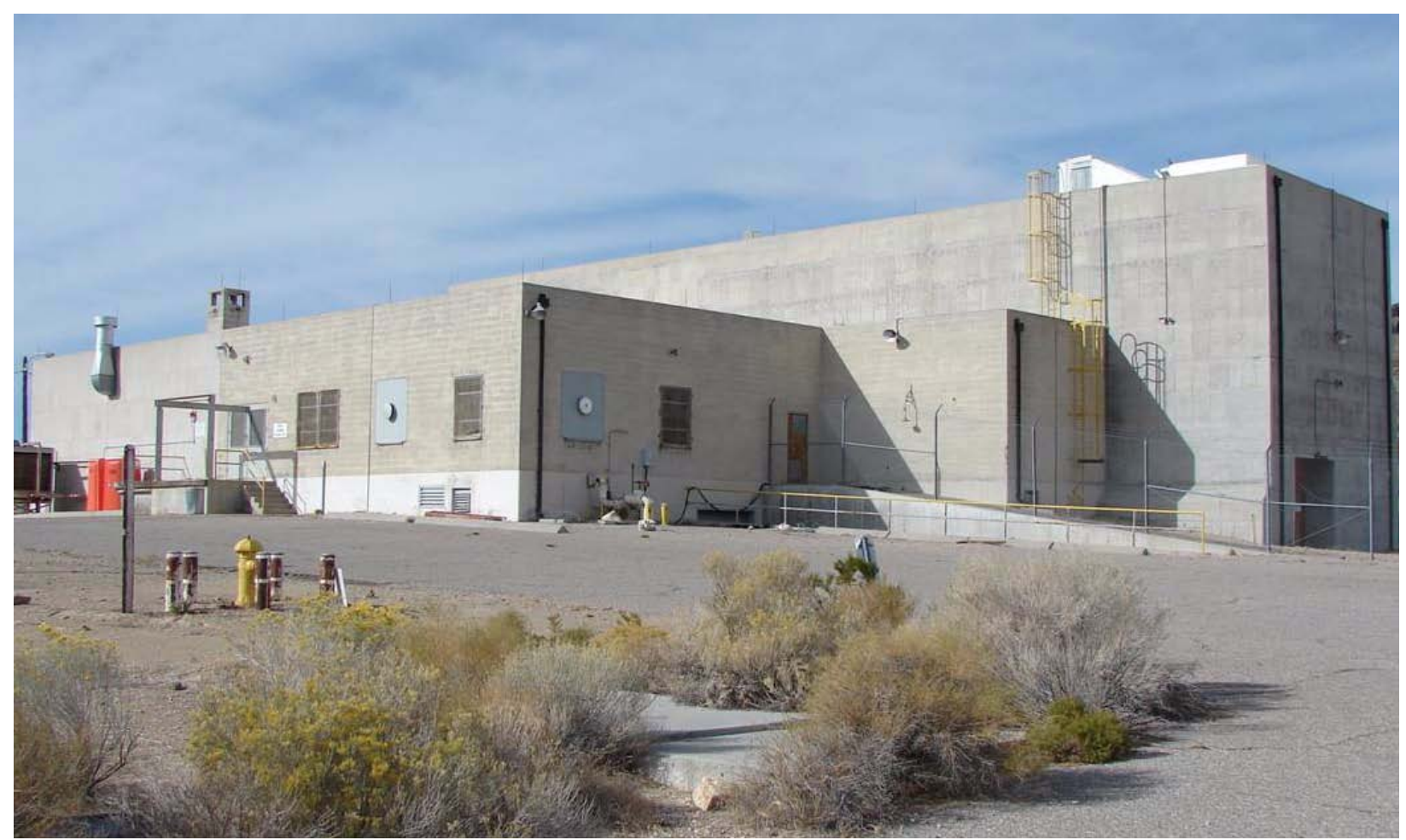

\subsection{Purpose}

With the radiological data and statistical analysis presented in this document, there is sufficient information to confirm that Building 2201 was successfully surveyed in accordance with Survey Plan 10015, "Final Status Survey Plan for Corrective Action Unit 117 - Pluto Disassembly Facility, Building 2201" (Attachment 1). By meeting the requirements within Survey Plan 10-015, NSTec personnel completed a Multi-Agency Radiation Survey and Site Investigation Manual (MARSSIM) Final Status Survey (FSS) for Building 2201.

This report provides a discussion on the limits for unrestricted release of real property, the MARSSIM process, a brief history of the building, actions taken to prepare the building for demolition, results of the FSS, and analysis and interpretation of those results. To successfully pass the FSS would mean that Building 2201 meets the established release criteria (Section 1.2) and may be disposed of as radiologically non-impacted, or "clean," rubble. 
Final Status Survey Report for Corrective Action Unit 117-Pluto Disassembly Facility, Building 2201

What will be left at the Pluto site after the rubble is removed will be the concrete slab and other rubble that will be buried in the basement, storage vaults, and other subsurface facility features. Residual radioactivity associated with the storage vaults or internal passageways within the remaining concrete foundation will be left in place for future end-use consideration. The underground drain lines will also remain. The storage vaults, concrete slab and underground drain lines will all be posted as Underground Radioactive Material Areas (URMAs). These areas will require further evaluation if there is a desire to remove all remaining radiological postings.

\subsection{Authorized Limits for Release}

The release criteria for Pluto were established by NNSA/NSO. The release criteria chosen by the NNSA/NSO for Building 2201 are the allowable total residual surface contamination values established by DOE O 5400.5, "Radiation Protection of the Public and the Environment." These pre-approved authorized limits for surface contamination values and corresponding assumptions were established by DOE 05400.5 and can be found in Table 1 of this report. The release criteria coincide with the acceptance criteria of the U10C landfill permit. The U10C landfill is the proposed location to dispose of the radiologically non-impacted, or "clean," building rubble following demolition. However, other disposition options that include the building and/or waste remaining at the NNSS may be considered providing that the same release limits apply.

Per the Final Status Survey Plan for CAU 117, the building will be considered "clean" if every survey unit has an average contamination value below the authorized limits. For this particular scenario, the surface contamination value of the most limiting nuclide within a mixture of nuclides was used as the release limit. The isotopes that were identified by in-situ measurements during the characterization phase were Cs-137, Am-241, Pu-239, U-235, and Th-231. This means that the average gross alpha contamination in the survey unit must be below the transuranic surface contamination value of 100 $\mathrm{dpm} / 100 \mathrm{~cm}^{2}$. The average gross beta/gamma contamination in the survey unit must be below the Cs137 surface contamination value of $5000 \mathrm{dpm} / 100 \mathrm{~cm}^{2}$.

Table 1. Table 4-2 from the NNSS Radiological Control Manual

\begin{tabular}{|c|c|c|c|}
\hline \multirow{2}{*}{ Radionuclides } & \multicolumn{3}{|c|}{ Contamination levels in $\mathrm{dpm} / 100 \mathrm{~cm}^{2}$} \\
\hline & Removable & Average & Maximum \\
\hline $\begin{array}{c}\text { Group } 1 \text { - Transuranics, I-125, I-129, Ac-227, Ra-226, Ra-228, } \\
\text { Th-228, Th-230, Pa-231 }\end{array}$ & 20 & 100 & 300 \\
\hline $\begin{array}{c}\text { Group } 2 \text { - Th-natural, Sr-90, I-126, I-131, I-133, Ra-223, Ra- } \\
2224, \text { U-232, Th-232 }\end{array}$ & 200 & 1,000 & 3,000 \\
\hline $\begin{array}{c}\text { Group } 3 \text { - U-natural, U-235, U-238, and associated decay } \\
\text { products, alpha emitters }\end{array}$ & 1,000 & 5,000 & 15,000 \\
\hline $\begin{array}{c}\text { Group } 4 \text { - Beta-gamma emitters (radionuclides with decay } \\
\text { modes other than alpha emission or spontaneous fission) } \\
\text { except Sr-90 and others noted above }\end{array}$ & 1,000 & 5,000 & 15,000 \\
\hline Tritium (applicable to surface and subsurface) & 10,000 & $\mathrm{~N} / \mathrm{A}$ & N/A \\
\hline
\end{tabular}


Final Status Survey Report for

Corrective Action Unit 117-Pluto

Disassembly Facility, Building 2201

According to National Security Technologies, LLC (NSTec), Organization Instruction Ol-0441.212, Revision 4, "Controlled and Unrestricted Release," items that have inaccessible surfaces and have been used in a Contamination Area, High Contamination Area, Airborne Radioactivity Area, have been decontaminated, or have an unknown history, require a review by either a Radiological Operations or Radiological Engineering Manager and approval from the Radiological Control Manager and the senior line manager of the project.

\subsection{Site Location and History}

CAU 117 is located north of Cane Spring Road in the southwestern portion of Area 26 of the NNSS. It comprises a single CAS, CAS 26-41-01, which consists of the Pluto Disassembly Facility, Building 2201.

Construction of Building 2201 began in May 1959 for Project Pluto, approximately four years after the project's initiation by the Department of Defense in 1955. After completion of the building in October 1960, the project was passed to Lawrence Radiation Laboratory (LRL), who managed Project Pluto, until its cancellation in 1964. The objective of Project Pluto was to design a nuclear reactor that could propel a missile through the atmosphere at altitudes ranging from sea level to several miles and at velocities up to three times the speed of sound (SNJV, 2009). As a result, the earthbound Tory II-A reactor and its flyable counterpart, the Tory II-C, were developed. The cores of these reactors incorporated several hundred thousand fuel elements consisting of a homogenous mixture of highly enriched uranium dioxide and beryllium oxide (SNJV, 2009). The propulsion system operated on the ramjet principle, in which large quantities of air were ingested, heated by the reactor, and expelled at a high temperature and pressure to provide thrust. Between 1961 and 1964, LRL conducted several tests of the Tory reactors, including four successful power runs with the Tory II-A and two power runs with the Tory II-C (SNJV, 2009).

Project Pluto was also associated with "Hot Box" tests performed in Building 2201. These tests consisted of using stacks of graphite blocks interspersed with a few oralloy (U-235) foils. Air was heated to high temperatures and circulated through the reactor to obtain initial test data. Results from these tests were used to design the Tory II-A reactor (SNJV, 2009).

Only the Tory II-A was disassembled in Building 2201 (SNJV, 2009). The Tory II-C reactor was stored in Building 2201 until 1974, when it was moved to the Reactor Maintenance, Assembly, and Disassembly (R-MAD) building for storage (SNJV, 2009). Actual disassembly of the Tory II-C was performed at the Engine Maintenance, Assembly, and Disassembly (E-MAD) building in 1976 (SNJV, 2009).

Building 2201 was designed specifically to perform remote adjustments, component replacement, and complete disassembly of the Tory II reactor systems. The Main Disassembly Bay (Room 102) housed the Tory II test vehicle when activities dictated that remote handling be used. Disassembly operations were viewed through 4-foot (ft)-thick leaded-glass observation windows immersed in oil (SNJV, 2009). During disassembly, the reactor core was removed from the railcar (used to transport the reactor to the test pad) with remotely operated manipulators. The heavily shielded postmortem hot cells adjacent to the disassembly bay were used to monitor control rod actuators during Project Pluto. Vaults within each cell were operated with remote manipulators for "fuel elements and classified core parts" (SNJV, 2009). The Cold Assembly Bay (Room 101) was used for storage and assembly of modular components for the reactor test vehicle (SNJV, 2009). A maintenance service pit and battery charger for the locomotive were also located in Room 101 (SNJV, 2009). 
Final Status Survey Report for

Corrective Action Unit 117-Pluto

Disassembly Facility, Building 2201

The disassembly bay was supported by a maintenance shop, darkroom, offices, and equipment storage rooms. All controls for Building 2201 operation were located in Room 105 (SNJV, 2009). The Warm \& Cold Storage Room (109) was used for repair and maintenance of equipment contaminated with lowactivity radiological contaminants and was also intended for low-activity glove-box work (SNJV, 2009). Both the Shower/Change Room (113) and Rad Safety Room (114) were designed as change rooms and check stations for personnel needing access to the hot cell and assembly areas (SNJV, 2009). Before it was converted into a restroom, Room 115 served as a darkroom for quickly developing photograph negatives (SNJV, 2009). Room 116 was originally used to store the many spare parts required for the facility. A small electronics maintenance area was later set up in Room 116.

During operation, Rooms 105 and 108 were air conditioned and maintained at a positive pressure so that air flowed into the Main Disassembly Bay (Room 102) and the hot cells (Rooms 104, 106, and 107) when equipment or services were passed through openings at each operating station (SNJV, 2009). These openings were plugged with lead plates or bagged shot when not in use (SNJV, 2009). The ventilation system in Room 102 was exhausted at the west end of the room through roughing and absolute filters before being vented to the atmosphere via the main exhaust stack in Room 103 (SNJV, 2009). In 1998, a portable air-conditioning system was installed by an unidentified "user." This user set up a portable system outside of the building with ducts running through external penetrations in the building that otherwise would have remained closed (SNJV, 2009).

The drainage system originating in the disassembly bay and postmortem cell area was designed to collect rinsate from gross decontamination efforts. Information from interviews with former personnel suggests that the septic drainage system was disconnected in 1964 (SNJV, 2009).

Following the cancellation of Project Pluto, Building 2201 was used for the Fuel Repackaging Operations Project conducted between 1971 and 1972 (SNJV, 2009). During this period, fuel elements from the Tory II reactors were removed from their original containers and placed in 6-liter containers that were then sealed, cleaned, and removed from the hot cells (Rooms 104, 106, and 107) of Building 2201. The containers were temporarily stored in the machine shop area of Building 2201, until they were taken to the decontamination pad in Area 6 for storage or potential future use (SNJV, 2009). The packaged fuel elements were eventually shipped to the Idaho National Engineering Laboratory (SNJV, 2009).

Starting in 1972, Building 2201 was used for a series of classified experiments following the fuel repackaging operations (SNJV, 2009). In the late 1970s and early 1980s, the Room 101 and Room 102 High Bays were modified to house the Hydrogen Content Test Facility (HCTF). The primary purpose of the HCTF was to simulate large holes for calibration of core logging instrumentation. The HCTF equipment consisted of a series of aluminum cells, each containing a different combination of water content and density. The cells contained sand, aluminum oxide, glass marbles, and varying water moisture content (SNJV, 2009).

As of 1986, Sandia National Laboratories (SNL) was using portions of Building 2201 to conduct weaponsrelated nondestructive testing of fast-acting closure systems (SNJV, 2009). Since 1996, SNL has performed activities in Building 2201 associated with non-nuclear rocket launching and other classified projects. Due to their sensitive nature, specific information on experiments conducted by SNL inside Building 2201 is not readily available (SNJV, 2009). In 1998, an unidentified "user" used Building 2201 for additional classified activities (SNJV, 2009).

Within the last decade, Building 2201 was included in an extensive Decontamination and Decommissioning (D\&D) effort for the U.S. Department of Energy, Environmental Restoration Division, 
Final Status Survey Report for

Corrective Action Unit 117-Pluto

Disassembly Facility, Building 2201

to identify possible sites that will need remediation within NNSS. The Preliminary Assessment investigation indicated that chemical, radiological, and environmental hazards were present at Building 2201, in particular lead, beryllium, PCBs, and chemicals associated with photographic development and routine maintenance and repair. Activities have been ongoing to sufficiently remediate chemical, toxic, environmental, and radiological hazards to meet acceptable regulatory requirements and prepare the building for final demolition.

Preparations for final demolition of Building 2201 have included:

- placing the building in a "cold and dark" configuration (de-energizing and disconnecting the building from plant power)

- sampling and draining of remaining piping systems

- sampling and grouting of drainage systems

- removal of wooden false floors in hot cells

- removal of ceiling tiles in office areas

- removal of HEPA filter plenum racks

- removal of leaded glass shield windows

- general equipment strip-out

- removal of Hydrogen Content Test Units

- asbestos abatement

- removal of the personnel platform in Room 102

Several individual campaigns to remove identified Asbestos Containing Material (ACM) have been completed with the most notable being the removal of paint, primer and skim coat on multiple building surfaces. Final Status Surveys were delayed until after the removal of paint, primer and skim coat was completed to ensure the building was in a "Final Status" condition. No fixatives were allowed to be used following removal of ACM. This was to ensure the FSS accurately measured remaining residual radioactive material on raw building surfaces.

\subsection{Multi Agency Radiation Survey and Site Investigation Manual (MARSSIM) Methodology and Final Status Survey Design}

Preparations for release of the building began in 2009 and followed the methodology described in MARSSIM. MARSSIM is the DOE approved process for release of Real Property (buildings and landmasses) to a set of established criteria or authorized limits.

MARSSIM recommends completion of the following process:

- Historical Site Assessment (HSA)

- Scoping Survey

- Characterization Survey

- Remedial Action Support Survey 
Final Status Survey Report for Corrective Action Unit 117-Pluto Disassembly Facility, Building 2201

- Final Status Survey (FSS)

- Verification Survey (not required for Building 2201)

The execution and results of the HSA, scoping survey, characterization survey, and remedial action support survey are discussed in Survey Plan 10-015 (Attachment 1). The FSS is the final validation using the most rigorous and formal survey methodology to prove that real property meets the desired release criteria. Based off the HSA, Scoping, Characterization, and Remedial Action Support survey, the criteria for the FSS is developed. Areas and rooms were placed into survey units and were classified into one of four risk classes:

- $\quad$ Class 1 (highest risk)

- $\quad$ Class 2

- $\quad$ Class 3

- Non Impacted (no risk)

Building 2201 was determined to contain areas from each risk class. The FSS design categorized these rooms as illustrated in Table 2.

Table 2. Building 2201 Room Classifications

\begin{tabular}{|c|c|c|}
\hline Survey Class & Room Number and Name & $\begin{array}{c}\text { Specific } \\
\text { Locations/Surfaces }\end{array}$ \\
\hline Class 1 & Room 102 - Main Disassembly Bay & Floor and Walls \\
\hline Class 1 & Room $104-$ Hot \& Warm Cell & Floor and Walls \\
\hline Class 1 & Room 106 - Kilo-Curie Hot Cell & Floor and Walls \\
\hline Class 1 & Room 107 - Hot Storage \& Packaging Room & Floor and Walls \\
\hline Class 2 & Room 102 - Main Disassembly Bay & Ceiling \\
\hline Class 2 & Room $104-$ Hot \& Warm Cell & Ceiling \\
\hline Class 2 & Room 106 - Kilo-Curie Hot Cell & Ceiling \\
\hline Class 2 & Room 107 - Hot Storage \& Packaging Room & Ceiling \\
\hline Class 3 & Room 101 - Cold Disassembly Bay & All \\
\hline Class 3 & Room 103 - Equipment Room & All \\
\hline Class 3 & Room 105 - Control Room & All \\
\hline Class 3 & Room 108 - Operating Area/Viewing Gallery & All \\
\hline Class 3 & Room 109 - Warm \& Cold Storage Room & All \\
\hline Class 3 & Room 110 - Main Entrance Hallway & All \\
\hline Class 3 & Room 111 - Janitor's Closet & All \\
\hline Class 3 & Room 112 - Restroom (Toilets and Sinks) & All \\
\hline
\end{tabular}


Final Status Survey Report for

Corrective Action Unit 117-Pluto

Disassembly Facility, Building 2201

\begin{tabular}{|c|c|c|}
\hline Survey Class & Room Number and Name & $\begin{array}{c}\text { Specific } \\
\text { Locations/Surfaces }\end{array}$ \\
\hline Class 3 & Room 113-Restroom (Showers) & All \\
\hline Class 3 & Room 114-Restroom (Lockers) & All \\
\hline Class 3 & Room 115-Storage & All \\
\hline Class 3 & Room 116-Supply \& Parts Room & All \\
\hline Class 3 & Room 117- Office & All \\
\hline Class 3 & Room 201-Equipment Room (lower) & All \\
\hline Class 3 & Room 301-Equipment Room (upper) & All \\
\hline Class 3 & Basement Room 1-Equipment Room & All \\
\hline Class 3 & Basement Room 2-Equipment Room & All \\
\hline Class 3 & Basement Room 3-Equipment Room & All \\
\hline Class 3 & Basement Room 4 - Equipment \& Storage Room & All \\
\hline Non Impacted & Building Exterior & \\
\hline
\end{tabular}

Each MARSSIM survey class has a specific set of criteria used to design the required surveys. The higher the risk class the more stringent the survey criteria and the smaller the allowable size of the survey unit. The requirements for Building 2201 were documented and approved through Survey Plan 10-015 (Attachment 1).

Once survey data was properly obtained, documented and approved, the data was run through a statistical test (the sign test). Based on the results of the statistical tests, a survey unit either passed or failed. Failed survey units would be areas of the building that contain surface contamination above the release criteria and would require further remediation to pass.

The next several sections explain in detail the intent of each step in the MARSSIM process, how it was accomplished, and any relevant data used or documented in the process.

\section{RADIOLOGICAL CONDITIONS}

\subsection{Historical Site Assessment (HSA)}

The HSA is the collection of data from historical documentation, observation, or personnel interviews, also known as "process knowledge." The HSA for Building 2201 is summarized in Section 1.3.

\subsection{Scoping Survey}

A scoping survey is a "big picture" type of survey performed if the HSA indicates an area may be impacted. It should also determine the specific contaminant(s) of concern. In the case of the Pluto Disassembly Facility, the HSA established that various rooms and systems were radiologically impacted. The intent of the scoping survey step in MARSSIM has been accomplished through review of multiple 
Final Status Survey Report for

Corrective Action Unit 117-Pluto

Disassembly Facility, Building 2201

documented pre-job, on-the-job, and post-job surveys performed over the previous years. The scoping survey was a culmination of several smaller surveys and analytical samples.

Various radiological surveys and decontamination activities took place in Building 2201 from 1971 to 1999. In 2008, radiological surveys were performed at various locations within the CAS. These radiological surveys were performed to identify the presence, the nature, and the extent of the remaining radiological contaminants. Various radiological walkover surveys were conducted at CAS 2641-01. The walkover surveys included the Main Disassembly Bay (Room 102), the hot cells (rooms 104, 106 , and 107), and an approximate 1,000-square-meter $\left(\mathrm{m}^{2}\right)$ area within the fenced area surrounding the exterior of Building 2201. Radiological walkover surveys were performed using an NE Technology Electra fitted with a DP6BD dual-alpha and beta/gamma radiation probe.

In 2009, Environmental Restoration performed a review of documented radiological survey results. This survey review revealed that some rooms in Building 2201 still contained low levels of residual radioactive material from previous operations.

\subsection{Characterization Survey}

A characterization survey is a more comprehensive survey and determines the nature and extent of the contamination, remedial alternatives, and provides data for the Final Status Survey. In the case of Building 2201, this survey was accomplished and documented in two separate phases based on accessibility of specific areas and surfaces in accordance with survey plans 09-022, "Pluto Disassembly Facility - Building Radiological Characterization," and 09-031, "Pluto Disassembly Facility - Radiological Characterization (Phase II) and Post Remediation Survey Requirements." These survey plans are included for reference as attachments in this document (Attachments 2 and 3).

Phase I of the characterization survey focused on areas and surfaces of the building that were readily accessible. The building was separated, or categorized, into "higher risk" and "lower risk" areas based off of scoping survey data and the HSA. Higher risk areas required a more thorough survey of the area where lower risk areas incorporated less rigorous survey requirements. The characterization survey included a random sampling of the building's exterior surfaces as well. In addition to building surfaces, any equipment remaining inside the building at the time of characterization was surveyed. This included the building stack, ventilation system, cranes, HTCUs, light fixtures, machine shop tools (drill press, lathe, work bench, etc.), electrical cabinets and auxiliary building support equipment (boiler, compressors, pumps, ventilation ducting, etc.) in the basement.

Upon completion of the Phase I characterization surveys, it was documented that multiple locations of fixed contamination above release criteria remained in Rooms 102, 104, 106 and 107. These locations were physically marked and noted for future action during the remediation phase. No removable contamination above release criteria was found.

Phase II of the characterization survey focused on additional areas inside Building 2201 that were inaccessible during Phase I surveys. These inaccessible areas were made accessible for RCT surveys. Once again, the remaining areas were separated, or categorized, into "higher risk" and "lower risk" areas based off of scoping survey data and the HSA. These areas were surveyed under varying degrees of rigor accordingly. Higher risk areas required a more thorough survey of the area where lower risk areas incorporated less rigorous survey requirements.

Phase II areas surveyed included: all four of the second stage filter plenums located in Room 103 and the basement, floor surfaces of Rooms 104, 106, and 107 following removal of a wooden flooring material, 
Final Status Survey Report for Corrective Action Unit 117-Pluto

Disassembly Facility, Building 2201

and the west mezzanine of Room 101. Upon completion of Phase II characterization surveys, no additional areas were found to be radiologically impacted.

Four vaults are located in Rooms 104 (1 vault), 106 (1 vault), and 107 (2 vaults) and were used for storage of nuclear fuel (uranium/beryllium). There is documentation that all remaining nuclear fuel was removed years ago. The vaults are illustrated in drawings as all being of similar size, design and construction. As stated in the approved Closure Report for CAU 117 (DOE/NV-1324), each of the four vault lids were core drilled, visually inspected with video, and had radiological monitoring performed. Radiological monitoring equipment was lowered into the vaults through the core drilled opening and "...no radiological contamination was found and all readings were indistinguishable from background levels" (SNJV, 2009). Although this information provided data to indicate the vaults were not radiologically impacted, MARSSIM does not recognize the methodology used to obtain the data based on the anticipated risk class (Class 1 ). The vaults are below grade and will remain after demolition is complete. Without any additional characterization or release surveys, these areas will be posted as Underground Radioactive Material Areas (URMAs) following the demolition of the above ground structure.

\subsection{Remedial Action Support Survey (Decontamination) Before FSS}

Remedial action support surveys were required prior to commencement of the FSS. Upon completion of the Phase I and Phase II characterization surveys, multiple isolated areas were identified that would exceed the waste acceptance criteria for the onsite landfill. These areas were physically marked and all were eventually decontaminated. The identified radiologically impacted and remediated areas were:

\section{Room 102 (Survey \#09-ER-A26-029)}

- North Wall - $796 \mathrm{dpm} / 100 \mathrm{~cm}^{2}$ alpha

- North Wall (Electrical panel) $-1,110 \mathrm{dpm} / 100 \mathrm{~cm}^{2}$ alpha

- South Wall - 1,200 dpm/100 $\mathrm{cm}^{2}$ beta

- Floor (Multiple locations) - 5,210 to $19,500 \mathrm{dpm} / 100 \mathrm{~cm}^{2}$ beta

\section{Room 104 (Survey \#09-ER-A26-032)}

- South Wall (Entire upper ledge) - 300 to $400 \mathrm{dpm} / 100 \mathrm{~cm}^{2}$ alpha

- South Wall (Inside penetration) - $354 \mathrm{dpm} / 100 \mathrm{~cm}^{2}$ alpha

- East Wall (Bottom, left side of filter rack) $-200 \mathrm{dpm} / 100 \mathrm{~cm}^{2}$ alpha

- East Wall (Bottom of window ledge) $-12,000 \mathrm{dpm} / 100 \mathrm{~cm}^{2}$ beta

\section{Room 106 (Survey \#09-ER-A26-033)}

- South Wall (Bottom, inside penetration) $-226 \mathrm{dpm} / 100 \mathrm{~cm}^{2}$ alpha

- South Wall (Inside plenum) - 210 to $710 \mathrm{dpm} / 100 \mathrm{~cm}^{2}$ alpha

- South Wall (Between grids A-3 and A-4) - 19,500 dpm/100 cm beta

- East Wall (3' high) $-2,640 \mathrm{dpm} / 100 \mathrm{~cm}^{2}$ beta

- East Wall ( $7^{\prime}$ high) $-1,644 \mathrm{dpm} / 100 \mathrm{~cm}^{2}$ beta 
Final Status Survey Report for

Corrective Action Unit 117-Pluto

Disassembly Facility, Building 2201

\section{Room 107 (Survey \#09-ER-A26-034)}

- Floor (Multiple locations) - 158 to $519 \mathrm{dpm} / 100 \mathrm{~cm}^{2}$ alpha

Typically, the contaminated areas were successfully decontaminated by removing the initial layer of concrete, approximately 1 to 3 inches in depth using various methods. The remedial action surveys were performed at each location during and immediately following decontamination. These surveys verified the success of decontamination efforts at each impacted location and that no contamination was spread to immediate surrounding areas during the decontamination process. The thin surface removal of impacted material provided reasonable assumptions that there was no basis for volumetrically contaminated concrete in the impacted areas as it was evident contamination did not migrate deep into any concrete surface. The survey requirements during this phase were captured in Survey Plan 09-031, "Pluto Disassembly Facility - Radiological Characterization (Phase II) and Post Remediation Survey Requirements" (Attachment 3).

\subsection{Remedial Action Support Survey (Decontamination) During FSS}

During the planning phase of the FSS, allowances were granted to perform remediation of areas with elevated activities during the execution of the FSS. Provisions for decontaminating "hot spots" and directions on re-surveying the area were provided in Survey Plan 10-015. Some "hot spots" were found and all were decontaminated to levels below the release limit. A further discussion on the details of these hot spots is found in Section 6.3.

\subsection{Volumetric Evaluation of Building Materials}

A review of historical documentation provided evidence that there were no known tests or experiments that involved operational nuclear reactors, neutron generating devices, or neutron sources capable of activating building materials. All porous materials analyzed, such as concrete and wood, contained in Building 2201 have shown no potential for volumetric contamination. Samples have been taken in concrete, paint, and wood and analyzed at both offsite laboratories and by onsite gamma spectroscopy. Prior to the commencement of the MARSSIM process, separate campaigns to analyze the paint in the four known impacted rooms $(102,104,106$, and 107) were conducted and the results are included in the Pluto Closure Report CAU 117 DOE/NV--1324 and the CAU 117 SAFER Plan DOE/NV--1228.

In addition to the above radiological assays, paint samples from the walls of Rooms 102, 104, 106, and 107 were collected and analyzed at an offsite laboratory in April 2010. These samples were analyzed for isotopic uranium, isotopic plutonium, gamma emitters, and for Strontium-90. The results showed that all samples had activities that were below detectable limits of gamma spectroscopy laboratory instrumentation. Strontium-90 concentration was not above laboratory instrument detection limits. The alpha spectroscopy instruments detected slightly elevated concentrations above the detection limits for uranium 233/234 and 238 (about 359 times lower than the U-10c landfill waste acceptance criteria) and for plutonium 239/240 (about 358 times lower than U-10c landfill waste acceptance criteria).

Another indication that volumetric contamination was not an issue was during remediation of the known contaminated areas found during the characterization survey. These areas were successfully decontaminated by removing the initial layer of concrete, no more than 1 to 3 inches in depth. This provided a reasonable assumption that there was no basis for volumetrically contaminated concrete in the impacted areas prior to initiating the FSS. 
Final Status Survey Report for

Corrective Action Unit 117-Pluto

Disassembly Facility, Building 2201

Following characterization surveys, the painted walls and ceiling surfaces of Rooms 102, 104, 106, and 107 were stripped during asbestos abatement activities. This process involved using a high pressure water system that effectively removed and collected all paint, primer, skim coat, and the initial layer of underlying concrete. This process occurred in the vast majority of all wall and ceiling surfaces in Rooms $102,104,106$, and 107. The FSS was performed following completion of this effort and in all instances where surveys were performed in areas where painted surfaces had been removed, no contamination was detected.

By performing the FSS after the removal of the paint, the possibility of the paint shielding the contamination from instrumentation was negated. Furthermore, the direct survey of the underlying concrete proved that the contamination did not migrate into the sub-surface concrete during historical or remediation activities. All evidence demonstrates that Building 2201 contains no volumetric contamination.

\section{REFERENCE AREAS (BACKGROUND RADIATION)}

Reference areas provide a location for background measurements which are used for comparisons with survey unit data. A site background reference area should have similar physical and radiological characteristics as the the survey unit being evaluated.

The reference areas were located in rooms 105 and 117 and are in a Class 3 area. Initially, room 117 was designated as the only reference area. Room 117 has an exterior wall with windows. Room 105 was added as a reference area after data showed that room 117 had a higher beta background than some of the hot cells. Natural background is lower in the hot cells because the walls and ceilings are composed of 4 feet of concrete. Room 105 was an ideal location for a reference area because it is surrounded on three sides by 4 -feet thick concrete walls, yet resides outside the Class 1 area. Room 105 served as the reference area for the survey units with thick concrete walls, while room 117 served as the reference area for survey units that are not heavily shielded.

Each detector used in the FSS had 20 measurements taken in the reference area on different materials. The averages of all the measurements in the reference area, by material type, are listed in Tables 3 and 4.

Table 3. Room 105 Background

\begin{tabular}{|c|c|c|}
\hline \multicolumn{3}{|c|}{ Combined Background Averages for Room 105} \\
\hline & $\mathrm{cpm}$ & $\mathrm{dpm} / 100 \mathrm{~cm}^{2}$ \\
\hline$\alpha$ Concrete & 4.3 & 20.9 \\
\hline Standard Deviation & 2.0 & 9.7 \\
\hline B Concrete & 1040.5 & 1735.9 \\
\hline Standard Deviation & 82.8 & 138.1 \\
\hline$\alpha$ Concrete Block & 4.8 & 23.4 \\
\hline Standard Deviation & 2.1 & 10.2 \\
\hline b Concrete Block & 696.7 & 1162.2 \\
\hline Standard Deviation & 46.8 & 78.0 \\
\hline
\end{tabular}


Final Status Survey Report for Corrective Action Unit 117-Pluto Disassembly Facility, Building 2201

Table 4. Room 117 Background

\begin{tabular}{|c|c|c|}
\hline \multicolumn{3}{|c|}{ Combined Background Averages for Room $\mathbf{1 1 7}$} \\
\hline & $\mathrm{cpm}$ & $\mathrm{dpm} / 100 \mathrm{~cm}^{2}$ \\
\hline$\alpha$ Concrete & 6.6 & 32.1 \\
\hline Standard Deviation & 3.7 & 18.0 \\
\hline 6 Concrete & 1665.3 & 2778.3 \\
\hline Standard Deviation & 79.3 & 132.2 \\
\hline$\alpha$ Concrete Block & 10.1 & 48.8 \\
\hline Standard Deviation & 3.5 & 16.9 \\
\hline 6 Concrete Block & 1386.5 & 2313.2 \\
\hline Standard Deviation & 87.7 & 146.4 \\
\hline
\end{tabular}

Two detectors were chosen to survey other materials such as drywall, wood, and metal. These materials are mainly found within Class 3 areas. The averages of all the measurements in the reference area, by material type, are listed in Tables 5 and 6.

Table 5. Electra 1120 Background for Different Materials

\begin{tabular}{|c|c|c|}
\hline \multicolumn{2}{|c|}{ Electra 1120 Background Averages for Different Materials } \\
\hline & $c p m$ & $d p m / 100 \mathrm{~cm}^{2}$ \\
\hline$\alpha$ Drywall & 11.5 & 54.1 \\
\hline Standard Deviation & 4.2 & 20.0 \\
\hline B Drywall & 1250.8 & 2004.5 \\
\hline Standard Deviation & 46.2 & 74.0 \\
\hline$\alpha$ Wood & 4.8 & 22.5 \\
\hline Standard Deviation & 2.1 & 10.2 \\
\hline 6 Wood & 1410.7 & 2260.7 \\
\hline Standard Deviation & 34.7 & 55.5 \\
\hline$\alpha$ Metal & 4.7 & 22.0 \\
\hline Standard Deviation & 1.9 & 8.8 \\
\hline 6 Metal & 1331.7 & 2134.1 \\
\hline Standard Deviation & 36.2 & 58.0 \\
\hline
\end{tabular}


Table 6. Electra 1504 Background for Different Materials

\begin{tabular}{|c|c|c|}
\hline \multicolumn{3}{|c|}{ Electra 1504 Background Averages for Different Materials } \\
\hline & $c p m$ & $d p m / 100 \mathrm{~cm}^{2}$ \\
\hline$\alpha$ Drywall & 8.8 & 45.2 \\
\hline Standard Deviation & 2.4 & 12.2 \\
\hline B Drywall & 1177.6 & 1982.4 \\
\hline Standard Deviation & 26.8 & 45.1 \\
\hline$\alpha$ Wood & 6.9 & 35.4 \\
\hline Standard Deviation & 3.5 & 17.9 \\
\hline B Wood & 1295.7 & 2181.2 \\
\hline Standard Deviation & 40.6 & 68.4 \\
\hline$\alpha$ Metal & 5.3 & 27.4 \\
\hline Standard Deviation & 2.4 & 12.6 \\
\hline B Metal & 1238.9 & 2085.6 \\
\hline Standard Deviation & 23.2 & 39.1 \\
\hline
\end{tabular}

\section{PERFORMANCE OF THE FINAL STATUS SURVEYS (Data Collection)}

Radiological surveys were performed by qualified Radiological Control Technicians (RCTs) utilizing the NE Electra specially fitted with the DP-8B $600 \mathrm{~cm}^{2}$ large-area, dual-scintillation probe. The addition of the DP-8B probes allowed RCTs to obtain the required MDA in a shorter amount of time (1.0 minute with the DP-8B versus 13.5 minutes with a standard DP-6 probe.) In addition, scan rate speeds were increased (one foot per second with the DP-8B versus 1 inch per second with a standard DP- 6 probe.) These increased efficiencies allowed for the actual data collection phase of the FSS to be done in an expedited manner.

Surveys were performed during the months of July and August 2010 and typically documented by individual rooms (for Class 3 areas) or survey units (for Class 1 and 2 areas). During this time the building was undergoing a large scale asbestos abatement effort. As rooms were cleared for asbestos and determined to be in their final configuration (a.k.a. final status), they would be turned over to Radiological Control personnel to perform the FSS. No fixatives were allowed to be used following the removal of asbestos. This was to ensure the FSS accurately measured remaining residual radioactive material on raw building surfaces.

Once RCTs began surveys in a room or area, as a protective measure to ensure no radioactive materials were introduced while surveys were in process or following completion of surveys in a particular room or area, administrative controls were used. These controls involved placing signage at all access points to a room or area that had been surveyed or was in the process of being surveyed. The signage stated:

"Building 2201 is currently undergoing a radiological MARSSIM Final Status Survey.

This area is either currently undergoing surveys or has been cleared. Absolutely no entry is allowed in this area of the building without prior Radcon authorization."

Once signage was in place, RCTs began collecting data (Figure 2). One of the major challenges of performing surveys was access to elevated areas. Many of the rooms in Building 2201 have walls and 
ceilings that were outside the reach of an RCT. In particular, the four Class 1 \& 2 rooms $(102,104,106$, $\& 107)$ all have 35 -foot high ceilings. These rooms required a $100 \%$ scan survey and were only accessible by the use of aerial lift platforms. RCTs were trained and qualified on the use of fall protection and aerial lift platforms. Scissor lifts and knuckle boom lifts provided the required accessibility (Figures 3 and 4). In addition, access to the roof to survey Rooms 201 and 301 required a "Roof Access / Elevated Work Plan" (Figure 5) due to the deteriorated condition of the existing roof.

Figure 2. RCTs performing scan surveys in Room 107.

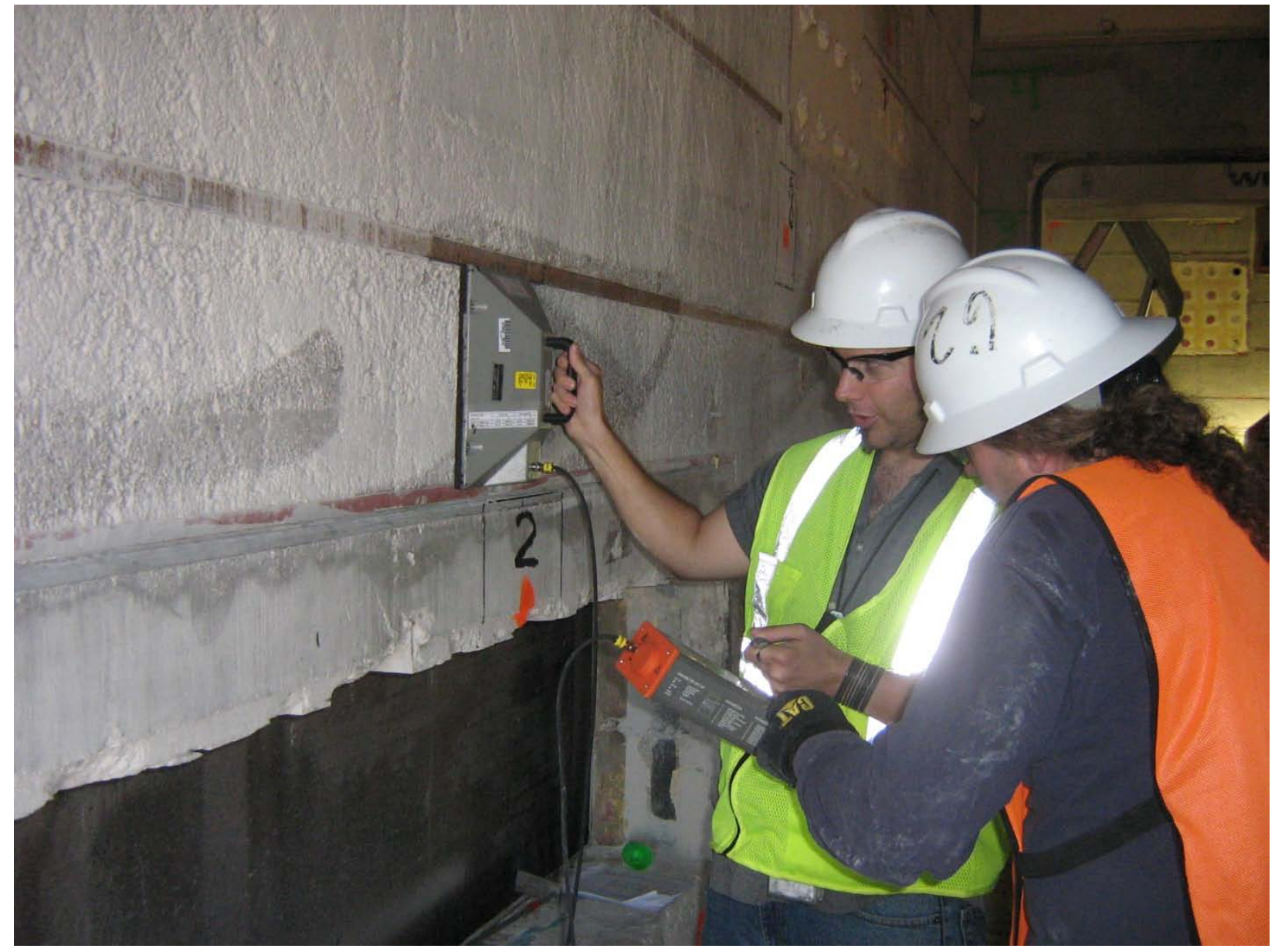


Final Status Survey Report for

Corrective Action Unit 117-Pluto

Disassembly Facility, Building 2201

Figure 3. RCTs perform surveys at height on scissor lift in Room 106

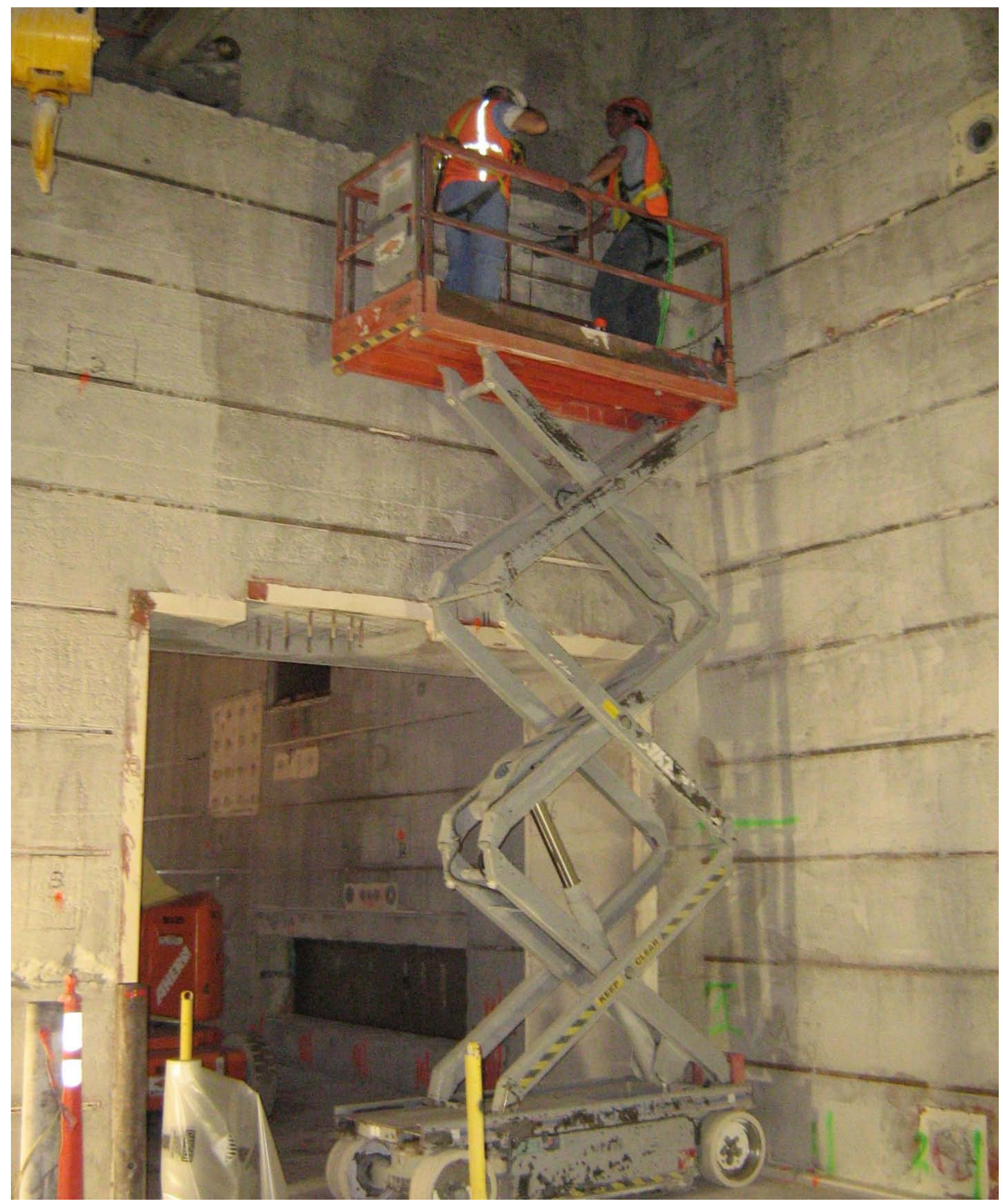


Final Status Survey Report for

Corrective Action Unit 117-Pluto

Disassembly Facility, Building 2201

Figure 4. RCTs perform surveys at height on a knuckle boom lift in Room 107

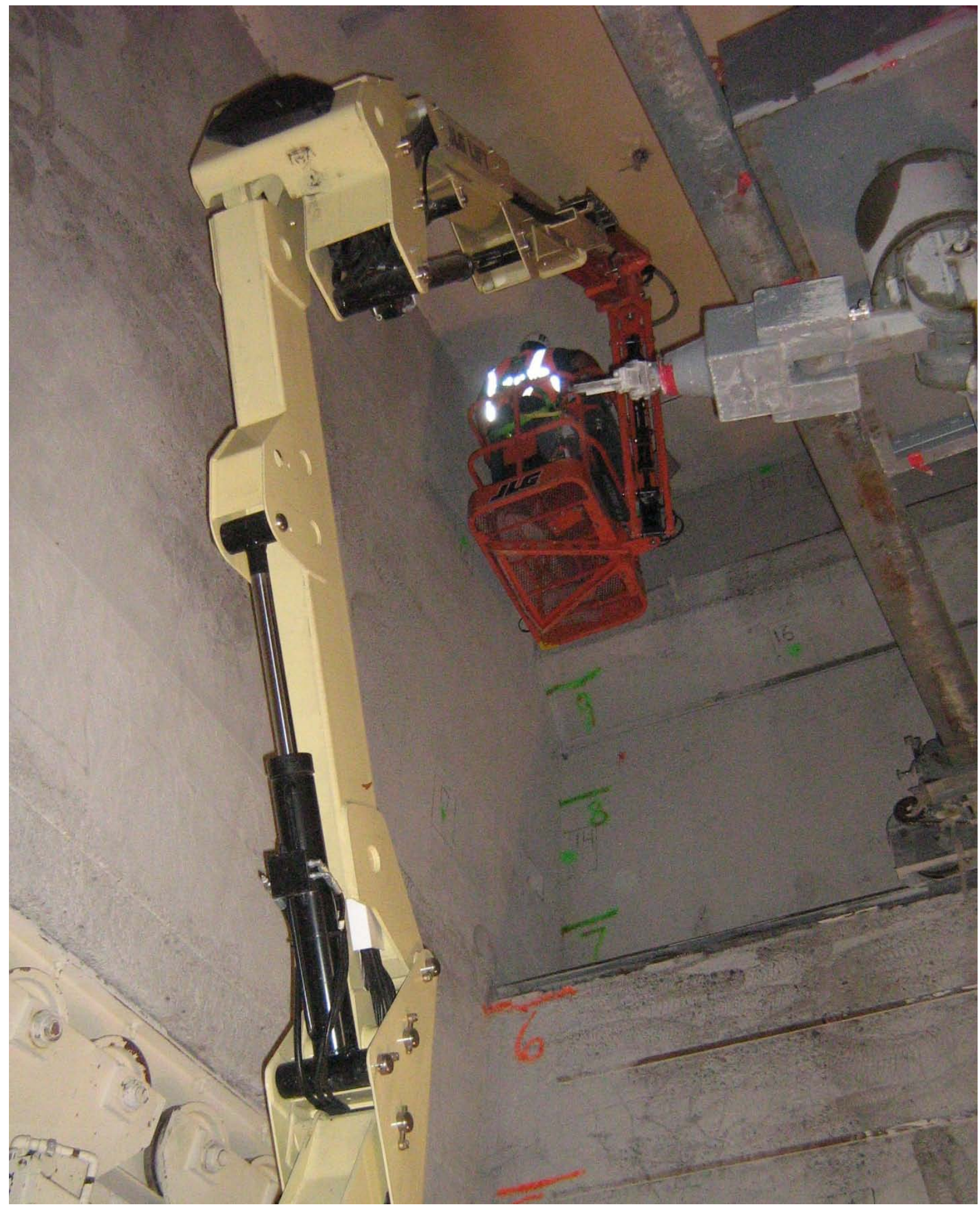


Figure 5. RCTs accessing roof in preparation for surveys in Rooms 201 and 301

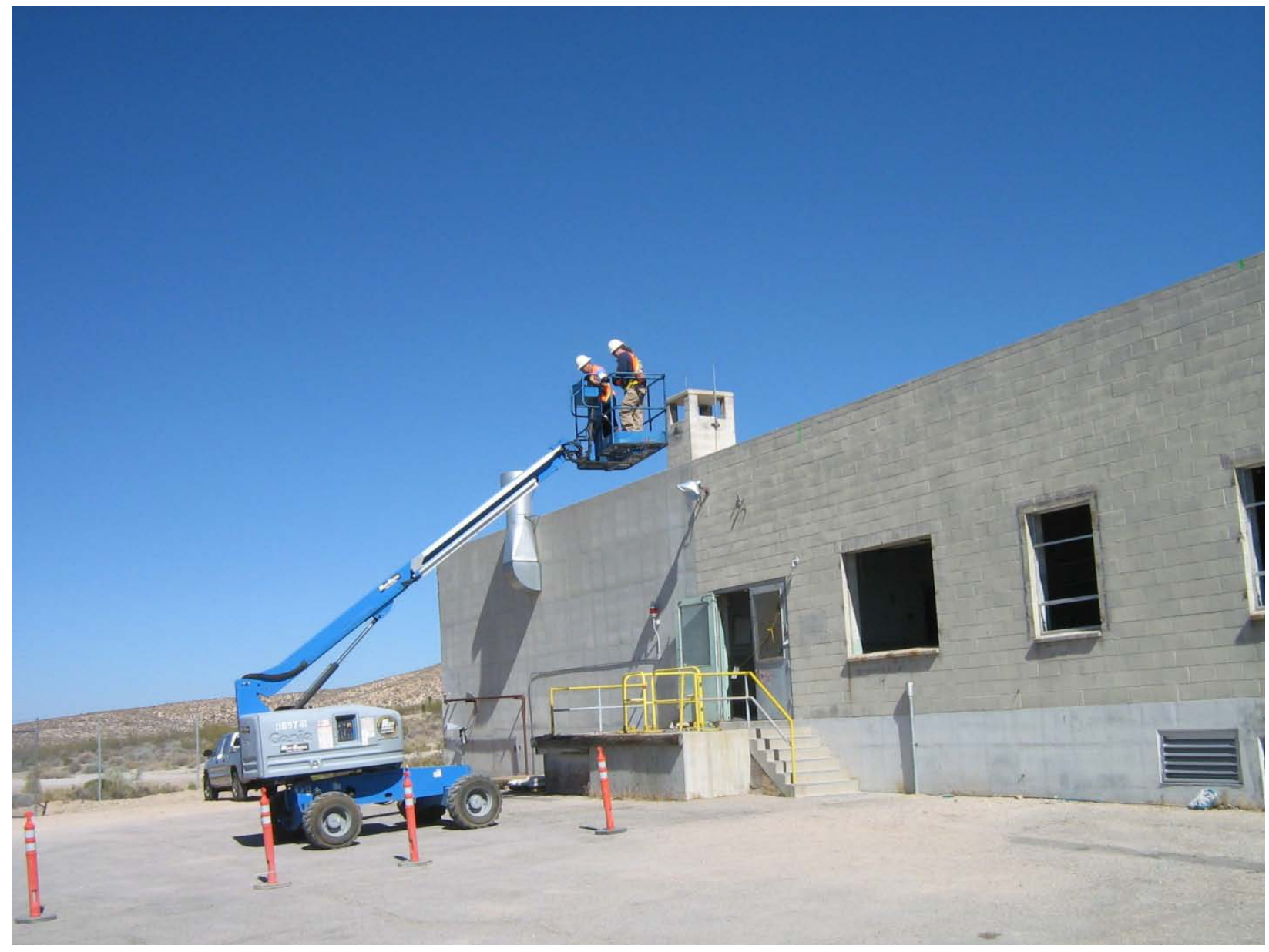

\section{DEVIATIONS FROM THE FINAL STATUS SURVEY PLAN}

Upon performing surveys, some minor errors were found in the survey maps and in some of the starting locations. These errors were corrected by the Radiological Engineer (RE) and Health Physics Supervisor (HPS). The corrections to Survey Plan 10-015 are documented in this section. These corrections fulfill the intent of Survey Plan 10-015.

\subsection{Incorrect Maps}

The survey map for the east wall of Room 102 was modified to accurately reflect the wall configuration. As a result, the survey unit was split into two separate Class 1 survey units, 102-EW-1 and 102-EW-2. The revised survey maps may be found in Appendix A.

Also, the ceiling above the external shield door in Room 102 was incorporated with the Class 2 ceiling survey unit, 102-C-1.

\subsection{Starting Locations}

Two of the random starting locations for two survey units in Room 107 were incorrectly calculated in Table 15 of Survey Plan 10-015. In addition, due to splitting the east wall of Room 102 into two separate 
survey units, the random starting locations had to be re-calculated. A corrected table was provided to the RCTs to perform the survey. The generated values are in Table 7, where $X$ and $Y$ are the starting coordinates (in meters), $A$ is the area (in square meters), $n$ is the number of samples per survey unit, and $L$ is the distance between the survey points (in meters).

Table 7. Updated Survey Unit Specific Parameters

\begin{tabular}{||c|c|c|c|c|c||}
\hline & $\mathbf{X}(\mathbf{m})$ & $\mathbf{Y}(\mathbf{m})$ & $\mathbf{A}\left(\mathbf{m}^{\mathbf{2}}\right)$ & $\mathbf{n}$ & $\mathbf{L}(\mathbf{m})$ \\
\hline Room 102, East Wall - Class 1 & & & & & \\
\hline Survey Unit 102-EW-1 & 3.64 & 3.79 & 70.7 & 11 & 2.72 \\
\hline $\begin{array}{c}\text { Survey Unit 102-EW-2 } \\
\text { Room 107, Floor - Class 1 }\end{array}$ & 4.2 & 3.01 & 61.2 & 11 & 2.53 \\
\hline $\begin{array}{c}\text { Survey Unit 107-F-1 } \\
\text { Room 107, Ceiling - Class 2 }\end{array}$ & 3.64 & 4.28 & 84.5 & 16 & 2.47 \\
\hline $\begin{array}{c}\text { Survey Unit 107-C-1 } \\
\text { Survel|| }\end{array}$ & 3.64 & 4.28 & 90.1 & 16 & 2.55 \\
\hline
\end{tabular}

\section{ASSESSMENT PHASE}

As each survey unit was completed, the average cpm value, standard deviation, median, maximum, and minimum was determined. Each sample with units of cpm was corrected for the appropriate background and converted to a value with units of $\mathrm{dpm} / 100 \mathrm{~cm}^{2}$. With a surface contamination value in the appropriate units, it was compared to the surface contamination limits, or in this case the derived concentration guideline level (DCGLw). The appropriate statistical test (sign test) was performed. If the survey unit was statistically determined to be below the DCGLw, then the survey unit passed and the null hypothesis (that the survey unit is above limits) was rejected. If the survey unit was equal to or below the critical value in the sign test, the null hypothesis was not rejected.

\subsection{Data Verification}

Data verification was performed to determine whether field personnel obtained data in accordance with the FSS plan. Daily quality control (QC) checks were performed for each field instrument. These QC checks were documented on a FRM-0077B, "Count Rate Survey Meter Operability Log" and reviewed by the HPS. RCTs then used the QC tested instruments to perform the survey. The survey results were documented on a FRM-0108B," Radiological Survey Report - Data." The HPS reviewed each FRM-0108B to ensure that the intent of the survey plan was fulfilled (i.e. number of samples was correct for each survey unit, proper instrumentation used, etc.). The FRM-0108B was then approved by the HPS and was then forwarded to the RE. See Appendix B for a compilation of all the approved survey results.

\subsection{Preliminary Data Review}

The RE performed a preliminary data review of the survey results. This data review was necessary in order to quickly identify survey units, or samples within a survey unit, that exceeded the DCGLw. Typically the survey results were reviewed by the RE within days of the survey being performed. The results of the data review were communicated to the HPS. 
Final Status Survey Report for Corrective Action Unit 117-Pluto

Disassembly Facility, Building 2201

When the RE received the survey results, a manipulation of data was needed to convert the results into usable data. The survey results were documented on the FRM-0108B in units of gross cpm. Essentially these results were in units of gross $\mathrm{cpm}$ per $600 \mathrm{~cm}^{2}$ (this area corresponds to the physical detector area).

The gross cpm value was then adjusted for the appropriate background material. The average alpha and beta backgrounds for different materials in reference areas were determined prior to the start of the FSS. If the sample was taken with the probe spanning more than one material, the background corresponding to the most abundant material was used. It should be noted that the background reference area in room 105 was used to adjust all samples taken on concrete surfaces. The background radiation levels were significantly lower in that reference area compared to room 117 . By adjusting the gross cpm value with the lower background, this intentionally biased surface activity to a larger value as a matter of conservatism. With the background adjusted cpm determined, the value was then converted to units of $\mathrm{dpm} / 100 \mathrm{~cm}^{2}$.

This conversion process was accomplished for both alpha and beta measurements by the following equation:

$$
\text { Surface Activity }\left(\frac{d p m}{100 \mathrm{~cm}^{2}}\right)=\frac{C_{g}-C_{b}}{E_{i} E_{s} \frac{A}{100}}
$$

Where: $C_{g}$ is gross counts (cpm), $C_{b}$ is the appropriate background counts (cpm), $E_{i}$ is the average instrument $(2 \pi)$ efficiency, $E_{s}$ is the surface efficiency (fraction of the decays that a detectable particle leaves the surface: default is 0.5 for betas with maximum energies above $400 \mathrm{keV}$ and 0.25 for alpha and betas with maximum energies between 150 and $400 \mathrm{keV})$, and $A$ is the physical probe area $\left(\mathrm{cm}^{2}\right)$.

With the sample values in the appropriate units $\left(\mathrm{dpm} / 100 \mathrm{~cm}^{2}\right)$, the data could then be manipulated and used for statistical testing. The concentration (known as the lower boundary of the gray region, or LBGR) was determined for each measurement by the following equation (MARSSIM):

$$
L B G R=\frac{\alpha \text { activity }}{100 \frac{\mathrm{dpm}}{100 \mathrm{~cm}^{2}}}+\frac{\beta \text { activity }}{5000 \frac{\mathrm{dpm}}{100 \mathrm{~cm}^{2}}}
$$

The LBGR of the survey unit was primarily driven by the alpha measurement because of the low surface activity limit of $100 \mathrm{dpm} / 100 \mathrm{~cm}^{2}$. The LBGR was compared to the DCGLw (which is unity) for each sample in the survey unit. A sample whose LBGR was greater than the DCGLw would constitute a sample that was above the release limits. The comparison of the LBGR to the DCGLw is the basis for the sign test and will be discussed further in Section 6.6.

The gross counts, background adjusted counts, counts to dpm conversion, average, standard deviation, median, maximum, minimum, and the LBGR (using the average surface activity) for each survey unit or room was determined. For a complete list of results, see Appendix C. All calculations were standardized into a Microsoft Excel spreadsheet template. This spreadsheet was verified by the HPS to contain the correct equations and, with the use of the spreadsheet, the equations were consistently applied to all data contained in the FRM- $0108 \mathrm{~B}$ received by the RE. 
Final Status Survey Report for Corrective Action Unit 117-Pluto Disassembly Facility, Building 2201

\subsection{Investigation of Elevated Readings}

In the Class 3 area, 587 static measurements (samples) were taken. These samples were background corrected with the Class 1 reference area (Room 105). By subtracting the lower background, the calculated surface activities were biased to an elevated activity. There was one sample in a Class 3 area that had an LBGR greater than the DCGL . This sample had an LBGR of 1.01 and was located in Room 4 of the basement. Some of the highest average surface activities in the Class 3 areas were in the basement. The HSA did not support any history of radioactive material or radiological/nuclear operations in the basement. This elevated reading was believed to be caused by natural radioactivity, specifically from radon. Higher radon levels were expected in the basements and in the vaults than in other areas of the facility. This was a specified concern in the FSS plan. Though the average activity was higher in the basements, no alpha or beta trigger levels were exceeded, the average LBGR was still below the $\mathrm{DCGL}_{w}$, and the survey unit passed the sign test based on 49 samples taken in Room 4.

If a hot spot was found during the FSS when performing a static or scan survey, the area was marked, decontaminated, resurveyed, and documented according to the provisions of Survey Plan 10-015. A list of all elevated scan surveys and their corresponding scan surveys after decontamination is provided in Table 8. After decontamination was performed, static measurements were taken within the hot spot area to verify that decontamination efforts were successful. Since Hot Spot $C$ in Room 106 was around 4 square feet, the hot spot was divided into four different areas (Hot Spot C-1, C-2, C-3, and C-4 in Table 9) and each area was surveyed after decontamination. The static measurements obtained after decontamination replaced the previous elevated measurements in the survey unit to obtain an average LBGR. A list of all elevated static measurements and their corresponding post decontamination static measurements is provided in Table 9.

Table 8. Elevated vs. Remediated Scans

\begin{tabular}{|c|c|c|c|c|}
\hline Location & Hot Spot Area & $\begin{array}{c}\alpha \text { Elevated } \\
\text { Reading } \\
\left(\mathrm{dpm} / 100 \mathrm{~cm}^{2}\right)\end{array}$ & $\begin{array}{c}\beta \text { Elevated } \\
\text { Reading } \\
\left(\mathrm{dpm} / 100 \mathrm{~cm}^{2}\right)\end{array}$ & $\begin{array}{c}\text { Post Decon } \\
\text { Reading } \\
\left(\mathrm{dpm} / 100 \mathrm{~cm}^{2}\right)\end{array}$ \\
\hline $\begin{array}{c}\text { Room 106 Floor - } \\
\text { Hot Spot A }\end{array}$ & $\sim 1$ square foot & 182.0 & 56675.8 & $<$ Trigger Levels \\
\hline $\begin{array}{c}\text { Room 106 Floor - } \\
\text { Hot Spot B }\end{array}$ & $\sim 1$ square foot & 172.3 & 18893.0 & $<$ Trigger Levels \\
\hline $\begin{array}{c}\text { Room 106 Floor - } \\
\text { Hot Spot C }\end{array}$ & $\sim 4$ square feet & 167.5 & 29667.1 & $<$ Trigger Levels \\
\hline $\begin{array}{c}\text { Room 106 Floor - } \\
\text { Hot Spot D }\end{array}$ & $\sim 1$ square foot & 104.7 & 5598.1 & $<$ Trigger Levels \\
\hline
\end{tabular}

Table 9. Elevated vs. Remediated Static Measurements

\begin{tabular}{|c|c|c|c|c|}
\hline Location & $\begin{array}{c}\alpha \text { Elevated } \\
\text { Measurement } \\
\left(\mathrm{dpm} / 100 \mathrm{~cm}^{2}\right)\end{array}$ & $\begin{array}{c}\alpha \text { Post Decon } \\
\text { Measurement } \\
\left(\mathrm{dpm} / 100 \mathrm{~cm}^{2}\right)\end{array}$ & $\begin{array}{c}\beta \text { Elevated } \\
\text { Measurement } \\
\left(\mathrm{dpm} / 100 \mathrm{~cm}^{2}\right)\end{array}$ & $\begin{array}{c}\beta \text { Post Decon } \\
\text { Measurement } \\
\left(\mathrm{dpm} / 100 \mathrm{~cm}^{2}\right)\end{array}$ \\
\hline $\begin{array}{c}\text { Room 106 Floor }- \\
\text { Hot Spot A }\end{array}$ & 182 & 17.8 & 56675.8 & -699.9 \\
\hline
\end{tabular}


Final Status Survey Report for Corrective Action Unit 117-Pluto Disassembly Facility, Building 2201

\begin{tabular}{|c|c|c|c|c|}
\hline Location & $\begin{array}{c}\alpha \text { Elevated } \\
\text { Measurement } \\
\left(\mathrm{dpm} / 100 \mathrm{~cm}^{2}\right)\end{array}$ & $\begin{array}{c}\alpha \text { Post Decon } \\
\text { Measurement } \\
\left(\mathrm{dpm} / 100 \mathrm{~cm}^{2}\right)\end{array}$ & $\begin{array}{c}\beta \text { Elevated } \\
\text { Measurement } \\
\left(\mathrm{dpm} / 100 \mathrm{~cm}^{2}\right)\end{array}$ & $\begin{array}{c}\beta \text { Post Decon } \\
\text { Measurement } \\
\left(\mathrm{dpm} / 100 \mathrm{~cm}^{2}\right)\end{array}$ \\
\hline $\begin{array}{c}\text { Room 106 Floor }- \\
\text { Hot Spot B }\end{array}$ & 172.3 & 32.2 & 18893.0 & 206.0 \\
\hline $\begin{array}{c}\text { Room 106 Floor } \\
\text { Hot Spot C-1 }\end{array}$ & 167.5 & 41.9 & 29667.1 & 262.7 \\
\hline $\begin{array}{c}\text { Room 106 Floor } \\
\text { Hot Spot C-2 }\end{array}$ & 167.5 & 37.1 & 29667.1 & 262.7 \\
\hline $\begin{array}{c}\text { Room 106 Floor - } \\
\text { Hot Spot C-3 }\end{array}$ & 167.5 & 37.1 & 29667.1 & -97.6 \\
\hline $\begin{array}{c}\text { Room 106 Floor } \\
\text { Hot Spot C-4 }\end{array}$ & 167.5 & 61.2 & 5598.1 & 241.0 \\
\hline $\begin{array}{c}\text { Room 106 Floor }- \\
\text { Hot Spot D }\end{array}$ & 104.7 & 30.9 \\
\hline
\end{tabular}

\subsection{Negative Beta Surface Activity}

In the Class 1 areas, the beta surface activities were consistently negative. This negative activity was a result of the background subtract with the average beta activity from the reference area in Room 105. Though Room 105 is surrounded on three sides with 4-foot thick concrete walls, the background in the area was higher than the Class 1 areas. The Class 1 areas, for the most part, are surrounded by 4 -feet thick concrete walls. Even if background was totally negated (i.e. background was not subtracted from the gross count) and every gross count was treated as actual surface activity, all survey units would still be lower than the DCGL . This is illustrated in Table 10 with the six survey units in Room 104. The highest non-background adjusted LBGR from a Class 1 or 2 survey unit was equal to 0.83 and was found in Room 106.

Table 10. Comparison of LBGR

\begin{tabular}{|c|c|c|}
\hline Survey Unit & Calculated LBGR & $\begin{array}{c}\text { LBGR without a subtracted } \\
\text { beta background }\end{array}$ \\
\hline $104-\mathrm{F}-1$ & 0.02 & 0.32 \\
\hline $104-\mathrm{NW}-1$ & 0.07 & 0.32 \\
\hline $104-\mathrm{EW}-1$ & 0.06 & 0.27 \\
\hline $104-\mathrm{SW}-1$ & 0.16 & 0.40 \\
\hline $104-W W-1$ & 0.15 & 0.40 \\
\hline $104-\mathrm{C}-1$ & 0.17 & 0.52 \\
\hline
\end{tabular}


Final Status Survey Report for Corrective Action Unit 117-Pluto Disassembly Facility, Building 2201

\subsection{Graphical Representation of Data}

To aid in the visualization of the hundreds of sample measurements, some graphs were plotted of the most relevant data (Figures 6-15). From the graphs, one can visualize the range of measurements. The alpha and beta surface activities within the survey units were sorted from least to greatest. Multiple Class 1 or Class 2 survey units were combined by room number. All Class 3 measurements were combined into one graph.

Figure 6. Class 3 Cumulative Alpha Measurements

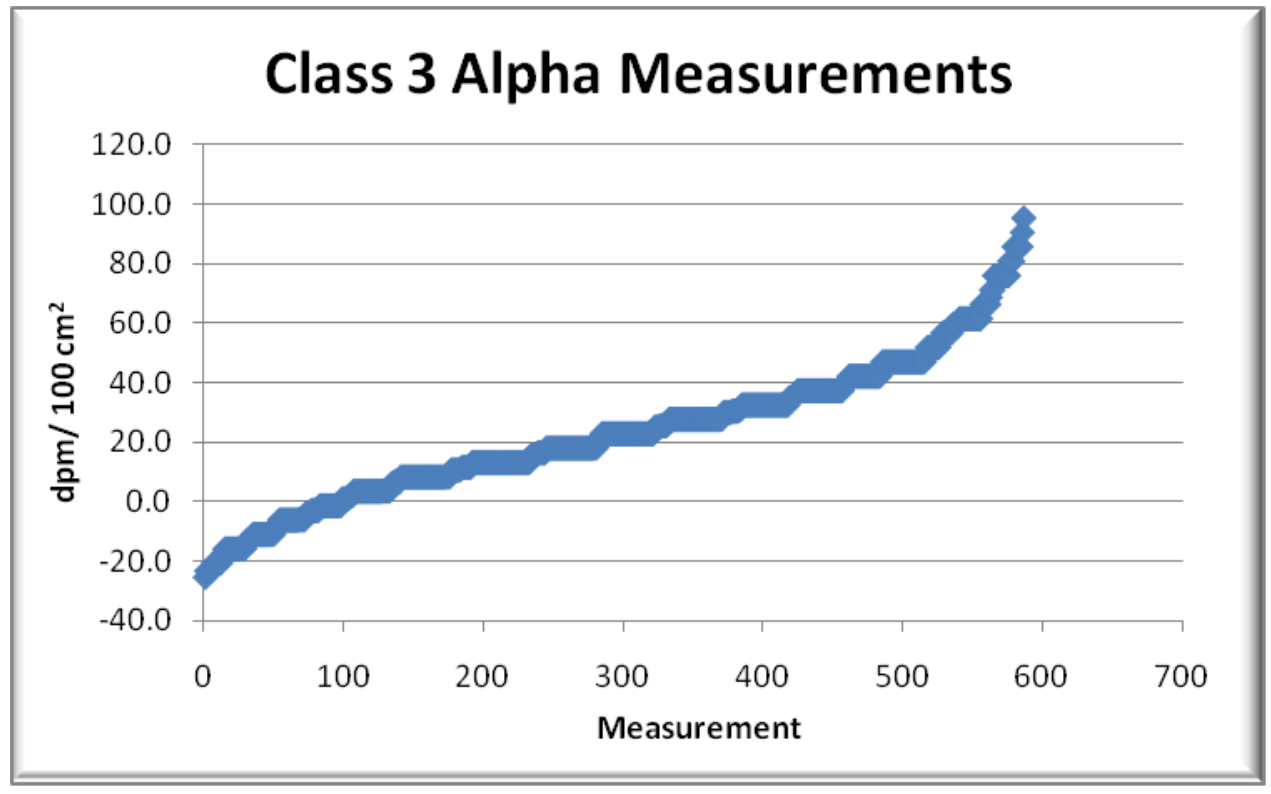

Figure 7. Class 3 Cumulative Beta Measurements

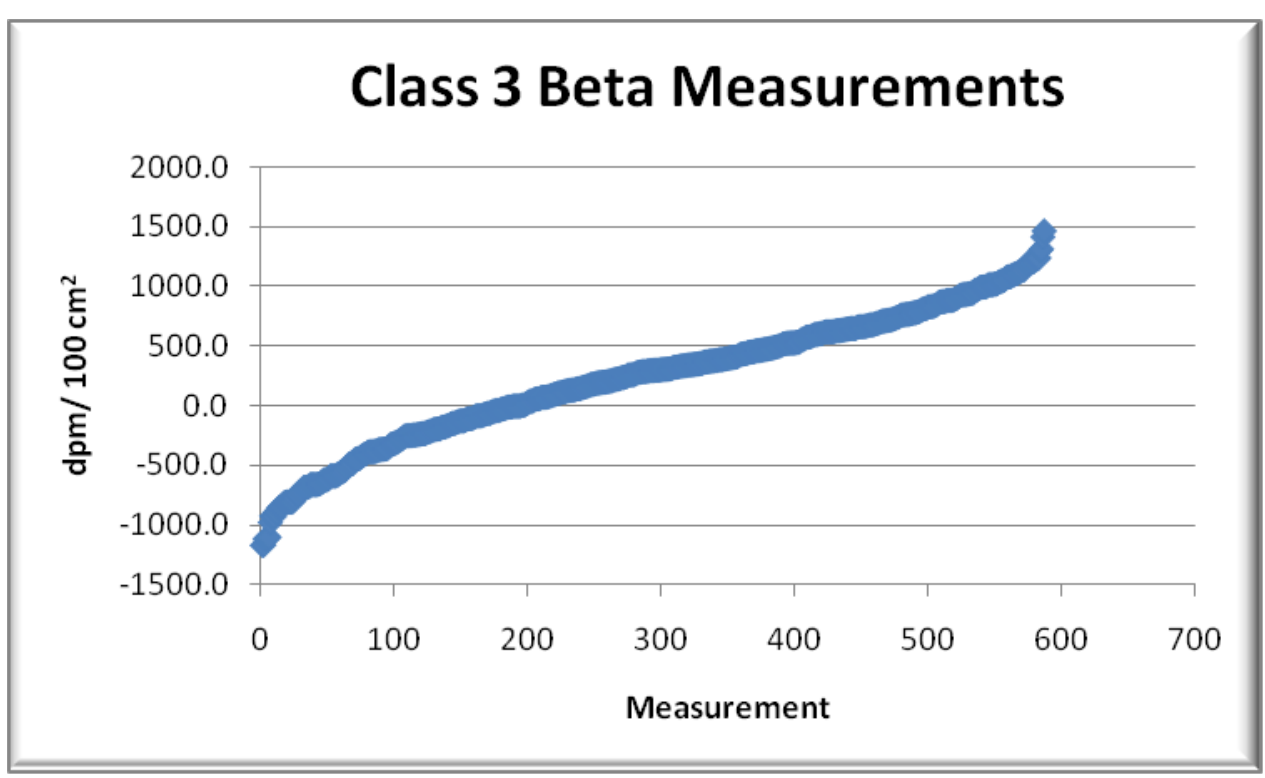


Final Status Survey Report for Corrective Action Unit 117-Pluto

Disassembly Facility, Building 2201

Figure 8. Room 102 Cumulative Alpha Measurements

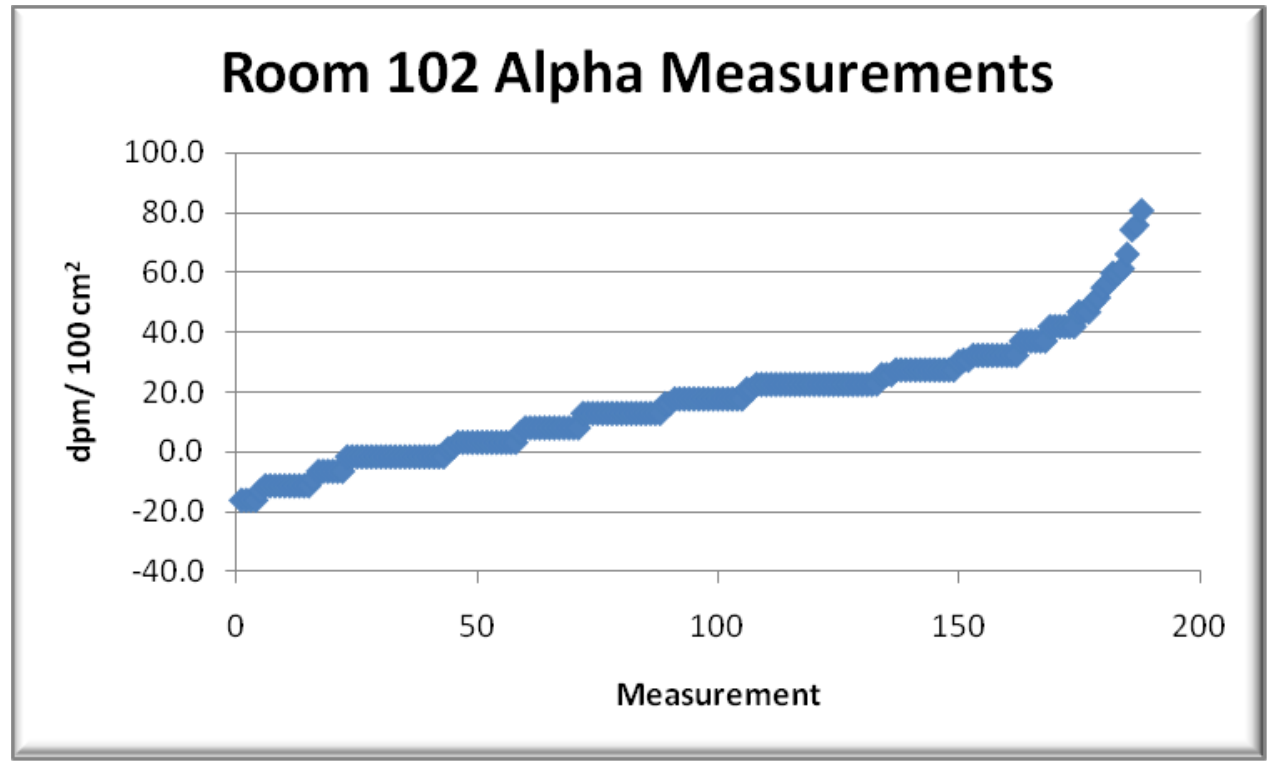

Figure 9. Room 102 Cumulative Beta Measurements

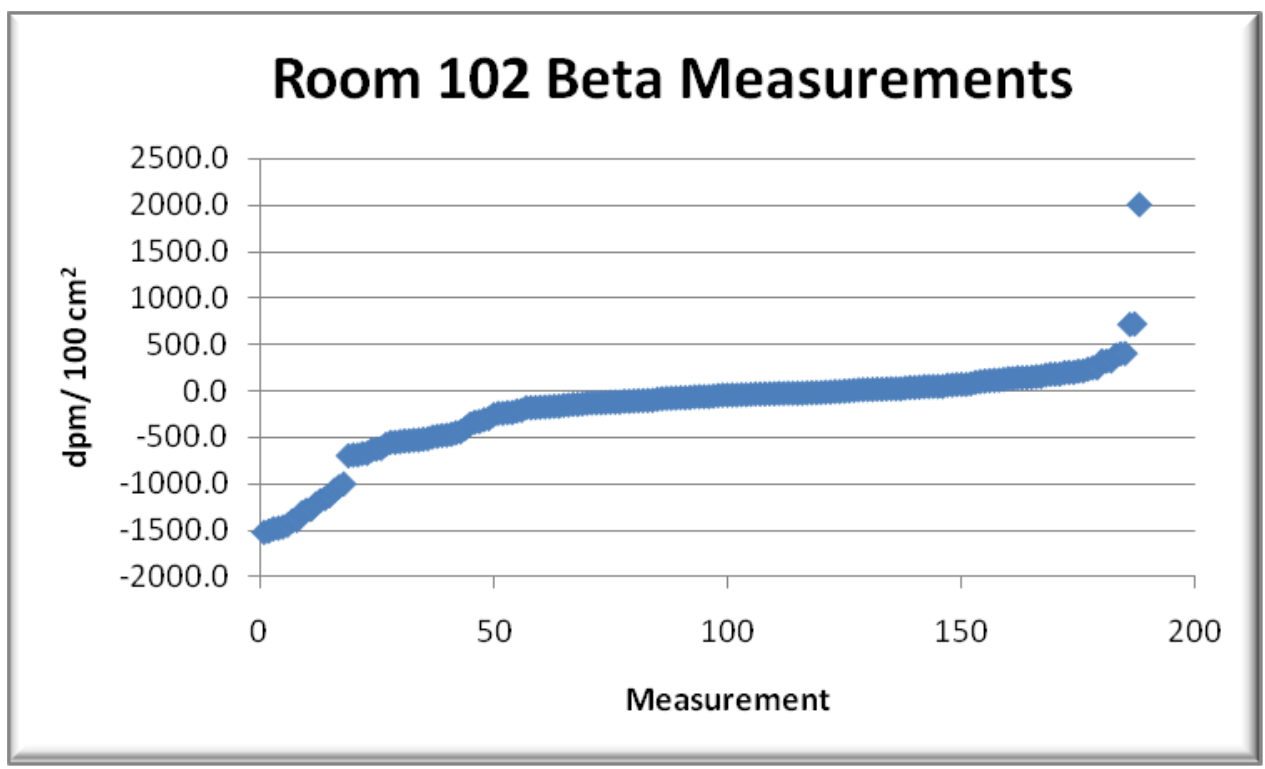


Final Status Survey Report for Corrective Action Unit 117-Pluto Disassembly Facility, Building 2201

Figure 10. Room 104 Cumulative Alpha Measurements

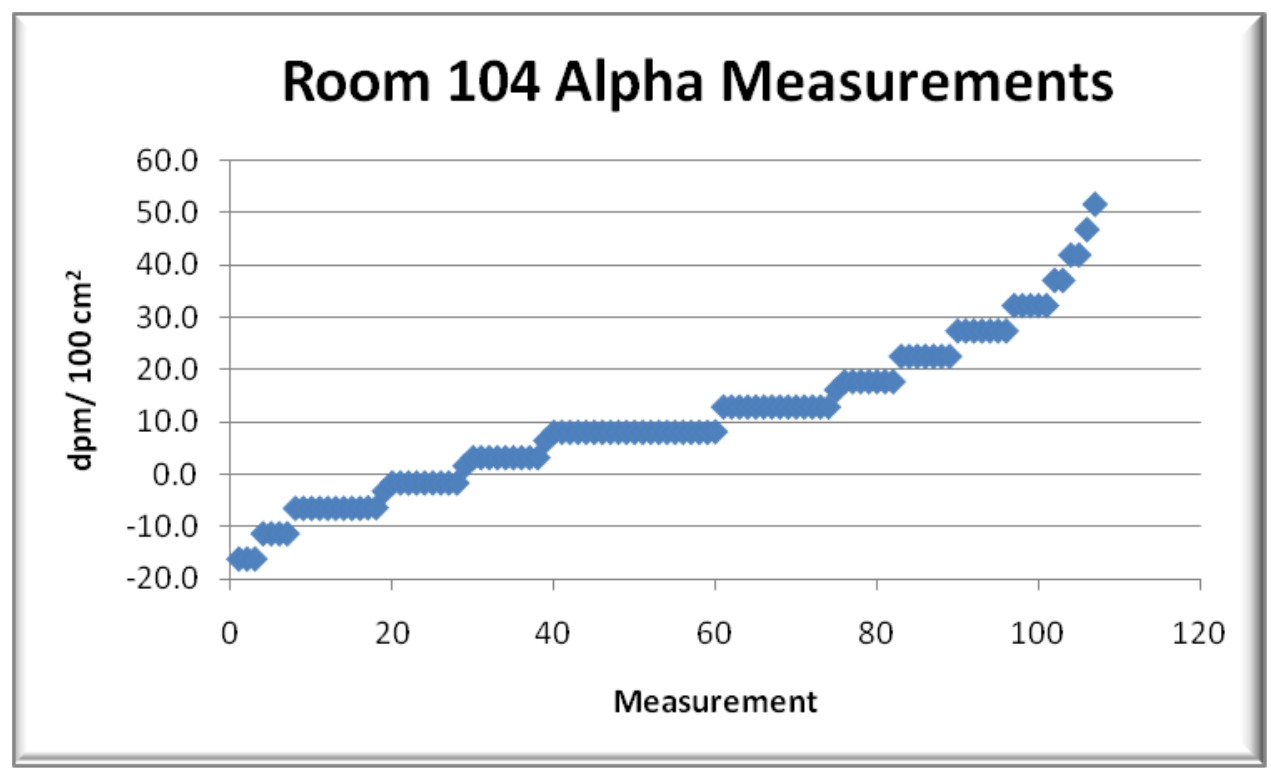

Figure 11. Room 104 Cumulative Beta Measurements

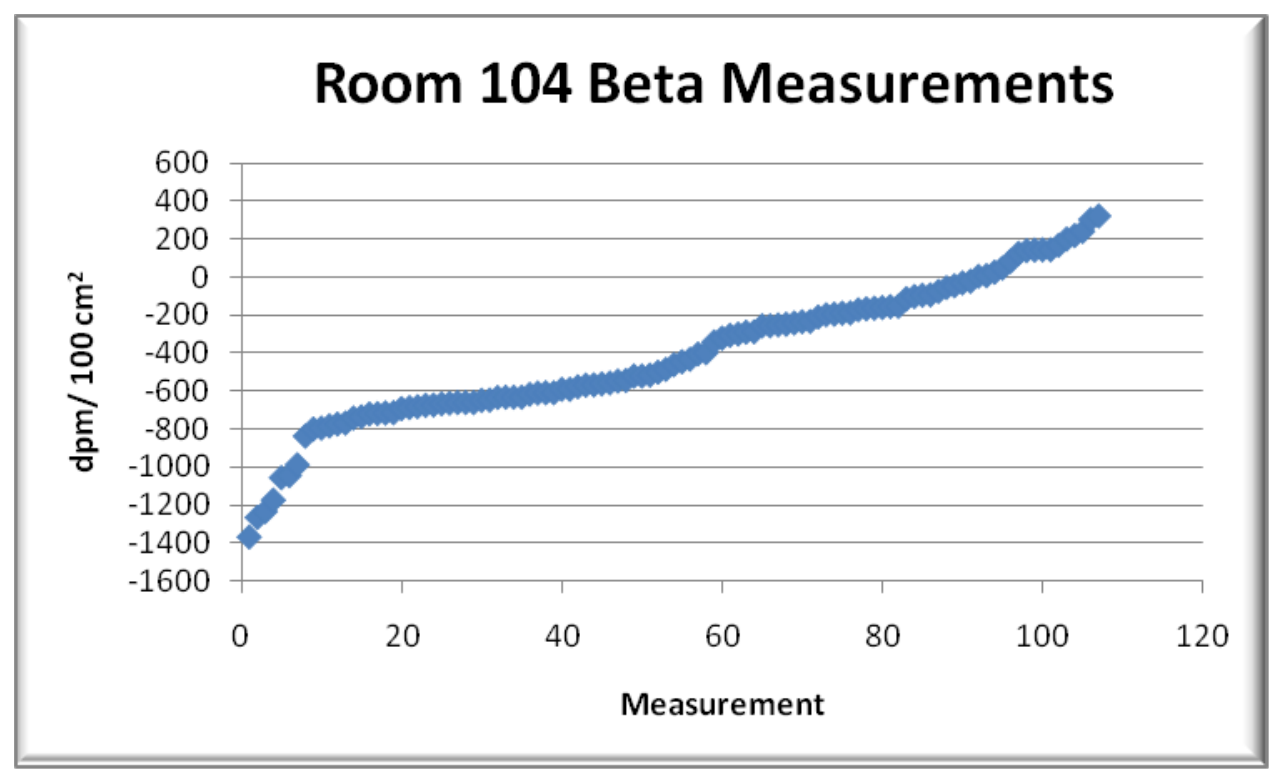


Final Status Survey Report for Corrective Action Unit 117-Pluto Disassembly Facility, Building 2201

Figure 12. Room 106 Cumulative Alpha Measurements

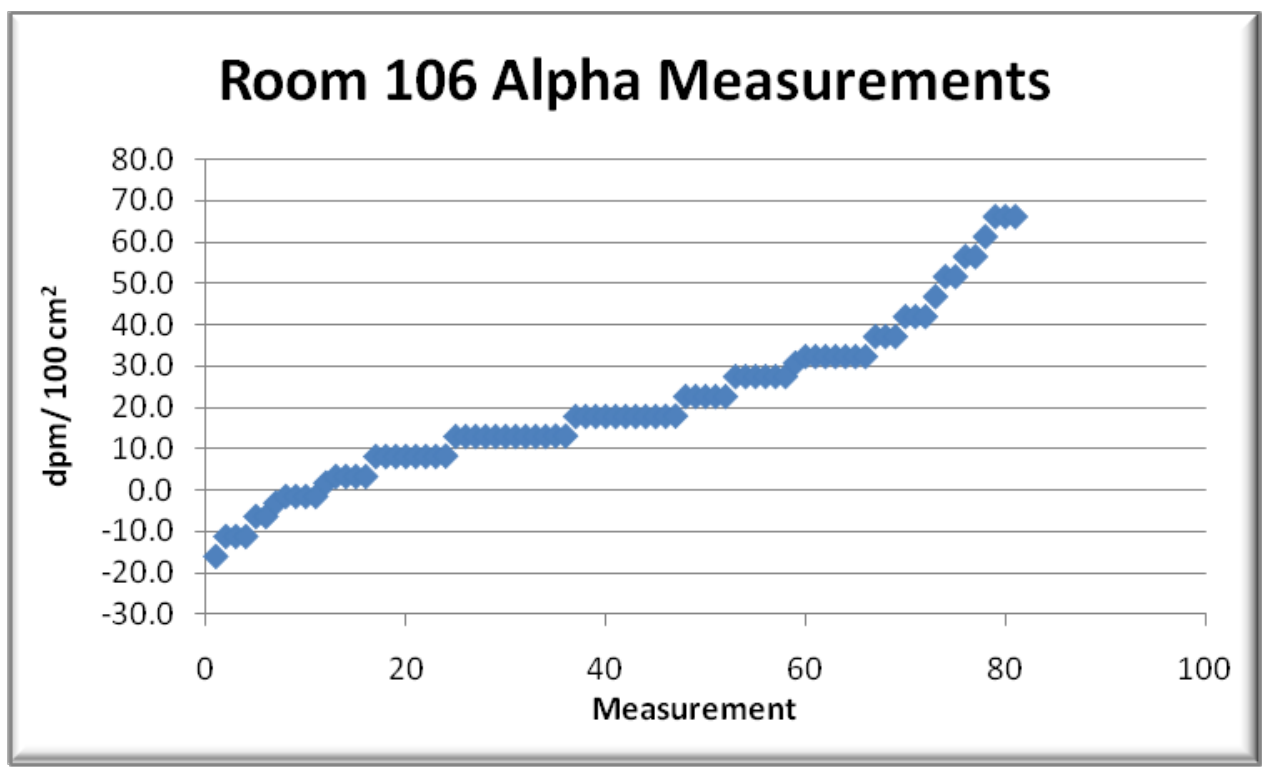

Figure 13. Room 106 Cumulative Beta Measurements

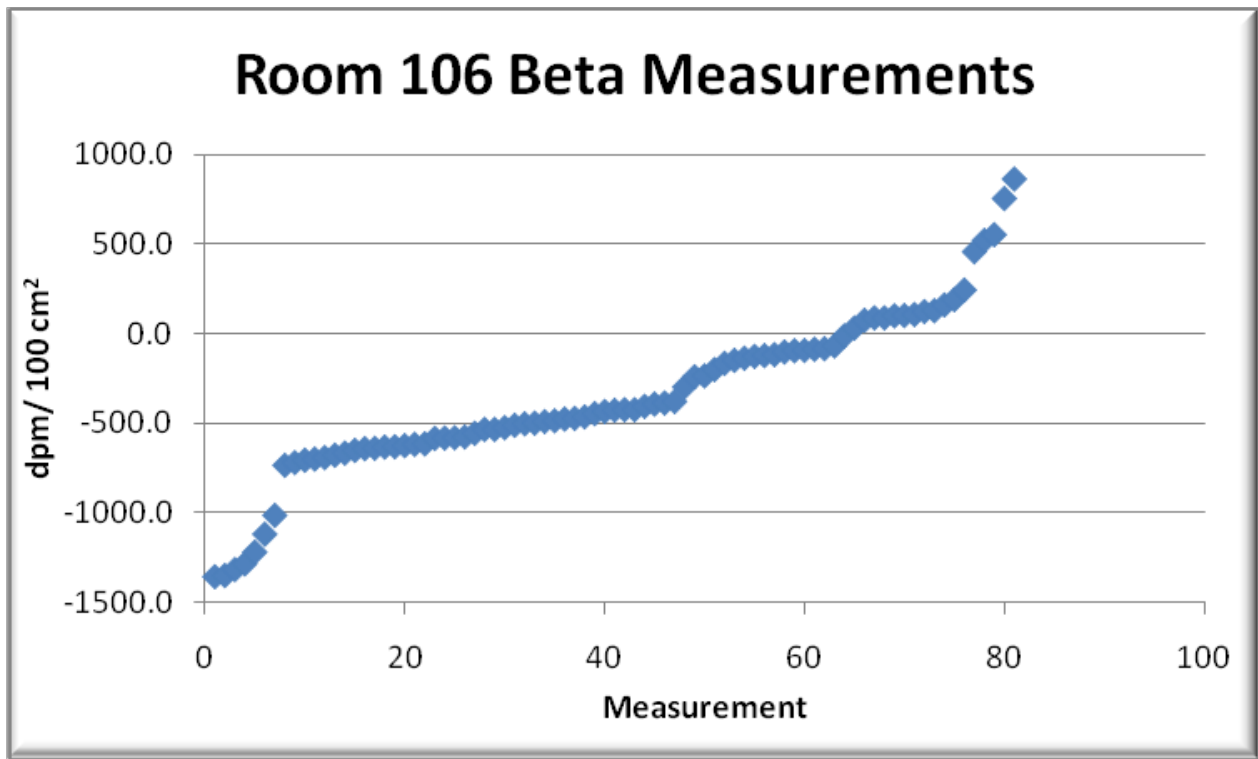


Final Status Survey Report for Corrective Action Unit 117-Pluto

Disassembly Facility, Building 2201

Figure 14. Room 107 Cumulative Alpha Measurements

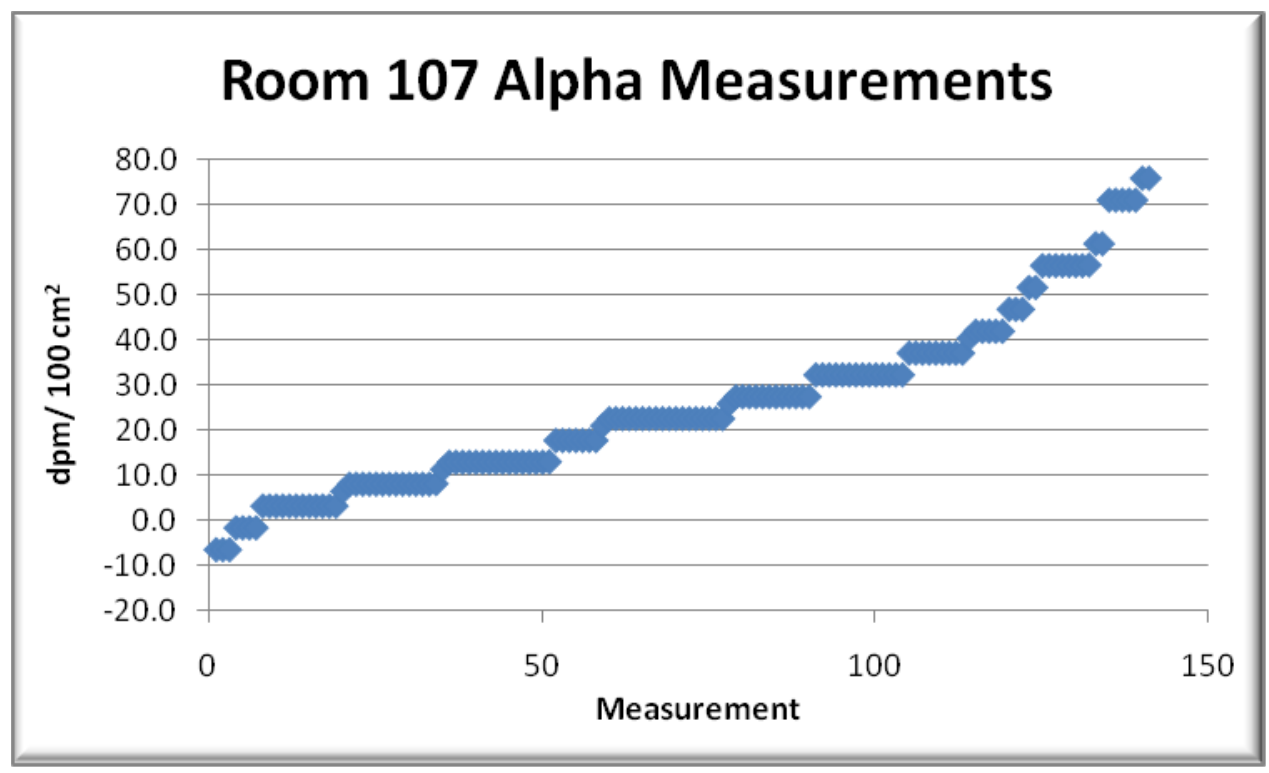

Figure 15. Room 107 Cumulative Beta Measurements

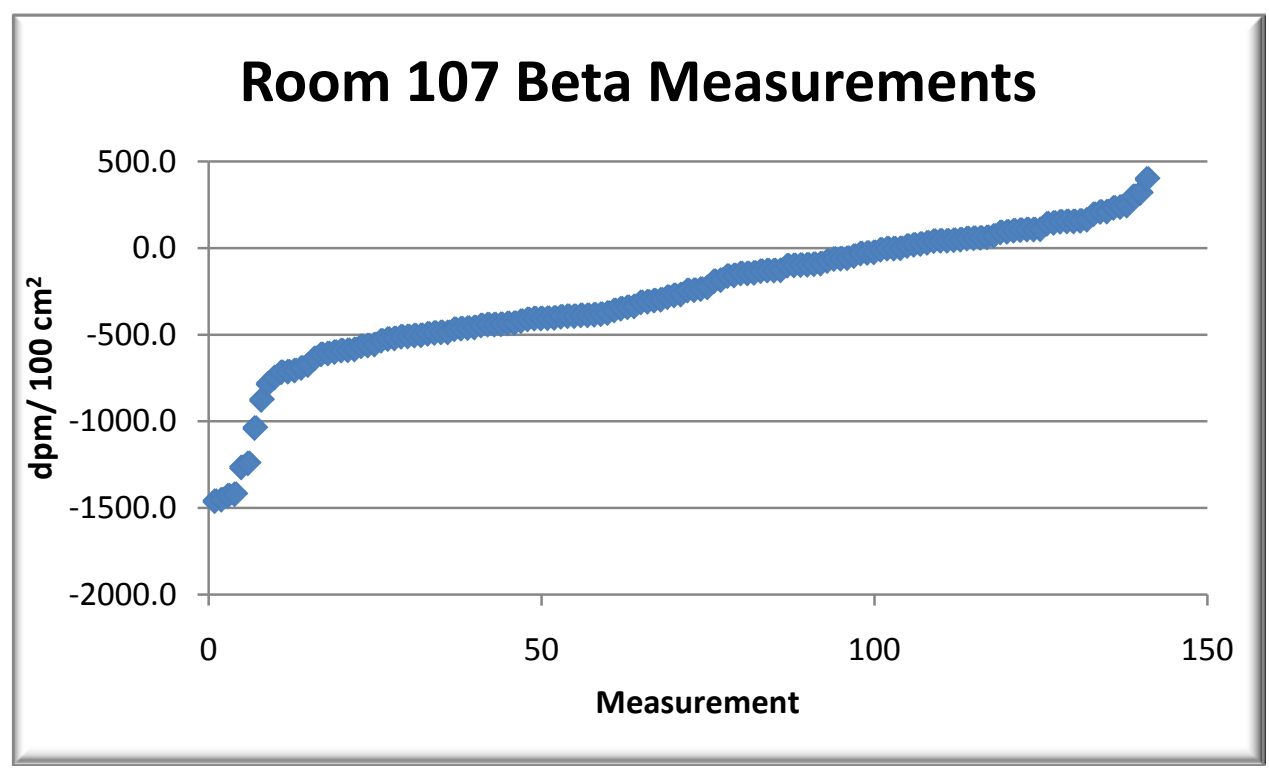


Final Status Survey Report for

Corrective Action Unit 117-Pluto

Disassembly Facility, Building 2201

\subsection{Sign Test}

It was determined in the planning phases of this project that the sign test would be used to evaluate the data since the measurements were not nuclide specific. In order to perform the sign test, the surface activities needed to be in a form that could be compared to the $D C G L_{w}$. For this cause, every sample was converted to units of $\mathrm{dpm} / 100 \mathrm{~cm}^{2}$ and then the LBGR was calculated (Section 6.2) for that particular sample.

The sign test was performed in the following manner:

1. Each measurement LBGR was subtracted from the $D C G L_{w}$ to obtain the difference, $D_{i}$ :

2. Each difference that was exactly zero was discarded and the sample size, $N$, was reduced by the number of such zero measurements.

3. The number of positive differences was counted. The result was the test statistic $S+$. (Note that a positive measurement corresponds to a measurement below the $\mathrm{DCGL}_{\mathrm{w}}$ and contributes evidence that the survey unit meets the release criterion.)

4. Large values of $S+$ indicated that the null hypothesis (i.e. that the survey unit exceeds the release criterion) was false. The value of $S+$ was compared to the critical value in MARSSIM Table I.3 (Appendix D). If $S+$ was greater than the critical value, $k$, in that table, then the null hypothesis was rejected (Abelquist, 2001).

\section{RESULTS}

The LBGR based on the average and median surface activities, number of measurements, and the statistical determination for each survey unit is provided in Sections 7.1 and 7.2. Because of the large amount of data generated, this section contains only a summary of the results. Since the LBGR is the basis for determining if a survey unit passes, each measurement in the survey unit had the LBGR calculated and compared to the $D C G L_{w}$. Both the average and median LBGR are listed in the summary tables. With $95 \%$ confidence, every average LBGR in each survey unit was less than the $D C G L_{w}$. The sign test was performed and the determination of either "PASS" or "FAIL" is listed for each room. See Appendices E-I for the detailed results of each room and/or survey unit.

\subsection{Class 3 Survey Unit Results}

The LBGR based on the average and median surface activities in the individual rooms of the Class 3 survey unit are provided in Table 11. Each measurement in the room had the LBGR calculated and compared to the DCGL . The sign test was performed and the determination of either "PASS" or "FAIL"

is listed for each room. Since the rooms are part of a Class 3 area with an unlimited surface area, all the static measurements were tallied together and the official Class 3 results are found in the bolded row entitled "Total." See Appendix E for the detailed results of each room in the Class 3 survey unit.

Only 1 of the 587 static measurements resulted in a LBGR greater than the DCGL $\mathrm{w}_{\mathrm{w}}$. This measurement was found in the Room 4 of the basement and was discussed in Section 6.3 "Investigation of Elevated Readings." All individual rooms within the Class 3 survey unit passed the sign test. The whole Class 3 area passed the sign test. 
Final Status Survey Report for Corrective Action Unit 117-Pluto Disassembly Facility, Building 2201

Table 11. Results of Rooms within a Class 3 Survey Unit

\begin{tabular}{|c|c|c|c|c|}
\hline $\begin{array}{c}\text { Class } 3 \text { Room } \\
\text { Number }\end{array}$ & $\begin{array}{l}\text { LBGR (based on } \\
\text { averages) }\end{array}$ & $\begin{array}{l}\text { LBGR (based } \\
\text { on median } \\
\text { values) }\end{array}$ & $\begin{array}{c}\text { Static } \\
\text { Measurements }\end{array}$ & $\begin{array}{l}\text { Statistical Test } \\
\text { Determination }\end{array}$ \\
\hline 101 & 0.18 & 0.18 & 112 & PASS \\
\hline 103 & 0.40 & 0.48 & 25 & PASS \\
\hline 105 & 0.00 & 0.00 & 16 & PASS \\
\hline 108 & 0.14 & 0.14 & 74 & PASS \\
\hline 109 & 0.26 & 0.25 & 80 & PASS \\
\hline 110 & 0.28 & 0.28 & 30 & PASS \\
\hline 111 & 0.25 & 0.27 & 10 & PASS \\
\hline $112,113,114$ & 0.43 & 0.48 & 24 & PASS \\
\hline 115 & 0.30 & 0.31 & 6 & PASS \\
\hline 116 & 0.32 & 0.33 & 45 & PASS \\
\hline 117 & 0.35 & 0.37 & 12 & PASS \\
\hline 201 & 0.49 & 0.54 & 12 & PASS \\
\hline 301 & 0.29 & 0.32 & 12 & PASS \\
\hline Basement 1 & 0.49 & 0.53 & 23 & PASS \\
\hline Basement 2 & 0.35 & 0.32 & 24 & PASS \\
\hline Basement 3 & 0.23 & 0.24 & 33 & PASS \\
\hline Basement 4 & 0.52 & 0.49 & 49 & PASS \\
\hline Total & 0.28 & 0.29 & 587 & PASS \\
\hline
\end{tabular}

The descriptive statistics for all the measurements in the Class 3 area are presented in Table 12.

Table 12. Class 3 Summary Table

\begin{tabular}{|c|c|c|}
\cline { 2 - 3 } \multicolumn{1}{c|}{} & \multicolumn{2}{c|}{$\mathbf{d p m} / \mathbf{1 0 0} \mathbf{~ c m} \mathbf{2}$} \\
\cline { 2 - 3 } Class 3 & alpha & beta \\
\hline Average & 22.9 & 239.4 \\
\hline Standard Deviation & 23.5 & 542.3 \\
\hline Median & 22.6 & 297.8 \\
\hline Maximum & 95.0 & 1467.1 \\
\hline Minimum & -25.6 & -1170.7 \\
\hline LBGR & 0.28 & Average \\
\hline LBGR & 0.29 & Median \\
\hline
\end{tabular}


Final Status Survey Report for Corrective Action Unit 117-Pluto Disassembly Facility, Building 2201

\begin{tabular}{|c|c|}
\multicolumn{2}{|c|}{ Descriptive Statistics } \\
\hline Total Samples: & 587 \\
\hline Negative LBGR: & 1 \\
\hline Class 3 Test Statistic: & 586 \\
\hline Critical Value (k): & 313 \\
\hline Sign Test Determination: & PASS \\
\hline
\end{tabular}

\subsection{Class 1 and 2 Survey Unit Results}

The LBGR based on the average and median surface activities for each Class 1 or 2 survey unit are provided in Tables 13-17 and are grouped by room number. Each measurement in the survey unit had the LBGR calculated and compared to the $\mathrm{DCGL}_{\mathrm{w}}$. The sign test was performed and the determination of either "PASS" or "FAIL" is listed for each survey unit. See Appendix F-I for the detailed results of each Class 1 or 2 survey unit.

After the hot spots in Room 106 were decontaminated, none of the 535 static measurements resulted in a LBGR greater than the DCGL .

All survey units in Rooms 102, 104, 106, and 107 passed the statistical test.

Table 13. Room 102 Survey Units

\begin{tabular}{|c|c|c|c|c|}
\hline $\begin{array}{c}\text { Class } 1 \text { or } 2 \\
\text { Survey Unit } \\
\text { Number }\end{array}$ & $\begin{array}{l}\text { LBGR (based on } \\
\text { averages) }\end{array}$ & $\begin{array}{l}\text { LBGR (based } \\
\text { on median } \\
\text { values) }\end{array}$ & $\begin{array}{c}\text { Static } \\
\text { Measurements }\end{array}$ & $\begin{array}{l}\text { Statistical Test } \\
\text { Determination }\end{array}$ \\
\hline $102-F-1$ & 0.03 & 0.04 & 14 & PASS \\
\hline $102-F-2$ & 0.07 & 0.09 & 11 & PASS \\
\hline $102-F-3$ & 0.06 & 0.01 & 12 & PASS \\
\hline $102-F-4$ & 0.05 & 0.03 & 15 & PASS \\
\hline $102-C-1$ & 0.21 & 0.20 & 12 & PASS \\
\hline 102-NW-1 & 0.17 & 0.18 & 14 & PASS \\
\hline $102-N W-2$ & 0.33 & 0.32 & 14 & PASS \\
\hline $102-N W-3$ & 0.24 & 0.27 & 13 & PASS \\
\hline 102-EW-1 & 0.23 & 0.23 & 13 & PASS \\
\hline 102-EW-2 & 0.43 & 0.37 & 12 & PASS \\
\hline 102-SW-1 & 0.16 & 0.18 & 13 & PASS \\
\hline $102-S W-2$ & 0.05 & 0.03 & 11 & PASS \\
\hline $102-S W-3$ & 0.10 & 0.11 & 12 & PASS \\
\hline 102-WW-1 & 0.16 & 0.18 & 11 & PASS \\
\hline
\end{tabular}


Final Status Survey Report for Corrective Action Unit 117-Pluto Disassembly Facility, Building 2201

\begin{tabular}{|c|c|c|c|c|}
\hline $\begin{array}{c}\text { Class } 1 \text { or } \mathbf{2} \\
\text { Survey Unit } \\
\text { Number }\end{array}$ & $\begin{array}{c}\text { LBGR (based on } \\
\text { averages) }\end{array}$ & $\begin{array}{c}\text { LBGR (based } \\
\text { on median } \\
\text { values) }\end{array}$ & $\begin{array}{c}\text { Static } \\
\text { Measurements }\end{array}$ & $\begin{array}{c}\text { Statistical Test } \\
\text { Determination }\end{array}$ \\
\hline 102-WW-2 & 0.30 & 0.25 & 11 & PASS \\
\hline $102-S D W-1$ & 0.31 & 0.26 & 12 & PASS \\
\hline
\end{tabular}

Table 14. Room 104 Survey Units

\begin{tabular}{|c|c|c|c|c|}
\hline $\begin{array}{c}\text { Class 1 or 2 } \\
\text { Survey Unit } \\
\text { Number }\end{array}$ & $\begin{array}{c}\text { LBGR (based on } \\
\text { averages) }\end{array}$ & $\begin{array}{c}\text { LBGR (based } \\
\text { on median } \\
\text { values) }\end{array}$ & $\begin{array}{c}\text { Static } \\
\text { Measurements }\end{array}$ & $\begin{array}{c}\text { Statistical Test } \\
\text { Determination }\end{array}$ \\
\hline $104-\mathrm{F}-1$ & 0.02 & 0.00 & 18 & PASS \\
\hline $104-\mathrm{C}-1$ & 0.17 & 0.16 & 17 & PASS \\
\hline $104-\mathrm{NW}-1$ & 0.07 & 0.08 & 17 & PASS \\
\hline $104-\mathrm{EW}-1$ & 0.06 & 0.03 & 16 & PASS \\
\hline $104-\mathrm{SW}-1$ & 0.16 & 0.15 & 18 & PASS \\
\hline $104-\mathrm{WW}-1$ & 0.15 & 0.08 & 21 & PASS \\
\hline
\end{tabular}

Table 15. Room 106 Survey Units

\begin{tabular}{|c|c|c|c|c|}
\hline $\begin{array}{c}\text { Class 1 or 2 } \\
\text { Survey Unit } \\
\text { Number }\end{array}$ & $\begin{array}{c}\text { LBGR (based on } \\
\text { averages) }\end{array}$ & $\begin{array}{c}\text { LBGR (based } \\
\text { on median } \\
\text { values) }\end{array}$ & $\begin{array}{c}\text { Static } \\
\text { Measurements }\end{array}$ & $\begin{array}{c}\text { Statistical Test } \\
\text { Determination }\end{array}$ \\
\hline $\begin{array}{c}\text { 106-F-1 (Post- } \\
\text { Decon) }\end{array}$ & 0.10 & 0.08 & 13 & PASS \\
\hline $106-C-1$ & 0.49 & 0.49 & 13 & PASS \\
\hline $106-N W-1$ & 0.12 & 0.15 & 14 & PASS \\
\hline $106-E W-1$ & 0.09 & 0.08 & 15 & PASS \\
\hline $106-S W-1$ & 0.23 & 0.18 & 13 & PASS \\
\hline $106-W W-1$ & 0.24 & 0.23 & 13 & PASS \\
\hline
\end{tabular}


Table 16. Room 107 Survey Units

\begin{tabular}{|c|c|c|c|c|}
\hline $\begin{array}{c}\text { Class 1 or 2 } \\
\text { Survey Unit } \\
\text { Number }\end{array}$ & $\begin{array}{c}\text { LBGR (based on } \\
\text { averages) }\end{array}$ & $\begin{array}{c}\text { LBGR (based } \\
\text { on median } \\
\text { values) }\end{array}$ & $\begin{array}{c}\text { Static } \\
\text { Measurements }\end{array}$ & $\begin{array}{c}\text { Statistical Test } \\
\text { Determination }\end{array}$ \\
\hline $107-F-1$ & 0.24 & 0.23 & 16 & PASS \\
\hline $107-C-1$ & 0.32 & 0.25 & 18 & PASS \\
\hline $107-N W-1$ & 0.42 & 0.42 & 16 & PASS \\
\hline $107-N W-2$ & 0.34 & 0.25 & 17 & PASS \\
\hline $107-E W-1$ & 0.22 & 0.20 & 20 & PASS \\
\hline $107-S W-1$ & 0.22 & 0.15 & 16 & PASS \\
\hline $107-S W-2$ & 0.16 & 0.13 & 18 & PASS \\
\hline $106-W W-1$ & 0.20 & 0.23 & 20 & PASS \\
\hline
\end{tabular}

Table 17. Combined Ledges Survey Unit

\begin{tabular}{|c|c|c|c|c|}
\hline $\begin{array}{c}\text { 102, 104, 106, } \\
\text { 107 Ledges }\end{array}$ & $\begin{array}{c}\text { LBGR (based on } \\
\text { average) }\end{array}$ & $\begin{array}{c}\text { LBGR (based } \\
\text { on median } \\
\text { value) }\end{array}$ & $\begin{array}{c}\text { Static } \\
\text { Measurements }\end{array}$ & $\begin{array}{c}\text { Statistical Test } \\
\text { Determination }\end{array}$ \\
\hline Ledges-1 & 0.33 & 0.34 & 17 & PASS \\
\hline
\end{tabular}

\subsection{Scanning Results}

Class 3 areas were subjectively scanned according to survey plan guidance. The scans focused on entryways and direct access points to Class 1 and 2 areas. No elevated measurements were detected during the scans in Class 3 areas.

Class 2 areas were scanned at $10 \%$ of the surface area of the survey unit. No elevated measurements were detected during the scans in Class 2 areas.

Class 1 areas were scanned at $100 \%$ of the surface area of the survey unit.

- Room 106 had a total of four locations on the floor ranging in size from $1 \mathrm{ft}^{2}$ to $4 \mathrm{ft}^{2}$ that were identified during scan surveys to have residual radioactivity greater than the release limits. These locations were all subsequently decontaminated and resurveyed. Results confirmed the decontamination efforts were successful (see Section 6.3).

- No other elevated measurements were detected in the remainder of the Class 1 survey units. 
Final Status Survey Report for

Corrective Action Unit 117-Pluto

Disassembly Facility, Building 2201

\section{CONCLUSIONS}

Building 2201 meets the release criteria commensurate with the Waste Acceptance Criteria (for radiological purposes) of the U10C landfill permit. Based on the thorough statistical sampling and scanning of the building's interior, Building 2201 may be considered radiologically "clean," or free of contamination.

- Every Class 3 designated room had an average surface contamination below the $\mathrm{DCGL}_{\mathrm{w}}$ and all rooms passed the statistical test. The null hypothesis (that the survey unit exceeds the release criteria) is rejected.

- Every survey unit in Room 102 had an average surface contamination below the $D_{C G L}$ and all survey units passed the statistical test. The null hypothesis (that each individual survey unit exceeds the release criteria) is rejected.

- Every survey unit in Room 104 had an average surface contamination below the $\mathrm{DCGL}_{\mathrm{w}}$ and all survey units passed the statistical test. The null hypothesis (that each individual survey unit exceeds the release criteria) is rejected.

- Every survey unit in Room 106 had an average surface contamination below the $\mathrm{DCGL}_{\mathrm{w}}$ and all survey units passed the statistical test. The null hypothesis (that each individual survey unit exceeds the release criteria) is rejected.

- Every survey unit in Room 107 had an average surface contamination below the $\mathrm{DCGL}_{\mathrm{w}}$ and all survey units passed the statistical test. The null hypothesis (that each individual survey unit exceeds the release criteria) is rejected.

Every survey unit in Building 2201 had an average surface contamination below the $\mathrm{DCGL}_{\mathrm{w}}$ and all survey units passed the statistical test. The null hypothesis (that each individual survey unit exceeds the release criteria) is rejected. Building 2201 meets the release criteria. 
Final Status Survey Report for

Corrective Action Unit 117-Pluto

Disassembly Facility, Building 2201

\section{REFERENCES}

Abelquist, E. W., 2001. Decommissioning Health Physics - A Handbook for MARSSIM Users. New York, NY: Taylor \& Francis Group LLC.

National Security Technologies, LLC, 2007. Streamlined Approach for Environmental Restoration (SAFER) Plan for Corrective Action Unit 117: Area 26 Pluto Disassembly Facility, Nevada Test Site, Nevada. DOE/NV--1228. Las Vegas, NV.

National Security Technologies, LLC, 2008. Company Directive CD-0441.004, "Material Release Program." Las Vegas, NV.

National Security Technologies, LLC, 2009. Final Status Survey Plan for Corrective Action Unit 117 - Pluto Disassembly Facility, Building 2201. Survey Plan 10-015. Las Vegas, NV.

National Security Technologies, LLC, 2008. Organization Instruction OI-0441.212, “Controlled and Unrestricted Release," Revision 4. Las Vegas, NV.

National Security Technologies, LLC, 2009. Pluto Disassembly Facility - Building Radiological Characterization. Survey Plan 09-022. Las Vegas, NV.

National Security Technologies, LLC, 2009. Pluto Disassembly Facility - RadiologicalCharacterization (Phase II) and Post Remediation Survey Requirements. Survey Plan 09-031. Las Vegas, NV.

Ott, R. L., M. T. Longnecker, 2001. Statistical Methods and Data Analysis. 5th ed. Duxbury: Wadsworth Group.

Stoller-Navarro Joint Venture (SNJV), 2009. Closure Report for Corrective Action Unit 117: Area 26 Pluto Disassembly Facility, Nevada Test Site, Nevada. DOE/NV--1324. Las Vegas, NV.

U.S. Nuclear Regulatory Commission, 2001. Multi-Agency Radiation Survey and Site Investigation Manual (MARSSIM). NUREG-1575 Rev. 1. August 2000. Washington, D.C.

U.S. Department of Energy, 1993. DOE O 5400.5, "Radiation Protection of the Public and the Environment." Washington, D.C.

U.S. Department of Energy, 1999. DOE Guidance 441.1-XX, "Control and Release of Property with Residual Radioactive Material for use with DOE 5400.5, Radiation Protection of the Public and the Environment." Washington, D.C.

U.S. Department of Energy, National Nuclear Security Administration Nevada Site Office, 2010. Nevada Test Site Radiological Control Manual, Revision 1. DOE/NV/25946--801. Las Vegas, NV. 
Final Status Survey Report for

Corrective Action Unit 117-Pluto

Disassembly Facility, Building 2201

APPENDIX A - Corrected Survey Maps 


\section{(n)}

\section{Survey \#}

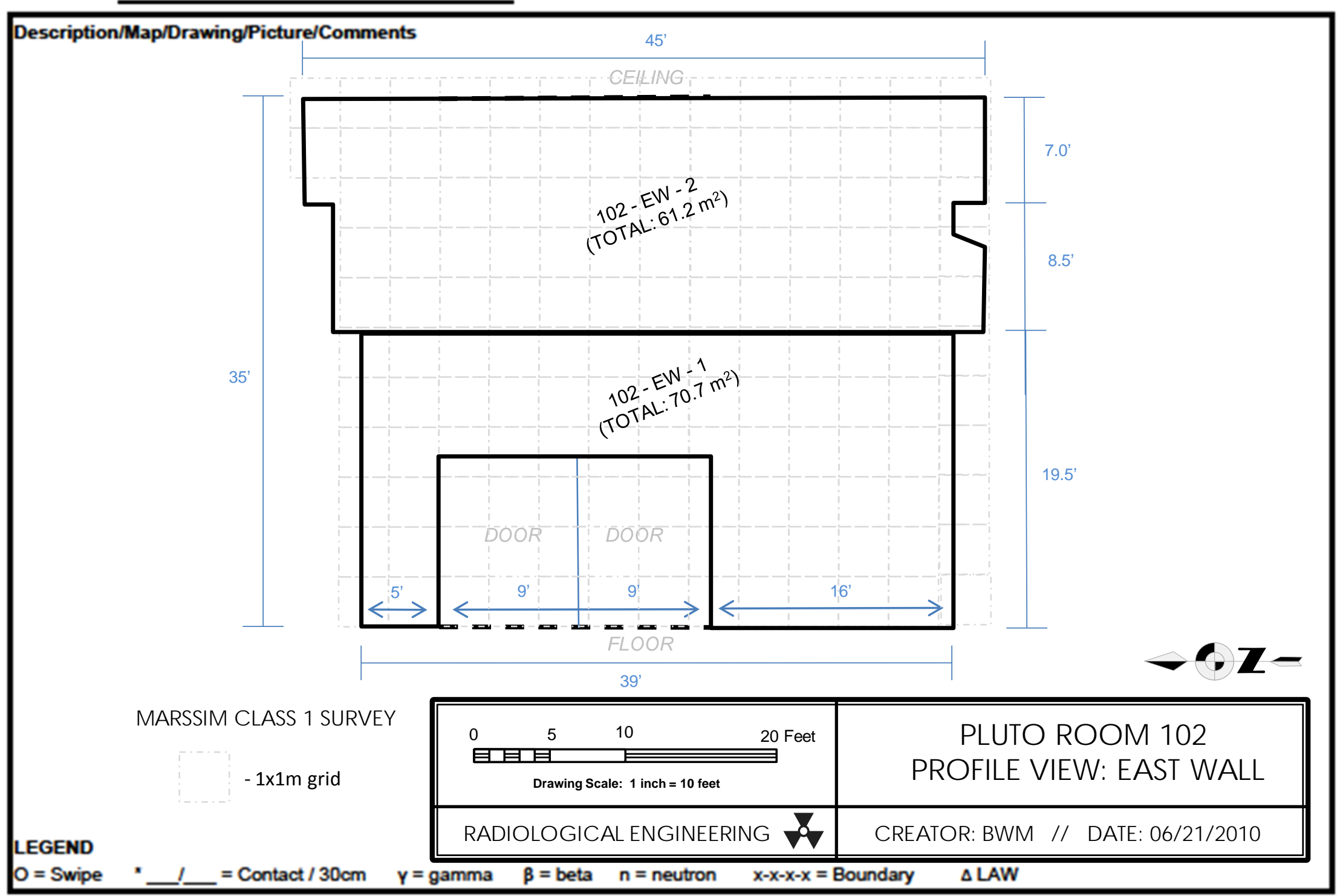




\section{RADIOLOG}

Survey \#

Description/Map/Drawing/Picture/Comments
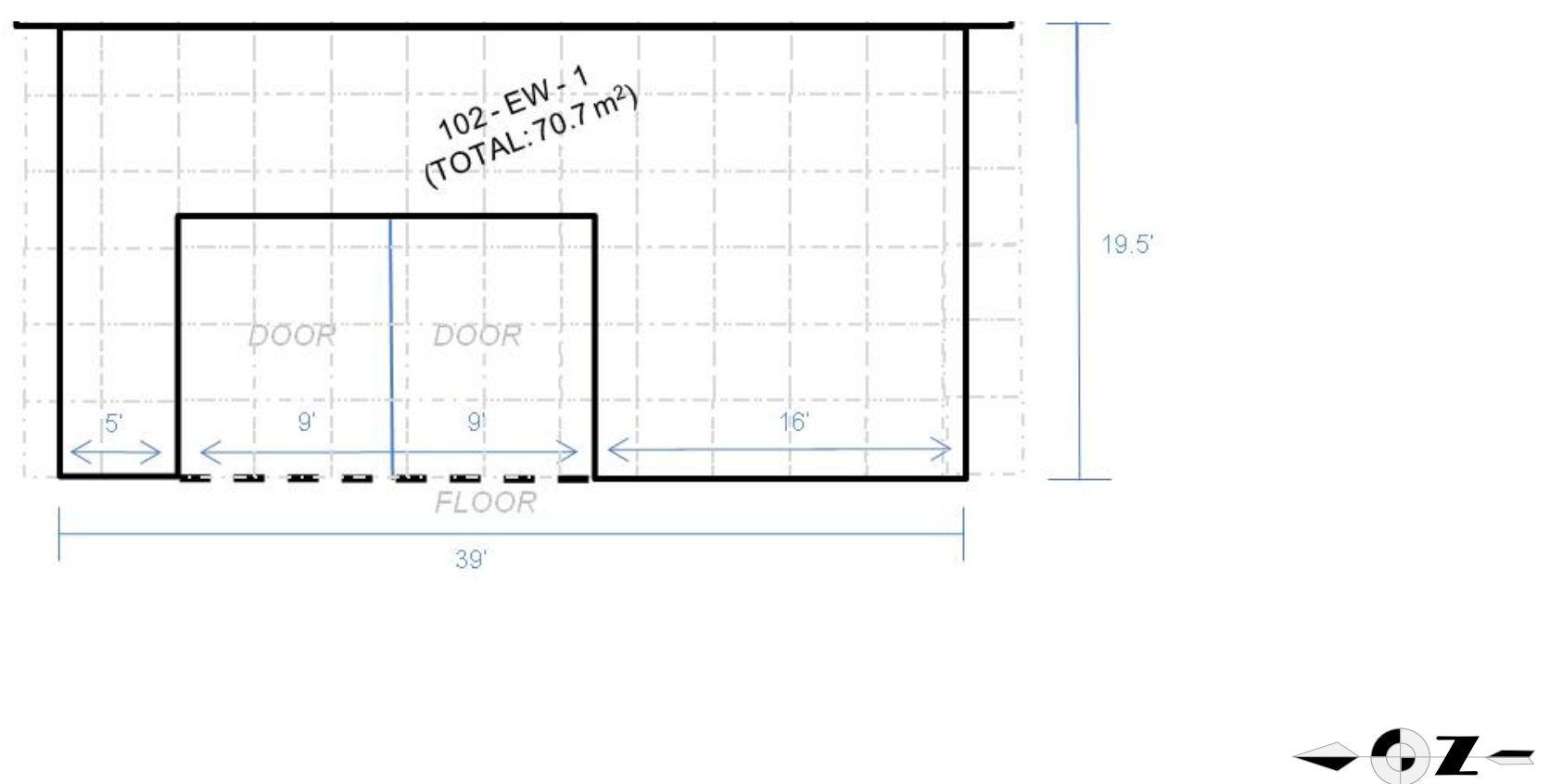

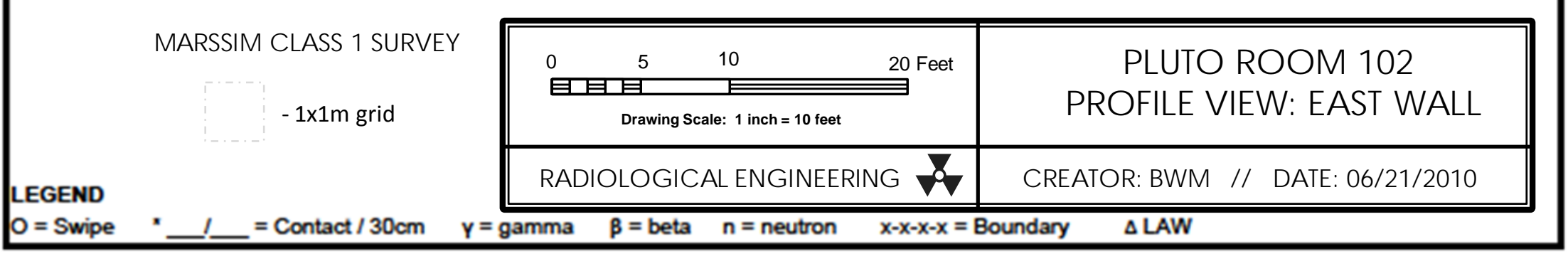




\section{RADIOLOG}

Survey \#

Description/Map/Drawing/Picture/Comments
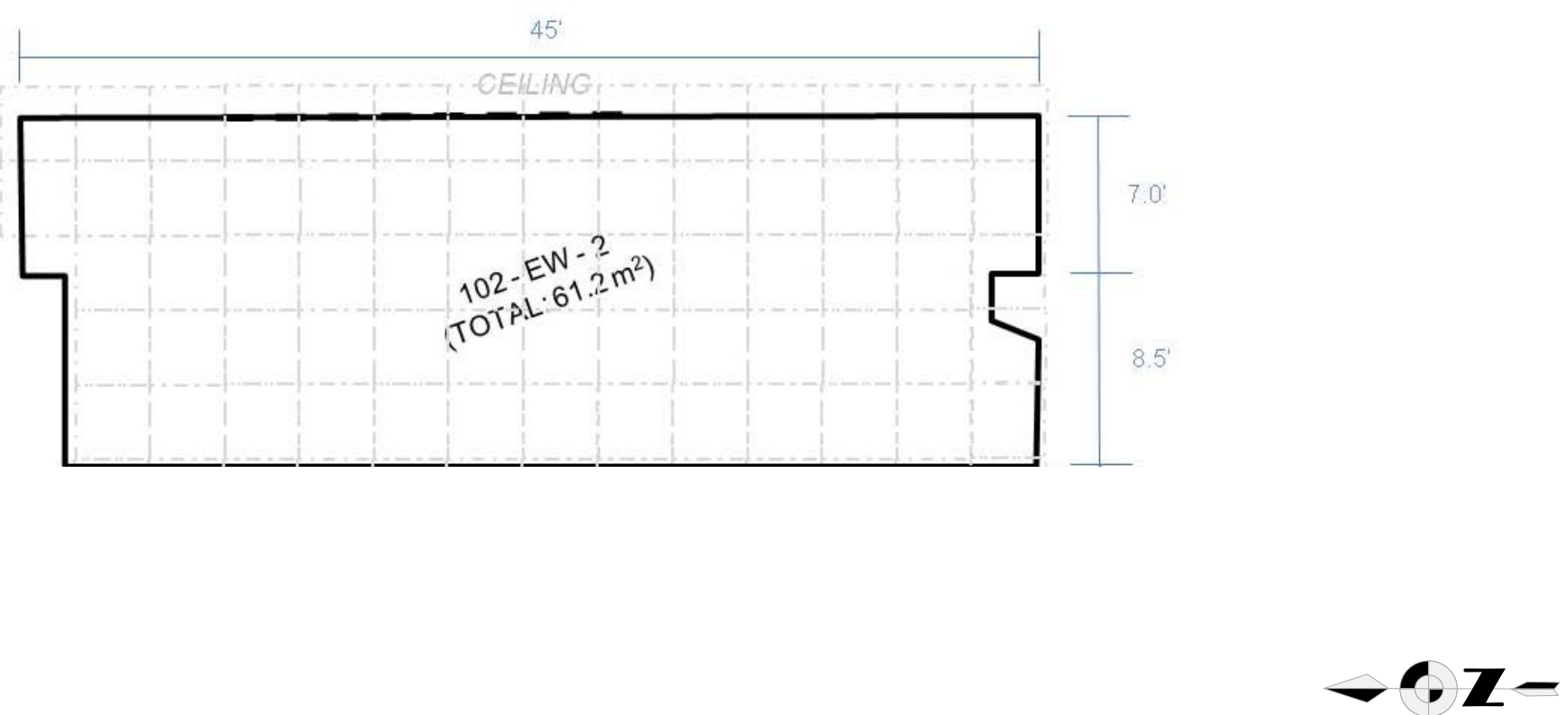

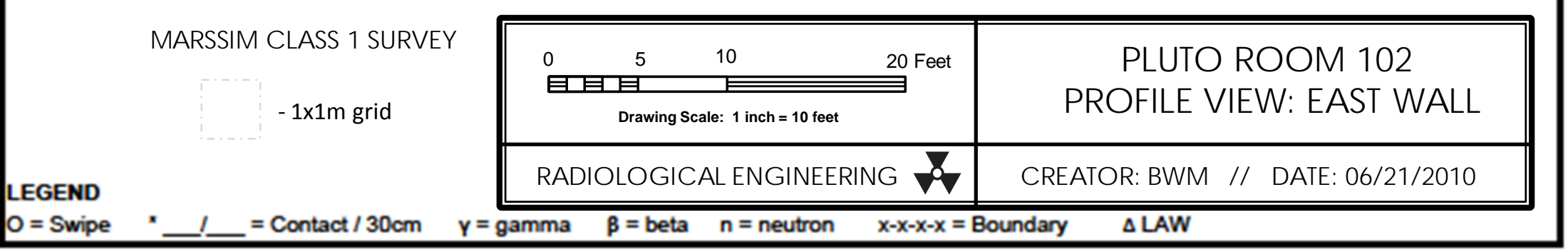


Final Status Survey Report for

Corrective Action Unit 117-Pluto

Disassembly Facility, Building 2201

\section{APPENDIX B - Approved Survey Results}




\section{Pluto Final Status Surveys - Completion Status (by room)}

\begin{tabular}{|c|c|c|c|c|c|c|c|}
\hline Room Number & Floor & Ceiling & North Wall & East Wall & South Wall & West Wall & Comments \\
\hline 101 & 157,158 & $158,161,165$ & $158,161,162$ & 164 & $158,161,163$ & 158,161 & Complete \\
\hline 102 & $\begin{array}{c}256,257,258, \\
259\end{array}$ & 278 & $261,262,263$ & 273,277 & $\begin{array}{c}267,268, \\
269,285\end{array}$ & 264,266 & Complete \\
\hline 103 & 190 & 189 & 188 & 188 & 188 & 188 & Complete \\
\hline 104 & 206 & 226 & 213 & 218 & 215 & 221 & Complete \\
\hline 105 & 168 & 168 & 168 & 168 & N/A & 168 & Complete \\
\hline 106 & $\begin{array}{c}\text { 275, } 276 \\
\text { (Post Decon) }\end{array}$ & 297 & 291 & 290 & 284 & 282 & Complete \\
\hline 107 & 279 & 296 & 280,281 & 288 & 283,289 & 286 & Complete \\
\hline $\begin{array}{c}\text { 102/104/106/107 } \\
\text { Ledges }\end{array}$ & \multicolumn{6}{|c|}{292} & Complete \\
\hline 108 & 173 & 172 & 171 & 170 & 170 & 171 & Complete \\
\hline 109 & 183 & 180 & 181 & 181 & 181 & 181 & Complete \\
\hline 110 & 228 & 228 & 228 & 228 & 228 & 228 & Complete \\
\hline 111 & 194 & 194 & 194 & 194 & 194 & 194 & Complete \\
\hline 112 & 204 & 205 & 203 & 203 & 203 & 203 & Complete \\
\hline 113 & 204 & 205 & 203 & 203 & 203 & 203 & Complete \\
\hline 114 & 204 & 205 & 203 & 203 & 203 & 203 & Complete \\
\hline 115 & 176 & 176 & 176 & 176 & 176 & 176 & Complete \\
\hline 116 & 177 & 178 & 179 & 179 & 179 & 179 & Complete \\
\hline 117 & 186 & 186 & 186 & 186 & 186 & 186 & Complete \\
\hline 201 & 239 & 239 & 239 & 239 & 239 & 239 & Complete \\
\hline 301 & 240 & 240 & 240 & 240 & 240 & 240 & Complete \\
\hline Basement \#1 & 191 & 193 & 192 & 192 & 192 & 192 & Complete \\
\hline Basement \#2 & 244 & 244 & 244 & 244 & 244 & 244 & Complete \\
\hline Basement \#3 & 246 & 246 & 246 & 246 & 246 & 246 & Complete \\
\hline Basement \#4 & 197 & 196 & 195 & 195 & 195 & 195 & Complete \\
\hline
\end{tabular}

Note: Judgemental scan surveys of Class 3 areas directly adjacent to Class 1 \& 2 areas performed on 7/29/10 and included rooms 105, 108, \& 109. Survey \# 10-ER-A26-230. Also Survey Unit 102-SDW-1 was included in the 102 South Wall completion (\#285) 
SURVEY \# 10-ER-A26-273

\begin{tabular}{|c|c|c|c|c|c|c|c|c|c|c|c|}
\hline \multicolumn{3}{|c|}{$\begin{array}{l}\text { Location: } \\
\text { Area } 26 \text { CAU117 Pluto }\end{array}$} & \multicolumn{2}{|c|}{$\begin{array}{l}\text { Purpose: } \\
\text { Final Status Survey, Room } 102 \\
\text { East Wall 102-EW-1 }\end{array}$} & \multicolumn{5}{|c|}{$\begin{array}{l}\text { Comments: } \\
\text { All one minute static counts are in cpm. Bkgd not subtracted } \\
\text { for Final Status Survey, Survey Plan \#10-015 }\end{array}$} & \multicolumn{2}{|c|}{$\begin{array}{l}\text { Date/Time: } \\
08 / 17 / 20101600\end{array}$} \\
\hline Instrument: & Serial \#: & Cal Due: & \begin{tabular}{|c|} 
Eff in \%: \\
Alpha / Beta \\
\end{tabular} & $\begin{array}{l}\text { BKG in dpm: } \\
\text { Alpha / Beta }\end{array}$ & $\begin{array}{l}\text { MDA in dpm: } \\
\text { Alpha / Beta }\end{array}$ & RWP\# & \multicolumn{5}{|l|}{$\mathrm{N} / \mathrm{A}$} \\
\hline Electra & 2125 & $4 / 6 / 2011$ & $14.4 / 18.2$ & $N / A$ & $N / A$ & \multicolumn{6}{|c|}{ ENV-10-RCT ENV SITE } \\
\hline Electra & 1120 & $4 / 6 / 2011$ & $14.1 / 20.8$ & $\mathrm{~N} / \mathrm{A}$ & $N / A$ & \multicolumn{3}{|c|}{ RCT Name: D.Cotroneo Jr. } & Signature: & \multicolumn{2}{|c|}{$-1 t$} \\
\hline Electra & 1201 & $4 / 6 / 2011$ & $14.4 / 20.3$ & $N / A$ & $N / A$ & \multicolumn{3}{|c|}{ RCT Name: K. Wilcox/M.Payne } & \multirow{3}{*}{\multicolumn{3}{|c|}{ Signature: $\mathrm{Ke} \cdot 20 \mathrm{P} / \mathrm{C}$. $\mathrm{ms}_{\mathrm{F}}$}} \\
\hline Electra & $N / A$ & $N / A$ & $N / A$ & $N / A$ & $N / A$ & RCT Name: & S.Brown/t & Bunce & & & \\
\hline Electra & $N / A$ & $N / A$ & $N / A$ & $\mathrm{~N} / \mathrm{A}$ & $N / A$ & RCT Name: & B. Fosket & Nelch & & & \\
\hline \multirow[t]{2}{*}{ Survey Point } & \multirow{2}{*}{\multicolumn{4}{|c|}{ Description/Comments }} & \multicolumn{2}{|c|}{$\begin{array}{l}\text { Removable } \\
\mathrm{dpm} / 100 \mathrm{~cm}^{2}\end{array}$} & \multicolumn{2}{|c|}{$\begin{array}{c}\text { Fixed + Removable } \\
\mathrm{dpm} / 100 \mathrm{~cm}^{2}\end{array}$} & \multirow{2}{*}{$\begin{array}{l}\text { Gamma } \\
\mathrm{mrem} / \mathrm{hr}\end{array}$} & \multirow{2}{*}{$\begin{array}{l}\text { Neutron } \\
\mathrm{mrem} / \mathrm{hr}\end{array}$} & \multirow{2}{*}{$\begin{array}{l}\text { Total } \\
\mathrm{mrem} / \mathrm{hr}\end{array}$} \\
\hline & & & & & Alpha & Beta & Alpha & Beta & & & \\
\hline 1 & \multicolumn{4}{|c|}{ Wall, Metal (Electra 1120) } & $N / A$ & $N / A$ & 9 & 689 & $N / A$ & $N / A$ & $N / A$ \\
\hline 2 & \multicolumn{4}{|c|}{ Wall, Metal (Electra 1120) } & $N / A$ & $N / A$ & 5 & 730 & $N / A$ & $N / A$ & $N / A$ \\
\hline 3 & \multicolumn{4}{|c|}{ Wall, Metal (Electra 1120) } & $N / A$ & $N / A$ & 15 & 1068 & $N / A$ & $N / A$ & $N / A$ \\
\hline 4 & \multicolumn{4}{|c|}{ Wall, Concrete (Electra 1201) } & $N / A$ & $N / A$ & 9 & 933 & $N / A$ & $N / A$ & $N / A$ \\
\hline 5 & \multicolumn{4}{|c|}{ Wall, Concrete (Electra 1201) } & $N / A$ & $N / A$ & 9 & 977 & $N / A$ & $N / A$ & $N / A$ \\
\hline 6 & \multicolumn{4}{|c|}{ Wall, Metal (Electra 1120) } & $N / A$ & $N / A$ & 17 & 1013 & $N / A$ & $N / A$ & $N / A$ \\
\hline 7 & \multicolumn{4}{|c|}{ Wall, Wood (Electra 1120) } & $N / A$ & $N / A$ & 5 & 715 & $N / A$ & $N / A$ & $N / A$ \\
\hline 8 & \multicolumn{4}{|c|}{ Wall, Metal (Electra 1120) } & $N / A$ & $N / A$ & 6 & 655 & $N / A$ & $N / A$ & $N / A$ \\
\hline 9 & \multicolumn{4}{|c|}{ Wall, Concrete (Electra 1120) } & $N / A$ & $N / A$ & 6 & 990 & $N / A$ & $N / A$ & $N / A$ \\
\hline 10 & \multicolumn{4}{|c|}{ Wall, Concrete (Electra 1120) } & $N / A$ & $N / A$ & 9 & 910 & $N / A$ & $\mathrm{~N} / \mathrm{A}$ & $N / A$ \\
\hline 11 & \multicolumn{4}{|c|}{ Wall, Concrete (Electra 1120) } & $N / A$ & $N / A$ & 13 & 968 & $N / A$ & $\mathrm{~N} / \mathrm{A}$ & $N / A$ \\
\hline \multicolumn{5}{|c|}{ Reviewed By (Print): } & Signature: & & & & & \multicolumn{2}{|c|}{$8 / 20 / 10$} \\
\hline
\end{tabular}


SURVEY \# 10-ER-A26-273

\begin{tabular}{|c|c|c|c|c|c|c|c|c|}
\hline \multirow[t]{2}{*}{$\begin{array}{l}\text { Survey } \\
\text { Point }\end{array}$} & \multirow[t]{2}{*}{ Description/Comments } & \multicolumn{2}{|c|}{$\begin{array}{l}\text { Removable } \\
\mathrm{dpm} / 100 \mathrm{~cm}^{2}\end{array}$} & \multicolumn{2}{|c|}{$\begin{array}{c}\text { Fixed + Removable } \\
\mathrm{dpm} / 100 \mathrm{~cm}^{2}\end{array}$} & \multirow{2}{*}{$\begin{array}{l}\text { Gamma } \\
\mathrm{mrem} / \mathrm{hr}\end{array}$} & \multirow{2}{*}{$\begin{array}{l}\text { Neutron } \\
\mathrm{mrem} / \mathrm{hr}\end{array}$} & \multirow{2}{*}{$\begin{array}{c}\text { Total } \\
\mathrm{mrem} / \mathrm{hr}\end{array}$} \\
\hline & & Alpha & Beta & Alpha & Beta & & & \\
\hline 12 & Wall, Concrete $(1120)$ & N/A & N/A & 7 & 931 & N/A & N/A & $\mathrm{N} / \mathrm{A}$ \\
\hline 13 & Wall, Concrete (1120) & N/A & $\mathrm{N} / \mathrm{A}$ & 10 & 937 & N/A & N/A & N/A \\
\hline N/A & ${ }^{\star *}$ Performed scan surveys for both alpha and beta** & N/A & N/A & $\mathrm{N} / \mathrm{A}$ & N/A & N/A & N/A & N/A \\
\hline N/A & ${ }^{\star \star}$ on $100 \%$ of survey unit. No trigger levels exceeded ${ }^{\star \star}$ & $\mathrm{N} / \mathrm{A}$ & $\mathrm{N} / \mathrm{A}$ & N/A & N/A & N/A & N/A & N/A \\
\hline N/A & $\mathrm{N} / \mathrm{A}$ & N/A & N/A & N/A & N/A & N/A & N/A & N/A \\
\hline $\mathrm{N} / \mathrm{A}$ & $N / A$ & $\mathrm{~N} / \mathrm{A}$ & N/A & N/A & $\mathrm{N} / \mathrm{A}$ & N/A & $\mathrm{N} / \mathrm{A}$ & N/A \\
\hline N/A & $N / A$ & $\mathrm{~N} / \mathrm{A}$ & N/A & N/A & $\mathrm{N} / \mathrm{A}$ & N/A & $\mathrm{N} / \mathrm{A}$ & N/A \\
\hline $\mathrm{N} / \mathrm{A}$ & $N / A$ & N/A & N/A & N/A & N/A & N/A & $\mathrm{N} / \mathrm{A}$ & N/A \\
\hline N/A & $N / A$ & N/A & N/A & N/A & N/A & N/A & N/A & N/A \\
\hline N/A & $\mathrm{N} / \mathrm{A}$ & N/A & $\mathrm{N} / \mathrm{A}$ & N/A & N/A & N/A & N/A & N/A \\
\hline N/A & $\mathrm{N} / \mathrm{A}$ & N/A & N/A & N/A & N/A & N/A & N/A & N/A \\
\hline $\mathrm{N} / \mathrm{A}$ & $N / A$ & N/A & $\mathrm{N} / \mathrm{A}$ & N/A & N/A & N/A & $\mathrm{N} / \mathrm{A}$ & N/A \\
\hline N/A & $\mathrm{N} / \mathrm{A}$ & $\mathrm{N} / \mathrm{A}$ & $\mathrm{N} / \mathrm{A}$ & N/A & $\mathrm{N} / \mathrm{A}$ & N/A & $\mathrm{N} / \mathrm{A}$ & $\mathrm{N} / \mathrm{A}$ \\
\hline N/A & $N / A$ & N/A & $\mathrm{N} / \mathrm{A}$ & N/A & $\mathrm{N} / \mathrm{A}$ & N/A & N/A & N/A \\
\hline N/A & $N / A$ & N/A & $\mathrm{N} / \mathrm{A}$ & N/A & $N / A$ & N/A & N/A & $\mathrm{N} / \mathrm{A}$ \\
\hline N/A & $N / A$ & N/A & $\mathrm{N} / \mathrm{A}$ & N/A & N/A & N/A & N/A & N/A \\
\hline N/A & $\mathrm{N} / \mathrm{A}$ & $\mathrm{N} / \mathrm{A}$ & N/A & N/A & N/A & N/A & N/A & N/A \\
\hline $\mathrm{N} / \mathrm{A}$ & $\mathrm{N} / \mathrm{A}$ & N/A & N/A & N/A & N/A & N/A & N/A & N/A \\
\hline N/A & $N / A$ & N/A & N/A & N/A & N/A & N/A & N/A & N/A \\
\hline $\mathrm{N} / \mathrm{A}$ & $\mathrm{N} / \mathrm{A}$ & N/A & N/A & N/A & N/A & N/A & N/A & $\mathrm{N} / \mathrm{A}$ \\
\hline N/A & $\mathrm{N} / \mathrm{A}$ & N/A & N/A & $\mathrm{N} / \mathrm{A}$ & N/A & N/A & N/A & N/A \\
\hline N/A & $N / A$ & N/A & N/A & N/A & N/A & N/A & N/A & N/A \\
\hline N/A & $N / A$ & N/A & $N / A$ & N/A & N/A & $\mathrm{N} / \mathrm{A}$ & N/A & $\mathrm{N} / \mathrm{A}$ \\
\hline
\end{tabular}


Survey $\# 10$ En A26 273

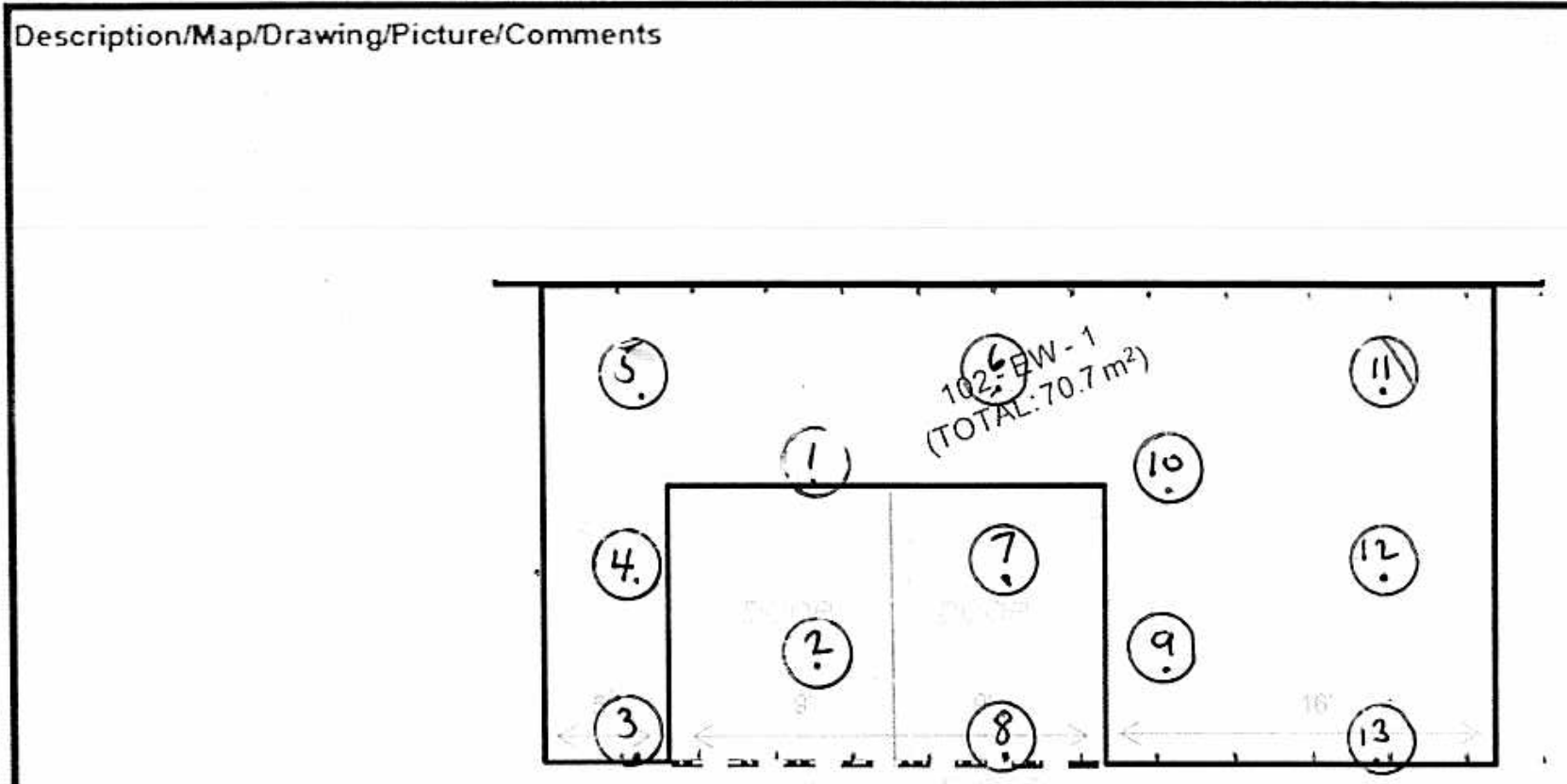

MARSSIM CLASS I SURVEY

$-1 \times 1 m$ grid

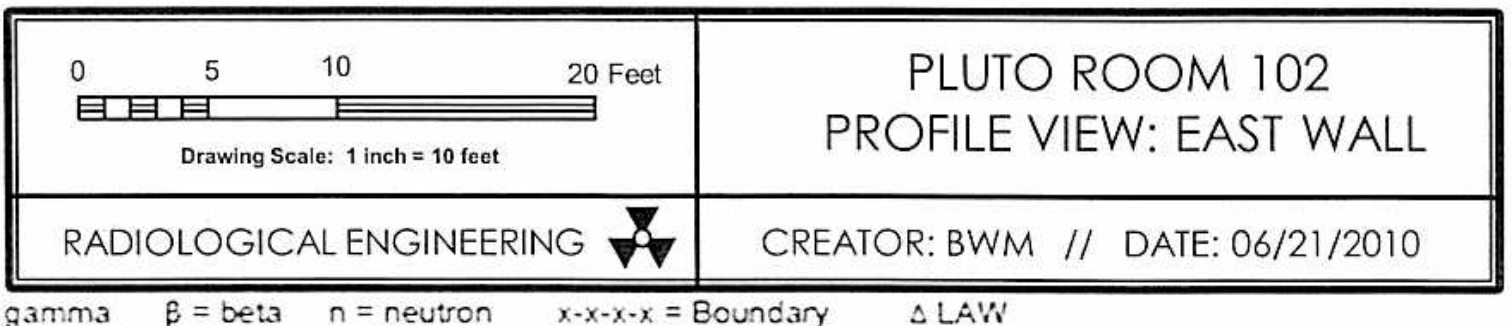




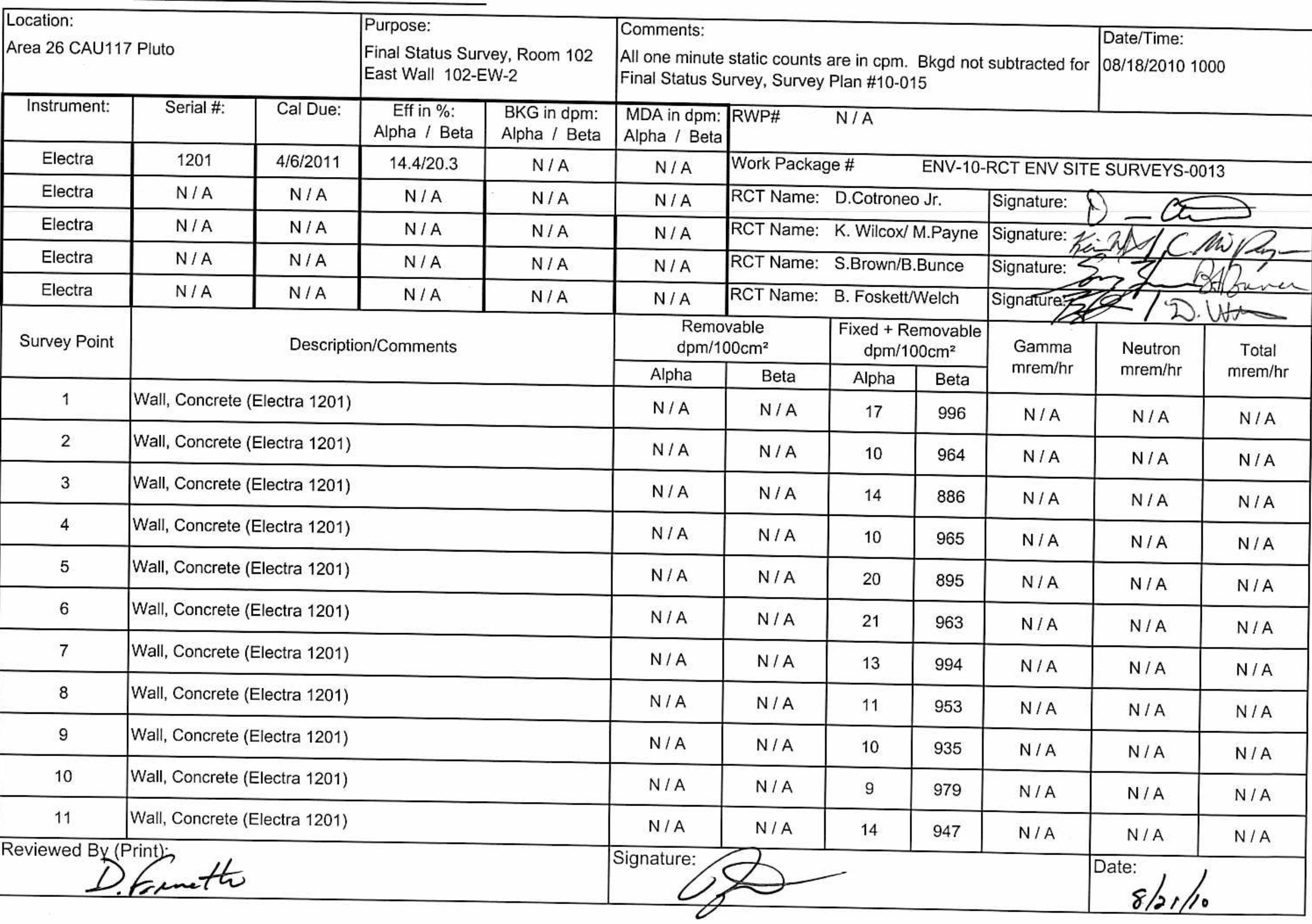


SURVEY \# 10-ER-A26-277

\begin{tabular}{|c|c|c|c|c|c|c|c|c|}
\hline \multirow[t]{2}{*}{ Survey Point } & \multirow{2}{*}{ Description/Comments } & \multicolumn{2}{|c|}{$\begin{array}{l}\text { Removable } \\
\mathrm{dpm} / 100 \mathrm{~cm}^{2}\end{array}$} & \multicolumn{2}{|c|}{$\begin{array}{c}\text { Fixed + Removable } \\
\mathrm{dpm} / 100 \mathrm{~cm}^{2}\end{array}$} & \multirow{2}{*}{$\begin{array}{l}\text { Gamma } \\
\mathrm{mrem} / \mathrm{hr}\end{array}$} & \multirow{2}{*}{$\begin{array}{l}\text { Neutron } \\
\mathrm{mrem} / \mathrm{hr}\end{array}$} & \multirow{2}{*}{$\begin{array}{c}\text { Total } \\
\text { mrem/hr }\end{array}$} \\
\hline & & Alpha & Beta & Alpha & Beta & & & \\
\hline 12 & Wall, Concrete (1201) & N/A & $\mathrm{N} / \mathrm{A}$ & 9 & 956 & N/A & N/A & N/A \\
\hline N/A & $N / A$ & N/A & $\mathrm{N} / \mathrm{A}$ & $\mathrm{N} / \mathrm{A}$ & N/A & N/A & N/A & N/A \\
\hline N/A & ${ }^{\star *}$ Performed scan surveys for both alpha and beta** & N/A & $\mathrm{N} / \mathrm{A}$ & N/A & $\mathrm{N} / \mathrm{A}$ & N/A & N/A & N/A \\
\hline N/A & ${ }^{* *}$ on $100 \%$ of survey unit. No trigger levels exceeded ${ }^{\star \star}$ & N/A & N/A & $\mathrm{N} / \mathrm{A}$ & N/A & $\mathrm{N} / \mathrm{A}$ & $\mathrm{N} / \mathrm{A}$ & $\mathrm{N} / \mathrm{A}$ \\
\hline N/A & $N / A$ & N/A & $\mathrm{N} / \mathrm{A}$ & $\mathrm{N} / \mathrm{A}$ & $\mathrm{N} / \mathrm{A}$ & N/A & N/A & N/A \\
\hline $\mathrm{N} / \mathrm{A}$ & $\mathrm{N} / \mathrm{A}$ & $\mathrm{N} / \mathrm{A}$ & N/A & $\mathrm{N} / \mathrm{A}$ & $\mathrm{N} / \mathrm{A}$ & N/A & $\mathrm{N} / \mathrm{A}$ & $\mathrm{N} / \mathrm{A}$ \\
\hline N/A & $\mathrm{N} / \mathrm{A}$ & $\mathrm{N} / \mathrm{A}$ & N/A & $\mathrm{N} / \mathrm{A}$ & $\mathrm{N} / \mathrm{A}$ & $\mathrm{N} / \mathrm{A}$ & N/A & N/A \\
\hline N/A & $\mathrm{N} / \mathrm{A}$ & $\mathrm{N} / \mathrm{A}$ & N/A & N/A & N/A & N/A & N/A & N/A \\
\hline N/A & $N / A$ & $\mathrm{~N} / \mathrm{A}$ & $\mathrm{N} / \mathrm{A}$ & $\mathrm{N} / \mathrm{A}$ & $\mathrm{N} / \mathrm{A}$ & N/A & $\mathrm{N} / \mathrm{A}$ & N/A \\
\hline N/A & $\mathrm{N} / \mathrm{A}$ & N/A & $\mathrm{N} / \mathrm{A}$ & $\mathrm{N} / \mathrm{A}$ & $\mathrm{N} / \mathrm{A}$ & $\mathrm{N} / \mathrm{A}$ & N/A & N/A \\
\hline N/A & $N / A$ & $\mathrm{~N} / \mathrm{A}$ & N/A & N/A & $\mathrm{N} / \mathrm{A}$ & N/A & N/A & $\mathrm{N} / \mathrm{A}$ \\
\hline N/A & $\mathrm{N} / \mathrm{A}$ & N/A & N/A & $\mathrm{N} / \mathrm{A}$ & N/A & N/A & N/A & N/A \\
\hline N/A & $\mathrm{N} / \mathrm{A}$ & $\mathrm{N} / \mathrm{A}$ & N/A & $\mathrm{N} / \mathrm{A}$ & $\mathrm{N} / \mathrm{A}$ & $\mathrm{N} / \mathrm{A}$ & N/A & N/A \\
\hline N/A & $N / A$ & N/A & N/A & N/A & N/A & N/A & $\mathrm{N} / \mathrm{A}$ & N/A \\
\hline N/A & $\mathrm{N} / \mathrm{A}$ & $\mathrm{N} / \mathrm{A}$ & $\mathrm{N} / \mathrm{A}$ & N/A & $\mathrm{N} / \mathrm{A}$ & $\mathrm{N} / \mathrm{A}$ & N/A & N/A \\
\hline N/A & $\mathrm{N} / \mathrm{A}$ & N/A & $\mathrm{N} / \mathrm{A}$ & N/A & $\mathrm{N} / \mathrm{A}$ & N/A & N/A & N/A \\
\hline N/A & $N / A$ & N/A & N/A & N/A & N/A & N/A & N/A & N/A \\
\hline N/A & $\mathrm{N} / \mathrm{A}$ & N/A & N/A & N/A & N/A & N/A & N/A & N/A \\
\hline N/A & $\mathrm{N} / \mathrm{A}$ & N/A & $\mathrm{N} / \mathrm{A}$ & N/A & $\mathrm{N} / \mathrm{A}$ & N/A & N/A & N/A \\
\hline N/A & $N / A$ & N/A & $\mathrm{N} / \mathrm{A}$ & N/A & N/A & $\mathrm{N} / \mathrm{A}$ & N/A & N/A \\
\hline N/A & $\mathrm{N} / \mathrm{A}$ & N/A & N/A & N/A & N/A & N/A & N/A & N/A \\
\hline N/A & $\mathrm{N} / \mathrm{A}$ & $\mathrm{N} / \mathrm{A}$ & $\mathrm{N} / \mathrm{A}$ & N/A & N/A & N/A & N/A & N/A \\
\hline N/A & $N / A$ & N/A & N/A & N/A & N/A & N/A & N/A & N/A \\
\hline
\end{tabular}


Survey $\# 10$ ER - A26-277

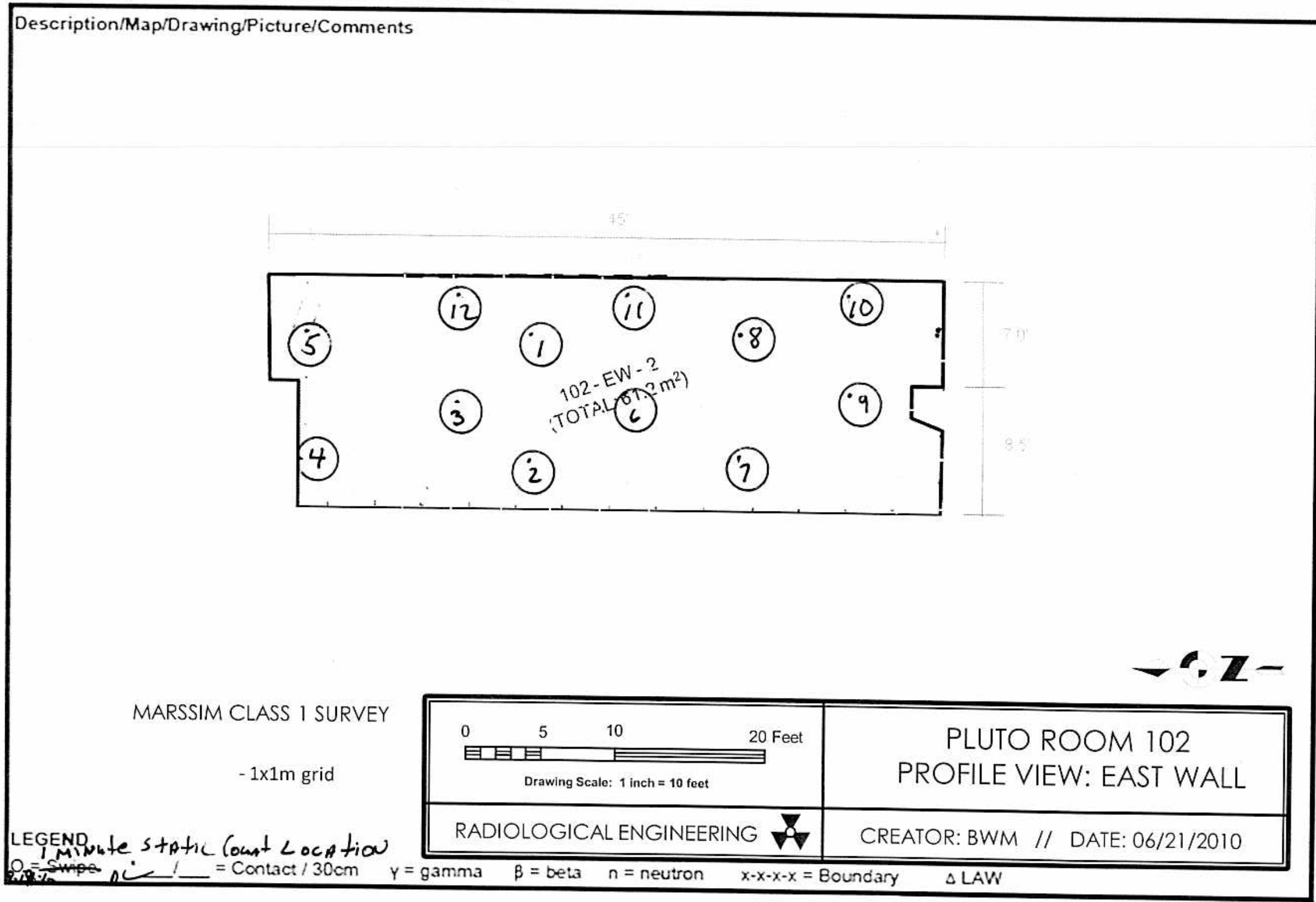


SURVEY \#

10-ER-A26-256

\begin{tabular}{|c|c|c|c|c|c|c|c|c|c|c|c|}
\hline \multicolumn{3}{|c|}{$\begin{array}{l}\text { Location: } \\
\text { Area } 26 \text { CAU117 Pluto }\end{array}$} & \multicolumn{2}{|c|}{$\begin{array}{l}\text { Purpose: } \\
\text { Final Status Survey, Room 102, } \\
\text { Floor / FSS-102-F-1 }\end{array}$} & \multicolumn{5}{|c|}{$\begin{array}{l}\text { Comments: } \\
\text { All one minute static counts are in } \mathrm{cpm} \text {. Bkgd not subtracted for } \\
\text { Final Status Survey, Survey Plan } \# 10-015\end{array}$} & \multicolumn{2}{|c|}{$\begin{array}{l}\text { Date/Time: } \\
08 / 12 / 20101000\end{array}$} \\
\hline Instrument: & Serial \#: & Cal Due: & $\begin{array}{c}\text { Eff in \%: } \\
\text { Alpha / Beta }\end{array}$ & $\begin{array}{l}\text { BKG in dpm: } \\
\text { Alpha / Beta }\end{array}$ & \begin{tabular}{|l|} 
MDA in dpm: \\
Alpha / Beta
\end{tabular} & RWP\# & \multicolumn{5}{|l|}{$\mathrm{N} / \mathrm{A}$} \\
\hline Electra & 4806 & $4 / 6 / 2011$ & $13.2 / 20.9$ & $\mathrm{~N} / \mathrm{A}$ & $\mathrm{N} / \mathrm{A}$ & \multicolumn{6}{|c|}{ Work Package \# $\quad$ ENV-10-RCT ENV SITE SURVEYS-0013 } \\
\hline Electra & 6697 & $4 / 6 / 2011$ & $13.8 / 19.9$ & $N / A$ & $N / A$ & \multicolumn{3}{|c|}{ RCT Name: D.Cotroneo Jr. } & \multicolumn{3}{|c|}{ Signature: $\theta-\&$} \\
\hline Electra & 1120 & $4 / 6 / 2011$ & $14.1 / 20.8$ & $\mathrm{~N} / \mathrm{A}$ & $\mathrm{N} / \mathrm{A}$ & \multicolumn{3}{|c|}{ RCT Name: Kevin Wilcox } & \multicolumn{3}{|c|}{ Signature: Ylai varo } \\
\hline Electra & 1201 & $4 / 6 / 2011$ & $14.4 / 20.3$ & $N / A$ & $N / A$ & \multicolumn{3}{|c|}{ RCT Name: S.Brown/B.Bunce } & \multirow{2}{*}{\multicolumn{3}{|c|}{ Signature: 5}} \\
\hline Electra & 1504 & $4 / 6 / 2011$ & $12.9 / 19.8$ & $\mathrm{~N} / \mathrm{A}$ & $N / A$ & RCT Name: & B. Fosket & & & & \\
\hline \multirow[t]{2}{*}{ Survey Point } & \multirow{2}{*}{\multicolumn{4}{|c|}{ Description/Comments }} & \multicolumn{2}{|c|}{$\begin{array}{l}\text { Removable } \\
\mathrm{dpm} / 100 \mathrm{~cm}^{2}\end{array}$} & \multicolumn{2}{|c|}{\begin{tabular}{|c|} 
Fixed + Removable \\
$\mathrm{dpm} / 100 \mathrm{~cm}^{2}$
\end{tabular}} & \multirow{2}{*}{$\begin{array}{l}\text { Gamma } \\
\mathrm{mrem} / \mathrm{hr}\end{array}$} & \multirow{2}{*}{$\begin{array}{l}\text { Neutron } \\
\mathrm{mrem} / \mathrm{hr}\end{array}$} & \multirow{2}{*}{$\begin{array}{c}\text { Total } \\
\mathrm{mrem} / \mathrm{hr}\end{array}$} \\
\hline & & & & & Alpha & Beta & Alpha & Beta & & & \\
\hline 1 & \multicolumn{4}{|c|}{ Floor, Concrete (Electra 4806) } & $N / A$ & $N / A$ & 2 & 1189 & $N / A$ & $N / A$ & $N / A$ \\
\hline 2 & \multicolumn{4}{|c|}{ Floor, Concrete (Electra 4806) } & $N / A$ & $N / A$ & 4 & 1107 & $\mathrm{~N} / \mathrm{A}$ & $N / A$ & $N / A$ \\
\hline 3 & \multicolumn{4}{|c|}{ Floor, Concrete (Electra 4806) } & $\mathrm{N} / \mathrm{A}$ & $N / A$ & 1 & 961 & $N / A$ & $N / A$ & $N / A$ \\
\hline 4 & \multicolumn{4}{|c|}{ Floor, Concrete (Electra 4806) } & $N / A$ & $N / A$ & 1 & 1148 & $N / A$ & $N / A$ & $N / A$ \\
\hline 5 & \multicolumn{4}{|c|}{ Floor, Concrete (Electra 4806) } & $N / A$ & $N / A$ & 2 & 1188 & $N / A$ & $\mathrm{~N} / \mathrm{A}$ & $N / A$ \\
\hline 6 & \multicolumn{4}{|c|}{ Floor, Concrete (Electra 4806) } & $N / A$ & $N / A$ & 2 & 1234 & $N / A$ & $N / A$ & $N / A$ \\
\hline 7 & \multicolumn{4}{|c|}{ Floor, Concrete (Electra 4806) } & $N / A$ & $N / A$ & 5 & 1078 & $N / A$ & $N / A$ & $N / A$ \\
\hline 8 & \multicolumn{4}{|c|}{ Floor, Concrete (Electra 4806) } & $N / A$ & $N / A$ & 5 & 1160 & $N / A$ & $N / A$ & $\mathrm{~N} / \mathrm{A}$ \\
\hline 9 & \multicolumn{4}{|c|}{ Floor, Concrete (Electra 4806) } & $N / A$ & $N / A$ & 11 & 1169 & $N / A$ & $N / A$ & $N / A$ \\
\hline 10 & \multicolumn{4}{|c|}{ Floor, Concrete (Electra 4806) } & $N / A$ & $N / A$ & 4 & 1233 & $N / A$ & $N / A$ & $N / A$ \\
\hline 11 & \multicolumn{4}{|c|}{ Floor, Concrete (Electra 4806) } & $\mathrm{N} / \mathrm{A}$ & $N / A$ & 3 & 1028 & $N / A$ & $N / A$ & $N / A$ \\
\hline \multicolumn{5}{|c|}{ Reviewed By (Pring): } & \multicolumn{7}{|c|}{ Signature: } \\
\hline
\end{tabular}


SURVEY \# 10-ER-A26-256

\begin{tabular}{|c|c|c|c|c|c|c|c|c|}
\hline \multirow[t]{2}{*}{ Survey Point } & \multirow[t]{2}{*}{ Description/Comments } & \multicolumn{2}{|c|}{$\begin{array}{l}\text { Removable } \\
\mathrm{dpm} / 100 \mathrm{~cm}^{2}\end{array}$} & \multicolumn{2}{|c|}{$\begin{array}{l}\text { Fixed + Removable } \\
\mathrm{dpm} / 100 \mathrm{~cm}^{2}\end{array}$} & \multirow{2}{*}{$\begin{array}{l}\text { Gamma } \\
\mathrm{mrem} / \mathrm{hr}\end{array}$} & \multirow{2}{*}{$\begin{array}{l}\text { Neutron } \\
\text { mrem/hr }\end{array}$} & \multirow{2}{*}{$\begin{array}{c}\text { Total } \\
\text { mrem/hr }\end{array}$} \\
\hline & & Alpha & Beta & Alpha & Beta & & & \\
\hline 12 & Floor, Concrete (Electra 4806\} & N/A & N/A & 9 & 1120 & $\mathrm{~N} / \mathrm{A}$ & N/A & $\mathrm{N} / \mathrm{A}$ \\
\hline 13 & Floor, Concrete (Electra 4806) & $\mathrm{N} / \mathrm{A}$ & N/A & 6 & 1148 & N/A & N/A & N/A \\
\hline 14 & Floor, Concrete (Electra 4806\} & N/A & N/A & 1 & 1054 & N/A & $\mathrm{N} / \mathrm{A}$ & N/A \\
\hline N/A & ${ }^{\star \star}$ Performed scan surveys for both alpha and beta** & $\mathrm{N} / \mathrm{A}$ & N/A & N/A & $\mathrm{N} / \mathrm{A}$ & $\mathrm{N} / \mathrm{A}$ & N/A & N/A \\
\hline N/A & ${ }^{\star \star}$ on $100 \%$ of survey unit. No trigger levels exceeded ${ }^{\star \star}$ & $\mathrm{N} / \mathrm{A}$ & N/A & N/A & $\mathrm{N} / \mathrm{A}$ & N/A & N/A & $\mathrm{N} / \mathrm{A}$ \\
\hline N/A & $N / A$ & N/A & N/A & N/A & $\mathrm{N} / \mathrm{A}$ & N/A & $\mathrm{N} / \mathrm{A}$ & N/A \\
\hline N/A & $\mathrm{N} / \mathrm{A}$ & $\mathrm{N} / \mathrm{A}$ & N/A & N/A & N/A & N/A & $\mathrm{N} / \mathrm{A}$ & N/A \\
\hline N/A & $N / A$ & $\mathrm{~N} / \mathrm{A}$ & N/A & N/A & $\mathrm{N} / \mathrm{A}$ & N/A & N/A & N/A \\
\hline N/A & $N / A$ & N/A & N/A & N/A & $\mathrm{N} / \mathrm{A}$ & N/A & N/A & $\mathrm{N} / \mathrm{A}$ \\
\hline $\mathrm{N} / \mathrm{A}$ & $\mathrm{N} / \mathrm{A}$ & N/A & N/A & N/A & $\mathrm{N} / \mathrm{A}$ & N/A & N/A & N/A \\
\hline N/A & $N / A$ & N/A & N/A & N/A & $\mathrm{N} / \mathrm{A}$ & N/A & N/A & $\mathrm{N} / \mathrm{A}$ \\
\hline $\mathrm{N} / \mathrm{A}$ & $\mathrm{N} / \mathrm{A}$ & $\mathrm{N} / \mathrm{A}$ & N/A & N/A & N/A & N/A & $\mathrm{N} / \mathrm{A}$ & $\mathrm{N} / \mathrm{A}$ \\
\hline N/A & $\mathrm{N} / \mathrm{A}$ & N/A & $\mathrm{N} / \mathrm{A}$ & $\mathrm{N} / \mathrm{A}$ & N/A & $\mathrm{N} / \mathrm{A}$ & $\mathrm{N} / \mathrm{A}$ & $\mathrm{N} / \mathrm{A}$ \\
\hline N/A & $N / A$ & $\mathrm{~N} / \mathrm{A}$ & N/A & N/A & $\mathrm{N} / \mathrm{A}$ & $\mathrm{N} / \mathrm{A}$ & $\mathrm{N} / \mathrm{A}$ & $\mathrm{N} / \mathrm{A}$ \\
\hline N/A & $\mathrm{N} / \mathrm{A}$ & $\mathrm{N} / \mathrm{A}$ & N/A & N/A & N/A & N/A & N/A & $\mathrm{N} / \mathrm{A}$ \\
\hline N/A & $N / A$ & N/A & N/A & N/A & N/A & N/A & N/A & N/A \\
\hline N/A & $\mathrm{N} / \mathrm{A}$ & N/A & N/A & N/A & N/A & N/A & N/A & N/A \\
\hline N/A & $N / A$ & N/A & N/A & N/A & $\mathrm{N} / \mathrm{A}$ & N/A & N/A & $\mathrm{N} / \mathrm{A}$ \\
\hline N/A & $\mathrm{N} / \mathrm{A}$ & N/A & N/A & N/A & N/A & N/A & N/A & N/A \\
\hline N/A & $N / A$ & N/A & $\mathrm{N} / \mathrm{A}$ & N/A & $\mathrm{N} / \mathrm{A}$ & N/A & N/A & N/A \\
\hline N/A & $\mathrm{N} / \mathrm{A}$ & N/A & N/A & N/A & $\mathrm{N} / \mathrm{A}$ & N/A & N/A & $\mathrm{N} / \mathrm{A}$ \\
\hline N/A & $\mathrm{N} / \mathrm{A}$ & N/A & $\mathrm{N} / \mathrm{A}$ & N/A & $\mathrm{N} / \mathrm{A}$ & N/A & N/A & $\mathrm{N} / \mathrm{A}$ \\
\hline N/A & $N / A$ & N/A & N/A & N/A & N/A & N/A & N/A & N/A \\
\hline
\end{tabular}


Survey \# 1O-ER-A26-256

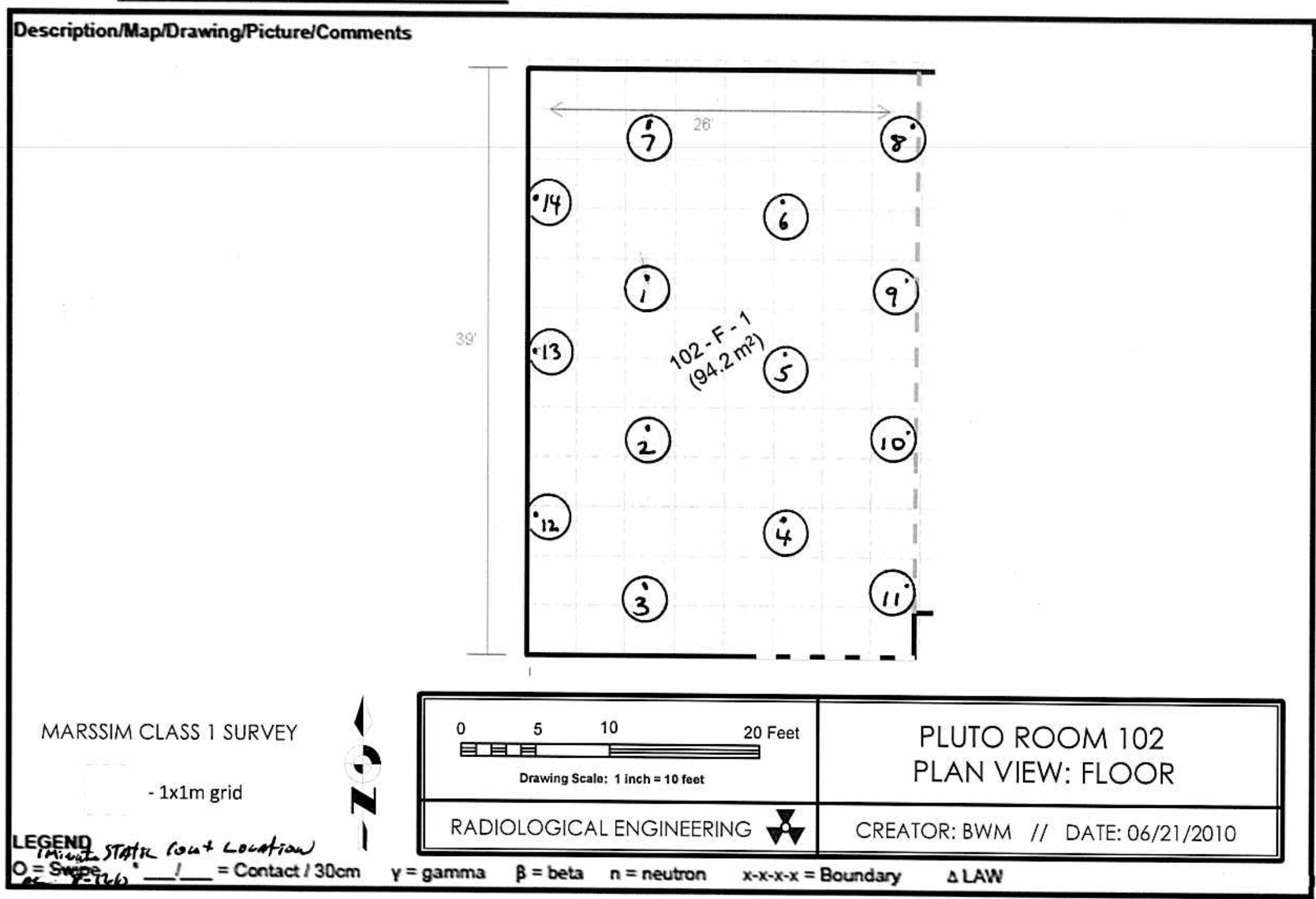


SURVEY \#

10-ER-A26-257

\begin{tabular}{|c|c|c|c|c|c|c|c|c|c|c|c|}
\hline \multicolumn{3}{|c|}{$\begin{array}{l}\text { Location: } \\
\text { Area } 26 \text { CAU117 Pluto }\end{array}$} & \multicolumn{2}{|c|}{$\begin{array}{l}\text { Purpose: } \\
\text { Final Status Survey, Room 102, } \\
\text { Floor / FSS-102-F-2 }\end{array}$} & \multicolumn{5}{|c|}{$\begin{array}{l}\text { Comments: } \\
\text { All one minute static counts are in cpm. Bkgd not subtracted for } \\
\text { Final Status Survey, Survey Plan \#10-015 }\end{array}$} & \multicolumn{2}{|c|}{$\begin{array}{l}\text { Date/Time: } \\
\text { 08/12/2010 } 1100\end{array}$} \\
\hline Instrument: & Serial \#: & Cal Due: & $\begin{array}{c}\text { Eff in \%: } \\
\text { Alpha / Beta }\end{array}$ & $\begin{array}{l}\text { BKG in dpm: } \\
\text { Alpha / Beta }\end{array}$ & \begin{tabular}{|l|} 
MDA in dpm: \\
Alpha / Beta \\
\end{tabular} & RWP\# & \multicolumn{5}{|l|}{$\mathrm{N} / \mathrm{A}$} \\
\hline Electra & 4806 & $4 / 6 / 2011$ & $13.2 / 20.9$ & $N / A$ & $N / A$ & \multicolumn{6}{|c|}{ Work Package \# $\quad$ ENV-10-RCT ENV SITE SURVEYS-0013 } \\
\hline Electra & 6697 & $4 / 6 / 2011$ & $13.8 / 19.9$ & $N / A$ & $\mathrm{~N} / \mathrm{A}$ & \multicolumn{3}{|c|}{ RCT Name: D.Cotroneo Jr. } & \multicolumn{3}{|c|}{ Signature: $0 \sim \infty$} \\
\hline Electra & 1120 & $4 / 6 / 2011$ & $14.1 / 20.8$ & $N / A$ & $N / A$ & \multicolumn{3}{|c|}{ RCT Name: Kevin Wilcox } & \multirow{2}{*}{\multicolumn{3}{|c|}{ Signature: $\mathrm{Ke}_{\mathrm{i}} \mathrm{NQ} \mathrm{Q}$}} \\
\hline Electra & 1201 & $4 / 6 / 2011$ & $14.4 / 20.3$ & $N / A$ & $\mathrm{~N} / \mathrm{A}$ & \multicolumn{3}{|c|}{ RCT Name: S.Brown/B.Bunce } & & & \\
\hline Electra & 1504 & $4 / 6 / 2011$ & $12.9 / 19.8$ & $N / A$ & $N / A$ & \multicolumn{3}{|c|}{ RCT Name: B. Foskett } & Signature: $\sum^{3}$ & & \\
\hline Survey Point & \multirow{2}{*}{\multicolumn{4}{|c|}{ Description/Comments }} & \multicolumn{2}{|c|}{$\begin{array}{l}\text { Removable } \\
\mathrm{dpm} / 100 \mathrm{~cm}^{2}\end{array}$} & \multicolumn{2}{|c|}{\begin{tabular}{|c|}
$\begin{array}{c}\text { Fixed + Removable } \\
\mathrm{dpm} / 100 \mathrm{~cm}^{2}\end{array}$ \\
\end{tabular}} & \multirow{2}{*}{$\begin{array}{l}\text { Gamma } \\
\mathrm{mrem} / \mathrm{hr}\end{array}$} & \multirow{2}{*}{$\begin{array}{l}\text { Neutron } \\
\mathrm{mrem} / \mathrm{hr}\end{array}$} & \multirow{2}{*}{$\begin{array}{l}\text { Total } \\
\mathrm{mrem} / \mathrm{hr}\end{array}$} \\
\hline & & & & & Alpha & Beta & Alpha & Beta & & & \\
\hline 1 & \multicolumn{4}{|c|}{ Floor, Concrete (Electra 6697) } & $N / A$ & $N / A$ & 7 & 1125 & $N / A$ & $N / A$ & $N / A$ \\
\hline 2 & \multicolumn{4}{|c|}{ Floor, Concrete (Electra 6697) } & $N / A$ & $N / A$ & 5 & 1168 & $N / A$ & $N / A$ & $N / A$ \\
\hline 3 & \multicolumn{4}{|c|}{ Floor, Concrete (Electra 6697) } & $N / A$ & $N / A$ & 3 & 931 & $\mathrm{~N} / \mathrm{A}$ & $\mathrm{N} / \mathrm{A}$ & $N / A$ \\
\hline 4 & \multicolumn{4}{|c|}{ Floor, Concrete (Electra 6697) } & $N / A$ & $N / A$ & 2 & 1051 & $N / A$ & $N / A$ & $\mathrm{~N} / \mathrm{A}$ \\
\hline 5 & \multicolumn{4}{|c|}{ Floor, Concrete (Electra 6697) } & $N / A$ & $N / A$ & 7 & 1054 & $N / A$ & $N / A$ & $N / A$ \\
\hline 6 & \multicolumn{4}{|c|}{ Floor, Concrete (Electra 6697) } & $N / A$ & $N / A$ & 2 & 1025 & $\mathrm{~N} / \mathrm{A}$ & $\mathrm{N} / \mathrm{A}$ & $N / A$ \\
\hline 7 & \multicolumn{4}{|c|}{ Floor, Concrete (Electra 6697) } & $N / A$ & $N / A$ & 9 & 1051 & $\mathrm{~N} / \mathrm{A}$ & $N / A$ & $N / A$ \\
\hline 8 & \multicolumn{4}{|c|}{ Floor, Concrete (Electra 6697) } & $N / A$ & $N / A$ & 10 & 1069 & $N / A$ & $\mathrm{~N} / \mathrm{A}$ & $N / A$ \\
\hline 9 & \multicolumn{4}{|c|}{ Floor, Concrete (Electra 6697) } & $N / A$ & $N / A$ & 4 & 1120 & $N / A$ & $N / A$ & $N / A$ \\
\hline 10 & \multicolumn{4}{|c|}{ Floor, Concrete (Electra 6697) } & $N / A$ & $N / A$ & 7 & 1110 & $N / A$ & $N / A$ & $\mathrm{~N} / \mathrm{A}$ \\
\hline 11 & \multicolumn{4}{|c|}{ Floor, Concrete (Electra 6697) } & $N / A$ & $N / A$ & 6 & 986 & $N / A$ & $N / A$ & $N / A$ \\
\hline \multicolumn{5}{|c|}{ Reviewed By (Print): } & Signature & & & & & Date: & \\
\hline
\end{tabular}


SURVEY \# 10-ER-A26-257

\begin{tabular}{|c|c|c|c|c|c|c|c|c|}
\hline \multirow[t]{2}{*}{ Survey Point } & \multirow[t]{2}{*}{ Description/Comments } & \multicolumn{2}{|c|}{$\begin{array}{l}\text { Removable } \\
\mathrm{dpm} / 100 \mathrm{~cm}^{2}\end{array}$} & \multicolumn{2}{|c|}{$\begin{array}{l}\text { Fixed + Removable } \\
\mathrm{dpm} / 100 \mathrm{~cm}^{2}\end{array}$} & \multirow{2}{*}{$\begin{array}{l}\text { Gamma } \\
\mathrm{mrem} / \mathrm{hr}\end{array}$} & \multirow{2}{*}{$\begin{array}{l}\text { Neutron } \\
\mathrm{mrem} / \mathrm{hr}\end{array}$} & \multirow{2}{*}{$\begin{array}{l}\text { Total } \\
\mathrm{mrem} / \mathrm{hr}\end{array}$} \\
\hline & & Alpha & Beta & Alpha & Beta & & & \\
\hline N/A & ${ }^{\star \star}$ Performed scan surveys for both alpha and beta** & N/A & N/A & N/A & N/A & N/A & N/A & N/A \\
\hline N/A & ${ }^{\star *}$ on $100 \%$ of survey unit. No trigger levels exceeded ${ }^{\star *}$ & N/A & N/A & N/A & N/A & N/A & N/A & $\mathrm{N} / \mathrm{A}$ \\
\hline N/A & $\mathrm{N} / \mathrm{A}$ & N/A & $\mathrm{N} / \mathrm{A}$ & N/A & N/A & N/A & N/A & N/A \\
\hline N/A & $\mathrm{N} / \mathrm{A}$ & N/A & N/A & N/A & N/A & N/A & N/A & N/A \\
\hline N/A & $\mathrm{N} / \mathrm{A}$ & N/A & $\mathrm{N} / \mathrm{A}$ & N/A & N/A & N/A & N/A & $\mathrm{N} / \mathrm{A}$ \\
\hline $\mathrm{N} / \mathrm{A}$ & $\mathrm{N} / \mathrm{A}$ & $\mathrm{N} / \mathrm{A}$ & $\mathrm{N} / \mathrm{A}$ & N/A & $\mathrm{N} / \mathrm{A}$ & N/A & N/A & N/A \\
\hline N/A & $\mathrm{N} / \mathrm{A}$ & $\mathrm{N} / \mathrm{A}$ & N/A & N/A & N/A & N/A & $\mathrm{N} / \mathrm{A}$ & $\mathrm{N} / \mathrm{A}$ \\
\hline N/A & $\mathrm{N} / \mathrm{A}$ & $\mathrm{N} / \mathrm{A}$ & N/A & N/A & N/A & N/A & N/A & N/A \\
\hline N/A & $\mathrm{N} / \mathrm{A}$ & N/A & N/A & N/A & N/A & N/A & N/A & $\mathrm{N} / \mathrm{A}$ \\
\hline N/A & $\mathrm{N} / \mathrm{A}$ & $\mathrm{N} / \mathrm{A}$ & N/A & N/A & N/A & N/A & N/A & N/A \\
\hline $\mathrm{N} / \mathrm{A}$ & $\mathrm{N} / \mathrm{A}$ & N/A & N/A & N/A & $\mathrm{N} / \mathrm{A}$ & N/A & N/A & N/A \\
\hline $\mathrm{N} / \mathrm{A}$ & $N / A$ & $\mathrm{~N} / \mathrm{A}$ & N/A & N/A & N/A & N/A & N/A & N/A \\
\hline N/A & $\mathrm{N} / \mathrm{A}$ & $\mathrm{N} / \mathrm{A}$ & N/A & N/A & N/A & N/A & N/A & N/A \\
\hline N/A & $\mathrm{N} / \mathrm{A}$ & N/A & N/A & N/A & N/A & N/A & N/A & N/A \\
\hline N/A & $\mathrm{N} / \mathrm{A}$ & N/A & N/A & N/A & $\mathrm{N} / \mathrm{A}$ & N/A & N/A & N/A \\
\hline N/A & $\mathrm{N} / \mathrm{A}$ & $\mathrm{N} / \mathrm{A}$ & N/A & N/A & $\mathrm{N} / \mathrm{A}$ & N/A & N/A & N/A \\
\hline $\mathrm{N} / \mathrm{A}$ & $\mathrm{N} / \mathrm{A}$ & N/A & N/A & N/A & N/A & N/A & $\mathrm{N} / \mathrm{A}$ & $\mathrm{N} / \mathrm{A}$ \\
\hline $\mathrm{N} / \mathrm{A}$ & $\mathrm{N} / \mathrm{A}$ & N/A & N/A & N/A & N/A & N/A & N/A & N/A \\
\hline $\mathrm{N} / \mathrm{A}$ & $\mathrm{N} / \mathrm{A}$ & $\mathrm{N} / \mathrm{A}$ & N/A & N/A & N/A & N/A & N/A & $\mathrm{N} / \mathrm{A}$ \\
\hline N/A & $N / A$ & N/A & $\mathrm{N} / \mathrm{A}$ & N/A & N/A & N/A & N/A & N/A \\
\hline N/A & $N / A$ & $\mathrm{~N} / \mathrm{A}$ & N/A & N/A & N/A & $\mathrm{N} / \mathrm{A}$ & N/A & $\mathrm{N} / \mathrm{A}$ \\
\hline N/A & $N / A$ & N/A & N/A & N/A & N/A & N/A & N/A & N/A \\
\hline N/A & $N / A$ & N/A & $\mathrm{N} / \mathrm{A}$ & N/A & $\mathrm{N} / \mathrm{A}$ & N/A & N/A & N/A \\
\hline
\end{tabular}


NSTec

Form

$05 / 24 / 10$

FRM-0108C

RADIOLOGICAL SURVEY REPORT - FULL MAP

Rev. 01

Survey $* 10-E R-A 26-257$

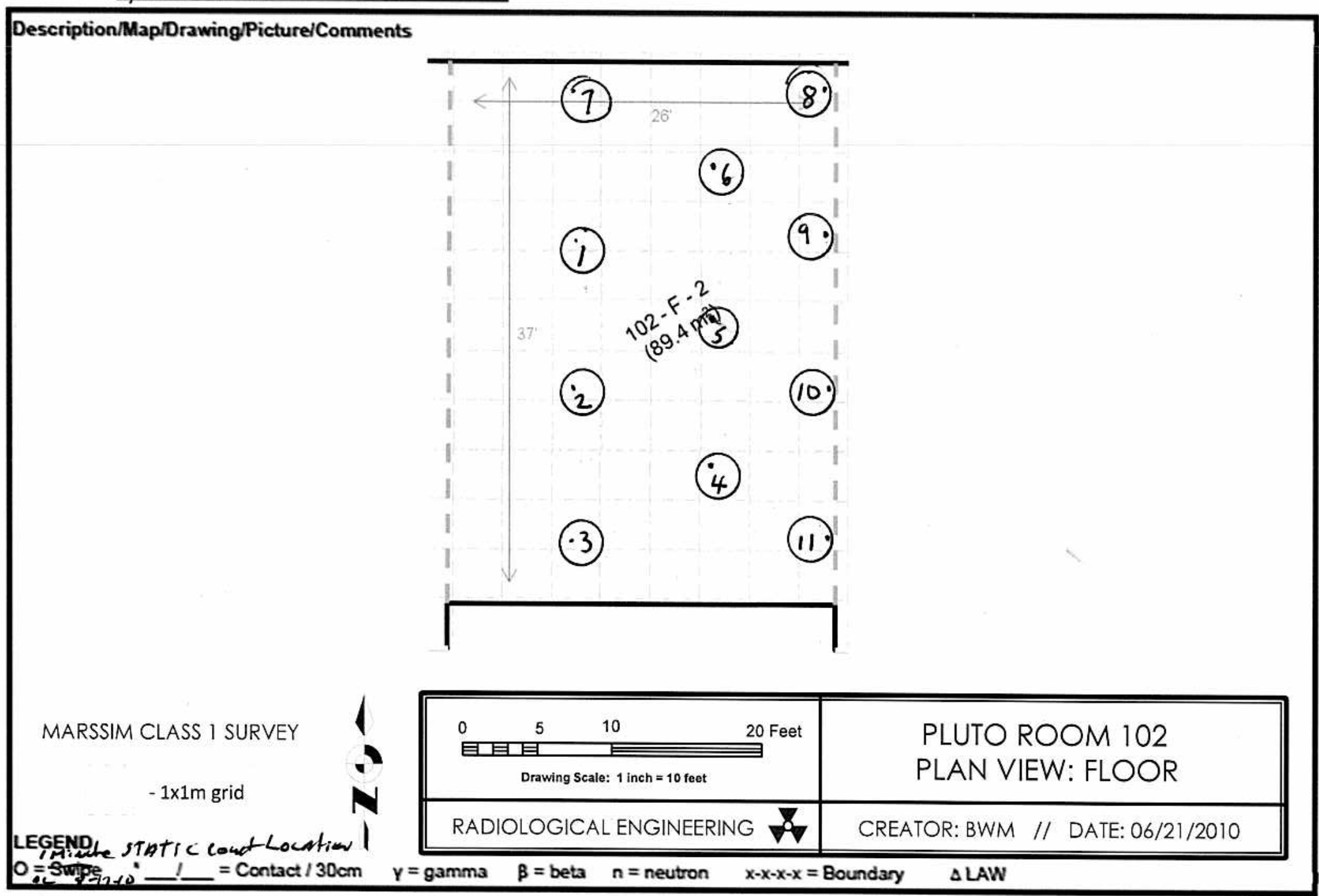


SURVEY \#

10-ER-A26-258

\begin{tabular}{|c|c|c|c|c|c|c|c|c|c|c|c|}
\hline \multicolumn{3}{|c|}{$\begin{array}{l}\text { Location: } \\
\text { Area } 26 \text { CAU117 Pluto }\end{array}$} & \multicolumn{2}{|c|}{$\begin{array}{l}\text { Purpose: } \\
\text { Final Status Survey, Room 102, } \\
\text { Floor / FSS-102-F-3 }\end{array}$} & \multicolumn{5}{|c|}{$\begin{array}{l}\text { Comments: } \\
\text { All one minute static counts are in cpm. Bkgd not subtracted } \\
\text { for Final Status Survey, Survey Plan \#10-015 }\end{array}$} & \multicolumn{2}{|c|}{$\begin{array}{l}\text { Date/Time: } \\
\text { 08/12/2010 } 1300\end{array}$} \\
\hline Instrument: & Serial \#: & Cal Due: & $\begin{array}{c}\text { Eff in \%: } \\
\text { Alpha / Beta }\end{array}$ & $\begin{array}{c}\text { BKG in dpm: } \\
\text { Alpha / Beta }\end{array}$ & $\begin{array}{l}\text { MDA in dpm: } \\
\text { Alpha / Beta } \\
\end{array}$ & RWP\# & \multicolumn{5}{|l|}{$\mathrm{N} / \mathrm{A}$} \\
\hline Electra & 4806 & $4 / 6 / 2011$ & $13.2 / 20.9$ & $N / A$ & $N / A$ & \multicolumn{6}{|c|}{ Work Package \# } \\
\hline Electra & 6697 & $4 / 6 / 2011$ & $13.8 / 19.9$ & $\mathrm{~N} / \mathrm{A}$ & $\mathrm{N} / \mathrm{A}$ & \multicolumn{3}{|c|}{ RCT Name: D.Cotroneo Jr. } & Signature: $Y$ & \multicolumn{2}{|c|}{$-H$} \\
\hline Electra & 1120 & $4 / 6 / 2011$ & $14.1 / 20.8$ & $N / A$ & $\mathrm{~N} / \mathrm{A}$ & \multicolumn{3}{|c|}{ RCT Name: Kevin Wilcox } & \multicolumn{3}{|c|}{ Signature: Ken: $2 . \mathrm{Cco}$} \\
\hline Electra & 1201 & $4 / 6 / 2011$ & $14.4 / 20.3$ & $N / A$ & $N / A$ & \multicolumn{3}{|c|}{ RCT Name: S.Brown/B.Bunce } & \multirow{2}{*}{\multicolumn{3}{|c|}{ Signature:5 5 signature: }} \\
\hline Electra & 1504 & $4 / 6 / 2011$ & $12.9 / 19.8$ & $\mathrm{~N} / \mathrm{A}$ & $\mathrm{N} / \mathrm{A}$ & RCT Name: & B. Foskett & & & & \\
\hline \multirow[t]{2}{*}{ Survey Point } & \multirow{2}{*}{\multicolumn{4}{|c|}{ Description/Comments }} & \multicolumn{2}{|c|}{$\begin{array}{l}\text { Removable } \\
\mathrm{dpm} / 100 \mathrm{~cm}^{2}\end{array}$} & \multicolumn{2}{|c|}{$\begin{array}{c}\text { Fixed + Removable } \\
\mathrm{dpm} / 100 \mathrm{~cm}^{2}\end{array}$} & \multirow{2}{*}{$\begin{array}{l}\text { Gamma } \\
\mathrm{mrem} / \mathrm{hr}\end{array}$} & \multirow{2}{*}{$\begin{array}{l}\text { Neutron } \\
\mathrm{mrem} / \mathrm{hr}\end{array}$} & \multirow{2}{*}{$\begin{array}{c}\text { Total } \\
\mathrm{mrem} / \mathrm{hr}\end{array}$} \\
\hline & & & & & Alpha & Beta & Alpha & Beta & & & \\
\hline 1 & \multicolumn{4}{|c|}{ Floor, Concrete (Electra 6697) } & $N / A$ & $N / A$ & 1 & 1132 & $N / A$ & $N / A$ & $N / A$ \\
\hline 2 & \multicolumn{4}{|c|}{ Floor, Concrete (Electra 6697) } & $N / A$ & $N / A$ & 4 & 1062 & $N / A$ & $N / A$ & $N / A$ \\
\hline 3 & \multicolumn{4}{|c|}{ Floor, Concrete (Electra 6697) } & $N / A$ & $N / A$ & 3 & 968 & $N / A$ & $N / A$ & $N / A$ \\
\hline 4 & \multicolumn{4}{|c|}{ Floor, Concrete (Electra 6697) } & $N / A$ & $N / A$ & 4 & 635 & $\mathrm{~N} / \mathrm{A}$ & $N / A$ & $N / A$ \\
\hline 5 & \multicolumn{4}{|c|}{ Floor, Concrete (Electra 6697) } & $N / A$ & $N / A$ & 4 & 1109 & $N / A$ & $N / A$ & $N / A$ \\
\hline 6 & \multicolumn{4}{|c|}{ Floor, Concrete (Electra 6697) } & $N / A$ & $N / A$ & 13 & 1126 & $N / A$ & $N / A$ & $N / A$ \\
\hline 7 & \multicolumn{4}{|c|}{ Floor, Concrete (Electra 6697) } & $N / A$ & $N / A$ & 11 & 1125 & $\mathrm{~N} / \mathrm{A}$ & $N / A$ & $N / A$ \\
\hline 8 & \multicolumn{4}{|c|}{ Floor, Concrete (Electra 6697) } & $N / A$ & $N / A$ & 6 & 1114 & $N / A$ & $N / A$ & $N / A$ \\
\hline 9 & \multicolumn{4}{|c|}{ Floor, Concrete (Electra 6697) } & $N / A$ & $N / A$ & 10 & 1025 & $N / A$ & $N / A$ & $N / A$ \\
\hline 10 & \multicolumn{4}{|c|}{ Floor, Concrete (Electra 6697) } & $N / A$ & $N / A$ & 2 & 1068 & $\mathrm{~N} / \mathrm{A}$ & $N / A$ & $N / A$ \\
\hline 11 & \multicolumn{4}{|c|}{ Floor, Concrete (Electra 6697) } & $N / A$ & $N / A$ & 4 & 1022 & $N / A$ & $N / A$ & $N / A$ \\
\hline \multicolumn{5}{|c|}{ Reviewed By (Print): } & Signature: & & & & & \multicolumn{2}{|c|}{ Date: $8 / 17 / 10$} \\
\hline
\end{tabular}


SURVEY \# 10-ER-A26-258

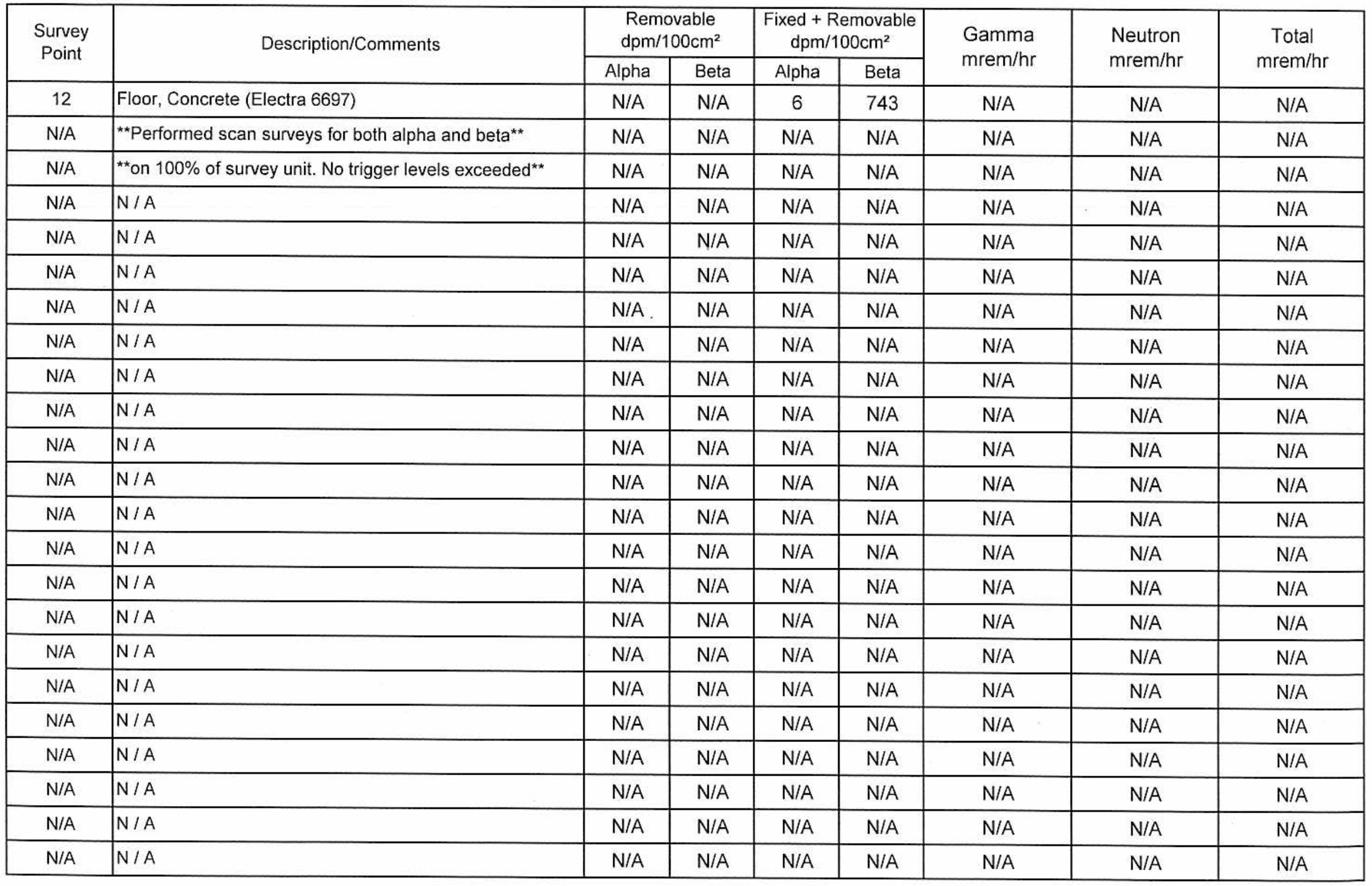


Survey $\#$ 10-ER-A26-258

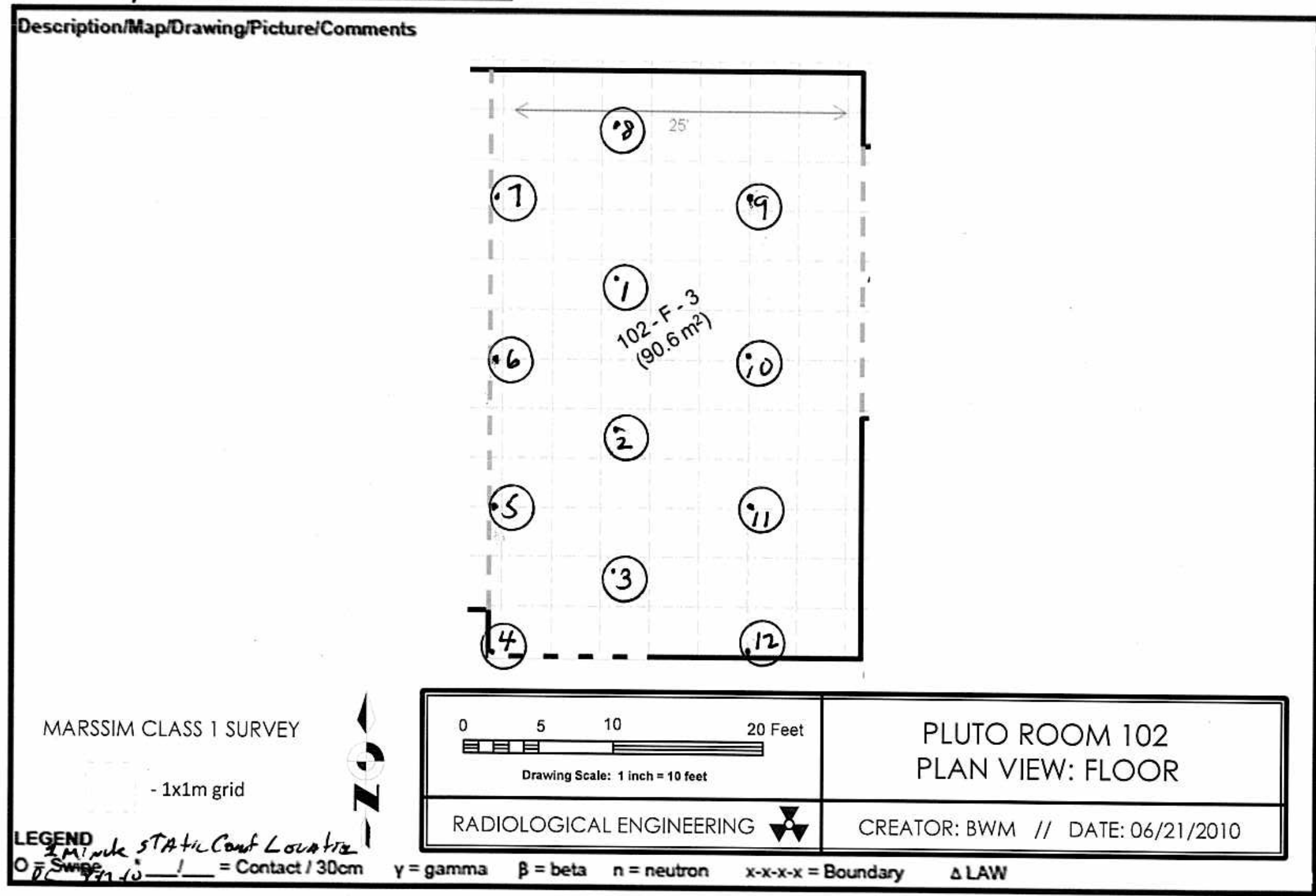


SURVEY\#

10-ER-A26-259

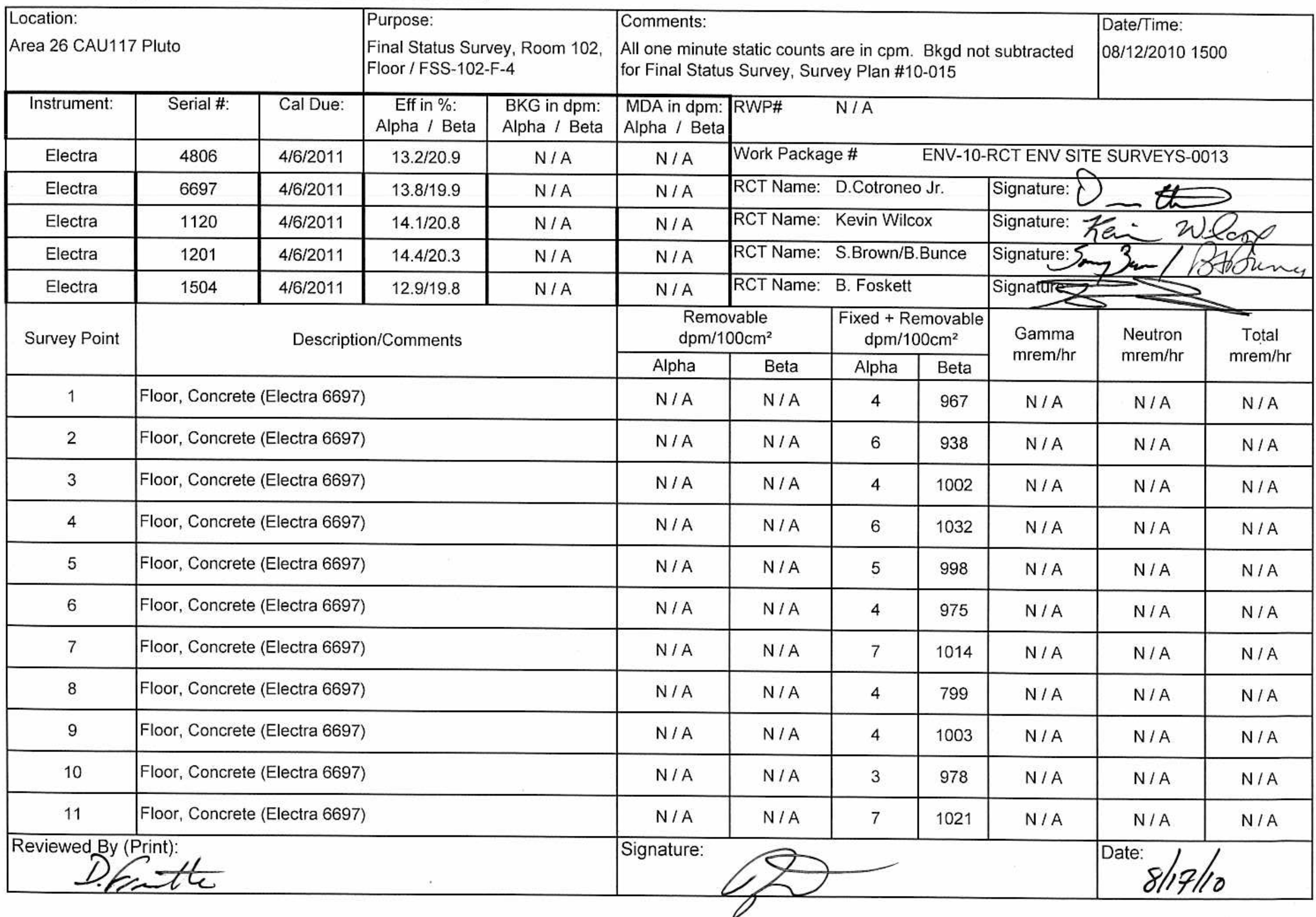


SURVEY \# 10-ER-A26-259

\begin{tabular}{|c|c|c|c|c|c|c|c|c|}
\hline \multirow{2}{*}{$\begin{array}{l}\text { Survey } \\
\text { Point }\end{array}$} & \multirow[t]{2}{*}{ Description/Comments } & \multicolumn{2}{|c|}{$\begin{array}{l}\text { Removable } \\
\mathrm{dpm} / 100 \mathrm{~cm}^{2}\end{array}$} & \multicolumn{2}{|c|}{$\begin{array}{c}\text { Fixed + Removable } \\
\text { dpm } / 100 \mathrm{~cm}^{2}\end{array}$} & \multirow{2}{*}{$\begin{array}{l}\text { Gamma } \\
\text { mrem } / \mathrm{hr}\end{array}$} & \multirow{2}{*}{$\begin{array}{l}\text { Neutron } \\
\text { mrem/hr }\end{array}$} & \multirow{2}{*}{$\begin{array}{c}\text { Total } \\
\text { mrem } / \mathrm{hr}\end{array}$} \\
\hline & & Alpha & Beta & Alpha & Beta & & & \\
\hline 12 & Floor, Concrete (Electra 6697) & N/A & N/A & 9 & 1003 & N/A & N/A & N/A \\
\hline 13 & Floor, Concrete (Electra 6697) & N/A & N/A & 4 & 1003 & N/A & N/A & N/A \\
\hline 14 & Floor, Concrete (Electra 6697) & N/A & N/A & 5 & 1069 & $\mathrm{~N} / \mathrm{A}$ & N/A & $\mathrm{N} / \mathrm{A}$ \\
\hline 15 & Floor, Concrete (Electra 6697) & N/A & N/A & 9 & 1095 & N/A & N/A & N/A \\
\hline N/A & ${ }^{\star \star}$ Performed scan surveys for both alpha and beta** & N/A & N/A & N/A & N/A & N/A & N/A & N/A \\
\hline N/A & ${ }^{\star *}$ on $100 \%$ of survey unit. No trigger levels exceeded ${ }^{\star *}$ & N/A & N/A & N/A & N/A & N/A & N/A & N/A \\
\hline N/A & $N / A$ & N/A & N/A & N/A & N/A & N/A & N/A & N/A \\
\hline N/A & $N / A$ & N/A & N/A & N/A & N/A & N/A & N/A & N/A \\
\hline N/A & $\mathrm{N} / \mathrm{A}$ & N/A & N/A & N/A & N/A & N/A & N/A & N/A \\
\hline N/A & $N / A$ & N/A & N/A & N/A & N/A & N/A & N/A & N/A \\
\hline N/A & $\mathrm{N} / \mathrm{A}$ & N/A & N/A & N/A & N/A & N/A & N/A & N/A \\
\hline N/A & $N / A$ & N/A & N/A & N/A & N/A & N/A & N/A & N/A \\
\hline N/A & $N / A$ & N/A & N/A & N/A & N/A & N/A & N/A & N/A \\
\hline N/A & $N / A$ & N/A & N/A & N/A & N/A & N/A & N/A & N/A \\
\hline N/A & $N / A$ & N/A & N/A & N/A & N/A & N/A & N/A & $\mathrm{N} / \mathrm{A}$ \\
\hline N/A & $N / A$ & N/A & N/A & N/A & N/A & N/A & N/A & N/A \\
\hline N/A & $N / A$ & N/A & N/A & N/A & N/A & N/A & N/A & N/A \\
\hline N/A & $\mathrm{N} / \mathrm{A}$ & N/A & N/A & N/A & N/A & $\mathrm{N} / \mathrm{A}$ & N/A & N/A \\
\hline N/A & $N / A$ & N/A & N/A & N/A & N/A & N/A & N/A & N/A \\
\hline N/A & $N / A$ & N/A & N/A & N/A & N/A & N/A & N/A & N/A \\
\hline N/A & $N / A$ & N/A & N/A & N/A & N/A & N/A & N/A & N/A \\
\hline N/A & $N / A$ & N/A & N/A & N/A & N/A & N/A & N/A & $\mathrm{N} / \mathrm{A}$ \\
\hline N/A & $N / A$ & N/A & N/A & N/A & N/A & N/A & N/A & N/A \\
\hline
\end{tabular}


Survey $\# 10-E R-A 26-259$

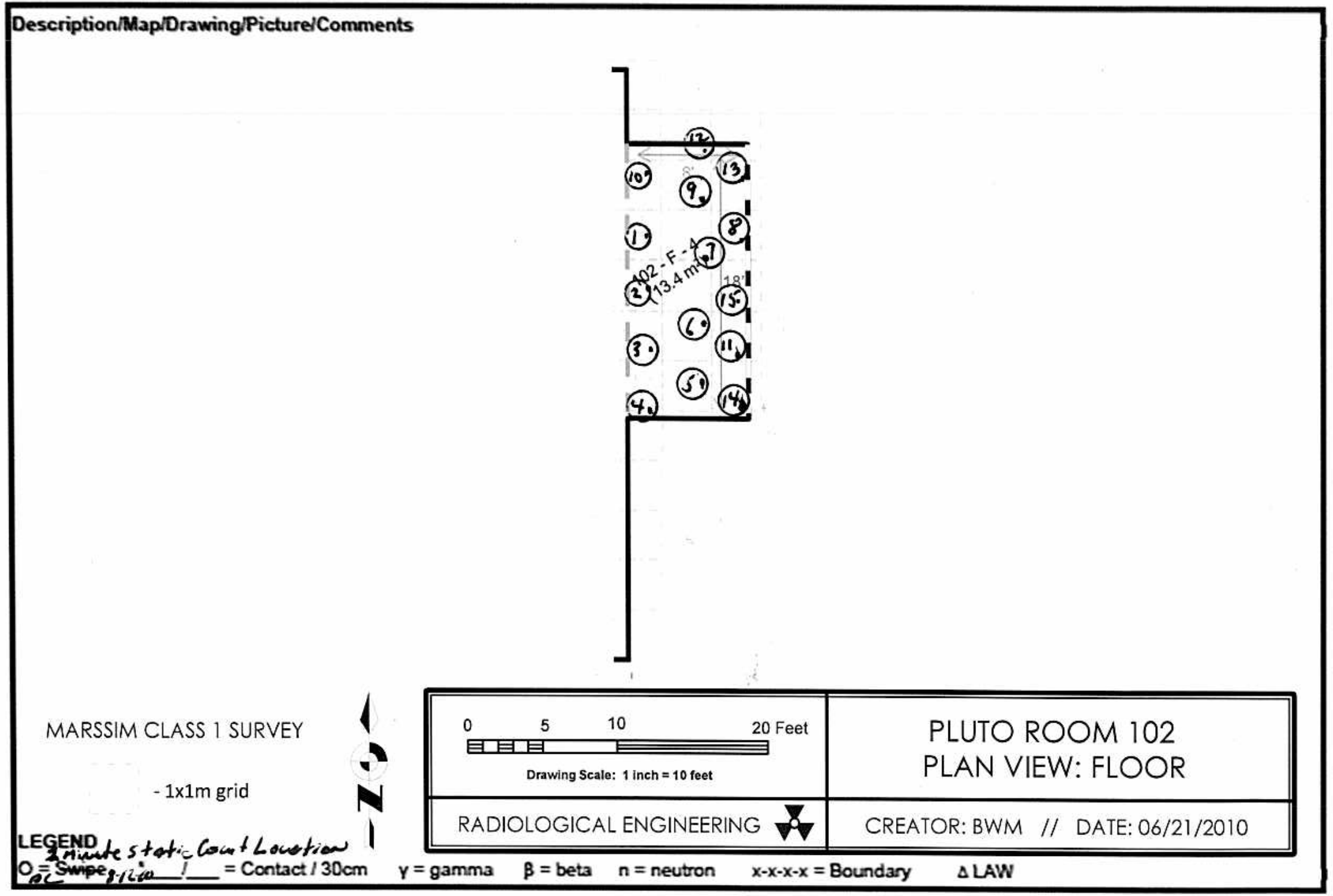


SURVEY \#

10-ER-A26-261

\begin{tabular}{|c|c|c|c|c|c|c|c|c|c|c|c|}
\hline \multicolumn{3}{|c|}{$\begin{array}{l}\text { Location: } \\
\text { Area } 26 \text { CAU117 Pluto }\end{array}$} & \multicolumn{2}{|c|}{$\begin{array}{l}\text { Purpose: } \\
\text { Final Status Survey, Room 102, } \\
\text { North Wall / FSS-102-NW-1 }\end{array}$} & \multicolumn{5}{|c|}{$\begin{array}{l}\text { Comments: } \\
\text { All one minute static counts are in cpm. Bkgd not subtracted } \\
\text { for Final Status Survey, Survey Plan \#10-015 }\end{array}$} & \multicolumn{2}{|c|}{$\begin{array}{l}\text { Date/Time: } \\
\text { 08/13/2010 } 1500\end{array}$} \\
\hline Instrument: & Serial \#: & Cal Due: & $\begin{array}{c}\text { Eff in \%: } \\
\text { Alpha / Beta }\end{array}$ & $\begin{array}{l}\text { BKG in dpm: } \\
\text { Alpha / Beta }\end{array}$ & \begin{tabular}{|l|} 
MDA in dpm: \\
Alpha / Beta \\
\end{tabular} & RWP\# & \multicolumn{5}{|l|}{$\mathrm{N} / \mathrm{A}$} \\
\hline Electra & 1120 & $4 / 6 / 2011$ & $14.1 / 20.8$ & $N / A$ & $N / A$ & \multicolumn{6}{|c|}{ 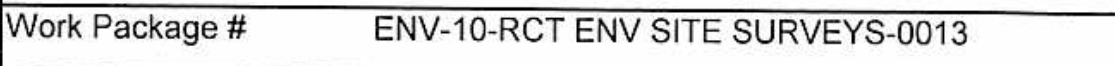 } \\
\hline Electra & 1504 & $4 / 6 / 2011$ & $12.9 / 19.8$ & $N / A$ & $N / A$ & \multicolumn{3}{|c|}{ RCT Name: D.Cotroneo Jr. } & \multicolumn{3}{|c|}{ Signature: $0-d$} \\
\hline Electra & $\mathrm{N} / \mathrm{A}$ & $N / A$ & $\mathrm{~N} / \mathrm{A}$ & $N / A$ & $N / A$ & \multicolumn{3}{|c|}{ RCT Name: Kevin Wilcox } & \multirow{3}{*}{\multicolumn{3}{|c|}{ Signature: $\mathrm{Yen} \mathrm{W}$ : }} \\
\hline Electra & $N / A$ & $N / A$ & $N / A$ & $N / A$ & $\mathrm{~N} / \mathrm{A}$ & RCT Name: & S.Brown/B & Bunce & & & \\
\hline Electra & $N / A$ & $N / A$ & $N / A$ & $N / A$ & $\mathrm{~N} / \mathrm{A}$ & RCT Name: & B. Foskett & & & & \\
\hline \multirow[t]{2}{*}{ Survey Point } & \multirow{2}{*}{\multicolumn{4}{|c|}{ Description/Comments }} & \multicolumn{2}{|c|}{$\begin{array}{l}\text { Removable } \\
\mathrm{dpm} / 100 \mathrm{~cm}^{2}\end{array}$} & \multicolumn{2}{|c|}{$\begin{array}{c}\text { Fixed + Removable } \\
\mathrm{dpm} / 100 \mathrm{~cm}^{2}\end{array}$} & \multirow{2}{*}{$\begin{array}{l}\text { Gamma } \\
\mathrm{mrem} / \mathrm{hr}\end{array}$} & \multirow{2}{*}{$\begin{array}{l}\text { Neutron } \\
\text { mrem } / \mathrm{hr}\end{array}$} & \multirow{2}{*}{$\begin{array}{c}\text { Total } \\
\mathrm{mrem} / \mathrm{hr}\end{array}$} \\
\hline & & & & & Alpha & Beta & Alpha & Beta & & & \\
\hline 1 & \multicolumn{4}{|c|}{ Wall, Concrete (Electra 1120) } & $N / A$ & $N / A$ & 7 & 1233 & $N / A$ & $N / A$ & $N / A$ \\
\hline 2 & \multicolumn{4}{|c|}{ Wall, Concrete (Electra 1120) } & $N / A$ & $N / A$ & 2 & 1059 & $N / A$ & $N / A$ & $N / A$ \\
\hline 3 & \multicolumn{4}{|c|}{ Wall, Concrete (Electra 1120) } & $N / A$ & $\mathrm{~N} / \mathrm{A}$ & 12 & 1024 & $N / A$ & $N / A$ & $\mathrm{~N} / \mathrm{A}$ \\
\hline 4 & \multicolumn{4}{|c|}{ Wall, Concrete (Electra 1120) } & $N / A$ & $N / A$ & 2 & 1025 & $N / A$ & $N / A$ & $N / A$ \\
\hline 5 & \multicolumn{4}{|c|}{ Wall, Concrete (Electra 1120) } & $N / A$ & $N / A$ & 9 & 1158 & $N / A$ & $N / A$ & $N / A$ \\
\hline 6 & \multicolumn{4}{|c|}{ Wall, Concrete (Electra 1120) } & $N / A$ & $N / A$ & 9 & 1180 & $N / A$ & $N / A$ & $N / A$ \\
\hline 7 & \multicolumn{4}{|c|}{ Wall, Concrete (Electra 1120) } & $N / A$ & $N / A$ & 8 & 1064 & $N / A$ & $N / A$ & $N / A$ \\
\hline 8 & \multicolumn{4}{|c|}{ Wall, Concrete (Electra 1120) } & $N / A$ & $N / A$ & 7 & 1054 & $N / A$ & $N / A$ & $N / A$ \\
\hline 9 & \multicolumn{4}{|c|}{ Wall, Concrete (Electra 1120) } & $N / A$ & $N / A$ & 8 & 1020 & $N / A$ & $N / A$ & $N / A$ \\
\hline 10 & \multicolumn{4}{|c|}{ Wall, Concrete (Electra 1120) } & $N / A$ & $N / A$ & 9 & 1048 & $N / A$ & $N / A$ & $N / A$ \\
\hline 11 & \multicolumn{4}{|c|}{ Wall, Concrete (Electra 1120) } & $N / A$ & $N / A$ & 10 & 1068 & $N / A$ & $N / A$ & $\mathrm{~N} / \mathrm{A}$ \\
\hline \multicolumn{5}{|c|}{ Reviewed By (Print): } & Signatur & & & & & \multicolumn{2}{|c|}{ Date: $8 / 17 / 10$} \\
\hline
\end{tabular}


SURVEY \# 10-ER-A26-261

\begin{tabular}{|c|c|c|c|c|c|c|c|c|}
\hline \multirow{2}{*}{$\begin{array}{l}\text { Survey } \\
\text { Point }\end{array}$} & \multirow[t]{2}{*}{ Description/Comments } & \multicolumn{2}{|c|}{$\begin{array}{l}\text { Removable } \\
\text { dpm } / 100 \mathrm{~cm}^{2}\end{array}$} & \multicolumn{2}{|c|}{$\begin{array}{c}\text { Fixed + Removable } \\
\mathrm{dpm} / 100 \mathrm{~cm}^{2}\end{array}$} & \multirow{2}{*}{$\begin{array}{l}\text { Gamma } \\
\mathrm{mrem} / \mathrm{hr}\end{array}$} & \multirow{2}{*}{$\begin{array}{l}\text { Neutron } \\
\mathrm{mrem} / \mathrm{hr}\end{array}$} & \multirow{2}{*}{$\begin{array}{c}\text { Total } \\
\mathrm{mrem} / \mathrm{hr}\end{array}$} \\
\hline & & Alpha & Beta & Alpha & Beta & & & \\
\hline 12 & Wall, Concrete (Electra 1120) & N/A & $\mathrm{N} / \mathrm{A}$ & 10 & 1041 & N/A & N/A & N/A \\
\hline 13 & Wall, Concrete (Electra 1120) & N/A & N/A & 7 & 1100 & N/A & N/A & N/A \\
\hline 14 & Wall, Concrete (Electra 1120) & N/A & $\mathrm{N} / \mathrm{A}$ & 6 & 992 & N/A & N/A & N/A \\
\hline N/A & $\mathrm{N} / \mathrm{A}$ & N/A & $\mathrm{N} / \mathrm{A}$ & N/A & $\mathrm{N} / \mathrm{A}$ & $\mathrm{N} / \mathrm{A}$ & N/A & N/A \\
\hline N/A & ${ }^{\star \star}$ Performed scan surveys for both alpha and beta ${ }^{\star *}$ & N/A & $\mathrm{N} / \mathrm{A}$ & $\mathrm{N} / \mathrm{A}$ & N/A & N/A & N/A & $\mathrm{N} / \mathrm{A}$ \\
\hline N/A & ${ }^{* *}$ on $100 \%$ of survey unit. No trigger levels exceeded ${ }^{\star *}$ & N/A & $\mathrm{N} / \mathrm{A}$ & N/A & N/A & N/A & N/A & N/A \\
\hline N/A & $\mathrm{N} / \mathrm{A}$ & N/A & $\mathrm{N} / \mathrm{A}$ & N/A & N/A & N/A & N/A & $\mathrm{N} / \mathrm{A}$ \\
\hline N/A & $\mathrm{N} / \mathrm{A}$ & N/A & $\mathrm{N} / \mathrm{A}$ & N/A & $\mathrm{N} / \mathrm{A}$ & $\mathrm{N} / \mathrm{A}$ & N/A & N/A \\
\hline N/A & $\mathrm{N} / \mathrm{A}$ & N/A & $\mathrm{N} / \mathrm{A}$ & N/A & N/A & N/A & N/A & N/A \\
\hline $\mathrm{N} / \mathrm{A}$ & $N / A$ & N/A & $\mathrm{N} / \mathrm{A}$ & $\mathrm{N} / \mathrm{A}$ & $\mathrm{N} / \mathrm{A}$ & $\mathrm{N} / \mathrm{A}$ & N/A & N/A \\
\hline N/A & $\mathrm{N} / \mathrm{A}$ & N/A & $\mathrm{N} / \mathrm{A}$ & N/A & $\mathrm{N} / \mathrm{A}$ & $\mathrm{N} / \mathrm{A}$ & $\mathrm{N} / \mathrm{A}$ & $\mathrm{N} / \mathrm{A}$ \\
\hline N/A & $\mathrm{N} / \mathrm{A}$ & $\mathrm{N} / \mathrm{A}$ & $\mathrm{N} / \mathrm{A}$ & $\mathrm{N} / \mathrm{A}$ & $\mathrm{N} / \mathrm{A}$ & $\mathrm{N} / \mathrm{A}$ & N/A & N/A \\
\hline $\mathrm{N} / \mathrm{A}$ & $\mathrm{N} / \mathrm{A}$ & N/A & N/A & $\mathrm{N} / \mathrm{A}$ & $\mathrm{N} / \mathrm{A}$ & $\mathrm{N} / \mathrm{A}$ & $\mathrm{N} / \mathrm{A}$ & N/A \\
\hline N/A & $\mathrm{N} / \mathrm{A}$ & N/A & $\mathrm{N} / \mathrm{A}$ & $\mathrm{N} / \mathrm{A}$ & $\mathrm{N} / \mathrm{A}$ & $\mathrm{N} / \mathrm{A}$ & N/A & N/A \\
\hline N/A & $\mathrm{N} / \mathrm{A}$ & N/A & N/A & $\mathrm{N} / \mathrm{A}$ & $\mathrm{N} / \mathrm{A}$ & $\mathrm{N} / \mathrm{A}$ & N/A & N/A \\
\hline $\mathrm{N} / \mathrm{A}$ & $\mathrm{N} / \mathrm{A}$ & $\mathrm{N} / \mathrm{A}$ & N/A & $\mathrm{N} / \mathrm{A}$ & $\mathrm{N} / \mathrm{A}$ & $\mathrm{N} / \mathrm{A}$ & N/A & N/A \\
\hline N/A & $\mathrm{N} / \mathrm{A}$ & $\mathrm{N} / \mathrm{A}$ & N/A & $\mathrm{N} / \mathrm{A}$ & $\mathrm{N} / \mathrm{A}$ & $\mathrm{N} / \mathrm{A}$ & N/A & N/A \\
\hline N/A & $\mathrm{N} / \mathrm{A}$ & N/A & N/A & N/A & N/A & $\mathrm{N} / \mathrm{A}$ & N/A & $\mathrm{N} / \mathrm{A}$ \\
\hline $\mathrm{N} / \mathrm{A}$ & $N / A$ & $\mathrm{~N} / \mathrm{A}$ & N/A & N/A & N/A & $\mathrm{N} / \mathrm{A}$ & $\mathrm{N} / \mathrm{A}$ & $\mathrm{N} / \mathrm{A}$ \\
\hline $\mathrm{N} / \mathrm{A}$ & $\mathrm{N} / \mathrm{A}$ & N/A & N/A & $\mathrm{N} / \mathrm{A}$ & N/A & N/A & N/A & N/A \\
\hline N/A & $N / A$ & N/A & N/A & N/A & $\mathrm{N} / \mathrm{A}$ & $\mathrm{N} / \mathrm{A}$ & N/A & $\mathrm{N} / \mathrm{A}$ \\
\hline N/A & $\mathrm{N} / \mathrm{A}$ & N/A & N/A & N/A & N/A & $\mathrm{N} / \mathrm{A}$ & N/A & N/A \\
\hline N/A & $N / A$ & N/A & N/A & $\mathrm{N} / \mathrm{A}$ & N/A & N/A & N/A & N/A \\
\hline
\end{tabular}


Survey $10-E R-A 26-261$

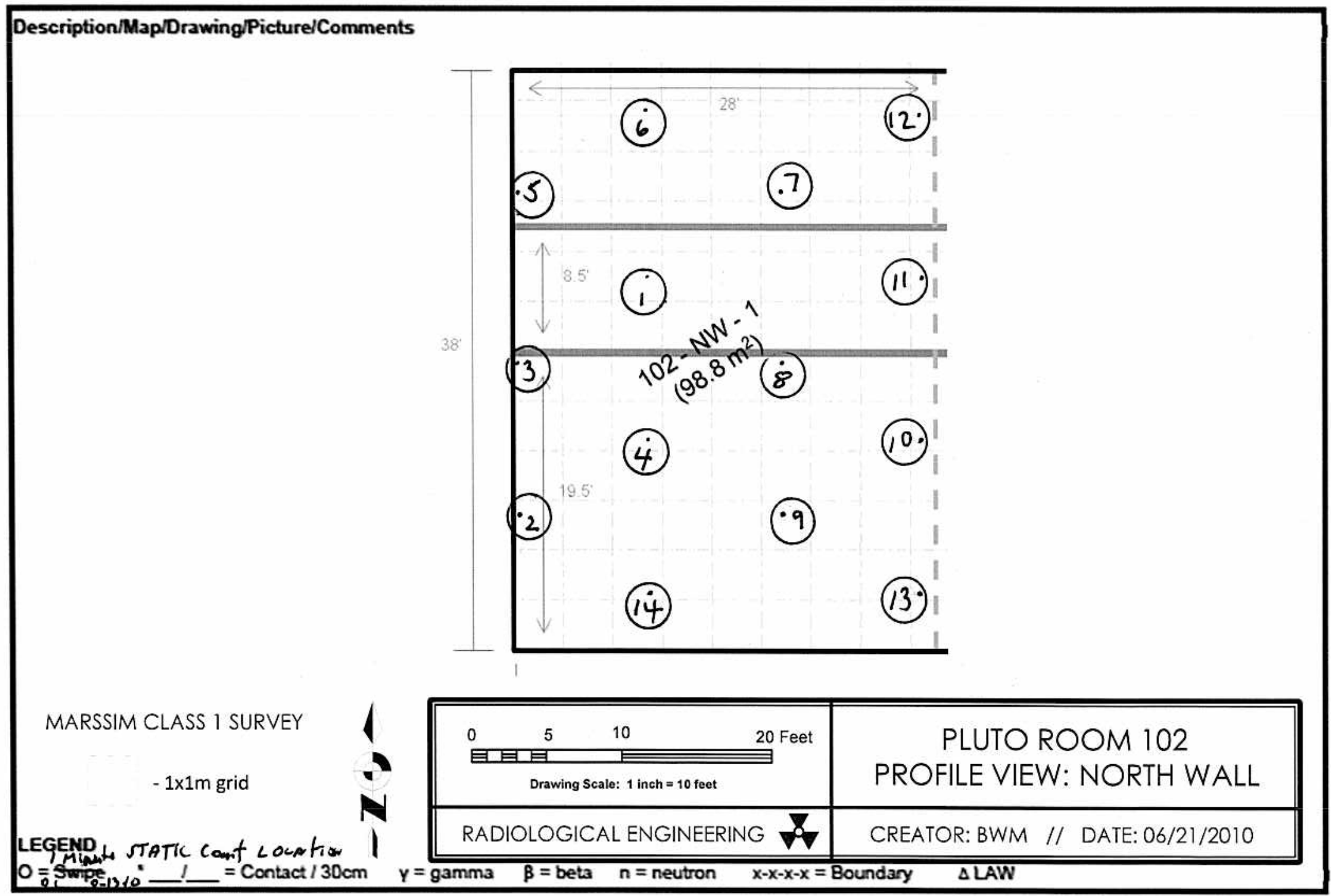


Form

FRM-0108B

\begin{tabular}{|c|c|c|c|c|c|c|c|c|c|c|c|}
\hline \multicolumn{3}{|c|}{$\begin{array}{l}\text { Location: } \\
\text { Area } 26 \text { CAU117 Pluto }\end{array}$} & \multicolumn{2}{|c|}{$\begin{array}{l}\text { Purpose: } \\
\text { Final Status Survey, Room 102, } \\
\text { North Wall / FSS-102-NW-2 }\end{array}$} & \multicolumn{5}{|c|}{$\begin{array}{l}\text { Comments: } \\
\text { All one minute static counts are in cpm. Bkgd not subtracted } \\
\text { for Final Status Survey, Survey Plan \#10-015 }\end{array}$} & \multicolumn{2}{|c|}{$\begin{array}{l}\text { Date/Time: } \\
\text { 08/16/2010 } 0900\end{array}$} \\
\hline Instrument: & Serial \#: & Cal Due: & $\begin{array}{c}\text { Eff in \%: } \\
\text { Alpha / Beta }\end{array}$ & $\begin{array}{l}\text { BKG in dpm: } \\
\text { Alpha / Beta }\end{array}$ & $\begin{array}{l}\text { MDA in dpm: } \\
\text { Alpha / Beta } \\
\end{array}$ & RWP\# & \multicolumn{5}{|l|}{$\mathrm{N} / \mathrm{A}$} \\
\hline Electra & 6697 & $4 / 6 / 2011$ & $13.8 / 19.9$ & $N / A$ & $N / A$ & \multicolumn{6}{|c|}{ ENV-10-RCT ENV SIT } \\
\hline Electra & 1504 & $4 / 6 / 2011$ & $12.9 / 19.8$ & $N / A$ & $N / A$ & \multicolumn{3}{|c|}{ RCT Name: D.Cotroneo Jr. } & \multicolumn{3}{|c|}{ Signature: $0-C$} \\
\hline Electra & $N / A$ & $\mathrm{~N} / \mathrm{A}$ & $N / A$ & $N / A$ & $\mathrm{~N} / \mathrm{A}$ & \multicolumn{3}{|c|}{ RCT Name: K. Wilcox/M.Payne } & \multirow{3}{*}{\multicolumn{3}{|c|}{ 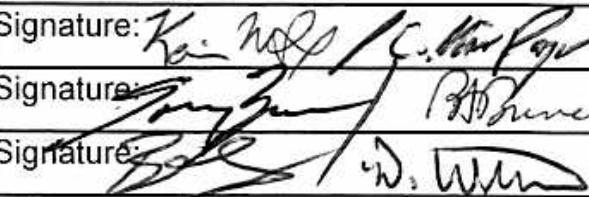 }} \\
\hline Electra & $N / A$ & $N / A$ & $\mathrm{~N} / \mathrm{A}$ & $N / A$ & $N / A$ & RCT Name: & S.Brown/t & Bunce & & & \\
\hline Electra & $N / A$ & $N / A$ & $N / A$ & $N / A$ & $N / A$ & RCT Name: & B. Fosket & Velch & & & \\
\hline \multirow[t]{2}{*}{ Survey Point } & \multirow{2}{*}{\multicolumn{4}{|c|}{ Description/Comments }} & \multicolumn{2}{|c|}{$\begin{array}{l}\text { Removable } \\
\mathrm{dpm} / 100 \mathrm{~cm}^{2}\end{array}$} & \multicolumn{2}{|c|}{\begin{tabular}{|c|}
$\begin{array}{c}\text { Fixed + Removable } \\
\mathrm{dpm} / 100 \mathrm{~cm}^{2}\end{array}$ \\
\end{tabular}} & \multirow{2}{*}{$\begin{array}{l}\text { Gamma } \\
\mathrm{mrem} / \mathrm{hr}\end{array}$} & \multirow{2}{*}{$\begin{array}{l}\text { Neutron } \\
\mathrm{mrem} / \mathrm{hr}\end{array}$} & \multirow{2}{*}{$\begin{array}{c}\text { Total } \\
\mathrm{mrem} / \mathrm{hr}\end{array}$} \\
\hline & & & & & Alpha & Beta & Alpha & Beta & & & \\
\hline 1 & \multicolumn{4}{|c|}{ Wall, Concrete (Electra 1504) } & $N / A$ & $N / A$ & 9 & 1027 & $N / A$ & $\mathrm{~N} / \mathrm{A}$ & $N / A$ \\
\hline 2 & \multicolumn{4}{|c|}{ Wall, Concrete (Electra 1504) } & $N / A$ & $N / A$ & 12 & 1271 & $N / A$ & $N / A$ & $N / A$ \\
\hline 3 & \multicolumn{4}{|c|}{ Wall, Concrete (Electra 1504) } & $N / A$ & $N / A$ & 8 & 1282 & $N / A$ & $N / A$ & $\mathrm{~N} / \mathrm{A}$ \\
\hline 4 & \multicolumn{4}{|c|}{ Wall, Concrete (Electra 1504) } & $N / A$ & $N / A$ & 12 & 1053 & $N / A$ & $N / A$ & $N / A$ \\
\hline 5 & \multicolumn{4}{|c|}{ Wall, Concrete (Electra 1504) } & $N / A$ & $N / A$ & 10 & 993 & $N / A$ & $N / A$ & $N / A$ \\
\hline 6 & \multicolumn{4}{|c|}{ Wall, Concrete (Electra 1504) } & $N / A$ & $N / A$ & 11 & 1081 & $N / A$ & $N / A$ & $N / A$ \\
\hline 7 & \multicolumn{4}{|c|}{ Wall, Concrete (Electra 1504) } & $N / A$ & $N / A$ & 11 & 1011 & $N / A$ & $N / A$ & $N / A$ \\
\hline 8 & \multicolumn{4}{|c|}{ Wall, Concrete (Electra 1504) } & $N / A$ & $N / A$ & 9 & 1022 & $N / A$ & $N / A$ & $N / A$ \\
\hline 9 & \multicolumn{4}{|c|}{ Wall, Concrete (Electra 1504) } & $N / A$ & $N / A$ & 13 & 1069 & $N / A$ & $N / A$ & $N / A$ \\
\hline 10 & \multicolumn{4}{|c|}{ Wall, Concrete (Electra 1504) } & $N / A$ & $N / A$ & 18 & 1013 & $N / A$ & $N / A$ & $N / A$ \\
\hline 11 & \multicolumn{4}{|c|}{ Wall, Concrete (Electra 1504) } & $N / A$ & $N / A$ & 11 & 1049 & $N / A$ & $N / A$ & $N / A$ \\
\hline \multicolumn{5}{|c|}{ Reviewed By (Print)i } & & & & & & & \\
\hline
\end{tabular}


SURVEY \# 10-ER-A26-263

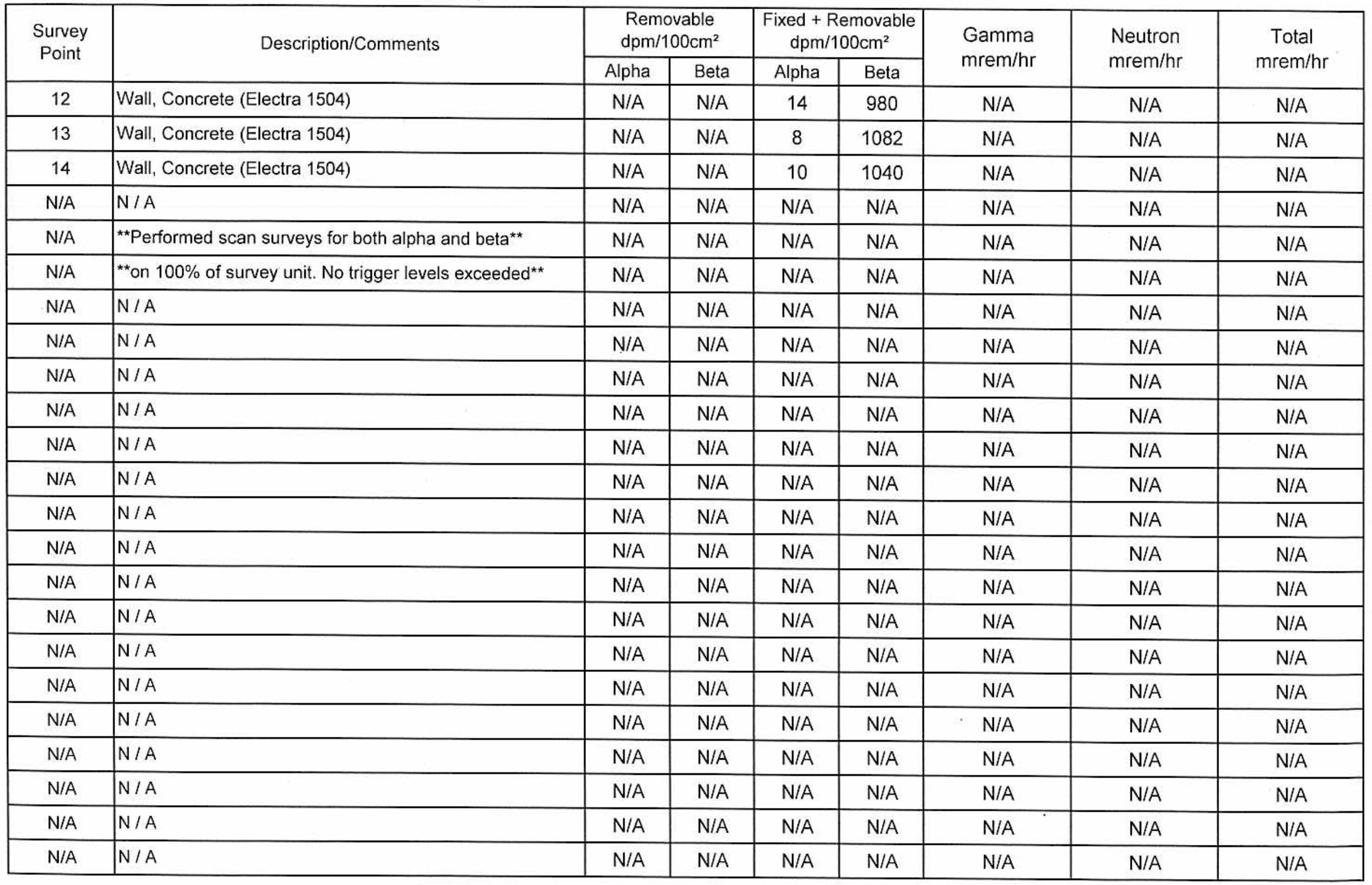


NSTec

Fom

RADIOLOGICAL SURVEY REPORT - FULL MAP

Survey $10-[D R-A 26-263$

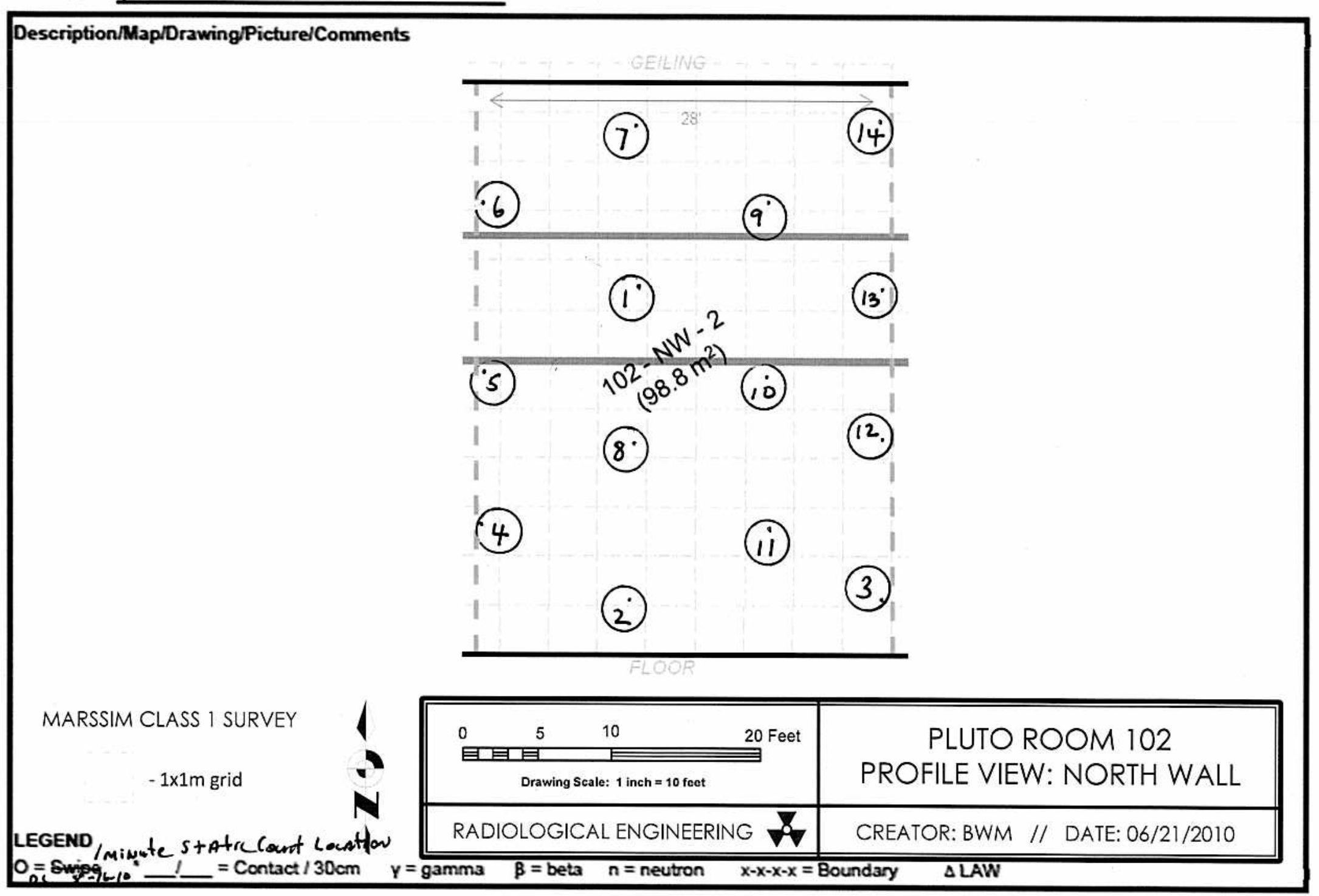


SURVEY \#

10-ER-A26-262

\begin{tabular}{|c|c|c|c|c|c|c|c|c|c|c|c|}
\hline \multicolumn{3}{|c|}{$\begin{array}{l}\text { Location: } \\
\text { Area } 26 \text { CAU117 Pluto }\end{array}$} & \multicolumn{2}{|c|}{$\begin{array}{l}\text { Purpose: } \\
\text { Final Status Survey, Room 102, } \\
\text { North Wall / FSS-102-NW-3 }\end{array}$} & \multicolumn{5}{|c|}{$\begin{array}{l}\text { Comments: } \\
\text { All one minute static counts are in cpm. Bkgd not subtracted } \\
\text { for Final Status Survey, Survey Plan \#10-015 }\end{array}$} & \multicolumn{2}{|c|}{$\begin{array}{l}\text { Date/Time: } \\
\text { 08/13/2010 } 1400\end{array}$} \\
\hline Instrument: & Serial \#: & Cal Due: & $\begin{array}{c}\text { Eff in \%: } \\
\text { Alpha / Beta }\end{array}$ & $\begin{array}{l}\text { BKG in dpm: } \\
\text { Alpha / Beta }\end{array}$ & $\begin{array}{l}\text { MDA in dpm: } \\
\text { Alpha / Beta }\end{array}$ & RWP\# & \multicolumn{5}{|l|}{$\mathrm{N} / \mathrm{A}$} \\
\hline Electra & 1120 & $4 / 6 / 2011$ & $14.1 / 20.8$ & $N / A$ & $\mathrm{~N} / \mathrm{A}$ & \multicolumn{6}{|c|}{ ENV-10-RCT ENV SIT } \\
\hline Electra & 1504 & $4 / 6 / 2011$ & $12.9 / 19.8$ & $N / A$ & $N / A$ & \multicolumn{3}{|c|}{ RCT Name: D.Cotroneo Jr. } & \multicolumn{3}{|c|}{ Signature: $0-x$} \\
\hline Electra & $N / A$ & $N / A$ & $\mathrm{~N} / \mathrm{A}$ & $N / A$ & $N / A$ & \multicolumn{3}{|c|}{ RCT Name: Kevin Wilcox } & \multicolumn{3}{|c|}{ signature: he. hano } \\
\hline Electra & $\mathrm{N} / \mathrm{A}$ & $N / A$ & $\mathrm{~N} / \mathrm{A}$ & $N / A$ & $N / A$ & \multicolumn{3}{|c|}{ RCT Name: S.Brown/B.Bunce } & \multicolumn{3}{|c|}{ Signatering 3} \\
\hline Electra & $N / A$ & $N / A$ & $N / A$ & $N / A$ & $N / A$ & \multicolumn{3}{|c|}{ RCT Name: B. Foskett } & Signature: 7 & 21 & \\
\hline Survey Point & \multirow{2}{*}{\multicolumn{4}{|c|}{ Description/Comments }} & \multicolumn{2}{|c|}{$\begin{array}{l}\text { Removable } \\
\mathrm{dpm} / 100 \mathrm{~cm}^{2}\end{array}$} & \multicolumn{2}{|c|}{$\begin{array}{c}\text { Fixed + Removable } \\
\mathrm{dpm} / 100 \mathrm{~cm}^{2}\end{array}$} & \multirow{2}{*}{$\begin{array}{l}\text { Gamma } \\
\mathrm{mrem} / \mathrm{hr}\end{array}$} & \multirow{2}{*}{$\begin{array}{l}\text { Neutron } \\
\mathrm{mrem} / \mathrm{hr}\end{array}$} & \multirow{2}{*}{$\begin{array}{c}\text { Total } \\
\mathrm{mrem} / \mathrm{hr}\end{array}$} \\
\hline & & & & & Alpha & Beta & Alpha & Beta & & & \\
\hline 1 & \multicolumn{4}{|c|}{ Wall, Concrete (Electra 1504) } & $N / A$ & $N / A$ & 10 & 1082 & $N / A$ & N/A & $N / A$ \\
\hline 2 & \multicolumn{4}{|c|}{ Wall, Concrete (Electra 1504) } & $N / A$ & $N / A$ & 12 & 1086 & $N / A$ & $N / A$ & $\mathrm{~N} / \mathrm{A}$ \\
\hline 3 & \multicolumn{4}{|c|}{ Wall, Concrete (Electra 1504) } & $N / A$ & $N / A$ & 13 & 953 & $N / A$ & $N / A$ & $\mathrm{~N} / \mathrm{A}$ \\
\hline 4 & \multicolumn{4}{|c|}{ Wall, Concrete (Electra 1504) } & $N / A$ & $N / A$ & 8 & 973 & $N / A$ & $N / A$ & $N / A$ \\
\hline 5 & \multicolumn{4}{|c|}{ Wall, Concrete (Electra 1504) } & $N / A$ & $N / A$ & 8 & 1039 & $N / A$ & $N / A$ & $N / A$ \\
\hline 6 & \multicolumn{4}{|c|}{ Wall, Concrete (Electra 1504) } & $N / A$ & $N / A$ & 5 & 1062 & $N / A$ & $\mathrm{~N} / \mathrm{A}$ & $\mathrm{N} / \mathrm{A}$ \\
\hline 7 & \multicolumn{4}{|c|}{ Wall, Concrete (Electra 1504) } & $N / A$ & $N / A$ & 4 & 1078 & $N / A$ & $N / A$ & $N / A$ \\
\hline 8 & \multicolumn{4}{|c|}{ Wall, Concrete (Electra 1504) } & $N / A$ & $N / A$ & 8 & 1143 & $N / A$ & $N / A$ & $N / A$ \\
\hline 9 & \multicolumn{4}{|c|}{ Wall, Concrete (Electra 1504) } & $N / A$ & $N / A$ & 7 & 1049 & $N / A$ & $N / A$ & $N / A$ \\
\hline 10 & \multicolumn{4}{|c|}{ Wall, Concrete (Electra 1504) } & $N / A$ & $N / A$ & 10 & 1020 & $N / A$ & $N / A$ & $N / A$ \\
\hline 11 & \multicolumn{4}{|c|}{ Wall, Concrete (Electra 1504) } & $N / A$ & $N / A$ & 11 & 941 & $N / A$ & $N / A$ & $N / A$ \\
\hline \multicolumn{5}{|c|}{ Reviewed By (Print) } & \multicolumn{3}{|l|}{ Signature: } & & & \multicolumn{2}{|c|}{ Date: $8 / 17 / 10$} \\
\hline
\end{tabular}


SURVEY \# 10-ER-A26-262

\begin{tabular}{|c|c|c|c|c|c|c|c|c|}
\hline \multirow[t]{2}{*}{$\begin{array}{l}\text { Survey } \\
\text { Point }\end{array}$} & \multirow[t]{2}{*}{ Description/Comments } & \multicolumn{2}{|c|}{$\begin{array}{l}\text { Removable } \\
\mathrm{dpm} / 100 \mathrm{~cm}^{2}\end{array}$} & \multicolumn{2}{|c|}{$\begin{array}{c}\text { Fixed + Removable } \\
\mathrm{dpm} / 100 \mathrm{~cm}^{2}\end{array}$} & \multirow{2}{*}{$\begin{array}{l}\text { Gamma } \\
\text { mrem/hr }\end{array}$} & \multirow{2}{*}{$\begin{array}{l}\text { Neutron } \\
\text { mrem/hr }\end{array}$} & \multirow{2}{*}{$\begin{array}{c}\text { Total } \\
\text { mrem/hr }\end{array}$} \\
\hline & & Alpha & Beta & Alpha & Beta & & & \\
\hline 12 & Wall, Concrete (Electra 1120) & N/A & $\mathrm{N} / \mathrm{A}$ & 13 & 941 & N/A & N/A & N/A \\
\hline 13 & Wall, Wood (Electra 1120) & N/A & $N / A$ & 11 & 701 & N/A & N/A & N/A \\
\hline N/A & $N / A$ & N/A & N/A & N/A & N/A & N/A & N/A & N/A \\
\hline N/A & $N / A$ & N/A & $\mathrm{N} / \mathrm{A}$ & N/A & N/A & N/A & N/A & N/A \\
\hline N/A & ${ }^{\star \star}$ Performed scan surveys for both alpha and beta** & N/A & $\mathrm{N} / \mathrm{A}$ & N/A & $\mathrm{N} / \mathrm{A}$ & N/A & N/A & N/A \\
\hline $\mathrm{N} / \mathrm{A}$ & ${ }^{* *}$ on $100 \%$ of survey unit. No trigger levels exceeded ${ }^{\star \star}$ & N/A & N/A & N/A & N/A & N/A & N/A & N/A \\
\hline N/A & $N / A$ & N/A & N/A & N/A & $\mathrm{N} / \mathrm{A}$ & N/A & N/A & N/A \\
\hline N/A & $N / A$ & N/A & N/A & N/A & $\mathrm{N} / \mathrm{A}$ & N/A & N/A & N/A \\
\hline N/A & $N / A$ & N/A & N/A & N/A & N/A & N/A & N/A & N/A \\
\hline N/A & $N / A$ & N/A & N/A & N/A & N/A & N/A & N/A & N/A \\
\hline N/A & $N / A$ & N/A & $\mathrm{N} / \mathrm{A}$ & N/A & $\mathrm{N} / \mathrm{A}$ & N/A & $N / A$ & N/A \\
\hline $\mathrm{N} / \mathrm{A}$ & $N / A$ & N/A & $\mathrm{N} / \mathrm{A}$ & N/A & $\mathrm{N} / \mathrm{A}$ & N/A & N/A & N/A \\
\hline N/A & $N / A$ & N/A & N/A & N/A & N/A & N/A & N/A & N/A \\
\hline N/A & $N / A$ & N/A & N/A & N/A & N/A & N/A & N/A & N/A \\
\hline N/A & $N / A$ & N/A & $\mathrm{N} / \mathrm{A}$ & N/A & $\mathrm{N} / \mathrm{A}$ & N/A & N/A & N/A \\
\hline N/A & $N / A$ & N/A & $\mathrm{N} / \mathrm{A}$ & N/A & $\mathrm{N} / \mathrm{A}$ & N/A & N/A & N/A \\
\hline N/A & $N / A$ & N/A & N/A & N/A & $\mathrm{N} / \mathrm{A}$ & N/A & N/A & N/A \\
\hline N/A & $N / A$ & N/A & N/A & N/A & $\mathrm{N} / \mathrm{A}$ & N/A & N/A & N/A \\
\hline N/A & $N / A$ & N/A & N/A & N/A & N/A & N/A & N/A & N/A \\
\hline N/A & $N / A$ & N/A & N/A & N/A & $\mathrm{N} / \mathrm{A}$ & N/A & N/A & N/A \\
\hline N/A & $\mathrm{N} / \mathrm{A}$ & N/A & $\mathrm{N} / \mathrm{A}$ & N/A & $\mathrm{N} / \mathrm{A}$ & N/A & N/A & N/A \\
\hline N/A & $N / A$ & N/A & $\mathrm{N} / \mathrm{A}$ & N/A & N/A & N/A & $N / A$ & N/A \\
\hline N/A & $N / A$ & N/A & $\mathrm{N} / \mathrm{A}$ & N/A & $\mathrm{N} / \mathrm{A}$ & N/A & N/A & N/A \\
\hline
\end{tabular}


NSTec

Form

05/24/10

FRM-0108C

RADIOLOGICAL SURVEY REPORT - FULL MAP

Rev. 01

Survey $=10-E R \cdot A 26-262$

Description/Map/Drawing/Picture/Comments

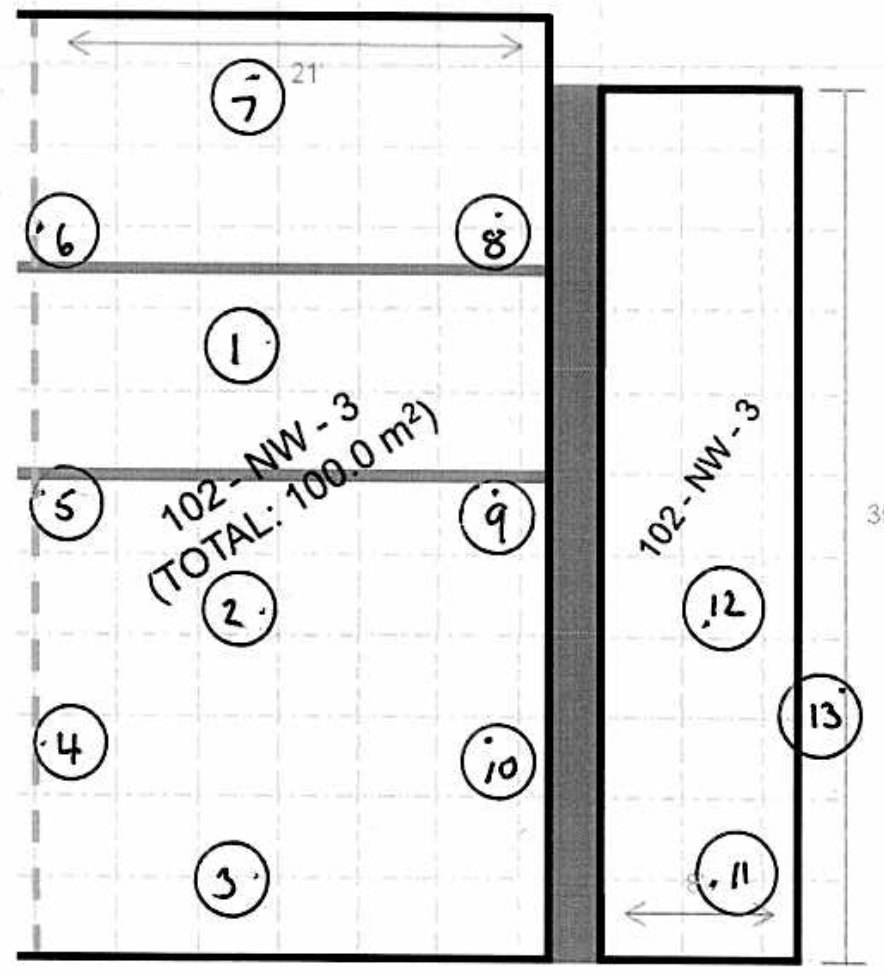

MARSSIM CLASS I SURVEY

$-1 \times 1 m$ grid

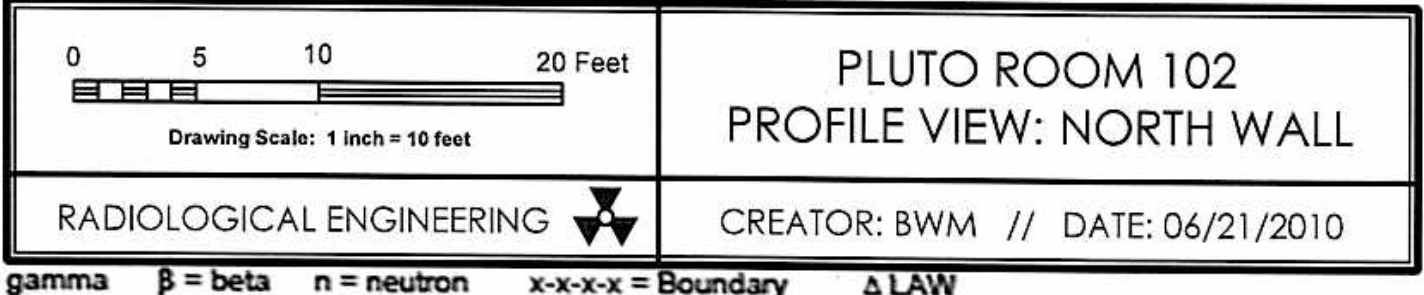

LEGEND

Minute statrc count Loustron I

$8=0$

$y=$ gamma

$\beta=$ beta $n=$ neutron

$x-x-x-x=$ Boundary

$\triangle$ LAW 
SURVEY \# 10-ER-A26-285

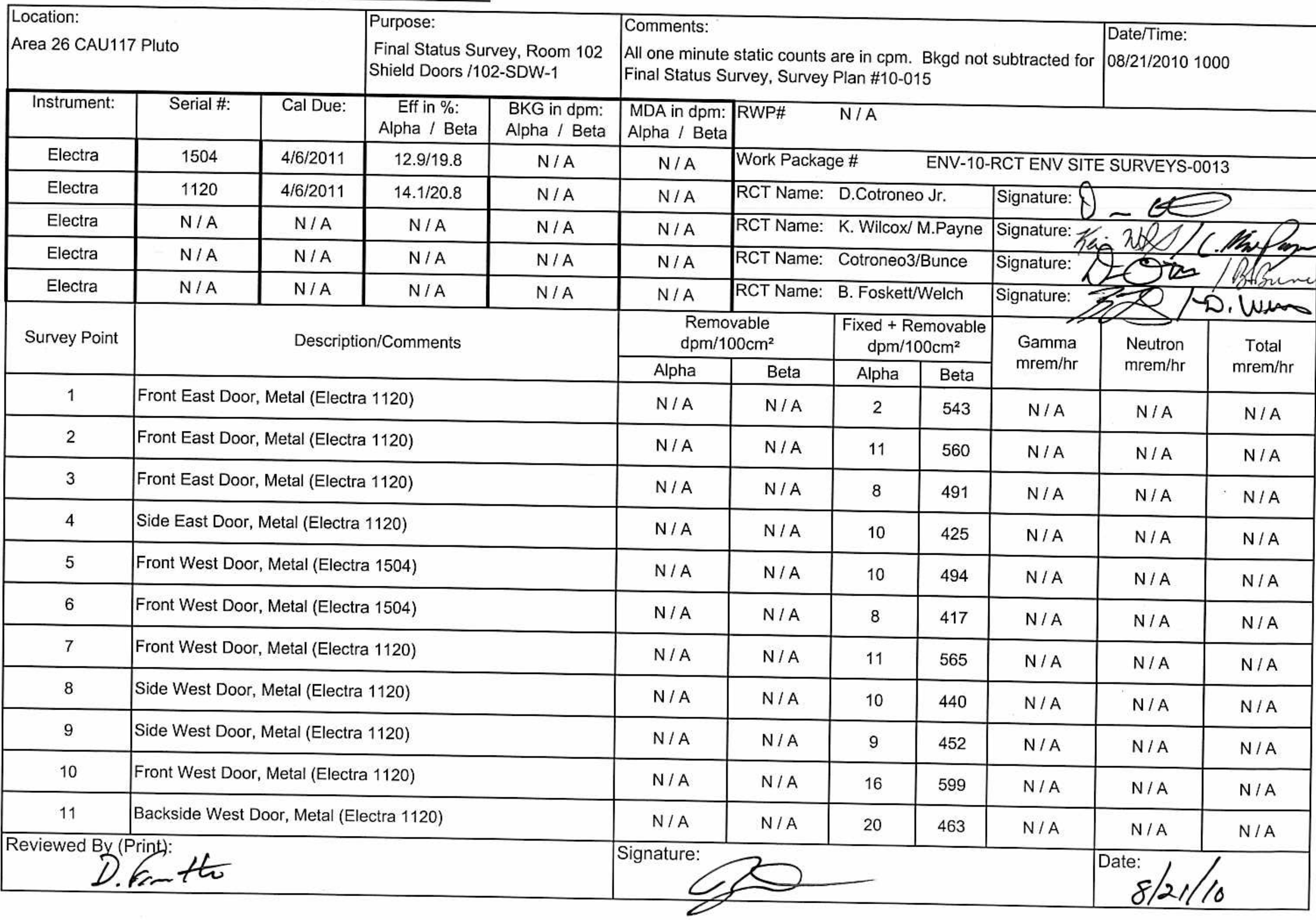


SURVEY \# 10-ER-A26-285

\begin{tabular}{|c|c|c|c|c|c|c|c|c|}
\hline \multirow[t]{2}{*}{ Survey Point } & \multirow[t]{2}{*}{ Description/Comments } & \multicolumn{2}{|c|}{$\begin{array}{l}\text { Removable } \\
\mathrm{dpm} / 100 \mathrm{~cm}^{2}\end{array}$} & \multicolumn{2}{|c|}{$\begin{array}{c}\text { Fixed + Removable } \\
\mathrm{dpm} / 100 \mathrm{~cm}^{2}\end{array}$} & \multirow{2}{*}{$\begin{array}{l}\text { Gamma } \\
\mathrm{mrem} / \mathrm{hr}\end{array}$} & \multirow{2}{*}{$\begin{array}{l}\text { Neutron } \\
\mathrm{mrem} / \mathrm{hr}\end{array}$} & \multirow{2}{*}{$\begin{array}{c}\text { Total } \\
\mathrm{mrem} / \mathrm{hr}\end{array}$} \\
\hline & & Alpha & Beta & Alpha & Beta & & & \\
\hline 12 & Backside East Door, Metal (1120) & N/A & $\mathrm{N} / \mathrm{A}$ & 17 & 444 & N/A & N/A & N/A \\
\hline N/A & $\mathrm{N} / \mathrm{A}$ & N/A & $\mathrm{N} / \mathrm{A}$ & N/A & $\mathrm{N} / \mathrm{A}$ & N/A & N/A & N/A \\
\hline N/A & ${ }^{\star *}$ Performed scan surveys for both alpha and beta** & N/A & $\mathrm{N} / \mathrm{A}$ & N/A & N/A & N/A & N/A & N/A \\
\hline N/A & ${ }^{* *}$ on $100 \%$ of survey unit. No trigger levels exceeded ${ }^{\star *}$ & N/A & N/A & N/A & N/A & N/A & N/A & $\mathrm{N} / \mathrm{A}$ \\
\hline N/A & $N / A$ & N/A & N/A & $\mathrm{N} / \mathrm{A}$ & N/A & N/A & N/A & N/A \\
\hline N/A & $\mathrm{N} / \mathrm{A}$ & N/A & N/A & $\mathrm{N} / \mathrm{A}$ & $\mathrm{N} / \mathrm{A}$ & N/A & N/A & N/A \\
\hline N/A & $\mathrm{N} / \mathrm{A}$ & N/A & N/A & $\mathrm{N} / \mathrm{A}$ & N/A & N/A & N/A & N/A \\
\hline$N / A$ & $\mathrm{~N} / \mathrm{A}$ & N/A & $\mathrm{N} / \mathrm{A}$ & $\mathrm{N} / \mathrm{A}$ & N/A & N/A & N/A & N/A \\
\hline N/A & $\mathrm{N} / \mathrm{A}$ & N/A & $\mathrm{N} / \mathrm{A}$ & $\mathrm{N} / \mathrm{A}$ & N/A & $\mathrm{N} / \mathrm{A}$ & N/A & N/A \\
\hline N/A & $\mathrm{N} / \mathrm{A}$ & N/A & $\mathrm{N} / \mathrm{A}$ & N/A & N/A & N/A & N/A & N/A \\
\hline N/A & $\mathrm{N} / \mathrm{A}$ & N/A & N/A & N/A & N/A & N/A & N/A & N/A \\
\hline N/A & $\mathrm{N} / \mathrm{A}$ & N/A & N/A & N/A & $\mathrm{N} / \mathrm{A}$ & $\mathrm{N} / \mathrm{A}$ & N/A & N/A \\
\hline $\mathrm{N} / \mathrm{A}$ & $\mathrm{N} / \mathrm{A}$ & N/A & N/A & $\mathrm{N} / \mathrm{A}$ & N/A & $\mathrm{N} / \mathrm{A}$ & N/A & N/A \\
\hline $\mathrm{N} / \mathrm{A}$ & $\mathrm{N} / \mathrm{A}$ & N/A & N/A & $\mathrm{N} / \mathrm{A}$ & $\mathrm{N} / \mathrm{A}$ & $\mathrm{N} / \mathrm{A}$ & N/A & N/A \\
\hline N/A & $\mathrm{N} / \mathrm{A}$ & N/A & $\mathrm{N} / \mathrm{A}$ & N/A & N/A & $\mathrm{N} / \mathrm{A}$ & N/A & N/A \\
\hline N/A & $\mathrm{N} / \mathrm{A}$ & N/A & $\mathrm{N} / \mathrm{A}$ & N/A & N/A & $\mathrm{N} / \mathrm{A}$ & N/A & N/A \\
\hline N/A & $\mathrm{N} / \mathrm{A}$ & N/A & $\mathrm{N} / \mathrm{A}$ & N/A & $\mathrm{N} / \mathrm{A}$ & N/A & N/A & N/A \\
\hline N/A & $N / A$ & N/A & N/A & N/A & $\mathrm{N} / \mathrm{A}$ & N/A & N/A & $\mathrm{N} / \mathrm{A}$ \\
\hline N/A & $\mathrm{N} / \mathrm{A}$ & N/A & N/A & N/A & $\mathrm{N} / \mathrm{A}$ & $\mathrm{N} / \mathrm{A}$ & N/A & N/A \\
\hline N/A & $\mathrm{N} / \mathrm{A}$ & N/A & N/A & N/A & $\mathrm{N} / \mathrm{A}$ & $\mathrm{N} / \mathrm{A}$ & N/A & N/A \\
\hline N/A & $\mathrm{N} / \mathrm{A}$ & N/A & $\mathrm{N} / \mathrm{A}$ & N/A & $\mathrm{N} / \mathrm{A}$ & N/A & N/A & N/A \\
\hline $\mathrm{N} / \mathrm{A}$ & $N / A$ & N/A & N/A & N/A & N/A & $\mathrm{N} / \mathrm{A}$ & N/A & N/A \\
\hline $\mathrm{N} / \mathrm{A}$ & $\mathrm{N} / \mathrm{A}$ & N/A & $\mathrm{N} / \mathrm{A}$ & N/A & N/A & N/A & N/A & N/A \\
\hline
\end{tabular}


FRM-0108C $10-E R-A 26-285$

Survey \# to flt? 6.285 DC 8.21-10

Description/Map/Drawing/Picture/Comments

"UNFOLDED" VIEW

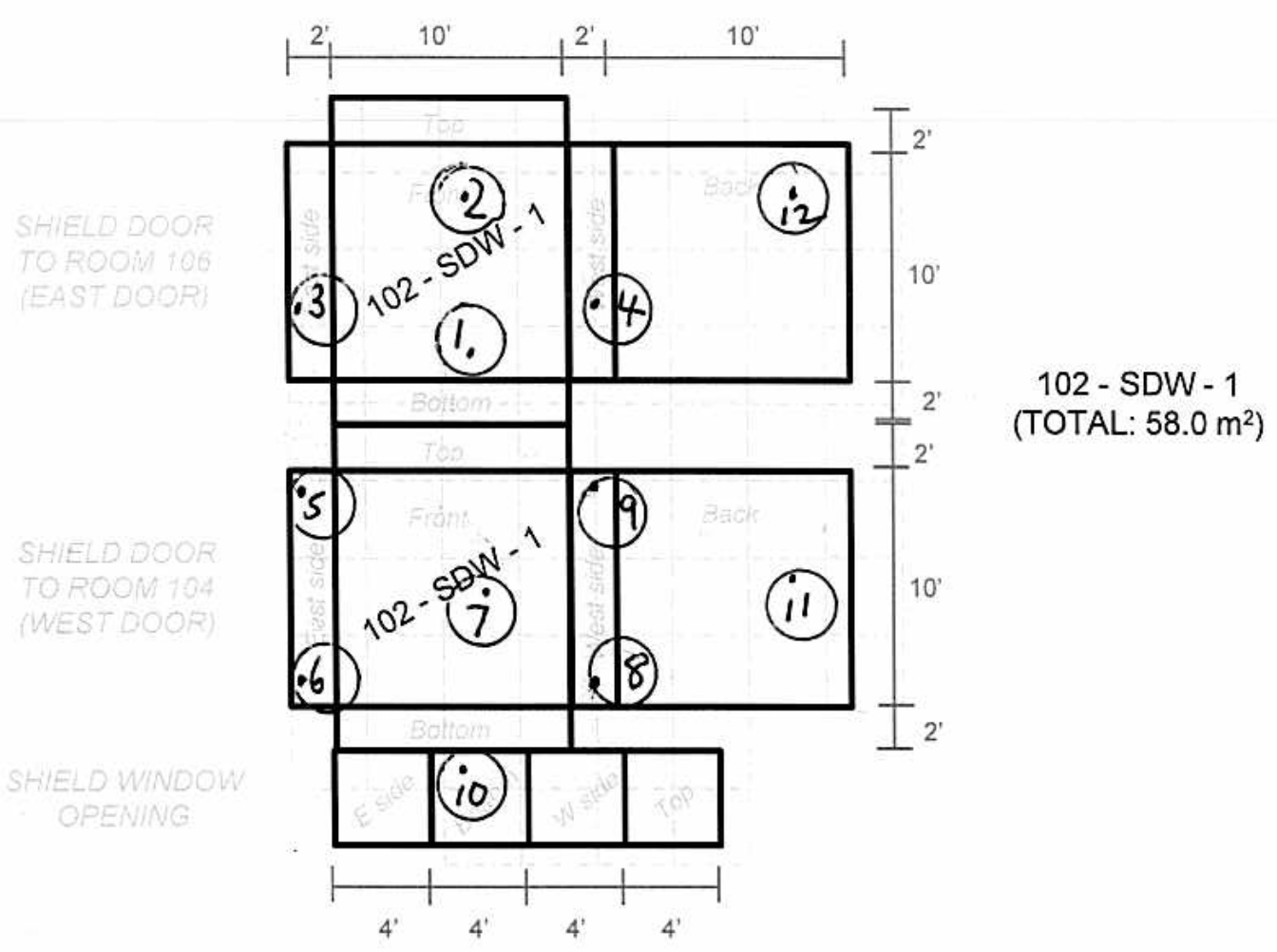

MARSSIM CLASS I SURVEY

$-1 \times 1 m$ grid

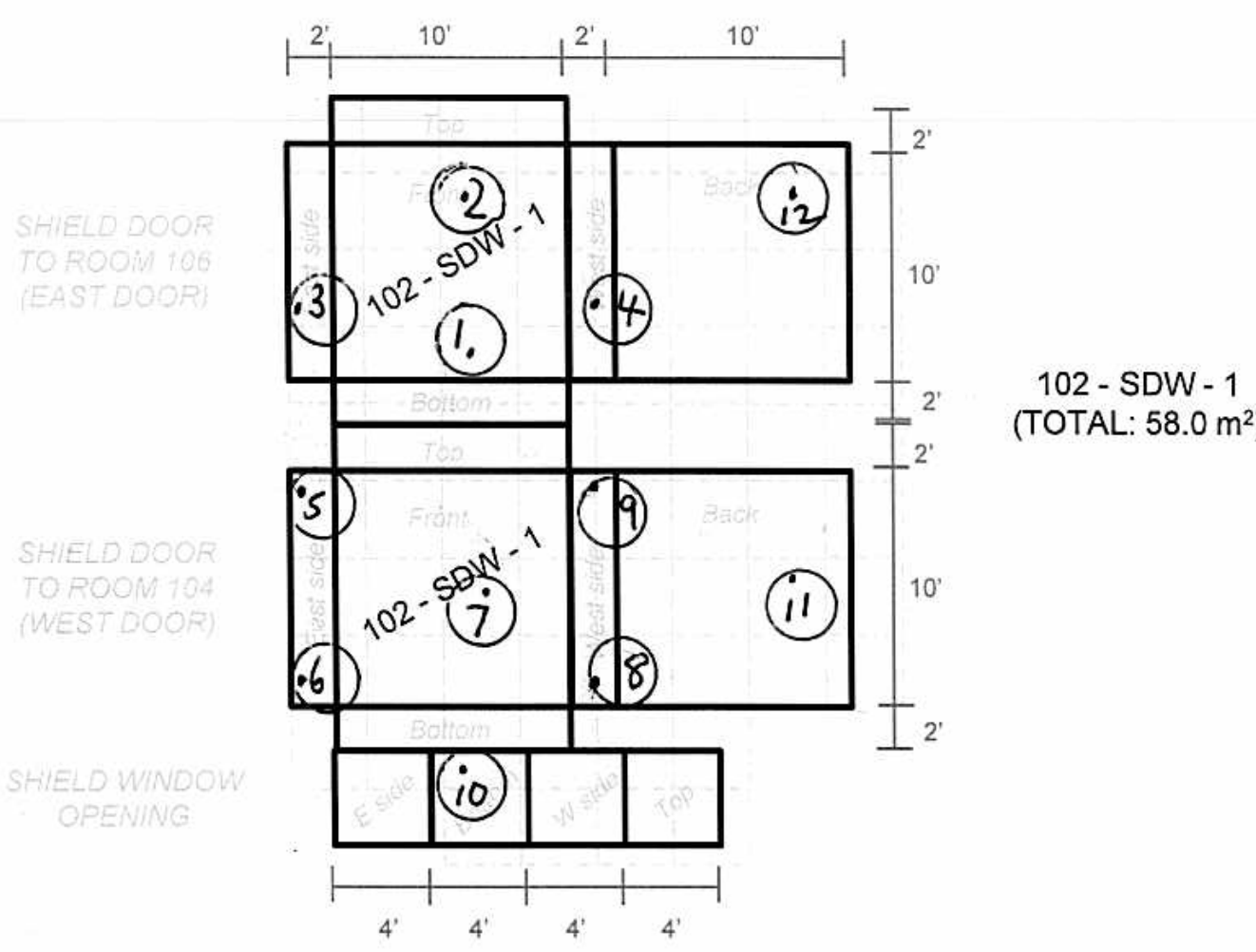

TOTAL: $58.0 \mathrm{~m}^{2}$

Page 3 of 3

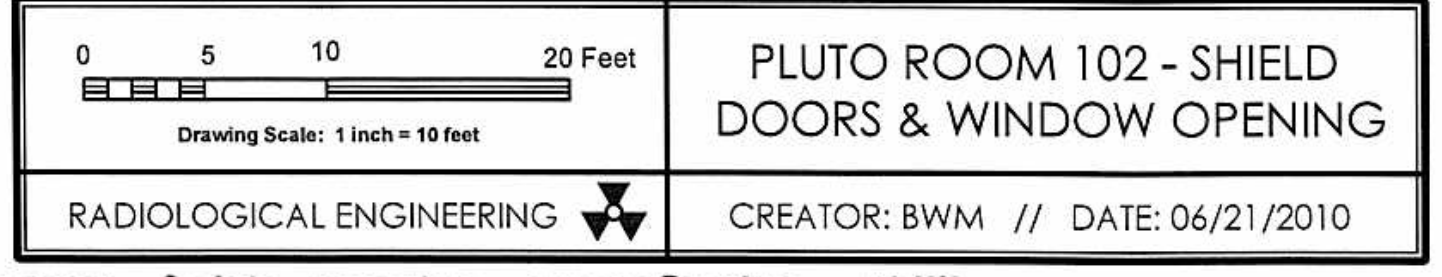

LEGEND Mimute STATIL Com'L OCA tions

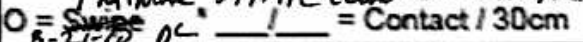


SURVEY \#

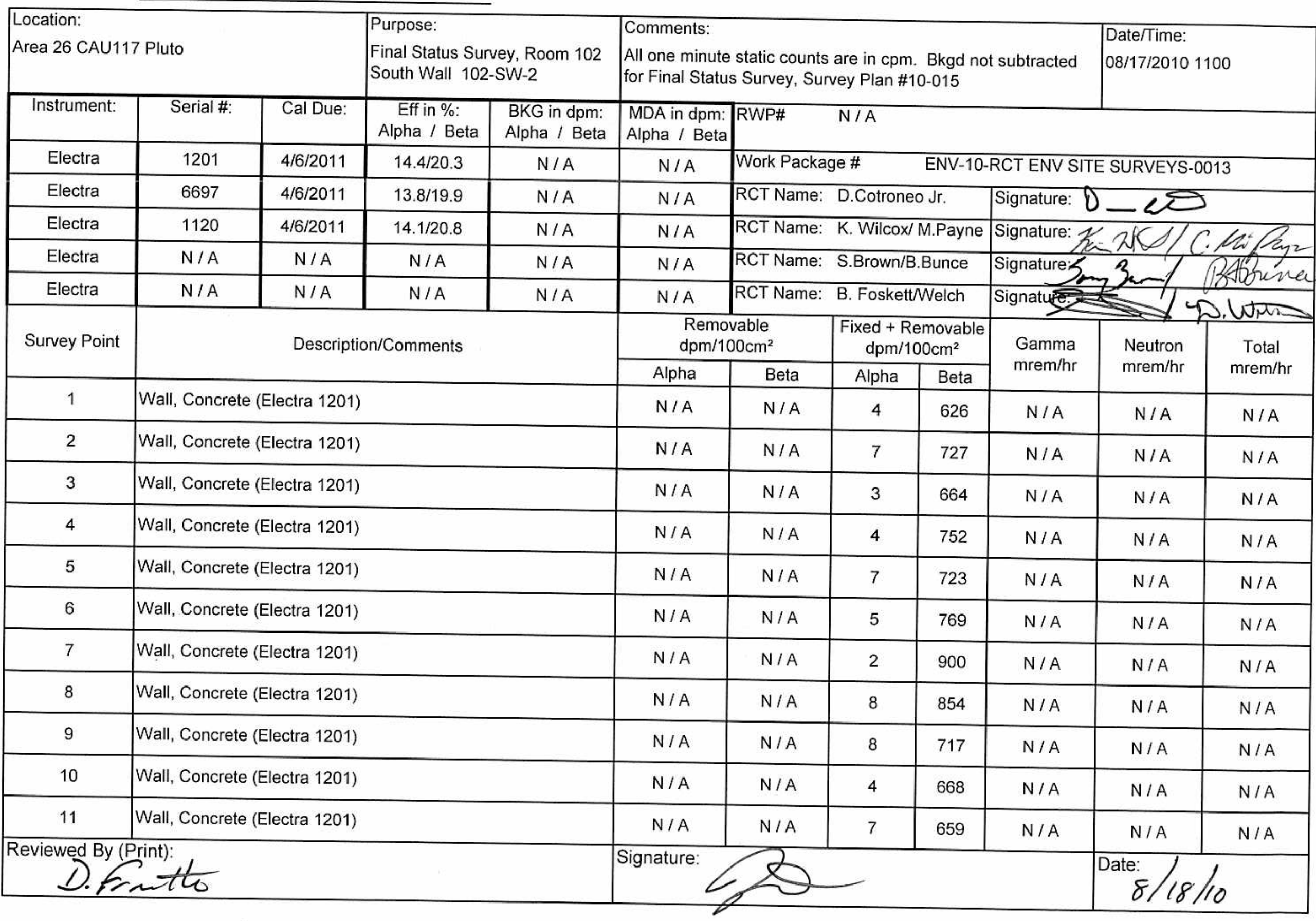


NSTec

Form

$05 / 24 / 10$

FRM-0108B

RADIOLOGICAL SURVEY REPORT - DATA (CONTINUATION)

$2_{\text {of }}^{\text {Rev }} 3^{0}$

SURVEY \# 10-ER-A26-268

\begin{tabular}{|c|c|c|c|c|c|c|c|c|}
\hline \multirow{2}{*}{$\begin{array}{c}\text { Survey } \\
\text { Point }\end{array}$} & \multirow[t]{2}{*}{ Description/Comments } & \multicolumn{2}{|c|}{$\begin{array}{l}\text { Removable } \\
\mathrm{dpm} / 100 \mathrm{~cm}^{2}\end{array}$} & \multicolumn{2}{|c|}{$\begin{array}{c}\text { Fixed + Removable } \\
\mathrm{dpm} / 100 \mathrm{~cm}^{2}\end{array}$} & \multirow{2}{*}{$\begin{array}{l}\text { Gamma } \\
\mathrm{mrem} / \mathrm{hr}\end{array}$} & \multirow{2}{*}{$\begin{array}{l}\text { Neutron } \\
\text { mrem } / \mathrm{hr}\end{array}$} & \multirow{2}{*}{$\begin{array}{c}\text { Total } \\
\text { mrem/hr }\end{array}$} \\
\hline & & Alpha & Beta & Alpha & Beta & & & \\
\hline $\mathrm{N} / \mathrm{A}$ & $N / A$ & N/A & N/A & N/A & N/A & $N / A$ & N/A & N/A \\
\hline N/A & ${ }^{\star \star}$ Performed scan surveys for both alpha and beta** & $\mathrm{N} / \mathrm{A}$ & N/A & $\mathrm{N} / \mathrm{A}$ & $\mathrm{N} / \mathrm{A}$ & N/A & N/A & N/A \\
\hline N/A & ${ }^{\star \star}$ on $100 \%$ of survey unit. No trigger levels exceeded ${ }^{\star \star}$ & $\mathrm{N} / \mathrm{A}$ & $\mathrm{N} / \mathrm{A}$ & N/A & N/A & N/A & N/A & N/A \\
\hline N/A & $N / A$ & N/A & N/A & N/A & $\mathrm{N} / \mathrm{A}$ & N/A & N/A & N/A \\
\hline N/A & $N / A$ & N/A & N/A & $\mathrm{N} / \mathrm{A}$ & N/A & N/A & N/A & N/A \\
\hline N/A & $N / A$ & N/A & N/A & $\mathrm{N} / \mathrm{A}$ & N/A & N/A & N/A & N/A \\
\hline N/A & $N / A$ & N/A & N/A & $N / A$ & N/A & N/A & N/A & N/A \\
\hline N/A & $N / A$ & N/A & $\mathrm{N} / \mathrm{A}$ & N/A & N/A & N/A & $N / A$ & $\mathrm{~N} / \mathrm{A}$ \\
\hline N/A & $N / A$ & N/A & $\mathrm{N} / \mathrm{A}$ & N/A & $\mathrm{N} / \mathrm{A}$ & N/A & N/A & N/A \\
\hline N/A & $\mathrm{N} / \mathrm{A}$ & N/A & N/A & N/A & N/A & N/A & $\mathrm{N} / \mathrm{A}$ & N/A \\
\hline N/A & $N / A$ & N/A & N/A & N/A & N/A & N/A & N/A & N/A \\
\hline $\mathrm{N} / \mathrm{A}$ & $N / A$ & N/A & N/A & N/A & N/A & N/A & N/A & N/A \\
\hline N/A & $N / A$ & N/A & N/A & N/A & N/A & N/A & $\mathrm{N} / \mathrm{A}$ & N/A \\
\hline N/A & $N / A$ & N/A & N/A & N/A & $\mathrm{N} / \mathrm{A}$ & N/A & N/A & N/A \\
\hline N/A & $N / A$ & N/A & $\mathrm{N} / \mathrm{A}$ & N/A & N/A & N/A & N/A & N/A \\
\hline $\mathrm{N} / \mathrm{A}$ & $N / A$ & $\mathrm{~N} / \mathrm{A}$ & N/A & N/A & $\mathrm{N} / \mathrm{A}$ & N/A & $\mathrm{N} / \mathrm{A}$ & $N / A$ \\
\hline $\mathrm{N} / \mathrm{A}$ & $N / A$ & N/A & N/A & N/A & N/A & $\mathrm{N} / \mathrm{A}$ & N/A & N/A \\
\hline N/A & $N / A$ & N/A & N/A & N/A & $\mathrm{N} / \mathrm{A}$ & $\mathrm{N} / \mathrm{A}$ & $\mathrm{N} / \mathrm{A}$ & N/A \\
\hline N/A & $N / A$ & N/A & N/A & $\mathrm{N} / \mathrm{A}$ & $\mathrm{N} / \mathrm{A}$ & $\mathrm{N} / \mathrm{A}$ & $\mathrm{N} / \mathrm{A}$ & N/A \\
\hline $\mathrm{N} / \mathrm{A}$ & $N / A$ & N/A & N/A & $\mathrm{N} / \mathrm{A}$ & $\mathrm{N} / \mathrm{A}$ & N/A & N/A & $\mathrm{N} / \mathrm{A}$ \\
\hline N/A & $N / A$ & N/A & $\mathrm{N} / \mathrm{A}$ & $\mathrm{N} / \mathrm{A}$ & $\mathrm{N} / \mathrm{A}$ & N/A & $\mathrm{N} / \mathrm{A}$ & $\mathrm{N} / \mathrm{A}$ \\
\hline N/A & $N / A$ & N/A & $\mathrm{N} / \mathrm{A}$ & N/A & N/A & N/A & N/A & N/A \\
\hline N/A & $N / A$ & N/A & N/A & N/A & N/A & $N / A$ & N/A & N/A \\
\hline
\end{tabular}


Survey $\# 10-E R-A 2 b-268$

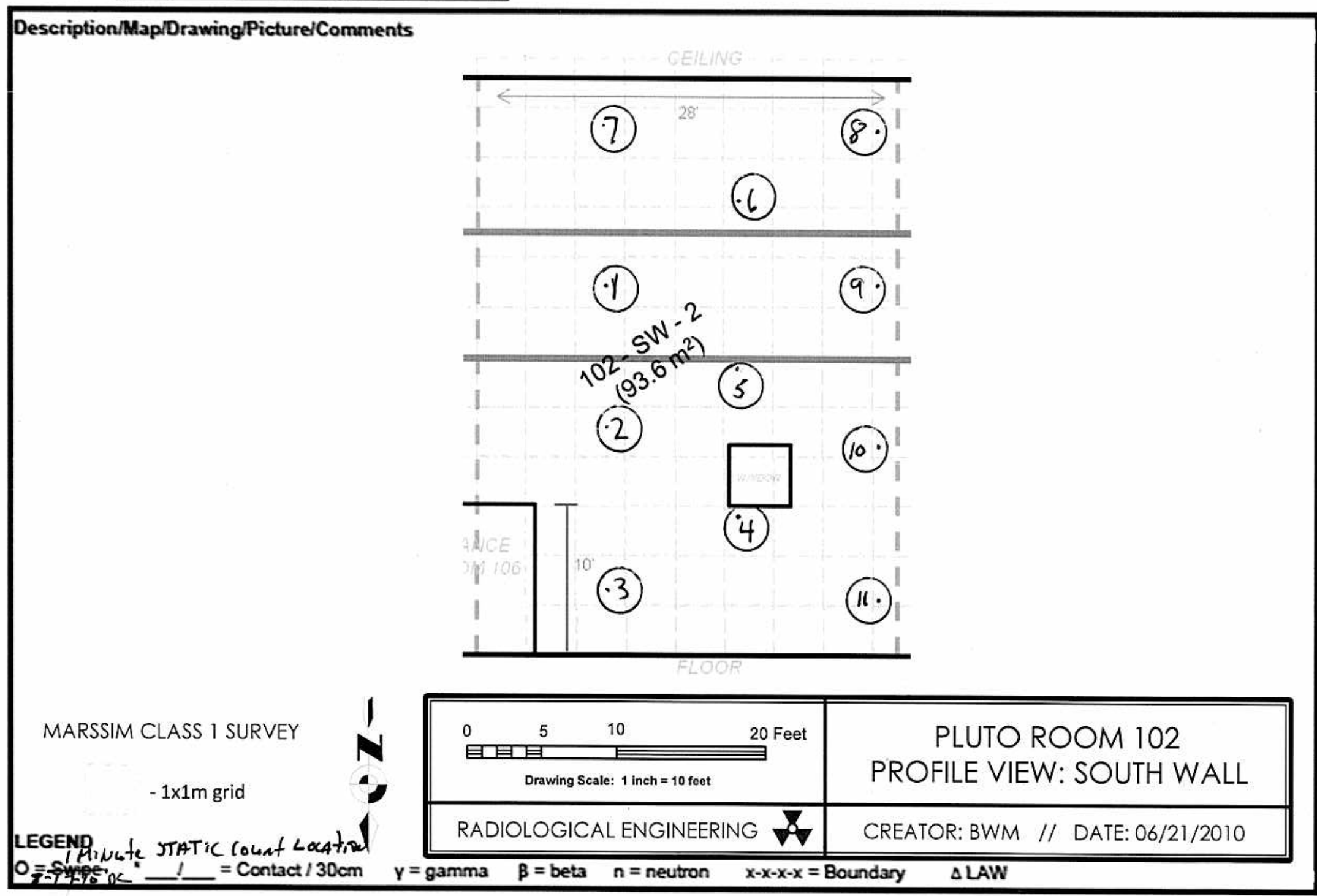


FRM-0108B

SURVEY \#

10-ER-A26-267

\begin{tabular}{|c|c|c|c|c|c|c|c|c|c|c|c|}
\hline \multicolumn{3}{|c|}{$\begin{array}{l}\text { Location: } \\
\text { Area } 26 \text { CAU117 Pluto }\end{array}$} & \multicolumn{2}{|c|}{$\begin{array}{l}\text { Purpose: } \\
\text { Final Status Survey, Room } 102 \\
\text { South Wall 102-SW-3 }\end{array}$} & \multicolumn{5}{|c|}{$\begin{array}{l}\text { Comments: } \\
\text { All one minute static counts are in cpm. Bkgd not subtracted } \\
\text { for Final Status Survey, Survey Plan \#10-015 }\end{array}$} & \multicolumn{2}{|c|}{$\begin{array}{l}\text { Date/Time: } \\
08 / 17 / 20100900\end{array}$} \\
\hline Instrument: & Serial \#: & Cal Due: & $\begin{array}{c}\text { Eff in \%: } \\
\text { Alpha / Beta }\end{array}$ & $\begin{array}{l}\text { BKG in dpm: } \\
\text { Alpha / Beta }\end{array}$ & $\begin{array}{l}\text { MDA in dpm: } \\
\text { Alpha / Beta }\end{array}$ & RWP\# & \multicolumn{5}{|l|}{$\mathrm{N} / \mathrm{A}$} \\
\hline Electra & 4806 & $4 / 6 / 2011$ & $13.2 / 20.9$ & $N / A$ & $N / A$ & \multicolumn{6}{|c|}{ ENV-10-RCT ENV SITE SURV } \\
\hline Electra & 1504 & $4 / 6 / 2011$ & $12.9 / 19.8$ & $N / A$ & $\mathrm{~N} / \mathrm{A}$ & \multicolumn{3}{|c|}{ RCT Name: D.Cotroneo Jr. } & \multicolumn{3}{|c|}{ Signature: 0 - } \\
\hline Electra & 1120 & $4 / 6 / 2011$ & $14.1 / 20.8$ & $N / A$ & $\mathrm{~N} / \mathrm{A}$ & \multicolumn{3}{|c|}{ RCT Name: K. Wilcox/M.Payne } & \multicolumn{3}{|c|}{ Signature: $K_{2}=200 / C \cdot \operatorname{lis} / a$} \\
\hline Electra & $N / A$ & $\mathrm{~N} / \mathrm{A}$ & $N / A$ & $\mathrm{~N} / \mathrm{A}$ & $N / A$ & \multicolumn{3}{|c|}{ RCT Name: S.Brown/B.Bunce } & \multirow{2}{*}{\multicolumn{3}{|c|}{ 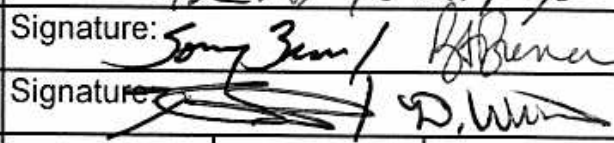 }} \\
\hline Electra & $N / A$ & $\mathrm{~N} / \mathrm{A}$ & $\mathrm{N} / \mathrm{A}$ & $\mathrm{N} / \mathrm{A}$ & $N / A$ & RCT Name: & B. Fosket & Velch & & & \\
\hline \multirow[t]{2}{*}{ Survey Point } & \multirow{2}{*}{\multicolumn{4}{|c|}{ Description/Comments }} & \multicolumn{2}{|c|}{$\begin{array}{l}\text { Removable } \\
\mathrm{dpm} / 100 \mathrm{~cm}^{2}\end{array}$} & \multicolumn{2}{|c|}{\begin{tabular}{|c|} 
Fixed + Removable \\
$\mathrm{dpm} / 100 \mathrm{~cm}^{2}$
\end{tabular}} & \multirow{2}{*}{$\begin{array}{l}\text { Gamma } \\
\mathrm{mrem} / \mathrm{hr}\end{array}$} & \multirow{2}{*}{$\begin{array}{l}\text { Neutron } \\
\text { mrem } / \mathrm{hr}\end{array}$} & \multirow{2}{*}{$\begin{array}{c}\text { Total } \\
\mathrm{mrem} / \mathrm{hr}\end{array}$} \\
\hline & & & & & Alpha & Beta & Alpha & Beta & & & \\
\hline 1 & \multicolumn{4}{|c|}{ Wall, Concrete (Electra 4806) } & $N / A$ & $N / A$ & 10 & 1029 & $\mathrm{~N} / \mathrm{A}$ & $N / A$ & $\mathrm{~N} / \mathrm{A}$ \\
\hline 2 & \multicolumn{4}{|c|}{ Wall, Concrete (Electra 4806) } & $N / A$ & $N / A$ & 6 & 621 & $N / A$ & $\mathrm{~N} / \mathrm{A}$ & $N / A$ \\
\hline 3 & \multicolumn{4}{|c|}{ Wall, Concrete (Electra 4806) } & $\mathrm{N} / \mathrm{A}$ & $N / A$ & 8 & 636 & $N / A$ & $N / A$ & $N / A$ \\
\hline 4 & \multicolumn{4}{|c|}{ Wall, Concrete (Electra 4806) } & $N / A$ & $N / A$ & 7 & 718 & $\mathrm{~N} / \mathrm{A}$ & $N / A$ & $N / A$ \\
\hline 5 & \multicolumn{4}{|c|}{ Wall, Concrete (Electra 4806) } & $N / A$ & $N / A$ & 2 & 825 & $N / A$ & $N / A$ & $N / A$ \\
\hline 6 & \multicolumn{4}{|c|}{ Wall, Concrete (Electra 4806) } & $N / A$ & $N / A$ & 8 & 1036 & $N / A$ & $N / A$ & $N / A$ \\
\hline 7 & \multicolumn{4}{|c|}{ Wall, Concrete (Electra 4806) } & $N / A$ & $N / A$ & 5 & 693 & $N / A$ & $N / A$ & $N / A$ \\
\hline 8 & \multicolumn{4}{|c|}{ Wall, Concrete (Electra 4806) } & $N / A$ & $N / A$ & 9 & 963 & $N / A$ & $N / A$ & $N / A$ \\
\hline 9 & \multicolumn{4}{|c|}{ Wall, Wood (Electra 1504) } & $N / A$ & $N / A$ & 5 & 676 & $N / A$ & $N / A$ & $N / A$ \\
\hline 10 & \multicolumn{4}{|c|}{ Wall, Concrete (Electra 4806) } & $N / A$ & $N / A$ & 5 & 1018 & $\mathrm{~N} / \mathrm{A}$ & $N / A$ & $N / A$ \\
\hline 11 & \multicolumn{4}{|c|}{ Wall, Concrete (Electra 4806) } & $N / A$ & $N / A$ & 9 & 1024 & $N / A$ & $N / A$ & $N / A$ \\
\hline \multicolumn{5}{|c|}{ Reviewed By,(Print): } & \multicolumn{5}{|l|}{ Signature: } & \multicolumn{2}{|c|}{$8 / 18 / 10$} \\
\hline
\end{tabular}


SURVEY \# 10-ER-A26-267

\begin{tabular}{|c|c|c|c|c|c|c|c|c|}
\hline \multirow{2}{*}{$\begin{array}{l}\text { Survey } \\
\text { Point }\end{array}$} & \multirow[t]{2}{*}{ Description/Comments } & \multicolumn{2}{|c|}{$\begin{array}{l}\text { Removable } \\
\mathrm{dpm} / 100 \mathrm{~cm}^{2}\end{array}$} & \multicolumn{2}{|c|}{$\begin{array}{c}\text { Fixed + Removable } \\
\mathrm{dpm} / 100 \mathrm{~cm}^{2}\end{array}$} & \multirow{2}{*}{$\begin{array}{l}\text { Gamma } \\
\text { mrem/hr }\end{array}$} & \multirow{2}{*}{$\begin{array}{l}\text { Neutron } \\
\mathrm{mrem} / \mathrm{hr}\end{array}$} & \multirow{2}{*}{$\begin{array}{c}\text { Total } \\
\text { mrem/hr }\end{array}$} \\
\hline & & Alpha & Beta & Alpha & Beta & & & \\
\hline 12 & Wall, Concrete (Electra 4806) & $\mathrm{N} / \mathrm{A}$ & N/A & 6 & 1033 & N/A & N/A & N/A \\
\hline N/A & ${ }^{* \star}$ Performed scan surveys for both alpha and beta** & N/A & $\mathrm{N} / \mathrm{A}$ & N/A & N/A & N/A & N/A & N/A \\
\hline N/A & ${ }^{\star \star}$ on $100 \%$ of survey unit. No trigger levels exceeded ${ }^{\star \star}$ & N/A & $\mathrm{N} / \mathrm{A}$ & N/A & N/A & N/A & N/A & N/A \\
\hline N/A & $N / A$ & N/A & N/A & N/A & N/A & N/A & N/A & N/A \\
\hline N/A & $N / A$ & N/A & N/A & N/A & N/A & N/A & N/A & N/A \\
\hline N/A & $N / A$ & N/A & N/A & N/A & N/A & N/A & N/A & N/A \\
\hline N/A & $N / A$ & N/A & $\mathrm{N} / \mathrm{A}$ & N/A & N/A & N/A & N/A & N/A \\
\hline N/A & $N / A$ & N/A & N/A & N/A & N/A & N/A & N/A & N/A \\
\hline N/A & $N / A$ & N/A & N/A & N/A & N/A & N/A & N/A & N/A \\
\hline N/A & $N / A$ & N/A & N/A & N/A & N/A & N/A & N/A & N/A \\
\hline N/A & $N / A$ & N/A & N/A & N/A & N/A & N/A & N/A & N/A \\
\hline N/A & $N / A$ & N/A & N/A & N/A & N/A & N/A & N/A & N/A \\
\hline N/A & $N / A$ & N/A & N/A & N/A & N/A & N/A & N/A & N/A \\
\hline N/A & $N / A$ & N/A & N/A & N/A & N/A & N/A & N/A & N/A \\
\hline N/A & $N / A$ & N/A & N/A & N/A & N/A & N/A & N/A & N/A \\
\hline N/A & $N / A$ & N/A & N/A & $\mathrm{N} / \mathrm{A}$ & N/A & N/A & $N / A$ & N/A \\
\hline $\mathrm{N} / \mathrm{A}$ & $N / A$ & N/A & N/A & N/A & N/A & N/A & N/A & N/A \\
\hline N/A & $N / A$ & N/A & N/A & N/A & N/A & N/A & N/A & N/A \\
\hline N/A & $N / A$ & N/A & N/A & N/A & N/A & N/A & N/A & N/A \\
\hline N/A & $N / A$ & N/A & N/A & N/A & N/A & N/A & N/A & N/A \\
\hline N/A & $N / A$ & N/A & N/A & N/A & N/A & N/A & N/A & N/A \\
\hline N/A & N/A & N/A & N/A & N/A & N/A & N/A & N/A & N/A \\
\hline N/A & $N / A$ & N/A & N/A & N/A & N/A & N/A & N/A & N/A \\
\hline
\end{tabular}




\section{7}

Page 3 of 3

Survey $=10-E R-A 26-267$

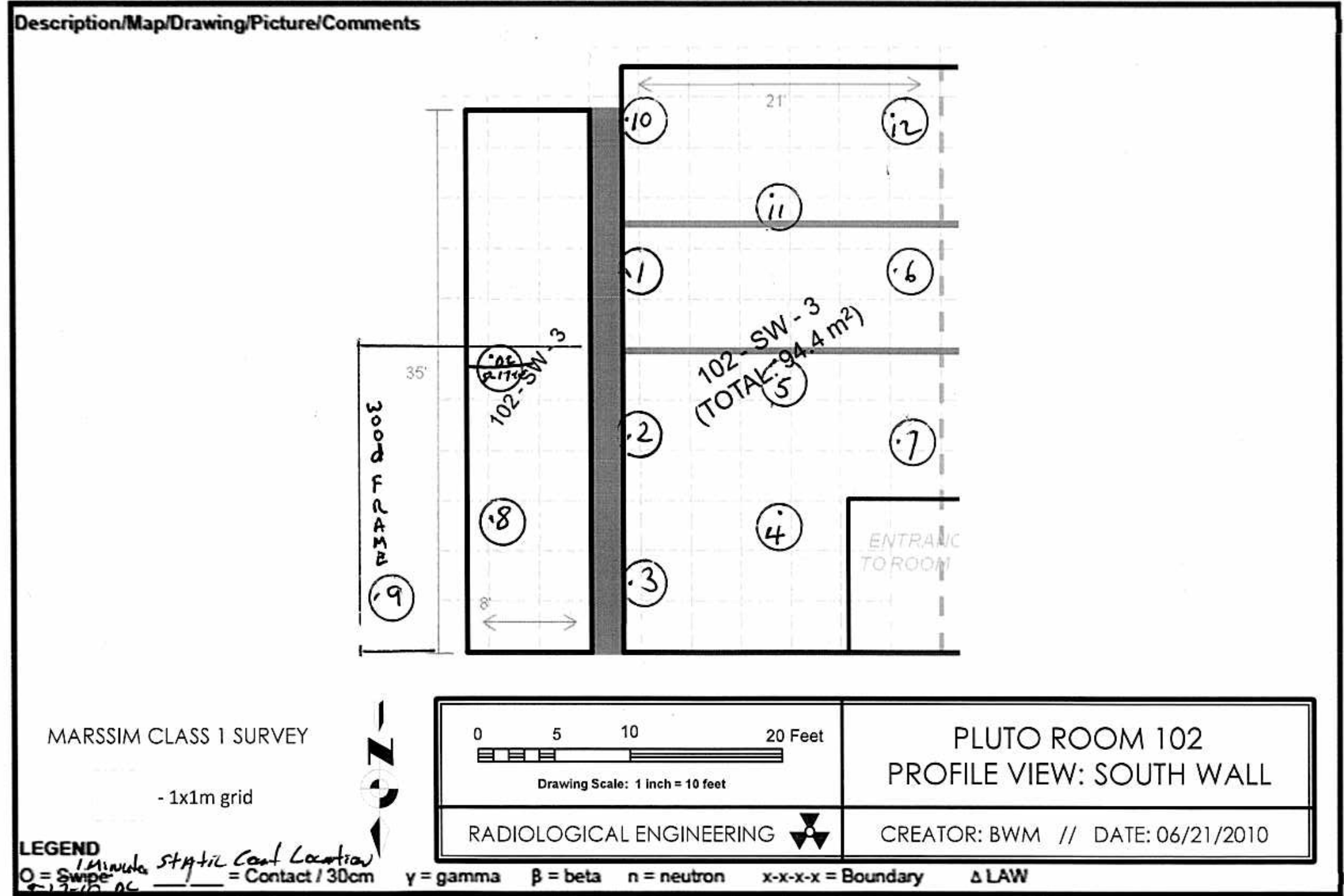


NSTec

Form

FRM-0108B

RADIOLOGICAL SURVEY REPORT - DATA

SURVEY \#

10-ER-A26-266

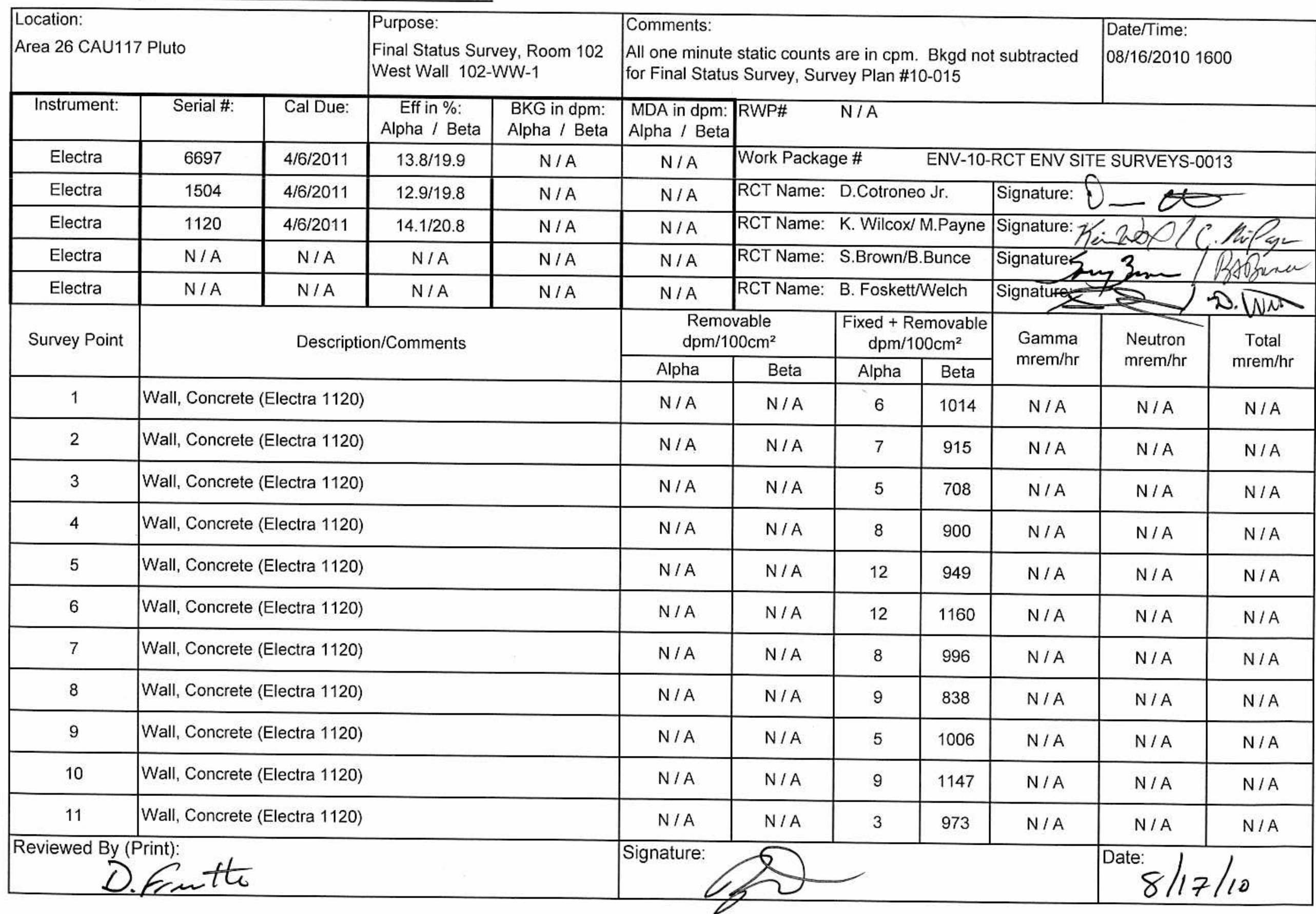


SURVEY \# 10-ER-A26-266

\begin{tabular}{|c|c|c|c|c|c|c|c|c|}
\hline \multirow{2}{*}{$\begin{array}{l}\text { Survey } \\
\text { Point }\end{array}$} & \multirow[t]{2}{*}{ Description/Comments } & \multicolumn{2}{|c|}{$\begin{array}{l}\text { Removable } \\
\mathrm{dpm} / 100 \mathrm{~cm}^{2}\end{array}$} & \multicolumn{2}{|c|}{$\begin{array}{c}\begin{array}{c}\text { Fixed + Removable } \\
\mathrm{dpm} / 100 \mathrm{~cm}^{2}\end{array} \\
\end{array}$} & \multirow{2}{*}{$\begin{array}{l}\text { Gamma } \\
\text { mrem/hr }\end{array}$} & \multirow{2}{*}{$\begin{array}{l}\text { Neutron } \\
\mathrm{mrem} / \mathrm{hr}\end{array}$} & \multirow{2}{*}{$\begin{array}{c}\text { Total } \\
\mathrm{mrem} / \mathrm{hr}\end{array}$} \\
\hline & & Alpha & Beta & Alpha & Beta & & & \\
\hline N/A & ${ }^{\star \star}$ Performed scan surveys for both alpha and beta** & N/A & N/A & $N / A$ & $\mathrm{~N} / \mathrm{A}$ & $N / A$ & N/A & $\mathrm{N} / \mathrm{A}$ \\
\hline N/A & ${ }^{\star *}$ on $100 \%$ of survey unit. No trigger levels exceeded ${ }^{* *}$ & N/A & N/A & N/A & $\mathrm{N} / \mathrm{A}$ & N/A & N/A & N/A \\
\hline N/A & $N / A$ & N/A & N/A & N/A & N/A & $\mathrm{N} / \mathrm{A}$ & N/A & N/A \\
\hline N/A & $N / A$ & $\mathrm{~N} / \mathrm{A}$ & N/A & $\mathrm{N} / \mathrm{A}$ & N/A & N/A & N/A & $N / A$ \\
\hline N/A & $N / A$ & N/A & N/A & N/A & N/A & N/A & N/A & $\mathrm{N} / \mathrm{A}$ \\
\hline N/A & $N / A$ & N/A & $\mathrm{N} / \mathrm{A}$ & N/A & N/A & N/A & N/A & N/A \\
\hline $\mathrm{N} / \mathrm{A}$ & $N / A$ & N/A & $\mathrm{N} / \mathrm{A}$ & N/A & $\mathrm{N} / \mathrm{A}$ & N/A & $\mathrm{N} / \mathrm{A}$ & N/A \\
\hline N/A & $N / A$ & N/A & N/A & N/A & N/A & N/A & N/A & $\mathrm{N} / \mathrm{A}$ \\
\hline N/A & $N / A$ & N/A & $\mathrm{N} / \mathrm{A}$ & $\mathrm{N} / \mathrm{A}$ & N/A & N/A & $\mathrm{N} / \mathrm{A}$ & N/A \\
\hline $\mathrm{N} / \mathrm{A}$ & $\mathrm{N} / \mathrm{A}$ & N/A & N/A & N/A & $\mathrm{N} / \mathrm{A}$ & N/A & N/A & N/A \\
\hline N/A & $N / A$ & N/A & $\mathrm{N} / \mathrm{A}$ & N/A & $\mathrm{N} / \mathrm{A}$ & N/A & $\mathrm{N} / \mathrm{A}$ & N/A \\
\hline N/A & $N / A$ & N/A & N/A & $\mathrm{N} / \mathrm{A}$ & $\mathrm{N} / \mathrm{A}$ & N/A & N/A & N/A \\
\hline N/A & $N / A$ & N/A & N/A & N/A & N/A & N/A & $\mathrm{N} / \mathrm{A}$ & N/A \\
\hline N/A & $\mathrm{N} / \mathrm{A}$ & $\mathrm{N} / \mathrm{A}$ & N/A & $\mathrm{N} / \mathrm{A}$ & N/A & N/A & N/A & N/A \\
\hline $\mathrm{N} / \mathrm{A}$ & $N / A$ & N/A & N/A & N/A & $\mathrm{N} / \mathrm{A}$ & N/A & N/A & N/A \\
\hline N/A & $\mathrm{N} / \mathrm{A}$ & N/A & $\mathrm{N} / \mathrm{A}$ & $\mathrm{N} / \mathrm{A}$ & N/A & N/A & $\mathrm{N} / \mathrm{A}$ & N/A \\
\hline N/A & $N / A$ & $\mathrm{~N} / \mathrm{A}$ & $\mathrm{N} / \mathrm{A}$ & N/A & $\mathrm{N} / \mathrm{A}$ & N/A & $\mathrm{N} / \mathrm{A}$ & N/A \\
\hline $\mathrm{N} / \mathrm{A}$ & $N / A$ & N/A & N/A & N/A & N/A & N/A & N/A & $N / A$ \\
\hline N/A & $\mathrm{N} / \mathrm{A}$ & N/A & N/A & N/A & N/A & N/A & N/A & $\mathrm{N} / \mathrm{A}$ \\
\hline N/A & $N / A$ & N/A & N/A & N/A & $\mathrm{N} / \mathrm{A}$ & N/A & N/A & N/A \\
\hline $\mathrm{N} / \mathrm{A}$ & $\mathrm{N} / \mathrm{A}$ & N/A & N/A & N/A & N/A & $\mathrm{N} / \mathrm{A}$ & N/A & N/A \\
\hline N/A & $N / A$ & N/A & N/A & $\mathrm{N} / \mathrm{A}$ & N/A & N/A & N/A & N/A \\
\hline N/A & $\mathrm{N} / \mathrm{A}$ & N/A & N/A & N/A & $\mathrm{N} / \mathrm{A}$ & N/A & $N / A$ & N/A \\
\hline
\end{tabular}


Survey \# 10. ER A25. 266

Description/Map/Drawing/Picture/Comments

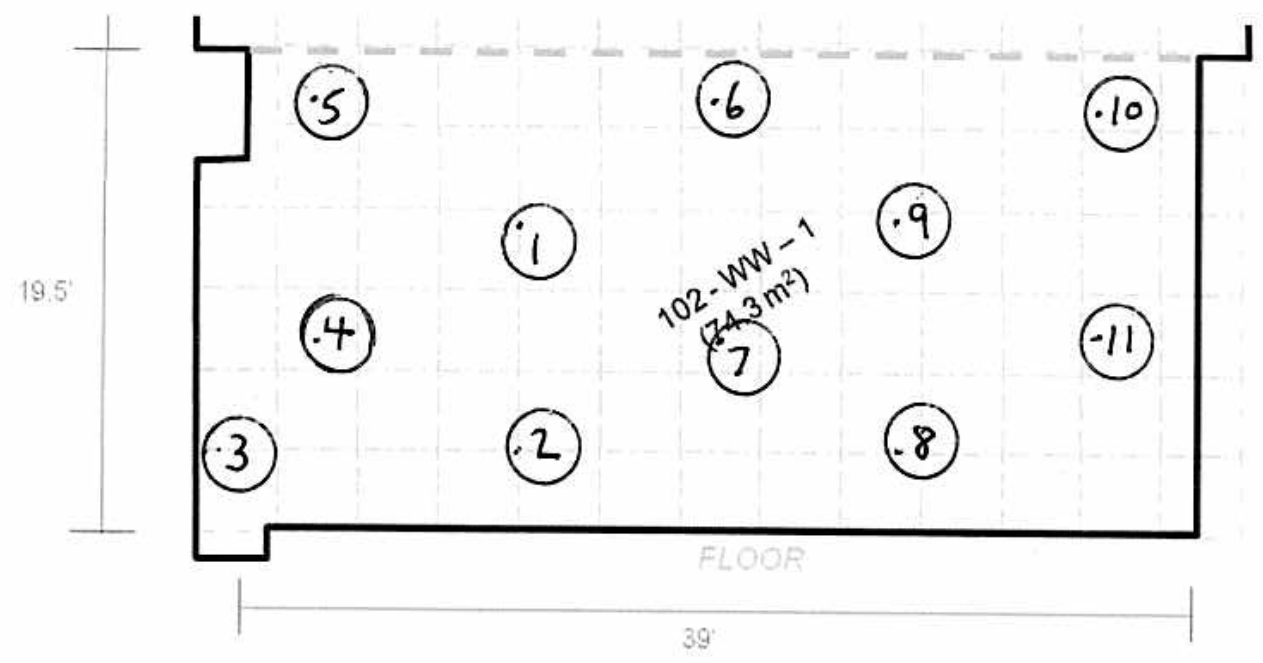

MARSSIM CLASS 1 SURVEY

$-1 \times 1 m$ grid

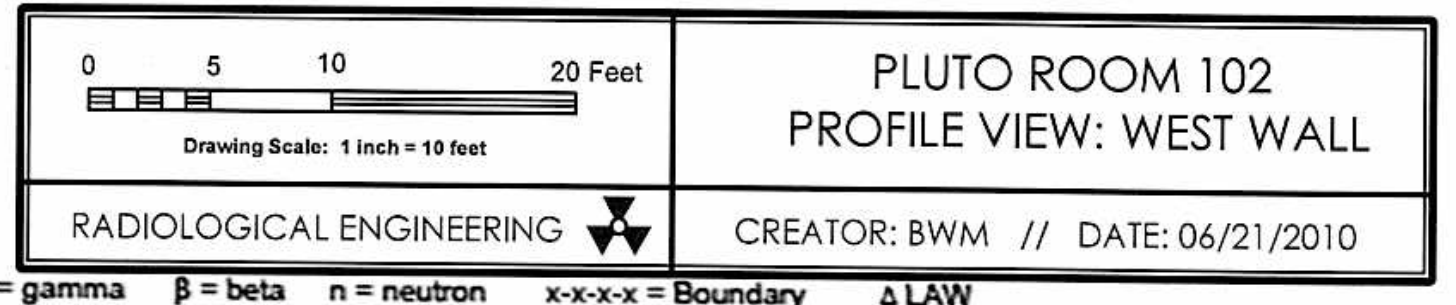

LEGEND

$0=$ Minamde, STATIC Count Locartiows $=$ Contact $/ 30 \mathrm{~cm}$ 


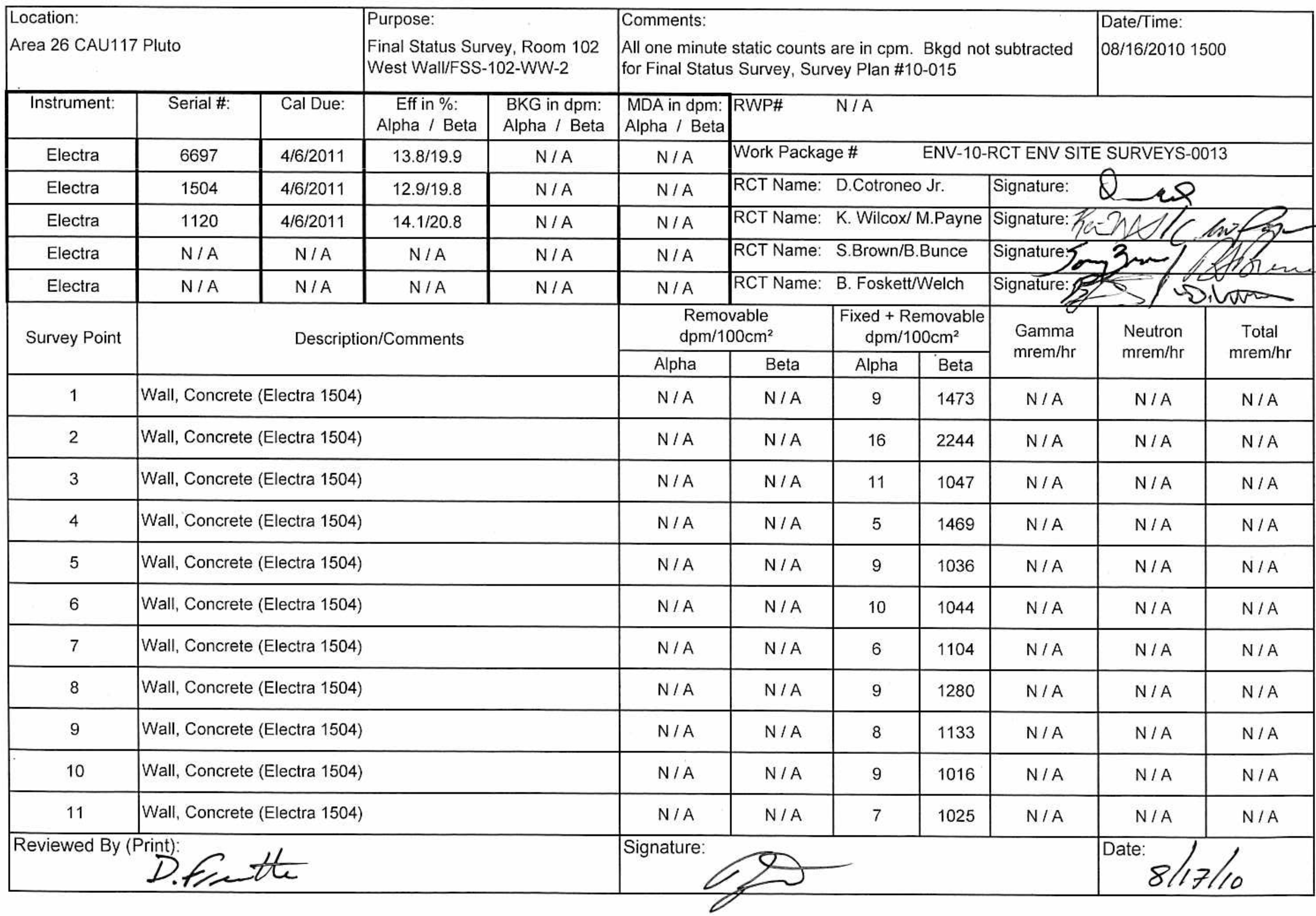


SURVEY \# 10-ER-A26-264

\begin{tabular}{|c|c|c|c|c|c|c|c|c|}
\hline \multirow{2}{*}{$\begin{array}{l}\text { Survey } \\
\text { Point }\end{array}$} & \multirow[t]{2}{*}{ Description/Comments } & \multicolumn{2}{|c|}{$\begin{array}{l}\text { Removable } \\
\mathrm{dpm} / 100 \mathrm{~cm}^{2}\end{array}$} & \multicolumn{2}{|c|}{$\begin{array}{c}\text { Fixed + Removable } \\
\mathrm{dpm} / 100 \mathrm{~cm}^{2}\end{array}$} & \multirow{2}{*}{$\begin{array}{l}\text { Gamma } \\
\mathrm{mrem} / \mathrm{hr}\end{array}$} & \multirow{2}{*}{$\begin{array}{l}\text { Neutron } \\
\mathrm{mrem} / \mathrm{hr}\end{array}$} & \multirow{2}{*}{$\begin{array}{c}\text { Total } \\
\text { mrem } / \mathrm{hr}\end{array}$} \\
\hline & & Alpha & Beta & Alpha & Beta & & & \\
\hline N/A & ${ }^{\star \star}$ Performed scan surveys for both alpha and beta ${ }^{\star \star}$ & N/A & N/A & N/A & $\mathrm{N} / \mathrm{A}$ & N/A & N/A & N/A \\
\hline N/A & ${ }^{* \star}$ on $100 \%$ of survey unit. No trigger levels exceeded ${ }^{\star *}$ & $\mathrm{~N} / \mathrm{A}$ & N/A & N/A & $\mathrm{N} / \mathrm{A}$ & N/A & N/A & N/A \\
\hline N/A & $N / A$ & N/A & N/A & N/A & N/A & $\mathrm{N} / \mathrm{A}$ & N/A & N/A \\
\hline N/A & $N / A$ & $\mathrm{~N} / \mathrm{A}$ & N/A & N/A & $\mathrm{N} / \mathrm{A}$ & N/A & $\mathrm{N} / \mathrm{A}$ & N/A \\
\hline N/A & $N / A$ & N/A & N/A & N/A & $\mathrm{N} / \mathrm{A}$ & N/A & $\mathrm{N} / \mathrm{A}$ & N/A \\
\hline N/A & $N / A$ & $\mathrm{~N} / \mathrm{A}$ & N/A & N/A & $\mathrm{N} / \mathrm{A}$ & N/A & N/A & N/A \\
\hline N/A & $N / A$ & N/A & N/A & N/A & N/A & N/A & N/A & $\mathrm{N} / \mathrm{A}$ \\
\hline N/A & $N / A$ & N/A & N/A & N/A & N/A & $\mathrm{N} / \mathrm{A}$ & N/A & N/A \\
\hline N/A & $\mathrm{N} / \mathrm{A}$ & N/A & N/A & $\mathrm{N} / \mathrm{A}$ & N/A & N/A & N/A & N/A \\
\hline N/A & $\mathrm{N} / \mathrm{A}$ & N/A & N/A & N/A & $\mathrm{N} / \mathrm{A}$ & N/A & N/A & N/A \\
\hline N/A & $\mathrm{N} / \mathrm{A}$ & $\mathrm{N} / \mathrm{A}$ & N/A & N/A & $\mathrm{N} / \mathrm{A}$ & $\mathrm{N} / \mathrm{A}$ & $\mathrm{N} / \mathrm{A}$ & N/A \\
\hline N/A & $N / A$ & N/A & N/A & $\mathrm{N} / \mathrm{A}$ & N/A & N/A & N/A & N/A \\
\hline N/A & $N / A$ & N/A & $\mathrm{N} / \mathrm{A}$ & N/A & N/A & $\mathrm{N} / \mathrm{A}$ & $\mathrm{N} / \mathrm{A}$ & $\mathrm{N} / \mathrm{A}$ \\
\hline N/A & $\mathrm{N} / \mathrm{A}$ & $\mathrm{N} / \mathrm{A}$ & $\mathrm{N} / \mathrm{A}$ & $\mathrm{N} / \mathrm{A}$ & N/A & $\mathrm{N} / \mathrm{A}$ & $\mathrm{N} / \mathrm{A}$ & N/A \\
\hline N/A & $\mathrm{N} / \mathrm{A}$ & N/A & N/A & $\mathrm{N} / \mathrm{A}$ & N/A & $\mathrm{N} / \mathrm{A}$ & $\mathrm{N} / \mathrm{A}$ & N/A \\
\hline N/A & $N / A$ & $\mathrm{~N} / \mathrm{A}$ & N/A & N/A & $\mathrm{N} / \mathrm{A}$ & $\mathrm{N} / \mathrm{A}$ & $\mathrm{N} / \mathrm{A}$ & $\mathrm{N} / \mathrm{A}$ \\
\hline N/A & $N / A$ & $\mathrm{~N} / \mathrm{A}$ & N/A & N/A & $\mathrm{N} / \mathrm{A}$ & N/A & N/A & $\mathrm{N} / \mathrm{A}$ \\
\hline N/A & $N / A$ & $\mathrm{~N} / \mathrm{A}$ & N/A & $\mathrm{N} / \mathrm{A}$ & N/A & $\mathrm{N} / \mathrm{A}$ & N/A & $\mathrm{N} / \mathrm{A}$ \\
\hline N/A & $N / A$ & $\mathrm{~N} / \mathrm{A}$ & $\mathrm{N} / \mathrm{A}$ & N/A & N/A & N/A & N/A & N/A \\
\hline N/A & $\mathrm{N} / \mathrm{A}$ & $\mathrm{N} / \mathrm{A}$ & $\mathrm{N} / \mathrm{A}$ & N/A & N/A & N/A & N/A & N/A \\
\hline N/A & $N / A$ & $\mathrm{~N} / \mathrm{A}$ & $\mathrm{N} / \mathrm{A}$ & N/A & $\mathrm{N} / \mathrm{A}$ & N/A & N/A & $\mathrm{N} / \mathrm{A}$ \\
\hline N/A & $\mathrm{N} / \mathrm{A}$ & $\mathrm{N} / \mathrm{A}$ & $\mathrm{N} / \mathrm{A}$ & N/A & N/A & N/A & N/A & N/A \\
\hline $\mathrm{N} / \mathrm{A}$ & $N / A$ & N/A & N/A & N/A & $\mathrm{N} / \mathrm{A}$ & N/A & N/A & $\mathrm{N} / \mathrm{A}$ \\
\hline
\end{tabular}


FRM-0108C

Survey $\# 10-[1 /-H 26-264$

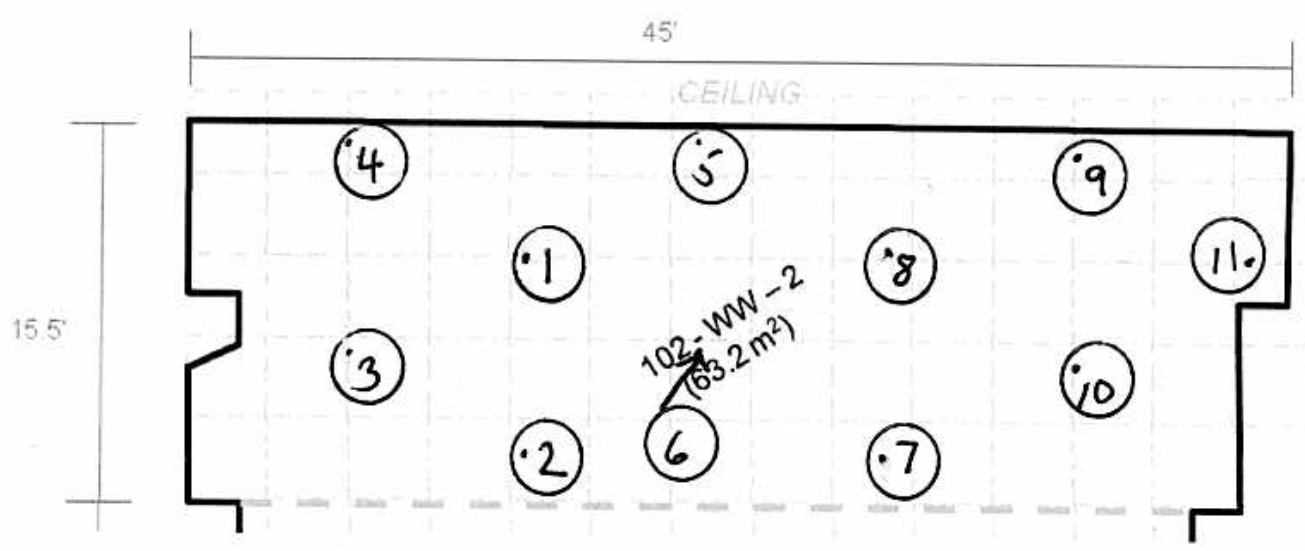

MARSSIM CLASS I SURVEY

$-1 \times 1 m$ grid

LEGEMD

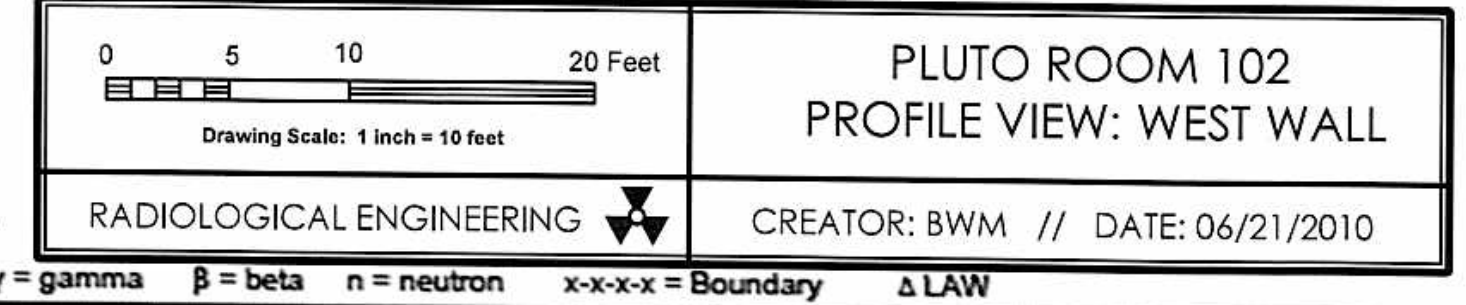


FRM-0108B

SURVEY \#

10-ER-A26-218

\begin{tabular}{|c|c|c|c|c|c|c|c|c|c|c|c|}
\hline \multicolumn{3}{|c|}{$\begin{array}{l}\text { Location: } \\
\text { Area } 26 \text { CAU117 Pluto }\end{array}$} & \multicolumn{2}{|c|}{$\begin{array}{l}\text { Purpose: } \\
\text { Final Status Survey, Room } 104 \\
\text { East Wall, Unit \# 104-EW-1 }\end{array}$} & \multicolumn{5}{|c|}{$\begin{array}{l}\text { Comments: } \\
\text { All one minute static counts are in cpm. Bkgd not subtracted } \\
\text { for Final Status Survey, Survey Plan \#10-015 }\end{array}$} & \multicolumn{2}{|c|}{$\begin{array}{l}\text { Date/Time: } \\
\text { 07/22/2010 } 1600\end{array}$} \\
\hline Instrument: & Serial \#: & Cal Due: & $\begin{array}{c}\text { Eff in \%: } \\
\text { Alpha / Beta }\end{array}$ & $\begin{array}{l}\text { BKG in dpm: } \\
\text { Alpha / Beta }\end{array}$ & $\begin{array}{l}\text { MDA in dpm: } \\
\text { Alpha / Beta }\end{array}$ & RWP\# & \multicolumn{5}{|l|}{$\mathrm{N} / \mathrm{A}$} \\
\hline Electra & 1504 & $4 / 6 / 2011$ & $12.9 / 19.8$ & $N / A$ & $N / A$ & \multicolumn{6}{|c|}{ ENV-10-RCT ENV SITE } \\
\hline Electra & 1120 & $4 / 6 / 2011$ & $14.1 / 20.8$ & $N / A$ & $N / A$ & \multicolumn{3}{|c|}{ RCT Name: D.Cotroneo Jr. } & \multicolumn{3}{|c|}{ Signature: } \\
\hline$N / A$ & $N / A$ & $N / A$ & $N / A$ & $N / A$ & $N / A$ & \multicolumn{3}{|c|}{ RCT Name: Kevin Wilcox } & \multicolumn{3}{|c|}{ Signature: Keri W.Oex } \\
\hline $\mathrm{N} / \mathrm{A}$ & $N / A$ & $N / A$ & $N / A$ & $\mathrm{~N} / \mathrm{A}$ & $N / A$ & \multicolumn{3}{|c|}{ RCT Name: S. Brown/B. Bunce } & \multirow{2}{*}{\multicolumn{3}{|c|}{ Signatures }} \\
\hline$N / A$ & $N / A$ & $N / A$ & $N / A$ & $N / A$ & $N / A$ & RCT Name: & Brian Fos & & & & \\
\hline \multirow[t]{2}{*}{ Survey Point } & \multirow{2}{*}{\multicolumn{4}{|c|}{ Description/Comments }} & \multicolumn{2}{|c|}{$\begin{array}{l}\text { Removable } \\
\mathrm{dpm} / 100 \mathrm{~cm}^{2}\end{array}$} & \multicolumn{2}{|c|}{$\begin{array}{c}\text { Fixed + Removable } \\
\mathrm{dpm} / 100 \mathrm{~cm}^{2}\end{array}$} & \multirow[t]{2}{*}{$\begin{array}{l}\text { Gamma } \\
\mathrm{mrem} / \mathrm{hr}\end{array}$} & \multirow[t]{2}{*}{$\begin{array}{l}\text { Neutron } \\
\mathrm{mrem} / \mathrm{hr}\end{array}$} & \multirow{2}{*}{$\begin{array}{c}\text { Total } \\
\mathrm{mrem} / \mathrm{hr}\end{array}$} \\
\hline & & & & & Alpha & Beta & Alpha & Beta & & & \\
\hline 1 & \multicolumn{4}{|c|}{ East Wall, Concrete (Electra 1120) } & $N / A$ & $N / A$ & 4 & 773 & $N / A$ & $N / A$ & $N / A$ \\
\hline 2 & \multicolumn{4}{|c|}{ East Wall, Concrete (Electra 1120) } & $N / A$ & $N / A$ & 7 & 699 & $N / A$ & $N / A$ & $N / A$ \\
\hline 3 & \multicolumn{4}{|c|}{ East Wall, Concrete (Electra 1504) } & $N / A$ & $N / A$ & 1 & 694 & $N / A$ & $N / A$ & $N / A$ \\
\hline 4 & \multicolumn{4}{|c|}{ East Wall, Concrete (Electra 1504) } & $N / A$ & $N / A$ & 4 & 599 & $N / A$ & $N / A$ & $N / A$ \\
\hline 5 & \multicolumn{4}{|c|}{ East Wall, Concrete (Electra 1120) } & $N / A$ & $N / A$ & 6 & 611 & $N / A$ & $N / A$ & $N / A$ \\
\hline 6 & \multicolumn{4}{|c|}{ East Wall, Concrete (Electra 1120) } & $N / A$ & $N / A$ & 10 & 661 & $N / A$ & $N / A$ & $N / A$ \\
\hline 7 & \multicolumn{4}{|c|}{ East Wall, Concrete (Electra 1120) } & $N / A$ & $N / A$ & 3 & 652 & $\mathrm{~N} / \mathrm{A}$ & $N / A$ & $\mathrm{~N} / \mathrm{A}$ \\
\hline 8 & \multicolumn{4}{|c|}{ East Wall, Concrete (Electra 1120) } & $N / A$ & $N / A$ & 4 & 740 & $N / A$ & $N / A$ & $N / A$ \\
\hline 9 & \multicolumn{4}{|c|}{ East Wall, Concrete (Electra 1120) } & $N / A$ & $N / A$ & 6 & 628 & $N / A$ & $N / A$ & $N / A$ \\
\hline 10 & \multicolumn{4}{|c|}{ East Wali, Concrete (Electra 1120) } & $N / A$ & $N / A$ & 3 & 728 & $N / A$ & $N / A$ & $N / A$ \\
\hline 11 & \multicolumn{4}{|c|}{ East Wall, Metal (Electra 1120) } & $N / A$ & $N / A$ & 6 & 509 & $N / A$ & $N / A$ & $N / A$ \\
\hline \multicolumn{5}{|c|}{ Reviewed By (Print): } & \multicolumn{5}{|l|}{ Signature: } & \multicolumn{2}{|c|}{ Date: $7 / 27 / 10$} \\
\hline
\end{tabular}


SURVEY \# 10-ER-A26-218

\begin{tabular}{|c|c|c|c|c|c|c|c|c|}
\hline \multirow{2}{*}{$\begin{array}{l}\text { Survey } \\
\text { Point }\end{array}$} & \multirow[t]{2}{*}{ Description/Comments } & \multicolumn{2}{|c|}{$\begin{array}{l}\text { Removable } \\
\mathrm{dpm} / 100 \mathrm{~cm}^{2}\end{array}$} & \multicolumn{2}{|c|}{$\begin{array}{c}\text { Fixed + Removable } \\
\mathrm{dpm} / 100 \mathrm{~cm}^{2}\end{array}$} & \multirow{2}{*}{$\begin{array}{l}\text { Gamma } \\
\mathrm{mrem} / \mathrm{hr}\end{array}$} & \multirow{2}{*}{$\begin{array}{l}\text { Neutron } \\
\mathrm{mrem} / \mathrm{hr}\end{array}$} & \multirow{2}{*}{$\begin{array}{c}\text { Total } \\
\text { mrem } / \mathrm{hr}\end{array}$} \\
\hline & & Alpha & Beta & Alpha & Beta & & & \\
\hline 12 & East Wall, Concrete (Electra 1504) & $\mathrm{N} / \mathrm{A}$ & N/A & 5 & 406 & N/A & $\mathrm{N} / \mathrm{A}$ & $N / A$ \\
\hline 13 & East Wall, Concrete (Electra 1504) & $\mathrm{N} / \mathrm{A}$ & $\mathrm{N} / \mathrm{A}$ & 5 & 661 & N/A & N/A & $\mathrm{N} / \mathrm{A}$ \\
\hline 14 & East Wall, Concrete (Electra 1120) & N/A & $\mathrm{N} / \mathrm{A}$ & 4 & 640 & N/A & $\mathrm{N} / \mathrm{A}$ & N/A \\
\hline 15 & East Wall, Concrete (Electra 1120) & $\mathrm{N} / \mathrm{A}$ & N/A & 9 & 703 & $\mathrm{~N} / \mathrm{A}$ & N/A & N/A \\
\hline 16 & East Wall, Concrete (Electra 1120) & N/A & N/A & 11 & 749 & N/A & N/A & $\mathrm{N} / \mathrm{A}$ \\
\hline $\mathrm{N} / \mathrm{A}$ & $N / A$ & $\mathrm{~N} / \mathrm{A}$ & N/A & $\mathrm{N} / \mathrm{A}$ & N/A & N/A & N/A & $\mathrm{N} / \mathrm{A}$ \\
\hline N/A & ${ }^{\star \star}$ Performed scan surveys for both alpha and beta & N/A & N/A & N/A & N/A & N/A & N/A & N/A \\
\hline N/A & ${ }^{* *}$ on $100 \%$ of survey unit. No trigger levels exceeded ${ }^{* *}$ & $\mathrm{~N} / \mathrm{A}$ & $\mathrm{N} / \mathrm{A}$ & $\mathrm{N} / \mathrm{A}$ & $\mathrm{N} / \mathrm{A}$ & N/A & $\mathrm{N} / \mathrm{A}$ & N/A \\
\hline N/A & $N / A$ & N/A & N/A & N/A & N/A & $\mathrm{N} / \mathrm{A}$ & $\mathrm{N} / \mathrm{A}$ & $\mathrm{N} / \mathrm{A}$ \\
\hline N/A & $N / A$ & N/A & $\mathrm{N} / \mathrm{A}$ & N/A & $\mathrm{N} / \mathrm{A}$ & N/A & $\mathrm{N} / \mathrm{A}$ & N/A \\
\hline N/A & $N / A$ & $\mathrm{~N} / \mathrm{A}$ & $\mathrm{N} / \mathrm{A}$ & $\mathrm{N} / \mathrm{A}$ & N/A & $\mathrm{N} / \mathrm{A}$ & N/A & $\mathrm{N} / \mathrm{A}$ \\
\hline N/A & $\mathrm{N} / \mathrm{A}$ & $\mathrm{N} / \mathrm{A}$ & N/A & $\mathrm{N} / \mathrm{A}$ & N/A & N/A & N/A & N/A \\
\hline $\mathrm{N} / \mathrm{A}$ & $\mathrm{N} / \mathrm{A}$ & $\mathrm{N} / \mathrm{A}$ & $\mathrm{N} / \mathrm{A}$ & $\mathrm{N} / \mathrm{A}$ & $\mathrm{N} / \mathrm{A}$ & N/A & $\mathrm{N} / \mathrm{A}$ & N/A \\
\hline $\mathrm{N} / \mathrm{A}$ & $\mathrm{N} / \mathrm{A}$ & $\mathrm{N} / \mathrm{A}$ & N/A & $\mathrm{N} / \mathrm{A}$ & $\mathrm{N} / \mathrm{A}$ & $\mathrm{N} / \mathrm{A}$ & $\mathrm{N} / \mathrm{A}$ & $\mathrm{N} / \mathrm{A}$ \\
\hline N/A & $\mathrm{N} / \mathrm{A}$ & N/A & N/A & $\mathrm{N} / \mathrm{A}$ & N/A & N/A & $\mathrm{N} / \mathrm{A}$ & $\mathrm{N} / \mathrm{A}$ \\
\hline N/A & $N / A$ & $\mathrm{~N} / \mathrm{A}$ & N/A & $\mathrm{N} / \mathrm{A}$ & N/A & N/A & N/A & $\mathrm{N} / \mathrm{A}$ \\
\hline N/A & $N / A$ & $\mathrm{~N} / \mathrm{A}$ & $\mathrm{N} / \mathrm{A}$ & $\mathrm{N} / \mathrm{A}$ & $\mathrm{N} / \mathrm{A}$ & N/A & $\mathrm{N} / \mathrm{A}$ & $\mathrm{N} / \mathrm{A}$ \\
\hline N/A & $N / A$ & $\mathrm{~N} / \mathrm{A}$ & N/A & N/A & $\mathrm{N} / \mathrm{A}$ & N/A & $\mathrm{N} / \mathrm{A}$ & N/A \\
\hline N/A & $N / A$ & $\mathrm{~N} / \mathrm{A}$ & $\mathrm{N} / \mathrm{A}$ & $\mathrm{N} / \mathrm{A}$ & N/A & N/A & N/A & $\mathrm{N} / \mathrm{A}$ \\
\hline N/A & $N / A$ & N/A & $\mathrm{N} / \mathrm{A}$ & N/A & N/A & N/A & N/A & $\mathrm{N} / \mathrm{A}$ \\
\hline N/A & $\mathrm{N} / \mathrm{A}$ & N/A & $\mathrm{N} / \mathrm{A}$ & $\mathrm{N} / \mathrm{A}$ & N/A & N/A & $\mathrm{N} / \mathrm{A}$ & $\mathrm{N} / \mathrm{A}$ \\
\hline N/A & $N / A$ & $\mathrm{~N} / \mathrm{A}$ & $\mathrm{N} / \mathrm{A}$ & $\mathrm{N} / \mathrm{A}$ & N/A & N/A & N/A & N/A \\
\hline N/A & $N / A$ & $\mathrm{~N} / \mathrm{A}$ & N/A & N/A & $\mathrm{N} / \mathrm{A}$ & N/A & N/A & N/A \\
\hline
\end{tabular}


Survey $=10-E R-A 26-218$

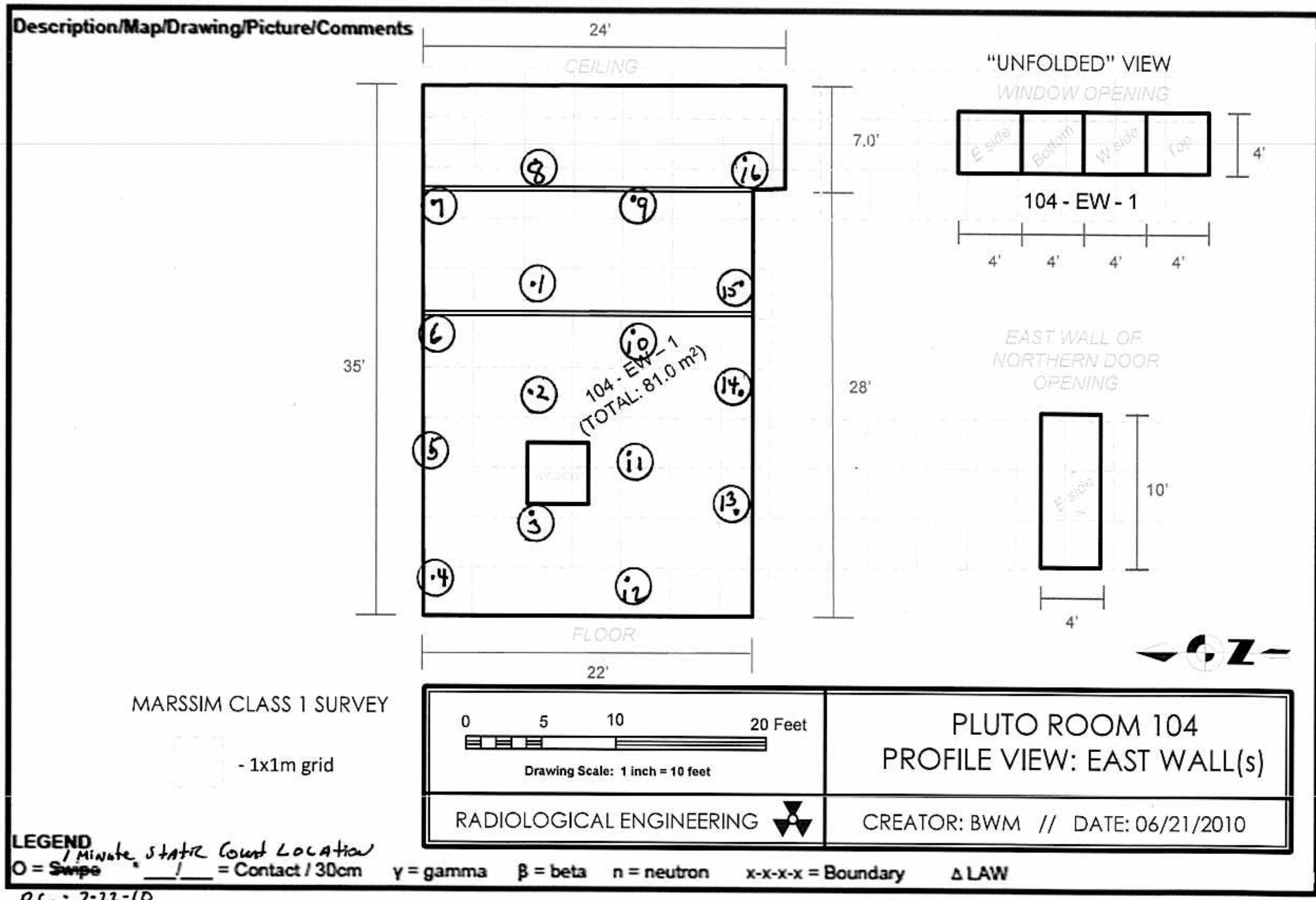




\begin{tabular}{|c|c|c|c|c|c|c|c|c|c|c|c|}
\hline \multicolumn{3}{|c|}{$\begin{array}{l}\text { Location: } \\
\text { Area } 26 \text { CAU117 Pluto }\end{array}$} & \multicolumn{2}{|c|}{$\begin{array}{l}\text { Purpose: } \\
\text { Final Status Survey, Room 104, } \\
\text { Floor }\end{array}$} & \multicolumn{5}{|c|}{$\begin{array}{l}\text { Comments: } \\
\text { All one minute static counts are in cpm. Bkgd not subtracted } \\
\text { for Final Status Survey, Survey Plan \#10-015 }\end{array}$} & \multicolumn{2}{|c|}{$\begin{array}{l}\text { Date/Time: } \\
\text { 07/14/2010 } 1400\end{array}$} \\
\hline Instrument: & Serial \#: & Cal Due: & $\begin{array}{c}\text { Eff in \%: } \\
\text { Alpha / Beta }\end{array}$ & $\begin{array}{l}\text { BKG in dpm: } \\
\text { Alpha / Beta }\end{array}$ & $\begin{array}{l}\text { MDA in dpm: } \\
\text { Alpha / Beta }\end{array}$ & RWP\# & \multicolumn{5}{|l|}{$N / A$} \\
\hline Electra & 1504 & $4 / 6 / 2011$ & $12.9 / 19.8$ & $N / A$ & $N / A$ & \multicolumn{6}{|c|}{ ENV-10-RCT ENV SITE SURVEYS-0013 } \\
\hline Electra & 2125 & $4 / 6 / 2011$ & $14.4 / 18.2$ & $N / A$ & $N / A$ & \multicolumn{3}{|c|}{ RCT Name: D.Cotroneo Jr. } & \multicolumn{3}{|c|}{ Signature: $\theta-\varepsilon$} \\
\hline$N / A$ & $\mathrm{~N} / \mathrm{A}$ & $N / A$ & $N / A$ & $N / A$ & $N / A$ & \multicolumn{3}{|c|}{ RCT Name: Kevin Wilcox } & \multicolumn{3}{|c|}{ Signature: Kesi W. Pon } \\
\hline $\mathrm{N} / \mathrm{A}$ & $\mathrm{N} / \mathrm{A}$ & $\mathrm{N} / \mathrm{A}$ & $N / A$ & $N / A$ & $N / A$ & \multicolumn{3}{|c|}{ RCT Name: Steve Munns } & \multicolumn{3}{|c|}{ Signature: } \\
\hline$N / A$ & $N / A$ & $N / A$ & $N / A$ & $N / A$ & $N / A$ & \multicolumn{3}{|c|}{ RCT Name: $\mathrm{N} / \mathrm{A}$} & \multicolumn{3}{|l|}{ Signature: } \\
\hline \multirow[t]{2}{*}{ Survey Point } & \multirow{2}{*}{\multicolumn{4}{|c|}{ Description/Comments }} & \multicolumn{2}{|c|}{$\begin{array}{l}\text { Removable } \\
\mathrm{dpm} / 100 \mathrm{~cm}^{2}\end{array}$} & \multicolumn{2}{|c|}{$\begin{array}{c}\text { Fixed + Removable } \\
\mathrm{dpm} / 100 \mathrm{~cm}^{2}\end{array}$} & \multirow{2}{*}{$\begin{array}{l}\text { Gamma } \\
\mathrm{mrem} / \mathrm{hr}\end{array}$} & \multirow{2}{*}{$\begin{array}{l}\text { Neutron } \\
\mathrm{mrem} / \mathrm{hr}\end{array}$} & \multirow{2}{*}{$\begin{array}{l}\text { Total } \\
\mathrm{mrem} / \mathrm{hr}\end{array}$} \\
\hline & & & & & Alpha & Beta & Alpha & Beta & & & \\
\hline 1 & \multicolumn{4}{|c|}{ Floor, Metal (Electra 1504) } & $N / A$ & $N / A$ & 4 & 611 & $N / A$ & $N / A$ & $N / A$ \\
\hline 2 & \multicolumn{4}{|c|}{ Floor, Concrete (Electra 1504) } & $\mathrm{N} / \mathrm{A}$ & $N / A$ & 2 & 1023 & $\mathrm{~N} / \mathrm{A}$ & $N / A$ & $N / A$ \\
\hline 3 & \multicolumn{4}{|c|}{ Floor, Concrete (Electra 1504) } & $N / A$ & $N / A$ & 3 & 1008 & $N / A$ & $N / A$ & $N / A$ \\
\hline 4 & \multicolumn{4}{|c|}{ Floor, Concrete (Electra 1504) } & $N / A$ & $N / A$ & 8 & 915 & $N / A$ & $N / A$ & $N / A$ \\
\hline 5 & \multicolumn{4}{|c|}{ Floor, Concrete (Electra 2125) } & $N / A$ & $N / A$ & 1 & 973 & $N / A$ & $N / A$ & $N / A$ \\
\hline 6 & \multicolumn{4}{|c|}{ Floor, Concrete (Electra 2125) } & $N / A$ & $N / A$ & 3 & 890 & $N / A$ & $N / A$ & $N / A$ \\
\hline 7 & \multicolumn{4}{|c|}{ Floor, Concrete (Electra 2125) } & $N / A$ & $N / A$ & 10 & 996 & $\mathrm{~N} / \mathrm{A}$ & $N / A$ & $N / A$ \\
\hline 8 & \multicolumn{4}{|c|}{ Floor, Concrete (Electra 2125) } & $N / A$ & $N / A$ & 6 & 1014 & $N / A$ & $N / A$ & $N / A$ \\
\hline 9 & \multicolumn{4}{|c|}{ Floor, Concrete (Electra 2125) } & $N / A$ & $N / A$ & 1 & 943 & $\mathrm{~N} / \mathrm{A}$ & $N / A$ & $N / A$ \\
\hline 10 & \multicolumn{4}{|c|}{ Floor, Concrete (Electra 2125) } & $\mathrm{N} / \mathrm{A}$ & $N / A$ & 2 & 712 & $N / A$ & $N / A$ & $\mathrm{~N} / \mathrm{A}$ \\
\hline 11 & \multicolumn{4}{|c|}{ Floor, Concrete (Electra 1504) } & $N / A$ & $N / A$ & 6 & 1046 & $N / A$ & $N / A$ & $N / A$ \\
\hline \multicolumn{5}{|c|}{ Reviewed By (Print): } & Signature: & & & & & Date: $7 / 2$ & \\
\hline
\end{tabular}


SURVEY \# 10-ER-A26-206

\begin{tabular}{|c|c|c|c|c|c|c|c|c|}
\hline \multirow{2}{*}{$\begin{array}{l}\text { Survey } \\
\text { Point }\end{array}$} & \multirow{2}{*}{ Description/Comments } & \multicolumn{2}{|c|}{$\begin{array}{l}\text { Removable } \\
\mathrm{dpm} / 100 \mathrm{~cm}^{2}\end{array}$} & \multicolumn{2}{|c|}{$\begin{array}{l}\text { Fixed + Removable } \\
\mathrm{dpm} / 100 \mathrm{~cm}^{2}\end{array}$} & \multirow{2}{*}{$\begin{array}{l}\text { Gamma } \\
\mathrm{mrem} / \mathrm{hr}\end{array}$} & \multirow{2}{*}{$\begin{array}{l}\text { Neutron } \\
\mathrm{mrem} / \mathrm{hr}\end{array}$} & \multirow{2}{*}{$\begin{array}{c}\text { Total } \\
\text { mrem } / \mathrm{hr}\end{array}$} \\
\hline & & Alpha & Beta & Alpha & Beta & & & \\
\hline 12 & Floor, Metal (Electra 1504) & N/A & N/A & 7 & 736 & N/A & N/A & $\mathrm{N} / \mathrm{A}$ \\
\hline 13 & Floor, Metal (Electra 1504) & N/A & N/A & 4 & 645 & $\mathrm{~N} / \mathrm{A}$ & N/A & $\mathrm{N} / \mathrm{A}$ \\
\hline 14 & Floor, Concrete (Electra 1504) & N/A & $\mathrm{N} / \mathrm{A}$ & 5 & 887 & N/A & N/A & N/A \\
\hline 15 & Floor, Concrete (Electra 1504) & N/A & $\mathrm{N} / \mathrm{A}$ & 4 & 928 & N/A & N/A & N/A \\
\hline 16 & Floor, Concrete (Electra 2125) & N/A & N/A & 9 & 945 & N/A & N/A & N/A \\
\hline 17 & Floor, Concrete (Electra 2125) & N/A & N/A & 4 & 892 & $\mathrm{~N} / \mathrm{A}$ & N/A & $\mathrm{N} / \mathrm{A}$ \\
\hline 18 & Floor, Concrete (Electra 2125) & N/A & N/A & 11 & 901 & $\mathrm{~N} / \mathrm{A}$ & N/A & $\mathrm{N} / \mathrm{A}$ \\
\hline N/A & $N / A$ & N/A & N/A & N/A & N/A & N/A & N/A & N/A \\
\hline N/A & ${ }^{\star \star P}$ erformed scan surveys for both alpha and beta** & N/A & N/A & N/A & N/A & N/A & $N / A$ & N/A \\
\hline N/A & ${ }^{\star *}$ on $100 \%$ of survey unit. No trigger levels exceeded ${ }^{\star \star}$ & N/A & N/A & N/A & N/A & N/A & N/A & N/A \\
\hline N/A & $\mathrm{N} / \mathrm{A}$ & N/A & N/A & N/A & N/A & N/A & $\mathrm{N} / \mathrm{A}$ & N/A \\
\hline N/A & N/A & N/A & N/A & N/A & N/A & N/A & $\mathrm{N} / \mathrm{A}$ & N/A \\
\hline $\mathrm{N} / \mathrm{A}$ & $N / A$ & N/A & N/A & N/A & N/A & N/A & N/A & N/A \\
\hline $\mathrm{N} / \mathrm{A}$ & $\mathrm{N} / \mathrm{A}$ & N/A & N/A & N/A & $\mathrm{N} / \mathrm{A}$ & N/A & N/A & N/A \\
\hline N/A & $N / A$ & N/A & N/A & N/A & N/A & $\mathrm{N} / \mathrm{A}$ & N/A & N/A \\
\hline $\mathrm{N} / \mathrm{A}$ & $N / A$ & N/A & $\mathrm{N} / \mathrm{A}$ & N/A & $\mathrm{N} / \mathrm{A}$ & N/A & N/A & $\mathrm{N} / \mathrm{A}$ \\
\hline $\mathrm{N} / \mathrm{A}$ & $N / A$ & N/A & $\mathrm{N} / \mathrm{A}$ & N/A & N/A & N/A & N/A & $\mathrm{N} / \mathrm{A}$ \\
\hline N/A & $\mathrm{N} / \mathrm{A}$ & N/A & N/A & N/A & $\mathrm{N} / \mathrm{A}$ & N/A & N/A & $\mathrm{N} / \mathrm{A}$ \\
\hline N/A & $\mathrm{N} / \mathrm{A}$ & N/A & N/A & N/A & $N / A$ & N/A & N/A & N/A \\
\hline N/A & $\mathrm{N} / \mathrm{A}$ & N/A & $\mathrm{N} / \mathrm{A}$ & N/A & N/A & N/A & $\mathrm{N} / \mathrm{A}$ & $\mathrm{N} / \mathrm{A}$ \\
\hline N/A & $\mathrm{N} / \mathrm{A}$ & $\mathrm{N} / \mathrm{A}$ & N/A & N/A & N/A & N/A & $\mathrm{N} / \mathrm{A}$ & $\mathrm{N} / \mathrm{A}$ \\
\hline N/A & $N / A$ & N/A & $\mathrm{N} / \mathrm{A}$ & N/A & $\mathrm{N} / \mathrm{A}$ & N/A & N/A & N/A \\
\hline N/A & $N / A$ & N/A & $\mathrm{N} / \mathrm{A}$ & N/A & $\mathrm{N} / \mathrm{A}$ & N/A & N/A & N/A \\
\hline
\end{tabular}


NSTec

Survey $\# 10-E R-A 26.206$

Description/Map/Drawing/Picture/Comments

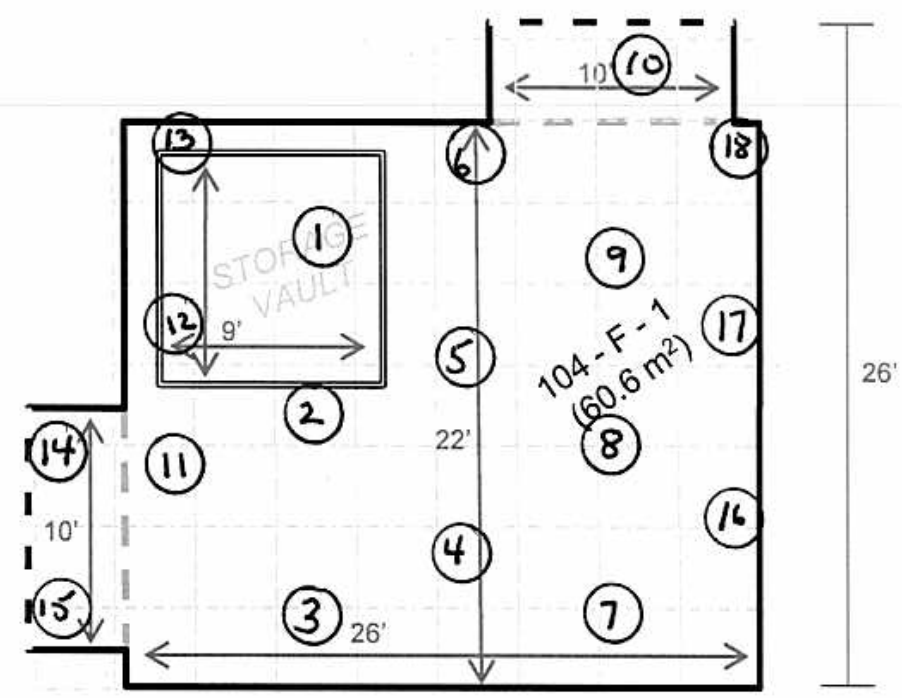

MARSSIM CLASS I SURVEY

$1 \times 1 m$ grid

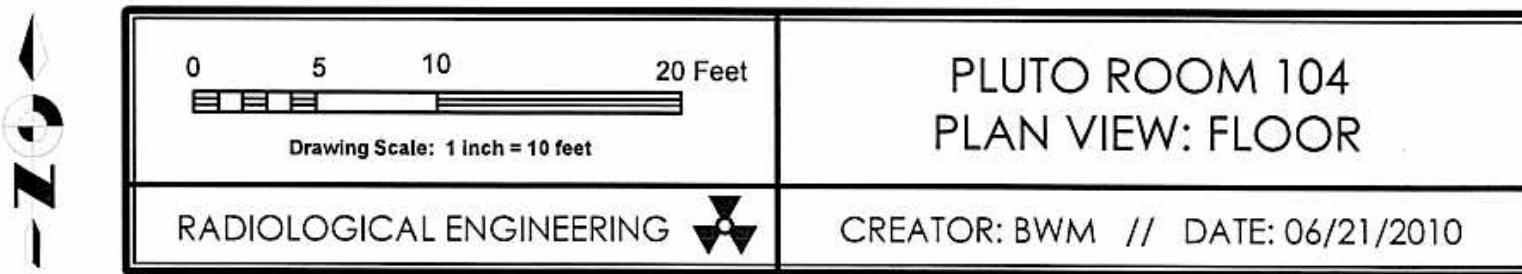

LEGEND

$0=$ Swipe $\quad 11=\operatorname{Contact~} 130 \mathrm{~cm}$

$y=$ gamma $\quad \beta=$ beta $n=$ neutron

$x-x-x-x=$ Boundary

$\triangle L A W$ 


\begin{tabular}{|c|c|c|c|c|c|c|c|c|c|c|c|}
\hline \multicolumn{3}{|c|}{$\begin{array}{l}\text { Location: } \\
\text { Area } 26 \text { CAU117 Pluto }\end{array}$} & \multicolumn{2}{|c|}{$\begin{array}{l}\text { Purpose: } \\
\text { Final Status Survey, Room } 104 \\
\text { North Wall, Unit \# 104-NW-1 }\end{array}$} & \multicolumn{5}{|c|}{$\begin{array}{l}\text { Comments: } \\
\text { All one minute static counts are in cpm. Bkgd not subtracted } \\
\text { for Final Status Survey, Survey Plan \#10-015 }\end{array}$} & \multicolumn{2}{|c|}{$\begin{array}{l}\text { Date/Time: } \\
\text { 07/21/2010 } 1400\end{array}$} \\
\hline Instrument: & Serial \#: & Cal Due: & $\begin{array}{c}\text { Eff in \%: } \\
\text { Alpha / Beta }\end{array}$ & $\begin{array}{l}\text { BKG in dpm: } \\
\text { Alpha / Beta }\end{array}$ & $\begin{array}{l}\text { MDA in dpm: } \\
\text { Alpha / Beta }\end{array}$ & RWP\# & \multicolumn{3}{|l|}{$\mathrm{N} / \mathrm{A}$} & & \\
\hline Electra & 1504 & $4 / 6 / 2011$ & $12.9 / 19.8$ & $\mathrm{~N} / \mathrm{A}$ & $N / A$ & \multicolumn{6}{|c|}{ Work Package \# $\quad$ ENV-10-RCT ENV SITE SURVEYS-0013 } \\
\hline $\mathrm{N} / \mathrm{A}$ & $\mathrm{N} / \mathrm{A}$ & $N / A$ & $N / A$ & $N / A$ & $N / A$ & \multicolumn{3}{|c|}{ RCT Name: D.Cotroneo Jr. } & Signature: & & \\
\hline$N / A$ & $\mathrm{~N} / \mathrm{A}$ & $N / A$ & $N / A$ & $N / A$ & $\mathrm{~N} / \mathrm{A}$ & \multicolumn{3}{|c|}{ RCT Name: Kevin Wilcox } & \multicolumn{3}{|c|}{ Signature: Kein W'Rese } \\
\hline$N / A$ & $N / A$ & $N / A$ & $N / A$ & $N / A$ & $N / A$ & \multicolumn{3}{|c|}{ RCT Name: S. Brown/B. Bunce } & \multirow{2}{*}{\multicolumn{3}{|c|}{ Signatures, $3+18+23 n$}} \\
\hline $\mathrm{N} / \mathrm{A}$ & $\mathrm{N} / \mathrm{A}$ & $N / A$ & $\mathrm{~N} / \mathrm{A}$ & $\mathrm{N} / \mathrm{A}$ & $N / A$ & RCT Name: & Brian Fos & & & & \\
\hline \multirow[t]{2}{*}{ Survey Point } & \multirow{2}{*}{\multicolumn{4}{|c|}{ Description/Comments }} & \multicolumn{2}{|c|}{$\begin{array}{l}\text { Removable } \\
\mathrm{dpm} / 100 \mathrm{~cm}^{2}\end{array}$} & \multicolumn{2}{|c|}{\begin{tabular}{|c|}
$\begin{array}{c}\text { Fixed + Removable } \\
\mathrm{dpm} / 100 \mathrm{~cm}^{2}\end{array}$ \\
\end{tabular}} & \multirow{2}{*}{$\begin{array}{l}\text { Gamma } \\
\mathrm{mrem} / \mathrm{hr}\end{array}$} & \multirow{2}{*}{$\begin{array}{l}\text { Neutron } \\
\text { mrem/hr }\end{array}$} & \multirow[t]{2}{*}{$\begin{array}{l}\text { Total } \\
\mathrm{mrem} / \mathrm{hr}\end{array}$} \\
\hline & & & & & Alpha & Beta & Alpha & Beta & & & \\
\hline 1 & \multicolumn{4}{|c|}{ North Wall, Concrete } & $N / A$ & $N / A$ & 4 & 896 & $N / A$ & $N / A$ & $N / A$ \\
\hline 2 & \multicolumn{4}{|c|}{ North Wall, Concrete } & $N / A$ & $N / A$ & 6 & 675 & $N / A$ & $N / A$ & $N / A$ \\
\hline 3 & \multicolumn{4}{|c|}{ North Wall, Concrete } & $N / A$ & $N / A$ & 2 & 609 & $N / A$ & $N / A$ & $N / A$ \\
\hline 4 & \multicolumn{4}{|c|}{ North Wall, Concrete } & $N / A$ & $N / A$ & 7 & 576 & $N / A$ & $N / A$ & $N / A$ \\
\hline 5 & \multicolumn{4}{|c|}{ North Wall, Concrete } & $N / A$ & $N / A$ & 6 & 636 & $N / A$ & $N / A$ & $N / A$ \\
\hline 6 & \multicolumn{4}{|c|}{ North Wall, Concrete } & $N / A$ & $N / A$ & 5 & 675 & $N / A$ & $N / A$ & $N / A$ \\
\hline 7 & \multicolumn{4}{|c|}{ North Wall, Concrete } & $N / A$ & $N / A$ & 6 & 922 & $N / A$ & $N / A$ & $N / A$ \\
\hline 8 & \multicolumn{4}{|c|}{ North Wall, Concrete } & $N / A$ & $N / A$ & 7 & 612 & $N / A$ & $N / A$ & $N / A$ \\
\hline 9 & \multicolumn{4}{|c|}{ North Wall, Concrete } & $N / A$ & $N / A$ & 9 & 661 & $N / A$ & $\mathrm{~N} / \mathrm{A}$ & $N / A$ \\
\hline 10 & \multicolumn{4}{|c|}{ North Wall, Concrete } & $N / A$ & $N / A$ & 7 & 644 & $N / A$ & $N / A$ & $N / A$ \\
\hline 11 & \multicolumn{4}{|c|}{ North Wall, Concrete } & $N / A$ & $N / A$ & 6 & 867 & $N / A$ & $N / A$ & $N / A$ \\
\hline \multicolumn{5}{|c|}{ Reviewed By (Print): } & \multicolumn{3}{|l|}{ Signature: } & & & \multicolumn{2}{|c|}{ Date: $7 / 27 / 10$} \\
\hline
\end{tabular}


SURVEY \# 10-ER-A26-213

\begin{tabular}{|c|c|c|c|c|c|c|c|c|}
\hline \multirow{2}{*}{$\begin{array}{l}\text { Survey } \\
\text { Point }\end{array}$} & \multirow[t]{2}{*}{ Description/Comments } & \multicolumn{2}{|c|}{$\begin{array}{l}\text { Removable } \\
\mathrm{dpm} / 100 \mathrm{~cm}^{2}\end{array}$} & \multicolumn{2}{|c|}{$\begin{array}{c}\text { Fixed + Removable } \\
\mathrm{dpm} / 100 \mathrm{~cm}^{2}\end{array}$} & \multirow{2}{*}{$\begin{array}{l}\text { Gamma } \\
\mathrm{mrem} / \mathrm{hr}\end{array}$} & \multirow{2}{*}{$\begin{array}{l}\text { Neutron } \\
\text { mrem/hr }\end{array}$} & \multirow{2}{*}{$\begin{array}{c}\text { Total } \\
\text { mrem } / \mathrm{hr}\end{array}$} \\
\hline & & Alpha & Beta & Alpha & Beta & & & \\
\hline 12 & North Wall, Concrete & N/A & $N / A$ & 7 & 924 & N/A & N/A & N/A \\
\hline 13 & North Wall, Concrete & N/A & N/A & 6 & 1044 & N/A & N/A & N/A \\
\hline 14 & North Wall, Concrete & N/A & N/A & 6 & 887 & N/A & N/A & N/A \\
\hline 15 & North Wall, Concrete & N/A & N/A & 6 & 980 & N/A & N/A & N/A \\
\hline 16 & North Wall, Concrete & N/A & N/A & 7 & 635 & N/A & N/A & N/A \\
\hline 17 & North Wall, Concrete $65 \%$, Metal $35 \%$ & N/A & N/A & 2 & 560 & N/A & N/A & N/A \\
\hline N/A & $N / A$ & N/A & N/A & N/A & N/A & N/A & N/A & $\mathrm{N} / \mathrm{A}$ \\
\hline N/A & **Performed scan surveys for both alpha and beta** & N/A & N/A & N/A & N/A & N/A & N/A & N/A \\
\hline N/A & ${ }^{\star \star}$ on $100 \%$ of survey unit. No trigger levels exceeded ${ }^{\star \star}$ & N/A & N/A & N/A & N/A & N/A & N/A & N/A \\
\hline N/A & $N / A$ & N/A & N/A & N/A & N/A & N/A & N/A & N/A \\
\hline N/A & $N / A$ & N/A & N/A & N/A & N/A & N/A & N/A & N/A \\
\hline N/A & $N / A$ & N/A & N/A & N/A & N/A & N/A & N/A & N/A \\
\hline N/A & $N / A$ & N/A & N/A & N/A & N/A & N/A & N/A & N/A \\
\hline N/A & $N / A$ & N/A & N/A & N/A & N/A & N/A & N/A & N/A \\
\hline N/A & $N / A$ & N/A & N/A & N/A & N/A & N/A & N/A & N/A \\
\hline N/A & $N / A$ & N/A & N/A & N/A & N/A & N/A & N/A & N/A \\
\hline N/A & $N / A$ & N/A & N/A & N/A & N/A & N/A & N/A & N/A \\
\hline N/A & $N / A$ & N/A & N/A & N/A & N/A & N/A & N/A & N/A \\
\hline N/A & $N / A$ & N/A & N/A & N/A & N/A & N/A & N/A & N/A \\
\hline N/A & $N / A$ & N/A & N/A & N/A & N/A & $\mathrm{N} / \mathrm{A}$ & N/A & N/A \\
\hline N/A & $N / A$ & N/A & N/A & N/A & N/A & N/A & N/A & N/A \\
\hline N/A & $N / A$ & N/A & N/A & N/A & N/A & N/A & N/A & N/A \\
\hline N/A & $N / A$ & N/A & N/A & N/A & N/A & N/A & N/A & N/A \\
\hline
\end{tabular}


Survey $\# 10-E R-A 26-213$

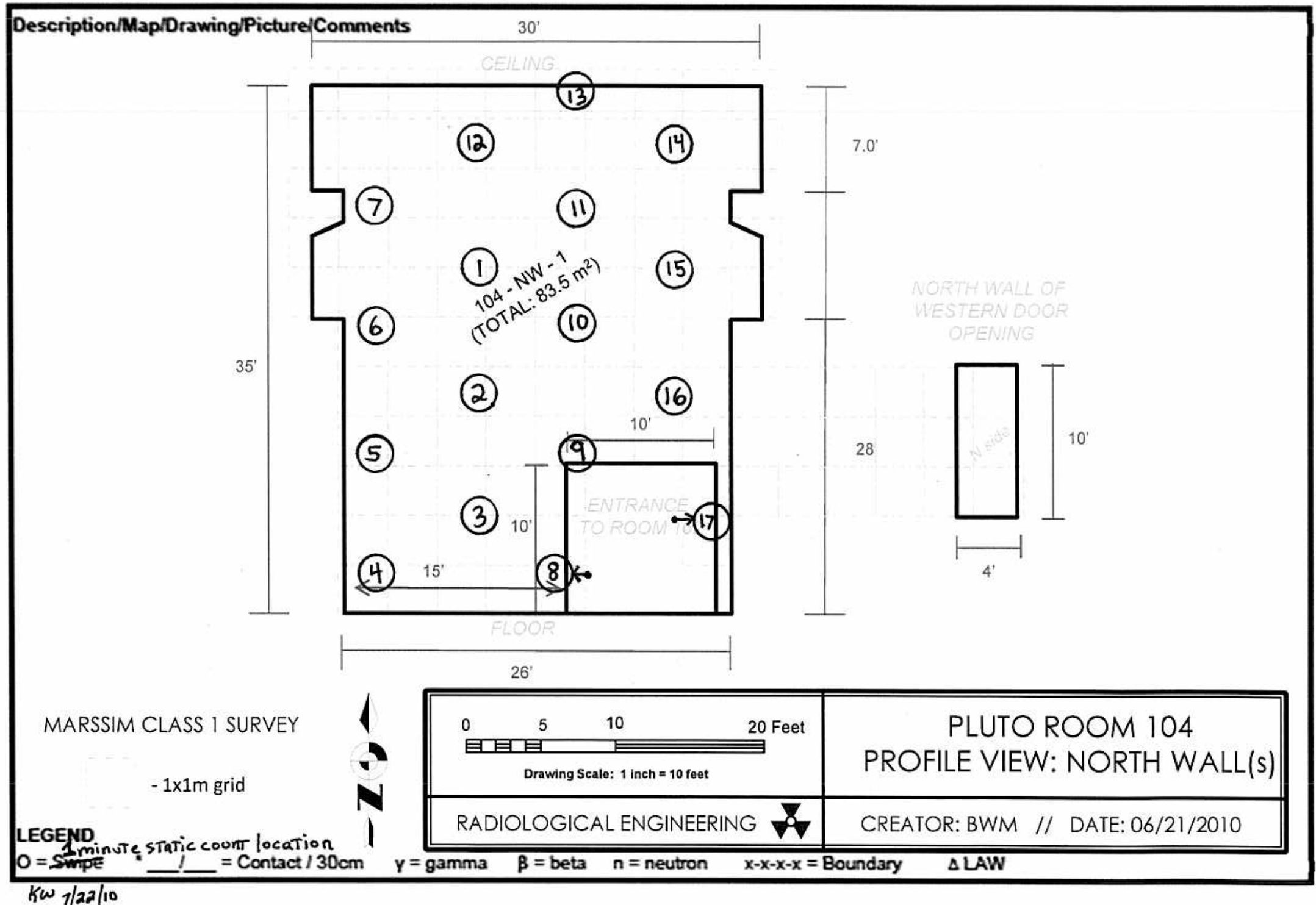


SURVEY \#

10-ER-A26-215

\begin{tabular}{|c|c|c|c|c|c|c|c|c|c|c|c|}
\hline \multicolumn{3}{|c|}{$\begin{array}{l}\text { Location: } \\
\text { Area } 26 \text { CAU117 Pluto }\end{array}$} & \multicolumn{2}{|c|}{$\begin{array}{l}\text { Purpose: } \\
\text { Final Status Survey, Room } 104 \\
\text { South Wall, Unit \# 104-SW-1 }\end{array}$} & \multicolumn{5}{|c|}{$\begin{array}{l}\text { Comments: } \\
\text { All one minute static counts are in cpm. Bkgd not subtracted } \\
\text { for Final Status Survey, Survey Plan \#10-015 }\end{array}$} & \multicolumn{2}{|c|}{$\begin{array}{l}\text { Date/Time: } \\
\text { 07/22/2010 } 1100\end{array}$} \\
\hline Instrument: & Serial \#: & Cal Due: & \begin{tabular}{|c|} 
Eff in \%: \\
Alpha / Beta
\end{tabular} & $\begin{array}{l}\text { BKG in dpm: } \\
\text { Alpha / Beta }\end{array}$ & \begin{tabular}{|l|} 
MDA in dpm: \\
Alpha / Beta
\end{tabular} & RWP\# & \multicolumn{3}{|l|}{$N / A$} & & \\
\hline Electra & 1504 & $4 / 6 / 2011$ & $12.9 / 19.8$ & $N / A$ & $N / A$ & \multicolumn{6}{|c|}{ 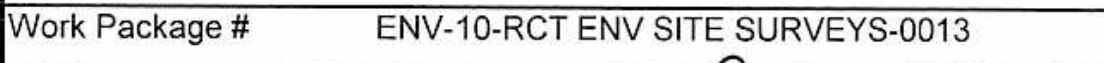 } \\
\hline Electra & 1120 & $4 / 6 / 2011$ & $14.1 / 20.8$ & $N / A$ & $N / A$ & \multicolumn{3}{|c|}{ RCT Name: D.Cotroneo Jr. } & \multirow{2}{*}{\multicolumn{3}{|c|}{ Signature: }} \\
\hline Electra & 4806 & $4 / 6 / 2011$ & $13.2 / 20.9$ & $N / A$ & $N / A$ & \multicolumn{3}{|c|}{ RCT Name: Kevin Wilcox } & & & \\
\hline$N / A$ & $N / A$ & $N / A$ & $N / A$ & $N / A$ & $N / A$ & RCT Name: & S. Brown & Bunce & \multirow{2}{*}{\multicolumn{3}{|c|}{ Signaturature: }} \\
\hline$N / A$ & $N / A$ & $N / A$ & $N / A$ & $N / A$ & $N / A$ & RCT Name: & Brian Fos & & & & \\
\hline \multirow[t]{2}{*}{ Survey Point } & \multirow{2}{*}{\multicolumn{4}{|c|}{ Description/Comments }} & \multicolumn{2}{|c|}{$\begin{array}{l}\text { Removable } \\
\mathrm{dpm} / 100 \mathrm{~cm}^{2}\end{array}$} & \multicolumn{2}{|c|}{$\begin{array}{c}\text { Fixed + Removable } \\
\mathrm{dpm} / 100 \mathrm{~cm}^{2}\end{array}$} & \multirow{2}{*}{$\begin{array}{l}\text { Gamma } \\
\mathrm{mrem} / \mathrm{hr}\end{array}$} & \multirow{2}{*}{$\begin{array}{l}\text { Neutron } \\
\mathrm{mrem} / \mathrm{hr}\end{array}$} & \multirow{2}{*}{$\begin{array}{c}\text { Total } \\
\mathrm{mrem} / \mathrm{hr}\end{array}$} \\
\hline & & & & & Alpha & Beta & Alpha & Beta & & & \\
\hline 1 & \multicolumn{4}{|c|}{ South Wall, Concrete (Electra 1120) } & $N / A$ & $N / A$ & 8 & 686 & $N / A$ & $N / A$ & $N / A$ \\
\hline 2 & \multicolumn{4}{|c|}{ South Wall, Concrete $15 \%$, Metal $85 \%$ (Electra 1120 ) } & $N / A$ & $N / A$ & 4 & 571 & $N / A$ & $N / A$ & $N / A$ \\
\hline 3 & \multicolumn{4}{|c|}{ South Wall, Concrete $90 \%$, Metal 10\% (Electra 1504) } & $N / A$ & $N / A$ & 6 & 660 & $N / A$ & $N / A$ & $N / A$ \\
\hline 4 & \multicolumn{4}{|c|}{ South Wall, Concrete (Electra 1504) } & $N / A$ & $N / A$ & 11 & 900 & $N / A$ & $N / A$ & $N / A$ \\
\hline 5 & \multicolumn{4}{|c|}{ South Wall, Concrete (Electra 1504) } & $N / A$ & $N / A$ & 8 & 631 & $N / A$ & $\mathrm{~N} / \mathrm{A}$ & $N / A$ \\
\hline 6 & \multicolumn{4}{|c|}{ South Wall, Concrete (Electra 1120) } & $N / A$ & $N / A$ & 4 & 644 & $N / A$ & $N / A$ & $\mathrm{~N} / \mathrm{A}$ \\
\hline 7 & \multicolumn{4}{|c|}{ South Wall, Concrete (Electra 1120) } & $N / A$ & $N / A$ & 15 & 802 & $N / A$ & $N / A$ & $N / A$ \\
\hline 8 & \multicolumn{4}{|c|}{ South Wall, Concrete (Electra 1120) } & $N / A$ & $N / A$ & 9 & 764 & $N / A$ & $N / A$ & $\mathrm{~N} / \mathrm{A}$ \\
\hline 9 & \multicolumn{4}{|c|}{ South Wall, Concrete (Electra 1120) } & $N / A$ & $N / A$ & 5 & 848 & $N / A$ & $N / A$ & $N / A$ \\
\hline 10 & \multicolumn{4}{|c|}{ South Wall, Concrete (Electra 1120) } & $N / A$ & $N / A$ & 7 & 836 & $N / A$ & $N / A$ & $N / A$ \\
\hline 11 & \multicolumn{4}{|c|}{ South Wall, Concrete (Electra 1120) } & $N / A$ & $N / A$ & 6 & 700 & $N / A$ & $N / A$ & $N / A$ \\
\hline \multicolumn{5}{|c|}{ Reviewed By (Print): } & \multicolumn{7}{|c|}{ Signature: } \\
\hline
\end{tabular}


SURVEY \# 10-ER-A26-215

\begin{tabular}{|c|c|c|c|c|c|c|c|c|}
\hline \multirow{2}{*}{$\begin{array}{l}\text { Survey } \\
\text { Point }\end{array}$} & \multirow[t]{2}{*}{ Description/Comments } & \multicolumn{2}{|c|}{$\begin{array}{l}\text { Removable } \\
\mathrm{dpm} / 100 \mathrm{~cm}^{2}\end{array}$} & \multicolumn{2}{|c|}{$\begin{array}{c}\text { Fixed + Removable } \\
\mathrm{dpm} / 100 \mathrm{~cm}^{2}\end{array}$} & \multirow{2}{*}{$\begin{array}{l}\text { Gamma } \\
\mathrm{mrem} / \mathrm{hr}\end{array}$} & \multirow{2}{*}{$\begin{array}{l}\text { Neutron } \\
\mathrm{mrem} / \mathrm{hr}\end{array}$} & \multirow{2}{*}{$\begin{array}{c}\text { Total } \\
\text { mrem } / \mathrm{hr}\end{array}$} \\
\hline & & Alpha & Beta & Alpha & Beta & & & \\
\hline 12 & South Wall, Concrete (Electra 1120) & N/A & N/A & 6 & 686 & N/A & N/A & $\mathrm{N} / \mathrm{A}$ \\
\hline 13 & South Wall, Concrete (Electra 1504) & N/A & N/A & 5 & 644 & N/A & N/A & $\mathrm{N} / \mathrm{A}$ \\
\hline 14 & South Wall, Concrete (Electra 1504) & $\mathrm{N} / \mathrm{A}$ & N/A & 4 & 781 & N/A & $N / A$ & N/A \\
\hline 15 & South Wall, Concrete (Electra 1504) & $\mathrm{N} / \mathrm{A}$ & N/A & 8 & 706 & N/A & N/A & N/A \\
\hline 16 & South Wall, Concrete (Electra 1120) & N/A & N/A & 8 & 595 & N/A & N/A & N/A \\
\hline 17 & South Wall, Concrete (Electra 1120) & $\mathrm{N} / \mathrm{A}$ & N/A & 10 & 857 & $N / A$ & N/A & N/A \\
\hline 18 & South Wall, Concrete (Electra 1120) & $\mathrm{N} / \mathrm{A}$ & N/A & 13 & 861 & N/A & N/A & $\mathrm{N} / \mathrm{A}$ \\
\hline N/A & $N / A$ & N/A & N/A & N/A & N/A & N/A & $\mathrm{N} / \mathrm{A}$ & N/A \\
\hline N/A & ${ }^{\star \star}$ Performed scan surveys for both alpha and beta** & N/A & N/A & N/A & $\mathrm{N} / \mathrm{A}$ & $\mathrm{N} / \mathrm{A}$ & N/A & N/A \\
\hline $\mathrm{N} / \mathrm{A}$ & ${ }^{\star \star}$ on $100 \%$ of survey unit. No trigger levels exceeded ${ }^{\star \star}$ & N/A & N/A & N/A & $\mathrm{N} / \mathrm{A}$ & N/A & N/A & N/A \\
\hline N/A & $\mathrm{N} / \mathrm{A}$ & N/A & N/A & N/A & $\mathrm{N} / \mathrm{A}$ & N/A & N/A & N/A \\
\hline N/A & $N / A$ & N/A & N/A & N/A & N/A & $N / A$ & N/A & N/A \\
\hline N/A & $N / A$ & N/A & N/A & N/A & N/A & N/A & N/A & $\mathrm{N} / \mathrm{A}$ \\
\hline $\mathrm{N} / \mathrm{A}$ & $N / A$ & N/A & N/A & N/A & N/A & $\mathrm{N} / \mathrm{A}$ & N/A & $\mathrm{N} / \mathrm{A}$ \\
\hline $\mathrm{N} / \mathrm{A}$ & $N / A$ & N/A & N/A & N/A & N/A & N/A & N/A & N/A \\
\hline N/A & $N / A$ & $\mathrm{~N} / \mathrm{A}$ & N/A & N/A & $\mathrm{N} / \mathrm{A}$ & $\mathrm{N} / \mathrm{A}$ & N/A & N/A \\
\hline N/A & $N / A$ & N/A & $\mathrm{N} / \mathrm{A}$ & $\mathrm{N} / \mathrm{A}$ & $\mathrm{N} / \mathrm{A}$ & N/A & N/A & N/A \\
\hline $\mathrm{N} / \mathrm{A}$ & $N / A$ & $\mathrm{~N} / \mathrm{A}$ & $\mathrm{N} / \mathrm{A}$ & N/A & $\mathrm{N} / \mathrm{A}$ & N/A & N/A & N/A \\
\hline N/A & $N / A$ & $\mathrm{~N} / \mathrm{A}$ & $\mathrm{N} / \mathrm{A}$ & N/A & $\mathrm{N} / \mathrm{A}$ & N/A & N/A & N/A \\
\hline N/A & $N / A$ & $\mathrm{~N} / \mathrm{A}$ & N/A & $\mathrm{N} / \mathrm{A}$ & $\mathrm{N} / \mathrm{A}$ & $\mathrm{N} / \mathrm{A}$ & $\mathrm{N} / \mathrm{A}$ & N/A \\
\hline $\mathrm{N} / \mathrm{A}$ & $\mathrm{N} / \mathrm{A}$ & $\mathrm{N} / \mathrm{A}$ & $\mathrm{N} / \mathrm{A}$ & N/A & N/A & N/A & N/A & $\mathrm{N} / \mathrm{A}$ \\
\hline $\mathrm{N} / \mathrm{A}$ & $N / A$ & N/A & N/A & N/A & $\mathrm{N} / \mathrm{A}$ & N/A & N/A & N/A \\
\hline N/A & $N / A$ & N/A & N/A & N/A & N/A & N/A & N/A & N/A \\
\hline
\end{tabular}


NSTec

Form

$05 / 24 / 10$

FRM-0108C

RADIOLOGICAL SURVEY REPORT - FULL MAP

Rev. 01

Survey \# $10-E R-A 26-215$

Page $\underline{3}$ of $\underline{3}$

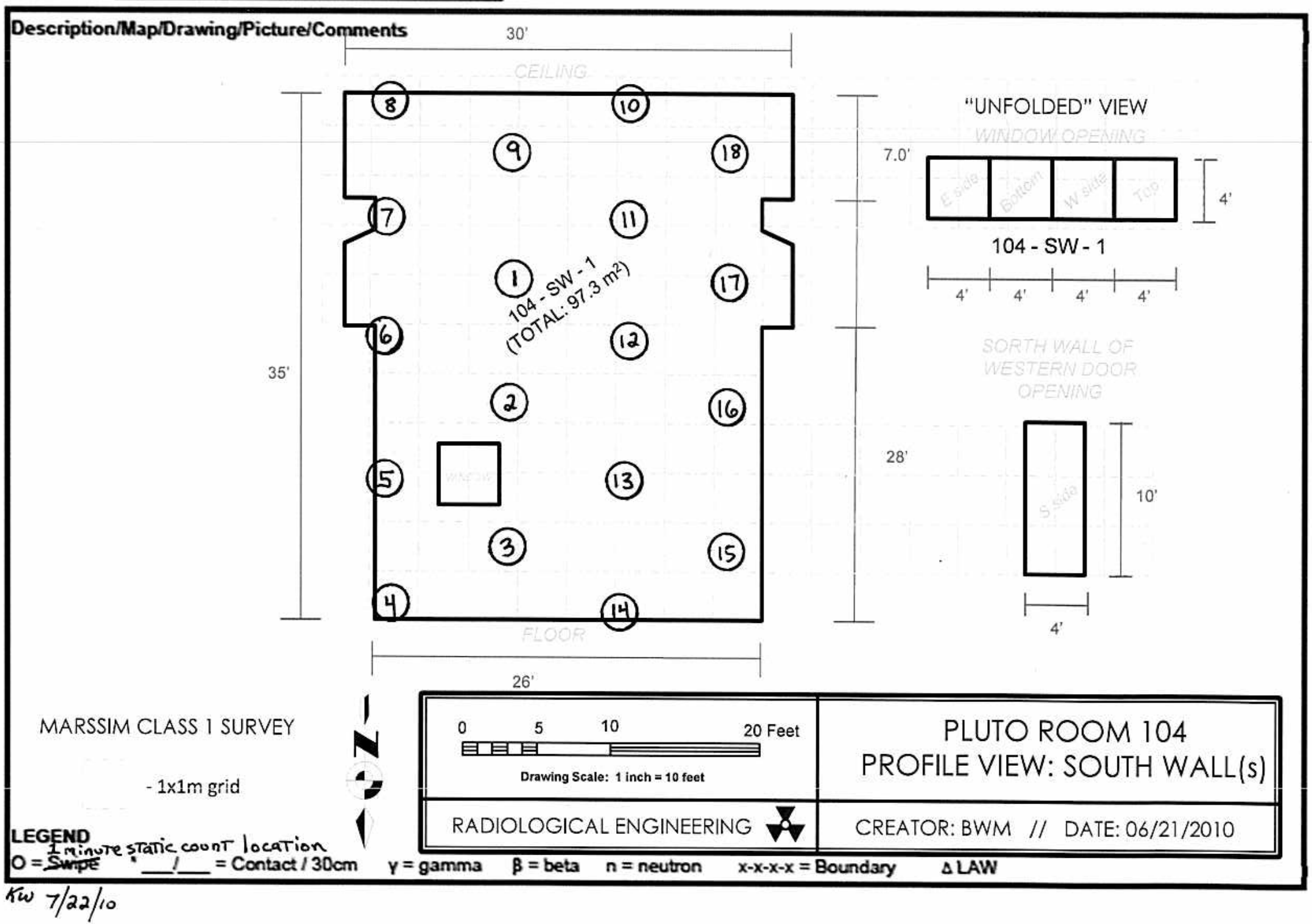


SURVEY \#

10-ER-A26-221

\begin{tabular}{|c|c|c|c|c|c|c|c|c|c|c|c|}
\hline \multicolumn{3}{|c|}{$\begin{array}{l}\text { Location: } \\
\text { Area } 26 \text { CAU117 Pluto }\end{array}$} & \multicolumn{2}{|c|}{$\begin{array}{l}\text { Purpose: } \\
\text { Final Status Survey, Room } 104 \\
\text { West Wall, Unit \# 104-WW-1 }\end{array}$} & \multicolumn{5}{|c|}{$\begin{array}{l}\text { Comments: } \\
\text { All one minute static counts are in cpm. Bkgd not subtracted } \\
\text { for Final Status Survey, Survey Plan \#10-015 }\end{array}$} & \multicolumn{2}{|c|}{$\begin{array}{l}\text { Date/Time: } \\
\text { 07/26/2010 } 1530\end{array}$} \\
\hline Instrument: & Serial \#: & Cal Due: & $\begin{array}{c}\text { Eff in \%: } \\
\text { Alpha / Beta } \\
\end{array}$ & $\begin{array}{l}\text { BKG in dpm: } \\
\text { Alpha / Beta }\end{array}$ & $\begin{array}{l}\text { MDA in dpm: } \\
\text { Alpha / Beta }\end{array}$ & RWP\# & \multicolumn{5}{|l|}{$\mathrm{N} / \mathrm{A}$} \\
\hline Electra & 6697 & $4 / 6 / 2011$ & $13.8 / 19.9$ & $\mathrm{~N} / \mathrm{A}$ & $N / A$ & \multicolumn{4}{|c|}{ Work Package \# } & SURVEYS- & \\
\hline Electra & 1120 & $4 / 6 / 2011$ & $14.1 / 20.8$ & $N / A$ & $\mathrm{~N} / \mathrm{A}$ & \multicolumn{3}{|c|}{ RCT Name: D.Cotroneo Jr. } & Signature: 8 & $\sim^{2}$ & \\
\hline$N / A$ & $N / A$ & $N / A$ & $N / A$ & $N / A$ & $N / A$ & \multicolumn{3}{|c|}{ RCT Name: Kevin Wilcox } & \multicolumn{3}{|c|}{ Signature: Kein WrlenO } \\
\hline$N / A$ & $N / A$ & $N / A$ & $N / A$ & $N / A$ & $N / A$ & \multicolumn{3}{|c|}{ RCT Name: S. Brown/B. Bunce } & \multirow{2}{*}{\multicolumn{3}{|c|}{ Signature: }} \\
\hline $\mathrm{N} / \mathrm{A}$ & $N / A$ & $\mathrm{~N} / \mathrm{A}$ & $\mathrm{N} / \mathrm{A}$ & $N / A$ & $N / A$ & RCT Name: & Brian Fos & & & & \\
\hline \multirow[t]{2}{*}{ Survey Point } & \multirow{2}{*}{\multicolumn{4}{|c|}{ Description/Comments }} & \multicolumn{2}{|c|}{$\begin{array}{l}\text { Removable } \\
\text { dpm } / 100 \mathrm{~cm}^{2}\end{array}$} & \multicolumn{2}{|c|}{\begin{tabular}{|c|}
$\begin{array}{c}\text { Fixed + Removable } \\
\mathrm{dpm} / 100 \mathrm{~cm}^{2}\end{array}$ \\
\end{tabular}} & \multirow{2}{*}{$\begin{array}{l}\text { Gamma } \\
\mathrm{mrem} / \mathrm{hr}\end{array}$} & \multirow{2}{*}{$\begin{array}{l}\text { Neutron } \\
\mathrm{mrem} / \mathrm{hr}\end{array}$} & \multirow{2}{*}{$\begin{array}{c}\text { Total } \\
\mathrm{mrem} / \mathrm{hr}\end{array}$} \\
\hline & & & & & Alpha & Beta & Alpha & Beta & & & \\
\hline 1 & \multicolumn{4}{|c|}{ West Wall, Concrete (Electra 1120) } & $N / A$ & $N / A$ & 12 & 868 & $N / A$ & $N / A$ & $N / A$ \\
\hline 2 & \multicolumn{4}{|c|}{ West Wall, Concrete (Electra 1120) } & $N / A$ & $N / A$ & 6 & 714 & $N / A$ & $N / A$ & $N / A$ \\
\hline 3 & \multicolumn{4}{|c|}{ West Wall, Concrete $80 \%$, Metal 20\% (Electra 1120) } & $\mathrm{N} / \mathrm{A}$ & $N / A$ & 8 & 590 & $N / A$ & $N / A$ & $N / A$ \\
\hline 4 & \multicolumn{4}{|c|}{ West Wall, Concrete $60 \%$, Metal $40 \%$ (Electra 1120 ) } & $N / A$ & $N / A$ & 5 & 626 & $N / A$ & $N / A$ & $N / A$ \\
\hline 5 & \multicolumn{4}{|c|}{ West Wall, Concrete (Electra 1120) } & $N / A$ & $N / A$ & 12 & 608 & $N / A$ & $N / A$ & $N / A$ \\
\hline 6 & \multicolumn{4}{|c|}{ West Wall, Concrete (Electra 1120) } & $N / A$ & $N / A$ & 7 & 670 & $N / A$ & $N / A$ & $N / A$ \\
\hline 7 & \multicolumn{4}{|c|}{ West Wall, Concrete (Electra 1120) } & $N / A$ & $N / A$ & 6 & 941 & $N / A$ & $N / A$ & $N / A$ \\
\hline 8 & \multicolumn{4}{|c|}{ West Wall, Concrete (Electra 1120) } & $N / A$ & $N / A$ & 10 & 985 & $N / A$ & $N / A$ & $N / A$ \\
\hline 9 & \multicolumn{4}{|c|}{ West Wall, Concrete (Electra 1120) } & $N / A$ & $N / A$ & 10 & 1028 & $N / A$ & $N / A$ & $N / A$ \\
\hline 10 & \multicolumn{4}{|c|}{ West Wall, Concrete (Electra 1120) } & $N / A$ & $N / A$ & 5 & 948 & $N / A$ & $N / A$ & $N / A$ \\
\hline 11 & \multicolumn{4}{|c|}{ West Wall, Concrete (Electra 1120) } & $N / A$ & $N / A$ & 8 & 938 & $\mathrm{~N} / \mathrm{A}$ & $N / A$ & $N / A$ \\
\hline \multicolumn{5}{|c|}{ Reviewed By (Print); } & \multicolumn{5}{|l|}{ Signature: } & \multicolumn{2}{|c|}{ Date: $7 / 27 / 10$} \\
\hline
\end{tabular}


SURVEY \# 10-ER-A26-221

\begin{tabular}{|c|c|c|c|c|c|c|c|c|}
\hline \multirow{2}{*}{$\begin{array}{l}\text { Survey } \\
\text { Point }\end{array}$} & \multirow[t]{2}{*}{ Description/Comments } & \multicolumn{2}{|c|}{$\begin{array}{l}\text { Removable } \\
\mathrm{dpm} / 100 \mathrm{~cm}^{2}\end{array}$} & \multicolumn{2}{|c|}{$\begin{array}{c}\text { Fixed + Removable } \\
\mathrm{dpm} / 100 \mathrm{~cm}^{2}\end{array}$} & \multirow{2}{*}{$\begin{array}{l}\text { Gamma } \\
\mathrm{mrem} / \mathrm{hr}\end{array}$} & \multirow{2}{*}{$\begin{array}{l}\text { Neutron } \\
\text { mrem } / \mathrm{hr}\end{array}$} & \multirow{2}{*}{$\begin{array}{c}\text { Total } \\
\text { mrem } / \mathrm{hr}\end{array}$} \\
\hline & & Alpha & Beta & Alpha & Beta & & & \\
\hline 12 & West Wall, Concrete (Electra 1120) & N/A & N/A & 5 & 731 & N/A & N/A & $\mathrm{N} / \mathrm{A}$ \\
\hline 13 & West Wall, Concrete (Electra 1120) & N/A & N/A & 3 & 579 & N/A & N/A & N/A \\
\hline 14 & West Wall, Concrete (Electra 1120) & N/A & N/A & 6 & 674 & N/A & N/A & N/A \\
\hline 15 & West Wall, Concrete 60\%, Metal 40\% (Electra 1120) & N/A & $N / A$ & 6 & 570 & N/A & N/A & N/A \\
\hline 16 & West Wall, Concrete (Electra 1120) & N/A & N/A & 5 & 625 & N/A & N/A & N/A \\
\hline 17 & West Wall, Concrete (Electra 1120) & N/A & $\mathrm{N} / \mathrm{A}$ & 6 & 651 & N/A & N/A & N/A \\
\hline 18 & West Wall, Concrete (Electra 1120) & N/A & N/A & 8 & 642 & N/A & N/A & N/A \\
\hline 19 & West Wall, Concrete (Electra 1120) & N/A & $\mathrm{N} / \mathrm{A}$ & 14 & 927 & N/A & N/A & N/A \\
\hline 20 & West Wall, Concrete (Electra 1120) & $\mathrm{N} / \mathrm{A}$ & N/A & 11 & 949 & N/A & N/A & N/A \\
\hline 21 & West Wall, Concrete (Electra 6697) & $\mathrm{N} / \mathrm{A}$ & N/A & 3 & 563 & N/A & N/A & N/A \\
\hline N/A & $N / A$ & N/A & N/A & N/A & $\mathrm{N} / \mathrm{A}$ & N/A & N/A & N/A \\
\hline N/A & ${ }^{\star *}$ Performed scan surveys for both alpha and beta** & N/A & N/A & $\mathrm{N} / \mathrm{A}$ & N/A & N/A & N/A & N/A \\
\hline N/A & ${ }^{\star *}$ on $100 \%$ of survey unit. No trigger levels exceeded ${ }^{\star \star}$ & N/A & N/A & N/A & N/A & N/A & N/A & N/A \\
\hline N/A & $N / A$ & N/A & $\mathrm{N} / \mathrm{A}$ & N/A & N/A & N/A & N/A & N/A \\
\hline N/A & $N / A$ & N/A & $\mathrm{N} / \mathrm{A}$ & N/A & N/A & N/A & N/A & N/A \\
\hline N/A & $N / A$ & N/A & $\mathrm{N} / \mathrm{A}$ & N/A & N/A & $\mathrm{N} / \mathrm{A}$ & N/A & N/A \\
\hline N/A & $N / A$ & $\mathrm{~N} / \mathrm{A}$ & N/A & N/A & N/A & N/A & N/A & $\mathrm{N} / \mathrm{A}$ \\
\hline N/A & $N / A$ & N/A & $\mathrm{N} / \mathrm{A}$ & N/A & N/A & N/A & N/A & N/A \\
\hline N/A & $N / A$ & N/A & N/A & N/A & N/A & N/A & N/A & N/A \\
\hline N/A & $N / A$ & N/A & N/A & N/A & N/A & N/A & N/A & N/A \\
\hline N/A & $N / A$ & N/A & $\mathrm{N} / \mathrm{A}$ & N/A & N/A & N/A & N/A & N/A \\
\hline N/A & $\mathrm{N} / \mathrm{A}$ & $\mathrm{N} / \mathrm{A}$ & N/A & N/A & N/A & N/A & N/A & N/A \\
\hline N/A & $\mathrm{N} / \mathrm{A}$ & $\mathrm{N} / \mathrm{A}$ & N/A & N/A & N/A & N/A & $\mathrm{N} / \mathrm{A}$ & N/A \\
\hline
\end{tabular}


NSTec

Form

FRM-0108C

Survey \# $10-E R-A 26-221$

RADIOLOGICAL SURVEY REPORT - FULL MAP

05/24/10

Rev. 01

Page $\underline{3}$ of $\underline{3}$

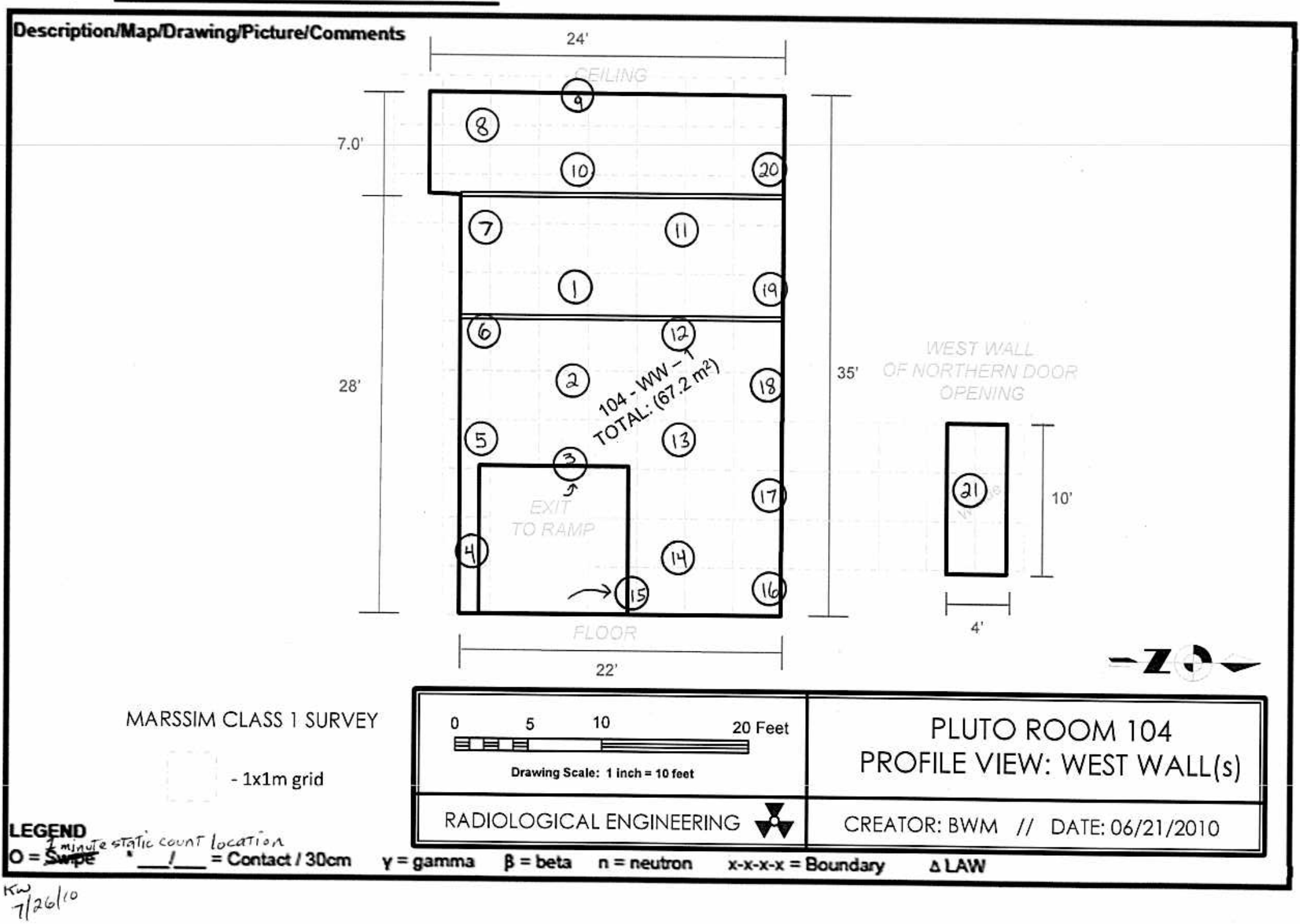


NSTec

SURVEY \# 10-ER-A26-290

\begin{tabular}{|c|c|c|c|c|c|c|c|c|c|c|c|}
\hline \multicolumn{3}{|c|}{$\begin{array}{l}\text { Location: } \\
\text { Area } 26 \text { CAU117 Pluto }\end{array}$} & \multicolumn{2}{|c|}{$\begin{array}{l}\text { Purpose: } \\
\text { Final Status Survey, Room } 106 \\
\text { East Wall / 106-EW-1 }\end{array}$} & \multicolumn{5}{|c|}{$\begin{array}{l}\text { Comments: } \\
\text { All one minute static counts are in cpm. Bkgd not subtracted for } \\
\text { Final Status Survey, Survey Plan \#10-015 }\end{array}$} & \multicolumn{2}{|c|}{$\begin{array}{l}\text { Date/Time: } \\
\text { 08/22/2010 } 1330\end{array}$} \\
\hline Instrument: & Serial \#: & Cal Due: & $\begin{array}{c}\text { Eff in \%: } \\
\text { Alpha / Beta }\end{array}$ & $\begin{array}{l}\text { BKG in dpm: } \\
\text { Alpha / Beta }\end{array}$ & \begin{tabular}{|l|} 
MDA in dpm: \\
Alpha / Beta \\
\end{tabular} & RWP\# & \multicolumn{5}{|l|}{$\mathrm{N} / \mathrm{A}$} \\
\hline Electra & 1120 & $4 / 6 / 2011$ & $14.1 / 20.8$ & $\mathrm{~N} / \mathrm{A}$ & $N / A$ & \multicolumn{6}{|c|}{ Work Package \# $\quad$ ENV-10-RCT ENV SITE SURVEYS-0013 } \\
\hline Electra & 1504 & $4 / 6 / 2011$ & $12.9 / 19.8$ & $\mathrm{~N} / \mathrm{A}$ & $N / A$ & \multicolumn{3}{|c|}{ RCT Name: D.Cotroneo Jr. } & \multicolumn{3}{|c|}{ Signature: $y-t$} \\
\hline Electra & $N / A$ & $N / A$ & $N / A$ & $N / A$ & $N / A$ & \multicolumn{3}{|c|}{ RCT Name: K. Wilcox/M.Payne } & \multicolumn{3}{|c|}{ Signature $\mathrm{Za}-\infty \mathrm{C}$ - } \\
\hline Electra & $\mathrm{N} / \mathrm{A}$ & $\mathrm{N} / \mathrm{A}$ & $N / A$ & $\mathrm{~N} / \mathrm{A}$ & $N / A$ & \multicolumn{3}{|c|}{ RCT Name: B. Bunce } & Signature: & \multicolumn{2}{|c|}{ Bothmen } \\
\hline Electra & $N / A$ & $N / A$ & $N / A$ & $N / A$ & $N / A$ & \multicolumn{3}{|c|}{ RCT Name: B. Foskett } & Signature: & \multicolumn{2}{|c|}{$\mathrm{S}$} \\
\hline \multirow[t]{2}{*}{ Survey Point } & \multirow{2}{*}{\multicolumn{4}{|c|}{ Description/Comments }} & \multicolumn{2}{|c|}{$\begin{array}{l}\text { Removable } \\
\mathrm{dpm} / 100 \mathrm{~cm}^{2}\end{array}$} & \multicolumn{2}{|c|}{\begin{tabular}{|c|}
$\begin{array}{c}\text { Fixed + Removable } \\
\mathrm{dpm} / 100 \mathrm{~cm}^{2}\end{array}$ \\
\end{tabular}} & \multirow{2}{*}{$\begin{array}{l}\text { Gamma } \\
\mathrm{mrem} / \mathrm{hr}\end{array}$} & \multirow{2}{*}{$\begin{array}{l}\text { Neutron } \\
\mathrm{mrem} / \mathrm{hr}\end{array}$} & \multirow{2}{*}{$\begin{array}{c}\text { Total } \\
\mathrm{mrem} / \mathrm{hr}\end{array}$} \\
\hline & & & & & Alpha & Beta & Alpha & Beta & & & \\
\hline 1 & \multicolumn{4}{|c|}{ Wall, Concrete (Electra 1504) } & $N / A$ & $N / A$ & 5 & 920 & $N / A$ & $N / A$ & $N / A$ \\
\hline 2 & \multicolumn{4}{|c|}{ Wall, Concrete (Electra 1504) } & $N / A$ & $N / A$ & 3 & 609 & $N / A$ & $N / A$ & $N / A$ \\
\hline 3 & \multicolumn{4}{|c|}{ Wall, Concrete (Electra 1504) } & $N / A$ & $N / A$ & 4 & 641 & $N / A$ & $N / A$ & $N / A$ \\
\hline 4 & \multicolumn{4}{|c|}{ Wall, Concrete (Electra 1504) } & $N / A$ & $N / A$ & 5 & 784 & $N / A$ & $N / A$ & $N / A$ \\
\hline 5 & \multicolumn{4}{|c|}{ Wall, Concrete (Electra 1504) } & $N / A$ & $N / A$ & 6 & 692 & $N / A$ & $N / A$ & $N / A$ \\
\hline 6 & \multicolumn{4}{|c|}{ Wall, Concrete (Electra 1504) } & $N / A$ & $N / A$ & 6 & 661 & $N / A$ & $N / A$ & $N / A$ \\
\hline 7 & \multicolumn{4}{|c|}{ Wall, Concrete (Electra 1504) } & $N / A$ & $N / A$ & 13 & 1033 & $\mathrm{~N} / \mathrm{A}$ & $\mathrm{N} / \mathrm{A}$ & $\mathrm{N} / \mathrm{A}$ \\
\hline 8 & \multicolumn{4}{|c|}{ Wall, Concrete (Electra 1504) } & $N / A$ & $N / A$ & 7 & 1085 & $\mathrm{~N} / \mathrm{A}$ & $N / A$ & $\mathrm{~N} / \mathrm{A}$ \\
\hline 9 & \multicolumn{4}{|c|}{ Wall, Concrete (Electra 1504) } & $N / A$ & $N / A$ & 4 & 964 & $N / A$ & $N / A$ & $N / A$ \\
\hline 10 & \multicolumn{4}{|c|}{ Wall, Concrete (Electra 1504) } & $N / A$ & $\mathrm{~N} / \mathrm{A}$ & 5 & 662 & $N / A$ & $N / A$ & $N / A$ \\
\hline 11 & \multicolumn{4}{|c|}{ Wall, Metal (Electra 1504) } & $N / A$ & $N / A$ & 7 & 430 & $N / A$ & $N / A$ & $N / A$ \\
\hline \multicolumn{5}{|c|}{ Reviewed By (Priat): } & & & & & & 81 & \\
\hline
\end{tabular}


NSTec

SURVEY \# 10-ER-A26-290

\begin{tabular}{|c|c|c|c|c|c|c|c|c|}
\hline \multirow[t]{2}{*}{ Survey Point } & \multirow[t]{2}{*}{ Description/Comments } & \multicolumn{2}{|c|}{$\begin{array}{l}\text { Removable } \\
\mathrm{dpm} / 100 \mathrm{~cm}^{2}\end{array}$} & \multicolumn{2}{|c|}{$\begin{array}{l}\text { Fixed + Removable } \\
\mathrm{dpm} / 100 \mathrm{~cm}^{2}\end{array}$} & \multirow{2}{*}{$\begin{array}{l}\text { Gamma } \\
\mathrm{mrem} / \mathrm{hr}\end{array}$} & \multirow{2}{*}{$\begin{array}{l}\text { Neutron } \\
\mathrm{mrem} / \mathrm{hr}\end{array}$} & \multirow{2}{*}{$\begin{array}{c}\text { Total } \\
\mathrm{mrem} / \mathrm{hr}\end{array}$} \\
\hline & & Alpha & Beta & Alpha & Beta & & & \\
\hline 12 & Wall, Metal (1504) & N/A & N/A & 8 & 426 & N/A & N/A & N/A \\
\hline 13 & Wall, Concrete (1504) & N/A & N/A & 7 & 690 & N/A & N/A & N/A \\
\hline 14 & Wall, Concrete (1504) & N/A & N/A & 6 & 617 & N/A & N/A & N/A \\
\hline 15 & Wall, Concrete (1504) & N/A & N/A & 10 & 786 & N/A & $\mathrm{N} / \mathrm{A}$ & N/A \\
\hline N/A & ${ }^{\star \star}$ Performed scan surveys for both alpha and beta ${ }^{\star \star}$ & N/A & N/A & $\mathrm{N} / \mathrm{A}$ & N/A & N/A & $\mathrm{N} / \mathrm{A}$ & N/A \\
\hline N/A & ${ }^{\star \star}$ on $100 \%$ of survey unit. No trigger levels exceeded ${ }^{\star \star}$ & N/A & N/A & N/A & N/A & N/A & N/A & N/A \\
\hline N/A & $\mathrm{N} / \mathrm{A}$ & N/A & N/A & N/A & N/A & N/A & N/A & N/A \\
\hline N/A & $\mathrm{N} / \mathrm{A}$ & N/A & N/A & N/A & N/A & N/A & N/A & N/A \\
\hline N/A & $\mathrm{N} / \mathrm{A}$ & N/A & N/A & N/A & N/A & N/A & N/A & N/A \\
\hline N/A & $N / A$ & N/A & $\mathrm{N} / \mathrm{A}$ & N/A & N/A & N/A & N/A & N/A \\
\hline N/A & $N / A$ & N/A & $\mathrm{N} / \mathrm{A}$ & $\mathrm{N} / \mathrm{A}$ & N/A & N/A & N/A & N/A \\
\hline N/A & $N / A$ & N/A & N/A & $\mathrm{N} / \mathrm{A}$ & N/A & N/A & N/A & N/A \\
\hline N/A & $\mathrm{N} / \mathrm{A}$ & N/A & $\mathrm{N} / \mathrm{A}$ & N/A & $\mathrm{N} / \mathrm{A}$ & N/A & N/A & N/A \\
\hline N/A & $N / A$ & N/A & N/A & N/A & N/A & N/A & N/A & N/A \\
\hline N/A & $\mathrm{N} / \mathrm{A}$ & N/A & N/A & N/A & $\mathrm{N} / \mathrm{A}$ & N/A & N/A & N/A \\
\hline N/A & $\mathrm{N} / \mathrm{A}$ & N/A & $\mathrm{N} / \mathrm{A}$ & N/A & N/A & N/A & N/A & N/A \\
\hline N/A & $N / A$ & N/A & N/A & N/A & $\mathrm{N} / \mathrm{A}$ & N/A & N/A & N/A \\
\hline N/A & $N / A$ & N/A & N/A & N/A & N/A & N/A & N/A & N/A \\
\hline N/A & $N / A$ & N/A & $\mathrm{N} / \mathrm{A}$ & N/A & $\mathrm{N} / \mathrm{A}$ & N/A & N/A & $\mathrm{N} / \mathrm{A}$ \\
\hline $\mathrm{N} / \mathrm{A}$ & $N / A$ & N/A & N/A & N/A & N/A & $\mathrm{N} / \mathrm{A}$ & N/A & $\mathrm{N} / \mathrm{A}$ \\
\hline N/A & $N / A$ & N/A & N/A & N/A & N/A & N/A & N/A & $\mathrm{N} / \mathrm{A}$ \\
\hline N/A & $N / A$ & N/A & $\mathrm{N} / \mathrm{A}$ & N/A & N/A & N/A & N/A & N/A \\
\hline $\mathrm{N} / \mathrm{A}$ & $N / A$ & N/A & N/A & N/A & $\mathrm{N} / \mathrm{A}$ & N/A & $\mathrm{N} / \mathrm{A}$ & N/A \\
\hline
\end{tabular}


FRM-0108C

Survey $\# 10-E R-A_{2} b-290$

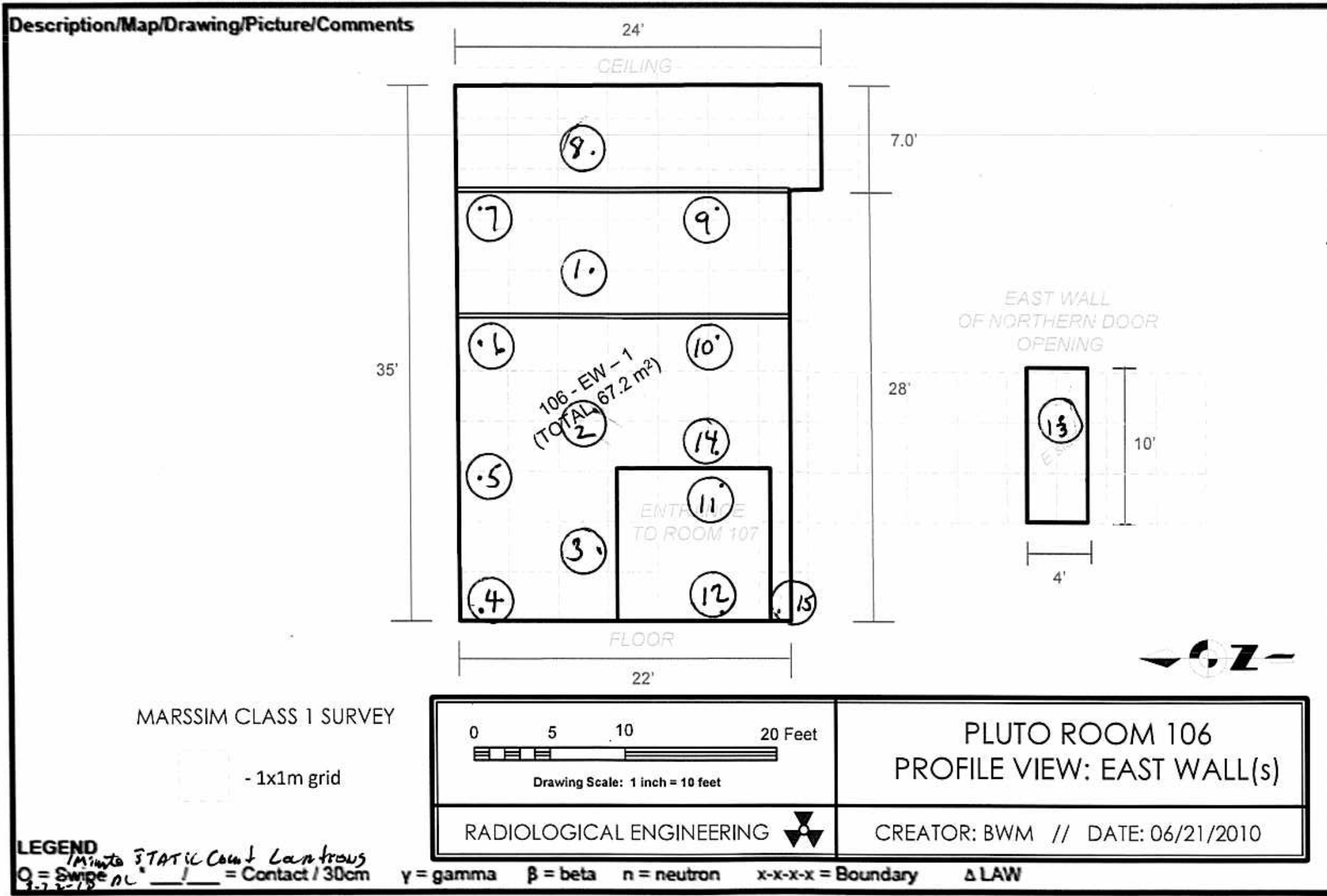




\begin{tabular}{|c|c|c|c|c|c|c|c|c|c|c|c|}
\hline \multicolumn{3}{|c|}{$\begin{array}{l}\text { Location: } \\
\text { Area } 26 \text { CAU117 Pluto }\end{array}$} & \multicolumn{2}{|c|}{$\begin{array}{l}\text { Purpose: } \\
\text { Final Status Survey, Room } 106 \\
\text { Floor, 106-F-1, Post-Decon }\end{array}$} & \multicolumn{5}{|c|}{$\begin{array}{l}\text { Comments: } \\
\text { All one minute static counts are in cpm. Bkgd not subtracted for } \\
\text { Final Status Survey, Survey Plan \#10-015 }\end{array}$} & \multicolumn{2}{|c|}{$\begin{array}{l}\text { Date/Time: } \\
08 / 19 / 20101400\end{array}$} \\
\hline Instrument: & Serial \#: & Cal Due: & $\begin{array}{c}\text { Eff in \%: } \\
\text { Alpha / Beta }\end{array}$ & $\begin{array}{l}\text { BKG in dpm: } \\
\text { Alpha / Beta }\end{array}$ & \begin{tabular}{|l|} 
MDA in dpm: \\
Alpha / Beta \\
\end{tabular} & RWP\# & \multicolumn{5}{|l|}{$\mathrm{N} / \mathrm{A}$} \\
\hline Electra & 1504 & $4 / 6 / 2011$ & $12.9 / 19.8$ & $\mathrm{~N} / \mathrm{A}$ & $\mathrm{N} / \mathrm{A}$ & \multicolumn{6}{|c|}{ Work Package \# ENV-10-RCT ENV SITE SURVEYS-0013 } \\
\hline Electra & 1120 & $4 / 6 / 2011$ & $14.1 / 20.8$ & $N / A$ & $\mathrm{~N} / \mathrm{A}$ & RCT Name: & \multicolumn{2}{|c|}{ D.Cotroneo Jr. } & \multicolumn{3}{|c|}{ Signature: $\mathrm{OW}$} \\
\hline Electra & 6697 & $4 / 6 / 2011$ & $13.8 / 19.9$ & $N / A$ & $N / A$ & RCT Name: & \multicolumn{2}{|c|}{ Kevin Wilcox } & \multirow{2}{*}{\multicolumn{3}{|c|}{ Signature: hen $2 \cdot \mathrm{Pcos}_{0}$}} \\
\hline $\mathrm{N} / \mathrm{A}$ & $N / A$ & $\mathrm{~N} / \mathrm{A}$ & $\mathrm{N} / \mathrm{A}$ & $\mathrm{N} / \mathrm{A}$ & $\mathrm{N} / \mathrm{A}$ & RCT Name: & \multicolumn{2}{|c|}{ Mike Payne } & & & \\
\hline$N / A$ & $\mathrm{~N} / \mathrm{A}$ & $\mathrm{N} / \mathrm{A}$ & $\mathrm{N} / \mathrm{A}$ & $\mathrm{N} / \mathrm{A}$ & $\mathrm{N} / \mathrm{A}$ & RCT Name: & $\mathrm{N} / \mathrm{A}$ & & \multicolumn{3}{|c|}{ Signature: $\quad v / A_{A}$} \\
\hline \multirow[t]{2}{*}{ Survey Point } & \multirow{2}{*}{\multicolumn{4}{|c|}{ Description/Comments }} & \multicolumn{2}{|c|}{$\begin{array}{l}\text { Removable } \\
\mathrm{dpm} / 100 \mathrm{~cm}^{2}\end{array}$} & \multicolumn{2}{|c|}{\begin{tabular}{|c|}
$\begin{array}{c}\text { Fixed + Removable } \\
\mathrm{dpm} / 100 \mathrm{~cm}^{2}\end{array}$ \\
\end{tabular}} & \multirow{2}{*}{$\begin{array}{l}\text { Gamma } \\
\mathrm{mrem} / \mathrm{hr}\end{array}$} & \multirow{2}{*}{$\begin{array}{l}\text { Neutron } \\
\mathrm{mrem} / \mathrm{hr}\end{array}$} & \multirow{2}{*}{$\begin{array}{l}\text { Total } \\
\mathrm{mrem} / \mathrm{hr}\end{array}$} \\
\hline & & & & & Alpha & Beta & Alpha & Beta & & & \\
\hline 1 & \multicolumn{4}{|c|}{ Floor, Concrete (Electra 1504) } & $N / A$ & $N / A$ & 8 & 621 & $N / A$ & $N / A$ & $N / A$ \\
\hline 2 & \multicolumn{4}{|c|}{ Floor, Concrete (Electra 1120) } & $N / A$ & $N / A$ & 11 & 1164 & $N / A$ & $N / A$ & $N / A$ \\
\hline 3 & \multicolumn{4}{|c|}{ Floor, Concrete (Electra 6697) } & $N / A$ & $N / A$ & 13 & 1198 & $N / A$ & $N / A$ & $N / A$ \\
\hline 4 & \multicolumn{4}{|c|}{ Floor, Concrete (Electra 6697) } & $N / A$ & $N / A$ & 19 & 1198 & $N / A$ & $N / A$ & $\mathrm{~N} / \mathrm{A}$ \\
\hline 5 & \multicolumn{4}{|c|}{ Floor, Concrete (Electra 6697) } & $N / A$ & $N / A$ & 12 & 982 & $N / A$ & $N / A$ & $\mathrm{~N} / \mathrm{A}$ \\
\hline 6 & \multicolumn{4}{|c|}{ Floor, Concrete (Electra 6697) } & $N / A$ & $N / A$ & 12 & 1022 & $N / A$ & $N / A$ & $N / A$ \\
\hline 7 & \multicolumn{4}{|c|}{ Floor, Concrete (Electra 6697) } & $\mathrm{N} / \mathrm{A}$ & $N / A$ & 17 & 1185 & $N / A$ & $N / A$ & $N / A$ \\
\hline$N / A$ & \multicolumn{4}{|c|}{$\begin{array}{l}{ }^{* \star} \text { Post decon survey of hot spots and surrounding areas }{ }^{\star *} \\
{ }^{\star *} \text { in Room } 106(106-\mathrm{F}-1) .^{\star \star}\end{array}$} & $N / A$ & $N / A$ & $\mathrm{~N} / \mathrm{A}$ & $N / A$ & $N / A$ & $N / A$ & $\mathrm{~N} / \mathrm{A}$ \\
\hline$N / A$ & \multicolumn{4}{|c|}{$\begin{array}{l}\star \star * \text { Re-survey of hot spots plus surrounding areas } \\
\star \star \\
\star(1 \text { square meter perimeter on each side of hot spot })^{\star \star}\end{array}$} & $\mathrm{N} / \mathrm{A}$ & $N / A$ & $N / A$ & $\mathrm{~N} / \mathrm{A}$ & $\mathrm{N} / \mathrm{A}$ & $\mathrm{N} / \mathrm{A}$ & $\mathrm{N} / \mathrm{A}$ \\
\hline $\mathrm{N} / \mathrm{A}$ & \multicolumn{4}{|c|}{$\begin{array}{l}* * \text { Performed scan surveys for both alpha and beta on }{ }^{\star *} \\
* * 100 \% \text { of surrounding areas. No trigger levels exceeded }\end{array}$} & $\mathrm{N} / \mathrm{A}$ & $\mathrm{N} / \mathrm{A}$ & $\mathrm{N} / \mathrm{A}$ & $N / A$ & $N / A$ & $N / A$ & $N / A$ \\
\hline$N / A$ & \multicolumn{4}{|l|}{$\mathrm{N} / \mathrm{A}$} & $N / A$ & $\mathrm{~N} / \mathrm{A}$ & $N / A$ & $N / A$ & $N / A$ & $\mathrm{~N} / \mathrm{A}$ & $N / A$ \\
\hline \multicolumn{5}{|c|}{ Reviewed By (Print): } & anoth & & & & & $\begin{array}{r}\text { Date: } \\
8\end{array}$ & \\
\hline
\end{tabular}


Survey $\# 10-E R-A 26-276$
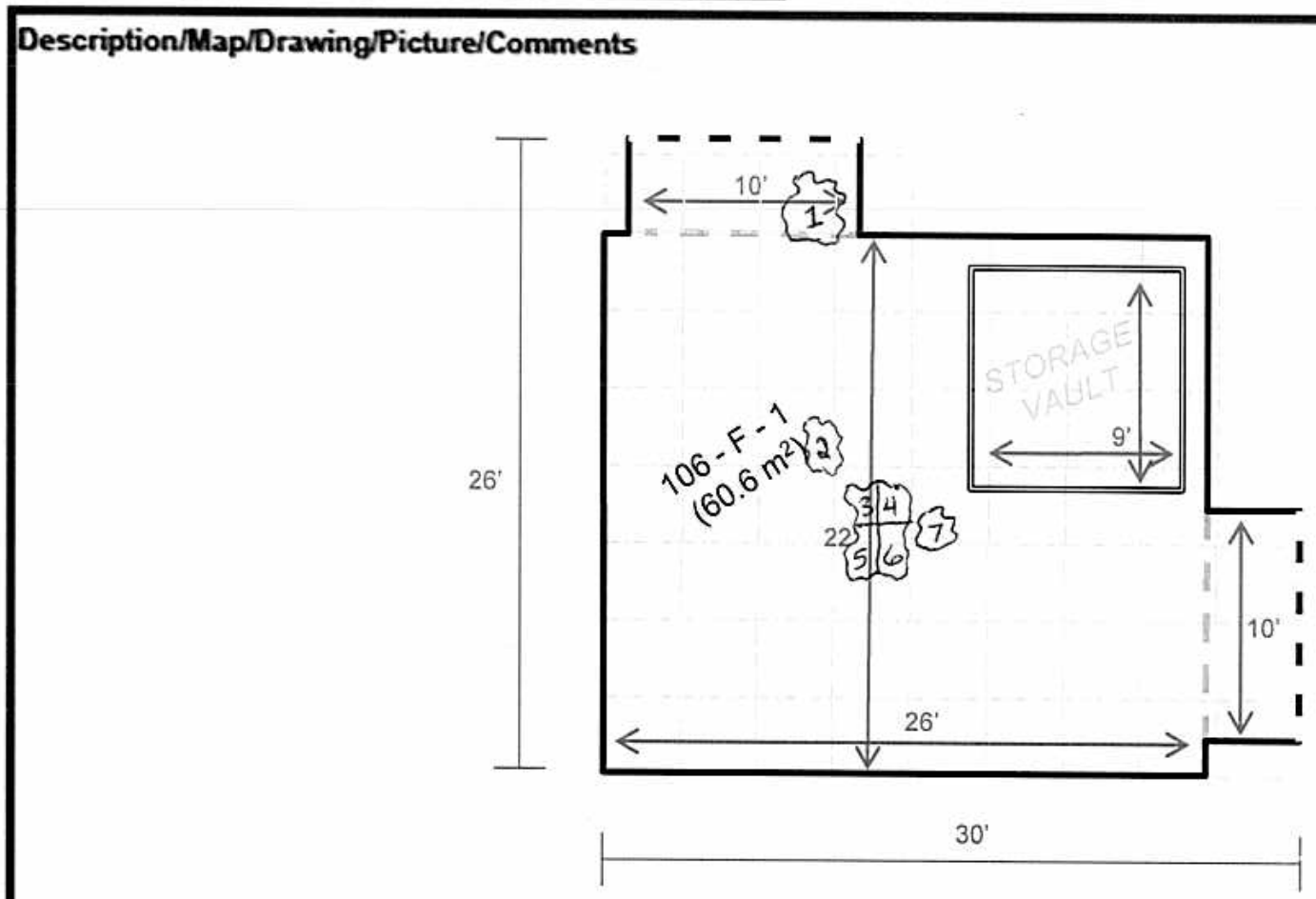

MARSSIM CLASS I SURVEY

$-1 \times 1 m$ grid

\begin{tabular}{||ccc|c||}
\hline 0 & 5 & 10 & 20 Feet \\
\hline
\end{tabular}

LEGEND 
Survey \# 10-ER-A26-276

Description/Map/Drawing/Picture/Comments

Building 2201 Pluto Disassembly Facility

Survey Plan 10-015

Room 106, Hot Spot A, Post Decon

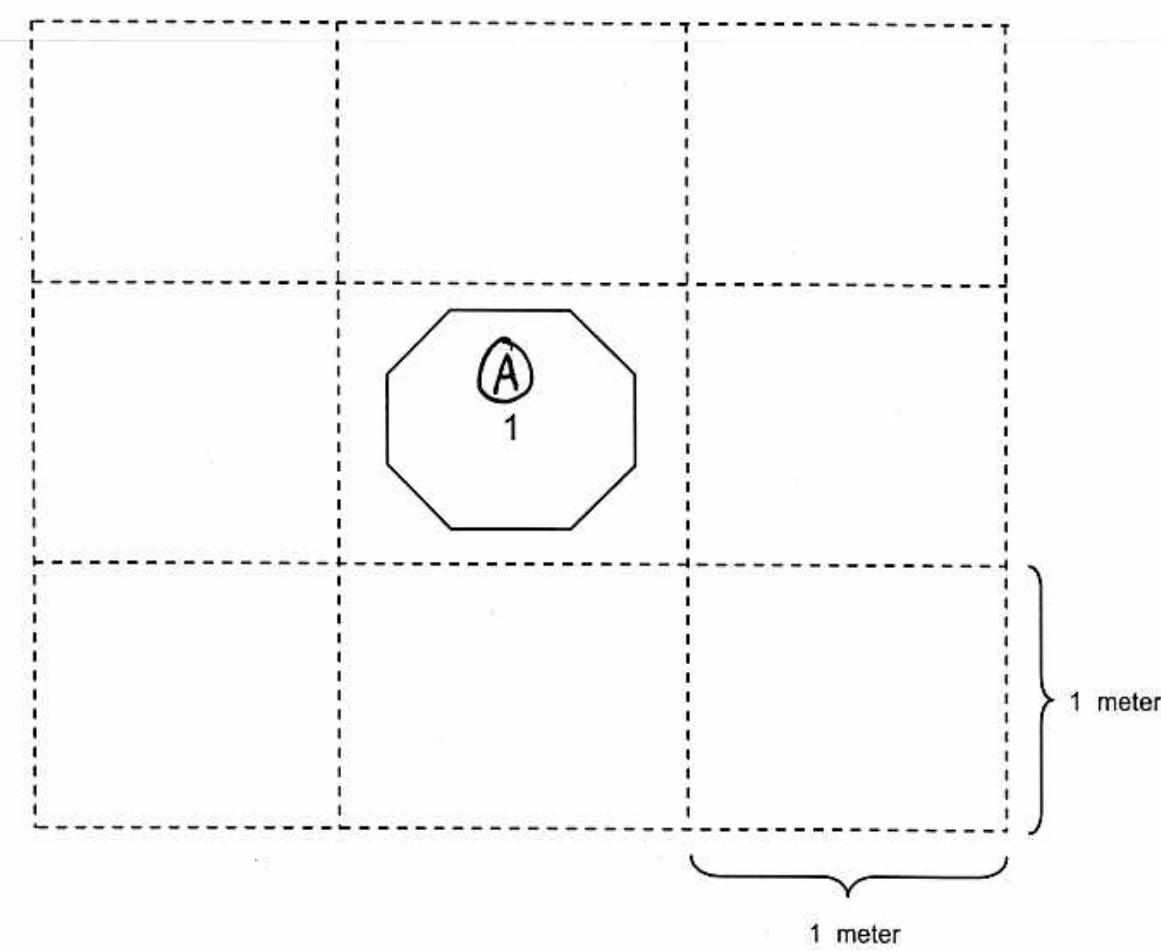

LEGEND

$O=-\operatorname{swpe}^{\infty}$ * 
Survey \# 10-ER-A26-276

Description/Map/Drawing/Picture/Comments

Building 2201 Pluto Disassembly Facility

Survey Plan 10-015

Room 106, Hot Spot B, Post Decon

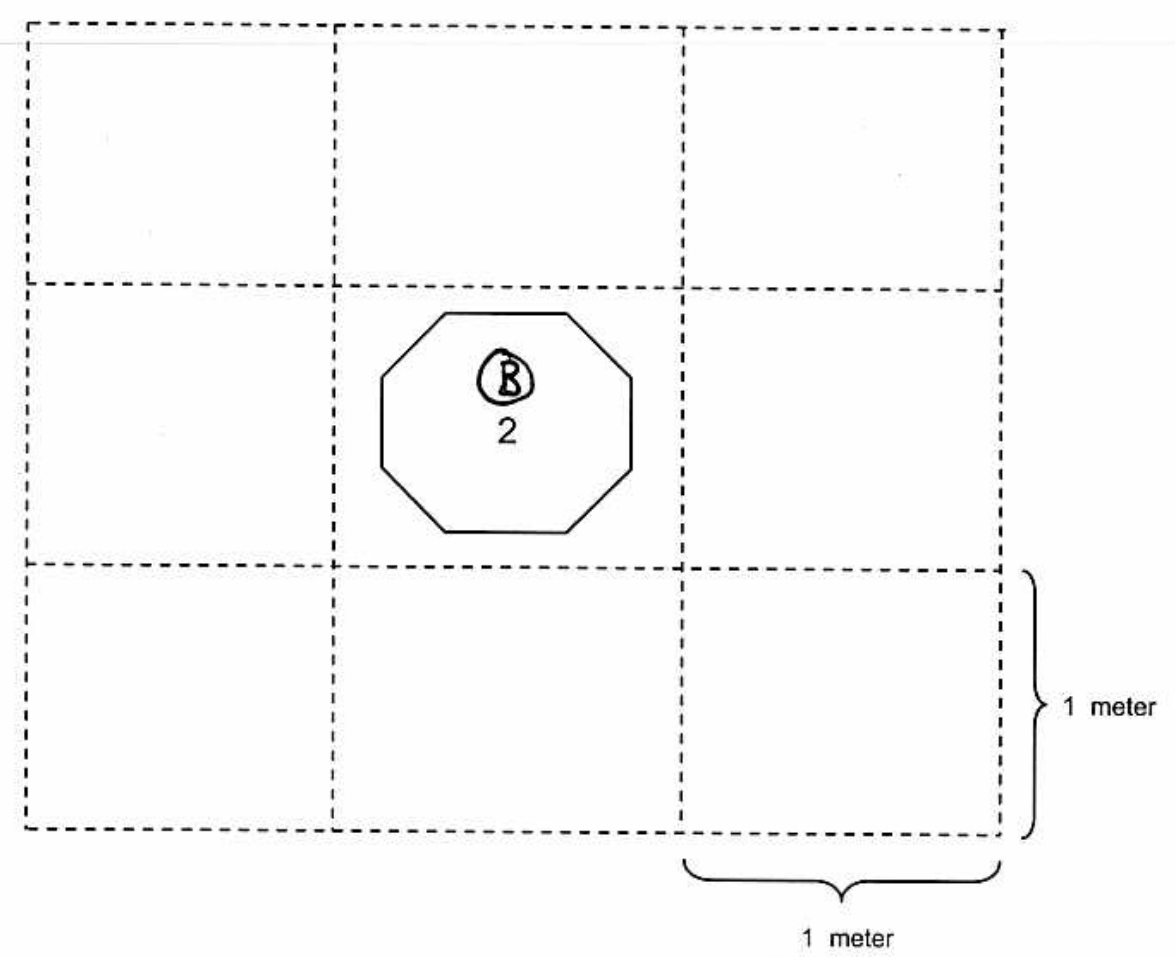

\section{LEGEND}

$O=$ Swipe $^{n} * 1 \ldots=$ Contact $/ 30 \mathrm{~cm}$ 
Survey \# 10-ER-A26-276

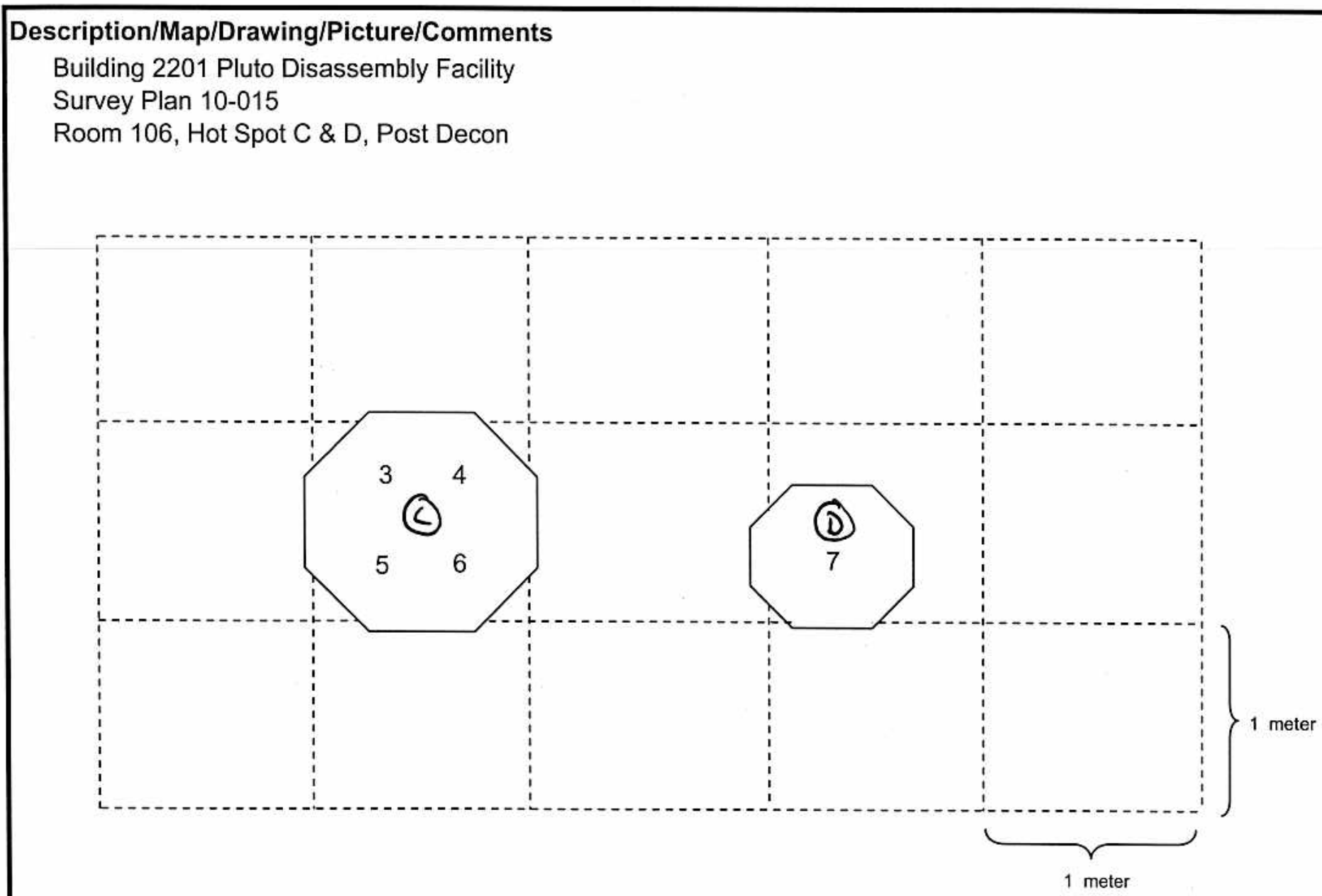

LEGEND

$O=$ Swipe $^{n} * 1 \quad=$ Contact $/ 30 \mathrm{~cm}$ 
SURVEY \#

10-ER-A26-275

\begin{tabular}{|c|c|c|c|c|c|c|c|c|c|c|c|}
\hline \multicolumn{3}{|c|}{$\begin{array}{l}\text { Location: } \\
\text { Area } 26 \text { CAU117 Pluto }\end{array}$} & \multicolumn{2}{|c|}{$\begin{array}{l}\text { Purpose: } \\
\text { Final Status Survey, Room 106, } \\
\text { Floor, 106-F-1 }\end{array}$} & \multicolumn{5}{|c|}{$\begin{array}{l}\text { Comments: } \\
\text { All one minute static counts are in cpm. Bkgd not subtracted for } \\
\text { Final Status Survey, Survey Plan \#10-015 }\end{array}$} & \multicolumn{2}{|c|}{$\begin{array}{l}\text { Date/Time: } \\
\text { 08/19/2010 } 1100\end{array}$} \\
\hline Instrument: & Serial \#: & Cal Due: & $\begin{array}{c}\text { Eff in \%: } \\
\text { Alpha / Beta }\end{array}$ & $\begin{array}{l}\text { BKG in dpm: } \\
\text { Alpha / Beta }\end{array}$ & $\begin{array}{l}\text { MDA in dpm: } \\
\text { Alpha / Beta } \\
\end{array}$ & RWP\# & \multicolumn{5}{|l|}{$\mathrm{N} / \mathrm{A}$} \\
\hline Electra & 1504 & $4 / 6 / 2011$ & $12.9 / 19.8$ & $N / A$ & $\mathrm{~N} / \mathrm{A}$ & \multicolumn{6}{|c|}{ ENV-10-RCT ENV SITE SL } \\
\hline Electra & 1120 & $4 / 6 / 2011$ & $14.1 / 20.8$ & $\mathrm{~N} / \mathrm{A}$ & $\mathrm{N} / \mathrm{A}$ & \multicolumn{3}{|c|}{ RCT Name: D.Cotroneo Jr. } & Signature: & \multicolumn{2}{|c|}{ 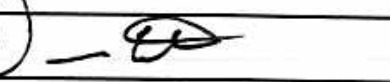 } \\
\hline$N / A$ & $N / A$ & $\mathrm{~N} / \mathrm{A}$ & $\mathrm{N} / \mathrm{A}$ & $\mathrm{N} / \mathrm{A}$ & $\mathrm{N} / \mathrm{A}$ & \multicolumn{3}{|c|}{ RCT Name: Kevin Wilcox } & \multicolumn{3}{|c|}{ Signature: Ker W. } \\
\hline$N / A$ & $N / A$ & $N / A$ & $\mathrm{~N} / \mathrm{A}$ & $\mathrm{N} / \mathrm{A}$ & $\mathrm{N} / \mathrm{A}$ & \multicolumn{3}{|c|}{ RCT Name: Mike Payne } & \multirow{2}{*}{\multicolumn{3}{|c|}{ Signature: $\mathrm{C} \cdot$ Signature: }} \\
\hline$N / A$ & $\mathrm{~N} / \mathrm{A}$ & $N / A$ & $N / A$ & $\mathrm{~N} / \mathrm{A}$ & $\mathrm{N} / \mathrm{A}$ & RCT Name: & $N / A$ & & & & \\
\hline \multirow[t]{2}{*}{ Survey Point } & \multirow{2}{*}{\multicolumn{4}{|c|}{ Description/Comments }} & \multicolumn{2}{|c|}{$\begin{array}{l}\text { Removable } \\
\mathrm{dpm} / 100 \mathrm{~cm}^{2}\end{array}$} & \multicolumn{2}{|c|}{\begin{tabular}{|c|} 
Fixed + Removable \\
$\mathrm{dpm} / 100 \mathrm{~cm}^{2}$
\end{tabular}} & \multirow{2}{*}{$\begin{array}{l}\text { Gamma } \\
\mathrm{mrem} / \mathrm{hr}\end{array}$} & \multirow{2}{*}{$\begin{array}{l}\text { Neutron } \\
\mathrm{mrem} / \mathrm{hr}\end{array}$} & \multirow{2}{*}{$\begin{array}{l}\text { Total } \\
\mathrm{mrem} / \mathrm{hr}\end{array}$} \\
\hline & & & & & Alpha & Beta & Alpha & Beta & & & \\
\hline 1 & \multicolumn{4}{|c|}{ Floor, Concrete (Electra 1120) } & $N / A$ & $N / A$ & 7 & 1100 & $N / A$ & $N / A$ & $N / A$ \\
\hline 2 & \multicolumn{4}{|c|}{ Floor, Concrete (Electra 1120) } & $N / A$ & $\mathrm{~N} / \mathrm{A}$ & 10 & 1314 & $N / A$ & $N / A$ & $N / A$ \\
\hline 3 & \multicolumn{4}{|c|}{ Floor, Concrete (Electra 1120) } & $N / A$ & $N / A$ & 2 & 958 & $\mathrm{~N} / \mathrm{A}$ & $N / A$ & $N / A$ \\
\hline 4 & \multicolumn{4}{|c|}{ Floor, Concrete (Electra 1120) } & $N / A$ & $N / A$ & 3 & 951 & $N / A$ & $N / A$ & $N / A$ \\
\hline 5 & \multicolumn{4}{|c|}{ Floor, Concrete (Electra 1120) } & $N / A$ & $N / A$ & 13 & 967 & $N / A$ & $\mathrm{~N} / \mathrm{A}$ & $N / A$ \\
\hline 6 & \multicolumn{4}{|c|}{ Floor, Concrete (Electra 1120) } & $N / A$ & $\mathrm{~N} / \mathrm{A}$ & 5 & 1352 & $N / A$ & $N / A$ & $\mathrm{~N} / \mathrm{A}$ \\
\hline 7 & \multicolumn{4}{|c|}{ Floor, Concrete (Electra 1120) } & $N / A$ & $N / A$ & 42 & 35012 & $N / A$ & $N / A$ & $N / A$ \\
\hline 8 & \multicolumn{4}{|c|}{ Floor, Metal (Electra 1120) } & $N / A$ & $N / A$ & 11 & 661 & $N / A$ & $N / A$ & $\mathrm{~N} / \mathrm{A}$ \\
\hline 9 & \multicolumn{4}{|c|}{ Floor, Metal (Electra 1120) } & $N / A$ & $N / A$ & 5 & 724 & $N / A$ & $\mathrm{~N} / \mathrm{A}$ & $\mathrm{N} / \mathrm{A}$ \\
\hline 10 & \multicolumn{4}{|c|}{ Floor, Concrete (Electra 1120) } & $N / A$ & $N / A$ & 9 & 999 & $N / A$ & $\mathrm{~N} / \mathrm{A}$ & $\mathrm{N} / \mathrm{A}$ \\
\hline 11 & \multicolumn{4}{|c|}{ Floor, Concrete (Electra 1120) } & $N / A$ & $\mathrm{~N} / \mathrm{A}$ & 2 & 896 & $N / A$ & $N / A$ & $N / A$ \\
\hline \multicolumn{5}{|c|}{ Reviewed By (Print): } & \multicolumn{7}{|c|}{ Signature: } \\
\hline
\end{tabular}




\section{NSTec}

SURVEY \# 10-ER-A26-275

\begin{tabular}{|c|c|c|c|c|c|c|c|c|}
\hline \multirow[t]{2}{*}{ Survey Point } & \multirow[t]{2}{*}{ Description/Comments } & \multicolumn{2}{|c|}{$\begin{array}{l}\text { Removable } \\
\mathrm{dpm} / 100 \mathrm{~cm}^{2}\end{array}$} & \multicolumn{2}{|c|}{$\begin{array}{c}\begin{array}{c}\text { Fixed + Removable } \\
\mathrm{dpm} / 100 \mathrm{~cm}^{2}\end{array} \\
\end{array}$} & \multirow{2}{*}{$\begin{array}{l}\text { Gamma } \\
\text { mrem/hr }\end{array}$} & \multirow{2}{*}{$\begin{array}{l}\text { Neutron } \\
\text { mrem/hr }\end{array}$} & \multirow{2}{*}{$\begin{array}{c}\text { Total } \\
\text { mrem/hr }\end{array}$} \\
\hline & & Alpha & Beta & Alpha & Beta & & & \\
\hline 12 & Floor, Concrete (Electra 1120) & N/A & N/A & 6 & 970 & $\mathrm{~N} / \mathrm{A}$ & N/A & N/A \\
\hline 13 & Floor, Metal (Electra 1120) & N/A & N/A & 4 & 601 & $\mathrm{~N} / \mathrm{A}$ & N/A & $\mathrm{N} / \mathrm{A}$ \\
\hline N/A & $\mathrm{N} / \mathrm{A}$ & N/A & N/A & N/A & N/A & $\mathrm{N} / \mathrm{A}$ & N/A & N/A \\
\hline N/A & ${ }^{\star}$ Performed scan surveys for both alpha and beta* & N/A & N/A & N/A & N/A & N/A & N/A & N/A \\
\hline N/A & *on $100 \%$ of survey unit. ${ }^{*}$ & N/A & N/A & N/A & N/A & N/A & N/A & N/A \\
\hline N/A & ${ }^{\star}$ Trigger levels exceeded in 4 spots as shown on map $^{\star}$ & N/A & N/A & N/A & N/A & N/A & N/A & N/A \\
\hline N/A & *and documented below: ${ }^{*}$ & N/A & N/A & N/A & N/A & N/A & N/A & N/A \\
\hline A & Floor, Concrete (Electra 1120), (size $\sim 1$ square foot) & N/A & $\mathrm{N} / \mathrm{A}$ & 42 & 35012 & N/A & N/A & N/A \\
\hline B & Floor, Concrete (Electra 1120), (size $\sim 1$ square foot) & N/A & N/A & 40 & 12365 & N/A & N/A & N/A \\
\hline $\mathrm{C}$ & Floor, Concrete (Electra 1120), (size $\sim 4$ square feet) & N/A & $\mathrm{N} / \mathrm{A}$ & 39 & 18823 & N/A & N/A & N/A \\
\hline $\mathrm{D}$ & Floor, Concrete (Electra 1120), (size $\sim 1$ square foot) & N/A & N/A & 26 & 4396 & $\mathrm{~N} / \mathrm{A}$ & N/A & N/A \\
\hline N/A & $\mathrm{N} / \mathrm{A}$ & N/A & $\mathrm{N} / \mathrm{A}$ & N/A & N/A & N/A & N/A & N/A \\
\hline N/A & $\mathrm{N} / \mathrm{A}$ & N/A & $\mathrm{N} / \mathrm{A}$ & N/A & N/A & $\mathrm{N} / \mathrm{A}$ & N/A & N/A \\
\hline $\mathrm{N} / \mathrm{A}$ & $\mathrm{N} / \mathrm{A}$ & N/A & N/A & N/A & N/A & N/A & N/A & N/A \\
\hline N/A & $\mathrm{N} / \mathrm{A}$ & N/A & N/A & N/A & N/A & N/A & N/A & N/A \\
\hline N/A & $\mathrm{N} / \mathrm{A}$ & N/A & N/A & N/A & N/A & N/A & N/A & N/A \\
\hline N/A & $\mathrm{N} / \mathrm{A}$ & N/A & $\mathrm{N} / \mathrm{A}$ & $\mathrm{N} / \mathrm{A}$ & N/A & N/A & N/A & N/A \\
\hline N/A & $\mathrm{N} / \mathrm{A}$ & N/A & $\mathrm{N} / \mathrm{A}$ & N/A & N/A & $\mathrm{N} / \mathrm{A}$ & N/A & N/A \\
\hline N/A & $N / A$ & $\mathrm{~N} / \mathrm{A}$ & N/A & N/A & N/A & N/A & N/A & N/A \\
\hline N/A & $\mathrm{N} / \mathrm{A}$ & N/A & $\mathrm{N} / \mathrm{A}$ & N/A & N/A & N/A & N/A & N/A \\
\hline N/A & $\mathrm{N} / \mathrm{A}$ & N/A & N/A & N/A & N/A & N/A & N/A & N/A \\
\hline N/A & $\mathrm{N} / \mathrm{A}$ & $\mathrm{N} / \mathrm{A}$ & $\mathrm{N} / \mathrm{A}$ & N/A & N/A & N/A & N/A & N/A \\
\hline N/A & $\mathrm{N} / \mathrm{A}$ & N/A & N/A & N/A & N/A & N/A & $\mathrm{N} / \mathrm{A}$ & N/A \\
\hline
\end{tabular}


NSTec

Form

05/24/10

FRM-0108C

RADIOLOGICAL SURVEY REPORT - FULL MAP

Rev. 01

Survey $\# 10-E R-A 26-275$

Description/Map/Drawing/Picture/Comments
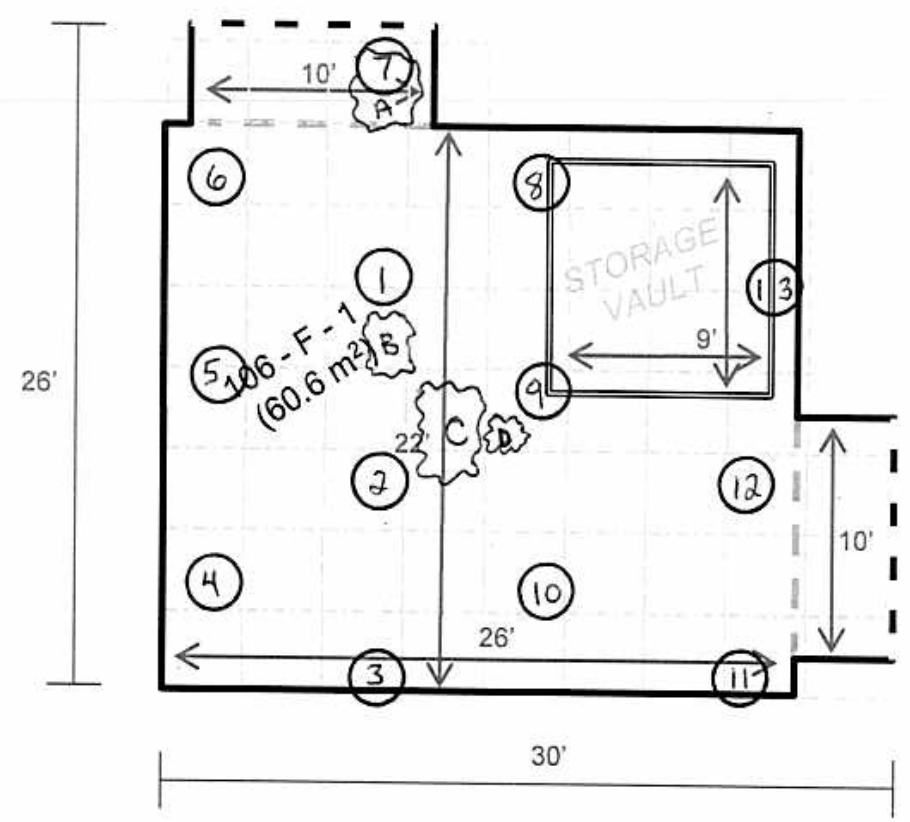

MARSSIM CLASS I SURVEY

$-1 \times 1 m$ grid

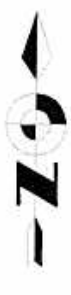

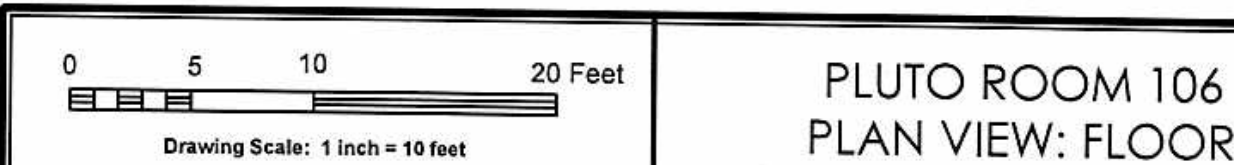

LEGEND

RADIOLOGICAL ENGINEERING

TREATOR: BWM

// DATE: $06 / 21 / 2010$

$0=\operatorname{siminuTe}$, STatic counT $=$ Contact $/ 30 \mathrm{~cm}$

$Y=$ gamma $\quad \beta=$ beta $n=$ neutron

$x-x-x-x=$ Boundary

DLAW EN= SPOT where Trigger 
SURVEY \#

10-ER-A26-291

\begin{tabular}{|c|c|c|c|c|c|c|c|c|c|c|c|}
\hline \multicolumn{3}{|c|}{$\begin{array}{l}\text { Location: } \\
\text { Area } 26 \text { CAU117 Pluto }\end{array}$} & \multicolumn{2}{|c|}{$\begin{array}{l}\text { Purpose: } \\
\text { Final Status Survey, Room } 106 \\
\text { North Wall / 106-NW-1 }\end{array}$} & \multicolumn{5}{|c|}{$\begin{array}{l}\text { Comments: } \\
\text { All one minute static counts are in cpm. Bkgd not subtracted for } \\
\text { Final Status Survey, Survey Plan \#10-015 }\end{array}$} & \multicolumn{2}{|c|}{$\begin{array}{l}\text { Date/Time: } \\
08 / 22 / 20101430\end{array}$} \\
\hline Instrument: & Serial \#: & Cal Due: & $\begin{array}{c}\text { Eff in \%: } \\
\text { Alpha / Beta } \\
\end{array}$ & $\begin{array}{l}\text { BKG in dpm: } \\
\text { Alpha / Beta }\end{array}$ & \begin{tabular}{|l|} 
MDA in dpm: \\
Alpha / Beta \\
\end{tabular} & RWP\# & \multicolumn{5}{|l|}{$\mathrm{N} / \mathrm{A}$} \\
\hline Electra & 1120 & $4 / 6 / 2011$ & $14.1 / 20.8$ & $N / A$ & $N / A$ & \multicolumn{6}{|c|}{ 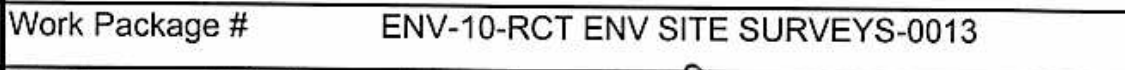 } \\
\hline Electra & 1504 & $4 / 6 / 2011$ & $12.9 / 19.8$ & $\mathrm{~N} / \mathrm{A}$ & $\mathrm{N} / \mathrm{A}$ & \multicolumn{3}{|c|}{ RCT Name: D.Cotroneo Jr. } & \multicolumn{3}{|c|}{ Signature: $\mathrm{O}$} \\
\hline Electra & $\mathrm{N} / \mathrm{A}$ & $\mathrm{N} / \mathrm{A}$ & $N / A$ & $N / A$ & $N / A$ & \multicolumn{3}{|c|}{ RCT Name: K. Wilcox/M.Payne } & \multirow{2}{*}{\multicolumn{3}{|c|}{ 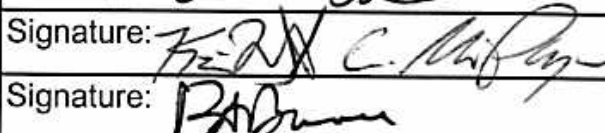 }} \\
\hline Electra & $\mathrm{N} / \mathrm{A}$ & $\mathrm{N} / \mathrm{A}$ & $\mathrm{N} / \mathrm{A}$ & $\mathrm{N} / \mathrm{A}$ & $\mathrm{N} / \mathrm{A}$ & \multicolumn{3}{|c|}{ RCT Name: B. Bunce } & & & \\
\hline Electra & $N / A$ & $N / A$ & $N / A$ & $N / A$ & $\mathrm{~N} / \mathrm{A}$ & RCT Name: & B. Fosket & & \multicolumn{3}{|c|}{ Signature: $\mathrm{S}$} \\
\hline \multirow[t]{2}{*}{ Survey Point } & \multirow{2}{*}{\multicolumn{4}{|c|}{ Description/Comments }} & \multicolumn{2}{|c|}{$\begin{array}{l}\text { Removable } \\
\mathrm{dpm} / 100 \mathrm{~cm}^{2}\end{array}$} & \multicolumn{2}{|c|}{$\begin{array}{c}\begin{array}{c}\text { Fixed + Removable } \\
\mathrm{dpm} / 100 \mathrm{~cm}^{2}\end{array} \\
\end{array}$} & \multirow{2}{*}{$\begin{array}{l}\text { Gamma } \\
\mathrm{mrem} / \mathrm{hr}\end{array}$} & \multirow{2}{*}{$\begin{array}{l}\text { Neutron } \\
\mathrm{mrem} / \mathrm{hr}\end{array}$} & \multirow{2}{*}{$\begin{array}{l}\text { Total } \\
\mathrm{mrem} / \mathrm{hr}\end{array}$} \\
\hline & & & & & Alpha & Beta & Alpha & Beta & & & \\
\hline 1 & \multicolumn{4}{|c|}{ Wall, Concrete (Electra 1504) } & $N / A$ & $N / A$ & 6 & 990 & $N / A$ & $N / A$ & $N / A$ \\
\hline 2 & \multicolumn{4}{|c|}{ Wall, Concrete (Electra 1504) } & $N / A$ & $N / A$ & 8 & 666 & $N / A$ & $N / A$ & $\mathrm{~N} / \mathrm{A}$ \\
\hline 3 & \multicolumn{4}{|c|}{ Wall, Concrete (Electra 1504) } & $N / A$ & $N / A$ & 8 & 657 & $N / A$ & $\mathrm{~N} / \mathrm{A}$ & $N / A$ \\
\hline 4 & \multicolumn{4}{|c|}{ Wall, Concrete (Electra 1504) } & $\mathrm{N} / \mathrm{A}$ & $N / A$ & 12 & 899 & $N / A$ & $N / A$ & $N / A$ \\
\hline 5 & \multicolumn{4}{|c|}{ Wall, Concrete (Electra 1120) } & $N / A$ & $N / A$ & 4 & 988 & $N / A$ & $N / A$ & $N / A$ \\
\hline 6 & \multicolumn{4}{|c|}{ Wall, Concrete (Electra 1120) } & $N / A$ & $N / A$ & 6 & 983 & $\mathrm{~N} / \mathrm{A}$ & $N / A$ & $N / A$ \\
\hline 7 & \multicolumn{4}{|c|}{ Wall, Concrete (Electra 1120) } & $N / A$ & $N / A$ & 8 & 656 & $N / A$ & $N / A$ & $N / A$ \\
\hline 8 & \multicolumn{4}{|c|}{ Wall, Concrete (Electra 1120) } & $N / A$ & $N / A$ & 9 & 600 & $N / A$ & $N / A$ & $N / A$ \\
\hline 9 & \multicolumn{4}{|c|}{ Wall, Concrete (Electra 1120) } & $N / A$ & $N / A$ & 2 & 786 & $N / A$ & $N / A$ & $N / A$ \\
\hline 10 & \multicolumn{4}{|c|}{ Wall, Concrete (Electra 1120) } & $\mathrm{N} / \mathrm{A}$ & $N / A$ & 1 & 749 & $\mathrm{~N} / \mathrm{A}$ & $N / A$ & $N / A$ \\
\hline 11 & \multicolumn{4}{|c|}{ Wall, Concrete (Electra 1504) } & $\mathrm{N} / \mathrm{A}$ & $N / A$ & 7 & 941 & $N / A$ & $N / A$ & $N / A$ \\
\hline \multicolumn{5}{|c|}{ Reviewed By (Print): } & \multicolumn{5}{|l|}{ Signature: } & \multicolumn{2}{|c|}{$8 / 23 / 10$} \\
\hline
\end{tabular}


SURVEY \# 10-ER-A26-291

\begin{tabular}{|c|c|c|c|c|c|c|c|c|}
\hline \multirow[t]{2}{*}{ Survey Point } & \multirow[t]{2}{*}{ Description/Comments } & \multicolumn{2}{|c|}{$\begin{array}{l}\text { Removable } \\
\mathrm{dpm} / 100 \mathrm{~cm}^{2}\end{array}$} & \multicolumn{2}{|c|}{$\begin{array}{c}\text { Fixed + Removable } \\
\text { dpm } / 100 \mathrm{~cm}^{2}\end{array}$} & \multirow{2}{*}{$\begin{array}{l}\text { Gamma } \\
\mathrm{mrem} / \mathrm{hr}\end{array}$} & \multirow{2}{*}{$\begin{array}{l}\text { Neutron } \\
\mathrm{mrem} / \mathrm{hr}\end{array}$} & \multirow{2}{*}{$\begin{array}{c}\text { Total } \\
\text { mrem/hr }\end{array}$} \\
\hline & & Alpha & Beta & Alpha & Beta & & & \\
\hline 12 & Wall, Concrete (1504) & N/A & $\mathrm{N} / \mathrm{A}$ & 8 & 984 & N/A & N/A & $\mathrm{N} / \mathrm{A}$ \\
\hline 13 & Wall, Concrete (1504) & N/A & $\mathrm{N} / \mathrm{A}$ & 11 & 1059 & N/A & N/A & N/A \\
\hline 14 & Wall, Concrete (1504) & N/A. & $\mathrm{N} / \mathrm{A}$ & 6 & 634 & N/A & N/A & N/A \\
\hline N/A & $N / A$ & N/A & $\mathrm{N} / \mathrm{A}$ & N/A & $\mathrm{N} / \mathrm{A}$ & N/A & N/A & $N / A$ \\
\hline N/A & ${ }^{* \star}$ Performed scan surveys for both alpha and beta** & N/A & $\mathrm{N} / \mathrm{A}$ & N/A & $\mathrm{N} / \mathrm{A}$ & N/A & N/A & $\mathrm{N} / \mathrm{A}$ \\
\hline $\mathrm{N} / \mathrm{A}$ & ${ }^{* *}$ on $100 \%$ of survey unit. No trigger levels exceeded ${ }^{\star \star}$ & N/A & $\mathrm{N} / \mathrm{A}$ & N/A & $\mathrm{N} / \mathrm{A}$ & N/A & N/A & N/A \\
\hline N/A & $N / A$ & N/A & $\mathrm{N} / \mathrm{A}$ & N/A & $\mathrm{N} / \mathrm{A}$ & N/A & $N / A$ & N/A \\
\hline N/A & $\mathrm{N} / \mathrm{A}$ & N/A & $\mathrm{N} / \mathrm{A}$ & $\mathrm{N} / \mathrm{A}$ & $\mathrm{N} / \mathrm{A}$ & N/A & $\mathrm{N} / \mathrm{A}$ & N/A \\
\hline N/A & $N / A$ & N/A & $\mathrm{N} / \mathrm{A}$ & N/A & $\mathrm{N} / \mathrm{A}$ & N/A & N/A & N/A \\
\hline N/A & $N / A$ & N/A & N/A & N/A & $\mathrm{N} / \mathrm{A}$ & $N / A$ & N/A & N/A \\
\hline N/A & $\mathrm{N} / \mathrm{A}$ & N/A & $\mathrm{N} / \mathrm{A}$ & N/A & $\mathrm{N} / \mathrm{A}$ & N/A & $N / A$ & N/A \\
\hline N/A & $\mathrm{N} / \mathrm{A}$ & N/A & N/A & N/A & $\mathrm{N} / \mathrm{A}$ & N/A & $N / A$ & N/A \\
\hline N/A & $N / A$ & N/A & $\mathrm{N} / \mathrm{A}$ & N/A & $\mathrm{N} / \mathrm{A}$ & N/A & N/A & $\mathrm{N} / \mathrm{A}$ \\
\hline N/A & $\mathrm{N} / \mathrm{A}$ & N/A & N/A & N/A & N/A & N/A & N/A & N/A \\
\hline N/A & $N / A$ & N/A & N/A & N/A & N/A & N/A & N/A & $\mathrm{N} / \mathrm{A}$ \\
\hline N/A & $N / A$ & N/A & N/A & N/A & $\mathrm{N} / \mathrm{A}$ & N/A & N/A & N/A \\
\hline N/A & $N / A$ & N/A & N/A & N/A & N/A & N/A & $\mathrm{N} / \mathrm{A}$ & N/A \\
\hline N/A & $N / A$ & N/A & N/A & N/A & $\mathrm{N} / \mathrm{A}$ & N/A & N/A & N/A \\
\hline N/A & $N / A$ & N/A & N/A & $\mathrm{N} / \mathrm{A}$ & N/A & N/A & N/A & N/A \\
\hline $\mathrm{N} / \mathrm{A}$ & $N / A$ & N/A & N/A & N/A & N/A & N/A & N/A & N/A \\
\hline N/A & $N / A$ & N/A & N/A & $\mathrm{N} / \mathrm{A}$ & N/A & $\mathrm{N} / \mathrm{A}$ & N/A & N/A \\
\hline N/A & $\mathrm{N} / \mathrm{A}$ & N/A & N/A & N/A & N/A & N/A & N/A & N/A \\
\hline N/A & $N / A$ & N/A & N/A & N/A & N/A & N/A & N/A & N/A \\
\hline
\end{tabular}




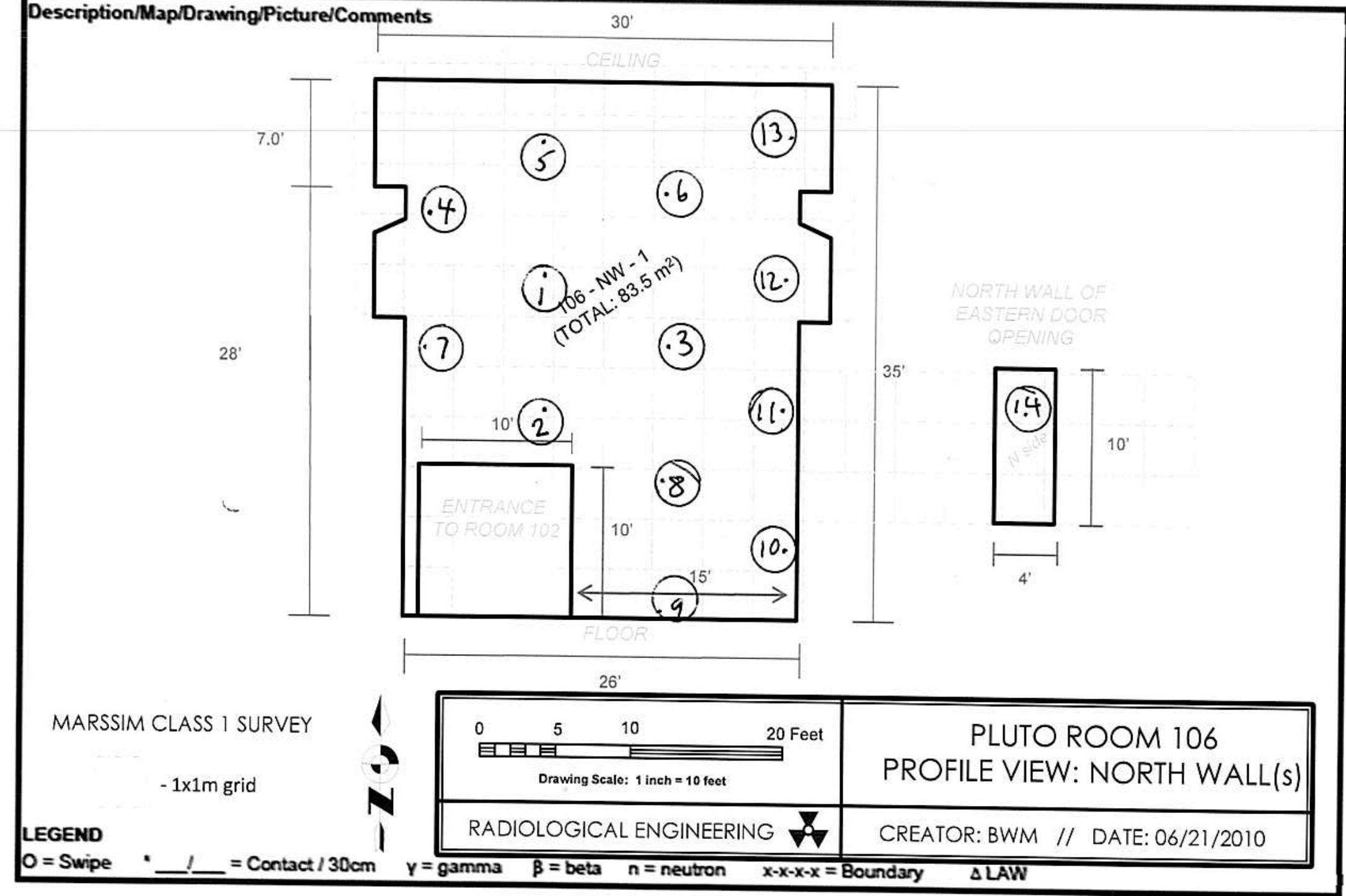




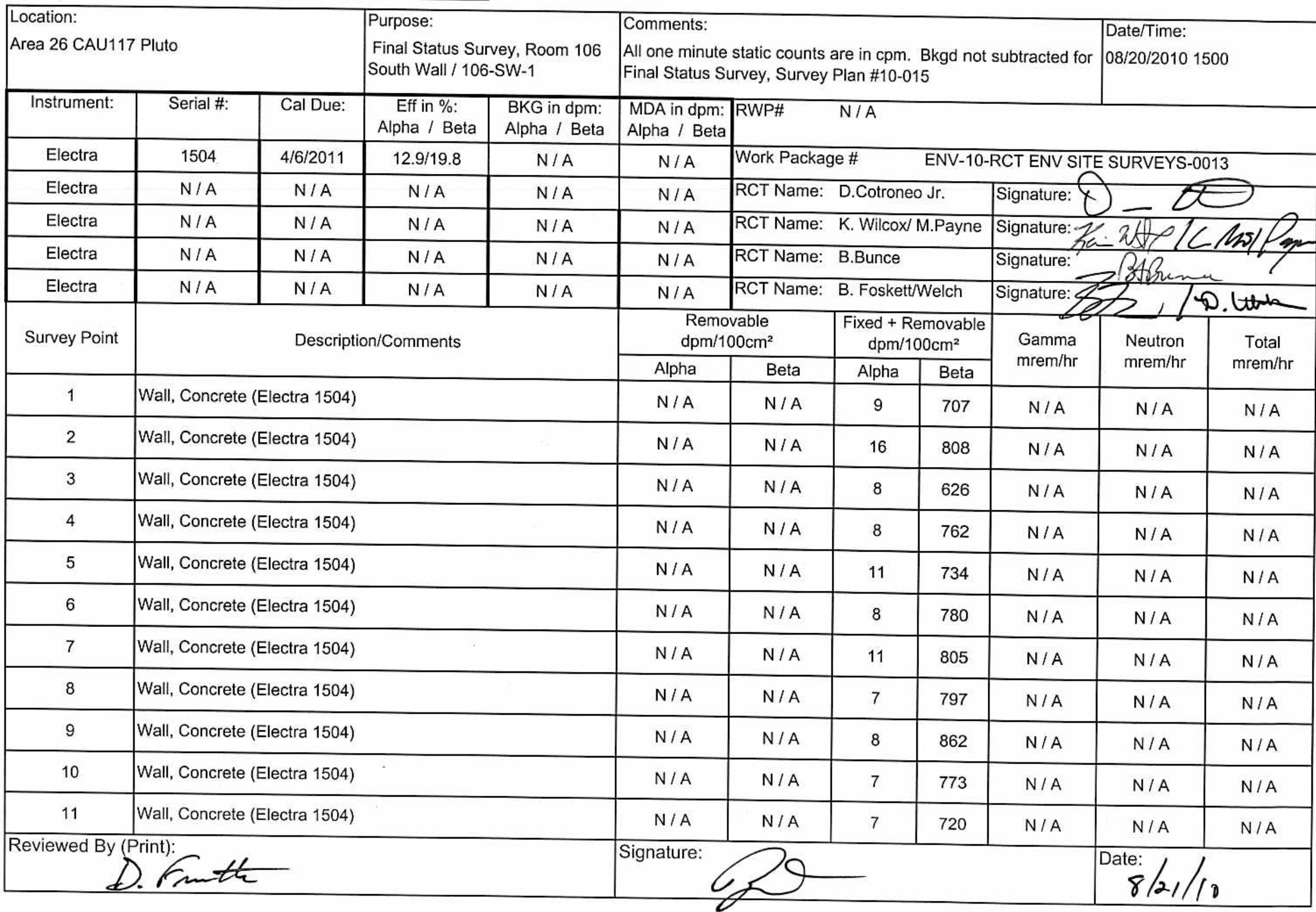




\section{NSTec}

\section{Form}

FRM-0108B

RADIOLOGICAL SURVEY REPORT - DATA (CONTINUATION)

SURVEY \# 10-ER-A26-284

\begin{tabular}{|c|c|c|c|c|c|c|c|c|}
\hline \multirow[t]{2}{*}{ Survey Point } & \multirow[t]{2}{*}{ Description/Comments } & \multicolumn{2}{|c|}{$\begin{array}{l}\text { Removable } \\
\mathrm{dpm} / 100 \mathrm{~cm}^{2}\end{array}$} & \multicolumn{2}{|c|}{$\begin{array}{c}\text { Fixed + Removable } \\
\mathrm{dpm} / 100 \mathrm{~cm}^{2}\end{array}$} & \multirow{2}{*}{$\begin{array}{l}\text { Gamma } \\
\mathrm{mrem} / \mathrm{hr}\end{array}$} & \multirow{2}{*}{$\begin{array}{l}\text { Neutron } \\
\mathrm{mrem} / \mathrm{hr}\end{array}$} & \multirow{2}{*}{$\begin{array}{c}\text { Total } \\
\text { mrem } / \mathrm{hr}\end{array}$} \\
\hline & & Alpha & Beta & Alpha & Beta & & & \\
\hline 12 & Wall, Concrete (1504) & N/A & $N / A$ & 10 & 755 & N/A & N/A & N/A \\
\hline 13 & Metal, Concrete (1504) & N/A & N/A & 8 & 469 & N/A & $\mathrm{N} / \mathrm{A}$ & N/A \\
\hline N/A & $\mathrm{N} / \mathrm{A}$ & N/A & N/A & N/A & $\mathrm{N} / \mathrm{A}$ & N/A & $\mathrm{N} / \mathrm{A}$ & N/A \\
\hline N/A & ${ }^{\star \star}$ Performed scan surveys for both alpha and beta** & N/A & N/A & N/A & $\mathrm{N} / \mathrm{A}$ & $N / A$ & N/A & N/A \\
\hline N/A & ${ }^{\star \star}$ on $100 \%$ of survey unit. No trigger levels exceeded ${ }^{\star \star}$ & $\mathrm{N} / \mathrm{A}$ & $\mathrm{N} / \mathrm{A}$ & N/A & N/A & N/A & N/A & N/A \\
\hline N/A & $N / A$ & $\mathrm{~N} / \mathrm{A}$ & $\mathrm{N} / \mathrm{A}$ & N/A & N/A & N/A & N/A & N/A \\
\hline N/A & $N / A$ & N/A & N/A & N/A & $\mathrm{N} / \mathrm{A}$ & N/A & $N / A$ & N/A \\
\hline N/A & $N / A$ & $\mathrm{~N} / \mathrm{A}$ & $\mathrm{N} / \mathrm{A}$ & $\mathrm{N} / \mathrm{A}$ & $\mathrm{N} / \mathrm{A}$ & N/A & N/A & N/A \\
\hline N/A & $\mathrm{N} / \mathrm{A}$ & N/A & N/A & N/A & $\mathrm{N} / \mathrm{A}$ & N/A & N/A & $\mathrm{N} / \mathrm{A}$ \\
\hline N/A & $N / A$ & N/A & N/A & N/A & N/A & N/A & N/A & N/A \\
\hline $\mathrm{N} / \mathrm{A}$ & $N / A$ & N/A & $\mathrm{N} / \mathrm{A}$ & N/A & $\mathrm{N} / \mathrm{A}$ & N/A & N/A & N/A \\
\hline N/A & $N / A$ & N/A & $\mathrm{N} / \mathrm{A}$ & N/A & $\mathrm{N} / \mathrm{A}$ & N/A & N/A & $\mathrm{N} / \mathrm{A}$ \\
\hline N/A & $\mathrm{N} / \mathrm{A}$ & N/A & N/A & N/A & $\mathrm{N} / \mathrm{A}$ & N/A & N/A & N/A \\
\hline N/A & $\mathrm{N} / \mathrm{A}$ & N/A & N/A & N/A & N/A & N/A & N/A & N/A \\
\hline N/A & $N / A$ & N/A & N/A & N/A & N/A & N/A & N/A & N/A \\
\hline N/A & $\mathrm{N} / \mathrm{A}$ & N/A & N/A & N/A & $\mathrm{N} / \mathrm{A}$ & N/A & N/A & N/A \\
\hline N/A & $N / A$ & N/A & $\mathrm{N} / \mathrm{A}$ & N/A & N/A & N/A & $\mathrm{N} / \mathrm{A}$ & N/A \\
\hline N/A & $N / A$ & N/A & N/A & N/A & N/A & N/A & N/A & N/A \\
\hline N/A & $\mathrm{N} / \mathrm{A}$ & N/A & N/A & N/A & $\mathrm{N} / \mathrm{A}$ & $\mathrm{N} / \mathrm{A}$ & N/A & $\mathrm{N} / \mathrm{A}$ \\
\hline N/A & $\mathrm{N} / \mathrm{A}$ & N/A & N/A & N/A & N/A & N/A & N/A & N/A \\
\hline N/A & $N / A$ & N/A & $\mathrm{N} / \mathrm{A}$ & N/A & $\mathrm{N} / \mathrm{A}$ & N/A & $\mathrm{N} / \mathrm{A}$ & N/A \\
\hline N/A & $\mathrm{N} / \mathrm{A}$ & N/A & N/A & N/A & N/A & N/A & N/A & N/A \\
\hline $\mathrm{N} / \mathrm{A}$ & $\mathrm{N} / \mathrm{A}$ & N/A & N/A & $\mathrm{N} / \mathrm{A}$ & N/A & N/A & N/A & $\mathrm{N} / \mathrm{A}$ \\
\hline
\end{tabular}


NSTec

Form

Survey $\# 10-E R-A 26-284$

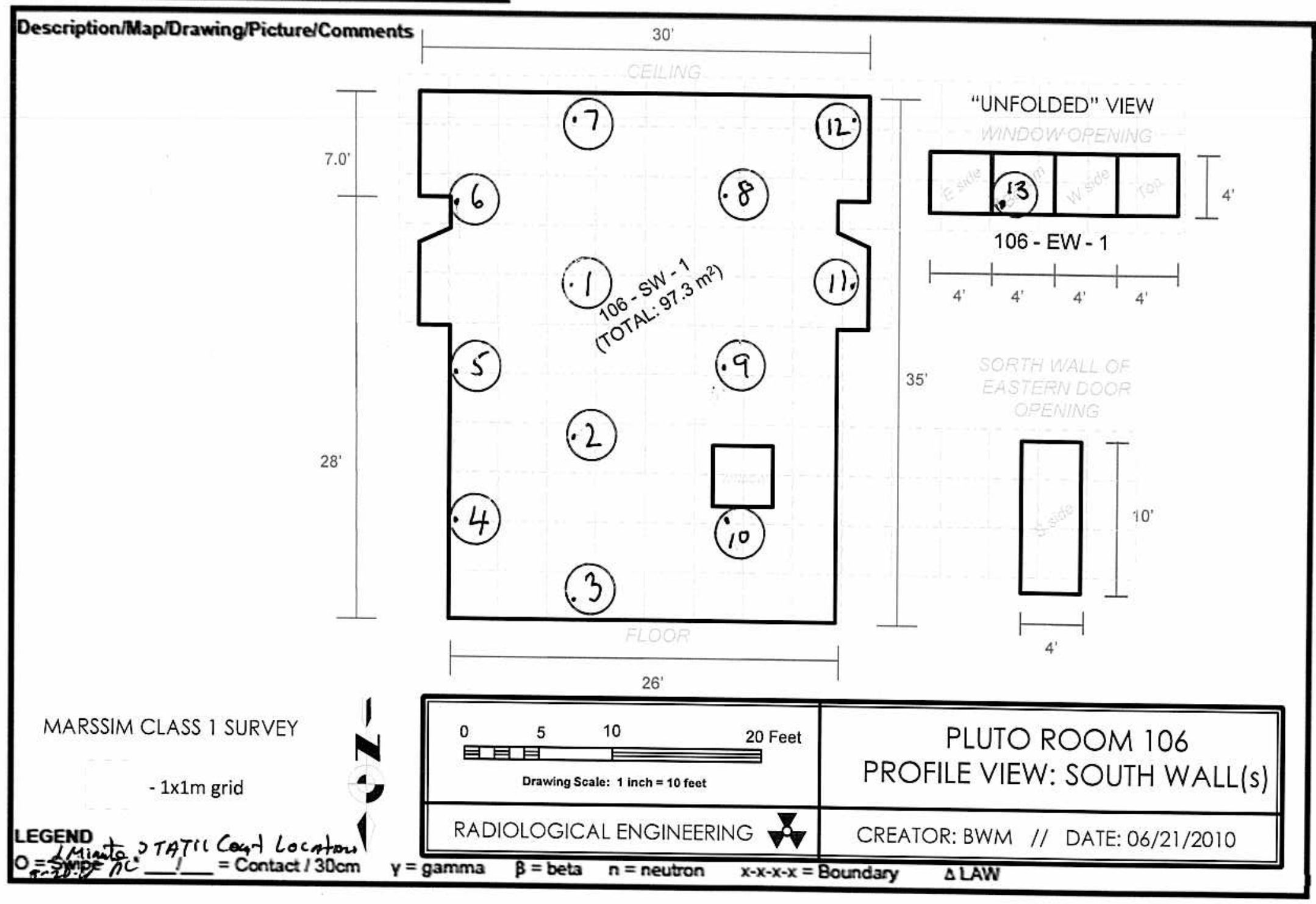


SURVEY \#

10-ER-A26-282

\begin{tabular}{|c|c|c|c|c|c|c|c|c|c|c|c|}
\hline \multicolumn{3}{|c|}{$\begin{array}{l}\text { Location: } \\
\text { Area } 26 \text { CAU117 Pluto }\end{array}$} & \multicolumn{2}{|c|}{$\begin{array}{l}\text { Purpose: } \\
\text { Final Status Survey, Room 106, } \\
\text { West Wall, 106-WW-1 }\end{array}$} & \multicolumn{5}{|c|}{$\begin{array}{l}\text { Comments: } \\
\text { All one minute static counts are in cpm. Bkgd not subtracted for } \\
\text { Final Status Survey, Survey Plan \#10-015 }\end{array}$} & \multicolumn{2}{|c|}{$\begin{array}{l}\text { Date/Time: } \\
\text { 08/20/2010 } 1100\end{array}$} \\
\hline Instrument: & Serial \#: & Cal Due: & $\begin{array}{c}\text { Eff in \%: } \\
\text { Alpha / Beta }\end{array}$ & $\begin{array}{l}\text { BKG in dpm: } \\
\text { Alpha / Beta }\end{array}$ & \begin{tabular}{|l|} 
MDA in dpm: \\
Alpha / Beta \\
\end{tabular} & RWP\# & \multicolumn{5}{|l|}{$\mathrm{N} / \mathrm{A}$} \\
\hline Electra & 1504 & $4 / 6 / 2011$ & $12.9 / 19.8$ & $N / A$ & $\mathrm{~N} / \mathrm{A}$ & \multicolumn{6}{|c|}{ Work Package \# $\quad$ ENV-10-RCT ENV SITE SURVEYS-0013 } \\
\hline$N / A$ & $N / A$ & $N / A$ & $\mathrm{~N} / \mathrm{A}$ & $N / A$ & $\mathrm{~N} / \mathrm{A}$ & \multicolumn{3}{|c|}{ RCT Name: D.Cotroneo Jr. } & Signature: & $\sim$ & \\
\hline$N / A$ & $\mathrm{~N} / \mathrm{A}$ & $N / A$ & $\mathrm{~N} / \mathrm{A}$ & $N / A$ & $N / A$ & \multicolumn{3}{|c|}{ RCT Name: Kevin Wilcox } & \multicolumn{3}{|c|}{ Signature: Keri 2leor } \\
\hline$N / A$ & $\mathrm{~N} / \mathrm{A}$ & $\mathrm{N} / \mathrm{A}$ & $\mathrm{N} / \mathrm{A}$ & $\mathrm{N} / \mathrm{A}$ & $\mathrm{N} / \mathrm{A}$ & \multicolumn{3}{|c|}{ RCT Name: Brian Bunce } & Signature: & \multicolumn{2}{|c|}{ Athine } \\
\hline $\mathrm{N} / \mathrm{A}$ & $\mathrm{N} / \mathrm{A}$ & $N / A$ & $N / A$ & $N / A$ & $\mathrm{~N} / \mathrm{A}$ & \multicolumn{3}{|c|}{ RCT Name: Dave Welch } & \multicolumn{3}{|c|}{ Signature: $\quad$ ¿D, Utom } \\
\hline \multirow[t]{2}{*}{ Survey Point } & \multirow{2}{*}{\multicolumn{4}{|c|}{ Description/Comments }} & \multicolumn{2}{|c|}{$\begin{array}{l}\text { Removable } \\
\mathrm{dpm} / 100 \mathrm{~cm}^{2}\end{array}$} & \multicolumn{2}{|c|}{\begin{tabular}{|c|} 
Fixed + Removable \\
$\mathrm{dpm} / 100 \mathrm{~cm}^{2}$
\end{tabular}} & \multirow{2}{*}{$\begin{array}{l}\text { Gamma } \\
\mathrm{mrem} / \mathrm{hr}\end{array}$} & \multirow{2}{*}{$\begin{array}{l}\text { Neutron } \\
\text { mrem } / \mathrm{hr}\end{array}$} & \multirow{2}{*}{$\begin{array}{l}\text { Total } \\
\mathrm{mrem} / \mathrm{hr}\end{array}$} \\
\hline & & & & & Alpha & Beta & Alpha & Beta & & & \\
\hline 1 & \multicolumn{4}{|l|}{ Wall, Concrete } & $N / A$ & $N / A$ & 7 & 739 & $N / A$ & $N / A$ & $N / A$ \\
\hline 2 & \multicolumn{4}{|l|}{ Wall, Concrete } & $N / A$ & $N / A$ & 7 & 1557 & $N / A$ & $\mathrm{~N} / \mathrm{A}$ & $N / A$ \\
\hline 3 & \multicolumn{4}{|l|}{ Wall, Concrete } & $N / A$ & $N / A$ & 7 & 746 & $N / A$ & $\mathrm{~N} / \mathrm{A}$ & $N / A$ \\
\hline 4 & \multicolumn{4}{|l|}{ Wall, Concrete } & $\mathrm{N} / \mathrm{A}$ & $N / A$ & 11 & 720 & $\mathrm{~N} / \mathrm{A}$ & $N / A$ & $\mathrm{~N} / \mathrm{A}$ \\
\hline 5 & \multicolumn{4}{|l|}{ Wall, Concrete } & $N / A$ & $N / A$ & 13 & 670 & $N / A$ & $N / A$ & $\mathrm{~N} / \mathrm{A}$ \\
\hline 6 & \multicolumn{4}{|l|}{ Wall, Concrete } & $N / A$ & $\mathrm{~N} / \mathrm{A}$ & 8 & 726 & $N / A$ & $N / A$ & $N / A$ \\
\hline 7 & \multicolumn{4}{|l|}{ Wall, Concrete } & $N / A$ & $N / A$ & 11 & 741 & $N / A$ & $N / A$ & $\mathrm{~N} / \mathrm{A}$ \\
\hline 8 & \multicolumn{4}{|l|}{ Wall, Concrete } & $N / A$ & $N / A$ & 4 & 757 & $\mathrm{~N} / \mathrm{A}$ & $\mathrm{N} / \mathrm{A}$ & $N / A$ \\
\hline 9 & \multicolumn{4}{|l|}{ Wall, Concrete } & $\mathrm{N} / \mathrm{A}$ & $\mathrm{N} / \mathrm{A}$ & 9 & 695 & $N / A$ & $\mathrm{~N} / \mathrm{A}$ & $N / A$ \\
\hline 10 & \multicolumn{4}{|l|}{ Wall, Concrete } & $N / A$ & $\mathrm{~N} / \mathrm{A}$ & 10 & 691 & $N / A$ & $\mathrm{~N} / \mathrm{A}$ & $N / A$ \\
\hline 11 & \multicolumn{4}{|l|}{ Wall, Concrete } & $N / A$ & $N / A$ & 16 & 672 & $N / A$ & $N / A$ & $N / A$ \\
\hline \multicolumn{5}{|c|}{ Reviewed By (Print): } & Signature: & & & & & \multicolumn{2}{|c|}{ Date: $8 / 20 / 10$} \\
\hline
\end{tabular}




\section{NSTec}

SURVEY \# 10-ER-A26-282

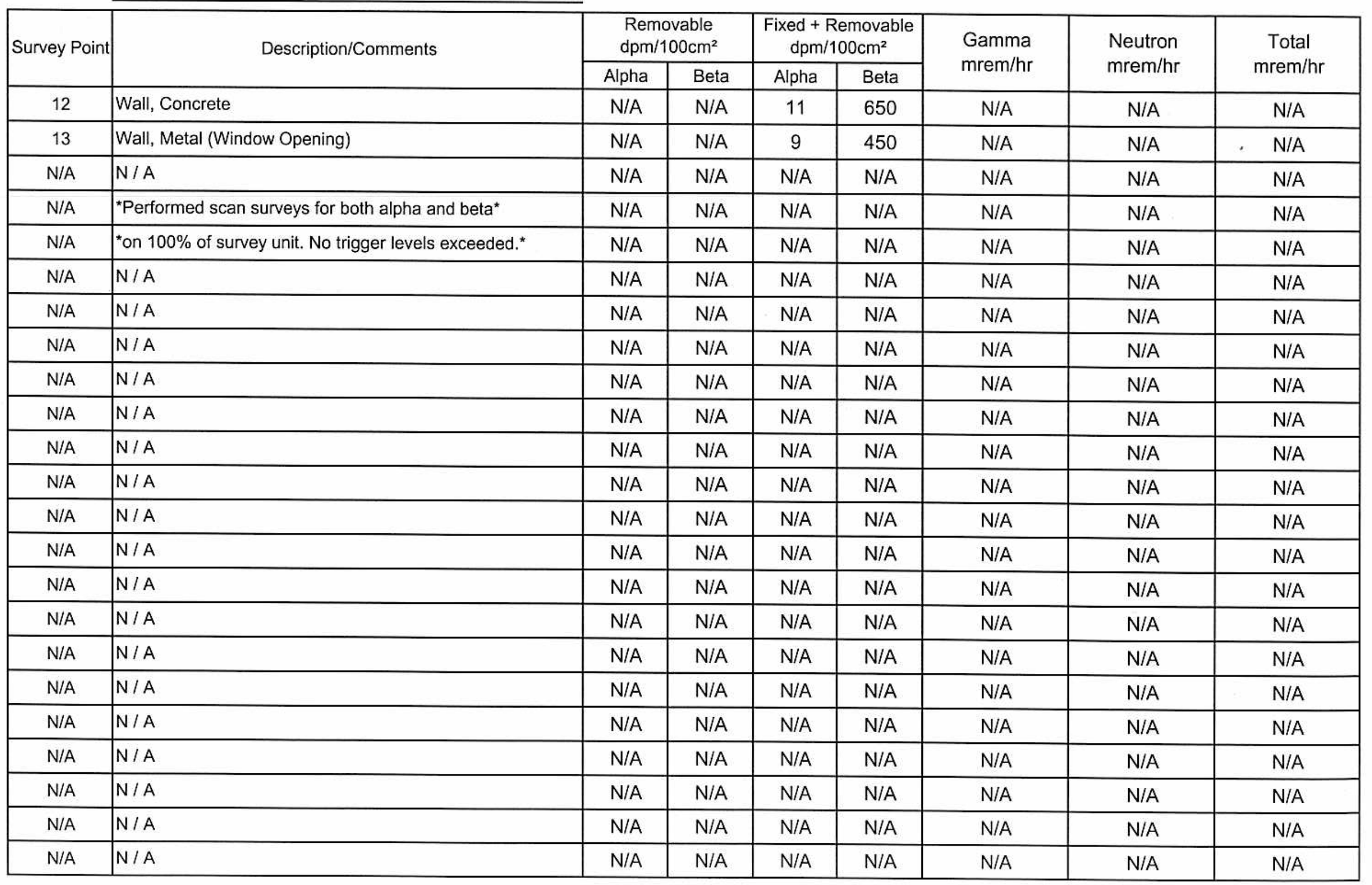


Survey $\# 10-E R-A 26-282$

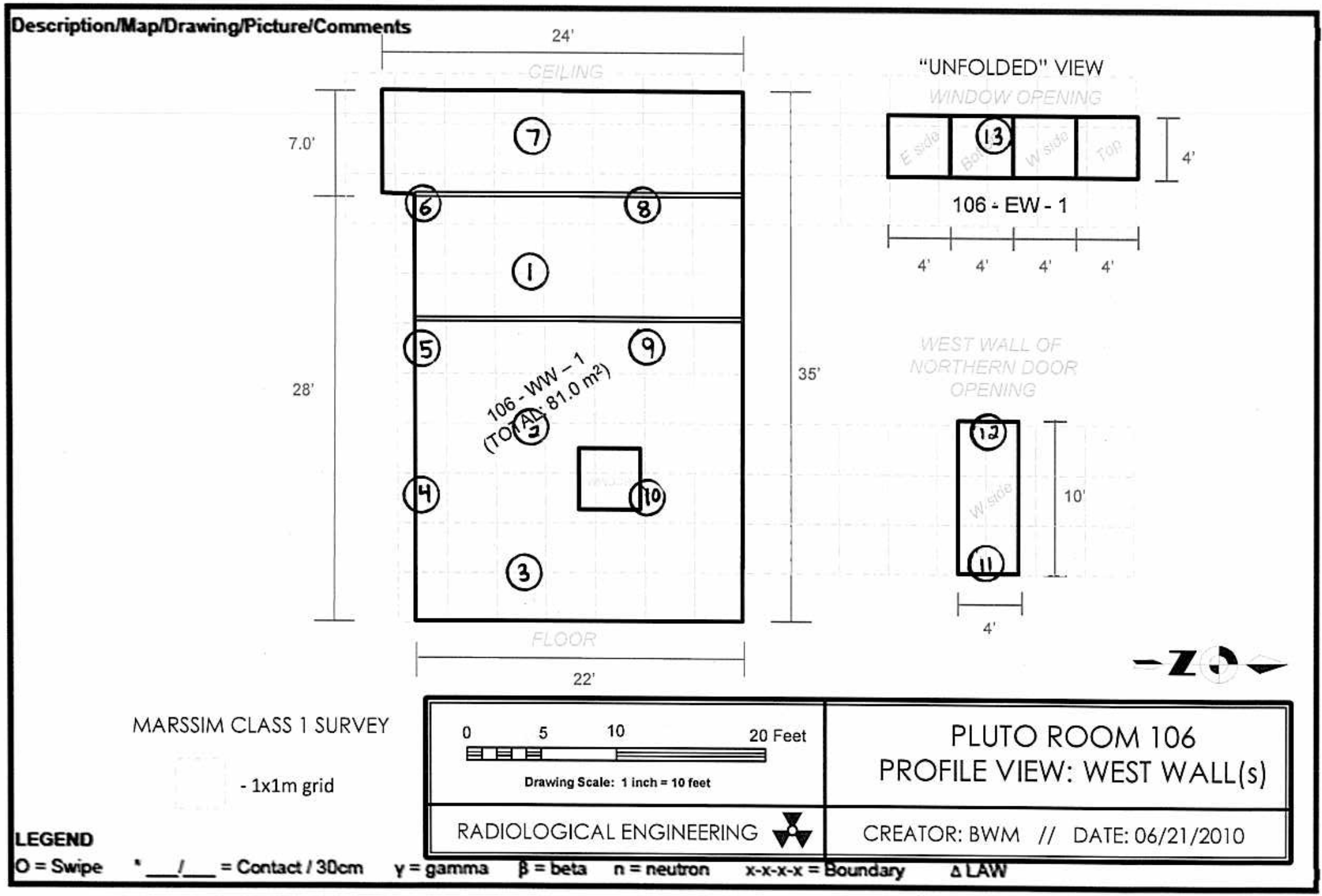




\begin{tabular}{|c|c|c|c|c|c|c|c|c|c|c|c|}
\hline \multicolumn{3}{|c|}{$\begin{array}{l}\text { Location: } \\
\text { Area } 26 \text { CAU117 Pluto }\end{array}$} & \multicolumn{2}{|c|}{$\begin{array}{l}\text { Purpose: } \\
\text { Final Status Survey, Room } 107 \\
\text { East Wall / 107-EW-1 }\end{array}$} & \multicolumn{5}{|c|}{$\begin{array}{l}\text { Comments: } \\
\text { All one minute static counts are in cpm. Bkgd not subtracted for } \\
\text { Final Status Survey, Survey Plan \#10-015 }\end{array}$} & \multicolumn{2}{|c|}{$\begin{array}{l}\text { Date/Time: } \\
\text { 08/22/2010 } 1000\end{array}$} \\
\hline Instrument: & Serial \#: & Cal Due: & $\begin{array}{c}\text { Eff in \%: } \\
\text { Alpha / Beta } \\
\end{array}$ & $\begin{array}{l}\text { BKG in dpm: } \\
\text { Alpha / Beta }\end{array}$ & \begin{tabular}{|l|} 
MDA in dpm: \\
Alpha / Beta \\
\end{tabular} & RWP\# & \multicolumn{5}{|l|}{$\mathrm{N} / \mathrm{A}$} \\
\hline Electra & 1120 & $4 / 6 / 2011$ & $14.1 / 20.8$ & $N / A$ & $N / A$ & \multicolumn{6}{|c|}{ Work Package \# $\quad$ ENV-10-RCT ENV SITE SURVEYS-0013 } \\
\hline Electra & 1504 & $4 / 6 / 2011$ & $12.9 / 19.8$ & $\mathrm{~N} / \mathrm{A}$ & $\mathrm{N} / \mathrm{A}$ & \multicolumn{3}{|c|}{ RCT Name: D.Cotroneo Jr. } & \multicolumn{3}{|c|}{ Signature: $y-\theta$} \\
\hline Electra & 2125 & $4 / 6 / 2011$ & $14.4 / 18.2$ & $\mathrm{~N} / \mathrm{A}$ & $\mathrm{N} / \mathrm{A}$ & \multicolumn{3}{|c|}{ RCT Name: K. Wilcox/M.Payne } & \multirow{2}{*}{\multicolumn{3}{|c|}{ 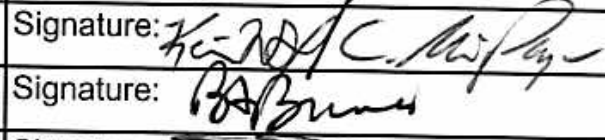 }} \\
\hline Electra & $\mathrm{N} / \mathrm{A}$ & $\mathrm{N} / \mathrm{A}$ & $\mathrm{N} / \mathrm{A}$ & $\mathrm{N} / \mathrm{A}$ & $\mathrm{N} / \mathrm{A}$ & \multicolumn{3}{|c|}{ RCT Name: B. Bunce } & & & \\
\hline Electra & $N / A$ & $\mathrm{~N} / \mathrm{A}$ & $\mathrm{N} / \mathrm{A}$ & $N / A$ & $N / A$ & RCT Name: & B. Foskett & & \multicolumn{3}{|c|}{ Signature: } \\
\hline \multirow[t]{2}{*}{ Survey Point } & \multirow{2}{*}{\multicolumn{4}{|c|}{ Description/Comments }} & \multicolumn{2}{|c|}{$\begin{array}{c}\begin{array}{c}\text { Removable } \\
\mathrm{dpm} / 100 \mathrm{~cm}^{2}\end{array} \\
\end{array}$} & \multicolumn{2}{|c|}{\begin{tabular}{|c|}
$\begin{array}{c}\text { Fixed + Removable } \\
\mathrm{dpm} / 100 \mathrm{~cm}^{2}\end{array}$ \\
\end{tabular}} & \multirow{2}{*}{$\begin{array}{c}\text { Gamma } \\
\mathrm{mrem} / \mathrm{hr}\end{array}$} & \multirow{2}{*}{$\begin{array}{l}\text { Neutron } \\
\mathrm{mrem} / \mathrm{hr}\end{array}$} & \multirow{2}{*}{$\begin{array}{l}\text { Total } \\
\mathrm{mrem} / \mathrm{hr}\end{array}$} \\
\hline & & & & & Alpha & Beta & Alpha & Beta & & & \\
\hline 1 & \multicolumn{4}{|c|}{ Wall, Concrete (Electra 1120) } & $N / A$ & $N / A$ & 8 & 1039 & $N / A$ & $N / A$ & $N / A$ \\
\hline 2 & \multicolumn{4}{|c|}{ Wall, Concrete (Electra 1120) } & $N / A$ & $N / A$ & 3 & 659 & $N / A$ & $N / A$ & $N / A$ \\
\hline 3 & \multicolumn{4}{|c|}{ Wall, Concrete (Electra 2125) } & $N / A$ & $N / A$ & 11 & 515 & $N / A$ & $N / A$ & $N / A$ \\
\hline 4 & \multicolumn{4}{|c|}{ Wall, Concrete (Electra 2125) } & $N / A$ & $N / A$ & 10 & 617 & $\mathrm{~N} / \mathrm{A}$ & $N / A$ & $N / A$ \\
\hline 5 & \multicolumn{4}{|c|}{ Wall, Metal (Electra 1120) } & $N / A$ & $N / A$ & 10 & 461 & $N / A$ & $N / A$ & $\mathrm{~N} / \mathrm{A}$ \\
\hline 6 & \multicolumn{4}{|c|}{ Wall, Metal (Electra 1504) } & $N / A$ & $N / A$ & 8 & 494 & $N / A$ & $N / A$ & $\mathrm{~N} / \mathrm{A}$ \\
\hline 7 & \multicolumn{4}{|c|}{ Wall, Concrete (Electra 2125) } & $\mathrm{N} / \mathrm{A}$ & $N / A$ & 5 & 690 & $N / A$ & $N / A$ & $\mathrm{~N} / \mathrm{A}$ \\
\hline 8 & \multicolumn{4}{|c|}{ Wall, Concrete (Electra 1504) } & $N / A$ & $N / A$ & 7 & 987 & $\mathrm{~N} / \mathrm{A}$ & $N / A$ & $N / A$ \\
\hline 9 & \multicolumn{4}{|c|}{ Wall, Concrete (Electra 1120) } & $N / A$ & $\mathrm{~N} / \mathrm{A}$ & 13 & 982 & $N / A$ & $N / A$ & $N / A$ \\
\hline 10 & \multicolumn{4}{|c|}{ Wall, Concrete (Electra 1120) } & $N / A$ & $N / A$ & 5 & 1022 & $N / A$ & $N / A$ & $N / A$ \\
\hline 11 & \multicolumn{4}{|c|}{ Wall, Metal (Electra 1504) } & $N / A$ & $N / A$ & 7 & 480 & $N / A$ & $N / A$ & $N / A$ \\
\hline \multicolumn{5}{|c|}{ Reviewed By (Print): } & $g n$ & & & & & $\begin{array}{r}\text { Date: } \\
8 / 2\end{array}$ & \\
\hline
\end{tabular}




\section{NSTec}

SURVEY \# 10-ER-A26-288

\begin{tabular}{|c|c|c|c|c|c|c|c|c|}
\hline \multirow[t]{2}{*}{ Survey Point } & \multirow[t]{2}{*}{ Description/Comments } & \multicolumn{2}{|c|}{$\begin{array}{l}\text { Removable } \\
\mathrm{dpm} / 100 \mathrm{~cm}^{2}\end{array}$} & \multicolumn{2}{|c|}{$\begin{array}{c}\text { Fixed + Removable } \\
\mathrm{dpm} / 100 \mathrm{~cm}^{2}\end{array}$} & \multirow{2}{*}{$\begin{array}{c}\text { Gamma } \\
\text { mrem/hr }\end{array}$} & \multirow{2}{*}{$\begin{array}{l}\text { Neutron } \\
\mathrm{mrem} / \mathrm{hr}\end{array}$} & \multirow{2}{*}{$\begin{array}{c}\text { Total } \\
\mathrm{mrem} / \mathrm{hr}\end{array}$} \\
\hline & & Alpha & Beta & Alpha & Beta & & & \\
\hline 12 & Wall, Metal (1504) & N/A & N/A & 17 & 902 & $\mathrm{~N} / \mathrm{A}$ & $\mathrm{N} / \mathrm{A}$ & N/A \\
\hline 13 & Wall, Concrete (1504) & N/A & N/A & 14 & 764 & $\mathrm{~N} / \mathrm{A}$ & N/A & N/A \\
\hline 14 & Wall, Concrete (2125) & N/A & $\mathrm{N} / \mathrm{A}$ & 11 & 1052 & $\mathrm{~N} / \mathrm{A}$ & N/A & N/A \\
\hline 15 & Wall, Concrete (1120) & N/A & $\mathrm{N} / \mathrm{A}$ & 8 & 747 & N/A & N/A & N/A \\
\hline 16 & Wall, Concrete (1120) & N/A & $\mathrm{N} / \mathrm{A}$ & 12 & 1128 & $\mathrm{~N} / \mathrm{A}$ & N/A & N/A \\
\hline 17 & Wall, Concrete (1504) & N/A & N/A & 6 & 593 & $\mathrm{~N} / \mathrm{A}$ & $\mathrm{N} / \mathrm{A}$ & N/A \\
\hline 18 & Wall, Concrete (1504) & N/A & $\mathrm{N} / \mathrm{A}$ & 9 & 611 & N/A & $\mathrm{N} / \mathrm{A}$ & N/A \\
\hline 19 & Wall, Concrete (1120) & $\mathrm{N} / \mathrm{A}$ & N/A & 9 & 1078 & $\mathrm{~N} / \mathrm{A}$ & N/A & N/A \\
\hline 20 & Wall, Concrete (1120) & N/A & $\mathrm{N} / \mathrm{A}$ & 7 & 1048 & N/A & $\mathrm{N} / \mathrm{A}$ & $\mathrm{N} / \mathrm{A}$ \\
\hline N/A & ${ }^{\star \star}$ Performed scan surveys for both alpha and beta ${ }^{\star \star}$ & N/A & N/A & N/A & N/A & N/A & N/A & N/A \\
\hline N/A & **on $100 \%$ of survey unit. No trigger levels exceeded ${ }^{\star *}$ & N/A & N/A & N/A & $\mathrm{N} / \mathrm{A}$ & N/A & N/A & $\mathrm{N} / \mathrm{A}$ \\
\hline N/A & $N / A$ & N/A & N/A & $\mathrm{N} / \mathrm{A}$ & $\mathrm{N} / \mathrm{A}$ & N/A & N/A & N/A \\
\hline N/A & $\mathrm{N} / \mathrm{A}$ & N/A & N/A & N/A & $\mathrm{N} / \mathrm{A}$ & N/A & N/A & N/A \\
\hline N/A & $\mathrm{N} / \mathrm{A}$ & N/A & N/A & N/A & N/A & $\mathrm{N} / \mathrm{A}$ & N/A & N/A \\
\hline N/A & $\mathrm{N} / \mathrm{A}$ & N/A & N/A & N/A & N/A & N/A & N/A & N/A \\
\hline N/A & $N / A$ & N/A & N/A & N/A & N/A & N/A & N/A & N/A \\
\hline N/A & $\mathrm{N} / \mathrm{A}$ & N/A & $\mathrm{N} / \mathrm{A}$ & N/A & N/A & N/A & N/A & $\mathrm{N} / \mathrm{A}$ \\
\hline N/A & $\mathrm{N} / \mathrm{A}$ & N/A & N/A & $\mathrm{N} / \mathrm{A}$ & N/A & N/A & N/A & N/A \\
\hline N/A & $\mathrm{N} / \mathrm{A}$ & N/A & N/A & $\mathrm{N} / \mathrm{A}$ & $\mathrm{N} / \mathrm{A}$ & $\mathrm{N} / \mathrm{A}$ & N/A & $\mathrm{N} / \mathrm{A}$ \\
\hline N/A & $N / A$ & N/A & N/A & N/A & N/A & N/A & $\mathrm{N} / \mathrm{A}$ & N/A \\
\hline N/A & $\mathrm{N} / \mathrm{A}$ & $\mathrm{N} / \mathrm{A}$ & N/A & N/A & $\mathrm{N} / \mathrm{A}$ & N/A & N/A & N/A \\
\hline N/A & $\mathrm{N} / \mathrm{A}$ & N/A & N/A & N/A & N/A & N/A & N/A & N/A \\
\hline N/A & $\mathrm{N} / \mathrm{A}$ & N/A & N/A & N/A & $\mathrm{N} / \mathrm{A}$ & N/A & N/A & N/A \\
\hline
\end{tabular}




\section{NSTec}

Form

05/24/10

Rev. 01

FRM-0108C

RADIOLOGICAL SURVEY REPORT - FULL MAP

Page 3 of 3

Survey $\# 10-F R-A 26-288$

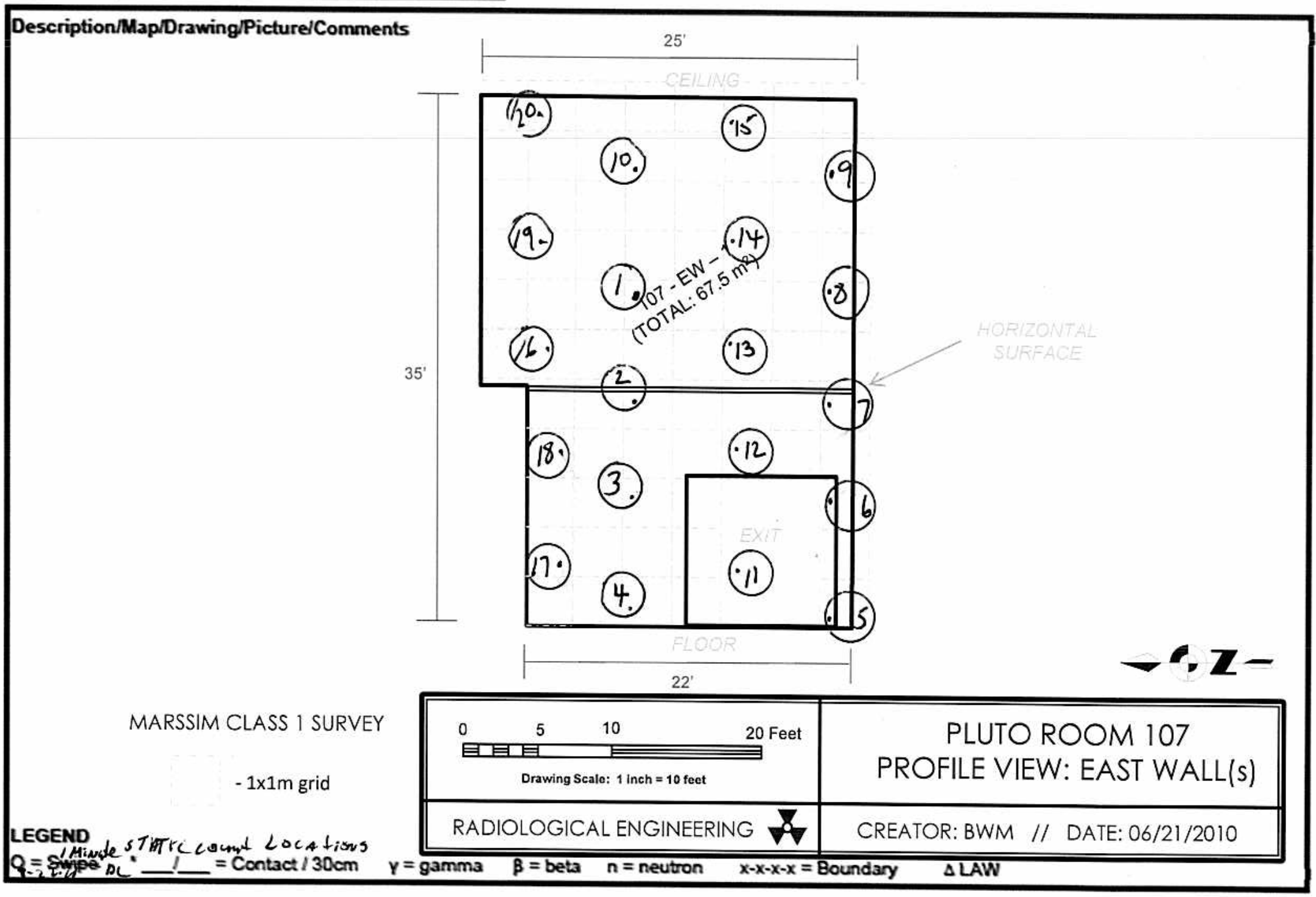




\begin{tabular}{|c|c|c|c|c|c|c|c|c|c|c|c|}
\hline \multicolumn{3}{|c|}{$\begin{array}{l}\text { Location: } \\
\text { Area } 26 \text { CAU117 Pluto }\end{array}$} & \multicolumn{2}{|c|}{$\begin{array}{l}\text { Purpose: } \\
\text { Final Status Survey, Room } 107 \\
\text { Floor 107-F-1 }\end{array}$} & \multicolumn{5}{|c|}{$\begin{array}{l}\text { Comments: } \\
\text { All one minute static counts are in cpm. Bkgd not subtracted for } \\
\text { Final Status Survey, Survey Plan \#10-015 }\end{array}$} & \multicolumn{2}{|c|}{$\begin{array}{l}\text { Date/Time: } \\
\text { 08/18/2010 } 1400\end{array}$} \\
\hline Instrument: & Serial \#: & Cal Due: & $\begin{array}{c}\text { Eff in \%: } \\
\text { Alpha / Beta }\end{array}$ & $\begin{array}{l}\text { BKG in dpm: } \\
\text { Alpha / Beta }\end{array}$ & \begin{tabular}{|l|} 
MDA in dpm: \\
Alpha / Beta \\
\end{tabular} & RWP\# & \multicolumn{5}{|l|}{$\mathrm{N} / \mathrm{A}$} \\
\hline Electra & 2125 & $4 / 6 / 2011$ & $14.4 / 18.2$ & $N / A$ & $N / A$ & \multicolumn{6}{|c|}{ Work Package \# } \\
\hline Electra & 1120 & $4 / 6 / 2011$ & $14.1 / 20.8$ & $\mathrm{~N} / \mathrm{A}$ & $N / A$ & RCT Name: & \multicolumn{2}{|c|}{ D.Cotroneo Jr. } & \multicolumn{3}{|l|}{ Signature: \& } \\
\hline Electra & 1504 & $4 / 6 / 2011$ & $12.9 / 19.8$ & $N / A$ & $N / A$ & RCT Name: & \multicolumn{2}{|c|}{ K. Wilcox/M.Payne } & \multirow{3}{*}{\multicolumn{3}{|c|}{ 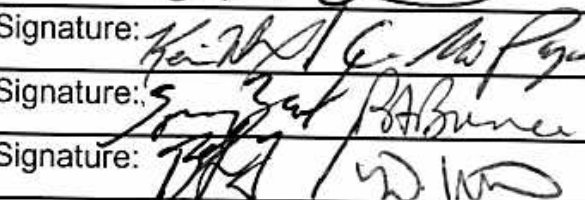 }} \\
\hline Electra & $N / A$ & $\mathrm{~N} / \mathrm{A}$ & $N / A$ & $N / A$ & $N / A$ & RCT Name: & S.Brown/t & Bunce & & & \\
\hline Electra & $\mathrm{N} / \mathrm{A}$ & $\mathrm{N} / \mathrm{A}$ & $N / A$ & $N / A$ & $N / A$ & RCT Name: & B. Fosket & Welch & & & \\
\hline \multirow[t]{2}{*}{ Survey Point } & \multirow{2}{*}{\multicolumn{4}{|c|}{ Description/Comments }} & \multicolumn{2}{|c|}{$\begin{array}{l}\text { Removable } \\
\mathrm{dpm} / 100 \mathrm{~cm}^{2}\end{array}$} & \multicolumn{2}{|c|}{\begin{tabular}{|c|}
$\begin{array}{c}\text { Fixed + Removable } \\
\mathrm{dpm} / 100 \mathrm{~cm}^{2}\end{array}$ \\
\end{tabular}} & \multirow{2}{*}{$\begin{array}{l}\text { Gamma } \\
\mathrm{mrem} / \mathrm{hr}\end{array}$} & \multirow{2}{*}{$\begin{array}{l}\text { Neutron } \\
\mathrm{mrem} / \mathrm{hr}\end{array}$} & \multirow{2}{*}{$\begin{array}{c}\text { Total } \\
\mathrm{mrem} / \mathrm{hr}\end{array}$} \\
\hline & & & & & Alpha & Beta & Alpha & Beta & & & \\
\hline 1 & \multicolumn{4}{|c|}{ Floor, Concrete (Electra 2125) } & $N / A$ & $N / A$ & 6 & 797 & $N / A$ & $\mathrm{~N} / \mathrm{A}$ & $N / A$ \\
\hline 2 & \multicolumn{4}{|c|}{ Floor, Concrete (Electra 2125) } & $N / A$ & $N / A$ & 7 & 1280 & $\mathrm{~N} / \mathrm{A}$ & $N / A$ & $\mathrm{~N} / \mathrm{A}$ \\
\hline 3 & \multicolumn{4}{|c|}{ Floor, Concrete (Electra 2125) } & $\mathrm{N} / \mathrm{A}$ & $N / A$ & 7 & 961 & $N / A$ & $N / A$ & $N / A$ \\
\hline 4 & \multicolumn{4}{|c|}{ Floor, Concrete (Electra 2125) } & $\mathrm{N} / \mathrm{A}$ & $N / A$ & 6 & 963 & $N / A$ & $N / A$ & $\mathrm{~N} / \mathrm{A}$ \\
\hline 5 & \multicolumn{4}{|c|}{ Floor, Concrete (Electra 2125) } & $N / A$ & $\mathrm{~N} / \mathrm{A}$ & 11 & 673 & $N / A$ & $\mathrm{~N} / \mathrm{A}$ & $N / A$ \\
\hline 6 & \multicolumn{4}{|c|}{ Floor, Concrete (Electra 2125) } & $N / A$ & $\mathrm{~N} / \mathrm{A}$ & 9 & 816 & $N / A$ & $N / A$ & $N / A$ \\
\hline 7 & \multicolumn{4}{|c|}{ Floor, Concrete (Electra 2125) } & $N / A$ & $\mathrm{~N} / \mathrm{A}$ & 10 & 812 & $N / A$ & $N / A$ & $N / A$ \\
\hline 8 & \multicolumn{4}{|c|}{ Floor, Concrete (Electra 2125) } & $N / A$ & $N / A$ & 11 & 996 & $\mathrm{~N} / \mathrm{A}$ & $N / A$ & $N / A$ \\
\hline 9 & \multicolumn{4}{|c|}{ Floor, Concrete (Electra 2125) } & $N / A$ & $N / A$ & 12 & 963 & $N / A$ & $N / A$ & $N / A$ \\
\hline 10 & \multicolumn{4}{|c|}{ Floor, Concrete (Electra 2125) } & $\mathrm{N} / \mathrm{A}$ & $N / A$ & 11 & 1231 & $N / A$ & $\mathrm{~N} / \mathrm{A}$ & $N / A$ \\
\hline 11 & \multicolumn{4}{|c|}{ Floor, Concrete (Electra 2125) } & $N / A$ & $N / A$ & 9 & 894 & $\mathrm{~N} / \mathrm{A}$ & $N / A$ & $N / A$ \\
\hline \multicolumn{5}{|c|}{ Reviewed By (Print)i } & antu & & & & & Date: & \\
\hline
\end{tabular}


SURVEY \# 10-ER-A26-279

\begin{tabular}{|c|c|c|c|c|c|c|c|c|}
\hline \multirow[t]{2}{*}{ Survey Point } & \multirow[t]{2}{*}{ Description/Comments } & \multicolumn{2}{|c|}{$\begin{array}{l}\text { Removable } \\
\mathrm{dpm} / 100 \mathrm{~cm}^{2}\end{array}$} & \multicolumn{2}{|c|}{$\begin{array}{c}\text { Fixed + Removable } \\
\mathrm{dpm} / 100 \mathrm{~cm}^{2}\end{array}$} & \multirow{2}{*}{$\begin{array}{l}\text { Gamma } \\
\mathrm{mrem} / \mathrm{hr}\end{array}$} & \multirow{2}{*}{$\begin{array}{l}\text { Neutron } \\
\text { mrem/hr }\end{array}$} & \multirow{2}{*}{$\begin{array}{c}\text { Total } \\
\text { mrem/hr }\end{array}$} \\
\hline & & Alpha & Beta & Alpha & Beta & & & \\
\hline 12 & Floor, Concrete (2125) & N/A & N/A & 5 & 831 & $\mathrm{~N} / \mathrm{A}$ & N/A & $\mathrm{N} / \mathrm{A}$ \\
\hline 13 & Floor, Metal (1120) & N/A & N/A & 7 & 709 & N/A & $\mathrm{N} / \mathrm{A}$ & N/A \\
\hline 14 & Floor, Concrete (2125) & N/A & N/A & 11 & 946 & N/A & N/A & $\mathrm{N} / \mathrm{A}$ \\
\hline 15 & Floor, Concrete (2125) & N/A & N/A & 7 & 955 & N/A & N/A & $\mathrm{N} / \mathrm{A}$ \\
\hline 16 & Floor, Concrete (2125) & N/A & N/A & 20 & 803 & N/A & N/A & $\mathrm{N} / \mathrm{A}$ \\
\hline N/A & ${ }^{\star *}$ Performed scan surveys for both alpha and beta** & N/A & N/A & N/A & N/A & N/A & $\mathrm{N} / \mathrm{A}$ & N/A \\
\hline N/A & ${ }^{* *}$ on $100 \%$ of survey unit. No trigger levels exceeded ${ }^{* *}$ & N/A & N/A & N/A & $\mathrm{N} / \mathrm{A}$ & N/A & N/A & $\mathrm{N} / \mathrm{A}$ \\
\hline $\mathrm{N} / \mathrm{A}$ & $\mathrm{N} / \mathrm{A}$ & N/A & $\mathrm{N} / \mathrm{A}$ & N/A & N/A & N/A & N/A & $\mathrm{N} / \mathrm{A}$ \\
\hline N/A & $N / A$ & N/A & N/A & $\mathrm{N} / \mathrm{A}$ & N/A & N/A & N/A & N/A \\
\hline N/A & $N / A$ & N/A & $\mathrm{N} / \mathrm{A}$ & N/A & N/A & N/A & N/A & $N / A$ \\
\hline N/A & $\mathrm{N} / \mathrm{A}$ & N/A & N/A & N/A & N/A & N/A & N/A & N/A \\
\hline $\mathrm{N} / \mathrm{A}$ & $N / A$ & N/A & N/A & N/A & N/A & N/A & N/A & $\mathrm{N} / \mathrm{A}$ \\
\hline N/A & $\mathrm{N} / \mathrm{A}$ & N/A & $\mathrm{N} / \mathrm{A}$ & N/A & N/A & $\mathrm{N} / \mathrm{A}$ & N/A & N/A \\
\hline N/A & $N / A$ & N/A & N/A & N/A & N/A & N/A & N/A & N/A \\
\hline N/A & $\mathrm{N} / \mathrm{A}$ & $\mathrm{N} / \mathrm{A}$ & N/A & $\mathrm{N} / \mathrm{A}$ & N/A & N/A & N/A & $\mathrm{N} / \mathrm{A}$ \\
\hline N/A & $\mathrm{N} / \mathrm{A}$ & $\mathrm{N} / \mathrm{A}$ & N/A & $\mathrm{N} / \mathrm{A}$ & $\mathrm{N} / \mathrm{A}$ & N/A & N/A & N/A \\
\hline N/A & $\mathrm{N} / \mathrm{A}$ & $\mathrm{N} / \mathrm{A}$ & $\mathrm{N} / \mathrm{A}$ & $\mathrm{N} / \mathrm{A}$ & N/A & $\mathrm{N} / \mathrm{A}$ & $\mathrm{N} / \mathrm{A}$ & N/A \\
\hline $\mathrm{N} / \mathrm{A}$ & $\mathrm{N} / \mathrm{A}$ & N/A & $\mathrm{N} / \mathrm{A}$ & N/A & N/A & N/A & N/A & N/A \\
\hline N/A & $\mathrm{N} / \mathrm{A}$ & N/A & N/A & N/A & N/A & N/A & N/A & N/A \\
\hline $\mathrm{N} / \mathrm{A}$ & $\mathrm{N} / \mathrm{A}$ & N/A & N/A & N/A & N/A & N/A & N/A & N/A \\
\hline N/A & $\mathrm{N} / \mathrm{A}$ & N/A & N/A & N/A & N/A & $\mathrm{N} / \mathrm{A}$ & N/A & N/A \\
\hline N/A & $\mathrm{N} / \mathrm{A}$ & N/A & N/A & $\mathrm{N} / \mathrm{A}$ & N/A & N/A & N/A & $\mathrm{N} / \mathrm{A}$ \\
\hline N/A & $\mathrm{N} / \mathrm{A}$ & N/A & N/A & N/A & N/A & N/A & N/A & N/A \\
\hline
\end{tabular}


NSTec

Form

05/24/10

FRM-0108C

RADIOLOGICAL SURVEY REPORT - FULL MAP

Survey $10-E R-A 26-279$

Description/Map/Drawing/Picture/Comments

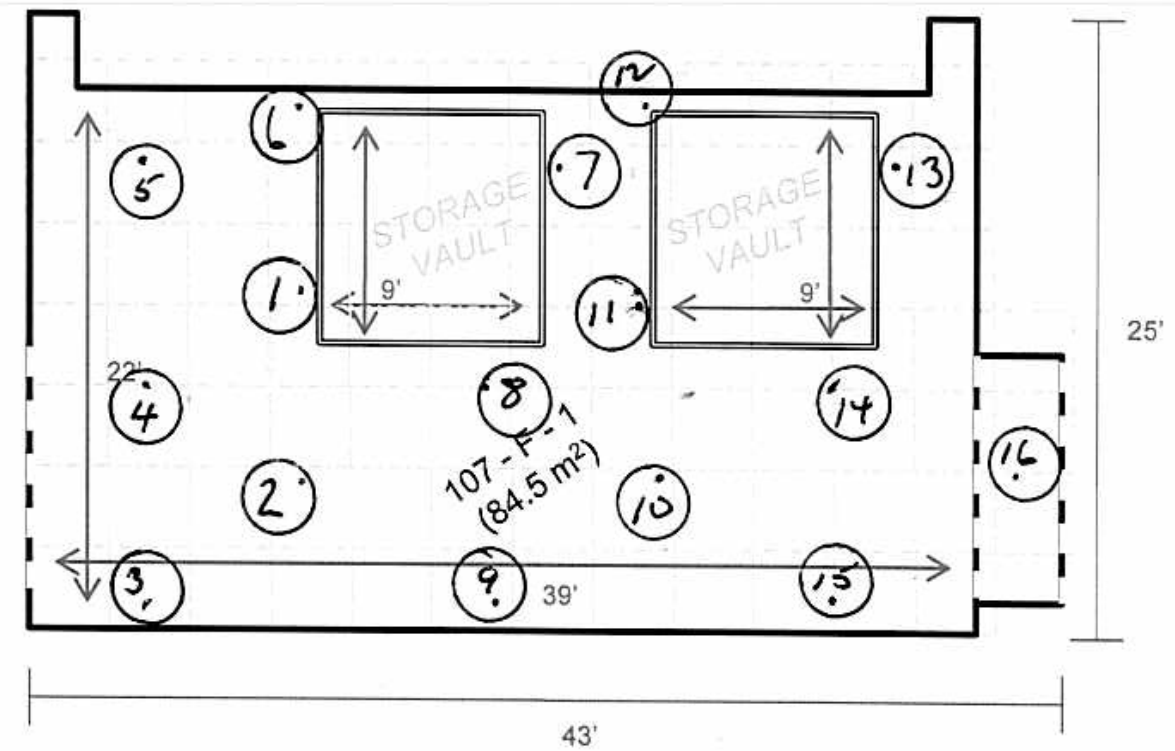

MARSSIM CLASS I SURVEY

$-1 \times 1 m$ grid

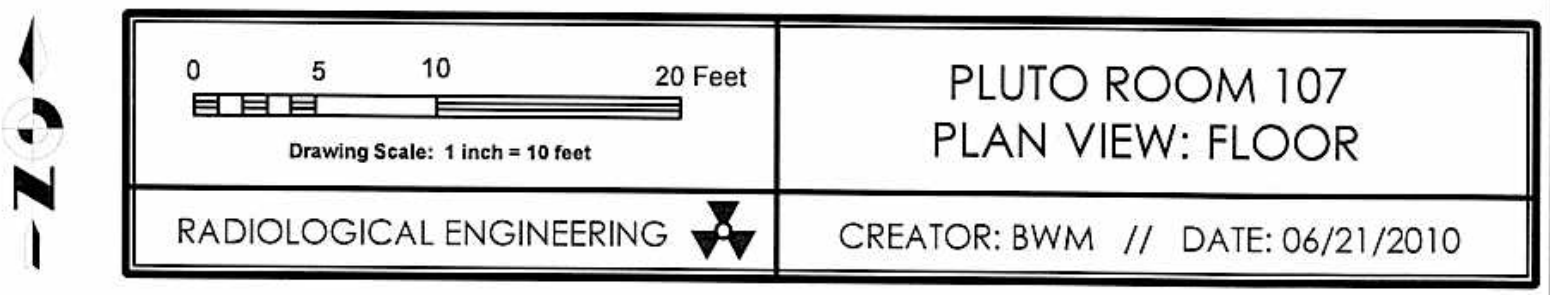

LEGEND $S$ STATR COu' LouAtron

o. SWipenc: $1=$ Contact $130 \mathrm{~cm}$ 
NSTec

SURVEY \#

10-ER-A26-280

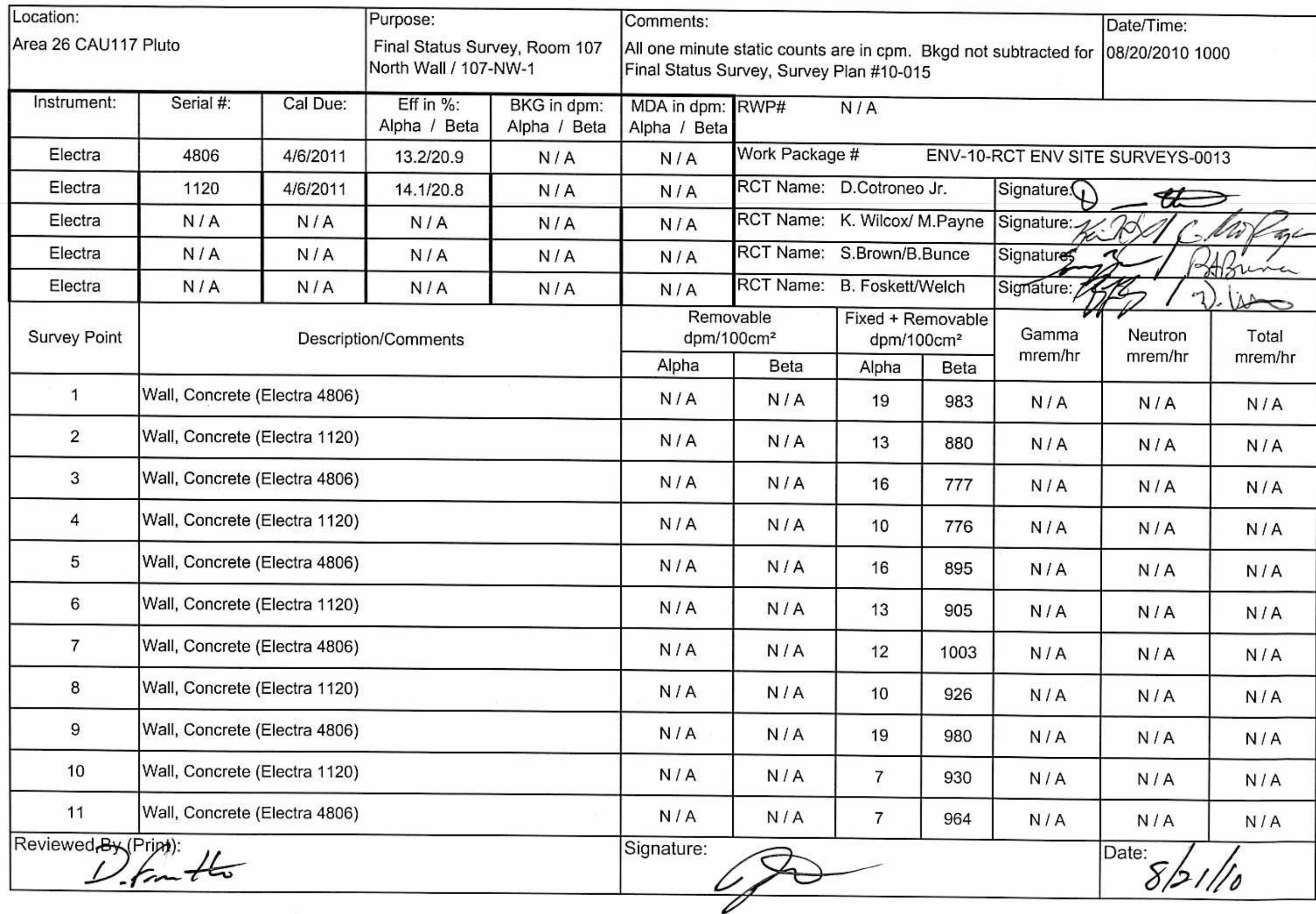


SURVEY \# 10-ER-A26-280

\begin{tabular}{|c|c|c|c|c|c|c|c|c|}
\hline \multirow[t]{2}{*}{ Survey Point } & \multirow[t]{2}{*}{ Description/Comments } & \multicolumn{2}{|c|}{$\begin{array}{l}\text { Removable } \\
\mathrm{dpm} / 100 \mathrm{~cm}^{2}\end{array}$} & \multicolumn{2}{|c|}{$\begin{array}{c}\begin{array}{c}\text { Fixed + Removable } \\
\mathrm{dpm} / 100 \mathrm{~cm}^{2}\end{array} \\
\end{array}$} & \multirow{2}{*}{$\begin{array}{l}\text { Gamma } \\
\mathrm{mrem} / \mathrm{hr}\end{array}$} & \multirow{2}{*}{$\begin{array}{l}\text { Neutron } \\
\mathrm{mrem} / \mathrm{hr}\end{array}$} & \multirow{2}{*}{$\begin{array}{c}\text { Total } \\
\text { mrem/hr }\end{array}$} \\
\hline & & Alpha & Beta & Alpha & Beta & & & \\
\hline 12 & Wall, Concrete (1120) & N/A & N/A & 13 & 877 & N/A & N/A & N/A \\
\hline 13 & Wall, Concrete (1120) & N/A & N/A & 16 & 805 & N/A & N/A & N/A \\
\hline 14 & Wall, Concrete (1120) & N/A & N/A & 17 & 839 & N/A & N/A & $\mathrm{N} / \mathrm{A}$ \\
\hline 15 & Wall, Concrete (4806) & N/A & N/A & 10 & 985 & N/A & N/A & N/A \\
\hline 16 & Wall, Concrete (4806) & $\mathrm{N} / \mathrm{A}$ & N/A & 10 & 953 & N/A & N/A & N/A \\
\hline N/A & 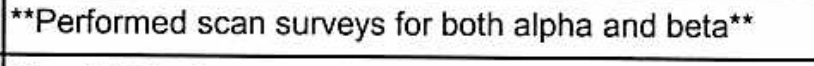 & N/A & N/A & N/A & N/A & N/A & N/A & N/A \\
\hline $\mathrm{N} / \mathrm{A}$ & ${ }^{\star \star}$ on $100 \%$ of survey unit. No trigger levels exceeded ${ }^{\star \star}$ & N/A & N/A & N/A & N/A & N/A & N/A & N/A \\
\hline $\mathrm{N} / \mathrm{A}$ & $\mathrm{N} / \mathrm{A}$ & N/A & N/A & N/A & $\mathrm{N} / \mathrm{A}$ & N/A & N/A & $\mathrm{N} / \mathrm{A}$ \\
\hline $\mathrm{N} / \mathrm{A}$ & $\mathrm{N} / \mathrm{A}$ & N/A & N/A & N/A & N/A & N/A & N/A & N/A \\
\hline N/A & $\mathrm{N} / \mathrm{A}$ & N/A & N/A & N/A & $\mathrm{N} / \mathrm{A}$ & N/A & N/A & N/A \\
\hline N/A & $\mathrm{N} / \mathrm{A}$ & N/A & $\mathrm{N} / \mathrm{A}$ & $\mathrm{N} / \mathrm{A}$ & $\mathrm{N} / \mathrm{A}$ & N/A & N/A & N/A \\
\hline N/A & $\mathrm{N} / \mathrm{A}$ & $\mathrm{N} / \mathrm{A}$ & N/A & N/A & $\mathrm{N} / \mathrm{A}$ & N/A & N/A & N/A \\
\hline N/A & $\mathrm{N} / \mathrm{A}$ & N/A & N/A & N/A & N/A & N/A & N/A & N/A \\
\hline N/A & $\mathrm{N} / \mathrm{A}$ & N/A & N/A & N/A & N/A & N/A & N/A & $\mathrm{N} / \mathrm{A}$ \\
\hline $\mathrm{N} / \mathrm{A}$ & $\mathrm{N} / \mathrm{A}$ & $\mathrm{N} / \mathrm{A}$ & N/A & N/A & N/A & N/A & N/A & $\mathrm{N} / \mathrm{A}$ \\
\hline $\mathrm{N} / \mathrm{A}$ & $\mathrm{N} / \mathrm{A}$ & N/A & N/A & N/A & N/A & N/A & N/A & N/A \\
\hline N/A & $N / A$ & N/A & N/A & N/A & $\mathrm{N} / \mathrm{A}$ & N/A & N/A & N/A \\
\hline $\mathrm{N} / \mathrm{A}$ & $\mathrm{N} / \mathrm{A}$ & N/A & N/A & N/A & $\mathrm{N} / \mathrm{A}$ & N/A & N/A & N/A \\
\hline N/A & $N / A$ & N/A & N/A & N/A & $\mathrm{N} / \mathrm{A}$ & N/A & N/A & N/A \\
\hline $\mathrm{N} / \mathrm{A}$ & $\mathrm{N} / \mathrm{A}$ & N/A & N/A & N/A & $\mathrm{N} / \mathrm{A}$ & N/A & N/A & N/A \\
\hline N/A & $\mathrm{N} / \mathrm{A}$ & $\mathrm{N} / \mathrm{A}$ & N/A & N/A & $\mathrm{N} / \mathrm{A}$ & N/A & N/A & $\mathrm{N} / \mathrm{A}$ \\
\hline N/A & $\mathrm{N} / \mathrm{A}$ & N/A & N/A & N/A & N/A & N/A & N/A & N/A \\
\hline N/A & $\mathrm{N} / \mathrm{A}$ & $\mathrm{N} / \mathrm{A}$ & N/A & N/A & N/A & N/A & N/A & N/A \\
\hline
\end{tabular}


Survey $\#+0 \cdot E R-A 26-280$

Description/Map/Drawing/Picture/Comments

(15)

(16)
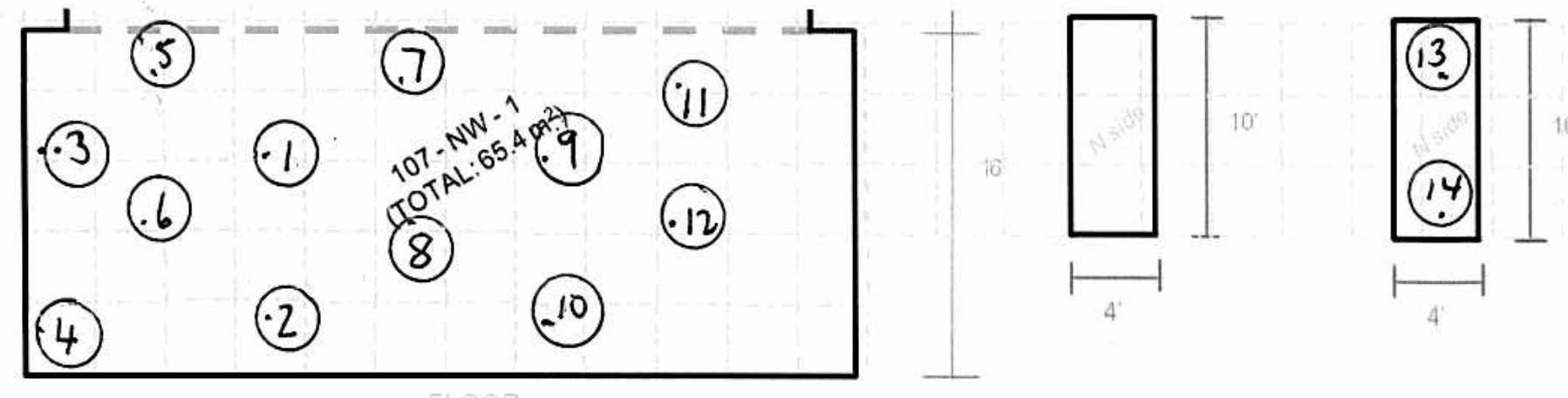

MARSSIM CLASS I SURVEY

$-1 \times 1 \mathrm{~m}$ grid

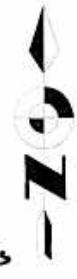

LEGEND JTATIC Cent Locntions

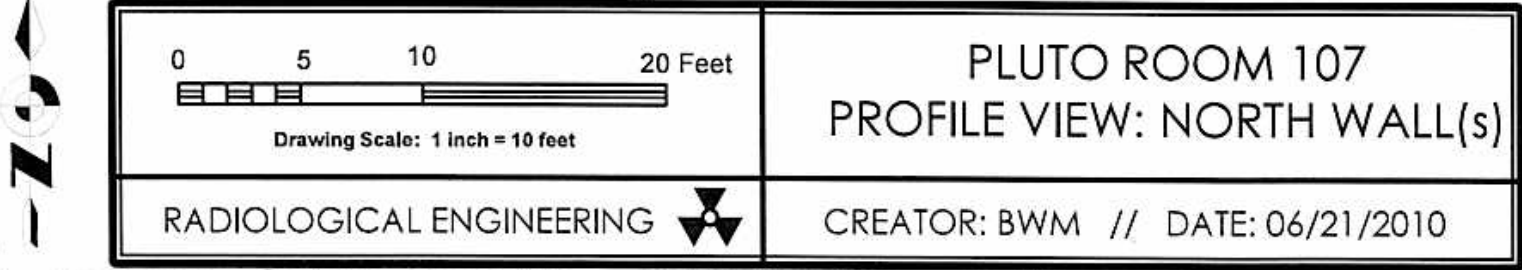

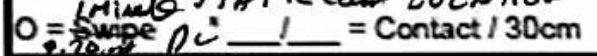


SURVEY \#

10-ER-A26-281

\begin{tabular}{|c|c|c|c|c|c|c|c|c|c|c|c|}
\hline \multicolumn{3}{|c|}{$\begin{array}{l}\text { Location: } \\
\text { Area } 26 \text { CAU117 Pluto }\end{array}$} & \multicolumn{2}{|c|}{$\begin{array}{l}\text { Purpose: } \\
\text { Final Status Survey, Room } 107 \\
\text { North Wall / 107-NW-2 }\end{array}$} & \multicolumn{5}{|c|}{$\begin{array}{l}\text { Comments: } \\
\text { All one minute static counts are in cpm. Bkgd not subtracted for } \\
\text { Final Status Survey, Survey Plan } \# 10-015\end{array}$} & \multicolumn{2}{|c|}{$\begin{array}{l}\text { Date/Time: } \\
\text { 08/20/2010 } 1300\end{array}$} \\
\hline Instrument: & Serial \#: & Cal Due: & $\begin{array}{c}\text { Eff in \%: } \\
\text { Alpha / Beta }\end{array}$ & $\begin{array}{l}\text { BKG in dpm: } \\
\text { Alpha / Beta }\end{array}$ & \begin{tabular}{|l|} 
MDA in dpm: \\
Alpha / Beta \\
\end{tabular} & RWP\# & \multicolumn{5}{|l|}{$\mathrm{N} / \mathrm{A}$} \\
\hline Electra & 1120 & $4 / 6 / 2011$ & $14.1 / 20.8$ & $N / A$ & $N / A$ & \multicolumn{6}{|c|}{$\begin{array}{ll}\text { Work Package \# } & \text { ENV-10-RCT ENV SITE SURVEYS-0013 }\end{array}$} \\
\hline Electra & $N / A$ & $\mathrm{~N} / \mathrm{A}$ & $\mathrm{N} / \mathrm{A}$ & $\mathrm{N} / \mathrm{A}$ & $\mathrm{N} / \mathrm{A}$ & \multicolumn{3}{|c|}{ RCT Name: D.Cotroneo Jr. } & \multicolumn{3}{|l|}{ Signature: $Q$} \\
\hline Electra & $N / A$ & $N / A$ & $N / A$ & $\mathrm{~N} / \mathrm{A}$ & $N / A$ & \multicolumn{3}{|c|}{ RCT Name: K. Wilcox/M.Payne } & \multirow{2}{*}{\multicolumn{3}{|c|}{ Signature: $\mathrm{Kein} 2 \mathrm{Cen}$}} \\
\hline Electra & $N / A$ & $N / A$ & $\mathrm{~N} / \mathrm{A}$ & $N / A$ & $N / A$ & RCT Name: & S.Brown/E & Bunce & & & \\
\hline Electra & $N / A$ & $N / A$ & $N / A$ & $N / A$ & $\mathrm{~N} / \mathrm{A}$ & RCT Name: & B. Fosket & Velch & atur & & \\
\hline \multirow[t]{2}{*}{ Survey Point } & \multirow{2}{*}{\multicolumn{4}{|c|}{ Description/Comments }} & \multicolumn{2}{|c|}{$\begin{array}{l}\text { Removable } \\
\mathrm{dpm} / 100 \mathrm{~cm}^{2}\end{array}$} & \multicolumn{2}{|c|}{\begin{tabular}{|c|}
$\begin{array}{c}\text { Fixed + Removable } \\
\mathrm{dpm} / 100 \mathrm{~cm}^{2}\end{array}$ \\
\end{tabular}} & \multirow{2}{*}{$\begin{array}{l}\text { Gamma } \\
\mathrm{mrem} / \mathrm{hr}\end{array}$} & \multirow{2}{*}{$\begin{array}{l}\text { Neutron } \\
\text { mrem/hr }\end{array}$} & \multirow{2}{*}{$\begin{array}{l}\text { Total } \\
\mathrm{mrem} / \mathrm{hr}\end{array}$} \\
\hline & & & & & Alpha & Beta & Alpha & Beta & & & \\
\hline 1 & \multicolumn{4}{|c|}{ Wall, Concrete (Electra 1120) } & $N / A$ & $N / A$ & 17 & 1065 & $\mathrm{~N} / \mathrm{A}$ & $N / A$ & $N / A$ \\
\hline 2 & \multicolumn{4}{|c|}{ Wall, Concrete (Electra 1120) } & $N / A$ & $N / A$ & 5 & 1039 & $N / A$ & $\mathrm{~N} / \mathrm{A}$ & $N / A$ \\
\hline 3 & \multicolumn{4}{|c|}{ Wall, Concrete (Electra 1120) } & $N / A$ & $N / A$ & 15 & 1105 & $N / A$ & $N / A$ & $N / A$ \\
\hline 4 & \multicolumn{4}{|c|}{ Wall, Concrete (Electra 1120) } & $N / A$ & $\mathrm{~N} / \mathrm{A}$ & 16 & 1040 & $N / A$ & $N / A$ & $N / A$ \\
\hline 5 & \multicolumn{4}{|c|}{ Wall, Concrete (Electra 1120) } & $N / A$ & $\mathrm{~N} / \mathrm{A}$ & 9 & 1095 & $N / A$ & $N / A$ & $N / A$ \\
\hline 6 & \multicolumn{4}{|c|}{ Wall, Concrete (Electra 1120) } & $N / A$ & $N / A$ & 9 & 1139 & $\mathrm{~N} / \mathrm{A}$ & $\mathrm{N} / \mathrm{A}$ & $N / A$ \\
\hline 7 & \multicolumn{4}{|c|}{ Wall, Concrete (Electra 1120) } & $N / A$ & $N / A$ & 4 & 1101 & $\mathrm{~N} / \mathrm{A}$ & $\mathrm{N} / \mathrm{A}$ & $N / A$ \\
\hline 8 & \multicolumn{4}{|c|}{ Wall, Concrete (Electra 1120) } & $N / A$ & $N / A$ & 4 & 1066 & $N / A$ & $N / A$ & $N / A$ \\
\hline 9 & \multicolumn{4}{|c|}{ Wall, Concrete (Electra 1120) } & $N / A$ & $N / A$ & 7 & 1158 & $N / A$ & $N / A$ & $N / A$ \\
\hline 10 & \multicolumn{4}{|c|}{ Wall, Concrete (Electra 1120) } & $\mathrm{N} / \mathrm{A}$ & $N / A$ & 9 & 1167 & $N / A$ & $N / A$ & $N / A$ \\
\hline 11 & \multicolumn{4}{|c|}{ Wall, Concrete (Electra 1120) } & $N / A$ & $N / A$ & 9 & 1083 & $N / A$ & $N / A$ & $N / A$ \\
\hline \multicolumn{5}{|c|}{ Reviewed By+(Print): } & \multicolumn{5}{|l|}{ Signature: } & Date: & \\
\hline
\end{tabular}


SURVEY \# 10-ER-A26-281

\begin{tabular}{|c|c|c|c|c|c|c|c|c|}
\hline \multirow[t]{2}{*}{ Survey Point } & \multirow[t]{2}{*}{ Description/Comments } & \multicolumn{2}{|c|}{$\begin{array}{l}\text { Removable } \\
\mathrm{dpm} / 100 \mathrm{~cm}^{2}\end{array}$} & \multicolumn{2}{|c|}{$\begin{array}{c}\text { Fixed + Removable } \\
\mathrm{dpm} / 100 \mathrm{~cm}^{2}\end{array}$} & \multirow{2}{*}{$\begin{array}{l}\text { Gamma } \\
\mathrm{mrem} / \mathrm{hr}\end{array}$} & \multirow{2}{*}{$\begin{array}{l}\text { Neutron } \\
\mathrm{mrem} / \mathrm{hr}\end{array}$} & \multirow{2}{*}{$\begin{array}{c}\text { Total } \\
\mathrm{mrem} / \mathrm{hr}\end{array}$} \\
\hline & & Alpha & Beta & Alpha & Beta & & & \\
\hline 12 & Wall, Concrete (1120) & N/A & N/A & 16 & 1183 & N/A & N/A & $\mathrm{N} / \mathrm{A}$ \\
\hline 13 & Wall, Concrete (1120) & N/A & N/A & 11 & 1132 & N/A & N/A & N/A \\
\hline 14 & Wall, Concrete (1120) & N/A & N/A & 14 & 1070 & N/A & $\mathrm{N} / \mathrm{A}$ & N/A \\
\hline 15 & Wall, Concrete (1120) & N/A & N/A & 12 & 1103 & N/A & N/A & N/A \\
\hline 16 & Wall, Concrete (1120) & N/A & N/A & 9 & 1125 & N/A & N/A & N/A \\
\hline 17 & Wall, Concrete (1120) & N/A & N/A & 19 & 1066 & N/A & N/A & N/A \\
\hline N/A & ${ }^{\star \star}$ Performed scan surveys for both alpha and beta** & N/A & $\mathrm{N} / \mathrm{A}$ & N/A & N/A & N/A & N/A & $\mathrm{N} / \mathrm{A}$ \\
\hline N/A & ${ }^{* *}$ on $100 \%$ of survey unit. No trigger levels exceeded ${ }^{* *}$ & N/A & N/A & N/A & N/A & N/A & N/A & N/A \\
\hline N/A & $\mathrm{N} / \mathrm{A}$ & N/A & N/A & N/A & $N / A$ & N/A & N/A & N/A \\
\hline$N / A$ & $N / A$ & $N / A$ & N/A & N/A & N/A & N/A & N/A & N/A \\
\hline N/A & $N / A$ & N/A & N/A & N/A & N/A & N/A & N/A & N/A \\
\hline $\mathrm{N} / \mathrm{A}$ & $\mathrm{N} / \mathrm{A}$ & N/A & N/A & N/A & N/A & N/A & N/A & N/A \\
\hline N/A & $N / A$ & $N / A$ & N/A & N/A & N/A & N/A & N/A & N/A \\
\hline N/A & $\mathrm{N} / \mathrm{A}$ & N/A & N/A & N/A & N/A & N/A & N/A & N/A \\
\hline N/A & $\mathrm{N} / \mathrm{A}$ & N/A & N/A & N/A & N/A & N/A & N/A & N/A \\
\hline N/A & $\mathrm{N} / \mathrm{A}$ & $\mathrm{N} / \mathrm{A}$ & N/A & N/A & N/A & N/A & N/A & N/A \\
\hline N/A & $N / A$ & N/A & N/A & N/A & N/A & N/A & N/A & $\mathrm{N} / \mathrm{A}$ \\
\hline N/A & $N / A$ & N/A & N/A & N/A & N/A & N/A & N/A & N/A \\
\hline N/A & $\mathrm{N} / \mathrm{A}$ & N/A & N/A & N/A & N/A & N/A & N/A & N/A \\
\hline N/A & $\mathrm{N} / \mathrm{A}$ & N/A & N/A & N/A & $\mathrm{N} / \mathrm{A}$ & N/A & N/A & N/A \\
\hline N/A & $N / A$ & N/A & N/A & N/A & N/A & N/A & N/A & N/A \\
\hline N/A & $\mathrm{N} / \mathrm{A}$ & N/A & N/A & N/A & N/A & N/A & N/A & N/A \\
\hline$N / A$ & $\mathrm{~N} / \mathrm{A}$ & N/A & N/A & N/A & N/A & N/A & N/A & $N / A$ \\
\hline
\end{tabular}


Survey $=10 E n A 26-281$

Description/Map/Drawing/Picture/Comments

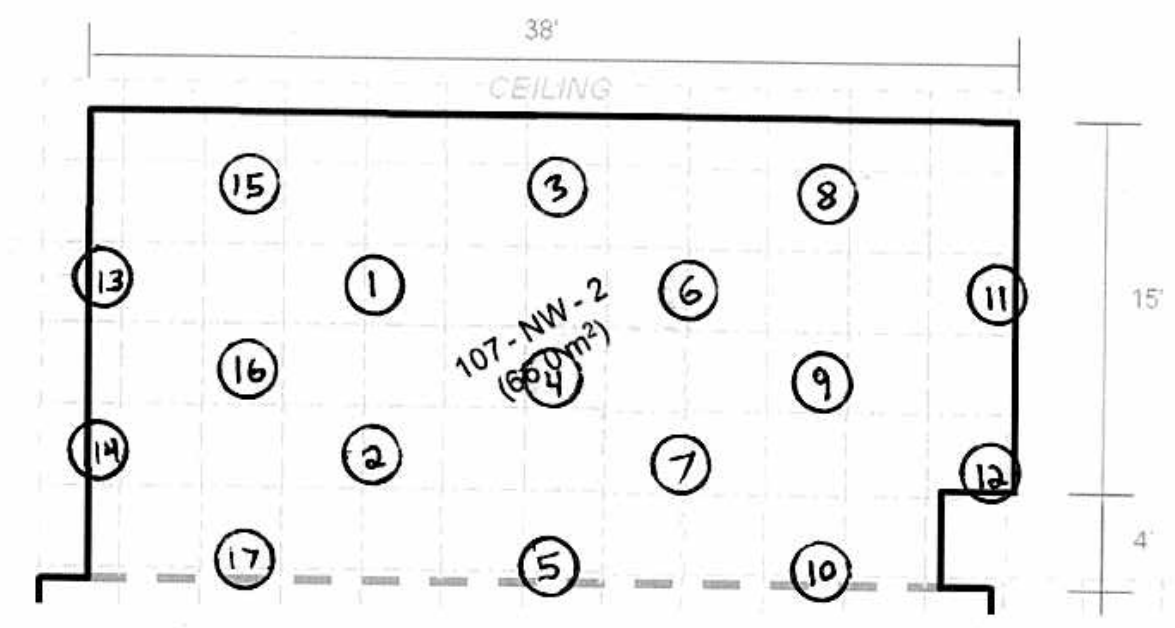

MARSSIM CLASS I SURVEY

$-1 \times 1 m$ grid

LEGEND

(

$0=$ Swipe $\because 1=\operatorname{Contact} / 30 \mathrm{~cm}$

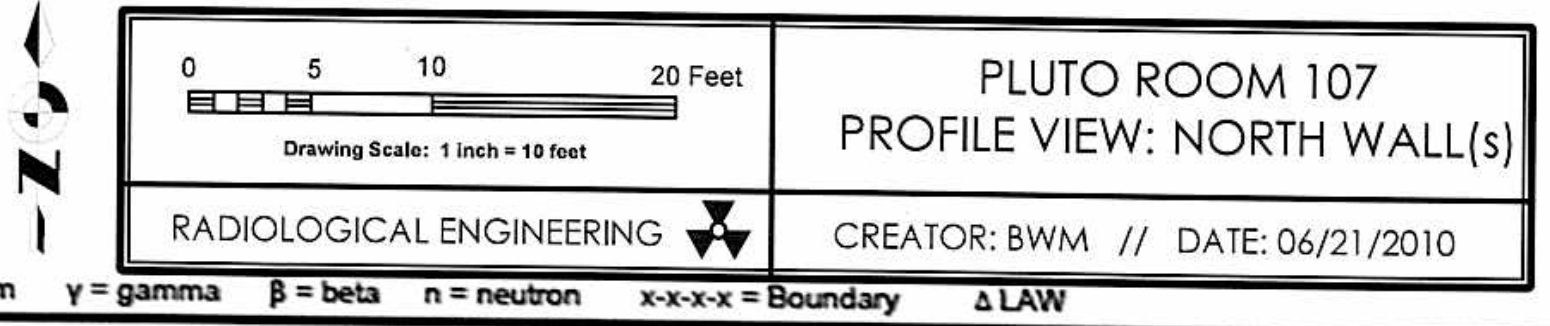




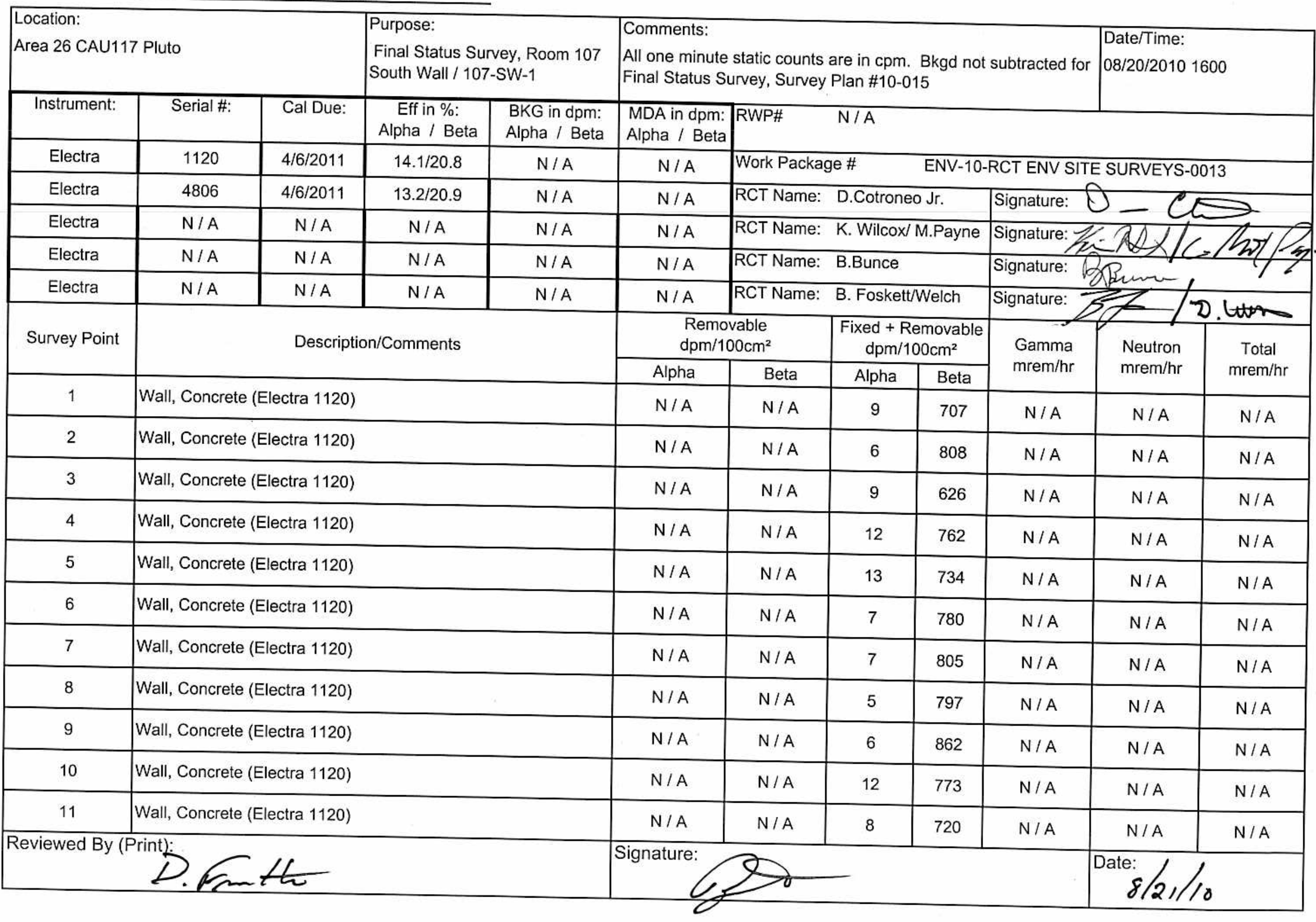


SURVEY \# 10-ER-A26-283

\begin{tabular}{|c|c|c|c|c|c|c|c|c|}
\hline \multirow[t]{2}{*}{ Survey Point } & \multirow[t]{2}{*}{ Description/Comments } & \multicolumn{2}{|c|}{$\begin{array}{l}\text { Removable } \\
\mathrm{dpm} / 100 \mathrm{~cm}^{2}\end{array}$} & \multicolumn{2}{|c|}{$\begin{array}{c}\text { Fixed + Removable } \\
\mathrm{dpm} / 100 \mathrm{~cm}^{2}\end{array}$} & \multirow{2}{*}{$\begin{array}{l}\text { Gamma } \\
\mathrm{mrem} / \mathrm{hr}\end{array}$} & \multirow{2}{*}{$\begin{array}{l}\text { Neutron } \\
\mathrm{mrem} / \mathrm{hr}\end{array}$} & \multirow{2}{*}{$\begin{array}{c}\text { Total } \\
\text { mrem } / \mathrm{hr}\end{array}$} \\
\hline & & Alpha & Beta & Alpha & Beta & & & \\
\hline 12 & Wall, Concrete (1120) & N/A & N/A & 7 & 749 & N/A & $\mathrm{N} / \mathrm{A}$ & N/A \\
\hline 13 & Wall, Concrete (1120) & $\mathrm{N} / \mathrm{A}$ & N/A & 6 & 735 & N/A & N/A & $\mathrm{N} / \mathrm{A}$ \\
\hline 14 & Wall, Concrete (1120) & $\mathrm{N} / \mathrm{A}$ & N/A & 9 & 760 & N/A & $\mathrm{N} / \mathrm{A}$ & N/A \\
\hline 15 & Wall, Concrete (1120) & $\mathrm{N} / \mathrm{A}$ & N/A & 20 & 728 & N/A & N/A & N/A \\
\hline 16 & Wall, Concrete (1120) & $\mathrm{N} / \mathrm{A}$ & $\mathrm{N} / \mathrm{A}$ & 6 & 686 & N/A & N/A & N/A \\
\hline N/A & $\mathrm{N} / \mathrm{A}$ & N/A & $\mathrm{N} / \mathrm{A}$ & $\mathrm{N} / \mathrm{A}$ & $\mathrm{N} / \mathrm{A}$ & $\mathrm{N} / \mathrm{A}$ & N/A & N/A \\
\hline N/A & ${ }^{\star *}$ Performed scan surveys for both alpha and beta** & N/A & $\mathrm{N} / \mathrm{A}$ & N/A & N/A & N/A & N/A & N/A \\
\hline N/A & ${ }^{\star \star}$ on $100 \%$ of survey unit. No trigger levels exceeded ${ }^{\star \star}$ & N/A & N/A & N/A & $\mathrm{N} / \mathrm{A}$ & N/A & N/A & N/A \\
\hline N/A & $N / A$ & N/A & $\mathrm{N} / \mathrm{A}$ & N/A & N/A & N/A & N/A & N/A \\
\hline N/A & $\mathrm{N} / \mathrm{A}$ & $\mathrm{N} / \mathrm{A}$ & $\mathrm{N} / \mathrm{A}$ & N/A & $\mathrm{N} / \mathrm{A}$ & N/A & N/A & N/A \\
\hline N/A & $\mathrm{N} / \mathrm{A}$ & N/A & N/A & N/A & N/A & N/A & $\mathrm{N} / \mathrm{A}$ & $\mathrm{N} / \mathrm{A}$ \\
\hline $\mathrm{N} / \mathrm{A}$ & $N / A$ & N/A & N/A & N/A & N/A & N/A & N/A & N/A \\
\hline N/A & $\mathrm{N} / \mathrm{A}$ & N/A & N/A & N/A & N/A & N/A & N/A & N/A \\
\hline N/A & $\mathrm{N} / \mathrm{A}$ & $\mathrm{N} / \mathrm{A}$ & N/A & N/A & N/A & N/A & N/A & N/A \\
\hline N/A & $\mathrm{N} / \mathrm{A}$ & N/A & $\mathrm{N} / \mathrm{A}$ & N/A & $\mathrm{N} / \mathrm{A}$ & N/A & N/A & N/A \\
\hline N/A & $N / A$ & N/A & N/A & N/A & N/A & N/A & N/A & $\mathrm{N} / \mathrm{A}$ \\
\hline N/A & $\mathrm{N} / \mathrm{A}$ & N/A & N/A & $\mathrm{N} / \mathrm{A}$ & $\mathrm{N} / \mathrm{A}$ & N/A & N/A & N/A \\
\hline N/A & $\mathrm{N} / \mathrm{A}$ & $\mathrm{N} / \mathrm{A}$ & N/A & N/A & N/A & N/A & N/A & N/A \\
\hline N/A & $N / A$ & $\mathrm{~N} / \mathrm{A}$ & N/A & $\mathrm{N} / \mathrm{A}$ & $\mathrm{N} / \mathrm{A}$ & N/A & N/A & N/A \\
\hline $\mathrm{N} / \mathrm{A}$ & $N / A$ & $\mathrm{~N} / \mathrm{A}$ & N/A & N/A & N/A & N/A & N/A & N/A \\
\hline N/A & $\mathrm{N} / \mathrm{A}$ & N/A & N/A & N/A & N/A & N/A & $\mathrm{N} / \mathrm{A}$ & $\mathrm{N} / \mathrm{A}$ \\
\hline N/A & $N / A$ & $\mathrm{~N} / \mathrm{A}$ & N/A & N/A & $\mathrm{N} / \mathrm{A}$ & N/A & $\mathrm{N} / \mathrm{A}$ & N/A \\
\hline $\mathrm{N} / \mathrm{A}$ & $\mathrm{N} / \mathrm{A}$ & $\mathrm{N} / \mathrm{A}$ & N/A & N/A & N/A & N/A & N/A & N/A \\
\hline
\end{tabular}


Survey 10 ERA26-283

Description/Map/Drawing/Picture/Comments

"UNFOLDED" VIEW

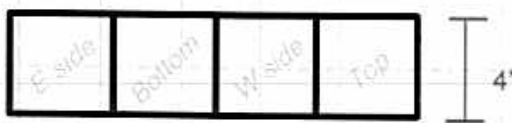

$107-\mathrm{SW}-1$

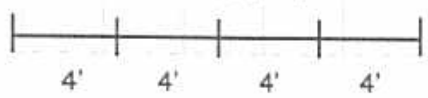

$16^{\prime}$
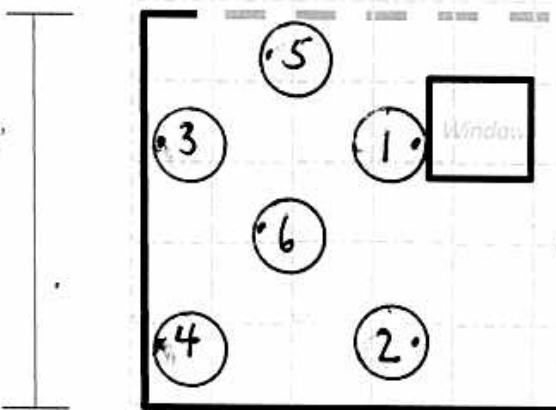

(6)
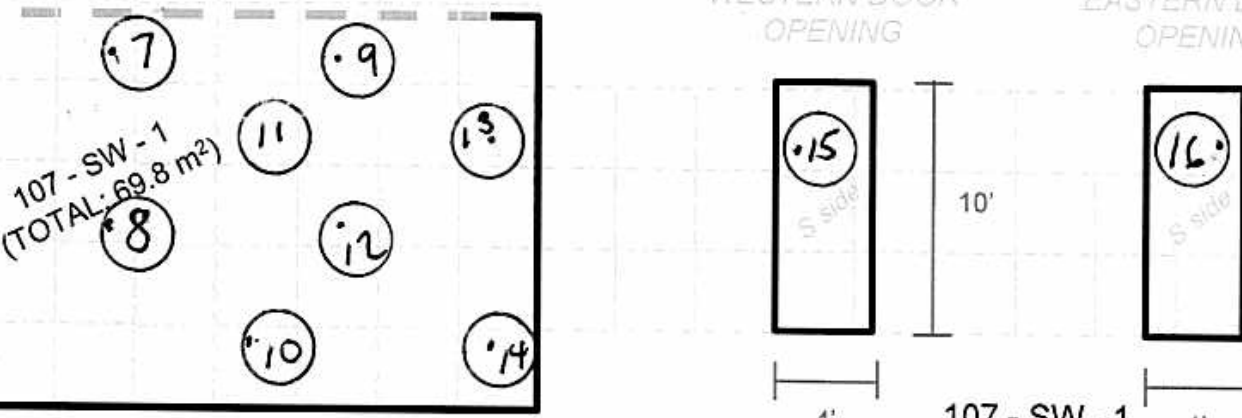

(4)

(2.)

14

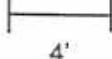

$107-$ SW - $1{ }^{\prime}$

MARSSIM CLASS 1 SURVEY

$-1 \times 1 m$ grid

LEGEND ITATIC Cont Locations$$
\text { - } x \text { im grid }
$$

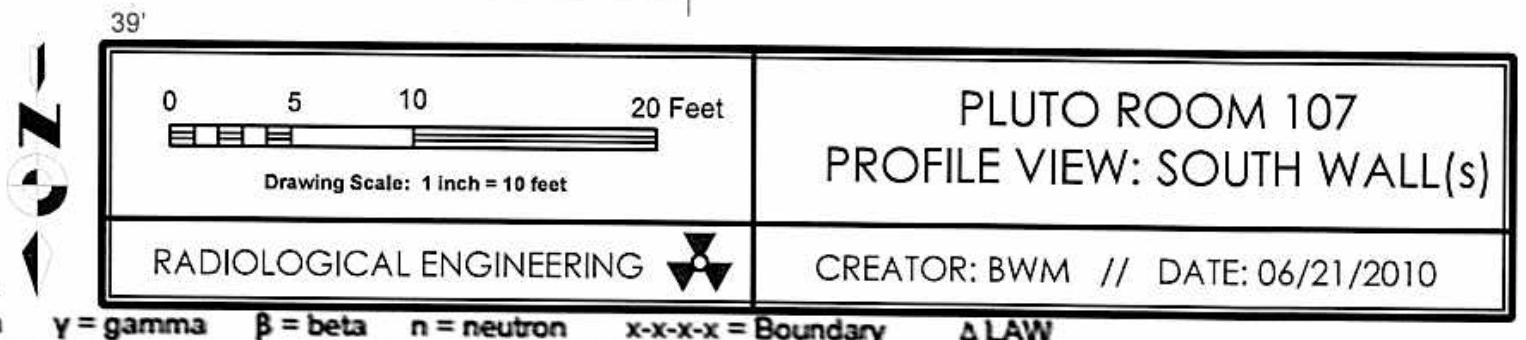


SURVEY \#

10-ER-A26-289

\begin{tabular}{|c|c|c|c|c|c|c|c|c|c|c|c|}
\hline \multicolumn{3}{|c|}{$\begin{array}{l}\text { Location: } \\
\text { Area } 26 \text { CAU117 Pluto }\end{array}$} & \multicolumn{2}{|c|}{$\begin{array}{l}\text { Purpose: } \\
\text { Final Status Survey, Room } 107 \\
\text { South Wall / 107-SW-2 }\end{array}$} & \multicolumn{5}{|c|}{$\begin{array}{l}\text { Comments: } \\
\text { All one minute static counts are in cpm. Bkgd not subtracted for } \\
\text { Final Status Survey, Survey Plan } \# 10-015\end{array}$} & \multicolumn{2}{|c|}{$\begin{array}{l}\text { Date/Time: } \\
\text { 08/22/2010 } 1200\end{array}$} \\
\hline Instrument: & Serial \#: & Cal Due: & $\begin{array}{c}\text { Eff in \%: } \\
\text { Alpha / Beta }\end{array}$ & $\begin{array}{l}\text { BKG in dpm: } \\
\text { Alpha / Beta }\end{array}$ & \begin{tabular}{|l|} 
MDA in dpm: \\
Alpha / Beta \\
\end{tabular} & RWP\# & \multicolumn{5}{|l|}{$\mathrm{N} / \mathrm{A}$} \\
\hline Electra & 1120 & $4 / 6 / 2011$ & $14.1 / 20.8$ & $N / A$ & $N / A$ & \multicolumn{6}{|c|}{ Work Package \# $\quad$ ENV-10-RCT ENV SITE SURVEYS-0013 } \\
\hline Electra & $\mathrm{N} / \mathrm{A}$ & $N / A$ & $N / A$ & $N / A$ & $\mathrm{~N} / \mathrm{A}$ & RCT Name: & \multicolumn{2}{|c|}{ D.Cotroneo Jr. } & \multicolumn{3}{|l|}{ Signature: } \\
\hline Electra & $N / A$ & $N / A$ & $N / A$ & $N / A$ & $N / A$ & RCT Name: & \multicolumn{2}{|c|}{ K. Wilcox/M.Payne } & \multirow{2}{*}{\multicolumn{3}{|c|}{ 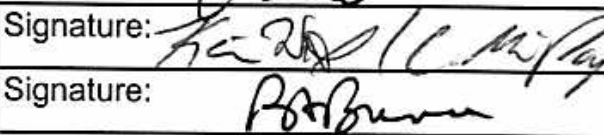 }} \\
\hline Electra & $N / A$ & $\mathrm{~N} / \mathrm{A}$ & $\mathrm{N} / \mathrm{A}$ & $\mathrm{N} / \mathrm{A}$ & $\mathrm{N} / \mathrm{A}$ & RCT Name: & \multicolumn{2}{|l|}{ B. Bunce } & & & \\
\hline Electra & $N / A$ & $N / A$ & $N / A$ & $N / A$ & $\mathrm{~N} / \mathrm{A}$ & RCT Name: & B. Fosket & & \multicolumn{3}{|c|}{ Signature } \\
\hline \multirow[t]{2}{*}{ Survey Point } & \multirow{2}{*}{\multicolumn{4}{|c|}{ Description/Comments }} & \multicolumn{2}{|c|}{$\begin{array}{l}\text { Removable } \\
\mathrm{dpm} / 100 \mathrm{~cm}^{2}\end{array}$} & \multicolumn{2}{|c|}{$\begin{array}{c}\text { Fixed + Removable } \\
\mathrm{dpm} / 100 \mathrm{~cm}^{2}\end{array}$} & \multirow{2}{*}{$\begin{array}{l}\text { Gamma } \\
\mathrm{mrem} / \mathrm{hr}\end{array}$} & \multirow{2}{*}{$\begin{array}{l}\text { Neutron } \\
\mathrm{mrem} / \mathrm{hr}\end{array}$} & \multirow{2}{*}{$\begin{array}{l}\text { Total } \\
\mathrm{mrem} / \mathrm{hr}\end{array}$} \\
\hline & & & & & Alpha & Beta & Alpha & Beta & & & \\
\hline 1 & \multicolumn{4}{|c|}{ Wall, Concrete (Electra 1120) } & $N / A$ & $N / A$ & 10 & 749 & $N / A$ & $\mathrm{~N} / \mathrm{A}$ & $N / A$ \\
\hline 2 & \multicolumn{4}{|c|}{ Wall, Concrete (Electra 1120) } & $N / A$ & $\mathrm{~N} / \mathrm{A}$ & 10 & 859 & $N / A$ & $N / A$ & $N / A$ \\
\hline 3 & \multicolumn{4}{|c|}{ Wall, Concrete (Electra 1120) } & $N / A$ & $N / A$ & 6 & 781 & $N / A$ & $N / A$ & $N / A$ \\
\hline 4 & \multicolumn{4}{|c|}{ Wall, Concrete (Electra 1120) } & $N / A$ & $N / A$ & 4 & 871 & $N / A$ & $N / A$ & $N / A$ \\
\hline 5 & \multicolumn{4}{|c|}{ Wall, Concrete (Electra 1120) } & $N / A$ & $N / A$ & 6 & 825 & $N / A$ & $N / A$ & $N / A$ \\
\hline 6 & \multicolumn{4}{|c|}{ Wall, Concrete (Electra 1120) } & $N / A$ & $N / A$ & 5 & 836 & $N / A$ & $N / A$ & $N / A$ \\
\hline 7 & \multicolumn{4}{|c|}{ Wall, Concrete (Electra 1120) } & $N / A$ & $N / A$ & 3 & 798 & $N / A$ & $N / A$ & $N / A$ \\
\hline 8 & \multicolumn{4}{|c|}{ Wall, Concrete (Electra 1120) } & $N / A$ & $N / A$ & 5 & 676 & $N / A$ & $N / A$ & $N / A$ \\
\hline 9 & \multicolumn{4}{|c|}{ Wall, Concrete (Electra 1120) } & $N / A$ & $N / A$ & 8 & 853 & $\mathrm{~N} / \mathrm{A}$ & $N / A$ & $N / A$ \\
\hline 10 & \multicolumn{4}{|c|}{ Wall, Concrete (Electra 1120) } & $N / A$ & $\mathrm{~N} / \mathrm{A}$ & 5 & 810 & $N / A$ & $N / A$ & $N / A$ \\
\hline 11 & \multicolumn{4}{|c|}{ Wall, Concrete (Electra 1120) } & $N / A$ & $N / A$ & 10 & 808 & $N / A$ & $N / A$ & $N / A$ \\
\hline \multicolumn{5}{|c|}{ Reviewed By (Print): } & \multicolumn{5}{|l|}{ Signature: } & \multicolumn{2}{|c|}{$8 / 23 / 10$} \\
\hline
\end{tabular}


SURVEY \# 10-ER-A26-289

\begin{tabular}{|c|c|c|c|c|c|c|c|c|}
\hline \multirow[t]{2}{*}{ Survey Point } & \multirow[t]{2}{*}{ Description/Comments } & \multicolumn{2}{|c|}{$\begin{array}{l}\text { Removable } \\
\mathrm{dpm} / 100 \mathrm{~cm}^{2}\end{array}$} & \multicolumn{2}{|c|}{$\begin{array}{c}\text { Fixed + Removable } \\
\mathrm{dpm} / 100 \mathrm{~cm}^{2}\end{array}$} & \multirow{2}{*}{$\begin{array}{l}\text { Gamma } \\
\mathrm{mrem} / \mathrm{hr}\end{array}$} & \multirow{2}{*}{$\begin{array}{l}\text { Neutron } \\
\mathrm{mrem} / \mathrm{hr}\end{array}$} & \multirow{2}{*}{$\begin{array}{c}\text { Total } \\
\text { mrem/hr }\end{array}$} \\
\hline & & Alpha & Beta & Alpha & Beta & & & \\
\hline 12 & Wall, Concrete (1120) & N/A & N/A & 4 & 744 & N/A & N/A & $\mathrm{N} / \mathrm{A}$ \\
\hline 13 & Wall, Concrete (1120) & N/A & N/A & 14 & 855 & N/A & N/A & N/A \\
\hline 14 & Wall, Concrete (1120) & N/A & N/A & 11 & 794 & $\mathrm{~N} / \mathrm{A}$ & N/A & N/A \\
\hline 15 & Wall, Concrete (1120) & $\mathrm{N} / \mathrm{A}$ & N/A & 8 & 788 & N/A & N/A & N/A \\
\hline 16 & Wall, Concrete (1120) & N/A & N/A & 12 & 776 & N/A & N/A & N/A \\
\hline 17 & Wall, Concrete (1120) & $\mathrm{N} / \mathrm{A}$ & N/A & 6 & 799 & $\mathrm{~N} / \mathrm{A}$ & N/A & N/A \\
\hline 18 & Wall, Concrete (1120) & $\mathrm{N} / \mathrm{A}$ & N/A & 9 & 701 & $\mathrm{~N} / \mathrm{A}$ & N/A & N/A \\
\hline N/A & ${ }^{\star \star}$ Performed scan surveys for both alpha and beta ${ }^{\star \star}$ & N/A & N/A & $\mathrm{N} / \mathrm{A}$ & $\mathrm{N} / \mathrm{A}$ & N/A & N/A & $\mathrm{N} / \mathrm{A}$ \\
\hline N/A & ${ }^{\star \star}$ on $100 \%$ of survey unit. No trigger levels exceeded ${ }^{\star \star}$ & N/A & N/A & $\mathrm{N} / \mathrm{A}$ & N/A & N/A & N/A & N/A \\
\hline N/A & $\mathrm{N} / \mathrm{A}$ & N/A & N/A & N/A & N/A & N/A & N/A & N/A \\
\hline N/A & $\mathrm{N} / \mathrm{A}$ & N/A & N/A & N/A & N/A & $\mathrm{N} / \mathrm{A}$ & N/A & N/A \\
\hline N/A & $\mathrm{N} / \mathrm{A}$ & N/A & N/A & N/A & $\mathrm{N} / \mathrm{A}$ & N/A & N/A & N/A \\
\hline N/A & $\mathrm{N} / \mathrm{A}$ & $\mathrm{N} / \mathrm{A}$ & $\mathrm{N} / \mathrm{A}$ & N/A & N/A & N/A & N/A & N/A \\
\hline $\mathrm{N} / \mathrm{A}$ & $N / A$ & N/A & N/A & N/A & N/A & N/A & N/A & N/A \\
\hline $\mathrm{N} / \mathrm{A}$ & $\mathrm{N} / \mathrm{A}$ & N/A & N/A & N/A & N/A & $\mathrm{N} / \mathrm{A}$ & N/A & $\mathrm{N} / \mathrm{A}$ \\
\hline N/A & $\mathrm{N} / \mathrm{A}$ & N/A & N/A & N/A & N/A & N/A & N/A & N/A \\
\hline $\mathrm{N} / \mathrm{A}$ & $\mathrm{N} / \mathrm{A}$ & N/A & N/A & N/A & $\mathrm{N} / \mathrm{A}$ & N/A & N/A & N/A \\
\hline N/A & $N / A$ & N/A & N/A & $\mathrm{N} / \mathrm{A}$ & N/A & N/A & N/A & N/A \\
\hline N/A & $\mathrm{N} / \mathrm{A}$ & N/A & N/A & N/A & N/A & $\mathrm{N} / \mathrm{A}$ & N/A & N/A \\
\hline $\mathrm{N} / \mathrm{A}$ & $\mathrm{N} / \mathrm{A}$ & $\mathrm{N} / \mathrm{A}$ & N/A & N/A & $\mathrm{N} / \mathrm{A}$ & N/A & N/A & N/A \\
\hline N/A & $\mathrm{N} / \mathrm{A}$ & $\mathrm{N} / \mathrm{A}$ & N/A & N/A & N/A & N/A & N/A & N/A \\
\hline N/A & $\mathrm{N} / \mathrm{A}$ & $\mathrm{N} / \mathrm{A}$ & N/A & $\mathrm{N} / \mathrm{A}$ & N/A & N/A & N/A & $\mathrm{N} / \mathrm{A}$ \\
\hline N/A & $N / A$ & N/A & N/A & N/A & N/A & $\mathrm{N} / \mathrm{A}$ & N/A & N/A \\
\hline
\end{tabular}


Survey $=10$ ER A26 289

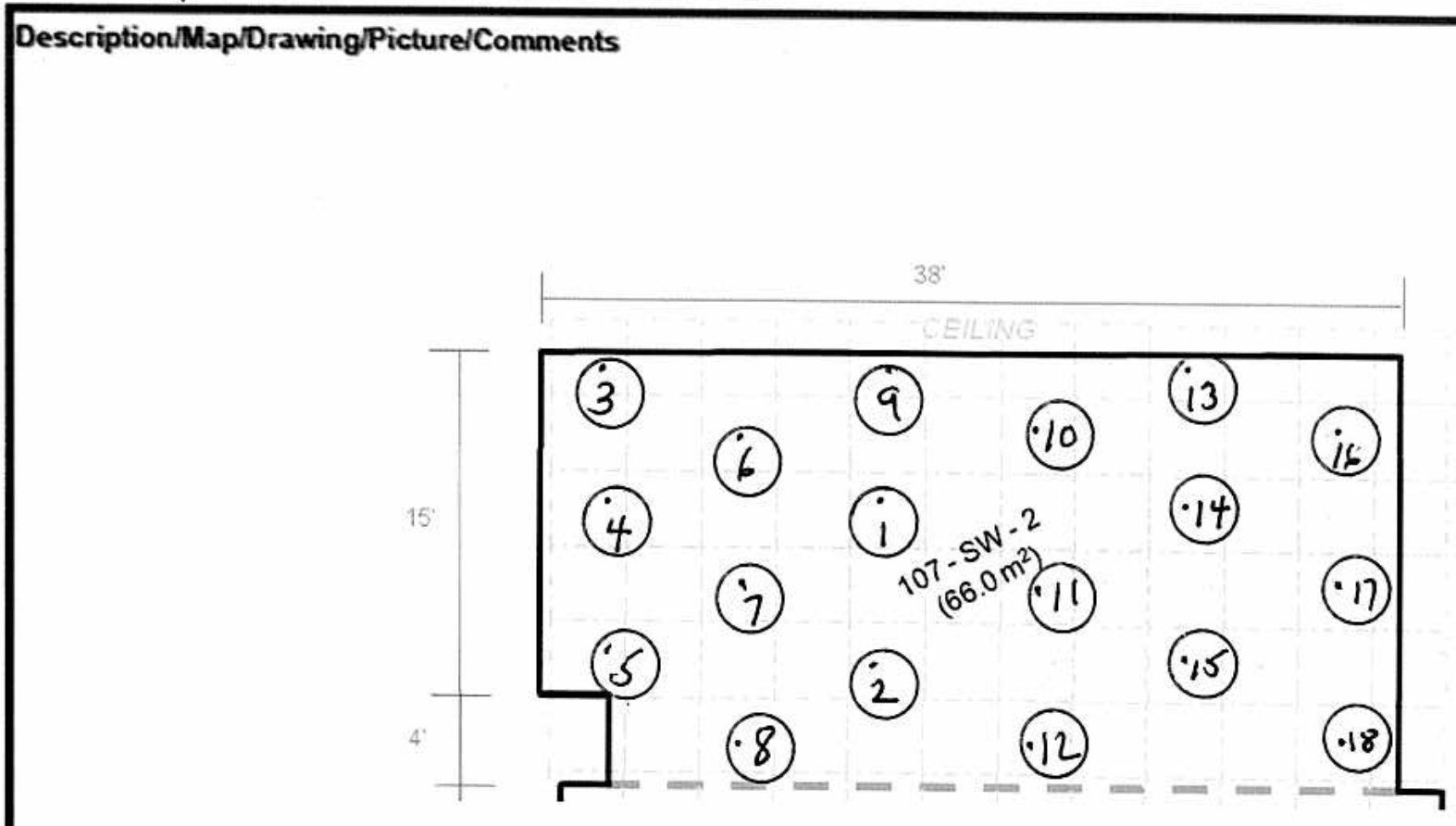

MARSSIM CLASS I SURVEY

$-1 \times 1 m$ grid

LEGEND

tark Count LOCA trons

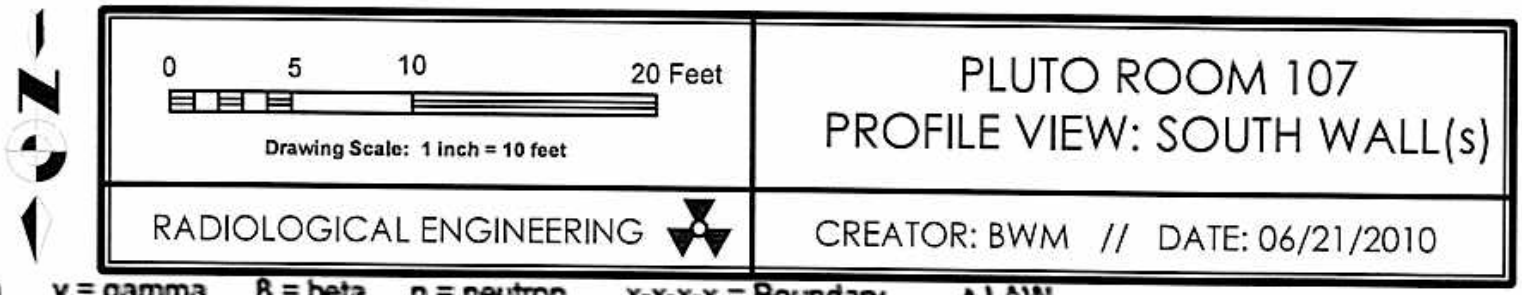

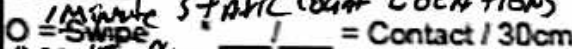




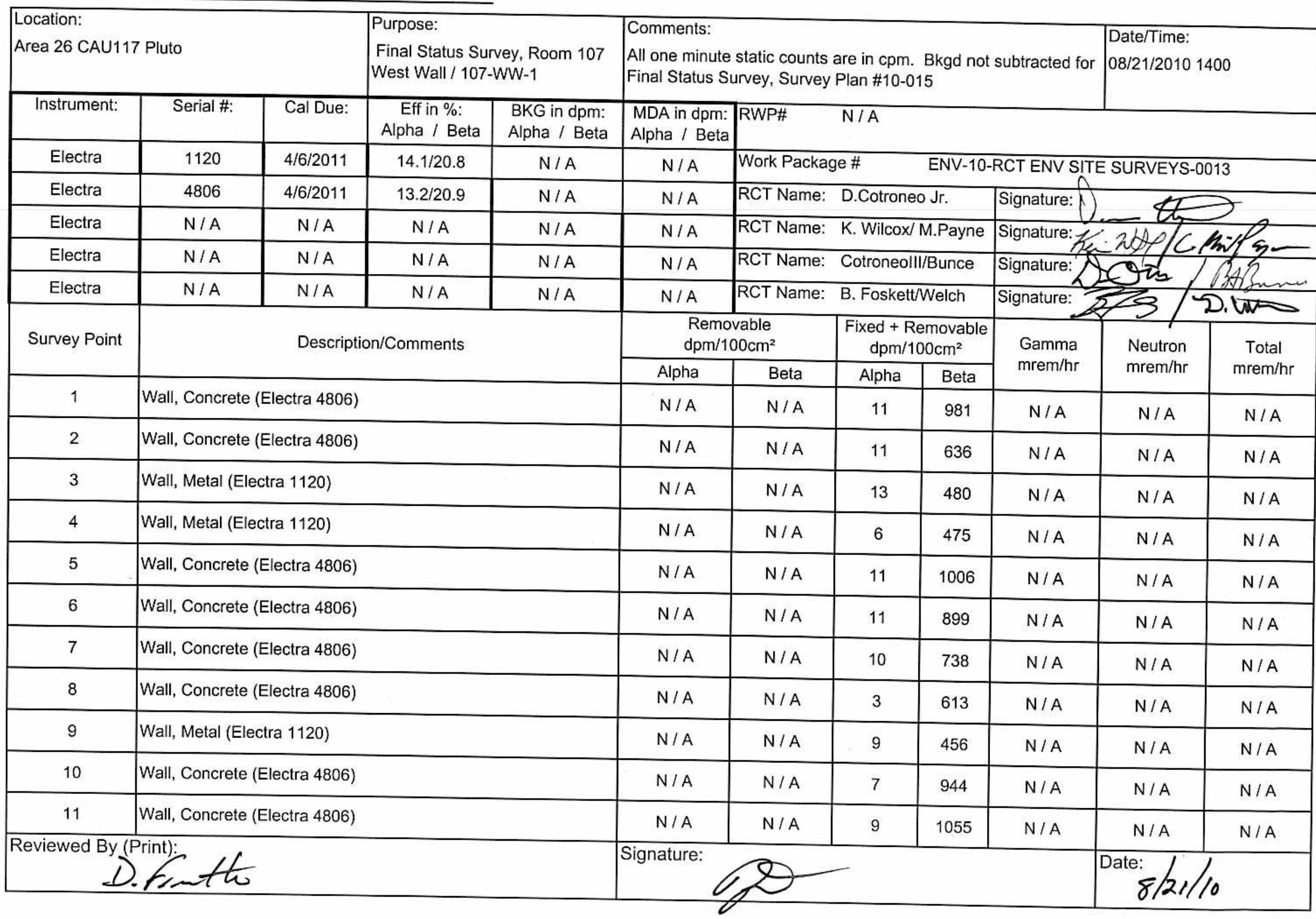


NSTec

Form

FRM-0108B

RADIOLOGICAL SURVEY REPORT - DATA (CONTINUATION)

SURVEY \# 10-ER-A26-286

\begin{tabular}{|c|c|c|c|c|c|c|c|c|}
\hline \multirow[t]{2}{*}{ Survey Point } & \multirow[t]{2}{*}{ Description/Comments } & \multicolumn{2}{|c|}{$\begin{array}{l}\text { Removable } \\
\mathrm{dpm} / 100 \mathrm{~cm}^{2}\end{array}$} & \multicolumn{2}{|c|}{$\begin{array}{c}\text { Fixed + Removable } \\
\mathrm{dpm} / 100 \mathrm{~cm}^{2}\end{array}$} & \multirow{2}{*}{$\begin{array}{l}\text { Gamma } \\
\mathrm{mrem} / \mathrm{hr}\end{array}$} & \multirow{2}{*}{$\begin{array}{l}\text { Neutron } \\
\mathrm{mrem} / \mathrm{hr}\end{array}$} & \multirow{2}{*}{$\begin{array}{c}\text { Total } \\
\text { mrem } / \mathrm{hr}\end{array}$} \\
\hline & & Alpha & Beta & Alpha & Beta & & & \\
\hline 12 & Wall, Concrete (4806) & $\mathrm{N} / \mathrm{A}$ & N/A & 9 & 1004 & $\mathrm{~N} / \mathrm{A}$ & N/A & N/A \\
\hline 13 & Wall, Concrete (4806) & N/A & $\mathrm{N} / \mathrm{A}$ & 10 & 739 & N/A & N/A & N/A \\
\hline 14 & Wall, Concrete (4806) & $\mathrm{N} / \mathrm{A}$ & $\mathrm{N} / \mathrm{A}$ & 5 & 571 & $\mathrm{~N} / \mathrm{A}$ & N/A & N/A \\
\hline 15 & Wall, Concrete (4806) & N/A & $\mathrm{N} / \mathrm{A}$ & 6 & 681 & N/A & N/A & $\mathrm{N} / \mathrm{A}$ \\
\hline 16 & Wall, Concrete (4806) & N/A & N/A & 5 & 1068 & $\mathrm{~N} / \mathrm{A}$ & N/A & N/A \\
\hline 17 & Wall, Concrete (4806) & $\mathrm{N} / \mathrm{A}$ & $\mathrm{N} / \mathrm{A}$ & 11 & 1059 & N/A & N/A & $\mathrm{N} / \mathrm{A}$ \\
\hline 18 & Wall, Concrete (4806) & N/A & N/A & 12 & 687 & N/A & N/A & $\mathrm{N} / \mathrm{A}$ \\
\hline 19 & Wall, Concrete (4806) & N/A & N/A & 5 & 726 & N/A & N/A & $\mathrm{N} / \mathrm{A}$ \\
\hline 20 & Wall, Concrete (4806) & N/A & N/A & 8 & 766 & N/A & N/A & $\mathrm{N} / \mathrm{A}$ \\
\hline N/A & ${ }^{\star \star}$ Performed scan surveys for both alpha and beta** & N/A & N/A & N/A & $\mathrm{N} / \mathrm{A}$ & N/A & N/A & N/A \\
\hline N/A & ${ }^{\star \star}$ on $100 \%$ of survey unit. No trigger levels exceeded ${ }^{\star \star}$ & N/A & $\mathrm{N} / \mathrm{A}$ & $\mathrm{N} / \mathrm{A}$ & N/A & $\mathrm{N} / \mathrm{A}$ & N/A & N/A \\
\hline N/A & $N / A$ & $\mathrm{~N} / \mathrm{A}$ & $\mathrm{N} / \mathrm{A}$ & $\mathrm{N} / \mathrm{A}$ & $\mathrm{N} / \mathrm{A}$ & N/A & N/A & N/A \\
\hline $\mathrm{N} / \mathrm{A}$ & $N / A$ & N/A & N/A & $\mathrm{N} / \mathrm{A}$ & $\mathrm{N} / \mathrm{A}$ & N/A & N/A & $\mathrm{N} / \mathrm{A}$ \\
\hline N/A & $\mathrm{N} / \mathrm{A}$ & N/A & N/A & N/A & $\mathrm{N} / \mathrm{A}$ & N/A & N/A & N/A \\
\hline N/A & $\mathrm{N} / \mathrm{A}$ & N/A & N/A & N/A & N/A & N/A & N/A & N/A \\
\hline N/A & $\mathrm{N} / \mathrm{A}$ & N/A & N/A & N/A & N/A & N/A & N/A & N/A \\
\hline N/A & $N / A$ & N/A & $\mathrm{N} / \mathrm{A}$ & N/A & N/A & N/A & N/A & N/A \\
\hline N/A & $\mathrm{N} / \mathrm{A}$ & N/A & N/A & N/A & N/A & N/A & N/A & $\mathrm{N} / \mathrm{A}$ \\
\hline $\mathrm{N} / \mathrm{A}$ & $\mathrm{N} / \mathrm{A}$ & N/A & N/A & $\mathrm{N} / \mathrm{A}$ & N/A & $\mathrm{N} / \mathrm{A}$ & N/A & N/A \\
\hline N/A & $\mathrm{N} / \mathrm{A}$ & N/A & N/A & N/A & $\mathrm{N} / \mathrm{A}$ & N/A & N/A & N/A \\
\hline $\mathrm{N} / \mathrm{A}$ & $\mathrm{N} / \mathrm{A}$ & N/A & N/A & N/A & N/A & N/A & N/A & $\mathrm{N} / \mathrm{A}$ \\
\hline N/A & $N / A$ & N/A & N/A & N/A & $\mathrm{N} / \mathrm{A}$ & N/A & N/A & $\mathrm{N} / \mathrm{A}$ \\
\hline N/A & $N / A$ & N/A & N/A & N/A & $\mathrm{N} / \mathrm{A}$ & N/A & N/A & N/A \\
\hline
\end{tabular}


Survey $\# 10-E R-A 26-286$

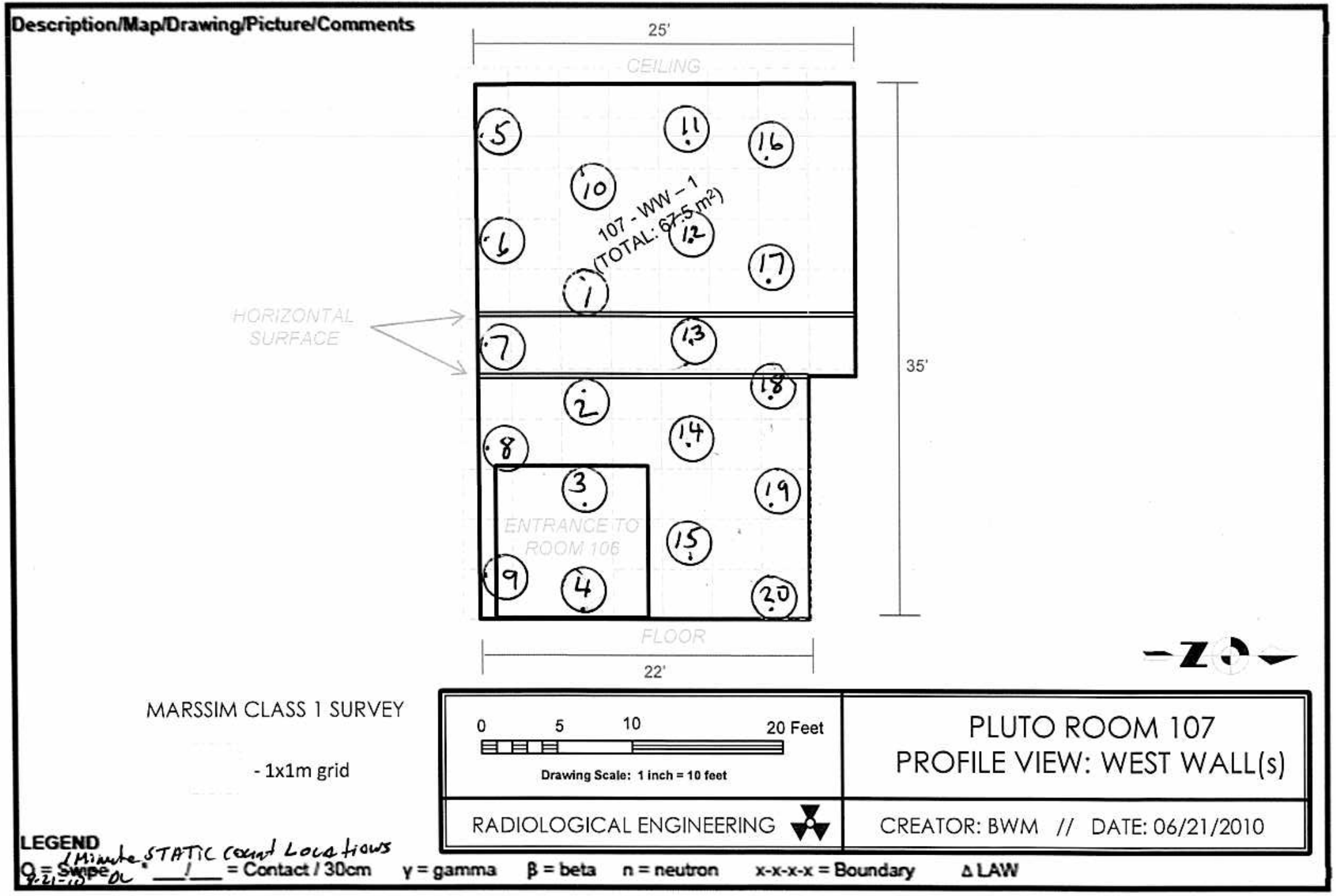


FRM-0108B

\begin{tabular}{|c|c|c|c|c|c|c|c|c|c|c|c|}
\hline \multicolumn{3}{|c|}{$\begin{array}{l}\text { Location: } \\
\text { Area } 26 \text { CAU117 Pluto }\end{array}$} & \multicolumn{2}{|c|}{$\begin{array}{l}\text { Purpose: } \\
\text { Final Status Survey, Room } 102 \\
\text { South Wall 102-SW-1 }\end{array}$} & \multicolumn{5}{|c|}{$\begin{array}{l}\text { Comments: } \\
\text { All one minute static counts are in cpm. Bkgd not subtracted } \\
\text { for Final Status Survey, Survey Plan \#10-015 }\end{array}$} & \multicolumn{2}{|c|}{$\begin{array}{l}\text { Date/Time: } \\
\text { 08/17/2010 } 1300\end{array}$} \\
\hline Instrument: & Serial \#: & Cal Due: & $\begin{array}{c}\text { Eff in \%: } \\
\text { Alpha / Beta }\end{array}$ & $\begin{array}{l}\text { BKG in dpm: } \\
\text { Alpha / Beta }\end{array}$ & \begin{tabular}{|l|} 
MDA in dpm: \\
Alpha / Beta
\end{tabular} & RWP\# & \multicolumn{5}{|l|}{$\mathrm{N} / \mathrm{A}$} \\
\hline Electra & 2125 & $4 / 6 / 2011$ & $14.4 / 18.2$ & $N / A$ & $\mathrm{~N} / \mathrm{A}$ & \multicolumn{6}{|c|}{ ENV-10-RCT ENV SITE } \\
\hline Electra & 6697 & $4 / 6 / 2011$ & $13.8 / 19.9$ & $N / A$ & $N / A$ & \multicolumn{3}{|c|}{ RCT Name: D.Cotroneo Jr. } & \multicolumn{3}{|c|}{ Signature: 0} \\
\hline Electra & 1201 & $4 / 6 / 2011$ & $14.4 / 20.3$ & $\mathrm{~N} / \mathrm{A}$ & $\mathrm{N} / \mathrm{A}$ & \multicolumn{3}{|c|}{ RCT Name: K. Wilcox/M.Payne } & \multirow{2}{*}{\multicolumn{3}{|c|}{ 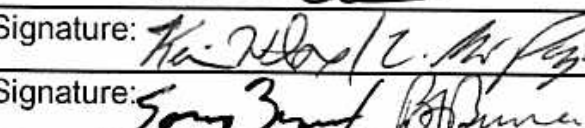 }} \\
\hline Electra & $N / A$ & $\mathrm{~N} / \mathrm{A}$ & $N / A$ & $\mathrm{~N} / \mathrm{A}$ & $N / A$ & \multicolumn{3}{|c|}{ RCT Name: S.Brown/B.Bunce } & & & \\
\hline Electra & $N / A$ & $N / A$ & $\mathrm{~N} / \mathrm{A}$ & $\mathrm{N} / \mathrm{A}$ & $N / A$ & RCT Name: & B. Fosket & Nelch & \multicolumn{3}{|c|}{ ignatife / D. Wt } \\
\hline \multirow[t]{2}{*}{ Survey Point } & \multirow{2}{*}{\multicolumn{4}{|c|}{ Description/Comments }} & \multicolumn{2}{|c|}{$\begin{array}{l}\text { Removable } \\
\mathrm{dpm} / 100 \mathrm{~cm}^{2}\end{array}$} & \multicolumn{2}{|c|}{$\begin{array}{c}\text { Fixed + Removable } \\
\mathrm{dpm} / 100 \mathrm{~cm}^{2}\end{array}$} & \multirow[t]{2}{*}{$\begin{array}{l}\mathrm{Gamma} \\
\mathrm{mrem} / \mathrm{hr}\end{array}$} & \multirow{2}{*}{$\begin{array}{l}\text { Neutron } \\
\mathrm{mrem} / \mathrm{hr}\end{array}$} & \multirow{2}{*}{$\begin{array}{c}\text { Total } \\
\mathrm{mrem} / \mathrm{hr}\end{array}$} \\
\hline & & & & & Alpha & Beta & Alpha & Beta & & & \\
\hline 1 & \multicolumn{4}{|c|}{ Wall, Concrete (Electra 2125) } & $N / A$ & $N / A$ & 15 & 895 & $N / A$ & $\mathrm{~N} / \mathrm{A}$ & $N / A$ \\
\hline 2 & \multicolumn{4}{|c|}{ Wall, Concrete (Electra 2125) } & $N / A$ & $N / A$ & 11 & 754 & $N / A$ & $N / A$ & $N / A$ \\
\hline 3 & \multicolumn{4}{|c|}{ Wall, Concrete (Electra 2125) } & $N / A$ & $N / A$ & 5 & 1129 & $N / A$ & $N / A$ & $\mathrm{~N} / \mathrm{A}$ \\
\hline 4 & \multicolumn{4}{|c|}{ Wall, Concrete (Electra 2125) } & $N / A$ & $N / A$ & 9 & 759 & $N / A$ & $N / A$ & $N / A$ \\
\hline 5 & \multicolumn{4}{|c|}{ Wall, Concrete (Electra 2125) } & $N / A$ & $N / A$ & 4 & 731 & $N / A$ & $N / A$ & $N / A$ \\
\hline 6 & \multicolumn{4}{|c|}{ Wall, Concrete (Electra 2125) } & $N / A$ & $N / A$ & 11 & 1030 & $N / A$ & $N / A$ & $N / A$ \\
\hline 7 & \multicolumn{4}{|c|}{ Wall, Concrete (Electra 2125) } & $N / A$ & $N / A$ & 9 & 1013 & $N / A$ & $N / A$ & $N / A$ \\
\hline 8 & \multicolumn{4}{|c|}{ Wall, Concrete (Electra 2125) } & $N / A$ & $N / A$ & 4 & 626 & $N / A$ & $N / A$ & $N / A$ \\
\hline 9 & \multicolumn{4}{|c|}{ Wall, Concrete (Electra 2125) } & $N / A$ & $N / A$ & 4 & 712 & $N / A$ & $N / A$ & $N / A$ \\
\hline 10 & \multicolumn{4}{|c|}{ Wall, Concrete (Electra 2125) } & $N / A$ & $N / A$ & 4 & 1016 & $N / A$ & $N / A$ & $N / A$ \\
\hline 11 & \multicolumn{4}{|c|}{ Wall, Concrete (Electra 2125) } & $N / A$ & $N / A$ & 9 & 861 & $N / A$ & $N / A$ & $N / A$ \\
\hline \multicolumn{5}{|c|}{ Reviewed By (Print): } & ignatu & & & & & \multicolumn{2}{|c|}{$8 / 18 / 10$} \\
\hline
\end{tabular}


NSTec

Form

FRM-0108B

RADIOLOGICAL SURVEY REPORT - DATA (CONTINUATION)

Rev. 0

SURVEY \# 10-ER-A26-269

\begin{tabular}{|c|c|c|c|c|c|c|c|c|}
\hline \multirow{2}{*}{$\begin{array}{c}\text { Survey } \\
\text { Point }\end{array}$} & \multirow[t]{2}{*}{ Description/Comments } & \multicolumn{2}{|c|}{$\begin{array}{l}\text { Removable } \\
\mathrm{dpm} / 100 \mathrm{~cm}^{2}\end{array}$} & \multicolumn{2}{|c|}{$\begin{array}{c}\text { Fixed + Removable } \\
\mathrm{dpm} / 100 \mathrm{~cm}^{2}\end{array}$} & \multirow{2}{*}{$\begin{array}{l}\text { Gamma } \\
\mathrm{mrem} / \mathrm{hr}\end{array}$} & \multirow{2}{*}{$\begin{array}{l}\text { Neutron } \\
\mathrm{mrem} / \mathrm{hr}\end{array}$} & \multirow{2}{*}{$\begin{array}{c}\text { Total } \\
\mathrm{mrem} / \mathrm{hr}\end{array}$} \\
\hline & & Alpha & Beta & Alpha & Beta & & & \\
\hline 12 & Wall, Concrete (2125) & N/A & N/A & 8 & 747 & $\mathrm{~N} / \mathrm{A}$ & N/A & N/A \\
\hline 13 & Wall, Concrete (2125) & $\mathrm{N} / \mathrm{A}$ & N/A & 7 & 708 & N/A & N/A & $\mathrm{N} / \mathrm{A}$ \\
\hline N/A & ${ }^{\star \star}$ Performed scan surveys for both alpha and beta** & $\mathrm{N} / \mathrm{A}$ & N/A & N/A & N/A & N/A & N/A & $\mathrm{N} / \mathrm{A}$ \\
\hline $\mathrm{N} / \mathrm{A}$ & ${ }^{\star \star}$ on $100 \%$ of survey unit. No trigger levels exceeded ${ }^{\star \star}$ & $\mathrm{N} / \mathrm{A}$ & N/A & N/A & $N / A$ & N/A & N/A & $\mathrm{N} / \mathrm{A}$ \\
\hline N/A & $N / A$ & N/A & N/A & $\mathrm{N} / \mathrm{A}$ & N/A & N/A & $N / A$ & $\mathrm{~N} / \mathrm{A}$ \\
\hline $\mathrm{N} / \mathrm{A}$ & $\mathrm{N} / \mathrm{A}$ & $\mathrm{N} / \mathrm{A}$ & N/A & N/A & N/A & $\mathrm{N} / \mathrm{A}$ & $\mathrm{N} / \mathrm{A}$ & $\mathrm{N} / \mathrm{A}$ \\
\hline $\mathrm{N} / \mathrm{A}$ & $N / A$ & N/A & N/A & N/A & $N / A$ & $\mathrm{~N} / \mathrm{A}$ & $\mathrm{N} / \mathrm{A}$ & $N / A$ \\
\hline $\mathrm{N} / \mathrm{A}$ & $\mathrm{N} / \mathrm{A}$ & N/A & N/A & N/A & N/A & N/A & N/A & $\mathrm{N} / \mathrm{A}$ \\
\hline $\mathrm{N} / \mathrm{A}$ & $N / A$ & N/A & N/A & N/A & N/A & N/A & $\mathrm{N} / \mathrm{A}$ & $\mathrm{N} / \mathrm{A}$ \\
\hline $\mathrm{N} / \mathrm{A}$ & $N / A$ & $\mathrm{~N} / \mathrm{A}$ & N/A & N/A & N/A & N/A & $\mathrm{N} / \mathrm{A}$ & $\mathrm{N} / \mathrm{A}$ \\
\hline N/A & $N / A$ & $\mathrm{~N} / \mathrm{A}$ & $\mathrm{N} / \mathrm{A}$ & N/A & N/A & N/A & N/A & N/A \\
\hline N/A & $N / A$ & N/A & N/A & N/A & $\mathrm{N} / \mathrm{A}$ & N/A & N/A & N/A \\
\hline $\mathrm{N} / \mathrm{A}$ & $N / A$ & $\mathrm{~N} / \mathrm{A}$ & N/A & N/A & $\mathrm{N} / \mathrm{A}$ & N/A & N/A & N/A \\
\hline $\mathrm{N} / \mathrm{A}$ & $N / A$ & N/A & $\mathrm{N} / \mathrm{A}$ & N/A & $\mathrm{N} / \mathrm{A}$ & N/A & N/A & $\mathrm{N} / \mathrm{A}$ \\
\hline $\mathrm{N} / \mathrm{A}$ & $N / A$ & N/A & $\mathrm{N} / \mathrm{A}$ & N/A & $\mathrm{N} / \mathrm{A}$ & N/A & $\mathrm{N} / \mathrm{A}$ & N/A \\
\hline N/A & $N / A$ & N/A & N/A & N/A & N/A & N/A & N/A & N/A \\
\hline $\mathrm{N} / \mathrm{A}$ & $N / A$ & N/A & $\mathrm{N} / \mathrm{A}$ & N/A & N/A & N/A & N/A & $\mathrm{N} / \mathrm{A}$ \\
\hline N/A & $N / A$ & $\mathrm{~N} / \mathrm{A}$ & $\mathrm{N} / \mathrm{A}$ & N/A & N/A & N/A & N/A & $\mathrm{N} / \mathrm{A}$ \\
\hline N/A & $N / A$ & N/A & $\mathrm{N} / \mathrm{A}$ & N/A & N/A & N/A & N/A & $\mathrm{N} / \mathrm{A}$ \\
\hline N/A & $N / A$ & N/A & N/A & N/A & N/A & N/A & N/A & $\mathrm{N} / \mathrm{A}$ \\
\hline N/A & $N / A$ & N/A & N/A & N/A & N/A & N/A & N/A & $\mathrm{N} / \mathrm{A}$ \\
\hline N/A & $N / A$ & $\mathrm{~N} / \mathrm{A}$ & N/A & N/A & N/A & N/A & N/A & $\mathrm{N} / \mathrm{A}$ \\
\hline N/A & $N / A$ & N/A & N/A & N/A & $\mathrm{N} / \mathrm{A}$ & N/A & $\mathrm{N} / \mathrm{A}$ & N/A \\
\hline
\end{tabular}


Survey $\# \angle 0-E R-A 26-269$

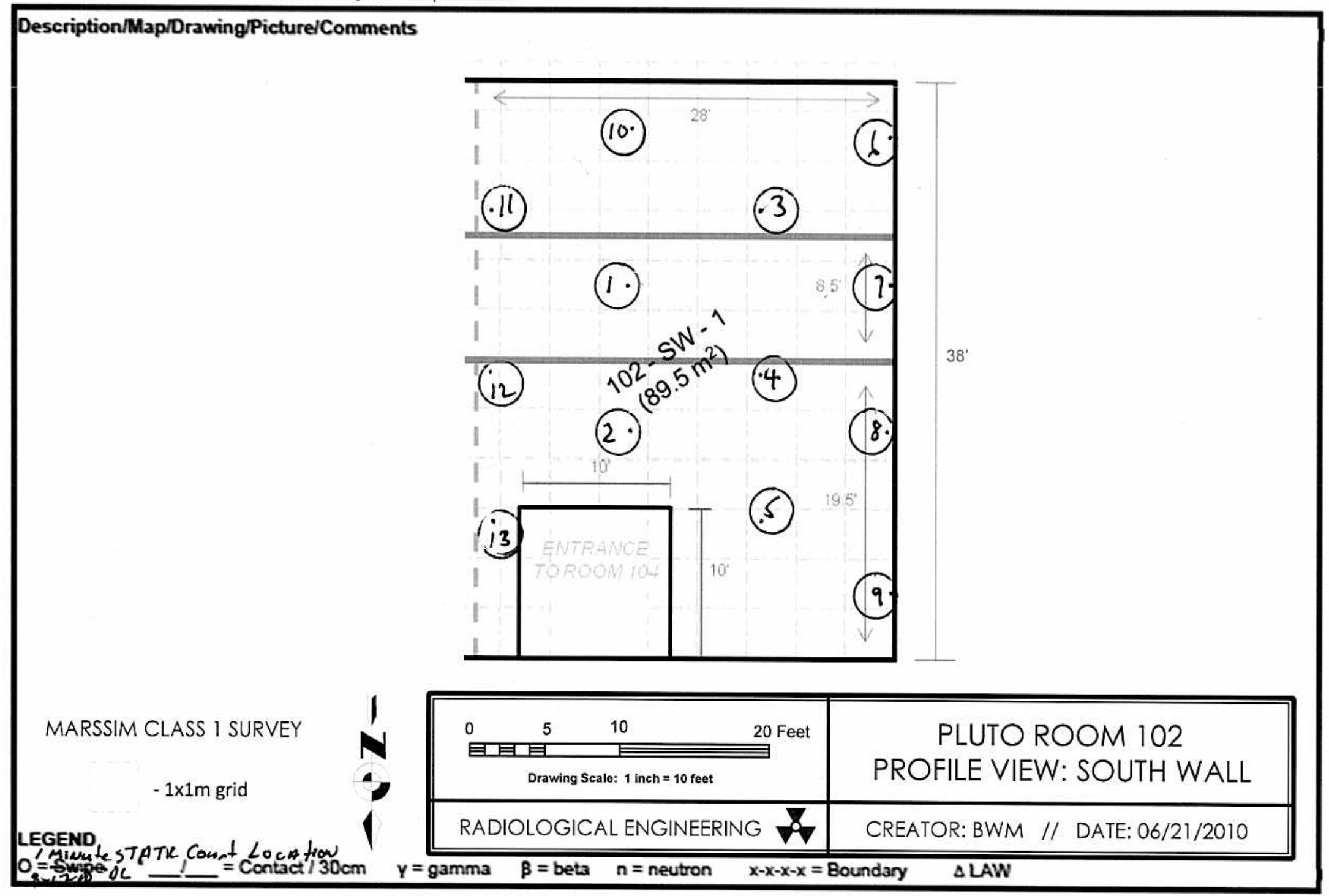


SURVEY \# 10-ER-A26-292

\begin{tabular}{|c|c|c|c|c|c|c|c|c|c|c|c|}
\hline $\begin{array}{l}\text { Location: } \\
\text { Area } 26 \text { CAU117 }\end{array}$ & Pluto & & $\begin{array}{l}\text { Purpose: } \\
\text { Final Status Su } \\
104,106,107 / L\end{array}$ & $\begin{array}{l}\text { ey, Rm 102, } \\
\text { dges-1 }\end{array}$ & $\begin{array}{l}\text { Comments: } \\
\text { All one minute s } \\
\text { Final Status Su }\end{array}$ & $\begin{array}{l}\text { static counts a } \\
\text { unvey, Survey F }\end{array}$ & $\begin{array}{l}\text { are in cpm } \\
\text { Plan \#10-C }\end{array}$ & $\begin{array}{l}\text { Bkgd not } \\
5\end{array}$ & subtracted for & $\begin{array}{l}\text { Date/Time: } \\
\text { 08/22/2010 1s }\end{array}$ & \\
\hline Instrument: & Serial \#: & Cal Due: & $\begin{array}{c}\text { Eff in \%: } \\
\text { Alpha / Beta }\end{array}$ & $\begin{array}{l}\text { BKG in dpm: } \\
\text { Alpha / Beta }\end{array}$ & \begin{tabular}{|l|} 
MDA in dpm: \\
Alpha / Beta
\end{tabular} & RWP\# & $\mathrm{N} / \mathrm{A}$ & & & & \\
\hline Electra & 6697 & $4 / 6 / 2011$ & $13.8 / 19.9$ & $\mathrm{~N} / \mathrm{A}$ & $N / A$ & Work Packag & ge \# & ENV-10-F & RCT ENV SITE & E SURVEYS- 0 & \\
\hline Electra & 1504 & $4 / 6 / 2011$ & $12.9 / 19.8$ & $\mathrm{~N} / \mathrm{A}$ & $N / A$ & RCT Name: & D.Cotron & Jr. & Signature: 8 & & \\
\hline $\mathrm{N} / \mathrm{A}$ & $\mathrm{N} / \mathrm{A}$ & $N / A$ & $N / A$ & $\mathrm{~N} / \mathrm{A}$ & $N / A$ & RCT Name: & K. Wilcox & M.Payne & Signature: & & \\
\hline $\mathrm{N} / \mathrm{A}$ & $N / A$ & $N / A$ & $N / A$ & $N / A$ & $N / A$ & RCT Name: & Cotroneo & /Bunce & Signature & & \\
\hline$N / A$ & $\mathrm{~N} / \mathrm{A}$ & $\mathrm{N} / \mathrm{A}$ & $\mathrm{N} / \mathrm{A}$ & $N / A$ & $N / A$ & RCT Name: & B. Fosket & Brown & Signature: & & \\
\hline Survey Point & & Descript & on/Comments & & $\begin{array}{r}\text { Remov } \\
\mathrm{dpm} / 10\end{array}$ & $\begin{array}{l}\text { vable } \\
00 \mathrm{~cm}^{2}\end{array}$ & $\begin{array}{r}\text { Fixed }+F \\
\mathrm{dpm} /\end{array}$ & $0 \mathrm{~cm}^{2}$ & Gamma & Neutron & Total \\
\hline & & & & & Alpha & Beta & Alpha & Beta & $\mathrm{mrem} / \mathrm{nr}$ & mrem/hr & rem/hr \\
\hline 1 & Ledge 102, C & rete (Electra & 6697) & & $\mathrm{N} / \mathrm{A}$ & $N / A$ & 11 & 1313 & $\mathrm{~N} / \mathrm{A}$ & $N / A$ & $N / A$ \\
\hline 2 & Ledge $102, \mathrm{C}$ & rete (Electra & 6697) & & $N / A$ & $N / A$ & 22 & 1246 & $\mathrm{~N} / \mathrm{A}$ & $N / A$ & $N / A$ \\
\hline 3 & Ledge $102, \mathrm{C}$ & rete (Electra & 6697) & & $\mathrm{N} / \mathrm{A}$ & $N / A$ & 6 & 1120 & $N / A$ & $\mathrm{~N} / \mathrm{A}$ & $\mathrm{N} / \mathrm{A}$ \\
\hline 4 & Ledge 102, C & ete (Electra & 6697) & & $N / A$ & $N / A$ & 12 & 1094 & $N / A$ & $N / A$ & $N / A$ \\
\hline 5 & Ledge 102, C & ete (Electra & 6697) & & $\mathrm{N} / \mathrm{A}$ & $\mathrm{N} / \mathrm{A}$ & 8 & 1003 & $N / A$ & $\mathrm{~N} / \mathrm{A}$ & $N / A$ \\
\hline 6 & Ledge $102, \mathrm{C}$ & ete (Electra & 6697) & & $N / A$ & $N / A$ & 12 & 959 & $N / A$ & $N / A$ & $\mathrm{~N} / \mathrm{A}$ \\
\hline 7 & Ledge $102, \mathrm{Cc}$ & ete (Electra & 6697) & & $\mathrm{N} / \mathrm{A}$ & $\mathrm{N} / \mathrm{A}$ & 11 & 1059 & $N / A$ & $N / A$ & $\mathrm{~N} / \mathrm{A}$ \\
\hline 8 & Ledge $102, \mathrm{C}$ & ete (Electra & 6697) & & $N / A$ & $N / A$ & 7 & 986 & $N / A$ & $N / A$ & $N / A$ \\
\hline 9 & Ledge $102, \mathrm{C}$ & ete (Electra & 6697) & & $N / A$ & $\mathrm{~N} / \mathrm{A}$ & 11 & 1224 & $N / A$ & $N / A$ & $N / A$ \\
\hline 10 & Ledge 102, Cc & ete (Electra & 6697) & & $N / A$ & $N / A$ & 10 & 1297 & $N / A$ & $N / A$ & $N / A$ \\
\hline 11 & Ledge $102, \mathrm{Cc}$ & ete (Electra & 6697) & & $\mathrm{N} / \mathrm{A}$ & $N / A$ & 15 & 1291 & $N / A$ & $N / A$ & $N / A$ \\
\hline Reviewed By ( & rint): & & & & Signature: & & & & & Date: $8 / 23$ & \\
\hline
\end{tabular}


SURVEY \# 10-ER-A26-292

\begin{tabular}{|c|c|c|c|c|c|c|c|c|}
\hline \multirow[t]{2}{*}{ Survey Point } & \multirow[t]{2}{*}{ Description/Comments } & \multicolumn{2}{|c|}{$\begin{array}{l}\text { Removable } \\
\mathrm{dpm} / 100 \mathrm{~cm}^{2}\end{array}$} & \multicolumn{2}{|c|}{$\begin{array}{c}\text { Fixed + Removable } \\
\mathrm{dpm} / 100 \mathrm{~cm}^{2}\end{array}$} & \multirow{2}{*}{$\begin{array}{l}\text { Gamma } \\
\text { mrem/hr }\end{array}$} & \multirow{2}{*}{$\begin{array}{l}\text { Neutron } \\
\mathrm{mrem} / \mathrm{hr}\end{array}$} & \multirow{2}{*}{$\begin{array}{c}\text { Total } \\
\text { mrem } / \mathrm{hr}\end{array}$} \\
\hline & & Alpha & Beta & Alpha & Beta & & & \\
\hline 12 & Ledge 104, Concrete (1504) & N/A & $\mathrm{N} / \mathrm{A}$ & 8 & 910 & N/A & N/A & N/A \\
\hline 13 & Ledge 104, Concrete (1504) & $\mathrm{N} / \mathrm{A}$ & N/A & 12 & 982 & $\mathrm{~N} / \mathrm{A}$ & $\mathrm{N} / \mathrm{A}$ & $\mathrm{N} / \mathrm{A}$ \\
\hline 14 & Ledge 104, Concrete (1504) & $\mathrm{N} / \mathrm{A}$ & N/A & 16 & 1104 & $\mathrm{~N} / \mathrm{A}$ & $\mathrm{N} / \mathrm{A}$ & N/A \\
\hline 15 & Ledge 106, Concrete (1504) & N/A & N/A & 9 & 1017 & N/A & $\mathrm{N} / \mathrm{A}$ & N/A \\
\hline 16 & Ledge 106, Concrete (1504) & $\mathrm{N} / \mathrm{A}$ & $\mathrm{N} / \mathrm{A}$ & 8 & 1241 & N/A & N/A & N/A \\
\hline 17 & Ledge 106 , Concrete (1504) & $\mathrm{N} / \mathrm{A}$ & N/A & 2 & 1287 & N/A & N/A & N/A \\
\hline N/A & ${ }^{* *}$ Performed scan surveys for both alpha and beta** & N/A & N/A & N/A & N/A & $\mathrm{N} / \mathrm{A}$ & N/A & N/A \\
\hline N/A & ${ }^{\star \star}$ on $100 \%$ of survey unit. No trigger levels exceeded ${ }^{\star \star}$ & $\mathrm{N} / \mathrm{A}$ & N/A & N/A & $\mathrm{N} / \mathrm{A}$ & N/A & N/A & N/A \\
\hline N/A & $\mathrm{N} / \mathrm{A}$ & N/A & N/A & N/A & N/A & $\mathrm{N} / \mathrm{A}$ & N/A & N/A \\
\hline N/A & $N / A$ & $\mathrm{~N} / \mathrm{A}$ & N/A & N/A & N/A & N/A & N/A & N/A \\
\hline N/A & N/A & N/A & N/A & N/A & N/A & $\mathrm{N} / \mathrm{A}$ & N/A & N/A \\
\hline N/A & $N / A$ & N/A & N/A & N/A & $\mathrm{N} / \mathrm{A}$ & N/A & N/A & N/A \\
\hline N/A & $\mathrm{N} / \mathrm{A}$ & N/A & $\mathrm{N} / \mathrm{A}$ & N/A & N/A & N/A & N/A & N/A \\
\hline N/A & $\mathrm{N} / \mathrm{A}$ & $\mathrm{N} / \mathrm{A}$ & N/A & N/A & N/A & N/A & N/A & N/A \\
\hline N/A & $\mathrm{N} / \mathrm{A}$ & N/A & N/A & N/A & N/A & N/A & N/A & N/A \\
\hline N/A & $N / A$ & N/A & N/A & $\mathrm{N} / \mathrm{A}$ & N/A & N/A & N/A & N/A \\
\hline N/A & $N / A$ & N/A & N/A & $\mathrm{N} / \mathrm{A}$ & N/A & N/A & N/A & N/A \\
\hline N/A & $N / A$ & N/A & N/A & N/A & N/A & N/A & N/A & N/A \\
\hline N/A & $\mathrm{N} / \mathrm{A}$ & $\mathrm{N} / \mathrm{A}$ & N/A & N/A & N/A & $\mathrm{N} / \mathrm{A}$ & $\mathrm{N} / \mathrm{A}$ & N/A \\
\hline N/A & $N / A$ & N/A & $\mathrm{N} / \mathrm{A}$ & $\mathrm{N} / \mathrm{A}$ & $\mathrm{N} / \mathrm{A}$ & N/A & N/A & $\mathrm{N} / \mathrm{A}$ \\
\hline N/A & $\mathrm{N} / \mathrm{A}$ & $\mathrm{N} / \mathrm{A}$ & N/A & N/A & N/A & N/A & N/A & N/A \\
\hline $\mathrm{N} / \mathrm{A}$ & $N / A$ & N/A & N/A & N/A & N/A & N/A & N/A & N/A \\
\hline N/A & $N / A$ & N/A & $\mathrm{N} / \mathrm{A}$ & N/A & N/A & N/A & N/A & N/A \\
\hline
\end{tabular}




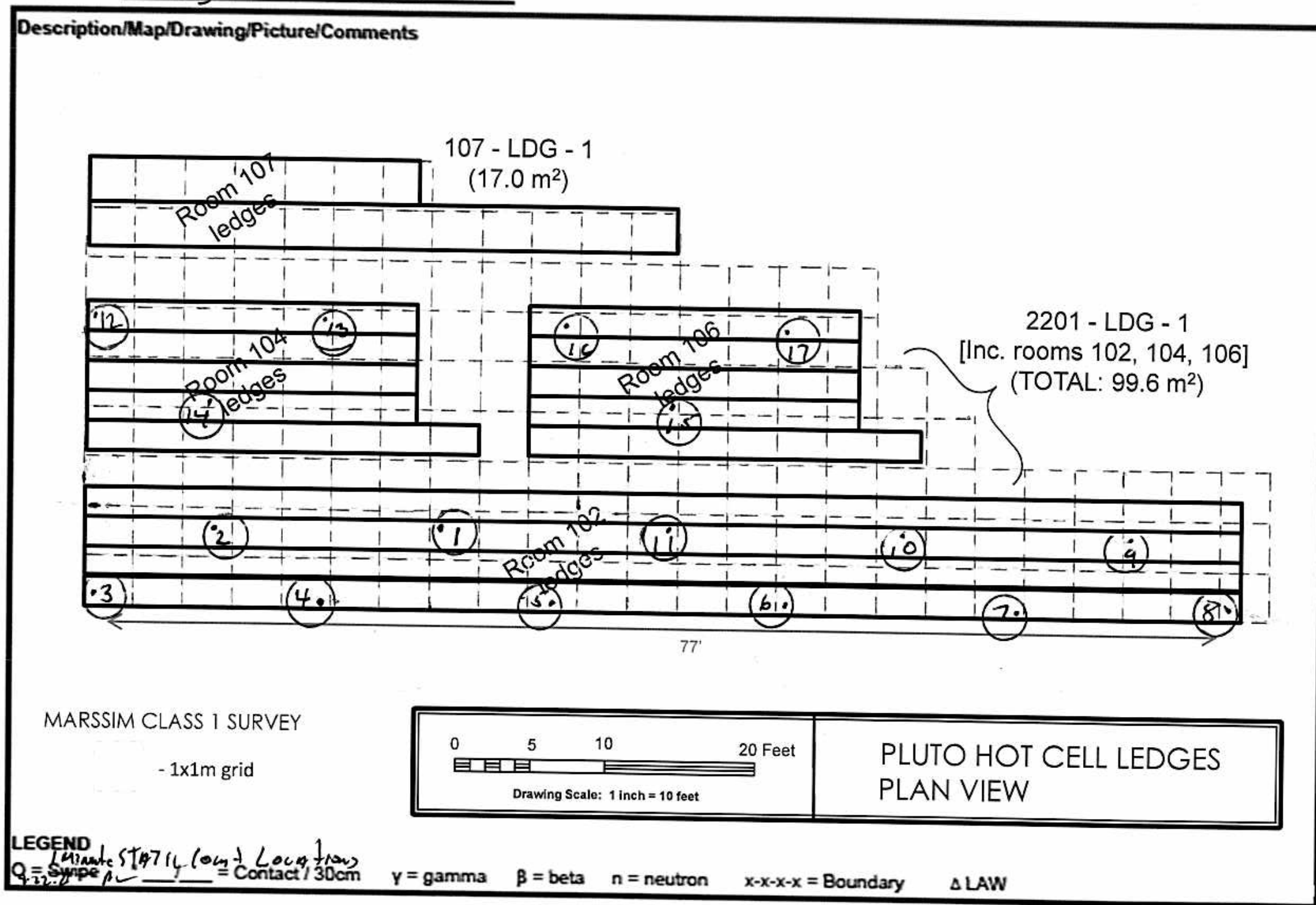


NSTec

Form

FRM-0108B

RADIOLOGICAL SURVEY REPORT - DATA

SURVEY \# 10-ER-A26-278

\begin{tabular}{|c|c|c|c|c|c|c|c|c|c|c|c|}
\hline \multicolumn{3}{|c|}{$\begin{array}{l}\text { Location: } \\
\text { Area } 26 \text { CAU117 Pluto }\end{array}$} & \multicolumn{2}{|c|}{$\begin{array}{l}\text { Purpose: } \\
\text { Final Status Survey, Room } 102 \\
\text { Ceiling } 102-C-1\end{array}$} & \multicolumn{5}{|c|}{$\begin{array}{l}\text { Comments: } \\
\text { All one minute static counts are in cpm. Bkgd not subtracted for } \\
\text { Final Status Survey, Survey Plan \#10-015 }\end{array}$} & \multicolumn{2}{|c|}{$\begin{array}{l}\text { Date/Time: } \\
\text { 08/19/2010 } 1000\end{array}$} \\
\hline Instrument: & Serial \#: & Cal Due: & $\begin{array}{c}\text { Eff in \%: } \\
\text { Alpha / Beta } \\
\end{array}$ & $\begin{array}{l}\text { BKG in dpm: } \\
\text { Alpha / Beta }\end{array}$ & \begin{tabular}{|l|} 
MDA in dpm: \\
Alpha / Beta
\end{tabular} & RWP\# & \multicolumn{5}{|l|}{$\mathrm{N} / \mathrm{A}$} \\
\hline Electra & 1201 & $4 / 6 / 2011$ & $14.4 / 20.3$ & $\mathrm{~N} / \mathrm{A}$ & $N / A$ & \multicolumn{4}{|c|}{ Work Package \# } & ESURVEY & \\
\hline Electra & 1120 & $4 / 6 / 2011$ & $14.1 / 20.8$ & $\mathrm{~N} / \mathrm{A}$ & $\mathrm{N} / \mathrm{A}$ & \multicolumn{3}{|c|}{ RCT Name: D.Cotroneo Jr. } & \multicolumn{3}{|c|}{ Signature: $\theta-\mathrm{C}$} \\
\hline Electra & $N / A$ & $N / A$ & $\mathrm{~N} / \mathrm{A}$ & $\mathrm{N} / \mathrm{A}$ & $\mathrm{N} / \mathrm{A}$ & \multicolumn{3}{|c|}{ RCT Name: K. Wilcox/M.Payne } & \multirow{3}{*}{\multicolumn{3}{|c|}{ 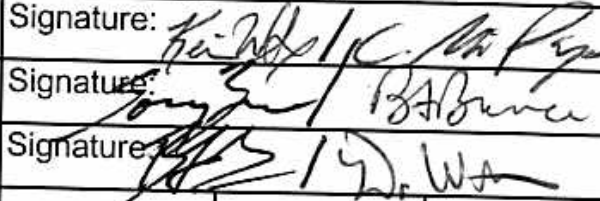 }} \\
\hline Electra & $N / A$ & $N / A$ & $\mathrm{~N} / \mathrm{A}$ & $\mathrm{N} / \mathrm{A}$ & $N / A$ & RCT Name: & S.Brown/E & Bunce & & & \\
\hline Electra & $N / A$ & $\mathrm{~N} / \mathrm{A}$ & $N / A$ & $N / A$ & $N / A$ & RCT Name: & B. Fosket & Nelch & & & \\
\hline \multirow[t]{2}{*}{ Survey Point } & \multirow{2}{*}{\multicolumn{4}{|c|}{ Description/Comments }} & \multicolumn{2}{|c|}{$\begin{array}{l}\text { Removable } \\
\mathrm{dpm} / 100 \mathrm{~cm}^{2}\end{array}$} & \multicolumn{2}{|c|}{\begin{tabular}{|c|}
$\begin{array}{c}\text { Fixed + Removable } \\
\mathrm{dpm} / 100 \mathrm{~cm}^{2}\end{array}$ \\
\end{tabular}} & \multirow{2}{*}{$\begin{array}{l}\text { Gamma } \\
\mathrm{mrem} / \mathrm{hr}\end{array}$} & \multirow{2}{*}{$\begin{array}{l}\text { Neutron } \\
\mathrm{mrem} / \mathrm{hr}\end{array}$} & \multirow{2}{*}{$\begin{array}{l}\text { Total } \\
\mathrm{mrem} / \mathrm{hr}\end{array}$} \\
\hline & & & & & Alpha & Beta & Alpha & Beta & & & \\
\hline 1 & \multicolumn{4}{|c|}{ Ceiling, Concrete (Electra 1201) } & $\mathrm{N} / \mathrm{A}$ & $N / A$ & 4 & 1006 & $N / A$ & $\mathrm{~N} / \mathrm{A}$ & $N / A$ \\
\hline 2 & \multicolumn{4}{|c|}{ Ceiling, Concrete (Electra 1201) } & $N / A$ & $N / A$ & 5 & 1044 & $N / A$ & $N / A$ & $N / A$ \\
\hline 3 & \multicolumn{4}{|c|}{ Ceiling, Concrete (Electra 1201) } & $N / A$ & $N / A$ & 12 & 1112 & $N / A$ & $N / A$ & $\mathrm{~N} / \mathrm{A}$ \\
\hline 4 & \multicolumn{4}{|c|}{ Ceiling, Concrete (Electra 1201) } & $N / A$ & $N / A$ & 8 & 993 & $N / A$ & $N / A$ & $\mathrm{~N} / \mathrm{A}$ \\
\hline 5 & \multicolumn{4}{|c|}{ Ceiling, Concrete (Electra 1201) } & $N / A$ & $\mathrm{~N} / \mathrm{A}$ & 5 & 1065 & $N / A$ & $\mathrm{~N} / \mathrm{A}$ & $\mathrm{N} / \mathrm{A}$ \\
\hline 6 & \multicolumn{4}{|c|}{ Ceiling, Concrete (Electra 1120) } & $N / A$ & $N / A$ & 7 & 1238 & $N / A$ & $N / A$ & $N / A$ \\
\hline 7 & \multicolumn{4}{|c|}{ Ceiling, Concrete (Electra 1120) } & $\mathrm{N} / \mathrm{A}$ & $N / A$ & 8 & 1097 & $N / A$ & $\mathrm{~N} / \mathrm{A}$ & $N / A$ \\
\hline 8 & \multicolumn{4}{|c|}{ Ceiling, Concrete (Electra 1120) } & $N / A$ & $N / A$ & 10 & 1122 & $N / A$ & $N / A$ & $N / A$ \\
\hline 9 & \multicolumn{4}{|c|}{ Ceiling, Concrete (Electra 1120) } & $\mathrm{N} / \mathrm{A}$ & $N / A$ & 8 & 1202 & $N / A$ & $N / A$ & $N / A$ \\
\hline 10 & \multicolumn{4}{|c|}{ Ceiling, Concrete (Electra 1120) } & $N / A$ & $\mathrm{~N} / \mathrm{A}$ & 13 & 1143 & $N / A$ & $N / A$ & $N / A$ \\
\hline 11 & \multicolumn{4}{|c|}{ Ceiling, Concrete (Electra 1120) } & $N / A$ & $N / A$ & 9 & 1223 & $N / A$ & $N / A$ & $\mathrm{~N} / \mathrm{A}$ \\
\hline \multicolumn{5}{|c|}{ Reviewed By (Print) } & Signature: & & & & & $\begin{array}{r}\text { Date: } \\
8 \%\end{array}$ & \\
\hline
\end{tabular}


SURVEY \# 10-ER-A26-278

\begin{tabular}{|c|c|c|c|c|c|c|c|c|}
\hline \multirow[t]{2}{*}{ Survey Point } & \multirow[t]{2}{*}{ Description/Comments } & \multicolumn{2}{|c|}{$\begin{array}{l}\text { Removable } \\
\mathrm{dpm} / 100 \mathrm{~cm}^{2}\end{array}$} & \multicolumn{2}{|c|}{$\begin{array}{c}\text { Fixed + Removable } \\
\mathrm{dpm} / 100 \mathrm{~cm}^{2}\end{array}$} & \multirow{2}{*}{$\begin{array}{l}\text { Gamma } \\
\mathrm{mrem} / \mathrm{hr}\end{array}$} & \multirow{2}{*}{$\begin{array}{l}\text { Neutron } \\
\mathrm{mrem} / \mathrm{hr}\end{array}$} & \multirow{2}{*}{$\begin{array}{c}\text { Total } \\
\text { mrem } / \mathrm{hr}\end{array}$} \\
\hline & & Alpha & Beta & Alpha & Beta & & & \\
\hline 12 & Ceiling, Concrete (1120) & N/A & $\mathrm{N} / \mathrm{A}$ & 9 & 1238 & N/A & N/A & N/A \\
\hline N/A & $N / A$ & N/A & N/A & N/A & N/A & N/A & N/A & N/A \\
\hline N/A & **Performed scan surveys for both alpha and beta** & N/A & N/A & N/A & N/A & $\mathrm{N} / \mathrm{A}$ & N/A & N/A \\
\hline N/A & ${ }^{* *}$ on $10 \%$ of survey unit. No trigger levels exceeded ${ }^{\star *}$ & N/A & N/A & N/A & N/A & N/A & N/A & N/A \\
\hline $\mathrm{N} / \mathrm{A}$ & $\mathrm{N} / \mathrm{A}$ & N/A & N/A & N/A & N/A & N/A & N/A & N/A \\
\hline $\mathrm{N} / \mathrm{A}$ & $N / A$ & N/A & N/A & $\mathrm{N} / \mathrm{A}$ & N/A & N/A & N/A & N/A \\
\hline $\mathrm{N} / \mathrm{A}$ & $N / A$ & N/A & N/A & N/A & N/A & N/A & $\mathrm{N} / \mathrm{A}$ & N/A \\
\hline N/A & $N / A$ & N/A & $\mathrm{N} / \mathrm{A}$ & N/A & N/A & N/A & N/A & N/A \\
\hline N/A & $N / A$ & N/A & $\mathrm{N} / \mathrm{A}$ & N/A & N/A & N/A & N/A & N/A \\
\hline N/A & $N / A$ & N/A & $\mathrm{N} / \mathrm{A}$ & N/A & N/A & N/A & N/A & N/A \\
\hline N/A & $N / A$ & N/A & $\mathrm{N} / \mathrm{A}$ & N/A & N/A & N/A & N/A & $\mathrm{N} / \mathrm{A}$ \\
\hline N/A & $N / A$ & N/A & $\mathrm{N} / \mathrm{A}$ & N/A & N/A & N/A & N/A & N/A \\
\hline N/A & $N / A$ & $\mathrm{~N} / \mathrm{A}$ & N/A & $\mathrm{N} / \mathrm{A}$ & N/A & N/A & N/A & N/A \\
\hline $\mathrm{N} / \mathrm{A}$ & $\mathrm{N} / \mathrm{A}$ & N/A & N/A & N/A & N/A & $\mathrm{N} / \mathrm{A}$ & N/A & N/A \\
\hline $\mathrm{N} / \mathrm{A}$ & $\mathrm{N} / \mathrm{A}$ & N/A & $\mathrm{N} / \mathrm{A}$ & N/A & N/A & N/A & N/A & N/A \\
\hline $\mathrm{N} / \mathrm{A}$ & $N / A$ & $\mathrm{~N} / \mathrm{A}$ & N/A & $\mathrm{N} / \mathrm{A}$ & N/A & N/A & N/A & N/A \\
\hline N/A & $N / A$ & N/A & N/A & $\mathrm{N} / \mathrm{A}$ & N/A & N/A & N/A & N/A \\
\hline N/A & $\mathrm{N} / \mathrm{A}$ & N/A & N/A & N/A & N/A & $\mathrm{N} / \mathrm{A}$ & N/A & N/A \\
\hline N/A & $\mathrm{N} / \mathrm{A}$ & N/A & N/A & $\mathrm{N} / \mathrm{A}$ & N/A & $\mathrm{N} / \mathrm{A}$ & N/A & N/A \\
\hline $\mathrm{N} / \mathrm{A}$ & $N / A$ & $\mathrm{~N} / \mathrm{A}$ & $\mathrm{N} / \mathrm{A}$ & N/A & $\mathrm{N} / \mathrm{A}$ & N/A & N/A & N/A \\
\hline N/A & $\mathrm{N} / \mathrm{A}$ & $\mathrm{N} / \mathrm{A}$ & $\mathrm{N} / \mathrm{A}$ & N/A & N/A & N/A & N/A & N/A \\
\hline N/A & $N / A$ & N/A & N/A & N/A & N/A & N/A & N/A & N/A \\
\hline N/A & $N / A$ & N/A & $\mathrm{N} / \mathrm{A}$ & $\mathrm{N} / \mathrm{A}$ & N/A & N/A & N/A & N/A \\
\hline
\end{tabular}




\section{NSTec}

Form

FRM-0108C

RADIOLOGICAL SURVEY REPORT - FULL MAP

05/24/10

Rev. 01

Survey $10-E R-A 26-278$

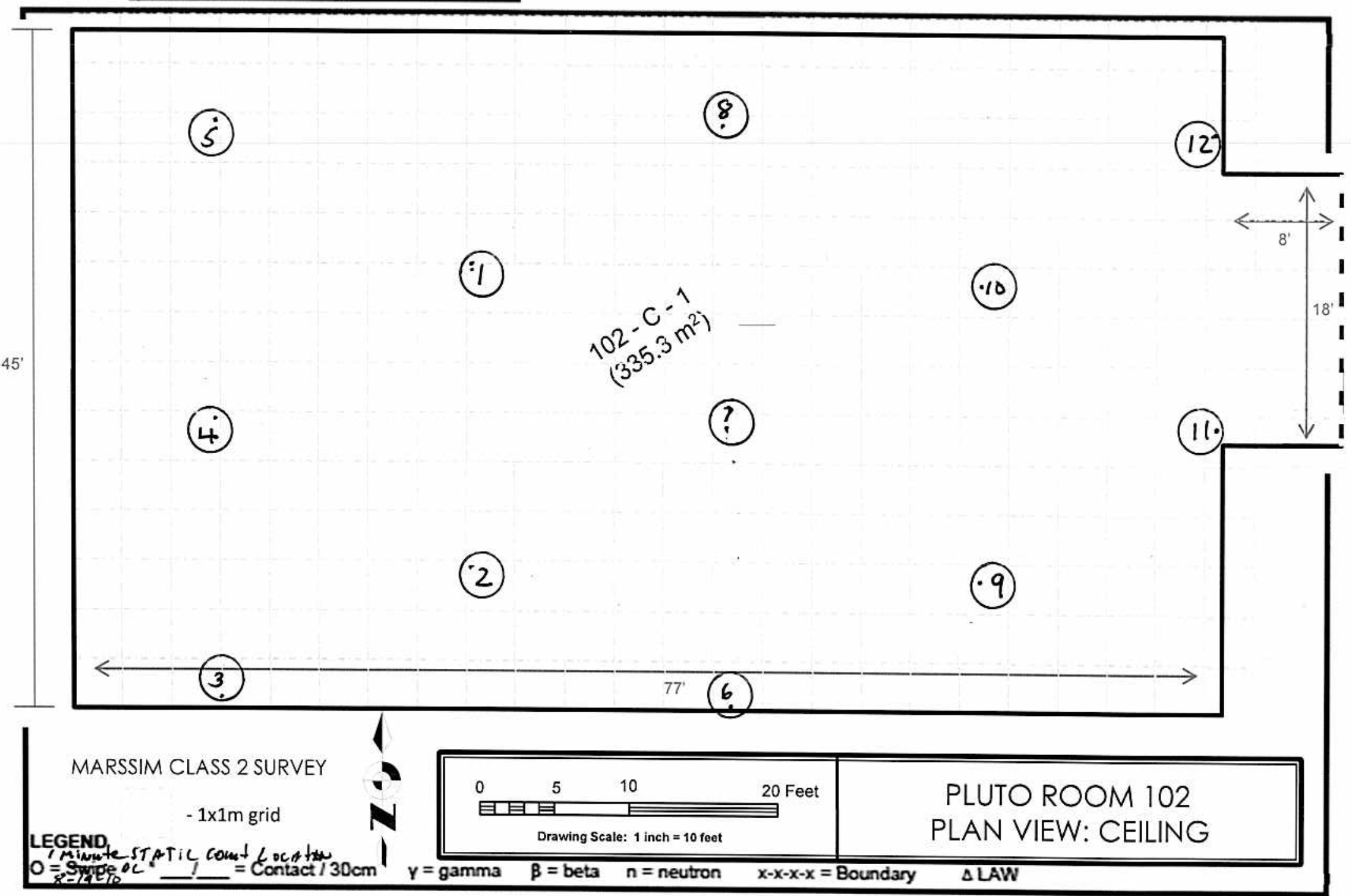


SURVEY \#

10-ER-A26-226

\begin{tabular}{|c|c|c|c|c|c|c|c|c|c|c|c|}
\hline \multicolumn{3}{|c|}{$\begin{array}{l}\text { Location: } \\
\text { Area } 26 \text { CAU117 Pluto }\end{array}$} & \multicolumn{2}{|c|}{$\begin{array}{l}\text { Purpose: } \\
\text { Final Status Survey, Room 104, } \\
\text { Ceiling, Unit \# 104-C-1 }\end{array}$} & \multicolumn{5}{|c|}{$\begin{array}{l}\text { Comments: } \\
\text { All one minute static counts are in cpm. Bkgd not subtracted for } \\
\text { Final Status Survey, Survey Plan } \# 10-015\end{array}$} & \multicolumn{2}{|c|}{$\begin{array}{l}\text { Date/Time: } \\
\text { 07/27/2010 } 1600\end{array}$} \\
\hline Instrument: & Serial \#: & Cal Due: & $\begin{array}{c}\text { Eff in \%: } \\
\text { Alpha / Beta }\end{array}$ & $\begin{array}{l}\text { BKG in dpm: } \\
\text { Alpha / Beta }\end{array}$ & \begin{tabular}{|l|} 
MDA in dpm: \\
Alpha / Beta
\end{tabular} & RWP\# & \multicolumn{5}{|l|}{$\mathrm{N} / \mathrm{A}$} \\
\hline Electra & 1120 & $4 / 6 / 2011$ & $14.1 / 20.8$ & $N / A$ & $\mathrm{~N} / \mathrm{A}$ & \multicolumn{6}{|c|}{ 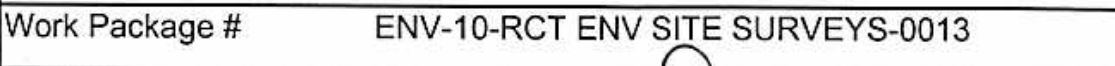 } \\
\hline$N / A$ & $\mathrm{~N} / \mathrm{A}$ & $N / A$ & $N / A$ & $N / A$ & $\mathrm{~N} / \mathrm{A}$ & \multicolumn{3}{|c|}{ RCT Name: D.Cotroneo Jr. } & Signature: & \multicolumn{2}{|c|}{ एक } \\
\hline $\mathrm{N} / \mathrm{A}$ & $\mathrm{N} / \mathrm{A}$ & $\mathrm{N} / \mathrm{A}$ & $\mathrm{N} / \mathrm{A}$ & $\mathrm{N} / \mathrm{A}$ & $\mathrm{N} / \mathrm{A}$ & \multicolumn{3}{|c|}{ RCT Name: Kevin Wilcox } & \multicolumn{3}{|c|}{ Signature: Kein W-Qcos } \\
\hline$N / A$ & $\mathrm{~N} / \mathrm{A}$ & $N / A$ & $N / A$ & $\mathrm{~N} / \mathrm{A}$ & $N / A$ & \multicolumn{3}{|c|}{ RCT Name: S. Brown/B. Bunce } & \multirow{2}{*}{\multicolumn{3}{|c|}{ Signaturesom 3 ignature: }} \\
\hline$N / A$ & $N / A$ & $N / A$ & $\mathrm{~N} / \mathrm{A}$ & $N / A$ & $N / A$ & RCT Name: & Brian Fost & & & & \\
\hline \multirow[t]{2}{*}{ Survey Point } & \multirow{2}{*}{\multicolumn{4}{|c|}{ Description/Comments }} & \multicolumn{2}{|c|}{$\begin{array}{l}\text { Removable } \\
\mathrm{dpm} / 100 \mathrm{~cm}^{2}\end{array}$} & \multicolumn{2}{|c|}{\begin{tabular}{|c|}
$\begin{array}{c}\text { Fixed + Removable } \\
\mathrm{dpm} / 100 \mathrm{~cm}^{2}\end{array}$ \\
\end{tabular}} & \multirow{2}{*}{$\begin{array}{l}\text { Gamma } \\
\mathrm{mrem} / \mathrm{hr}\end{array}$} & \multirow{2}{*}{$\begin{array}{l}\text { Neutron } \\
\mathrm{mrem} / \mathrm{hr}\end{array}$} & \multirow{2}{*}{$\begin{array}{l}\text { Total } \\
\mathrm{mrem} / \mathrm{hr}\end{array}$} \\
\hline & & & & & Alpha & Beta & Alpha & Beta & & & \\
\hline 1 & \multicolumn{4}{|c|}{ Ceiling, Concrete } & $N / A$ & $N / A$ & 7 & 1235 & $N / A$ & $N / A$ & $N / A$ \\
\hline 2 & \multicolumn{4}{|c|}{ Ceiling, Concrete } & $N / A$ & $N / A$ & 13 & 1142 & $N / A$ & $N / A$ & $N / A$ \\
\hline 3 & \multicolumn{4}{|c|}{ Ceiling, Concrete } & $N / A$ & $\mathrm{~N} / \mathrm{A}$ & 10 & 1117 & $N / A$ & $N / A$ & $N / A$ \\
\hline 4 & \multicolumn{4}{|c|}{ Ceiling, Concrete } & $N / A$ & $N / A$ & 7 & 1057 & $N / A$ & $\mathrm{~N} / \mathrm{A}$ & $N / A$ \\
\hline 5 & \multicolumn{4}{|c|}{ Ceiling, Concrete } & $N / A$ & $N / A$ & 9 & 1127 & $N / A$ & $N / A$ & $N / A$ \\
\hline 6 & \multicolumn{4}{|c|}{ Ceiling, Concrete } & $N / A$ & $\mathrm{~N} / \mathrm{A}$ & 7 & 1125 & $N / A$ & $N / A$ & $N / A$ \\
\hline 7 & \multicolumn{4}{|c|}{ Ceiling, Concrete } & $N / A$ & $N / A$ & 7 & 1173 & $\mathrm{~N} / \mathrm{A}$ & $N / A$ & $N / A$ \\
\hline 8 & \multicolumn{4}{|c|}{ Ceiling, Concrete } & $\mathrm{N} / \mathrm{A}$ & $\mathrm{N} / \mathrm{A}$ & 7 & 1128 & $N / A$ & $N / A$ & $N / A$ \\
\hline 9 & \multicolumn{4}{|c|}{ Ceiling, Concrete } & $N / A$ & $N / A$ & 9 & 984 & $N / A$ & $N / A$ & $N / A$ \\
\hline 10 & \multicolumn{4}{|c|}{ Ceiling, Concrete } & $N / A$ & $\mathrm{~N} / \mathrm{A}$ & 9 & 1092 & $N / A$ & $\mathrm{~N} / \mathrm{A}$ & $N / A$ \\
\hline 11 & \multicolumn{4}{|c|}{ Ceiling, Concrete } & $N / A$ & $N / A$ & 3 & 1128 & $N / A$ & $N / A$ & $N / A$ \\
\hline \multicolumn{5}{|c|}{ Reviewed By (Print): } & \multicolumn{7}{|c|}{ Signature: } \\
\hline
\end{tabular}


SURVEY \# 10-ER-A26-226

\begin{tabular}{|c|c|c|c|c|c|c|c|c|}
\hline \multirow[t]{2}{*}{ Survey Point } & \multirow[t]{2}{*}{ Description/Comments } & \multicolumn{2}{|c|}{$\begin{array}{l}\text { Removable } \\
\mathrm{dpm} / 100 \mathrm{~cm}^{2}\end{array}$} & \multicolumn{2}{|c|}{$\begin{array}{c}\text { Fixed + Removable } \\
\mathrm{dpm} / 100 \mathrm{~cm}^{2}\end{array}$} & \multirow{2}{*}{$\begin{array}{l}\text { Gamma } \\
\text { mrem/hr }\end{array}$} & \multirow{2}{*}{$\begin{array}{l}\text { Neutron } \\
\mathrm{mrem} / \mathrm{hr}\end{array}$} & \multirow{2}{*}{$\begin{array}{c}\text { Total } \\
\mathrm{mrem} / \mathrm{hr}\end{array}$} \\
\hline & & Alpha & Beta & Alpha & Beta & & & \\
\hline 12 & Ceiling, Concrete & $\mathrm{N} / \mathrm{A}$ & $\mathrm{N} / \mathrm{A}$ & 10 & 1186 & N/A & N/A & N/A \\
\hline 13 & Ceiling, Concrete & N/A & N/A & 6 & 1224 & N/A & N/A & N/A \\
\hline 14 & Ceiling, Concrete & N/A & N/A & 11 & 728 & N/A & N/A & N/A \\
\hline 15 & Ceiling, Concrete & N/A & N/A & 3 & 1070 & N/A & N/A & N/A \\
\hline 16 & Ceiling, Concrete & $\mathrm{N} / \mathrm{A}$ & N/A & 7 & 1162 & N/A & N/A & N/A \\
\hline 17 & Ceiling, Concrete & $\mathrm{N} / \mathrm{A}$ & N/A & 3 & 798 & $\mathrm{~N} / \mathrm{A}$ & N/A & N/A \\
\hline 18 & $N / A$ & N/A & $\mathrm{N} / \mathrm{A}$ & N/A & $\mathrm{N} / \mathrm{A}$ & N/A & N/A & N/A \\
\hline N/A & $N / A$ & N/A & N/A & N/A & $\mathrm{N} / \mathrm{A}$ & N/A & N/A & N/A \\
\hline N/A & **Performed scan surveys for both alpha and beta** & $\mathrm{N} / \mathrm{A}$ & $\mathrm{N} / \mathrm{A}$ & N/A & $\mathrm{N} / \mathrm{A}$ & $\mathrm{N} / \mathrm{A}$ & N/A & N/A \\
\hline N/A & ${ }^{\star \star}$ on $10 \%$ of survey unit. No trigger levels exceeded ${ }^{\star \star}$ & N/A & N/A & N/A & N/A & N/A & N/A & $\mathrm{N} / \mathrm{A}$ \\
\hline N/A & $N / A$ & $\mathrm{~N} / \mathrm{A}$ & N/A & N/A & $\mathrm{N} / \mathrm{A}$ & N/A & N/A & N/A \\
\hline N/A & $\mathrm{N} / \mathrm{A}$ & N/A & N/A & N/A & N/A & N/A & $\mathrm{N} / \mathrm{A}$ & $\mathrm{N} / \mathrm{A}$ \\
\hline N/A & $N / A$ & N/A & $\mathrm{N} / \mathrm{A}$ & N/A & $\mathrm{N} / \mathrm{A}$ & N/A & N/A & N/A \\
\hline N/A & $N / A$ & N/A & N/A & N/A & $\mathrm{N} / \mathrm{A}$ & N/A & $\mathrm{N} / \mathrm{A}$ & N/A \\
\hline N/A & $\mathrm{N} / \mathrm{A}$ & N/A & N/A & N/A & $\mathrm{N} / \mathrm{A}$ & N/A & N/A & N/A \\
\hline N/A & $N / A$ & N/A & N/A & N/A & $\mathrm{N} / \mathrm{A}$ & N/A & $N / A$ & N/A \\
\hline N/A & $N / A$ & N/A & N/A & N/A & $\mathrm{N} / \mathrm{A}$ & N/A & N/A & N/A \\
\hline $\mathrm{N} / \mathrm{A}$ & $N / A$ & N/A & N/A & N/A & $\mathrm{N} / \mathrm{A}$ & N/A & N/A & N/A \\
\hline $\mathrm{N} / \mathrm{A}$ & $N / A$ & N/A & N/A & N/A & $\mathrm{N} / \mathrm{A}$ & N/A & N/A & N/A \\
\hline $\mathrm{N} / \mathrm{A}$ & $\mathrm{N} / \mathrm{A}$ & $\mathrm{N} / \mathrm{A}$ & N/A & N/A & $\mathrm{N} / \mathrm{A}$ & N/A & N/A & N/A \\
\hline N/A & $N / A$ & N/A & N/A & N/A & $\mathrm{N} / \mathrm{A}$ & N/A & N/A & N/A \\
\hline N/A & $N / A$ & N/A & $\mathrm{N} / \mathrm{A}$ & N/A & $\mathrm{N} / \mathrm{A}$ & N/A & N/A & $\mathrm{N} / \mathrm{A}$ \\
\hline $\mathrm{N} / \mathrm{A}$ & $N / A$ & N/A & N/A & N/A & $\mathrm{N} / \mathrm{A}$ & $N / A$ & $\mathrm{~N} / \mathrm{A}$ & $\mathrm{N} / \mathrm{A}$ \\
\hline
\end{tabular}


Survey $=10-E R-A 26-226$

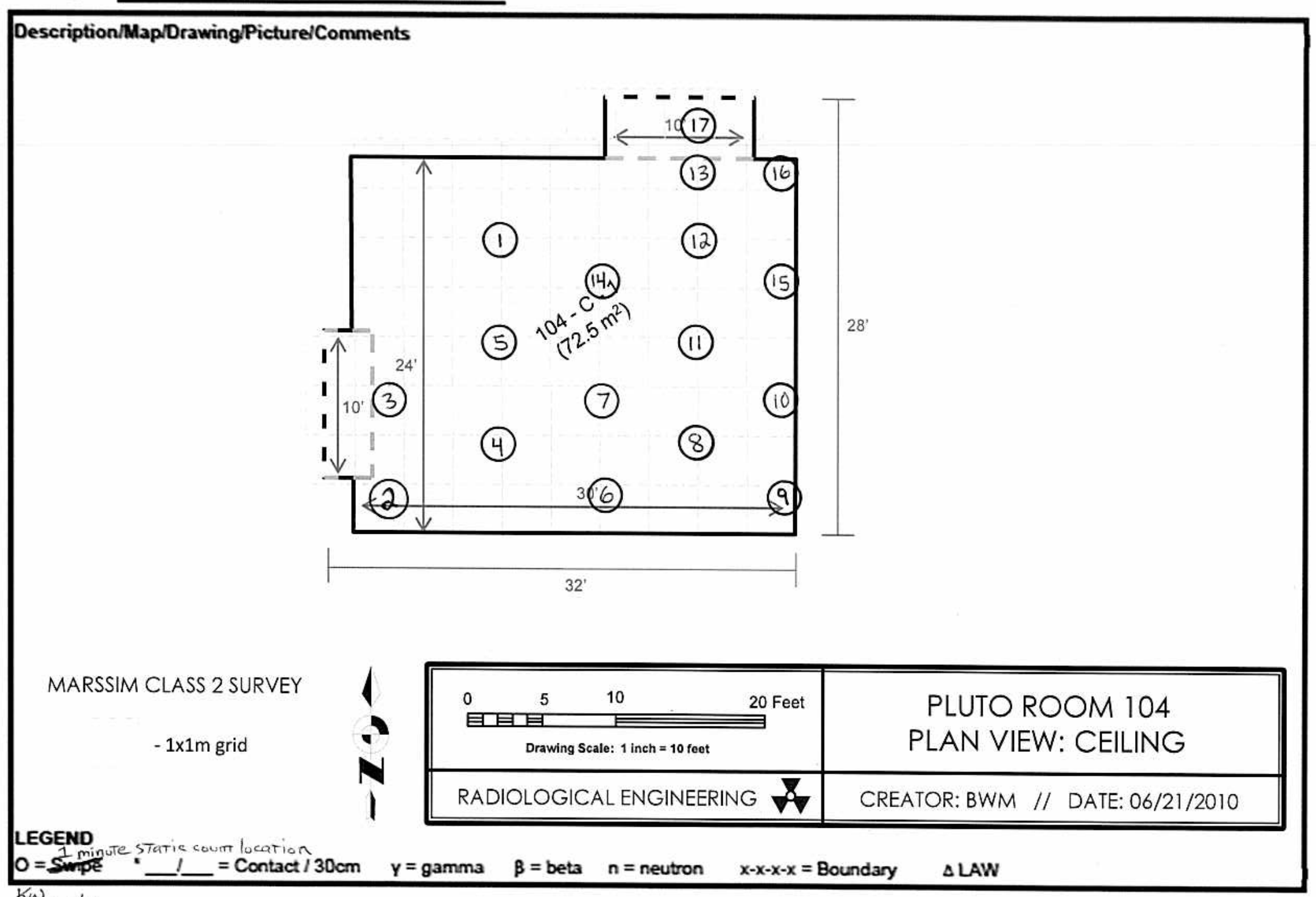




\section{NSTec}

Form

FRM-0108B

RADIOLOGICAL SURVEY REPORT - DATA

SURVEY \# 10-ER-A26-297

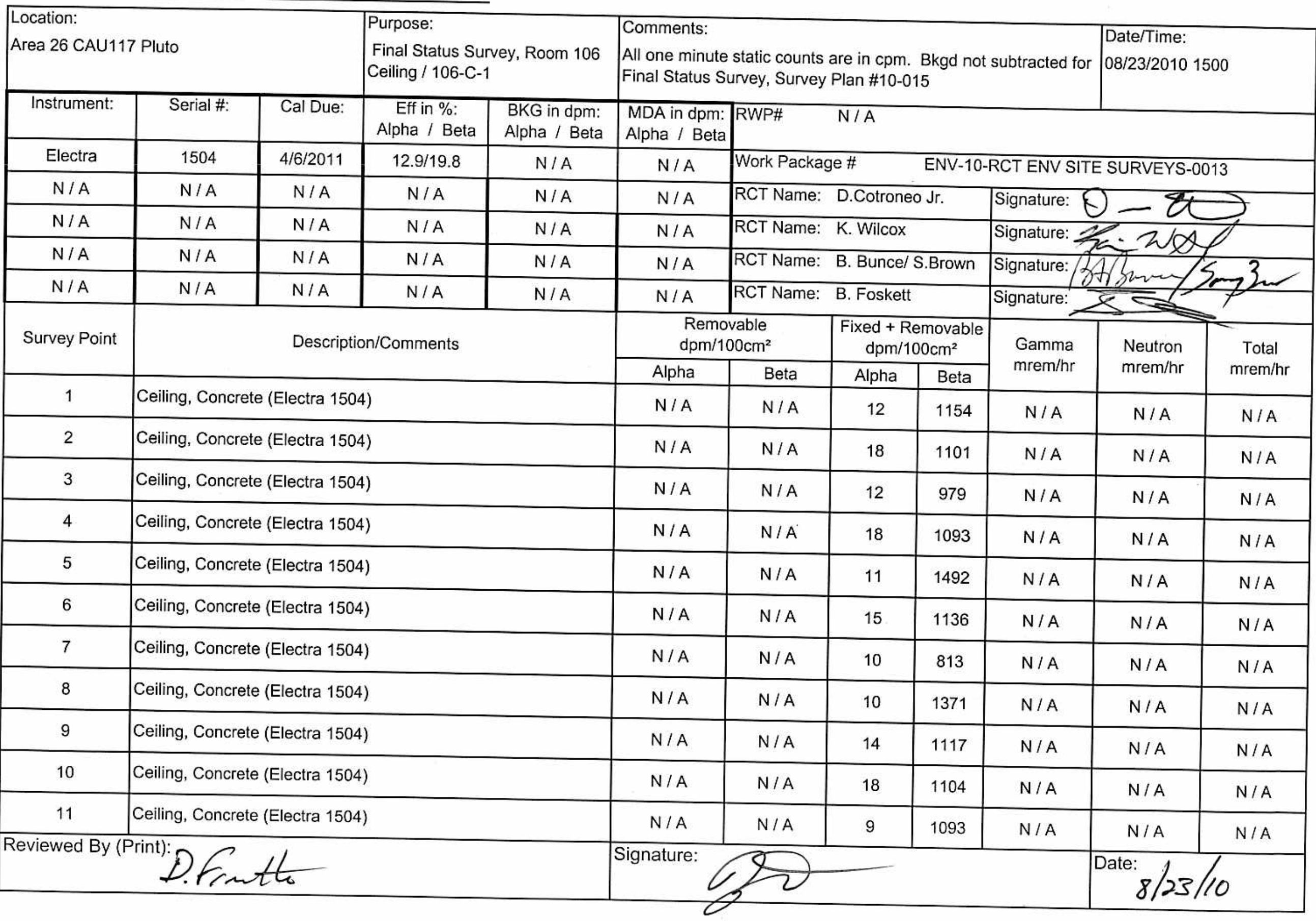


SURVEY \# 10-ER-A26-297

\begin{tabular}{|c|c|c|c|c|c|c|c|c|}
\hline \multirow[t]{2}{*}{ Survey Point } & \multirow[t]{2}{*}{ Description/Comments } & \multicolumn{2}{|c|}{$\begin{array}{l}\text { Removable } \\
\mathrm{dpm} / 100 \mathrm{~cm}^{2}\end{array}$} & \multicolumn{2}{|c|}{$\begin{array}{c}\text { Fixed + Removable } \\
\mathrm{dpm} / 100 \mathrm{~cm}^{2}\end{array}$} & \multirow{2}{*}{$\begin{array}{l}\text { Gamma } \\
\mathrm{mrem} / \mathrm{hr}\end{array}$} & \multirow{2}{*}{$\begin{array}{l}\text { Neutron } \\
\text { mrem/hr }\end{array}$} & \multirow{2}{*}{$\begin{array}{c}\text { Total } \\
\mathrm{mrem} / \mathrm{hr}\end{array}$} \\
\hline & & Alpha & Beta & Alpha & Beta & & & \\
\hline 12 & Ceiling, Concrete (1504) & N/A & N/A & 15 & 1114 & N/A & N/A & N/A \\
\hline 13 & Ceiling, Concrete (1504) & N/A & N/A & 17 & 1186 & N/A & N/A & N/A \\
\hline N/A & ${ }^{\star \star}$ Performed scan surveys for both alpha and beta** & N/A & N/A & N/A & N/A & N/A & N/A & N/A \\
\hline N/A & ${ }^{\star \star}$ on $100 \%$ of survey unit. No trigger levels exceeded ${ }^{\star \star}$ & N/A & N/A & N/A & N/A & N/A & N/A & N/A \\
\hline N/A & $\mathrm{N} / \mathrm{A}$ & N/A & N/A & N/A & N/A & N/A & N/A & N/A \\
\hline $\mathrm{N} / \mathrm{A}$ & $N / A$ & N/A & N/A & N/A & $\mathrm{N} / \mathrm{A}$ & N/A & N/A & N/A \\
\hline N/A & $N / A$ & N/A & N/A & N/A & N/A & N/A & N/A & N/A \\
\hline N/A & $\mathrm{N} / \mathrm{A}$ & N/A & N/A & N/A & N/A & N/A & N/A & N/A \\
\hline N/A & $\mathrm{N} / \mathrm{A}$ & N/A & N/A & N/A & N/A & N/A & N/A & $\mathrm{N} / \mathrm{A}$ \\
\hline N/A & N/A & N/A & N/A & N/A & N/A & N/A & N/A & N/A \\
\hline N/A & $\mathrm{N} / \mathrm{A}$ & N/A & N/A & N/A & N/A & N/A & N/A & N/A \\
\hline N/A & $\mathrm{N} / \mathrm{A}$ & N/A & N/A & N/A & $\mathrm{N} / \mathrm{A}$ & N/A & N/A & N/A \\
\hline N/A & $\mathrm{N} / \mathrm{A}$ & N/A & N/A & N/A & N/A & N/A & N/A & N/A \\
\hline N/A & $\mathrm{N} / \mathrm{A}$ & N/A & N/A & N/A & N/A & N/A & N/A & N/A \\
\hline N/A & $\mathrm{N} / \mathrm{A}$ & N/A & N/A & N/A & N/A & N/A & N/A & N/A \\
\hline N/A & $\mathrm{N} / \mathrm{A}$ & N/A & N/A & N/A & N/A & N/A & N/A & N/A \\
\hline N/A & $\mathrm{N} / \mathrm{A}$ & N/A & N/A & N/A & N/A & N/A & N/A & N/A \\
\hline N/A & $\mathrm{N} / \mathrm{A}$ & N/A & $\mathrm{N} / \mathrm{A}$ & N/A & $\mathrm{N} / \mathrm{A}$ & N/A & N/A & N/A \\
\hline N/A & $\mathrm{N} / \mathrm{A}$ & N/A & N/A & N/A & N/A & N/A & N/A & N/A \\
\hline N/A & $N / A$ & N/A & N/A & N/A & N/A & N/A & N/A & N/A \\
\hline N/A & $\mathrm{N} / \mathrm{A}$ & N/A & N/A & N/A & N/A & N/A & N/A & N/A \\
\hline N/A & $\mathrm{N} / \mathrm{A}$ & N/A & N/A & N/A & N/A & N/A & N/A & N/A \\
\hline N/A & $N / A$ & N/A & N/A & N/A & N/A & N/A & N/A & N/A \\
\hline
\end{tabular}


Survey $\# 10-E R-A 26-297$

Description/Map/Drawing/Picture/Comments

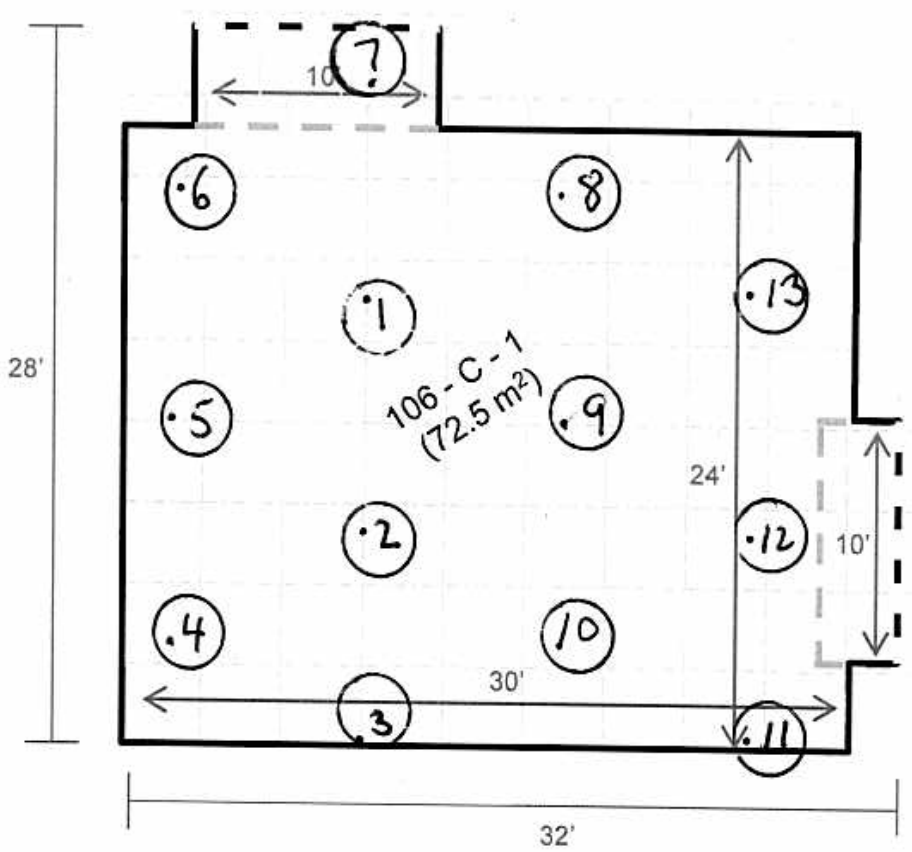

MARSSIM CLASS 2 SURVEY

$-1 \times 1 m$ grid

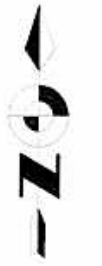

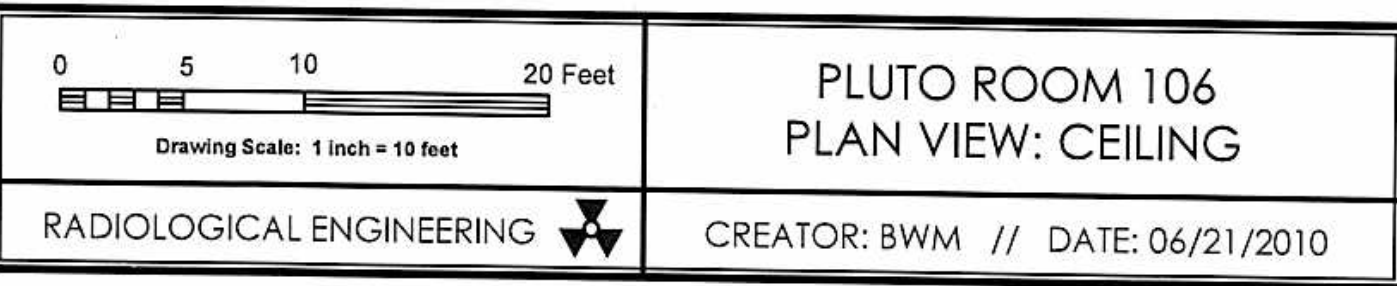

LEGEND 


\section{NSTec}

SURVEY \#

10-ER-A26-296

\begin{tabular}{|c|c|c|c|c|c|c|c|c|c|c|c|}
\hline \multicolumn{3}{|c|}{$\begin{array}{l}\text { Location: } \\
\text { Area } 26 \text { CAU117 Pluto }\end{array}$} & \multicolumn{2}{|c|}{$\begin{array}{l}\text { Purpose: } \\
\text { Final Status Survey, Room } 107 \\
\text { Ceiling / 107-C-1 }\end{array}$} & \multicolumn{5}{|c|}{$\begin{array}{l}\text { Comments: } \\
\text { All one minute static counts are in cpm. Bkgd not subtracted for } \\
\text { Final Status Survey, Survey Plan \#10-015 }\end{array}$} & \multicolumn{2}{|c|}{$\begin{array}{l}\text { Date/Time: } \\
\text { 08/23/2010 } 1000\end{array}$} \\
\hline Instrument: & Serial \#: & Cal Due: & $\begin{array}{c}\text { Eff in \%: } \\
\text { Alpha / Beta }\end{array}$ & $\begin{array}{l}\text { BKG in dpm: } \\
\text { Alpha / Beta }\end{array}$ & $\begin{array}{l}\text { MDA in dpm: } \\
\text { Alpha / Beta }\end{array}$ & RWP\# & \multicolumn{5}{|l|}{$\mathrm{N} / \mathrm{A}$} \\
\hline Electra & 1504 & $4 / 6 / 2011$ & $12.9 / 19.8$ & $N / A$ & $\mathrm{~N} / \mathrm{A}$ & \multicolumn{6}{|c|}{$\begin{array}{ll}\text { Work Package \# } \quad \text { ENV-10-RCT ENV SITE SURVEYS-0013 } \\
\end{array}$} \\
\hline $\mathrm{N} / \mathrm{A}$ & $\mathrm{N} / \mathrm{A}$ & $N / A$ & $\mathrm{~N} / \mathrm{A}$ & $\mathrm{N} / \mathrm{A}$ & $N / A$ & RCT Name: & \multicolumn{2}{|c|}{ D.Cotroneo Jr. } & \multicolumn{3}{|c|}{ Signature: 8} \\
\hline$N / A$ & $N / A$ & $N / A$ & $\mathrm{~N} / \mathrm{A}$ & $N / A$ & $N / A$ & RCT Name: & \multicolumn{2}{|c|}{ K. Wilcox/M.Payne } & \multicolumn{3}{|c|}{ Signature: He: W.ONol } \\
\hline$N / A$ & $N / A$ & $N / A$ & $N / A$ & $N / A$ & $N / A$ & RCT Name: & \multicolumn{2}{|c|}{ B. Bunce/ S.Brown } & \multicolumn{3}{|c|}{ Signature: Sflunce S } \\
\hline$N / A$ & $\mathrm{~N} / \mathrm{A}$ & $N / A$ & $\mathrm{~N} / \mathrm{A}$ & $N / A$ & $N / A$ & RCT Name: & B. Foskett & & Signature: & 6 & \\
\hline \multirow[t]{2}{*}{ Survey Point } & \multirow{2}{*}{\multicolumn{4}{|c|}{ Description/Comments }} & \multicolumn{2}{|c|}{$\begin{array}{l}\text { Removable } \\
\mathrm{dpm} / 100 \mathrm{~cm}^{2}\end{array}$} & \multicolumn{2}{|c|}{\begin{tabular}{|c|} 
Fixed + Removable \\
$\mathrm{dpm} / 100 \mathrm{~cm}^{2}$
\end{tabular}} & \multirow{2}{*}{$\begin{array}{l}\text { Gamma } \\
\mathrm{mrem} / \mathrm{hr}\end{array}$} & \multirow{2}{*}{$\begin{array}{l}\text { Neutron } \\
\mathrm{mrem} / \mathrm{hr}\end{array}$} & \multirow{2}{*}{$\begin{array}{l}\text { Total } \\
\mathrm{mrem} / \mathrm{hr}\end{array}$} \\
\hline & & & & & Alpha & Beta & Alpha & Beta & & & \\
\hline 1 & \multicolumn{4}{|c|}{ Ceiling, Concrete (Electra 1504) } & $N / A$ & $N / A$ & 11 & 1133 & $\mathrm{~N} / \mathrm{A}$ & $N / A$ & $N / A$ \\
\hline 2 & \multicolumn{4}{|c|}{ Ceiling, Concrete (Electra 1504) } & $N / A$ & $N / A$ & 9 & 1024 & $N / A$ & $N / A$ & $N / A$ \\
\hline 3 & \multicolumn{4}{|c|}{ Ceiling, Concrete (Electra 1504) } & $N / A$ & $N / A$ & 9 & 1105 & $N / A$ & $N / A$ & $N / A$ \\
\hline 4 & \multicolumn{4}{|c|}{ Ceiling, Concrete (Electra 1504) } & $N / A$ & $N / A$ & 9 & 1135 & $N / A$ & $N / A$ & $N / A$ \\
\hline 5 & \multicolumn{4}{|c|}{ Ceiling, Concrete (Electra 1504) } & $N / A$ & $N / A$ & 16 & 1188 & $N / A$ & $N / A$ & $N / A$ \\
\hline 6 & \multicolumn{4}{|c|}{ Ceiling, Concrete (Electra 1504) } & $N / A$ & $N / A$ & 8 & 1166 & $N / A$ & $N / A$ & $N / A$ \\
\hline 7 & \multicolumn{4}{|c|}{ Ceiling, Concrete (Electra 1504) } & $N / A$ & $\mathrm{~N} / \mathrm{A}$ & 19 & 1106 & $N / A$ & $N / A$ & $N / A$ \\
\hline 8 & \multicolumn{4}{|c|}{ Ceiling, Concrete (Electra 1504) } & $N / A$ & $N / A$ & 10 & 1076 & $N / A$ & $\mathrm{~N} / \mathrm{A}$ & $N / A$ \\
\hline 9 & \multicolumn{4}{|c|}{ Ceiling, Concrete (Electra 1504) } & $N / A$ & $N / A$ & 7 & 1180 & $N / A$ & $N / A$ & $N / A$ \\
\hline 10 & \multicolumn{4}{|c|}{ Ceiling, Concrete (Electra 1504) } & $N / A$ & $\mathrm{~N} / \mathrm{A}$ & 15 & 1075 & $N / A$ & $N / A$ & $N / A$ \\
\hline 11 & \multicolumn{4}{|c|}{ Ceiling, Concrete (Electra 1504) } & $N / A$ & $N / A$ & 16 & 1026 & $N / A$ & $N / A$ & $N / A$ \\
\hline \multicolumn{5}{|c|}{ Reviewed By (Print): } & Signature: & & & & & Date: & \\
\hline
\end{tabular}


NSTec

Form

FRM-0108B

RADIOLOGICAL SURVEY REPORT - DATA (CONTINUATION)

Rev. 0

SURVEY \# 10-ER-A26-296

Page $\leq$ of 3

\begin{tabular}{|c|c|c|c|c|c|c|c|c|}
\hline \multirow[t]{2}{*}{ Survey Point } & \multirow[t]{2}{*}{ Description/Comments } & \multicolumn{2}{|c|}{$\begin{array}{l}\text { Removable } \\
\mathrm{dpm} / 100 \mathrm{~cm}^{2}\end{array}$} & \multicolumn{2}{|c|}{$\begin{array}{c}\text { Fixed + Removable } \\
\mathrm{dpm} / 100 \mathrm{~cm}^{2}\end{array}$} & \multirow{2}{*}{$\begin{array}{l}\text { Gamma } \\
\mathrm{mrem} / \mathrm{hr}\end{array}$} & \multirow{2}{*}{$\begin{array}{l}\text { Neutron } \\
\mathrm{mrem} / \mathrm{hr}\end{array}$} & \multirow{2}{*}{$\begin{array}{c}\text { Total } \\
\mathrm{mrem} / \mathrm{hr}\end{array}$} \\
\hline & & Alpha & Beta & Alpha & Beta & & & \\
\hline 12 & Ceiling, Concrete (1504) & N/A & N/A & 19 & 1097 & N/A & N/A & N/A \\
\hline 13 & Ceiling, Concrete (1504) & N/A & $\mathrm{N} / \mathrm{A}$ & 5 & 1035 & N/A & $\mathrm{N} / \mathrm{A}$ & N/A \\
\hline 14 & Ceiling, Concrete (1504) & $\mathrm{N} / \mathrm{A}$ & N/A & 7 & 953 & N/A & N/A & N/A \\
\hline 15 & Ceiling, Concrete (1504) & N/A & $\mathrm{N} / \mathrm{A}$ & 6 & 1013 & N/A & N/A & N/A \\
\hline 16 & Ceiling, Concrete (1504) & N/A & $\mathrm{N} / \mathrm{A}$ & 6 & 1074 & N/A & N/A & N/A \\
\hline 17 & Ceiling, Concrete (1504) & $\mathrm{N} / \mathrm{A}$ & N/A & 7 & 1133 & $\mathrm{~N} / \mathrm{A}$ & $\mathrm{N} / \mathrm{A}$ & N/A \\
\hline 18 & Ceiling, Concrete (1504) & N/A & N/A & 12 & 1220 & N/A & $\mathrm{N} / \mathrm{A}$ & N/A \\
\hline N/A & N/A & $\mathrm{N} / \mathrm{A}$ & $\mathrm{N} / \mathrm{A}$ & N/A & N/A & N/A & N/A & N/A \\
\hline N/A & $\mathrm{N} / \mathrm{A}$ & N/A & N/A & N/A & $\mathrm{N} / \mathrm{A}$ & N/A & N/A & N/A \\
\hline $\mathrm{N} / \mathrm{A}$ & ${ }^{* *}$ Performed scan surveys for both alpha and beta** & N/A & N/A & N/A & N/A & N/A & N/A & N/A \\
\hline N/A & ${ }^{\star \star}$ on $100 \%$ of survey unit. No trigger levels exceeded ${ }^{\star \star}$ & N/A & N/A & N/A & N/A & N/A & N/A & N/A \\
\hline N/A & $\mathrm{N} / \mathrm{A}$ & N/A & N/A & N/A & $\mathrm{N} / \mathrm{A}$ & N/A & N/A & $\mathrm{N} / \mathrm{A}$ \\
\hline N/A & $\mathrm{N} / \mathrm{A}$ & $\mathrm{N} / \mathrm{A}$ & N/A & N/A & $\mathrm{N} / \mathrm{A}$ & N/A & N/A & N/A \\
\hline N/A & $\mathrm{N} / \mathrm{A}$ & N/A & $\mathrm{N} / \mathrm{A}$ & $\mathrm{N} / \mathrm{A}$ & N/A & $\mathrm{N} / \mathrm{A}$ & N/A & N/A \\
\hline N/A & $\mathrm{N} / \mathrm{A}$ & N/A & N/A & N/A & N/A & N/A & N/A & N/A \\
\hline $\mathrm{N} / \mathrm{A}$ & $\mathrm{N} / \mathrm{A}$ & N/A & N/A & N/A & N/A & N/A & N/A & N/A \\
\hline N/A & $N / A$ & N/A & N/A & $\mathrm{N} / \mathrm{A}$ & N/A & N/A & N/A & N/A \\
\hline N/A & $\mathrm{N} / \mathrm{A}$ & N/A & N/A & $\mathrm{N} / \mathrm{A}$ & N/A & N/A & N/A & N/A \\
\hline N/A & N/A & N/A & N/A & N/A & N/A & N/A & N/A & N/A \\
\hline N/A & $\mathrm{N} / \mathrm{A}$ & N/A & $\mathrm{N} / \mathrm{A}$ & N/A & N/A & N/A & N/A & $\mathrm{N} / \mathrm{A}$ \\
\hline N/A & $\mathrm{N} / \mathrm{A}$ & N/A & N/A & N/A & N/A & N/A & N/A & N/A \\
\hline N/A & N/A & N/A & N/A & N/A & N/A & N/A & N/A & N/A \\
\hline N/A & $N / A$ & N/A & N/A & N/A & N/A & N/A & N/A & N/A \\
\hline
\end{tabular}


NSTec

Form

05/21/10

FRM-0108C

RADIOLOGICAL SURVEY REPORT - FULL MAP

Rev. 01

Survey $=10-E R-A 26-296$

Description/Map/Drawing/Picture/Comments

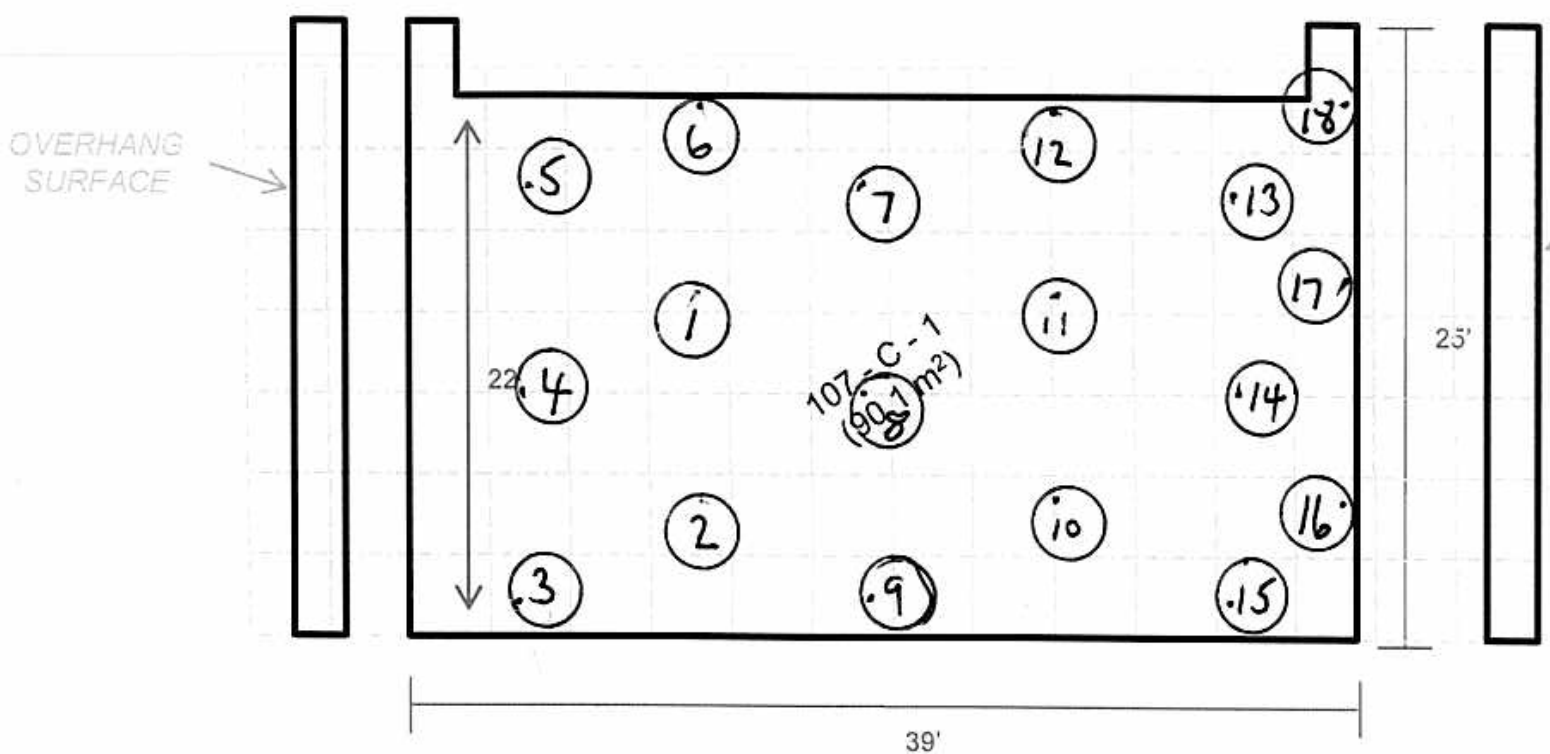

MARSSIM CLASS 2 SURVEY

$-1 \times 1 m$ grid

\begin{tabular}{|c|c|}
\hline$\underbrace{20}_{\substack{0 \\
\text { Drawing Scale: } 1 \text { inch = } 10 \text { foet }}}$ & $\begin{array}{l}\text { PLUTO ROOM } 107 \\
\text { PLAN VIEW: CEILING }\end{array}$ \\
\hline RADIOLOGICAL ENGINEERING & CREATOR: BWM // DATE: 06/21/2010 \\
\hline
\end{tabular}

LEGEND

S STATR cout Loc 1 i ias

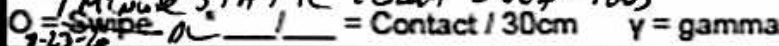

$\beta=$ beta $n=$ neutron

$x-x-x-x=$ Boundary

$\triangle L A W$ 


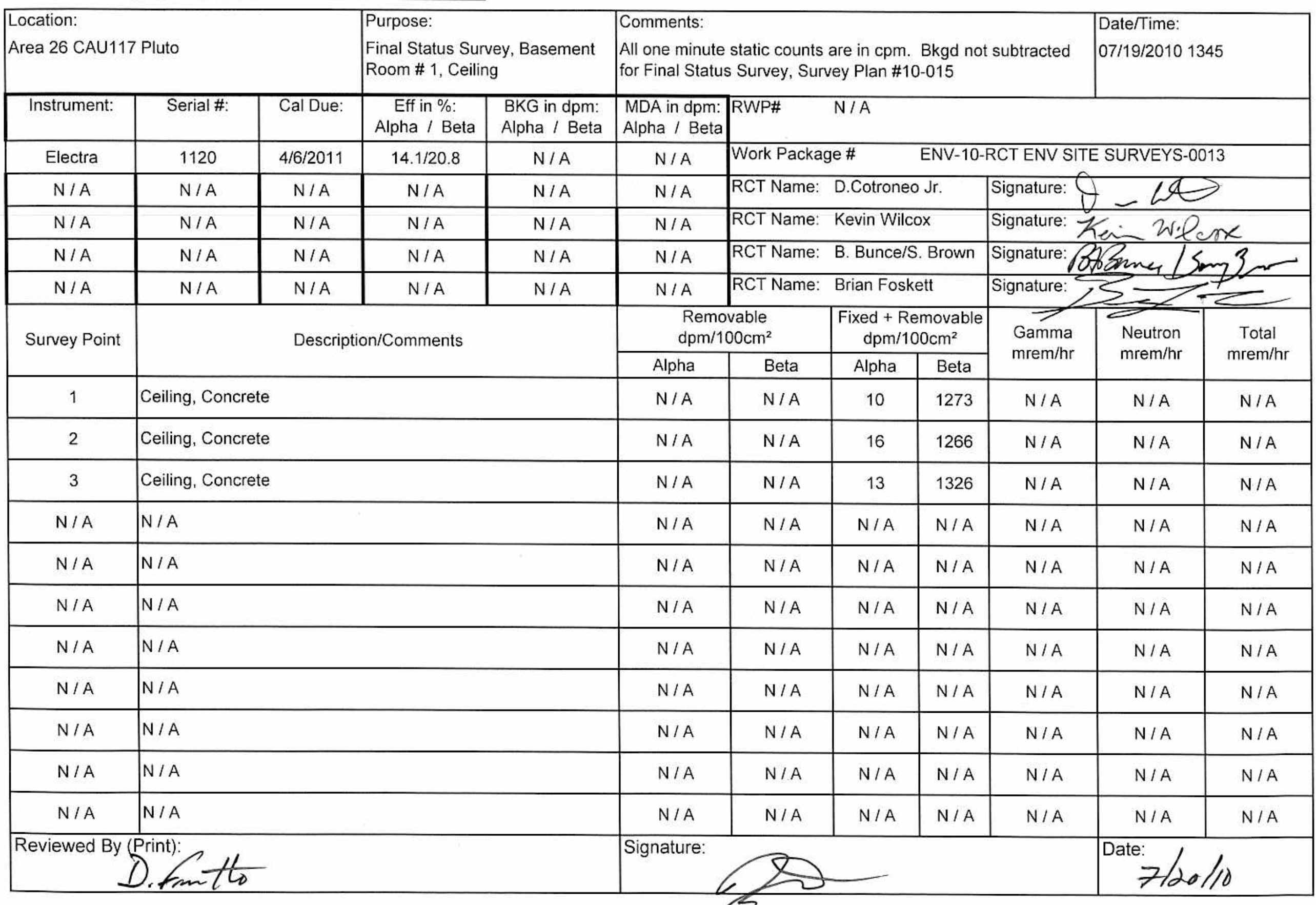


Survey \# 10-ER-A26-193

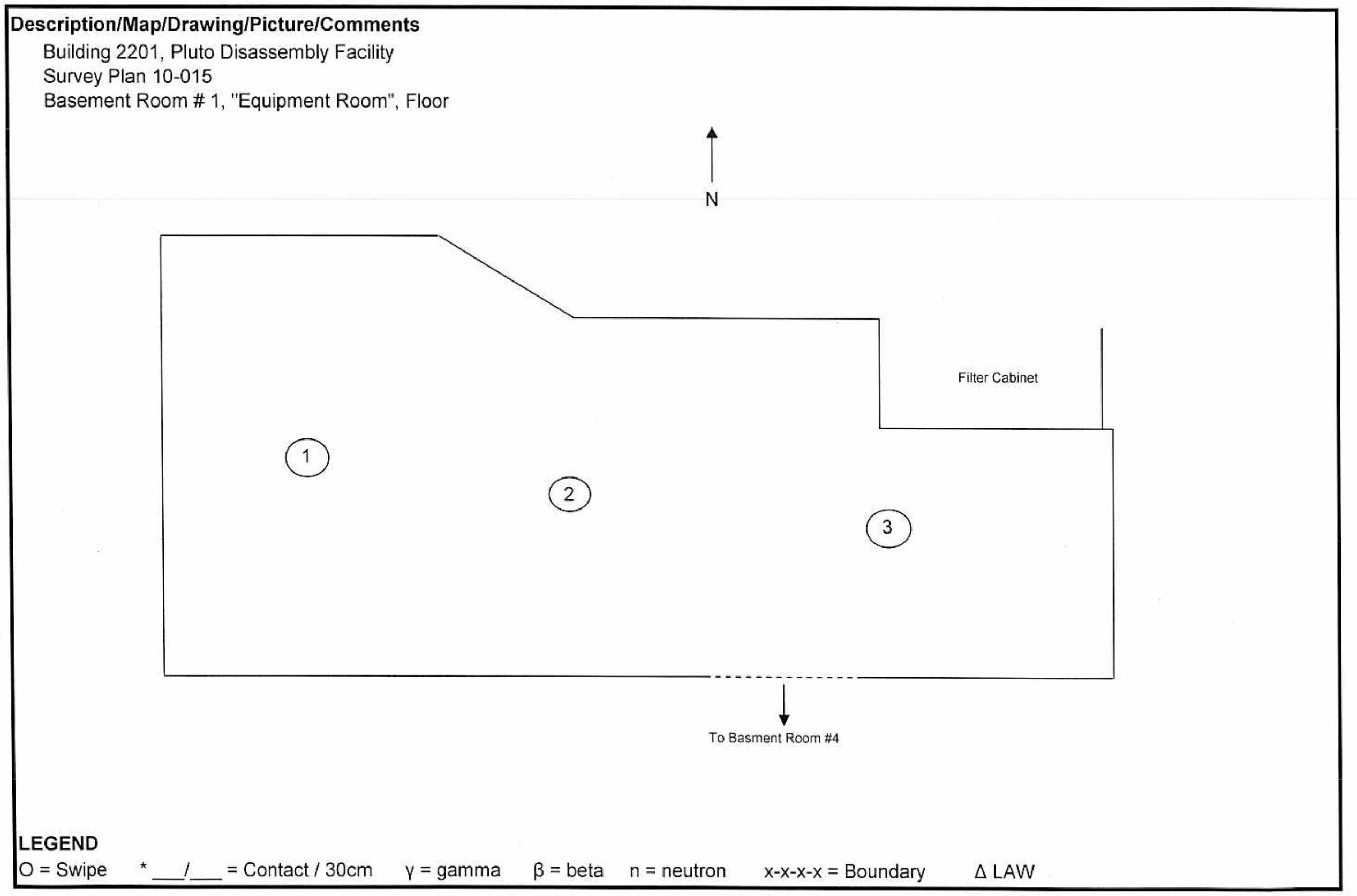




\begin{tabular}{|c|c|c|c|c|c|c|c|c|c|c|c|}
\hline \multicolumn{3}{|c|}{$\begin{array}{l}\text { Location: } \\
\text { Area } 26 \text { CAU117 Pluto }\end{array}$} & \multicolumn{2}{|c|}{$\begin{array}{l}\text { Purpose: } \\
\text { Final Status Survey, Basement } \\
\text { Room \# 1, Floor }\end{array}$} & \multicolumn{5}{|c|}{$\begin{array}{l}\text { Comments: } \\
\text { All one minute static counts are in cpm. Bkgd not subtracted } \\
\text { for Final Status Survey, Survey Plan \#10-015 }\end{array}$} & \multicolumn{2}{|c|}{$\begin{array}{l}\text { Date/Time: } \\
\text { 07/19/2010 } 1300\end{array}$} \\
\hline Instrument: & Serial \#: & Cal Due: & $\begin{array}{c}\text { Eff in \%: } \\
\text { Alpha / Beta }\end{array}$ & $\begin{array}{l}\text { BKG in dpm: } \\
\text { Alpha / Beta }\end{array}$ & \begin{tabular}{|l|} 
MDA in dpm: \\
Alpha / Beta \\
\end{tabular} & RWP\# & \multicolumn{5}{|l|}{$N / A$} \\
\hline Electra & 1120 & $4 / 6 / 2011$ & $14.1 / 20.8$ & $N / A$ & $N / A$ & \multicolumn{6}{|c|}{ Work Package \# } \\
\hline $\mathrm{N} / \mathrm{A}$ & $N / A$ & $\mathrm{~N} / \mathrm{A}$ & $\mathrm{N} / \mathrm{A}$ & $N / A$ & $N / A$ & \multicolumn{3}{|c|}{ RCT Name: D.Cotroneo Jr. } & \multicolumn{3}{|c|}{ Signature. $+E$} \\
\hline$N / A$ & $N / A$ & $N / A$ & $N / A$ & $N / A$ & $\mathrm{~N} / \mathrm{A}$ & \multicolumn{3}{|c|}{ RCT Name: Kevin Wilcox } & \multicolumn{3}{|c|}{ Signature: Ker W.leot } \\
\hline$N / A$ & $N / A$ & $N / A$ & $N / A$ & $N / A$ & $N / A$ & \multicolumn{3}{|c|}{ RCT Name: B. Bunce/S. Brown } & \multirow{2}{*}{\multicolumn{3}{|c|}{ Signature: Aflang $15 \mathrm{sm}$}} \\
\hline$N / A$ & $N / A$ & $N / A$ & $N / A$ & $N / A$ & $\mathrm{~N} / \mathrm{A}$ & RCT Name: & Brian Fos & & & & \\
\hline \multirow[t]{2}{*}{ Survey Point } & \multirow{2}{*}{\multicolumn{4}{|c|}{ Description/Comments }} & \multicolumn{2}{|c|}{$\begin{array}{l}\text { Removable } \\
\mathrm{dpm} / 100 \mathrm{~cm}^{2}\end{array}$} & \multicolumn{2}{|c|}{\begin{tabular}{|c|}
$\begin{array}{c}\text { Fixed + Removable } \\
\mathrm{dpm} / 100 \mathrm{~cm}^{2}\end{array}$ \\
\end{tabular}} & \multirow{2}{*}{$\begin{array}{l}\text { Gamma } \\
\mathrm{mrem} / \mathrm{hr}\end{array}$} & \multirow{2}{*}{$\begin{array}{l}\text { Neutron } \\
\mathrm{mrem} / \mathrm{hr}\end{array}$} & \multirow{2}{*}{$\begin{array}{c}\text { Total } \\
\mathrm{mrem} / \mathrm{hr}\end{array}$} \\
\hline & & & & & Alpha & Beta & Alpha & Beta & & & \\
\hline 1 & \multicolumn{4}{|l|}{ Floor, Concrete } & $N / A$ & $N / A$ & 15 & 1465 & $N / A$ & $N / A$ & $N / A$ \\
\hline 2 & \multicolumn{4}{|l|}{ Floor, Concrete } & $N / A$ & $N / A$ & 22 & 1419 & $N / A$ & $N / A$ & $N / A$ \\
\hline 3 & \multicolumn{4}{|c|}{ Floor, Concrete } & $N / A$ & $N / A$ & 16 & 1324 & $N / A$ & $N / A$ & $N / A$ \\
\hline 4 & \multicolumn{4}{|c|}{ Floor, Concrete } & $N / A$ & $N / A$ & 14 & 1531 & $N / A$ & $N / A$ & $N / A$ \\
\hline 5 & \multicolumn{4}{|c|}{ Floor, Concrete } & $N / A$ & $N / A$ & 14 & 1466 & $N / A$ & $N / A$ & $N / A$ \\
\hline 6 & \multicolumn{4}{|l|}{ Floor, Concrete } & $N / A$ & $N / A$ & 14 & 1375 & $N / A$ & $N / A$ & $N / A$ \\
\hline 7 & \multicolumn{4}{|c|}{ Floor, Concrete } & $\mathrm{N} / \mathrm{A}$ & $N / A$ & 12 & 1421 & $N / A$ & $N / A$ & $N / A$ \\
\hline 8 & \multicolumn{4}{|c|}{ Floor, Concrete } & $N / A$ & $N / A$ & 14 & 1470 & $N / A$ & $N / A$ & $N / A$ \\
\hline$N / A$ & \multicolumn{4}{|l|}{$N / A$} & $N / A$ & $N / A$ & $N / A$ & $N / A$ & $N / A$ & $N / A$ & $N / A$ \\
\hline $\mathrm{N} / \mathrm{A}$ & \multicolumn{4}{|l|}{$N / A$} & $N / A$ & $N / A$ & $N / A$ & $N / A$ & $N / A$ & $N / A$ & $N / A$ \\
\hline$N / A$ & \multicolumn{4}{|l|}{$N / A$} & $\mathrm{~N} / \mathrm{A}$ & $N / A$ & $N / A$ & $N / A$ & $\mathrm{~N} / \mathrm{A}$ & $N / A$ & $N / A$ \\
\hline \multicolumn{5}{|c|}{ Reviewed By (Print): } & \multicolumn{5}{|l|}{ Signature: } & Date: $7 / 2$ & \\
\hline
\end{tabular}


Survey \# 10-ER-A26-191

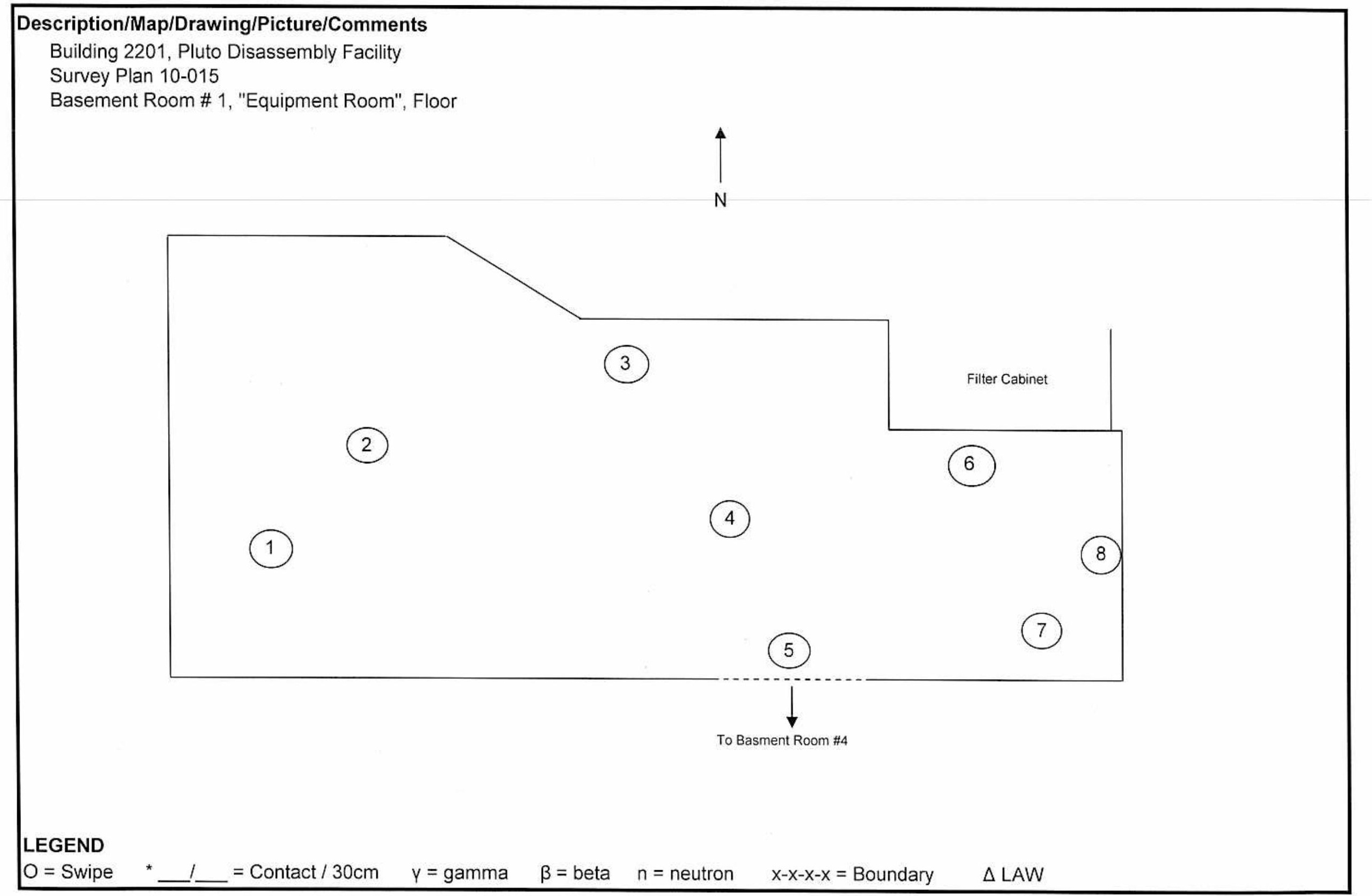




\begin{tabular}{|c|c|c|c|c|c|c|c|c|c|c|c|}
\hline \multicolumn{3}{|c|}{$\begin{array}{l}\text { Location: } \\
\text { Area } 26 \text { CAU117 Pluto }\end{array}$} & \multicolumn{2}{|c|}{$\begin{array}{l}\text { Purpose: } \\
\text { Final Status Survey, Basement } \\
\text { Room \# } 1 \text {, Walls }\end{array}$} & \multicolumn{5}{|c|}{$\begin{array}{l}\text { Comments: } \\
\text { All one minute static counts are in cpm. Bkgd not subtracted } \\
\text { for Final Status Survey, Survey Plan \#10-015 }\end{array}$} & \multicolumn{2}{|c|}{$\begin{array}{l}\text { Date/Time: } \\
\text { 07/19/2010 } 1320\end{array}$} \\
\hline Instrument: & Serial \#: & Cal Due: & \begin{tabular}{|c|} 
Eff in \%: \\
Alpha / Beta
\end{tabular} & $\begin{array}{l}\text { BKG in dpm: } \\
\text { Alpha / Beta }\end{array}$ & $\begin{array}{l}\text { MDA in dpm: } \\
\text { Alpha / Beta }\end{array}$ & RWP\# & \multicolumn{5}{|l|}{$\mathrm{N} / \mathrm{A}$} \\
\hline Electra & 1120 & $4 / 6 / 2011$ & $14.1 / 20.8$ & $N / A$ & $N / A$ & \multicolumn{6}{|c|}{ Work Package \# $\quad$ ENV-10-RCT ENV SITE SURVEYS-0013 } \\
\hline$N / A$ & $N / A$ & $\mathrm{~N} / \mathrm{A}$ & $N / A$ & $\mathrm{~N} / \mathrm{A}$ & $N / A$ & RCT Name: & \multicolumn{2}{|c|}{ D.Cotroneo Jr. } & \multicolumn{3}{|c|}{ Signature. $\theta-\omega$} \\
\hline$N / A$ & $N / A$ & $N / A$ & $N / A$ & N/A & $N / A$ & RCT Name: & \multicolumn{2}{|c|}{ Kevin Wilcox } & \multicolumn{3}{|c|}{ Signature: Yer 2 UCox } \\
\hline$N / A$ & $N / A$ & $N / A$ & $N / A$ & $N / A$ & N/A & RCT Name: & \multicolumn{2}{|c|}{ B. Bunce/S. Brown } & \multicolumn{3}{|c|}{ 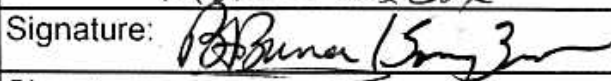 } \\
\hline$N / A$ & $N / A$ & $N / A$ & $N / A$ & $N / A$ & $N / A$ & RCT Name: & Brian Fos & & Signature: & 2 & \\
\hline \multirow[t]{2}{*}{ Survey Point } & \multirow{2}{*}{\multicolumn{4}{|c|}{ Description/Comments }} & \multicolumn{2}{|c|}{$\begin{array}{l}\text { Removable } \\
\mathrm{dpm} / 100 \mathrm{~cm}^{2}\end{array}$} & \multicolumn{2}{|c|}{\begin{tabular}{|c|} 
Fixed + Removable \\
$\mathrm{dpm} / 100 \mathrm{~cm}^{2}$
\end{tabular}} & \multirow{2}{*}{$\begin{array}{l}\text { Gamma } \\
\mathrm{mrem} / \mathrm{hr}\end{array}$} & \multirow{2}{*}{$\begin{array}{l}\text { Neutron } \\
\text { mrem } / \mathrm{hr}\end{array}$} & \multirow{2}{*}{$\begin{array}{c}\text { Total } \\
\mathrm{mrem} / \mathrm{hr}\end{array}$} \\
\hline & & & & & Alpha & Beta & Alpha & Beta & & & \\
\hline 1 & \multicolumn{4}{|c|}{ West Wall, Concrete } & $N / A$ & $N / A$ & 23 & 1224 & $N / A$ & $N / A$ & $N / A$ \\
\hline 2 & \multicolumn{4}{|c|}{ West Wall, Concrete } & $\mathrm{N} / \mathrm{A}$ & $N / A$ & 13 & 1207 & $N / A$ & $N / A$ & $\mathrm{~N} / \mathrm{A}$ \\
\hline 3 & \multicolumn{4}{|c|}{ North Wall, Concrete } & $N / A$ & $N / A$ & 17 & 894 & $N / A$ & $N / A$ & $N / A$ \\
\hline 4 & \multicolumn{4}{|c|}{ North Wall, Concrete } & $N / A$ & $N / A$ & 14 & 965 & $N / A$ & $N / A$ & $N / A$ \\
\hline 5 & \multicolumn{4}{|c|}{ North Wall, Metal } & $N / A$ & $N / A$ & 4 & 841 & $N / A$ & $N / A$ & $N / A$ \\
\hline 6 & \multicolumn{4}{|c|}{ East Wall, Concrete } & $N / A$ & $N / A$ & 18 & 1183 & $N / A$ & $N / A$ & $N / A$ \\
\hline 7 & \multicolumn{4}{|c|}{ East Wall, Concrete } & $N / A$ & $N / A$ & 13 & 1225 & $N / A$ & $N / A$ & $N / A$ \\
\hline 8 & \multicolumn{4}{|c|}{ South Wall, Concrete } & $N / A$ & $\mathrm{~N} / \mathrm{A}$ & 13 & 1159 & $\mathrm{~N} / \mathrm{A}$ & $N / A$ & $N / A$ \\
\hline 9 & \multicolumn{4}{|c|}{ South Wall (Door), Metal } & $N / A$ & $N / A$ & 14 & 948 & $N / A$ & $N / A$ & $N / A$ \\
\hline 10 & \multicolumn{4}{|c|}{ South Wall, Concrete } & $N / A$ & $N / A$ & 10 & 1235 & $N / A$ & $N / A$ & $N / A$ \\
\hline 11 & \multicolumn{4}{|c|}{ South Wall, Concrete } & $N / A$ & $N / A$ & 14 & 1120 & $N / A$ & $N / A$ & $N / A$ \\
\hline \multicolumn{5}{|c|}{ Reviewed By (Print): } & \multicolumn{5}{|l|}{ Signature: } & \multicolumn{2}{|c|}{ Date: $7 / 20 / 10$} \\
\hline
\end{tabular}


Form

SURVEY \# 10-ER-A26-192

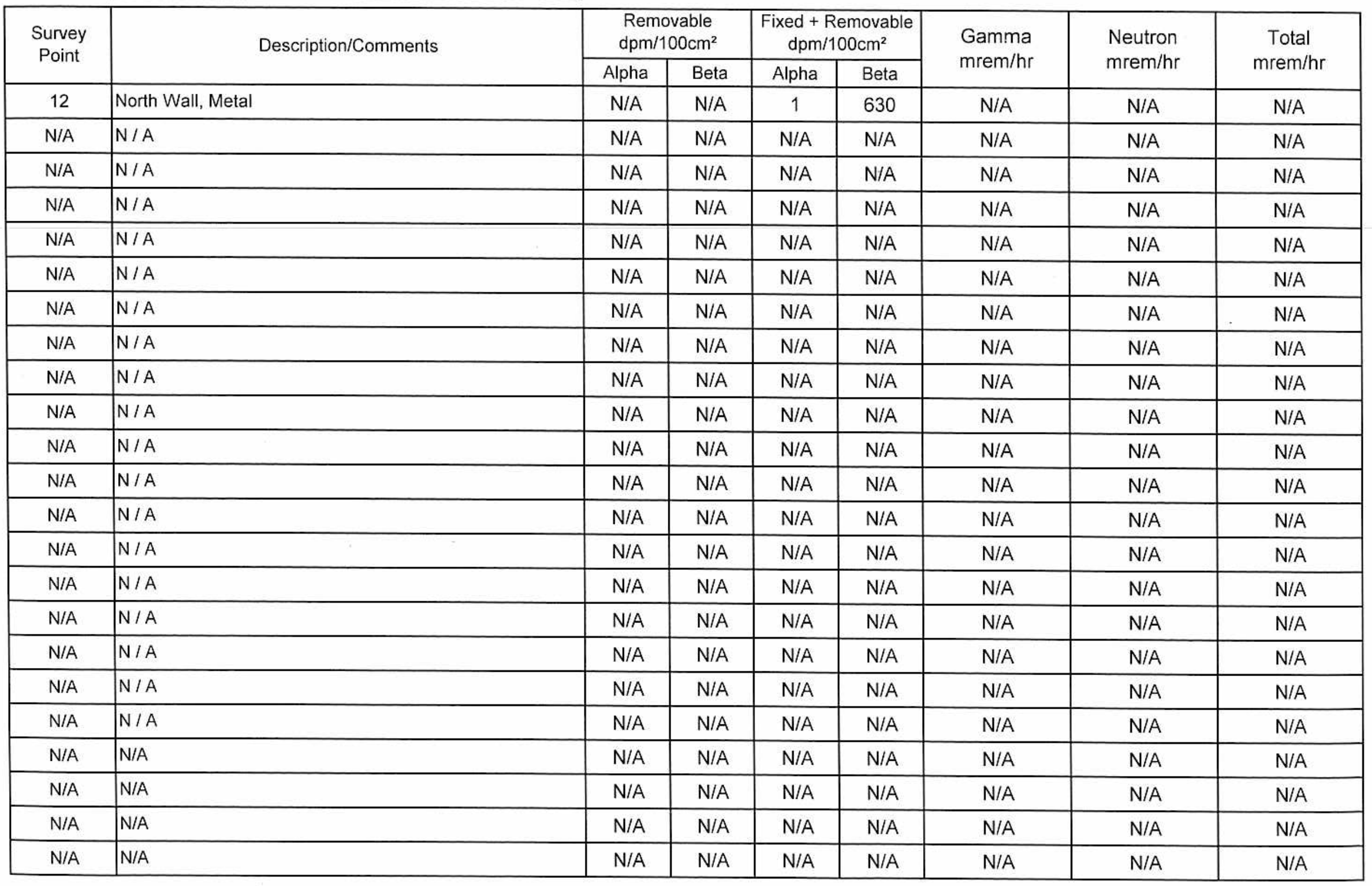


Survey \# 10-ER-A26-192

\section{Description/Map/Drawing/Picture/Comments}

Building 2201, Pluto Disassembly Facility

Survey Plan 10-015

Basement Room \# 1, "Equipment Room"

Walls
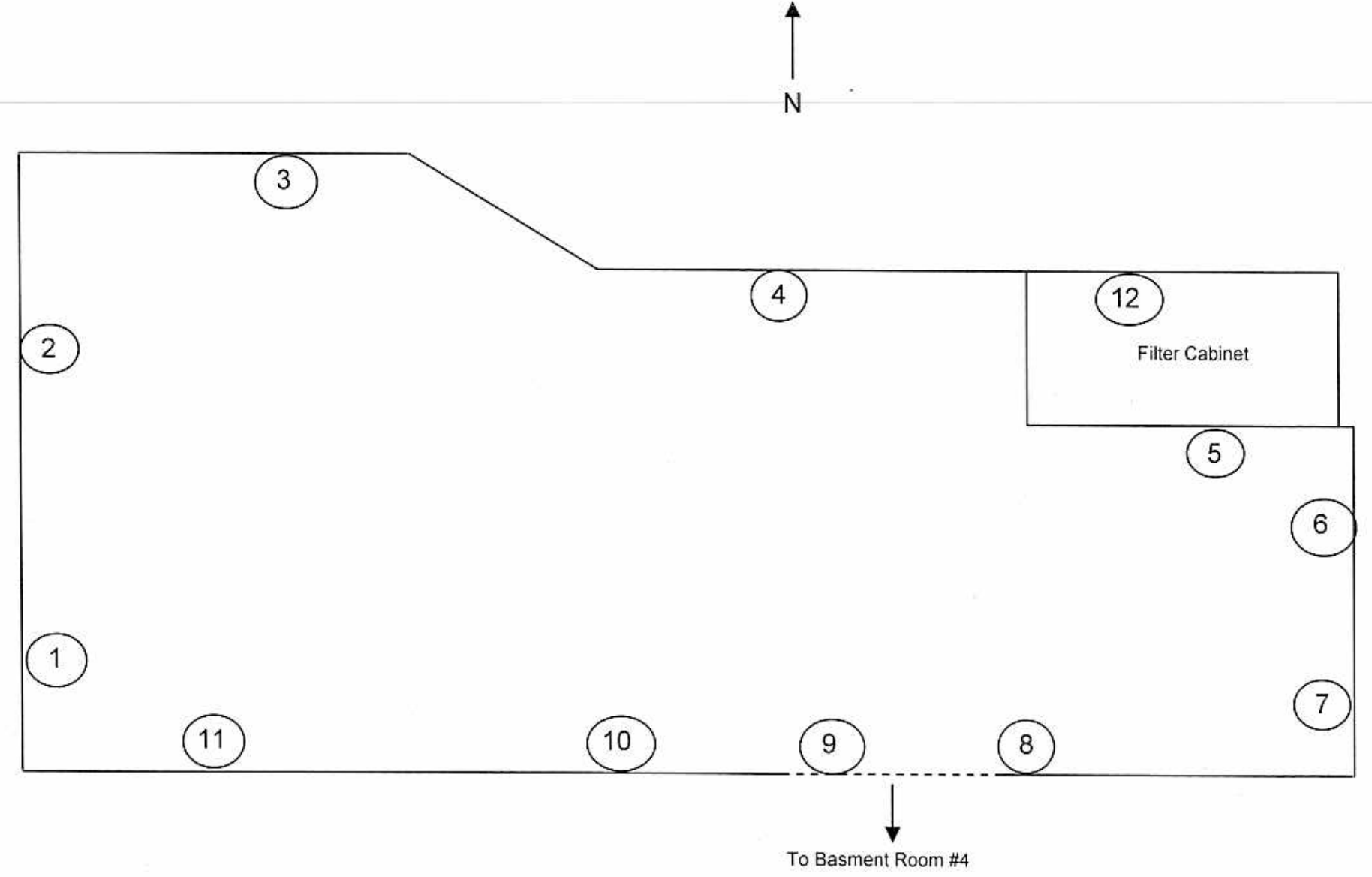

LEGEND

$\mathrm{O}=$ Swipe 
SURVEY \#

10-ER-A26-244

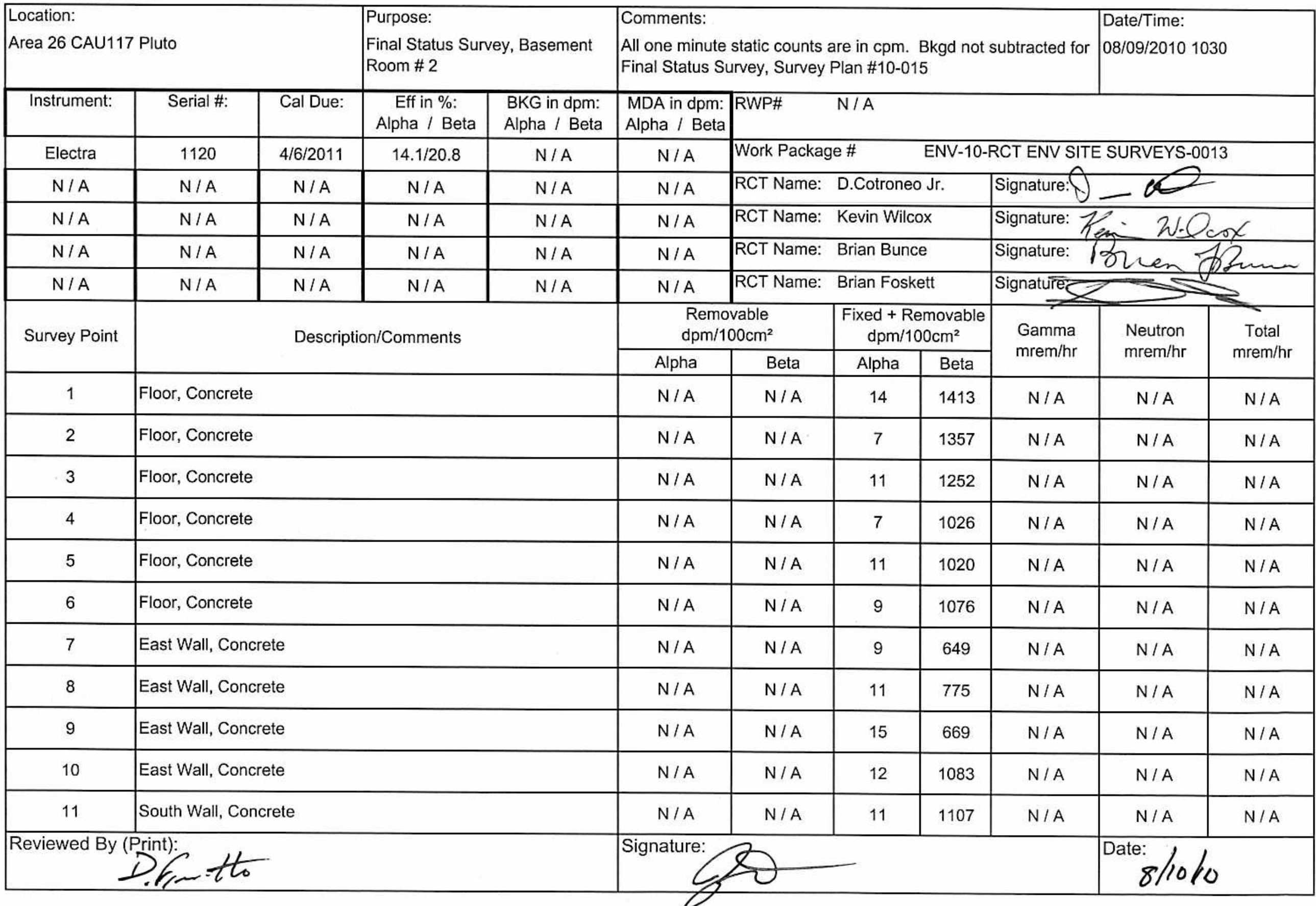


NSTec

SURVEY \# 10-ER-A26-244

\begin{tabular}{|c|c|c|c|c|c|c|c|c|}
\hline \multirow[t]{2}{*}{ Survey Point } & \multirow[t]{2}{*}{ Description/Comments } & \multicolumn{2}{|c|}{$\begin{array}{l}\text { Removable } \\
\mathrm{dpm} / 100 \mathrm{~cm}^{2}\end{array}$} & \multicolumn{2}{|c|}{$\begin{array}{c}\text { Fixed + Removable } \\
\mathrm{dpm} / 100 \mathrm{~cm}^{2}\end{array}$} & \multirow{2}{*}{$\begin{array}{l}\text { Gamma } \\
\mathrm{mrem} / \mathrm{hr}\end{array}$} & \multirow{2}{*}{$\begin{array}{l}\text { Neutron } \\
\text { mrem/hr }\end{array}$} & \multirow{2}{*}{$\begin{array}{c}\text { Total } \\
\text { mrem/hr }\end{array}$} \\
\hline & & Alpha & Beta & Alpha & Beta & & & \\
\hline 12 & South Wall, Concrete & $\mathrm{N} / \mathrm{A}$ & N/A & 14 & 1099 & N/A & N/A & N/A \\
\hline 13 & West Wall, Concrete & $\mathrm{N} / \mathrm{A}$ & $\mathrm{N} / \mathrm{A}$ & 9 & 1148 & N/A & N/A & $\mathrm{N} / \mathrm{A}$ \\
\hline 14 & West Wall, Concrete & $\mathrm{N} / \mathrm{A}$ & N/A & 5 & 1099 & N/A & N/A & N/A \\
\hline 15 & West Wall, Concrete & $\mathrm{N} / \mathrm{A}$ & N/A & 14 & 671 & N/A & N/A & N/A \\
\hline 16 & West Wall, Concrete & $\mathrm{N} / \mathrm{A}$ & N/A & 17 & 611 & N/A & N/A & N/A \\
\hline 17 & North Wall, Concrete & $\mathrm{N} / \mathrm{A}$ & N/A & 8 & 630 & N/A & N/A & N/A \\
\hline 18 & North Wall, Concrete & $\mathrm{N} / \mathrm{A}$ & N/A & 12 & 609 & N/A & N/A & N/A \\
\hline 19 & Ceiling, Concrete & $\mathrm{N} / \mathrm{A}$ & N/A & 10 & 1227 & N/A & N/A & N/A \\
\hline 20 & Ceiling, Concrete & $\mathrm{N} / \mathrm{A}$ & $\mathrm{N} / \mathrm{A}$ & 10 & 1039 & N/A & N/A & $\mathrm{N} / \mathrm{A}$ \\
\hline 21 & Ceiling, Concrete & $\mathrm{N} / \mathrm{A}$ & N/A & 17 & 998 & $\mathrm{~N} / \mathrm{A}$ & N/A & $\mathrm{N} / \mathrm{A}$ \\
\hline 22 & Ceiling, Concrete & $\mathrm{N} / \mathrm{A}$ & N/A & 13 & 953 & $\mathrm{~N} / \mathrm{A}$ & N/A & N/A \\
\hline 23 & Ceiling, Concrete & $\mathrm{N} / \mathrm{A}$ & N/A & 12 & 869 & N/A & N/A & N/A \\
\hline 24 & Ceiling, Concrete & $\mathrm{N} / \mathrm{A}$ & N/A & 22 & 1235 & $\mathrm{~N} / \mathrm{A}$ & N/A & N/A \\
\hline $\mathrm{N} / \mathrm{A}$ & $\mathrm{N} / \mathrm{A}$ & $\mathrm{N} / \mathrm{A}$ & N/A & N/A & N/A & N/A & N/A & N/A \\
\hline $\mathrm{N} / \mathrm{A}$ & $\mathrm{N} / \mathrm{A}$ & N/A & N/A & N/A & $\mathrm{N} / \mathrm{A}$ & $\mathrm{N} / \mathrm{A}$ & N/A & N/A \\
\hline N/A & $\mathrm{N} / \mathrm{A}$ & $\mathrm{N} / \mathrm{A}$ & N/A & N/A & $\mathrm{N} / \mathrm{A}$ & N/A & N/A & N/A \\
\hline $\mathrm{N} / \mathrm{A}$ & $\mathrm{N} / \mathrm{A}$ & $\mathrm{N} / \mathrm{A}$ & N/A & N/A & N/A & N/A & N/A & N/A \\
\hline $\mathrm{N} / \mathrm{A}$ & $\mathrm{N} / \mathrm{A}$ & $\mathrm{N} / \mathrm{A}$ & N/A & N/A & N/A & N/A & N/A & $\mathrm{N} / \mathrm{A}$ \\
\hline N/A & $\mathrm{N} / \mathrm{A}$ & $\mathrm{N} / \mathrm{A}$ & N/A & N/A & N/A & N/A & N/A & N/A \\
\hline $\mathrm{N} / \mathrm{A}$ & $\mathrm{N} / \mathrm{A}$ & $\mathrm{N} / \mathrm{A}$ & N/A & N/A & N/A & $\mathrm{N} / \mathrm{A}$ & N/A & $\mathrm{N} / \mathrm{A}$ \\
\hline N/A & $\mathrm{N} / \mathrm{A}$ & $\mathrm{N} / \mathrm{A}$ & N/A & N/A & N/A & $\mathrm{N} / \mathrm{A}$ & $\mathrm{N} / \mathrm{A}$ & $\mathrm{N} / \mathrm{A}$ \\
\hline N/A & $\mathrm{N} / \mathrm{A}$ & $\mathrm{N} / \mathrm{A}$ & N/A & N/A & N/A & $\mathrm{N} / \mathrm{A}$ & N/A & N/A \\
\hline N/A & $N / A$ & $\mathrm{~N} / \mathrm{A}$ & N/A & N/A & N/A & N/A & N/A & N/A \\
\hline
\end{tabular}


Survey \# 10-ER-A26-244

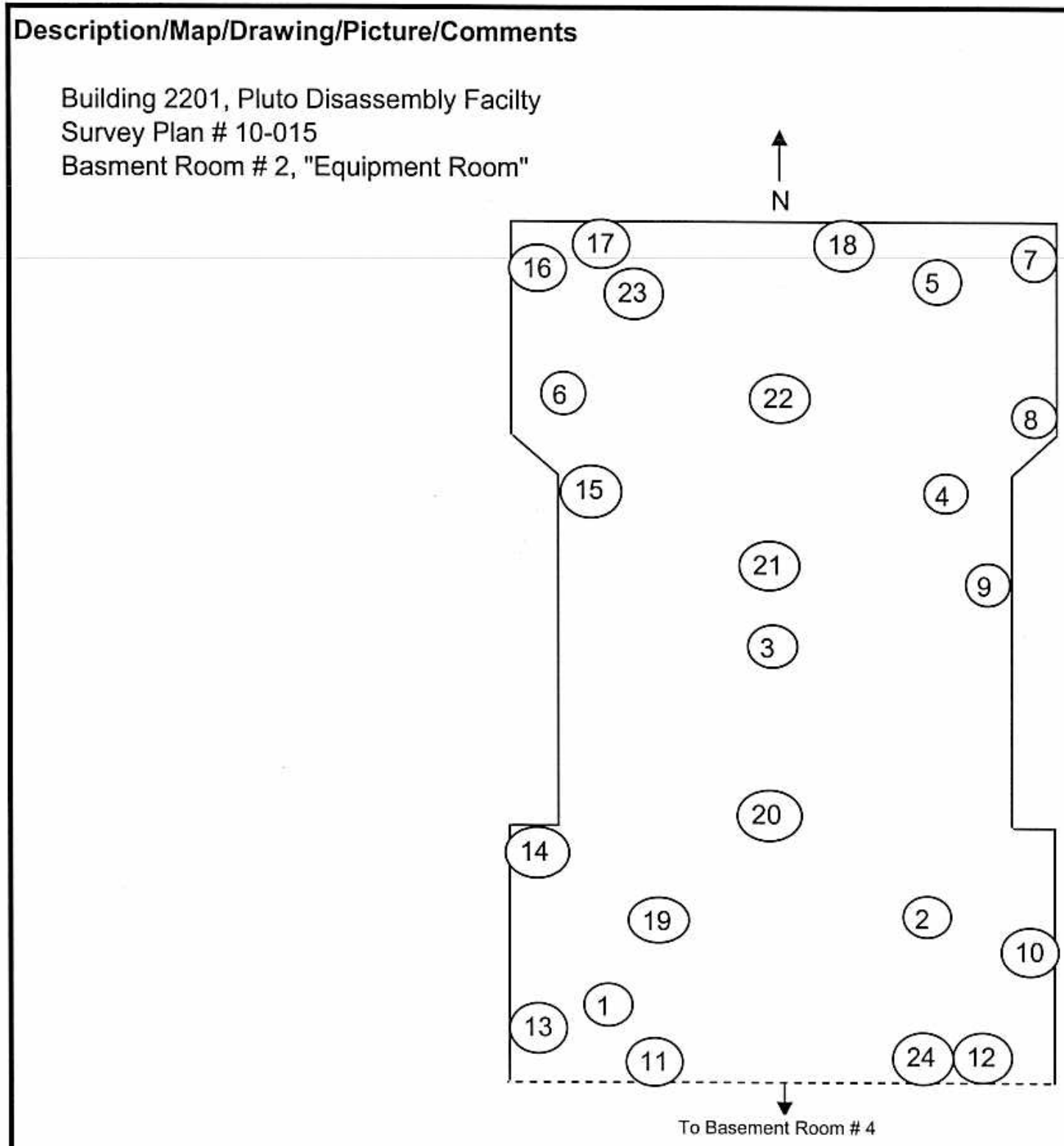

\section{LEGEND}


SURVEY \#

10-ER-A26-246

\begin{tabular}{|c|c|c|c|c|c|c|c|c|c|c|c|}
\hline \multicolumn{3}{|c|}{$\begin{array}{l}\text { Location: } \\
\text { Area } 26 \text { CAU117 Pluto }\end{array}$} & \multicolumn{2}{|c|}{$\begin{array}{l}\text { Purpose: } \\
\text { Final Status Survey, Basement } \\
\text { Room \# } 3\end{array}$} & \multicolumn{5}{|c|}{$\begin{array}{l}\text { Comments: } \\
\text { All one minute static counts are in } \mathrm{cpm} \text {. Bkgd not subtracted for } \\
\text { Final Status Survey, Survey Plan } \# 10-015\end{array}$} & \multicolumn{2}{|c|}{$\begin{array}{l}\text { Date/Time: } \\
\text { 08/09/2010 } 1300\end{array}$} \\
\hline Instrument: & Serial \#: & Cal Due: & $\begin{array}{c}\text { Eff in \%: } \\
\text { Alpha / Beta }\end{array}$ & $\begin{array}{l}\text { BKG in dpm: } \\
\text { Alpha / Beta }\end{array}$ & \begin{tabular}{|l|} 
MDA in dpm: \\
Alpha / Beta
\end{tabular} & RWP\# & \multicolumn{5}{|l|}{$\mathrm{N} / \mathrm{A}$} \\
\hline Electra & 1504 & $4 / 6 / 2011$ & $12.9 / 19.8$ & $\mathrm{~N} / \mathrm{A}$ & $\mathrm{N} / \mathrm{A}$ & \multicolumn{6}{|c|}{ 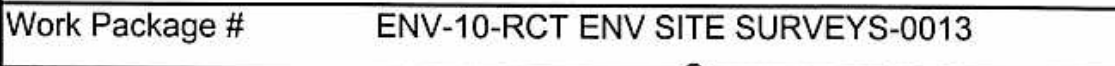 } \\
\hline $\mathrm{N} / \mathrm{A}$ & N/A & N/A & $N / A$ & $N / A$ & $\mathrm{~N} / \mathrm{A}$ & \multicolumn{3}{|c|}{ RCT Name: D.Cotroneo Jr. } & \multicolumn{3}{|l|}{ Signature: \{} \\
\hline$N / A$ & $N / A$ & N/A & $N / A$ & $\mathrm{~N} / \mathrm{A}$ & $\mathrm{N} / \mathrm{A}$ & \multicolumn{3}{|c|}{ RCT Name: Kevin Wilcox } & \multicolumn{3}{|c|}{ Signature: Keni $\mathrm{K} \cdot \mathrm{los}$} \\
\hline$N / A$ & N/A & $\mathrm{N} / \mathrm{A}$ & $N / A$ & $\mathrm{~N} / \mathrm{A}$ & $\mathrm{N} / \mathrm{A}$ & \multicolumn{3}{|c|}{ RCT Name: Brian Bunce } & \multicolumn{3}{|c|}{ Signature: 180 hrum } \\
\hline $\mathrm{N} / \mathrm{A}$ & $\mathrm{N} / \mathrm{A}$ & $N / A$ & $\mathrm{~N} / \mathrm{A}$ & $\mathrm{N} / \mathrm{A}$ & $\mathrm{N} / \mathrm{A}$ & \multicolumn{3}{|c|}{ RCT Name: Brian Foskett } & \multirow{3}{*}{\multicolumn{3}{|c|}{\begin{tabular}{|c|c|c|} 
Signature: & \\
$\begin{array}{c}\text { Gamma } \\
\mathrm{mrem} / \mathrm{hr}\end{array}$ & $\begin{array}{c}\text { Neutron } \\
\mathrm{mrem} / \mathrm{hr}\end{array}$ & $\begin{array}{c}\text { Total } \\
\mathrm{mrem} / \mathrm{hr}\end{array}$ \\
\end{tabular}}} \\
\hline \multirow[t]{2}{*}{ Survey Point } & \multirow{2}{*}{\multicolumn{4}{|c|}{ Description/Comments }} & \multicolumn{2}{|c|}{$\begin{array}{c}\text { Removable } \\
\mathrm{dpm} / 100 \mathrm{~cm}^{2}\end{array}$} & \multicolumn{2}{|c|}{\begin{tabular}{|c|}
$\begin{array}{c}\text { Fixed + Removable } \\
\mathrm{dpm} / 100 \mathrm{~cm}^{2}\end{array}$ \\
\end{tabular}} & & & \\
\hline & & & & & Alpha & Beta & Alpha & Beta & & & \\
\hline 1 & \multicolumn{4}{|c|}{ Floor, Concrete } & $N / A$ & $\mathrm{~N} / \mathrm{A}$ & 4 & 1470 & $N / A$ & $N / A$ & $N / A$ \\
\hline 2 & \multicolumn{4}{|c|}{ Floor, Concrete } & $N / A$ & $N / A$ & 8 & 1430 & $N / A$ & $\mathrm{~N} / \mathrm{A}$ & $\mathrm{N} / \mathrm{A}$ \\
\hline 3 & \multicolumn{4}{|c|}{ Floor, Concrete } & $N / A$ & $N / A$ & 11 & 1248 & $N / A$ & $\mathrm{~N} / \mathrm{A}$ & $N / A$ \\
\hline 4 & \multicolumn{4}{|c|}{ Floor, Concrete } & $N / A$ & $N / A$ & 4 & 1312 & $N / A$ & $N / A$ & $N / A$ \\
\hline 5 & \multicolumn{4}{|c|}{ Floor, Concrete } & $N / A$ & $N / A$ & 7 & 1269 & $N / A$ & $N / A$ & $N / A$ \\
\hline 6 & \multicolumn{4}{|c|}{ Floor, Concrete } & $N / A$ & $N / A$ & 11 & 1376 & $N / A$ & $N / A$ & $N / A$ \\
\hline 7 & \multicolumn{4}{|c|}{ Floor, Concrete } & $N / A$ & $\mathrm{~N} / \mathrm{A}$ & 9 & 1373 & $N / A$ & $N / A$ & $N / A$ \\
\hline 8 & \multicolumn{4}{|c|}{ Floor, Concrete } & $N / A$ & $N / A$ & 10 & 1356 & $N / A$ & $N / A$ & $N / A$ \\
\hline 9 & \multicolumn{4}{|c|}{ Floor, Concrete } & $\mathrm{N} / \mathrm{A}$ & $N / A$ & 12 & 1311 & $\mathrm{~N} / \mathrm{A}$ & $\mathrm{N} / \mathrm{A}$ & $N / A$ \\
\hline 10 & \multicolumn{4}{|c|}{ Floor, Concrete } & $N / A$ & $N / A$ & 7 & 1312 & $\mathrm{~N} / \mathrm{A}$ & $\mathrm{N} / \mathrm{A}$ & $N / A$ \\
\hline 11 & \multicolumn{4}{|c|}{ North Wall, Metal Plenum } & $N / A$ & $N / A$ & 4 & 578 & $N / A$ & $N / A$ & $N / A$ \\
\hline \multicolumn{5}{|c|}{ Reviewed By (Print): Flentth } & Signature: & & & & & \multicolumn{2}{|c|}{$8 / 10 / 10$} \\
\hline
\end{tabular}


SURVEY \# 10-ER-A26-246

\begin{tabular}{|c|c|c|c|c|c|c|c|c|}
\hline \multirow[t]{2}{*}{ Survey Point } & \multirow[t]{2}{*}{ Description/Comments } & \multicolumn{2}{|c|}{$\begin{array}{l}\text { Removable } \\
\mathrm{dpm} / 100 \mathrm{~cm}^{2}\end{array}$} & \multicolumn{2}{|c|}{$\begin{array}{c}\text { Fixed + Removable } \\
\mathrm{dpm} / 100 \mathrm{~cm}^{2}\end{array}$} & \multirow{2}{*}{$\begin{array}{l}\text { Gamma } \\
\mathrm{mrem} / \mathrm{hr}\end{array}$} & \multirow{2}{*}{$\begin{array}{l}\text { Neutron } \\
\mathrm{mrem} / \mathrm{hr}\end{array}$} & \multirow{2}{*}{$\begin{array}{c}\text { Total } \\
\mathrm{mrem} / \mathrm{h}\end{array}$} \\
\hline & & Alpha & Beta & Alpha & Beta & & & \\
\hline 12 & North Wall, Metal Duct & N/A & N/A & 7 & 706 & $\mathrm{~N} / \mathrm{A}$ & N/A & N/A \\
\hline 13 & North Wall, Concrete & N/A & N/A & 8 & 934 & $\mathrm{~N} / \mathrm{A}$ & N/A & N/A \\
\hline 14 & North Wall, Metal Duct & N/A & N/A & 6 & 796 & $\mathrm{~N} / \mathrm{A}$ & $\mathrm{N} / \mathrm{A}$ & $\mathrm{N} / \mathrm{A}$ \\
\hline 15 & North Wall, Metal Plenum & N/A & $\mathrm{N} / \mathrm{A}$ & 3 & 578 & N/A & N/A & N/A \\
\hline 16 & East Wall, Concrete & N/A & N/A & 14 & 1216 & $\mathrm{~N} / \mathrm{A}$ & N/A & $\mathrm{N} / \mathrm{A}$ \\
\hline 17 & East Wall, Concrete & N/A & N/A & 14 & 1254 & N/A & N/A & N/A \\
\hline 18 & South Wall, Concrete & $\mathrm{N} / \mathrm{A}$ & $\mathrm{N} / \mathrm{A}$ & 10 & 1172 & $\mathrm{~N} / \mathrm{A}$ & N/A & $\mathrm{N} / \mathrm{A}$ \\
\hline 19 & South Wall, Concrete & N/A & N/A & 9 & 1112 & N/A & N/A & $\mathrm{N} / \mathrm{A}$ \\
\hline 20 & South Wall, Concrete & $\mathrm{N} / \mathrm{A}$ & $\mathrm{N} / \mathrm{A}$ & 6 & 1116 & N/A & $\mathrm{N} / \mathrm{A}$ & N/A \\
\hline 21 & South Wall, Concrete & N/A & N/A & 5 & 1064 & N/A & N/A & $\mathrm{N} / \mathrm{A}$ \\
\hline 22 & South Wall, Concrete & N/A & $\mathrm{N} / \mathrm{A}$ & 14 & 1131 & N/A & N/A & N/A \\
\hline 23 & West Wall, Concrete & N/A & N/A & 21 & 1148 & N/A & $N / A$ & $\mathrm{~N} / \mathrm{A}$ \\
\hline 24 & West Wall, Metal & $\mathrm{N} / \mathrm{A}$ & $\mathrm{N} / \mathrm{A}$ & 6 & 704 & N/A & N/A & $\mathrm{N} / \mathrm{A}$ \\
\hline 25 & Ceiling, Concrete & N/A & $\mathrm{N} / \mathrm{A}$ & 8 & 1319 & N/A & N/A & $\mathrm{N} / \mathrm{A}$ \\
\hline 26 & Ceiling, Metal & N/A & N/A & 4 & 843 & N/A & $\mathrm{N} / \mathrm{A}$ & $\mathrm{N} / \mathrm{A}$ \\
\hline 27 & Ceiling, Concrete & $\mathrm{N} / \mathrm{A}$ & $\mathrm{N} / \mathrm{A}$ & 14 & 1213 & N/A & N/A & N/A \\
\hline 28 & Ceiling, Concrete & N/A & $\mathrm{N} / \mathrm{A}$ & 8 & 1325 & N/A & N/A & $\mathrm{N} / \mathrm{A}$ \\
\hline 29 & Ceiling, Concrete & $N / A$ & N/A & 12 & 1250 & N/A & $\mathrm{N} / \mathrm{A}$ & $\mathrm{N} / \mathrm{A}$ \\
\hline 30 & Ceiling, Concrete & N/A & N/A & 17 & 1226 & N/A & N/A & $\mathrm{N} / \mathrm{A}$ \\
\hline 31 & Ceiling, Concrete & N/A & N/A & 7 & 1273 & N/A & N/A & N/A \\
\hline 32 & Ceiling, Metal & N/A & N/A & 6 & 753 & N/A & N/A & N/A \\
\hline 33 & Ceiling, Concrete & N/A & N/A & 12 & 1261 & N/A & N/A & N/A \\
\hline N/A & $\mathrm{N} / \mathrm{A}$ & N/A & N/A & N/A & $\mathrm{N} / \mathrm{A}$ & N/A & N/A & N/A \\
\hline
\end{tabular}


Survey \# 10-ER-A26-246

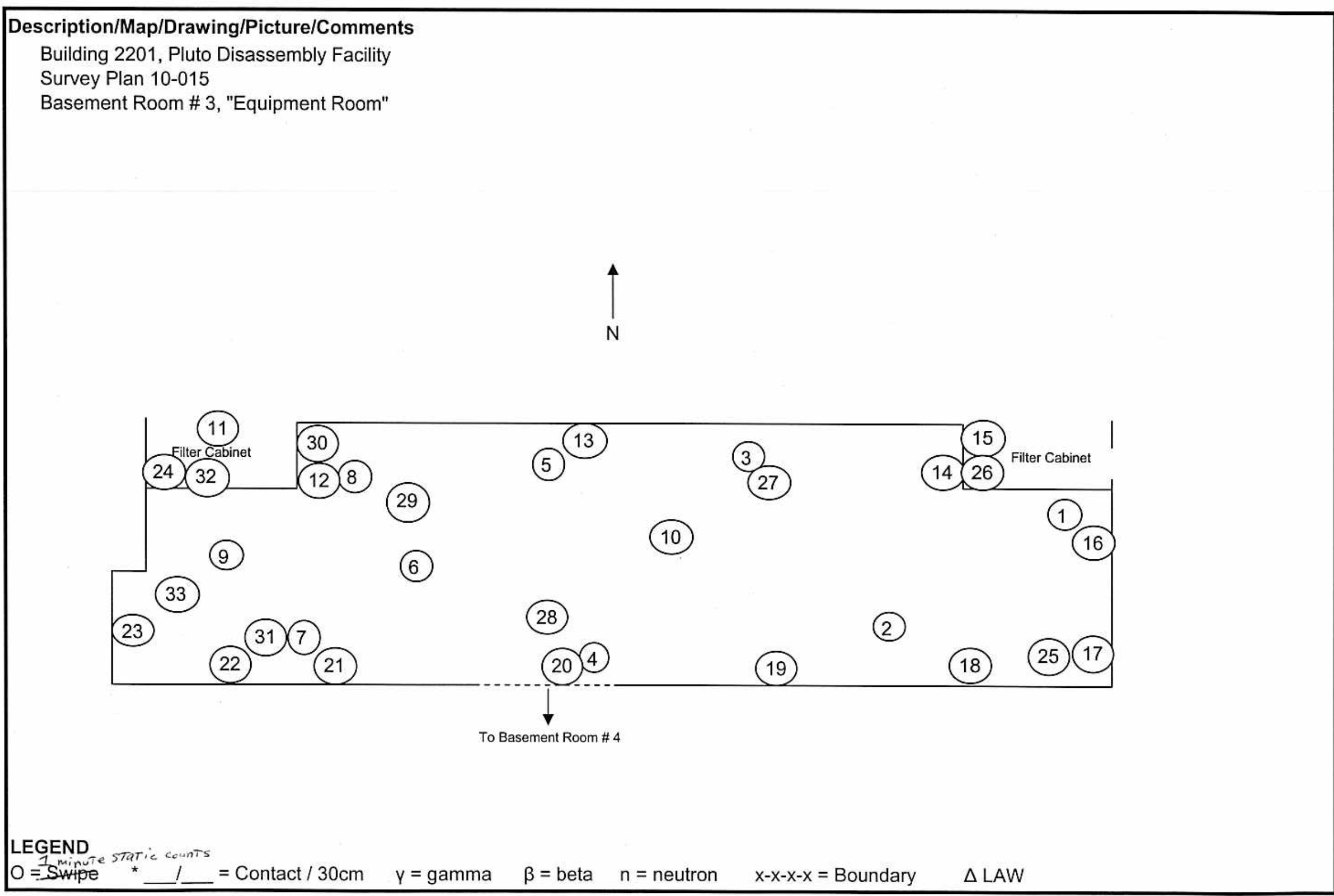




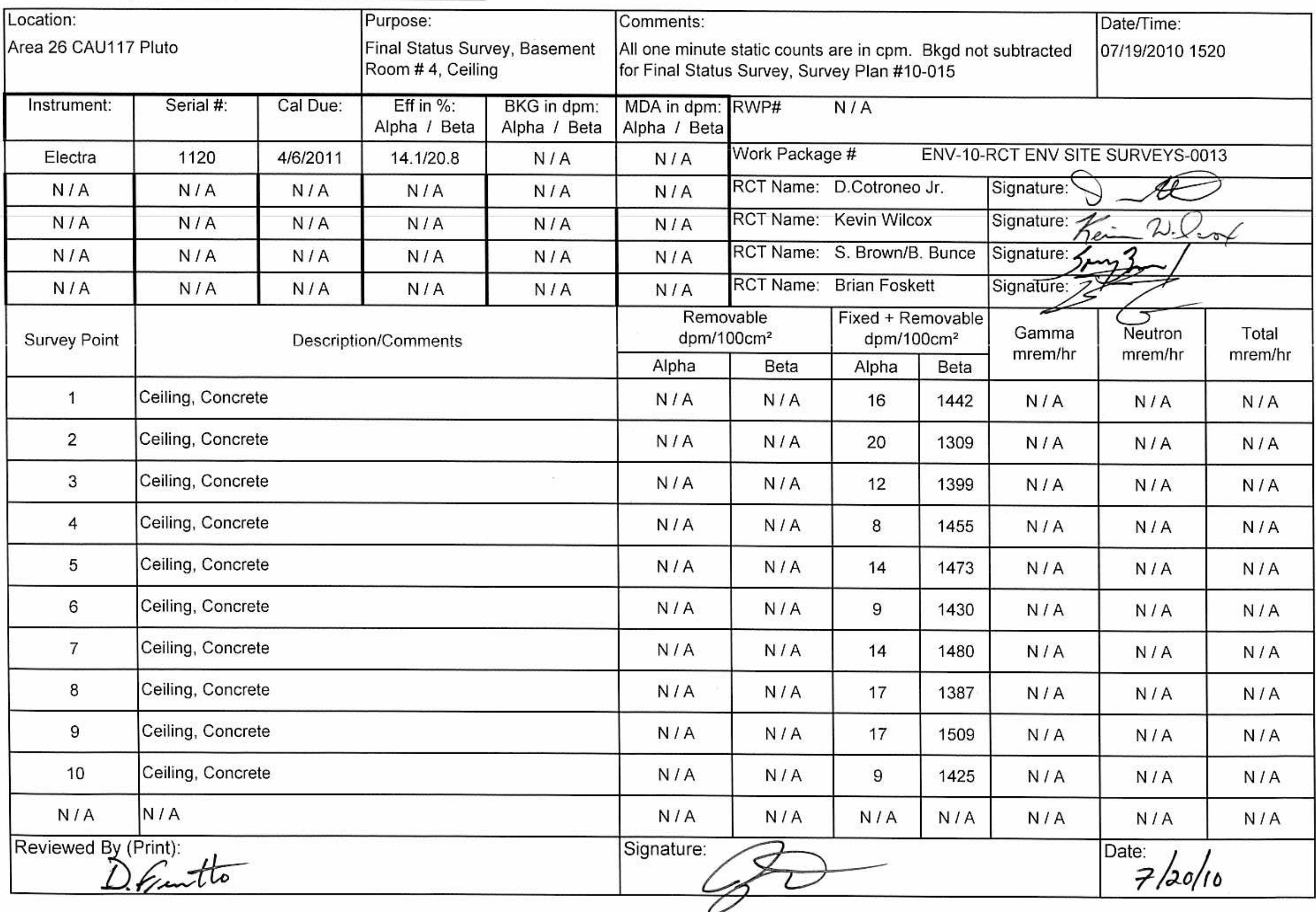


Survey \# 10-ER-A26-196

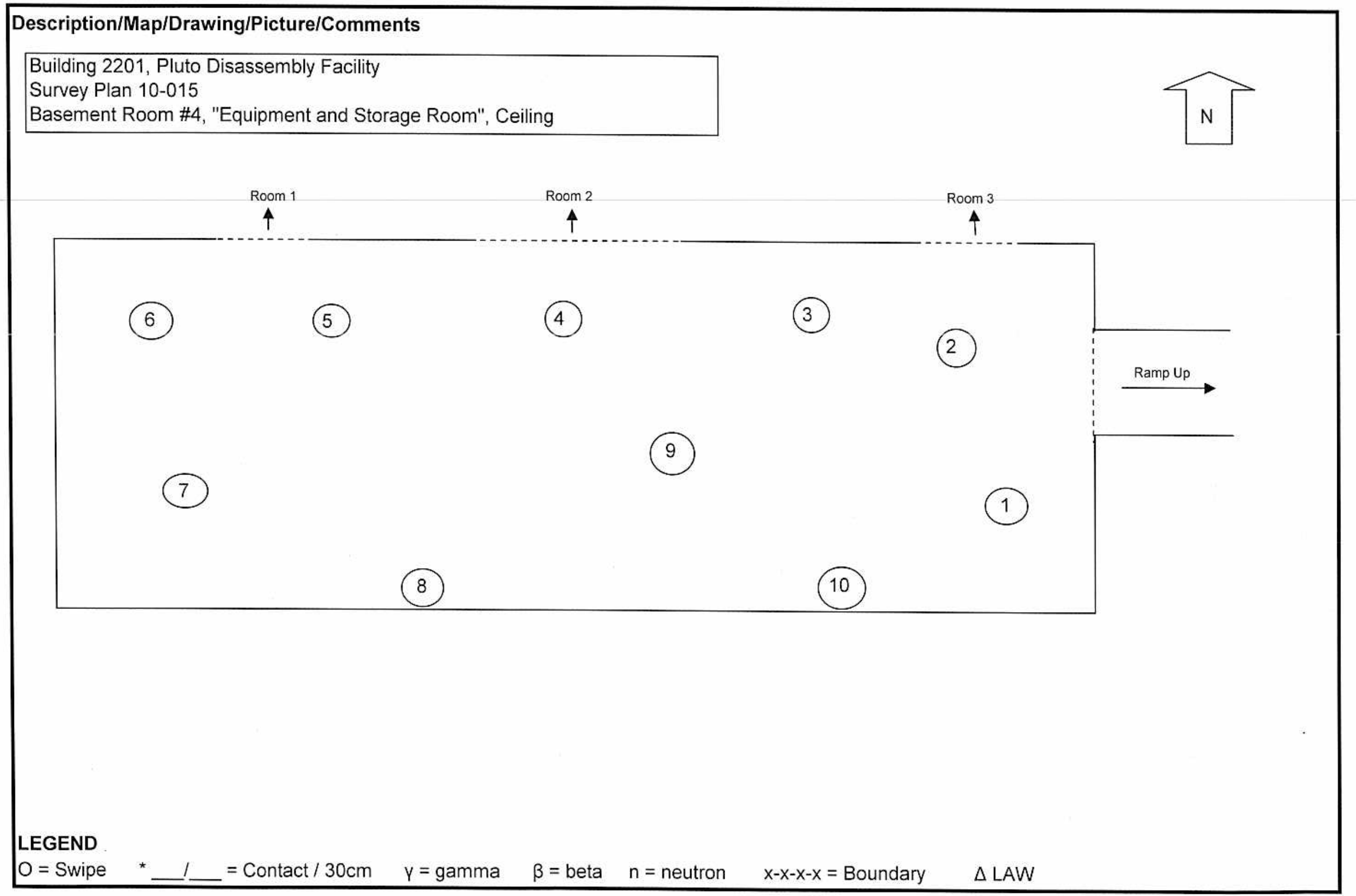




\begin{tabular}{|c|c|c|c|c|c|c|c|c|c|c|c|}
\hline \multicolumn{3}{|c|}{$\begin{array}{l}\text { Location: } \\
\text { Area } 26 \text { CAU117 Pluto }\end{array}$} & \multicolumn{2}{|c|}{$\begin{array}{l}\text { Purpose: } \\
\text { Final Status Survey, Basement } \\
\text { Room \# 4, Floor }\end{array}$} & \multicolumn{5}{|c|}{$\begin{array}{l}\text { Comments: } \\
\text { All one minute static counts are in cpm. Bkgd not subtracted } \\
\text { for Final Status Survey, Survey Plan \#10-015 }\end{array}$} & \multicolumn{2}{|c|}{$\begin{array}{l}\text { Date/Time: } \\
\text { 07/19/2010 } 1530\end{array}$} \\
\hline Instrument: & Serial \#: & Cal Due: & $\begin{array}{c}\text { Eff in \%: } \\
\text { Alpha / Beta }\end{array}$ & $\begin{array}{l}\text { BKG in dpm: } \\
\text { Alpha / Beta }\end{array}$ & \begin{tabular}{|l|} 
MDA in dpm: \\
Alpha / Beta \\
\end{tabular} & RWP\# & \multicolumn{5}{|l|}{$N / A$} \\
\hline Electra & 1504 & $4 / 6 / 2011$ & $12.9 / 19.8$ & $N / A$ & $\mathrm{~N} / \mathrm{A}$ & \multicolumn{4}{|c|}{ ENV-10-RCT ENV SI } & E SURVEYS- & \\
\hline$N / A$ & $N / A$ & $N / A$ & $N / A$ & $N / A$ & $N / A$ & \multicolumn{3}{|c|}{ RCT Name: D.Cotroneo Jr. } & Signature: & \multicolumn{2}{|c|}{$\theta-100$} \\
\hline$N / A$ & $N / A$ & $N / A$ & $N / A$ & $N / A$ & $N / A$ & \multicolumn{3}{|c|}{ RCT Name: Kevin Wilcox } & \multicolumn{3}{|c|}{ Signature: Ken W-Quex } \\
\hline$N / A$ & $N / A$ & $N / A$ & $N / A$ & $N / A$ & $N / A$ & \multicolumn{3}{|c|}{ RCT Name: S. Brown/B. Bunce } & \multicolumn{3}{|c|}{ Signature: } \\
\hline$N / A$ & $N / A$ & $N / A$ & $N / A$ & $N / A$ & $N / A$ & \multicolumn{3}{|c|}{ RCT Name: Brian Foskett } & Signature: & & \\
\hline Survey Point & & \multirow{2}{*}{\multicolumn{3}{|c|}{ Description/Comments }} & \multicolumn{2}{|c|}{$\begin{array}{l}\text { Removable } \\
\mathrm{dpm} / 100 \mathrm{~cm}^{2}\end{array}$} & \multicolumn{2}{|c|}{$\begin{array}{c}\text { Fixed + Removable } \\
\mathrm{dpm} / 100 \mathrm{~cm}^{2}\end{array}$} & \multirow{2}{*}{$\begin{array}{l}\text { Gamma } \\
\mathrm{mrem} / \mathrm{hr}\end{array}$} & \multirow{2}{*}{$\begin{array}{l}\text { Neutron } \\
\mathrm{mrem} / \mathrm{hr}\end{array}$} & \multirow{2}{*}{$\begin{array}{l}\text { Total } \\
\mathrm{mrem} / \mathrm{hr}\end{array}$} \\
\hline & & & & & Alpha & Beta & Alpha & Beta & & & \\
\hline 1 & \multicolumn{4}{|c|}{ Floor, Concrete } & $N / A$ & $N / A$ & 9 & 1304 & $N / A$ & $N / A$ & $N / A$ \\
\hline 2 & \multicolumn{4}{|c|}{ Floor, Concrete } & $N / A$ & $N / A$ & 9 & 1565 & $N / A$ & $\mathrm{~N} / \mathrm{A}$ & $N / A$ \\
\hline 3 & \multicolumn{4}{|c|}{ Floor, Concrete } & $\mathrm{N} / \mathrm{A}$ & $N / A$ & 10 & 1508 & $N / A$ & $N / A$ & $N / A$ \\
\hline 4 & \multicolumn{4}{|c|}{ Floor, Concrete } & $N / A$ & $N / A$ & 11 & 1509 & $N / A$ & $N / A$ & $N / A$ \\
\hline 5 & \multicolumn{4}{|c|}{ Floor, Concrete } & $N / A$ & $N / A$ & 7 & 1447 & $N / A$ & $N / A$ & $N / A$ \\
\hline 6 & \multicolumn{4}{|c|}{ Floor, Concrete } & $N / A$ & $N / A$ & 5 & 1486 & $N / A$ & $N / A$ & $N / A$ \\
\hline 7 & \multicolumn{4}{|c|}{ Floor, Concrete } & $N / A$ & $\mathrm{~N} / \mathrm{A}$ & 4 & 1386 & $\mathrm{~N} / \mathrm{A}$ & $N / A$ & $N / A$ \\
\hline 8 & \multicolumn{4}{|c|}{ Floor, Concrete } & $N / A$ & $N / A$ & 14 & 1467 & $N / A$ & $N / A$ & $N / A$ \\
\hline 9 & \multicolumn{4}{|c|}{ Floor, Concrete } & $N / A$ & $N / A$ & 13 & 1525 & $N / A$ & $N / A$ & $\mathrm{~N} / \mathrm{A}$ \\
\hline 10 & \multicolumn{4}{|c|}{ Floor, Concrete } & $N / A$ & $N / A$ & 6 & 1439 & $\mathrm{~N} / \mathrm{A}$ & $N / A$ & $N / A$ \\
\hline 11 & \multicolumn{4}{|c|}{ Floor, Concrete } & $N / A$ & $N / A$ & 11 & 1410 & $N / A$ & $N / A$ & $N / A$ \\
\hline \multicolumn{5}{|c|}{ Reviewed By(Print): } & \multicolumn{5}{|l|}{ Signature: } & \multicolumn{2}{|c|}{ Date: $/ 20 / 10$} \\
\hline
\end{tabular}


SURVEY \# 10-ER-A26-197

\begin{tabular}{|c|c|c|c|c|c|c|c|c|}
\hline \multirow{2}{*}{$\begin{array}{l}\text { Survey } \\
\text { Point }\end{array}$} & \multirow[t]{2}{*}{ Description/Comments } & \multicolumn{2}{|c|}{$\begin{array}{l}\text { Removable } \\
\mathrm{dpm} / 100 \mathrm{~cm}^{2}\end{array}$} & \multicolumn{2}{|c|}{$\begin{array}{l}\text { Fixed + Removable } \\
\text { dpm } / 100 \mathrm{~cm}^{2}\end{array}$} & \multirow{2}{*}{$\begin{array}{l}\text { Gamma } \\
\mathrm{mrem} / \mathrm{hr}\end{array}$} & \multirow{2}{*}{$\begin{array}{l}\text { Neutron } \\
\text { mrem } / \mathrm{hr}\end{array}$} & \multirow{2}{*}{$\begin{array}{c}\text { Total } \\
\text { mrem } / \mathrm{h}\end{array}$} \\
\hline & & Alpha & Beta & Alpha & Beta & & & \\
\hline 12 & Floor, Concrete & N/A & N/A & 13 & 1541 & $\mathrm{~N} / \mathrm{A}$ & N/A & $\mathrm{N} / \mathrm{A}$ \\
\hline 13 & Floor, Concrete & $\mathrm{N} / \mathrm{A}$ & N/A & 12 & 1444 & $\mathrm{~N} / \mathrm{A}$ & $\mathrm{N} / \mathrm{A}$ & $\mathrm{N} / \mathrm{A}$ \\
\hline 14 & Floor, Concrete & N/A & N/A & 10 & 1423 & $\mathrm{~N} / \mathrm{A}$ & N/A & $\mathrm{N} / \mathrm{A}$ \\
\hline 15 & Floor, Concrete & N/A & $\mathrm{N} / \mathrm{A}$ & 8 & 1513 & $\mathrm{~N} / \mathrm{A}$ & N/A & $\mathrm{N} / \mathrm{A}$ \\
\hline 16 & Floor, Concrete & N/A & $\mathrm{N} / \mathrm{A}$ & 9 & 1477 & $\mathrm{~N} / \mathrm{A}$ & N/A & N/A \\
\hline 17 & Floor, Concrete & N/A & $\mathrm{N} / \mathrm{A}$ & 9 & 1448 & N/A & N/A & $\mathrm{N} / \mathrm{A}$ \\
\hline 18 & Floor, Concrete & N/A & N/A & 18 & 1449 & N/A & $\mathrm{N} / \mathrm{A}$ & $\mathrm{N} / \mathrm{A}$ \\
\hline 19 & Floor, Concrete & $\mathrm{N} / \mathrm{A}$ & $\mathrm{N} / \mathrm{A}$ & 12 & 1440 & N/A & N/A & $\mathrm{N} / \mathrm{A}$ \\
\hline 20 & Floor, Concrete & $\mathrm{N} / \mathrm{A}$ & $\mathrm{N} / \mathrm{A}$ & 8 & 1422 & $\mathrm{~N} / \mathrm{A}$ & N/A & N/A \\
\hline $\mathrm{N} / \mathrm{A}$ & $N / A$ & $\mathrm{~N} / \mathrm{A}$ & $\mathrm{N} / \mathrm{A}$ & $\mathrm{N} / \mathrm{A}$ & $\mathrm{N} / \mathrm{A}$ & $\mathrm{N} / \mathrm{A}$ & N/A & $\mathrm{N} / \mathrm{A}$ \\
\hline $\mathrm{N} / \mathrm{A}$ & $N / A$ & $\mathrm{~N} / \mathrm{A}$ & $\mathrm{N} / \mathrm{A}$ & N/A & $\mathrm{N} / \mathrm{A}$ & $\mathrm{N} / \mathrm{A}$ & N/A & $\mathrm{N} / \mathrm{A}$ \\
\hline N/A & $N / A$ & $\mathrm{~N} / \mathrm{A}$ & $\mathrm{N} / \mathrm{A}$ & N/A & $\mathrm{N} / \mathrm{A}$ & N/A & $\mathrm{N} / \mathrm{A}$ & N/A \\
\hline N/A & $N / A$ & $\mathrm{~N} / \mathrm{A}$ & $\mathrm{N} / \mathrm{A}$ & $\mathrm{N} / \mathrm{A}$ & $\mathrm{N} / \mathrm{A}$ & N/A & $\mathrm{N} / \mathrm{A}$ & $\mathrm{N} / \mathrm{A}$ \\
\hline N/A & $N / A$ & $\mathrm{~N} / \mathrm{A}$ & N/A & N/A & $\mathrm{N} / \mathrm{A}$ & N/A & $\mathrm{N} / \mathrm{A}$ & $\mathrm{N} / \mathrm{A}$ \\
\hline N/A & $N / A$ & $\mathrm{~N} / \mathrm{A}$ & N/A & N/A & $\mathrm{N} / \mathrm{A}$ & N/A & N/A & $\mathrm{N} / \mathrm{A}$ \\
\hline $\mathrm{N} / \mathrm{A}$ & $\mathrm{N} / \mathrm{A}$ & $\mathrm{N} / \mathrm{A}$ & N/A & N/A & N/A & N/A & N/A & N/A \\
\hline $\mathrm{N} / \mathrm{A}$ & $\mathrm{N} / \mathrm{A}$ & N/A & $\mathrm{N} / \mathrm{A}$ & N/A & N/A & N/A & $\mathrm{N} / \mathrm{A}$ & $\mathrm{N} / \mathrm{A}$ \\
\hline $\mathrm{N} / \mathrm{A}$ & $N / A$ & $\mathrm{~N} / \mathrm{A}$ & $\mathrm{N} / \mathrm{A}$ & $\mathrm{N} / \mathrm{A}$ & $\mathrm{N} / \mathrm{A}$ & $\mathrm{N} / \mathrm{A}$ & $\mathrm{N} / \mathrm{A}$ & $\mathrm{N} / \mathrm{A}$ \\
\hline N/A & $N / A$ & $\mathrm{~N} / \mathrm{A}$ & N/A & $\mathrm{N} / \mathrm{A}$ & $\mathrm{N} / \mathrm{A}$ & $\mathrm{N} / \mathrm{A}$ & N/A & N/A \\
\hline N/A & N/A & $\mathrm{N} / \mathrm{A}$ & N/A & N/A & $\mathrm{N} / \mathrm{A}$ & $\mathrm{N} / \mathrm{A}$ & $\mathrm{N} / \mathrm{A}$ & N/A \\
\hline N/A & N/A & $\mathrm{N} / \mathrm{A}$ & $\mathrm{N} / \mathrm{A}$ & $\mathrm{N} / \mathrm{A}$ & N/A & $\mathrm{N} / \mathrm{A}$ & $\mathrm{N} / \mathrm{A}$ & N/A \\
\hline $\mathrm{N} / \mathrm{A}$ & N/A & N/A & N/A & N/A & N/A & N/A & N/A & N/A \\
\hline N/A & N/A & N/A & $\mathrm{N} / \mathrm{A}$ & N/A & $\mathrm{N} / \mathrm{A}$ & N/A & N/A & $\mathrm{N} / \mathrm{A}$ \\
\hline
\end{tabular}




\section{Survey \# 10-ER-A26-197}

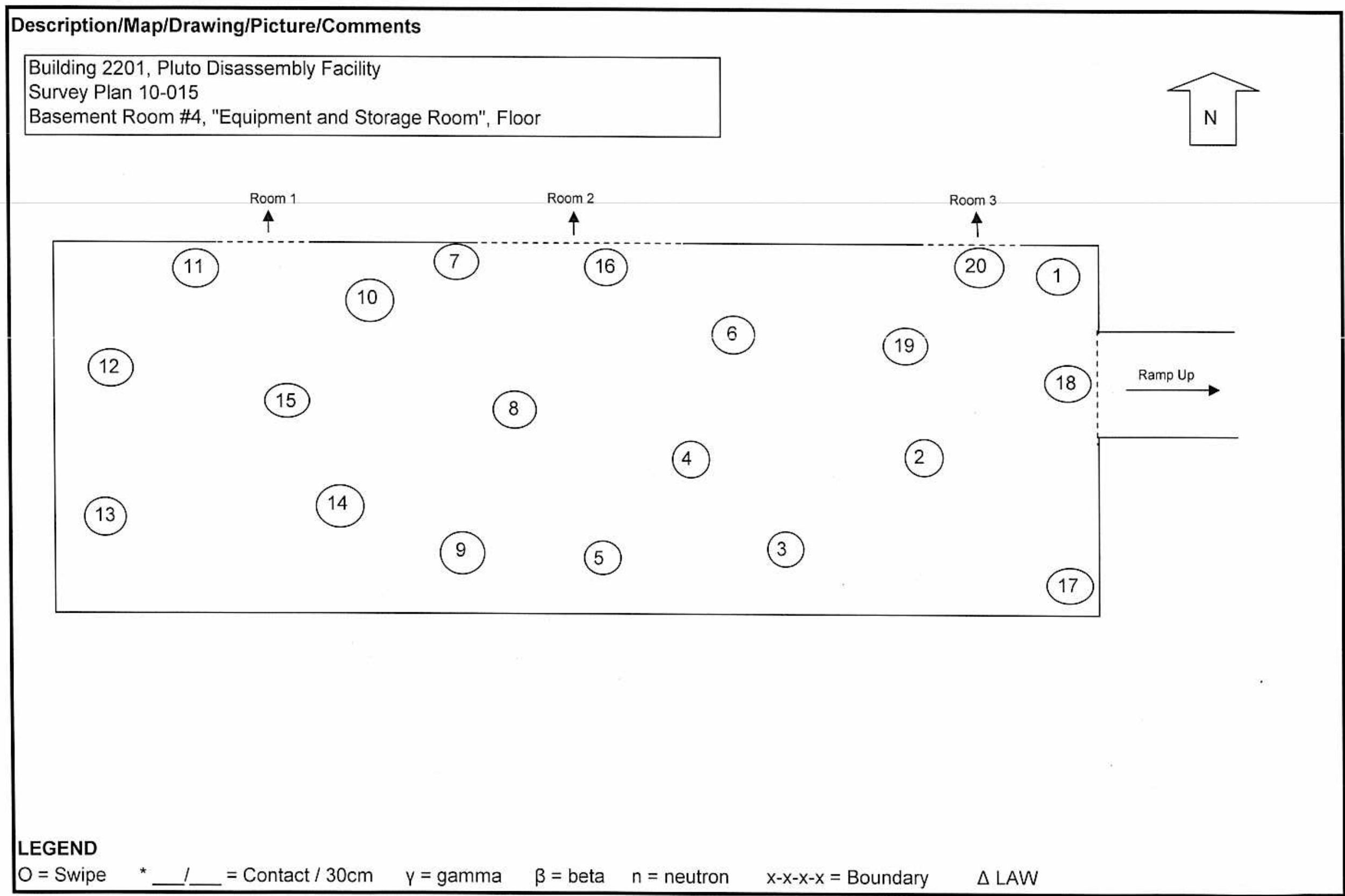


SURVEY \# 10-ER-A26-195

\begin{tabular}{|c|c|c|c|c|c|c|c|c|c|c|c|}
\hline \multicolumn{3}{|c|}{$\begin{array}{l}\text { Location: } \\
\text { Area } 26 \text { CAU117 Pluto }\end{array}$} & \multicolumn{2}{|c|}{$\begin{array}{l}\text { Purpose: } \\
\text { Final Status Survey, Basement } \\
\text { Room \# 4, Walls }\end{array}$} & \multicolumn{5}{|c|}{$\begin{array}{l}\text { Comments: } \\
\text { All one minute static counts are in cpm. Bkgd not subtracted } \\
\text { for Final Status Survey, Survey Plan \#10-015 }\end{array}$} & \multicolumn{2}{|c|}{$\begin{array}{l}\text { Date/Time: } \\
\text { 07/19/2010 } 1500\end{array}$} \\
\hline Instrument: & Serial \#: & Cal Due: & $\begin{array}{c}\text { Eff in \%: } \\
\text { Alpha / Beta }\end{array}$ & $\begin{array}{l}\text { BKG in dpm: } \\
\text { Alpha / Beta }\end{array}$ & \begin{tabular}{|l|} 
MDA in dpm: \\
Alpha / Beta
\end{tabular} & RWP\# & \multicolumn{5}{|l|}{$\mathrm{N} / \mathrm{A}$} \\
\hline Electra & 1120 & $4 / 6 / 2011$ & $14.1 / 20.8$ & $N / A$ & $N / A$ & \multicolumn{4}{|c|}{ Work Package \# } & E SURVEYS & \\
\hline$N / A$ & $N / A$ & $N / A$ & $N / A$ & $\mathrm{~N} / \mathrm{A}$ & $N / A$ & \multicolumn{3}{|c|}{ RCT Name: D.Cotroneo Jr. } & \multicolumn{3}{|c|}{ Signature: U $-W$} \\
\hline$N / A$ & $N / A$ & $\mathrm{~N} / \mathrm{A}$ & $N / A$ & $N / A$ & $N / A$ & \multicolumn{3}{|c|}{ RCT Name: Kevin Wilcox } & \multicolumn{3}{|c|}{ Signature: Ken W.leox } \\
\hline$N / A$ & $N / A$ & $N / A$ & $N / A$ & $\mathrm{~N} / \mathrm{A}$ & $N / A$ & \multicolumn{3}{|c|}{ RCT Name: S. Brown/B. Bunce } & \multirow{2}{*}{\multicolumn{3}{|c|}{ Signature: }} \\
\hline $\mathrm{N} / \mathrm{A}$ & $N / A$ & $N / A$ & $N / A$ & $N / A$ & $\mathrm{~N} / \mathrm{A}$ & RCT Name: & Brian Fos & & & & \\
\hline \multirow[t]{2}{*}{ Survey Point } & \multirow{2}{*}{\multicolumn{4}{|c|}{ Description/Comments }} & \multicolumn{2}{|c|}{$\begin{array}{l}\text { Removable } \\
\mathrm{dpm} / 100 \mathrm{~cm}^{2}\end{array}$} & \multicolumn{2}{|c|}{$\begin{array}{c}\text { Fixed + Removable } \\
\mathrm{dpm} / 100 \mathrm{~cm}^{2}\end{array}$} & \multirow{2}{*}{$\begin{array}{l}\text { Gamma } \\
\mathrm{mrem} / \mathrm{hr}\end{array}$} & \multirow{2}{*}{$\begin{array}{l}\text { Neutron } \\
\mathrm{mrem} / \mathrm{hr}\end{array}$} & \multirow{2}{*}{$\begin{array}{c}\text { Total } \\
\mathrm{mrem} / \mathrm{hr}\end{array}$} \\
\hline & & & & & Alpha & Beta & Alpha & Beta & & & \\
\hline 1 & \multicolumn{4}{|c|}{ West Wall, Concrete } & $N / A$ & $N / A$ & 24 & 1221 & $N / A$ & $N / A$ & $N / A$ \\
\hline 2 & \multicolumn{4}{|c|}{ West Wall, Concrete } & $N / A$ & $N / A$ & 20 & 1209 & $N / A$ & $\mathrm{~N} / \mathrm{A}$ & $N / A$ \\
\hline 3 & \multicolumn{4}{|c|}{ West Wall, Concrete } & $N / A$ & $\mathrm{~N} / \mathrm{A}$ & 6 & 1265 & $\mathrm{~N} / \mathrm{A}$ & $\mathrm{N} / \mathrm{A}$ & $N / A$ \\
\hline 4 & \multicolumn{4}{|c|}{ West Wall, Concrete } & $N / A$ & $N / A$ & 20 & 1220 & $N / A$ & $N / A$ & $N / A$ \\
\hline 5 & \multicolumn{4}{|c|}{ North Wall, Concrete } & $N / A$ & $N / A$ & 19 & 1266 & $N / A$ & $N / A$ & $\mathrm{~N} / \mathrm{A}$ \\
\hline 6 & \multicolumn{4}{|c|}{ North Wall, Concrete } & $\mathrm{N} / \mathrm{A}$ & $\mathrm{N} / \mathrm{A}$ & 10 & 1154 & $N / A$ & $N / A$ & $N / A$ \\
\hline 7 & \multicolumn{4}{|c|}{ North Wall, Concrete } & $N / A$ & $N / A$ & 20 & 1196 & $N / A$ & $N / A$ & $\mathrm{~N} / \mathrm{A}$ \\
\hline 8 & \multicolumn{4}{|c|}{ North Wall, Concrete } & $\mathrm{N} / \mathrm{A}$ & $N / A$ & 12 & 1276 & $N / A$ & $N / A$ & $N / A$ \\
\hline 9 & \multicolumn{4}{|c|}{ North Wall, Concrete } & $N / A$ & $N / A$ & 17 & 1172 & $N / A$ & $N / A$ & $N / A$ \\
\hline 10 & \multicolumn{4}{|c|}{ North Wall, Concrete } & $N / A$ & $N / A$ & 7 & 1197 & $N / A$ & $N / A$ & $N / A$ \\
\hline 11 & \multicolumn{4}{|c|}{ East Wall, Concrete } & $N / A$ & $N / A$ & 8 & 1198 & $N / A$ & $\mathrm{~N} / \mathrm{A}$ & $N / A$ \\
\hline \multicolumn{5}{|c|}{ Reviewed By (Print): } & Signatur & & & & & \multicolumn{2}{|c|}{ Date: $7 / 20 / 10$} \\
\hline
\end{tabular}


SURVEY \# 10-ER-A26-195

\begin{tabular}{|c|c|c|c|c|c|c|c|c|}
\hline \multirow{2}{*}{$\begin{array}{l}\text { Survey } \\
\text { Point }\end{array}$} & \multirow[t]{2}{*}{ Description/Comments } & \multicolumn{2}{|c|}{$\begin{array}{l}\text { Removable } \\
\mathrm{dpm} / 100 \mathrm{~cm}^{2}\end{array}$} & \multicolumn{2}{|c|}{$\begin{array}{c}\text { Fixed + Removable } \\
\mathrm{dpm} / 100 \mathrm{~cm}^{2}\end{array}$} & \multirow{2}{*}{$\begin{array}{c}\text { Gamma } \\
\mathrm{mrem} / \mathrm{hr}\end{array}$} & \multirow{2}{*}{$\begin{array}{l}\text { Neutron } \\
\mathrm{mrem} / \mathrm{hr}\end{array}$} & \multirow{2}{*}{$\begin{array}{c}\text { Total } \\
\text { mrem/hr }\end{array}$} \\
\hline & & Alpha & Beta & Alpha & Beta & & & \\
\hline 12 & East Wall, Concrete & N/A & N/A & 16 & 1272 & $\mathrm{~N} / \mathrm{A}$ & $\mathrm{N} / \mathrm{A}$ & N/A \\
\hline 13 & East Wall, Concrete & N/A & N/A & 20 & 1288 & N/A & $\mathrm{N} / \mathrm{A}$ & $\mathrm{N} / \mathrm{A}$ \\
\hline 14 & East Wall, Concrete & N/A & N/A & 17 & 1301 & N/A & $\mathrm{N} / \mathrm{A}$ & $\mathrm{N} / \mathrm{A}$ \\
\hline 15 & South Wall, Concrete & $\mathrm{N} / \mathrm{A}$ & N/A & 12 & 1355 & $\mathrm{~N} / \mathrm{A}$ & $\mathrm{N} / \mathrm{A}$ & $\mathrm{N} / \mathrm{A}$ \\
\hline 16 & South Wall, Concrete & $\mathrm{N} / \mathrm{A}$ & $\mathrm{N} / \mathrm{A}$ & 22 & 1261 & N/A & N/A & N/A \\
\hline 17 & South Wall, Concrete & $\mathrm{N} / \mathrm{A}$ & $\mathrm{N} / \mathrm{A}$ & 12 & 1241 & N/A & N/A & $\mathrm{N} / \mathrm{A}$ \\
\hline 18 & South Wall, Concrete & N/A & $\mathrm{N} / \mathrm{A}$ & 15 & 1220 & $\mathrm{~N} / \mathrm{A}$ & N/A & $\mathrm{N} / \mathrm{A}$ \\
\hline 19 & South Wall, Concrete & N/A & N/A & 14 & 1250 & N/A & $\mathrm{N} / \mathrm{A}$ & N/A \\
\hline N/A & $\mathrm{N} / \mathrm{A}$ & N/A & $\mathrm{N} / \mathrm{A}$ & $\mathrm{N} / \mathrm{A}$ & $\mathrm{N} / \mathrm{A}$ & $\mathrm{N} / \mathrm{A}$ & $\mathrm{N} / \mathrm{A}$ & N/A \\
\hline N/A & $N / A$ & N/A & $\mathrm{N} / \mathrm{A}$ & N/A & $\mathrm{N} / \mathrm{A}$ & N/A & N/A & N/A \\
\hline N/A & $N / A$ & $\mathrm{~N} / \mathrm{A}$ & $\mathrm{N} / \mathrm{A}$ & $\mathrm{N} / \mathrm{A}$ & $\mathrm{N} / \mathrm{A}$ & N/A & N/A & $\mathrm{N} / \mathrm{A}$ \\
\hline N/A & $N / A$ & $\mathrm{~N} / \mathrm{A}$ & $\mathrm{N} / \mathrm{A}$ & $\mathrm{N} / \mathrm{A}$ & $\mathrm{N} / \mathrm{A}$ & $\mathrm{N} / \mathrm{A}$ & $\mathrm{N} / \mathrm{A}$ & $\mathrm{N} / \mathrm{A}$ \\
\hline N/A & $N / A$ & N/A & N/A & $\mathrm{N} / \mathrm{A}$ & N/A & N/A & $\mathrm{N} / \mathrm{A}$ & $\mathrm{N} / \mathrm{A}$ \\
\hline N/A & $\mathrm{N} / \mathrm{A}$ & N/A & N/A & $\mathrm{N} / \mathrm{A}$ & $\mathrm{N} / \mathrm{A}$ & N/A & N/A & N/A \\
\hline $\mathrm{N} / \mathrm{A}$ & $N / A$ & N/A & N/A & $\mathrm{N} / \mathrm{A}$ & N/A & N/A & N/A & N/A \\
\hline $\mathrm{N} / \mathrm{A}$ & $N / A$ & $\mathrm{~N} / \mathrm{A}$ & N/A & $\mathrm{N} / \mathrm{A}$ & N/A & N/A & N/A & $\mathrm{N} / \mathrm{A}$ \\
\hline $\mathrm{N} / \mathrm{A}$ & $N / A$ & $\mathrm{~N} / \mathrm{A}$ & $\mathrm{N} / \mathrm{A}$ & N/A & $\mathrm{N} / \mathrm{A}$ & N/A & N/A & N/A \\
\hline $\mathrm{N} / \mathrm{A}$ & $N / A$ & N/A & $\mathrm{N} / \mathrm{A}$ & N/A & $\mathrm{N} / \mathrm{A}$ & N/A & $\mathrm{N} / \mathrm{A}$ & $\mathrm{N} / \mathrm{A}$ \\
\hline N/A & $N / A$ & N/A & $\mathrm{N} / \mathrm{A}$ & $\mathrm{N} / \mathrm{A}$ & $\mathrm{N} / \mathrm{A}$ & N/A & $\mathrm{N} / \mathrm{A}$ & N/A \\
\hline $\mathrm{N} / \mathrm{A}$ & N/A & N/A & $\mathrm{N} / \mathrm{A}$ & $\mathrm{N} / \mathrm{A}$ & $\mathrm{N} / \mathrm{A}$ & $\mathrm{N} / \mathrm{A}$ & $\mathrm{N} / \mathrm{A}$ & N/A \\
\hline N/A & N/A & N/A & $\mathrm{N} / \mathrm{A}$ & N/A & N/A & N/A & N/A & $\mathrm{N} / \mathrm{A}$ \\
\hline N/A & N/A & $\mathrm{N} / \mathrm{A}$ & $\mathrm{N} / \mathrm{A}$ & $\mathrm{N} / \mathrm{A}$ & N/A & N/A & $\mathrm{N} / \mathrm{A}$ & $\mathrm{N} / \mathrm{A}$ \\
\hline $\mathrm{N} / \mathrm{A}$ & N/A & N/A & $\mathrm{N} / \mathrm{A}$ & $\mathrm{N} / \mathrm{A}$ & $\mathrm{N} / \mathrm{A}$ & $\mathrm{N} / \mathrm{A}$ & $\mathrm{N} / \mathrm{A}$ & N/A \\
\hline
\end{tabular}


Survey \# 10-ER-A26-195

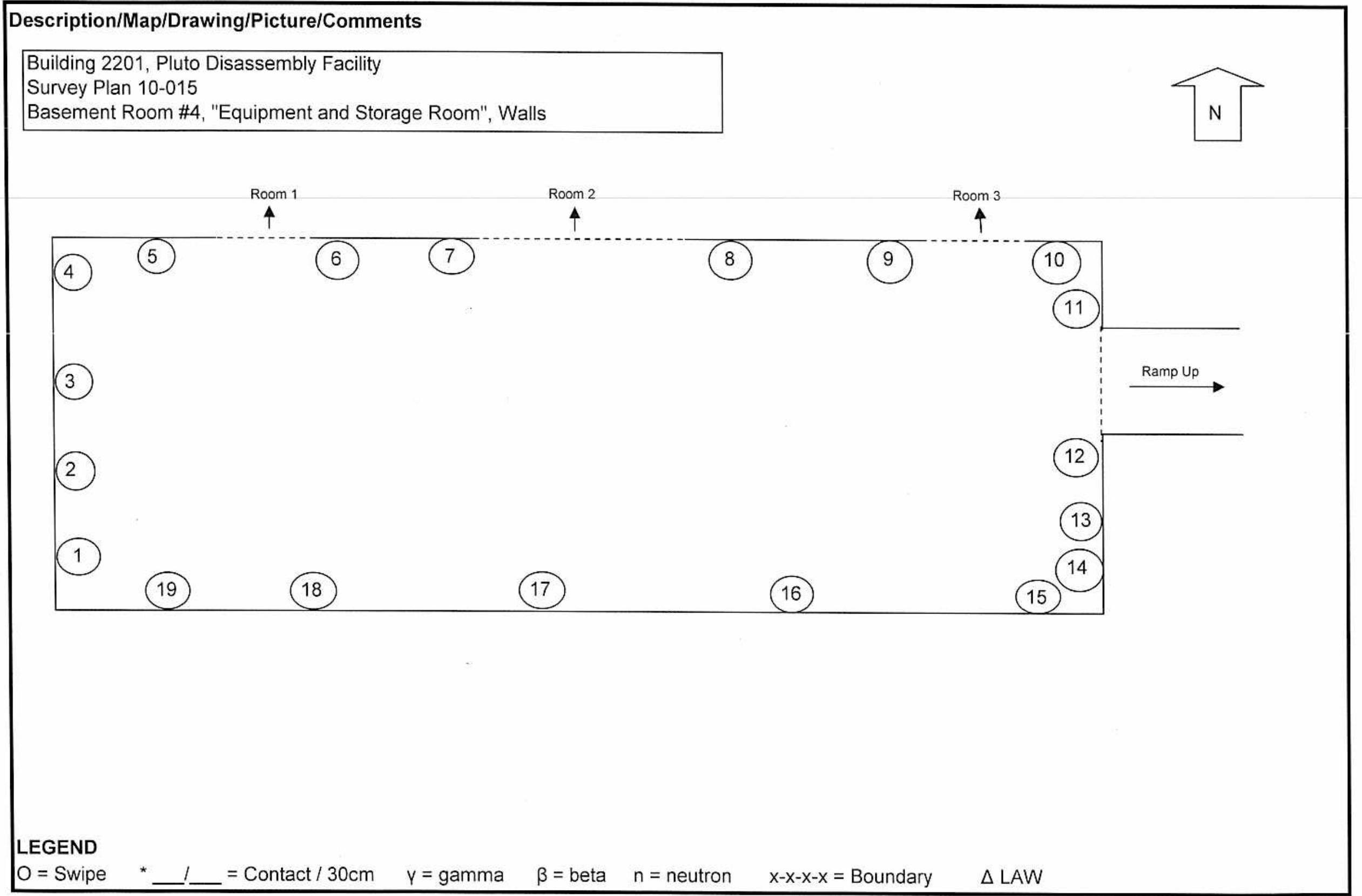


SURVEY \#

10-ER-A26-230

\begin{tabular}{|c|c|c|c|c|c|c|c|c|c|c|c|}
\hline \multicolumn{3}{|c|}{$\begin{array}{l}\text { Location: } \\
\text { Area } 26 \text { CAU117 Pluto }\end{array}$} & \multicolumn{2}{|c|}{$\begin{array}{l}\text { Purpose: } \\
\text { Final Status Survey, Class } 3 \\
\text { Areas, Rooms 105, 108, } 109\end{array}$} & \multicolumn{5}{|c|}{$\begin{array}{l}\text { Comments: } \\
\text { All one minute static counts are in cpm. Bkgd not subtracted for } \\
\text { Final Status Survey, Survey Plan } \# 10-015\end{array}$} & \multicolumn{2}{|c|}{$\begin{array}{l}\text { Date/Time: } \\
\text { 07/29/2010 } 1400\end{array}$} \\
\hline Instrument: & Serial \#: & Cal Due: & $\begin{array}{c}\text { Eff in \%: } \\
\text { Alpha / Beta }\end{array}$ & $\begin{array}{l}\text { BKG in dpm: } \\
\text { Alpha / Beta }\end{array}$ & \begin{tabular}{|l|} 
MDA in dpm: \\
Alpha / Beta \\
\end{tabular} & RWP\# & \multicolumn{5}{|l|}{$\mathrm{N} / \mathrm{A}$} \\
\hline Electra & 2125 & $4 / 6 / 2011$ & $14.4 / 18.2$ & $\mathrm{~N} / \mathrm{A}$ & $\mathrm{N} / \mathrm{A}$ & \multicolumn{6}{|c|}{ 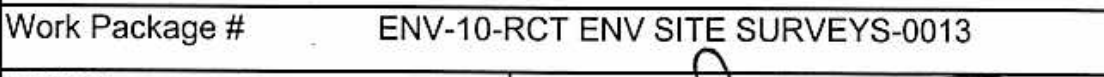 } \\
\hline$N / A$ & $N / A$ & $\mathrm{~N} / \mathrm{A}$ & $\mathrm{N} / \mathrm{A}$ & $\mathrm{N} / \mathrm{A}$ & $\mathrm{N} / \mathrm{A}$ & \multicolumn{3}{|c|}{ RCT Name: D.Cotroneo Jr. } & Signature: $\quad$ & & $\Rightarrow$ \\
\hline$N / A$ & $N / A$ & $N / A$ & $\mathrm{~N} / \mathrm{A}$ & $\mathrm{N} / \mathrm{A}$ & $\mathrm{N} / \mathrm{A}$ & \multicolumn{3}{|c|}{ RCT Name: Kevin Wilcox } & \multicolumn{3}{|c|}{ Signature: $z_{i}=2$ Weof } \\
\hline$N / A$ & $N / A$ & $N / A$ & $N / A$ & $N / A$ & $N / A$ & \multicolumn{3}{|c|}{ RCT Name: $\mathrm{N} / \mathrm{A}$} & Signature: & \multicolumn{2}{|l|}{$N / A$} \\
\hline $\mathrm{N} / \mathrm{A}$ & $\mathrm{N} / \mathrm{A}$ & $N / A$ & $N / A$ & $\mathrm{~N} / \mathrm{A}$ & $\mathrm{N} / \mathrm{A}$ & \multicolumn{3}{|c|}{ RCT Name: N/A } & Signature: & \multicolumn{2}{|l|}{$N / A$} \\
\hline \multirow[t]{2}{*}{ Survey Point } & \multirow{2}{*}{\multicolumn{4}{|c|}{ Description/Comments }} & \multicolumn{2}{|c|}{$\begin{array}{l}\text { Removable } \\
\text { dpm } / 100 \mathrm{~cm}^{2}\end{array}$} & \multicolumn{2}{|c|}{\begin{tabular}{|c|} 
Fixed + Removable \\
$\mathrm{dpm} / 100 \mathrm{~cm}^{2}$
\end{tabular}} & \multirow{2}{*}{$\begin{array}{l}\text { Gamma } \\
\mathrm{mrem} / \mathrm{hr}\end{array}$} & \multirow{2}{*}{$\begin{array}{l}\text { Neutron } \\
\mathrm{mrem} / \mathrm{hr}\end{array}$} & \multirow{2}{*}{$\begin{array}{l}\text { Total } \\
\mathrm{mrem} / \mathrm{hr}\end{array}$} \\
\hline & & & & & Alpha & Beta & Alpha & Beta & & & \\
\hline$N / A$ & \multicolumn{4}{|c|}{ **Judgemental Scans of Class 3 Areas...** } & $N / A$ & $N / A$ & $N / A$ & $N / A$ & $N / A$ & $N / A$ & $N / A$ \\
\hline $\mathrm{N} / \mathrm{A}$ & \multicolumn{4}{|c|}{ **...Adjacent to Class 1 and Class 2 Areas $^{* *}$} & $\mathrm{~N} / \mathrm{A}$ & $N / A$ & $N / A$ & $N / A$ & $N / A$ & N/A & $N / A$ \\
\hline 1 & \multicolumn{4}{|c|}{ Room 109, Bottom of Ramp, Concrete Floor } & $N / A$ & $N / A$ & $N / A$ & $N / A$ & $N / A$ & $N / A$ & $N / A$ \\
\hline$N / A$ & \multicolumn{4}{|c|}{${ }^{\star \star}$ Performed scan surveys for both Alpha and Beta...** } & $N / A$ & $N / A$ & $N / A$ & $N / A$ & $N / A$ & $N / A$ & $N / A$ \\
\hline$N / A$ & \multicolumn{4}{|c|}{$\star \star \ldots$ on $100 \%$ of survey unit. No trigger levels exceded ${ }^{\star \star}$} & $N / A$ & $N / A$ & $N / A$ & $N / A$ & $N / A$ & $N / A$ & $N / A$ \\
\hline 2 & \multicolumn{4}{|c|}{ Room 105, Floor, Concrete } & $N / A$ & $N / A$ & $N / A$ & $N / A$ & $N / A$ & $N / A$ & $N / A$ \\
\hline$N / A$ & \multicolumn{4}{|c|}{ **Performed scan surveys for both Alpha and Beta... ${ }^{\star \star}$} & $N / A$ & $\mathrm{~N} / \mathrm{A}$ & $N / A$ & $N / A$ & $N / A$ & $N / A$ & $N / A$ \\
\hline $\mathrm{N} / \mathrm{A}$ & \multicolumn{4}{|c|}{$\star \star \ldots$ on $100 \%$ of survey unit. No trigger levels exceded ${ }^{\star \star}$} & $N / A$ & $\mathrm{~N} / \mathrm{A}$ & $N / A$ & $N / A$ & $N / A$ & $N / A$ & $N / A$ \\
\hline 3 & \multicolumn{4}{|c|}{ Room 108, Floor, Concrete } & $N / A$ & $N / A$ & $N / A$ & $N / A$ & $N / A$ & $N / A$ & $N / A$ \\
\hline$N / A$ & \multicolumn{4}{|c|}{${ }^{\star *}$ Performed scan surveys for both Alpha and Beta...** } & $N / A$ & $N / A$ & $N / A$ & $N / A$ & $N / A$ & $N / A$ & $N / A$ \\
\hline$N / A$ & \multicolumn{4}{|c|}{${ }^{\star \star} \ldots$ on $100 \%$ of survey unit. No trigger levels exceded ${ }^{\star \star}$} & $N / A$ & $N / A$ & $N / A$ & $N / A$ & $N / A$ & $N / A$ & $N / A$ \\
\hline \multicolumn{5}{|c|}{ Reviewed By (Print): } & \multicolumn{5}{|l|}{ Signature: } & $\begin{array}{r}\text { Date: } \\
8 / 2 /\end{array}$ & \\
\hline
\end{tabular}


SURVEY \#

10-ER-A26-165

\begin{tabular}{|c|c|c|c|c|c|c|c|c|c|c|c|}
\hline \multicolumn{3}{|c|}{$\begin{array}{l}\text { Location: } \\
\text { Area } 26 \text { CAU117 Pluto }\end{array}$} & \multicolumn{2}{|c|}{$\begin{array}{l}\text { Purpose: } \\
\text { Final Status Status, Ceiling of } \\
\text { Main Room } 101\end{array}$} & Comments: & \multicolumn{4}{|c|}{$\begin{array}{l}\text { All one minute static counts are in } \mathrm{cpm} \text { Bkgd not subtracted } \\
\text { for Final Status Survey, Survey Plan \#10-015 }\end{array}$} & \multicolumn{2}{|c|}{$\begin{array}{l}\text { Date/Time: } \\
\text { 07/08/2010 } 1300\end{array}$} \\
\hline Instrument: & Serial \#: & Cal Due: & $\begin{array}{c}\text { Eff in \%: } \\
\text { Alpha / Beta }\end{array}$ & $\begin{array}{l}\text { BKG in dpm: } \\
\text { Alpha / Beta }\end{array}$ & \begin{tabular}{|l|} 
MDA in dpm: \\
Alpha / Beta
\end{tabular} & RWP\# & \multicolumn{5}{|l|}{$\mathrm{N} / \mathrm{A}$} \\
\hline Electra & 1120 & $4 / 6 / 2011$ & $14.1 / 20.8$ & $N / A$ & $N / A$ & \multicolumn{6}{|c|}{ Work Package \# $\quad$ ENV-10-RCT ENV SITE SURVEYS-0013 } \\
\hline$N / A$ & $N / A$ & $N / A$ & $N / A$ & $N / A$ & $N / A$ & \multicolumn{3}{|c|}{ RCT Name: D.Cotroneo Jr. } & \multicolumn{3}{|c|}{ Signature: $f-C E$} \\
\hline$N / A$ & $N / A$ & $N / A$ & $N / A$ & $N / A$ & $N / A$ & \multicolumn{3}{|c|}{ RCT Name: Kevin Wilcox } & \multirow{2}{*}{\multicolumn{3}{|c|}{ Signature:Ter Wheof }} \\
\hline$N / A$ & $N / A$ & $N / A$ & $N / A$ & $N / A$ & $N / A$ & \multicolumn{3}{|c|}{ RCT Name: S.Brown/B.Bunce } & & & \\
\hline$N / A$ & $N / A$ & $N / A$ & $N / A$ & $N / A$ & $N / A$ & \multicolumn{3}{|c|}{ RCT Name: Brian Foskett } & Signature: & & \\
\hline \multirow[t]{2}{*}{ Survey Point } & \multirow[t]{2}{*}{ Description/Comments } & \multirow{2}{*}{\multicolumn{3}{|c|}{ Description/Comments }} & \multicolumn{2}{|c|}{$\begin{array}{l}\text { Removable } \\
\mathrm{dpm} / 100 \mathrm{~cm}^{2}\end{array}$} & \multicolumn{2}{|c|}{$\begin{array}{c}\text { Fixed + Removable } \\
\mathrm{dpm} / 100 \mathrm{~cm}^{2}\end{array}$} & \multirow{2}{*}{$\begin{array}{l}\text { Gamma } \\
\text { mrem/hr }\end{array}$} & \multirow{2}{*}{$\begin{array}{l}\text { Neutron } \\
\mathrm{mrem} / \mathrm{hr}\end{array}$} & \multirow{2}{*}{$\begin{array}{c}\text { Total } \\
\mathrm{mrem} / \mathrm{hr}\end{array}$} \\
\hline & & & & & Alpha & Beta & Alpha & Beta & & & \\
\hline$N / A$ & \multicolumn{4}{|c|}{ Ceiling of Main Room (east of mezzanine to roll-up door) } & $N / A$ & $N / A$ & $\mathrm{~N} / \mathrm{A}$ & $N / A$ & $N / A$ & $N / A$ & $N / A$ \\
\hline 1 & \multicolumn{4}{|c|}{ Ceiling Concrete } & $N / A$ & $N / A$ & 8 & 1413 & $N / A$ & $N / A$ & $N / A$ \\
\hline 2 & \multicolumn{4}{|c|}{ Ceiling Concrete } & $N / A$ & $N / A$ & 9 & 1427 & $N / A$ & $N / A$ & $N / A$ \\
\hline 3 & \multicolumn{4}{|c|}{ Ceiling Concrete } & $N / A$ & $N / A$ & 10 & 1309 & $N / A$ & $N / A$ & $N / A$ \\
\hline 4 & \multicolumn{4}{|c|}{ Ceiling Concrete } & $N / A$ & $N / A$ & 1 & 1389 & $N / A$ & $N / A$ & $N / A$ \\
\hline 5 & \multicolumn{4}{|c|}{ Ceiling Concrete } & $N / A$ & $N / A$ & 22 & 1279 & $N / A$ & $N / A$ & $N / A$ \\
\hline 6 & \multicolumn{4}{|c|}{ Ceiling Concrete } & $N / A$ & $N / A$ & 11 & 1317 & $\mathrm{~N} / \mathrm{A}$ & $N / A$ & $N / A$ \\
\hline$N / A$ & \multicolumn{4}{|l|}{ N/A } & $N / A$ & $N / A$ & $\mathrm{~N} / \mathrm{A}$ & $N / A$ & $N / A$ & $N / A$ & $N / A$ \\
\hline$N / A$ & \multicolumn{4}{|l|}{ N/A } & $N / A$ & $N / A$ & $N / A$ & $N / A$ & $N / A$ & $N / A$ & $N / A$ \\
\hline$N / A$ & N/A & & & & $N / A$ & $N / A$ & $N / A$ & $N / A$ & $N / A$ & $N / A$ & $N / A$ \\
\hline$N / A$ & N/A & & & & $N / A$ & $\mathrm{~N} / \mathrm{A}$ & $N / A$ & $N / A$ & $N / A$ & $N / A$ & $N / A$ \\
\hline Reviewed By & Print): & & & & $\mathrm{Sig}$ & & & & & Date: & \\
\hline
\end{tabular}


10-ER-A26-165

\section{Description/Map/Drawing/Picture/Comments}

Building 2201, Pluto Disassembly Facilty

Survey Plan \# 10-015

Room 101 "Cold Assembly Bay"

- Main Room (east of mezzanine)

-Ceiling (from roll-up door to crane)

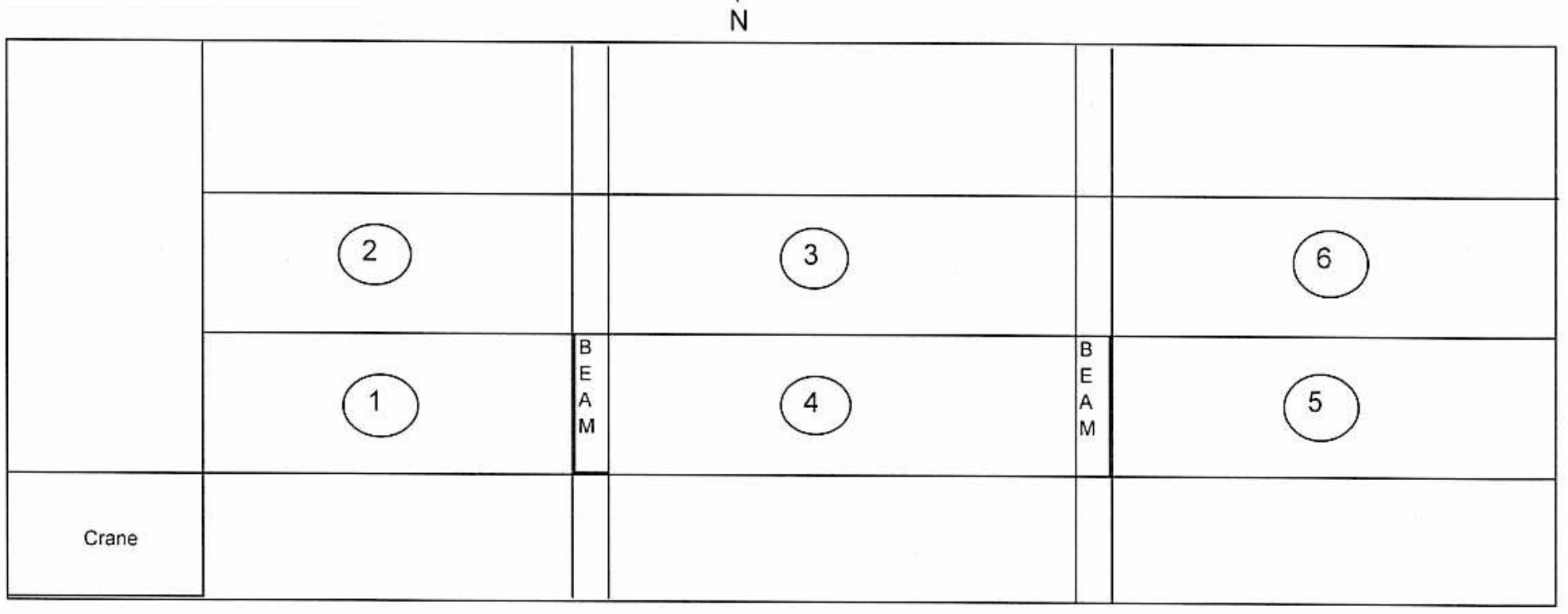


SURVEY \#

10-ER-A26-164

\begin{tabular}{|c|c|c|c|c|c|c|c|c|c|c|c|}
\hline \multicolumn{3}{|c|}{$\begin{array}{l}\text { Location: } \\
\text { Area } 26 \text { CAU117 Pluto }\end{array}$} & \multicolumn{2}{|c|}{$\begin{array}{l}\text { Purpose: } \\
\text { Final Status Survey, East Wall } \\
\text { Main Room } 101\end{array}$} & \multicolumn{5}{|c|}{$\begin{array}{l}\text { Comments: } \\
\text { All one minute static counts are in cpm Bkgd not subtracted } \\
\text { for Final Status Survey, Survey Plan \#10-015 }\end{array}$} & \multicolumn{2}{|c|}{$\begin{array}{l}\text { Date/Time: } \\
\text { 07/08/2010 } 1500\end{array}$} \\
\hline Instrument: & Serial \#: & Cal Due: & $\begin{array}{c}\text { Eff in \%: } \\
\text { Alpha / Beta }\end{array}$ & $\begin{array}{l}\text { BKG in dpm: } \\
\text { Alpha / Beta }\end{array}$ & \begin{tabular}{|l|} 
MDA in dpm: \\
Alpha / Beta
\end{tabular} & RWP\# & \multicolumn{5}{|l|}{$\mathrm{N} / \mathrm{A}$} \\
\hline Electra & 1504 & $4 / 6 / 2011$ & $12.9 / 19.8$ & $N / A$ & $N / A$ & \multicolumn{6}{|c|}{ ENV-10-RCT ENV SITE } \\
\hline$N / A$ & $N / A$ & $N / A$ & $N / A$ & $N / A$ & \multirow{2}{*}{$\begin{array}{l}N / A \\
N / A\end{array}$} & RCT Name: & \multicolumn{2}{|c|}{ D.Cotroneo Jr. } & \multicolumn{3}{|c|}{ Signature: $\mathrm{O}-\mathrm{C}$} \\
\hline $\mathrm{N} / \mathrm{A}$ & $\mathrm{N} / \mathrm{A}$ & $N / A$ & $N / A$ & $N / A$ & & RCT Name: & \multicolumn{2}{|c|}{ Kevin Wilcox } & \multicolumn{3}{|c|}{ Signature:Z7ein welcos } \\
\hline$N / A$ & $N / A$ & $N / A$ & $N / A$ & $N / A$ & $N / A$ & RCT Name: & \multicolumn{2}{|c|}{ S.Brown/B.Bunce } & \multicolumn{3}{|c|}{ Signature $\% 3$ Pabrume } \\
\hline$N / A$ & $N / A$ & $\mathrm{~N} / \mathrm{A}$ & $N / A$ & $N / A$ & $N / A$ & RCT Name: & Brian Fos & & Sigrrature: & & \\
\hline \multirow[t]{2}{*}{ Survey Point } & \multirow{2}{*}{\multicolumn{4}{|c|}{ Description/Comments }} & \multicolumn{2}{|c|}{$\begin{array}{l}\text { Removable } \\
\mathrm{dpm} / 100 \mathrm{~cm}^{2}\end{array}$} & \multicolumn{2}{|c|}{$\begin{array}{c}\text { Fixed + Removable } \\
\mathrm{dpm} / 100 \mathrm{~cm}^{2}\end{array}$} & \multirow[t]{2}{*}{$\begin{array}{l}\text { Gamma } \\
\mathrm{mrem} / \mathrm{hr}\end{array}$} & \multirow[t]{2}{*}{$\begin{array}{l}\text { Neutron } \\
\mathrm{mrem} / \mathrm{hr}\end{array}$} & \multirow[t]{2}{*}{$\begin{array}{l}\text { Total } \\
\mathrm{mrem} / \mathrm{hr}\end{array}$} \\
\hline & & & & & Alpha & Beta & Alpha & Beta & & & \\
\hline$N / A$ & \multicolumn{4}{|c|}{ Main Room (east of mezzanine) East Wall } & $N / A$ & $N / A$ & $N / A$ & $N / A$ & $N / A$ & $N / A$ & $N / A$ \\
\hline 1 & \multicolumn{4}{|c|}{ East Wall Concrete (Above roll-up door) } & $\mathrm{N} / \mathrm{A}$ & $N / A$ & 1 & 1164 & $N / A$ & $N / A$ & $N / A$ \\
\hline 2 & \multicolumn{4}{|c|}{ East Wall Concrete (Above roll-up door) } & $N / A$ & $N / A$ & 9 & 1151 & $N / A$ & $N / A$ & $N / A$ \\
\hline 3 & \multicolumn{4}{|c|}{ East Wall Metal (Roll-up door) } & $N / A$ & $N / A$ & 4 & 852 & $N / A$ & $N / A$ & $N / A$ \\
\hline 4 & \multicolumn{4}{|c|}{ East Wall Metal (Roll-up door) } & $N / A$ & $N / A$ & 5 & 925 & $N / A$ & $N / A$ & $N / A$ \\
\hline$N / A$ & \multicolumn{4}{|l|}{ N/A } & $\mathrm{N} / \mathrm{A}$ & $N / A$ & $N / A$ & $N / A$ & $\mathrm{~N} / \mathrm{A}$ & $N / A$ & $N / A$ \\
\hline $\mathrm{N} / \mathrm{A}$ & \multicolumn{4}{|l|}{ N/A } & $N / A$ & $N / A$ & $N / A$ & $N / A$ & $\mathrm{~N} / \mathrm{A}$ & $N / A$ & $N / A$ \\
\hline$N / A$ & \multicolumn{4}{|l|}{ N/A } & $N / A$ & $N / A$ & $N / A$ & $N / A$ & $N / A$ & $N / A$ & $N / A$ \\
\hline$N / A$ & \multicolumn{4}{|l|}{ N/A } & $\mathrm{N} / \mathrm{A}$ & $N / A$ & $N / A$ & $N / A$ & $\mathrm{~N} / \mathrm{A}$ & $N / A$ & $N / A$ \\
\hline$N / A$ & \multicolumn{4}{|l|}{ N/A } & $N / A$ & $N / A$ & $N / A$ & $N / A$ & $N / A$ & $N / A$ & $N / A$ \\
\hline$N / A$ & \multicolumn{4}{|l|}{ N/A } & $N / A$ & $N / A$ & $N / A$ & $N / A$ & $N / A$ & $N / A$ & $N / A$ \\
\hline \multicolumn{5}{|c|}{ Reviewed By (Print): } & Signature: & & & & & \multicolumn{2}{|c|}{$7 / 12 / 10$} \\
\hline
\end{tabular}


Survey \# 10-ER-A26-164

\section{Description/Map/Drawing/Picture/Comments}

Building 2201, Pluto Disassembly Facilty

Survey Plan \# 10-015

Room 101 "Cold Assembly Bay"

- East Wall with Roll-up Door closed

- letters appear on actual building structure, corresponding numbers used on Survey Data Form

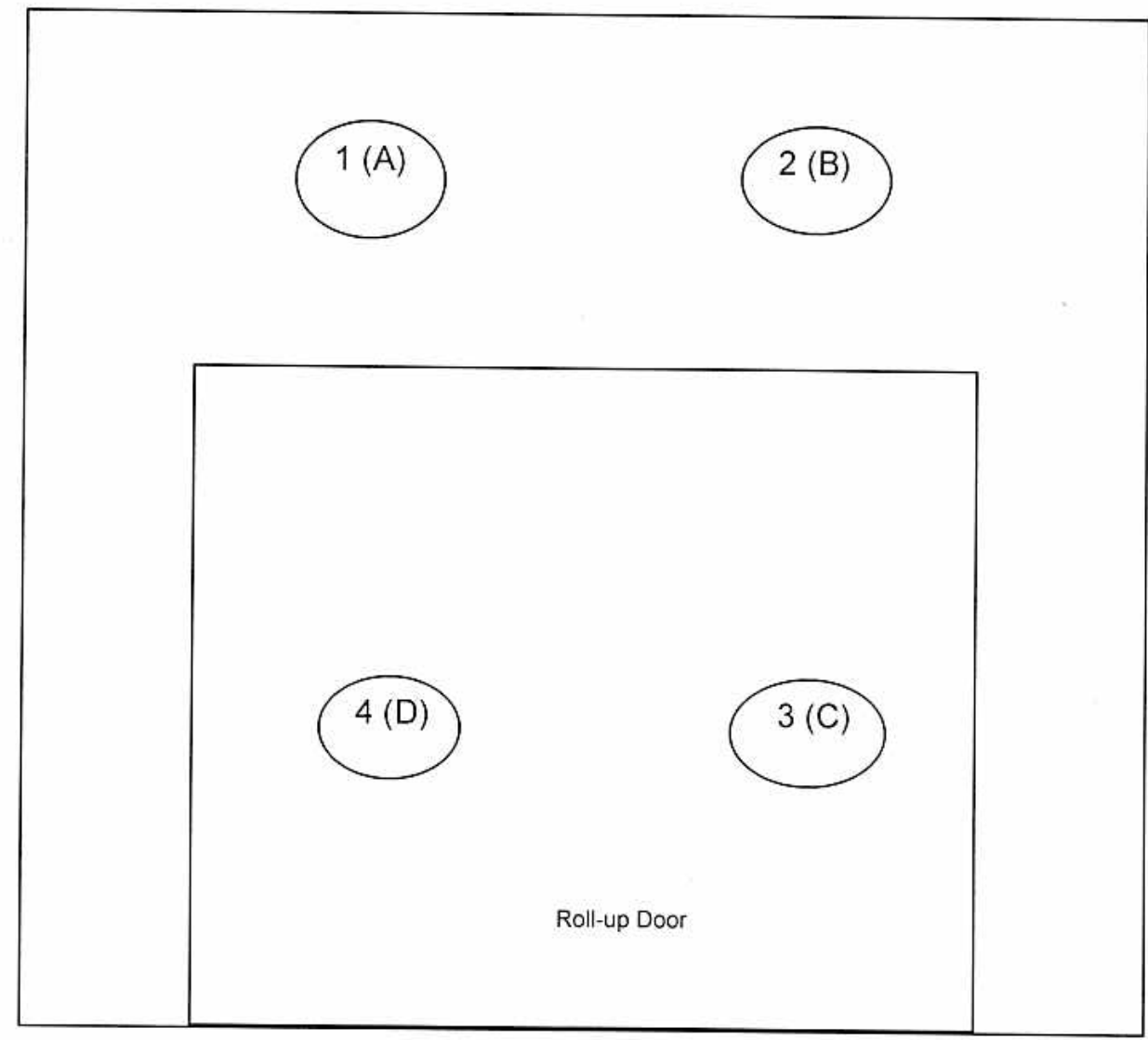


SURVEY\#

10-ER-A26-157

\begin{tabular}{|c|c|c|c|c|c|c|c|c|c|c|c|}
\hline \multicolumn{3}{|c|}{$\begin{array}{l}\text { Location: } \\
\text { Area } 26 \text { CAU117 Pluto }\end{array}$} & \multicolumn{2}{|c|}{$\begin{array}{l}\text { Purpose: } \\
\text { Final Status Survey Plan \# 10- } \\
\text { 015, Room 101, Floor }\end{array}$} & \multicolumn{5}{|c|}{$\begin{array}{l}\text { Comments: } \\
\text { Bkgd not subtracted for Final Status Survey, MDA is } \\
\text { determined by Rad Engineering, Sample counts in CPM }\end{array}$} & \multicolumn{2}{|c|}{$\begin{array}{l}\text { Date/Time: } \\
\text { 07/07/2010 } 1400\end{array}$} \\
\hline Instrument: & Serial \#: & Cal Due: & \begin{tabular}{|c|} 
Eff in \%: \\
Alpha / Beta \\
\end{tabular} & $\begin{array}{l}\text { BKG in dpm: } \\
\text { Alpha / Beta }\end{array}$ & \begin{tabular}{|l|} 
MDA in dpm: \\
Alpha / Beta \\
\end{tabular} & RWP\# & \multicolumn{5}{|c|}{$\mathrm{N} / \mathrm{A}$} \\
\hline Electra & 4806 & $4 / 6 / 2011$ & $13.2 / 20.9$ & $\mathrm{~N} / \mathrm{A}$ & $N / A$ & \multicolumn{6}{|c|}{ ENV-10-RCT ENV SITE SURVEYS-C } \\
\hline Electra & 1120 & $4 / 6 / 2011$ & $14.1 / 20.8$ & $N / A$ & $\mathrm{~N} / \mathrm{A}$ & \multicolumn{3}{|c|}{ RCT Name: D.Cotroneo Jr. } & \multicolumn{3}{|c|}{ Signature: 8 . } \\
\hline$N / A$ & $N / A$ & $N / A$ & $N / A$ & $\mathrm{~N} / \mathrm{A}$ & $\mathrm{N} / \mathrm{A}$ & \multicolumn{3}{|c|}{ RCT Name: Brian Foskett } & \multicolumn{3}{|c|}{ Signature. $\rightleftharpoons$} \\
\hline$N / A$ & $N / A$ & $N / A$ & $N / A$ & $N / A$ & $\mathrm{~N} / \mathrm{A}$ & \multicolumn{3}{|c|}{ RCT Name: Sonny Brown } & \multicolumn{3}{|c|}{ Signature: Somy 3 mm } \\
\hline $\mathrm{N} / \mathrm{A}$ & $N / A$ & $N / A$ & $N / A$ & $N / A$ & $N / A$ & \multicolumn{3}{|c|}{ RCT Name: Kevin Wilcox } & \multicolumn{3}{|c|}{ Signature: Kein Wilcot } \\
\hline \multirow[t]{2}{*}{ Survey Point } & \multirow{2}{*}{\multicolumn{4}{|c|}{ Description/Comments }} & \multicolumn{2}{|c|}{$\begin{array}{l}\text { Removable } \\
\mathrm{dpm} / 100 \mathrm{~cm}^{2}\end{array}$} & \multicolumn{2}{|c|}{$\begin{array}{c}\text { Fixed + Removable } \\
d p m / 100 \mathrm{~cm}^{2}\end{array}$} & \multirow{2}{*}{$\begin{array}{l}\text { Gamma } \\
\mathrm{mrem} / \mathrm{hr}\end{array}$} & \multirow{2}{*}{$\begin{array}{l}\text { Neutron } \\
\text { mrem/hr }\end{array}$} & \multirow{2}{*}{$\begin{array}{c}\text { Total } \\
\mathrm{mrem} / \mathrm{hr}\end{array}$} \\
\hline & & & & & Alpha & Beta & Alpha & Beta & & & \\
\hline 1 & \multicolumn{4}{|c|}{ Concrete Floor used Electra \#4806 } & $N / A$ & $N / A$ & 8 & 1413 & $N / A$ & $N / A$ & $N / A$ \\
\hline 2 & \multicolumn{4}{|c|}{ Concrete Floor used Electra \#4806 } & $N / A$ & $N / A$ & 3 & 1437 & $N / A$ & $N / A$ & $N / A$ \\
\hline 3 & \multicolumn{4}{|c|}{ Concrete Floor used Electra \#4806 } & $N / A$ & $N / A$ & 1 & 1403 & $N / A$ & $N / A$ & $N / A$ \\
\hline 4 & \multicolumn{4}{|c|}{ Concrete Floor used Electra \#4806 } & $N / A$ & $N / A$ & 9 & 1441 & $N / A$ & $N / A$ & $N / A$ \\
\hline 5 & \multicolumn{4}{|c|}{ Concrete Floor used Electra \#4806 } & $N / A$ & $N / A$ & 5 & 1439 & $N / A$ & $N / A$ & $N / A$ \\
\hline 6 & \multicolumn{4}{|c|}{ Concrete Floor used Electra \#4806 } & $N / A$ & $N / A$ & 0 & 531 & $N / A$ & $N / A$ & $N / A$ \\
\hline 7 & \multicolumn{4}{|c|}{ Concrete Floor used Electra \#4806 } & $N / A$ & $N / A$ & 4 & 1293 & $N / A$ & $\mathrm{~N} / \mathrm{A}$ & $N / A$ \\
\hline 8 & \multicolumn{4}{|c|}{ Concrete Floor used Electra \#4806 } & $N / A$ & $N / A$ & 1 & 570 & $N / A$ & $N / A$ & $\mathrm{~N} / \mathrm{A}$ \\
\hline 9 & \multicolumn{4}{|c|}{ Concrete Floor used Electra \#4806 } & $N / A$ & $N / A$ & 5 & 1405 & $N / A$ & $N / A$ & $N / A$ \\
\hline 10 & \multicolumn{4}{|c|}{ Concrete Floor used Electra \#4806 } & $N / A$ & $N / A$ & 10 & 1357 & $N / A$ & $N / A$ & $N / A$ \\
\hline 11 & \multicolumn{4}{|c|}{ Concrete Floor used Electra \#4806 } & $N / A$ & $N / A$ & 7 & 1360 & $N / A$ & $N / A$ & $N / A$ \\
\hline \multicolumn{5}{|c|}{ Reviewed By (Print): } & Signature: & & & & & $\begin{array}{l}\text { Date: } \\
\quad 7 / \\
\end{array}$ & \\
\hline
\end{tabular}


SURVEY \# 10-ER-A26-157

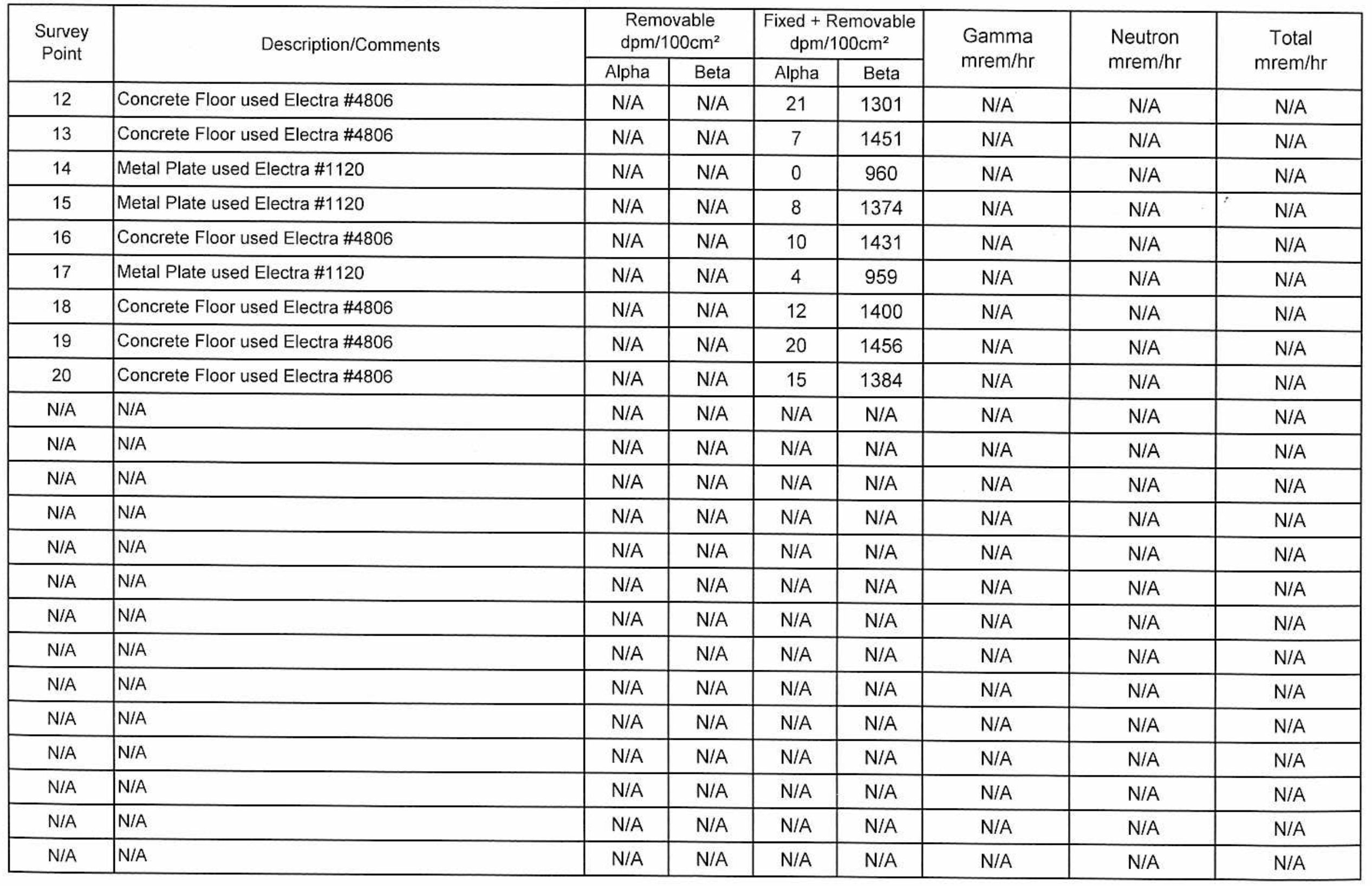


Description/Map/Drawing/Picture/Comments

Building 2201, Pluto Disassembly Facility

Survey Plan \# 10-015

Room 101 "Cold Assembly Bay"

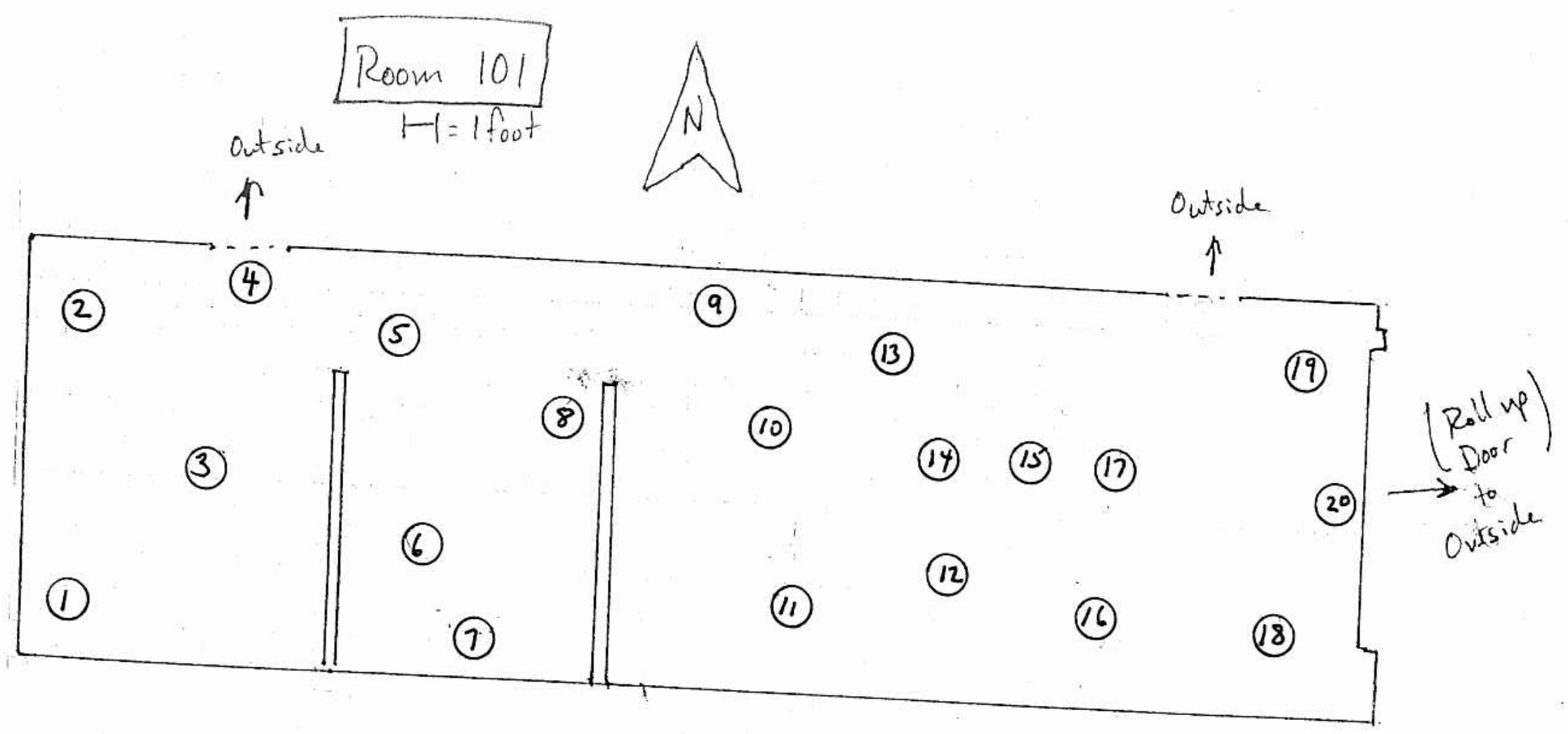


SURVEY \#

10-ER-A26-158

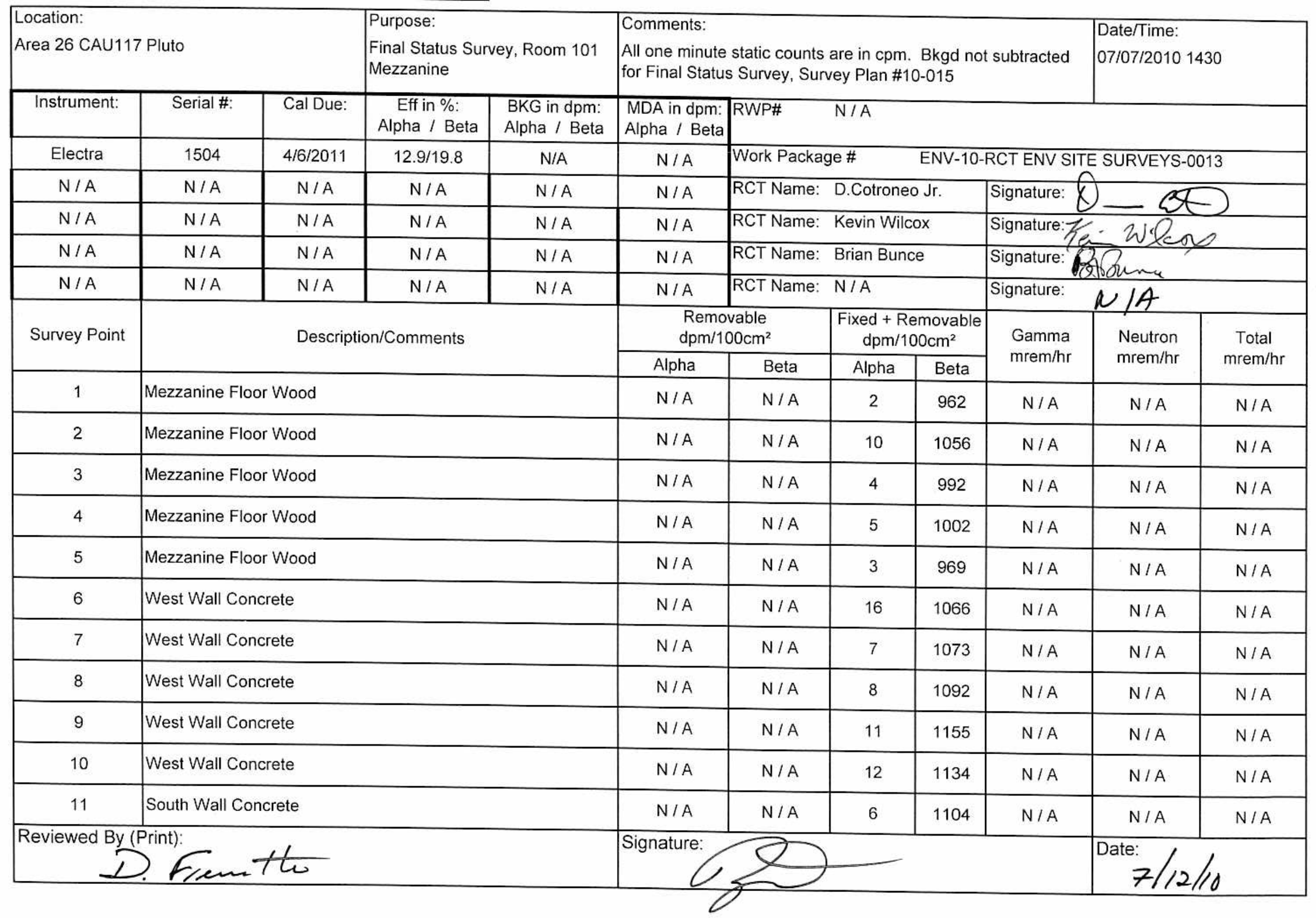


SURVEY \# 10-ER-A26-158

\begin{tabular}{|c|c|c|c|c|c|c|c|c|}
\hline \multirow{2}{*}{$\begin{array}{l}\text { Survey } \\
\text { Point }\end{array}$} & \multirow[t]{2}{*}{ Description/Comments } & \multicolumn{2}{|c|}{$\begin{array}{l}\text { Removable } \\
\mathrm{dpm} / 100 \mathrm{~cm}^{2}\end{array}$} & \multicolumn{2}{|c|}{$\begin{array}{c}\text { Fixed + Removable } \\
\mathrm{dpm} / 100 \mathrm{~cm}^{2}\end{array}$} & \multirow{2}{*}{$\begin{array}{l}\text { Gamma } \\
\mathrm{mrem} / \mathrm{hr}\end{array}$} & \multirow{2}{*}{$\begin{array}{l}\text { Neutron } \\
\mathrm{mrem} / \mathrm{hr}\end{array}$} & \multirow{2}{*}{$\begin{array}{c}\text { Total } \\
\text { mrem/hr }\end{array}$} \\
\hline & & Alpha & Beta & Alpha & Beta & & & \\
\hline 12 & South Wall Concrete & N/A & N/A & 7 & 991 & N/A & N/A & $\mathrm{N} / \mathrm{A}$ \\
\hline 13 & South Wall Concrete & $\mathrm{N} / \mathrm{A}$ & N/A & 10 & 977 & N/A & N/A & $\mathrm{N} / \mathrm{A}$ \\
\hline 14 & South Wall Concrete & N/A & $\mathrm{N} / \mathrm{A}$ & 14 & 948 & N/A & N/A & $\mathrm{N} / \mathrm{A}$ \\
\hline 15 & South Wall Concrete & $\mathrm{N} / \mathrm{A}$ & N/A & 14 & 997 & $\mathrm{~N} / \mathrm{A}$ & $\mathrm{N} / \mathrm{A}$ & $\mathrm{N} / \mathrm{A}$ \\
\hline 16 & North Wall Concrete & N/A & N/A & 8 & 1121 & N/A & $\mathrm{N} / \mathrm{A}$ & $\mathrm{N} / \mathrm{A}$ \\
\hline 17 & North Wall Concrete & $\mathrm{N} / \mathrm{A}$ & $\mathrm{N} / \mathrm{A}$ & 6 & 1062 & $\mathrm{~N} / \mathrm{A}$ & $\mathrm{N} / \mathrm{A}$ & $\mathrm{N} / \mathrm{A}$ \\
\hline 18 & North Wall Concrete & $\mathrm{N} / \mathrm{A}$ & N/A & 6 & 1044 & $\mathrm{~N} / \mathrm{A}$ & $\mathrm{N} / \mathrm{A}$ & $\mathrm{N} / \mathrm{A}$ \\
\hline 19 & North Wall Concrete & N/A & N/A & 7 & 1096 & N/A & $\mathrm{N} / \mathrm{A}$ & $\mathrm{N} / \mathrm{A}$ \\
\hline 20 & North Wall Concrete & N/A & $\mathrm{N} / \mathrm{A}$ & 10 & 1043 & N/A & N/A & N/A \\
\hline 21 & Craneveyor Platform/Track Metal & N/A & N/A & 8 & 875 & $\mathrm{~N} / \mathrm{A}$ & $\mathrm{N} / \mathrm{A}$ & $\mathrm{N} / \mathrm{A}$ \\
\hline 22 & Craneveyor Platform/Track Metal & N/A & N/A & 4 & 793 & N/A & N/A & $\mathrm{N} / \mathrm{A}$ \\
\hline 23 & Ceiling Concrete & $\mathrm{N} / \mathrm{A}$ & N/A & 1 & 1262 & $\mathrm{~N} / \mathrm{A}$ & N/A & $\mathrm{N} / \mathrm{A}$ \\
\hline 24 & Ceiling Concrete & $\mathrm{N} / \mathrm{A}$ & N/A & 3 & 1279 & $\mathrm{~N} / \mathrm{A}$ & $\mathrm{N} / \mathrm{A}$ & $\mathrm{N} / \mathrm{A}$ \\
\hline 25 & Ceiling Concrete & $\mathrm{N} / \mathrm{A}$ & N/A & 3 & 1248 & $\mathrm{~N} / \mathrm{A}$ & $\mathrm{N} / \mathrm{A}$ & N/A \\
\hline 26 & Ceiling Concrete & $\mathrm{N} / \mathrm{A}$ & N/A & 2 & 1241 & $\mathrm{~N} / \mathrm{A}$ & $\mathrm{N} / \mathrm{A}$ & N/A \\
\hline 27 & Ceiling Concrete & N/A & N/A & 3 & 1327 & N/A & N/A & $\mathrm{N} / \mathrm{A}$ \\
\hline 28 & Mezzanine Floor Wood & $\mathrm{N} / \mathrm{A}$ & $\mathrm{N} / \mathrm{A}$ & 8 & 964 & N/A & N/A & $\mathrm{N} / \mathrm{A}$ \\
\hline N/A & N/A & $\mathrm{N} / \mathrm{A}$ & N/A & N/A & N/A & N/A & $\mathrm{N} / \mathrm{A}$ & N/A \\
\hline N/A & N/A & N/A & N/A & N/A & $\mathrm{N} / \mathrm{A}$ & N/A & $\mathrm{N} / \mathrm{A}$ & N/A \\
\hline N/A & N/A & N/A & N/A & N/A & $\mathrm{N} / \mathrm{A}$ & N/A & $\mathrm{N} / \mathrm{A}$ & N/A \\
\hline $\mathrm{N} / \mathrm{A}$ & N/A & N/A & N/A & N/A & $\mathrm{N} / \mathrm{A}$ & $\mathrm{N} / \mathrm{A}$ & $\mathrm{N} / \mathrm{A}$ & N/A \\
\hline N/A & N/A & N/A & N/A & N/A & $\mathrm{N} / \mathrm{A}$ & N/A & N/A & N/A \\
\hline N/A & N/A & N/A & N/A & N/A & $\mathrm{N} / \mathrm{A}$ & $\mathrm{N} / \mathrm{A}$ & $\mathrm{N} / \mathrm{A}$ & N/A \\
\hline
\end{tabular}


FRM-0108B

SURVEY \#

10-ER-A26-162

\begin{tabular}{|c|c|c|c|c|c|c|c|c|c|c|c|}
\hline \multicolumn{3}{|c|}{$\begin{array}{l}\text { Location: } \\
\text { Area } 26 \text { CAU117 Pluto }\end{array}$} & \multicolumn{2}{|c|}{$\begin{array}{l}\text { Purpose: } \\
\text { Final Status Survey, North Wall } \\
\text { Main Room } 101\end{array}$} & \multicolumn{5}{|c|}{$\begin{array}{l}\text { All one minute static counts are in } \mathrm{cpm} \text { Bkgd not subtracted } \\
\text { for Final Status Survey, Survey Plan \#10-015 }\end{array}$} & \multicolumn{2}{|c|}{$\begin{array}{l}\text { Date/Time: } \\
\text { 07/08/2010 } 1000\end{array}$} \\
\hline Instrument: & Serial \#: & Cal Due: & $\begin{array}{c}\text { Eff in \%: } \\
\text { Alpha / Beta }\end{array}$ & $\begin{array}{l}\text { BKG in dpm: } \\
\text { Alpha / Beta }\end{array}$ & $\begin{array}{l}\text { MDA in dpm: } \\
\text { Alpha / Beta }\end{array}$ & RWP\# & \multicolumn{5}{|c|}{$\mathrm{N} / \mathrm{A}$} \\
\hline Electra & 4806 & $4 / 6 / 2011$ & $13.2 / 20.9$ & $N / A$ & $N / A$ & \multicolumn{6}{|c|}{ 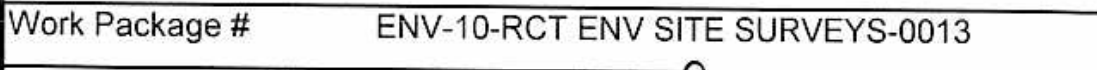 } \\
\hline Electra & 1120 & $4 / 6 / 2011$ & $12.9 / 19.8$ & $\mathrm{~N} / \mathrm{A}$ & \multirow{2}{*}{$\begin{array}{l}N / A \\
N / A\end{array}$} & RCT Name: D.Cotroneo Jr. & \multicolumn{2}{|c|}{ D.Cotroneo Jr. } & \multicolumn{3}{|c|}{ Signature: 0} \\
\hline$N / A$ & $\mathrm{~N} / \mathrm{A}$ & $N / A$ & $\mathrm{~N} / \mathrm{A}$ & $N / A$ & & \multicolumn{3}{|c|}{ RCT Name: Kevin Wilcox } & \multicolumn{3}{|c|}{ Signature: Kei. W.leox } \\
\hline $\mathrm{N} / \mathrm{A}$ & $N / A$ & $N / A$ & $\mathrm{~N} / \mathrm{A}$ & $N / A$ & $\mathrm{~N} / \mathrm{A}$ & RCT Name: & \multicolumn{2}{|c|}{ S.Brown/B.Bunce } & \multirow{2}{*}{\multicolumn{3}{|c|}{ Signature:5ngature: }} \\
\hline $\mathrm{N} / \mathrm{A}$ & $\mathrm{N} / \mathrm{A}$ & $\mathrm{N} / \mathrm{A}$ & $\mathrm{N} / \mathrm{A}$ & $N / A$ & $N / A$ & RCT Name: & Brian Fos & & & & \\
\hline \multirow[t]{2}{*}{ Survey Point } & \multirow{2}{*}{\multicolumn{4}{|c|}{ Description/Comments }} & \multicolumn{2}{|c|}{$\begin{array}{l}\text { Removable } \\
\mathrm{dpm} / 100 \mathrm{~cm}^{2}\end{array}$} & \multicolumn{2}{|c|}{$\begin{array}{c}\text { Fixed + Removable } \\
\mathrm{dpm} / 100 \mathrm{~cm}^{2}\end{array}$} & \multirow{2}{*}{$\begin{array}{l}\text { Gamma } \\
\mathrm{mrem} / \mathrm{hr}\end{array}$} & \multirow{2}{*}{$\begin{array}{l}\text { Neutron } \\
\mathrm{mrem} / \mathrm{hr}\end{array}$} & \multirow{2}{*}{$\begin{array}{l}\text { Total } \\
\mathrm{mrem} / \mathrm{hr}\end{array}$} \\
\hline & & & & & Alpha & Beta & Alpha & Beta & & & \\
\hline$N / A$ & \multicolumn{4}{|c|}{ Main Room (east of mezzanine) North Wall } & $N / A$ & $N / A$ & $N / A$ & $N / A$ & $N / A$ & $N / A$ & $N / A$ \\
\hline 1 & \multicolumn{4}{|c|}{ North Wall Concrete (Electra 4806) } & $N / A$ & $N / A$ & 10 & 1210 & $N / A$ & $N / A$ & $N / A$ \\
\hline 2 & \multicolumn{4}{|c|}{ North Wall Concrete (Electra 4806) } & $N / A$ & $N / A$ & 7 & 1176 & $N / A$ & $N / A$ & $N / A$ \\
\hline 3 & \multicolumn{4}{|c|}{ North Wall Concrete (Electra 4806) } & $N / A$ & $N / A$ & 12 & 1124 & $N / A$ & $N / A$ & $N / A$ \\
\hline 4 & \multicolumn{4}{|c|}{ North Wall Concrete (Electra 4806) } & $N / A$ & $N / A$ & 6 & 1195 & $N / A$ & $N / A$ & $N / A$ \\
\hline 5 & \multicolumn{4}{|c|}{ North Wall Concrete (Electra 1120) } & $N / A$ & $N / A$ & 9 & 1213 & $N / A$ & $N / A$ & $N / A$ \\
\hline 6 & \multicolumn{4}{|c|}{ North Wall Concrete (Electra 1120) } & $N / A$ & $N / A$ & 8 & 1127 & $N / A$ & $N / A$ & $N / A$ \\
\hline 7 & \multicolumn{4}{|c|}{ North Wall Concrete (Electra 1120) } & $N / A$ & $N / A$ & 11 & 1156 & $N / A$ & $N / A$ & $N / A$ \\
\hline$N / A$ & \multicolumn{4}{|l|}{ N/A } & $N / A$ & $N / A$ & $N / A$ & $N / A$ & $N / A$ & $N / A$ & $N / A$ \\
\hline$N / A$ & \multicolumn{4}{|l|}{ N/A } & $N / A$ & $N / A$ & $N / A$ & $N / A$ & $N / A$ & $N / A$ & $N / A$ \\
\hline$N / A$ & \multicolumn{4}{|l|}{ N/A } & $N / A$ & $N / A$ & $N / A$ & $N / A$ & $N / A$ & $N / A$ & $N / A$ \\
\hline Reviewed By & & & & & Signature: & & & & & Date: $7 / 10$ & \\
\hline
\end{tabular}




\section{RADIOLOG}

Survey \#10-ER.A26-162

\section{Description/Map/Drawing/Picture/Comments}

Building 2201, Pluto Disassembly Facilty

Survey Plan \# 10-015

Room 101 "Cold Assembly Bay"

- Main Room (east of mezzanine), North Wall

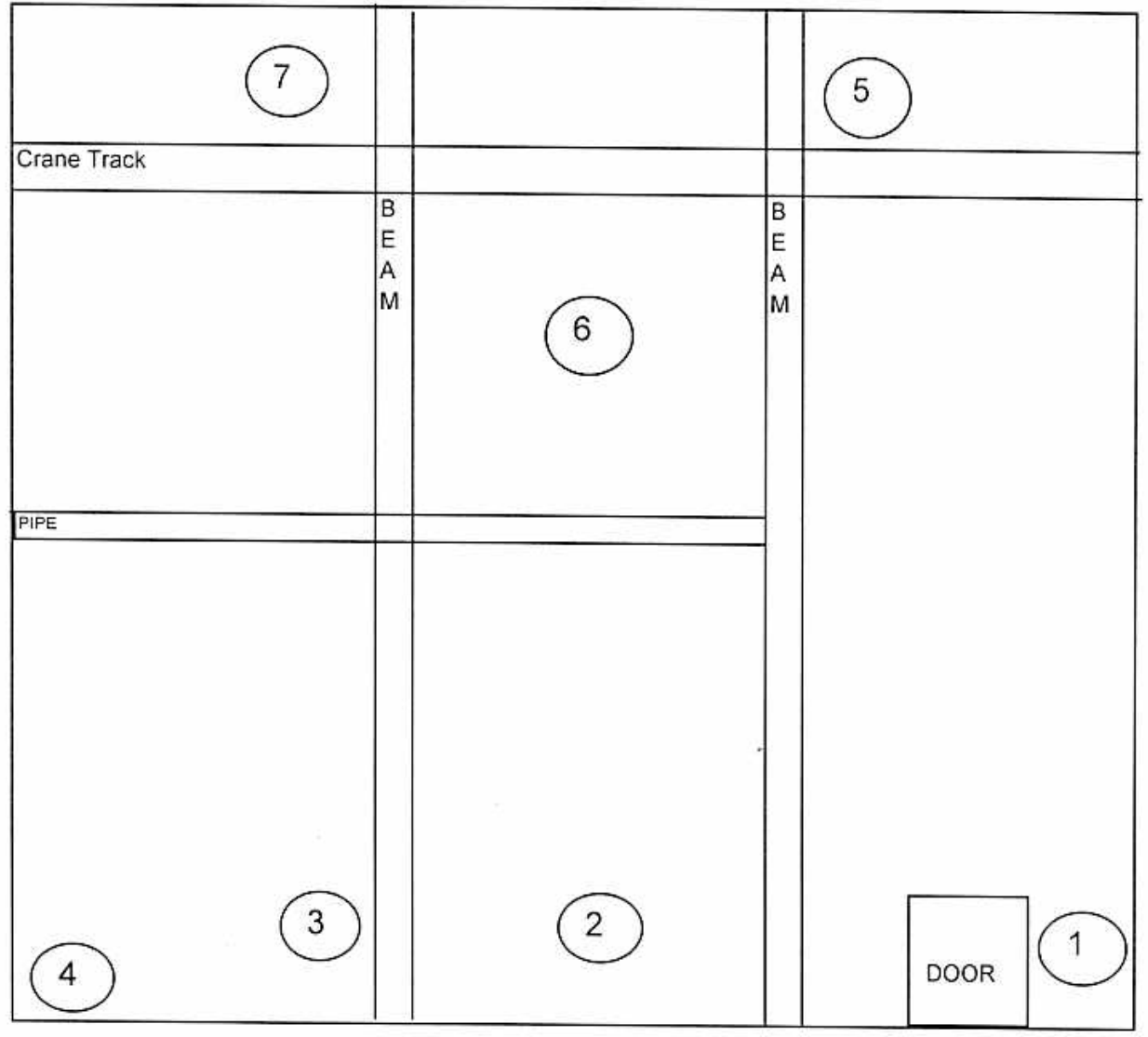

LEGEND,

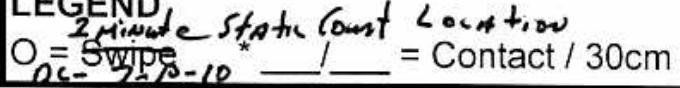

$Y=$ gamma

$\beta=$ beta 
FRM-0108B

SURVEY \#

10-ER-A26-163

\begin{tabular}{|c|c|c|c|c|c|c|c|c|c|c|c|}
\hline \multicolumn{3}{|c|}{$\begin{array}{l}\text { Location: } \\
\text { Area } 26 \text { CAU117 Pluto }\end{array}$} & \multicolumn{2}{|c|}{$\begin{array}{l}\text { Purpose: } \\
\text { Final Status Survey, South Wall } \\
\text { Main Room } 101\end{array}$} & \multicolumn{5}{|c|}{$\begin{array}{l}\text { Comments: } \\
\text { All one minute static counts are in } \mathrm{cpm} \quad B k g d \text { not subtracted }\end{array}$} & \multicolumn{2}{|c|}{$\begin{array}{l}\text { Date/Time: } \\
\text { 07/08/2010 } 1000\end{array}$} \\
\hline Instrument: & Serial \#: & Cal Due: & $\begin{array}{c}\text { Eff in \%: } \\
\text { Alpha / Beta }\end{array}$ & $\begin{array}{l}\text { BKG in dpm: } \\
\text { Alpha / Beta }\end{array}$ & \begin{tabular}{|l|} 
MDA in dpm: \\
Alpha / Beta \\
\end{tabular} & RWP\# & \multicolumn{5}{|l|}{$\mathrm{N} / \mathrm{A}$} \\
\hline Electra & 6697 & $4 / 6 / 2011$ & $13.8 / 19.9$ & $N / A$ & $N / A$ & \multicolumn{6}{|c|}{ Work Package \# $\quad$ ENV-10-RCT ENV SITE SURVEYS-0013 } \\
\hline Electra & 1120 & $4 / 6 / 2011$ & $12.9 / 19.8$ & $\mathrm{~N} / \mathrm{A}$ & $N / A$ & \multicolumn{3}{|c|}{ RCT Name: D.Cotroneo Jr. } & Signature: & \multicolumn{2}{|c|}{$-\mathrm{Cs}$} \\
\hline$N / A$ & $N / A$ & $N / A$ & $\mathrm{~N} / \mathrm{A}$ & $\mathrm{N} / \mathrm{A}$ & $\mathrm{N} / \mathrm{A}$ & \multicolumn{3}{|c|}{ RCT Name: Kevin Wilcox } & \multicolumn{3}{|c|}{ Signature: Kei-Welove } \\
\hline$N / A$ & $N / A$ & $N / A$ & $N / A$ & $N / A$ & $\mathrm{~N} / \mathrm{A}$ & \multicolumn{3}{|c|}{ RCT Name: S.Brown/B.Bunce } & \multicolumn{3}{|c|}{ 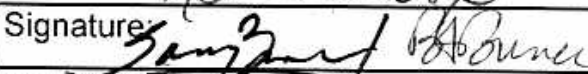 } \\
\hline $\mathrm{N} / \mathrm{A}$ & $\mathrm{N} / \mathrm{A}$ & $\mathrm{N} / \mathrm{A}$ & $N / A$ & $\mathrm{~N} / \mathrm{A}$ & $N / A$ & \multicolumn{3}{|c|}{ RCT Name: Brian Foskett } & Signature: & & \\
\hline Survey Point & \multirow{2}{*}{\multicolumn{4}{|c|}{ Description/Comments }} & \multicolumn{2}{|c|}{$\begin{array}{l}\text { Removable } \\
\mathrm{dpm} / 100 \mathrm{~cm}^{2}\end{array}$} & \multicolumn{2}{|c|}{$\begin{array}{c}\text { Fixed + Removable } \\
\mathrm{dpm} / 100 \mathrm{~cm}^{2}\end{array}$} & \multirow{2}{*}{$\begin{array}{l}\text { Gamma } \\
\mathrm{mrem} / \mathrm{hr}\end{array}$} & \multirow{2}{*}{$\begin{array}{l}\text { Neutron } \\
\mathrm{mrem} / \mathrm{hr}\end{array}$} & \multirow{2}{*}{$\begin{array}{l}\text { Total } \\
\mathrm{mrem} / \mathrm{hr}\end{array}$} \\
\hline & & & & & Alpha & Beta & Alpha & Beta & & & \\
\hline$N / A$ & \multicolumn{4}{|c|}{ Main Room (east of mezzanine) South Wall } & $N / A$ & $N / A$ & $N / A$ & $N / A$ & $\mathrm{~N} / \mathrm{A}$ & $N / A$ & $N / A$ \\
\hline 1 & \multicolumn{4}{|c|}{ South Wall Concrete (Electra 1120) } & $N / A$ & $N / A$ & 18 & 1031 & $\mathrm{~N} / \mathrm{A}$ & $N / A$ & $N / A$ \\
\hline 2 & \multicolumn{4}{|c|}{ South Wall Concrete (Electra 1120) } & $N / A$ & $N / A$ & 18 & 965 & $N / A$ & $N / A$ & $N / A$ \\
\hline 3 & \multicolumn{4}{|c|}{ South Wall Concrete (Electra 1120) } & $N / A$ & $N / A$ & 20 & 990 & $N / A$ & $N / A$ & $N / A$ \\
\hline 4 & \multicolumn{4}{|c|}{ South Wall Concrete (Electra 1120) } & $N / A$ & $N / A$ & 17 & 925 & $N / A$ & $N / A$ & $N / A$ \\
\hline 5 & \multicolumn{4}{|c|}{ South Wall Concrete (Electra 6697) } & $N / A$ & $N / A$ & 11 & 1075 & $N / A$ & $N / A$ & $N / A$ \\
\hline 6 & \multicolumn{4}{|c|}{ South Wall Upper Ledge Concrete (Electra 6697) } & $N / A$ & $N / A$ & 6 & 1318 & $\mathrm{~N} / \mathrm{A}$ & $N / A$ & $N / A$ \\
\hline 7 & \multicolumn{4}{|c|}{ South Wall Concrete (Electra 6697) } & $N / A$ & $N / A$ & 9 & 1109 & $N / A$ & $N / A$ & $N / A$ \\
\hline 8 & \multicolumn{4}{|c|}{ South Wall Concrete (Electra 6697) } & $N / A$ & $N / A$ & 10 & 1013 & $N / A$ & $N / A$ & $N / A$ \\
\hline$N / A$ & \multicolumn{4}{|l|}{ N/A } & $N / A$ & $N / A$ & $N / A$ & $N / A$ & $N / A$ & $N / A$ & $N / A$ \\
\hline$N / A$ & \multicolumn{4}{|l|}{ N/A } & $N / A$ & $N / A$ & $N / A$ & $N / A$ & $\mathrm{~N} / \mathrm{A}$ & $N / A$ & $N / A$ \\
\hline \multicolumn{5}{|c|}{ Reviewed By (Print): } & \multicolumn{5}{|l|}{ Signature: } & $\begin{array}{r}\text { Date: } \\
7\end{array}$ & \\
\hline
\end{tabular}


Survey \# 10-ER-A26-163

\section{Description/Map/Drawing/Picture/Comments}

Building 2201, Pluto Disassembly Facilty

Survey Plan \# 10-015

Room 101 "Cold Assembly Bay"

- Main Room (east of mezzanine), South Wall

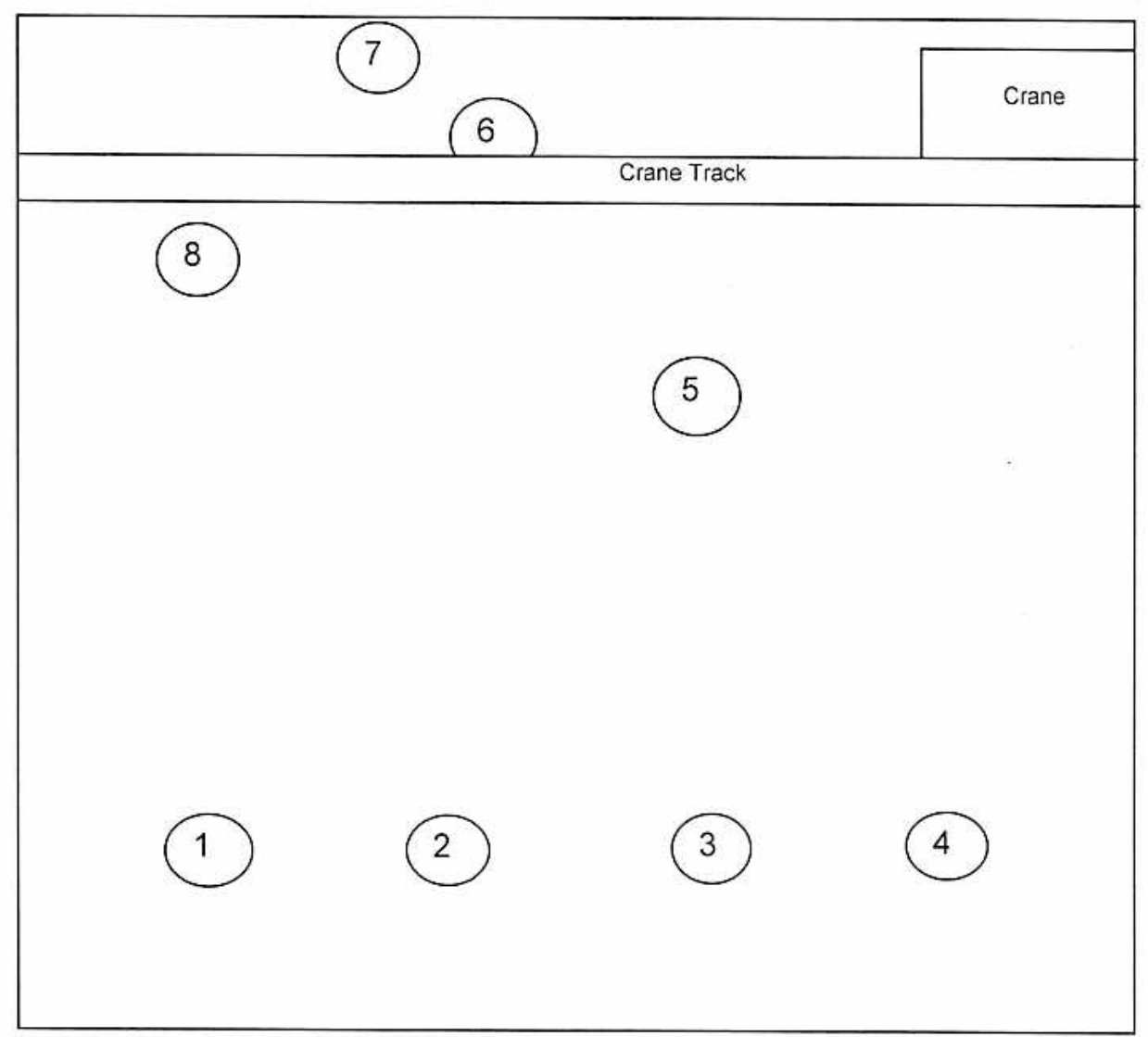


SURVEY \#

10-ER-A26-161

\begin{tabular}{|c|c|c|c|c|c|c|c|c|c|c|c|}
\hline \multicolumn{3}{|c|}{$\begin{array}{l}\text { Location: } \\
\text { Area } 26 \text { CAU117 Pluto }\end{array}$} & \multicolumn{2}{|c|}{$\begin{array}{l}\text { Purpose: } \\
\text { Final Status (walls \& ceilings) } \\
\text { West end of room } 101\end{array}$} & \multicolumn{5}{|c|}{$\begin{array}{l}\text { Comments: } \\
\text { All one minute static counts are in cpm Bkgd not subtracted } \\
\text { for Final Status Survey, Survey Plan \#10-015 }\end{array}$} & \multicolumn{2}{|c|}{$\begin{array}{l}\text { Date/Time: } \\
\text { 07/07/2010 } 1000\end{array}$} \\
\hline Instrument: & Serial \#: & Cal Due: & $\begin{array}{c}\text { Eff in \%: } \\
\text { Alpha / Beta }\end{array}$ & $\begin{array}{l}\text { BKG in dpm: } \\
\text { Alpha / Beta }\end{array}$ & \begin{tabular}{|l|} 
MDA in dpm: \\
Alpha / Beta \\
\end{tabular} & RWP\# & \multicolumn{5}{|l|}{$\mathrm{N} / \mathrm{A}$} \\
\hline Electra & 4806 & $4 / 6 / 2011$ & $13.2 / 20.9$ & $\mathrm{~N} / \mathrm{A}$ & $N / A$ & \multicolumn{6}{|c|}{ ENV-10-RCT ENV SI } \\
\hline Electra & 1504 & $4 / 6 / 2011$ & $12.9 / 19.8$ & $\mathrm{~N} / \mathrm{A}$ & $N / A$ & \multicolumn{3}{|c|}{ RCT Name: D.Cotroneo Jr. } & \multicolumn{3}{|l|}{ Signature: } \\
\hline Electra & 1120 & $4 / 6 / 2011$ & $14.1 / 20.8$ & $N / A$ & $\mathrm{~N} / \mathrm{A}$ & \multicolumn{3}{|c|}{ RCT Name: Kevin Wilcox } & \multicolumn{3}{|c|}{ Signature: Kei Wleof } \\
\hline$N / A$ & $N / A$ & $N / A$ & $N / A$ & $\mathrm{~N} / \mathrm{A}$ & $N / A$ & \multicolumn{3}{|c|}{ RCT Name: Sonny Brown } & \multirow{2}{*}{\multicolumn{3}{|c|}{ Signature: }} \\
\hline$N / A$ & $N / A$ & $N / A$ & $\mathrm{~N} / \mathrm{A}$ & $N / A$ & $N / A$ & RCT Name: & Brian Fos & & & & \\
\hline \multirow[t]{2}{*}{ Survey Point } & \multirow{2}{*}{\multicolumn{4}{|c|}{ Description/Comments }} & \multicolumn{2}{|c|}{$\begin{array}{l}\text { Removable } \\
\mathrm{dpm} / 100 \mathrm{~cm}^{2}\end{array}$} & \multicolumn{2}{|c|}{\begin{tabular}{|c|} 
Fixed + Removable \\
$\mathrm{dpm} / 100 \mathrm{~cm}^{2}$
\end{tabular}} & \multirow{2}{*}{$\begin{array}{c}\text { Gamma } \\
\mathrm{mrem} / \mathrm{hr}\end{array}$} & \multirow{2}{*}{$\begin{array}{l}\text { Neutron } \\
\mathrm{mrem} / \mathrm{hr}\end{array}$} & \multirow{2}{*}{$\begin{array}{l}\text { Total } \\
\mathrm{mrem} / \mathrm{hr}\end{array}$} \\
\hline & & & & & Alpha & Beta & Alpha & Beta & & & \\
\hline$N / A$ & \multicolumn{4}{|c|}{ West end room (West of Cinderblock wall) } & $\mathrm{N} / \mathrm{A}$ & $N / A$ & $N / A$ & $N / A$ & $N / A$ & $N / A$ & $N / A$ \\
\hline 1 & \multicolumn{4}{|c|}{ Concrete Wall South (Electra 4806) } & $N / A$ & $N / A$ & 7 & 989 & $N / A$ & $\mathrm{~N} / \mathrm{A}$ & $N / A$ \\
\hline 2 & \multicolumn{4}{|c|}{ Concrete Wall West (Electra 4806) } & $N / A$ & $N / A$ & 6 & 1112 & $N / A$ & $N / A$ & $N / A$ \\
\hline 3 & \multicolumn{4}{|c|}{ Concrete Wall North (Electra 4806) } & $N / A$ & $N / A$ & 6 & 1076 & $N / A$ & $N / A$ & $N / A$ \\
\hline 4 & \multicolumn{4}{|c|}{ Cinderblock wall (Electra 4806) } & $N / A$ & $N / A$ & 5 & 1279 & $N / A$ & $N / A$ & $N / A$ \\
\hline 11 & \multicolumn{4}{|c|}{ Wood ceiling (Electra 1120) } & $N / A$ & $N / A$ & 2 & 1001 & $N / A$ & $N / A$ & $N / A$ \\
\hline 12 & \multicolumn{4}{|c|}{ Wood ceiling (Electra 1120) } & $N / A$ & $N / A$ & 13 & 1000 & $\mathrm{~N} / \mathrm{A}$ & $N / A$ & $N / A$ \\
\hline 13 & \multicolumn{4}{|c|}{ Wood ceiling (Electra 1120) } & $N / A$ & $N / A$ & 13 & 910 & $N / A$ & $\mathrm{~N} / \mathrm{A}$ & $N / A$ \\
\hline$N / A$ & \multicolumn{4}{|c|}{ West end room (West of Wood framed wall) } & $N / A$ & $N / A$ & $N / A$ & $N / A$ & $N / A$ & $N / A$ & $N / A$ \\
\hline 5 & \multicolumn{4}{|c|}{ Concrete Wall North (Electra 4806) } & $N / A$ & $N / A$ & 11 & 1035 & $N / A$ & $N / A$ & $N / A$ \\
\hline 6 & \multicolumn{4}{|c|}{ Cinderblock wall (Electra 4806) } & $N / A$ & $N / A$ & 5 & 1319 & $N / A$ & $N / A$ & $N / A$ \\
\hline \multicolumn{5}{|c|}{ Reviewed By (Print): } & \multicolumn{5}{|l|}{ Signature: } & \multicolumn{2}{|c|}{ Date: $7 / 12 / 10$} \\
\hline
\end{tabular}


SURVEY \# 10-ER-A26-161

\begin{tabular}{|c|c|c|c|c|c|c|c|c|c|c|c|c|}
\hline \multirow{2}{*}{$\begin{array}{l}\text { Survey } \\
\text { Point }\end{array}$} & \multirow{2}{*}{\multicolumn{5}{|c|}{ Description/Comments }} & \multicolumn{2}{|c|}{$\begin{array}{l}\text { Removable } \\
\mathrm{dpm} / 100 \mathrm{~cm}^{2}\end{array}$} & \multicolumn{2}{|c|}{$\begin{array}{c}\text { Fixed + Removable } \\
\text { dpm } / 100 \mathrm{~cm}^{2}\end{array}$} & \multirow{2}{*}{$\begin{array}{l}\text { Gamma } \\
\text { mrem/hr }\end{array}$} & \multirow{2}{*}{$\begin{array}{l}\text { Neutron } \\
\text { mrem/hr }\end{array}$} & \multirow{2}{*}{$\begin{array}{c}\text { Total } \\
\text { mrem } / \mathrm{hr}\end{array}$} \\
\hline & & & & & & Alpha & Beta & Alpha & Beta & & & \\
\hline 7 & \multicolumn{5}{|c|}{ Concrete wall south (Electra 4806) } & N/A & N/A & 11 & 1039 & $N / A$ & N/A & N/A \\
\hline 8 & \multicolumn{5}{|c|}{ Wood ceiling (Electra 1120) } & N/A & N/A & 17 & 927 & N/A & N/A & N/A \\
\hline 9 & \multicolumn{5}{|c|}{ Wood ceiling (Electra 1120) } & N/A & N/A & 10 & 1058 & N/A & N/A & N/A \\
\hline 10 & \multicolumn{5}{|c|}{ Wood ceiling (Electra 1120) } & N/A & N/A & 11 & 1015 & N/A & N/A & N/A \\
\hline 14 & \multicolumn{5}{|c|}{ Wood Framed west wall (Electra 1504) } & N/A & N/A & 2 & 741 & N/A & N/A & N/A \\
\hline 15 & \multicolumn{5}{|c|}{ Wood Framed west wall (Electra 1504) } & N/A & N/A & 6 & 1065 & N/A & N/A & $\mathrm{N} / \mathrm{A}$ \\
\hline N/A & N/A & N/A & N/A & N/A & $\mathrm{N} / \mathrm{A}$ & N/A & N/A & N/A & N/A & N/A & N/A & N/A \\
\hline N/A & N/A & N/A & N/A & N/A & $\mathrm{N} / \mathrm{A}$ & N/A & N/A & N/A & N/A & $\mathrm{N} / \mathrm{A}$ & N/A & N/A \\
\hline N/A & N/A & N/A & N/A & N/A & N/A & N/A & N/A & N/A & N/A & N/A & N/A & N/A \\
\hline N/A & N/A & N/A & $\mathrm{N} / \mathrm{A}$ & N/A & N/A & N/A & N/A & N/A & N/A & $\mathrm{N} / \mathrm{A}$ & N/A & N/A \\
\hline N/A & N/A & N/A & N/A & N/A & N/A & N/A & N/A & N/A & N/A & $\mathrm{N} / \mathrm{A}$ & N/A & N/A \\
\hline N/A & N/A & N/A & N/A & N/A & N/A & N/A & N/A & N/A & N/A & N/A & N/A & N/A \\
\hline N/A & N/A & N/A & N/A & N/A & N/A & N/A & N/A & N/A & N/A & N/A & N/A & N/A \\
\hline N/A & N/A & N/A & $\mathrm{N} / \mathrm{A}$ & N/A & N/A & N/A & N/A & N/A & N/A & $\mathrm{N} / \mathrm{A}$ & N/A & N/A \\
\hline N/A & N/A & $N / A$ & N/A & N/A & $N / A$ & $N / A$ & N/A & $N / A$ & N/A & $\mathrm{N} / \mathrm{A}$ & N/A & N/A \\
\hline N/A & N/A & $\mathrm{N} / \mathrm{A}$ & N/A & N/A & $\mathrm{N} / \mathrm{A}$ & N/A & N/A & N/A & N/A & $\mathrm{N} / \mathrm{A}$ & N/A & N/A \\
\hline N/A & N/A & N/A & N/A & N/A & N/A & N/A & N/A & N/A & $\mathrm{N} / \mathrm{A}$ & N/A & N/A & N/A \\
\hline N/A & N/A & N/A & $\mathrm{N} / \mathrm{A}$ & N/A & N/A & N/A & N/A & N/A & N/A & N/A & N/A & N/A \\
\hline N/A & N/A & N/A & N/A & N/A & $\mathrm{N} / \mathrm{A}$ & $\mathrm{N} / \mathrm{A}$ & N/A & N/A & N/A & N/A & N/A & N/A \\
\hline N/A & N/A & N/A & N/A & N/A & $\mathrm{N} / \mathrm{A}$ & $\mathrm{N} / \mathrm{A}$ & N/A & N/A & N/A & N/A & N/A & N/A \\
\hline N/A & N/A & $\mathrm{N} / \mathrm{A}$ & N/A & N/A & N/A & N/A & N/A & $\mathrm{N} / \mathrm{A}$ & N/A & $\mathrm{N} / \mathrm{A}$ & N/A & N/A \\
\hline N/A & N/A & $\mathrm{N} / \mathrm{A}$ & $\mathrm{N} / \mathrm{A}$ & N/A & $\mathrm{N} / \mathrm{A}$ & N/A & N/A & N/A & N/A & N/A & N/A & N/A \\
\hline N/A & N/A & $\mathrm{N} / \mathrm{A}$ & N/A & N/A & $\mathrm{N} / \mathrm{A}$ & N/A & $\mathrm{N} / \mathrm{A}$ & $\mathrm{N} / \mathrm{A}$ & N/A & N/A & $N / A$ & N/A \\
\hline
\end{tabular}




\section{RaDioloct}

Survey $10 E R \cdot A 26-161$

\section{Description/Map/Drawing/Picture/Comments}

Building 2201, Pluto Disassembly Facilty

Survey Plan \# 10-015

Room 101 "Cold Assembly Bay"

- Back Rooms West End (below mezzanine)
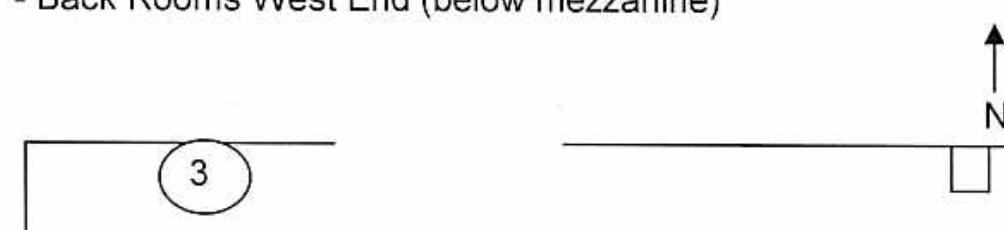

$\uparrow_{N}$

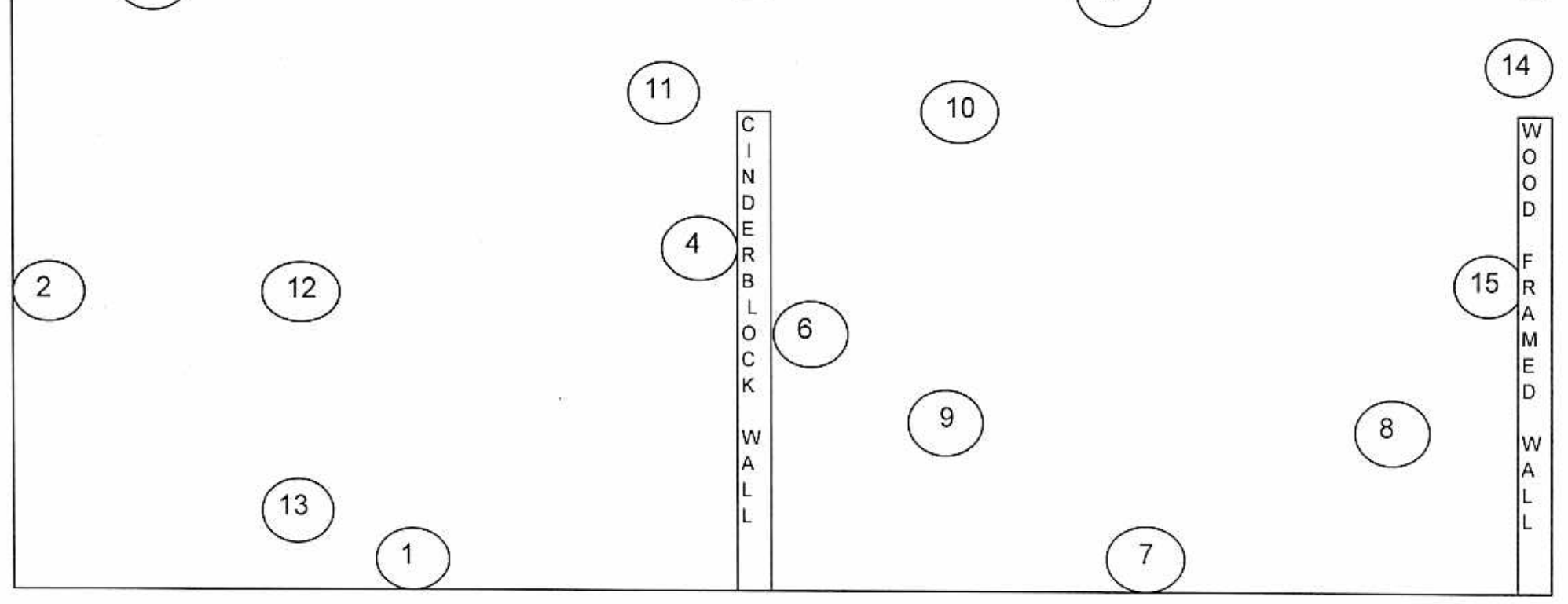

LEGEND

te Static Count LoCatian

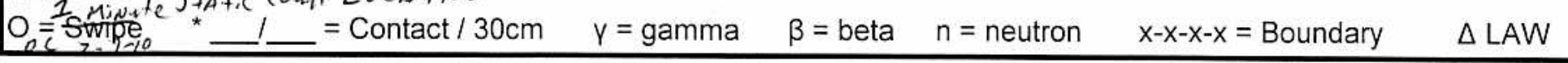


SURVEY \#

10-ER-A26-189

\begin{tabular}{|c|c|c|c|c|c|c|c|c|c|c|c|}
\hline \multicolumn{3}{|c|}{$\begin{array}{l}\text { Location: } \\
\text { Area } 26 \text { CAU117 Pluto }\end{array}$} & \multicolumn{2}{|c|}{$\begin{array}{l}\text { Purpose: } \\
\text { Final Status Survey, Room 103, } \\
\text { Ceiling }\end{array}$} & \multicolumn{5}{|c|}{$\begin{array}{l}\text { Comments: } \\
\text { All one minute static counts are in cpm. Bkgd not subtracted } \\
\text { for Final Status Survey, Survey Plan \#10-015 }\end{array}$} & \multicolumn{2}{|c|}{$\begin{array}{l}\text { Date/Time: } \\
\text { 07/19/2010 } 1100\end{array}$} \\
\hline Instrument: & Serial \#: & Cal Due: & \begin{tabular}{|c|} 
Eff in \%: \\
Alpha / Beta
\end{tabular} & $\begin{array}{l}\text { BKG in dpm: } \\
\text { Alpha / Beta }\end{array}$ & \begin{tabular}{|l|} 
MDA in dpm: \\
Alpha / Beta \\
\end{tabular} & RWP\# & \multicolumn{5}{|l|}{$\mathrm{N} / \mathrm{A}$} \\
\hline Electra & 1120 & $4 / 6 / 2011$ & $14.1 / 20.8$ & $N / A$ & $\mathrm{~N} / \mathrm{A}$ & \multicolumn{6}{|c|}{ Work Package \# $\quad$ ENV-10-RCT ENV SITE SURVEYS-0013 } \\
\hline $\mathrm{N} / \mathrm{A}$ & $N / A$ & $N / A$ & $\mathrm{~N} / \mathrm{A}$ & $N / A$ & $N / A$ & \multicolumn{3}{|c|}{ RCT Name: D.Cotroneo Jr. } & \multicolumn{3}{|c|}{ Signature: $(\theta)$} \\
\hline$N / A$ & $N / A$ & $N / A$ & $N / A$ & $\mathrm{~N} / \mathrm{A}$ & $\mathrm{N} / \mathrm{A}$ & \multicolumn{3}{|c|}{ RCT Name: Kevin Wilcox } & \multicolumn{3}{|c|}{ Signature: Kevi Wheos } \\
\hline$N / A$ & $N / A$ & $N / A$ & $N / A$ & $\mathrm{~N} / \mathrm{A}$ & $N / A$ & \multicolumn{3}{|c|}{ RCT Name: B. Bunce/S. Brown } & \multirow{2}{*}{\multicolumn{3}{|c|}{ Signature: कीforna/Smis }} \\
\hline$N / A$ & $\mathrm{~N} / \mathrm{A}$ & $\mathrm{N} / \mathrm{A}$ & $\mathrm{N} / \mathrm{A}$ & $N / A$ & $\mathrm{~N} / \mathrm{A}$ & RCT Name: & Brian Fos & & & & \\
\hline \multirow[t]{2}{*}{ Survey Point } & \multirow{2}{*}{\multicolumn{4}{|c|}{ Description/Comments }} & \multicolumn{2}{|c|}{$\begin{array}{l}\text { Removable } \\
\mathrm{dpm} / 100 \mathrm{~cm}^{2}\end{array}$} & \multicolumn{2}{|c|}{\begin{tabular}{|c|}
$\begin{array}{c}\text { Fixed + Removable } \\
\mathrm{dpm} / 100 \mathrm{~cm}^{2}\end{array}$ \\
\end{tabular}} & \multirow{2}{*}{$\begin{array}{l}\text { Gamma } \\
\mathrm{mrem} / \mathrm{hr}\end{array}$} & \multirow{2}{*}{$\begin{array}{l}\text { Neutron } \\
\mathrm{mrem} / \mathrm{hr}\end{array}$} & \multirow{2}{*}{$\begin{array}{c}\text { Total } \\
\mathrm{mrem} / \mathrm{hr}\end{array}$} \\
\hline & & & & & Alpha & Beta & Alpha & Beta & & & \\
\hline 1 & \multicolumn{4}{|l|}{ Ceiling, Metal } & $N / A$ & $N / A$ & 11 & 1303 & $N / A$ & $N / A$ & $N / A$ \\
\hline 2 & \multicolumn{4}{|l|}{ Ceiling, Metal } & $N / A$ & $N / A$ & 3 & 1211 & $N / A$ & $N / A$ & $N / A$ \\
\hline$N / A$ & \multicolumn{4}{|l|}{$N / A$} & $N / A$ & $N / A$ & $N / A$ & $N / A$ & $N / A$ & $\mathrm{~N} / \mathrm{A}$ & $N / A$ \\
\hline $\mathrm{N} / \mathrm{A}$ & \multicolumn{4}{|l|}{$N / A$} & $N / A$ & $N / A$ & $N / A$ & $N / A$ & $\mathrm{~N} / \mathrm{A}$ & $\mathrm{N} / \mathrm{A}$ & $N / A$ \\
\hline$N / A$ & \multicolumn{4}{|l|}{$N / A$} & $N / A$ & $N / A$ & $N / A$ & $N / A$ & $\mathrm{~N} / \mathrm{A}$ & $\mathrm{N} / \mathrm{A}$ & $N / A$ \\
\hline $\mathrm{N} / \mathrm{A}$ & \multicolumn{4}{|l|}{$N / A$} & $N / A$ & $N / A$ & $\mathrm{~N} / \mathrm{A}$ & $N / A$ & $N / A$ & $N / A$ & $N / A$ \\
\hline$N / A$ & \multicolumn{4}{|l|}{$N / A$} & $N / A$ & $\mathrm{~N} / \mathrm{A}$ & $N / A$ & $N / A$ & $N / A$ & $\mathrm{~N} / \mathrm{A}$ & $N / A$ \\
\hline$N / A$ & \multicolumn{4}{|l|}{$\mathrm{N} / \mathrm{A}$} & $N / A$ & $\mathrm{~N} / \mathrm{A}$ & $N / A$ & $N / A$ & $N / A$ & $N / A$ & $N / A$ \\
\hline$N / A$ & \multicolumn{4}{|l|}{$N / A$} & $N / A$ & $N / A$ & $N / A$ & $N / A$ & $\mathrm{~N} / \mathrm{A}$ & $N / A$ & $N / A$ \\
\hline$N / A$ & \multicolumn{4}{|l|}{$N / A$} & $N / A$ & $N / A$ & $N / A$ & $N / A$ & $N / A$ & $N / A$ & $N / A$ \\
\hline$N / A$ & \multicolumn{4}{|l|}{$N / A$} & $N / A$ & $N / A$ & $N / A$ & $N / A$ & $N / A$ & $N / A$ & $N / A$ \\
\hline \multicolumn{5}{|c|}{ Reviewed By (Print): } & \multicolumn{5}{|l|}{ Signature: } & \multicolumn{2}{|c|}{ Date: $7 / 20 / 10$} \\
\hline
\end{tabular}


Survey \# 10-ER-A26-189

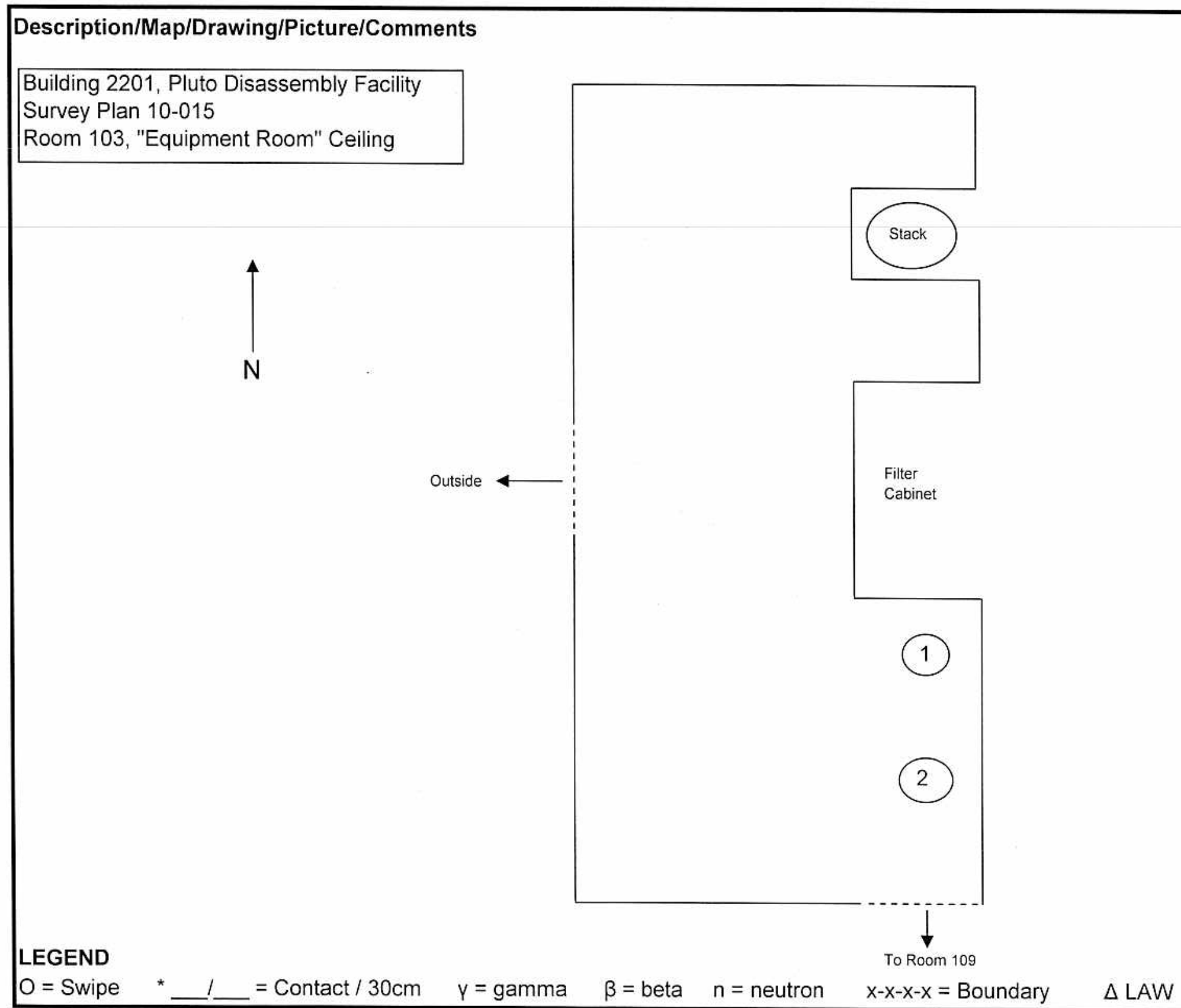




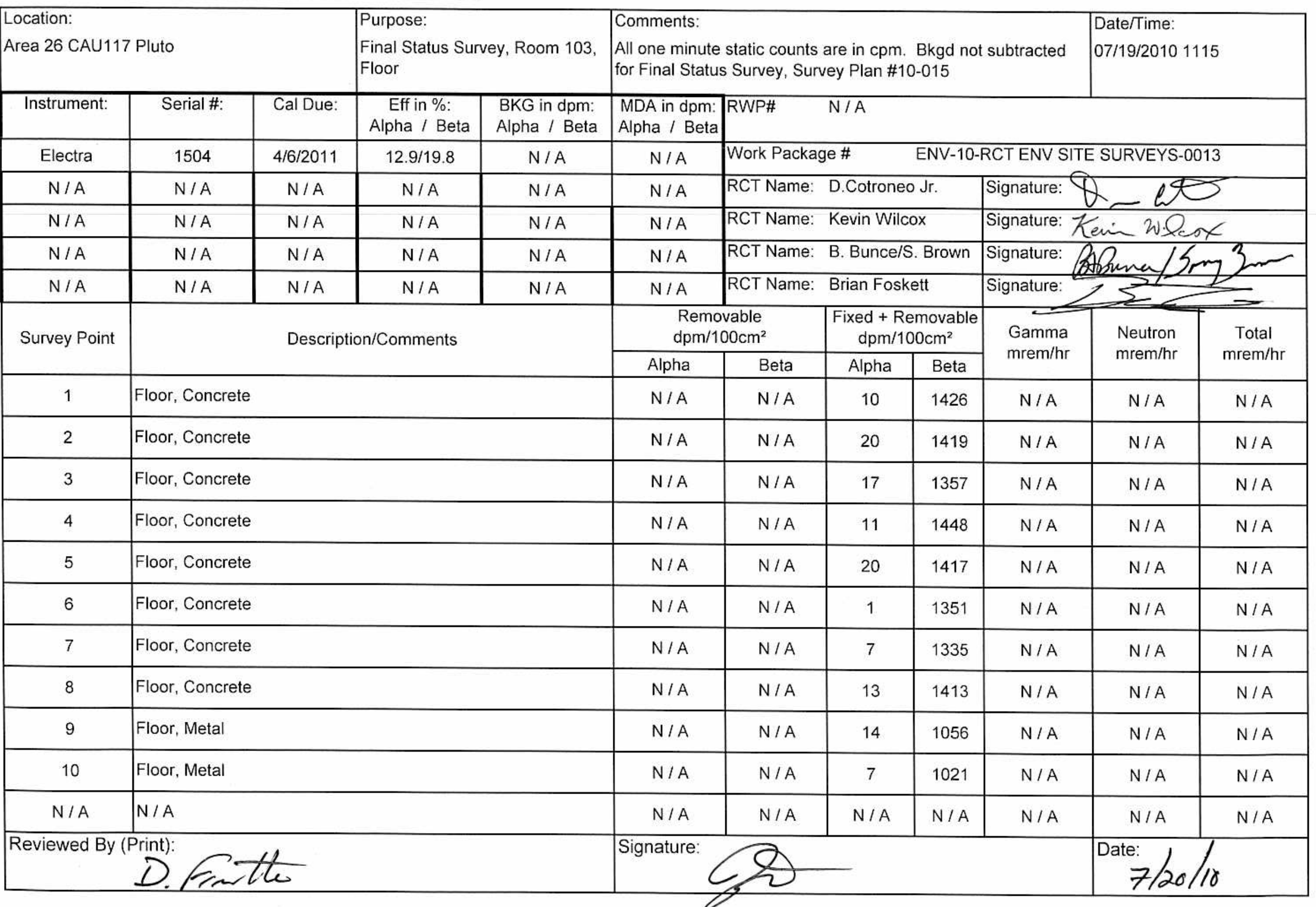


Survey \# 10-ER-A26-190

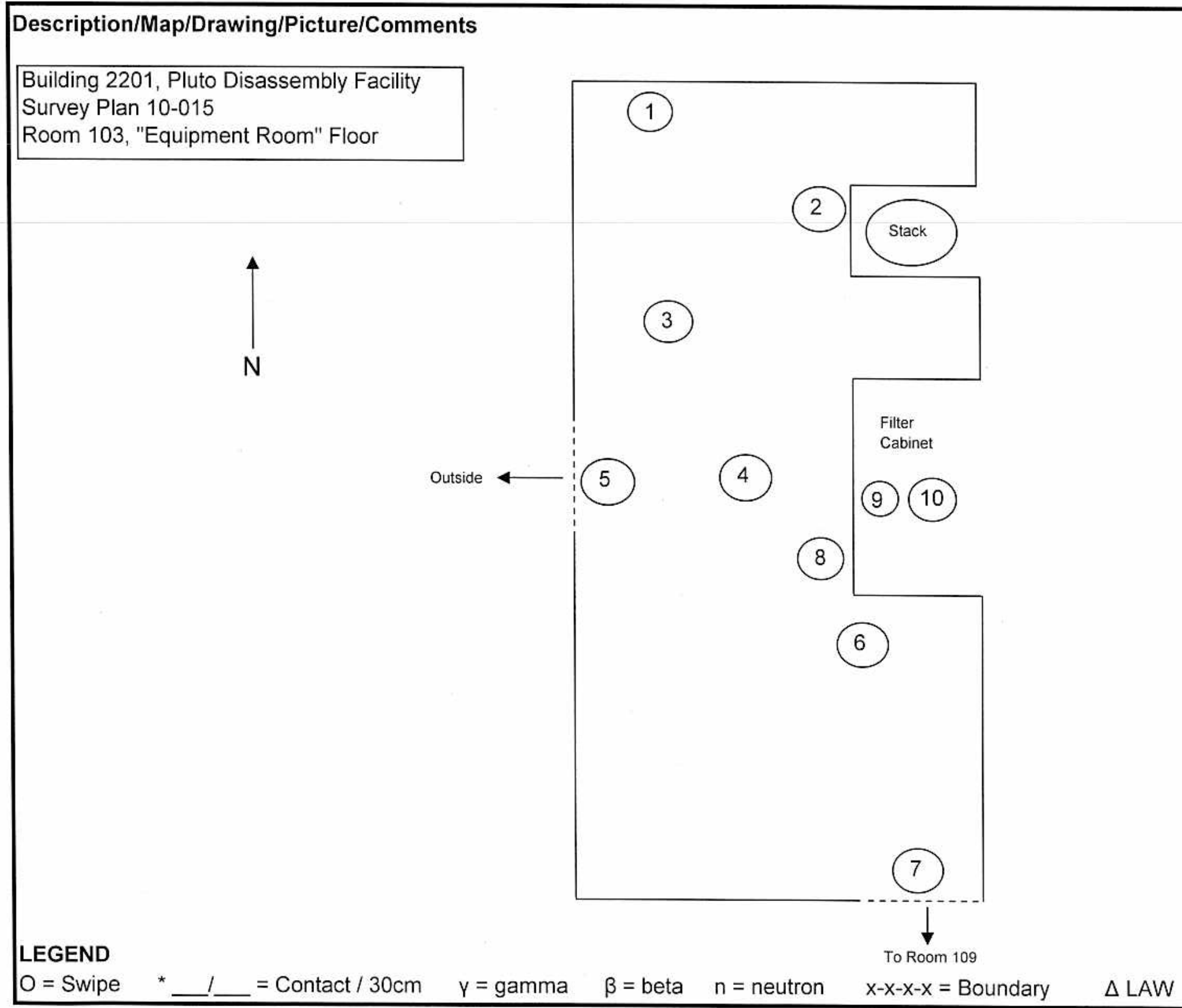


SURVEY \# 10-ER-A26-188

\begin{tabular}{|c|c|c|c|c|c|c|c|c|c|c|c|}
\hline \multicolumn{3}{|c|}{$\begin{array}{l}\text { Location: } \\
\text { Area } 26 \text { CAU117 Pluto }\end{array}$} & \multicolumn{2}{|c|}{$\begin{array}{l}\text { Purpose: } \\
\text { Final Status Survey, Room 103, } \\
\text { Walls }\end{array}$} & \multicolumn{5}{|c|}{$\begin{array}{l}\text { Comments: } \\
\text { All one minute static counts are in cpm. Bkgd not subtracted } \\
\text { for Final Status Survey, Survey Plan \#10-015 }\end{array}$} & \multicolumn{2}{|c|}{$\begin{array}{l}\text { Date/Time: } \\
\text { 07/19/2010 } 1030\end{array}$} \\
\hline Instrument: & Serial\#: & Cal Due: & $\begin{array}{c}\text { Eff in \%: } \\
\text { Alpha / Beta }\end{array}$ & $\begin{array}{l}\text { BKG in dpm: } \\
\text { Alpha / Beta }\end{array}$ & \begin{tabular}{|l|} 
MDA in dpm: \\
Alpha / Beta
\end{tabular} & RWP\# & \multicolumn{5}{|l|}{$\mathrm{N} / \mathrm{A}$} \\
\hline Electra & 1120 & $4 / 6 / 2011$ & $14.1 / 20.8$ & $N / A$ & $N / A$ & \multicolumn{6}{|c|}{ Work Package \# $\quad$ ENV-10-RCT ENV SITE SURVEYS-0013 } \\
\hline$N / A$ & $\mathrm{~N} / \mathrm{A}$ & $N / A$ & $N / A$ & $N / A$ & $\mathrm{~N} / \mathrm{A}$ & \multicolumn{3}{|c|}{ RCT Name: D.Cotroneo Jr. } & \multicolumn{3}{|c|}{ Signature: $Q-\omega$} \\
\hline$N / A$ & $\mathrm{~N} / \mathrm{A}$ & $N / A$ & $N / A$ & $N / A$ & $\mathrm{~N} / \mathrm{A}$ & \multicolumn{3}{|c|}{ RCT Name: Kevin Wilcox } & \multicolumn{3}{|c|}{ Signature: Treni Ws loos } \\
\hline$N / A$ & $\mathrm{~N} / \mathrm{A}$ & $N / A$ & $N / A$ & $N / A$ & $N / A$ & \multicolumn{3}{|c|}{ RCT Name: B. Bunce/S. Brown } & \multirow{2}{*}{\multicolumn{3}{|c|}{ Signature: 24 huna/5rman }} \\
\hline $\mathrm{N} / \mathrm{A}$ & $\mathrm{N} / \mathrm{A}$ & $N / A$ & $N / A$ & $\mathrm{~N} / \mathrm{A}$ & $N / A$ & RCT Name: & Brian Fos & & & & \\
\hline \multirow[t]{2}{*}{ Survey Point } & \multirow{2}{*}{\multicolumn{4}{|c|}{ Description/Comments }} & \multicolumn{2}{|c|}{$\begin{array}{l}\text { Removable } \\
\mathrm{dpm} / 100 \mathrm{~cm}^{2}\end{array}$} & \multicolumn{2}{|c|}{\begin{tabular}{|c|} 
Fixed + Removable \\
$\mathrm{dpm} / 100 \mathrm{~cm}^{2}$
\end{tabular}} & \multirow{2}{*}{$\begin{array}{l}\text { Gamma } \\
\mathrm{mrem} / \mathrm{hr}\end{array}$} & \multirow{2}{*}{$\begin{array}{l}\text { Neutron } \\
\text { mrem } / \mathrm{hr}\end{array}$} & \multirow{2}{*}{$\begin{array}{l}\text { Total } \\
\mathrm{mrem} / \mathrm{hr}\end{array}$} \\
\hline & & & & & Alpha & Beta & Alpha & Beta & & & \\
\hline 1 & \multicolumn{4}{|c|}{ North Wall, Concrete } & $N / A$ & $N / A$ & 8 & 1219 & $N / A$ & $N / A$ & $N / A$ \\
\hline 2 & \multicolumn{4}{|c|}{ North Wall, Concrete } & $\mathrm{N} / \mathrm{A}$ & $N / A$ & 13 & 1213 & $N / A$ & $N / A$ & $N / A$ \\
\hline 3 & \multicolumn{4}{|c|}{ East Wall, Concrete } & $N / A$ & $N / A$ & 22 & 1214 & $N / A$ & $N / A$ & $N / A$ \\
\hline 4 & \multicolumn{4}{|c|}{ East Wall, Concrete } & $N / A$ & $N / A$ & 14 & 1220 & $N / A$ & $N / A$ & $N / A$ \\
\hline 5 & \multicolumn{4}{|c|}{ East Wall, Metal } & $N / A$ & $N / A$ & 6 & 824 & $N / A$ & $N / A$ & $N / A$ \\
\hline 6 & \multicolumn{4}{|c|}{ East Wall, Metal } & $N / A$ & $\mathrm{~N} / \mathrm{A}$ & 7 & 869 & $N / A$ & $N / A$ & $N / A$ \\
\hline 7 & \multicolumn{4}{|c|}{ East Wall, Concrete } & $N / A$ & $N / A$ & 15 & 1157 & $N / A$ & $N / A$ & $N / A$ \\
\hline 8 & \multicolumn{4}{|c|}{ South Wall, Concrete } & $N / A$ & $N / A$ & 13 & 1141 & $N / A$ & $N / A$ & $N / A$ \\
\hline 9 & \multicolumn{4}{|c|}{ West Wall, Concrete } & $N / A$ & $N / A$ & 12 & 1179 & $\mathrm{~N} / \mathrm{A}$ & $N / A$ & $N / A$ \\
\hline 10 & \multicolumn{4}{|c|}{ West Wall, Concrete } & $N / A$ & $N / A$ & 13 & 1255 & $N / A$ & $N / A$ & $N / A$ \\
\hline 11 & \multicolumn{4}{|c|}{ West Wall, Concrete } & $N / A$ & $N / A$ & 17 & 1166 & $N / A$ & $N / A$ & $N / A$ \\
\hline \multicolumn{5}{|c|}{ Reviewed By (Print): } & \multicolumn{7}{|c|}{ Signature: } \\
\hline
\end{tabular}


SURVEY \# 10-ER-A26-188

\begin{tabular}{|c|c|c|c|c|c|c|c|c|}
\hline \multirow{2}{*}{$\begin{array}{l}\text { Survey } \\
\text { Point }\end{array}$} & \multirow[t]{2}{*}{ Description/Comments } & \multicolumn{2}{|c|}{$\begin{array}{l}\text { Removable } \\
\mathrm{dpm} / 100 \mathrm{~cm}^{2}\end{array}$} & \multicolumn{2}{|c|}{$\begin{array}{c}\text { Fixed + Removable } \\
\mathrm{dpm} / 100 \mathrm{~cm}^{2}\end{array}$} & \multirow{2}{*}{$\begin{array}{l}\text { Gamma } \\
\mathrm{mrem} / \mathrm{hr}\end{array}$} & \multirow{2}{*}{$\begin{array}{l}\text { Neutron } \\
\text { mrem/hr }\end{array}$} & \multirow{2}{*}{$\begin{array}{c}\text { Total } \\
\text { mrem/h }\end{array}$} \\
\hline & & Alpha & Beta & Alpha & Beta & & & \\
\hline 12 & South Wall, Concrete & N/A & $\mathrm{N} / \mathrm{A}$ & 13 & 1157 & N/A & $\mathrm{N} / \mathrm{A}$ & $\mathrm{N} / \mathrm{A}$ \\
\hline 13 & West Wall, Concrete & N/A & N/A & 11 & 1244 & N/A & $\mathrm{N} / \mathrm{A}$ & $\mathrm{N} / \mathrm{A}$ \\
\hline $\mathrm{N} / \mathrm{A}$ & $\mathrm{N} / \mathrm{A}$ & N/A & $\mathrm{N} / \mathrm{A}$ & N/A & $\mathrm{N} / \mathrm{A}$ & N/A & N/A & N/A \\
\hline N/A & $\mathrm{N} / \mathrm{A}$ & N/A & N/A & N/A & $\mathrm{N} / \mathrm{A}$ & N/A & $\mathrm{N} / \mathrm{A}$ & $\mathrm{N} / \mathrm{A}$ \\
\hline N/A & $\mathrm{N} / \mathrm{A}$ & N/A & N/A & N/A & $\mathrm{N} / \mathrm{A}$ & N/A & $\mathrm{N} / \mathrm{A}$ & N/A \\
\hline N/A & $\mathrm{N} / \mathrm{A}$ & N/A & $\mathrm{N} / \mathrm{A}$ & N/A & $\mathrm{N} / \mathrm{A}$ & $\mathrm{N} / \mathrm{A}$ & $\mathrm{N} / \mathrm{A}$ & N/A \\
\hline $\mathrm{N} / \mathrm{A}$ & $\mathrm{N} / \mathrm{A}$ & N/A & $\mathrm{N} / \mathrm{A}$ & N/A & N/A & N/A & $\mathrm{N} / \mathrm{A}$ & N/A \\
\hline N/A & $N / A$ & N/A & $\mathrm{N} / \mathrm{A}$ & N/A & $\mathrm{N} / \mathrm{A}$ & N/A & $\mathrm{N} / \mathrm{A}$ & $\mathrm{N} / \mathrm{A}$ \\
\hline N/A & $\mathrm{N} / \mathrm{A}$ & N/A & $\mathrm{N} / \mathrm{A}$ & N/A & $\mathrm{N} / \mathrm{A}$ & N/A & $\mathrm{N} / \mathrm{A}$ & $\mathrm{N} / \mathrm{A}$ \\
\hline $\mathrm{N} / \mathrm{A}$ & $N / A$ & N/A & N/A & N/A & $\mathrm{N} / \mathrm{A}$ & $\mathrm{N} / \mathrm{A}$ & N/A & N/A \\
\hline $\mathrm{N} / \mathrm{A}$ & $\mathrm{N} / \mathrm{A}$ & N/A & $\mathrm{N} / \mathrm{A}$ & N/A & N/A & N/A & $\mathrm{N} / \mathrm{A}$ & $\mathrm{N} / \mathrm{A}$ \\
\hline N/A & $\mathrm{N} / \mathrm{A}$ & N/A & N/A & N/A & $\mathrm{N} / \mathrm{A}$ & $\mathrm{N} / \mathrm{A}$ & N/A & $\mathrm{N} / \mathrm{A}$ \\
\hline $\mathrm{N} / \mathrm{A}$ & $N / A$ & N/A & $\mathrm{N} / \mathrm{A}$ & N/A & $\mathrm{N} / \mathrm{A}$ & N/A & N/A & $\mathrm{N} / \mathrm{A}$ \\
\hline $\mathrm{N} / \mathrm{A}$ & $\mathrm{N} / \mathrm{A}$ & N/A & $\mathrm{N} / \mathrm{A}$ & N/A & N/A & N/A & N/A & N/A \\
\hline N/A & $N / A$ & N/A & $\mathrm{N} / \mathrm{A}$ & $\mathrm{N} / \mathrm{A}$ & $\mathrm{N} / \mathrm{A}$ & N/A & $\mathrm{N} / \mathrm{A}$ & $\mathrm{N} / \mathrm{A}$ \\
\hline N/A & $N / A$ & N/A & $\mathrm{N} / \mathrm{A}$ & N/A & $\mathrm{N} / \mathrm{A}$ & N/A & $\mathrm{N} / \mathrm{A}$ & N/A \\
\hline N/A & $N / A$ & N/A & N/A & N/A & $\mathrm{N} / \mathrm{A}$ & N/A & $\mathrm{N} / \mathrm{A}$ & N/A \\
\hline N/A & $\mathrm{N} / \mathrm{A}$ & $\mathrm{N} / \mathrm{A}$ & $\mathrm{N} / \mathrm{A}$ & N/A & $\mathrm{N} / \mathrm{A}$ & N/A & $\mathrm{N} / \mathrm{A}$ & N/A \\
\hline $\mathrm{N} / \mathrm{A}$ & $N / A$ & $\mathrm{~N} / \mathrm{A}$ & N/A & N/A & $\mathrm{N} / \mathrm{A}$ & N/A & N/A & N/A \\
\hline $\mathrm{N} / \mathrm{A}$ & $\mathrm{N} / \mathrm{A}$ & $\mathrm{N} / \mathrm{A}$ & $\mathrm{N} / \mathrm{A}$ & N/A & $\mathrm{N} / \mathrm{A}$ & $\mathrm{N} / \mathrm{A}$ & N/A & N/A \\
\hline N/A & N/A & N/A & $\mathrm{N} / \mathrm{A}$ & N/A & $\mathrm{N} / \mathrm{A}$ & N/A & $\mathrm{N} / \mathrm{A}$ & $\mathrm{N} / \mathrm{A}$ \\
\hline N/A & N/A & N/A & $\mathrm{N} / \mathrm{A}$ & N/A & $\mathrm{N} / \mathrm{A}$ & N/A & $\mathrm{N} / \mathrm{A}$ & N/A \\
\hline N/A & N/A & N/A & N/A & N/A & $\mathrm{N} / \mathrm{A}$ & N/A & $\mathrm{N} / \mathrm{A}$ & $\mathrm{N} / \mathrm{A}$ \\
\hline
\end{tabular}


Survey \# 10-ER-A26-188

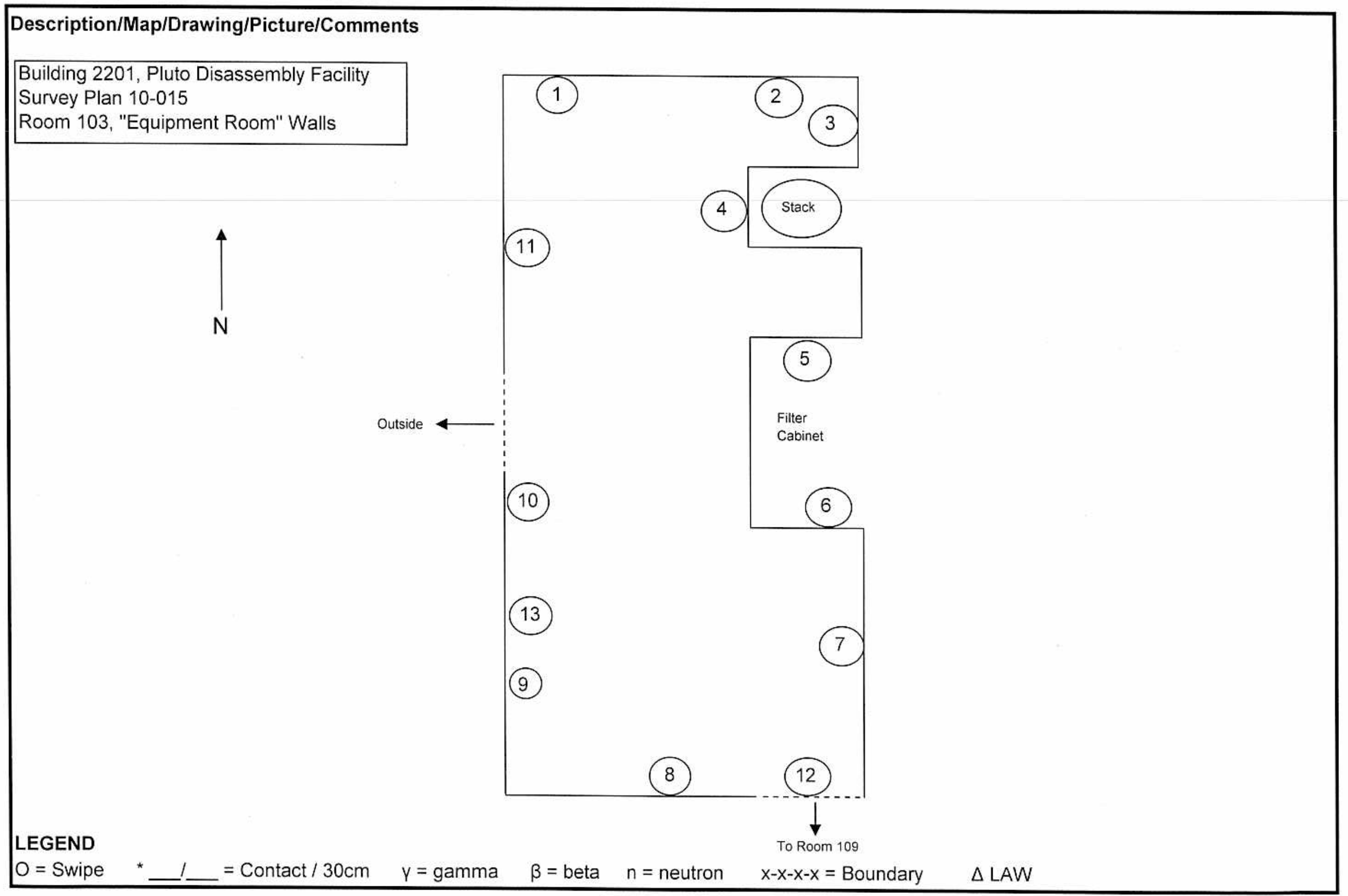


FRM-0108B

SURVEY \#

10-ER-A26-168

\begin{tabular}{|c|c|c|c|c|c|c|c|c|c|c|c|}
\hline \multicolumn{3}{|c|}{$\begin{array}{l}\text { Location: } \\
\text { Area } 26 \text { CAU117 Pluto }\end{array}$} & \multicolumn{2}{|c|}{$\begin{array}{l}\text { Purpose: } \\
\text { Final Status Survey, Room } 105\end{array}$} & & $\begin{array}{l}\text { All one minute static counts are in } \mathrm{cpm} \text { Bkgd not subtracted } \\
\text { for Final Status Survey, Survey Plan \#10-015 }\end{array}$ & \multicolumn{2}{|c|}{$\begin{array}{l}\text { Date/Time: } \\
\text { 07/08/2010 } 1600\end{array}$} \\
\hline Instrument: & Serial \#: & Cal Due: & \begin{tabular}{|c|} 
Eff in \%: \\
Alpha / Beta \\
\end{tabular} & $\begin{array}{l}\text { BKG in dpm: } \\
\text { Alpha / Beta }\end{array}$ & $\begin{array}{l}\text { MDA in dpm: } \\
\text { Alpha / Beta } \\
\end{array}$ & RWP\# & \multicolumn{5}{|l|}{$\mathrm{N} / \mathrm{A}$} \\
\hline Electra & 1504 & $4 / 6 / 2011$ & $12.9 / 19.8$ & $N / A$ & $N / A$ & \multicolumn{6}{|c|}{ Work Package \# $\quad$ ENV-10-RCT ENV SITE SURVEYS-0013 } \\
\hline $\mathrm{N} / \mathrm{A}$ & $\mathrm{N} / \mathrm{A}$ & $N / A$ & $\mathrm{~N} / \mathrm{A}$ & $N / A$ & \multirow{2}{*}{$\begin{array}{l}N / A \\
N / A\end{array}$} & RCT Name: D.Cotroneo Jr. & \multicolumn{2}{|c|}{ D.Cotroneo Jr. } & \multicolumn{3}{|c|}{ Signature: $(0$} \\
\hline$N / A$ & $N / A$ & $N / A$ & $N / A$ & $N / A$ & & \multicolumn{3}{|c|}{ RCT Name: Kevin Wilcox } & \multicolumn{3}{|c|}{ Signature: $\mathrm{Ze}=\mathrm{W}_{\mathrm{C}} \mathrm{Cos}$} \\
\hline$N / A$ & $\mathrm{~N} / \mathrm{A}$ & $N / A$ & $N / A$ & $N / A$ & $N / A$ & RCT Name: & S.Brown/E & Bunce & \multirow{2}{*}{\multicolumn{3}{|c|}{ Signature: }} \\
\hline $\mathrm{N} / \mathrm{A}$ & $N / A$ & $N / A$ & $\mathrm{~N} / \mathrm{A}$ & $N / A$ & $N / A$ & RCT Name: & Brian Fos & & & & \\
\hline \multirow[t]{2}{*}{ Survey Point } & \multirow{2}{*}{\multicolumn{4}{|c|}{ Description/Comments }} & \multicolumn{2}{|c|}{$\begin{array}{l}\text { Removable } \\
\mathrm{dpm} / 100 \mathrm{~cm}^{2}\end{array}$} & \multicolumn{2}{|c|}{$\begin{array}{c}\text { Fixed + Removable } \\
\mathrm{dpm} / 100 \mathrm{~cm}^{2}\end{array}$} & \multirow{2}{*}{$\begin{array}{l}\text { Gamma } \\
\mathrm{mrem} / \mathrm{hr}\end{array}$} & \multirow{2}{*}{$\begin{array}{l}\text { Neutron } \\
\mathrm{mrem} / \mathrm{hr}\end{array}$} & \multirow{2}{*}{$\begin{array}{c}\text { Total } \\
\mathrm{mrem} / \mathrm{hr}\end{array}$} \\
\hline & & & & & Alpha & Beta & Alpha & Beta & & & \\
\hline 1 & \multicolumn{4}{|c|}{ Floor Concrete } & $N / A$ & $N / A$ & 2 & 977 & $\mathrm{~N} / \mathrm{A}$ & $N / A$ & $N / A$ \\
\hline 2 & \multicolumn{4}{|c|}{ Floor Concrete } & $N / A$ & $N / A$ & 6 & 1150 & $\mathrm{~N} / \mathrm{A}$ & $N / A$ & $N / A$ \\
\hline 3 & \multicolumn{4}{|c|}{ Floor Concrete } & $N / A$ & $N / A$ & 7 & 1137 & $N / A$ & $N / A$ & $N / A$ \\
\hline 4 & \multicolumn{4}{|c|}{ Floor Concrete } & $N / A$ & $N / A$ & 2 & 1283 & $N / A$ & $N / A$ & $N / A$ \\
\hline 5 & \multicolumn{4}{|c|}{ East Wall Concrete } & $N / A$ & $N / A$ & 5 & 729 & $N / A$ & $N / A$ & $N / A$ \\
\hline 6 & \multicolumn{4}{|c|}{ East Wall Concrete } & $N / A$ & $N / A$ & 2 & 751 & $N / A$ & $N / A$ & $N / A$ \\
\hline 7 & \multicolumn{4}{|c|}{ East Wall Concrete } & $N / A$ & $N / A$ & 3 & 618 & $N / A$ & $N / A$ & $N / A$ \\
\hline 8 & \multicolumn{4}{|c|}{ West Wall Concrete } & $N / A$ & $N / A$ & 3 & 1029 & $N / A$ & $N / A$ & $N / A$ \\
\hline 9 & \multicolumn{4}{|c|}{ West Wall Concrete } & $N / A$ & $N / A$ & 3 & 703 & $N / A$ & $N / A$ & $N / A$ \\
\hline 10 & \multicolumn{4}{|c|}{ West Wall Concrete } & $N / A$ & $N / A$ & 0 & 647 & $N / A$ & $N / A$ & $N / A$ \\
\hline 11 & \multicolumn{4}{|c|}{ North Wall Concrete } & $N / A$ & $\mathrm{~N} / \mathrm{A}$ & 2 & 1166 & $N / A$ & $N / A$ & $N / A$ \\
\hline \multicolumn{5}{|c|}{ Reviewed By (Print): } & Signature & & & & & Date: $7 / 12$ & \\
\hline
\end{tabular}


NSTec

SURVEY \# 10-ER-A26-168

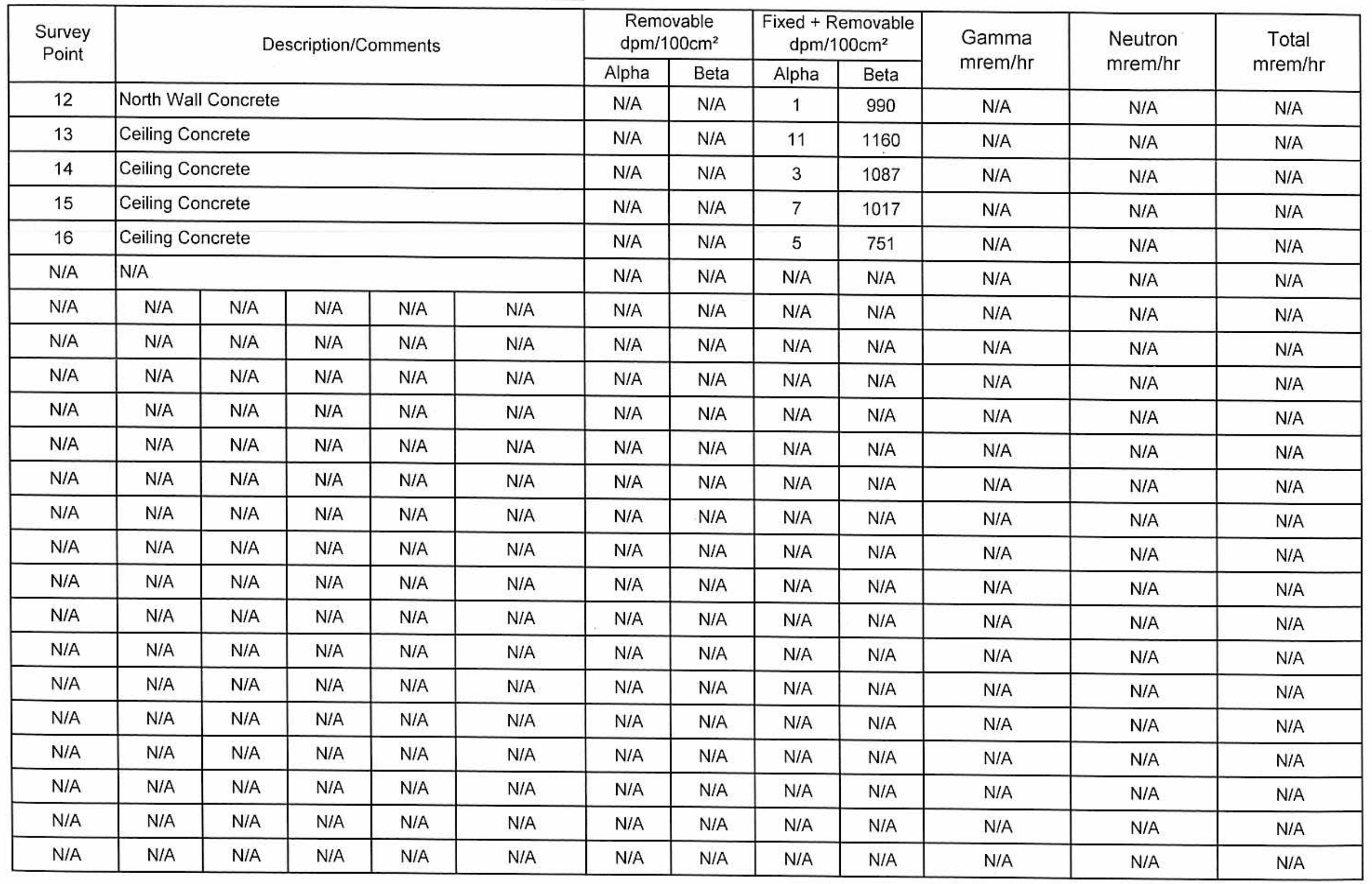




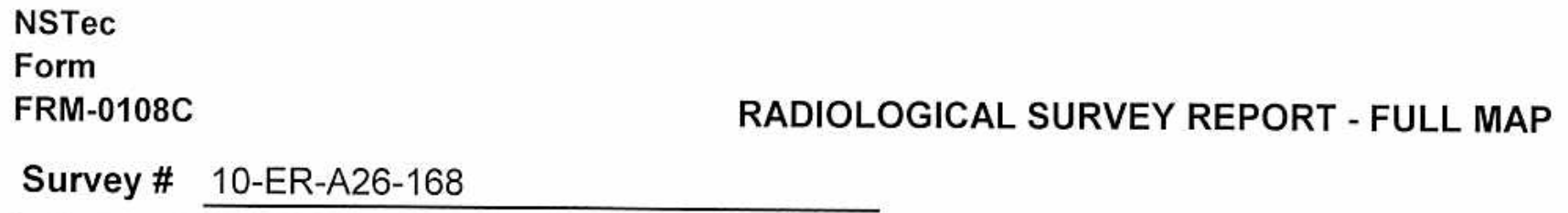

05/24/10

Rev. 01

Page 3 of 3

Description/Map/Drawing/Picture/Comments

Building 2201 Pluto Disassembly Facility

Survey Plan 10-015

Room 105 "Control Room"

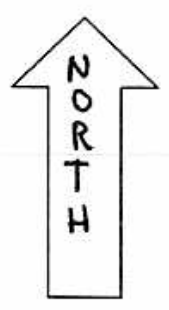

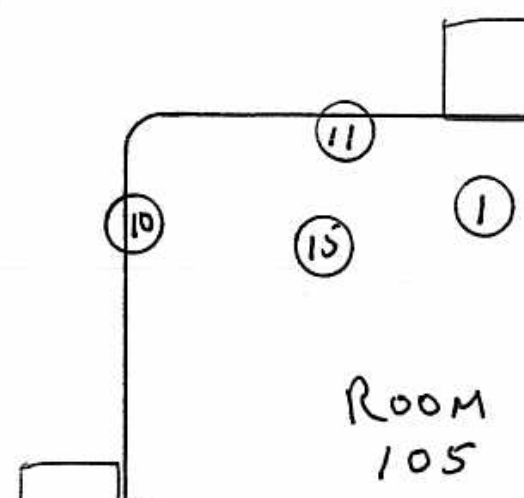

(9)

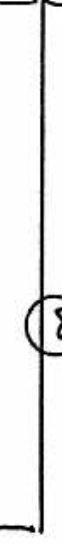

(2) (14)

(4)

Room 108
(12)

(16)

(6)

(3)

(13)

Room 108

LEGEND STATC Cont Lout iou

$\mathrm{O}_{n=3}=30$

$=$ Contact $/ 30 \mathrm{~cm}$

$Y=$ gamma

$\beta=$ beta $n=$ neutron

$x-x-x-x=$ Boundary

$\triangle$ LAW 
FRM-0108B

SURVEY \#

10-ER-A26-172

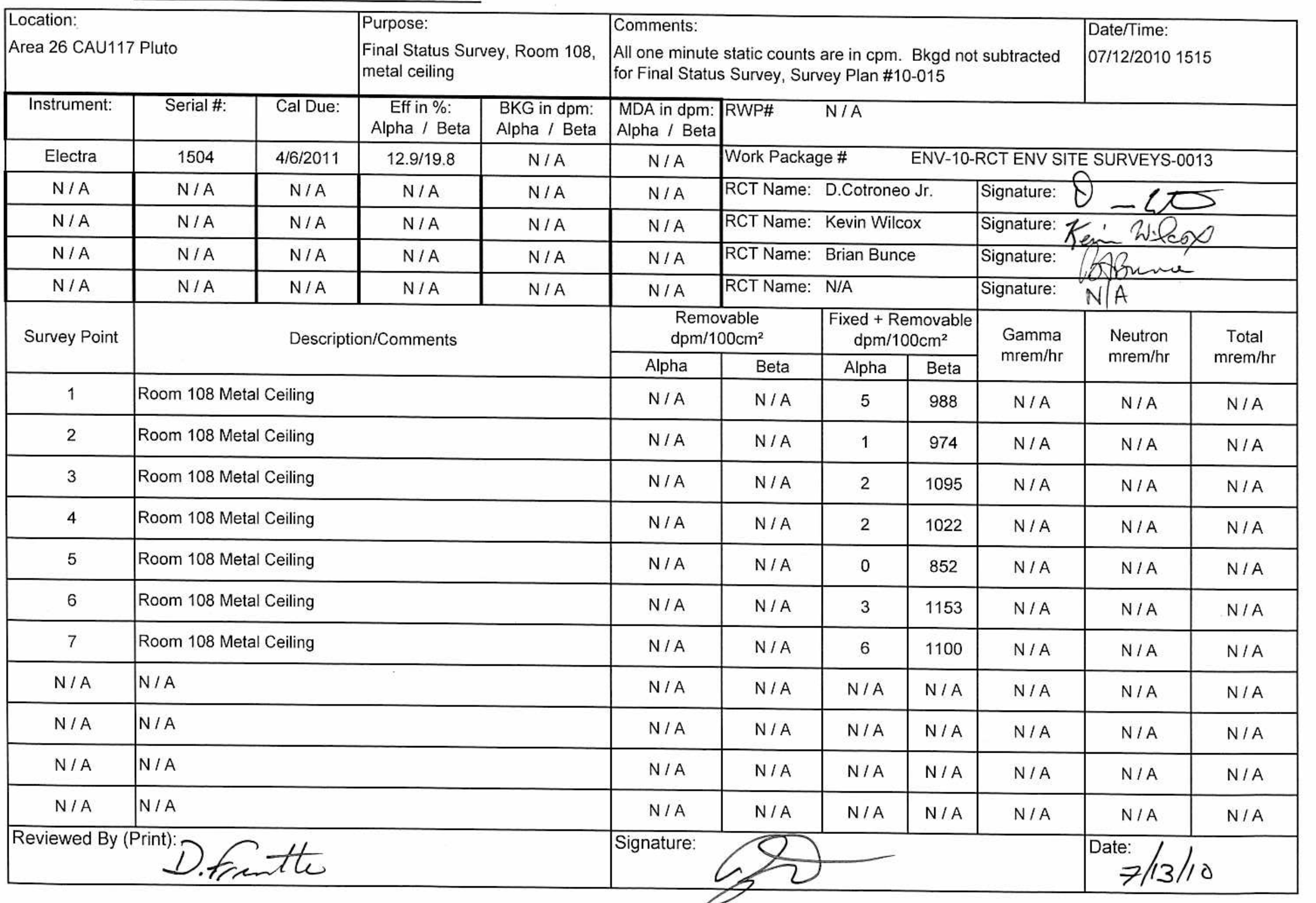


Desc iption/Map/Drawing/Picture/Comments

Building 2201, Pluto Disassembly Facility, Room 108 "Operating Area"

Survey Plan 10-015 Room 108 Metal Ceiling

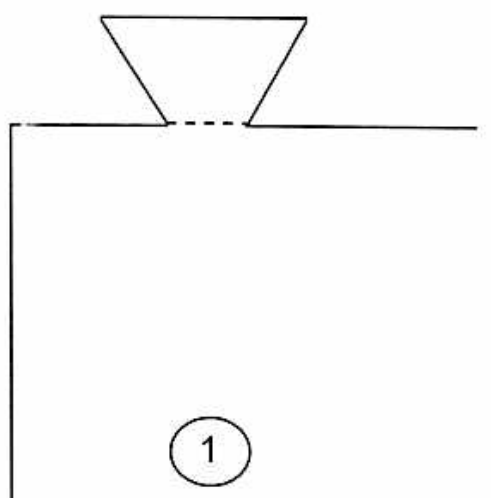

Room 105

$\uparrow$

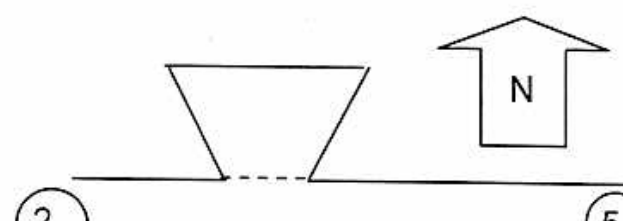

(5)

(4)
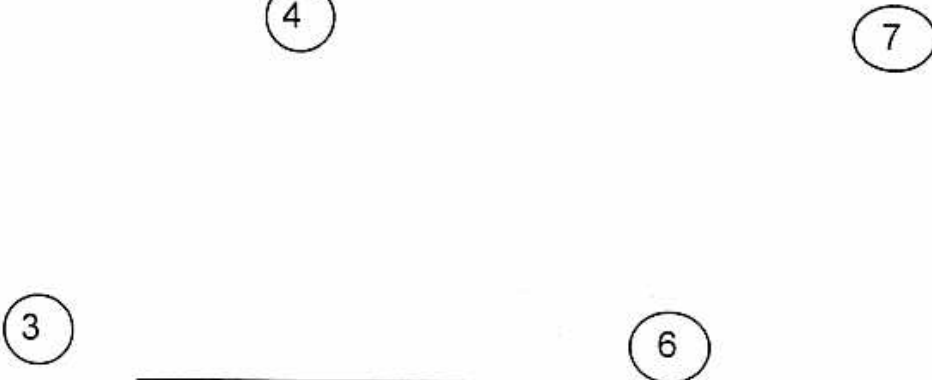

LEGIEND

$\mathrm{O}=$ Swipe

* $\quad 1 \quad=$ Contact $/ 30 \mathrm{~cm}$

$Y=$ gamma

$\beta=$ beta

$n=$ neutron 
SURVEY \#

10-ER-A26-173

\begin{tabular}{|c|c|c|c|c|c|c|c|c|c|c|c|}
\hline \multicolumn{3}{|c|}{$\begin{array}{l}\text { Location: } \\
\text { Area } 26 \text { CAU117 Pluto }\end{array}$} & \multicolumn{2}{|c|}{$\begin{array}{l}\text { Purpose: } \\
\text { Final Status Survey, Room 108, } \\
\text { Concrete Floor }\end{array}$} & \multicolumn{5}{|c|}{$\begin{array}{l}\text { Comments: } \\
\text { All one minute static counts are in cpm. Bkgd not subtracted } \\
\text { for Final Status Survey, Survey Plan \#10-015 }\end{array}$} & \multicolumn{2}{|c|}{$\begin{array}{l}\text { Date/Time: } \\
\text { 07/12/2010 } 1530\end{array}$} \\
\hline Instrument: & Serial \#: & Cal Due: & $\begin{array}{c}\text { Eff in \%: } \\
\text { Alpha / Beta }\end{array}$ & $\begin{array}{l}\text { BKG in dpm: } \\
\text { Alpha / Beta }\end{array}$ & $\begin{array}{l}\text { MDA in dpm: } \\
\text { Alpha / Beta }\end{array}$ & RWP\# & \multicolumn{5}{|c|}{$\mathrm{N} / \mathrm{A}$} \\
\hline Electra & 1504 & $4 / 6 / 2011$ & $12.9 / 19.8$ & $N / A$ & $N / A$ & \multicolumn{6}{|c|}{ ENV-10-RCT ENV SITE } \\
\hline Electra & 1120 & $4 / 6 / 2011$ & $14.1 / 20.8$ & $\mathrm{~N} / \mathrm{A}$ & $\mathrm{N} / \mathrm{A}$ & \multicolumn{3}{|c|}{ RCT Name: D.Cotroneo Jr. } & \multicolumn{3}{|c|}{ Signature: $\forall \sim t$} \\
\hline$N / A$ & $N / A$ & $\mathrm{~N} / \mathrm{A}$ & $N / A$ & $\mathrm{~N} / \mathrm{A}$ & $N / A$ & \multicolumn{3}{|c|}{ RCT Name: Kevin Wilcox } & \multicolumn{3}{|c|}{ Signature: Kein W.Poor } \\
\hline$N / A$ & $N / A$ & $N / A$ & $N / A$ & $N / A$ & $\mathrm{~N} / \mathrm{A}$ & \multicolumn{3}{|c|}{ RCT Name: S. Brown/B. Bunce } & \multicolumn{3}{|c|}{ 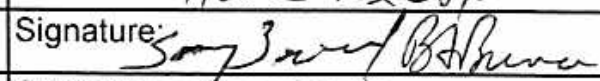 } \\
\hline $\mathrm{N} / \mathrm{A}$ & $N / A$ & $N / A$ & $\mathrm{~N} / \mathrm{A}$ & $\mathrm{N} / \mathrm{A}$ & $\mathrm{N} / \mathrm{A}$ & \multicolumn{3}{|c|}{ RCT Name: Brian Foskett } & \multicolumn{3}{|c|}{ Signature. $<\leq$} \\
\hline \multirow[t]{2}{*}{ Survey Point } & \multirow{2}{*}{\multicolumn{4}{|c|}{ Description/Comments }} & \multicolumn{2}{|c|}{$\begin{array}{l}\text { Removable } \\
\mathrm{dpm} / 100 \mathrm{~cm}^{2}\end{array}$} & \multicolumn{2}{|c|}{$\begin{array}{c}\text { Fixed + Removable } \\
\mathrm{dpm} / 100 \mathrm{~cm}^{2}\end{array}$} & \multirow{2}{*}{$\begin{array}{l}\text { Gamma } \\
\mathrm{mrem} / \mathrm{hr}\end{array}$} & \multirow{2}{*}{$\begin{array}{l}\text { Neutron } \\
\mathrm{mrem} / \mathrm{hr}\end{array}$} & \multirow{2}{*}{$\begin{array}{c}\text { Total } \\
\mathrm{mrem} / \mathrm{hr}\end{array}$} \\
\hline & & & & & Alpha & Beta & Alpha & Beta & & & \\
\hline 1 & \multicolumn{4}{|c|}{ Room 108 Concrete Floor (Electra 1120) } & $N / A$ & $N / A$ & 12 & 1530 & $N / A$ & $N / A$ & $N / A$ \\
\hline 2 & \multicolumn{4}{|c|}{ Room 108 Concrete Floor (Electra 1120) } & $N / A$ & $\mathrm{~N} / \mathrm{A}$ & 10 & 1573 & $N / A$ & $N / A$ & $N / A$ \\
\hline 3 & \multicolumn{4}{|c|}{ Room 108 Concrete Floor (Electra 1120) } & $N / A$ & $N / A$ & 9 & 1501 & $N / A$ & $N / A$ & $N / A$ \\
\hline 4 & \multicolumn{4}{|c|}{ Room 108 Concrete Floor (Electra 1120) } & $N / A$ & $N / A$ & 1 & 1428 & $N / A$ & $N / A$ & $N / A$ \\
\hline 5 & \multicolumn{4}{|c|}{ Room 108 Concrete Floor (Electra 1120) } & $N / A$ & $N / A$ & 1 & 1343 & $N / A$ & $N / A$ & $N / A$ \\
\hline 6 & \multicolumn{4}{|c|}{ Room 108 Concrete Floor (Electra 1120) } & $N / A$ & $N / A$ & 8 & 1501 & $\mathrm{~N} / \mathrm{A}$ & $N / A$ & $N / A$ \\
\hline 7 & \multicolumn{4}{|c|}{ Room 108 Concrete Floor (Electra 1120) } & $N / A$ & $N / A$ & 1 & 1230 & $N / A$ & $N / A$ & $N / A$ \\
\hline 8 & \multicolumn{4}{|c|}{ Room 108 Concrete Floor (Electra 1120) } & $N / A$ & $N / A$ & 10 & 1498 & $\mathrm{~N} / \mathrm{A}$ & $N / A$ & $N / A$ \\
\hline 9 & \multicolumn{4}{|c|}{ Room 108 Concrete Floor (Electra 1120) } & $N / A$ & $N / A$ & 10 & 1517 & $N / A$ & $N / A$ & $N / A$ \\
\hline 10 & \multicolumn{4}{|c|}{ Room 108 Concrete Floor (Electra 1504) } & $N / A$ & $N / A$ & 7 & 1285 & $N / A$ & $N / A$ & $N / A$ \\
\hline 11 & \multicolumn{4}{|c|}{ Room 108 Concrete Floor (Electra 1120) } & $N / A$ & $N / A$ & 7 & 1506 & $N / A$ & $N / A$ & $N / A$ \\
\hline \multicolumn{5}{|c|}{ Reviewed By (Print): } & Signature: & & & & & Date: & \\
\hline
\end{tabular}


SURVEY \# 10-ER-A26-173

\begin{tabular}{|c|c|c|c|c|c|c|c|c|}
\hline \multirow{2}{*}{$\begin{array}{l}\text { Survey } \\
\text { Point }\end{array}$} & \multirow[t]{2}{*}{ Description/Comments } & \multicolumn{2}{|c|}{$\begin{array}{l}\text { Removable } \\
\mathrm{dpm} / 100 \mathrm{~cm}^{2}\end{array}$} & \multicolumn{2}{|c|}{$\begin{array}{c}\text { Fixed + Removable } \\
\mathrm{dpm} / 100 \mathrm{~cm}^{2}\end{array}$} & \multirow{2}{*}{$\begin{array}{l}\text { Gamma } \\
\mathrm{mrem} / \mathrm{hr}\end{array}$} & \multirow{2}{*}{$\begin{array}{l}\text { Neutron } \\
\text { mrem } / \mathrm{hr}\end{array}$} & \multirow{2}{*}{$\begin{array}{c}\text { Total } \\
\mathrm{mrem} / \mathrm{hr}\end{array}$} \\
\hline & & Alpha & Beta & Alpha & Beta & & & \\
\hline 12 & Room 108 Concrete Floor (Electra 1504) & N/A & N/A & 0 & 1262 & N/A & N/A & N/A \\
\hline 13 & Room 108 Concrete Floor (Electra 1504) & N/A & $\mathrm{N} / \mathrm{A}$ & 2 & 1359 & N/A & N/A & N/A \\
\hline 14 & Room 108 Concrete Floor (Electra 1504) & N/A & $\mathrm{N} / \mathrm{A}$ & 2 & 1413 & N/A & N/A & N/A \\
\hline 15 & Room 108 Concrete Floor (Electra 1504) & N/A & $\mathrm{N} / \mathrm{A}$ & 4 & 1315 & N/A & N/A & $\mathrm{N} / \mathrm{A}$ \\
\hline 16 & Room 108 Concrete Floor (Electra 1120) & N/A & N/A & 8 & 1414 & N/A & N/A & N/A \\
\hline 17 & Room 108 Concrete Floor (Electra 1504) & N/A & N/A & 0 & 1352 & N/A & N/A & N/A \\
\hline N/A & $\mathrm{N} / \mathrm{A}$ & N/A & N/A & N/A & N/A & N/A & $\mathrm{N} / \mathrm{A}$ & N/A \\
\hline $\mathrm{N} / \mathrm{A}$ & $\mathrm{N} / \mathrm{A}$ & N/A & N/A & N/A & N/A & N/A & $\mathrm{N} / \mathrm{A}$ & N/A \\
\hline N/A & $N / A$ & N/A & $\mathrm{N} / \mathrm{A}$ & $\mathrm{N} / \mathrm{A}$ & N/A & N/A & N/A & N/A \\
\hline N/A & $\mathrm{N} / \mathrm{A}$ & N/A & $\mathrm{N} / \mathrm{A}$ & N/A & $\mathrm{N} / \mathrm{A}$ & N/A & N/A & $\mathrm{N} / \mathrm{A}$ \\
\hline N/A & $N / A$ & N/A & $\mathrm{N} / \mathrm{A}$ & N/A & $\mathrm{N} / \mathrm{A}$ & N/A & N/A & N/A \\
\hline N/A & $N / A$ & N/A & $\mathrm{N} / \mathrm{A}$ & N/A & $\mathrm{N} / \mathrm{A}$ & N/A & N/A & $\mathrm{N} / \mathrm{A}$ \\
\hline N/A & $\mathrm{N} / \mathrm{A}$ & N/A & $\mathrm{N} / \mathrm{A}$ & N/A & $\mathrm{N} / \mathrm{A}$ & N/A & N/A & N/A \\
\hline N/A & $\mathrm{N} / \mathrm{A}$ & N/A & N/A & N/A & N/A & N/A & N/A & $\mathrm{N} / \mathrm{A}$ \\
\hline N/A & $N / A$ & N/A & N/A & N/A & N/A & N/A & $\mathrm{N} / \mathrm{A}$ & N/A \\
\hline N/A & $N / A$ & N/A & N/A & N/A & N/A & N/A & N/A & N/A \\
\hline $\mathrm{N} / \mathrm{A}$ & $\mathrm{N} / \mathrm{A}$ & $\mathrm{N} / \mathrm{A}$ & $\mathrm{N} / \mathrm{A}$ & N/A & N/A & $\mathrm{N} / \mathrm{A}$ & N/A & N/A \\
\hline N/A & $N / A$ & $\mathrm{~N} / \mathrm{A}$ & $\mathrm{N} / \mathrm{A}$ & N/A & $\mathrm{N} / \mathrm{A}$ & N/A & N/A & N/A \\
\hline $\mathrm{N} / \mathrm{A}$ & $\mathrm{N} / \mathrm{A}$ & $\mathrm{N} / \mathrm{A}$ & $\mathrm{N} / \mathrm{A}$ & N/A & N/A & N/A & N/A & $\mathrm{N} / \mathrm{A}$ \\
\hline N/A & N/A & N/A & $\mathrm{N} / \mathrm{A}$ & $\mathrm{N} / \mathrm{A}$ & $\mathrm{N} / \mathrm{A}$ & N/A & N/A & N/A \\
\hline $\mathrm{N} / \mathrm{A}$ & N/A & N/A & $\mathrm{N} / \mathrm{A}$ & $\mathrm{N} / \mathrm{A}$ & $\mathrm{N} / \mathrm{A}$ & N/A & N/A & N/A \\
\hline N/A & N/A & N/A & N/A & $\mathrm{N} / \mathrm{A}$ & N/A & $\mathrm{N} / \mathrm{A}$ & N/A & N/A \\
\hline N/A & N/A & N/A & N/A & N/A & $\mathrm{N} / \mathrm{A}$ & $\mathrm{N} / \mathrm{A}$ & N/A & N/A \\
\hline
\end{tabular}


Survey \# $10-E R-A 26-173$

\section{Description/Map/Drawing/Picture/Comments}

Building 2201, Pluto Disassembly Facility, Room 108 "Operating Area"

Survey Plan 10-015 Room 108 Concrete Floor
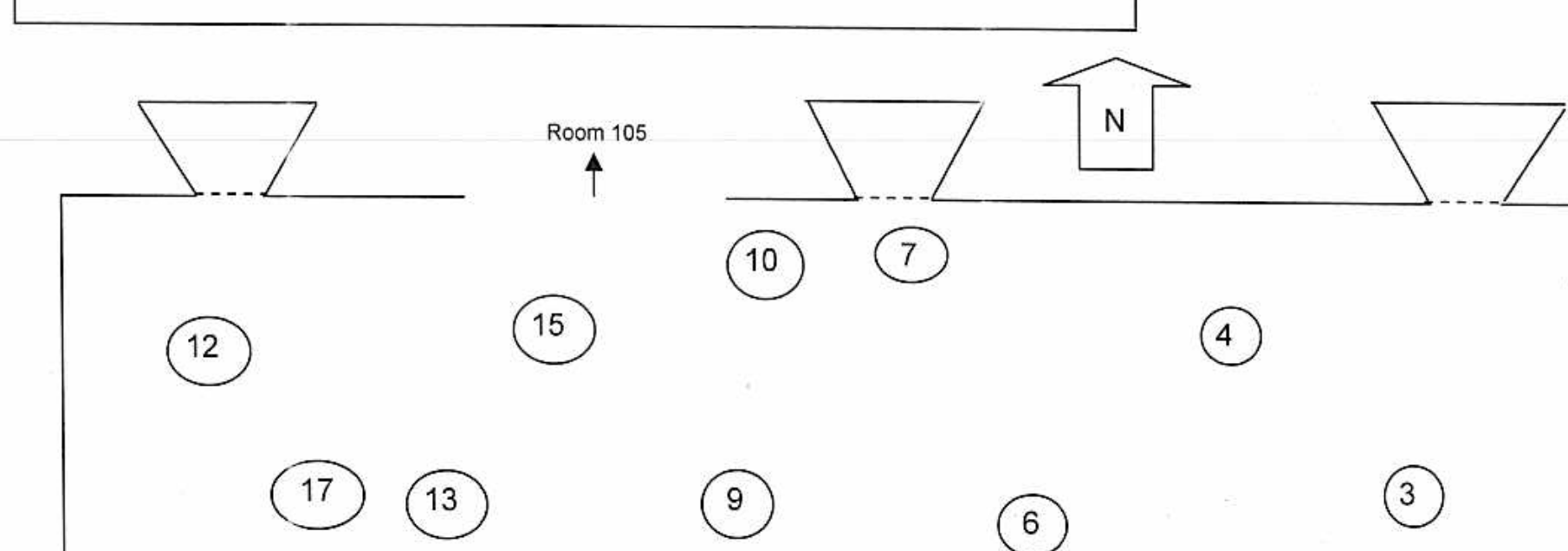

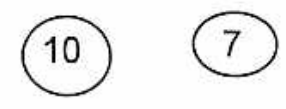

(9)

16

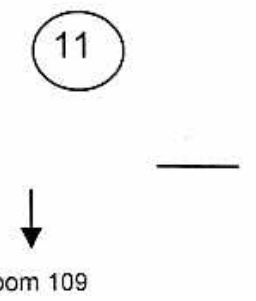

\section{4}

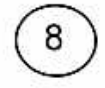

(4)

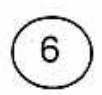

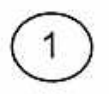

(3)

(5)

LEGEND

$\mathrm{O}=$ Swipe 
SURVEY \#

10-ER-A26-171

\begin{tabular}{|c|c|c|c|c|c|c|c|c|c|c|c|}
\hline \multicolumn{3}{|c|}{$\begin{array}{l}\text { Location: } \\
\text { Area } 26 \text { CAU117 Pluto }\end{array}$} & \multicolumn{2}{|c|}{$\begin{array}{l}\text { Purpose: } \\
\text { Final Status Survey, Room 108, } \\
\text { North Wall \& West Wall }\end{array}$} & \multicolumn{5}{|c|}{$\begin{array}{l}\text { Comments: } \\
\text { All one minute static counts are in cpm. Bkgd not subtracted } \\
\text { for Final Status Survey, Survey Plan \#10-015 }\end{array}$} & \multicolumn{2}{|c|}{$\begin{array}{l}\text { Date/Time: } \\
07 / 12 / 20101445\end{array}$} \\
\hline Instrument: & Serial \#: & Cal Due: & $\begin{array}{c}\text { Eff in \%: } \\
\text { Alpha / Beta }\end{array}$ & $\begin{array}{l}\text { BKG in dpm: } \\
\text { Alpha / Beta }\end{array}$ & \begin{tabular}{|l|} 
MDA in dpm: \\
Alpha / Beta
\end{tabular} & \multicolumn{6}{|c|}{ RWP\# $\quad \mathrm{N} / \mathrm{A}$} \\
\hline Electra & 6697 & $4 / 6 / 2011$ & $13.8 / 19.9$ & $N / A$ & $\mathrm{~N} / \mathrm{A}$ & \multicolumn{6}{|c|}{ Work Package \# ENV-10-RCT ENV SITE SURVEYS-0013 } \\
\hline Electra & 1504 & $4 / 6 / 2011$ & $12.9 / 19.8$ & $\mathrm{~N} / \mathrm{A}$ & $N / A$ & \multicolumn{3}{|c|}{ RCT Name: D.Cotroneo Jr. } & Signature: & \multicolumn{2}{|c|}{$0 \quad \Leftrightarrow$} \\
\hline$N / A$ & $\mathrm{~N} / \mathrm{A}$ & $\mathrm{N} / \mathrm{A}$ & $\mathrm{N} / \mathrm{A}$ & $N / A$ & $N / A$ & \multicolumn{3}{|c|}{ RCT Name: Kevin Wilcox } & Signature: & \multicolumn{2}{|c|}{ Reri Wilor } \\
\hline $\mathrm{N} / \mathrm{A}$ & $N / A$ & $N / A$ & $N / A$ & $N / A$ & $N / A$ & \multicolumn{3}{|c|}{ RCT Name: Brian Bunce } & Signature: & \multicolumn{2}{|c|}{ Baknu } \\
\hline$N / A$ & $N / A$ & $N / A$ & $N / A$ & $N / A$ & $\mathrm{~N} / \mathrm{A}$ & \multicolumn{3}{|c|}{ RCT Name: N/A } & Signature: & \multicolumn{2}{|c|}{$N / A$} \\
\hline \multirow[t]{2}{*}{ Survey Point } & \multirow{2}{*}{\multicolumn{4}{|c|}{ Description/Comments }} & \multicolumn{2}{|c|}{\begin{tabular}{l|} 
Removable \\
$\mathrm{dpm} / 100 \mathrm{~cm}^{2}$
\end{tabular}} & \multicolumn{2}{|c|}{$\begin{array}{c}\text { Fixed + Removable } \\
\mathrm{dpm} / 100 \mathrm{~cm}^{2}\end{array}$} & \multirow{2}{*}{$\begin{array}{l}\text { Gamma } \\
\mathrm{mrem} / \mathrm{hr}\end{array}$} & \multirow{2}{*}{$\begin{array}{l}\text { Neutron } \\
\mathrm{mrem} / \mathrm{hr}\end{array}$} & \multirow{2}{*}{$\begin{array}{l}\text { Total } \\
\mathrm{mrem} / \mathrm{hr}\end{array}$} \\
\hline & & & & & Alpha & Beta & Alpha & Beta & & & \\
\hline 1 & \multicolumn{4}{|c|}{ West Wall Concrete (Electra 6697) } & $\mathrm{N} / \mathrm{A}$ & $N / A$ & 13 & 1047 & $N / A$ & $N / A$ & $\mathrm{~N} / \mathrm{A}$ \\
\hline 2 & \multicolumn{4}{|c|}{ West Wall Concrete (Electra 6697) } & $N / A$ & $N / A$ & 8 & 1078 & $\mathrm{~N} / \mathrm{A}$ & $N / A$ & $N / A$ \\
\hline 3 & \multicolumn{4}{|c|}{ West Wall Concrete (Electra 6697) } & $N / A$ & $N / A$ & 8 & 1044 & $N / A$ & $N / A$ & $N / A$ \\
\hline 4 & \multicolumn{4}{|c|}{ West Wall Concrete (Electra 6697) } & $N / A$ & $N / A$ & 9 & 984 & $\mathrm{~N} / \mathrm{A}$ & $N / A$ & $N / A$ \\
\hline 5 & \multicolumn{4}{|c|}{ West Wall Concrete (Electra 6697) } & $\mathrm{N} / \mathrm{A}$ & $N / A$ & 8 & 890 & $N / A$ & $N / A$ & $N / A$ \\
\hline 6 & \multicolumn{4}{|c|}{ West Wall Concrete (Electra 6697) } & $N / A$ & $N / A$ & 9 & 828 & $N / A$ & $N / A$ & $N / A$ \\
\hline 7 & \multicolumn{4}{|c|}{ West Wall Concrete (Electra 6697) } & $N / A$ & $N / A$ & 11 & 1055 & $\mathrm{~N} / \mathrm{A}$ & $N / A$ & $N / A$ \\
\hline 8 & \multicolumn{4}{|c|}{ West Wall Concrete (Electra 6697) } & $N / A$ & $N / A$ & 5 & 976 & $N / A$ & $N / A$ & $N / A$ \\
\hline 9 & \multicolumn{4}{|c|}{ North Wall Concrete (Electra 1504) } & $N / A$ & $N / A$ & 4 & 795 & $N / A$ & $N / A$ & $N / A$ \\
\hline 10 & \multicolumn{4}{|c|}{ North Wall Concrete (Electra 1504) } & $N / A$ & $N / A$ & 8 & 822 & $N / A$ & $\mathrm{~N} / \mathrm{A}$ & $N / A$ \\
\hline 11 & \multicolumn{4}{|c|}{ North Wall Concrete (Electra 1504) } & $N / A$ & $N / A$ & 8 & 912 & $N / A$ & $N / A$ & $N / A$ \\
\hline \multicolumn{5}{|c|}{ Reviewed By (Rrint): } & Signature: & & & & & $\begin{aligned} & \text { Date: } \\
& 7 \\
&\end{aligned}$ & \\
\hline
\end{tabular}


SURVEY \# 10-ER-A26-171

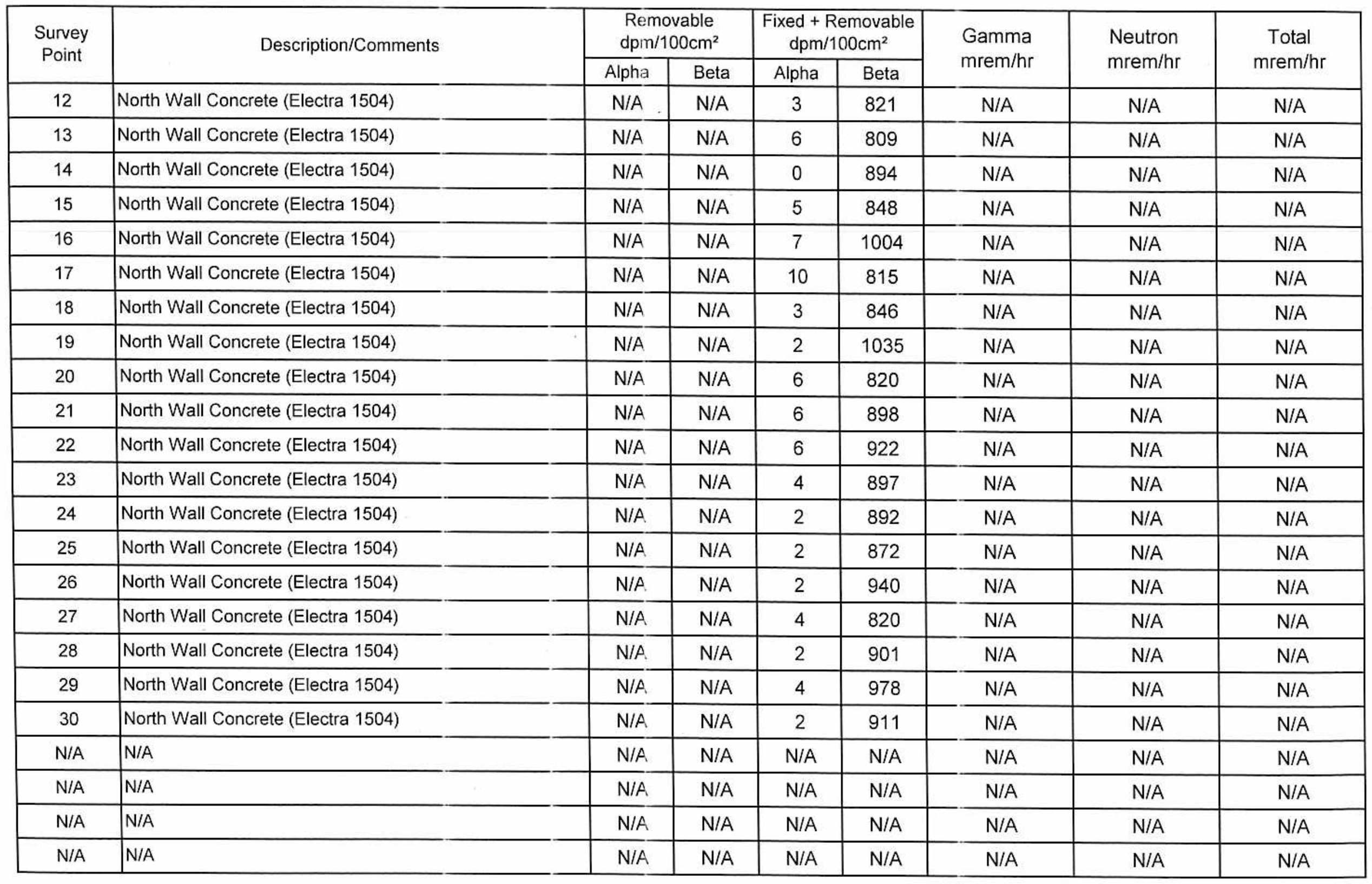


Survey \# $10-E R-A 26-171$

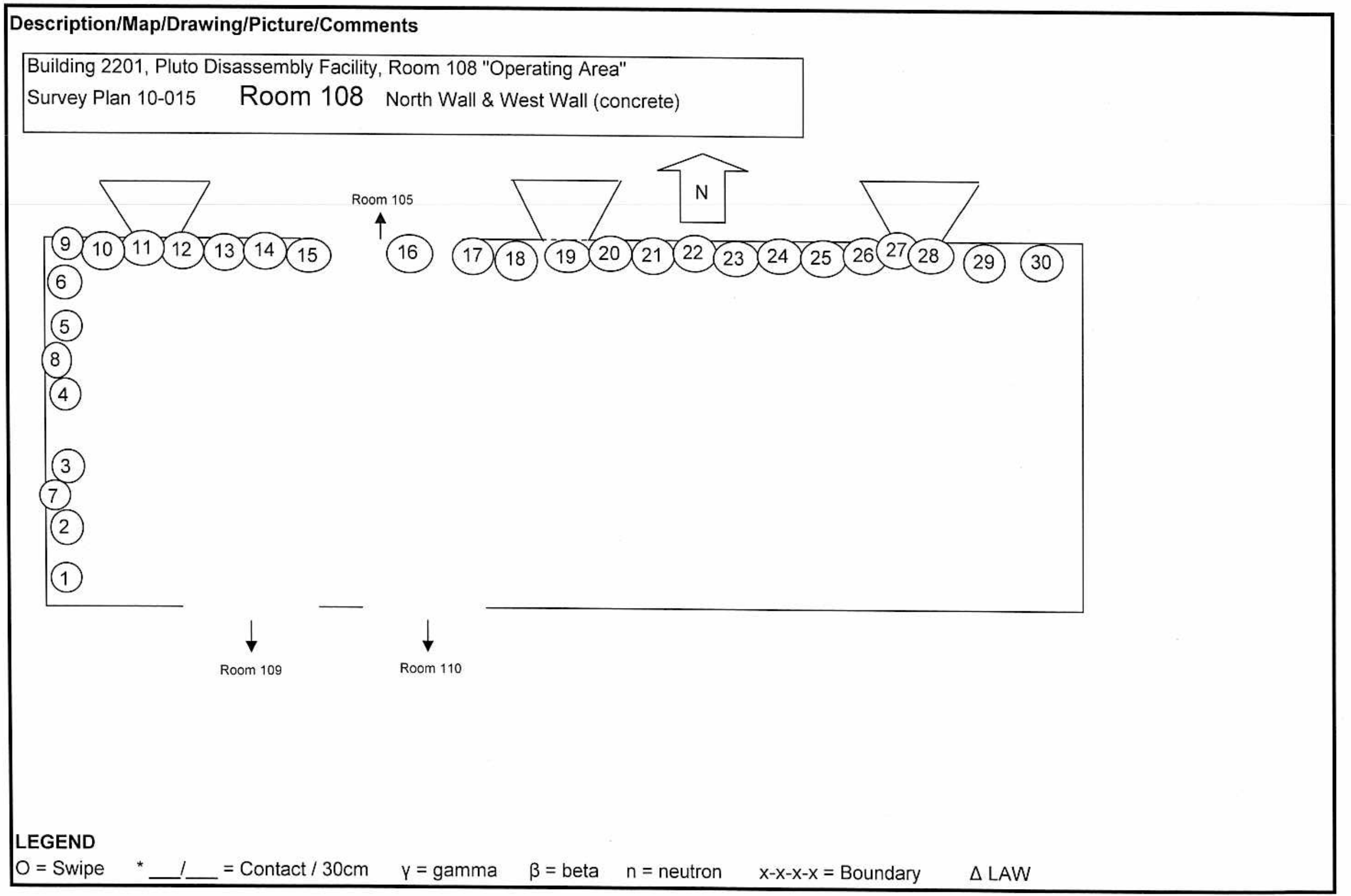


SURVEY \#

10-ER-A26-170

\begin{tabular}{|c|c|c|c|c|c|c|c|c|c|c|c|}
\hline \multicolumn{3}{|c|}{$\begin{array}{l}\text { Location: } \\
\text { Area } 26 \text { CAU117 Pluto }\end{array}$} & \multicolumn{2}{|c|}{$\begin{array}{l}\text { Purpose: } \\
\text { Final Status Survey, Room 108, } \\
\text { South Wall \& East Wall }\end{array}$} & \multicolumn{5}{|c|}{$\begin{array}{l}\text { Comments: } \\
\text { All one minute static counts are in cpm. Bkgd not subtracted } \\
\text { for Final Status Survey, Survey Plan \#10-015 }\end{array}$} & \multicolumn{2}{|c|}{$\begin{array}{l}\text { Date/Time: } \\
\text { 07/12/2010 } 1430\end{array}$} \\
\hline Instrument: & Serial \#: & Cal Due: & $\begin{array}{c}\text { Eff in \%: } \\
\text { Alpha / Beta }\end{array}$ & $\begin{array}{l}\text { BKG in dpm: } \\
\text { Alpha / Beta }\end{array}$ & \begin{tabular}{|l|} 
MDA in dpm: \\
Alpha / Beta \\
\end{tabular} & RWP\# & \multicolumn{5}{|l|}{$\mathrm{N} / \mathrm{A}$} \\
\hline Electra & 1120 & $4 / 6 / 2011$ & $14.1 / 20.8$ & N/A & $N / A$ & \multicolumn{6}{|c|}{ ENV-10-RCT ENV SITE SURVEYS-0013 } \\
\hline$N / A$ & $N / A$ & $\mathrm{~N} / \mathrm{A}$ & $N / A$ & $\mathrm{~N} / \mathrm{A}$ & $\mathrm{N} / \mathrm{A}$ & \multicolumn{3}{|c|}{ RCT Name: D.Cotroneo Jr. } & \multicolumn{3}{|c|}{ Signature: $0 \rightarrow t$} \\
\hline$N / A$ & $N / A$ & $\mathrm{~N} / \mathrm{A}$ & $N / A$ & $\mathrm{~N} / \mathrm{A}$ & $\mathrm{N} / \mathrm{A}$ & \multicolumn{3}{|c|}{ RCT Name: Kevin Wilcox } & \multicolumn{3}{|c|}{ Signature: Keni $2 \sqrt{5}$ cox } \\
\hline$N / A$ & $N / A$ & $N / A$ & $N / A$ & $\mathrm{~N} / \mathrm{A}$ & $\mathrm{N} / \mathrm{A}$ & \multicolumn{3}{|c|}{ RCT Name: Brian Foskett } & \multirow{2}{*}{\multicolumn{3}{|c|}{ Signature: }} \\
\hline$N / A$ & $N / A$ & $\mathrm{~N} / \mathrm{A}$ & $N / A$ & $N / A$ & $N / A$ & RCT Name: & Sonny Brc & & & & \\
\hline \multirow[t]{2}{*}{ Survey Point } & \multirow{2}{*}{\multicolumn{4}{|c|}{ Description/Comments }} & \multicolumn{2}{|c|}{$\begin{array}{l}\text { Removable } \\
\mathrm{dpm} / 100 \mathrm{~cm}^{2}\end{array}$} & \multicolumn{2}{|c|}{$\begin{array}{c}\text { Fixed + Removable } \\
\mathrm{dpm} / 100 \mathrm{~cm}^{2}\end{array}$} & \multirow{2}{*}{$\begin{array}{l}\text { Gamma } \\
\mathrm{mrem} / \mathrm{hr}\end{array}$} & \multirow{2}{*}{$\begin{array}{l}\text { Neutron } \\
\mathrm{mrem} / \mathrm{hr}\end{array}$} & \multirow{2}{*}{$\begin{array}{l}\text { Total } \\
\mathrm{mrem} / \mathrm{hr}\end{array}$} \\
\hline & & & & & Alpha & Beta & Alpha & Beta & & & \\
\hline 1 & \multicolumn{4}{|c|}{ South Wall Concrete } & $N / A$ & $N / A$ & 8 & 1218 & $N / A$ & $N / A$ & $N / A$ \\
\hline 2 & \multicolumn{4}{|c|}{ South Wall Concrete } & $N / A$ & $N / A$ & 12 & 1223 & $N / A$ & $N / A$ & $N / A$ \\
\hline 3 & \multicolumn{4}{|c|}{ South Wall Concrete } & $N / A$ & $N / A$ & 9 & 1251 & $\mathrm{~N} / \mathrm{A}$ & $N / A$ & $N / A$ \\
\hline 4 & \multicolumn{4}{|c|}{ South Wall Metal } & $N / A$ & $N / A$ & 8 & 745 & $N / A$ & $N / A$ & $N / A$ \\
\hline 5 & \multicolumn{4}{|c|}{ South Wall Concrete } & $N / A$ & $N / A$ & 9 & 1269 & $N / A$ & $N / A$ & $\mathrm{~N} / \mathrm{A}$ \\
\hline 6 & \multicolumn{4}{|c|}{ South Wall Concrete } & $N / A$ & $N / A$ & 9 & 1218 & $N / A$ & $N / A$ & $N / A$ \\
\hline 7 & \multicolumn{4}{|c|}{ South Wall Concrete } & $N / A$ & $N / A$ & 13 & 1219 & $N / A$ & $N / A$ & $N / A$ \\
\hline 8 & \multicolumn{4}{|c|}{ South Wall Concrete } & $N / A$ & $N / A$ & 14 & 1093 & $N / A$ & $N / A$ & $N / A$ \\
\hline 9 & \multicolumn{4}{|c|}{ South Wall Concrete } & $N / A$ & $N / A$ & 5 & 1216 & $\mathrm{~N} / \mathrm{A}$ & $N / A$ & $N / A$ \\
\hline 10 & \multicolumn{4}{|c|}{ South Wall Concrete } & $N / A$ & $N / A$ & 9 & 1106 & $N / A$ & $N / A$ & $N / A$ \\
\hline 11 & \multicolumn{4}{|c|}{ South Wall Concrete } & $N / A$ & $\mathrm{~N} / \mathrm{A}$ & 5 & 1118 & $N / A$ & $N / A$ & $N / A$ \\
\hline \multicolumn{5}{|c|}{ Reviewed By (Print): } & \multicolumn{7}{|c|}{ Signature: } \\
\hline
\end{tabular}


SURVEY \# 10-ER-A26-170

\begin{tabular}{|c|c|c|c|c|c|c|c|c|}
\hline \multirow{2}{*}{$\begin{array}{c}\text { Survey } \\
\text { Point }\end{array}$} & \multirow[t]{2}{*}{ Description/Comments } & \multicolumn{2}{|c|}{$\begin{array}{l}\text { Removable } \\
\mathrm{dpm} / 100 \mathrm{~cm}^{2}\end{array}$} & \multicolumn{2}{|c|}{\begin{tabular}{|c|}
$\begin{array}{c}\text { Fixed + Removable } \\
\mathrm{dpm} / 100 \mathrm{~cm}^{2}\end{array}$ \\
\end{tabular}} & \multirow{2}{*}{$\begin{array}{l}\text { Gamma } \\
\mathrm{mrem} / \mathrm{hr}\end{array}$} & \multirow{2}{*}{$\begin{array}{l}\text { Neutron } \\
\mathrm{mrem} / \mathrm{hr}\end{array}$} & \multirow{2}{*}{$\begin{array}{c}\text { Total } \\
\text { mrem } / \mathrm{hr}\end{array}$} \\
\hline & & Alpha & Beta & Alpha & Beta & & & \\
\hline 12 & South Wall Concrete & N/A & $\mathrm{N} / \mathrm{A}$ & 8 & 1196 & N/A & N/A & N/A \\
\hline 13 & South Wall Concrete & N/A & $\mathrm{N} / \mathrm{A}$ & 16 & 1318 & N/A & $\mathrm{N} / \mathrm{A}$ & N/A \\
\hline 14 & South Wall Concrete & $\mathrm{N} / \mathrm{A}$ & $\mathrm{N} / \mathrm{A}$ & 17 & 1357 & $\mathrm{~N} / \mathrm{A}$ & N/A & N/A \\
\hline 15 & East Wall Concrete & N/A & $\mathrm{N} / \mathrm{A}$ & 17 & 1187 & N/A & $\mathrm{N} / \mathrm{A}$ & N/A \\
\hline 16 & East Wall Concrete & N/A & $\mathrm{N} / \mathrm{A}$ & 12 & 1247 & $\mathrm{~N} / \mathrm{A}$ & $\mathrm{N} / \mathrm{A}$ & N/A \\
\hline 17 & East Wall Concrete & N/A & $\mathrm{N} / \mathrm{A}$ & 17 & 1187 & $\mathrm{~N} / \mathrm{A}$ & N/A & $\mathrm{N} / \mathrm{A}$ \\
\hline 18 & East Wall Concrete & N/A & $\mathrm{N} / \mathrm{A}$ & 12 & 1247 & N/A & $\mathrm{N} / \mathrm{A}$ & N/A \\
\hline 19 & East Wall Concrete & $\mathrm{N} / \mathrm{A}$ & $\mathrm{N} / \mathrm{A}$ & 10 & 1321 & N/A & N/A & N/A \\
\hline 20 & East Wall Concrete & N/A & $\mathrm{N} / \mathrm{A}$ & 21 & 1331 & $\mathrm{~N} / \mathrm{A}$ & $\mathrm{N} / \mathrm{A}$ & N/A \\
\hline 21 & N/A & N/A & $\mathrm{N} / \mathrm{A}$ & N/A & $\mathrm{N} / \mathrm{A}$ & N/A & $\mathrm{N} / \mathrm{A}$ & N/A \\
\hline 22 & N/A & $\mathrm{N} / \mathrm{A}$ & $\mathrm{N} / \mathrm{A}$ & N/A & $\mathrm{N} / \mathrm{A}$ & N/A & $\mathrm{N} / \mathrm{A}$ & N/A \\
\hline 23 & N/A & N/A & $\mathrm{N} / \mathrm{A}$ & N/A & $\mathrm{N} / \mathrm{A}$ & N/A & $\mathrm{N} / \mathrm{A}$ & N/A \\
\hline 24 & N/A & N/A & $\mathrm{N} / \mathrm{A}$ & $\mathrm{N} / \mathrm{A}$ & $\mathrm{N} / \mathrm{A}$ & $\mathrm{N} / \mathrm{A}$ & $\mathrm{N} / \mathrm{A}$ & N/A \\
\hline 25 & N/A & $\mathrm{N} / \mathrm{A}$ & $\mathrm{N} / \mathrm{A}$ & N/A & $\mathrm{N} / \mathrm{A}$ & N/A & $\mathrm{N} / \mathrm{A}$ & N/A \\
\hline 26 & $\mathrm{~N} / \mathrm{A}$ & $\mathrm{N} / \mathrm{A}$ & $\mathrm{N} / \mathrm{A}$ & $\mathrm{N} / \mathrm{A}$ & $\mathrm{N} / \mathrm{A}$ & $\mathrm{N} / \mathrm{A}$ & $\mathrm{N} / \mathrm{A}$ & N/A \\
\hline 27 & N/A & $\mathrm{N} / \mathrm{A}$ & $\mathrm{N} / \mathrm{A}$ & N/A & $\mathrm{N} / \mathrm{A}$ & N/A & N/A & N/A \\
\hline 28 & N/A & N/A & $\mathrm{N} / \mathrm{A}$ & N/A & $\mathrm{N} / \mathrm{A}$ & N/A & $\mathrm{N} / \mathrm{A}$ & N/A \\
\hline N/A & N/A & N/A & N/A & N/A & $\mathrm{N} / \mathrm{A}$ & N/A & N/A & N/A \\
\hline N/A & $\mathrm{N} / \mathrm{A}$ & N/A & N/A & N/A & $\mathrm{N} / \mathrm{A}$ & N/A & $\mathrm{N} / \mathrm{A}$ & $\mathrm{N} / \mathrm{A}$ \\
\hline N/A & $\mathrm{N} / \mathrm{A}$ & N/A & $\mathrm{N} / \mathrm{A}$ & N/A & N/A & N/A & N/A & N/A \\
\hline $\mathrm{N} / \mathrm{A}$ & $\mathrm{N} / \mathrm{A}$ & $\mathrm{N} / \mathrm{A}$ & $\mathrm{N} / \mathrm{A}$ & N/A & N/A & N/A & N/A & $\mathrm{N} / \mathrm{A}$ \\
\hline N/A & N/A & N/A & N/A & $\mathrm{N} / \mathrm{A}$ & N/A & N/A & N/A & $\mathrm{N} / \mathrm{A}$ \\
\hline N/A & $\mathrm{N} / \mathrm{A}$ & $\mathrm{N} / \mathrm{A}$ & $\mathrm{N} / \mathrm{A}$ & N/A & $\mathrm{N} / \mathrm{A}$ & $\mathrm{N} / \mathrm{A}$ & N/A & $N / A$ \\
\hline
\end{tabular}


Survey \#

$10-E R-A 26-170$

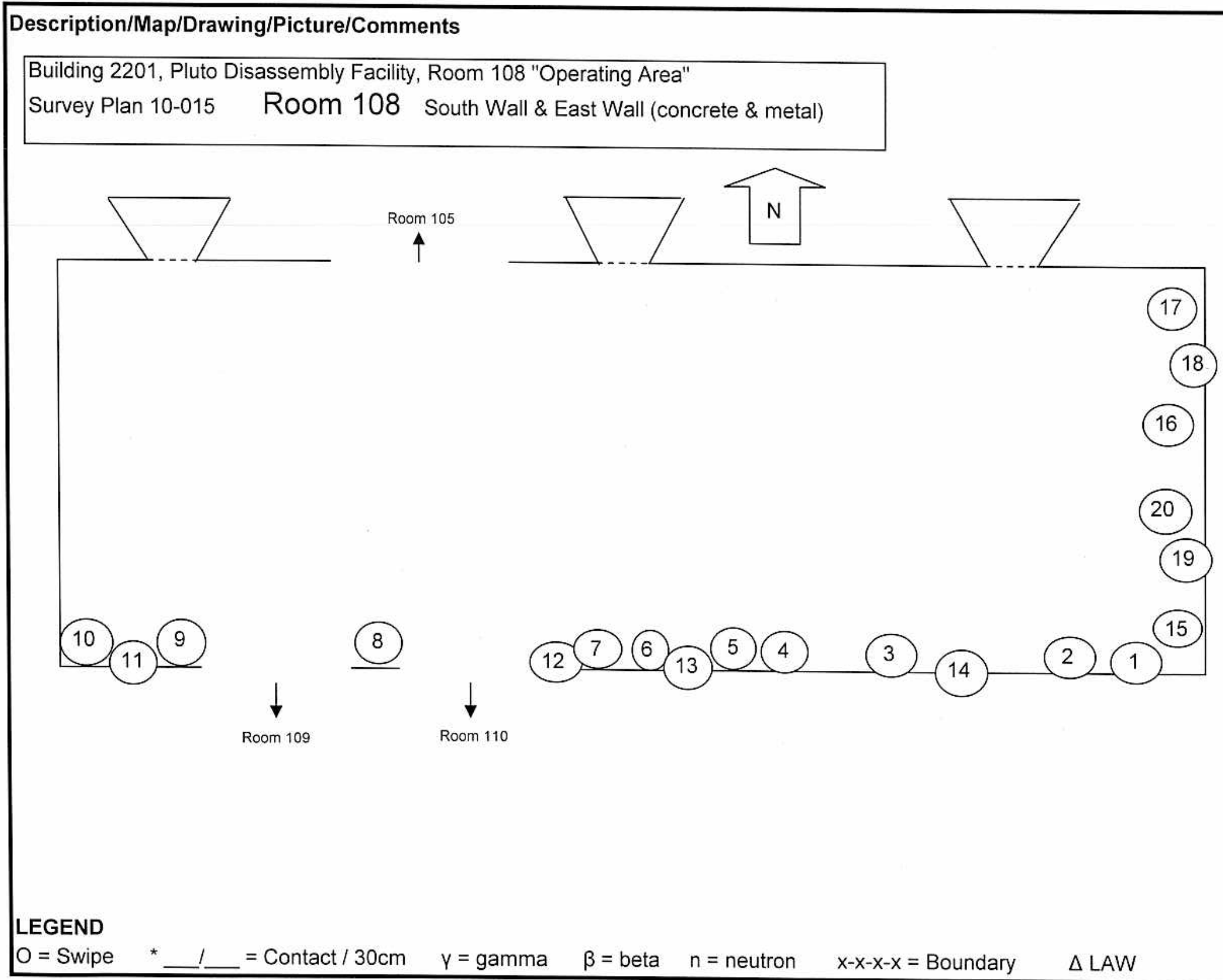




\begin{tabular}{|c|c|c|c|c|c|c|c|c|c|c|c|}
\hline \multicolumn{3}{|c|}{$\begin{array}{l}\text { Location: } \\
\text { Area } 26 \text { CAU117 Pluto }\end{array}$} & \multicolumn{2}{|c|}{$\begin{array}{l}\text { Purpose: } \\
\text { Final Status Survey, Room 109, } \\
\text { Ceiling }\end{array}$} & \multicolumn{5}{|c|}{$\begin{array}{l}\text { Comments: } \\
\text { All one minute static counts are in cpm. Bkgd not subtracted } \\
\text { for Final Status Survey, Survey Plan \#10-015 }\end{array}$} & \multicolumn{2}{|c|}{$\begin{array}{l}\text { Date/Time: } \\
\text { 07/14/2010 } 1545\end{array}$} \\
\hline Instrument: & Serial \#: & Cal Due: & $\begin{array}{c}\text { Eff in \%: } \\
\text { Alpha / Beta }\end{array}$ & $\begin{array}{l}\text { BKG in dpm: } \\
\text { Alpha / Beta }\end{array}$ & $\begin{array}{l}\text { MDA in dpm: } \\
\text { Alpha / Beta }\end{array}$ & RWP\# & \multicolumn{5}{|l|}{$N / A$} \\
\hline Electra & 1120 & $4 / 6 / 2011$ & $14.1 / 20.8$ & $N / A$ & $N / A$ & \multicolumn{6}{|c|}{ Work Package \# $\quad$ ENV-10-RCT ENV SITE SURVEYS-0013 } \\
\hline$N / A$ & $N / A$ & $N / A$ & $N / A$ & $N / A$ & $N / A$ & \multicolumn{3}{|c|}{ RCT Name: D.Cotroneo Jr. } & \multicolumn{3}{|c|}{ Signature: $\mathbb{C} \square$} \\
\hline$N / A$ & $N / A$ & $N / A$ & $N / A$ & $N / A$ & $N / A$ & \multicolumn{3}{|c|}{ RCT Name: Kevin Wilcox } & \multicolumn{3}{|c|}{ Signature:Keni Wolow } \\
\hline $\mathrm{N} / \mathrm{A}$ & $\mathrm{N} / \mathrm{A}$ & $\mathrm{N} / \mathrm{A}$ & $N / A$ & $\mathrm{~N} / \mathrm{A}$ & $N / A$ & \multicolumn{3}{|c|}{ RCT Name: Brian Bunce } & \multicolumn{3}{|c|}{ Signature: 146fume } \\
\hline$N / A$ & $N / A$ & $N / A$ & $N / A$ & $N / A$ & $N / A$ & \multicolumn{3}{|c|}{ RCT Name: Sonny Brown } & \multicolumn{3}{|c|}{ Signature:Som 3} \\
\hline \multirow[t]{2}{*}{ Survey Point } & \multirow{2}{*}{\multicolumn{4}{|c|}{ Description/Comments }} & \multicolumn{2}{|c|}{$\begin{array}{l}\text { Removable } \\
\mathrm{dpm} / 100 \mathrm{~cm}^{2}\end{array}$} & \multicolumn{2}{|c|}{\begin{tabular}{|c|}
$\begin{array}{c}\text { Fixed + Removable } \\
\mathrm{dpm} / 100 \mathrm{~cm}^{2}\end{array}$ \\
\end{tabular}} & \multirow{2}{*}{$\begin{array}{l}\text { Gamma } \\
\mathrm{mrem} / \mathrm{hr}\end{array}$} & \multirow{2}{*}{$\begin{array}{l}\text { Neutron } \\
\mathrm{mrem} / \mathrm{hr}\end{array}$} & \multirow{2}{*}{$\begin{array}{l}\text { Total } \\
\mathrm{mrem} / \mathrm{hr}\end{array}$} \\
\hline & & & & & Alpha & Beta & Alpha & Beta & & & \\
\hline 1 & \multicolumn{4}{|l|}{ Ceiling Metal } & $N / A$ & $N / A$ & 3 & 1185 & $N / A$ & $N / A$ & $\mathrm{~N} / \mathrm{A}$ \\
\hline 2 & \multicolumn{4}{|l|}{ Ceiling Metal } & $N / A$ & $N / A$ & 4 & 1182 & $N / A$ & $N / A$ & $N / A$ \\
\hline 3 & \multicolumn{4}{|l|}{ Ceiling Metal } & $N / A$ & $N / A$ & 5 & 1253 & $\mathrm{~N} / \mathrm{A}$ & $N / A$ & $N / A$ \\
\hline 4 & \multicolumn{4}{|l|}{ Ceiling Metal } & $N / A$ & $N / A$ & 4 & 1166 & $N / A$ & $N / A$ & $N / A$ \\
\hline 5 & \multicolumn{4}{|l|}{ Ceiling Metal } & $N / A$ & $N / A$ & 6 & 1207 & $N / A$ & $N / A$ & $N / A$ \\
\hline 6 & \multicolumn{4}{|l|}{ Ceiling Metal } & $N / A$ & $N / A$ & 7 & 1243 & $N / A$ & $N / A$ & $N / A$ \\
\hline 7 & \multicolumn{4}{|l|}{ Ceiling Metal } & $N / A$ & $N / A$ & 4 & 1215 & $N / A$ & $N / A$ & $N / A$ \\
\hline 8 & \multicolumn{4}{|l|}{ Ceiling Metal } & $N / A$ & $N / A$ & 2 & 1251 & $N / A$ & $N / A$ & $N / A$ \\
\hline 9 & \multicolumn{4}{|l|}{ Ceiling Metal } & $N / A$ & $N / A$ & 6 & 1145 & $N / A$ & $N / A$ & $N / A$ \\
\hline$N / A$ & \multicolumn{4}{|l|}{$N / A$} & $N / A$ & $N / A$ & $N / A$ & $N / A$ & $N / A$ & $N / A$ & $N / A$ \\
\hline$N / A$ & \multicolumn{4}{|l|}{$N / A$} & $N / A$ & $N / A$ & $N / A$ & $N / A$ & $N / A$ & $N / A$ & $N / A$ \\
\hline \multicolumn{5}{|c|}{ Reviewed By (Print): } & Signature: & & & & & Date: & \\
\hline
\end{tabular}


Survey \# 10-ER-A26-180

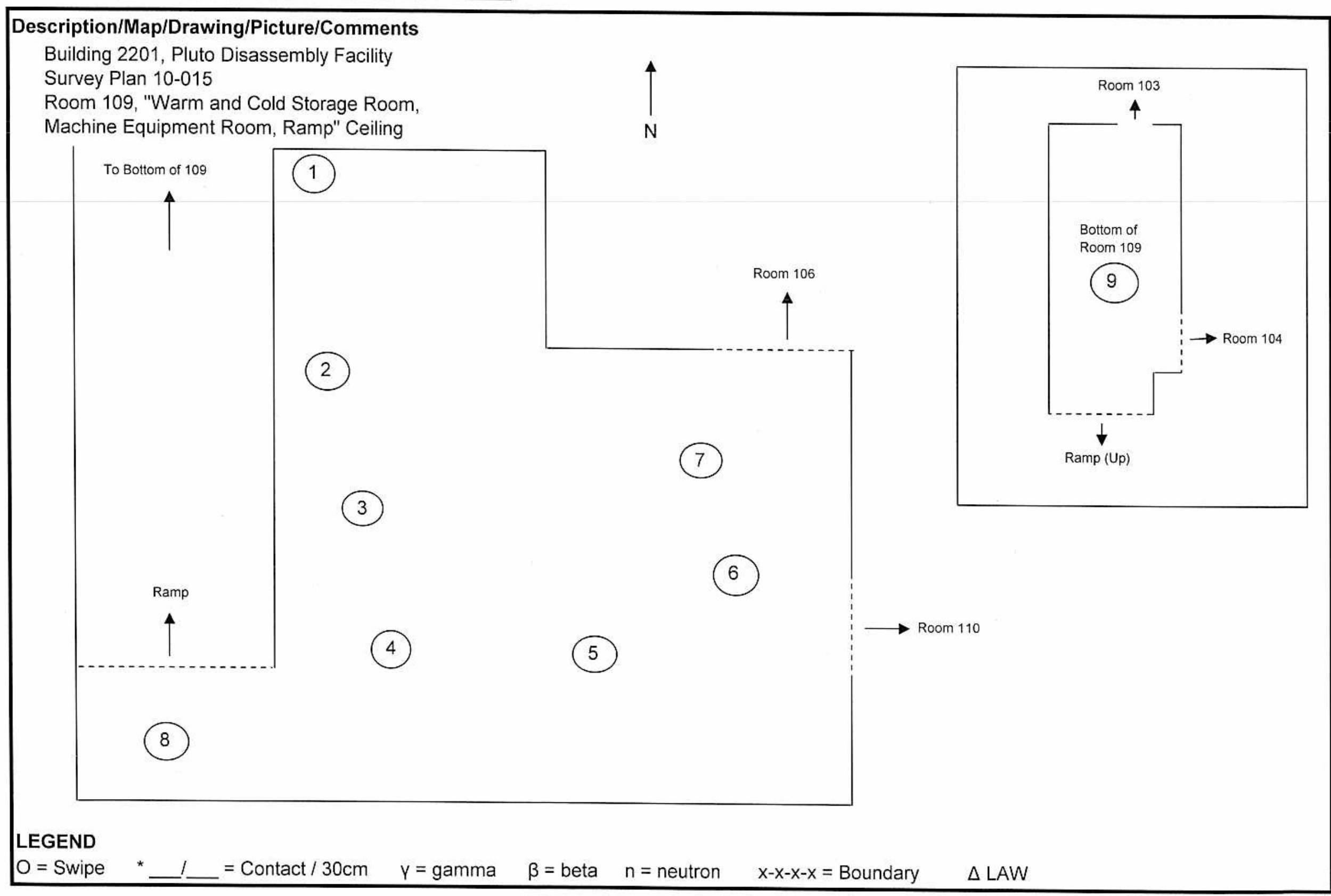


SURVEY \# 10-ER-A26-183

\begin{tabular}{|c|c|c|c|c|c|c|c|c|c|c|c|}
\hline \multicolumn{3}{|c|}{$\begin{array}{l}\text { Location: } \\
\text { Area } 26 \text { CAU117 Pluto }\end{array}$} & \multicolumn{2}{|c|}{$\begin{array}{l}\text { Purpose: } \\
\text { Final Status Survey, Room 109, } \\
\text { Floor }\end{array}$} & \multicolumn{5}{|c|}{$\begin{array}{l}\text { Comments: } \\
\text { All one minute static counts are in cpm. Bkgd not subtracted } \\
\text { for Final Status Survey, Survey Plan \#10-015 }\end{array}$} & \multicolumn{2}{|c|}{$\begin{array}{l}\text { Date/Time: } \\
07 / 14 / 20101430\end{array}$} \\
\hline Instrument: & Serial \#: & Cal Due: & \begin{tabular}{|c|} 
Eff in \%: \\
Alpha / Beta \\
\end{tabular} & $\begin{array}{l}\text { BKG in dpm: } \\
\text { Alpha / Beta }\end{array}$ & $\begin{array}{l}\text { MDA in dpm: } \\
\text { Alpha / Beta }\end{array}$ & RWP\# & \multicolumn{5}{|c|}{$\mathrm{N} / \mathrm{A}$} \\
\hline Electra & 1120 & $4 / 6 / 2011$ & $14.1 / 20.8$ & $N / A$ & $\mathrm{~N} / \mathrm{A}$ & \multicolumn{6}{|c|}{ Work Package \# $\quad$ ENV-10-RCT ENV SITE SURVEYS-0013 } \\
\hline$N / A$ & $\mathrm{~N} / \mathrm{A}$ & $N / A$ & $N / A$ & $N / A$ & $\mathrm{~N} / \mathrm{A}$ & \multicolumn{3}{|c|}{ RCT Name: D.Cotroneo Jr. } & \multicolumn{3}{|c|}{ Signature: $O-C$} \\
\hline$N / A$ & $N / A$ & $N / A$ & $N / A$ & $N / A$ & $N / A$ & \multicolumn{3}{|c|}{ RCT Name: Kevin Wilcox } & \multicolumn{3}{|c|}{ Signature: Ken Weles } \\
\hline$N / A$ & $N / A$ & $N / A$ & $N / A$ & $N / A$ & $N / A$ & \multicolumn{3}{|c|}{ RCT Name: S. Brown/B. Bunce } & \multirow{2}{*}{\multicolumn{3}{|c|}{ Signature: }} \\
\hline$N / A$ & $N / A$ & $\mathrm{~N} / \mathrm{A}$ & $\mathrm{N} / \mathrm{A}$ & $\mathrm{N} / \mathrm{A}$ & $N / A$ & RCT Name: & Brian Fos & & & & \\
\hline \multirow[t]{2}{*}{ Survey Point } & \multirow{2}{*}{\multicolumn{4}{|c|}{ Description/Comments }} & \multicolumn{2}{|c|}{$\begin{array}{l}\text { Removable } \\
\mathrm{dpm} / 100 \mathrm{~cm}^{2}\end{array}$} & \multicolumn{2}{|c|}{\begin{tabular}{|c|}
$\begin{array}{c}\text { Fixed + Removable } \\
\mathrm{dpm} / 100 \mathrm{~cm}^{2}\end{array}$ \\
\end{tabular}} & \multirow{2}{*}{$\begin{array}{l}\text { Gamma } \\
\mathrm{mrem} / \mathrm{hr}\end{array}$} & \multirow{2}{*}{$\begin{array}{l}\text { Neutron } \\
\text { mrem/hr }\end{array}$} & \multirow{2}{*}{$\begin{array}{c}\text { Total } \\
\mathrm{mrem} / \mathrm{hr}\end{array}$} \\
\hline & & & & & Alpha & Beta & Alpha & Beta & & & \\
\hline 1 & \multicolumn{4}{|l|}{ Floor Concrete } & $N / A$ & $N / A$ & 9 & 1273 & $N / A$ & $N / A$ & $N / A$ \\
\hline 2 & \multicolumn{4}{|l|}{ Floor Concrete } & $N / A$ & $N / A$ & 10 & 1286 & $N / A$ & $N / A$ & $N / A$ \\
\hline 3 & \multicolumn{4}{|l|}{ Floor Concrete } & $N / A$ & $N / A$ & 9 & 940 & $N / A$ & $N / A$ & $N / A$ \\
\hline 4 & \multicolumn{4}{|l|}{ Floor Concrete } & $N / A$ & $N / A$ & 13 & 909 & $N / A$ & $N / A$ & $N / A$ \\
\hline 5 & \multicolumn{4}{|l|}{ Floor Concrete } & $N / A$ & $N / A$ & 3 & 942 & $N / A$ & $N / A$ & $N / A$ \\
\hline 6 & \multicolumn{4}{|l|}{ Floor Concrete } & $\mathrm{N} / \mathrm{A}$ & $N / A$ & 7 & 922 & $N / A$ & $N / A$ & $\mathrm{~N} / \mathrm{A}$ \\
\hline 7 & \multicolumn{4}{|l|}{ Floor Concrete } & $N / A$ & $N / A$ & 7 & 1386 & $N / A$ & $N / A$ & $N / A$ \\
\hline 8 & \multicolumn{4}{|l|}{ Floor Concrete } & $N / A$ & $N / A$ & 12 & 1419 & $N / A$ & $N / A$ & $N / A$ \\
\hline 9 & \multicolumn{4}{|l|}{ Floor Concrete } & $N / A$ & $N / A$ & 10 & 1322 & $N / A$ & $N / A$ & $N / A$ \\
\hline 10 & \multicolumn{4}{|l|}{ Floor Concrete } & $N / A$ & $N / A$ & 5 & 1400 & $N / A$ & $N / A$ & $N / A$ \\
\hline 11 & \multicolumn{4}{|l|}{ Floor Concrete } & $N / A$ & $\mathrm{~N} / \mathrm{A}$ & 11 & 1438 & $\mathrm{~N} / \mathrm{A}$ & $N / A$ & $N / A$ \\
\hline \multicolumn{5}{|c|}{ Reviewed By (Print): } & Signature & & & & & \multicolumn{2}{|c|}{ Date: $7 / 19 / 10$} \\
\hline
\end{tabular}


SURVEY \# 10-ER-A26-183

\begin{tabular}{|c|c|c|c|c|c|c|c|c|}
\hline \multirow{2}{*}{$\begin{array}{l}\text { Survey } \\
\text { Point }\end{array}$} & \multirow[t]{2}{*}{ Description/Comments } & \multicolumn{2}{|c|}{$\begin{array}{l}\text { Removable } \\
\mathrm{dpm} / 100 \mathrm{~cm}^{2}\end{array}$} & \multicolumn{2}{|c|}{\begin{tabular}{|c|} 
Fixed + Removable \\
$\mathrm{dpm} / 100 \mathrm{~cm}^{2}$
\end{tabular}} & \multirow{2}{*}{$\begin{array}{l}\text { Gamma } \\
\text { mrem/hr }\end{array}$} & \multirow{2}{*}{$\begin{array}{l}\text { Neutron } \\
\mathrm{mrem} / \mathrm{hr}\end{array}$} & \multirow{2}{*}{$\begin{array}{c}\text { Total } \\
\text { mrem/hr }\end{array}$} \\
\hline & & Alpha & Beta & Alpha & Beta & & & \\
\hline 12 & Floor Concrete & N/A & $N / A$ & 6 & 1439 & N/A & N/A & N/A \\
\hline 13 & Floor Concrete & N/A & $\mathrm{N} / \mathrm{A}$ & 6 & 1483 & N/A & N/A & $\mathrm{N} / \mathrm{A}$ \\
\hline 14 & Floor Concrete & N/A & N/A & 11 & 1505 & N/A & N/A & $N / A$ \\
\hline 15 & Floor Concrete & N/A & N/A & 9 & 1539 & $\mathrm{~N} / \mathrm{A}$ & $\mathrm{N} / \mathrm{A}$ & $\mathrm{N} / \mathrm{A}$ \\
\hline 16 & Floor Concrete & $\mathrm{N} / \mathrm{A}$ & $\mathrm{N} / \mathrm{A}$ & 6 & 1601 & N/A & $\mathrm{N} / \mathrm{A}$ & $\mathrm{N} / \mathrm{A}$ \\
\hline 17 & Floor Concrete & N/A & N/A & 10 & 1625 & N/A & $\mathrm{N} / \mathrm{A}$ & $\mathrm{N} / \mathrm{A}$ \\
\hline 18 & Floor Concrete & $\mathrm{N} / \mathrm{A}$ & N/A & 8 & 1530 & N/A & N/A & $\mathrm{N} / \mathrm{A}$ \\
\hline 19 & Floor Concrete & N/A & N/A & 7 & 1494 & N/A & N/A & $\mathrm{N} / \mathrm{A}$ \\
\hline 20 & Floor Concrete & N/A & N/A & 9 & 1565 & N/A & $\mathrm{N} / \mathrm{A}$ & $\mathrm{N} / \mathrm{A}$ \\
\hline 21 & Floor Concrete & N/A & $N / A$ & 4 & 1547 & N/A & N/A & N/A \\
\hline 22 & Floor Concrete & N/A & $\mathrm{N} / \mathrm{A}$ & 14 & 1497 & N/A & $\mathrm{N} / \mathrm{A}$ & N/A \\
\hline 23 & Floor Concrete & N/A & $\mathrm{N} / \mathrm{A}$ & 6 & 1578 & N/A & $\mathrm{N} / \mathrm{A}$ & N/A \\
\hline 24 & Floor Concrete & N/A & $\mathrm{N} / \mathrm{A}$ & 7 & 1541 & N/A & N/A & $\mathrm{N} / \mathrm{A}$ \\
\hline 25 & Floor Concrete & N/A & $\mathrm{N} / \mathrm{A}$ & 11 & 1581 & N/A & N/A & $\mathrm{N} / \mathrm{A}$ \\
\hline 26 & Floor Concrete & N/A & $\mathrm{N} / \mathrm{A}$ & 8 & 1637 & N/A & N/A & $\mathrm{N} / \mathrm{A}$ \\
\hline 27 & Floor Concrete & N/A & N/A & 12 & 1524 & $\mathrm{~N} / \mathrm{A}$ & N/A & N/A \\
\hline 28 & Floor Concrete & N/A & N/A & 14 & 1552 & N/A & $N / A$ & N/A \\
\hline 29 & Floor Concrete & N/A & N/A & 12 & 1571 & N/A & N/A & $\mathrm{N} / \mathrm{A}$ \\
\hline 30 & Floor Concrete & N/A & $\mathrm{N} / \mathrm{A}$ & 12 & 1514 & N/A & $\mathrm{N} / \mathrm{A}$ & $\mathrm{N} / \mathrm{A}$ \\
\hline 31 & Floor Concrete & N/A & N/A & 1 & 1544 & N/A & N/A & $\mathrm{N} / \mathrm{A}$ \\
\hline 32 & Floor Concrete & N/A & $\mathrm{N} / \mathrm{A}$ & 7 & 1415 & N/A & N/A & N/A \\
\hline 33 & Floor Concrete & N/A & $\mathrm{N} / \mathrm{A}$ & 9 & 1598 & N/A & N/A & N/A \\
\hline 34 & Floor Concrete & N/A & $N / A$ & 7 & 1458 & N/A & $N / A$ & N/A \\
\hline
\end{tabular}


SURVEY \# 10-ER-A26-183

\begin{tabular}{|c|c|c|c|c|c|c|c|c|}
\hline \multirow{2}{*}{$\begin{array}{l}\text { Survey } \\
\text { Point }\end{array}$} & \multirow[t]{2}{*}{ Description/Comments } & \multicolumn{2}{|c|}{$\begin{array}{l}\text { Removable } \\
\mathrm{dpm} / 100 \mathrm{~cm}^{2}\end{array}$} & \multicolumn{2}{|c|}{$\begin{array}{c}\text { Fixed + Removable } \\
\mathrm{dpm} / 100 \mathrm{~cm}^{2}\end{array}$} & \multirow{2}{*}{$\begin{array}{l}\text { Gamma } \\
\mathrm{mrem} / \mathrm{hr}\end{array}$} & \multirow{2}{*}{$\begin{array}{l}\text { Neutron } \\
\text { mrem/hr }\end{array}$} & \multirow{2}{*}{$\begin{array}{c}\text { Total } \\
\text { mrem/hr }\end{array}$} \\
\hline & & Alpha & Beta & Alpha & Beta & & & \\
\hline 35 & Floor Concrete & N/A & N/A & 5 & 1641 & N/A & N/A & N/A \\
\hline 36 & Floor Concrete & $\mathrm{N} / \mathrm{A}$ & N/A & 7 & 1686 & $\mathrm{~N} / \mathrm{A}$ & N/A & N/A \\
\hline 37 & Floor Concrete & $\mathrm{N} / \mathrm{A}$ & N/A & 10 & 1487 & N/A & N/A & $\mathrm{N} / \mathrm{A}$ \\
\hline 38 & Floor Concrete & N/A & $\mathrm{N} / \mathrm{A}$ & 9 & 1430 & N/A & N/A & N/A \\
\hline 39 & Floor Concrete & N/A & N/A & 5 & 1243 & N/A & $\mathrm{N} / \mathrm{A}$ & N/A \\
\hline 40 & Floor Concrete & N/A & $\mathrm{N} / \mathrm{A}$ & 6 & 1403 & N/A & $\mathrm{N} / \mathrm{A}$ & $\mathrm{N} / \mathrm{A}$ \\
\hline N/A & N/A & $N / A$ & N/A & N/A & $N / A$ & $\mathrm{~N} / \mathrm{A}$ & $\mathrm{N} / \mathrm{A}$ & N/A \\
\hline $\mathrm{N} / \mathrm{A}$ & N/A & N/A & $\mathrm{N} / \mathrm{A}$ & N/A & $\mathrm{N} / \mathrm{A}$ & N/A & N/A & N/A \\
\hline $\mathrm{N} / \mathrm{A}$ & N/A & N/A & $\mathrm{N} / \mathrm{A}$ & N/A & $\mathrm{N} / \mathrm{A}$ & N/A & N/A & $\mathrm{N} / \mathrm{A}$ \\
\hline $\mathrm{N} / \mathrm{A}$ & N/A & N/A & $\mathrm{N} / \mathrm{A}$ & $\mathrm{N} / \mathrm{A}$ & N/A & N/A & N/A & $\mathrm{N} / \mathrm{A}$ \\
\hline N/A & N/A & $\mathrm{N} / \mathrm{A}$ & $\mathrm{N} / \mathrm{A}$ & $\mathrm{N} / \mathrm{A}$ & N/A & N/A & $\mathrm{N} / \mathrm{A}$ & N/A \\
\hline N/A & N/A & $\mathrm{N} / \mathrm{A}$ & N/A & $\mathrm{N} / \mathrm{A}$ & N/A & N/A & N/A & N/A \\
\hline $\mathrm{N} / \mathrm{A}$ & N/A & $\mathrm{N} / \mathrm{A}$ & N/A & $\mathrm{N} / \mathrm{A}$ & $\mathrm{N} / \mathrm{A}$ & N/A & N/A & $\mathrm{N} / \mathrm{A}$ \\
\hline $\mathrm{N} / \mathrm{A}$ & N/A & N/A & $\mathrm{N} / \mathrm{A}$ & N/A & N/A & $\mathrm{N} / \mathrm{A}$ & N/A & N/A \\
\hline $\mathrm{N} / \mathrm{A}$ & N/A & N/A & $\mathrm{N} / \mathrm{A}$ & N/A & $\mathrm{N} / \mathrm{A}$ & N/A & N/A & N/A \\
\hline N/A & N/A & $\mathrm{N} / \mathrm{A}$ & N/A & N/A & $\mathrm{N} / \mathrm{A}$ & N/A & N/A & N/A \\
\hline $\mathrm{N} / \mathrm{A}$ & N/A & N/A & N/A & N/A & N/A & N/A & N/A & N/A \\
\hline $\mathrm{N} / \mathrm{A}$ & N/A & N/A & N/A & N/A & $\mathrm{N} / \mathrm{A}$ & N/A & N/A & N/A \\
\hline N/A & N/A & N/A & $\mathrm{N} / \mathrm{A}$ & N/A & $\mathrm{N} / \mathrm{A}$ & N/A & N/A & N/A \\
\hline $\mathrm{N} / \mathrm{A}$ & N/A & N/A & $\mathrm{N} / \mathrm{A}$ & N/A & $\mathrm{N} / \mathrm{A}$ & N/A & N/A & $\mathrm{N} / \mathrm{A}$ \\
\hline N/A & N/A & N/A & N/A & N/A & N/A & N/A & N/A & N/A \\
\hline N/A & N/A & N/A & N/A & N/A & N/A & N/A & N/A & N/A \\
\hline $\mathrm{N} / \mathrm{A}$ & N/A & N/A & N/A & N/A & N/A & N/A & N/A & N/A \\
\hline
\end{tabular}


RADIOLOGICAL SURVEY REPORT -FULL MAP

Survey \# 10-ER-A26-183

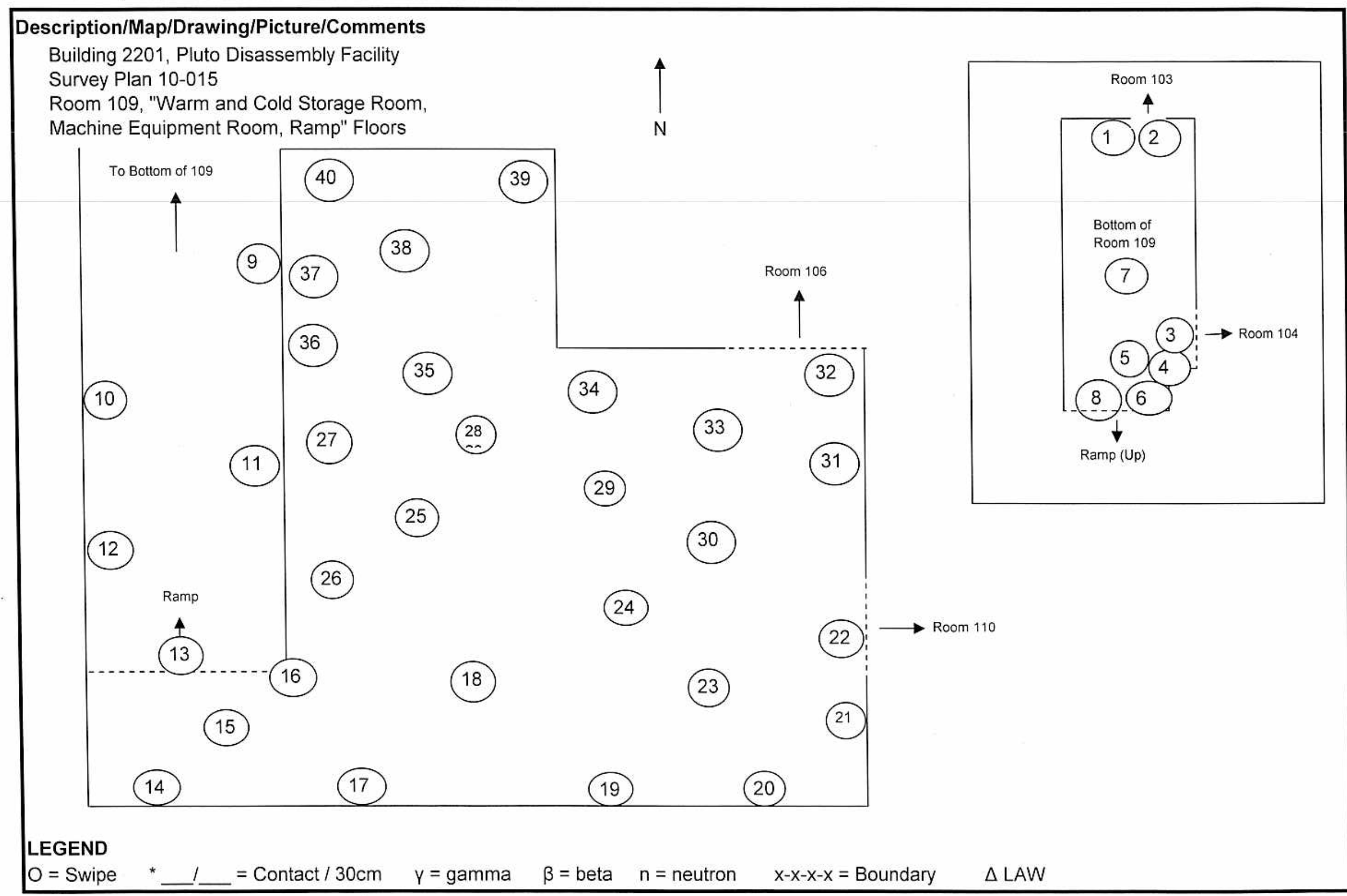




\begin{tabular}{|c|c|c|c|c|c|c|c|c|c|c|c|}
\hline \multicolumn{3}{|c|}{$\begin{array}{l}\text { Location: } \\
\text { Area } 26 \text { CAU117 Pluto }\end{array}$} & \multicolumn{2}{|c|}{$\begin{array}{l}\text { Purpose: } \\
\text { Final Status Survey, Room 109, } \\
\text { Walls }\end{array}$} & \multicolumn{5}{|c|}{$\begin{array}{l}\text { Comments: } \\
\text { All one minute static counts are in cpm. Bkgd not subtracted } \\
\text { for Final Status Survey, Survey Plan \#10-015 }\end{array}$} & \multicolumn{2}{|c|}{$\begin{array}{l}\text { Date/Time: } \\
07 / 14 / 20101615\end{array}$} \\
\hline Instrument: & Serial \#: & Cal Due: & $\begin{array}{c}\text { Eff in \%: } \\
\text { Alpha / Beta }\end{array}$ & $\begin{array}{l}\text { BKG in dpm: } \\
\text { Alpha / Beta }\end{array}$ & $\begin{array}{l}\text { MDA in dpm: } \\
\text { Alpha / Beta }\end{array}$ & RWP\# & \multicolumn{3}{|l|}{$N / A$} & & \\
\hline Electra & 1120 & $4 / 6 / 2011$ & $14.1 / 20.8$ & $N / A$ & $N / A$ & \multicolumn{6}{|c|}{ Work Package \# $\quad$ ENV-10-RCT ENV SITE SURVEYS-0013 } \\
\hline$N / A$ & $N / A$ & $\mathrm{~N} / \mathrm{A}$ & $N / A$ & $N / A$ & $N / A$ & \multicolumn{3}{|c|}{ RCT Name: D.Cotroneo Jr. } & Signature: & & \\
\hline $\mathrm{N} / \mathrm{A}$ & $\mathrm{N} / \mathrm{A}$ & $\mathrm{N} / \mathrm{A}$ & $\mathrm{N} / \mathrm{A}$ & $\mathrm{N} / \mathrm{A}$ & $N / A$ & \multicolumn{3}{|c|}{ RCT Name: Kevin Wilcox } & \multicolumn{3}{|c|}{ Signature: $\mathrm{Kes}=\mathrm{W} \cdot \mathrm{Oen}$} \\
\hline$N / A$ & $\mathrm{~N} / \mathrm{A}$ & $\mathrm{N} / \mathrm{A}$ & $\mathrm{N} / \mathrm{A}$ & $N / A$ & $N / A$ & \multicolumn{3}{|c|}{ RCT Name: Brian Bunce } & \multicolumn{3}{|c|}{ Signature: } \\
\hline$N / A$ & $N / A$ & $\mathrm{~N} / \mathrm{A}$ & $N / A$ & $N / A$ & $N / A$ & \multicolumn{3}{|c|}{ RCT Name: Sonny Brown } & \multirow{3}{*}{\multicolumn{2}{|c|}{\begin{tabular}{|c|c|} 
Signature: & $\begin{array}{l}\text { Neutron } \\
\text { mrem } / \mathrm{hr}\end{array}$ \\
$\mathrm{mrem} / \mathrm{hr}$ &
\end{tabular}}} & \\
\hline \multirow{2}{*}{ Survey Point } & \multirow{2}{*}{\multicolumn{4}{|c|}{ Description/Comments }} & \multicolumn{2}{|c|}{$\begin{array}{l}\text { Removable } \\
\mathrm{dpm} / 100 \mathrm{~cm}^{2}\end{array}$} & \multicolumn{2}{|c|}{\begin{tabular}{|c|}
$\begin{array}{c}\text { Fixed + Removable } \\
\mathrm{dpm} / 100 \mathrm{~cm}^{2}\end{array}$ \\
\end{tabular}} & & & $\begin{array}{c}\text { Total } \\
\text { mrem } / \mathrm{hr}\end{array}$ \\
\hline & & & & & Alpha & Beta & Alpha & Beta & & & \\
\hline 1 & \multicolumn{4}{|c|}{ North Wall Concrete } & $N / A$ & $N / A$ & 9 & 900 & $N / A$ & $N / A$ & $N / A$ \\
\hline 2 & \multicolumn{4}{|c|}{ North Wall Concrete } & $N / A$ & $N / A$ & 7 & 906 & $N / A$ & $N / A$ & $N / A$ \\
\hline 3 & \multicolumn{4}{|c|}{ East Wall Concrete } & $N / A$ & $N / A$ & 6 & 1119 & $N / A$ & $N / A$ & $N / A$ \\
\hline 4 & \multicolumn{4}{|c|}{ East Wall Concrete } & $N / A$ & $N / A$ & 8 & 1120 & $N / A$ & $N / A$ & $N / A$ \\
\hline 5 & \multicolumn{4}{|c|}{ North Wall Metal } & $N / A$ & $N / A$ & 7 & 816 & $N / A$ & $N / A$ & $N / A$ \\
\hline 6 & \multicolumn{4}{|c|}{ East Wall Concrete } & $N / A$ & $N / A$ & 3 & 1138 & $N / A$ & $N / A$ & $N / A$ \\
\hline 7 & \multicolumn{4}{|c|}{ East Wall Concrete } & $N / A$ & $N / A$ & 9 & 1236 & $N / A$ & $N / A$ & $N / A$ \\
\hline 8 & \multicolumn{4}{|c|}{ East Wall Metal } & $N / A$ & $N / A$ & 9 & 924 & $N / A$ & $N / A$ & $N / A$ \\
\hline 9 & \multicolumn{4}{|c|}{ East Wall Concrete } & $N / A$ & $\mathrm{~N} / \mathrm{A}$ & 15 & 1239 & $N / A$ & $N / A$ & $N / A$ \\
\hline 10 & \multicolumn{4}{|c|}{ South Wall Concrete } & $N / A$ & $N / A$ & 4 & 1298 & $N / A$ & $N / A$ & $N / A$ \\
\hline 11 & \multicolumn{4}{|c|}{ South Wall Concrete } & $N / A$ & $N / A$ & 15 & 1163 & $N / A$ & $N / A$ & $N / A$ \\
\hline \multicolumn{5}{|c|}{ Reviewed By (Print): } & \multicolumn{5}{|l|}{ Signature: } & Date: & \\
\hline
\end{tabular}


SURVEY \# 10-ER-A26-181

\begin{tabular}{|c|c|c|c|c|c|c|c|c|}
\hline \multirow{2}{*}{$\begin{array}{l}\text { Survey } \\
\text { Point }\end{array}$} & \multirow[t]{2}{*}{ Description/Comments } & \multicolumn{2}{|c|}{$\begin{array}{l}\text { Removable } \\
\mathrm{dpm} / 100 \mathrm{~cm}^{2}\end{array}$} & \multicolumn{2}{|c|}{\begin{tabular}{|c|}
$\begin{array}{c}\text { Fixed + Removable } \\
\mathrm{dpm} / 100 \mathrm{~cm}^{2}\end{array}$ \\
\end{tabular}} & \multirow{2}{*}{$\begin{array}{l}\text { Gamma } \\
\mathrm{mrem} / \mathrm{hr}\end{array}$} & \multirow{2}{*}{$\begin{array}{l}\text { Neutron } \\
\text { mrem/hr }\end{array}$} & \multirow{2}{*}{$\begin{array}{c}\text { Total } \\
\text { mrem } / \mathrm{hr}\end{array}$} \\
\hline & & Alpha & Beta & Alpha & Beta & & & \\
\hline 12 & South Wall Concrete & N/A & N/A & 4 & 1224 & N/A & $\mathrm{N} / \mathrm{A}$ & N/A \\
\hline 13 & South Wall Concrete & N/A & N/A & 4 & 1254 & N/A & $\mathrm{N} / \mathrm{A}$ & N/A \\
\hline 14 & South Wall Concrete & N/A & $\mathrm{N} / \mathrm{A}$ & 6 & 1198 & N/A & N/A & N/A \\
\hline 15 & South Wall Concrete & N/A & $\mathrm{N} / \mathrm{A}$ & 11 & 1181 & $\mathrm{~N} / \mathrm{A}$ & $\mathrm{N} / \mathrm{A}$ & $\mathrm{N} / \mathrm{A}$ \\
\hline 16 & South Wall Concrete & N/A & $\mathrm{N} / \mathrm{A}$ & 6 & 1159 & $\mathrm{~N} / \mathrm{A}$ & $\mathrm{N} / \mathrm{A}$ & $\mathrm{N} / \mathrm{A}$ \\
\hline 17 & West Wall Concrete & N/A & N/A & 8 & 1211 & N/A & $\mathrm{N} / \mathrm{A}$ & $\mathrm{N} / \mathrm{A}$ \\
\hline 18 & West Wall Concrete & $\mathrm{N} / \mathrm{A}$ & $\mathrm{N} / \mathrm{A}$ & 12 & 1174 & N/A & $\mathrm{N} / \mathrm{A}$ & N/A \\
\hline 19 & West Wall (Ramp) Concrete & N/A & $N / A$ & 14 & 1223 & N/A & $\mathrm{N} / \mathrm{A}$ & N/A \\
\hline 20 & West Wall (Ramp) Concrete & N/A & $\mathrm{N} / \mathrm{A}$ & 12 & 1238 & N/A & $\mathrm{N} / \mathrm{A}$ & $\mathrm{N} / \mathrm{A}$ \\
\hline 21 & West Wall (Ramp) Concrete & $\mathrm{N} / \mathrm{A}$ & $\mathrm{N} / \mathrm{A}$ & 8 & 1277 & N/A & $\mathrm{N} / \mathrm{A}$ & $\mathrm{N} / \mathrm{A}$ \\
\hline 22 & West Wall (Ramp) Concrete & N/A & $\mathrm{N} / \mathrm{A}$ & 11 & 1246 & N/A & $\mathrm{N} / \mathrm{A}$ & $\mathrm{N} / \mathrm{A}$ \\
\hline 23 & West Wall (Ramp) Concrete & $\mathrm{N} / \mathrm{A}$ & $\mathrm{N} / \mathrm{A}$ & 7 & 1282 & N/A & N/A & $\mathrm{N} / \mathrm{A}$ \\
\hline 24 & West Wall (Ramp) Concrete & $\mathrm{N} / \mathrm{A}$ & N/A & 19 & 1205 & $\mathrm{~N} / \mathrm{A}$ & N/A & N/A \\
\hline 25 & West Wall Concrete & N/A & $\mathrm{N} / \mathrm{A}$ & 13 & 1175 & N/A & N/A & N/A \\
\hline 26 & West Wall Concrete & N/A & $\mathrm{N} / \mathrm{A}$ & 17 & 1084 & N/A & $\mathrm{N} / \mathrm{A}$ & N/A \\
\hline 27 & West Wall Concrete & $\mathrm{N} / \mathrm{A}$ & $\mathrm{N} / \mathrm{A}$ & 15 & 1132 & N/A & N/A & N/A \\
\hline 28 & North Wall Concrete & $\mathrm{N} / \mathrm{A}$ & $\mathrm{N} / \mathrm{A}$ & 13 & 1116 & N/A & N/A & $\mathrm{N} / \mathrm{A}$ \\
\hline 29 & East Wall Metal & N/A & $\mathrm{N} / \mathrm{A}$ & 7 & 632 & $\mathrm{~N} / \mathrm{A}$ & $\mathrm{N} / \mathrm{A}$ & $\mathrm{N} / \mathrm{A}$ \\
\hline 30 & East Wall Concrete & $\mathrm{N} / \mathrm{A}$ & N/A & 7 & 853 & $\mathrm{~N} / \mathrm{A}$ & $\mathrm{N} / \mathrm{A}$ & N/A \\
\hline 31 & East Wall (Ramp) Concrete & N/A & N/A & 14 & 1162 & $\mathrm{~N} / \mathrm{A}$ & $\mathrm{N} / \mathrm{A}$ & N/A \\
\hline N/A & N/A & N/A & $\mathrm{N} / \mathrm{A}$ & $\mathrm{N} / \mathrm{A}$ & $\mathrm{N} / \mathrm{A}$ & N/A & N/A & $\mathrm{N} / \mathrm{A}$ \\
\hline $\mathrm{N} / \mathrm{A}$ & N/A & N/A & $\mathrm{N} / \mathrm{A}$ & $\mathrm{N} / \mathrm{A}$ & $\mathrm{N} / \mathrm{A}$ & N/A & $\mathrm{N} / \mathrm{A}$ & $\mathrm{N} / \mathrm{A}$ \\
\hline N/A & N/A & N/A & N/A & N/A & N/A & N/A & $\mathrm{N} / \mathrm{A}$ & N/A \\
\hline
\end{tabular}




\section{Survey \# 10-ER-A26-181}

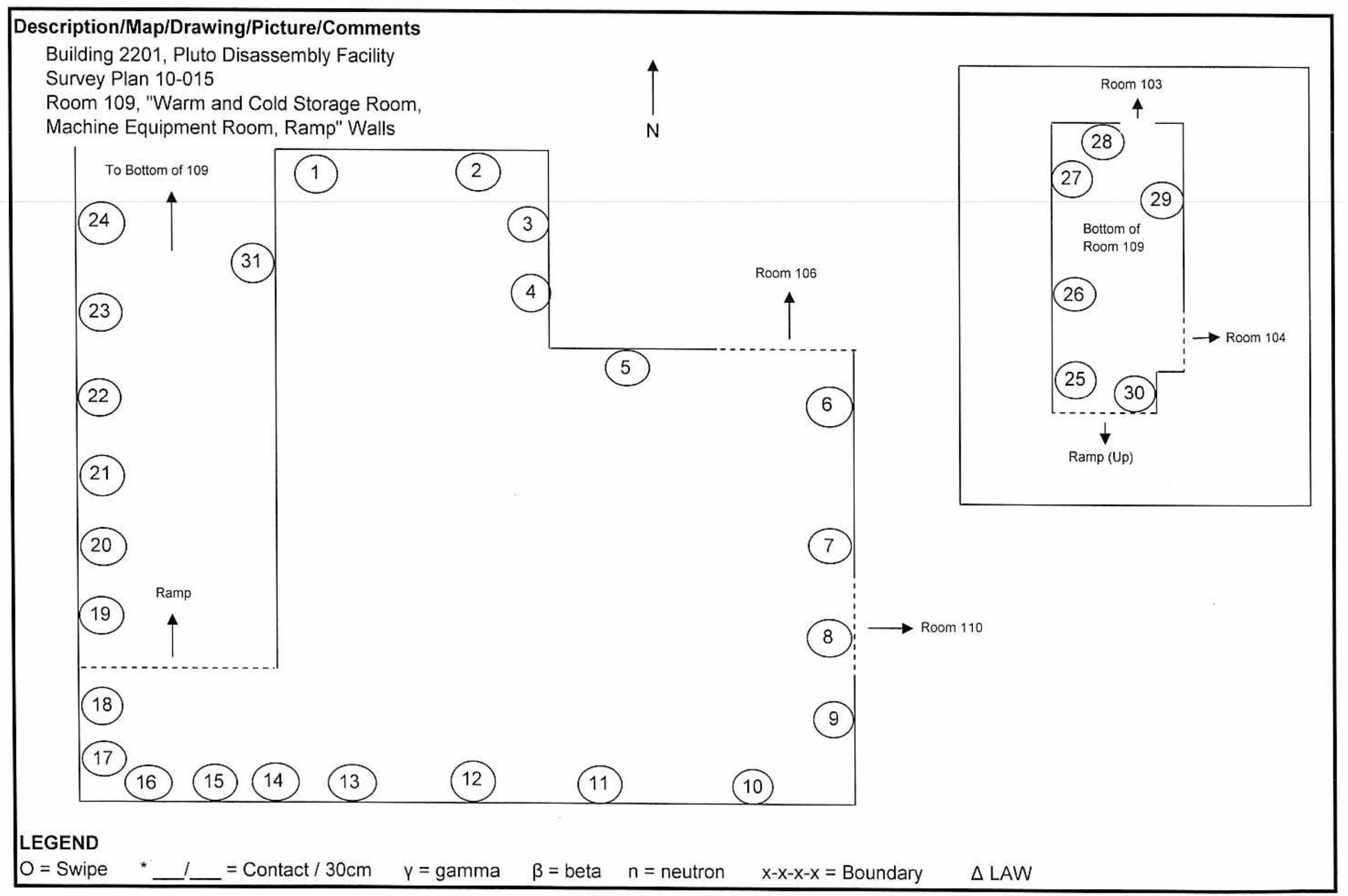


SURVEY \#

10-ER-A26-228

\begin{tabular}{|c|c|c|c|c|c|c|c|c|c|c|c|}
\hline \multicolumn{3}{|c|}{$\begin{array}{l}\text { Location: } \\
\text { Area } 26 \text { CAU117 Pluto }\end{array}$} & \multicolumn{2}{|c|}{$\begin{array}{l}\text { Purpose: } \\
\text { Final Status Survey, Room 110, } \\
\text { Floor, Ceiling and Walls }\end{array}$} & \multicolumn{5}{|c|}{$\begin{array}{l}\text { Comments: } \\
\text { All one minute static counts are in cpm. Bkgd not subtracted for } \\
\text { Final Status Survey, Survey Plan } \# 10-015\end{array}$} & \multicolumn{2}{|c|}{$\begin{array}{l}\text { Date/Time: } \\
\text { 07/28/2010 } 1300\end{array}$} \\
\hline Instrument: & Serial \#: & Cal Due: & $\begin{array}{c}\text { Eff in \%: } \\
\text { Alpha / Beta }\end{array}$ & $\begin{array}{l}\text { BKG in dpm: } \\
\text { Alpha / Beta }\end{array}$ & \begin{tabular}{|l|} 
MDA in dpm: \\
Alpha / Beta
\end{tabular} & RWP\# & \multicolumn{5}{|l|}{$\mathrm{N} / \mathrm{A}$} \\
\hline Electra & 1504 & $4 / 6 / 2011$ & $12.9 / 19.8$ & $N / A$ & $N / A$ & \multicolumn{6}{|c|}{ Work Package \# $\quad$ ENV-10-RCT ENV SITE SURVEYS-0013 } \\
\hline$N / A$ & $N / A$ & $\mathrm{~N} / \mathrm{A}$ & $\mathrm{N} / \mathrm{A}$ & $N / A$ & $N / A$ & \multicolumn{3}{|c|}{ RCT Name: D.Cotroneo Jr. } & \multicolumn{3}{|c|}{ Signature: $-\infty$} \\
\hline $\mathrm{N} / \mathrm{A}$ & $\mathrm{N} / \mathrm{A}$ & $\mathrm{N} / \mathrm{A}$ & $\mathrm{N} / \mathrm{A}$ & $\mathrm{N} / \mathrm{A}$ & $\mathrm{N} / \mathrm{A}$ & \multicolumn{3}{|c|}{ RCT Name: Kevin Wilcox } & \multicolumn{3}{|c|}{ Signature: Fein W.leox } \\
\hline $\mathrm{N} / \mathrm{A}$ & $N / A$ & $N / A$ & $\mathrm{~N} / \mathrm{A}$ & $\mathrm{N} / \mathrm{A}$ & $N / A$ & \multicolumn{3}{|c|}{ RCT Name: B. Bunce/S. Brown } & \multicolumn{3}{|c|}{ 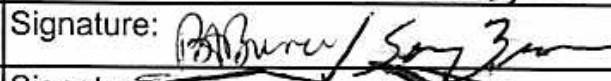 } \\
\hline $\mathrm{N} / \mathrm{A}$ & $N / A$ & $\mathrm{~N} / \mathrm{A}$ & $N / A$ & $N / A$ & $\mathrm{~N} / \mathrm{A}$ & \multicolumn{3}{|c|}{ RCT Name: Brian Foskett } & \multirow{3}{*}{\multicolumn{3}{|c|}{ 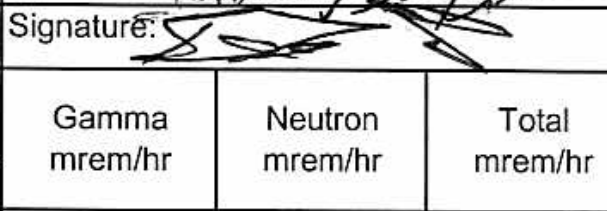 }} \\
\hline \multirow[t]{2}{*}{ Survey Point } & \multirow{2}{*}{\multicolumn{4}{|c|}{ Description/Comments }} & \multicolumn{2}{|c|}{$\begin{array}{l}\text { Removable } \\
\mathrm{dpm} / 100 \mathrm{~cm}^{2}\end{array}$} & \multicolumn{2}{|c|}{$\begin{array}{c}\text { Fixed + Removable } \\
\mathrm{dpm} / 100 \mathrm{~cm}^{2}\end{array}$} & & & \\
\hline & & & & & Alpha & Beta & Alpha & Beta & & & \\
\hline 1 & \multicolumn{4}{|c|}{ Floor Concrete } & $N / A$ & $N / A$ & 11 & 1564 & $N / A$ & $N / A$ & $\mathrm{~N} / \mathrm{A}$ \\
\hline 2 & \multicolumn{4}{|c|}{ Floor Concrete } & $N / A$ & $N / A$ & 6 & 1707 & $N / A$ & $N / A$ & $\mathrm{~N} / \mathrm{A}$ \\
\hline 3 & \multicolumn{4}{|l|}{ Floor Concrete } & $N / A$ & $\mathrm{~N} / \mathrm{A}$ & 5 & 1607 & $N / A$ & $N / A$ & $N / A$ \\
\hline 4 & \multicolumn{4}{|l|}{ Floor Concrete } & $N / A$ & $N / A$ & 5 & 1724 & $\mathrm{~N} / \mathrm{A}$ & $N / A$ & $N / A$ \\
\hline 5 & \multicolumn{4}{|l|}{ Floor Concrete } & $N / A$ & $N / A$ & 5 & 1562 & $N / A$ & $\mathrm{~N} / \mathrm{A}$ & $N / A$ \\
\hline 6 & \multicolumn{4}{|c|}{ Floor Concrete } & $N / A$ & $N / A$ & 5 & 1638 & $N / A$ & $N / A$ & $N / A$ \\
\hline 7 & \multicolumn{4}{|c|}{ West Wall, Concrete Block } & $N / A$ & $N / A$ & 12 & 1227 & $N / A$ & $N / A$ & $N / A$ \\
\hline 8 & \multicolumn{4}{|c|}{ West Wall, Concrete Block } & $N / A$ & $N / A$ & 7 & 1155 & $N / A$ & $\mathrm{~N} / \mathrm{A}$ & $N / A$ \\
\hline 9 & \multicolumn{4}{|c|}{ West Wall, Concrete Block } & $N / A$ & $N / A$ & 10 & 1226 & $N / A$ & $N / A$ & $N / A$ \\
\hline 10 & \multicolumn{4}{|c|}{ West Wall, Concrete Block } & $N / A$ & $N / A$ & 4 & 1227 & $N / A$ & $N / A$ & $N / A$ \\
\hline 11 & \multicolumn{4}{|c|}{ West Wall, Concrete Block } & $N / A$ & $N / A$ & 7 & 1240 & $N / A$ & $N / A$ & $N / A$ \\
\hline \multicolumn{5}{|c|}{ Reviewed By (Print): } & \multicolumn{5}{|l|}{ Signature: } & \multicolumn{2}{|c|}{ Date: $7 / 29 / 10$} \\
\hline
\end{tabular}


SURVEY \# 10-ER-A26-228

\begin{tabular}{|c|c|c|c|c|c|c|c|c|}
\hline \multirow[t]{2}{*}{ Survey Point } & \multirow[t]{2}{*}{ Description/Comments } & \multicolumn{2}{|c|}{$\begin{array}{l}\text { Removable } \\
\mathrm{dpm} / 100 \mathrm{~cm}^{2}\end{array}$} & \multicolumn{2}{|c|}{$\begin{array}{c}\text { Fixed + Removable } \\
\mathrm{dpm} / 100 \mathrm{~cm}^{2}\end{array}$} & \multirow{2}{*}{$\begin{array}{c}\text { Gamma } \\
\mathrm{mrem} / \mathrm{hr}\end{array}$} & \multirow{2}{*}{$\begin{array}{l}\text { Neutron } \\
\mathrm{mrem} / \mathrm{hr}\end{array}$} & \multirow{2}{*}{$\begin{array}{c}\text { Total } \\
\mathrm{mrem} / \mathrm{hr}\end{array}$} \\
\hline & & Alpha & Beta & Alpha & Beta & & & \\
\hline 12 & West Wall, Concrete Block & $\mathrm{N} / \mathrm{A}$ & N/A & 7 & 1326 & N/A & N/A & N/A \\
\hline 13 & North Wall, Concrete Block & $N / A$ & N/A & 11 & 1265 & N/A & N/A & N/A \\
\hline 14 & North Wall, Metal & $N / A$ & N/A & 7 & 915 & N/A & N/A & N/A \\
\hline 15 & North Wall, Concrete Block & N/A & N/A & 8 & 1576 & N/A & $\mathrm{N} / \mathrm{A}$ & N/A \\
\hline 16 & East Wall, Concrete Block & $\mathrm{N} / \mathrm{A}$ & N/A & 7 & 1362 & N/A & N/A & N/A \\
\hline 17 & East Wall, Concrete Block & $N / A$ & $N / A$ & 15 & 1264 & N/A & N/A & N/A \\
\hline 18 & East Wall, Metal & N/A & N/A & 4 & 1023 & N/A & $\mathrm{N} / \mathrm{A}$ & N/A \\
\hline 19 & East Wall, Concrete Block & $\mathrm{N} / \mathrm{A}$ & N/A & 16 & 1354 & N/A & $\mathrm{N} / \mathrm{A}$ & N/A \\
\hline 20 & East Wall, Concrete Block & N/A & N/A & 13 & 1306 & N/A & $\mathrm{N} / \mathrm{A}$ & N/A \\
\hline 21 & East Wall, Concrete Block & $\mathrm{N} / \mathrm{A}$ & N/A & 11 & 1371 & N/A & N/A & $\mathrm{N} / \mathrm{A}$ \\
\hline 22 & South Wall, Concrete Block & N/A & N/A & 7 & 1379 & N/A & N/A & N/A \\
\hline 23 & South Wall, Concrete Block & N/A & N/A & 11 & 1295 & N/A & N/A & N/A \\
\hline 24 & South Wall, Concrete Block & N/A & N/A & 3 & 1217 & $\mathrm{~N} / \mathrm{A}$ & $\mathrm{N} / \mathrm{A}$ & $\mathrm{N} / \mathrm{A}$ \\
\hline 25 & Ceiling, Metal & N/A & N/A & 4 & 1163 & N/A & $\mathrm{N} / \mathrm{A}$ & N/A \\
\hline 26 & Ceiling, Metal & $\mathrm{N} / \mathrm{A}$ & N/A & 2 & 1234 & $\mathrm{~N} / \mathrm{A}$ & $\mathrm{N} / \mathrm{A}$ & $\mathrm{N} / \mathrm{A}$ \\
\hline 27 & Ceiling, Metal & $\mathrm{N} / \mathrm{A}$ & N/A & 9 & 1189 & N/A & $\mathrm{N} / \mathrm{A}$ & $\mathrm{N} / \mathrm{A}$ \\
\hline 28 & Ceiling, Metal & $\mathrm{N} / \mathrm{A}$ & N/A & 11 & 1202 & N/A & $\mathrm{N} / \mathrm{A}$ & N/A \\
\hline 29 & Ceiling, Metal & $\mathrm{N} / \mathrm{A}$ & $\mathrm{N} / \mathrm{A}$ & 2 & 1172 & $\mathrm{~N} / \mathrm{A}$ & N/A & N/A \\
\hline 30 & Ceiling, Metal & $\mathrm{N} / \mathrm{A}$ & N/A & 9 & 1225 & $\mathrm{~N} / \mathrm{A}$ & N/A & N/A \\
\hline N/A & N/A & $\mathrm{N} / \mathrm{A}$ & N/A & N/A & N/A & N/A & N/A & N/A \\
\hline N/A & $\mathrm{N} / \mathrm{A}$ & $\mathrm{N} / \mathrm{A}$ & N/A & N/A & N/A & N/A & $\mathrm{N} / \mathrm{A}$ & $\mathrm{N} / \mathrm{A}$ \\
\hline N/A & N/A & $\mathrm{N} / \mathrm{A}$ & N/A & N/A & N/A & N/A & N/A & N/A \\
\hline$N / A$ & N/A & $\mathrm{N} / \mathrm{A}$ & N/A & N/A & N/A & N/A & N/A & N/A \\
\hline
\end{tabular}


Survey \# 10-ER-A26-228

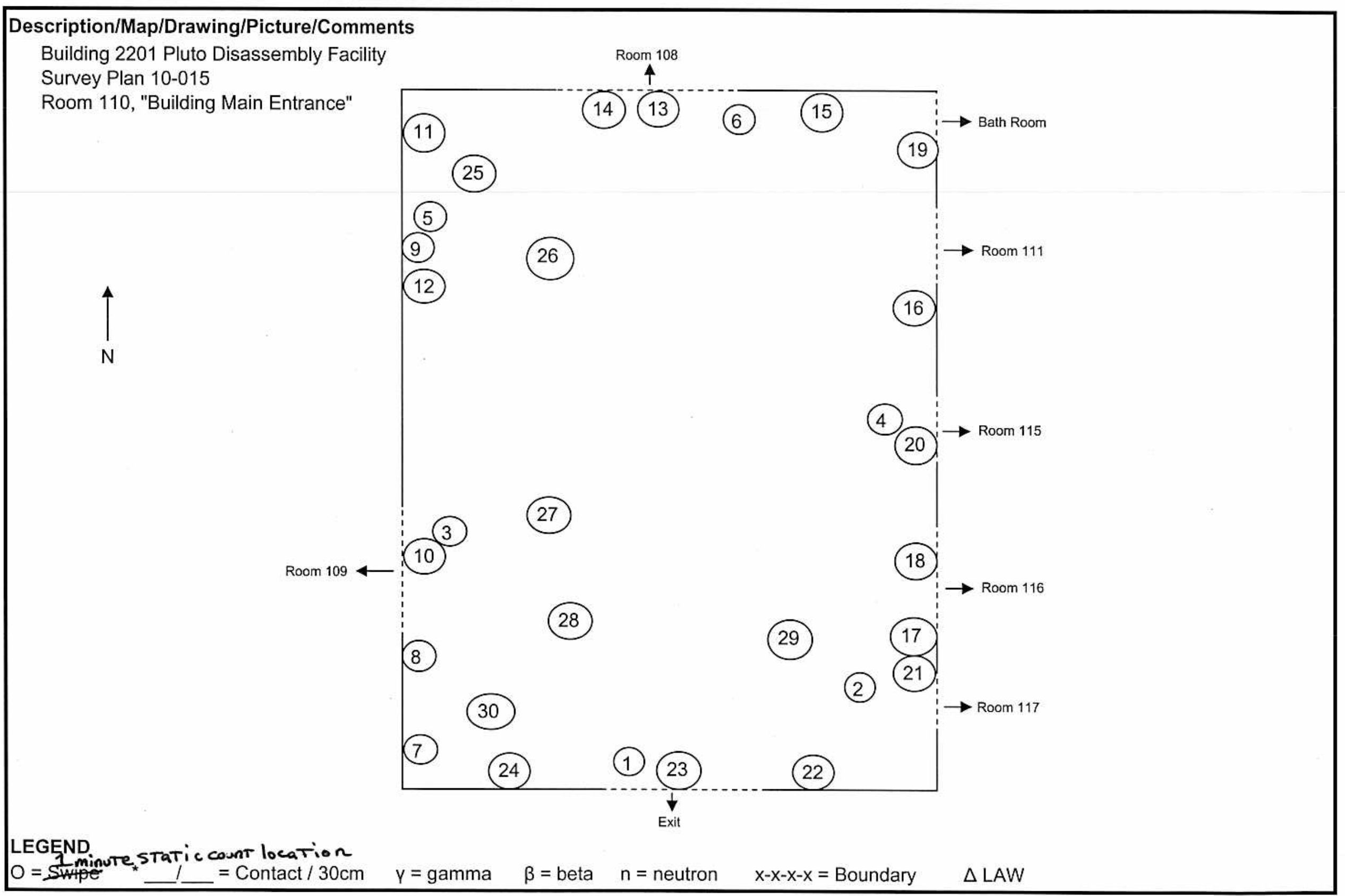


SURVEY \# 10-ER-A26-194

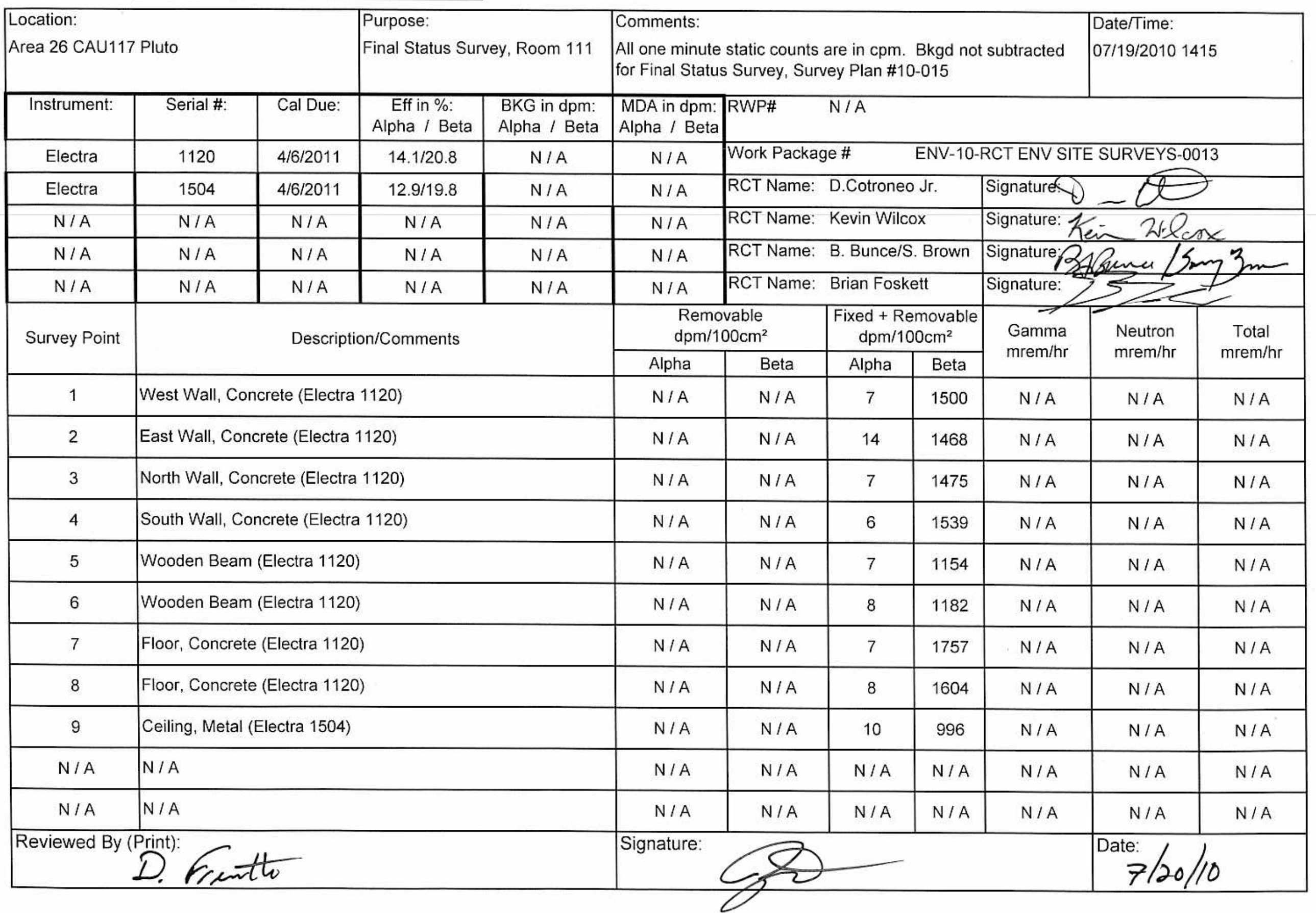


Survey \# 10-ER-A26-194

\section{Description/Map/Drawing/Picture/Comments}

Building 2201 Pluto Disassembly Facility

Survey Plan 10-015

Room 111

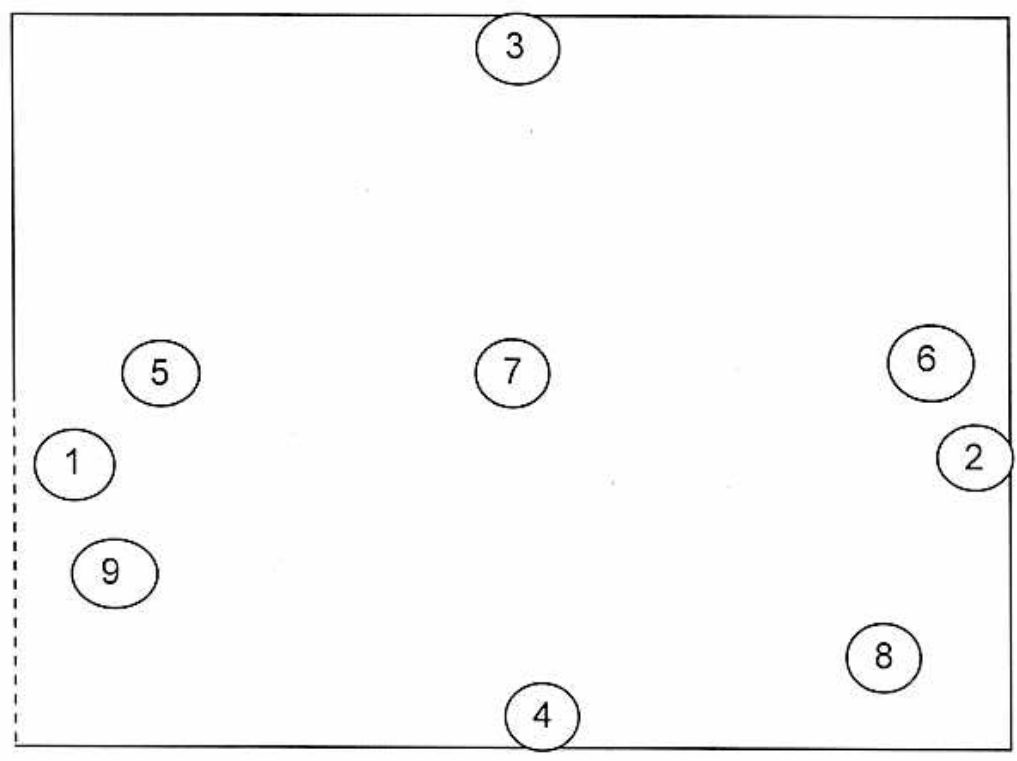

LEGEND

$\mathrm{O}=$ Swipe 


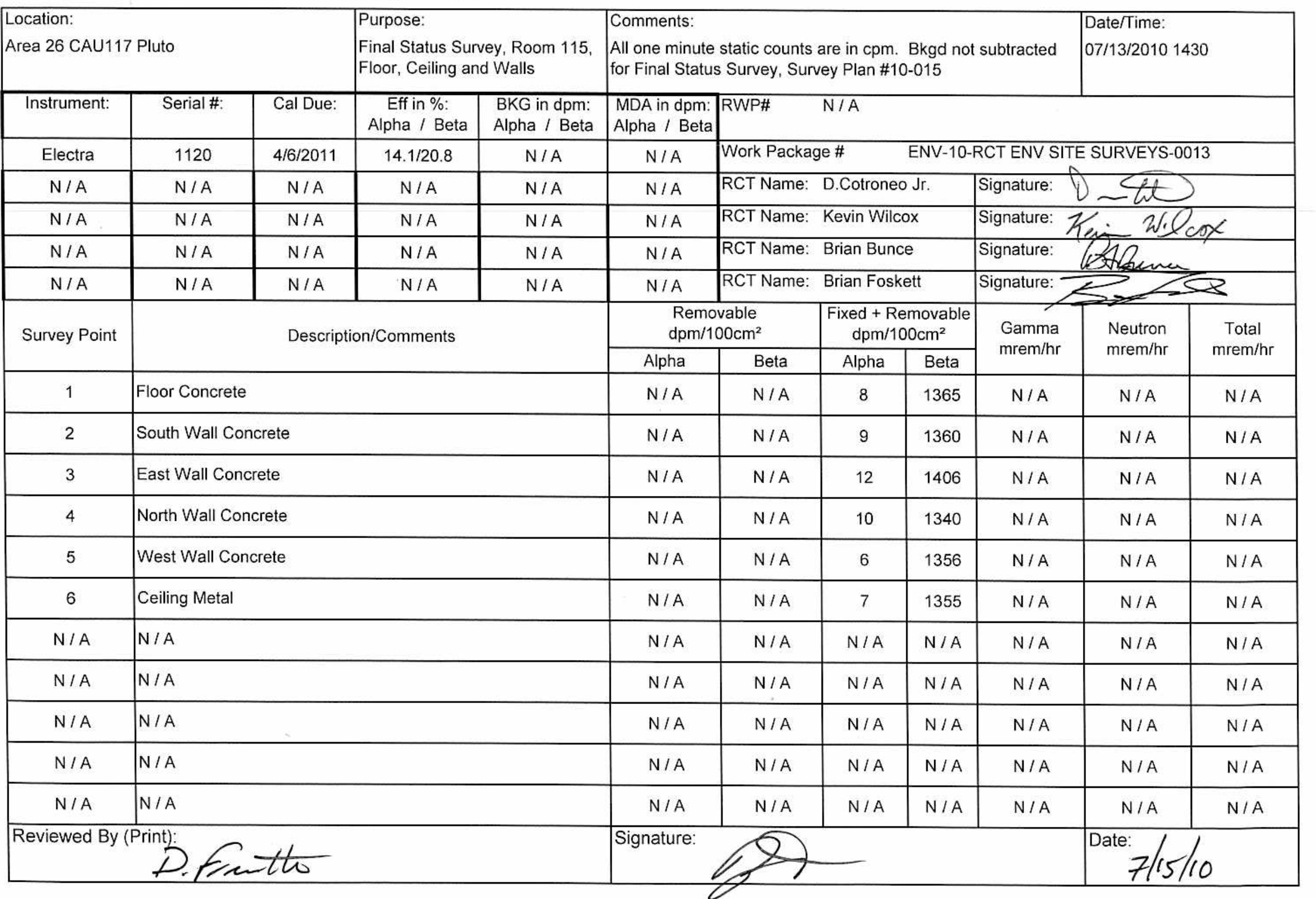


Survey \# 10-ER-A26-176

Description/Map/Drawing/Picture/Comments

Building 2201 Pluto Disassembly Facility

Survey Plan 10-015

Room 115

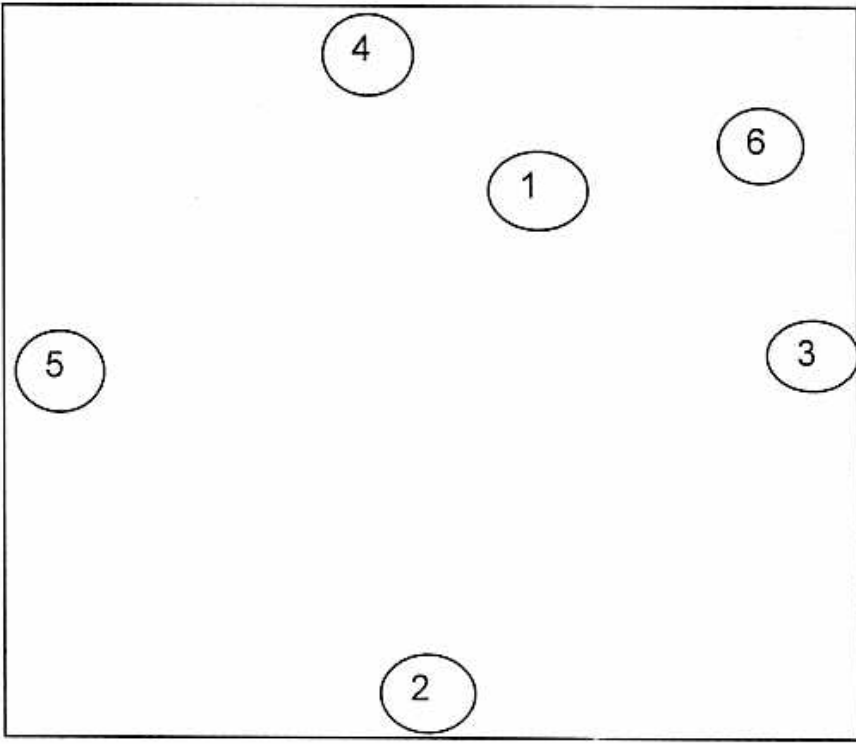

\section{LEGEND}

$\mathrm{O}=$ Swipe 


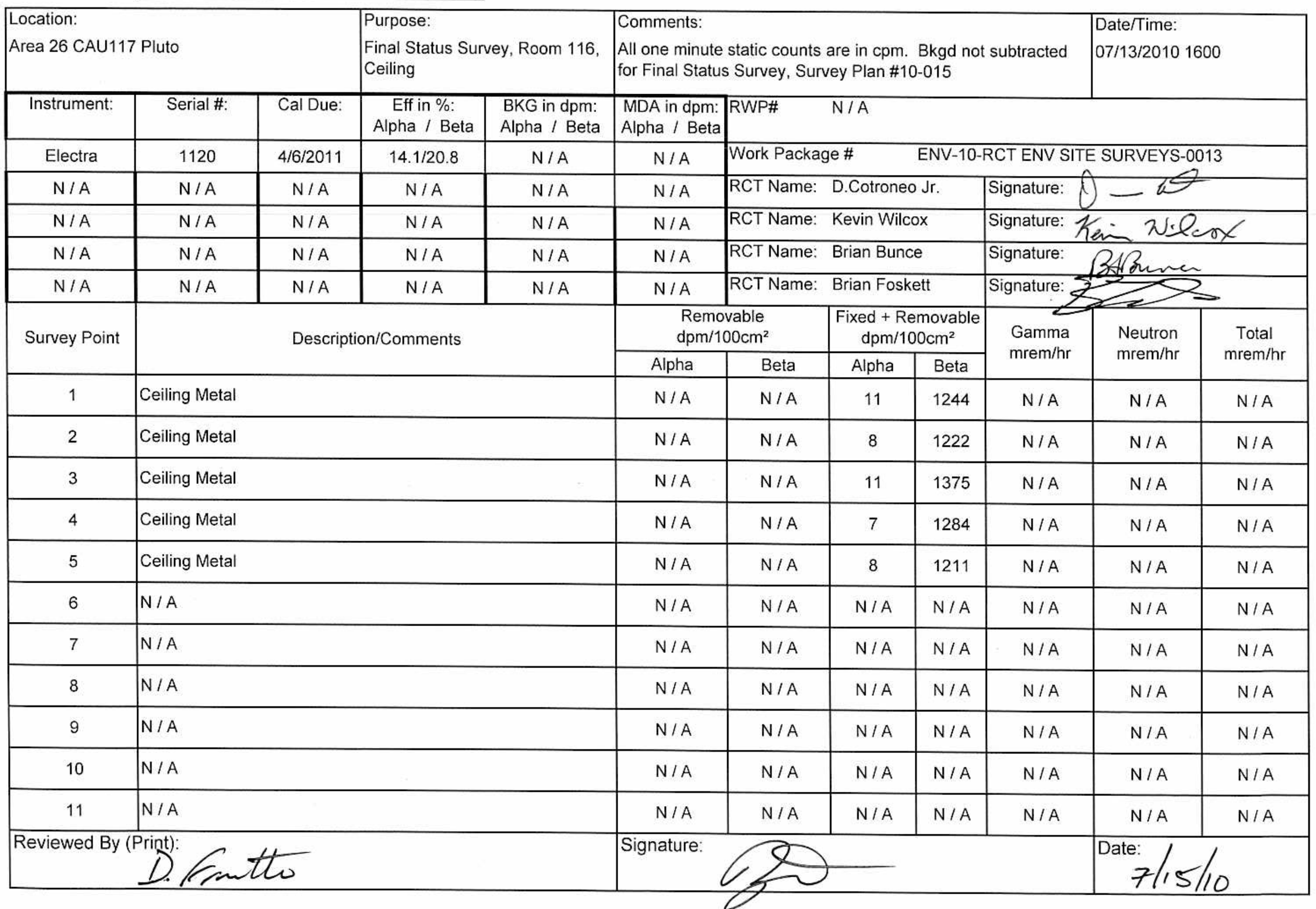


Survey \# 10-ER-A26-178

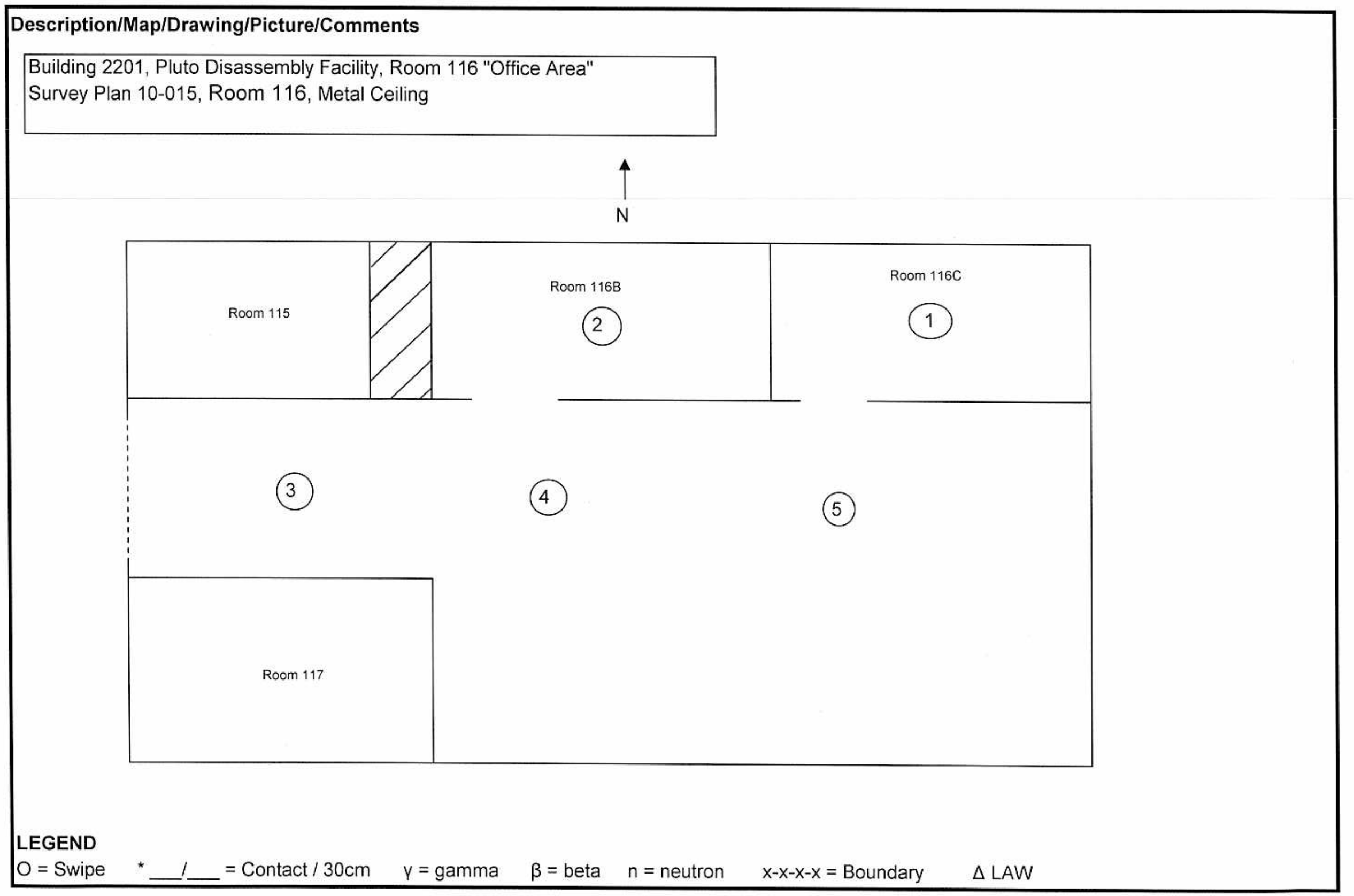




\begin{tabular}{|c|c|c|c|c|c|c|c|c|c|c|c|}
\hline \multicolumn{3}{|c|}{$\begin{array}{l}\text { Location: } \\
\text { Area } 26 \text { CAU117 Pluto }\end{array}$} & \multicolumn{2}{|c|}{$\begin{array}{l}\text { Purpose: } \\
\text { Final Status Survey, Room 116, } \\
\text { Floor }\end{array}$} & \multicolumn{5}{|c|}{$\begin{array}{l}\text { Comments: } \\
\text { All one minute static counts are in cpm. Bkgd not subtracted } \\
\text { for Final Status Survey, Survey Plan \#10-015 }\end{array}$} & \multicolumn{2}{|c|}{$\begin{array}{l}\text { Date/Time: } \\
\text { 07/13/2010 } 1530\end{array}$} \\
\hline Instrument: & Serial \#: & Cal Due: & \begin{tabular}{|c|} 
Eff in \%: \\
Alpha / Beta \\
\end{tabular} & $\begin{array}{l}\text { BKG in dpm: } \\
\text { Alpha / Beta }\end{array}$ & \begin{tabular}{|l|} 
MDA in dpm: \\
Alpha / Beta \\
\end{tabular} & RWP\# & \multicolumn{5}{|l|}{$N / A$} \\
\hline Electra & 1120 & $4 / 6 / 2011$ & $14.1 / 20.8$ & $\mathrm{~N} / \mathrm{A}$ & $\mathrm{N} / \mathrm{A}$ & \multicolumn{6}{|c|}{ Work Package \# $\quad$ ENV-10-RCT ENV SITE SURVEYS-0013 } \\
\hline$N / A$ & $\mathrm{~N} / \mathrm{A}$ & $\mathrm{N} / \mathrm{A}$ & $\mathrm{N} / \mathrm{A}$ & $\mathrm{N} / \mathrm{A}$ & $N / A$ & \multicolumn{3}{|c|}{ RCT Name: D.Cotroneo Jr. } & Signature: $\lambda$ & \multicolumn{2}{|c|}{ 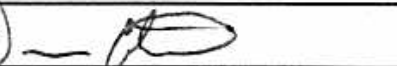 } \\
\hline$N / A$ & $N / A$ & $N / A$ & $N / A$ & $\mathrm{~N} / \mathrm{A}$ & $N / A$ & \multicolumn{3}{|c|}{ RCT Name: Kevin Wilcox } & \multicolumn{3}{|c|}{ Signature: Keni W.Leol } \\
\hline$N / A$ & $\mathrm{~N} / \mathrm{A}$ & $N / A$ & $N / A$ & $N / A$ & $N / A$ & \multicolumn{3}{|c|}{ RCT Name: S. Brown/B. Bunce } & \multirow{2}{*}{\multicolumn{3}{|c|}{ Signatureinge: }} \\
\hline$N / A$ & $N / A$ & $N / A$ & $N / A$ & $N / A$ & $\mathrm{~N} / \mathrm{A}$ & RCT Name: & Brian Fos & & & & \\
\hline \multirow[t]{2}{*}{ Survey Point } & \multirow{2}{*}{\multicolumn{4}{|c|}{ Description/Comments }} & \multicolumn{2}{|c|}{$\begin{array}{l}\text { Removable } \\
\mathrm{dpm} / 100 \mathrm{~cm}^{2}\end{array}$} & \multicolumn{2}{|c|}{\begin{tabular}{|c|}
$\begin{array}{c}\text { Fixed + Removable } \\
\mathrm{dpm} / 100 \mathrm{~cm}^{2}\end{array}$ \\
\end{tabular}} & \multirow{2}{*}{$\begin{array}{c}\text { Gamma } \\
\mathrm{mrem} / \mathrm{hr}\end{array}$} & \multirow{2}{*}{$\begin{array}{l}\text { Neutron } \\
\mathrm{mrem} / \mathrm{hr}\end{array}$} & \multirow{2}{*}{$\begin{array}{c}\text { Total } \\
\text { mrem } / \mathrm{hr}\end{array}$} \\
\hline & & & & & Alpha & Beta & Alpha & Beta & & & \\
\hline 1 & \multicolumn{4}{|c|}{ Floor Concrete } & $N / A$ & $N / A$ & 10 & 1668 & $N / A$ & $N / A$ & $\mathrm{~N} / \mathrm{A}$ \\
\hline 2 & \multicolumn{4}{|l|}{ Floor Concrete } & $\mathrm{N} / \mathrm{A}$ & $N / A$ & 9 & 1666 & $N / A$ & $N / A$ & $N / A$ \\
\hline 3 & \multicolumn{4}{|l|}{ Floor Concrete } & $N / A$ & $N / A$ & 9 & 1653 & $N / A$ & $N / A$ & $N / A$ \\
\hline 4 & \multicolumn{4}{|l|}{ Floor Concrete } & $N / A$ & $N / A$ & 6 & 1649 & $N / A$ & $N / A$ & $N / A$ \\
\hline 5 & \multicolumn{4}{|c|}{ Floor Concrete } & $N / A$ & $N / A$ & 5 & 1622 & $N / A$ & $N / A$ & $N / A$ \\
\hline 6 & \multicolumn{4}{|c|}{ Floor Concrete } & $N / A$ & $N / A$ & 8 & 1651 & $N / A$ & $N / A$ & $N / A$ \\
\hline 7 & \multicolumn{4}{|c|}{ Floor Concrete } & $N / A$ & $N / A$ & 3 & 1650 & $N / A$ & $N / A$ & $N / A$ \\
\hline 8 & \multicolumn{4}{|c|}{ Floor Concrete } & $N / A$ & $N / A$ & 3 & 1636 & $N / A$ & $\mathrm{~N} / \mathrm{A}$ & $\mathrm{N} / \mathrm{A}$ \\
\hline 9 & \multicolumn{4}{|c|}{ Floor Concrete } & $N / A$ & $N / A$ & 7 & 1690 & $N / A$ & $N / A$ & $N / A$ \\
\hline 10 & \multicolumn{4}{|c|}{ Floor Concrete } & $N / A$ & $N / A$ & 8 & 1683 & $\mathrm{~N} / \mathrm{A}$ & $N / A$ & $N / A$ \\
\hline 11 & \multicolumn{4}{|c|}{ Floor Concrete } & $N / A$ & $N / A$ & 11 & 1650 & $N / A$ & $N / A$ & $N / A$ \\
\hline \multicolumn{5}{|c|}{ Reviewed By (Print): } & \multicolumn{5}{|l|}{ Signature: } & \multicolumn{2}{|c|}{ Date: $7 / 15 / 10$} \\
\hline
\end{tabular}


SURVEY \# 10-ER-A26-177

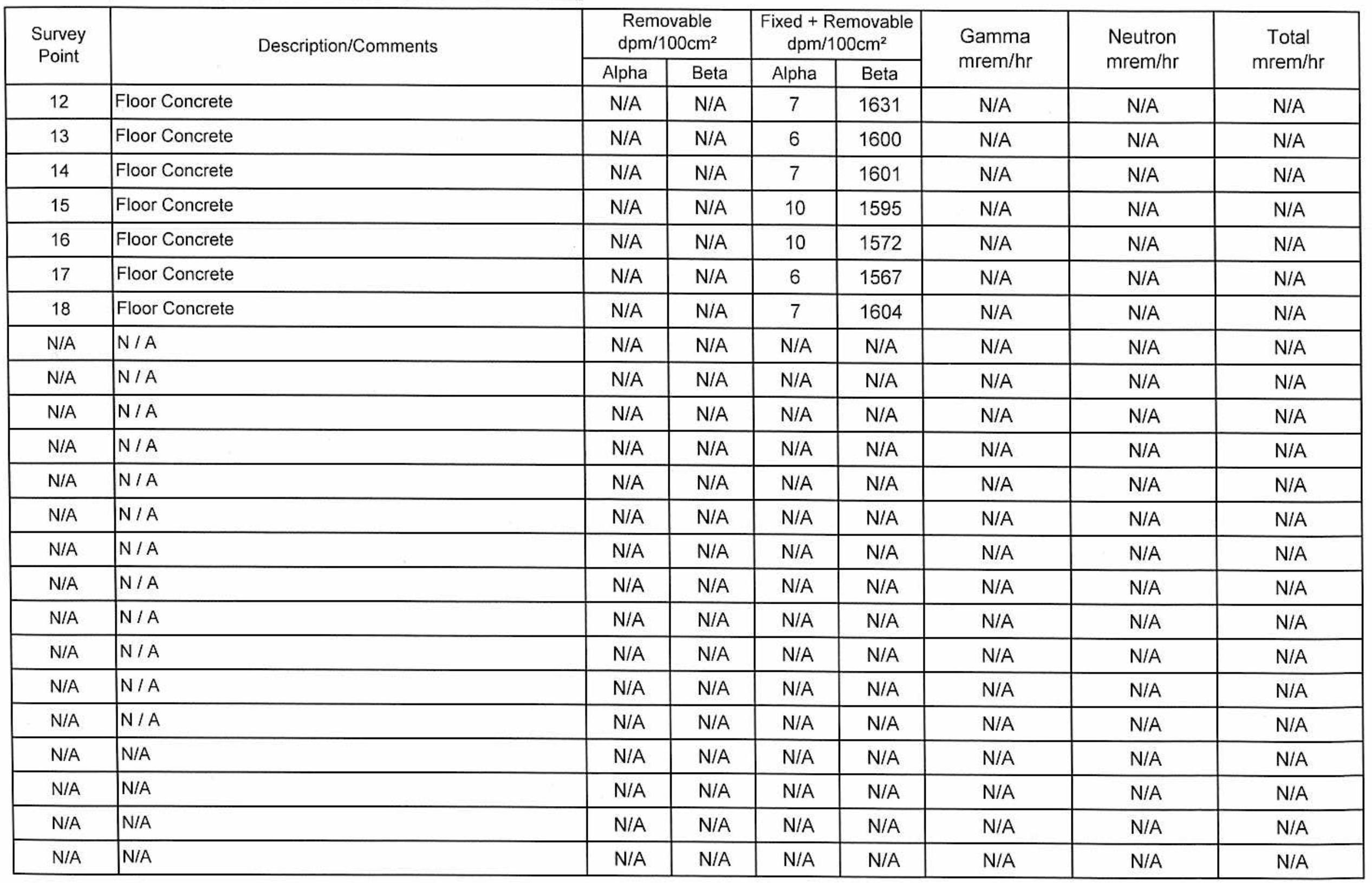


Survey \# 10-ER-A26-177

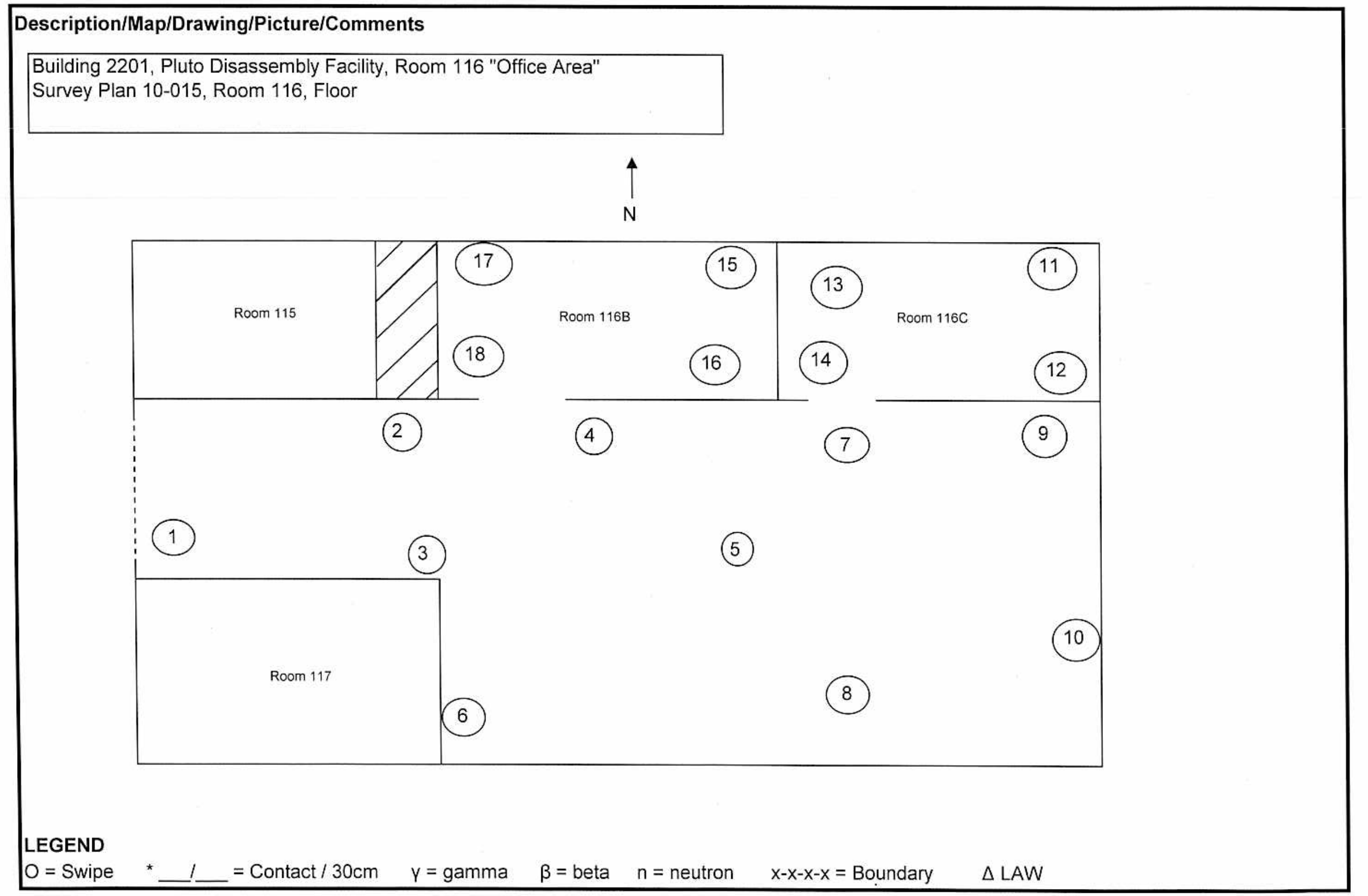




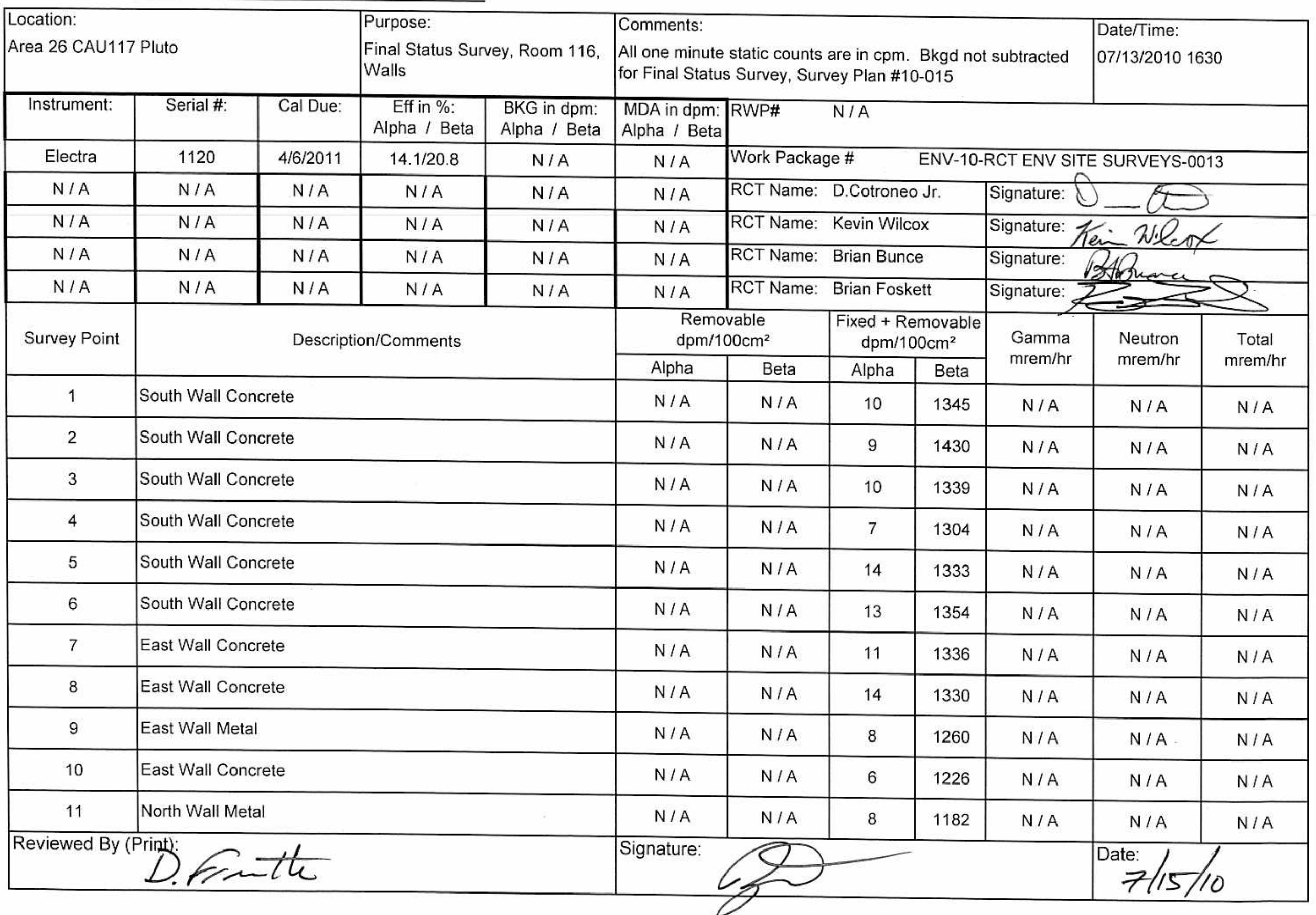


SURVEY \# 10-ER-A26-179

\begin{tabular}{|c|c|c|c|c|c|c|c|c|}
\hline \multirow{2}{*}{$\begin{array}{l}\text { Survey } \\
\text { Point }\end{array}$} & \multirow{2}{*}{ Description/Comments } & \multicolumn{2}{|c|}{$\begin{array}{l}\text { Removable } \\
\mathrm{dpm} / 100 \mathrm{~cm}^{2}\end{array}$} & \multicolumn{2}{|c|}{$\begin{array}{l}\text { Fixed + Removable } \\
\mathrm{dpm} / 100 \mathrm{~cm}^{2}\end{array}$} & \multirow{2}{*}{$\begin{array}{l}\text { Gamma } \\
\text { mrem/hr }\end{array}$} & \multirow{2}{*}{$\begin{array}{l}\text { Neutron } \\
\text { mrem } / \mathrm{hr}\end{array}$} & \multirow{2}{*}{$\begin{array}{c}\text { Total } \\
\text { mrem } / \mathrm{hr}\end{array}$} \\
\hline & & Alpha & Beta & Alpha & Beta & & & \\
\hline 12 & North Wall Metal & N/A & $\mathrm{N} / \mathrm{A}$ & 9 & 1293 & N/A & $\mathrm{N} / \mathrm{A}$ & N/A \\
\hline 13 & North Wall Concrete & N/A & N/A & 12 & 1003 & N/A & N/A & $\mathrm{N} / \mathrm{A}$ \\
\hline 14 & North Wall Concrete & N/A & $\mathrm{N} / \mathrm{A}$ & 11 & 980 & N/A & $\mathrm{N} / \mathrm{A}$ & $\mathrm{N} / \mathrm{A}$ \\
\hline 15 & North Wall Concrete & N/A & $\mathrm{N} / \mathrm{A}$ & 7 & 1392 & $\mathrm{~N} / \mathrm{A}$ & N/A & N/A \\
\hline 16 & North Wall Concrete & N/A & N/A & 11 & 1284 & N/A & N/A & N/A \\
\hline 17 & West Wall Concrete & N/A & $\mathrm{N} / \mathrm{A}$ & 14 & 1303 & N/A & $\mathrm{N} / \mathrm{A}$ & $\mathrm{N} / \mathrm{A}$ \\
\hline 18 & West Wall Concrete & N/A & N/A & 13 & 1472 & $\mathrm{~N} / \mathrm{A}$ & N/A & $\mathrm{N} / \mathrm{A}$ \\
\hline 19 & West Wall Metal & $\mathrm{N} / \mathrm{A}$ & $\mathrm{N} / \mathrm{A}$ & 6 & 1179 & $\mathrm{~N} / \mathrm{A}$ & N/A & N/A \\
\hline 20 & West Wall Metal & $\mathrm{N} / \mathrm{A}$ & $\mathrm{N} / \mathrm{A}$ & 12 & 1058 & N/A & N/A & N/A \\
\hline 21 & South Wall Metal & N/A & $\mathrm{N} / \mathrm{A}$ & 12 & 1068 & N/A & $\mathrm{N} / \mathrm{A}$ & $\mathrm{N} / \mathrm{A}$ \\
\hline 22 & South Wall Metal & N/A & N/A & 10 & 1098 & N/A & $\mathrm{N} / \mathrm{A}$ & $\mathrm{N} / \mathrm{A}$ \\
\hline $\mathrm{N} / \mathrm{A}$ & $N / A$ & N/A & $\mathrm{N} / \mathrm{A}$ & N/A & N/A & $\mathrm{N} / \mathrm{A}$ & $\mathrm{N} / \mathrm{A}$ & N/A \\
\hline N/A & $N / A$ & N/A & $\mathrm{N} / \mathrm{A}$ & $\mathrm{N} / \mathrm{A}$ & $\mathrm{N} / \mathrm{A}$ & N/A & $\mathrm{N} / \mathrm{A}$ & $\mathrm{N} / \mathrm{A}$ \\
\hline $\mathrm{N} / \mathrm{A}$ & $\mathrm{N} / \mathrm{A}$ & N/A & N/A & $\mathrm{N} / \mathrm{A}$ & N/A & N/A & N/A & $\mathrm{N} / \mathrm{A}$ \\
\hline N/A & $N / A$ & N/A & $\mathrm{N} / \mathrm{A}$ & N/A & N/A & N/A & N/A & N/A \\
\hline N/A & $N / A$ & N/A & $\mathrm{N} / \mathrm{A}$ & $\mathrm{N} / \mathrm{A}$ & N/A & $\mathrm{N} / \mathrm{A}$ & $\mathrm{N} / \mathrm{A}$ & $\mathrm{N} / \mathrm{A}$ \\
\hline N/A & $N / A$ & N/A & $\mathrm{N} / \mathrm{A}$ & N/A & N/A & $\mathrm{N} / \mathrm{A}$ & $\mathrm{N} / \mathrm{A}$ & N/A \\
\hline$N / A$ & $\mathrm{~N} / \mathrm{A}$ & N/A & N/A & N/A & N/A & $\mathrm{N} / \mathrm{A}$ & $\mathrm{N} / \mathrm{A}$ & $\mathrm{N} / \mathrm{A}$ \\
\hline N/A & $N / A$ & N/A & N/A & N/A & $\mathrm{N} / \mathrm{A}$ & N/A & N/A & $\mathrm{N} / \mathrm{A}$ \\
\hline N/A & N/A & N/A & N/A & N/A & N/A & N/A & $\mathrm{N} / \mathrm{A}$ & $\mathrm{N} / \mathrm{A}$ \\
\hline N/A & N/A & N/A & $\mathrm{N} / \mathrm{A}$ & N/A & N/A & $\mathrm{N} / \mathrm{A}$ & $\mathrm{N} / \mathrm{A}$ & $\mathrm{N} / \mathrm{A}$ \\
\hline N/A & $\mathrm{N} / \mathrm{A}$ & N/A & $\mathrm{N} / \mathrm{A}$ & N/A & $\mathrm{N} / \mathrm{A}$ & $\mathrm{N} / \mathrm{A}$ & $\mathrm{N} / \mathrm{A}$ & $\mathrm{N} / \mathrm{A}$ \\
\hline N/A & N/A & $\mathrm{N} / \mathrm{A}$ & $\mathrm{N} / \mathrm{A}$ & N/A & N/A & N/A & N/A & N/A \\
\hline
\end{tabular}


Survey \# 10-ER-A26-179

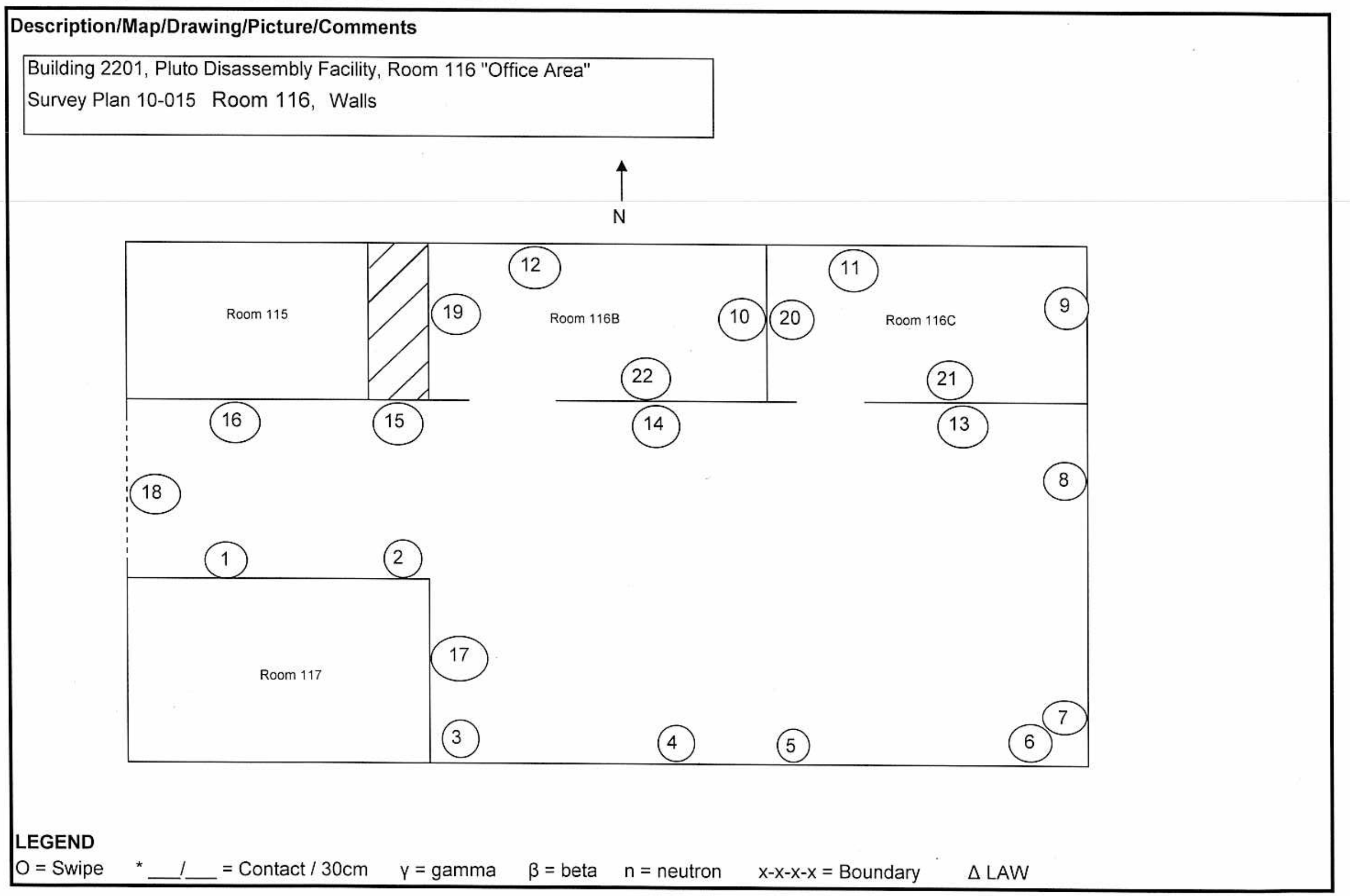




\begin{tabular}{|c|c|c|c|c|c|c|c|c|c|c|c|}
\hline \multicolumn{3}{|c|}{$\begin{array}{l}\text { Location: } \\
\text { Area } 26 \text { CAU117 Pluto }\end{array}$} & \multicolumn{2}{|c|}{$\begin{array}{l}\text { Purpose: } \\
\text { Final Status Survey, Room } 117\end{array}$} & \multicolumn{5}{|c|}{$\begin{array}{l}\text { Comments: } \\
\text { All one minute static counts are in cpm. Bkgd not subtracted } \\
\text { for Final Status Survey, Survey Plan \#10-015 }\end{array}$} & \multicolumn{2}{|c|}{$\begin{array}{l}\text { Date/Time: } \\
\text { 07/19/2010 } 0930\end{array}$} \\
\hline Instrument: & Serial \#: & Cal Due: & \begin{tabular}{|c|} 
Eff in \%: \\
Alpha / Beta
\end{tabular} & $\begin{array}{l}\text { BKG in dpm: } \\
\text { Alpha / Beta }\end{array}$ & \begin{tabular}{|l|} 
MDA in dpm: \\
Alpha / Beta
\end{tabular} & RWP\# & \multicolumn{5}{|l|}{$\mathrm{N} / \mathrm{A}$} \\
\hline Electra & 1120 & $4 / 6 / 2011$ & $14.1 / 20.8$ & $N / A$ & $N / A$ & \multicolumn{6}{|c|}{ Work Package \# $\quad$ ENV-10-RCT ENV SITE SURVEYS-0013 } \\
\hline$N / A$ & $N / A$ & $\mathrm{~N} / \mathrm{A}$ & $N / A$ & $N / A$ & $N / A$ & \multicolumn{3}{|c|}{ RCT Name: D.Cotroneo Jr. } & \multicolumn{3}{|c|}{ Signature: $\left(0 \_\right.$l } \\
\hline$N / A$ & $N / A$ & $N / A$ & $N / A$ & $N / A$ & $N / A$ & \multicolumn{3}{|c|}{ RCT Name: Kevin Wilcox } & \multicolumn{3}{|c|}{ Signature: Keni Welcox } \\
\hline$N / A$ & $N / A$ & $N / A$ & $N / A$ & $N / A$ & $N / A$ & \multicolumn{3}{|c|}{ RCT Name: B. Bunce/S. Brown } & \multirow{2}{*}{\multicolumn{3}{|c|}{ Signature: Stama/ $/ 5$}} \\
\hline$N / A$ & $N / A$ & $N / A$ & $N / A$ & $N / A$ & $N / A$ & RCT Name: & Brian Fosk & & & & \\
\hline \multirow[t]{2}{*}{ Survey Point } & \multirow{2}{*}{\multicolumn{4}{|c|}{ Description/Comments }} & \multicolumn{2}{|c|}{$\begin{array}{l}\text { Removable } \\
\mathrm{dpm} / 100 \mathrm{~cm}^{2}\end{array}$} & \multicolumn{2}{|c|}{$\begin{array}{c}\text { Fixed + Removable } \\
\mathrm{dpm} / 100 \mathrm{~cm}^{2}\end{array}$} & \multirow{2}{*}{$\begin{array}{l}\text { Gamma } \\
\mathrm{mrem} / \mathrm{hr}\end{array}$} & \multirow{2}{*}{$\begin{array}{l}\text { Neutron } \\
\text { mrem/hr }\end{array}$} & \multirow{2}{*}{$\begin{array}{l}\text { Total } \\
\mathrm{mrem} / \mathrm{hr}\end{array}$} \\
\hline & & & & & Alpha & Beta & Alpha & Beta & & & \\
\hline 1 & \multicolumn{4}{|c|}{ Floor Concrete } & $N / A$ & $N / A$ & 5 & 1752 & $N / A$ & $N / A$ & $N / A$ \\
\hline 2 & \multicolumn{4}{|c|}{ Floor Concrete } & $N / A$ & $N / A$ & 6 & 1690 & $N / A$ & $N / A$ & $N / A$ \\
\hline 3 & \multicolumn{4}{|c|}{ East Wall Concrete Block } & $N / A$ & $N / A$ & 5 & 1355 & $N / A$ & $N / A$ & $N / A$ \\
\hline 4 & \multicolumn{4}{|c|}{ East Wall Concrete Block } & $N / A$ & $N / A$ & 13 & 1421 & $N / A$ & $N / A$ & $N / A$ \\
\hline 5 & \multicolumn{4}{|c|}{ North Wall Concrete Block } & $N / A$ & $N / A$ & 11 & 1419 & $N / A$ & $N / A$ & $N / A$ \\
\hline 6 & \multicolumn{4}{|c|}{ North Wall Concrete Block } & $N / A$ & $N / A$ & 5 & 1479 & $N / A$ & $N / A$ & $N / A$ \\
\hline 7 & \multicolumn{4}{|c|}{ West Wall Concrete Block } & $N / A$ & $\mathrm{~N} / \mathrm{A}$ & 9 & 1436 & $N / A$ & $\mathrm{~N} / \mathrm{A}$ & $N / A$ \\
\hline 8 & \multicolumn{4}{|c|}{ West Wall Concrete Block } & $N / A$ & $N / A$ & 8 & 1485 & $N / A$ & $N / A$ & $N / A$ \\
\hline 9 & \multicolumn{4}{|c|}{ South Wall Concrete Block } & $N / A$ & $N / A$ & 11 & 1436 & $N / A$ & $\mathrm{~N} / \mathrm{A}$ & $N / A$ \\
\hline 10 & \multicolumn{4}{|c|}{ South Wall Concrete Block } & $N / A$ & $\mathrm{~N} / \mathrm{A}$ & 10 & 1347 & $N / A$ & $N / A$ & $N / A$ \\
\hline 11 & \multicolumn{4}{|l|}{ Ceiling Metal } & $N / A$ & $N / A$ & 5 & 1308 & $\mathrm{~N} / \mathrm{A}$ & $N / A$ & $N / A$ \\
\hline \multicolumn{5}{|c|}{ Reviewed By(Print): } & Signature: & & & & & \multicolumn{2}{|c|}{$\begin{array}{l}\text { Date: } \\
7 / 20 / 10\end{array}$} \\
\hline
\end{tabular}


SURVEY \# 10-ER-A26-186

\begin{tabular}{|c|c|c|c|c|c|c|c|c|}
\hline \multirow{2}{*}{$\begin{array}{l}\text { Survey } \\
\text { Point }\end{array}$} & \multirow[t]{2}{*}{ Description/Comments } & \multicolumn{2}{|c|}{$\begin{array}{l}\text { Removable } \\
\mathrm{dpm} / 100 \mathrm{~cm}^{2}\end{array}$} & \multicolumn{2}{|c|}{\begin{tabular}{|c|}
$\begin{array}{c}\text { Fixed + Removable } \\
\mathrm{dpm} / 100 \mathrm{~cm}^{2}\end{array}$ \\
\end{tabular}} & \multirow{2}{*}{$\begin{array}{l}\text { Gamma } \\
\mathrm{mrem} / \mathrm{hr}\end{array}$} & \multirow{2}{*}{$\begin{array}{l}\text { Neutron } \\
\text { mrem } / \mathrm{hr}\end{array}$} & \multirow{2}{*}{$\begin{array}{l}\text { Total } \\
\mathrm{mrem} / \mathrm{hr}\end{array}$} \\
\hline & & Alpha & Beta & Alpha & Beta & & & \\
\hline 12 & Ceiling Metal & N/A & N/A & 7 & 1325 & N/A & N/A & N/A \\
\hline N/A & $N / A$ & N/A & $\mathrm{N} / \mathrm{A}$ & N/A & $\mathrm{N} / \mathrm{A}$ & $\mathrm{N} / \mathrm{A}$ & N/A & N/A \\
\hline N/A & $N / A$ & N/A & N/A & N/A & $\mathrm{N} / \mathrm{A}$ & $\mathrm{N} / \mathrm{A}$ & N/A & N/A \\
\hline N/A & $N / A$ & N/A & $\mathrm{N} / \mathrm{A}$ & N/A & $\mathrm{N} / \mathrm{A}$ & N/A & $\mathrm{N} / \mathrm{A}$ & N/A \\
\hline N/A & $N / A$ & N/A & $\mathrm{N} / \mathrm{A}$ & $\mathrm{N} / \mathrm{A}$ & $\mathrm{N} / \mathrm{A}$ & N/A & N/A & N/A \\
\hline $\mathrm{N} / \mathrm{A}$ & $N / A$ & N/A & $\mathrm{N} / \mathrm{A}$ & N/A & $\mathrm{N} / \mathrm{A}$ & $\mathrm{N} / \mathrm{A}$ & N/A & $\mathrm{N} / \mathrm{A}$ \\
\hline N/A & $N / A$ & $\mathrm{~N} / \mathrm{A}$ & N/A & N/A & N/A & N/A & $\mathrm{N} / \mathrm{A}$ & N/A \\
\hline N/A & $N / A$ & N/A & N/A & N/A & $\mathrm{N} / \mathrm{A}$ & $\mathrm{N} / \mathrm{A}$ & $\mathrm{N} / \mathrm{A}$ & $\mathrm{N} / \mathrm{A}$ \\
\hline N/A & $N / A$ & N/A & N/A & N/A & $\mathrm{N} / \mathrm{A}$ & $\mathrm{N} / \mathrm{A}$ & $\mathrm{N} / \mathrm{A}$ & N/A \\
\hline $\mathrm{N} / \mathrm{A}$ & $N / A$ & N/A & N/A & N/A & $\mathrm{N} / \mathrm{A}$ & N/A & N/A & N/A \\
\hline N/A & $N / A$ & N/A & $\mathrm{N} / \mathrm{A}$ & N/A & $\mathrm{N} / \mathrm{A}$ & N/A & N/A & N/A \\
\hline N/A & $N / A$ & N/A & $\mathrm{N} / \mathrm{A}$ & $\mathrm{N} / \mathrm{A}$ & $\mathrm{N} / \mathrm{A}$ & N/A & N/A & N/A \\
\hline N/A & $N / A$ & N/A & $\mathrm{N} / \mathrm{A}$ & $\mathrm{N} / \mathrm{A}$ & $\mathrm{N} / \mathrm{A}$ & $\mathrm{N} / \mathrm{A}$ & $\mathrm{N} / \mathrm{A}$ & N/A \\
\hline N/A & $N / A$ & N/A & N/A & N/A & $\mathrm{N} / \mathrm{A}$ & N/A & N/A & $\mathrm{N} / \mathrm{A}$ \\
\hline N/A & $N / A$ & N/A & N/A & $\mathrm{N} / \mathrm{A}$ & $\mathrm{N} / \mathrm{A}$ & N/A & N/A & N/A \\
\hline$N / A$ & $N / A$ & N/A & $\mathrm{N} / \mathrm{A}$ & N/A & N/A & N/A & $\mathrm{N} / \mathrm{A}$ & N/A \\
\hline$N / A$ & $N / A$ & N/A & $\mathrm{N} / \mathrm{A}$ & N/A & N/A & N/A & N/A & N/A \\
\hline $\mathrm{N} / \mathrm{A}$ & $N / A$ & N/A & N/A & N/A & N/A & N/A & N/A & N/A \\
\hline $\mathrm{N} / \mathrm{A}$ & $N / A$ & N/A & $\mathrm{N} / \mathrm{A}$ & N/A & N/A & N/A & N/A & N/A \\
\hline N/A & N/A & N/A & $\mathrm{N} / \mathrm{A}$ & N/A & N/A & N/A & N/A & N/A \\
\hline$N / A$ & N/A & N/A & $\mathrm{N} / \mathrm{A}$ & N/A & $\mathrm{N} / \mathrm{A}$ & N/A & N/A & N/A \\
\hline N/A & N/A & N/A & $\mathrm{N} / \mathrm{A}$ & N/A & N/A & N/A & N/A & N/A \\
\hline N/A & N/A & N/A & $\mathrm{N} / \mathrm{A}$ & N/A & N/A & N/A & $N / A$ & $N / A$ \\
\hline
\end{tabular}


Survey \# 10-ER-A26-186

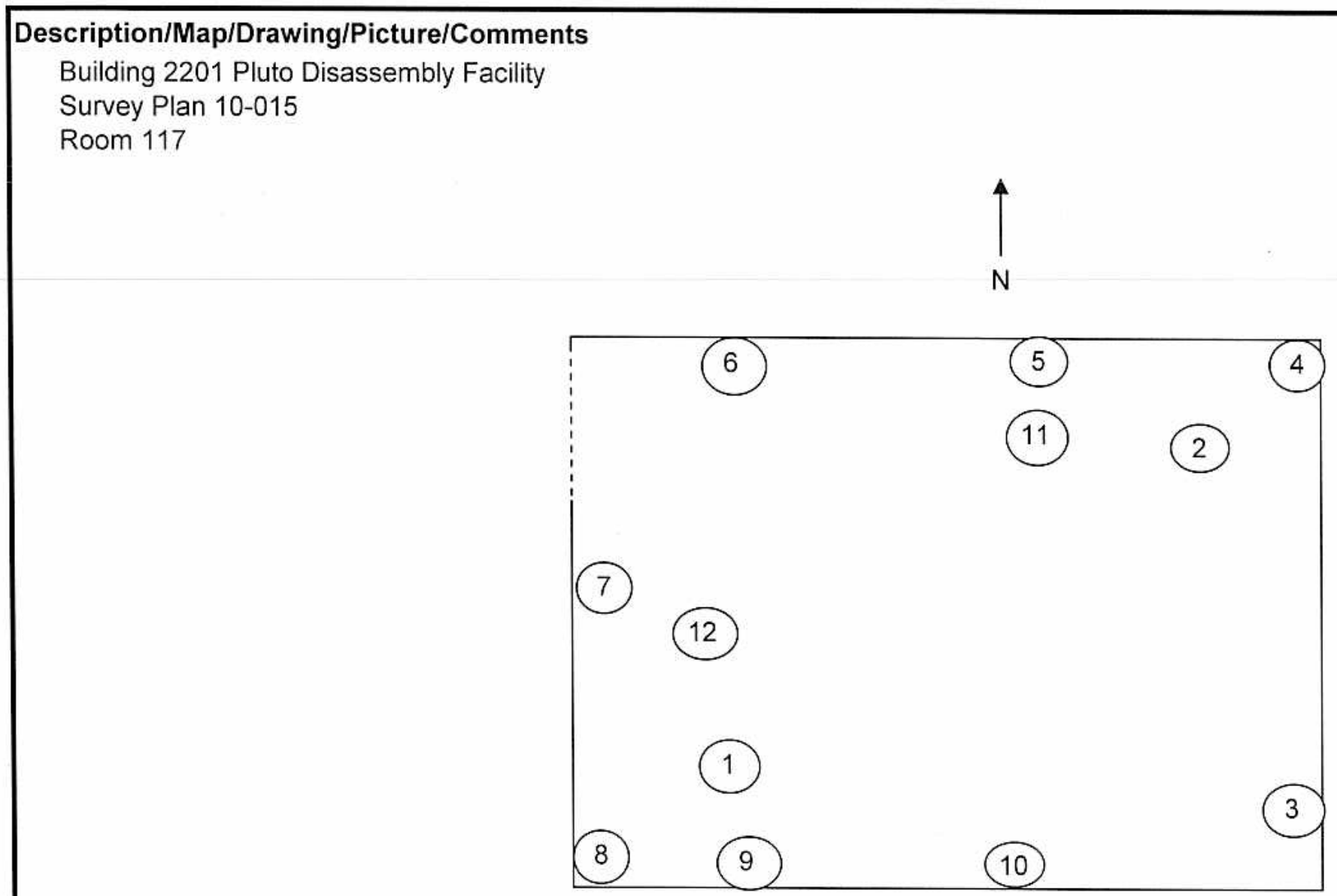


SURVEY \#

10-ER-A26-239

\begin{tabular}{|c|c|c|c|c|c|c|c|c|c|c|c|}
\hline \multicolumn{3}{|c|}{$\begin{array}{l}\text { Location: } \\
\text { Area } 26 \text { CAU117 Pluto }\end{array}$} & \multicolumn{2}{|c|}{$\begin{array}{l}\text { Purpose: } \\
\text { Final Status Survey, Room 201, } \\
\text { Floor, Ceiling and Walls }\end{array}$} & \multicolumn{5}{|c|}{$\begin{array}{l}\text { Comments: } \\
\text { All one minute static counts are in cpm. Bkgd not subtracted } \\
\text { for Final Status Survey, Survey Plan \#10-015 }\end{array}$} & \multicolumn{2}{|c|}{$\begin{array}{l}\text { Date/Time: } \\
08 / 4 / 20100900\end{array}$} \\
\hline Instrument: & Serial \#: & Cal Due: & $\begin{array}{c}\text { Eff in \%: } \\
\text { Alpha / Beta } \\
\end{array}$ & $\begin{array}{l}\text { BKG in dpm: } \\
\text { Alpha / Beta }\end{array}$ & \begin{tabular}{|l|} 
MDA in dpm: \\
Alpha / Beta \\
\end{tabular} & RWP\# & \multicolumn{3}{|l|}{$\mathrm{N} / \mathrm{A}$} & & \\
\hline Electra & 1120 & $4 / 6 / 2011$ & $14.1 / 20.8$ & $\mathrm{~N} / \mathrm{A}$ & $N / A$ & \multicolumn{6}{|c|}{ Work Package \# $\quad$ ENV-10-RCT ENV SITE SURVEYS-0013 } \\
\hline $\mathrm{N} / \mathrm{A}$ & $\mathrm{N} / \mathrm{A}$ & $N / A$ & $N / A$ & $N / A$ & $\mathrm{~N} / \mathrm{A}$ & \multicolumn{3}{|c|}{ RCT Name: D.Cotroneo Jr. } & Signature: & \multicolumn{2}{|c|}{$\rightarrow 2$} \\
\hline$N / A$ & $N / A$ & $N / A$ & N/A & $\mathrm{N} / \mathrm{A}$ & $N / A$ & \multicolumn{3}{|c|}{ RCT Name: Kevin Wilcox } & \multicolumn{3}{|c|}{ Signature: Keis $2 . \mathrm{Cos}$} \\
\hline$N / A$ & $N / A$ & $N / A$ & $\mathrm{~N} / \mathrm{A}$ & $\mathrm{N} / \mathrm{A}$ & $\mathrm{N} / \mathrm{A}$ & \multicolumn{3}{|c|}{ RCT Name: Brian Bunce } & \multicolumn{3}{|c|}{ Signature: } \\
\hline $\mathrm{N} / \mathrm{A}$ & $N / A$ & $N / A$ & $N / A$ & $\mathrm{~N} / \mathrm{A}$ & $N / A$ & \multicolumn{3}{|c|}{ RCT Name: Brian Foskett } & \multirow{3}{*}{\multicolumn{3}{|c|}{ 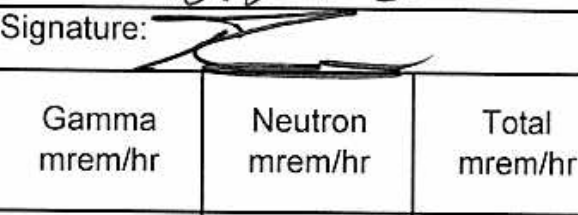 }} \\
\hline \multirow[t]{2}{*}{ Survey Point } & \multirow{2}{*}{\multicolumn{4}{|c|}{ Description/Comments }} & \multicolumn{2}{|c|}{$\begin{array}{l}\text { Removable } \\
\mathrm{dpm} / 100 \mathrm{~cm}^{2}\end{array}$} & \multicolumn{2}{|c|}{\begin{tabular}{|c|}
$\begin{array}{c}\text { Fixed + Removable } \\
\mathrm{dpm} / 100 \mathrm{~cm}^{2}\end{array}$ \\
\end{tabular}} & & & \\
\hline & & & & & Alpha & Beta & Alpha & Beta & & & \\
\hline 1 & \multicolumn{4}{|c|}{ Floor Concrete } & $N / A$ & $N / A$ & 9 & 1183 & $N / A$ & $N / A$ & $N / A$ \\
\hline 2 & \multicolumn{4}{|c|}{ Floor Concrete } & $N / A$ & $N / A$ & 10 & 1268 & $N / A$ & $N / A$ & $N / A$ \\
\hline 3 & \multicolumn{4}{|c|}{ East Wall Concrete } & $N / A$ & $N / A$ & 20 & 925 & $N / A$ & $N / A$ & $N / A$ \\
\hline 4 & \multicolumn{4}{|c|}{ East Wall Concrete } & $\mathrm{N} / \mathrm{A}$ & $\mathrm{N} / \mathrm{A}$ & 6 & 886 & $N / A$ & $N / A$ & $N / A$ \\
\hline 5 & \multicolumn{4}{|c|}{ North Wall Concrete } & $\mathrm{N} / \mathrm{A}$ & $N / A$ & 8 & 772 & $\mathrm{~N} / \mathrm{A}$ & $N / A$ & $N / A$ \\
\hline 6 & \multicolumn{4}{|c|}{ North Wall Concrete } & $N / A$ & $N / A$ & 20 & 751 & $N / A$ & $N / A$ & $N / A$ \\
\hline 7 & \multicolumn{4}{|c|}{ West Wall Concrete } & $\mathrm{N} / \mathrm{A}$ & $\mathrm{N} / \mathrm{A}$ & 11 & 964 & $N / A$ & $N / A$ & $N / A$ \\
\hline 8 & \multicolumn{4}{|c|}{ West Wall Concrete } & $N / A$ & $N / A$ & 14 & 849 & $N / A$ & $N / A$ & $N / A$ \\
\hline 9 & \multicolumn{4}{|c|}{ South Wall Concrete } & $N / A$ & $\mathrm{~N} / \mathrm{A}$ & 17 & 1083 & $\mathrm{~N} / \mathrm{A}$ & $N / A$ & $N / A$ \\
\hline 10 & \multicolumn{4}{|c|}{ South Wall Concrete } & $\mathrm{N} / \mathrm{A}$ & $N / A$ & 22 & 1087 & $N / A$ & $N / A$ & $\mathrm{~N} / \mathrm{A}$ \\
\hline 11 & \multicolumn{4}{|c|}{ Ceiling Concrete } & $N / A$ & $N / A$ & 18 & 1233 & $N / A$ & $N / A$ & $N / A$ \\
\hline \multicolumn{5}{|c|}{ Reviewed By (Print): } & Signature: & & & & & \multicolumn{2}{|c|}{ Date: $8 / 4 / 10$} \\
\hline
\end{tabular}


SURVEY \# 10-ER-A26-239

\begin{tabular}{|c|c|c|c|c|c|c|c|c|}
\hline \multirow{2}{*}{$\begin{array}{l}\text { Survey } \\
\text { Point }\end{array}$} & \multirow[t]{2}{*}{ Description/Comments } & \multicolumn{2}{|c|}{$\begin{array}{l}\text { Removable } \\
\mathrm{dpm} / 100 \mathrm{~cm}^{2}\end{array}$} & \multicolumn{2}{|c|}{$\begin{array}{c}\text { Fixed + Removable } \\
\text { dpm } / 100 \mathrm{~cm}^{2}\end{array}$} & \multirow{2}{*}{$\begin{array}{l}\text { Gamma } \\
\mathrm{mrem} / \mathrm{hr}\end{array}$} & \multirow{2}{*}{$\begin{array}{l}\text { Neutron } \\
\text { mrem/hr }\end{array}$} & \multirow{2}{*}{$\begin{array}{c}\text { Total } \\
\text { mrem } / \mathrm{hr}\end{array}$} \\
\hline & & Alpha & Beta & Alpha & Beta & & & \\
\hline 12 & Ceiling Concrete & N/A & N/A & 18 & 1127 & N/A & N/A & N/A \\
\hline N/A & $N / A$ & N/A & N/A & N/A & $\mathrm{N} / \mathrm{A}$ & N/A & N/A & N/A \\
\hline N/A & $N / A$ & N/A & N/A & N/A & $\mathrm{N} / \mathrm{A}$ & N/A & N/A & N/A \\
\hline N/A & $N / A$ & N/A & N/A & N/A & $N / A$ & N/A & N/A & N/A \\
\hline N/A & $N / A$ & N/A & N/A & N/A & N/A & N/A & N/A & N/A \\
\hline N/A & $N / A$ & N/A & N/A & N/A & $\mathrm{N} / \mathrm{A}$ & N/A & N/A & N/A \\
\hline N/A & $N / A$ & N/A & N/A & N/A & $\mathrm{N} / \mathrm{A}$ & N/A & N/A & N/A \\
\hline N/A & $N / A$ & N/A & N/A & N/A & $\mathrm{N} / \mathrm{A}$ & N/A & N/A & N/A \\
\hline N/A & $N / A$ & N/A & N/A & N/A & $\mathrm{N} / \mathrm{A}$ & N/A & N/A & N/A \\
\hline N/A & $N / A$ & N/A & N/A & N/A & N/A & N/A & N/A & N/A \\
\hline N/A & $N / A$ & N/A & N/A & N/A & N/A & N/A & N/A & N/A \\
\hline N/A & $\mathrm{N} / \mathrm{A}$ & N/A & N/A & N/A & N/A & N/A & N/A & N/A \\
\hline N/A & $N / A$ & N/A & N/A & N/A & N/A & N/A & N/A & N/A \\
\hline N/A & $N / A$ & N/A & $\mathrm{N} / \mathrm{A}$ & N/A & $\mathrm{N} / \mathrm{A}$ & N/A & N/A & N/A \\
\hline N/A & $N / A$ & N/A & N/A & N/A & N/A & N/A & N/A & N/A \\
\hline N/A & $N / A$ & N/A & N/A & N/A & $\mathrm{N} / \mathrm{A}$ & N/A & N/A & N/A \\
\hline N/A & $N / A$ & N/A & N/A & N/A & N/A & N/A & N/A & N/A \\
\hline N/A & $N / A$ & N/A & N/A & N/A & N/A & N/A & N/A & N/A \\
\hline N/A & $N / A$ & N/A & N/A & N/A & N/A & N/A & N/A & N/A \\
\hline N/A & N/A & N/A & N/A & N/A & N/A & N/A & N/A & N/A \\
\hline N/A & N/A & N/A & N/A & N/A & N/A & N/A & N/A & N/A \\
\hline N/A & N/A & N/A & N/A & N/A & N/A & N/A & N/A & N/A \\
\hline N/A & N/A & N/A & N/A & N/A & N/A & N/A & N/A & N/A \\
\hline
\end{tabular}


Survey \# 10-ER-A26-239

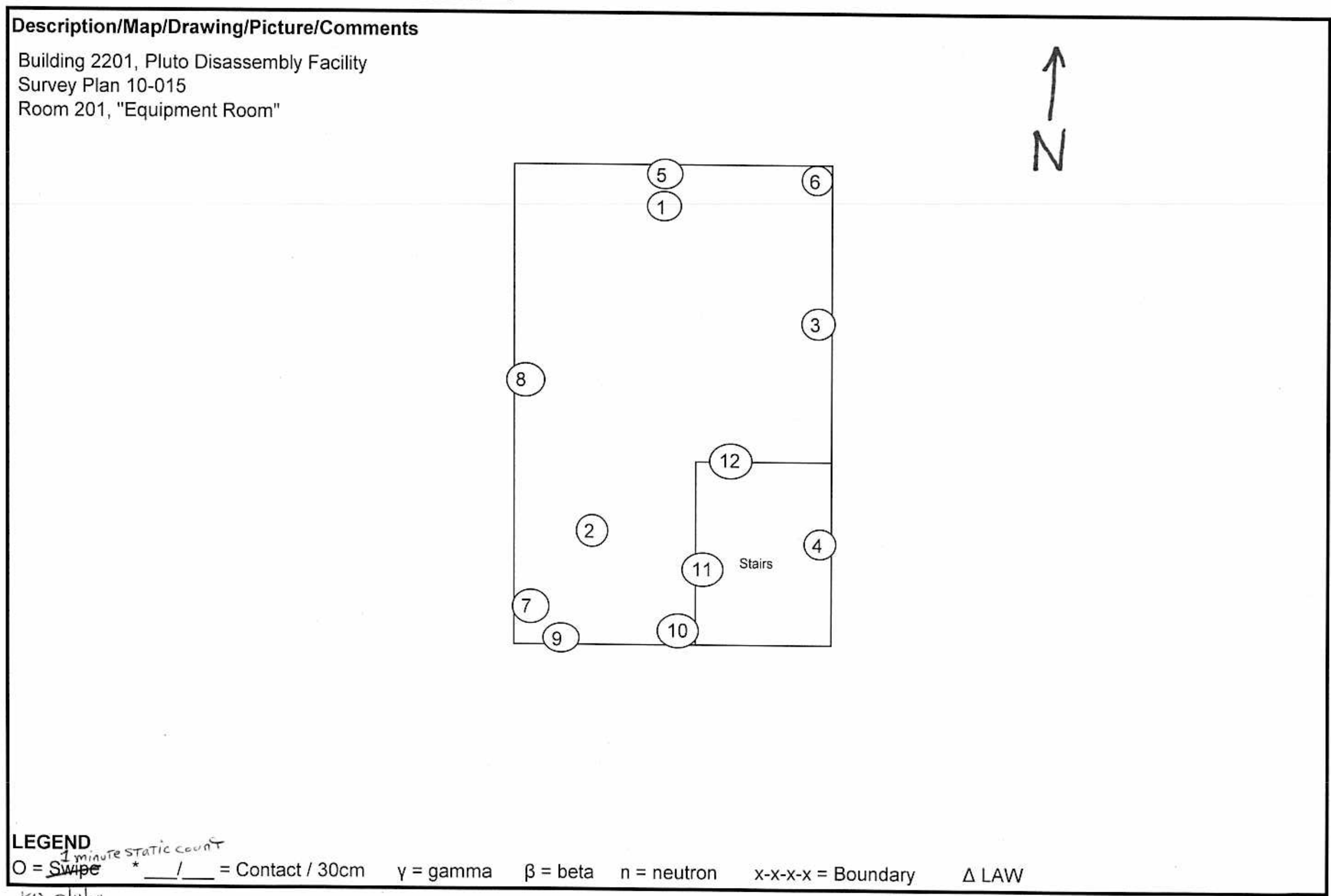


SURVEY \#

10-ER-A26-240

\begin{tabular}{|c|c|c|c|c|c|c|c|c|c|c|c|}
\hline \multicolumn{3}{|c|}{$\begin{array}{l}\text { Location: } \\
\text { Area } 26 \text { CAU117 Pluto }\end{array}$} & \multicolumn{2}{|c|}{$\begin{array}{l}\text { Purpose: } \\
\text { Final Status Survey, Room 301, } \\
\text { Floor, Ceiling and Walls }\end{array}$} & \multicolumn{5}{|c|}{$\begin{array}{l}\text { Comments: } \\
\text { All one minute static counts are in cpm. Bkgd not subtracted } \\
\text { for Final Status Survey, Survey Plan \#10-015 }\end{array}$} & \multicolumn{2}{|c|}{$\begin{array}{l}\text { Date/Time: } \\
\text { 08/4/2010 } 0930\end{array}$} \\
\hline Instrument: & Serial \#: & Cal Due: & $\begin{array}{c}\text { Eff in \%: } \\
\text { Alpha / Beta }\end{array}$ & $\begin{array}{l}\text { BKG in dpm: } \\
\text { Alpha / Beta }\end{array}$ & \begin{tabular}{|l|} 
MDA in dpm: \\
Alpha / Beta \\
\end{tabular} & RWP\# & \multicolumn{5}{|l|}{$\mathrm{N} / \mathrm{A}$} \\
\hline Electra & 1504 & $4 / 6 / 2011$ & $12.9 / 19.8$ & $N / A$ & $N / A$ & \multicolumn{6}{|c|}{ Work Package \# } \\
\hline$N / A$ & $N / A$ & $\mathrm{~N} / \mathrm{A}$ & $N / A$ & $\mathrm{~N} / \mathrm{A}$ & $\mathrm{N} / \mathrm{A}$ & \multicolumn{3}{|c|}{ RCT Name: D.Cotroneo Jr. } & \multicolumn{3}{|c|}{ Signature: $8-\pi$} \\
\hline $\mathrm{N} / \mathrm{A}$ & $N / A$ & $N / A$ & $N / A$ & $\mathrm{~N} / \mathrm{A}$ & $N / A$ & \multicolumn{3}{|c|}{ RCT Name: Kevin Wilcox } & \multicolumn{3}{|c|}{ Signature: Kein Wose } \\
\hline$N / A$ & $N / A$ & $N / A$ & $\mathrm{~N} / \mathrm{A}$ & $N / A$ & $N / A$ & \multicolumn{3}{|c|}{ RCT Name: Brian Bunce } & \multicolumn{3}{|c|}{ Signature: } \\
\hline$N / A$ & $N / A$ & $N / A$ & $\mathrm{~N} / \mathrm{A}$ & $\mathrm{N} / \mathrm{A}$ & $\mathrm{N} / \mathrm{A}$ & \multicolumn{3}{|c|}{ RCT Name: Brian Foskett } & \multicolumn{3}{|c|}{ Signature: 2} \\
\hline \multirow[t]{2}{*}{ Survey Point } & \multirow{2}{*}{\multicolumn{4}{|c|}{ Description/Comments }} & \multicolumn{2}{|c|}{$\begin{array}{l}\text { Removable } \\
\mathrm{dpm} / 100 \mathrm{~cm}^{2}\end{array}$} & \multicolumn{2}{|c|}{\begin{tabular}{|c|}
$\begin{array}{c}\text { Fixed + Removable } \\
\mathrm{dpm} / 100 \mathrm{~cm}^{2}\end{array}$ \\
\end{tabular}} & \multirow{2}{*}{$\begin{array}{l}\text { Gamma } \\
\mathrm{mrem} / \mathrm{hr}\end{array}$} & \multirow{2}{*}{$\begin{array}{l}\text { Neutron } \\
\mathrm{mrem} / \mathrm{hr}\end{array}$} & \multirow{2}{*}{$\begin{array}{l}\text { Total } \\
\mathrm{mrem} / \mathrm{hr}\end{array}$} \\
\hline & & & & & Alpha & Beta & Alpha & Beta & & & \\
\hline 1 & \multicolumn{4}{|c|}{ Floor Concrete } & $\mathrm{N} / \mathrm{A}$ & $N / A$ & 12 & 1329 & $N / A$ & $N / A$ & $N / A$ \\
\hline 2 & \multicolumn{4}{|c|}{ Floor Concrete } & $N / A$ & $N / A$ & 7 & 1242 & $N / A$ & $N / A$ & $N / A$ \\
\hline 3 & \multicolumn{4}{|c|}{ East Wall Concrete } & $N / A$ & $N / A$ & 10 & 863 & $N / A$ & $N / A$ & $N / A$ \\
\hline 4 & \multicolumn{4}{|c|}{ East Wall Concrete } & $N / A$ & $N / A$ & 10 & 891 & $N / A$ & $N / A$ & $\mathrm{~N} / \mathrm{A}$ \\
\hline 5 & \multicolumn{4}{|c|}{ North Wall Concrete } & $N / A$ & $N / A$ & 12 & 812 & $N / A$ & $N / A$ & $N / A$ \\
\hline 6 & \multicolumn{4}{|c|}{ North Wall Concrete } & $N / A$ & $N / A$ & 7 & 933 & $N / A$ & $N / A$ & $N / A$ \\
\hline 7 & \multicolumn{4}{|c|}{ West Wall Concrete } & $N / A$ & $N / A$ & 15 & 836 & $N / A$ & $N / A$ & $N / A$ \\
\hline 8 & \multicolumn{4}{|c|}{ West Wall Concrete } & $N / A$ & $N / A$ & 13 & 934 & $N / A$ & $N / A$ & $N / A$ \\
\hline 9 & \multicolumn{4}{|c|}{ South Wall Concrete } & $N / A$ & $N / A$ & 5 & 1143 & $N / A$ & $N / A$ & $N / A$ \\
\hline 10 & \multicolumn{4}{|c|}{ South Wall Concrete } & $N / A$ & $N / A$ & 16 & 1017 & $N / A$ & $N / A$ & $\mathrm{~N} / \mathrm{A}$ \\
\hline 11 & \multicolumn{4}{|c|}{ Ceiling Concrete } & $\mathrm{N} / \mathrm{A}$ & $N / A$ & 13 & 1265 & $N / A$ & $N / A$ & $N / A$ \\
\hline \multicolumn{5}{|c|}{ Reviewed By (Print): } & Signature: & & & & & Date: 8 & \\
\hline
\end{tabular}


SURVEY \# 10-ER-A26-240

\begin{tabular}{|c|c|c|c|c|c|c|c|c|}
\hline \multirow{2}{*}{$\begin{array}{l}\text { Survey } \\
\text { Point }\end{array}$} & \multirow[t]{2}{*}{ Description/Comments } & \multicolumn{2}{|c|}{$\begin{array}{l}\text { Removable } \\
\mathrm{dpm} / 100 \mathrm{~cm}^{2}\end{array}$} & \multicolumn{2}{|c|}{$\begin{array}{c}\text { Fixed + Removable } \\
\text { dpm } / 100 \mathrm{~cm}^{2}\end{array}$} & \multirow{2}{*}{$\begin{array}{l}\text { Gamma } \\
\mathrm{mrem} / \mathrm{hr}\end{array}$} & \multirow{2}{*}{$\begin{array}{l}\text { Neutron } \\
\text { mrem } / \mathrm{hr}\end{array}$} & \multirow{2}{*}{$\begin{array}{c}\text { Total } \\
\text { mrem/hr }\end{array}$} \\
\hline & & Alpha & Beta & Alpha & Beta & & & \\
\hline 12 & Ceiling Concrete & N/A & N/A & 4 & 1282 & N/A & N/A & N/A \\
\hline N/A & $N / A$ & N/A & N/A & N/A & $\mathrm{N} / \mathrm{A}$ & N/A & N/A & N/A \\
\hline N/A & $N / A$ & N/A & $\mathrm{N} / \mathrm{A}$ & N/A & N/A & N/A & N/A & N/A \\
\hline N/A & $N / A$ & N/A & $N / A$ & N/A & $N / A$ & N/A & N/A & N/A \\
\hline N/A & $N / A$ & N/A & $\mathrm{N} / \mathrm{A}$ & N/A & N/A & N/A & N/A & N/A \\
\hline N/A & $\mathrm{N} / \mathrm{A}$ & N/A & $N / A$ & N/A & N/A & N/A & N/A & N/A \\
\hline N/A & $N / A$ & N/A & $\mathrm{N} / \mathrm{A}$ & N/A & N/A & N/A & N/A & N/A \\
\hline N/A & $N / A$ & N/A & $\mathrm{N} / \mathrm{A}$ & N/A & N/A & N/A & $N / A$ & N/A \\
\hline N/A & $N / A$ & N/A & $N / A$ & N/A & N/A & N/A & N/A & N/A \\
\hline N/A & $N / A$ & N/A & $\mathrm{N} / \mathrm{A}$ & N/A & N/A & N/A & N/A & N/A \\
\hline N/A & $N / A$ & N/A & $\mathrm{N} / \mathrm{A}$ & N/A & N/A & N/A & N/A & N/A \\
\hline N/A & $N / A$ & N/A & N/A & N/A & N/A & N/A & N/A & N/A \\
\hline N/A & $N / A$ & N/A & N/A & N/A & N/A & N/A & N/A & N/A \\
\hline N/A & $N / A$ & N/A & N/A & N/A & N/A & N/A & N/A & N/A \\
\hline N/A & $N / A$ & N/A & N/A & N/A & $\mathrm{N} / \mathrm{A}$ & N/A & N/A & N/A \\
\hline N/A & $N / A$ & N/A & $\mathrm{N} / \mathrm{A}$ & N/A & $\mathrm{N} / \mathrm{A}$ & N/A & N/A & N/A \\
\hline N/A & $N / A$ & N/A & N/A & N/A & $N / A$ & N/A & N/A & N/A \\
\hline $\mathrm{N} / \mathrm{A}$ & $N / A$ & N/A & $\mathrm{N} / \mathrm{A}$ & N/A & $\mathrm{N} / \mathrm{A}$ & N/A & N/A & N/A \\
\hline N/A & $N / A$ & N/A & N/A & N/A & $\mathrm{N} / \mathrm{A}$ & N/A & N/A & N/A \\
\hline N/A & $N / A$ & N/A & N/A & N/A & N/A & N/A & N/A & N/A \\
\hline N/A & $N / A$ & N/A & N/A & N/A & $\mathrm{N} / \mathrm{A}$ & N/A & N/A & N/A \\
\hline N/A & $N / A$ & N/A & $\mathrm{N} / \mathrm{A}$ & N/A & $\mathrm{N} / \mathrm{A}$ & N/A & N/A & N/A \\
\hline N/A & $N / A$ & N/A & N/A & N/A & $N / A$ & N/A & N/A & N/A \\
\hline
\end{tabular}


Survey \# 10-ER-A26-240

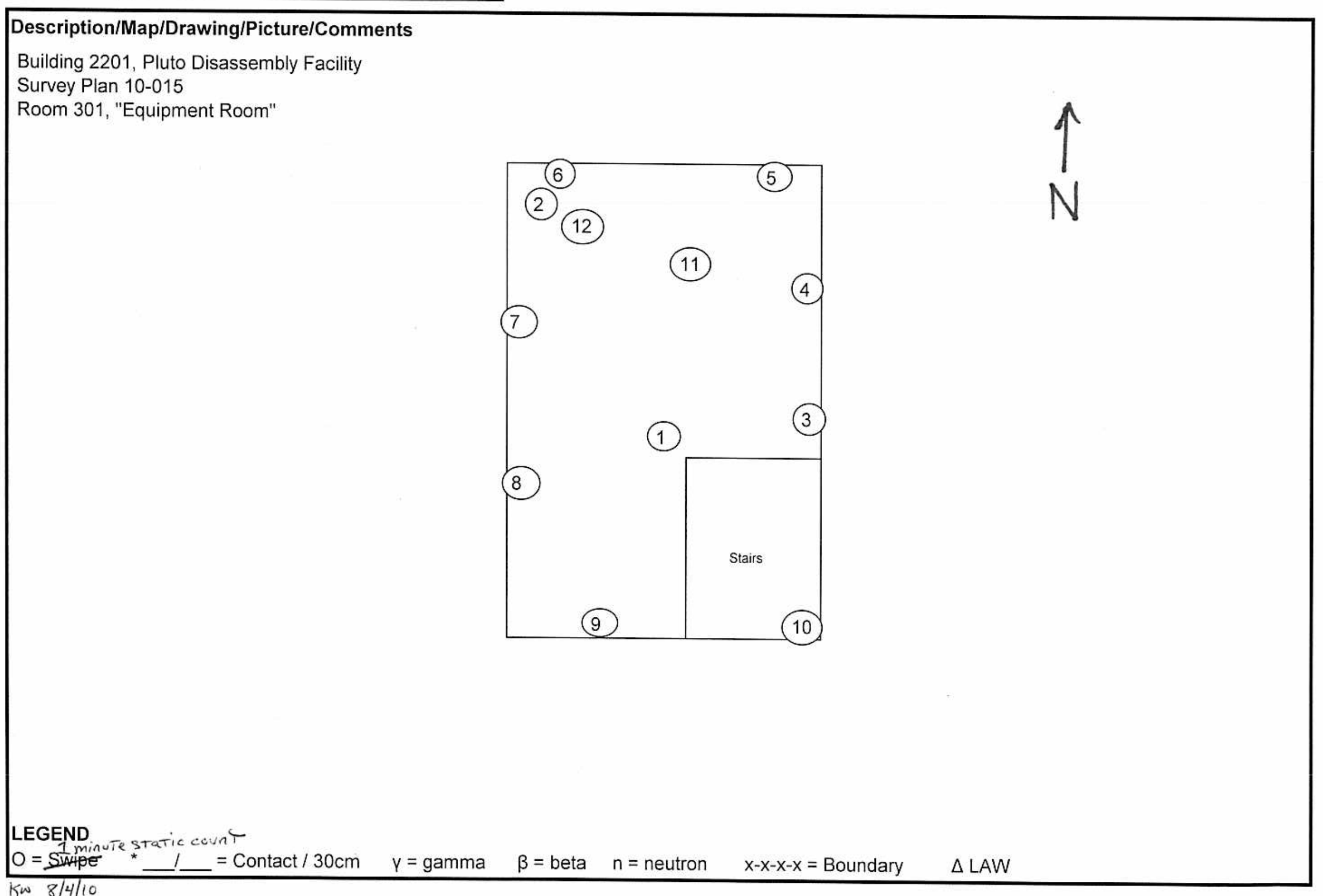


SURVEY\# 10-ER-A26-205

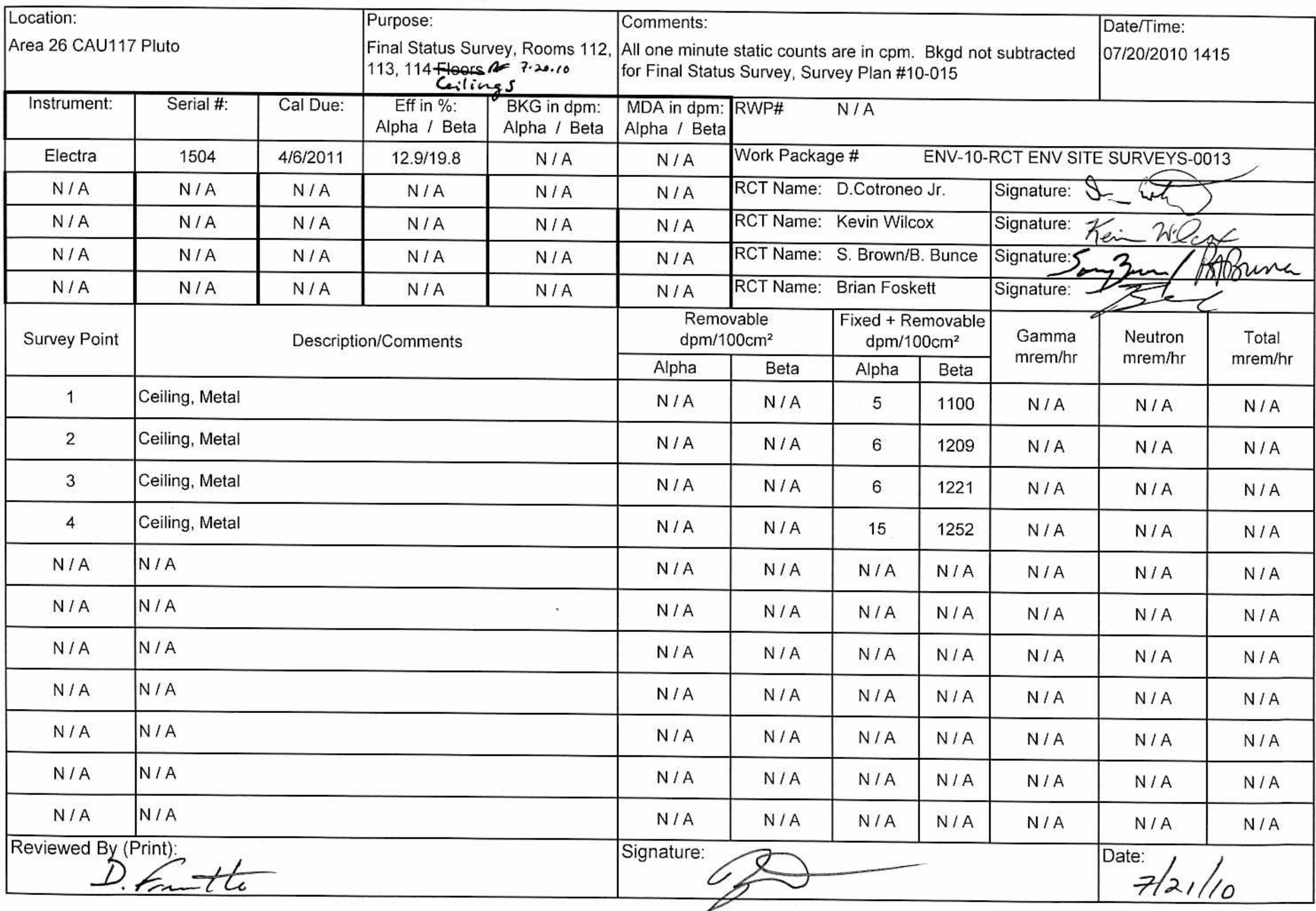




\section{Survey \# 10-ER-A26-205}

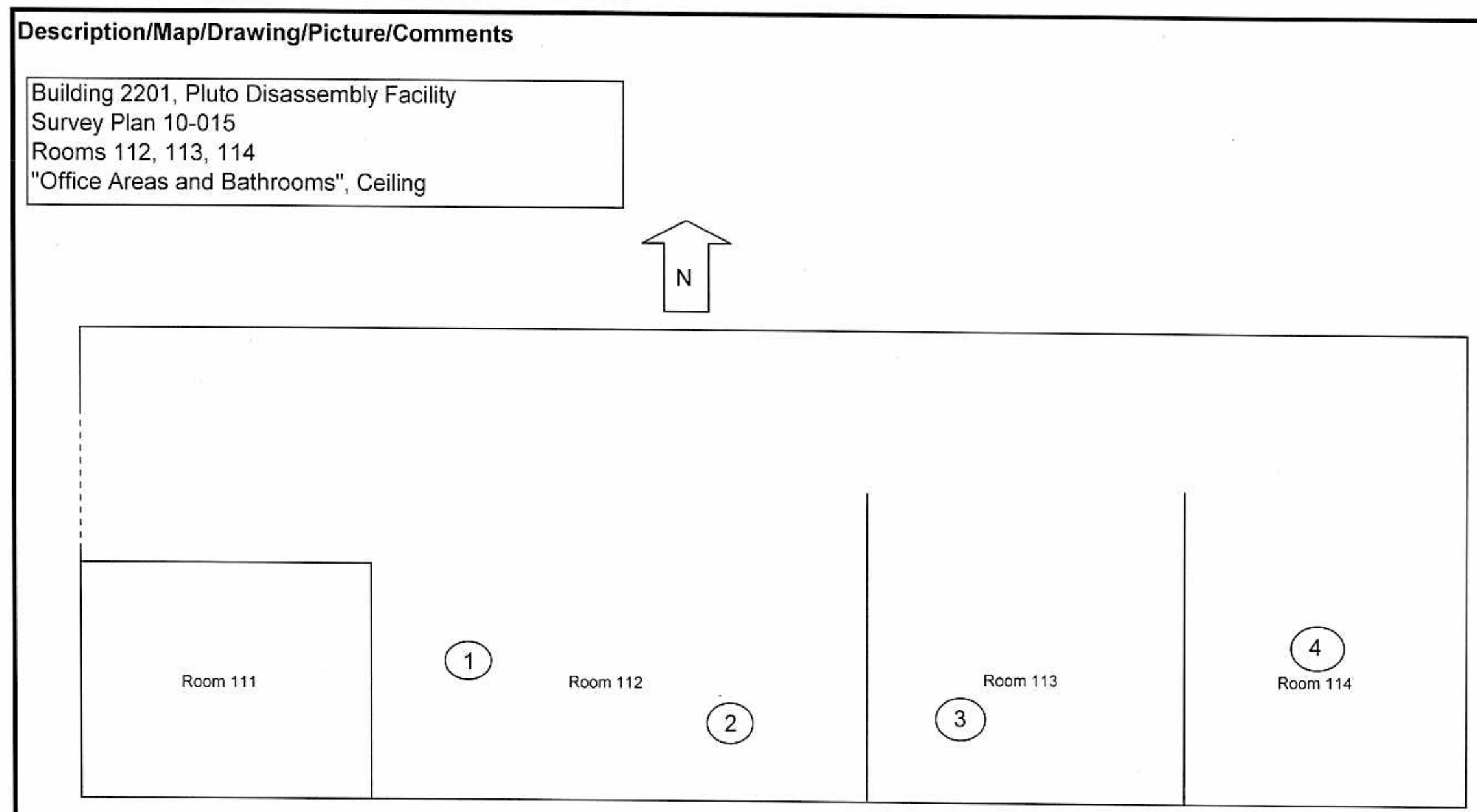

LEGEND

O = Swipe 


\begin{tabular}{|c|c|c|c|c|c|c|c|c|c|c|c|}
\hline \multicolumn{3}{|c|}{$\begin{array}{l}\text { Location: } \\
\text { Area } 26 \text { CAU117 Pluto }\end{array}$} & \multicolumn{2}{|c|}{$\begin{array}{l}\text { Purpose: } \\
\text { Final Status Survey, Rooms 112, } \\
113,114 \text { Floors }\end{array}$} & \multicolumn{5}{|c|}{$\begin{array}{l}\text { Comments: } \\
\text { All one minute static counts are in cpm. Bkgd not subtracted } \\
\text { for Final Status Survey, Survey Plan \#10-015 }\end{array}$} & \multicolumn{2}{|c|}{$\begin{array}{l}\text { Date/Time: } \\
\text { 07/20/2010 } 1400\end{array}$} \\
\hline Instrument: & Serial \#: & Cal Due: & $\begin{array}{c}\text { Eff in \%: } \\
\text { Alpha / Beta }\end{array}$ & $\begin{array}{l}\text { BKG in dpm: } \\
\text { Alpha / Beta }\end{array}$ & $\begin{array}{l}\text { MDA in dpm: } \\
\text { Alpha / Beta }\end{array}$ & RWP\# & \multicolumn{5}{|l|}{$N / A$} \\
\hline Electra & 1504 & $4 / 6 / 2011$ & $12.9 / 19.8$ & $N / A$ & $N / A$ & \multicolumn{6}{|c|}{ 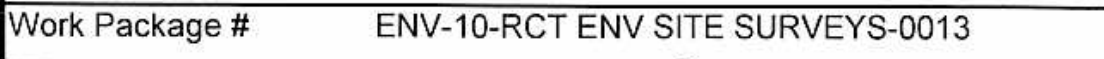 } \\
\hline$N / A$ & $N / A$ & $\mathrm{~N} / \mathrm{A}$ & $N / A$ & $N / A$ & $N / A$ & \multicolumn{3}{|c|}{ RCT Name: D.Cotroneo Jr. } & \multicolumn{3}{|c|}{ Signature: $y \ldots$} \\
\hline$N / A$ & $N / A$ & $N / A$ & $N / A$ & $N / A$ & $N / A$ & \multicolumn{3}{|c|}{ RCT Name: Kevin Wilcox } & \multicolumn{3}{|c|}{ Signature: Kein W.ON } \\
\hline$N / A$ & $\mathrm{~N} / \mathrm{A}$ & $N / A$ & $N / A$ & $N / A$ & $N / A$ & \multicolumn{3}{|c|}{ RCT Name: S. Brown/B. Bunce } & \multirow{2}{*}{\multicolumn{3}{|c|}{ Signature:Sar 3 Signature: }} \\
\hline$N / A$ & $N / A$ & $N / A$ & $N / A$ & $N / A$ & $N / A$ & RCT Name: & Brian Fos & & & & \\
\hline \multirow[t]{2}{*}{ Survey Point } & \multirow{2}{*}{\multicolumn{4}{|c|}{ Description/Comments }} & \multicolumn{2}{|c|}{$\begin{array}{l}\text { Removable } \\
\mathrm{dpm} / 100 \mathrm{~cm}^{2}\end{array}$} & \multicolumn{2}{|c|}{$\begin{array}{c}\text { Fixed + Removable } \\
\mathrm{dpm} / 100 \mathrm{~cm}^{2}\end{array}$} & \multirow{2}{*}{$\begin{array}{l}\text { Gamma } \\
\mathrm{mrem} / \mathrm{hr}\end{array}$} & \multirow{2}{*}{$\begin{array}{l}\text { Neutron } \\
\mathrm{mrem} / \mathrm{hr}\end{array}$} & \multirow{2}{*}{$\begin{array}{l}\text { Total } \\
\mathrm{mrem} / \mathrm{hr}\end{array}$} \\
\hline & & & & & Alpha & Beta & Alpha & Beta & & & \\
\hline 1 & \multicolumn{4}{|c|}{ Floor, Concrete } & $N / A$ & $N / A$ & 10 & 1412 & $N / A$ & $N / A$ & $N / A$ \\
\hline 2 & \multicolumn{4}{|c|}{ Floor, Concrete } & $N / A$ & $N / A$ & 11 & 1502 & $N / A$ & $\mathrm{~N} / \mathrm{A}$ & $N / A$ \\
\hline 3 & \multicolumn{4}{|c|}{ Floor, Concrete } & $N / A$ & $N / A$ & 8 & 1396 & $N / A$ & $N / A$ & $N / A$ \\
\hline 4 & \multicolumn{4}{|c|}{ Floor, Concrete } & $N / A$ & $N / A$ & 13 & 1712 & $N / A$ & $N / A$ & $N / A$ \\
\hline 5 & \multicolumn{4}{|c|}{ Floor, Concrete } & $N / A$ & $N / A$ & 10 & 1461 & $N / A$ & $N / A$ & $N / A$ \\
\hline 6 & \multicolumn{4}{|c|}{ Floor, Concrete } & $N / A$ & $N / A$ & 9 & 1653 & $N / A$ & $N / A$ & $N / A$ \\
\hline 7 & \multicolumn{4}{|c|}{ Floor, Concrete } & $N / A$ & $N / A$ & 12 & 1592 & $N / A$ & $N / A$ & $N / A$ \\
\hline 8 & \multicolumn{4}{|c|}{ Floor, Concrete } & $N / A$ & $N / A$ & 12 & 1540 & $N / A$ & $N / A$ & $N / A$ \\
\hline$N / A$ & \multicolumn{4}{|l|}{$N / A$} & $N / A$ & $N / A$ & $N / A$ & $N / A$ & $N / A$ & $\mathrm{~N} / \mathrm{A}$ & $N / A$ \\
\hline$N / A$ & \multicolumn{4}{|l|}{$N / A$} & $N / A$ & $\mathrm{~N} / \mathrm{A}$ & $N / A$ & $N / A$ & $N / A$ & $N / A$ & $N / A$ \\
\hline $\mathrm{N} / \mathrm{A}$ & \multicolumn{4}{|l|}{$N / A$} & $\mathrm{~N} / \mathrm{A}$ & $N / A$ & $N / A$ & $N / A$ & $\mathrm{~N} / \mathrm{A}$ & $N / A$ & $N / A$ \\
\hline \multicolumn{5}{|c|}{ Reviewed By (Print): } & \multicolumn{5}{|l|}{ Signature: } & \multicolumn{2}{|c|}{ Date: $7 / 2, / 10$} \\
\hline
\end{tabular}


Survey \# 10-ER-A26-204

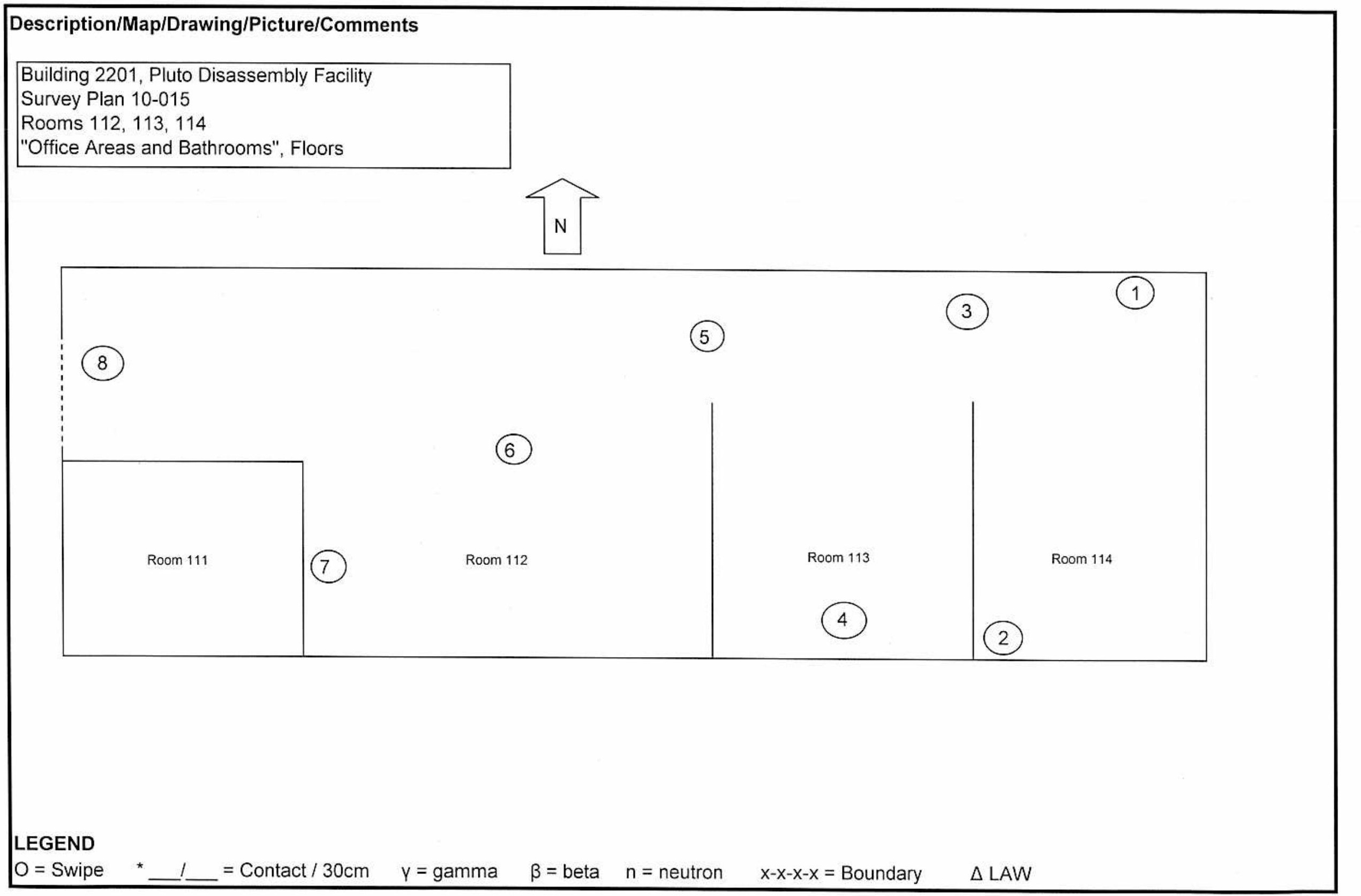


SURVEY \#

10-ER-A26-203

\begin{tabular}{|c|c|c|c|c|c|c|c|c|c|c|c|}
\hline \multicolumn{3}{|c|}{$\begin{array}{l}\text { Location: } \\
\text { Area } 26 \text { CAU117 Pluto }\end{array}$} & \multicolumn{2}{|c|}{$\begin{array}{l}\text { Purpose: } \\
\text { Final Status Survey, Rooms 112, } \\
113,114 \text { Walls }\end{array}$} & \multicolumn{5}{|c|}{$\begin{array}{l}\text { Comments: } \\
\text { All one minute static counts are in cpm. Bkgd not subtracted } \\
\text { for Final Status Survey, Survey Plan \#10-015 }\end{array}$} & \multicolumn{2}{|c|}{$\begin{array}{l}\text { Date/Time: } \\
\text { 07/20/2010 } 1315\end{array}$} \\
\hline Instrument: & Serial \#: & Cal Due: & $\begin{array}{c}\text { Eff in \%: } \\
\text { Alpha / Beta }\end{array}$ & $\begin{array}{l}\text { BKG in dpm: } \\
\text { Alpha / Beta }\end{array}$ & \begin{tabular}{|l|} 
MDA in dpm: \\
Alpha / Beta \\
\end{tabular} & RWP\# & \multicolumn{5}{|l|}{$\mathrm{N} / \mathrm{A}$} \\
\hline Electra & 1504 & $4 / 6 / 2011$ & $12.9 / 19.8$ & $N / A$ & $N / A$ & \multicolumn{6}{|c|}{ Work Package \# $\quad$ ENV-10-RCT ENV SITE SURVEYS-0013 } \\
\hline$N / A$ & $N / A$ & $\mathrm{~N} / \mathrm{A}$ & $\mathrm{N} / \mathrm{A}$ & $\mathrm{N} / \mathrm{A}$ & $\mathrm{N} / \mathrm{A}$ & \multicolumn{3}{|c|}{ RCT Name: D.Cotroneo Jr. } & \multicolumn{3}{|c|}{ Signature: $y-\infty$} \\
\hline$N / A$ & $\mathrm{~N} / \mathrm{A}$ & $N / A$ & $N / A$ & $\mathrm{~N} / \mathrm{A}$ & $N / A$ & \multicolumn{3}{|c|}{ RCT Name: Kevin Wilcox } & \multicolumn{3}{|c|}{ Signature: Kén W. Doof } \\
\hline$N / A$ & $\mathrm{~N} / \mathrm{A}$ & $N / A$ & $\mathrm{~N} / \mathrm{A}$ & $\mathrm{N} / \mathrm{A}$ & $N / A$ & \multicolumn{3}{|c|}{ RCT Name: S. Brown/B. Bunce } & \multirow{2}{*}{\multicolumn{3}{|c|}{ Signature: }} \\
\hline$N / A$ & $N / A$ & $N / A$ & $N / A$ & $N / A$ & $N / A$ & RCT Name: & Brian Fos & & & & \\
\hline \multirow[t]{2}{*}{ Survey Point } & \multirow{2}{*}{\multicolumn{4}{|c|}{ Description/Comments }} & \multicolumn{2}{|c|}{$\begin{array}{l}\text { Removable } \\
\mathrm{dpm} / 100 \mathrm{~cm}^{2}\end{array}$} & \multicolumn{2}{|c|}{$\begin{array}{c}\text { Fixed + Removable } \\
\mathrm{dpm} / 100 \mathrm{~cm}^{2}\end{array}$} & \multirow{2}{*}{$\begin{array}{l}\text { Gamma } \\
\mathrm{mrem} / \mathrm{hr}\end{array}$} & \multirow{2}{*}{$\begin{array}{l}\text { Neutron } \\
\mathrm{mrem} / \mathrm{hr}\end{array}$} & \multirow{2}{*}{$\begin{array}{c}\text { Total } \\
\mathrm{mrem} / \mathrm{hr}\end{array}$} \\
\hline & & & & & Alpha & Beta & Alpha & Beta & & & \\
\hline 1 & \multicolumn{4}{|c|}{ Room 114, East Wall, Cinderblock } & $N / A$ & $N / A$ & 10 & 1393 & $N / A$ & $N / A$ & $N / A$ \\
\hline 2 & \multicolumn{4}{|c|}{ Room 113, East Wall, Cinderblock } & $N / A$ & $N / A$ & 19 & 1420 & $N / A$ & $N / A$ & $N / A$ \\
\hline 3 & \multicolumn{4}{|c|}{ Room 112, East Wall, Cinderblock } & $N / A$ & $N / A$ & 10 & 1420 & $N / A$ & $N / A$ & $N / A$ \\
\hline 4 & \multicolumn{4}{|c|}{ Room 112, South Wall, Cinderblock } & $N / A$ & $N / A$ & 12 & 1329 & $N / A$ & $N / A$ & $N / A$ \\
\hline 5 & \multicolumn{4}{|c|}{ Room 112 , South Wall, Cinderblock } & $N / A$ & $N / A$ & 5 & 1295 & $N / A$ & $N / A$ & $N / A$ \\
\hline 6 & \multicolumn{4}{|c|}{ Room 112, West Wall, Cinderblock } & $N / A$ & $N / A$ & 12 & 1428 & $N / A$ & $N / A$ & $N / A$ \\
\hline 7 & \multicolumn{4}{|c|}{ Room 112, North Wall, Cinderblock } & $N / A$ & $N / A$ & 8 & 1441 & $N / A$ & $N / A$ & $N / A$ \\
\hline 8 & \multicolumn{4}{|c|}{ Room 112, North Wall, Cinderblock } & $N / A$ & $N / A$ & 16 & 1256 & $N / A$ & $N / A$ & $N / A$ \\
\hline 9 & \multicolumn{4}{|c|}{ Room 112, North Wall, Cinderblock } & $N / A$ & $N / A$ & 7 & 1313 & $N / A$ & $N / A$ & $N / A$ \\
\hline 10 & \multicolumn{4}{|c|}{ Room 113, North Wall, Cinderblock } & $N / A$ & $N / A$ & 7 & 1359 & $N / A$ & $N / A$ & $N / A$ \\
\hline 11 & \multicolumn{4}{|c|}{ Room 114, South Wall, Cinderblock } & $N / A$ & $N / A$ & 12 & 1381 & $N / A$ & $N / A$ & $N / A$ \\
\hline \multicolumn{5}{|c|}{ Reviewed By (Print): } & \multicolumn{5}{|l|}{ Signature: } & \multicolumn{2}{|c|}{ Date: $7 / 2, / 10$} \\
\hline
\end{tabular}


SURVEY \# 10-ER-A26-203

\begin{tabular}{|c|c|c|c|c|c|c|c|c|}
\hline \multirow{2}{*}{$\begin{array}{l}\text { Survey } \\
\text { Point }\end{array}$} & \multirow[t]{2}{*}{ Description/Comments } & \multicolumn{2}{|c|}{$\begin{array}{l}\text { Removable } \\
\mathrm{dpm} / 100 \mathrm{~cm}^{2}\end{array}$} & \multicolumn{2}{|c|}{$\begin{array}{c}\text { Fixed + Removable } \\
\mathrm{dpm} / 100 \mathrm{~cm}^{2}\end{array}$} & \multirow{2}{*}{$\begin{array}{l}\text { Gamma } \\
\text { mrem/hr }\end{array}$} & \multirow{2}{*}{$\begin{array}{l}\text { Neutron } \\
\mathrm{mrem} / \mathrm{hr}\end{array}$} & \multirow{2}{*}{$\begin{array}{c}\text { Total } \\
\text { mrem/hr }\end{array}$} \\
\hline & & Alpha & Beta & Alpha & Beta & & & \\
\hline 12 & Room 113, West Wall, Cinderblock & N/A & $N / A$ & 11 & 1546 & N/A & N/A & N/A \\
\hline N/A & $\mathrm{N} / \mathrm{A}$ & N/A & N/A & N/A & N/A & N/A & $\mathrm{N} / \mathrm{A}$ & N/A \\
\hline N/A & $\mathrm{N} / \mathrm{A}$ & N/A & $\mathrm{N} / \mathrm{A}$ & N/A & N/A & N/A & N/A & $\mathrm{N} / \mathrm{A}$ \\
\hline N/A & $N / A$ & N/A & $N / A$ & $\mathrm{~N} / \mathrm{A}$ & $N / A$ & N/A & $\mathrm{N} / \mathrm{A}$ & N/A \\
\hline N/A & $\mathrm{N} / \mathrm{A}$ & N/A & $\mathrm{N} / \mathrm{A}$ & N/A & N/A & N/A & N/A & N/A \\
\hline $\mathrm{N} / \mathrm{A}$ & $\mathrm{N} / \mathrm{A}$ & $\mathrm{N} / \mathrm{A}$ & N/A & N/A & N/A & N/A & $\mathrm{N} / \mathrm{A}$ & $\mathrm{N} / \mathrm{A}$ \\
\hline N/A & $\mathrm{N} / \mathrm{A}$ & N/A & $\mathrm{N} / \mathrm{A}$ & N/A & N/A & N/A & $\mathrm{N} / \mathrm{A}$ & N/A \\
\hline $\mathrm{N} / \mathrm{A}$ & $N / A$ & N/A & $\mathrm{N} / \mathrm{A}$ & N/A & $\mathrm{N} / \mathrm{A}$ & N/A & $\mathrm{N} / \mathrm{A}$ & N/A \\
\hline $\mathrm{N} / \mathrm{A}$ & $\mathrm{N} / \mathrm{A}$ & $\mathrm{N} / \mathrm{A}$ & N/A & N/A & $N / A$ & N/A & $\mathrm{N} / \mathrm{A}$ & N/A \\
\hline $\mathrm{N} / \mathrm{A}$ & $\mathrm{N} / \mathrm{A}$ & N/A & $\mathrm{N} / \mathrm{A}$ & N/A & N/A & $\mathrm{N} / \mathrm{A}$ & $\mathrm{N} / \mathrm{A}$ & $\mathrm{N} / \mathrm{A}$ \\
\hline N/A & $\mathrm{N} / \mathrm{A}$ & N/A & $\mathrm{N} / \mathrm{A}$ & N/A & $\mathrm{N} / \mathrm{A}$ & N/A & N/A & N/A \\
\hline N/A & $\mathrm{N} / \mathrm{A}$ & $\mathrm{N} / \mathrm{A}$ & $\mathrm{N} / \mathrm{A}$ & N/A & N/A & N/A & N/A & N/A \\
\hline N/A & $\mathrm{N} / \mathrm{A}$ & N/A & N/A & N/A & N/A & N/A & $\mathrm{N} / \mathrm{A}$ & N/A \\
\hline N/A & $\mathrm{N} / \mathrm{A}$ & N/A & $\mathrm{N} / \mathrm{A}$ & N/A & $\mathrm{N} / \mathrm{A}$ & $\mathrm{N} / \mathrm{A}$ & N/A & N/A \\
\hline $\mathrm{N} / \mathrm{A}$ & $\mathrm{N} / \mathrm{A}$ & N/A & $\mathrm{N} / \mathrm{A}$ & N/A & $\mathrm{N} / \mathrm{A}$ & $\mathrm{N} / \mathrm{A}$ & N/A & N/A \\
\hline N/A & $\mathrm{N} / \mathrm{A}$ & N/A & $\mathrm{N} / \mathrm{A}$ & N/A & $\mathrm{N} / \mathrm{A}$ & N/A & N/A & N/A \\
\hline $\mathrm{N} / \mathrm{A}$ & $\mathrm{N} / \mathrm{A}$ & N/A & $\mathrm{N} / \mathrm{A}$ & N/A & N/A & $\mathrm{N} / \mathrm{A}$ & N/A & N/A \\
\hline N/A & $\mathrm{N} / \mathrm{A}$ & N/A & $\mathrm{N} / \mathrm{A}$ & N/A & N/A & N/A & N/A & N/A \\
\hline N/A & $\mathrm{N} / \mathrm{A}$ & N/A & $\mathrm{N} / \mathrm{A}$ & N/A & N/A & N/A & N/A & N/A \\
\hline N/A & N/A & N/A & N/A & N/A & N/A & $\mathrm{N} / \mathrm{A}$ & $\mathrm{N} / \mathrm{A}$ & N/A \\
\hline N/A & N/A & N/A & N/A & N/A & N/A & N/A & $\mathrm{N} / \mathrm{A}$ & N/A \\
\hline $\mathrm{N} / \mathrm{A}$ & N/A & N/A & $\mathrm{N} / \mathrm{A}$ & N/A & N/A & $\mathrm{N} / \mathrm{A}$ & $\mathrm{N} / \mathrm{A}$ & N/A \\
\hline $\mathrm{N} / \mathrm{A}$ & N/A & N/A & N/A & N/A & $\mathrm{N} / \mathrm{A}$ & N/A & $\mathrm{N} / \mathrm{A}$ & $\mathrm{N} / \mathrm{A}$ \\
\hline
\end{tabular}


Survey \# 10-ER-A26-203

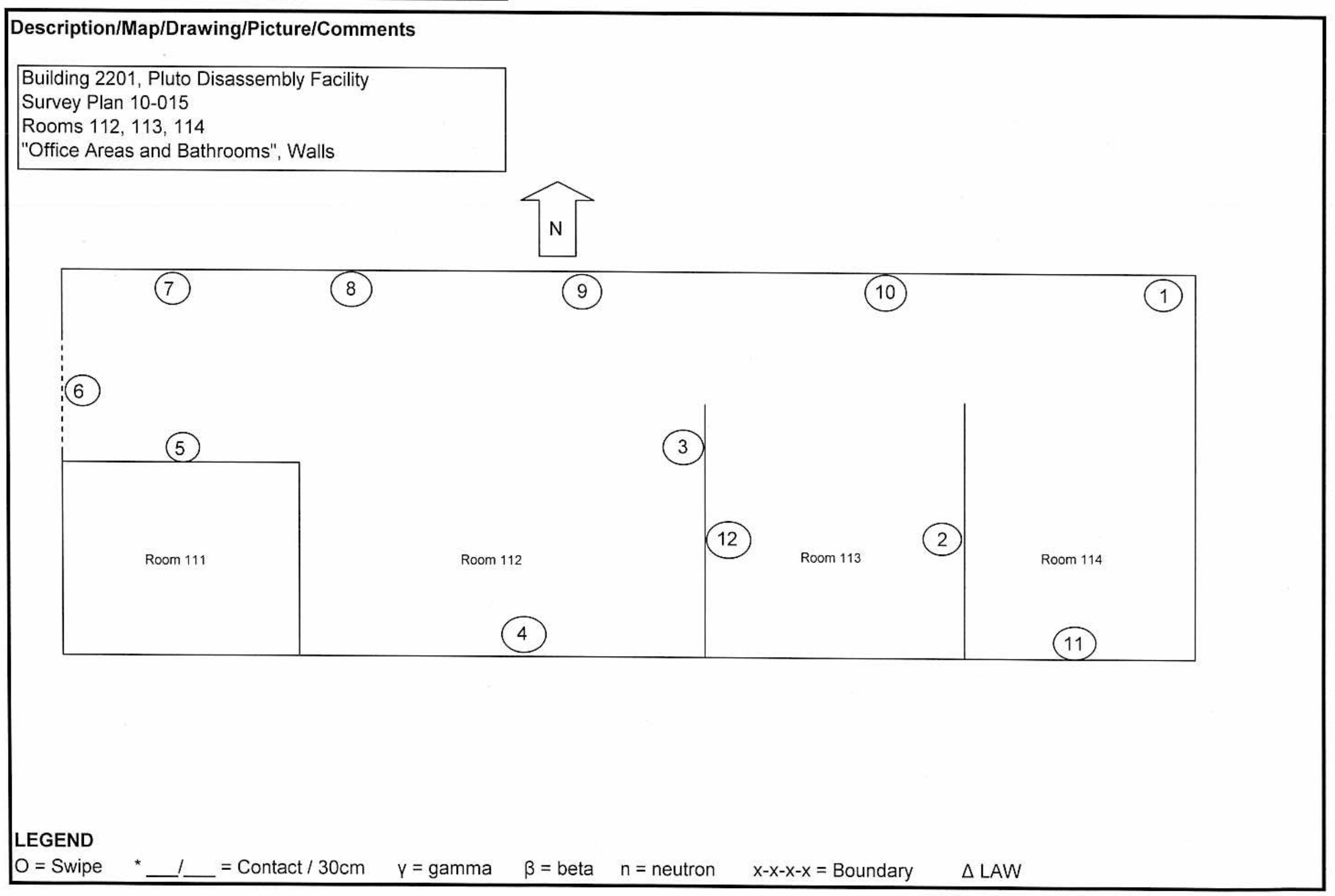


Final Status Survey Report for

Corrective Action Unit 117-Pluto

Disassembly Facility, Building 2201

\section{APPENDIX C - Data for Each Room or Survey Unit}


Data for the Class 3 Area by Room 
10-ER-A26-157

Final Status Survey Plan \# 10-015, Room 101, Floor

\begin{tabular}{|c|c|c|c|c|c|c|c|}
\hline & & \multicolumn{2}{|c|}{ RAW DATA cpm } & \multicolumn{2}{|c|}{ BKG ADJUSTED cpm } & \multicolumn{2}{|c|}{$\mathrm{dpm} / 100 \mathrm{~cm} 2$} \\
\hline & & Alpha & Beta & Alpha & Beta & Alpha & Beta \\
\hline 1 & Concrete Floor used Electra \#4806 & 8 & 1413 & 3.7 & 372.5 & 17.8 & 621.4 \\
\hline 2 & Concrete Floor used Electra \#4806 & 3 & 1437 & -1.3 & 396.5 & -6.4 & 661.5 \\
\hline 3 & Concrete Floor used Electra \#4806 & 1 & 1403 & -3.3 & 362.5 & -16.1 & 604.7 \\
\hline 4 & Concrete Floor used Electra \#4806 & 9 & 1441 & 4.7 & 400.5 & 22.6 & 668.1 \\
\hline 5 & Concrete Floor used Electra \#4806 & 5 & 1439 & 0.7 & 398.5 & 3.3 & 664.8 \\
\hline 6 & Concrete Floor used Electra \#4806 & 0 & 531 & -4.3 & -509.5 & -20.9 & -850.1 \\
\hline 7 & Concrete Floor used Electra \#4806 & 4 & 1293 & -0.3 & 252.5 & -1.6 & 421.2 \\
\hline 8 & Concrete Floor used Electra \#4806 & 1 & 570 & -3.3 & -470.5 & -16.1 & -785.0 \\
\hline 9 & Concrete Floor used Electra \#4806 & 5 & 1405 & 0.7 & 364.5 & 3.3 & 608.1 \\
\hline 10 & Concrete Floor used Electra \#4806 & 10 & 1357 & 5.7 & 316.5 & 27.4 & 528.0 \\
\hline 11 & Concrete Floor used Electra \#4806 & 7 & 1360 & 2.7 & 319.5 & 12.9 & 533.0 \\
\hline 12 & Concrete Floor used Electra \#4806 & 21 & 1301 & 16.7 & 260.5 & 80.6 & 434.6 \\
\hline 13 & Concrete Floor used Electra \#4806 & 7 & 1451 & 2.7 & 410.5 & 12.9 & 684.8 \\
\hline 14 & Metal Plate used Electra $\# 1120$ & 0 & 960 & -4.7 & -371.7 & -22.5 & -620.1 \\
\hline 15 & Metal Plate used Electra \#1120 & 8 & 1374 & 3.4 & 42.3 & 16.2 & 70.6 \\
\hline 16 & Concrete Floor used Electra \#4806 & 10 & 1431 & 5.7 & 390.5 & 27.4 & 651.4 \\
\hline 17 & Metal Plate used Electra \#1120 & 4 & 959 & -0.7 & -372.7 & -3.1 & -621.8 \\
\hline 18 & Concrete Floor used Electra \#4806 & 12 & 1400 & 7.7 & 359.5 & 37.1 & 599.7 \\
\hline 19 & Concrete Floor used Electra \#4806 & 20 & 1456 & 15.7 & 415.5 & 75.7 & 693.2 \\
\hline 20 & Concrete Floor used Electra \#4806 & 15 & 1384 & 10.7 & 343.5 & 51.6 & 573.0 \\
\hline & & & & & Averages & 15.1 & 307.1 \\
\hline & & & & & StDev & 29.2 & 546.2 \\
\hline & & & & & Median & 12.9 & 586.4 \\
\hline & & & & & Max & 80.6 & 693.2 \\
\hline & & & & & Min & -22.5 & -850.1 \\
\hline & & & & & *LBGR & 0.21 & \\
\hline & & & & & *Negative & les not us & GBR \\
\hline
\end{tabular}


10-ER-A26-165

Final Status Status, Ceiling of Main Room 101

\begin{tabular}{|c|c|c|c|c|c|c|c|}
\hline & & \multicolumn{2}{|c|}{ RAW DATA cpm } & \multicolumn{2}{|c|}{ BKG ADJUSTED cpm } & \multicolumn{2}{|c|}{$\mathrm{dpm} / 100 \mathrm{~cm} 2$} \\
\hline & & Alpha & Beta & Alpha & Beta & Alpha & Beta \\
\hline 1 & Ceiling Concrete & 8 & 1413 & 3.7 & 372.5 & 17.8 & 621.4 \\
\hline 2 & Ceiling Concrete & 9 & 1427 & 4.7 & 386.5 & 22.6 & 644.8 \\
\hline 3 & Ceiling Concrete & 10 & 1309 & 5.7 & 268.5 & 27.4 & 447.9 \\
\hline 4 & Ceiling Concrete & 1 & 1389 & -3.3 & 348.5 & -16.1 & 581.4 \\
\hline 5 & Ceiling Concrete & 22 & 1279 & 17.7 & 238.5 & 85.4 & 397.9 \\
\hline 6 & Ceiling Concrete & 11 & 1317 & 6.7 & 276.5 & 32.2 & 461.3 \\
\hline 7 & Concrete wall south(electra 4806) & 11 & 1039 & 6.7 & -1.5 & 32.2 & -2.5 \\
\hline 8 & Wood ceiling(electra1120) & 17 & 927 & 12.3 & -483.7 & 59.2 & -807.0 \\
\hline 9 & Wood ceiling(electra1120) & 10 & 1058 & 5.3 & -352.7 & 25.4 & -588.4 \\
\hline 10 & Wood ceiling(electra1120) & 11 & 1015 & 6.3 & -395.7 & 30.2 & -660.2 \\
\hline 14 & Wood Framed west wall (electra 1504) & 2 & 741 & -2.8 & -669.7 & -13.3 & -1117.3 \\
\hline 15 & Wood Framed west wall (electra 1504) & 6 & 1065 & 1.3 & -345.7 & 6.0 & -576.7 \\
\hline & & & & & Averages & 25.8 & -49.8 \\
\hline & & & & & StDev & 27.8 & 653.4 \\
\hline & & & & & Median & 26.4 & 197.7 \\
\hline & & & & & Max & 85.4 & 644.8 \\
\hline & & & & & Min & -16.1 & -1117.3 \\
\hline & & & & & *LBGR & 0.26 & \\
\hline & & & & & ${ }^{*}$ Negative & vales not u & LGBR \\
\hline
\end{tabular}


10-ER-A26-164

Final Status Survey, East Wall Main Room 101

\begin{tabular}{|c|c|c|c|c|c|c|c|}
\hline & & \multicolumn{2}{|c|}{ RAW DATA cpm } & \multicolumn{2}{|c|}{ BKG ADJUSTED cpm } & \multicolumn{2}{|c|}{$\mathrm{dpm} / 100 \mathrm{~cm} 2$} \\
\hline & & Alpha & Beta & Alpha & Beta & Alpha & Beta \\
\hline 1 & East Wall Concrete (Above roll-up door) & 1 & 1164 & -3.3 & 123.5 & -16.1 & 206.0 \\
\hline 2 & East Wall Concrete (Above roll-up door) & 9 & 1151 & 4.7 & 110.5 & 22.6 & 184.3 \\
\hline 3 & East Wall Metal (Roll-up door) & 4 & 852 & -0.7 & -479.7 & -3.1 & -800.3 \\
\hline 4 & East Wall Metal (Roll-up door) & 5 & 925 & 0.4 & -406.7 & 1.7 & -678.5 \\
\hline 7 & Concrete wall south(electra 4806) & 11 & 1039 & 6.7 & -1.5 & 32.2 & -2.5 \\
\hline 8 & Wood ceiling(electra1120) & 17 & 927 & 12.3 & -483.7 & 59.2 & -807.0 \\
\hline 9 & Wood ceiling(electra1120) & 10 & 1058 & 5.3 & -352.7 & 25.4 & -588.4 \\
\hline 10 & Wood ceiling(electra1120) & 11 & 1015 & 6.3 & -395.7 & 30.2 & -660.2 \\
\hline 14 & Wood Framed west wall (electra 1504) & 2 & 741 & -2.8 & -669.7 & -13.3 & -1117.3 \\
\hline \multirow[t]{8}{*}{15} & Wood Framed west wall (electra 1504) & 6 & 1065 & 1.3 & -345.7 & 6.0 & -576.7 \\
\hline & & & & & Averages & 14.5 & -484.1 \\
\hline & & & & & StDev & 23.6 & 453.2 \\
\hline & & & & & Median & 14.3 & -624.3 \\
\hline & & & & & Max & 59.2 & 206.0 \\
\hline & & & & & Min & -16.1 & -1117.3 \\
\hline & & & & & *LBGR & 0.14 & \\
\hline & & & & & \multicolumn{3}{|c|}{ *Negative vales not used in LGBR } \\
\hline
\end{tabular}


10-ER-A26-158

Final Status Survey, Room 101 Mezzanine

\begin{tabular}{|c|c|c|c|c|c|c|c|}
\hline & & \multicolumn{2}{|c|}{ RAW DATA cpm } & \multicolumn{2}{|c|}{ BKG ADJUSTED cpm } & \multicolumn{2}{|c|}{$\mathrm{dpm} / 100 \mathrm{~cm} 2$} \\
\hline & & Alpha & Beta & Alpha & Beta & Alpha & Beta \\
\hline 1 & Mezzanine Floor Wood & 2 & 962 & -4.9 & -333.7 & -23.4 & -556.6 \\
\hline 2 & Mezzanine Floor Wood & 10 & 1056 & 3.2 & -239.7 & 15.2 & -399.8 \\
\hline 3 & Mezzanine Floor Wood & 4 & 992 & -2.9 & -303.7 & -13.8 & -506.6 \\
\hline 4 & Mezzanine Floor Wood & 5 & 1002 & -1.9 & -293.7 & -8.9 & -489.9 \\
\hline 5 & Mezzanine Floor Wood & 3 & 969 & -3.9 & -326.7 & -18.6 & -545.0 \\
\hline 6 & West Wall Concrete & 16 & 1066 & 11.7 & 25.5 & 56.4 & 42.5 \\
\hline 7 & West Wall Concrete & 7 & 1073 & 2.7 & 32.5 & 12.9 & 54.2 \\
\hline 8 & West Wall Concrete & 8 & 1092 & 3.7 & 51.5 & 17.8 & 85.9 \\
\hline 9 & West Wall Concrete & 11 & 1155 & 6.7 & 114.5 & 32.2 & 191.0 \\
\hline 10 & West Wall Concrete & 12 & 1134 & 7.7 & 93.5 & 37.1 & 155.9 \\
\hline 11 & South Wall Concrete & 6 & 1104 & 1.7 & 63.5 & 8.1 & 105.9 \\
\hline 12 & South Wall Concrete & 7 & 991 & 2.7 & -49.5 & 12.9 & -82.6 \\
\hline 13 & South Wall Concrete & 10 & 977 & 5.7 & -63.5 & 27.4 & -106.0 \\
\hline 14 & South Wall Concrete & 14 & 948 & 9.7 & -92.5 & 46.7 & -154.4 \\
\hline 15 & South Wall Concrete & 14 & 997 & 9.7 & -43.5 & 46.7 & -72.6 \\
\hline 16 & North Wall Concrete & 8 & 1121 & 3.7 & 80.5 & 17.8 & 134.3 \\
\hline 17 & North Wall Concrete & 6 & 1062 & 1.7 & 21.5 & 8.1 & 35.8 \\
\hline 18 & North Wall Concrete & 6 & 1044 & 1.7 & 3.5 & 8.1 & 5.8 \\
\hline 19 & North Wall Concrete & 7 & 1096 & 2.7 & 55.5 & 12.9 & 92.6 \\
\hline 20 & North Wall Concrete & 10 & 1043 & 5.7 & 2.5 & 27.4 & 4.1 \\
\hline 21 & Craneveyor Platform/Track Metal & 8 & 875 & 2.7 & -363.9 & 13.0 & -607.0 \\
\hline 22 & Craneveyor Platform/Track Metal & 4 & 793 & -1.3 & -445.9 & -6.3 & -743.8 \\
\hline 23 & Ceiling Concrete & 1 & 1262 & -3.3 & 221.5 & -16.1 & 369.5 \\
\hline 24 & Ceiling Concrete & 3 & 1279 & -1.3 & 238.5 & -6.4 & 397.9 \\
\hline 25 & Ceiling Concrete & 3 & 1248 & -1.3 & 207.5 & -6.4 & 346.1 \\
\hline 26 & Ceiling Concrete & 2 & 1241 & -2.3 & 200.5 & -11.2 & 334.5 \\
\hline 27 & Ceiling Concrete & 3 & 1327 & -1.3 & 286.5 & -6.4 & 477.9 \\
\hline
\end{tabular}




\begin{tabular}{|c|c|c|}
\hline-331.7 & 5.6 & -553.3 \\
\hline Averages & 10.3 & -70.9 \\
\hline StDev & 21.0 & 347.7 \\
\hline Median & 10.5 & 20.8 \\
\hline Max & 56.4 & 477.9 \\
\hline Min & -23.4 & -743.8 \\
\hline *LBGR & 0.10 & \multicolumn{2}{|l}{} \\
\hline * Negative
\end{tabular}

*Negative vales not used in LGBR

\section{0-ER-A26-162}

Final Status Survey, North Wall Main Room 101

\begin{tabular}{|c|c|c|c|c|c|c|c|}
\hline & & \multicolumn{2}{|c|}{ RAW DATA cpm } & \multicolumn{2}{|c|}{ BKG ADJUSTED cpm } & \multicolumn{2}{|c|}{$\mathrm{dpm} / 100 \mathrm{~cm} 2$} \\
\hline & & Alpha & Beta & Alpha & Beta & Alpha & Beta \\
\hline 1 & North Wall Concrete (Electra 4806) & 10 & 1210 & 5.7 & 169.5 & 27.4 & 282.7 \\
\hline 2 & North Wall Concrete (Electra 4806) & 7 & 1176 & 2.7 & 135.5 & 12.9 & 226.0 \\
\hline 3 & North Wall Concrete (Electra 4806) & 12 & 1124 & 7.7 & 83.5 & 37.1 & 139.3 \\
\hline 4 & North Wall Concrete (Electra 4806) & 6 & 1195 & 1.7 & 154.5 & 8.1 & 257.7 \\
\hline 5 & North Wall Concrete (Electra 1120) & 9 & 1213 & 4.7 & 172.5 & 22.6 & 287.7 \\
\hline 6 & North Wall Concrete (Electra 1120) & 8 & 1127 & 3.7 & 86.5 & 17.8 & 144.3 \\
\hline 7 & North Wall Concrete (Electra 1120) & 11 & 1156 & 6.7 & 115.5 & 32.2 & 192.7 \\
\hline 7 & Concrete wall south(electra 4806) & 11 & 1039 & 6.7 & -1.5 & 32.2 & -2.5 \\
\hline 8 & Wood ceiling(electra1120) & 17 & 927 & 12.3 & -483.7 & 59.2 & -807.0 \\
\hline 9 & Wood ceiling(electra1120) & 10 & 1058 & 5.3 & -352.7 & 25.4 & -588.4 \\
\hline 10 & Wood ceiling(electra1120) & 11 & 1015 & 6.3 & -395.7 & 30.2 & -660.2 \\
\hline 14 & Wood Framed west wall (electra 1504) & 2 & 741 & -4.9 & -554.7 & -23.4 & -925.3 \\
\hline 15 & Wood Framed west wall (electra 1504) & 6 & 1065 & -0.9 & -230.7 & -4.1 & -384.8 \\
\hline & & & & & Averages & 21.3 & -141.4 \\
\hline & & & & & StDev & 20.3 & 459.7 \\
\hline & & & & & Median & 25.4 & 139.3 \\
\hline & & & & & Max & 59.2 & 287.7 \\
\hline & & & & & Min & -23.4 & -925.3 \\
\hline & & & & & $*$ LBGR & 0.21 & \\
\hline
\end{tabular}


10-ER-A26-163

Final Status Survey, South Wall Main Room 101

\begin{tabular}{|c|c|c|c|c|c|c|c|}
\hline & \multicolumn{2}{|c|}{ RAW DATA cpm } & \multicolumn{2}{|c|}{ BKG ADJUSTED cpm } & \multicolumn{2}{|c|}{$\mathrm{dpm} / 100 \mathrm{~cm} 2$} \\
\hline & & Alpha & Beta & Alpha & Beta & Alpha & Beta \\
\hline 1 & South Wall Concrete (Electra 1120) & 18 & 1031 & 13.7 & -9.5 & 66.1 & -15.9 \\
\hline 2 & South Wall Concrete (Electra 1120) & 18 & 965 & 13.7 & -75.5 & 66.1 & -126.0 \\
\hline 3 & South Wall Concrete (Electra 1120) & 20 & 990 & 15.7 & -50.5 & 75.7 & -84.3 \\
\hline 4 & South Wall Concrete (Electra 1120) & 17 & 925 & 12.7 & -115.5 & 61.2 & -192.7 \\
\hline 5 & South Wall Concrete (Electra 6697) & 11 & 1075 & 6.7 & 34.5 & 32.2 & 57.5 \\
\hline 6 & South Wall Upper Ledge Concrete (Electra 6697) & 6 & 1318 & 1.7 & 277.5 & 8.1 & 462.9 \\
\hline 7 & South Wall Concrete (Electra 6697) & 9 & 1109 & 4.7 & 68.5 & 22.6 & 114.2 \\
\hline 8 & South Wall Concrete (Electra 6697) & 10 & 1013 & 5.7 & -27.5 & 27.4 & -45.9 \\
\hline 7 & Concrete wall south(electra 4806) & 11 & 1039 & 6.7 & -1.5 & 32.2 & -2.5 \\
\hline 8 & Wood ceiling(electra1120) & 17 & 927 & 12.3 & -483.7 & 59.2 & -807.0 \\
\hline 9 & Wood ceiling(electra1120) & 10 & 1058 & 5.3 & -352.7 & 25.4 & -588.4 \\
\hline 10 & Wood ceiling(electra1120) & 11 & 1015 & 6.3 & -395.7 & 30.2 & -660.2 \\
\hline 14 & Wood Framed west wall (electra 1504) & 2 & 741 & -4.9 & -554.7 & -23.4 & -925.3 \\
\hline 15 & Wood Framed west wall (electra 1504) & 6 & 1065 & -0.9 & -230.7 & -4.1 & -384.8 \\
\hline & & & & & Averages & 34.2 & -228.5 \\
\hline & & & & & StDev & 28.9 & 392.2 \\
\hline & & & & & Median & 31.2 & -105.1 \\
\hline & & & & & Max & 75.7 & 462.9 \\
\hline & & & & & Min & -23.4 & -925.3 \\
\hline & & & & & *LBGR & 0.34 & \\
\hline & & & & & *Negative & ales not us & LGBR \\
\hline
\end{tabular}


10-ER-A26-161

Final Status (walls \& ceilings) West end of room 101

\begin{tabular}{|c|c|c|c|c|c|c|c|}
\hline & & \multicolumn{2}{|c|}{ RAW DATA cpm } & \multicolumn{2}{|c|}{ BKG ADJUSTED cpm } & \multicolumn{2}{|c|}{$\mathrm{dpm} / 100 \mathrm{~cm} 2$} \\
\hline & & Alpha & Beta & Alpha & Beta & Alpha & Beta \\
\hline 1 & Concrete Wall South (Electra 4806) & 7 & 989 & 2.7 & -51.5 & 12.9 & -86.0 \\
\hline 2 & Concrete Wall West (Electra 4806) & 6 & 1112 & 1.7 & 71.5 & 8.1 & 119.2 \\
\hline 3 & Concrete Wall North (Electra 4806) & 6 & 1076 & 1.7 & 35.5 & 8.1 & 59.2 \\
\hline 4 & Cinderblock wall (Electra 4806) & 5 & 1279 & 0.2 & 582.4 & 0.8 & 971.6 \\
\hline 11 & Wood ceiling (Electra 1120) & 2 & 1001 & -2.8 & -409.7 & -13.3 & -683.5 \\
\hline 12 & Wood ceiling (Electra 1120) & 13 & 1000 & 8.3 & -410.7 & 39.9 & -685.2 \\
\hline 13 & Wood ceiling (Electra 1120) & 13 & 910 & 8.3 & -500.7 & 39.9 & -835.3 \\
\hline 5 & Concrete Wall North (Electra 4806) & 11 & 1035 & 6.7 & -5.5 & 32.2 & -9.2 \\
\hline 6 & Cinderblock wall (Electra 4806) & 5 & 1319 & 0.2 & 622.4 & 0.8 & 1038.3 \\
\hline 7 & Concrete wall south (Electra 4806) & 11 & 1039 & 6.7 & -1.5 & 32.2 & -2.5 \\
\hline 8 & Wood ceiling (Electra 1120) & 17 & 927 & 12.3 & -483.7 & 59.2 & -807.0 \\
\hline 9 & Wood ceiling (Electra 1120) & 10 & 1058 & 5.3 & -352.7 & 25.4 & -588.4 \\
\hline 10 & Wood ceiling (Electra 1120) & 11 & 1015 & 6.3 & -395.7 & 30.2 & -660.2 \\
\hline 14 & Wood Framed west wall (Electra 1504) & 2 & 741 & -4.9 & -554.7 & -23.4 & -925.3 \\
\hline \multirow[t]{8}{*}{13} & Wood Framed west wall (Electra 1504) & 6 & 1065 & -0.9 & -230.7 & -4.1 & -384.8 \\
\hline & & & & & Averages & 16.6 & -231.9 \\
\hline & & & & & StDev & 22.7 & 616.0 \\
\hline & & & & & Median & 12.9 & -384.8 \\
\hline & & & & & Max & 59.2 & 1038.3 \\
\hline & & & & & Min & -23.4 & -925.3 \\
\hline & & & & & *LBGR & 0.17 & \\
\hline & & & & & \multicolumn{3}{|c|}{ *Negative vales not used in LGBR } \\
\hline
\end{tabular}


10-ER-A26-188

Final Status Survey, Room 103, Walls

\begin{tabular}{|c|c|c|c|c|c|c|c|}
\hline & & \multicolumn{2}{|c|}{ RAW DATA cpm } & \multicolumn{2}{|c|}{ BKG ADJUSTED cpm } & \multicolumn{2}{|c|}{$\mathrm{dpm} / 100 \mathrm{~cm} 2$} \\
\hline & & Alpha & Beta & Alpha & Beta & Alpha & Beta \\
\hline 1 & North Wall, Concrete & 8 & 1219 & 3.7 & 178.5 & 17.8 & 297.8 \\
\hline 2 & North Wall, Concrete & 13 & 1213 & 8.7 & 172.5 & 41.9 & 287.7 \\
\hline 3 & East Wall, Concrete & 22 & 1214 & 17.7 & 173.5 & 85.4 & 289.4 \\
\hline 4 & East Wall, Concrete & 14 & 1220 & 9.7 & 179.5 & 46.7 & 299.4 \\
\hline 5 & East Wall, Metal & 6 & 824 & 1.4 & -507.7 & 6.5 & -847.0 \\
\hline 6 & East Wall, Metal & 7 & 869 & 2.4 & -462.7 & 11.4 & -771.9 \\
\hline 7 & East Wall, Concrete & 15 & 1157 & 10.7 & 116.5 & 51.6 & 194.3 \\
\hline 8 & South Wall, Concrete & 13 & 1141 & 8.7 & 100.5 & 41.9 & 167.6 \\
\hline 9 & West Wall, Concrete & 12 & 1179 & 7.7 & 138.5 & 37.1 & 231.0 \\
\hline 10 & West Wall, Concrete & 13 & 1255 & 8.7 & 214.5 & 41.9 & 357.8 \\
\hline 11 & West Wall, Concrete & 17 & 1166 & 12.7 & 125.5 & 61.2 & 209.3 \\
\hline 12 & South Wall, Concrete & 13 & 1157 & 8.7 & 116.5 & 41.9 & 194.3 \\
\hline \multirow[t]{8}{*}{13} & West Wall, Concrete & 11 & 1244 & 6.7 & 203.5 & 32.2 & 339.5 \\
\hline & & & & & Averages & 39.8 & 96.1 \\
\hline & & & & & StDev & 20.8 & 406.4 \\
\hline & & & & & Median & 41.9 & 231.0 \\
\hline & & & & & Max & 85.4 & 357.8 \\
\hline & & & & & Min & 6.5 & -847.0 \\
\hline & & & & & $*$ LBGR & 0.42 & \\
\hline & & & & & *Negative & vales not $\mathrm{c}$ & $\mathrm{n}$ LGBR \\
\hline
\end{tabular}




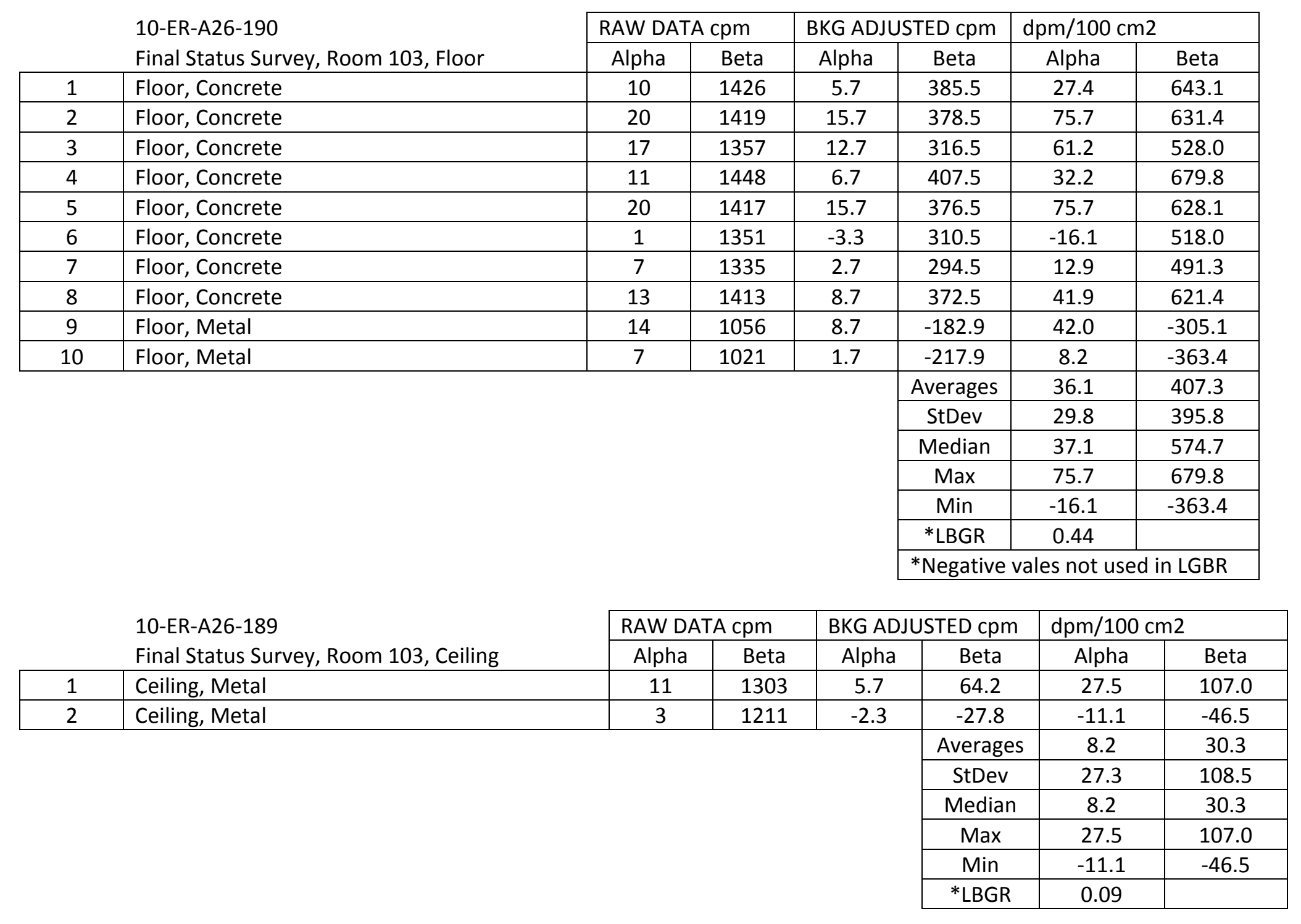


10-ER-A26-168

Final Status Survey, Room 105

\begin{tabular}{|c|c|c|c|c|c|c|c|}
\hline & & \multicolumn{2}{|c|}{ RAW DATA cpm } & \multicolumn{2}{|c|}{ BKG ADJUSTED cpm } & \multicolumn{2}{|c|}{$\mathrm{dpm} / 100 \mathrm{~cm} 2$} \\
\hline & & Alpha & Beta & Alpha & Beta & Alpha & Beta \\
\hline 1 & Floor Concrete & 2 & 977 & -2.3 & -63.5 & -11.2 & -106.0 \\
\hline 2 & Floor Concrete & 6 & 1150 & 1.7 & 109.5 & 8.1 & 182.6 \\
\hline 3 & Floor Concrete & 7 & 1137 & 2.7 & 96.5 & 12.9 & 161.0 \\
\hline 4 & Floor Concrete & 2 & 1283 & -2.3 & 242.5 & -11.2 & 404.5 \\
\hline 5 & East Wall Concrete & 5 & 729 & 0.7 & -311.5 & 3.3 & -519.7 \\
\hline 6 & East Wall Concrete & 2 & 751 & -2.3 & -289.5 & -11.2 & -483.0 \\
\hline 7 & East Wall Concrete & 3 & 618 & -1.3 & -422.5 & -6.4 & -704.9 \\
\hline 8 & West Wall Concrete & 3 & 1029 & -1.3 & -11.5 & -6.4 & -19.2 \\
\hline 9 & West Wall Concrete & 3 & 703 & -1.3 & -337.5 & -6.4 & -563.1 \\
\hline 10 & West Wall Concrete & 0 & 647 & -4.3 & -393.5 & -20.9 & -656.5 \\
\hline 11 & North Wall Concrete & 2 & 1166 & -2.3 & 125.5 & -11.2 & 209.3 \\
\hline 12 & North Wall Concrete & 1 & 990 & -3.3 & -50.5 & -16.1 & -84.3 \\
\hline 13 & Ceiling Concrete & 11 & 1160 & 6.7 & 119.5 & 32.2 & 199.3 \\
\hline 14 & Ceiling Concrete & 3 & 1087 & -1.3 & 46.5 & -6.4 & 77.5 \\
\hline 15 & Ceiling Concrete & 7 & 1017 & 2.7 & -23.5 & 12.9 & -39.2 \\
\hline \multirow[t]{8}{*}{16} & Ceiling Concrete & 5 & 751 & 0.7 & -289.5 & 3.3 & -483.0 \\
\hline & & & & & Averages & -2.2 & -151.5 \\
\hline & & & & & StDev & 13.5 & 360.3 \\
\hline & & & & & Median & -6.4 & -61.8 \\
\hline & & & & & Max & 32.2 & 404.5 \\
\hline & & & & & Min & -20.9 & -704.9 \\
\hline & & & & & $*$ LBGR & 0.00 & \\
\hline & & & & & \multicolumn{3}{|c|}{ *Negative vales not used in LGBR } \\
\hline
\end{tabular}


10-ER-A26-172

Final Status Survey, Room 108, metal ceiling

\begin{tabular}{|c|c|c|c|c|c|c|c|}
\hline & \multirow[t]{2}{*}{ 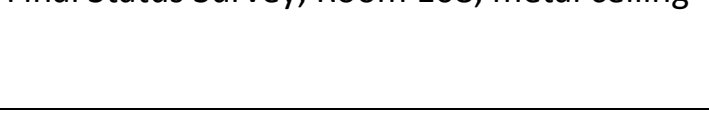 } & \multicolumn{2}{|c|}{ RAW DATA cpm } & \multicolumn{2}{|c|}{ BKG ADJUSTED cpm } & \multicolumn{2}{|c|}{$\mathrm{dpm} / 100 \mathrm{~cm} 2$} \\
\hline & & Alpha & Beta & Alpha & Beta & Alpha & Beta \\
\hline 1 & Room 108 Metal Ceiling & 5 & 988 & -0.3 & -250.9 & -1.4 & -418.5 \\
\hline 2 & Room 108 Metal Ceiling & 1 & 974 & -4.3 & -264.9 & -20.8 & -441.9 \\
\hline 3 & Room 108 Metal Ceiling & 2 & 1095 & -3.3 & -143.9 & -15.9 & -240.0 \\
\hline 4 & Room 108 Metal Ceiling & 2 & 1022 & -3.3 & -216.9 & -15.9 & -361.8 \\
\hline 5 & Room 108 Metal Ceiling & 0 & 852 & -5.3 & -386.9 & -25.6 & -645.4 \\
\hline 6 & Room 108 Metal Ceiling & 3 & 1153 & -2.3 & -85.8 & -11.1 & -143.2 \\
\hline 7 & Room 108 Metal Ceiling & 6 & 1100 & 0.7 & -138.9 & 3.4 & -231.6 \\
\hline & & & & & Averages & -12.5 & -354.6 \\
\hline & & & & & StDev & 10.3 & 167.9 \\
\hline & & & & & Median & -15.9 & -361.8 \\
\hline & & & & & Max & 3.4 & -143.2 \\
\hline & & & & & Min & -25.6 & -645.4 \\
\hline & & & & & $*$ LBGR & 0.00 & \\
\hline & & & & & *Negativ & es not & in LGBR \\
\hline
\end{tabular}


10-ER-A26-173

Final Status Survey, Room 108, Concrete Floor

\begin{tabular}{|c|c|c|c|c|c|c|c|}
\hline & & \multicolumn{2}{|c|}{ RAW DATA cpm } & \multicolumn{2}{|c|}{ BKG ADJUSTED cpm } & \multicolumn{2}{|c|}{$\mathrm{dpm} / 100 \mathrm{~cm} 2$} \\
\hline & & \multirow{2}{*}{$\begin{array}{c}\text { Alpha } \\
12\end{array}$} & \multirow{2}{*}{$\begin{array}{l}\text { Beta } \\
1530\end{array}$} & \multirow{2}{*}{$\begin{array}{c}\text { Alpha } \\
7.7\end{array}$} & \multirow{2}{*}{$\begin{array}{c}\text { Beta } \\
489.5\end{array}$} & \multirow{2}{*}{$\begin{array}{c}\text { Alpha } \\
37.1\end{array}$} & \multirow{2}{*}{$\begin{array}{c}\text { Beta } \\
816.6\end{array}$} \\
\hline 1 & Room 108 Concrete Floor (Electra 1120) & & & & & & \\
\hline 2 & Room 108 Concrete Floor (Electra 1120) & 10 & 1573 & 5.7 & 532.5 & 27.4 & 888.3 \\
\hline 4 & Room 108 Concrete Floor (Electra 1120) & 1 & 1428 & -3.3 & 387.5 & -16.1 & 646.4 \\
\hline 5 & Room 108 Concrete Floor (Electra 1120) & 1 & 1343 & -3.3 & 302.5 & -16.1 & 504.6 \\
\hline 8 & Room 108 Concrete Floor (Electra 1120) & 10 & 1498 & 5.7 & 457.5 & 27.4 & 763.2 \\
\hline 9 & Room 108 Concrete Floor (Electra 1120) & 10 & 1517 & 5.7 & 476.5 & 27.4 & 794.9 \\
\hline 10 & Room 108 Concrete Floor (Electra 1504) & 7 & 1285 & 2.7 & 244.5 & 12.9 & 407.9 \\
\hline 11 & Room 108 Concrete Floor (Electra 1120) & 7 & 1506 & 2.7 & 465.5 & 12.9 & 776.6 \\
\hline 12 & Room 108 Concrete Floor (Electra 1504) & 0 & 1262 & -4.3 & 221.5 & -20.9 & 369.5 \\
\hline 16 & Room 108 Concrete Floor (Electra 1120) & 8 & 1414 & 3.7 & 373.5 & 17.8 & 623.1 \\
\hline \multirow[t]{8}{*}{17} & Room 108 Concrete Floor (Electra 1504) & 0 & 1352 & -4.3 & 311.5 & -20.9 & 519.6 \\
\hline & & & & & Averages & 5.3 & 622.0 \\
\hline & & & & & StDev & 20.2 & 174.7 \\
\hline & & & & & Median & 12.9 & 623.1 \\
\hline & & & & & Max & 37.1 & 888.3 \\
\hline & & & & & Min & -20.9 & 316.1 \\
\hline & & & & & $*$ LBGR & 0.18 & \\
\hline & & & & & *Negative & vales not $\mathrm{c}$ & in LGBR \\
\hline
\end{tabular}


10-ER-A26-171

Final Status Survey, Room 108, North Wall \& West Wall

\begin{tabular}{|c|c|c|c|c|c|c|c|}
\hline & & \multicolumn{2}{|c|}{ RAW DATA cpm } & \multicolumn{2}{|c|}{ BKG ADJUSTED cpm } & \multicolumn{2}{|c|}{$\mathrm{dpm} / 100 \mathrm{~cm} 2$} \\
\hline & & Alpha & Beta & Alpha & Beta & Alpha & Beta \\
\hline 1 & West Wall Concrete (Electra 6697) & 13 & 1047 & 8.7 & 6.5 & 41.9 & 10.8 \\
\hline 2 & West Wall Concrete (Electra 6697) & 8 & 1078 & 3.7 & 37.5 & 17.8 & 62.5 \\
\hline 3 & West Wall Concrete (Electra 6697) & 8 & 1044 & 3.7 & 3.5 & 17.8 & 5.8 \\
\hline 4 & West Wall Concrete (Electra 6697) & 9 & 984 & 4.7 & -56.5 & 22.6 & -94.3 \\
\hline 5 & West Wall Concrete (Electra 6697) & 8 & 890 & 3.7 & -150.5 & 17.8 & -251.1 \\
\hline 6 & West Wall Concrete (Electra 6697) & 9 & 828 & 4.7 & -212.5 & 22.6 & -354.6 \\
\hline 7 & West Wall Concrete (Electra 6697) & 11 & 1055 & 6.7 & 14.5 & 32.2 & 24.1 \\
\hline 8 & West Wall Concrete (Electra 6697) & 5 & 976 & 0.7 & -64.5 & 3.3 & -107.6 \\
\hline 9 & North Wall Concrete (Electra 1504) & 4 & 795 & -0.3 & -245.5 & -1.6 & -409.6 \\
\hline 10 & North Wall Concrete (Electra 1504) & 8 & 822 & 3.7 & -218.5 & 17.8 & -364.6 \\
\hline 11 & North Wall Concrete (Electra 1504) & 8 & 912 & 3.7 & -128.5 & 17.8 & -214.4 \\
\hline 12 & North Wall Concrete (Electra 1504) & 3 & 821 & -1.3 & -219.5 & -6.4 & -366.2 \\
\hline 13 & North Wall Concrete (Electra 1504) & 6 & 809 & 1.7 & -231.5 & 8.1 & -386.3 \\
\hline 14 & North Wall Concrete (Electra 1504) & 0 & 894 & -4.3 & -146.5 & -20.9 & -244.5 \\
\hline 15 & North Wall Concrete (Electra 1504) & 5 & 848 & 0.7 & -192.5 & 3.3 & -321.2 \\
\hline 16 & North Wall Concrete (Electra 1504) & 7 & 1004 & 2.7 & -36.5 & 12.9 & -60.9 \\
\hline 17 & North Wall Concrete (Electra 1504) & 10 & 815 & 5.7 & -225.5 & 27.4 & -376.3 \\
\hline 18 & North Wall Concrete (Electra 1504) & 3 & 846 & -1.3 & -194.5 & -6.4 & -324.5 \\
\hline 19 & North Wall Concrete (Electra 1504) & 2 & 1035 & -2.3 & -5.5 & -11.2 & -9.2 \\
\hline 20 & North Wall Concrete (Electra 1504) & 6 & 820 & 1.7 & -220.5 & 8.1 & -367.9 \\
\hline 21 & North Wall Concrete (Electra 1504) & 6 & 898 & 1.7 & -142.5 & 8.1 & -237.8 \\
\hline 22 & North Wall Concrete (Electra 1504) & 6 & 922 & 1.7 & -118.5 & 8.1 & -197.7 \\
\hline 23 & North Wall Concrete (Electra 1504) & 4 & 897 & -0.3 & -143.5 & -1.6 & -239.4 \\
\hline
\end{tabular}




\begin{tabular}{|c|c|c|c|c|c|c|c|}
\hline 24 & North Wall Concrete (Electra 1504) & 2 & 892 & -2.3 & -148.5 & -11.2 & -247.8 \\
\hline 25 & North Wall Concrete (Electra 1504) & 2 & 872 & -2.3 & -168.5 & -11.2 & -281.2 \\
\hline 26 & North Wall Concrete (Electra 1504) & 2 & 940 & -2.3 & -100.5 & -11.2 & -167.7 \\
\hline 27 & North Wall Concrete (Electra 1504) & 4 & 820 & -0.3 & -220.5 & -1.6 & -367.9 \\
\hline 28 & North Wall Concrete (Electra 1504) & 2 & 901 & -2.3 & -139.5 & -11.2 & -232.8 \\
\hline 29 & North Wall Concrete (Electra 1504) & 4 & 978 & -0.3 & -62.5 & -1.6 & -104.3 \\
\hline 30 & North Wall Concrete (Electra 1504) & 2 & 911 & -2.3 & -129.5 & -11.2 & -216.1 \\
\hline & & & & & Averages & 6.0 & -214.8 \\
\hline & & & & & StDev & 15.3 & 141.2 \\
\hline & & & & & Median & 5.7 & -238.6 \\
\hline & & & & & Max & 41.9 & 62.5 \\
\hline & & & & & Min & -20.9 & -409.6 \\
\hline & & & & & *LBGR & 0.06 & \\
\hline & & & & & \multicolumn{3}{|c|}{ *Negative vales not used in LGBR } \\
\hline
\end{tabular}


10-ER-A26-170

Final Status Survey, Room 108, South Wall \& East Wall

\begin{tabular}{|c|c|c|c|c|c|c|c|}
\hline & & \multicolumn{2}{|c|}{ RAW DATA cpm } & \multicolumn{2}{|c|}{ BKG ADJUSTED cpm } & \multicolumn{2}{|c|}{$\mathrm{dpm} / 100 \mathrm{~cm} 2$} \\
\hline & & Alpha & Beta & Alpha & Beta & Alpha & Beta \\
\hline 1 & South Wall Concrete & 8 & 1218 & 3.7 & 177.5 & 17.8 & 296.1 \\
\hline 2 & South Wall Concrete & 12 & 1223 & 7.7 & 182.5 & 37.1 & 304.4 \\
\hline 3 & South Wall Concrete & 9 & 1251 & 4.7 & 210.5 & 22.6 & 351.1 \\
\hline 4 & South Wall Metal & 8 & 745 & 3.4 & -586.7 & 16.2 & -978.8 \\
\hline 5 & South Wall Concrete & 9 & 1269 & 4.7 & 228.5 & 22.6 & 381.2 \\
\hline 6 & South Wall Concrete & 9 & 1218 & 4.7 & 177.5 & 22.6 & 296.1 \\
\hline 7 & South Wall Concrete & 13 & 1219 & 8.7 & 178.5 & 41.9 & 297.8 \\
\hline 8 & South Wall Concrete & 14 & 1093 & 9.7 & 52.5 & 46.7 & 87.5 \\
\hline 9 & South Wall Concrete & 5 & 1216 & 0.7 & 175.5 & 3.3 & 292.8 \\
\hline 10 & South Wall Concrete & 9 & 1106 & 4.7 & 65.5 & 22.6 & 109.2 \\
\hline 11 & South Wall Concrete & 5 & 1118 & 0.7 & 77.5 & 3.3 & 129.3 \\
\hline 12 & South Wall Concrete & 8 & 1196 & 3.7 & 155.5 & 17.8 & 259.4 \\
\hline 13 & South Wall Concrete & 16 & 1318 & 11.7 & 277.5 & 56.4 & 462.9 \\
\hline 14 & South Wall Concrete & 17 & 1357 & 12.7 & 316.5 & 61.2 & 528.0 \\
\hline 15 & East Wall Concrete & 17 & 1187 & 12.7 & 146.5 & 61.2 & 244.4 \\
\hline 16 & East Wall Concrete & 12 & 1247 & 7.7 & 206.5 & 37.1 & 344.5 \\
\hline 17 & East Wall Concrete & 17 & 1187 & 12.7 & 146.5 & 61.2 & 244.4 \\
\hline 18 & East Wall Concrete & 12 & 1247 & 7.7 & 206.5 & 37.1 & 344.5 \\
\hline 19 & East Wall Concrete & 10 & 1321 & 5.7 & 280.5 & 27.4 & 467.9 \\
\hline 20 & East Wall Concrete & 21 & 1331 & 16.7 & 290.5 & 80.6 & 484.6 \\
\hline & & & & & Averages & 34.8 & 247.4 \\
\hline & & & & & StDev & 21.1 & 312.3 \\
\hline & & & & & Median & 32.2 & 296.9 \\
\hline & & & & & Max & 80.6 & 528.0 \\
\hline & & & & & Min & 3.3 & -978.8 \\
\hline & & & & & $*$ LBGR & 0.40 & \\
\hline
\end{tabular}


10-ER-A26-181

Final Status Survey, Room 109, Walls

\begin{tabular}{|c|c|c|c|c|c|c|c|}
\hline & & \multicolumn{2}{|c|}{ RAW DATA cpm } & \multicolumn{2}{|c|}{ BKG ADJUSTED cpm } & \multicolumn{2}{|c|}{$\mathrm{dpm} / 100 \mathrm{~cm} 2$} \\
\hline & & Alpha & Beta & Alpha & Beta & Alpha & Beta \\
\hline 1 & North Wall Concrete & 9 & 900 & 4.7 & -140.5 & 22.6 & -234.4 \\
\hline 2 & North Wall Concrete & 7 & 906 & 2.7 & -134.5 & 12.9 & -224.4 \\
\hline 3 & East Wall Concrete & 6 & 1119 & 1.7 & 78.5 & 8.1 & 130.9 \\
\hline 4 & East Wall Concrete & 8 & 1120 & 3.7 & 79.5 & 17.8 & 132.6 \\
\hline 5 & North Wall Metal & 7 & 816 & 2.4 & -515.7 & 11.4 & -860.4 \\
\hline 6 & East Wall Concrete & 3 & 1138 & -1.3 & 97.5 & -6.4 & 162.6 \\
\hline 7 & East Wall Concrete & 9 & 1236 & 4.7 & 195.5 & 22.6 & 326.1 \\
\hline 8 & East Wall Metal & 9 & 924 & 4.4 & -407.7 & 21.0 & -680.2 \\
\hline 9 & East Wall Concrete & 15 & 1239 & 10.7 & 198.5 & 51.6 & 331.1 \\
\hline 10 & South Wall Concrete & 4 & 1298 & -0.3 & 257.5 & -1.6 & 429.6 \\
\hline 11 & South Wall Concrete & 15 & 1163 & 10.7 & 122.5 & 51.6 & 204.3 \\
\hline 12 & South Wall Concrete & 4 & 1224 & -0.3 & 183.5 & -1.6 & 306.1 \\
\hline 13 & South Wall Concrete & 4 & 1254 & -0.3 & 213.5 & -1.6 & 356.1 \\
\hline 14 & South Wall Concrete & 6 & 1198 & 1.7 & 157.5 & 8.1 & 262.7 \\
\hline 15 & South Wall Concrete & 11 & 1181 & 6.7 & 140.5 & 32.2 & 234.4 \\
\hline 16 & South Wall Concrete & 6 & 1159 & 1.7 & 118.5 & 8.1 & 197.7 \\
\hline 17 & West Wall Concrete & 8 & 1211 & 3.7 & 170.5 & 17.8 & 284.4 \\
\hline 18 & West Wall Concrete & 12 & 1174 & 7.7 & 133.5 & 37.1 & 222.7 \\
\hline 19 & West Wall (Ramp) Concrete & 14 & 1223 & 9.7 & 182.5 & 46.7 & 304.4 \\
\hline 20 & West Wall (Ramp) Concrete & 12 & 1238 & 7.7 & 197.5 & 37.1 & 329.5 \\
\hline 21 & West Wall (Ramp) Concrete & 8 & 1277 & 3.7 & 236.5 & 17.8 & 394.5 \\
\hline 22 & West Wall (Ramp) Concrete & 11 & 1246 & 6.7 & 205.5 & 32.2 & 342.8 \\
\hline 23 & West Wall (Ramp) Concrete & 7 & 1282 & 2.7 & 241.5 & 12.9 & 402.9 \\
\hline 24 & West Wall (Ramp) Concrete & 19 & 1205 & 14.7 & 164.5 & 70.9 & 274.4 \\
\hline 25 & West Wall Concrete & 13 & 1175 & 8.7 & 134.5 & 41.9 & 224.3 \\
\hline 26 & West Wall Concrete & 17 & 1084 & 12.7 & 43.5 & 61.2 & 72.5 \\
\hline 27 & West Wall Concrete & 15 & 1132 & 10.7 & 91.5 & 51.6 & 152.6 \\
\hline
\end{tabular}




\begin{tabular}{|c|c|c|c|c|c|c|c|}
\hline 28 & North Wall Concrete & 13 & 1116 & 8.7 & 75.5 & 41.9 & 125.9 \\
\hline 29 & East Wall Metal & 7 & 632 & 2.4 & -699.7 & 11.4 & -1167.3 \\
\hline 30 & East Wall Concrete & 7 & 853 & 2.7 & -187.5 & 12.9 & -312.9 \\
\hline 31 & East Wall (Ramp) Concrete & 14 & 1162 & 9.7 & 121.5 & 46.7 & 202.7 \\
\hline & & & & & Averages & 25.7 & 94.5 \\
\hline & & & & & StDev & 20.3 & 381.1 \\
\hline & & & & & Median & 21.0 & 222.7 \\
\hline & & & & & Max & 70.9 & 429.6 \\
\hline & & & & & Min & -6.4 & -1167.3 \\
\hline & & & & & *LBGR & 0.28 & \\
\hline & & & & & \multicolumn{3}{|c|}{ *Negative vales not used in LGBR } \\
\hline
\end{tabular}

10-ER-A26-180

Final Status Survey, Room 109, Ceiling

\begin{tabular}{|c|c|c|c|c|c|c|c|}
\hline & & \multicolumn{2}{|c|}{ RAW DATA cpm } & \multicolumn{2}{|c|}{ BKG ADJUSTED cpm } & \multicolumn{2}{|c|}{$\mathrm{dpm} / 100 \mathrm{~cm} 2$} \\
\hline & & Alpha & Beta & Alpha & Beta & Alpha & Beta \\
\hline 1 & Ceiling Metal & 3 & 1185 & -1.7 & -146.7 & -8.0 & -244.7 \\
\hline 2 & Ceiling Metal & 4 & 1182 & -0.7 & -149.7 & -3.1 & -249.7 \\
\hline 3 & Ceiling Metal & 5 & 1253 & 0.4 & -78.7 & 1.7 & -131.3 \\
\hline 4 & Ceiling Metal & 4 & 1166 & -0.7 & -165.7 & -3.1 & -276.4 \\
\hline 5 & Ceiling Metal & 6 & 1207 & 1.4 & -124.7 & 6.5 & -208.0 \\
\hline 6 & Ceiling Metal & 7 & 1243 & 2.4 & -88.7 & 11.4 & -148.0 \\
\hline 7 & Ceiling Metal & 4 & 1215 & -0.7 & -116.7 & -3.1 & -194.7 \\
\hline 8 & Ceiling Metal & 2 & 1251 & -2.7 & -80.7 & -12.8 & -134.6 \\
\hline 9 & Ceiling Metal & 6 & 1145 & 1.4 & -186.7 & 6.5 & -311.5 \\
\hline & & & & & Averages & -0.5 & -211.0 \\
\hline & & & & & StDev & 7.7 & 64.7 \\
\hline & & & & & Median & -3.1 & -208.0 \\
\hline & & & & & Max & 11.4 & -131.3 \\
\hline & & & & & Min & -12.8 & -311.5 \\
\hline
\end{tabular}


10-ER-A26-183

\begin{tabular}{|c|c|c|}
\hline *LBGR & 0.00 & \\
\hline *Negative vales not used in LGBR
\end{tabular}

Final Status Survey, Room 109, Floor pg1

\begin{tabular}{|c|c|c|c|c|c|c|c|}
\hline & & \multicolumn{2}{|c|}{ RAW DATA cpm } & \multicolumn{2}{|c|}{ BKG ADJUSTED cpm } & \multicolumn{2}{|c|}{$\mathrm{dpm} / 100 \mathrm{~cm} 2$} \\
\hline & & Alpha & Beta & Alpha & Beta & Alpha & Beta \\
\hline 1 & Floor Concrete & 9 & 1273 & 4.7 & 232.5 & 22.6 & 387.8 \\
\hline 2 & Floor Concrete & 10 & 1286 & 5.7 & 245.5 & 27.4 & 409.5 \\
\hline 3 & Floor Concrete & 9 & 940 & 4.7 & -100.5 & 22.6 & -167.7 \\
\hline 4 & Floor Concrete & 13 & 909 & 8.7 & -131.5 & 41.9 & -219.4 \\
\hline 5 & Floor Concrete & 3 & 942 & -1.3 & -98.5 & -6.4 & -164.4 \\
\hline 6 & Floor Concrete & 7 & 922 & 2.7 & -118.5 & 12.9 & -197.7 \\
\hline 7 & Floor Concrete & 7 & 1386 & 2.7 & 345.5 & 12.9 & 576.4 \\
\hline 8 & Floor Concrete & 12 & 1419 & 7.7 & 378.5 & 37.1 & 631.4 \\
\hline 9 & Floor Concrete & 10 & 1322 & 5.7 & 281.5 & 27.4 & 469.6 \\
\hline 10 & Floor Concrete & 5 & 1400 & 0.7 & 359.5 & 3.3 & 599.7 \\
\hline 11 & Floor Concrete & 11 & 1438 & 6.7 & 397.5 & 32.2 & 663.1 \\
\hline 12 & Floor Concrete & 6 & 1439 & 1.7 & 398.5 & 8.1 & 664.8 \\
\hline 13 & Floor Concrete & 6 & 1483 & 1.7 & 442.5 & 8.1 & 738.2 \\
\hline 14 & Floor Concrete & 11 & 1505 & 6.7 & 464.5 & 32.2 & 774.9 \\
\hline 15 & Floor Concrete & 9 & 1539 & 4.7 & 498.5 & 22.6 & 831.6 \\
\hline 16 & Floor Concrete & 6 & 1601 & 1.7 & 560.5 & 8.1 & 935.1 \\
\hline 17 & Floor Concrete & 10 & 1625 & 5.7 & 584.5 & 27.4 & 975.1 \\
\hline 18 & Floor Concrete & 8 & 1530 & 3.7 & 489.5 & 17.8 & 816.6 \\
\hline 19 & Floor Concrete & 7 & 1494 & 2.7 & 453.5 & 12.9 & 756.5 \\
\hline 20 & Floor Concrete & 9 & 1565 & 4.7 & 524.5 & 22.6 & 875.0 \\
\hline 21 & Floor Concrete & 4 & 1547 & -0.3 & 506.5 & -1.6 & 845.0 \\
\hline 22 & Floor Concrete & 14 & 1497 & 9.7 & 456.5 & 46.7 & 761.6 \\
\hline 23 & Floor Concrete & 6 & 1578 & 1.7 & 537.5 & 8.1 & 896.7 \\
\hline 24 & Floor Concrete & 7 & 1541 & 2.7 & 500.5 & 12.9 & 835.0 \\
\hline 25 & Floor Concrete & 11 & 1581 & 6.7 & 540.5 & 32.2 & 901.7 \\
\hline 26 & Floor Concrete & 8 & 1637 & 3.7 & 596.5 & 17.8 & 995.1 \\
\hline 27 & Floor Concrete & 12 & 1524 & 7.7 & 483.5 & 37.1 & 806.6 \\
\hline
\end{tabular}




\begin{tabular}{|c|c|c|c|c|c|c|c|}
\hline 28 & Floor Concrete & 14 & 1552 & 9.7 & 511.5 & 46.7 & 853.3 \\
\hline 29 & Floor Concrete & 12 & 1571 & 7.7 & 530.5 & 37.1 & 885.0 \\
\hline 30 & Floor Concrete & 12 & 1514 & 7.7 & 473.5 & 37.1 & 789.9 \\
\hline 31 & Floor Concrete & 1 & 1544 & -3.3 & 503.5 & -16.1 & 840.0 \\
\hline 32 & Floor Concrete & 7 & 1415 & 2.7 & 374.5 & 12.9 & 624.7 \\
\hline 33 & Floor Concrete & 9 & 1598 & 4.7 & 557.5 & 22.6 & 930.1 \\
\hline 34 & Floor Concrete & 7 & 1458 & 2.7 & 417.5 & 12.9 & 696.5 \\
\hline & & & & & Averages & 20.6 & 647.6 \\
\hline & & & & & StDev & 14.9 & 343.5 \\
\hline & & & & & Median & 22.6 & 768.2 \\
\hline & & & & & Max & 46.7 & 995.1 \\
\hline & & & & & Min & -16.1 & -219.4 \\
\hline & & & & & *LBGR & 0.34 & \\
\hline & & & & & \multicolumn{3}{|c|}{ *Negative vales not used in LGBR } \\
\hline
\end{tabular}

10-ER-A26-183

Final Status Survey, Room 109, Floor pg3

\begin{tabular}{|c|c|c|c|c|c|c|c|}
\hline & & \multicolumn{2}{|c|}{ RAW DATA cpm } & \multicolumn{2}{|c|}{ BKG ADJUSTED cpm } & \multicolumn{2}{|c|}{$\mathrm{dpm} / 100 \mathrm{~cm} 2$} \\
\hline & & Alpha & Beta & Alpha & Beta & Alpha & Beta \\
\hline 35 & Floor Concrete & 5 & 1641 & 0.7 & 600.5 & 3.3 & 1001.8 \\
\hline 36 & Floor Concrete & 7 & 1686 & 2.7 & 645.5 & 12.9 & 1076.9 \\
\hline 37 & Floor Concrete & 10 & 1487 & 5.7 & 446.5 & 27.4 & 744.9 \\
\hline 38 & Floor Concrete & 9 & 1430 & 4.7 & 389.5 & 22.6 & 649.8 \\
\hline 39 & Floor Concrete & 5 & 1243 & 0.7 & 202.5 & 3.3 & 337.8 \\
\hline 40 & Floor Concrete & 6 & 1403 & 1.7 & 362.5 & 8.1 & 604.7 \\
\hline & & & & & Averages & 12.9 & 736.0 \\
\hline & & & & & StDev & 10.1 & 272.1 \\
\hline & & & & & Median & 10.5 & 697.3 \\
\hline & & & & & Max & 27.4 & 1076.9 \\
\hline & & & & & Min & 3.3 & 337.8 \\
\hline
\end{tabular}


10-ER-A26-194

\begin{tabular}{c|c|c|}
$*$ LBGR & 0.28 & \\
$*$ *Negative vales not used in LGBR
\end{tabular}

Final Status Survey, Room 111

\begin{tabular}{|c|c|c|c|c|c|c|c|}
\hline & & \multicolumn{2}{|c|}{ RAW DATA cpm } & \multicolumn{2}{|c|}{ BKG ADJUSTED cpm } & \multicolumn{2}{|c|}{$\mathrm{dpm} / 100 \mathrm{~cm} 2$} \\
\hline & & Alpha & Beta & Alpha & Beta & Alpha & Beta \\
\hline 1 & West Wall, Concrete (Electra 1120) & 7 & 1500 & 2.7 & 459.5 & 12.9 & 766.6 \\
\hline 2 & East Wall, Concrete (Electra 1120) & 14 & 1468 & 9.7 & 427.5 & 46.7 & 713.2 \\
\hline 3 & North Wall, Concrete (Electra 1120) & 7 & 1475 & 2.7 & 434.5 & 12.9 & 724.8 \\
\hline 4 & South Wall, Concrete (Electra 1120) & 6 & 1539 & 1.7 & 498.5 & 8.1 & 831.6 \\
\hline 5 & Wooden Beam (Electra 1120) & 7 & 1154 & 2.3 & -256.7 & 10.9 & -428.3 \\
\hline 6 & Wooden Beam (Electra 1120) & 8 & 1182 & 3.3 & -228.7 & 15.7 & -381.5 \\
\hline 7 & Floor, Concrete (Electra 1120) & 7 & 1757 & 2.7 & 716.5 & 12.9 & 1195.3 \\
\hline 8 & Floor, Concrete (Electra 1120) & 8 & 1604 & 3.7 & 563.5 & 17.8 & 940.1 \\
\hline 9 & Ceiling, Metal (Electra 1504) & 10 & 996 & 4.7 & -242.9 & 22.7 & -405.2 \\
\hline 12 & Ceiling Metal & 7 & 1325 & 1.7 & 86.2 & 8.2 & 143.7 \\
\hline & & & & & Averages & 16.9 & 410.0 \\
\hline & & & & & StDev & 11.4 & 619.9 \\
\hline & & & & & Median & 12.9 & 719.0 \\
\hline & & & & & Max & 46.7 & 1195.3 \\
\hline & & & & & Min & 8.1 & -428.3 \\
\hline & & & & & $*$ LBGR & 0.25 & \\
\hline & & & & & *Negative & vales not & in LGBR \\
\hline
\end{tabular}




\section{0-ER-A26-176}

Final Status Survey, Room 115, Floor, Ceiling and Walls

\begin{tabular}{|c|c|c|c|c|c|c|c|}
\hline & & \multicolumn{2}{|c|}{ RAW DATA cpm } & \multicolumn{2}{|c|}{ BKG ADJUSTED cpm } & \multicolumn{2}{|c|}{$\mathrm{dpm} / 100 \mathrm{~cm} 2$} \\
\hline & & Alpha & Beta & Alpha & Beta & Alpha & Beta \\
\hline 1 & Floor Concrete & 8 & 1365 & 3.7 & 324.5 & 17.8 & 541.3 \\
\hline 2 & South Wall Concrete & 9 & 1360 & 4.7 & 319.5 & 22.6 & 533.0 \\
\hline 3 & East Wall Concrete & 12 & 1406 & 7.7 & 365.5 & 37.1 & 609.7 \\
\hline 4 & North Wall Concrete & 10 & 1340 & 5.7 & 299.5 & 27.4 & 499.6 \\
\hline 5 & West Wall Concrete & 6 & 1356 & 1.7 & 315.5 & 8.1 & 526.3 \\
\hline 6 & Ceiling Metal & 7 & 1355 & 2.4 & 23.3 & 11.4 & 38.9 \\
\hline & & & & & Averages & 20.7 & 458.1 \\
\hline & & & & & StDev & 10.7 & 208.6 \\
\hline & & & & & Median & 20.2 & 529.7 \\
\hline & & & & & Max & 37.1 & 609.7 \\
\hline & & & & & Min & 8.1 & 38.9 \\
\hline & & & & & $*$ LBGR & 0.30 & \\
\hline & & & & & *Negative & ales not $\mathrm{u}$ & n LGBR \\
\hline
\end{tabular}

\begin{tabular}{|c|c|c|c|c|c|c|c|}
\hline & \multirow{2}{*}{$\begin{array}{l}\text { 10-ER-A26-178 } \\
\text { Final Status Survey, Room 116, Ceiling }\end{array}$} & \multicolumn{2}{|c|}{ RAW DATA cpm } & \multicolumn{2}{|c|}{ BKG ADJUSTED cpm } & \multicolumn{2}{|c|}{$\mathrm{dpm} / 100 \mathrm{~cm} 2$} \\
\hline & & Alpha & Beta & Alpha & Beta & Alpha & Beta \\
\hline 1 & Ceiling Metal & 11 & 1244 & 6.4 & -87.7 & 30.7 & -146.3 \\
\hline 2 & Ceiling Metal & 8 & 1222 & 3.4 & -109.7 & 16.2 & -183.0 \\
\hline 3 & Ceiling Metal & 11 & 1375 & 6.4 & 43.3 & 30.7 & 72.2 \\
\hline 4 & Ceiling Metal & 7 & 1284 & 2.4 & -47.7 & 11.4 & -79.6 \\
\hline 5 & Ceiling Metal & 8 & 1211 & 3.4 & -120.7 & 16.2 & -201.4 \\
\hline & & & & & Averages & 21.0 & -107.6 \\
\hline & & & & & StDev & 9.0 & 110.8 \\
\hline & & & & & Median & 16.2 & -146.3 \\
\hline & & & & & Max & 30.7 & 72.2 \\
\hline & & & & & Min & 11.4 & -201.4 \\
\hline & & & & & *LBGR & 0.2 & \\
\hline
\end{tabular}


${ }^{*}$ Negative vales not used in LGBR

10-ER-A26-179

Final Status Survey, Room 116, Walls

\begin{tabular}{|c|c|c|c|c|c|c|c|}
\hline & & \multirow{2}{*}{\multicolumn{2}{|c|}{ RAW DATA cpm }} & \multirow{2}{*}{\multicolumn{2}{|c|}{ BKG ADJUSTED cpm }} & \multirow{2}{*}{\multicolumn{2}{|c|}{$\mathrm{dpm} / 100 \mathrm{~cm} 2$}} \\
\hline & & & & & & & \\
\hline & & Alpha & Beta & Alpha & Beta & Alpha & Beta \\
\hline 1 & South Wall Concrete & 10 & 1345 & 5.7 & 304.5 & 27.4 & 508.0 \\
\hline 2 & South Wall Concrete & 9 & 1430 & 4.7 & 389.5 & 22.6 & 649.8 \\
\hline 3 & South Wall Concrete & 10 & 1339 & 5.7 & 298.5 & 27.4 & 498.0 \\
\hline 4 & South Wall Concrete & 7 & 1304 & 2.7 & 263.5 & 12.9 & 439.6 \\
\hline 5 & South Wall Concrete & 14 & 1333 & 9.7 & 292.5 & 46.7 & 487.9 \\
\hline 6 & South Wall Concrete & 13 & 1354 & 8.7 & 313.5 & 41.9 & 523.0 \\
\hline 7 & East Wall Concrete & 11 & 1336 & 6.7 & 295.5 & 32.2 & 493.0 \\
\hline 8 & East Wall Concrete & 14 & 1330 & 9.7 & 289.5 & 46.7 & 482.9 \\
\hline 9 & East Wall Metal & 8 & 1260 & 3.4 & -71.7 & 16.2 & -119.6 \\
\hline 10 & East Wall Concrete & 6 & 1226 & 1.7 & 185.5 & 8.1 & 309.4 \\
\hline 11 & North Wall Metal & 8 & 1182 & 3.4 & -149.7 & 16.2 & -249.7 \\
\hline 12 & North Wall Metal & 9 & 1293 & 4.4 & -38.7 & 21.0 & -64.6 \\
\hline 13 & North Wall Concrete & 12 & 1003 & 7.7 & -37.5 & 37.1 & -62.6 \\
\hline 14 & North Wall Concrete & 11 & 980 & 6.7 & -60.5 & 32.2 & -101.0 \\
\hline 15 & North Wall Concrete & 7 & 1392 & 2.7 & 351.5 & 12.9 & 586.4 \\
\hline 16 & North Wall Concrete & 11 & 1284 & 6.7 & 243.5 & 32.2 & 406.2 \\
\hline 17 & West Wall Concrete & 14 & 1303 & 9.7 & 262.5 & 46.7 & 437.9 \\
\hline 18 & West Wall Concrete & 13 & 1472 & 8.7 & 431.5 & 41.9 & 719.8 \\
\hline 19 & West Wall Metal & 6 & 1179 & 1.4 & -152.7 & 6.5 & -254.8 \\
\hline 20 & West Wall Metal & 12 & 1058 & 7.4 & -273.7 & 35.5 & -456.6 \\
\hline 21 & South Wall Metal & 12 & 1068 & 7.4 & -263.7 & 35.5 & -439.9 \\
\hline 22 & South Wall Metal & 10 & 1098 & 5.4 & -233.7 & 25.8 & -389.9 \\
\hline & & & & & Averages & 28.5 & 200.1 \\
\hline & & & & & StDev & 12.6 & 393.8 \\
\hline & & & & & Median & 29.8 & 422.0 \\
\hline & & & & & Max & 46.7 & 719.8 \\
\hline & & & & & Min & 6.5 & -456.6 \\
\hline
\end{tabular}




\begin{tabular}{|c|c|c|}
\hline$*$ LBGR & 0.32 & \\
\hline *Negative vales not used in LGBR \\
\hline
\end{tabular}

10-ER-A26-177

Final Status Survey, Room 116, Floor

\begin{tabular}{|c|c|c|c|c|c|c|c|}
\hline & & \multicolumn{2}{|c|}{ RAW DATA cpm } & \multicolumn{2}{|c|}{ BKG ADJUSTED cpm } & \multicolumn{2}{|c|}{$\mathrm{dpm} / 100 \mathrm{~cm} 2$} \\
\hline & & Alpha & Beta & Alpha & Beta & Alpha & Beta \\
\hline 1 & Floor Concrete & 10 & 1668 & 5.7 & 627.5 & 27.4 & 1046.8 \\
\hline 2 & Floor Concrete & 9 & 1666 & 4.7 & 625.5 & 22.6 & 1043.5 \\
\hline 3 & Floor Concrete & 9 & 1653 & 4.7 & 612.5 & 22.6 & 1021.8 \\
\hline 4 & Floor Concrete & 6 & 1649 & 1.7 & 608.5 & 8.1 & 1015.1 \\
\hline 5 & Floor Concrete & 5 & 1622 & 0.7 & 581.5 & 3.3 & 970.1 \\
\hline 6 & Floor Concrete & 8 & 1651 & 3.7 & 610.5 & 17.8 & 1018.5 \\
\hline 7 & Floor Concrete & 3 & 1650 & -1.3 & 609.5 & -6.4 & 1016.8 \\
\hline 8 & Floor Concrete & 3 & 1636 & -1.3 & 595.5 & -6.4 & 993.5 \\
\hline 9 & Floor Concrete & 7 & 1690 & 2.7 & 649.5 & 12.9 & 1083.5 \\
\hline 10 & Floor Concrete & 8 & 1683 & 3.7 & 642.5 & 17.8 & 1071.9 \\
\hline 11 & Floor Concrete & 11 & 1650 & 6.7 & 609.5 & 32.2 & 1016.8 \\
\hline 12 & Floor Concrete & 7 & 1631 & 2.7 & 590.5 & 12.9 & 985.1 \\
\hline 13 & Floor Concrete & 6 & 1600 & 1.7 & 559.5 & 8.1 & 933.4 \\
\hline 14 & Floor Concrete & 7 & 1601 & 2.7 & 560.5 & 12.9 & 935.1 \\
\hline 15 & Floor Concrete & 10 & 1595 & 5.7 & 554.5 & 27.4 & 925.1 \\
\hline 16 & Floor Concrete & 10 & 1572 & 5.7 & 531.5 & 27.4 & 886.7 \\
\hline 17 & Floor Concrete & 6 & 1567 & 1.7 & 526.5 & 8.1 & 878.3 \\
\hline 18 & Floor Concrete & 7 & 1604 & 2.7 & 563.5 & 12.9 & 940.1 \\
\hline & & & & & Averages & 14.5 & 987.9 \\
\hline & & & & & StDev & 11.1 & 60.3 \\
\hline & & & & & Median & 12.9 & 1004.3 \\
\hline & & & & & Max & 32.2 & 1083.5 \\
\hline & & & & & Min & -6.4 & 878.3 \\
\hline
\end{tabular}




\section{0-ER-A26-186}

*LBGR

0.34

*Negative vales not used in LGBR

Final Status Survey, Room 117

\begin{tabular}{|c|c|c|c|c|c|c|c|}
\hline & & \multicolumn{2}{|c|}{ RAW DATA cpm } & \multicolumn{2}{|c|}{ BKG ADJUSTED cpm } & \multicolumn{2}{|c|}{$\mathrm{dpm} / 100 \mathrm{~cm} 2$} \\
\hline & & Alpha & Beta & Alpha & Beta & Alpha & Beta \\
\hline 1 & Floor Concrete & 5 & 1752 & 0.7 & 711.5 & 3.3 & 1187.0 \\
\hline 2 & Floor Concrete & 6 & 1690 & 1.7 & 649.5 & 8.1 & 1083.5 \\
\hline 3 & East Wall Concrete Block & 5 & 1355 & 0.2 & 658.4 & 0.8 & 1098.3 \\
\hline 4 & East Wall Concrete Block & 13 & 1421 & 8.2 & 724.4 & 39.4 & 1208.5 \\
\hline 5 & North Wall Concrete Block & 11 & 1419 & 6.2 & 722.4 & 29.8 & 1205.1 \\
\hline 6 & North Wall Concrete Block & 5 & 1479 & 0.2 & 782.4 & 0.8 & 1305.2 \\
\hline 7 & West Wall Concrete Block & 9 & 1436 & 4.2 & 739.4 & 20.1 & 1233.5 \\
\hline 8 & West Wall Concrete Block & 8 & 1485 & 3.2 & 788.4 & 15.3 & 1315.2 \\
\hline 9 & South Wall Concrete Block & 11 & 1436 & 6.2 & 739.4 & 29.8 & 1233.5 \\
\hline 10 & South Wall Concrete Block & 10 & 1347 & 5.2 & 650.4 & 24.9 & 1085.0 \\
\hline 11 & Ceiling Metal & 5 & 1308 & 0.4 & -23.7 & 1.7 & -39.5 \\
\hline 12 & Ceiling Metal & 7 & 1325 & 2.4 & -6.7 & 11.4 & -11.2 \\
\hline & & & & & Averages & 15.4 & 992.0 \\
\hline & & & & & StDev & 13.3 & 481.3 \\
\hline & & & & & Median & 13.3 & 1196.1 \\
\hline & & & & & Max & 39.4 & 1315.2 \\
\hline & & & & & Min & 0.8 & -39.5 \\
\hline & & & & & $*$ LBGR & 0.35 & \\
\hline & & & & & *Negative & vales not u & in LGBR \\
\hline
\end{tabular}


10-ER-A26-193

Final Status Survey, Basement Room \# 1, Ceiling

\begin{tabular}{|c|c|c|c|c|c|c|c|}
\hline & & \multicolumn{2}{|c|}{ RAW DATA cpm } & \multicolumn{2}{|c|}{ BKG ADJUSTED cpm } & \multicolumn{2}{|c|}{$\mathrm{dpm} / 100 \mathrm{~cm} 2$} \\
\hline & & Alpha & Beta & Alpha & Beta & Alpha & Beta \\
\hline 1 & Ceiling, Concrete & 10 & 1273 & 5.7 & 232.5 & 27.4 & 387.8 \\
\hline 2 & Ceiling, Concrete & 16 & 1266 & 11.7 & 225.5 & 56.4 & 376.2 \\
\hline 3 & Ceiling, Concrete & 13 & 1326 & 8.7 & 285.5 & 41.9 & 476.3 \\
\hline & & & & & Averages & 41.9 & 413.4 \\
\hline & & & & & StDev & 14.5 & 54.7 \\
\hline & & & & & Median & 41.9 & 387.8 \\
\hline & & & & & Max & 56.4 & 476.3 \\
\hline & & & & & Min & 27.4 & 376.2 \\
\hline & & & & & *LBGR & 0.50 & \\
\hline & & & & & \multicolumn{3}{|c|}{ *Negative vales not used in LGBR } \\
\hline
\end{tabular}

\begin{tabular}{|c|c|c|c|c|c|c|c|}
\hline & \multirow{2}{*}{$\begin{array}{l}\text { 10-ER-A26-191 } \\
\text { Final Status Survey, Basement Room \# 1, Floor }\end{array}$} & \multicolumn{2}{|c|}{ RAW DATA cpm } & \multicolumn{2}{|c|}{ BKG ADJUSTED cpm } & \multicolumn{2}{|c|}{$\mathrm{dpm} / 100 \mathrm{~cm} 2$} \\
\hline & & Alpha & Beta & Alpha & Beta & Alpha & Beta \\
\hline 1 & Floor, Concrete & 15 & 1465 & 10.7 & 424.5 & 51.6 & 708.2 \\
\hline 2 & Floor, Concrete & 22 & 1419 & 17.7 & 378.5 & 85.4 & 631.4 \\
\hline 3 & Floor, Concrete & 16 & 1324 & 11.7 & 283.5 & 56.4 & 472.9 \\
\hline 4 & Floor, Concrete & 14 & 1531 & 9.7 & 490.5 & 46.7 & 818.3 \\
\hline 5 & Floor, Concrete & 14 & 1466 & 9.7 & 425.5 & 46.7 & 709.8 \\
\hline 6 & Floor, Concrete & 14 & 1375 & 9.7 & 334.5 & 46.7 & 558.0 \\
\hline 7 & Floor, Concrete & 12 & 1421 & 7.7 & 380.5 & 37.1 & 634.8 \\
\hline 8 & Floor, Concrete & 14 & 1470 & 9.7 & 429.5 & 46.7 & 716.5 \\
\hline & & & & & Averages & 52.2 & 656.2 \\
\hline & & & & & StDev & 14.5 & 106.8 \\
\hline & & & & & Median & 46.7 & 671.5 \\
\hline & & & & & Max & 85.4 & 818.3 \\
\hline
\end{tabular}




\section{0-ER-A26-192}

\begin{tabular}{|c|c|c|} 
Min & 37.1 & 472.9 \\
\hline *LBGR & 0.65 & \\
\hline \multicolumn{3}{|c|}{ * Negative vales not used in LGBR } \\
\hline
\end{tabular}

Final Status Survey, Basement Room \# 1, Walls

\begin{tabular}{|c|c|c|c|c|c|c|c|}
\hline & & \multicolumn{2}{|c|}{ RAW DATA cpm } & \multicolumn{2}{|c|}{ BKG ADJUSTED cpm } & \multicolumn{2}{|c|}{$\mathrm{dpm} / 100 \mathrm{~cm} 2$} \\
\hline & & \multirow{2}{*}{$\begin{array}{c}\text { Alpha } \\
23\end{array}$} & \multirow{2}{*}{$\begin{array}{l}\text { Beta } \\
1224\end{array}$} & \multirow{2}{*}{$\begin{array}{c}\text { Alpha } \\
18.7\end{array}$} & \multirow{2}{*}{$\begin{array}{c}\text { Beta } \\
183.5\end{array}$} & \multirow{2}{*}{$\begin{array}{c}\text { Alpha } \\
90.2\end{array}$} & \multirow{2}{*}{$\begin{array}{c}\text { Beta } \\
306.1\end{array}$} \\
\hline 1 & West Wall, Concrete & & & & & & \\
\hline 2 & West Wall, Concrete & 13 & 1207 & 8.7 & 166.5 & 41.9 & 277.7 \\
\hline 3 & North Wall, Concrete & 17 & 894 & 12.7 & -146.5 & 61.2 & -244.5 \\
\hline 4 & North Wall, Concrete & 14 & 965 & 9.7 & -75.5 & 46.7 & -126.0 \\
\hline 5 & North Wall, Metal & 4 & 841 & -0.7 & -490.7 & -3.1 & -818.7 \\
\hline 6 & East Wall, Concrete & 18 & 1183 & 13.7 & 142.5 & 66.1 & 237.7 \\
\hline 7 & East Wall, Concrete & 13 & 1225 & 8.7 & 184.5 & 41.9 & 307.8 \\
\hline 8 & South Wall, Concrete & 13 & 1159 & 8.7 & 118.5 & 41.9 & 197.7 \\
\hline 9 & South Wall (Door), Metal & 14 & 948 & 9.4 & -383.7 & 45.2 & -640.1 \\
\hline 10 & South Wall, Concrete & 10 & 1235 & 5.7 & 194.5 & 27.4 & 324.4 \\
\hline 11 & South Wall, Concrete & 14 & 1120 & 9.7 & 79.5 & 46.7 & 132.6 \\
\hline \multirow[t]{8}{*}{12} & North Wall, Metal & 1 & 630 & -3.7 & -701.7 & -17.6 & -1170.7 \\
\hline & & & & & Averages & 40.7 & -101.3 \\
\hline & & & & & StDev & 28.8 & 512.3 \\
\hline & & & & & Median & 43.5 & 165.1 \\
\hline & & & & & Max & 90.2 & 324.4 \\
\hline & & & & & Min & -17.6 & -1170.7 \\
\hline & & & & & $*$ LBGR & 0.41 & \\
\hline & & & & & *Negative & vales not & in LGBR \\
\hline
\end{tabular}


10-ER-A26-196

Final Status Survey, Basement Room \# 4, Ceiling

\begin{tabular}{|c|c|c|c|c|c|c|c|}
\hline & & \multicolumn{2}{|c|}{ RAW DATA cpm } & \multicolumn{2}{|c|}{ BKG ADJUSTED cpm } & \multicolumn{2}{|c|}{$\mathrm{dpm} / 100 \mathrm{~cm} 2$} \\
\hline & & Alpha & Beta & Alpha & Beta & Alpha & Beta \\
\hline 1 & Ceiling, Concrete & 16 & 1442 & 11.7 & 401.5 & 56.4 & 669.8 \\
\hline 2 & Ceiling, Concrete & 20 & 1309 & 15.7 & 268.5 & 75.7 & 447.9 \\
\hline 3 & Ceiling, Concrete & 12 & 1399 & 7.7 & 358.5 & 37.1 & 598.1 \\
\hline 4 & Ceiling, Concrete & 8 & 1455 & 3.7 & 414.5 & 17.8 & 691.5 \\
\hline 5 & Ceiling, Concrete & 14 & 1473 & 9.7 & 432.5 & 46.7 & 721.5 \\
\hline 6 & Ceiling, Concrete & 9 & 1430 & 4.7 & 389.5 & 22.6 & 649.8 \\
\hline 7 & Ceiling, Concrete & 14 & 1480 & 9.7 & 439.5 & 46.7 & 733.2 \\
\hline 8 & Ceiling, Concrete & 17 & 1387 & 12.7 & 346.5 & 61.2 & 578.0 \\
\hline 9 & Ceiling, Concrete & 17 & 1509 & 12.7 & 468.5 & 61.2 & 781.6 \\
\hline 10 & Ceiling, Concrete & 9 & 1425 & 4.7 & 384.5 & 22.6 & 641.4 \\
\hline & & & & & Averages & 44.8 & 651.3 \\
\hline & & & & & StDev & 19.5 & 94.4 \\
\hline & & & & & Median & 46.7 & 659.8 \\
\hline & & & & & Max & 75.7 & 781.6 \\
\hline & & & & & Min & 17.8 & 447.9 \\
\hline & & & & & *LBGR & 0.58 & \\
\hline & & & & & ${ }^{*}$ Negative & vales not & n LGBR \\
\hline
\end{tabular}




\begin{tabular}{|c|c|c|c|c|c|c|c|}
\hline & \multirow{2}{*}{$\begin{array}{l}\text { 10-ER-A26-197 } \\
\text { Final Status Survey, Basement Room \# 4, Floor }\end{array}$} & \multicolumn{2}{|c|}{ RAW DATA cpm } & \multicolumn{2}{|c|}{ BKG ADJUSTED cpm } & \multicolumn{2}{|c|}{$\mathrm{dpm} / 100 \mathrm{~cm} 2$} \\
\hline & & Alpha & Beta & Alpha & Beta & Alpha & Beta \\
\hline 1 & Floor, Concrete & 9 & 1304 & 4.7 & 263.5 & 22.6 & 439.6 \\
\hline 2 & Floor, Concrete & 9 & 1565 & 4.7 & 524.5 & 22.6 & 875.0 \\
\hline 3 & Floor, Concrete & 10 & 1508 & 5.7 & 467.5 & 27.4 & 779.9 \\
\hline 4 & Floor, Concrete & 11 & 1509 & 6.7 & 468.5 & 32.2 & 781.6 \\
\hline 5 & Floor, Concrete & 7 & 1447 & 2.7 & 406.5 & 12.9 & 678.1 \\
\hline 6 & Floor, Concrete & 5 & 1486 & 0.7 & 445.5 & 3.3 & 743.2 \\
\hline 7 & Floor, Concrete & 4 & 1386 & -0.3 & 345.5 & -1.6 & 576.4 \\
\hline 8 & Floor, Concrete & 14 & 1467 & 9.7 & 426.5 & 46.7 & 711.5 \\
\hline 9 & Floor, Concrete & 13 & 1525 & 8.7 & 484.5 & 41.9 & 808.3 \\
\hline 10 & Floor, Concrete & 6 & 1439 & 1.7 & 398.5 & 8.1 & 664.8 \\
\hline 11 & Floor, Concrete & 11 & 1410 & 6.7 & 369.5 & 32.2 & 616.4 \\
\hline 12 & Floor, Concrete & 13 & 1541 & 8.7 & 500.5 & 41.9 & 835.0 \\
\hline 13 & Floor, Concrete & 12 & 1444 & 7.7 & 403.5 & 37.1 & 673.1 \\
\hline 14 & Floor, Concrete & 10 & 1423 & 5.7 & 382.5 & 27.4 & 638.1 \\
\hline 15 & Floor, Concrete & 8 & 1513 & 3.7 & 472.5 & 17.8 & 788.2 \\
\hline 16 & Floor, Concrete & 9 & 1477 & 4.7 & 436.5 & 22.6 & 728.2 \\
\hline 17 & Floor, Concrete & 9 & 1448 & 4.7 & 407.5 & 22.6 & 679.8 \\
\hline 18 & Floor, Concrete & 18 & 1449 & 13.7 & 408.5 & 66.1 & 681.5 \\
\hline 19 & Floor, Concrete & 12 & 1440 & 7.7 & 399.5 & 37.1 & 666.5 \\
\hline 20 & Floor, Concrete & 8 & 1422 & 3.7 & 381.5 & 17.8 & 636.4 \\
\hline & & & & & Averages & 26.9 & 700.1 \\
\hline & & & & & StDev & 15.9 & 99.1 \\
\hline & & & & & Median & 25.0 & 680.6 \\
\hline & & & & & Max & 66.1 & 875.0 \\
\hline & & & & & Min & -1.6 & 439.6 \\
\hline
\end{tabular}




\begin{tabular}{|c|c|c|}
\hline *LBGR & 0.41 & \\
\hline *Negative vales not used in LGBR
\end{tabular}

10-ER-A26-195

Final Status Survey, Basement Room \# 4, Walls

\begin{tabular}{|c|c|c|c|c|c|c|c|}
\hline & & \multicolumn{2}{|c|}{ RAW DATA cpm } & \multicolumn{2}{|c|}{ BKG ADJUSTED cpm } & \multicolumn{2}{|c|}{$\mathrm{dpm} / 100 \mathrm{~cm} 2$} \\
\hline & & Alpha & Beta & Alpha & Beta & Alpha & Beta \\
\hline 1 & West Wall, Concrete & 24 & 1221 & 19.7 & 180.5 & 95.0 & 301.1 \\
\hline 2 & West Wall, Concrete & 20 & 1209 & 15.7 & 168.5 & 75.7 & 281.1 \\
\hline 3 & West Wall, Concrete & 6 & 1265 & 1.7 & 224.5 & 8.1 & 374.5 \\
\hline 4 & West Wall, Concrete & 20 & 1220 & 15.7 & 179.5 & 75.7 & 299.4 \\
\hline 5 & North Wall, Concrete & 19 & 1266 & 14.7 & 225.5 & 70.9 & 376.2 \\
\hline 6 & North Wall, Concrete & 10 & 1154 & 5.7 & 113.5 & 27.4 & 189.3 \\
\hline 7 & North Wall, Concrete & 20 & 1196 & 15.7 & 155.5 & 75.7 & 259.4 \\
\hline 8 & North Wall, Concrete & 12 & 1276 & 7.7 & 235.5 & 37.1 & 392.9 \\
\hline 9 & North Wall, Concrete & 17 & 1172 & 12.7 & 131.5 & 61.2 & 219.3 \\
\hline 10 & North Wall, Concrete & 7 & 1197 & 2.7 & 156.5 & 12.9 & 261.1 \\
\hline 11 & East Wall, Concrete & 8 & 1198 & 3.7 & 157.5 & 17.8 & 262.7 \\
\hline 12 & East Wall, Concrete & 16 & 1272 & 11.7 & 231.5 & 56.4 & 386.2 \\
\hline 13 & East Wall, Concrete & 20 & 1288 & 15.7 & 247.5 & 75.7 & 412.9 \\
\hline 14 & East Wall, Concrete & 17 & 1301 & 12.7 & 260.5 & 61.2 & 434.6 \\
\hline 15 & South Wall, Concrete & 12 & 1355 & 7.7 & 314.5 & 37.1 & 524.6 \\
\hline 16 & South Wall, Concrete & 22 & 1261 & 17.7 & 220.5 & 85.4 & 367.8 \\
\hline 17 & South Wall, Concrete & 12 & 1241 & 7.7 & 200.5 & 37.1 & 334.5 \\
\hline 18 & South Wall, Concrete & 15 & 1220 & 10.7 & 179.5 & 51.6 & 299.4 \\
\hline 19 & South Wall, Concrete & 14 & 1250 & 9.7 & 209.5 & 46.7 & 349.5 \\
\hline & & & & & Averages & 53.1 & 333.0 \\
\hline & & & & & StDev & 25.5 & 81.6 \\
\hline & & & & & Median & 56.4 & 334.5 \\
\hline & & & & & Max & 95.0 & 524.6 \\
\hline
\end{tabular}


10-ER-A26-205

Final Status Survey, Rooms 112, 113, 114

Ceiling

\begin{tabular}{|c|c|c|c|c|c|c|c|}
\hline & 10-ER-A26-205 & \multicolumn{2}{|c|}{ RAW DATA cpm } & \multicolumn{2}{|c|}{ BKG ADJUSTED cpm } & \multicolumn{2}{|c|}{$\mathrm{dpm} / 100 \mathrm{~cm} 2$} \\
\hline & $\begin{array}{l}\text { Final Status Survey, Rooms 112, 113, } 114 \\
\text { Ceiling }\end{array}$ & \multirow{2}{*}{$\begin{array}{c}\text { Alpha } \\
5\end{array}$} & \multirow{2}{*}{$\begin{array}{l}\text { Beta } \\
1100\end{array}$} & \multirow{2}{*}{$\begin{array}{c}\text { Alpha } \\
-0.3\end{array}$} & \multirow{2}{*}{$\begin{array}{c}\text { Beta } \\
-138.9 \\
\end{array}$} & \multirow{2}{*}{$\begin{array}{c}\text { Alpha } \\
-1.4\end{array}$} & \multirow{2}{*}{$\begin{array}{c}\text { Beta } \\
-231.6\end{array}$} \\
\hline 1 & Ceiling, Metal & & & & & & \\
\hline 2 & Ceiling, Metal & 6 & 1209 & 0.7 & -29.8 & 3.4 & -49.8 \\
\hline 3 & Ceiling, Metal & 6 & 1221 & 0.7 & -17.8 & 3.4 & -29.8 \\
\hline 4 & Ceiling, Metal & 15 & 1252 & 9.7 & 13.2 & 46.9 & 21.9 \\
\hline & \multirow{9}{*}{$\begin{array}{l}\text { 10-ER-A26-204 } \\
\text { Final Status Survey, Rooms 112, 113, } 114 \\
\text { Floors }\end{array}$} & & & & Averages & 13.0 & -72.3 \\
\hline & & & & & StDev & 22.7 & 110.4 \\
\hline & & & & & Median & 3.4 & -39.8 \\
\hline & & & & & Max & 46.9 & 21.9 \\
\hline & & & & & Min & -1.4 & -231.6 \\
\hline & & & & & *LBGR & 0.13 & \\
\hline & & & & & \multicolumn{3}{|c|}{ *Negative vales not used in LGBR } \\
\hline & & \multicolumn{2}{|c|}{ RAW DATA cpm } & \multicolumn{2}{|c|}{ BKG ADJUSTED cpm } & \multicolumn{2}{|c|}{$\mathrm{dpm} / 100 \mathrm{~cm} 2$} \\
\hline & & Alpha & Beta & Alpha & Beta & Alpha & Beta \\
\hline 1 & Floor, Concrete & 10 & 1412 & 5.7 & 371.5 & 27.4 & 619.7 \\
\hline 2 & Floor, Concrete & 11 & 1502 & 6.7 & 461.5 & 32.2 & 769.9 \\
\hline 3 & Floor, Concrete & 8 & 1396 & 3.7 & 355.5 & 17.8 & 593.1 \\
\hline 4 & Floor, Concrete & 13 & 1712 & 8.7 & 671.5 & 41.9 & 1120.2 \\
\hline 5 & Floor, Concrete & 10 & 1461 & 5.7 & 420.5 & 27.4 & 701.5 \\
\hline 6 & Floor, Concrete & 9 & 1653 & 4.7 & 612.5 & 22.6 & 1021.8 \\
\hline 7 & Floor, Concrete & 12 & 1592 & 7.7 & 551.5 & 37.1 & 920.0 \\
\hline 8 & Floor, Concrete & 12 & 1540 & 7.7 & 499.5 & 37.1 & 833.3 \\
\hline & & & & & Averages & 30.4 & 822.4 \\
\hline & & & & & StDev & 8.1 & 188.7 \\
\hline & & & & & Median & 29.8 & 801.6 \\
\hline & & & & & Max & 41.9 & 1120.2 \\
\hline
\end{tabular}

\begin{tabular}{|c|c|c|c|c|c|c|c|}
\hline & 10-ER-A26-205 & \multicolumn{2}{|c|}{ RAW DATA cpm } & \multicolumn{2}{|c|}{ BKG ADJUSTED cpm } & \multicolumn{2}{|c|}{$\mathrm{dpm} / 100 \mathrm{~cm} 2$} \\
\hline & $\begin{array}{l}\text { Final Status Survey, Rooms 112, 113, } 114 \\
\text { Ceiling }\end{array}$ & \multirow{2}{*}{$\begin{array}{c}\text { Alpha } \\
5\end{array}$} & \multirow{2}{*}{$\begin{array}{l}\text { Beta } \\
1100\end{array}$} & \multirow{2}{*}{$\begin{array}{c}\text { Alpha } \\
-0.3\end{array}$} & \multirow{2}{*}{$\begin{array}{c}\text { Beta } \\
-138.9 \\
\end{array}$} & \multirow{2}{*}{$\begin{array}{c}\text { Alpha } \\
-1.4\end{array}$} & \multirow{2}{*}{$\begin{array}{c}\text { Beta } \\
-231.6\end{array}$} \\
\hline 1 & Ceiling, Metal & & & & & & \\
\hline 2 & Ceiling, Metal & 6 & 1209 & 0.7 & -29.8 & 3.4 & -49.8 \\
\hline 3 & Ceiling, Metal & 6 & 1221 & 0.7 & -17.8 & 3.4 & -29.8 \\
\hline 4 & Ceiling, Metal & 15 & 1252 & 9.7 & 13.2 & 46.9 & 21.9 \\
\hline & \multirow{9}{*}{$\begin{array}{l}\text { 10-ER-A26-204 } \\
\text { Final Status Survey, Rooms 112, 113, } 114 \\
\text { Floors }\end{array}$} & & & & Averages & 13.0 & -72.3 \\
\hline & & & & & StDev & 22.7 & 110.4 \\
\hline & & & & & Median & 3.4 & -39.8 \\
\hline & & & & & Max & 46.9 & 21.9 \\
\hline & & & & & Min & -1.4 & -231.6 \\
\hline & & & & & *LBGR & 0.13 & \\
\hline & & & & & \multicolumn{3}{|c|}{ *Negative vales not used in LGBR } \\
\hline & & \multicolumn{2}{|c|}{ RAW DATA cpm } & \multicolumn{2}{|c|}{ BKG ADJUSTED cpm } & \multicolumn{2}{|c|}{$\mathrm{dpm} / 100 \mathrm{~cm} 2$} \\
\hline & & Alpha & Beta & Alpha & Beta & Alpha & Beta \\
\hline 1 & Floor, Concrete & 10 & 1412 & 5.7 & 371.5 & 27.4 & 619.7 \\
\hline 2 & Floor, Concrete & 11 & 1502 & 6.7 & 461.5 & 32.2 & 769.9 \\
\hline 3 & Floor, Concrete & 8 & 1396 & 3.7 & 355.5 & 17.8 & 593.1 \\
\hline 4 & Floor, Concrete & 13 & 1712 & 8.7 & 671.5 & 41.9 & 1120.2 \\
\hline 5 & Floor, Concrete & 10 & 1461 & 5.7 & 420.5 & 27.4 & 701.5 \\
\hline 6 & Floor, Concrete & 9 & 1653 & 4.7 & 612.5 & 22.6 & 1021.8 \\
\hline 7 & Floor, Concrete & 12 & 1592 & 7.7 & 551.5 & 37.1 & 920.0 \\
\hline 8 & Floor, Concrete & 12 & 1540 & 7.7 & 499.5 & 37.1 & 833.3 \\
\hline & & & & & Averages & 30.4 & 822.4 \\
\hline & & & & & StDev & 8.1 & 188.7 \\
\hline & & & & & Median & 29.8 & 801.6 \\
\hline & & & & & Max & 41.9 & 1120.2 \\
\hline
\end{tabular}

0.60

*Negative vales not used in LGBR 


\begin{tabular}{|c|c|c|}
\hline Min & 17.8 & 593.1 \\
\hline *LBGR & 0.47 & \\
\hline
\end{tabular}

10-ER-A26-203

Final Status Survey, Rooms 112, 113, 114 Walls

\begin{tabular}{|c|c|c|c|c|c|c|c|}
\hline & & \multicolumn{2}{|c|}{ RAW DATA cpm } & \multicolumn{2}{|c|}{ BKG ADJUSTED cpm } & \multicolumn{2}{|c|}{$\mathrm{dpm} / 100 \mathrm{~cm} 2$} \\
\hline & & Alpha & Beta & Alpha & Beta & Alpha & Beta \\
\hline 1 & Room 114, East Wall, Cinderblock & 10 & 1393 & 5.2 & 696.4 & 24.9 & 1161.7 \\
\hline 2 & Room 113, East Wall, Cinderblock & 19 & 1420 & 14.2 & 723.4 & 68.4 & 1206.8 \\
\hline 3 & Room 112, East Wall, Cinderblock & 10 & 1420 & 5.2 & 723.4 & 24.9 & 1206.8 \\
\hline 4 & Room 112, South Wall, Cinderblock & 12 & 1329 & 7.2 & 632.4 & 34.6 & 1055.0 \\
\hline 5 & Room 112, South Wall, Cinderblock & 5 & 1295 & 0.2 & 598.4 & 0.8 & 998.2 \\
\hline 6 & Room 112, West Wall, Cinderblock & 12 & 1428 & 7.2 & 731.4 & 34.6 & 1220.1 \\
\hline 7 & Room 112, North Wall, Cinderblock & 8 & 1441 & 3.2 & 744.4 & 15.3 & 1241.8 \\
\hline 8 & Room 112, North Wall, Cinderblock & 16 & 1256 & 11.2 & 559.4 & 53.9 & 933.2 \\
\hline 9 & Room 112, North Wall, Cinderblock & 7 & 1313 & 2.2 & 616.4 & 10.4 & 1028.3 \\
\hline 10 & Room 113, North Wall, Cinderblock & 7 & 1359 & 2.2 & 662.4 & 10.4 & 1105.0 \\
\hline 11 & Room 114, South Wall, Cinderblock & 12 & 1381 & 7.2 & 684.4 & 34.6 & 1141.7 \\
\hline 12 & Room 113, West Wall, Cinderblock & 11 & 1546 & 6.2 & 849.4 & 29.8 & 1417.0 \\
\hline & & & & & Averages & 28.5 & 1143.0 \\
\hline & & & & & StDev & 19.0 & 130.2 \\
\hline & & & & & Median & 27.3 & 1151.7 \\
\hline & & & & & Max & 68.4 & 1417.0 \\
\hline & & & & & Min & 0.8 & 933.2 \\
\hline & & & & & $*$ LBGR & 0.51 & \\
\hline & & & & & \multicolumn{3}{|c|}{${ }^{*}$ Negative vales not used in LGBR } \\
\hline
\end{tabular}


10-ER-A26-228

Final Status Survey, Room 110, Floor, Ceiling and Walls

\begin{tabular}{|c|c|c|c|c|c|c|c|}
\hline & & \multicolumn{2}{|c|}{ RAW DATA cpm } & \multicolumn{2}{|c|}{ BKG ADJUSTED cpm } & \multicolumn{2}{|c|}{$\mathrm{dpm} / 100 \mathrm{~cm} 2$} \\
\hline & & Alpha & Beta & Alpha & Beta & Alpha & Beta \\
\hline 1 & Floor Concrete & 11 & 1564 & 6.7 & 523.5 & 32.2 & 873.3 \\
\hline 2 & Floor Concrete & 6 & 1707 & 1.7 & 666.5 & 8.1 & 1111.9 \\
\hline 3 & Floor Concrete & 5 & 1607 & 0.7 & 566.5 & 3.3 & 945.1 \\
\hline 4 & Floor Concrete & 5 & 1724 & 0.7 & 683.5 & 3.3 & 1140.3 \\
\hline 5 & Floor Concrete & 5 & 1562 & 0.7 & 521.5 & 3.3 & 870.0 \\
\hline 6 & Floor Concrete & 5 & 1638 & 0.7 & 597.5 & 3.3 & 996.8 \\
\hline 7 & West Wall, Concrete Block & 12 & 1227 & 7.2 & 530.4 & 34.6 & 884.8 \\
\hline 8 & West Wall, Concrete Block & 7 & 1155 & 2.2 & 458.4 & 10.4 & 764.7 \\
\hline 9 & West Wall, Concrete Block & 10 & 1226 & 5.2 & 529.4 & 24.9 & 883.1 \\
\hline 10 & West Wall, Concrete Block & 4 & 1227 & -0.8 & 530.4 & -4.1 & 884.8 \\
\hline 11 & West Wall, Concrete Block & 7 & 1240 & 2.2 & 543.4 & 10.4 & 906.5 \\
\hline 12 & West Wall, Concrete Block & 7 & 1326 & 2.2 & 629.4 & 10.4 & 1050.0 \\
\hline 13 & North Wall, Concrete Block & 11 & 1265 & 6.2 & 568.4 & 29.8 & 948.2 \\
\hline 14 & North Wall, Metal & 7 & 915 & 1.7 & -323.9 & 8.2 & -540.3 \\
\hline 15 & North Wall, Concrete Block & 8 & 1576 & 3.2 & 879.4 & 15.3 & 1467.1 \\
\hline 16 & East Wall, Concrete Block & 7 & 1362 & 2.2 & 665.4 & 10.4 & 1110.0 \\
\hline 17 & East Wall, Concrete Block & 15 & 1264 & 10.2 & 567.4 & 49.1 & 946.5 \\
\hline 18 & East Wall, Metal & 4 & 1023 & -1.3 & -215.9 & -6.3 & -360.1 \\
\hline 19 & East Wall, Concrete Block & 16 & 1354 & 11.2 & 657.4 & 53.9 & 1096.7 \\
\hline 20 & East Wall, Concrete Block & 13 & 1306 & 8.2 & 609.4 & 39.4 & 1016.6 \\
\hline 21 & East Wall, Concrete Block & 11 & 1371 & 6.2 & 674.4 & 29.8 & 1125.0 \\
\hline 22 & South Wall, Concrete Block & 7 & 1379 & 2.2 & 682.4 & 10.4 & 1138.4 \\
\hline 23 & South Wall, Concrete Block & 11 & 1295 & 6.2 & 598.4 & 29.8 & 998.2 \\
\hline
\end{tabular}




\begin{tabular}{|c|c|c|c|c|c|c|c|}
\hline 24 & South Wall, Concrete Block & 3 & 1217 & -1.8 & 520.4 & -8.9 & 868.1 \\
\hline 25 & Ceiling, Metal & 4 & 1163 & -1.3 & -75.8 & -6.3 & -126.5 \\
\hline 26 & Ceiling, Metal & 2 & 1234 & -3.3 & -4.8 & -15.9 & -8.1 \\
\hline 27 & Ceiling, Metal & 9 & 1189 & 3.7 & -49.8 & 17.9 & -83.2 \\
\hline 28 & Ceiling, Metal & 11 & 1202 & 5.7 & -36.8 & 27.5 & -61.5 \\
\hline 29 & Ceiling, Metal & 2 & 1172 & -3.3 & -66.8 & -15.9 & -111.5 \\
\hline 30 & Ceiling, Metal & 9 & 1225 & 3.7 & -13.8 & 17.9 & -23.1 \\
\hline & & & & & Averages & 14.2 & 690.4 \\
\hline & & & & & StDev & 18.0 & 547.3 \\
\hline & & & & & Median & 10.4 & 895.6 \\
\hline & & & & & Max & 53.9 & 1467.1 \\
\hline & & & & & Min & -15.9 & -540.3 \\
\hline & & & & & *LBGR & 0.28 & \\
\hline & & & & & \multicolumn{3}{|c|}{ *Negative vales not used in LGBR } \\
\hline
\end{tabular}


10-ER-A26-239

Final Status Survey, Room 201, Floor, Ceiling and Walls

\begin{tabular}{|c|c|c|c|c|c|c|c|}
\hline & & \multicolumn{2}{|c|}{ RAW DATA cpm } & \multicolumn{2}{|c|}{ BKG ADJUSTED cpm } & \multicolumn{2}{|c|}{$\mathrm{dpm} / 100 \mathrm{~cm} 2$} \\
\hline & & Alpha & Beta & Alpha & Beta & Alpha & Beta \\
\hline 1 & Floor Concrete & 9 & 1183 & 4.7 & 142.5 & 22.6 & 237.7 \\
\hline 2 & Floor Concrete & 10 & 1268 & 5.7 & 227.5 & 27.4 & 379.5 \\
\hline 3 & East Wall Concrete & 20 & 925 & 15.7 & -115.5 & 75.7 & -192.7 \\
\hline 4 & East Wall Concrete & 6 & 886 & 1.7 & -154.5 & 8.1 & -257.8 \\
\hline 5 & North Wall Concrete & 8 & 772 & 3.7 & -268.5 & 17.8 & -448.0 \\
\hline 6 & North Wall Concrete & 20 & 751 & 15.7 & -289.5 & 75.7 & -483.0 \\
\hline 7 & West Wall Concrete & 11 & 964 & 6.7 & -76.5 & 32.2 & -127.7 \\
\hline 8 & West Wall Concrete & 14 & 849 & 9.7 & -191.5 & 46.7 & -319.5 \\
\hline 9 & South Wall Concrete & 17 & 1083 & 12.7 & 42.5 & 61.2 & 70.9 \\
\hline 10 & South Wall Concrete & 22 & 1087 & 17.7 & 46.5 & 85.4 & 77.5 \\
\hline 11 & Ceiling Concrete & 18 & 1233 & 13.7 & 192.5 & 66.1 & 321.1 \\
\hline 12 & Ceiling Concrete & 18 & 1127 & 13.7 & 86.5 & 66.1 & 144.3 \\
\hline & & & & & Averages & 48.8 & -49.8 \\
\hline & & & & & StDev & 26.3 & 295.7 \\
\hline & & & & & Median & 54.0 & -28.4 \\
\hline & & & & & Max & 85.4 & 379.5 \\
\hline & & & & & Min & 8.1 & -483.0 \\
\hline & & & & & $*$ LBGR & 0.49 & \\
\hline
\end{tabular}


10-ER-A26-240

Final Status Survey, Room 301, Floor, Ceiling and Walls

\begin{tabular}{|c|c|c|c|c|c|c|c|}
\hline & & \multicolumn{2}{|c|}{ RAW DATA cpm } & \multicolumn{2}{|c|}{ BKG ADJUSTED cpm } & \multicolumn{2}{|c|}{$\mathrm{dpm} / 100 \mathrm{~cm} 2$} \\
\hline & & Alpha & Beta & Alpha & Beta & Alpha & Beta \\
\hline 1 & Floor Concrete & 12 & 1329 & 7.7 & 288.5 & 37.1 & 481.3 \\
\hline 2 & Floor Concrete & 7 & 1242 & 2.7 & 201.5 & 12.9 & 336.1 \\
\hline 3 & East Wall Concrete & 10 & 863 & 5.7 & -177.5 & 27.4 & -296.2 \\
\hline 4 & East Wall Concrete & 10 & 891 & 5.7 & -149.5 & 27.4 & -249.5 \\
\hline 5 & North Wall Concrete & 12 & 812 & 7.7 & -228.5 & 37.1 & -381.3 \\
\hline 6 & North Wall Concrete & 7 & 933 & 2.7 & -107.5 & 12.9 & -179.4 \\
\hline 7 & West Wall Concrete & 15 & 836 & 10.7 & -204.5 & 51.6 & -341.2 \\
\hline 8 & West Wall Concrete & 13 & 934 & 8.7 & -106.5 & 41.9 & -177.7 \\
\hline 9 & South Wall Concrete & 5 & 1143 & 0.7 & 102.5 & 3.3 & 171.0 \\
\hline 10 & South Wall Concrete & 16 & 1017 & 11.7 & -23.5 & 56.4 & -39.2 \\
\hline 11 & Ceiling Concrete & 13 & 1265 & 8.7 & 224.5 & 41.9 & 374.5 \\
\hline 12 & Ceiling Concrete & 4 & 1282 & -0.3 & 241.5 & -1.6 & 402.9 \\
\hline & & & & & Averages & 29.0 & 8.4 \\
\hline & & & & & StDev & 18.7 & 323.7 \\
\hline & & & & & Median & 32.2 & -108.5 \\
\hline & & & & & Max & 56.4 & 481.3 \\
\hline & & & & & Min & -1.6 & -381.3 \\
\hline & & & & & $*$ LBGR & 0.29 & \\
\hline & & & & & *Negativ & vales not & n LGBR \\
\hline
\end{tabular}


Data for Survey Units in Room 102 
10-ER-A26-269

Final Status Survey, Room 102 South Wall 102-SW-1

\begin{tabular}{|c|c|c|c|c|c|c|c|c|c|}
\hline & & \multicolumn{2}{|c|}{ RAW DATA cpm } & \multicolumn{2}{|c|}{ BKG ADJUSTED cpm } & \multicolumn{2}{|l|}{$\mathrm{dpm} / 100 \mathrm{~cm} 2$} & \multirow[b]{2}{*}{ LBGR } & \multirow[b]{2}{*}{ Sign } \\
\hline & & Alpha & Beta & Alpha & Beta & Alpha & Beta & & \\
\hline 1 & Wall, Concrete (Electra 2125) & 15 & 895 & 10.7 & -145.5 & 51.6 & -242.8 & 0.52 & 1 \\
\hline 2 & Wall, Concrete (Electra 2125) & 11 & 754 & 6.7 & -286.5 & 32.2 & -478.0 & 0.32 & 1 \\
\hline 3 & Wall, Concrete (Electra 2125) & 5 & 1129 & 0.7 & 88.5 & 3.3 & 147.6 & 0.06 & 1 \\
\hline 4 & Wall, Concrete (Electra 2125) & 9 & 759 & 4.7 & -281.5 & 22.6 & -469.7 & 0.23 & 1 \\
\hline 5 & Wall, Concrete (Electra 2125) & 4 & 731 & -0.3 & -309.5 & -1.6 & -516.4 & 0.00 & 1 \\
\hline 6 & Wall, Concrete (Electra 2125) & 11 & 1030 & 6.7 & -10.5 & 32.2 & -17.6 & 0.32 & 1 \\
\hline 7 & Wall, Concrete (Electra 2125) & 9 & 1013 & 4.7 & -27.5 & 22.6 & -45.9 & 0.23 & 1 \\
\hline 8 & Wall, Concrete (Electra 2125) & 4 & 626 & -0.3 & -414.5 & -1.6 & -691.6 & 0.00 & 1 \\
\hline 9 & Wall, Concrete (Electra 2125) & 4 & 712 & -0.3 & -328.5 & -1.6 & -548.1 & 0.00 & 1 \\
\hline 10 & Wall, Concrete (Electra 2125) & 4 & 1016 & -0.3 & -24.5 & -1.6 & -40.9 & 0.00 & 1 \\
\hline 11 & Wall, Concrete (Electra 2125) & 9 & 861 & 4.7 & -179.5 & 22.6 & -299.5 & 0.23 & 1 \\
\hline 12 & Wall, Concrete (2125) & 8 & 747 & 3.7 & -293.5 & 17.8 & -489.7 & 0.18 & 1 \\
\hline 13 & Wall, Concrete (2125) & 7 & 708 & 2.7 & -332.5 & 12.9 & -554.8 & 0.13 & 1 \\
\hline & & & & & Averages & 16.3 & -326.7 & & \\
\hline & & & & & StDev & 16.7 & 262.8 & & \\
\hline & & & & & Median & 17.8 & -469.7 & & \\
\hline & & & & & Max & 51.6 & 147.6 & & \\
\hline & & & & & Min & -1.6 & -691.6 & & \\
\hline & & & & & *LBGR & 0.16 & & & \\
\hline & & & & & \multicolumn{3}{|c|}{ *Negative vales not used in LGBR } & & \\
\hline & & & & & & \# of samples: & 13 & & \\
\hline & & & & & & Sign test stat: & 13 & & \\
\hline
\end{tabular}


10-ER-A26-268

Final Status Survey, Room 102 South Wall 102-SW-2

\begin{tabular}{|c|c|c|c|c|c|c|c|c|c|}
\hline & & \multicolumn{2}{|c|}{ RAW DATA cpm } & \multicolumn{2}{|c|}{ BKG ADJUSTED cpm } & \multicolumn{2}{|l|}{$\mathrm{dpm} / 100 \mathrm{~cm} 2$} & \multirow[b]{2}{*}{ LBGR } & \multirow[b]{2}{*}{ Sign } \\
\hline & & Alpha & Beta & Alpha & Beta & Alpha & Beta & & \\
\hline 1 & Wall, Concrete (Electra 1201) & 4 & 626 & -0.3 & -414.5 & -1.6 & -691.6 & 0.00 & 1 \\
\hline 2 & Wall, Concrete (Electra 1201) & 7 & 727 & 2.7 & -313.5 & 12.9 & -523.1 & 0.13 & 1 \\
\hline 3 & Wall, Concrete (Electra 1201) & 3 & 664 & -1.3 & -376.5 & -6.4 & -628.2 & 0.00 & 1 \\
\hline 4 & Wall, Concrete (Electra 1201) & 4 & 752 & -0.3 & -288.5 & -1.6 & -481.4 & 0.00 & 1 \\
\hline 5 & Wall, Concrete (Electra 1201) & 7 & 723 & 2.7 & -317.5 & 12.9 & -529.7 & 0.13 & 1 \\
\hline 6 & Wall, Concrete (Electra 1201) & 5 & 769 & 0.7 & -271.5 & 3.3 & -453.0 & 0.03 & 1 \\
\hline 7 & Wall, Concrete (Electra 1201) & 2 & 900 & -2.3 & -140.5 & -11.2 & -234.4 & 0.00 & 1 \\
\hline 8 & Wall, Concrete (Electra 1201) & 8 & 854 & 3.7 & -186.5 & 17.8 & -311.2 & 0.18 & 1 \\
\hline 9 & Wall, Concrete (Electra 1201) & 8 & 717 & 3.7 & -323.5 & 17.8 & -539.7 & 0.18 & 1 \\
\hline 10 & Wall, Concrete (Electra 1201) & 4 & 668 & -0.3 & -372.5 & -1.6 & -621.5 & 0.00 & 1 \\
\hline 11 & Wall, Concrete (Electra 1201) & 7 & 659 & 2.7 & -381.5 & 12.9 & -636.5 & 0.13 & 1 \\
\hline & & & & & Averages & 5.0 & -513.7 & & \\
\hline & & & & & StDev & 10.2 & 140.1 & & \\
\hline & & & & & Median & 3.3 & -529.7 & & \\
\hline & & & & & Max & 17.8 & -234.4 & & \\
\hline & & & & & Min & -11.2 & -691.6 & & \\
\hline & & & & & $*$ LBGR & 0.05 & & & \\
\hline & & & & & \multicolumn{3}{|c|}{ *Negative vales not used in LGBR } & & \\
\hline & & & & & & \# of samples: & 11 & & \\
\hline & & & & & & Sign test stat: & 11 & & \\
\hline
\end{tabular}




\section{0-ER-A26-267}

Final Status Survey, Room 102 South Wall 102-SW-3

\begin{tabular}{|c|c|c|c|c|c|c|c|c|c|}
\hline & & \multicolumn{2}{|c|}{ RAW DATA cpm } & \multicolumn{2}{|c|}{ BKG ADJUSTED cpm } & \multicolumn{2}{|l|}{$\mathrm{dpm} / 100 \mathrm{~cm} 2$} & \multirow[b]{2}{*}{ LBGR } & \multirow[b]{2}{*}{ Sign } \\
\hline & & Alpha & Beta & Alpha & Beta & Alpha & Beta & & \\
\hline 1 & Wall, Concrete (Electra 4806) & 10 & 1029 & 5.7 & -11.5 & 27.4 & -19.2 & 0.3 & 1 \\
\hline 2 & Wall, Concrete (Electra 4806) & 6 & 621 & 1.7 & -419.5 & 8.1 & -699.9 & 0.1 & 1 \\
\hline 3 & Wall, Concrete (Electra 4806) & 8 & 636 & 3.7 & -404.5 & 17.8 & -674.9 & 0.2 & 1 \\
\hline 4 & Wall, Concrete (Electra 4806) & 7 & 718 & 2.7 & -322.5 & 12.9 & -538.1 & 0.1 & 1 \\
\hline 5 & Wall, Concrete (Electra 4806) & 2 & 825 & -2.3 & -215.5 & -11.2 & -359.6 & 0.0 & 1 \\
\hline 6 & Wall, Concrete (Electra 4806) & 8 & 1036 & 3.7 & -4.5 & 17.8 & -7.5 & 0.2 & 1 \\
\hline 7 & Wall, Concrete (Electra 4806) & 5 & 693 & 0.7 & -347.5 & 3.3 & -579.8 & 0.0 & 1 \\
\hline 8 & Wall, Concrete (Electra 4806) & 9 & 963 & 4.7 & -77.5 & 22.6 & -129.3 & 0.2 & 1 \\
\hline 9 & Wall, Wood (Electra 1504) & 5 & 676 & -1.9 & -619.7 & -8.9 & -1033.8 & 0.0 & 1 \\
\hline 10 & Wall, Concrete (Electra 4806) & 5 & 1018 & 0.7 & -22.5 & 3.3 & -37.6 & 0.0 & 1 \\
\hline 11 & Wall, Concrete (Electra 4806) & 9 & 1024 & 4.7 & -16.5 & 22.6 & -27.6 & 0.2 & 1 \\
\hline 12 & Wall, Concrete (Electra 4806) & 6 & 1033 & 1.7 & -7.5 & 8.1 & -12.6 & 0.1 & 1 \\
\hline & & & & & Averages & 10.3 & -343.3 & & \\
\hline & & & & & StDev & 12.3 & 353.4 & & \\
\hline & & & & & Median & 10.5 & -244.5 & & \\
\hline & & & & & Max & 27.4 & -7.5 & & \\
\hline & & & & & Min & -11.2 & -1033.8 & & \\
\hline & & & & & *LBGR & 0.10 & & & \\
\hline & & & & & \multicolumn{3}{|c|}{ *Negative vales not used in LGBR } & & \\
\hline & & & & & & \# of samples: & 12 & & \\
\hline & & & & & & Sign test stat: & 12 & & \\
\hline
\end{tabular}


10-ER-A26-266

Final Status Survey, Room 102 West Wall 102-WW-1

\begin{tabular}{|c|c|c|c|c|c|c|c|c|c|}
\hline & & \multicolumn{2}{|c|}{ RAW DATA cpm } & \multicolumn{2}{|c|}{ BKG ADJUSTED cpm } & \multicolumn{2}{|l|}{$\mathrm{dpm} / 100 \mathrm{~cm} 2$} & \multirow[b]{2}{*}{ LBGR } & \multirow[b]{2}{*}{ Sign } \\
\hline & & Alpha & Beta & Alpha & Beta & Alpha & Beta & & \\
\hline 1 & Wall, Concrete (Electra 1120) & 6 & 1014 & 1.7 & -26.5 & 8.1 & -44.3 & 0.08 & 1 \\
\hline 2 & Wall, Concrete (Electra 1120) & 7 & 915 & 2.7 & -125.5 & 12.9 & -209.4 & 0.13 & 1 \\
\hline 3 & Wall, Concrete (Electra 1120) & 5 & 708 & 0.7 & -332.5 & 3.3 & -554.8 & 0.03 & 1 \\
\hline 4 & Wall, Concrete (Electra 1120) & 8 & 900 & 3.7 & -140.5 & 17.8 & -234.4 & 0.18 & 1 \\
\hline 5 & Wall, Concrete (Electra 1120) & 12 & 949 & 7.7 & -91.5 & 37.1 & -152.7 & 0.37 & 1 \\
\hline 6 & Wall, Concrete (Electra 1120) & 12 & 1160 & 7.7 & 119.5 & 37.1 & 199.3 & 0.41 & 1 \\
\hline 7 & Wall, Concrete (Electra 1120) & 8 & 996 & 3.7 & -44.5 & 17.8 & -74.3 & 0.18 & 1 \\
\hline 8 & Wall, Concrete (Electra 1120) & 9 & 838 & 4.7 & -202.5 & 22.6 & -337.9 & 0.23 & 1 \\
\hline 9 & Wall, Concrete (Electra 1120) & 5 & 1006 & 0.7 & -34.5 & 3.3 & -57.6 & 0.03 & 1 \\
\hline 10 & Wall, Concrete (Electra 1120) & 9 & 1147 & 4.7 & 106.5 & 22.6 & 177.6 & 0.26 & 1 \\
\hline 11 & Wall, Concrete (Electra 1120) & 3 & 973 & -1.3 & -67.5 & -6.4 & -112.7 & 0.00 & 1 \\
\hline & & & & & Averages & 16.0 & -127.4 & & \\
\hline & & & & & StDev & 13.7 & 214.6 & & \\
\hline & & & & & Median & 17.8 & -112.7 & & \\
\hline & & & & & Max & 37.1 & 199.3 & & \\
\hline & & & & & Min & -6.4 & -554.8 & & \\
\hline & & & & & *LBGR & 0.16 & & & \\
\hline & & & & & \multicolumn{3}{|c|}{ *Negative vales not used in LGBR } & & \\
\hline & & & & & & \# of samples: & 11 & & \\
\hline & & & & & & Sign test stat: & 11 & & \\
\hline
\end{tabular}


10-ER-A26-264

Final Status Survey, Room 102 West Wall/FSS-102-WW-2

\begin{tabular}{|c|c|c|c|c|c|c|c|c|c|}
\hline & & \multicolumn{2}{|c|}{ RAW DATA cpm } & \multicolumn{2}{|c|}{ BKG ADJUSTED cpm } & \multicolumn{2}{|l|}{$\mathrm{dpm} / 100 \mathrm{~cm} 2$} & \multirow[b]{2}{*}{ LBGR } & \multirow[b]{2}{*}{ Sign } \\
\hline & & Alpha & Beta & Alpha & Beta & Alpha & Beta & & \\
\hline 1 & Wall, Concrete (Electra 1504) & 9 & 1473 & 4.7 & 432.5 & 22.6 & 721.5 & 0.37 & 1 \\
\hline 2 & Wall, Concrete (Electra 1504) & 16 & 2244 & 11.7 & 1203.5 & 56.4 & 2007.8 & 0.97 & 1 \\
\hline 3 & Wall, Concrete (Electra 1504) & 11 & 1047 & 6.7 & 6.5 & 32.2 & 10.8 & 0.32 & 1 \\
\hline 4 & Wall, Concrete (Electra 1504) & 5 & 1469 & 0.7 & 428.5 & 3.3 & 714.8 & 0.18 & 1 \\
\hline 5 & Wall, Concrete (Electra 1504) & 9 & 1036 & 4.7 & -4.5 & 22.6 & -7.5 & 0.23 & 1 \\
\hline 6 & Wall, Concrete (Electra 1504) & 10 & 1044 & 5.7 & 3.5 & 27.4 & 5.8 & 0.28 & 1 \\
\hline 7 & Wall, Concrete (Electra 1504) & 6 & 1104 & 1.7 & 63.5 & 8.1 & 105.9 & 0.10 & 1 \\
\hline 8 & Wall, Concrete (Electra 1504) & 9 & 1280 & 4.7 & 239.5 & 22.6 & 399.5 & 0.31 & 1 \\
\hline 9 & Wall, Concrete (Electra 1504) & 8 & 1133 & 3.7 & 92.5 & 17.8 & 154.3 & 0.21 & 1 \\
\hline 10 & Wall, Concrete (Electra 1504) & 9 & 1016 & 4.7 & -24.5 & 22.6 & -40.9 & 0.23 & 1 \\
\hline 11 & Wall, Concrete (Electra 1504) & 7 & 1025 & 2.7 & -15.5 & 12.9 & -25.9 & 0.13 & 1 \\
\hline & & & & & Averages & 22.6 & 367.8 & & \\
\hline & & & & & StDev & 14.0 & 614.1 & & \\
\hline & & & & & Median & 22.6 & 105.9 & & \\
\hline & & & & & Max & 56.4 & 2007.8 & & \\
\hline & & & & & Min & 3.3 & -40.9 & & \\
\hline & & & & & *LBGR & 0.30 & & & \\
\hline & & & & & \multicolumn{3}{|c|}{ *Negative vales not used in LGBR } & & \\
\hline & & & & & & \# of samples: & 11 & & \\
\hline & & & & & & Sign test stat: & 11 & & \\
\hline
\end{tabular}




\section{0-ER-A26-261}

Final Status Survey, Room 102, North Wall / FSS-102-NW-1

\begin{tabular}{|c|c|c|c|c|c|c|c|c|c|}
\hline & & \multicolumn{2}{|c|}{ RAW DATA cpm } & \multicolumn{2}{|c|}{ BKG ADJUSTED cpm } & \multicolumn{2}{|l|}{$\mathrm{dpm} / 100 \mathrm{~cm} 2$} & \multirow[b]{2}{*}{ LBGR } & \multirow[b]{2}{*}{ Sign } \\
\hline & & Alpha & Beta & Alpha & Beta & Alpha & Beta & & \\
\hline 1 & Wall, Concrete (Electra 1120) & 7 & 1233 & 2.7 & 192.5 & 12.9 & 321.1 & 0.19 & 1 \\
\hline 2 & Wall, Concrete (Electra 1120) & 2 & 1059 & -2.3 & 18.5 & -11.2 & 30.8 & 0.01 & 1 \\
\hline 3 & Wall, Concrete (Electra 1120) & 12 & 1024 & 7.7 & -16.5 & 37.1 & -27.6 & 0.37 & 1 \\
\hline 4 & Wall, Concrete (Electra 1120) & 2 & 1025 & -2.3 & -15.5 & -11.2 & -25.9 & 0.00 & 1 \\
\hline 5 & Wall, Concrete (Electra 1120) & 9 & 1158 & 4.7 & 117.5 & 22.6 & 196.0 & 0.27 & 1 \\
\hline 6 & Wall, Concrete (Electra 1120) & 9 & 1180 & 4.7 & 139.5 & 22.6 & 232.7 & 0.27 & 1 \\
\hline 7 & Wall, Concrete (Electra 1120) & 8 & 1064 & 3.7 & 23.5 & 17.8 & 39.2 & 0.19 & 1 \\
\hline 8 & Wall, Concrete (Electra 1120) & 7 & 1054 & 2.7 & 13.5 & 12.9 & 22.5 & 0.13 & 1 \\
\hline 9 & Wall, Concrete (Electra 1120) & 8 & 1020 & 3.7 & -20.5 & 17.8 & -34.2 & 0.18 & 1 \\
\hline 10 & Wall, Concrete (Electra 1120) & 9 & 1048 & 4.7 & 7.5 & 22.6 & 12.5 & 0.23 & 1 \\
\hline 11 & Wall, Concrete (Electra 1120) & 10 & 1068 & 5.7 & 27.5 & 27.4 & 45.8 & 0.28 & 1 \\
\hline 12 & Wall, Concrete (Electra 1120) & 10 & 1041 & 5.7 & 0.5 & 27.4 & 0.8 & 0.27 & 1 \\
\hline 13 & Wall, Concrete (Electra 1120) & 7 & 1100 & 2.7 & 59.5 & 12.9 & 99.2 & 0.15 & 1 \\
\hline 14 & Wall, Concrete (Electra 1120) & 6 & 992 & 1.7 & -48.5 & 8.1 & -81.0 & 0.08 & 1 \\
\hline & & & & & Averages & 15.7 & 59.4 & & \\
\hline & & & & & StDev & 13.6 & 114.5 & & \\
\hline & & & & & Median & 17.8 & 26.7 & & \\
\hline & & & & & Max & 37.1 & 321.1 & & \\
\hline & & & & & Min & -11.2 & -81.0 & & \\
\hline & & & & & *LBGR & 0.17 & & & \\
\hline & & & & & *Negative & vales not used $\mathrm{i}$ & GBR & & \\
\hline & & & & & & \# of samples: & 14 & & \\
\hline & & & & & & Sign test stat: & 14 & & \\
\hline
\end{tabular}




\section{0-ER-A26-263}

Final Status Survey, Room 102, North Wall / FSS-102-NW-2

\begin{tabular}{|c|c|c|c|c|c|c|c|c|c|}
\hline & & \multicolumn{2}{|c|}{ RAW DATA cpm } & \multicolumn{2}{|c|}{ BKG ADJUSTED cpm } & \multicolumn{2}{|l|}{$\mathrm{dpm} / 100 \mathrm{~cm} 2$} & \multirow{2}{*}{ LBGR } & \multirow[b]{2}{*}{ Sign } \\
\hline & & Alpha & Beta & Alpha & Beta & Alpha & Beta & & \\
\hline 1 & Wall, Concrete (Electra 1504) & 9 & 1027 & 4.7 & -13.5 & 22.6 & -22.6 & 0.23 & 1 \\
\hline 2 & Wall, Concrete (Electra 1504) & 12 & 1271 & 7.7 & 230.5 & 37.1 & 384.5 & 0.45 & 1 \\
\hline 3 & Wall, Concrete (Electra 1504) & 8 & 1282 & 3.7 & 241.5 & 17.8 & 402.9 & 0.26 & 1 \\
\hline 4 & Wall, Concrete (Electra 1504) & 12 & 1053 & 7.7 & 12.5 & 37.1 & 20.8 & 0.37 & 1 \\
\hline 5 & Wall, Concrete (Electra 1504) & 10 & 993 & 5.7 & -47.5 & 27.4 & -79.3 & 0.27 & 1 \\
\hline 6 & Wall, Concrete (Electra 1504) & 11 & 1081 & 6.7 & 40.5 & 32.2 & 67.5 & 0.34 & 1 \\
\hline 7 & Wall, Concrete (Electra 1504) & 11 & 1011 & 6.7 & -29.5 & 32.2 & -49.3 & 0.32 & 1 \\
\hline 8 & Wall, Concrete (Electra 1504) & 9 & 1022 & 4.7 & -18.5 & 22.6 & -30.9 & 0.23 & 1 \\
\hline 9 & Wall, Concrete (Electra 1504) & 13 & 1069 & 8.7 & 28.5 & 41.9 & 47.5 & 0.43 & 1 \\
\hline 10 & Wall, Concrete (Electra 1504) & 18 & 1013 & 13.7 & -27.5 & 66.1 & -45.9 & 0.66 & 1 \\
\hline 11 & Wall, Concrete (Electra 1504) & 11 & 1049 & 6.7 & 8.5 & 32.2 & 14.1 & 0.33 & 1 \\
\hline 12 & Wall, Concrete (Electra 1504) & 14 & 980 & 9.7 & -60.5 & 46.7 & -101.0 & 0.47 & 1 \\
\hline 13 & Wall, Concrete (Electra 1504) & 8 & 1082 & 3.7 & 41.5 & 17.8 & 69.2 & 0.19 & 1 \\
\hline \multirow[t]{10}{*}{14} & Wall, Concrete (Electra 1504) & 10 & 1040 & 4.7 & -198.9 & 22.7 & -331.7 & 0.23 & 1 \\
\hline & & & & & Averages & 32.6 & 24.7 & & \\
\hline & & & & & StDev & 13.0 & 185.3 & & \\
\hline & & & & & Median & 32.2 & -4.2 & & \\
\hline & & & & & Max & 66.1 & 402.9 & & \\
\hline & & & & & Min & 17.8 & -331.7 & & \\
\hline & & & & & $*$ LBGR & 0.33 & & & \\
\hline & & & & & \multicolumn{3}{|c|}{${ }^{*}$ Negative vales not used in LGBR } & & \\
\hline & & & & & & \# of samples: & 14 & & \\
\hline & & & & & & Sign test stat: & 14 & & \\
\hline
\end{tabular}




\section{0-ER-A26-262}

Final Status Survey, Room 102, North Wall / FSS-102-NW-3

\begin{tabular}{|c|c|c|c|c|c|c|c|c|c|}
\hline & & \multicolumn{2}{|c|}{ RAW DATA cpm } & \multicolumn{2}{|c|}{ BKG ADJUSTED cpm } & \multicolumn{2}{|l|}{$\mathrm{dpm} / 100 \mathrm{~cm} 2$} & \multirow[b]{2}{*}{ LBGR } & \multirow[b]{2}{*}{ Sign } \\
\hline & & Alpha & Beta & Alpha & Beta & Alpha & Beta & & \\
\hline 1 & Wall, Concrete (Electra 1504) & 10 & 1082 & 5.7 & 41.5 & 27.4 & 69.2 & 0.29 & 1 \\
\hline 2 & Wall, Concrete (Electra 1504) & 12 & 1086 & 7.7 & 45.5 & 37.1 & 75.9 & 0.39 & 1 \\
\hline 3 & Wall, Concrete (Electra 1504) & 13 & 953 & 8.7 & -87.5 & 41.9 & -146.0 & 0.42 & 1 \\
\hline 4 & Wall, Concrete (Electra 1504) & 8 & 973 & 3.7 & -67.5 & 17.8 & -112.7 & 0.18 & 1 \\
\hline 5 & Wall, Concrete (Electra 1504) & 8 & 1039 & 3.7 & -1.5 & 17.8 & -2.5 & 0.18 & 1 \\
\hline 6 & Wall, Concrete (Electra 1504) & 5 & 1062 & 0.7 & 21.5 & 3.3 & 35.8 & 0.04 & 1 \\
\hline 7 & Wall, Concrete (Electra 1504) & 4 & 1078 & -0.3 & 37.5 & -1.6 & 62.5 & 0.01 & 1 \\
\hline 8 & Wall, Concrete (Electra 1504) & 8 & 1143 & 3.7 & 102.5 & 17.8 & 171.0 & 0.21 & 1 \\
\hline 9 & Wall, Concrete (Electra 1504) & 7 & 1049 & 2.7 & 8.5 & 12.9 & 14.1 & 0.13 & 1 \\
\hline 10 & Wall, Concrete (Electra 1504) & 10 & 1020 & 5.7 & -20.5 & 27.4 & -34.2 & 0.27 & 1 \\
\hline 11 & Wall, Concrete (Electra 1504) & 11 & 941 & 6.7 & -99.5 & 32.2 & -166.0 & 0.32 & 1 \\
\hline 12 & Wall, Concrete (Electra 1120) & 13 & 941 & 8.7 & -99.5 & 41.9 & -166.0 & 0.42 & 1 \\
\hline 13 & Wall, Wood (Electra 1120) & 11 & 701 & 6.3 & -709.7 & 30.2 & -1184.0 & 0.30 & 1 \\
\hline & & & & & Averages & 23.5 & -106.4 & & \\
\hline & & & & & StDev & 13.7 & 340.4 & & \\
\hline & & & & & Median & 27.4 & -2.5 & & \\
\hline & & & & & Max & 41.9 & 171.0 & & \\
\hline & & & & & Min & -1.6 & -1184.0 & & \\
\hline & & & & & $*$ LBGR & 0.24 & & & \\
\hline & & & & & *Negative & vales not used ir & LGBR & & \\
\hline & & & & & & \# of samples: & 13 & & \\
\hline & & & & & & Sign test stat: & 13 & & \\
\hline
\end{tabular}




\section{0-ER-A26-256}

Final Status Survey, Room 102, Floor / FSS-102-F-1

\begin{tabular}{|c|c|c|c|c|c|c|c|c|c|}
\hline & & \multicolumn{2}{|c|}{ RAW DATA cpm } & \multicolumn{2}{|c|}{ BKG ADJUSTED cpm } & \multicolumn{2}{|l|}{$\mathrm{dpm} / 100 \mathrm{~cm} 2$} & \multirow{2}{*}{ LBGR } & \multirow[b]{2}{*}{ Sign } \\
\hline & & Alpha & Beta & Alpha & Beta & Alpha & Beta & & \\
\hline 1 & Floor, Concrete (Electra 4806) & 2 & 1189 & -2.3 & 148.5 & -11.2 & 247.7 & 0.05 & 1 \\
\hline 2 & Floor, Concrete (Electra 4806) & 4 & 1107 & -0.3 & 66.5 & -1.6 & 110.9 & 0.02 & 1 \\
\hline 3 & Floor, Concrete (Electra 4806) & 1 & 961 & -3.3 & -79.5 & -16.1 & -132.7 & 0.00 & 1 \\
\hline 4 & Floor, Concrete (Electra 4806) & 1 & 1148 & -3.3 & 107.5 & -16.1 & 179.3 & 0.04 & 1 \\
\hline 5 & Floor, Concrete (Electra 4806) & 2 & 1188 & -2.3 & 147.5 & -11.2 & 246.0 & 0.05 & 1 \\
\hline 6 & Floor, Concrete (Electra 4806) & 2 & 1234 & -2.3 & 193.5 & -11.2 & 322.8 & 0.06 & 1 \\
\hline 7 & Floor, Concrete (Electra 4806) & 5 & 1078 & 0.7 & 37.5 & 3.3 & 62.5 & 0.05 & 1 \\
\hline 8 & Floor, Concrete (Electra 4806) & 5 & 1160 & 0.7 & 119.5 & 3.3 & 199.3 & 0.07 & 1 \\
\hline 9 & Floor, Concrete (Electra 4806) & 11 & 1169 & 6.7 & 128.5 & 32.2 & 214.3 & 0.37 & 1 \\
\hline 10 & Floor, Concrete (Electra 4806) & 4 & 1233 & -0.3 & 192.5 & -1.6 & 321.1 & 0.06 & 1 \\
\hline 11 & Floor, Concrete (Electra 4806) & 3 & 1028 & -1.3 & -12.5 & -6.4 & -20.9 & 0.00 & 1 \\
\hline 12 & Floor, Concrete (Electra 4806\} & 9 & 1120 & 4.7 & 79.5 & 22.6 & 132.6 & 0.25 & 1 \\
\hline 13 & Floor, Concrete (Electra 4806\} & 6 & 1148 & 1.7 & 107.5 & 8.1 & 179.3 & 0.12 & 1 \\
\hline 14 & Floor, Concrete (Electra 4806\} & 1 & 1054 & -3.3 & 13.5 & -16.1 & 22.5 & 0.00 & 1 \\
\hline & & & & & Averages & -1.6 & 148.9 & & \\
\hline & & & & & StDev & 14.7 & 130.4 & & \\
\hline & & & & & Median & -4.0 & 179.3 & & \\
\hline & & & & & Max & 32.2 & 322.8 & & \\
\hline & & & & & Min & -16.1 & -132.7 & & \\
\hline & & & & & *LBGR & 0.03 & & & \\
\hline & & & & & *Negative & vales not used $\mathrm{i}$ & LGBR & & \\
\hline & & & & & & \# of samples: & 14 & & \\
\hline & & & & & & Sign test stat: & 14 & & \\
\hline
\end{tabular}


10-ER-A26-257

Final Status Survey, Room 102, Floor / FSS-102-F-2

\begin{tabular}{|c|c|c|c|c|c|c|c|c|c|}
\hline & & \multicolumn{2}{|c|}{ RAW DATA cpm } & \multicolumn{2}{|c|}{ BKG ADJUSTED cpm } & \multicolumn{2}{|l|}{$\mathrm{dpm} / 100 \mathrm{~cm} 2$} & \multirow[b]{2}{*}{ LBGR } & \multirow[b]{2}{*}{ Sign } \\
\hline & & Alpha & Beta & Alpha & Beta & Alpha & Beta & & \\
\hline 1 & Floor, Concrete (Electra 6697) & 7 & 1125 & 2.7 & 84.5 & 12.9 & 140.9 & 0.16 & 1 \\
\hline 2 & Floor, Concrete (Electra 6697) & 5 & 1168 & 0.7 & 127.5 & 3.3 & 212.7 & 0.08 & 1 \\
\hline 3 & Floor, Concrete (Electra 6697) & 3 & 931 & -1.3 & -109.5 & -6.4 & -182.7 & 0.00 & 1 \\
\hline 4 & Floor, Concrete (Electra 6697) & 2 & 1051 & -2.3 & 10.5 & -11.2 & 17.5 & 0.00 & 1 \\
\hline 5 & Floor, Concrete (Electra 6697) & 7 & 1054 & 2.7 & 13.5 & 12.9 & 22.5 & 0.13 & 1 \\
\hline 6 & Floor, Concrete (Electra 6697) & 2 & 1025 & -2.3 & -15.5 & -11.2 & -25.9 & 0.00 & 1 \\
\hline 7 & Floor, Concrete (Electra 6697) & 9 & 1051 & 4.7 & 10.5 & 22.6 & 17.5 & 0.23 & 1 \\
\hline 8 & Floor, Concrete (Electra 6697) & 10 & 1069 & 5.7 & 28.5 & 27.4 & 47.5 & 0.28 & 1 \\
\hline 9 & Floor, Concrete (Electra 6697) & 4 & 1120 & -0.3 & 79.5 & -1.6 & 132.6 & 0.03 & 1 \\
\hline 10 & Floor, Concrete (Electra 6697) & 7 & 1110 & 2.7 & 69.5 & 12.9 & 115.9 & 0.15 & 1 \\
\hline 11 & Floor, Concrete (Electra 6697) & 6 & 986 & 1.7 & -54.5 & 8.1 & -91.0 & 0.08 & 1 \\
\hline & & & & & Averages & 6.3 & 37.0 & & \\
\hline & & & & & StDev & 13.0 & 112.6 & & \\
\hline & & & & & Median & 8.1 & 22.5 & & \\
\hline & & & & & Max & 27.4 & 212.7 & & \\
\hline & & & & & Min & -11.2 & -182.7 & & \\
\hline & & & & & $*$ LBGR & 0.07 & & & \\
\hline & & & & & \multicolumn{3}{|c|}{ *Negative vales not used in LGBR } & & \\
\hline & & & & & & \# of samples: & 11 & & \\
\hline & & & & & & Sign test stat: & 11 & & \\
\hline
\end{tabular}




\section{0-ER-A26-258}

Final Status Survey, Room 102, Floor / FSS-102-F-3

\begin{tabular}{|c|c|c|c|c|c|c|c|c|c|}
\hline & & \multicolumn{2}{|c|}{ RAW DATA cpm } & \multicolumn{2}{|c|}{ BKG ADJUSTED cpm } & \multicolumn{2}{|l|}{$\mathrm{dpm} / 100 \mathrm{~cm} 2$} & \multirow[b]{2}{*}{ LBGR } & \multirow[b]{2}{*}{ Sign } \\
\hline & & Alpha & Beta & Alpha & Beta & Alpha & Beta & & \\
\hline 1 & Floor, Concrete (Electra 6697) & 1 & 1132 & -3.3 & 91.5 & -16.1 & 152.6 & 0.03 & 1 \\
\hline 2 & Floor, Concrete (Electra 6697) & 4 & 1062 & -0.3 & 21.5 & -1.6 & 35.8 & 0.01 & 1 \\
\hline 3 & Floor, Concrete (Electra 6697) & 3 & 968 & -1.3 & -72.5 & -6.4 & -121.0 & 0.00 & 1 \\
\hline 4 & Floor, Concrete (Electra 6697) & 4 & 635 & -0.3 & -405.5 & -1.6 & -676.6 & 0.00 & 1 \\
\hline 5 & Floor, Concrete (Electra 6697) & 4 & 1109 & -0.3 & 68.5 & -1.6 & 114.2 & 0.02 & 1 \\
\hline 6 & Floor, Concrete (Electra 6697) & 13 & 1126 & 8.7 & 85.5 & 41.9 & 142.6 & 0.45 & 1 \\
\hline 7 & Floor, Concrete (Electra 6697) & 11 & 1125 & 6.7 & 84.5 & 32.2 & 140.9 & 0.35 & 1 \\
\hline 8 & Floor, Concrete (Electra 6697) & 6 & 1114 & 1.7 & 73.5 & 8.1 & 122.6 & 0.11 & 1 \\
\hline 9 & Floor, Concrete (Electra 6697) & 10 & 1025 & 5.7 & -15.5 & 27.4 & -25.9 & 0.27 & 1 \\
\hline 10 & Floor, Concrete (Electra 6697) & 2 & 1068 & -2.3 & 27.5 & -11.2 & 45.8 & 0.01 & 1 \\
\hline 11 & Floor, Concrete (Electra 6697) & 4 & 1022 & -0.3 & -18.5 & -1.6 & -30.9 & 0.00 & 1 \\
\hline 12 & Floor, Concrete (Electra 6697) & 6 & 743 & 1.7 & -297.5 & 8.1 & -496.4 & 0.08 & 1 \\
\hline & & & & & Averages & 6.5 & -49.7 & & \\
\hline & & & & & StDev & 18.1 & 267.3 & & \\
\hline & & & & & Median & -1.6 & 40.8 & & \\
\hline & & & & & Max & 41.9 & 152.6 & & \\
\hline & & & & & Min & -16.1 & -676.6 & & \\
\hline & & & & & *LBGR & 0.06 & & & \\
\hline & & & & & \multicolumn{3}{|c|}{ *Negative vales not used in LGBR } & & \\
\hline & & & & & & \# of samples: & 12 & & \\
\hline & & & & & & Sign test stat: & 12 & & \\
\hline
\end{tabular}




\section{0-ER-A26-259}

Final Status Survey, Room 102, Floor / FSS-102-F-4

\begin{tabular}{|c|c|c|c|c|c|c|c|c|c|}
\hline & & \multicolumn{2}{|c|}{ RAW DATA cpm } & \multicolumn{2}{|c|}{ BKG ADJUSTED cpm } & \multicolumn{2}{|l|}{$\mathrm{dpm} / 100 \mathrm{~cm} 2$} & \multirow[b]{2}{*}{ LBGR } & \multirow[b]{2}{*}{ Sign } \\
\hline & & Alpha & Beta & Alpha & Beta & Alpha & Beta & & \\
\hline 1 & Floor, Concrete (Electra 6697) & 4 & 967 & -0.3 & -73.5 & -1.6 & -122.7 & 0.00 & 1 \\
\hline 2 & Floor, Concrete (Electra 6697) & 6 & 938 & 1.7 & -102.5 & 8.1 & -171.0 & 0.08 & 1 \\
\hline 3 & Floor, Concrete (Electra 6697) & 4 & 1002 & -0.3 & -38.5 & -1.6 & -64.3 & 0.00 & 1 \\
\hline 4 & Floor, Concrete (Electra 6697) & 6 & 1032 & 1.7 & -8.5 & 8.1 & -14.2 & 0.08 & 1 \\
\hline 5 & Floor, Concrete (Electra 6697) & 5 & 998 & 0.7 & -42.5 & 3.3 & -70.9 & 0.03 & 1 \\
\hline 6 & Floor, Concrete (Electra 6697) & 4 & 975 & -0.3 & -65.5 & -1.6 & -109.3 & 0.00 & 1 \\
\hline 7 & Floor, Concrete (Electra 6697) & 7 & 1014 & 2.7 & -26.5 & 12.9 & -44.3 & 0.13 & 1 \\
\hline 8 & Floor, Concrete (Electra 6697) & 4 & 799 & -0.3 & -241.5 & -1.6 & -402.9 & 0.00 & 1 \\
\hline 9 & Floor, Concrete (Electra 6697) & 4 & 1003 & -0.3 & -37.5 & -1.6 & -62.6 & 0.00 & 1 \\
\hline 10 & Floor, Concrete (Electra 6697) & 3 & 978 & -1.3 & -62.5 & -6.4 & -104.3 & 0.00 & 1 \\
\hline 11 & Floor, Concrete (Electra 6697) & 7 & 1021 & 2.7 & -19.5 & 12.9 & -32.6 & 0.13 & 1 \\
\hline 12 & Floor, Concrete (Electra 6697) & 9 & 1003 & 4.7 & -37.5 & 22.6 & -62.6 & 0.23 & 1 \\
\hline 13 & Floor, Concrete (Electra 6697) & 4 & 1003 & -0.3 & -37.5 & -1.6 & -62.6 & 0.00 & 1 \\
\hline 14 & Floor, Concrete (Electra 6697) & 5 & 1069 & 0.7 & 28.5 & 3.3 & 47.5 & 0.04 & 1 \\
\hline 15 & Floor, Concrete (Electra 6697) & 9 & 1095 & 4.7 & 54.5 & 22.6 & 90.9 & 0.24 & 1 \\
\hline & & & & & Averages & 5.2 & -79.1 & & \\
\hline & & & & & StDev & 9.1 & 110.3 & & \\
\hline & & & & & Median & 3.3 & -62.6 & & \\
\hline & & & & & Max & 22.6 & 90.9 & & \\
\hline & & & & & Min & -6.4 & -402.9 & & \\
\hline & & & & & *LBGR & 0.05 & & & \\
\hline & & & & & *Negative & vales not used $\mathrm{i}$ & LGBR & & \\
\hline & & & & & & \# of samples: & 15 & & \\
\hline & & & & & & Sign test stat: & 15 & & \\
\hline
\end{tabular}




\section{0-ER-A26-273}

Final Status Survey, Room 102 East Wall 102-EW-1

\begin{tabular}{|c|c|c|c|c|c|c|c|c|c|}
\hline & & \multicolumn{2}{|c|}{ RAW DATA cpm } & \multicolumn{2}{|c|}{ BKG ADJUSTED cpm } & \multicolumn{2}{|l|}{$\mathrm{dpm} / 100 \mathrm{~cm} 2$} & \multirow[b]{2}{*}{ LBGR } & \multirow[b]{2}{*}{ Sigr } \\
\hline & & Alpha & Beta & Alpha & Beta & Alpha & Beta & & \\
\hline 1 & Wall, Metal (Electra 1120) & 9 & 689 & 4.4 & -642.7 & 21.0 & -1072.2 & 0.21 & 1 \\
\hline 2 & Wall, Metal (Electra 1120) & 5 & 730 & 0.4 & -601.7 & 1.7 & -1003.8 & 0.02 & 1 \\
\hline 3 & Wall, Metal (Electra 1120) & 15 & 1068 & 10.4 & -263.7 & 50.0 & -439.9 & 0.50 & 1 \\
\hline 4 & Wall, Concrete (Electra 1201) & 9 & 933 & 4.7 & -107.5 & 22.6 & -179.4 & 0.23 & 1 \\
\hline 5 & Wall, Concrete (Electra 1201) & 9 & 977 & 4.7 & -63.5 & 22.6 & -106.0 & 0.23 & 1 \\
\hline 6 & Wall, Metal (Electra 1120) & 17 & 1013 & 12.4 & -318.7 & 59.7 & -531.7 & 0.60 & 1 \\
\hline 7 & Wall, Wood (Electra 1120) & 5 & 715 & 0.3 & -695.7 & 1.2 & -1160.7 & 0.01 & 1 \\
\hline 8 & Wall, Metal (Electra 1120) & 6 & 655 & 1.4 & -676.7 & 6.5 & -1129.0 & 0.07 & 1 \\
\hline 9 & Wall, Concrete (Electra 1120) & 6 & 990 & 1.7 & -50.5 & 8.1 & -84.3 & 0.08 & 1 \\
\hline 10 & Wall, Concrete (Electra 1120) & 9 & 910 & 4.7 & -130.5 & 22.6 & -217.8 & 0.23 & 1 \\
\hline 11 & Wall, Concrete (Electra 1120) & 13 & 968 & 8.7 & -72.5 & 41.9 & -121.0 & 0.42 & 1 \\
\hline 12 & Wall, Concrete (1120) & 7 & 931 & 2.7 & -109.5 & 12.9 & -182.7 & 0.13 & 1 \\
\hline 13 & Wall, Concrete (1120) & 10 & 937 & 5.7 & -103.5 & 27.4 & -172.7 & 0.27 & 1 \\
\hline & & & & & Averages & 22.9 & -492.4 & & \\
\hline & & & & & StDev & 18.2 & 435.8 & & \\
\hline & & & & & Median & 22.6 & -217.8 & & \\
\hline & & & & & Max & 59.7 & -84.3 & & \\
\hline & & & & & Min & 1.2 & -1160.7 & & \\
\hline & & & & & *LBGR & 0.23 & & & \\
\hline & & & & & *Negative & vales not used in & LGBR & & \\
\hline & & & & & & \# of samples: & 13 & & \\
\hline & & & & & & Sign test stat: & 13 & & \\
\hline
\end{tabular}


10-ER-A26-277

Final Status Survey, Room 102 East Wall 102-EW-2

\begin{tabular}{|c|c|c|c|c|c|c|c|c|c|}
\hline & & \multicolumn{2}{|c|}{ RAW DATA cpm } & \multicolumn{2}{|c|}{ BKG ADJUSTED cpm } & \multicolumn{2}{|l|}{$\mathrm{dpm} / 100 \mathrm{~cm} 2$} & \multirow[b]{2}{*}{ LBGR } & \multirow[b]{2}{*}{ Sigr } \\
\hline & & Alpha & Beta & Alpha & Beta & Alpha & Beta & & \\
\hline 1 & Wall, Concrete (Electra 1201) & 17 & 996 & 12.7 & -44.5 & 61.2 & -74.3 & 0.61 & 1 \\
\hline 2 & Wall, Concrete (Electra 1201) & 10 & 964 & 5.7 & -76.5 & 27.4 & -127.7 & 0.27 & 1 \\
\hline 3 & Wall, Concrete (Electra 1201) & 14 & 886 & 9.7 & -154.5 & 46.7 & -257.8 & 0.47 & 1 \\
\hline 4 & Wall, Concrete (Electra 1201) & 10 & 965 & 5.7 & -75.5 & 27.4 & -126.0 & 0.27 & 1 \\
\hline 5 & Wall, Concrete (Electra 1201) & 20 & 895 & 15.7 & -145.5 & 75.7 & -242.8 & 0.76 & 1 \\
\hline 6 & Wall, Concrete (Electra 1201) & 21 & 963 & 16.7 & -77.5 & 80.6 & -129.3 & 0.81 & 1 \\
\hline 7 & Wall, Concrete (Electra 1201) & 13 & 994 & 8.7 & -46.5 & 41.9 & -77.6 & 0.42 & 1 \\
\hline 8 & Wall, Concrete (Electra 1201) & 11 & 953 & 6.7 & -87.5 & 32.2 & -146.0 & 0.32 & 1 \\
\hline 9 & Wall, Concrete (Electra 1201) & 10 & 935 & 5.7 & -105.5 & 27.4 & -176.1 & 0.27 & 1 \\
\hline 10 & Wall, Concrete (Electra 1201) & 9 & 979 & 4.7 & -61.5 & 22.6 & -102.6 & 0.23 & 1 \\
\hline 11 & Wall, Concrete (Electra 1201) & 14 & 947 & 9.7 & -93.5 & 46.7 & -156.0 & 0.47 & 1 \\
\hline 12 & Wall, Concrete (1201) & 9 & 956 & 4.7 & -84.5 & 22.6 & -141.0 & 0.23 & 1 \\
\hline & & & & & Averages & 42.7 & -146.4 & & \\
\hline & & & & & StDev & 20.3 & 56.9 & & \\
\hline & & & & & Median & 37.1 & -135.2 & & \\
\hline & & & & & Max & 80.6 & -74.3 & & \\
\hline & & & & & Min & 22.6 & -257.8 & & \\
\hline & & & & & ${ }^{*}$ LBGR & 0.43 & & & \\
\hline & & & & & *Negative & vales not used in & LGBR & & \\
\hline & & & & & & \# of samples: & 12 & & \\
\hline & & & & & & Sign test stat: & 12 & & \\
\hline
\end{tabular}




\section{0-ER-A26-278}

Final Status Survey, Room 102 Ceiling 102-C-1

\begin{tabular}{|c|c|c|c|c|c|c|c|c|c|}
\hline & & \multicolumn{2}{|c|}{ RAW DATA cpm } & \multicolumn{2}{|c|}{ BKG ADJUSTED cpm } & \multicolumn{2}{|l|}{$\mathrm{dpm} / 100 \mathrm{~cm} 2$} & \multirow[b]{2}{*}{ LBGR } & \multirow[b]{2}{*}{ Sign } \\
\hline & & Alpha & Beta & Alpha & Beta & Alpha & Beta & & \\
\hline 1 & Ceiling, Concrete (Electra 1201) & 4 & 1006 & -0.3 & -34.5 & -1.6 & -57.6 & 0.00 & 1 \\
\hline 2 & Ceiling, Concrete (Electra 1201) & 5 & 1044 & 0.7 & 3.5 & 3.3 & 5.8 & 0.03 & 1 \\
\hline 3 & Ceiling, Concrete (Electra 1201) & 12 & 1112 & 7.7 & 71.5 & 37.1 & 119.2 & 0.39 & 1 \\
\hline 4 & Ceiling, Concrete (Electra 1201) & 8 & 993 & 3.7 & -47.5 & 17.8 & -79.3 & 0.18 & 1 \\
\hline 5 & Ceiling, Concrete (Electra 1201) & 5 & 1065 & 0.7 & 24.5 & 3.3 & 40.8 & 0.04 & 1 \\
\hline 6 & Ceiling, Concrete (Electra 1120) & 7 & 1238 & 2.7 & 197.5 & 12.9 & 329.5 & 0.20 & 1 \\
\hline 7 & Ceiling, Concrete (Electra 1120) & 8 & 1097 & 3.7 & 56.5 & 17.8 & 94.2 & 0.20 & 1 \\
\hline 8 & Ceiling, Concrete (Electra 1120) & 10 & 1122 & 5.7 & 81.5 & 27.4 & 135.9 & 0.30 & 1 \\
\hline 9 & Ceiling, Concrete (Electra 1120) & 8 & 1202 & 3.7 & 161.5 & 17.8 & 269.4 & 0.23 & 1 \\
\hline 10 & Ceiling, Concrete (Electra 1120) & 13 & 1143 & 8.7 & 102.5 & 41.9 & 171.0 & 0.45 & 1 \\
\hline 11 & Ceiling, Concrete (Electra 1120) & 9 & 1223 & 4.7 & 182.5 & 22.6 & 304.4 & 0.29 & 1 \\
\hline 12 & Ceiling, Concrete (1120) & 9 & 1238 & 4.7 & 197.5 & 22.6 & 329.5 & 0.29 & 1 \\
\hline & & & & & Averages & 18.6 & 138.6 & & \\
\hline & & & & & StDev & 13.2 & 145.9 & & \\
\hline & & & & & Median & 17.8 & 127.6 & & \\
\hline & & & & & Max & 41.9 & 329.5 & & \\
\hline & & & & & Min & -1.6 & -79.3 & & \\
\hline & & & & & ${ }^{*}$ LBGR & 0.21 & & & \\
\hline & & & & & \multicolumn{3}{|c|}{ *Negative vales not used in LGBR } & & \\
\hline & & & & & & \# of samples: & 12 & & \\
\hline & & & & & & Sign test stat: & 12 & & \\
\hline
\end{tabular}


10-ER-A26-285

Final Status Survey, Room 102 Shield Doors /102-SDW-1

\begin{tabular}{|c|c|c|c|c|c|c|c|c|c|}
\hline & & \multicolumn{2}{|c|}{ RAW DATA cpm } & \multicolumn{2}{|c|}{ BKG ADJUSTED cpm } & \multicolumn{2}{|l|}{$\mathrm{dpm} / 100 \mathrm{~cm} 2$} & \multirow[b]{2}{*}{ LBGR } & \multirow[b]{2}{*}{ Sign } \\
\hline & & Alpha & Beta & Alpha & Beta & Alpha & Beta & & \\
\hline 1 & Front East Door, Metal (Electra 1120) & 2 & 543 & -2.7 & -788.7 & -12.8 & -1315.8 & 0.00 & 1 \\
\hline 2 & Front East Door, Metal (Electra 1120) & 11 & 560 & 6.4 & -771.7 & 30.7 & -1287.5 & 0.31 & 1 \\
\hline 3 & Front East Door, Metal (Electra 1120) & 8 & 491 & 3.4 & -840.7 & 16.2 & -1402.6 & 0.16 & 1 \\
\hline 4 & Side East Door, Metal (Electra 1120) & 10 & 425 & 5.4 & -906.7 & 25.8 & -1512.7 & 0.26 & 1 \\
\hline 5 & Front West Door, Metal (Electra 1504) & 10 & 494 & 5.4 & -837.7 & 25.8 & -1397.6 & 0.26 & 1 \\
\hline 6 & Front West Door, Metal (Electra 1504) & 8 & 417 & 3.4 & -914.7 & 16.2 & -1526.0 & 0.16 & 1 \\
\hline 7 & Front West Door, Metal (Electra 1120) & 11 & 565 & 6.4 & -766.7 & 30.7 & -1279.1 & 0.31 & 1 \\
\hline 8 & Side West Door, Metal (Electra 1120) & 10 & 440 & 5.4 & -891.7 & 25.8 & -1487.7 & 0.26 & 1 \\
\hline 9 & Side West Door, Metal (Electra 1120) & 9 & 452 & 4.4 & -879.7 & 21.0 & -1467.6 & 0.21 & 1 \\
\hline 10 & Front West Door, Metal (Electra 1120) & 16 & 599 & 11.4 & -732.7 & 54.8 & -1222.4 & 0.55 & 1 \\
\hline 11 & Backside West Door, Metal (Electra 1120) & 20 & 463 & 15.4 & -868.7 & 74.2 & -1449.3 & 0.74 & 1 \\
\hline 12 & Backside East Door, Metal (1120) & 17 & 444 & 12.4 & -887.7 & 59.7 & -1481.0 & 0.60 & 1 \\
\hline & & & & & Averages & 30.7 & -1402.4 & & \\
\hline & & & & & StDev & 22.9 & 102.5 & & \\
\hline & & & & & Median & 25.8 & -1425.9 & & \\
\hline & & & & & Max & 74.2 & -1222.4 & & \\
\hline & & & & & Min & -12.8 & -1526.0 & & \\
\hline & & & & & $*$ LBGR & 0.31 & & & \\
\hline & & & & & \multicolumn{3}{|c|}{ *Negative vales not used in LGBR } & & \\
\hline & & & & & & \# of samples: & 12 & & \\
\hline & & & & & & Sign test stat: & 12 & & \\
\hline
\end{tabular}


Data for Survey Units in Room 104 
10-ER-A26-206

\begin{tabular}{|c|c|c|c|c|c|c|c|}
\hline \multicolumn{2}{|c|}{ RAW DATA cpm } & \multicolumn{2}{|c|}{ BKG ADJUSTED cpm } & \multicolumn{2}{|l|}{$\mathrm{dpm} / 100 \mathrm{~cm} 2$} & \multirow[b]{2}{*}{ LBGR } & \multirow[b]{2}{*}{ Sign } \\
\hline Alpha & Beta & Alpha & Beta & Alpha & Beta & & \\
\hline 4 & 611 & -1.3 & -627.9 & -6.3 & -1047.5 & 0.00 & 1 \\
\hline 2 & 1023 & -2.3 & -17.5 & -11.2 & -29.2 & 0.00 & 1 \\
\hline 3 & 1008 & -1.3 & -32.5 & -6.4 & -54.3 & 0.00 & 1 \\
\hline 8 & 915 & 3.7 & -125.5 & 17.8 & -209.4 & 0.18 & 1 \\
\hline 1 & 973 & -3.3 & -67.5 & -16.1 & -112.7 & 0.00 & 1 \\
\hline 3 & 890 & -1.3 & -150.5 & -6.4 & -251.1 & 0.00 & 1 \\
\hline 10 & 996 & 5.7 & -44.5 & 27.4 & -74.3 & 0.27 & 1 \\
\hline 6 & 1014 & 1.7 & -26.5 & 8.1 & -44.3 & 0.08 & 1 \\
\hline 1 & 943 & -3.3 & -97.5 & -16.1 & -162.7 & 0.00 & 1 \\
\hline 2 & 712 & -2.3 & -328.5 & -11.2 & -548.1 & 0.00 & 1 \\
\hline 6 & 1046 & 1.7 & 5.5 & 8.1 & 9.1 & 0.08 & 1 \\
\hline 7 & 736 & 1.7 & -502.9 & 8.2 & -838.9 & 0.08 & 1 \\
\hline 4 & 645 & -1.3 & -593.9 & -6.3 & -990.7 & 0.00 & 1 \\
\hline 5 & 887 & 0.7 & -153.5 & 3.3 & -256.1 & 0.03 & 1 \\
\hline 4 & 928 & -0.3 & -112.5 & -1.6 & -187.7 & 0.00 & 1 \\
\hline 9 & 945 & 4.7 & -95.5 & 22.6 & -159.4 & 0.23 & 1 \\
\hline 4 & 892 & -0.3 & -148.5 & -1.6 & -247.8 & 0.00 & 1 \\
\hline 11 & 901 & 6.7 & -139.5 & 32.2 & -232.8 & 0.32 & 1 \\
\hline & & & Averages & 2.5 & -302.1 & & \\
\hline & & & StDev & 14.7 & 328.9 & & \\
\hline & & & Median & -1.6 & -198.6 & & \\
\hline & & & Max & 32.2 & 9.1 & & \\
\hline & & & Min & -16.1 & -1047.5 & & \\
\hline & & & $*$ LBGR & 0.02 & & & \\
\hline & & & \multicolumn{3}{|c|}{ *Negative vales not used in LGBR } & & \\
\hline & & & & \# of samples: & 18 & & \\
\hline & & & & Sign test stat: & 18 & & \\
\hline
\end{tabular}




\section{0-ER-A26-213}

Final Status Survey, Room 104 North Wall, Unit \# 104-NW-1

\begin{tabular}{|c|c|c|c|c|c|c|c|c|c|}
\hline & & \multicolumn{2}{|c|}{ RAW DATA cpm } & \multicolumn{2}{|c|}{ BKG ADJUSTED cpm } & \multicolumn{2}{|l|}{$\mathrm{dpm} / 100 \mathrm{~cm} 2$} & \multirow[b]{2}{*}{ LBGR } & \multirow[b]{2}{*}{ Sign } \\
\hline & & Alpha & Beta & Alpha & Beta & \multicolumn{2}{|l|}{ Alpha } & & \\
\hline 1 & North Wall, Concrete & 4 & 896 & -0.3 & -144.5 & -1.6 & -241.1 & 0.00 & 1 \\
\hline 2 & North Wall, Concrete & 6 & 675 & 1.7 & -365.5 & 8.1 & -609.8 & 0.08 & 1 \\
\hline 3 & North Wall, Concrete & 2 & 609 & -2.3 & -431.5 & -11.2 & -719.9 & 0.00 & 1 \\
\hline 4 & North Wall, Concrete & 7 & 576 & 2.7 & -464.5 & 12.9 & -775.0 & 0.13 & 1 \\
\hline 5 & North Wall, Concrete & 6 & 636 & 1.7 & -404.5 & 8.1 & -674.9 & 0.08 & 1 \\
\hline 6 & North Wall, Concrete & 5 & 675 & 0.7 & -365.5 & 3.3 & -609.8 & 0.03 & 1 \\
\hline 7 & North Wall, Concrete & 6 & 922 & 1.7 & -118.5 & 8.1 & -197.7 & 0.08 & 1 \\
\hline 8 & North Wall, Concrete & 7 & 612 & 2.7 & -428.5 & 12.9 & -714.9 & 0.13 & 1 \\
\hline 9 & North Wall, Concrete & 9 & 661 & 4.7 & -379.5 & 22.6 & -633.2 & 0.23 & 1 \\
\hline 10 & North Wall, Concrete & 7 & 644 & 2.7 & -396.5 & 12.9 & -661.5 & 0.13 & 1 \\
\hline 11 & North Wall, Concrete & 6 & 867 & 1.7 & -173.5 & 8.1 & -289.5 & 0.08 & 1 \\
\hline 12 & North Wall, Concrete & 7 & 924 & 2.7 & -116.5 & 12.9 & -194.4 & 0.13 & 1 \\
\hline 13 & North Wall, Concrete & 6 & 1044 & 1.7 & 3.5 & 8.1 & 5.8 & 0.08 & 1 \\
\hline 14 & North Wall, Concrete & 6 & 887 & 1.7 & -153.5 & 8.1 & -256.1 & 0.08 & 1 \\
\hline 15 & North Wall, Concrete & 6 & 980 & 1.7 & -60.5 & 8.1 & -101.0 & 0.08 & 1 \\
\hline 16 & North Wall, Concrete & 7 & 635 & 2.7 & -405.5 & 12.9 & -676.6 & 0.13 & 1 \\
\hline 17 & North Wall, Concrete 65\%, Metal 35\% & 2 & 560 & -2.3 & -480.5 & -11.2 & -801.7 & 0.00 & 1 \\
\hline & & & & & Averages & 7.2 & -479.5 & & \\
\hline & & & & & StDev & 8.6 & 268.6 & & \\
\hline & & & & & Median & 8.1 & -609.8 & & \\
\hline & & & & & Max & 22.6 & 5.8 & & \\
\hline & & & & & Min & -11.2 & -801.7 & & \\
\hline & & & & & *LBGR & 0.07 & & & \\
\hline & & & & & *Negative $\mathrm{v}$ & s not used in LGBR & & & \\
\hline & & & & & & \# of samples: & 17 & & \\
\hline & & & & & & Sign test stat: & 17 & & \\
\hline
\end{tabular}


10-ER-A26-218

RAW DATA cpm

Final Status Survey, Room 104 East Wall, Unit \# 104-EW-1

\begin{tabular}{|l|l}
1 & East Wall, Concrete (Electra 1120) \\
\hline 2 & East Wall Concrete (Electra 1120)
\end{tabular}

2 East Wall, Concrete (Electra 1120)

3 East Wall, Concrete (Electra 1504)

4 East Wall, Concrete (Electra 1504)

5 East Wall, Concrete (Electra 1120)

6 East Wall, Concrete (Electra 1120)

7 East Wall, Concrete (Electra 1120)

8 East Wall, Concrete (Electra 1120)

9 East Wall, Concrete (Electra 1120)

10 East Wall, Concrete (Electra 1120)

11 East Wall, Metal (Electra 1120)

12 East Wall, Concrete (Electra 1504)

13 East Wall, Concrete (Electra 1504)

14 East Wall, Concrete (Electra 1120)

15 East Wall, Concrete (Electra 1120)

16 East Wall, Concrete (Electra 1120)

\begin{tabular}{|c|c|c|c|c|c|c|c|}
\hline \multicolumn{2}{|c|}{ RAW DATA cpm } & \multicolumn{2}{|c|}{ BKG ADJUSTED cpm } & \multicolumn{2}{|c|}{$\mathrm{dpm} / 100 \mathrm{~cm} 2$} & \multirow[b]{2}{*}{ LBGR } & \multirow[b]{2}{*}{ Sign } \\
\hline Alpha & Beta & Alpha & Beta & Alpha & Beta & & \\
\hline 4 & 773 & -0.3 & -267.5 & -1.6 & -446.3 & 0.00 & 1 \\
\hline 7 & 699 & 2.7 & -341.5 & 12.9 & -569.8 & 0.13 & 1 \\
\hline 1 & 694 & -3.3 & -346.5 & -16.1 & -578.1 & 0.00 & 1 \\
\hline 4 & 599 & -0.3 & -441.5 & -1.6 & -736.6 & 0.00 & 1 \\
\hline 6 & 611 & 1.7 & -429.5 & 8.1 & -716.6 & 0.08 & 1 \\
\hline 10 & 661 & 5.7 & -379.5 & 27.4 & -633.2 & 0.27 & 1 \\
\hline 3 & 652 & -1.3 & -388.5 & -6.4 & -648.2 & 0.00 & 1 \\
\hline 4 & 740 & -0.3 & -300.5 & -1.6 & -501.4 & 0.00 & 1 \\
\hline 6 & 628 & 1.7 & -412.5 & 8.1 & -688.2 & 0.08 & 1 \\
\hline 3 & 728 & -1.3 & -312.5 & -6.4 & -521.4 & 0.00 & 1 \\
\hline 6 & 509 & 1.4 & -822.7 & 6.5 & -1372.5 & 0.07 & 1 \\
\hline 5 & 406 & 0.7 & -634.5 & 3.3 & -1058.6 & 0.03 & 1 \\
\hline 5 & 661 & 0.7 & -379.5 & 3.3 & -633.2 & 0.03 & 1 \\
\hline 4 & 640 & -0.3 & -400.5 & -1.6 & -668.2 & 0.00 & 1 \\
\hline 9 & 703 & 4.7 & -337.5 & 22.6 & -563.1 & 0.23 & 1 \\
\hline 11 & 749 & 6.7 & -291.5 & 32.2 & -486.4 & 0.32 & 1 \\
\hline & & & Averages & 5.6 & -676.4 & & \\
\hline & & & StDev & 12.9 & 233.6 & & \\
\hline & & & Median & 3.3 & -633.2 & & \\
\hline & & & Max & 32.2 & -446.3 & & \\
\hline & & & Min & -16.1 & -1372.5 & & \\
\hline & & & *LBGR & 0.06 & & & \\
\hline & & & \multicolumn{3}{|c|}{ *Negative vales not used in LGBR } & & \\
\hline & & & \multicolumn{2}{|c|}{ \# of samples: } & 16 & & \\
\hline & & & \multicolumn{2}{|c|}{ Sign test stat: } & 16 & & \\
\hline
\end{tabular}


10-ER-A26-215

Final Status Survey, Room 104 South Wall, Unit \# 104-SW-1

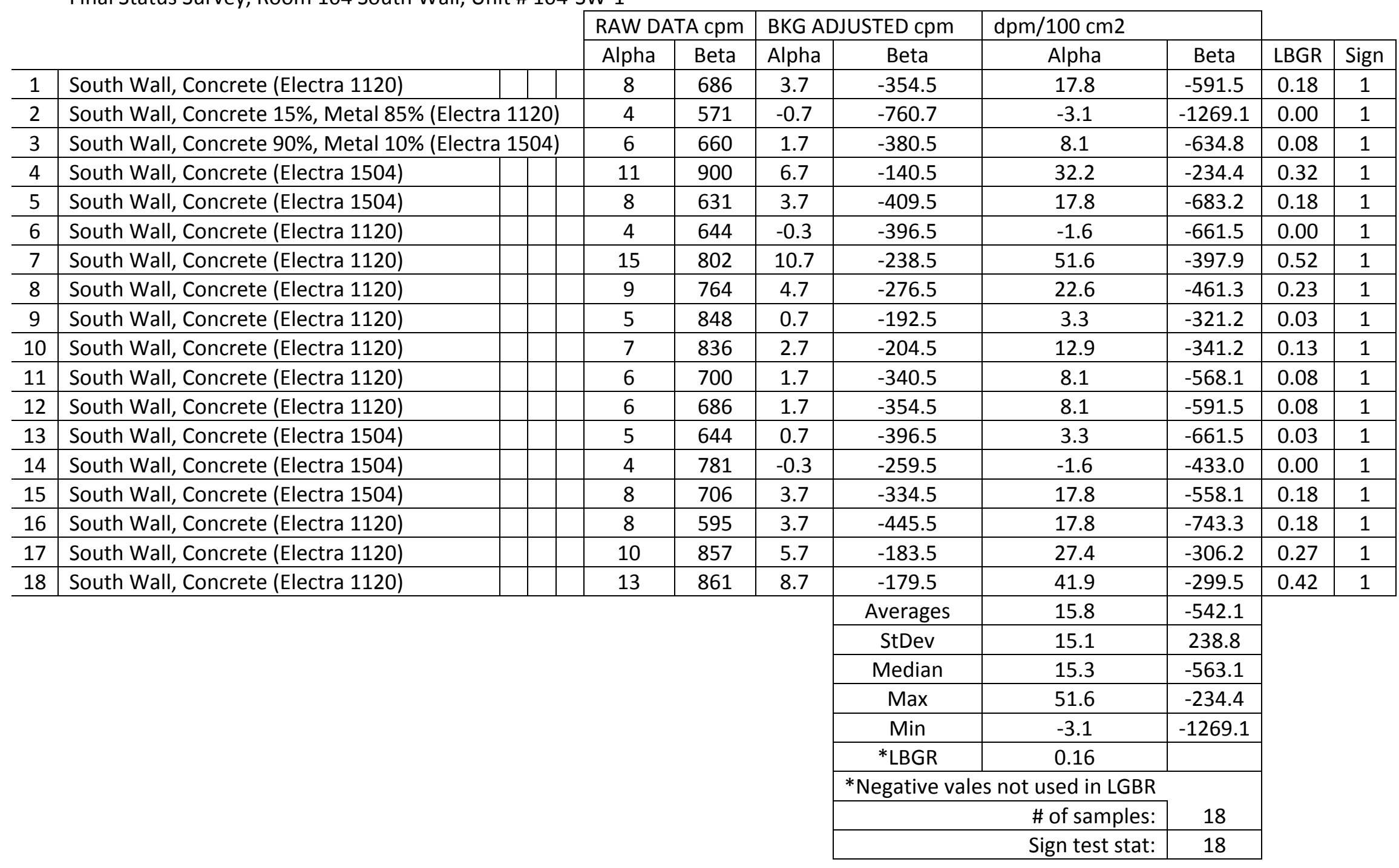


10-ER-A26-221

\begin{tabular}{|c|c|c|c|c|c|c|c|}
\hline \multicolumn{2}{|c|}{ RAW DATA cpm } & \multicolumn{2}{|c|}{ BKG ADJUSTED cpm } & \multicolumn{2}{|c|}{$\mathrm{dpm} / 100 \mathrm{~cm} 2$} & \multirow[b]{2}{*}{ LBGR } & \multirow[b]{2}{*}{ Sign } \\
\hline Alpha & Beta & Alpha & Beta & Alpha & Beta & & \\
\hline 12 & 868 & 7.7 & -172.5 & 37.1 & -287.8 & 0.37 & 1 \\
\hline 6 & 714 & 1.7 & -326.5 & 8.1 & -544.8 & 0.08 & 1 \\
\hline 8 & 590 & 3.4 & -741.7 & 16.2 & -1237.4 & 0.16 & 1 \\
\hline 5 & 626 & 0.4 & -705.7 & 1.7 & -1177.3 & 0.02 & 1 \\
\hline 12 & 608 & 7.7 & -432.5 & 37.1 & -721.6 & 0.37 & 1 \\
\hline 7 & 670 & 2.7 & -370.5 & 12.9 & -618.2 & 0.13 & 1 \\
\hline 6 & 941 & 1.7 & -99.5 & 8.1 & -166.0 & 0.08 & 1 \\
\hline 10 & 985 & 5.7 & -55.5 & 27.4 & -92.6 & 0.27 & 1 \\
\hline 10 & 1028 & 5.7 & -12.5 & 27.4 & -20.9 & 0.27 & 1 \\
\hline 5 & 948 & 0.7 & -92.5 & 3.3 & -154.4 & 0.03 & 1 \\
\hline 8 & 938 & 3.7 & -102.5 & 17.8 & -171.0 & 0.18 & 1 \\
\hline 5 & 731 & 0.7 & -309.5 & 3.3 & -516.4 & 0.03 & 1 \\
\hline 3 & 579 & -1.3 & -461.5 & -6.4 & -770.0 & 0.00 & 1 \\
\hline 6 & 674 & 1.7 & -366.5 & 8.1 & -611.5 & 0.08 & 1 \\
\hline 6 & 570 & 1.7 & -470.5 & 8.1 & -785.0 & 0.08 & 1 \\
\hline 5 & 625 & 0.7 & -415.5 & 3.3 & -693.2 & 0.03 & 1 \\
\hline 6 & 651 & 1.7 & -389.5 & 8.1 & -649.9 & 0.08 & 1 \\
\hline 8 & 642 & 3.7 & -398.5 & 17.8 & -664.9 & 0.18 & 1 \\
\hline 14 & 927 & 9.7 & -113.5 & 46.7 & -189.4 & 0.47 & 1 \\
\hline 11 & 949 & 6.7 & -91.5 & 32.2 & -152.7 & 0.32 & 1 \\
\hline 3 & 563 & -1.3 & -477.5 & -6.4 & -796.7 & 0.00 & 1 \\
\hline & & & Averages & 14.8 & -524.8 & & \\
\hline & & & StDev & 14.8 & 345.9 & & \\
\hline & & & Median & 8.1 & -611.5 & & \\
\hline & & & Max & 46.7 & -20.9 & & \\
\hline & & & Min & -6.4 & -1237.4 & & \\
\hline & & & *LBGR & 0.15 & & & \\
\hline & & & ${ }^{*}$ Negative va & not used & $n$ LGBR & & \\
\hline & & & & samples: & 21 & & \\
\hline & & & & est stat: & 21 & & \\
\hline
\end{tabular}




\begin{tabular}{|c|c|c|c|c|c|c|c|c|c|}
\hline \multicolumn{2}{|r|}{ 10-ER-A26-226 } & \multicolumn{2}{|c|}{ RAW DATA cpm } & \multicolumn{2}{|c|}{ BKG ADJUSTED cpm } & \multicolumn{2}{|l|}{$\mathrm{dpm} / 100 \mathrm{~cm} 2$} & \multirow[b]{2}{*}{ LBGR } & \multirow[b]{2}{*}{ Sign } \\
\hline \multicolumn{2}{|c|}{ Final Status Survey, Room 104, Ceiling, Unit \# 104-C-1 } & Alpha & Beta & Alpha & Beta & Alpha & Beta & & \\
\hline 1 & Ceiling, Concrete & 7 & 1235 & 2.7 & 194.5 & 12.9 & 324.4 & 0.19 & 1 \\
\hline 2 & Ceiling, Concrete & 13 & 1142 & 8.7 & 101.5 & 41.9 & 169.3 & 0.45 & 1 \\
\hline 3 & Ceiling, Concrete & 10 & 1117 & 5.7 & 76.5 & 27.4 & 127.6 & 0.30 & 1 \\
\hline 4 & Ceiling, Concrete & 7 & 1057 & 2.7 & 16.5 & 12.9 & 27.5 & 0.13 & 1 \\
\hline 5 & Ceiling, Concrete & 9 & 1127 & 4.7 & 86.5 & 22.6 & 144.3 & 0.25 & 1 \\
\hline 6 & Ceiling, Concrete & 7 & 1125 & 2.7 & 84.5 & 12.9 & 140.9 & 0.16 & 1 \\
\hline 7 & Ceiling, Concrete & 7 & 1173 & 2.7 & 132.5 & 12.9 & 221.0 & 0.17 & 1 \\
\hline 8 & Ceiling, Concrete & 7 & 1128 & 2.7 & 87.5 & 12.9 & 145.9 & 0.16 & 1 \\
\hline 9 & Ceiling, Concrete & 9 & 984 & 4.7 & -56.5 & 22.6 & -94.3 & 0.23 & 1 \\
\hline 10 & Ceiling, Concrete & 9 & 1092 & 4.7 & 51.5 & 22.6 & 85.9 & 0.24 & 1 \\
\hline 11 & Ceiling, Concrete & 3 & 1128 & -1.3 & 87.5 & -6.4 & 145.9 & 0.03 & 1 \\
\hline 12 & Ceiling, Concrete & 10 & 1186 & 5.7 & 145.5 & 27.4 & 242.7 & 0.32 & 1 \\
\hline 13 & Ceiling, Concrete & 6 & 1224 & 1.7 & 183.5 & 8.1 & 306.1 & 0.14 & 1 \\
\hline 14 & Ceiling, Concrete & 11 & 728 & 6.7 & -312.5 & 32.2 & -521.4 & 0.32 & 1 \\
\hline 15 & Ceiling, Concrete & 3 & 1070 & -1.3 & 29.5 & -6.4 & 49.2 & 0.01 & 1 \\
\hline 16 & Ceiling, Concrete & 7 & 1162 & 2.7 & 121.5 & 12.9 & 202.7 & 0.17 & 1 \\
\hline 17 & Ceiling, Concrete & 3 & 798 & -1.3 & -242.5 & -6.4 & -404.6 & 0.00 & 1 \\
\hline & & & & & Averages & 15.5 & 77.2 & & \\
\hline & & & & & StDev & 13.6 & 227.7 & & \\
\hline & & & & & Median & 12.9 & 144.3 & & \\
\hline & & & & & Max & 41.9 & 324.4 & & \\
\hline & & & & & Min & -6.4 & -521.4 & & \\
\hline & & & & & *LBGR & 0.17 & & & \\
\hline & & & & & *Negative & vales not used $\mathrm{i}$ & LGBR & & \\
\hline & & & & & & \# of samples: & 17 & & \\
\hline & & & & & & Sign test stat: & 17 & & \\
\hline
\end{tabular}


Data for Survey Units in Room 106 


\section{0-ER-A26-275}

Final Status Survey, Room 106, Floor, 106-F-1

POST-DECON

\begin{tabular}{|c|c|c|c|c|c|c|c|c|c|}
\hline & & & \\
\hline & & Alpha & Beta & Alpha & Beta & Alpha & Beta & LBGR & Sign \\
\hline 1 & Floor, Concrete (Electra 1120) & 7 & 1100 & 2.7 & 59.5 & 12.9 & 99.2 & 0.15 & 1 \\
\hline 2 & Floor, Concrete (Electra 1120) & 10 & 1314 & 5.7 & 273.5 & 27.4 & 456.2 & 0.37 & 1 \\
\hline 3 & Floor, Concrete (Electra 1120) & 2 & 958 & -2.3 & -82.5 & -11.2 & -137.7 & 0.00 & 1 \\
\hline 4 & Floor, Concrete (Electra 1120) & 3 & 951 & -1.3 & -89.5 & -6.4 & -149.4 & 0.00 & 1 \\
\hline 5 & Floor, Concrete (Electra 1120) & 13 & 967 & 8.7 & -73.5 & 41.9 & -122.7 & 0.42 & 1 \\
\hline 6 & Floor, Concrete (Electra 1120) & 5 & 1352 & 0.7 & 311.5 & 3.3 & 519.6 & 0.14 & 1 \\
\hline 7 & Floor, Concrete (Electra 1120) post decon & 8 & 621 & 3.7 & -419.5 & 17.8 & -699.9 & 0.18 & 1 \\
\hline 8 & Floor, Metal (Electra 1120) & 11 & 661 & 6.4 & -670.7 & 30.7 & -1119.0 & 0.31 & 1 \\
\hline 9 & Floor, Metal (Electra 1120) & 5 & 724 & 0.4 & -607.7 & 1.7 & -1013.8 & 0.02 & 1 \\
\hline 10 & Floor, Concrete (Electra 1120) & 9 & 999 & 4.7 & -41.5 & 22.6 & -69.3 & 0.23 & 1 \\
\hline 11 & Floor, Concrete (Electra 1120) & 2 & 896 & -2.3 & -144.5 & -11.2 & -241.1 & 0.00 & 1 \\
\hline 12 & Floor, Concrete (Electra 1120) & 6 & 970 & 1.7 & -70.5 & 8.1 & -117.7 & 0.08 & 1 \\
\hline 13 & Floor, Metal (Electra 1120) & 4 & 601 & -0.7 & -730.7 & -3.1 & -1219.1 & 0.00 & 1 \\
\hline & & & & & Averages & 10.3 & -293.4 & & \\
\hline & & & & & StDev & 16.9 & 559.6 & & \\
\hline & & & & & Median & 8.1 & -137.7 & & \\
\hline & & & & & Max & 41.9 & 519.6 & & \\
\hline & & & & & Min & -11.2 & -1219.1 & & \\
\hline & & & & & *LBGR & 0.10 & & & \\
\hline & & & & & *Negative & ales not used & LGBR & & \\
\hline & & & & & & \# of samples: & 13 & & \\
\hline & & & & & & Sign test stat: & 13 & & \\
\hline
\end{tabular}


10-ER-A26-276

Final Status Survey, Room 106 Floor, Hot Spot, Post-Decon

\begin{tabular}{|c|c|c|c|c|c|c|c|c|c|}
\hline & & \multicolumn{2}{|c|}{ RAW DATA cpm } & \multicolumn{2}{|c|}{ BKG ADJUSTED cpm } & \multicolumn{2}{|l|}{$\mathrm{dpm} / 100 \mathrm{~cm} 2$} & \multirow[b]{2}{*}{ LBGR } & \multirow[b]{2}{*}{ Sign } \\
\hline & & Alpha & Beta & Alpha & Beta & Alpha & Beta & & \\
\hline 1 & Floor, Concrete (Electra 1504) & 8 & 621 & 3.7 & -419.5 & 17.8 & -699.9 & 0.18 & 1 \\
\hline 2 & Floor, Concrete (Electra 1120) & 11 & 1164 & 6.7 & 123.5 & 32.2 & 206.0 & 0.36 & 1 \\
\hline 3 & Floor, Concrete (Electra 6697) & 13 & 1198 & 8.7 & 157.5 & 41.9 & 262.7 & 0.47 & 1 \\
\hline 4 & Floor, Concrete (Electra 6697) & 19 & 1198 & 14.7 & 157.5 & 70.9 & 262.7 & 0.76 & 1 \\
\hline 5 & Floor, Concrete (Electra 6697) & 12 & 982 & 7.7 & -58.5 & 37.1 & -97.6 & 0.37 & 1 \\
\hline 6 & Floor, Concrete (Electra 6697) & 12 & 1022 & 7.7 & -18.5 & 37.1 & -30.9 & 0.37 & 1 \\
\hline 7 & Floor, Concrete (Electra 6697) & 17 & 1185 & 12.7 & 144.5 & 61.2 & 241.0 & 0.66 & 1 \\
\hline & & & & & Averages & 42.6 & 20.6 & & \\
\hline & & & & & StDev & 18.0 & 350.2 & & \\
\hline & & & & & Median & 37.1 & 206.0 & & \\
\hline & & & & & Max & 70.9 & 262.7 & & \\
\hline & & & & & Min & 17.8 & -699.9 & & \\
\hline & & & & & *LBGR & 0.43 & & & \\
\hline & & & & & *Negative & vales not used & LGBR & & \\
\hline & & & & & & \# of samples: & 7 & & \\
\hline & & & & & & Sign test stat: & 7 & & \\
\hline
\end{tabular}




\section{0-ER-A26-290}

Final Status Survey, Room 106 East Wall / 106-EW-1

\begin{tabular}{|c|c|c|c|c|c|c|c|c|c|}
\hline & & \multicolumn{2}{|c|}{ RAW DATA cpm } & \multicolumn{2}{|c|}{ BKG ADJUSTED cpm } & \multicolumn{2}{|l|}{$\mathrm{dpm} / 100 \mathrm{~cm} 2$} & \multirow[b]{2}{*}{ LBGR } & \multirow[b]{2}{*}{ Sign } \\
\hline & & Alpha & Beta & Alpha & Beta & Alpha & Beta & & \\
\hline 1 & Wall, Concrete (Electra 1504) & 5 & 920 & 0.7 & -120.5 & 3.3 & -201.1 & 0.03 & 1 \\
\hline 2 & Wall, Concrete (Electra 1504) & 3 & 609 & -1.3 & -431.5 & -6.4 & -719.9 & 0.00 & 1 \\
\hline 3 & Wall, Concrete (Electra 1504) & 4 & 641 & -0.3 & -399.5 & -1.6 & -666.5 & 0.00 & 1 \\
\hline 4 & Wall, Concrete (Electra 1504) & 5 & 784 & 0.7 & -256.5 & 3.3 & -428.0 & 0.03 & 1 \\
\hline 5 & Wall, Concrete (Electra 1504) & 6 & 692 & 1.7 & -348.5 & 8.1 & -581.5 & 0.08 & 1 \\
\hline 6 & Wall, Concrete (Electra 1504) & 6 & 661 & 1.7 & -379.5 & 8.1 & -633.2 & 0.08 & 1 \\
\hline 7 & Wall, Concrete (Electra 1504) & 13 & 1033 & 8.7 & -7.5 & 41.9 & -12.6 & 0.42 & 1 \\
\hline 8 & Wall, Concrete (Electra 1504) & 7 & 1085 & 2.7 & 44.5 & 12.9 & 74.2 & 0.14 & 1 \\
\hline 9 & Wall, Concrete (Electra 1504) & 4 & 964 & -0.3 & -76.5 & -1.6 & -127.7 & 0.00 & 1 \\
\hline 10 & Wall, Concrete (Electra 1504) & 5 & 662 & 0.7 & -378.5 & 3.3 & -631.5 & 0.03 & 1 \\
\hline 11 & Wall, Metal (Electra 1504) & 7 & 430 & 1.7 & -808.9 & 8.2 & -1349.4 & 0.08 & 1 \\
\hline 12 & Wall, Metal (1504) & 8 & 426 & 2.7 & -812.9 & 13.0 & -1356.1 & 0.13 & 1 \\
\hline 13 & Wall, Concrete (1504) & 7 & 690 & 2.7 & -350.5 & 12.9 & -584.8 & 0.13 & 1 \\
\hline 14 & Wall, Concrete (1504) & 6 & 617 & 1.7 & -423.5 & 8.1 & -706.6 & 0.08 & 1 \\
\hline 15 & Wall, Concrete (1504) & 10 & 786 & 5.7 & -254.5 & 27.4 & -424.6 & 0.27 & 1 \\
\hline & & & & & Averages & 9.4 & -556.6 & & \\
\hline & & & & & StDev & 12.0 & 412.3 & & \\
\hline & & & & & Median & 8.1 & -584.8 & & \\
\hline & & & & & Max & 41.9 & 74.2 & & \\
\hline & & & & & Min & -6.4 & -1356.1 & & \\
\hline & & & & & *LBGR & 0.09 & & & \\
\hline & & & & & \multicolumn{3}{|c|}{ *Negative vales not used in LGBR } & & \\
\hline & & & & & & \# of samples: & 15 & & \\
\hline & & & & & & Sign test stat: & 15 & & \\
\hline
\end{tabular}




\section{0-ER-A26-291}

Final Status Survey, Room 106 North Wall / 106-NW-1

\begin{tabular}{|c|c|c|c|c|c|c|c|c|c|}
\hline & & \multicolumn{2}{|c|}{ RAW DATA cpm } & \multicolumn{2}{|c|}{ BKG ADJUSTED cpm } & \multicolumn{2}{|l|}{$\mathrm{dpm} / 100 \mathrm{~cm} 2$} & \multirow[b]{2}{*}{ LBGR } & \multirow[b]{2}{*}{ Sign } \\
\hline & & Alpha & Beta & Alpha & Beta & Alpha & Beta & & \\
\hline 1 & Wall, Concrete (Electra 1504) & 6 & 990 & 1.7 & -50.5 & 8.1 & -84.3 & 0.08 & 1 \\
\hline 2 & Wall, Concrete (Electra 1504) & 8 & 666 & 3.7 & -374.5 & 17.8 & -624.8 & 0.18 & 1 \\
\hline 3 & Wall, Concrete (Electra 1504) & 8 & 657 & 3.7 & -383.5 & 17.8 & -639.8 & 0.18 & 1 \\
\hline 4 & Wall, Concrete (Electra 1504) & 12 & 899 & 7.7 & -141.5 & 37.1 & -236.1 & 0.37 & 1 \\
\hline 5 & Wall, Concrete (Electra 1120) & 4 & 988 & -0.3 & -52.5 & -1.6 & -87.6 & 0.00 & 1 \\
\hline 6 & Wall, Concrete (Electra 1120) & 6 & 983 & 1.7 & -57.5 & 8.1 & -96.0 & 0.08 & 1 \\
\hline 7 & Wall, Concrete (Electra 1120) & 8 & 656 & 3.7 & -384.5 & 17.8 & -641.5 & 0.18 & 1 \\
\hline 8 & Wall, Concrete (Electra 1120) & 9 & 600 & 4.7 & -440.5 & 22.6 & -734.9 & 0.23 & 1 \\
\hline 9 & Wall, Concrete (Electra 1120) & 2 & 786 & -2.3 & -254.5 & -11.2 & -424.6 & 0.00 & 1 \\
\hline 10 & Wall, Concrete (Electra 1120) & 1 & 749 & -3.3 & -291.5 & -16.1 & -486.4 & 0.00 & 1 \\
\hline 11 & Wall, Concrete (Electra 1504) & 7 & 941 & 2.7 & -99.5 & 12.9 & -166.0 & 0.13 & 1 \\
\hline 12 & Wall, Concrete (1504) & 8 & 984 & 3.7 & -56.5 & 17.8 & -94.3 & 0.18 & 1 \\
\hline 13 & Wall, Concrete (1504) & 11 & 1059 & 6.7 & 18.5 & 32.2 & 30.8 & 0.33 & 1 \\
\hline 14 & Wall, Concrete (1504) & 6 & 634 & 1.7 & -406.5 & 8.1 & -678.2 & 0.08 & 1 \\
\hline & & & & & Averages & 12.2 & -354.6 & & \\
\hline & & & & & StDev & 14.8 & 275.3 & & \\
\hline & & & & & Median & 15.3 & -330.4 & & \\
\hline & & & & & Max & 37.1 & 30.8 & & \\
\hline & & & & & Min & -16.1 & -734.9 & & \\
\hline & & & & & $*$ LBGR & 0.12 & & & \\
\hline & & & & & \multicolumn{3}{|c|}{ *Negative vales not used in LGBR } & & \\
\hline & & & & & & \# of samples: & 14 & & \\
\hline & & & & & & Sign test stat: & 14 & & \\
\hline
\end{tabular}




\section{0-ER-A26-284}

Final Status Survey, Room 106 South Wall / 106-SW-1

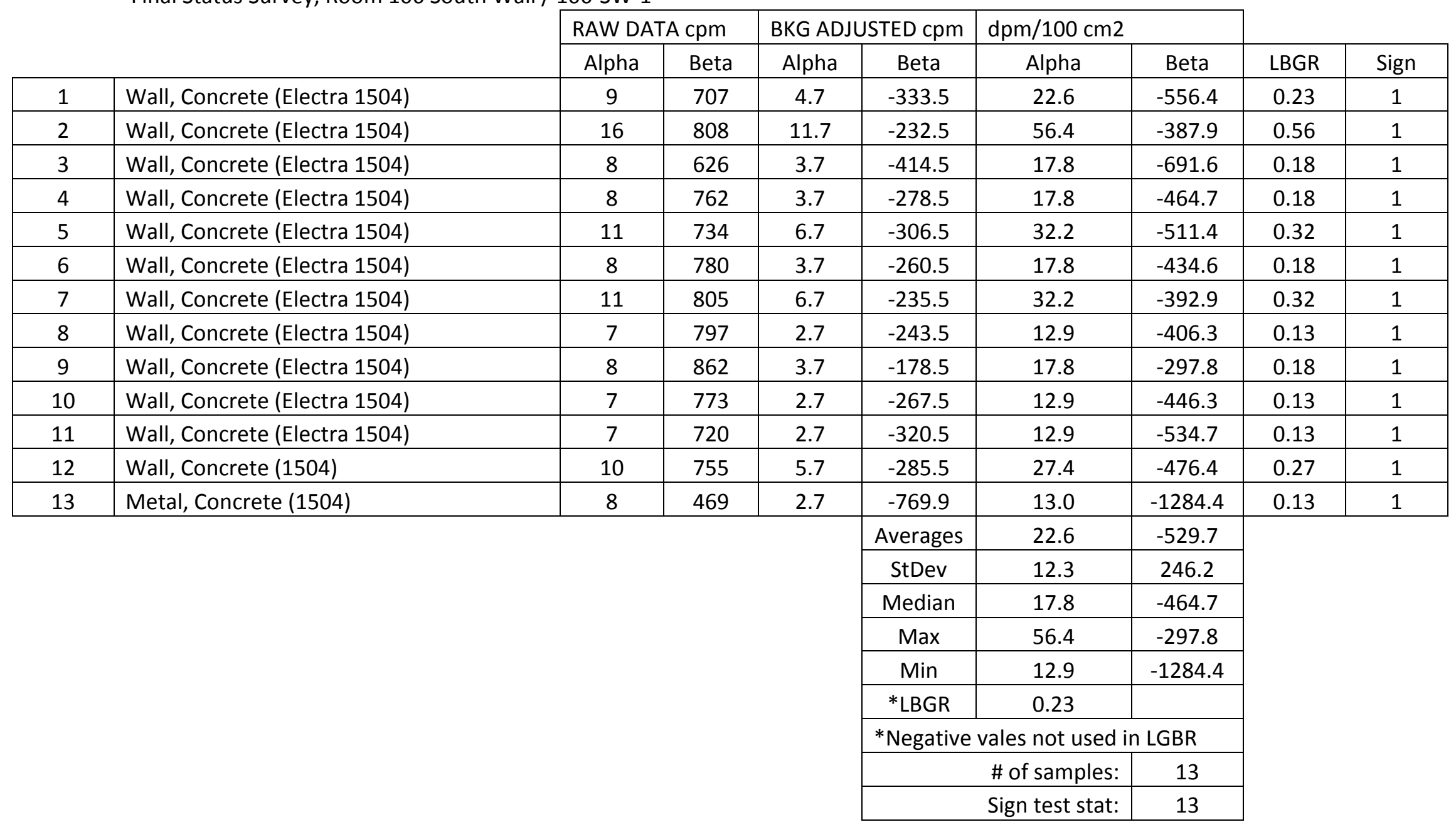




\section{0-ER-A26-282}

Final Status Survey, Room 106, West Wall, 106-WW-1

\begin{tabular}{|c|c|c|c|c|c|c|c|c|c|}
\hline & & \multicolumn{2}{|c|}{ RAW DATA cpm } & \multicolumn{2}{|c|}{ BKG ADJUSTED cpm } & \multicolumn{2}{|l|}{$\mathrm{dpm} / 100 \mathrm{~cm} 2$} & \multirow[b]{2}{*}{ LBGR } & \multirow[b]{2}{*}{ Sign } \\
\hline & & Alpha & Beta & Alpha & Beta & Alpha & Beta & & \\
\hline 1 & Wall, Concrete & 7 & 739 & 2.7 & -301.5 & 12.9 & -503.0 & 0.13 & 1 \\
\hline 2 & Wall, Concrete & 7 & 1557 & 2.7 & 516.5 & 12.9 & 861.7 & 0.30 & 1 \\
\hline 3 & Wall, Concrete & 7 & 746 & 2.7 & -294.5 & 12.9 & -491.4 & 0.13 & 1 \\
\hline 4 & Wall, Concrete & 11 & 720 & 6.7 & -320.5 & 32.2 & -534.7 & 0.32 & 1 \\
\hline 5 & Wall, Concrete & 13 & 670 & 8.7 & -370.5 & 41.9 & -618.2 & 0.42 & 1 \\
\hline 6 & Wall, Concrete & 8 & 726 & 3.7 & -314.5 & 17.8 & -524.7 & 0.18 & 1 \\
\hline 7 & Wall, Concrete & 11 & 741 & 6.7 & -299.5 & 32.2 & -499.7 & 0.32 & 1 \\
\hline 8 & Wall, Concrete & 4 & 757 & -0.3 & -283.5 & -1.6 & -473.0 & 0.00 & 1 \\
\hline 9 & Wall, Concrete & 9 & 695 & 4.7 & -345.5 & 22.6 & -576.5 & 0.23 & 1 \\
\hline 10 & Wall, Concrete & 10 & 691 & 5.7 & -349.5 & 27.4 & -583.1 & 0.27 & 1 \\
\hline 11 & Wall, Concrete & 16 & 672 & 11.7 & -368.5 & 56.4 & -614.8 & 0.56 & 1 \\
\hline 12 & Wall, Concrete & 11 & 650 & 6.7 & -390.5 & 32.2 & -651.5 & 0.32 & 1 \\
\hline 13 & Wall, Metal (Window Opening) & 9 & 450 & 3.7 & -788.9 & 17.9 & -1316.1 & 0.18 & 1 \\
\hline & & & & & Averages & 24.5 & -501.9 & & \\
\hline & & & & & StDev & 15.0 & 464.2 & & \\
\hline & & & & & Median & 22.6 & -534.7 & & \\
\hline & & & & & Max & 56.4 & 861.7 & & \\
\hline & & & & & Min & -1.6 & -1316.1 & & \\
\hline & & & & & *LBGR & 0.24 & & & \\
\hline & & & & & *Negative & Iales not used i & LGBR & & \\
\hline & & & & & & \# of samples: & 13 & & \\
\hline & & & & & & Sign test stat: & 13 & & \\
\hline
\end{tabular}




\section{0_ER-A26-296}

Final Status Survey, Room 106, Ceiling / 106-C-1

\begin{tabular}{|c|c|c|c|c|c|c|c|c|c|}
\hline & & \multicolumn{2}{|c|}{ RAW DATA cpm } & \multicolumn{2}{|c|}{ BKG ADJUSTED cpm } & \multicolumn{2}{|l|}{$\mathrm{dpm} / 100 \mathrm{~cm} 2$} & \multirow[b]{2}{*}{ LBGR } & \multirow[b]{2}{*}{ Sign } \\
\hline & & Alpha & Beta & Alpha & Beta & Alpha & Beta & & \\
\hline 1 & Ceiling, Concrete (Electra 1504) & 12 & 1154 & 7.7 & 113.5 & 37.1 & 189.3 & 0.41 & 1 \\
\hline 2 & Ceiling, Concrete (Electra 1504) & 18 & 1101 & 13.7 & 60.5 & 66.1 & 100.9 & 0.68 & 1 \\
\hline 3 & Ceiling, Concrete (Electra 1504) & 12 & 979 & 7.7 & -61.5 & 37.1 & -102.6 & 0.37 & 1 \\
\hline 4 & Ceiling, Concrete (Electra 1504) & 18 & 1093 & 13.7 & 52.5 & 66.1 & 87.5 & 0.68 & 1 \\
\hline 5 & Ceiling, Concrete (Electra 1504) & 11 & 1492 & 6.7 & 451.5 & 32.2 & 753.2 & 0.47 & 1 \\
\hline 6 & Ceiling, Concrete (Electra 1504) & 15 & 1136 & 10.7 & 95.5 & 51.6 & 159.3 & 0.55 & 1 \\
\hline 7 & Ceiling, Concrete (Electra 1504) & 10 & 813 & 5.7 & -227.5 & 27.4 & -379.6 & 0.27 & 1 \\
\hline 8 & Ceiling, Concrete (Electra 1504) & 10 & 1371 & 5.7 & 330.5 & 27.4 & 551.3 & 0.38 & 1 \\
\hline 9 & Ceiling, Concrete (Electra 1504) & 14 & 1117 & 9.7 & 76.5 & 46.7 & 127.6 & 0.49 & 1 \\
\hline 10 & Ceiling, Concrete (Electra 1504) & 18 & 1104 & 13.7 & 63.5 & 66.1 & 105.9 & 0.68 & 1 \\
\hline 11 & Ceiling, Concrete (Electra 1504) & 9 & 1093 & 4.7 & 52.5 & 22.6 & 87.5 & 0.24 & 1 \\
\hline 12 & Ceiling, Concrete (Electra 1504) & 15 & 1114 & 10.7 & 73.5 & 51.6 & 122.6 & 0.54 & 1 \\
\hline 13 & Ceiling, Concrete (Electra 1504) & 17 & 1186 & 12.7 & 145.5 & 61.2 & 242.7 & 0.66 & 1 \\
\hline & & & & & Averages & 45.6 & 157.4 & & \\
\hline & & & & & StDev & 16.0 & 272.7 & & \\
\hline & & & & & Median & 46.7 & 122.6 & & \\
\hline & & & & & Max & 66.1 & 753.2 & & \\
\hline & & & & & Min & 22.6 & -379.6 & & \\
\hline & & & & & *LBGR & 0.49 & & & \\
\hline & & & & & *Negative & vales not used ir & LGBR & & \\
\hline & & & & & & \# of samples: & 13 & & \\
\hline & & & & & & Sign test stat: & 13 & & \\
\hline
\end{tabular}


Data for Survey Units in Room 107 and Ledges-1 
10-ER-A26-279

Final Status Survey, Room 107 Floor 107-F-1

\begin{tabular}{|c|c|c|c|c|c|c|c|c|c|}
\hline & & \multicolumn{2}{|c|}{ RAW DATA cpm } & \multicolumn{2}{|c|}{ BKG ADJUSTED cpm } & \multicolumn{2}{|l|}{$\mathrm{dpm} / 100 \mathrm{~cm} 2$} & \multirow[b]{2}{*}{ LBGR } & \multirow[b]{2}{*}{ Sign } \\
\hline & & Alpha & Beta & Alpha & Beta & Alpha & Beta & & \\
\hline 1 & Floor, Concrete (Electra 2125) & 6 & 797 & 1.7 & -243.5 & 8.1 & -406.3 & 0.08 & 1 \\
\hline 2 & Floor, Concrete (Electra 2125) & 7 & 1280 & 2.7 & 239.5 & 12.9 & 399.5 & 0.21 & 1 \\
\hline 3 & Floor, Concrete (Electra 2125) & 7 & 961 & 2.7 & -79.5 & 12.9 & -132.7 & 0.13 & 1 \\
\hline 4 & Floor, Concrete (Electra 2125) & 6 & 963 & 1.7 & -77.5 & 8.1 & -129.3 & 0.08 & 1 \\
\hline 5 & Floor, Concrete (Electra 2125) & 11 & 673 & 6.7 & -367.5 & 32.2 & -613.2 & 0.32 & 1 \\
\hline 6 & Floor, Concrete (Electra 2125) & 9 & 816 & 4.7 & -224.5 & 22.6 & -374.6 & 0.23 & 1 \\
\hline 7 & Floor, Concrete (Electra 2125) & 10 & 812 & 5.7 & -228.5 & 27.4 & -381.3 & 0.27 & 1 \\
\hline 8 & Floor, Concrete (Electra 2125) & 11 & 996 & 6.7 & -44.5 & 32.2 & -74.3 & 0.32 & 1 \\
\hline 9 & Floor, Concrete (Electra 2125) & 12 & 963 & 7.7 & -77.5 & 37.1 & -129.3 & 0.37 & 1 \\
\hline 10 & Floor, Concrete (Electra 2125) & 11 & 1231 & 6.7 & 190.5 & 32.2 & 317.8 & 0.39 & 1 \\
\hline 11 & Floor, Concrete (Electra 2125) & 9 & 894 & 4.7 & -146.5 & 22.6 & -244.5 & 0.23 & 1 \\
\hline 12 & Floor, Concrete (2125) & 5 & 831 & 0.7 & -209.5 & 3.3 & -349.6 & 0.03 & 1 \\
\hline 13 & Floor, Metal (1120) & 7 & 709 & 2.4 & -622.7 & 11.4 & -1038.9 & 0.11 & 1 \\
\hline 14 & Floor, Concrete (2125) & 11 & 946 & 6.7 & -94.5 & 32.2 & -157.7 & 0.32 & 1 \\
\hline 15 & Floor, Concrete (2125) & 7 & 955 & 2.7 & -85.5 & 12.9 & -142.7 & 0.13 & 1 \\
\hline \multirow[t]{10}{*}{16} & Floor, Concrete (2125) & 20 & 803 & 15.7 & -237.5 & 75.7 & -396.3 & 0.76 & 1 \\
\hline & & & & & Averages & 24.0 & -240.8 & & \\
\hline & & & & & StDev & 17.5 & 334.6 & & \\
\hline & & & & & Median & 22.6 & -201.1 & & \\
\hline & & & & & Max & 75.7 & 399.5 & & \\
\hline & & & & & Min & 3.3 & -1038.9 & & \\
\hline & & & & & $*$ LBGR & 0.24 & & & \\
\hline & & & & & \multicolumn{3}{|c|}{ *Negative vales not used in LGBR } & & \\
\hline & & & & & & \# of samples: & 16 & & \\
\hline & & & & & & Sign test stat: & 16 & & \\
\hline
\end{tabular}




\section{0-ER-A26-280}

Final Status Survey, Room 107 North Wall / 107-NW-1

\begin{tabular}{|c|c|c|c|c|c|c|c|c|c|}
\hline & & \multicolumn{2}{|c|}{ RAW DATA cpm } & \multicolumn{2}{|c|}{ BKG ADJUSTED cpm } & \multicolumn{2}{|l|}{$\mathrm{dpm} / 100 \mathrm{~cm} 2$} & \multirow[b]{2}{*}{ LBGR } & \multirow[b]{2}{*}{ Sign } \\
\hline & & Alpha & Beta & Alpha & Beta & Alpha & Beta & & \\
\hline 1 & Wall, Concrete (Electra 4806) & 19 & 983 & 14.7 & -57.5 & 70.9 & -96.0 & 0.71 & 1 \\
\hline 2 & Wall, Concrete (Electra 1120) & 13 & 880 & 8.7 & -160.5 & 41.9 & -267.8 & 0.42 & 1 \\
\hline 3 & Wall, Concrete (Electra 4806) & 16 & 777 & 11.7 & -263.5 & 56.4 & -439.6 & 0.56 & 1 \\
\hline 4 & Wall, Concrete (Electra 1120) & 10 & 776 & 5.7 & -264.5 & 27.4 & -441.3 & 0.27 & 1 \\
\hline 5 & Wall, Concrete (Electra 4806) & 16 & 895 & 11.7 & -145.5 & 56.4 & -242.8 & 0.56 & 1 \\
\hline 6 & Wall, Concrete (Electra 1120) & 13 & 905 & 8.7 & -135.5 & 41.9 & -226.1 & 0.42 & 1 \\
\hline 7 & Wall, Concrete (Electra 4806) & 12 & 1003 & 7.7 & -37.5 & 37.1 & -62.6 & 0.37 & 1 \\
\hline 8 & Wall, Concrete (Electra 1120) & 10 & 926 & 5.7 & -114.5 & 27.4 & -191.1 & 0.27 & 1 \\
\hline 9 & Wall, Concrete (Electra 4806) & 19 & 980 & 14.7 & -60.5 & 70.9 & -101.0 & 0.71 & 1 \\
\hline 10 & Wall, Concrete (Electra 1120) & 7 & 930 & 2.7 & -110.5 & 12.9 & -184.4 & 0.13 & 1 \\
\hline 11 & Wall, Concrete (Electra 4806) & 7 & 964 & 2.7 & -76.5 & 12.9 & -127.7 & 0.13 & 1 \\
\hline 12 & Wall, Concrete (1120) & 13 & 877 & 8.7 & -163.5 & 41.9 & -272.8 & 0.42 & 1 \\
\hline 13 & Wall, Concrete (1120) & 16 & 805 & 11.7 & -235.5 & 56.4 & -392.9 & 0.56 & 1 \\
\hline 14 & Wall, Concrete (1120) & 17 & 839 & 12.7 & -201.5 & 61.2 & -336.2 & 0.61 & 1 \\
\hline 15 & Wall, Concrete (4806) & 10 & 985 & 5.7 & -55.5 & 27.4 & -92.6 & 0.27 & 1 \\
\hline 16 & Wall, Concrete (4806) & 10 & 953 & 5.7 & -87.5 & 27.4 & -146.0 & 0.27 & 1 \\
\hline & & & & & Averages & 41.9 & -226.3 & & \\
\hline & & & & & StDev & 18.7 & 124.4 & & \\
\hline & & & & & Median & 41.9 & -208.6 & & \\
\hline & & & & & Max & 70.9 & -62.6 & & \\
\hline & & & & & Min & 12.9 & -441.3 & & \\
\hline & & & & & *LBGR & 0.42 & & & \\
\hline & & & & & *Negative & vales not used i & LGBR & & \\
\hline & & & & & & \# of samples: & 16 & & \\
\hline & & & & & & Sign test stat: & 16 & & \\
\hline
\end{tabular}




\section{0-ER-A26-281}

Final Status Survey, Room 107 North Wall / 107-NW-2

\begin{tabular}{|c|c|c|c|c|c|c|c|c|c|}
\hline & & \multicolumn{2}{|c|}{ RAW DATA cpm } & \multicolumn{2}{|c|}{ BKG ADJUSTED cpm } & \multicolumn{2}{|l|}{$\mathrm{dpm} / 100 \mathrm{~cm} 2$} & \multirow[b]{2}{*}{ LBGR } & \multirow[b]{2}{*}{ Sign } \\
\hline & & Alpha & Beta & Alpha & Beta & Alpha & Beta & & \\
\hline 1 & Wall, Concrete (Electra 1120) & 17 & 1065 & 12.7 & 24.5 & 61.2 & 40.8 & 0.62 & 1 \\
\hline 2 & Wall, Concrete (Electra 1120) & 5 & 1039 & 0.7 & -1.5 & 3.3 & -2.5 & 0.03 & 1 \\
\hline 3 & Wall, Concrete (Electra 1120) & 15 & 1105 & 10.7 & 64.5 & 51.6 & 107.6 & 0.54 & 1 \\
\hline 4 & Wall, Concrete (Electra 1120) & 16 & 1040 & 11.7 & -0.5 & 56.4 & -0.9 & 0.56 & 1 \\
\hline 5 & Wall, Concrete (Electra 1120) & 9 & 1095 & 4.7 & 54.5 & 22.6 & 90.9 & 0.24 & 1 \\
\hline 6 & Wall, Concrete (Electra 1120) & 9 & 1139 & 4.7 & 98.5 & 22.6 & 164.3 & 0.26 & 1 \\
\hline 7 & Wall, Concrete (Electra 1120) & 4 & 1101 & -0.3 & 60.5 & -1.6 & 100.9 & 0.02 & 1 \\
\hline 8 & Wall, Concrete (Electra 1120) & 4 & 1066 & -0.3 & 25.5 & -1.6 & 42.5 & 0.01 & 1 \\
\hline 9 & Wall, Concrete (Electra 1120) & 7 & 1158 & 2.7 & 117.5 & 12.9 & 196.0 & 0.17 & 1 \\
\hline 10 & Wall, Concrete (Electra 1120) & 9 & 1167 & 4.7 & 126.5 & 22.6 & 211.0 & 0.27 & 1 \\
\hline 11 & Wall, Concrete (Electra 1120) & 9 & 1083 & 4.7 & 42.5 & 22.6 & 70.9 & 0.24 & 1 \\
\hline 12 & Wall, Concrete (1120) & 16 & 1183 & 11.7 & 142.5 & 56.4 & 237.7 & 0.61 & 1 \\
\hline 13 & Wall, Concrete (1120) & 11 & 1132 & 6.7 & 91.5 & 32.2 & 152.6 & 0.35 & 1 \\
\hline 14 & Wall, Concrete (1120) & 14 & 1070 & 9.7 & 29.5 & 46.7 & 49.2 & 0.48 & 1 \\
\hline 15 & Wall, Concrete (1120) & 12 & 1103 & 7.7 & 62.5 & 37.1 & 104.2 & 0.39 & 1 \\
\hline 16 & Wall, Concrete (1120) & 9 & 1125 & 4.7 & 84.5 & 22.6 & 140.9 & 0.25 & 1 \\
\hline 17 & Wall, Concrete (1120) & 19 & 1066 & 14.7 & 25.5 & 70.9 & 42.5 & 0.72 & 1 \\
\hline & & & & & Averages & 31.7 & 102.9 & & \\
\hline & & & & & StDev & 22.5 & 72.2 & & \\
\hline & & & & & Median & 22.6 & 100.9 & & \\
\hline & & & & & Max & 70.9 & 237.7 & & \\
\hline & & & & & Min & -1.6 & -2.5 & & \\
\hline & & & & & *LBGR & 0.34 & & & \\
\hline & & & & & \multicolumn{3}{|c|}{ *Negative vales not used in LGBR } & & \\
\hline & & & & & & \# of samples: & 17 & & \\
\hline & & & & & & Sign test stat: & 17 & & \\
\hline
\end{tabular}




\section{0-ER-A26-283}

Final Status Survey, Room 107 South Wall / 107-SW-1

\begin{tabular}{|c|c|c|c|c|c|c|c|c|c|}
\hline & & \multicolumn{2}{|c|}{ RAW DATA cpm } & \multicolumn{2}{|c|}{ BKG ADJUSTED cpm } & \multicolumn{2}{|l|}{$\mathrm{dpm} / 100 \mathrm{~cm} 2$} & \multirow[b]{2}{*}{ LBGR } & \multirow[b]{2}{*}{ Sign } \\
\hline & & Alpha & Beta & Alpha & Beta & Alpha & Beta & & \\
\hline 1 & Wall, Concrete (Electra 1120) & 9 & 707 & 4.7 & -333.5 & 22.6 & -556.4 & 0.23 & 1 \\
\hline 2 & Wall, Concrete (Electra 1120) & 6 & 808 & 1.7 & -232.5 & 8.1 & -387.9 & 0.08 & 1 \\
\hline 3 & Wall, Concrete (Electra 1120) & 9 & 626 & 4.7 & -414.5 & 22.6 & -691.6 & 0.23 & 1 \\
\hline 4 & Wall, Concrete (Electra 1120) & 12 & 762 & 7.7 & -278.5 & 37.1 & -464.7 & 0.37 & 1 \\
\hline 5 & Wall, Concrete (Electra 1120) & 13 & 734 & 8.7 & -306.5 & 41.9 & -511.4 & 0.42 & 1 \\
\hline 6 & Wall, Concrete (Electra 1120) & 7 & 780 & 2.7 & -260.5 & 12.9 & -434.6 & 0.13 & 1 \\
\hline 7 & Wall, Concrete (Electra 1120) & 7 & 805 & 2.7 & -235.5 & 12.9 & -392.9 & 0.13 & 1 \\
\hline 8 & Wall, Concrete (Electra 1120) & 5 & 797 & 0.7 & -243.5 & 3.3 & -406.3 & 0.03 & 1 \\
\hline 9 & Wall, Concrete (Electra 1120) & 6 & 862 & 1.7 & -178.5 & 8.1 & -297.8 & 0.08 & 1 \\
\hline 10 & Wall, Concrete (Electra 1120) & 12 & 773 & 7.7 & -267.5 & 37.1 & -446.3 & 0.37 & 1 \\
\hline 11 & Wall, Concrete (Electra 1120) & 8 & 720 & 3.7 & -320.5 & 17.8 & -534.7 & 0.18 & 1 \\
\hline 12 & Wall, Concrete (1120) & 7 & 749 & 2.7 & -291.5 & 12.9 & -486.4 & 0.13 & 1 \\
\hline 13 & Wall, Concrete (1120) & 6 & 735 & 1.7 & -305.5 & 8.1 & -509.7 & 0.08 & 1 \\
\hline 14 & Wall, Concrete (1120) & 9 & 760 & 4.7 & -280.5 & 22.6 & -468.0 & 0.23 & 1 \\
\hline 15 & Wall, Concrete (1120) & 20 & 728 & 15.7 & -312.5 & 75.7 & -521.4 & 0.76 & 1 \\
\hline 16 & Wall, Concrete (1120) & 6 & 686 & 1.7 & -354.5 & 8.1 & -591.5 & 0.08 & 1 \\
\hline & & & & & Averages & 22.0 & -481.4 & & \\
\hline & & & & & StDev & 18.5 & 92.3 & & \\
\hline & & & & & Median & 15.3 & -477.2 & & \\
\hline & & & & & Max & 75.7 & -297.8 & & \\
\hline & & & & & Min & 3.3 & -691.6 & & \\
\hline & & & & & *LBGR & 0.22 & & & \\
\hline & & & & & \multicolumn{3}{|c|}{ *Negative vales not used in LGBR } & & \\
\hline & & & & & & \# of samples: & 16 & & \\
\hline & & & & & & Sign test stat: & 16 & & \\
\hline
\end{tabular}


10-ER-A26-289

Final Status Survey, Room 107 South Wall / 107-SW-2

\begin{tabular}{|c|c|c|c|c|c|c|c|c|c|}
\hline \multirow{2}{*}{\multicolumn{2}{|c|}{ Final Status Survey, Room 107 South Wall / 107-SW-2 }} & & & & \\
\hline & & Alpha & Beta & Alpha & Beta & Alpha & Beta & LBGR & Sign \\
\hline 1 & Wall, Concrete (Electra 1120) & 10 & 749 & 5.7 & -291.5 & 27.4 & -486.4 & 0.27 & 1 \\
\hline 2 & Wall, Concrete (Electra 1120) & 10 & 859 & 5.7 & -181.5 & 27.4 & -302.8 & 0.27 & 1 \\
\hline 3 & Wall, Concrete (Electra 1120) & 6 & 781 & 1.7 & -259.5 & 8.1 & -433.0 & 0.08 & 1 \\
\hline 4 & Wall, Concrete (Electra 1120) & 4 & 871 & -0.3 & -169.5 & -1.6 & -282.8 & 0.00 & 1 \\
\hline 5 & Wall, Concrete (Electra 1120) & 6 & 825 & 1.7 & -215.5 & 8.1 & -359.6 & 0.08 & 1 \\
\hline 6 & Wall, Concrete (Electra 1120) & 5 & 836 & 0.7 & -204.5 & 3.3 & -341.2 & 0.03 & 1 \\
\hline 7 & Wall, Concrete (Electra 1120) & 3 & 798 & -1.3 & -242.5 & -6.4 & -404.6 & 0.00 & 1 \\
\hline 8 & Wall, Concrete (Electra 1120) & 5 & 676 & 0.7 & -364.5 & 3.3 & -608.1 & 0.03 & 1 \\
\hline 9 & Wall, Concrete (Electra 1120) & 8 & 853 & 3.7 & -187.5 & 17.8 & -312.9 & 0.18 & 1 \\
\hline 10 & Wall, Concrete (Electra 1120) & 5 & 810 & 0.7 & -230.5 & 3.3 & -384.6 & 0.03 & 1 \\
\hline 11 & Wall, Concrete (Electra 1120) & 10 & 808 & 5.7 & -232.5 & 27.4 & -387.9 & 0.27 & 1 \\
\hline 12 & Wall, Concrete (1120) & 4 & 744 & -0.3 & -296.5 & -1.6 & -494.7 & 0.00 & 1 \\
\hline 13 & Wall, Concrete (1120) & 14 & 855 & 9.7 & -185.5 & 46.7 & -309.5 & 0.47 & 1 \\
\hline 14 & Wall, Concrete (1120) & 11 & 794 & 6.7 & -246.5 & 32.2 & -411.3 & 0.32 & 1 \\
\hline 15 & Wall, Concrete (1120) & 8 & 788 & 3.7 & -252.5 & 17.8 & -421.3 & 0.18 & 1 \\
\hline 16 & Wall, Concrete (1120) & 12 & 776 & 7.7 & -264.5 & 37.1 & -441.3 & 0.37 & 1 \\
\hline 17 & Wall, Concrete (1120) & 6 & 799 & 1.7 & -241.5 & 8.1 & -402.9 & 0.08 & 1 \\
\hline 18 & Wall, Concrete (1120) & 9 & 701 & 4.7 & -339.5 & 22.6 & -566.4 & 0.23 & 1 \\
\hline & & & & & Averages & 15.6 & -408.4 & & \\
\hline & & & & & StDev & 15.1 & 88.8 & & \\
\hline & & & & & Median & 12.9 & -403.8 & & \\
\hline & & & & & Max & 46.7 & -282.8 & & \\
\hline & & & & & Min & -6.4 & -608.1 & & \\
\hline & & & & & *LBGR & 0.16 & & & \\
\hline & & & & & *Negative & vales not used & LGBR & & \\
\hline & & & & & & \# of samples: & 18 & & \\
\hline & & & & & & Sign test stat: & 18 & & \\
\hline
\end{tabular}




\section{0-ER-A26-286}

Final Status Survey, Room 107 West Wall / 107-WW-1

\begin{tabular}{|c|c|c|c|c|c|c|c|c|c|}
\hline & & \multicolumn{2}{|c|}{ RAW DATA cpm } & \multicolumn{2}{|c|}{ BKG ADJUSTED cpm } & \multicolumn{2}{|c|}{$\mathrm{dpm} / 100 \mathrm{~cm} 2$} & \multirow[b]{2}{*}{ LBGR } & \multirow[b]{2}{*}{ Sign } \\
\hline & & Alpha & Beta & Alpha & Beta & Alpha & Beta & & \\
\hline 1 & Wall, Concrete (Electra 4806) & 11 & 981 & 6.7 & -59.5 & 32.2 & -99.3 & 0.32 & 1 \\
\hline 2 & Wall, Concrete (Electra 4806) & 11 & 636 & 6.7 & -404.5 & 32.2 & -674.9 & 0.32 & 1 \\
\hline 3 & Wall, Metal (Electra 1120) & 13 & 480 & 8.4 & -851.7 & 40.3 & -1420.9 & 0.40 & 1 \\
\hline 4 & Wall, Metal (Electra 1120) & 6 & 475 & 1.4 & -856.7 & 6.5 & -1429.3 & 0.07 & 1 \\
\hline 5 & Wall, Concrete (Electra 4806) & 11 & 1006 & 6.7 & -34.5 & 32.2 & -57.6 & 0.32 & 1 \\
\hline 6 & Wall, Concrete (Electra 4806) & 11 & 899 & 6.7 & -141.5 & 32.2 & -236.1 & 0.32 & 1 \\
\hline 7 & Wall, Concrete (Electra 4806) & 10 & 738 & 5.7 & -302.5 & 27.4 & -504.7 & 0.27 & 1 \\
\hline 8 & Wall, Concrete (Electra 4806) & 3 & 613 & -1.3 & -427.5 & -6.4 & -713.3 & 0.00 & 1 \\
\hline 9 & Wall, Metal (Electra 1120) & 9 & 456 & 4.4 & -875.7 & 21.0 & -1461.0 & 0.21 & 1 \\
\hline 10 & Wall, Concrete (Electra 4806) & 7 & 944 & 2.7 & -96.5 & 12.9 & -161.0 & 0.13 & 1 \\
\hline 11 & Wall, Concrete (Electra 4806) & 9 & 1055 & 4.7 & 14.5 & 22.6 & 24.1 & 0.23 & 1 \\
\hline 12 & Wall, Concrete (4806) & 9 & 1004 & 4.7 & -36.5 & 22.6 & -60.9 & 0.23 & 1 \\
\hline 13 & Wall, Concrete (4806) & 10 & 739 & 5.7 & -301.5 & 27.4 & -503.0 & 0.27 & 1 \\
\hline 14 & Wall, Concrete (4806) & 5 & 571 & 0.7 & -469.5 & 3.3 & -783.3 & 0.03 & 1 \\
\hline 15 & Wall, Concrete (4806) & 6 & 681 & 1.7 & -359.5 & 8.1 & -599.8 & 0.08 & 1 \\
\hline 16 & Wall, Concrete (4806) & 5 & 1068 & 0.7 & 27.5 & 3.3 & 45.8 & 0.04 & 1 \\
\hline 17 & Wall, Concrete (4806) & 11 & 1059 & 6.7 & 18.5 & 32.2 & 30.8 & 0.33 & 1 \\
\hline 18 & Wall, Concrete (4806) & 12 & 687 & 7.7 & -353.5 & 37.1 & -589.8 & 0.37 & 1 \\
\hline 19 & Wall, Concrete (4806) & 5 & 726 & 0.7 & -314.5 & 3.3 & -524.7 & 0.03 & 1 \\
\hline 20 & Wall, Concrete (4806) & 8 & 766 & 3.7 & -274.5 & 17.8 & -458.0 & 0.18 & 1 \\
\hline & & & & & Averages & 20.4 & -508.8 & & \\
\hline & & & & & StDev & 13.6 & 481.1 & & \\
\hline & & & & & Median & 22.6 & -503.9 & & \\
\hline & & & & & Max & 40.3 & 45.8 & & \\
\hline & & & & & Min & -6.4 & -1461.0 & & \\
\hline
\end{tabular}


*LBGR

0.20

*Negative vales not used in LGBR \# of samples: Sign test stat: 
10-ER-A26-288

Final Status Survey, Room 107 East Wall / 107-EW-1

\begin{tabular}{|c|c|c|c|c|c|c|c|c|c|}
\hline & & \multicolumn{2}{|c|}{ RAW DATA cpm } & \multicolumn{2}{|c|}{ BKG ADJUSTED cpm } & \multicolumn{2}{|c|}{$\mathrm{dpm} / 100 \mathrm{~cm} 2$} & \multirow[b]{2}{*}{ LBGR } & \multirow[b]{2}{*}{ Sign } \\
\hline & & Alpha & Beta & Alpha & Beta & Alpha & Beta & & \\
\hline 1 & Wall, Concrete (Electra 1120) & 8 & 1039 & 3.7 & -1.5 & 17.8 & -2.5 & 0.18 & 1 \\
\hline 2 & Wall, Concrete (Electra 1120) & 3 & 659 & -1.3 & -381.5 & -6.4 & -636.5 & 0.00 & 1 \\
\hline 3 & Wall, Concrete (Electra 2125) & 11 & 515 & 6.7 & -525.5 & 32.2 & -876.8 & 0.32 & 1 \\
\hline 4 & Wall, Concrete (Electra 2125) & 10 & 617 & 5.7 & -423.5 & 27.4 & -706.6 & 0.27 & 1 \\
\hline 5 & Wall, Metal (Electra 1120) & 10 & 461 & 5.4 & -870.7 & 25.8 & -1452.6 & 0.26 & 1 \\
\hline 6 & Wall, Metal (Electra 1504) & 8 & 494 & 2.7 & -744.9 & 13.0 & -1242.7 & 0.13 & 1 \\
\hline 7 & Wall, Concrete (Electra 2125) & 5 & 690 & 0.7 & -350.5 & 3.3 & -584.8 & 0.03 & 1 \\
\hline 8 & Wall, Concrete (Electra 1504) & 7 & 987 & 2.7 & -53.5 & 12.9 & -89.3 & 0.13 & 1 \\
\hline 9 & Wall, Concrete (Electra 1120) & 13 & 982 & 8.7 & -58.5 & 41.9 & -97.6 & 0.42 & 1 \\
\hline 10 & Wall, Concrete (Electra 1120) & 5 & 1022 & 0.7 & -18.5 & 3.3 & -30.9 & 0.03 & 1 \\
\hline 11 & Wall, Metal (Electra 1504) & 7 & 480 & 1.7 & -758.9 & 8.2 & -1266.0 & 0.08 & 1 \\
\hline 12 & Wall, Metal (1504) & 17 & 902 & 11.7 & -336.9 & 56.5 & -562.0 & 0.57 & 1 \\
\hline 13 & Wall, Concrete (1504) & 14 & 764 & 9.7 & -276.5 & 46.7 & -461.3 & 0.47 & 1 \\
\hline 14 & Wall, Concrete (2125) & 11 & 1052 & 6.7 & 11.5 & 32.2 & 19.1 & 0.33 & 1 \\
\hline 15 & Wall, Concrete (1120) & 8 & 747 & 3.7 & -293.5 & 17.8 & -489.7 & 0.18 & 1 \\
\hline 16 & Wall, Concrete (1120) & 12 & 1128 & 7.7 & 87.5 & 37.1 & 145.9 & 0.40 & 1 \\
\hline 17 & Wall, Concrete (1504) & 6 & 593 & 1.7 & -447.5 & 8.1 & -746.6 & 0.08 & 1 \\
\hline 18 & Wall, Concrete (1504) & 9 & 611 & 4.7 & -429.5 & 22.6 & -716.6 & 0.23 & 1 \\
\hline 19 & Wall, Concrete (1120) & 9 & 1078 & 4.7 & 37.5 & 22.6 & 62.5 & 0.24 & 1 \\
\hline 20 & Wall, Concrete (1120) & 7 & 1048 & 2.7 & 7.5 & 12.9 & 12.5 & 0.13 & 1 \\
\hline & & & & & Averages & 21.8 & -486.1 & & \\
\hline & & & & & StDev & 16.0 & 482.8 & & \\
\hline & & & & & Median & 20.2 & -525.8 & & \\
\hline & & & & & Max & 56.5 & 145.9 & & \\
\hline & & & & & Min & -6.4 & -1452.6 & & \\
\hline
\end{tabular}


* LBGR

0.22

*Negative vales not used in LGBR \# of samples: Sign test stat: 


\begin{tabular}{|c|c|c|c|c|c|c|c|c|c|}
\hline & \multirow{2}{*}{$\begin{array}{l}\text { 10_ER-A26-296 } \\
\text { Final Status Survey, Room 107, Ceiling / 107-C-1 }\end{array}$} & \multicolumn{2}{|c|}{ RAW DATA cpm } & \multicolumn{2}{|c|}{ BKG ADJUSTED cpm } & \multicolumn{2}{|l|}{$\mathrm{dpm} / 100 \mathrm{~cm} 2$} & \multirow[b]{2}{*}{ LBGR } & \multirow[b]{2}{*}{ Sign } \\
\hline & & Alpha & Beta & Alpha & Beta & Alpha & Beta & & \\
\hline 1 & Ceiling, Concrete (Electra 1504) & 11 & 1133 & 6.7 & 92.5 & 32.2 & 154.3 & 0.35 & 1 \\
\hline 2 & Ceiling, Concrete (Electra 1504) & 9 & 1024 & 4.7 & -16.5 & 22.6 & -27.6 & 0.23 & 1 \\
\hline 3 & Ceiling, Concrete (Electra 1504) & 9 & 1105 & 4.7 & 64.5 & 22.6 & 107.6 & 0.25 & 1 \\
\hline 4 & Ceiling, Concrete (Electra 1504) & 9 & 1135 & 4.7 & 94.5 & 22.6 & 157.6 & 0.26 & 1 \\
\hline 5 & Ceiling, Concrete (Electra 1504) & 16 & 1188 & 11.7 & 147.5 & 56.4 & 246.0 & 0.61 & 1 \\
\hline 6 & Ceiling, Concrete (Electra 1504) & 8 & 1166 & 3.7 & 125.5 & 17.8 & 209.3 & 0.22 & 1 \\
\hline 7 & Ceiling, Concrete (Electra 1504) & 19 & 1106 & 14.7 & 65.5 & 70.9 & 109.2 & 0.73 & 1 \\
\hline 8 & Ceiling, Concrete (Electra 1504) & 10 & 1076 & 5.7 & 35.5 & 27.4 & 59.2 & 0.29 & 1 \\
\hline 9 & Ceiling, Concrete (Electra 1504) & 7 & 1180 & 2.7 & 139.5 & 12.9 & 232.7 & 0.18 & 1 \\
\hline 10 & Ceiling, Concrete (Electra 1504) & 15 & 1075 & 10.7 & 34.5 & 51.6 & 57.5 & 0.53 & 1 \\
\hline 11 & Ceiling, Concrete (Electra 1504) & 16 & 1026 & 11.7 & -14.5 & 56.4 & -24.2 & 0.56 & 1 \\
\hline 12 & Ceiling, Concrete (Electra 1504) & 19 & 1097 & 14.7 & 56.5 & 70.9 & 94.2 & 0.73 & 1 \\
\hline 13 & Ceiling, Concrete (Electra 1504) & 5 & 1035 & 0.7 & -5.5 & 3.3 & -9.2 & 0.03 & 1 \\
\hline 14 & Ceiling, Concrete (Electra 1504) & 7 & 953 & 2.7 & -87.5 & 12.9 & -146.0 & 0.13 & 1 \\
\hline 15 & Ceiling, Concrete (Electra 1504) & 6 & 1013 & 1.7 & -27.5 & 8.1 & -45.9 & 0.08 & 1 \\
\hline 16 & Ceiling, Concrete (Electra 1504) & 6 & 1074 & 1.7 & 33.5 & 8.1 & 55.8 & 0.09 & 1 \\
\hline 17 & Ceiling, Concrete (Electra 1504) & 7 & 1133 & 2.7 & 92.5 & 12.9 & 154.3 & 0.16 & 1 \\
\hline 18 & Ceiling, Concrete (Electra 1504) & 12 & 1220 & 7.7 & 179.5 & 37.1 & 299.4 & 0.43 & 1 \\
\hline & & & & & Averages & 30.4 & 93.6 & & \\
\hline & & & & & StDev & 21.9 & 116.4 & & \\
\hline & & & & & Median & 22.6 & 100.9 & & \\
\hline & & & & & Max & 70.9 & 299.4 & & \\
\hline & & & & & Min & 3.3 & -146.0 & & \\
\hline & & & & & $*$ LBGR & 0.32 & & & \\
\hline & & & & & \multicolumn{3}{|c|}{${ }^{*}$ Negative vales not used in LGBR } & & \\
\hline & & & & & & \# of samples: & 18 & & \\
\hline & & & & & & Sign test stat: & 18 & & \\
\hline
\end{tabular}




\begin{tabular}{|c|c|c|c|c|c|c|c|c|c|}
\hline & \multirow{2}{*}{$\begin{array}{l}\text { 10-ER-A26-292 } \\
\text { Final Status Survey, Rm 102, 104,106, } 107 \text { / Ledges-1 }\end{array}$} & \multicolumn{2}{|c|}{ RAW DATA cpm } & \multicolumn{2}{|c|}{ BKG ADJUSTED cpm } & \multicolumn{2}{|l|}{$\mathrm{dpm} / 100 \mathrm{~cm} 2$} & \multirow[b]{2}{*}{ LBGR } & \multirow[b]{2}{*}{ Sign } \\
\hline & & Alpha & Beta & Alpha & Beta & Alpha & Beta & & \\
\hline 1 & Ledge 102, Concrete (Electra 6697) & 11 & 1313 & 6.7 & 272.5 & 32.2 & 454.6 & 0.41 & 1 \\
\hline 2 & Ledge 102, Concrete (Electra 6697) & 22 & 1246 & 17.7 & 205.5 & 85.4 & 342.8 & 0.92 & 1 \\
\hline 3 & Ledge 102, Concrete (Electra 6697) & 6 & 1120 & 1.7 & 79.5 & 8.1 & 132.6 & 0.11 & 1 \\
\hline 4 & Ledge 102, Concrete (Electra 6697) & 12 & 1094 & 7.7 & 53.5 & 37.1 & 89.2 & 0.39 & 1 \\
\hline 5 & Ledge 102, Concrete (Electra 6697) & 8 & 1003 & 3.7 & -37.5 & 17.8 & -62.6 & 0.18 & 1 \\
\hline 6 & Ledge 102, Concrete (Electra 6697) & 12 & 959 & 7.7 & -81.5 & 37.1 & -136.0 & 0.37 & 1 \\
\hline 7 & Ledge 102, Concrete (Electra 6697) & 11 & 1059 & 6.7 & 18.5 & 32.2 & 30.8 & 0.33 & 1 \\
\hline 8 & Ledge 102, Concrete (Electra 6697) & 7 & 986 & 2.7 & -54.5 & 12.9 & -91.0 & 0.13 & 1 \\
\hline 9 & Ledge 102, Concrete (Electra 6697) & 11 & 1224 & 6.7 & 183.5 & 32.2 & 306.1 & 0.38 & 1 \\
\hline 10 & Ledge 102, Concrete (Electra 6697) & 10 & 1297 & 5.7 & 256.5 & 27.4 & 427.9 & 0.36 & 1 \\
\hline 11 & Ledge 102, Concrete (Electra 6697) & 15 & 1291 & 10.7 & 250.5 & 51.6 & 417.9 & 0.60 & 1 \\
\hline 12 & Ledge 104, Concrete (1504) & 8 & 910 & 3.7 & -130.5 & 17.8 & -217.8 & 0.18 & 1 \\
\hline 13 & Ledge 104, Concrete (1504) & 12 & 982 & 7.7 & -58.5 & 37.1 & -97.6 & 0.37 & 1 \\
\hline 14 & Ledge 104, Concrete (1504) & 16 & 1104 & 11.7 & 63.5 & 56.4 & 105.9 & 0.59 & 1 \\
\hline 15 & Ledge 106, Concrete (1504) & 9 & 1017 & 4.7 & -23.5 & 22.6 & -39.2 & 0.23 & 1 \\
\hline 16 & Ledge 106, Concrete (1504) & 8 & 1241 & 3.7 & 200.5 & 17.8 & 334.5 & 0.24 & 1 \\
\hline \multirow[t]{10}{*}{17} & Ledge 106, Concrete (1504) & 2 & 1287 & -2.3 & 246.5 & -11.2 & 411.2 & 0.08 & 1 \\
\hline & & & & & Averages & 30.3 & 141.7 & & \\
\hline & & & & & StDev & 21.5 & 229.4 & & \\
\hline & & & & & Median & 32.2 & 105.9 & & \\
\hline & & & & & Max & 85.4 & 454.6 & & \\
\hline & & & & & Min & -11.2 & -217.8 & & \\
\hline & & & & & $*$ LBGR & 0.33 & & & \\
\hline & & & & & \multicolumn{3}{|c|}{ *Negative vales not used in LGBR } & & \\
\hline & & & & & & \# of samples: & 17 & & \\
\hline & & & & & & Sign test stat: & 17 & & \\
\hline
\end{tabular}


Final Status Survey Report for

Corrective Action Unit 117-Pluto

Disassembly Facility, Building 2201

\section{APPENDIX D - MARSSIM Table I.3}


Appendix I

\section{I.3 Critical Values for the SignTest}

Table I.3 Critical Values for the Sign Test Statistic S+

\begin{tabular}{|c|c|c|c|c|c|c|c|c|c|}
\hline \multirow[b]{2}{*}{$N$} & \multicolumn{9}{|c|}{ Alpha } \\
\hline & 0.005 & 0.01 & 0.025 & 0.05 & 0.1 & 0.2 & 0.3 & 0.4 & 0.5 \\
\hline 4 & 4 & 4 & 4 & 4 & 3 & 3 & 3 & 2 & 2 \\
\hline 5 & 5 & 5 & 5 & 4 & 4 & 3 & 3 & 3 & 2 \\
\hline 6 & 6 & 6 & 5 & 5 & 5 & 4 & 4 & 3 & 3 \\
\hline 7 & 7 & 6 & 6 & 6 & 5 & 5 & 4 & 4 & 3 \\
\hline 8 & 7 & 7 & 7 & 6 & 6 & 5 & 5 & 4 & 4 \\
\hline 9 & 8 & 8 & 7 & 7 & 6 & 6 & 5 & 5 & 4 \\
\hline 10 & 9 & 9 & 8 & 8 & 7 & 6 & 6 & 5 & 5 \\
\hline 11 & 10 & 9 & 9 & 8 & 8 & 7 & 6 & 6 & 5 \\
\hline 12 & 10 & 10 & 9 & 9 & 8 & 7 & 7 & 6 & 6 \\
\hline 13 & 11 & 11 & 10 & 9 & 9 & 8 & 7 & 7 & 6 \\
\hline 14 & 12 & 11 & 11 & 10 & 9 & 9 & 8 & 7 & 7 \\
\hline 15 & 12 & 12 & 11 & 11 & 10 & 9 & 9 & 8 & 7 \\
\hline 16 & 13 & 13 & 12 & 11 & 11 & 10 & 9 & 9 & 8 \\
\hline 17 & 14 & 13 & 12 & 12 & 11 & 10 & 10 & 9 & 8 \\
\hline 18 & 14 & 14 & 13 & 12 & 12 & 11 & 10 & 10 & 9 \\
\hline 19 & 15 & 14 & 14 & 13 & 12 & 11 & 11 & 10 & 9 \\
\hline 20 & 16 & 15 & 14 & 14 & 13 & 12 & 11 & 11 & 10 \\
\hline 21 & 16 & 16 & 15 & 14 & 13 & 12 & 12 & 11 & 10 \\
\hline 22 & 17 & 16 & 16 & 15 & 14 & 13 & 12 & 12 & 11 \\
\hline 23 & 18 & 17 & 16 & 15 & 15 & 14 & 13 & 12 & 11 \\
\hline 24 & 18 & 18 & 17 & 16 & 15 & 14 & 13 & 13 & 12 \\
\hline 25 & 19 & 18 & 17 & 17 & 16 & 15 & 14 & 13 & 12 \\
\hline 26 & 19 & 19 & 18 & 17 & 16 & 15 & 14 & 14 & 13 \\
\hline 27 & 20 & 19 & 19 & 18 & 17 & 16 & 15 & 14 & 13 \\
\hline 28 & 21 & 20 & 19 & 18 & 17 & 16 & 15 & 15 & 14 \\
\hline 29 & 21 & 21 & 20 & 19 & 18 & 17 & 16 & 15 & 14 \\
\hline 30 & 22 & 21 & 20 & 19 & 19 & 17 & 16 & 16 & 15 \\
\hline
\end{tabular}


Table I.3 Critical Values for the Sign Test Statistic S+ (continued)

\begin{tabular}{|c|c|c|c|c|c|c|c|c|c|}
\hline \multirow[b]{2}{*}{$N$} & \multicolumn{9}{|c|}{ Alpha } \\
\hline & 0.005 & 0.01 & 0.025 & 0.05 & 0.1 & 0.2 & 0.3 & 0.4 & 0.5 \\
\hline 31 & 23 & 22 & 21 & 20 & 19 & 18 & 17 & 16 & 15 \\
\hline 32 & 23 & 23 & 22 & 21 & 20 & 18 & 17 & 17 & 16 \\
\hline 33 & 24 & 23 & 22 & 21 & 20 & 19 & 18 & 17 & 16 \\
\hline 34 & 24 & 24 & 23 & 22 & 21 & 19 & 19 & 18 & 17 \\
\hline 35 & 25 & 24 & 23 & 22 & 21 & 20 & 19 & 18 & 17 \\
\hline 36 & 26 & 25 & 24 & 23 & 22 & 21 & 20 & 19 & 18 \\
\hline 37 & 26 & 26 & 24 & 23 & 22 & 21 & 20 & 19 & 18 \\
\hline 38 & 27 & 26 & 25 & 24 & 23 & 22 & 21 & 20 & 19 \\
\hline 39 & 27 & 27 & 26 & 25 & 23 & 22 & 21 & 20 & 19 \\
\hline 40 & 28 & 27 & 26 & 25 & 24 & 23 & 22 & 21 & 20 \\
\hline 41 & 29 & 28 & 27 & 26 & 25 & 23 & 22 & 21 & 20 \\
\hline 42 & 29 & 28 & 27 & 26 & 25 & 24 & 23 & 22 & 21 \\
\hline 43 & 30 & 29 & 28 & 27 & 26 & 24 & 23 & 22 & 21 \\
\hline 44 & 30 & 30 & 28 & 27 & 26 & 25 & 24 & 23 & 22 \\
\hline 45 & 31 & 30 & 29 & 28 & 27 & 25 & 24 & 23 & 22 \\
\hline 46 & 32 & 31 & 30 & 29 & 27 & 26 & 25 & 24 & 23 \\
\hline 47 & 32 & 31 & 30 & 29 & 28 & 26 & 25 & 24 & 23 \\
\hline 48 & 33 & 32 & 31 & 30 & 28 & 27 & 26 & 25 & 24 \\
\hline 49 & 33 & 33 & 31 & 30 & 29 & 27 & 26 & 25 & 24 \\
\hline 50 & 34 & 33 & 32 & 31 & 30 & 28 & 27 & 26 & 25 \\
\hline
\end{tabular}

For $\mathrm{N}$ greater than 50 , the table (critical) value can be calculated from:

$$
\frac{N}{2}+\frac{z}{2} \sqrt{N}
$$

$\mathrm{z}$ is the $(1-\alpha)$ percentile of a standard normal distribution, which can be found on page I-10 or on page 5-28 in Table 5.2. 
Final Status Survey Report for

Corrective Action Unit 117-Pluto

Disassembly Facility, Building 2201

APPENDIX E - Class 3 Detailed Results by Room 


\begin{tabular}{|c|c|c|}
\cline { 2 - 3 } \multicolumn{1}{c|}{} & \multicolumn{2}{c|}{ dpm/100 cm2 } \\
\cline { 2 - 3 } Room 101 & alpha & beta \\
\hline Average & 18.3 & -87.5 \\
\hline StDev & 25.3 & 525.6 \\
\hline Median & 17.8 & -2.5 \\
\hline Max & 85.4 & 1038.3 \\
\hline Min & -23.4 & -1117.3 \\
\hline *LBGR & 0.18 & average \\
\hline *LBGR & 0.18 & median \\
\hline *Negative vales not used in LBGR \\
\hline
\end{tabular}

\begin{tabular}{|c|c|c|}
\cline { 2 - 3 } \multicolumn{1}{c|}{} & \multicolumn{2}{c|}{ dpm/100 cm2 } \\
\cline { 2 - 3 } \multicolumn{1}{c|}{ Room 103 } & alpha & beta \\
\hline Average & 35.8 & 215.3 \\
\hline StDev & 25.5 & 409.6 \\
\hline Median & 41.9 & 289.4 \\
\hline Max & 85.4 & 679.8 \\
\hline Min & -16.1 & -847.0 \\
\hline *LBGR & 0.40 & average \\
\hline *LBGR & 0.48 & median \\
\hline *Negative vales not used in LBGR \\
\hline
\end{tabular}

\begin{tabular}{|c|c|c|}
\cline { 2 - 3 } \multicolumn{1}{c|}{} & \multicolumn{2}{c|}{ dpm/100 cm2 } \\
\cline { 2 - 3 } Room 105 & alpha & beta \\
\hline Average & -2.2 & -151.5 \\
\hline StDev & 13.5 & 360.3 \\
\hline Median & -6.4 & -61.8 \\
\hline Max & 32.2 & 404.5 \\
\hline Min & -20.9 & -704.9 \\
\hline *LBGR & 0.00 & average \\
\hline *LBGR & 0.00 & median \\
\hline *Negative vales not used in LBGR \\
\hline
\end{tabular}

\begin{tabular}{|c|c|c|}
\cline { 2 - 3 } \multicolumn{1}{c|}{} & \multicolumn{2}{c|}{$\mathbf{d p m} / \mathbf{1 0 0} \mathbf{~ c m} \mathbf{2}$} \\
\cline { 2 - 3 } Room 109 & alpha & beta \\
\hline Average & 19.6 & 343.3 \\
\hline StDev & 18.1 & 469.5 \\
\hline Median & 17.8 & 340.3 \\
\hline Max & 70.9 & 1076.9 \\
\hline Min & -16.1 & -1167.3 \\
\hline *LBGR & 0.26 & average \\
\hline *LBGR & 0.25 & median \\
\hline *Negative vales not used in LBGR \\
\hline
\end{tabular}

\begin{tabular}{|c|c|c|}
\cline { 2 - 3 } \multicolumn{1}{c|}{} & \multicolumn{2}{c|}{$\mathbf{d p m} / \mathbf{1 0 0} \mathbf{~ c m} \mathbf{2}$} \\
\cline { 2 - 3 } Room 108 & alpha & beta \\
\hline Average & 11.9 & 89.1 \\
\hline StDev & 23.1 & 414.9 \\
\hline Median & 12.9 & 43.3 \\
\hline Max & 80.6 & 888.3 \\
\hline Min & -25.6 & -978.8 \\
\hline *LBGR & 0.14 & average \\
\hline *LBGR & 0.14 & median \\
\hline *Negative vales not used in LBGR \\
\hline
\end{tabular}

\begin{tabular}{|c|c|c|}
\hline \multirow[b]{2}{*}{ Room 110} & \multicolumn{2}{|c|}{$\mathrm{dpm} / 100 \mathrm{~cm} 2$} \\
\hline & alpha & beta \\
\hline Average & 14.2 & 690.4 \\
\hline StDev & 18.0 & 547.3 \\
\hline Median & 10.4 & 895.6 \\
\hline Max & 53.9 & 1467.1 \\
\hline Min & -15.9 & -540.3 \\
\hline *LBGR & 0.28 & average \\
\hline *LBGR & 0.28 & median \\
\hline \multicolumn{3}{|c|}{ *Negative vales not used in LBGR } \\
\hline
\end{tabular}




\begin{tabular}{|c|c|c|}
\cline { 2 - 3 } \multicolumn{1}{c|}{} & \multicolumn{2}{c|}{ dpm/100 cm2 } \\
\cline { 2 - 3 } Room 111 & alpha & beta \\
\hline Average & 16.9 & 410.0 \\
\hline StDev & 11.4 & 619.9 \\
\hline Median & 12.9 & 719.0 \\
\hline Max & 46.7 & 1195.3 \\
\hline Min & 8.1 & -428.3 \\
\hline *LBGR & 0.25 & average \\
\hline *LBGR & 0.27 & median \\
\hline *Negative vales not used in LBGR \\
\hline
\end{tabular}

\begin{tabular}{|c|c|c|}
\cline { 2 - 3 } \multicolumn{1}{c|}{} & \multicolumn{2}{c|}{$\mathrm{dpm} / \mathbf{1 0 0} \mathbf{~ c m} \mathbf{2}$} \\
\cline { 2 - 3 } Room 115 & alpha & beta \\
\hline Average & 20.7 & 458.1 \\
\hline StDev & 10.7 & 208.6 \\
\hline Median & 20.2 & 529.7 \\
\hline Max & 37.1 & 609.7 \\
\hline Min & 8.1 & 38.9 \\
\hline *LBGR & 0.30 & average \\
\hline *LBGR & 0.31 & median \\
\hline *Negative vales not used in LBGR \\
\hline
\end{tabular}

\begin{tabular}{|c|c|c|}
\cline { 2 - 3 } \multicolumn{1}{c|}{} & \multicolumn{2}{c|}{$\mathrm{dpm} / \mathbf{1 0 0} \mathbf{~ c m} \mathbf{2}$} \\
\cline { 2 - 3 } Room 117 & alpha & beta \\
\hline Average & 15.4 & 992.0 \\
\hline StDev & 13.3 & 481.3 \\
\hline Median & 13.3 & 1196.1 \\
\hline Max & 39.4 & 1315.2 \\
\hline Min & 0.8 & -39.5 \\
\hline *LBGR & 0.35 & average \\
\hline *LBGR & 0.37 & median \\
\hline *Negative vales not used in LBGR \\
\hline
\end{tabular}

\begin{tabular}{|c|c|c|}
\hline \multirow[b]{2}{*}{$\begin{array}{c}\text { Rooms 112, } \\
113,114 \\
\end{array}$} & \multicolumn{2}{|c|}{$\mathrm{dpm} / 100 \mathrm{~cm} 2$} \\
\hline & alpha & beta \\
\hline Average & 26.6 & 833.6 \\
\hline StDev & 17.3 & 461.8 \\
\hline Median & 27.4 & 1010.0 \\
\hline Max & 68.4 & 1417.0 \\
\hline Min & -1.4 & -231.6 \\
\hline *LBGR & 0.43 & average \\
\hline *LBGR & 0.48 & median \\
\hline
\end{tabular}

\begin{tabular}{|c|c|c|}
\cline { 2 - 3 } \multicolumn{1}{c|}{} & \multicolumn{2}{c|}{ dpm/100 cm2 } \\
\cline { 2 - 3 } Room 116 & alpha & Beta \\
\hline Average & 22.1 & 481.0 \\
\hline StDev & 13.2 & 510.3 \\
\hline Median & 22.6 & 508.0 \\
\hline Max & 46.7 & 1083.5 \\
\hline Min & -6.4 & -456.6 \\
\hline *LBGR & 0.32 & Average \\
\hline *LBGR & 0.33 & Median \\
\hline *Negative vales not used in LBGR \\
\hline
\end{tabular}

\begin{tabular}{|c|c|c|}
\hline \multirow[b]{2}{*}{ Room 201} & \multicolumn{2}{|c|}{$\mathrm{dpm} / 100 \mathrm{~cm} 2$} \\
\hline & alpha & beta \\
\hline Average & 48.8 & -49.8 \\
\hline StDev & 26.3 & 295.7 \\
\hline Median & 54.0 & -28.4 \\
\hline Max & 85.4 & 379.5 \\
\hline Min & 8.1 & -483.0 \\
\hline *LBGR & 0.49 & average \\
\hline *LBGR & 0.54 & median \\
\hline
\end{tabular}




\begin{tabular}{|c|c|c|}
\cline { 2 - 3 } \multicolumn{1}{c|}{} & \multicolumn{2}{c|}{ dpm/100 cm2 } \\
\cline { 2 - 3 } Room 301 & alpha & beta \\
\hline Average & 29.0 & 8.4 \\
\hline StDev & 18.7 & 323.7 \\
\hline Median & 32.2 & -108.5 \\
\hline Max & 56.4 & 481.3 \\
\hline Min & -1.6 & -381.3 \\
\hline *LBGR & 0.29 & average \\
\hline *LBGR & 0.32 & median \\
\hline *Negative vales not used in LBGR \\
\hline
\end{tabular}

\begin{tabular}{|c|c|c|}
\hline \multirow[b]{2}{*}{ Basement 1} & \multicolumn{2}{|c|}{$\mathrm{dpm} / 100 \mathrm{~cm} 2$} \\
\hline & alpha & beta \\
\hline Average & 44.9 & 229.3 \\
\hline StDev & 23.0 & 515.4 \\
\hline Median & 46.7 & 324.4 \\
\hline Max & 90.2 & 818.3 \\
\hline Min & -17.6 & -1170.7 \\
\hline *LBGR & 0.49 & average \\
\hline *LBGR & 0.53 & median \\
\hline
\end{tabular}

\begin{tabular}{|c|c|c|}
\cline { 2 - 3 } \multicolumn{1}{c|}{} & \multicolumn{2}{c|}{ dpm/100 $\mathbf{~ c m} \mathbf{2}$} \\
\cline { 2 - 3 } Basement 2 & alpha & beta \\
\hline Average & 35.5 & -94.4 \\
\hline StDev & 18.1 & 409.0 \\
\hline Median & 32.2 & -13.4 \\
\hline Max & 85.4 & 621.4 \\
\hline Min & 3.3 & -719.9 \\
\hline *LBGR & 0.35 & average \\
\hline *LBGR & 0.32 & median \\
\hline *Negative vales not used in LBGR \\
\hline
\end{tabular}

\begin{tabular}{|c|c|c|}
\cline { 2 - 3 } \multicolumn{1}{c|}{} & \multicolumn{2}{c|}{ dpm/100 cm2 } \\
\cline { 2 - 3 } Basement 4 & alpha & beta \\
\hline Average & 40.7 & 547.8 \\
\hline StDev & 23.6 & 195.5 \\
\hline Median & 37.1 & 616.4 \\
\hline Max & 95.0 & 875.0 \\
\hline Min & -1.6 & 189.3 \\
\hline *LBGR & 0.52 & average \\
\hline *LBGR & 0.49 & median \\
\hline *Negative vales not used in LBGR \\
\hline
\end{tabular}

\begin{tabular}{|c|c|c|}
\hline \multirow[b]{2}{*}{ Basement 3} & \multicolumn{2}{|c|}{$\mathrm{dpm} / 100 \mathrm{~cm} 2$} \\
\hline & alpha & Beta \\
\hline Average & 21.7 & 86.1 \\
\hline StDev & 21.1 & 545.7 \\
\hline Median & 17.8 & 309.4 \\
\hline Max & 80.6 & 716.5 \\
\hline Min & -11.1 & -1102.5 \\
\hline *LBGR & 0.23 & average \\
\hline *LBGR & 0.24 & median \\
\hline
\end{tabular}


Final Status Survey Report for

Corrective Action Unit 117-Pluto

Disassembly Facility, Building 2201

APPENDIX F - Room 102 and Ledges Detailed Results by Survey Unit 


\begin{tabular}{|c|c|c|}
\hline \multirow[b]{2}{*}{ SU\# 102-SW-1 } & \multicolumn{2}{|c|}{$\mathrm{dpm} / 100 \mathrm{~cm} 2$} \\
\hline & alpha & beta \\
\hline Average & 16.3 & -326.7 \\
\hline StDev & 16.7 & 262.8 \\
\hline Median & 17.8 & -469.7 \\
\hline Max & 51.6 & 147.6 \\
\hline Min & -1.6 & -691.6 \\
\hline *LBGR & 0.16 & average \\
\hline *LBGR & 0.18 & median \\
\hline \multicolumn{3}{|c|}{ *Negative vales not used in LBGR } \\
\hline \multicolumn{2}{|c|}{ \# of samples: } & 13 \\
\hline \multicolumn{2}{|c|}{ Sign test stat $(\mathrm{S}+)$} & 13 \\
\hline \multicolumn{2}{|c|}{ critical value (k): } & 9 \\
\hline \multicolumn{2}{|c|}{ determination: } & PASS \\
\hline
\end{tabular}

\begin{tabular}{|c|c|c|}
\hline \multirow[b]{2}{*}{ SU\# 102-SW-2 } & \multicolumn{2}{|c|}{$\mathrm{dpm} / 100 \mathrm{~cm} 2$} \\
\hline & alpha & beta \\
\hline Average & 5.0 & -513.7 \\
\hline StDev & 10.2 & 140.1 \\
\hline Median & 3.3 & -529.7 \\
\hline Max & 17.8 & -234.4 \\
\hline Min & -11.2 & -691.6 \\
\hline *LBGR & 0.05 & average \\
\hline *LBGR & 0.03 & median \\
\hline \multicolumn{3}{|c|}{ *Negative vales not used in LBGR } \\
\hline \multicolumn{2}{|c|}{ \# of samples: } & 11 \\
\hline \multicolumn{2}{|c|}{ Sign test stat $(S+)$} & 11 \\
\hline \multicolumn{2}{|c|}{ critical value (k): } & 8 \\
\hline \multicolumn{2}{|c|}{ determination: } & PASS \\
\hline
\end{tabular}

\begin{tabular}{|c|c|c|}
\hline \multirow[b]{2}{*}{ SU\# 102-SW-3 } & \multicolumn{2}{|c|}{$\mathrm{dpm} / 100 \mathrm{~cm} 2$} \\
\hline & alpha & beta \\
\hline Average & 10.3 & -343.3 \\
\hline StDev & 12.3 & 353.4 \\
\hline Median & 10.5 & -244.5 \\
\hline Max & 27.4 & -7.5 \\
\hline Min & -11.2 & -1033.8 \\
\hline *LBGR & 0.10 & average \\
\hline *LBGR & 0.11 & median \\
\hline \multicolumn{3}{|c|}{ *Negative vales not used in LBGR } \\
\hline \multicolumn{2}{|c|}{ \# of samples: } & 12 \\
\hline \multicolumn{2}{|c|}{ Sign test stat $(S+)$} & 12 \\
\hline \multicolumn{2}{|c|}{ critical value (k): } & 9 \\
\hline \multicolumn{2}{|c|}{ determination: } & PASS \\
\hline
\end{tabular}




\begin{tabular}{|c|c|c|}
\hline \multirow[b]{2}{*}{ SU\# 102-WW-1 } & \multicolumn{2}{|c|}{$\mathrm{dpm} / 100 \mathrm{~cm} 2$} \\
\hline & alpha & beta \\
\hline Average & 16.0 & -127.4 \\
\hline StDev & 13.7 & 214.6 \\
\hline Median & 17.8 & -112.7 \\
\hline Max & 37.1 & 199.3 \\
\hline Min & -6.4 & -554.8 \\
\hline *LBGR & 0.16 & average \\
\hline *LBGR & 0.18 & median \\
\hline \multicolumn{3}{|c|}{ *Negative vales not used in LBGR } \\
\hline \multicolumn{2}{|c|}{ \# of samples: } & 11 \\
\hline \multicolumn{2}{|c|}{ Sign test stat $(\mathrm{S}+)$} & 11 \\
\hline \multicolumn{2}{|c|}{ critical value (k): } & 8 \\
\hline \multicolumn{2}{|c|}{ determination: } & PASS \\
\hline
\end{tabular}

\begin{tabular}{|c|c|c|}
\hline \multirow[b]{2}{*}{ SU\# 102-WW-2 } & \multicolumn{2}{|c|}{$\mathrm{dpm} / 100 \mathrm{~cm} 2$} \\
\hline & alpha & beta \\
\hline Average & 22.6 & 367.8 \\
\hline StDev & 14.0 & 614.1 \\
\hline Median & 22.6 & 105.9 \\
\hline Max & 56.4 & 2007.8 \\
\hline Min & 3.3 & -40.9 \\
\hline *LBGR & 0.30 & average \\
\hline *LBGR & 0.25 & median \\
\hline \multicolumn{3}{|c|}{ *Negative vales not used in LBGR } \\
\hline \multicolumn{2}{|c|}{ \# of samples: } & 11 \\
\hline \multicolumn{2}{|c|}{ Sign test stat $(\mathrm{S}+)$} & 11 \\
\hline \multicolumn{2}{|c|}{ critical value (k): } & 8 \\
\hline \multicolumn{2}{|c|}{ determination: } & PASS \\
\hline
\end{tabular}

\begin{tabular}{|c|c|c|}
\hline \multirow[b]{2}{*}{ SU\# 102-NW-1 } & \multicolumn{2}{|c|}{$\mathrm{dpm} / 100 \mathrm{~cm} 2$} \\
\hline & alpha & beta \\
\hline Average & 15.7 & 59.4 \\
\hline StDev & 13.6 & 114.5 \\
\hline Median & 17.8 & 26.7 \\
\hline Max & 37.1 & 321.1 \\
\hline Min & -11.2 & -81.0 \\
\hline *LBGR & 0.17 & average \\
\hline *LBGR & 0.18 & median \\
\hline \multicolumn{3}{|c|}{ *Negative vales not used in LBGR } \\
\hline \multicolumn{2}{|c|}{ \# of samples: } & 14 \\
\hline \multicolumn{2}{|c|}{ Sign test stat $(\mathrm{S}+)$} & 14 \\
\hline \multicolumn{2}{|c|}{ critical value (k): } & 10 \\
\hline \multicolumn{2}{|c|}{ determination: } & PASS \\
\hline
\end{tabular}




\begin{tabular}{|c|c|c|}
\hline \multirow[b]{2}{*}{ SU\# 102-NW-2 } & \multicolumn{2}{|c|}{$\mathrm{dpm} / 100 \mathrm{~cm} 2$} \\
\hline & alpha & beta \\
\hline Average & 32.6 & 24.7 \\
\hline StDev & 13.0 & 185.3 \\
\hline Median & 32.2 & -4.2 \\
\hline Max & 66.1 & 402.9 \\
\hline Min & 17.8 & -331.7 \\
\hline *LBGR & 0.33 & average \\
\hline *LBGR & 0.32 & median \\
\hline \multicolumn{3}{|c|}{ *Negative vales not used in LBGR } \\
\hline \multicolumn{2}{|c|}{ \# of samples: } & 14 \\
\hline \multicolumn{2}{|c|}{ Sign test stat $(S+)$} & 14 \\
\hline \multicolumn{2}{|c|}{ critical value (k): } & 10 \\
\hline \multicolumn{2}{|c|}{ determination: } & PASS \\
\hline
\end{tabular}

\begin{tabular}{|c|c|c|}
\hline \multirow[b]{2}{*}{ SU\# 102-NW-3 } & \multicolumn{2}{|c|}{$\mathrm{dpm} / 100 \mathrm{~cm} 2$} \\
\hline & alpha & beta \\
\hline Average & 23.5 & -106.4 \\
\hline StDev & 13.7 & 340.4 \\
\hline Median & 27.4 & -2.5 \\
\hline Max & 41.9 & 171.0 \\
\hline Min & -1.6 & -1184.0 \\
\hline *LBGR & 0.24 & average \\
\hline *LBGR & 0.27 & median \\
\hline \multicolumn{3}{|c|}{${ }^{*}$ Negative vales not used in LBGR } \\
\hline \multicolumn{2}{|c|}{ \# of samples: } & 13 \\
\hline \multicolumn{2}{|c|}{ Sign test stat $(\mathrm{S}+)$} & 13 \\
\hline \multicolumn{2}{|c|}{ critical value (k): } & 9 \\
\hline \multicolumn{2}{|c|}{ determination: } & PASS \\
\hline
\end{tabular}

\begin{tabular}{|c|c|c|}
\hline \multirow[b]{2}{*}{ SU\# 102-F-1 } & \multicolumn{2}{|c|}{$\mathrm{dpm} / 100 \mathrm{~cm} 2$} \\
\hline & alpha & beta \\
\hline Average & -1.6 & 148.9 \\
\hline StDev & 14.7 & 130.4 \\
\hline Median & -4.0 & 179.3 \\
\hline Max & 32.2 & 322.8 \\
\hline Min & -16.1 & -132.7 \\
\hline *LBGR & 0.03 & average \\
\hline *LBGR & 0.04 & median \\
\hline \multicolumn{3}{|c|}{ *Negative vales not used in LBGR } \\
\hline \multicolumn{2}{|c|}{ \# of samples: } & 14 \\
\hline \multicolumn{2}{|c|}{ Sign test stat $(\mathrm{S}+)$} & 14 \\
\hline \multicolumn{2}{|c|}{ critical value (k): } & 10 \\
\hline \multicolumn{2}{|c|}{ determination: } & PASS \\
\hline
\end{tabular}




\begin{tabular}{|c|c|c|}
\hline \multirow[b]{2}{*}{ SU\# 102-F-2 } & \multicolumn{2}{|c|}{$\mathrm{dpm} / 100 \mathrm{~cm} 2$} \\
\hline & alpha & beta \\
\hline Average & 6.3 & 37.0 \\
\hline StDev & 13.0 & 112.6 \\
\hline Median & 8.1 & 22.5 \\
\hline Max & 27.4 & 212.7 \\
\hline Min & -11.2 & -182.7 \\
\hline *LBGR & 0.07 & average \\
\hline *LBGR & 0.09 & median \\
\hline \multicolumn{3}{|c|}{ *Negative vales not used in LBGR } \\
\hline \multicolumn{2}{|c|}{ \# of samples: } & 11 \\
\hline \multicolumn{2}{|c|}{ Sign test stat $(S+)$} & 11 \\
\hline \multicolumn{2}{|c|}{ critical value (k): } & 8 \\
\hline \multicolumn{2}{|c|}{ determination: } & PASS \\
\hline
\end{tabular}

\begin{tabular}{|c|c|c|}
\hline \multirow[b]{2}{*}{ SU\# 102-F-3 } & \multicolumn{2}{|c|}{$\mathrm{dpm} / 100 \mathrm{~cm} 2$} \\
\hline & alpha & beta \\
\hline Average & 6.5 & -49.7 \\
\hline StDev & 18.1 & 267.3 \\
\hline Median & -1.6 & 40.8 \\
\hline Max & 41.9 & 152.6 \\
\hline Min & -16.1 & -676.6 \\
\hline *LBGR & 0.06 & average \\
\hline *LBGR & 0.01 & median \\
\hline \multicolumn{3}{|c|}{ *Negative vales not used in LBGR } \\
\hline \multicolumn{2}{|c|}{ \# of samples: } & 12 \\
\hline \multicolumn{2}{|c|}{ Sign test stat $(S+)$} & 12 \\
\hline \multicolumn{2}{|c|}{ critical value (k): } & 9 \\
\hline \multicolumn{2}{|c|}{ determination: } & PASS \\
\hline
\end{tabular}

\begin{tabular}{|c|c|c|}
\hline \multirow[b]{2}{*}{ SU\# 102-F-4 } & \multicolumn{2}{|c|}{$\mathrm{dpm} / 100 \mathrm{~cm} 2$} \\
\hline & alpha & beta \\
\hline Average & 5.2 & -79.1 \\
\hline StDev & 9.1 & 110.3 \\
\hline Median & 3.3 & -62.6 \\
\hline Max & 22.6 & 90.9 \\
\hline Min & -6.4 & -402.9 \\
\hline *LBGR & 0.05 & average \\
\hline *LBGR & 0.03 & median \\
\hline \multicolumn{3}{|c|}{ *Negative vales not used in LBGR } \\
\hline \multicolumn{2}{|c|}{ \# of samples: } & 15 \\
\hline \multicolumn{2}{|c|}{ Sign test stat $(S+)$} & 15 \\
\hline \multicolumn{2}{|c|}{ critical value (k): } & 11 \\
\hline \multicolumn{2}{|c|}{ determination: } & PASS \\
\hline
\end{tabular}




\begin{tabular}{|c|c|c|}
\hline \multirow[b]{2}{*}{ SU\# 102-EW-1 } & \multicolumn{2}{|c|}{$\mathrm{dpm} / 100 \mathrm{~cm} 2$} \\
\hline & alpha & beta \\
\hline Average & 22.9 & -492.4 \\
\hline StDev & 18.2 & 435.8 \\
\hline Median & 22.6 & -217.8 \\
\hline Max & 59.7 & -84.3 \\
\hline Min & 1.2 & -1160.7 \\
\hline *LBGR & 0.23 & average \\
\hline *LBGR & 0.23 & median \\
\hline \multicolumn{3}{|c|}{ *Negative vales not used in LBGR } \\
\hline \multicolumn{2}{|c|}{ \# of samples: } & 13 \\
\hline \multicolumn{2}{|c|}{ Sign test stat $(\mathrm{S}+)$} & 13 \\
\hline \multicolumn{2}{|c|}{ critical value (k): } & 9 \\
\hline \multicolumn{2}{|c|}{ determination: } & PASS \\
\hline
\end{tabular}

\begin{tabular}{|c|c|c|}
\hline \multirow[b]{2}{*}{ SU\# 102-EW-2 } & \multicolumn{2}{|c|}{$\mathrm{dpm} / 100 \mathrm{~cm} 2$} \\
\hline & alpha & beta \\
\hline Average & 42.7 & -146.4 \\
\hline StDev & 20.3 & 56.9 \\
\hline Median & 37.1 & -135.2 \\
\hline Max & 80.6 & -74.3 \\
\hline Min & 22.6 & -257.8 \\
\hline *LBGR & 0.43 & average \\
\hline *LBGR & 0.37 & median \\
\hline \multicolumn{3}{|c|}{ *Negative vales not used in LBGR } \\
\hline \multicolumn{2}{|c|}{ \# of samples: } & 12 \\
\hline \multicolumn{2}{|c|}{ Sign test stat $(\mathrm{S}+)$} & 12 \\
\hline \multicolumn{2}{|c|}{ critical value (k): } & 9 \\
\hline \multicolumn{2}{|c|}{ determination: } & PASS \\
\hline
\end{tabular}

\begin{tabular}{|c|c|c|}
\hline \multirow[b]{2}{*}{ SU\# 102-C-1 } & \multicolumn{2}{|c|}{$\mathrm{dpm} / 100 \mathrm{~cm} 2$} \\
\hline & alpha & beta \\
\hline Average & 18.6 & 138.6 \\
\hline StDev & 13.2 & 145.9 \\
\hline Median & 17.8 & 127.6 \\
\hline Max & 41.9 & 329.5 \\
\hline Min & -1.6 & -79.3 \\
\hline *LBGR & 0.21 & average \\
\hline *LBGR & 0.20 & median \\
\hline \multicolumn{3}{|c|}{ *Negative vales not used in LBGR } \\
\hline \multicolumn{2}{|c|}{ \# of samples: } & 12 \\
\hline \multicolumn{2}{|c|}{ Sign test stat $(\mathrm{S}+)$} & 12 \\
\hline \multicolumn{2}{|c|}{ critical value (k): } & 9 \\
\hline \multicolumn{2}{|c|}{ determination: } & PASS \\
\hline
\end{tabular}




\begin{tabular}{|c|c|c|}
\hline \multirow[b]{2}{*}{ SU\# 102-SDW-1 } & \multicolumn{2}{|c|}{$\mathrm{dpm} / 100 \mathrm{~cm} 2$} \\
\hline & alpha & beta \\
\hline Average & 30.7 & -1402.4 \\
\hline StDev & 22.9 & 102.5 \\
\hline Median & 25.8 & -1425.9 \\
\hline Max & 74.2 & -1222.4 \\
\hline Min & -12.8 & -1526.0 \\
\hline *LBGR & 0.31 & average \\
\hline *LBGR & 0.26 & median \\
\hline \multicolumn{3}{|c|}{ *Negative vales not used in LBGR } \\
\hline \multicolumn{2}{|c|}{ \# of samples: } & 12 \\
\hline \multicolumn{2}{|c|}{ Sign test stat $(S+)$} & 12 \\
\hline \multicolumn{2}{|c|}{ critical value (k): } & 9 \\
\hline \multicolumn{2}{|c|}{ determination: } & PASS \\
\hline
\end{tabular}

\begin{tabular}{|c|c|c|}
\hline \multirow[b]{2}{*}{ SU\# Ledges-1 } & \multicolumn{2}{|c|}{$\mathrm{dpm} / 100 \mathrm{~cm} 2$} \\
\hline & alpha & beta \\
\hline Average & 30.3 & 141.7 \\
\hline StDev & 21.5 & 229.4 \\
\hline Median & 32.2 & 105.9 \\
\hline Max & 85.4 & 454.6 \\
\hline Min & -11.2 & -217.8 \\
\hline *LBGR & 0.33 & average \\
\hline *LBGR & 0.34 & median \\
\hline \multicolumn{3}{|c|}{ *Negative vales not used in LBGR } \\
\hline \multicolumn{2}{|c|}{ \# of samples: } & 17 \\
\hline \multicolumn{2}{|c|}{ Sign test stat $(\mathrm{S}+)$} & 17 \\
\hline \multicolumn{2}{|c|}{ critical value (k): } & 12 \\
\hline \multicolumn{2}{|c|}{ determination: } & PASS \\
\hline
\end{tabular}


Final Status Survey Report for

Corrective Action Unit 117-Pluto

Disassembly Facility, Building 2201

\section{APPENDIX G - Room 104 Detailed Results by Survey Unit}




\begin{tabular}{|c|c|c|}
\hline \multirow[b]{2}{*}{ SU\# 104-F-1 } & \multicolumn{2}{|c|}{$\mathrm{dpm} / 100 \mathrm{~cm} 2$} \\
\hline & alpha & beta \\
\hline Average & 2.5 & -302.1 \\
\hline StDev & 14.7 & 328.9 \\
\hline Median & -1.6 & -198.6 \\
\hline Max & 32.2 & 9.1 \\
\hline Min & -16.1 & -1047.5 \\
\hline *LBGR & 0.02 & average \\
\hline *LBGR & 0.00 & median \\
\hline \multicolumn{3}{|c|}{${ }^{*}$ Negative vales not used in LBGR } \\
\hline \multicolumn{2}{|c|}{ \# of samples: } & 18 \\
\hline \multicolumn{2}{|c|}{ Sign test stat $\left(S_{+}\right)$} & 18 \\
\hline \multicolumn{2}{|c|}{ critical value (k): } & 12 \\
\hline \multicolumn{2}{|c|}{ determination: } & PASS \\
\hline
\end{tabular}

\begin{tabular}{|c|c|c|}
\hline \multirow[b]{2}{*}{ SU\# 104-EW-1 } & \multicolumn{2}{|c|}{$\mathrm{dpm} / 100 \mathrm{~cm} 2$} \\
\hline & alpha & beta \\
\hline Average & 5.6 & -676.4 \\
\hline StDev & 12.9 & 233.6 \\
\hline Median & 3.3 & -633.2 \\
\hline Max & 32.2 & -446.3 \\
\hline Min & -16.1 & -1372.5 \\
\hline *LBGR & 0.06 & average \\
\hline *LBGR & 0.03 & median \\
\hline \multicolumn{3}{|c|}{ *Negative vales not used in LBGR } \\
\hline \multicolumn{2}{|c|}{ \# of samples: } & 16 \\
\hline \multicolumn{2}{|c|}{ Sign test stat $(S+)$} & 16 \\
\hline \multicolumn{2}{|c|}{ critical value (k): } & 11 \\
\hline \multicolumn{2}{|c|}{ determination: } & PASS \\
\hline
\end{tabular}

\begin{tabular}{|c|c|c|}
\hline \multirow[b]{2}{*}{ SU\# 104-SW-1 } & \multicolumn{2}{|c|}{$\mathrm{dpm} / 100 \mathrm{~cm} 2$} \\
\hline & alpha & beta \\
\hline Average & 15.8 & -542.1 \\
\hline StDev & 15.1 & 238.8 \\
\hline Median & 15.3 & -563.1 \\
\hline Max & 51.6 & -234.4 \\
\hline Min & -3.1 & -1269.1 \\
\hline *LBGR & 0.16 & average \\
\hline *LBGR & 0.15 & median \\
\hline \multicolumn{3}{|c|}{${ }^{*}$ Negative vales not used in LBGR } \\
\hline \multicolumn{2}{|c|}{ \# of samples: } & 18 \\
\hline \multicolumn{2}{|c|}{ Sign test stat $(\mathrm{S}+)$} & 18 \\
\hline \multicolumn{2}{|c|}{ critical value (k): } & 12 \\
\hline \multicolumn{2}{|c|}{ determination: } & PASS \\
\hline
\end{tabular}




\begin{tabular}{|c|c|c|}
\hline \multirow[b]{2}{*}{ SU\# 104-WW-1 } & \multicolumn{2}{|c|}{$\mathrm{dpm} / 100 \mathrm{~cm} 2$} \\
\hline & alpha & beta \\
\hline Average & 14.8 & -524.8 \\
\hline StDev & 14.8 & 345.9 \\
\hline Median & 8.1 & -611.5 \\
\hline Max & 46.7 & -20.9 \\
\hline Min & -6.4 & -1237.4 \\
\hline *LBGR & 0.15 & average \\
\hline *LBGR & 0.08 & median \\
\hline \multicolumn{3}{|c|}{${ }^{*}$ Negative vales not used in LBGR } \\
\hline \multicolumn{2}{|c|}{ \# of samples: } & 21 \\
\hline \multicolumn{2}{|c|}{ Sign test stat $(\mathrm{S}+)$} & 21 \\
\hline \multicolumn{2}{|c|}{ critical value (k): } & 14 \\
\hline \multicolumn{2}{|c|}{ determination: } & PASS \\
\hline
\end{tabular}

\begin{tabular}{|c|c|c|}
\hline \multirow[b]{2}{*}{ SU\# 104-C-1 } & \multicolumn{2}{|c|}{$\mathrm{dpm} / 100 \mathrm{~cm} 2$} \\
\hline & alpha & beta \\
\hline Average & 15.5 & 77.2 \\
\hline StDev & 13.6 & 227.7 \\
\hline Median & 12.9 & 144.3 \\
\hline Max & 41.9 & 324.4 \\
\hline Min & -6.4 & -521.4 \\
\hline *LBGR & 0.17 & average \\
\hline *LBGR & 0.16 & median \\
\hline \multicolumn{3}{|c|}{ *Negative vales not used in LBGR } \\
\hline \multicolumn{2}{|c|}{ \# of samples: } & 17 \\
\hline \multicolumn{2}{|c|}{ Sign test stat $(\mathrm{S}+)$} & 17 \\
\hline \multicolumn{2}{|c|}{ critical value (k): } & 12 \\
\hline \multicolumn{2}{|c|}{ determination: } & PASS \\
\hline
\end{tabular}

\begin{tabular}{|c|c|c|}
\hline \multirow[b]{2}{*}{ SU\# 104-NW-1 } & \multicolumn{2}{|c|}{$\mathrm{dpm} / 100 \mathrm{~cm} 2$} \\
\hline & alpha & beta \\
\hline Average & 7.2 & -479.5 \\
\hline StDev & 8.6 & 268.6 \\
\hline Median & 8.1 & -609.8 \\
\hline Max & 22.6 & 5.8 \\
\hline Min & -11.2 & -801.7 \\
\hline *LBGR & 0.07 & average \\
\hline *LBGR & 0.08 & median \\
\hline \multicolumn{3}{|c|}{ *Negative vales not used in LBGR } \\
\hline \multicolumn{2}{|c|}{ \# of samples: } & 17 \\
\hline \multicolumn{2}{|c|}{ Sign test stat $(S+)$} & 17 \\
\hline \multicolumn{2}{|c|}{ critical value (k): } & 12 \\
\hline \multicolumn{2}{|c|}{ determination: } & PASS \\
\hline
\end{tabular}


Final Status Survey Report for

Corrective Action Unit 117-Pluto

Disassembly Facility, Building 2201

\section{APPENDIX H - Room 106 Detailed Results by Survey Unit}




\begin{tabular}{|c|c|c|}
\hline \multirow[b]{2}{*}{ SU\# 106-SW-1 } & \multicolumn{2}{|c|}{$\mathrm{dpm} / 100 \mathrm{~cm} 2$} \\
\hline & alpha & beta \\
\hline Average & 22.6 & -529.7 \\
\hline StDev & 12.3 & 246.2 \\
\hline Median & 17.8 & -464.7 \\
\hline Max & 56.4 & -297.8 \\
\hline Min & 12.9 & -1284.4 \\
\hline *LBGR & 0.23 & average \\
\hline *LBGR & 0.18 & median \\
\hline \multicolumn{3}{|c|}{ *Negative vales not used in LBGR } \\
\hline \multicolumn{2}{|c|}{ \# of samples: } & 13 \\
\hline \multicolumn{2}{|c|}{ Sign test stat $(\mathrm{S}+)$} & 13 \\
\hline \multicolumn{2}{|c|}{ critical value ( $k$ ): } & 9 \\
\hline \multicolumn{2}{|c|}{ determination: } & PASS \\
\hline
\end{tabular}

\begin{tabular}{|c|c|c|}
\hline \multirow[b]{2}{*}{ SU\# 106-EW-1 } & \multicolumn{2}{|c|}{$\mathrm{dpm} / 100 \mathrm{~cm} 2$} \\
\hline & alpha & beta \\
\hline Average & 9.4 & -556.6 \\
\hline StDev & 12.0 & 412.3 \\
\hline Median & 8.1 & -584.8 \\
\hline Max & 41.9 & 74.2 \\
\hline Min & -6.4 & -1356.1 \\
\hline *LBGR & 0.09 & average \\
\hline *LBGR & 0.08 & median \\
\hline \multicolumn{3}{|c|}{${ }^{*}$ Negative vales not used in LBGR } \\
\hline \multicolumn{2}{|c|}{ \# of samples: } & 15 \\
\hline \multicolumn{2}{|c|}{ Sign test stat $(S+)$} & 15 \\
\hline \multicolumn{2}{|c|}{ critical value (k): } & 11 \\
\hline \multicolumn{2}{|c|}{ determination: } & PASS \\
\hline
\end{tabular}

\begin{tabular}{|c|c|c|}
\hline \multirow[b]{2}{*}{ SU\# 106-NW-1 } & \multicolumn{2}{|c|}{$\mathrm{dpm} / 100 \mathrm{~cm} 2$} \\
\hline & alpha & beta \\
\hline Average & 12.2 & -354.6 \\
\hline StDev & 14.8 & 275.3 \\
\hline Median & 15.3 & -330.4 \\
\hline Max & 37.1 & 30.8 \\
\hline Min & -16.1 & -734.9 \\
\hline *LBGR & 0.12 & average \\
\hline *LBGR & 0.15 & median \\
\hline \multicolumn{3}{|c|}{ *Negative vales not used in LBGR } \\
\hline \multicolumn{2}{|c|}{ \# of samples: } & 14 \\
\hline \multicolumn{2}{|c|}{ Sign test stat $(\mathrm{S}+)$} & 14 \\
\hline \multicolumn{2}{|c|}{ critical value (k): } & 10 \\
\hline \multicolumn{2}{|c|}{ determination: } & PASS \\
\hline
\end{tabular}




\begin{tabular}{|c|c|c|}
\hline \multirow{2}{*}{$\begin{array}{l}\text { (Post-Decon) } \\
\text { SU\# 106-F-1 }\end{array}$} & \multicolumn{2}{|c|}{$\mathrm{dpm} / 100 \mathrm{~cm} 2$} \\
\hline & alpha & beta \\
\hline Average & 10.3 & -293.4 \\
\hline StDev & 16.9 & 559.6 \\
\hline Median & 8.1 & -137.7 \\
\hline Max & 41.9 & 519.6 \\
\hline Min & -11.2 & -1219.1 \\
\hline *LBGR & 0.10 & average \\
\hline *LBGR & 0.08 & median \\
\hline \multicolumn{3}{|c|}{ *Negative vales not used in LBGR } \\
\hline \multicolumn{2}{|c|}{ \# of samples: } & 13 \\
\hline \multicolumn{2}{|c|}{ Sign test stat $(S+)$} & 13 \\
\hline \multicolumn{2}{|c|}{ critical value (k): } & 9 \\
\hline \multicolumn{2}{|c|}{ determination: } & PASS \\
\hline
\end{tabular}

\begin{tabular}{|c|c|c|}
\hline \multirow[b]{2}{*}{ SU\# 106-WW-1 } & \multicolumn{2}{|c|}{$\mathrm{dpm} / 100 \mathrm{~cm} 2$} \\
\hline & alpha & beta \\
\hline Average & 24.5 & -501.9 \\
\hline StDev & 15.0 & 464.2 \\
\hline Median & 22.6 & -534.7 \\
\hline Max & 56.4 & 861.7 \\
\hline Min & -1.6 & -1316.1 \\
\hline *LBGR & 0.24 & average \\
\hline *LBGR & 0.23 & median \\
\hline \multicolumn{3}{|c|}{${ }^{*}$ Negative vales not used in LBGR } \\
\hline \multicolumn{2}{|c|}{ \# of samples: } & 13 \\
\hline \multicolumn{2}{|c|}{ Sign test stat $(S+)$} & 13 \\
\hline \multicolumn{2}{|c|}{ critical value (k): } & 9 \\
\hline \multicolumn{2}{|c|}{ determination: } & PASS \\
\hline
\end{tabular}

\begin{tabular}{|c|c|c|}
\hline \multirow[b]{2}{*}{ SU\# 106-C-1 } & \multicolumn{2}{|c|}{$\mathrm{dpm} / 100 \mathrm{~cm} 2$} \\
\hline & alpha & beta \\
\hline Average & 45.6 & 157.4 \\
\hline StDev & 16.0 & 272.7 \\
\hline Median & 46.7 & 122.6 \\
\hline Max & 66.1 & 753.2 \\
\hline Min & 22.6 & -379.6 \\
\hline *LBGR & 0.49 & average \\
\hline *LBGR & 0.49 & median \\
\hline \multicolumn{3}{|c|}{ *Negative vales not used in LBGR } \\
\hline \multicolumn{2}{|c|}{ \# of samples: } & 13 \\
\hline \multicolumn{2}{|c|}{ Sign test stat $(S+)$} & 13 \\
\hline \multicolumn{2}{|c|}{ critical value (k): } & 9 \\
\hline \multicolumn{2}{|c|}{ determination: } & PASS \\
\hline
\end{tabular}


Final Status Survey Report for

Corrective Action Unit 117-Pluto

Disassembly Facility, Building 2201

\section{APPENDIX I - Room 107 Detailed Results by Survey Unit}




\begin{tabular}{|c|c|c|}
\hline \multirow[b]{2}{*}{ SU\# 107-F-1 } & \multicolumn{2}{|c|}{$\mathrm{dpm} / 100 \mathrm{~cm} 2$} \\
\hline & alpha & beta \\
\hline Average & 24.0 & -240.8 \\
\hline StDev & 17.5 & 334.6 \\
\hline Median & 22.6 & -201.1 \\
\hline Max & 75.7 & 399.5 \\
\hline Min & 3.3 & -1038.9 \\
\hline *LBGR & 0.24 & average \\
\hline *LBGR & 0.23 & median \\
\hline \multicolumn{3}{|c|}{ *Negative vales not used in LBGR } \\
\hline \multicolumn{2}{|c|}{ \# of samples: } & 16 \\
\hline \multicolumn{2}{|c|}{ Sign test stat $\left(\mathrm{S}_{+}\right)$} & 16 \\
\hline \multicolumn{2}{|c|}{ critical value (k): } & 11 \\
\hline \multicolumn{2}{|c|}{ determination: } & PASS \\
\hline
\end{tabular}

\begin{tabular}{|c|c|c|}
\hline \multirow[b]{2}{*}{ SU\# 107-NW-1 } & \multicolumn{2}{|c|}{$\mathrm{dpm} / 100 \mathrm{~cm} 2$} \\
\hline & alpha & beta \\
\hline Average & 41.9 & -226.3 \\
\hline StDev & 18.7 & 124.4 \\
\hline Median & 41.9 & -208.6 \\
\hline Max & 70.9 & -62.6 \\
\hline Min & 12.9 & -441.3 \\
\hline *LBGR & 0.42 & average \\
\hline *LBGR & 0.42 & median \\
\hline \multicolumn{3}{|c|}{ *Negative vales not used in LBGR } \\
\hline \multicolumn{2}{|c|}{ \# of samples: } & 16 \\
\hline \multicolumn{2}{|c|}{ Sign test stat $(\mathrm{S}+)$} & 16 \\
\hline \multicolumn{2}{|c|}{ critical value (k): } & 11 \\
\hline \multicolumn{2}{|c|}{ determination: } & PASS \\
\hline
\end{tabular}

\begin{tabular}{|c|c|c|}
\hline \multirow[b]{2}{*}{ SU\# 107-NW-2 } & \multicolumn{2}{|c|}{$\mathrm{dpm} / 100 \mathrm{~cm} 2$} \\
\hline & alpha & beta \\
\hline Average & 31.7 & 102.9 \\
\hline StDev & 22.5 & 72.2 \\
\hline Median & 22.6 & 100.9 \\
\hline Max & 70.9 & 237.7 \\
\hline Min & -1.6 & -2.5 \\
\hline *LBGR & 0.34 & average \\
\hline *LBGR & 0.25 & median \\
\hline \multicolumn{3}{|c|}{ *Negative vales not used in LBGR } \\
\hline \multicolumn{2}{|c|}{ \# of samples: } & 17 \\
\hline \multicolumn{2}{|c|}{ Sign test stat $(\mathrm{S}+)$} & 17 \\
\hline \multicolumn{2}{|c|}{ critical value (k): } & 12 \\
\hline \multicolumn{2}{|c|}{ determination: } & PASS \\
\hline
\end{tabular}




\begin{tabular}{|c|c|c|}
\hline \multirow[b]{2}{*}{ SU\# 107-SW-1 } & \multicolumn{2}{|c|}{$\mathrm{dpm} / 100 \mathrm{~cm} 2$} \\
\hline & alpha & beta \\
\hline Average & 22.0 & -481.4 \\
\hline StDev & 18.5 & 92.3 \\
\hline Median & 15.3 & -477.2 \\
\hline Max & 75.7 & -297.8 \\
\hline Min & 3.3 & -691.6 \\
\hline *LBGR & 0.22 & average \\
\hline *LBGR & 0.15 & median \\
\hline \multicolumn{3}{|c|}{ *Negative vales not used in LBGR } \\
\hline \multicolumn{2}{|c|}{ \# of samples: } & 16 \\
\hline \multicolumn{2}{|c|}{ Sign test stat $(\mathrm{S}+)$} & 16 \\
\hline \multicolumn{2}{|c|}{ critical value (k): } & 11 \\
\hline \multicolumn{2}{|c|}{ determination: } & PASS \\
\hline
\end{tabular}

\begin{tabular}{|c|c|c|}
\hline \multirow[b]{2}{*}{ SU\# 107-SW-2 } & \multicolumn{2}{|c|}{$\mathrm{dpm} / 100 \mathrm{~cm} 2$} \\
\hline & alpha & beta \\
\hline Average & 15.6 & -408.4 \\
\hline StDev & 15.1 & 88.8 \\
\hline Median & 12.9 & -403.8 \\
\hline Max & 46.7 & -282.8 \\
\hline Min & -6.4 & -608.1 \\
\hline *LBGR & 0.16 & average \\
\hline *LBGR & 0.13 & median \\
\hline \multicolumn{3}{|c|}{ *Negative vales not used in LBGR } \\
\hline \multicolumn{2}{|c|}{ \# of samples: } & 18 \\
\hline \multicolumn{2}{|c|}{ Sign test stat $(\mathrm{S}+)$} & 18 \\
\hline \multicolumn{2}{|c|}{ critical value (k): } & 12 \\
\hline \multicolumn{2}{|c|}{ determination: } & PASS \\
\hline
\end{tabular}

\begin{tabular}{|c|c|c|}
\hline \multirow[b]{2}{*}{ SU\# 107-WW-1 } & \multicolumn{2}{|c|}{$\mathrm{dpm} / 100 \mathrm{~cm} 2$} \\
\hline & alpha & beta \\
\hline Average & 20.4 & -508.8 \\
\hline StDev & 13.6 & 481.1 \\
\hline Median & 22.6 & -503.9 \\
\hline Max & 40.3 & 45.8 \\
\hline Min & -6.4 & -1461.0 \\
\hline *LBGR & 0.20 & average \\
\hline *LBGR & 0.23 & median \\
\hline \multicolumn{3}{|c|}{ *Negative vales not used in LBGR } \\
\hline \multicolumn{2}{|c|}{ \# of samples: } & 20 \\
\hline \multicolumn{2}{|c|}{ Sign test stat $(S+)$} & 20 \\
\hline \multicolumn{2}{|c|}{ critical value (k): } & 14 \\
\hline \multicolumn{2}{|c|}{ determination: } & PASS \\
\hline
\end{tabular}




\begin{tabular}{|c|c|c|}
\hline \multirow[b]{2}{*}{ SU\# 107-EW-1 } & \multicolumn{2}{|c|}{$\mathrm{dpm} / 100 \mathrm{~cm} 2$} \\
\hline & alpha & beta \\
\hline Average & 21.8 & -486.1 \\
\hline StDev & 16.0 & 482.8 \\
\hline Median & 20.2 & -525.8 \\
\hline Max & 56.5 & 145.9 \\
\hline Min & -6.4 & -1452.6 \\
\hline *LBGR & 0.22 & average \\
\hline *LBGR & 0.20 & median \\
\hline \multicolumn{3}{|c|}{ *Negative vales not used in LBGR } \\
\hline \multicolumn{2}{|c|}{ \# of samples: } & 20 \\
\hline \multicolumn{2}{|c|}{ Sign test stat $(\mathrm{S}+)$} & 20 \\
\hline \multicolumn{2}{|c|}{ critical value (k): } & 14 \\
\hline \multicolumn{2}{|c|}{ determination: } & PASS \\
\hline
\end{tabular}

\begin{tabular}{|c|c|c|}
\hline \multirow[b]{2}{*}{ SU\# 107-C-1 } & \multicolumn{2}{|c|}{$\mathrm{dpm} / 100 \mathrm{~cm} 2$} \\
\hline & alpha & beta \\
\hline Average & 30.4 & 93.6 \\
\hline StDev & 21.9 & 116.4 \\
\hline Median & 22.6 & 100.9 \\
\hline Max & 70.9 & 299.4 \\
\hline Min & 3.3 & -146.0 \\
\hline *LBGR & 0.32 & average \\
\hline *LBGR & 0.25 & median \\
\hline \multicolumn{3}{|c|}{ *Negative vales not used in LBGR } \\
\hline \multicolumn{2}{|c|}{ \# of samples: } & 18 \\
\hline \multicolumn{2}{|c|}{ Sign test stat $(S+)$} & 18 \\
\hline \multicolumn{2}{|c|}{ critical value ( $k$ ): } & 12 \\
\hline \multicolumn{2}{|c|}{ determination: } & PASS \\
\hline
\end{tabular}


Final Status Survey Report for Corrective Action Unit 117-Pluto

Disassembly Facility, Building 2201

\section{ATTACHMENT 1}

Survey Plan 10-015, "Final Status Survey Plan for Corrective Action Unit 117 Pluto Disassembly Facility, Building 2201" 


\section{National Security Technologies LLC}

Radiological Operations

Survey Plan

FINAL STATUS SURVEY PLAN FOR CORRECTIVE ACTION UNIT 117 - PLUTO DISASSEMBLY FACILITY, BUILDING 2201

\section{$10-015$}

June 22, 2010

Prepared by:

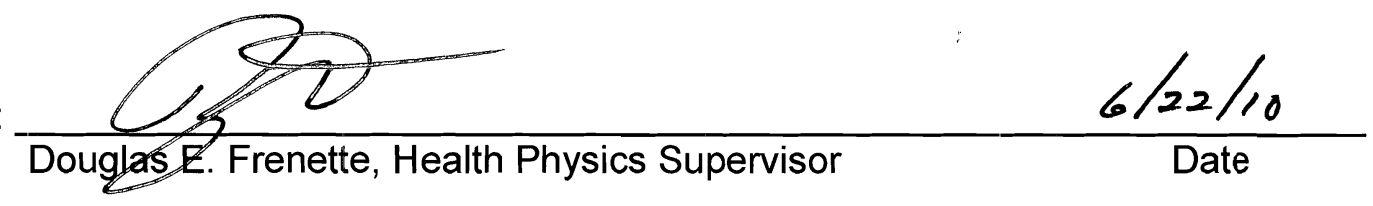

Reviewed by:

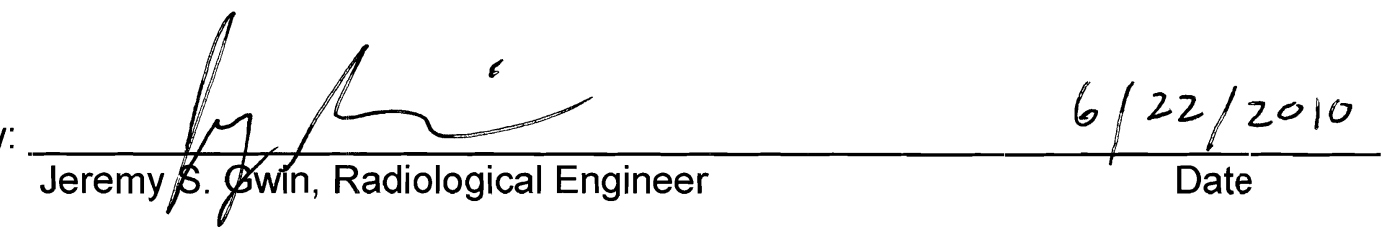

Concurrence by:

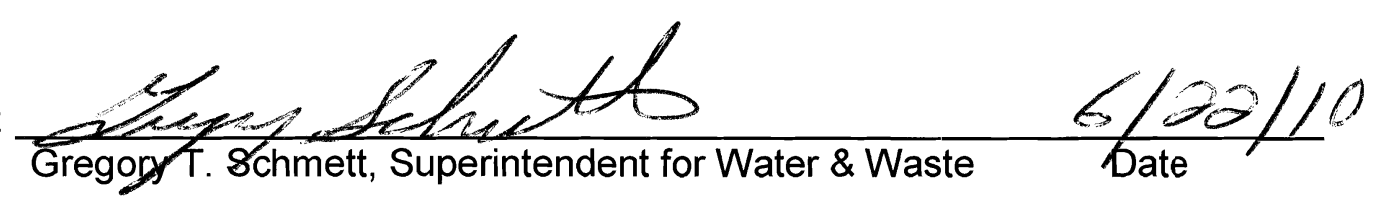

Concurrence by: $\frac{D}{\text { for Annette L. Primrose, ER Program Manager }} \quad 0 / 2.2 / 10$
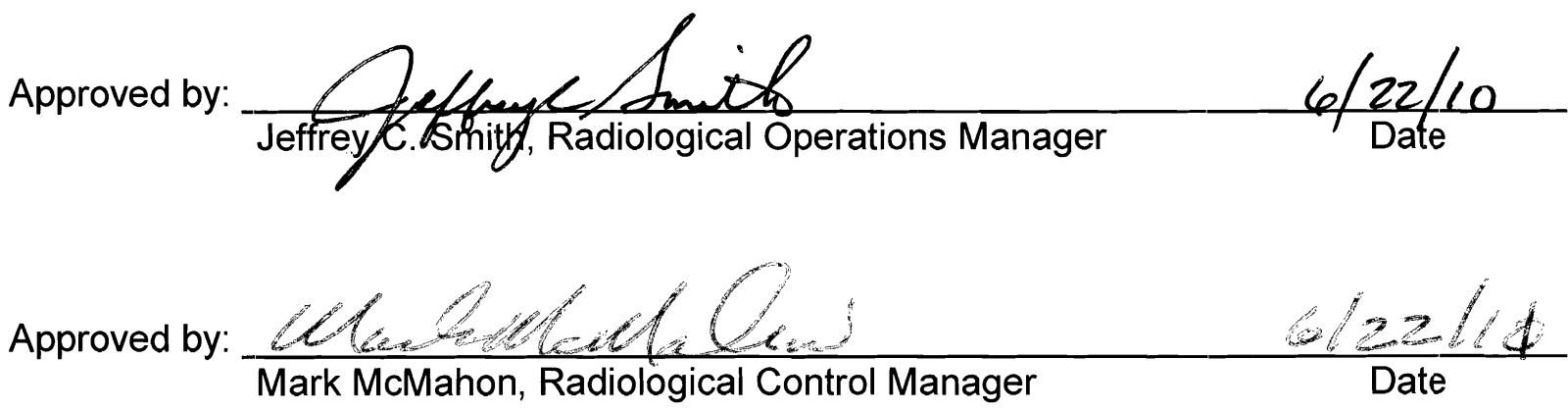


\section{Purpose}

This survey plan is to provide Radiological Control Technicians (RCTs) with the survey criteria required to perform the Final Status Survey using the Multi-Agency Radiation Survey and Site Investigation Manual (MARSSIM) process at the Pluto Disassembly Facility, Building 2201.

\section{Scope}

This survey plan presents information supporting closure of Corrective Action Unit (CAU) 117, Area 26 Pluto Disassembly Facility, Nevada Test Site (NTS), Nevada. The NTS is located approximately 65 miles northwest of Las Vegas, Nevada (Figures $1 \& 2$ ). CAU 117 is located approximately 10 miles (mi) northwest of Mercury, Nevada, in the southwest region of Area 26 at the NTS and is comprised of one Corrective Action Site (CAS) 26-41-01, Pluto Disassembly Facility.

The survey plan pertains to the entire Building 2201 structure. The term "building" in reference to this survey plan applies to the physical structure (floors, ceilings, walls) and all permanently installed equipment contained within the structure. This survey plan does not address the four underground storage vaults and buried piping. The survey plan does not include the soils surrounding the building, nor the above or below ground facility support systems (e.g., electrical, water, sewer) exterior to the building.

The end goal of this MARSSIM Final Status Survey (FSS) is to collect sufficient data to statistically prove that all the survey units in the Pluto facility meet the release criteria of U.S. Department of Energy (DOE) Order DOE O 5400.5, "Radiation Protection of the Public and the Environment," for disposal at the onsite construction debris landfill U10c. After the survey units are proven clean by being less than DOE 05400.5 release limits, the building will be demolished. The rubble is a remnant of the surveyed building, so by default the rubble will be assumed clean and able to be released to the U10C landfill. The rubble will not need to be resurveyed for release (as it was previously surveyed and passed the MARSSIM FSS).

What will be left at the Pluto site after the rubble is removed will be the concrete slab and other rubble that will be buried in the basement. Residual radioactivity associated with the storage vaults or internal passageways within the remaining concrete foundation, will be left in place for future end-use consideration. The underground drain lines will also remain. The storage vaults, concrete slab and underground drain lines will all be posted as Underground Radioactive Material Areas (URMAs). These areas will require further evaluation if there is a desire to remove all remaining radiological postings.

Public involvement with National Security Technologies, LLC (NSTec) and the National Nuclear Security Administration Nevada Site Office (NNSA/NSO) site-related activities are captured in a variety of ways. For more information, see the Environmental Technical Services Annual Report as found on http://www.nv.doe.gov/library/publications/environmental.aspx.

\section{Implementing the MARSSIM process}

As part of the MARSSIM approach to releasing Building 2201 to the levels required by the applicable onsite landfill's waste acceptance criteria, the manual recommends the following process: 
1. Historical Site Assessment (HSA) - This is the collection of data from historical documentation, observation, or personnel interviews, also known as "process knowledge." A brief synopsis of the facility and operations is included in this section.

2. Scoping Survey - A scoping survey is a "big picture" type of survey performed if the HSA indicates an area may be impacted. It should also determine the specific contaminant(s) of concern. In the case of the Pluto Disassembly Facility, it has already been established that various rooms and systems are or may be impacted. The intent of this step has been accomplished through review of pre-job and job coverage surveys performed over the previous years and during Decontamination and Decommissioning (D\&D) preparations last year. It is a compilation of numerous smaller surveys and analytical samples.

3. Characterization Survey - This is a more comprehensive survey and determines the nature and extent of the contamination, remedial alternatives, and provides data for the Final Status Survey. In the case of Pluto Disassembly Facility, this survey was accomplished and documented in two separate phases based on accessibility of specific areas and surfaces in accordance with survey plans \#09-022, "Pluto Disassembly Facility - Building Radiological Characterization," and \#09-031, "Pluto Disassembly Facility - Radiological Characterization (Phase II) and Post Remediation Survey Requirements."

4. Remedial Action Support Survey - This survey is more commonly referred to at the NTS as "in-process" or "job coverage" survey. Its purpose is to provide real time data that will help guide remedial action operations (i.e. decontamination and contaminated equipment removal). In the case of Pluto Disassembly Facility, based off of the characterization surveys performed, four rooms in the facility (rooms 102, 104, 106, and 107) were found to have residual contamination and required remediation. Remediation efforts were successfully completed in Spring of 2010 and postremediation surveys were documented in accordance with Survey Plan \#09-031.

5. Final Status Survey - This survey is used to demonstrate that the potential dose from residual contamination is below release criteria. Release criteria depend on several factors, but primarily on what the final disposition is (e.g., release to the public, release to an onsite landfill, below FFACO use restriction). This survey is the most comprehensive survey performed and has the most stringent requirements built into it for the type, quantity, and quality of data acquired. This survey requires a detailed survey plan with a high level of approval that may include regulatory agencies representatives. Because the facility will be demolished and released to an onsite landfill, no regulatory approval is required per NSTec procedures. The FSS is the intent of this survey plan.

6. Verification Survey - To verify that the site is acceptable for release, the regulatory agency might bring in an independent contractor to perform this survey. It is typically a survey with limited scope that might duplicate $10 \%$ of the final status survey measurements. Based on formal discussions between NSTec (the site Maintenance and Operating (M\&O) contractor) and NNSA/NSO Environmental Management managers, no verification survey will be required. (See Appendix B) 


\section{History (Historical Site Analysis)}

CAU 117 is located north of Cane Spring Road in the southwestern portion of Area 26 of the NTS. It includes a single CAS, CAS 26-41-01, which consists of the Pluto Disassembly Facility (also known as Building 2201), a facility water tower, and a nearby wood shed. The water tower and wood shed have been demolished and removed as part of site closure. Current access to CAS 26-41-01 is limited by locked entry ways to Building 2201.

Construction of Building 2201 began in May 1959 for Project Pluto, approximately four years after the project's initiation by the Department of Defense in 1955. After completion of the building in October 1960, the project was passed to Lawrence Radiation Laboratory (LRL), who managed the Project Pluto until its cancellation in 1964. The objective of Project Pluto was to design a nuclear reactor that could propel a missile through the atmosphere at altitudes ranging from sea level to several miles and at velocities up to three times the speed of sound (SNJV, 2009). The earthbound Tory II-A reactor and its flyable counterpart, the Tory II-C, were developed for Project Pluto. The cores of these reactors incorporated several hundred thousand fuel elements consisting of a homogenous mixture of highly enriched uranium dioxide and beryllium oxide (SNJV, 2009). The propulsion system operated on the ramjet principle, in which large quantities of air were ingested, heated by the reactor, and expelled at a high temperature and pressure to provide thrust. Between 1961 and 1964, LRL conducted several tests of the Tory reactors, including four successful power runs with the Tory II-A and two power runs with the Tory II-C (SNJV, 2009).

Project Pluto was also associated with "Hot Box" tests performed in Building 2201. These tests consisted of using stacks of graphite blocks interspersed with a few oralloy (U-235) foils. Air was heated to high temperatures and circulated through the reactor to obtain initial test data. Results from these tests were used to design the Tory II-A reactor (SNJV, 2009).

Only the Tory II-A was disassembled in Building 2201 (SNJV, 2009). The Tory II-C reactor was stored in Building 2201 until 1974, when it was moved to the Reactor Maintenance, Assembly, and Disassembly (R-MAD) building for storage (Author Unknown, Date Unknown). Actual disassembly of the Tory II-C was performed at the Engine Maintenance, Assembly, and Disassembly (E-MAD) building in 1976 (SNJV, 2009).

Building 2201 was designed specifically to perform remote adjustments, component replacement, and complete disassembly of the Tory II reactor systems. The Main Disassembly Bay (Room 102) housed the Tory II test vehicle when activities dictated that remote handling be used. Disassembly operations were viewed through 4-foot (ft)-thick leaded-glass observation windows immersed in oil (SNJV, 2009). During disassembly, the reactor core was removed from the railcar (used to transport the reactor to the test pad) with remotely operated manipulators. The heavily shielded postmortem hot cells adjacent to the disassembly bay were used to monitor control rod actuators during Project Pluto. Vaults within each cell were used to store "fuel elements and classified core parts" (SNJV, 2009). The Cold Assembly Bay (Room 101) was used for storage and assembly of modular components for the reactor test vehicle (SNJV, 2009). A maintenance service pit and battery charger for the locomotive were also located in Room 101 (SNJV, 2009). 
The disassembly bay was supported by a maintenance shop, darkroom, offices, and equipment storage rooms. All controls for Building 2201 operation were located in Room 105 (SNJV, 2009). The Warm \& Cold Storage Room (109) was used for repair and maintenance of equipment contaminated with low-activity radiological contaminants and was also intended for low-activity glove-box work (SNJV, 2009). Both the Shower/Change Room (113) and Rad Safety Room (114) were designed as change rooms and check stations for personnel needing access to the hot cell and assembly areas (SNJV, 2009). Before it was converted into a restroom, Room 115 served as a darkroom for quickly developing photograph negatives (SNJV, 2009). Room 116 was originally used to store the many spare parts required for the facility. A small electronics maintenance area was later set up in Room 116.

During operation, Rooms 105 and 108 were air conditioned and maintained at a positive pressure so that air flowed into the Main Disassembly Bay (Room 102) and the hot cells (Rooms 104, 106, and 107) when equipment or services were passed through openings at each operating station (SNJV, 2009). These openings were plugged with lead plates or bagged shot when not in use (SNJV, 2009). The ventilation system in Room 102 was exhausted at the west end of the room through roughing and absolute filters before being vented to the atmosphere via the main exhaust stack in Room 103 (SNJV, 2009). In 1998, a portable air-conditioning system was installed by an unidentified "user." This user set up a portable system outside of the building with ducts running through pre-existing penetrations in the external wall of the building that otherwise would have remained closed (SNJV, 2009). There is no documentation that additional radioactive materials were introduced during this time at this location.

The drainage system originating in the disassembly bay and postmortem cell area was designed to collect rinsate from gross decontamination efforts ending in the external septic system. Information from interviews with former personnel suggests that the septic drainage system was disconnected in 1964 (SNJV, 2009).

Following the cancellation of Project Pluto, Building 2201 was used for the Fuel Repackaging Operations Project conducted between 1971 and 1972 (SNJV, 2009). During this period, fuel elements from the Tory II reactors were removed from their original containers and placed in 6liter containers that were then sealed, cleaned, and removed from the hot cells (rooms 104, 106, and 107) of Building 2201. The containers were temporarily stored in the machine shop area of Building 2201 until they were taken to the decontamination pad in Area 6 for storage or potential future use (SNJV, 2009). The packaged fuel elements were eventually shipped to the Idaho National Engineering Laboratory (SNJV, 2009). 
Figure 1. Location of the Nevada Test Site

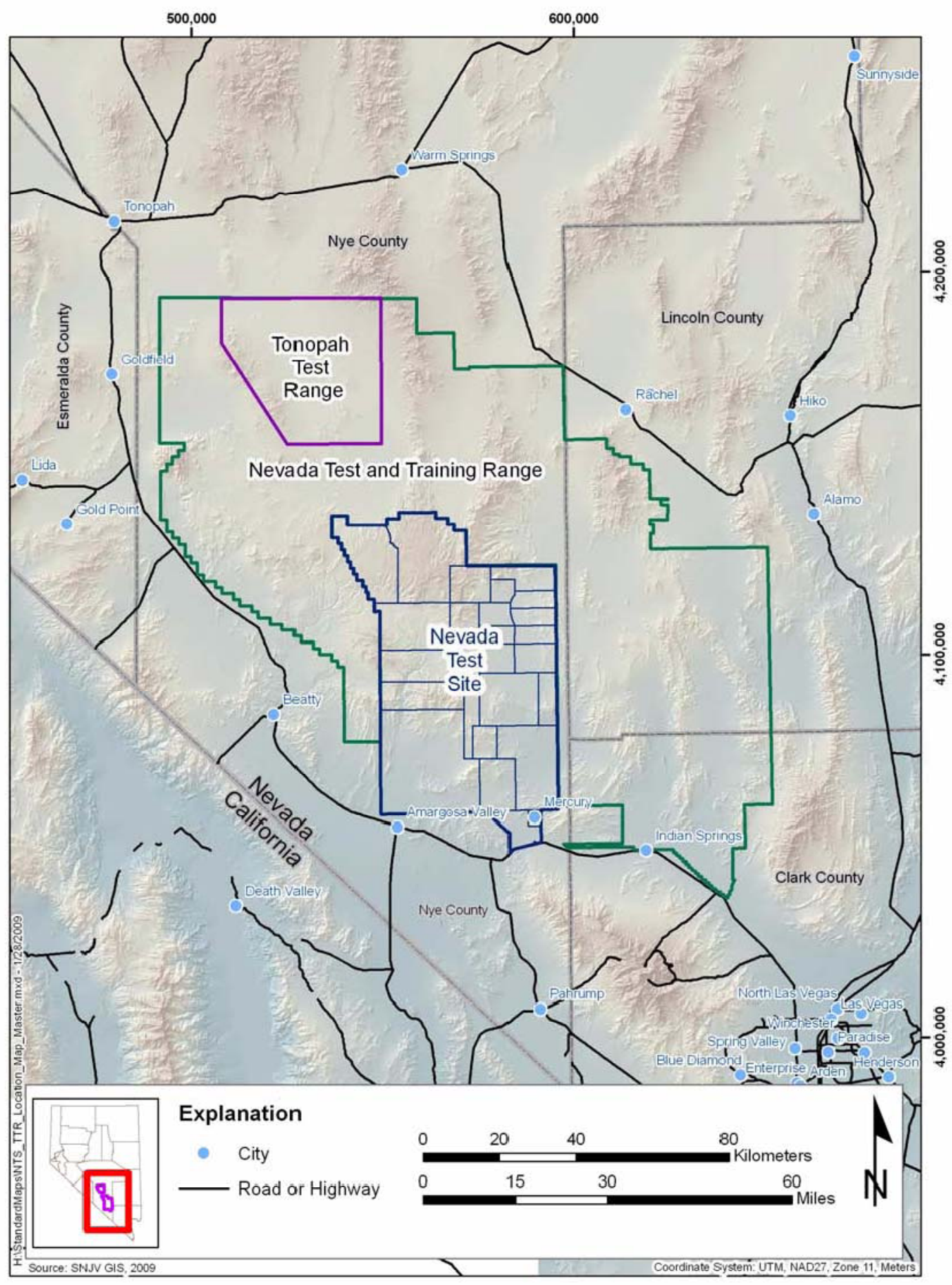


Figure 2. Location of the Pluto Facility

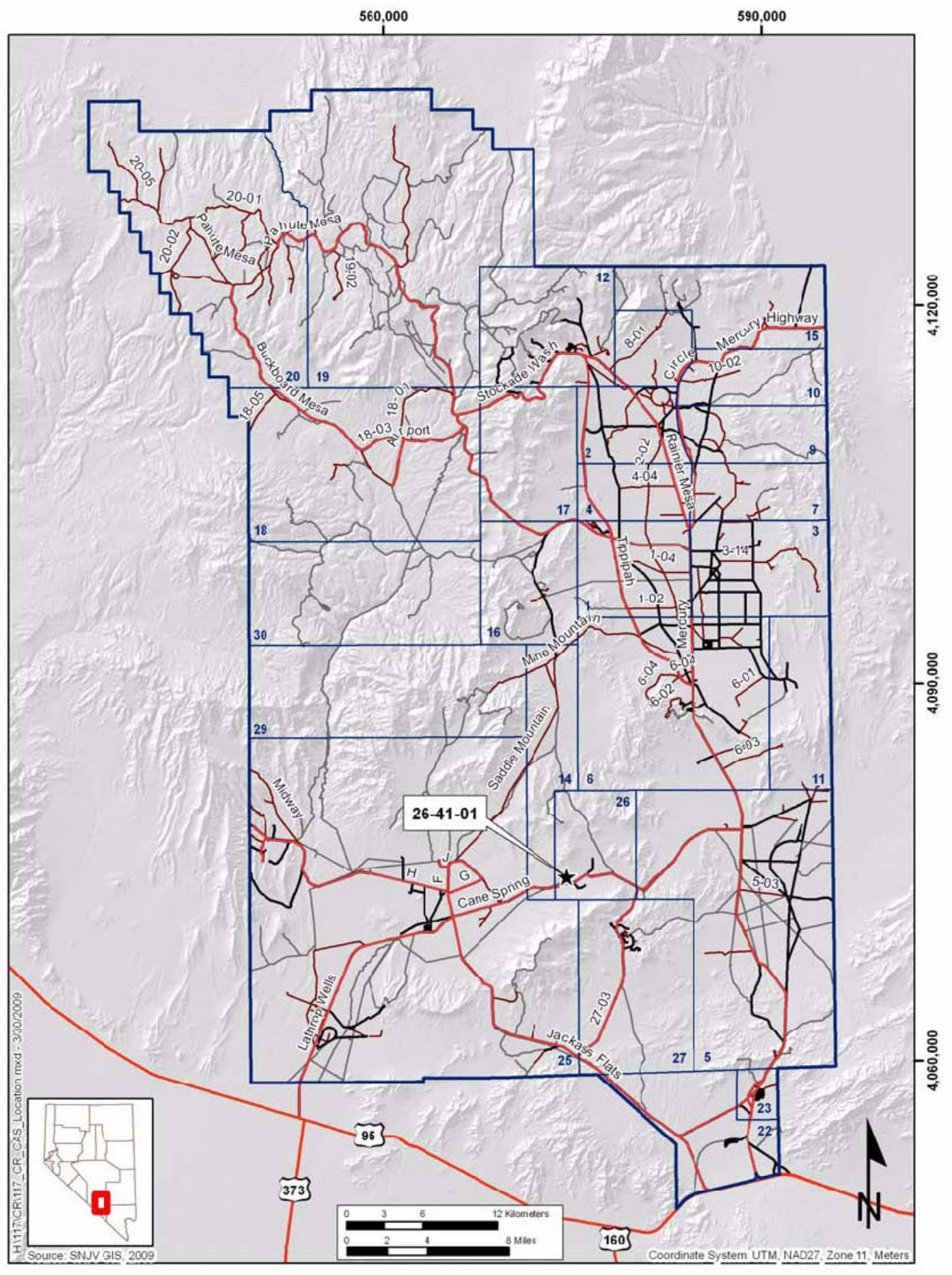


Starting in 1972, Building 2201 was used for a series of classified experiments following the fuel repackaging operations (SNJV, 2009). Although the nature of these experiments is classified, it is suspected that additional radioactive materials (transuranics) were introduced into the Hot Cells at this time. In the late 1970s and early 1980s, the Room 101 and Room 102 High Bays were modified to house the Hydrogen Content Test Facility (HCTF). The primary purpose of the HCTF was to simulate large dry boreholes for calibration of core logging instrumentation. The HCTF equipment consisted of a series of aluminum cells, each containing a different combination of water content and density. The cells contain sand, aluminum oxide, glass marbles, and varying water moisture content. The HCTF equipment will be dismantled and dispositioned during the demolition of Building 2201.

As of 1986, Sandia National Laboratories (SNL) was using portions of Building 2201 to conduct weapons-related nondestructive testing of fast-acting closure systems (SNJV, 2009). Since 1996, SNL has performed activities in Building 2201 associated with non-nuclear rocket launching and other classified projects. Due to their sensitive nature, specific information on experiments conducted by SNL inside Building 2201 is not readily available (SNJV, 2009). In 1998, Building 2201 was used for additional classified activities (SNJV, 2009).

\section{Radiological Scoping Surveys}

Various radiological surveys and decontamination activities took place in Building 2201 from 1971 to 1999 . In 2008, radiological surveys were performed at various locations within the CAS. These radiological surveys were performed to identify the presence, the nature, and the extent of the remaining radiological contaminants. Various radiological walkover surveys were conducted at CAS 26-41-01. The walkover surveys included the Main Disassembly Bay (Room 102), the hot cells (rooms 104, 106, and 107), and an approximate 1,000-square-meter (m2) area within the fenced area surrounding the exterior of Building 2201. Radiological walkover surveys were performed using an NE Technology Electra fitted with a DP6BD dualalpha and beta/gamma radiation probe.

In order to characterize the ventilation system, surveys were performed for fixed and removable radiological contamination. The High Efficiency Particulate Air (HEPA) pre-filters and 2-by-2-by-1-ft box-type HEPA filters were sampled as Potential Source Material (PSM) and removed from the Building 2201 ventilation system. A thorough survey of the accessible areas of the HEPA ventilation system revealed that all HEPA banks/plenums in Rooms 102, $103,104,106$, and 107 showed varying levels of removable and fixed alpha contamination. As a result of the survey, the contamination on the frames was fixed in place. The 2 nd stage filter banks in Room 103 and the basement are enclosed behind a set of double doors. No contamination was noted in any part of the system downstream of the second stage filter banks. This includes the main building exhaust stack. The current plan is to grout in place the remaining below grade internal ventilation system passageways. Filter racks and frames that were above the surface of the remaining concrete slab were removed and disposed of. 


\section{Characterization Survey (Phase I)}

The survey plan for this phase of characterization can be found as Radiological Operations Survey Plan 09-022, "Pluto Disassembly Facility-Building 2201 Radiological Characterization," dated July 22, 2009.

During this phase of characterization, the building was divided into "higher risk" and "lower risk" areas based off the known operational history in each area/room. The "higher risk" areas had known or potential contamination from prior scoping surveys and the HSA. "Higher risk" floors were gridded off with $1 \times 1$ meter grids, while the walls had grids that were $3 \times 3$ meters. The higher risk rooms $(102,104,106$, and 107) were surveyed using the following guidelines:

- Direct surveys - $100 \%$ of accessible surfaces on all walls, floors, ceilings and equipment were scanned with an NE Electra and DP6 Probe (or equivalent) followed with a direct static measurement at the highest direct scanned reading in each grid location, or "survey unit" documented for both alpha and beta.

- Removable surveys - At each location where direct readings were greater $100 \mathrm{dpm} / 100$ $\mathrm{cm}^{2}$ alpha or $5,000 \mathrm{dpm} / 100 \mathrm{~cm}^{2}$ beta, a swipe was taken and documented. Otherwise, swipes were not required.

All other rooms, equipment, and systems not listed as "higher risk" were considered "lower risk", and surveyed under the following guidelines:

Direct Surveys -

- Rooms - RCTs estimated $10 \mathrm{~m}^{2}$ sections of rooms and took one direct reading per section. The reading was documented for both alpha and beta results.

- Building Exterior - $10 \mathrm{~m}^{2}$ sections of the building exterior surfaces (walls and roof) were estimated and RCTs took one direct reading per section. Both alpha and beta results were documented.

- Stack and Ventilation System - RCTs opened any and all readily accessible portions of the ventilation system and performed direct surveys of these areas to the greatest extent possible. The building's stack had three access ports at the bottom of the stack, halfway from the top and at the top. Direct survey readings were taken at each of these access ports. Both alpha and beta results were documented. Survey results showed no contamination above Table 4-2 of the DOE/NV/25946--801 Revison 1, "Nevada Test Site Radiological Control Manual," and no further survey points were obtained. The intake openings to the ventilation system in rooms 102, 104, 106, and 107 were considered "Higher Risk," and therefore the survey requirements of that section were applicable.

- Underground Storage Vaults - Due to their current configuration and inaccessibility, these are not applicable to this survey plan.

- Equipment - RCTs performed a direct scan of $100 \%$ accessible surfaces on remaining equipment. RCTs were instructed to pay particular attention to those areas where staining and discoloration have occurred or areas where contamination would likely accumulate.

Note: These were the minimum required surveys. The RCT was encouraged to take more if an area looked suspicious or if direct readings indicated a potential for contamination exists. 
Removable surveys -

- At each location where direct readings were greater $100 \mathrm{dpm} / 100 \mathrm{~cm}^{2}$ alpha or 5,000 $\mathrm{dpm} / 100 \mathrm{~cm}^{2}$ beta, a swipe was taken and documented. Otherwise, swipes were not required.

Hot spots and the associated grid area were marked by RCTs for easy identification for subsequent remediation. These hot spots were later characterized by ISOCS and radionuclides were identified. The radionuclides that were identified were Cs-137, Am-241, Pu-239, U-235, and Th-231.

\section{Characterization Survey (Phase II)}

The survey plan for this phase of characterization is Radiological Operations Survey Plan 09031, "Pluto Disassembly Facility-Building 2201 Radiological Characterization (Phase II) and Post Remediation Survey Requirements," dated November 12, 2009.

During the initial phase of radiological characterization surveys it was noted that some areas suspected to be radiologically impacted were physically inaccessible to the RCTs to perform surveys. During the second phase of characterization these areas were made accessible for RCTs. Again, they were grouped into "Higher risk" and "Lower Risk" categories and surveyed as follows:

Higher Risk - As part of the second phase of Pluto's radiological characterization, the following areas in Building 2201 were considered higher risk:

- Room 104 (Under the false floor) - Hot and Warm Cell

- Room 106 (Under the false floor) - Kilo-Curie Hot Cell

- Room 107 (Under the false floor) - Hot Storage and Packaging Room

- Each of the four underground vaults (rooms 104, 106, and 107)

- Each of the four second stage filter plenums (Room 103 and facility basement)

Each of these areas were surveyed using the following guidelines:

Direct surveys - $100 \%$ of accessible surfaces were scanned with an NE Electra and DP6 Probe (or equivalent) followed with a direct static measurement at the highest direct scanned reading in each grid location, or "survey unit," and documented for both alpha and beta measurements.

Removable surveys - At each location where direct readings were greater than $100 \mathrm{dpm} / 100$ $\mathrm{cm}^{2}$ alpha or $5,000 \mathrm{dpm} / 100 \mathrm{~cm}^{2}$ beta, a swipe was taken and documented. All swipes were counted on a performance tested Tennelec, Protean, 3030 or 2929 scalar. Otherwise, swipes were not required. 
Lower Risk - As part of the second phase of Pluto's radiological characterization, the following areas were considered lower risk, and surveyed under the following guidelines:

Direct Surveys - Room 101 West mezzanine wood ceiling (two sections) - 100\% of newly exposed accessible surfaces were scanned with an NE Electra and DP6 Probe (or equivalent) followed with a direct static measurement at the highest direct scanned reading in both ceiling sections and documented for both alpha and beta.

In addition to the above radiological surveys, paint samples from the walls of rooms 102, 104, 106, and 107 were collected and analyzed at an offsite laboratory in April 2010. These samples were analyzed for isotopic uranium, isotopic plutonium, gamma emitters, and for Strontium-90. The results showed that all samples had activities that were below detectable limits of gamma spectroscopy lab instrumentation. Strontium-90 concentration was not above lab instrument detection limits. The alpha spectroscopy instruments detected slightly elevated concentrations above the detection limits for uranium 233,234, 235, and 238 and for plutonium 239 and 240 . Other paint samples were collected in previous years and yielded similar results.

\section{Remedial Action Surveys}

Upon completion of the both Phase I and Phase II characterization surveys, multiple isolated areas were identified that would exceed the waste acceptance criteria for the construction waste onsite landfill. These areas were physically marked and all were eventually decontaminated. The identified radiologically impacted and remediated areas were:

Room 102 (Survey \#09-ER-A26-029)

- North Wall - $796 \mathrm{dpm} / 100 \mathrm{~cm}^{2}$ alpha

- North Wall (Electrical panel) $-1,110 \mathrm{dpm} / 100 \mathrm{~cm}^{2}$ alpha

- South Wall $-1,200 \mathrm{dpm} / 100 \mathrm{~cm}^{2}$ beta

- Floor (Multiple locations) $-5,210$ to $19,500 \mathrm{dpm} / 100 \mathrm{~cm}^{2}$ beta

Room 104 (Survey \#09-ER-A26-032)

- South Wall (Entire upper ledge) - 300 to $400 \mathrm{dpm} / 100 \mathrm{~cm}^{2}$ alpha

- South Wall (Inside penetration) - $354 \mathrm{dpm} / 100 \mathrm{~cm}^{2}$ alpha

- East Wall (Bottom, left side of filter rack) $-200 \mathrm{dpm} / 100 \mathrm{~cm}^{2}$ alpha

- East Wall (Bottom of window ledge) $-12,000 \mathrm{dpm} / 100 \mathrm{~cm}^{2}$ beta

Room 106 (Survey \#09-ER-A26-033)

- South Wall (Bottom, inside penetration) $-226 \mathrm{dpm} / 100 \mathrm{~cm}^{2}$ alpha

- South Wall (Inside plenum) - 210 to $710 \mathrm{dpm} / 100 \mathrm{~cm}^{2}$ alpha

- South Wall (Between grids A-3 and A-4) - 19,500 dpm/100 $\mathrm{cm}^{2}$ beta

- East Wall (3' high) - 2,640 dpm/100 $\mathrm{cm}^{2}$ beta

- East Wall ( $7^{\prime}$ high) $-1,644 \mathrm{dpm} / 100 \mathrm{~cm}^{2}$ beta

Room 107 (Survey \#09-ER-A26-034)

- Floor (Multiple locations) - 158 to $519 \mathrm{dpm} / 100 \mathrm{~cm}^{2}$ alpha 
Typically, the contaminated areas identified were successfully decontaminated by removing the surface layer of concrete, approximately 1 to 3 inches in depth. This provided reasonable proof that there was no volumetrically contaminated concrete in the impacted areas.

The painted wall and ceiling surfaces of rooms 102, 104, 106, and 107 were stripped during asbestos abatement activities. This eliminated the possibility of paint shielding contamination measurements during the final status survey.

Samples of the wood slats obtained from Room 101 were surveyed and analyzed in a high purity germanium (HPGe) detector to ensure no activation or fission products were present. The wood mezzanine in room 101 will be disposed of during the final demolition of the building.

In addition to the removal of contaminated surfaces, the first stage HEPA filter plenum racks in rooms 102, 104, 106, and 107 were removed and disposed of as low level waste. Upon removal of the racks, the RCTs performed surveys on the newly exposed surfaces behind the plenum racks. No contamination was found.

\section{Release Criteria}

The release criteria for Pluto were established by the NNSA/NSO. The release criteria chosen by the regulator for Building 2201 are the allowable total residual surface contamination values established by DOE O 5400.5. These pre-approved authorized limits for surface contamination values and corresponding assumptions were established by DOE O 5400.5 and can be found in Table 2 of this survey plan.

The release criteria are compatible with the acceptance criteria of the U10C landfill permit. The U10C landfill is an on-site landfill (i.e., the landfill resides within the NTS boundaries). The $\mathrm{U} 10 \mathrm{C}$ landfill is the proposed location to dispose of the building rubble after demolition.

The building will be considered "clean" if every survey unit has an average contamination value below the authorized limits. The surface contamination value of the most limiting nuclide within a mixture of nuclides will be used as the release limit. The isotopes that were identified by insitu measurements during the characterization phase were Cs-137, Am-241, Pu-239, U-235, and Th-231. Therefore, the average gross alpha contamination in the survey unit must be below the transuranic surface contamination value of $100 \mathrm{dpm} / 100 \mathrm{~cm}^{2}$. The average gross beta/gamma contamination in the survey unit must be below the Cs-137 surface contamination value of $5000 \mathrm{dpm} / 100 \mathrm{~cm}^{2}$.

Per regulator guidance, requesting dose-based alternate authorized limits (i.e. limits that result in less than 25 mrem/year with ALARA considerations) will be required if any of the following conditions are observed:

- Unable to demonstrate that the DOE O 5400.5 surface contamination limits are met

- Potential for volumetric contamination

- Contamination in inaccessible areas.

To date, all recent porous materials analyzed, such as concrete and wood, contained in Building 2201 have shown no potential for volumetric contamination. Samples have been taken in concrete, paint, and wood and analyzed at both offsite laboratories and by onsite gamma spectroscopy. Furthermore, during remediation activities (decontamination) of 
impacted areas discovered during characterization surveys, remediation of each of these areas was easily accomplished by simply removing the initial layer of surface material. Once this top layer of material was removed, no further contamination was detected. This provided further evidence that volumetric contamination of porous materials at Pluto does not exist.

If the building cannot be considered clean based on the surface activity results, a dose-based authorized limit may be generated using the DOE approved dose/risk modeling code RESRAD. RESRAD is the only code designated in DOE O 5400.5 for the evaluation of radioactively contaminated sites. RESRAD uses a pathway analysis method in which the relationship between radionuclide concentrations in soil and the dose to a member of the critical population group is expressed as a pathway sum, which is the sum of products of "pathway factor" products. Nine environmental pathways are considered: direct exposure, inhalation of particulates and radon, and ingestion of plant foods, meat, milk, aquatic foods, water, and soil. (Argonne National Laboratory, 2001)

The RESRAD-Build code was used to perform a dose based calculation based off the release limits selected by the regulator. All defaults in the code were selected except the square footage of the contaminated floor was increased to $100 \mathrm{~m}^{2}$ (so the floor area was increased and source area was increased) and the removable fraction of contamination was lowered to $10 \%$ versus $50 \%$ as the default value. The removable fraction of contamination was lowered to reflect the characterization survey results of mainly fixed contamination in the survey unit. Two calculations were performed. The first calculation assumed a contaminated floor with Cs137 as the contaminant uniformly distributed over the entire floor surface $\left(100 \mathrm{~m}^{2}\right)$ with a surface activity of $5000 \mathrm{dpm} / 100 \mathrm{~cm}^{2}$. The second calculation assumed a contaminated floor with $\mathrm{Pu}-239$ as the contaminant uniformly distributed over the entire floor surface $\left(100 \mathrm{~m}^{2}\right)$ with a surface activity of $100 \mathrm{dpm} / 100 \mathrm{~cm}^{2}$. The results are as follows:

Table 1. RESRAD Results

\begin{tabular}{|c|c|c|}
\hline & \multicolumn{2}{|c|}{$\begin{array}{c}\text { Maximum Dose in a } \\
\text { Single Year }\end{array}$} \\
\hline Cs-137 & 2.38 & mrem \\
\hline Pu-239 & 3.69 & mrem \\
\hline Total & 6.07 & mrem \\
\hline
\end{tabular}

In terms of preliminary dose estimates, based on DOE O 5400.5 surface contamination values and using site specific parameters, it is reasonable to state that the dose to a maximally exposed individual in the survey unit will meet the dose constraint of less than $25 \mathrm{mrem} / \mathrm{year}$ with the ALARA goal of a few millirem per year. It is important to note that from characterization surveys, only spotty fixed contamination has been found. No large areas of general contamination were discovered.

\section{Instructions}

All surveys shall be performed by qualified personnel. Personnel must use their experience, knowledge of the area in which they are working, and workplace indicators to ensure adequate surveys are being performed, in addition to the information provided in this document. 
1) Determining the Derived Concentration Guideline Limits (DCGL)

a) As mentioned previously, the authorized release limits for surface contamination established by DOE O 5400.5 are the DCGLs for this project. It should be noted that these release criteria are generally more restrictive than a dose-based DCGL computed from RESRAD.

2) Determining the Gross $\mathrm{DCGL}_{w}$ for Multiple Nuclides when Performing Gross Alpha and Beta Measurements (MARSSIM 4.3.4)

a) We will use the most restrictive DCGLs, for both alpha and beta emitters, from the identified radionuclides. From isotopic analysis of hot spots found in the facility during the characterization phase, the radionuclides identified were Cs-137, Am-241, Pu-239, U-235, and Th-231. The DCGLs for these radionuclides were compared and the most restrictive DCGL was selected. For the Pluto facility, we will use the DCGL of Pu-239 for gross alpha contamination and the DCGL of Cs-137 for gross beta contamination. From DOE O 5400.5, the release limit for Pu-239 is $100 \mathrm{dpm} / 100 \mathrm{~cm}^{2}$ and Cs-137 is $5,000 \mathrm{dpm} / 100 \mathrm{~cm}^{2}$ (Table 2).

Table 2. Allowable Total Residual Surface Contamination Values in $\mathrm{dpm} / 100 \mathrm{~cm}^{2}$ (from DOE O 5400.5)

\begin{tabular}{|l|l|l|l|}
\hline RADIONUCLIDE & REMOVABLE & $\begin{array}{l}\text { AVERAGE } \\
\text { (FIXED \& } \\
\text { REMOVABLE) }\end{array}$ & $\begin{array}{l}\text { MAXIMUM } \\
\text { ALLOWABLE } \\
\text { (FIXED \& } \\
\text { REMOVABLE) }\end{array}$ \\
\hline $\begin{array}{l}\text { Transuranics, }{ }^{125} \mathrm{I},{ }^{129} \mathrm{I},{ }^{226} \mathrm{Ra},{ }^{227} \mathrm{Ac}, \\
{ }^{228} \mathrm{Ra},{ }^{228} \mathrm{Th},{ }^{230} \mathrm{Th},{ }^{231} \mathrm{~Pa},\end{array}$ & 20 & 100 & 300 \\
\hline $\begin{array}{l}\mathrm{Th}-\mathrm{nat},{ }^{90} \mathrm{Sr},{ }^{226} \mathrm{I},{ }^{131} \mathrm{I},{ }^{133} \mathrm{I},{ }^{223} \mathrm{Ra},{ }^{224} \mathrm{Ra}, \\
{ }^{232} \mathrm{U},{ }^{232} \mathrm{Th},\end{array}$ & 200 & 1,000 & 3,000 \\
\hline $\begin{array}{l}\mathrm{U}-\mathrm{natural},{ }^{235} \mathrm{U},{ }^{238} \mathrm{U} \text { and associated } \\
\text { decay products, alpha emitters }\end{array}$ & $1,000 \alpha$ & $5,000 \alpha$ & $15,000 \alpha$ \\
\hline $\begin{array}{l}\beta+\gamma \text { emitters (radionuclides with } \\
\text { decay modes other than } \alpha-e m i s s i o n \\
\text { or spontaneous fission) except } 90 \mathrm{Sr} \\
\text { and others noted above. }\end{array}$ & $1,000 \beta+\gamma$ & $5,000 \beta+\gamma$ & $15,000 \beta+\gamma$ \\
\hline Tritium and Tritiated Compounds & 10,000 & N/A & N/A \\
\hline
\end{tabular}

b) Since release limits from DOE 05400.5 are being used, the assumptions used to derive the surface contamination values will also be used. These assumptions are found as notes to the table in Appendix $A$.

c) The most notable assumptions are that alpha and beta emitters are treated separately if the surface is contaminated with both types of contamination and that average contamination values can be averaged over an area of no more than $1 \mathrm{~m}^{2}$. 
3) Determine the DCGL $L_{\text {emc }}$ (NUREG-1505 Chapter 8)

a) The $\mathrm{DCGL}_{\mathrm{emc}}$ is the maximum permitted average concentration in a hot spot. It is the concentration of a specified nuclide in a specified area (area is smaller than the survey unit) that is assumed to result in 25 mrem a year (i.e. the release criterion). Hot spots containing alpha contamination will be assumed as Pu-239. Hot spots containing beta contamination will be assumed as Cs-137. The DCGL $L_{e m c}$ is calculated as follows:

$$
D C G L_{\mathrm{EMC}}=D C G L_{\mathrm{W}} \times A F
$$

Where: $A F$ is the area factor that is specific to the nuclide and the area.

b) There are no default values for AF. The area factor is calculated using the RESRAD family of codes. To obtain the AF, divide the dose predicted with RESRAD for the survey unit (or default unit) by the dose predicted for the area of the hot spot.

c) A worst case hot spot must be assumed for the planning phase. This area is the area that is bounded by four measurement/sampling points. During the data assessment, actual hot spot areas are used to determine the DCGL $\mathrm{emc}_{\text {. }}$. Derived area factors, using RESRAD, are listed in Table 3.

Table 3. Area Factors

\begin{tabular}{||c|c|c|c||}
\hline \multicolumn{4}{||c||}{$100 \mathrm{~m}^{2}$ Room with Defaults } \\
\hline \multicolumn{4}{||}{ Pu-239 $\begin{array}{c}100 \mathrm{dpm} / 100 \mathrm{~cm}^{2} \\
\text { Contamination }\end{array}$} \\
\hline $\mathbf{m}^{\mathbf{2}}$ & mrem & Area Factor & DCGL \\
\hline 100 & 18.10 & 1.00 & 138.12 \\
\hline 50 & 9.06 & 2.00 & $2.76 \mathrm{E}+02$ \\
\hline 36 & 6.53 & 2.77 & $3.83 \mathrm{E}+02$ \\
\hline 25 & 4.53 & 4.00 & $5.52 \mathrm{E}+02$ \\
\hline 16 & 2.90 & 6.24 & $8.62 \mathrm{E}+02$ \\
\hline 9 & 1.63 & 11.10 & $1.53 \mathrm{E}+03$ \\
\hline 3 & 0.54 & 33.27 & $4.60 \mathrm{E}+03$ \\
\hline 1 & 0.18 & 100.00 & $1.38 \mathrm{E}+04$ \\
\hline 0.1 & 0.02 & 1000.00 & $1.38 \mathrm{E}+05$ \\
\hline \hline
\end{tabular}

4) Classify Site According to Contamination Potential (MARSSIM 4.4)

a) Class 1 impacted areas either contain or have a potential for individual measurements above the DCGL. Remediated areas are also considered Class 1 areas. The following rooms (excluding ceilings) have been determined by characterization data to be Class 1 impacted areas:

i) Room 102 - Main Disassembly

ii) Room 104 - Hot and Warm Cell

iii) Room 106 - Kilo Curie Hot Cell

iv) Room 107 - Hot Storage and Packaging Room 
b) Class 2 impacted areas

i) Room 102 Ceiling - Main Disassembly

ii) Room 104 Ceiling - Hot and Warm Cell

iii) Room 106 Ceiling - Kilo Curie Hot Cell

iv) Room 107 Ceiling - Hot Storage and Packaging Room

c) Class 3 impacted areas

i) All other areas inside the facility that are not Class 1 or Class 2 areas

d) Non-impacted areas

i) Exterior surfaces of the facility

5) Establish Survey Units (MARSSIM 4.6)

a) Class 1 impacted areas will have a maximum survey unit area of $100 \mathrm{~m}^{2}$

b) Class 2 impacted areas will have a maximum survey unit area of $1000 \mathrm{~m}^{2}$

c) Class 3 impacted areas do not have a maximum survey unit area

d) Non-Impacted areas do not require surveys or survey units

6) Determine Whether Scenario A or B will be Used

a) Scenario $A$ is the most commonly employed approach in final status surveys and the only approach described in MARSSIM. The object is to demonstrate that the average/median level of residual radioactivity in a survey unit is less than the DCGL. We will be utilizing Scenario $A$ for this final status survey.

7) Determine Which Statistical Test will be Used to Assess the Data

a) Since the analysis is not nuclide specific (gross beta and gross alpha measurements on surfaces), the Sign Test will be utilized.

b) We will subtract average background from survey unit gross measurements and use the Sign Test on net values due to the gross alpha background is a significant fraction of the DCGL.

8) Determine if the Unity Rule will be Used in the Statistical Tests (NUREG 1505 Chapter 11)

a) The Unity Rule will be employed. The unity rule is used when two or more radionuclides are analyzed in each sample (alpha and beta measurements are performed at each location). The unity rule can be thought as a sum of the fractions where "concentrations" of multiple radionuclides are expressed as fractions of the DCGLs. When a DCGL for multiple nuclides is used in these tests, it is assigned a value of 1 .

Combined concentration of multiple nuclides $=\frac{\text { Conc. nuclide } 1}{D C G L 1}+\frac{\text { Conc. nuclide } 2}{D C G L 2}+$ etc. 
b) When the unity rule is employed, the DCGL becomes one. The sum of the surface activities of the survey unit compared to the most limiting DCGL for that emitter, whether alpha or beta, must be less than unity to prove that the survey unit passes. This is mathematically described below:

$$
1>\frac{\text { Gross alpha activity }}{100 \frac{\mathrm{dpm}}{100 \mathrm{~cm}^{2}}}+\frac{\text { Gross beta activity }}{5000 \frac{\mathrm{dpm}}{100 \mathrm{~cm}^{2}}}
$$

9) Select Type of Detection Equipment (MARSSIM Chapter 8)

a) Detection equipment for performing scans and static measurements on structural surfaces must be able to detect alpha and beta surface activity. The contamination detector that will be used is the NE Electra which utilizes a dual phosphor scintillation probe.

i) The standard NE Electra uses a Thermo Scientific DP6B detector probe with a physical probe area of $100 \mathrm{~cm}^{2}$. After estimating the time needed to achieve the minimum detectable concentrations Minimum Detectable Concentrations (MDCs) using this probe, a larger physical probe area was desired (See Step 10).

ii) The Thermo Scientific DP8B detector probe for the NE Electra has a physical probe area of $600 \mathrm{~cm}^{2}$. This probe allows for achieving the desired MDC for transuranics with a reasonable sample time.

iii) The NE Electra with either type of probe may be used during the FSS.

b) The NE Electras with their associated DP8B probes were calibrated by Pacific Northwest National Laboratories (PNNL) in Richland, Washington.

c) The NE Electra is operated in accordance with Organizational Instruction Ol-0441.211, "Direct and Indirect Surveys."

d) Specific instructions for samples and scans with the DP8B probes will be discussed in the pre-field briefing at the job site by the Environmental Restoration (ER) Health Physics Supervisor (HPS) and Radiological Engineer (RE).

10) Determine Measurement Protocols (MARSSIM 5.5.3)

a) All Class 1 survey units will have $100 \%$ scan coverage. Static measurements will be collected in systematic (triangular) pattern according to the survey maps (Appendix E).

b) All Class 2 survey units will have scan coverage of $10 \%$ of the survey unit. These scans should also bias towards suspect areas such as cracks, discoloration, and stains. Static measurements will be collected in systematic (triangular) pattern according to the survey maps (Appendix E).

c) All Class 3 survey units will have judgmental scan coverage. Those scans should focus on areas immediately adjacent or in close proximity to Class 1 or 2 entry points. Static measurements will be distributed randomly.

d) For non-impacted areas, no scan coverage or static measurements are required.

11) Determine the Measurement and Scan MDC (MARSSIM 6.7)

a) Measurement/Sample MDC 
One of the few absolute requirements in MARSSIM is that the measurement MDC be below the $D_{C G L_{w}}$. Nevertheless, MARSSIM recommends an MDC that is $10-50 \%$ of the $\mathrm{DCGL}_{\mathrm{w}}$.

$$
\text { Measurement } M D C\left(\frac{d p m}{100 \mathrm{~cm}^{2}}\right)=\frac{3+4.65 \sqrt{C_{b}}}{t E_{i} E_{s} \frac{A}{100}}
$$

Where: $C_{b}$ is background count (counts), $t$ is the count time (minutes: this equation assumes that the sample and background count times are equal), $E_{i}$ is the instrument $(2 \pi)$ efficiency, $E_{s}$ is the surface efficiency (fraction of the decays that a detectable particle leaves the surface: default is 0.5 for betas with maximum energies above 400 $\mathrm{keV}$ and 0.25 for alpha and betas with maximum energies between 150 and $400 \mathrm{keV}$ ), and $A$ is the physical probe area $\left(\mathrm{cm}^{2}\right)$.

For the calculation of an MDC with different background and measurement count times the following equation may be used:

$$
\text { Measurement } M D C\left(\frac{d p m}{100 \mathrm{~cm}^{2}}\right)=\frac{3+3.29 \sqrt{R_{b} T_{s+b}\left(1+\frac{T_{s+b}}{T_{b}}\right)}}{E_{i} E_{s} \frac{A}{100} T_{s+b}}
$$

Where: $R_{b}$ is background count rate (counts per minute), $T_{s+b}$ is the sample count time (minutes), $T_{b}$ is the background count time, $E_{i}$ is the instrument (2m) efficiency, $E_{s}$ is the surface efficiency (fraction of the decays that a detectable particle leaves the surface: default is 0.5 for betas with maximum energies above $400 \mathrm{keV}$ and 0.25 for alpha and betas with maximum energies between 150 and $400 \mathrm{keV}$ ), and $A$ is the physical probe area $\left(\mathrm{cm}^{2}\right)$ (Abelquist, 2001). This equation is more versatile and will be used for the MDC calculations in this FSS.

To illustrate the basic calculation, a sampling of NE Electra parameters was taken from characterization surveys and average values for alpha and beta background and efficiencies were derived. The average values for the NE Electra with a DP6B and DP8B probe are in Table 4 below.

Table 4. Average NE Electra Parameters

\begin{tabular}{|c|c|c|c|c|}
\hline $\begin{array}{c}\text { NE Electra } \\
\text { Probe }\end{array}$ & $\begin{array}{c}\text { alpha bkg } \\
(\mathrm{dpm})\end{array}$ & $\begin{array}{c}\text { beta bkg } \\
(\mathrm{dpm})\end{array}$ & $\begin{array}{c}\text { Alpha } \\
\text { efficiency \% }\end{array}$ & $\begin{array}{c}\text { Beta } \\
\text { efficiency \% }\end{array}$ \\
\hline DP6B & 19.00 & 1675.17 & 14.72 & 24.30 \\
\hline DP8B & 22.10 & 7835.34 & 13.80 & 19.98 \\
\hline
\end{tabular}

Using the average parameter values in Table 4 into the MDC calculation for different sample and background count times, the MDC was determined for different probe areas. The results are in the following four tables. Table 5 and Table 6 are static measurement MDCs for alpha detection. Table 7 and Table 8 are static measurement MDCs for beta detection. 
Table 5. Alpha MDC of $100 \mathrm{~cm}^{2}$ Probe

\begin{tabular}{|l|l|l|}
\hline $\begin{array}{l}\text { Static } \mathbf{M D C} \\
\boldsymbol{d p m} / \mathbf{1 0 0} \mathbf{c m}^{\mathbf{2}}\end{array}$ & 2.7968 & Rb: background count rate (cpm) \\
\cline { 2 - 3 } $\mathbf{4 8 . 6 0}$ & 15 & Ts+b: sample count time in minutes \\
\hline & 60 & Tb: background count time in minutes \\
\hline & 0.1472 & $\mathrm{E}_{\mathrm{i}}$ : instrument 2 pi eff \\
\hline & 0.25 & $\mathrm{E}_{\mathrm{s}}$ : surface efficiency \\
\hline & 100 & A: probe area in $100 \mathrm{~cm}^{2}$ \\
\hline
\end{tabular}

Table 6. Alpha MDC of $600 \mathrm{~cm}^{2}$ Probe

\begin{tabular}{|l|l|l|}
\hline $\begin{array}{l}\text { Static } \mathbf{M D C} \\
\boldsymbol{d p m} / \mathbf{1 0 0} \mathbf{c m}^{\mathbf{2}}\end{array}$ & 3.05 & Rb: background count rate $(\mathrm{cpm})$ \\
\cline { 2 - 3 } & 1 & $\begin{array}{l}\text { Ts+b: sample count time in } \\
\text { minutes }\end{array}$ \\
\hline $\mathbf{4 3 . 6 0}$ & 10 & $\begin{array}{l}\text { Tb: background count time in } \\
\text { minutes }\end{array}$ \\
\hline & 0.138 & $\mathrm{E}_{\mathrm{i}}$ : instrument 2 pi eff \\
\hline & 0.25 & $\mathrm{E}_{\mathrm{s}}$ : surface efficiency \\
\hline & 600 & A: probe area in $600 \mathrm{~cm}^{2}$ \\
\hline
\end{tabular}

Table 7. Beta MDC of $100 \mathrm{~cm}^{2}$ Probe

\begin{tabular}{|l|l|l|}
\hline $\begin{array}{l}\text { Static } \mathbf{M D C} \\
\boldsymbol{d p m} / \mathbf{1 0 0} \mathbf{c m}^{\mathbf{2}}\end{array}$ & 407.025 & Rb: background count rate (cpm) \\
\hline $\mathbf{5 9 7 . 6 8}$ & 1 & Ts+b: sample count time in minutes \\
\hline & 10 & Tb: background count time in minutes \\
\hline & 0.243 & $\mathrm{E}_{\mathrm{i}}:$ instrument 2 pi eff \\
\hline & 0.5 & $\mathrm{E}_{\mathrm{s}}$ : surface efficiency \\
\hline & 100 & A: probe area in $100 \mathrm{~cm}^{2}$ \\
\hline
\end{tabular}

Table 8. Beta MDC of $600 \mathrm{~cm}^{2}$ Probe

\begin{tabular}{|l|l|l|}
\hline \multirow{2}{*}{$\begin{array}{l}\text { Static } \mathbf{M D C} \\
\boldsymbol{d p m} / \mathbf{1 0 0} \mathbf{c m}^{\mathbf{2}}\end{array}$} & 1565.5 & Rb: background count rate $(\mathrm{cpm})$ \\
\hline & 1 & Ts+b: sample count time in minutes \\
\hline $\mathbf{2 3 2 . 7 8}$ & 10 & $\begin{array}{l}\text { Tb: background count time in } \\
\text { minutes }\end{array}$ \\
\hline & 0.1998 & $\mathrm{E}_{\mathrm{i}}$ : instrument 2 pi eff \\
\hline & 0.5 & $\mathrm{E}_{\mathrm{s}}$ : surface efficiency \\
\hline & 600 & A: probe area in $600 \mathrm{~cm}^{2}$ \\
\hline
\end{tabular}

b) Scan MDC

A scan MDC may be calculated per the following equation (MARSSIM):

$$
\operatorname{Scan} M D C\left(\frac{d p m}{100 \mathrm{~cm}^{2}}\right)=\frac{60 d^{\prime} \sqrt{C_{b i}}}{i \sqrt{p} E i E s \frac{A}{100}}
$$


Where: $C_{b i}$ is the background count during time interval $i, i$ is the time interval (seconds) that the probe is over the hot spot (of an assumed size), $d^{\prime}$ is 2.32 if the acceptable probability of false positives is 0.25 and the acceptable probability of a correct detection is 0.95 (MARSSIM Table 6.5), $E_{i}$ is the instrument (2 pi) efficiency, $E_{s}$ is the surface efficiency (fraction of the decays that a detectable particle leaves the surface: default is 0.5 for betas with maximum energies above $400 \mathrm{keV}$ and 0.25 for alpha and betas with maximum energies between 150 and $400 \mathrm{keV})$, and $A$ is the physical probe area $\left(\mathrm{cm}^{2}\right)$.

Using the average parameter values in Table into the scan MDC calculation, the scan MDC was determined for different probe areas. The assumed scan rate for the $600 \mathrm{~cm}^{2}$ probe is $30 \mathrm{~cm} / \mathrm{s}$ and for the $100 \mathrm{~cm}^{2}$ probe the scan rate is $5 \mathrm{~cm} / \mathrm{s}$. The assumed hot spot for planning purposes is $5 \mathrm{~m}^{2}$, or the area bounded by four static measurement points. The scan MDCs are in the following four tables. Table 9 and Table 10 are scan MDCs for alpha detection. Table 11 and Table 12 are scan MDCs for beta detection.

Table 9. Alpha Scan MDC for $100 \mathrm{~cm}^{2}$ Probe

\begin{tabular}{|l|l|l|}
\hline $\begin{array}{l}\text { Scan } \mathbf{M D C} \\
\text { dpm/100 } \mathbf{c m}^{\mathbf{2}}\end{array}$ & 2.32 & D \\
\cline { 2 - 3 } $\mathbf{1 7 2 . 7 1}$ & 2.08 & $\mathrm{C}_{\mathrm{bi}}$ : bkg count during time interval I \\
\hline & 44.72 & i: time interval in seconds that probe is over hot spot \\
\hline & 0.5 & p: surveyor efficiency $\sim .5$ \\
\hline & 0.1472 & $\mathrm{E}_{\mathrm{i}}$ : instrument 2 pi eff \\
\hline & 0.25 & $\mathrm{E}_{\mathrm{s}}$ : surface efficiency \\
\hline & 100 & A: physical probe area in $100 \mathrm{~cm}^{2}$ \\
\hline
\end{tabular}

Table 10. Alpha Scan MDC for $600 \mathrm{~cm}^{2}$ Probe

\begin{tabular}{|l|l|l|}
\hline $\begin{array}{l}\text { Scan } \mathbf{M D C} \\
\boldsymbol{d p m} / \mathbf{1 0 0} \mathbf{c m}^{2}\end{array}$ & 2.32 & $\mathrm{~d}$ \\
\cline { 2 - 3 } & 0.38 & $\mathrm{C}_{\mathrm{bi}}$ : bkg count during time interval I \\
\hline $\mathbf{7 8 . 5 4}$ & 7.45 & i: time interval in seconds that probe is over hot spot \\
\hline & 0.5 & p: surveyor efficiency $~ .5$ \\
\hline & 0.138 & $\mathrm{E}_{\mathrm{i}}$ : instrument 2 pi eff \\
\hline & 0.25 & $\mathrm{E}_{\mathrm{s}}$ : surface efficiency \\
\hline & 600 & A: physical probe area in $600 \mathrm{~cm}^{2}$ \\
\hline
\end{tabular}

Table 11. Beta Scan MDC for $100 \mathrm{~cm}^{2}$ Probe

\begin{tabular}{|l|l|l|}
\hline $\begin{array}{l}\text { Scan } \mathbf{M D C} \\
\mathbf{d p m} / \mathbf{1 0 0} \mathbf{c m}^{2}\end{array}$ & 2.48 & $\mathrm{~d}$ \\
\cline { 2 - 3 } $\mathbf{6 7 4 . 6 0}$ & 303.40 & $\mathrm{C}_{\mathrm{bi}}$ : bkg count during time interval I \\
\hline & 44.72 & i: time interval in seconds that probe is over hot spot \\
\hline & 0.5 & p: surveyor efficiency $\sim .5$ \\
\hline & 0.243 & $\mathrm{E}_{\mathrm{i}}$ : instrument 2 pi eff \\
\hline & 0.5 & $\mathrm{E}_{\mathrm{s}}$ : surface efficiency \\
\hline & 100 & A: physical probe area in $100 \mathrm{~cm}^{2}$ \\
\hline
\end{tabular}


Table 12. Beta Scan MDC for $600 \mathrm{~cm}^{2}$ Probe

\begin{tabular}{|l|l|l|}
\hline $\begin{array}{l}\text { Scan } \mathbf{M D C} \\
\boldsymbol{d p m} / \mathbf{1 0 0} \mathbf{c m}^{2}\end{array}$ & 2.48 & $\mathrm{D}$ \\
\cline { 2 - 3 } & 50.57 & $\mathrm{C}_{\mathrm{bi}}$ : bkg count during time interval I \\
\hline $\mathbf{3 3 4 . 9 5}$ & 7.45 & i: time interval in seconds that probe is over hot spot \\
\hline & 0.5 & p: surveyor efficiency $\sim .5$ \\
\hline & 0.1998 & $\mathrm{E}_{\mathrm{i}}$ : instrument 2 pi eff \\
\hline & 0.5 & $\mathrm{E}_{\mathrm{s}}$ : surface efficiency \\
\hline & 600 & A: physical probe area in $600 \mathrm{~cm}^{2}$ \\
\hline
\end{tabular}

12) Determine the Scan and Measurement Investigation Levels (MARSSIM 5.5.2.6)

a) The investigation level is the instrument response that triggers an investigation when exceeded. MARSSIM suggests the following as investigation levels:

i) For a Class 1 survey unit the instrument response corresponding to the $D C G L_{e m c}$ for the area bounded by four measurement points.

ii) For a Class 2 survey unit the instrument response corresponding to the $D C G L_{w}$. If this is exceeded, the survey unit may have been misclassified.

iii) For a Class 3 survey unit the instrument response corresponding to some fraction of the $D C G L_{w}$. If the scan MDC exceeds the $D_{C G L}$, the instrument response at the scan MDC might be used.

b) For our purpose in this survey, all classes will have investigation levels (trigger levels) that correspond to the $D C G L_{w}$. These trigger levels are instrument specific and each NE Electra with the DP8B probes will have the trigger levels attached for RCT reference. An example of the general trigger levels is provided in Table 13.

Table 13. Range of Trigger Levels in Different Media

\begin{tabular}{|c|r|r|}
\cline { 2 - 3 } \multicolumn{1}{c|}{} & \multicolumn{2}{c|}{ Trigger Levels (cpm) } \\
\cline { 2 - 3 } \multicolumn{1}{c|}{} & Class 1 and 2 & \multicolumn{1}{c|}{ Class 3 } \\
\hline Minimum Concrete $\alpha$ & 23.50 & 25.35 \\
\hline Maximum Concrete $\alpha$ & 26.25 & 29.50 \\
\hline Minimum Concrete $\beta$ & 3698.00 & 4284.00 \\
\hline Maximum Concrete $\beta$ & 4230.00 & 4868.00 \\
\hline Minimum Concrete Block $\alpha$ & 24.00 & 27.90 \\
\hline Maximum Concrete Block $\alpha$ & 26.80 & 33.40 \\
\hline Minimum Concrete Block $\beta$ & 3430.00 & 4030.00 \\
\hline Maximum Concrete Block $\beta$ & 3846.00 & 4615.00 \\
\hline
\end{tabular}


13) Determine Acceptability of Type I and Type II Errors and Set the Lower Boundary of the Gray Region (LBGR) (MARSSIM Appendix D)

a) For the purpose of the statistical tests, the Null (working) hypothesis is that the median level of contamination in the survey unit exceeds the DCGL $L_{w}$. For correct statistical terminology, if the survey unit "passes" (i.e. the median level of contamination is lower than the DCGL), then we "reject the null hypothesis." If the median level of contamination in the survey unit exceeds the $D C G L_{w}$, then we "fail to reject the null hypothesis."

b) The regulator establishes maximum acceptable probability $(\alpha)$ of the statistical test falsely concluding that the median level of contamination (above background) in the survey unit is below the DCGL ${ }_{w}$ when it is actually above it. "Alpha" is known as a Type I error. A Type I error is committed if one rejects the null hypothesis when it is true (Ott \& Longnecker, 2001). The Type I error value was approved at 0.05 , i.e. $5 \%$ (see Appendix B - Approval of parameters (via email string).

c) The licensee establishes acceptable probability (beta or Type II error) of the statistical test falsely concluding that the median level of contamination (above background) exceeds the DCGL $L_{w}$ when it is at a concentration known as the LBGR. The Type II error value is 0.10 , (i.e., $10 \%$ ).

d) The licensee sets the LBGR at some concentration below the DCGL $L_{w}$. In general, the LBGR should be set at the expected average/median concentration in the survey unit. The LBGR for the survey units will be determined by step $14 \mathrm{~b}$.

14) Determine the Appropriate Number of Measurements or Samples (MARSSIM 5.5.2)

a) In order to calculate the required number of measurements, one must first calculate the relative shift. The relative shift is a unitless number (often between 1 and 4 ) related to the chance that individual measurements will exceed the $\mathrm{DCGL}_{\mathrm{w}}$. The smaller the relative shift, the greater the likelihood some measurements exceed the $\mathrm{DCGL}_{\mathrm{w}}$ and the greater the number of measurements that should be made. Calculate the relative shift by the following equation:

$$
\text { Relative Shift }=\frac{D C G L_{w}-L B G R}{\sigma}
$$

Where: $\sigma$ is the expected variability of the measurements (based off earlier characterizations). Since the unity rule is employed, 1 is used as the value for the $\mathrm{DCGL}_{\mathrm{w}}$ in the relative shift calculation (MARSSIM).

b) The following equations are used to calculate the LGBR and $\sigma$ (the expected variability of the measurements) when the unity rule is employed:

$$
L G B R=\frac{\text { Expected Conc. nuclide } 1}{D C G L 1}+\frac{\text { Expected Conc } . \text { nuclide } 2}{D C G L 2}+\text { etc. }
$$




$$
\sigma=\sqrt{\left(\frac{\sigma}{D C G L}\right)_{\text {nuclide } 1}^{2}+\left(\frac{\sigma}{D C G L}\right)_{\text {nuclide } 2}^{2}+\text { etc. }}
$$

Where: $\sigma$ is the expected variability of the measurements (Abelquist, 2001).

c) Given the relative shift and values for Type I and Type II error, the required number of measurements/samples can then be found in Table 5.5 of MARSSIM (when the sign test is employed). It is important to note that the table value has already been increased by an additional $20 \%$ to account for missing or unusable data and uncertainty in the calculated value of the required number of samples (MARSSIM, NUREG-1575).

d) Another way to calculate the number of required samples in a survey unit is by using appropriate software tools, such as COMPASS. The COMPASS software was designed to facilitate the use of MARSSIM and guide the user into making informed decisions in designing final status surveys. COMPASS also simplifies the application of statistical tests by performing the calculations and providing prospective power curves that help determine what level of confidence the user is willing to accept for a particular number of measurements or samples for a survey unit. After performing the final status survey, COMPASS assesses the data for comparison to the release criteria (ORISE). See appendix D for COMPASS results.

15) Establish Reference Areas and Appropriate Background Measurements (MARSSIM 4.5)

a) Reference areas provide a location for background measurements which are used for comparisons with survey unit data. A site background reference area should have similar physical and radiological characteristics as the the survey unit being evaluated. Background reference areas are normally selected from non-impacted areas. In some situations, a reference area may be associated with the survey unit being evaluated, but cannot be potentially contaminated by site activities... this option should be discussed with the responsible regulatory agency during survey planning (MARSSIM). The option of having a reference area inside a Class 3 survey unit was discussed with the regulator and approved (See Appendix B - Approval of Parameters).

The reference area will receive an adequate amount of measurements to establish an average background value per instrument/probe type. It will not be necessary to reestablish an average background reference area every day. The reference area should be viewed as a survey unit, which requires a full survey to be complete.

Depending on the time of the day and characteristics of the building material, radon may substantially influence the background count rate. This is a concern for concrete surfaces, namely in the storage vaults in rooms 104, 106, and 107. Radon may accumulate in the vaults due to pressure/air circulation differences. Background measurements performed in the early morning hours may be influenced to a greater degree by radon and its progeny due to less mixing of radon in the atmosphere, or less mixing of the building atmosphere. The background radiation levels for materials that have no significant naturally occurring radioactivity component (e.g., metals, wood, drywall) are driven by the ambient gamma radiation background and radon levels (Abelquist, 2001). 
b) The reference areas will be located in rooms 105 and 117, which reside in a Class 3 area. Initially, Room 117 was designated as the only reference area. Room 117 has an exterior wall with windows. Room 105 was added as a reference area after data showed that Room 117 had a higher beta background than some of the hot cells. Natural background is lower in the hot cells because the walls and ceilings are composed of 4 feet of concrete. Room 105 was an ideal location for a reference area because it is surrounded on three sides by 4 feet thick concrete walls, yet resides outside the Class 1 area. Room 105 will serve as the reference area for the survey units with thick concrete walls, while Room 117 will serve as the reference area for survey units that are not heavily shielded.

c) Another background consideration is the fundamentally different way that the MARSSIM deals with background measurements in comparison to the standard D\&D approach. That is, many D\&D professionals use the conventional survey approach of subtracting background levels from gross measurements of surface activity. However, the MARSSIM statistical approach compares background measurements to the survey unit measurements, rather than subtracting the background from the survey unit measurements (Abelquist, 2001).

The average of 20 background measurements from each detector was used to establish the reference area for each material type. The material types that will be included into the reference areas are: concrete, cinderblock, drywall, wood, and metal. The concrete reference area is of particular importance because most of the Class 1 areas consist primarily of this material. For ease of access during required background count times, sections of wood and metal were cut from the ceilings in rooms 109,110, and 101. These sections of material were placed in Room 117 where they could easily be counted by technicians.

An example of the average background readings for concrete and concrete block in reference Room 105 using the NE Electra with the DP8B probes is found in Table 14.

Table 14. Combined Background Averages for Room 105

\begin{tabular}{||c|c|c||}
\hline & $c p m$ & $d p m / 100 \mathrm{~cm} 2$ \\
\hline$\alpha$ Concrete & 4.3 & 20.9 \\
\hline $\begin{array}{c}\text { Standard } \\
\text { Deviation }\end{array}$ & 2.0 & 9.7 \\
\hline$\beta$ Concrete & 1040.5 & 1735.9 \\
\hline $\begin{array}{c}\text { Standard } \\
\text { Deviation }\end{array}$ & 82.8 & 138.1 \\
\hline$\alpha$ Concrete Block & 4.8 & 23.4 \\
\hline $\begin{array}{c}\text { Standard } \\
\text { Deviation }\end{array}$ & 2.1 & 10.2 \\
\hline$\beta$ Concrete Block & 696.7 & 1162.2 \\
\hline $\begin{array}{c}\text { Standard } \\
\text { Deviation }\end{array}$ & 46.8 & 78.0 \\
\hline \multicolumn{2}{|c|}{} \\
\hline
\end{tabular}


Only those detectors used to establish a reference area for a material type will be used in the corresponding survey unit. That is to say, some detectors will be primarily used in Class 1 areas which consist of mainly concrete surfaces. The detectors which are used to survey those concrete surfaces should also be used in establishing the concrete reference area. A detector that has no reference area for a certain material type may not be used to survey the material type in a survey unit.

The average background of a specific material in the reference area will be subtracted from the survey unit measurement of the same material type after all data is collected. The survey unit measurements recorded by the RCT will not have the background subtracted, (i.e. electronically stored in instrumentation). During the data review and analysis, the average background from the reference area will be subtracted from the survey unit measurement and then the statistical test (Sign Test) will be performed.

Again, the reference area is established once and the results are used in data analysis and statistical comparisons. The reference area should not be confused with daily instrument background checks performed by RCTs.

16) For Class 1 Survey Units, the Number of Measurements May Need to be Increased (MARSSIM 5.5.2.4)

a) The number of measurements within a survey unit may need to be increased because the scan rate must be sufficiently sensitive to detect a hot spot exceeding the DCGL $L_{e m c}$. The largest (worst case) potential hot spot area is assumed to be that bounded by four measurement points (the survey unit area divided by the number of measurements/samples).

b) If the scan MDC is below the $D C G L_{w}$, then the scan MDC is also below the $D C G L_{e m c}$ and there is no need to adjust the amount of measurements. If the scan MDC is above the $\mathrm{DCGL}_{w}$, it must be compared with the $D C G L_{e m c}$ for a hot spot of that area. Then, if the scan MDC is above the DCGL ${ }_{e m c}$ for that area, the number of fixed measurements must be increased so that the increased $D C G L_{e m c}$ equals the scan MDC.

c) To increase the number of measurements, we first divide the actual scan MDC by the $\mathrm{DCGL}_{w}$. This gives the the area factor for the new, smaller hot spot where the scan MDC equals the DCGL $L_{e m c}$. Then the hot spot area corresponding to this area factor is determined. Dividing this new area into the total survey unit area gives the new required number of measurements/samples.

17) Establish reference grid and determine measurement/sample locations (MARSSIM 5.5.2.5)

a) MARSSIM does not recommend a particular type of reference grid. When measurements/samples are to be distributed in a systematic pattern (Class 1 and 2 survey units), MARSSIM recommends a triangular (equilateral) pattern. The reference grid coordinates of the starting point for a systematic pattern are determined using random numbers. If the measurement/samples are to be distributed randomly (Class 3 survey units), the coordinates for all locations are selected using random numbers. 
b) The procedure for selecting a starting point for the unit grid survey uses a two page random number table located in Appendix $\mathrm{C}$. Two random numbers between zero and one are selected to locate the random start for the sampling grid. These same two random numbers will be used for each survey unit. Using these numbers, the random start for a triangular sampling pattern is found by multiplying these numbers by the length and width of the reference grid for that specific survey units' $X$ and $Y$ axes:

$$
\begin{aligned}
& \left.X=\left(1^{\text {st }} \text { random number }\right) \times \text { (length of reference grid }\right) \\
& \left.Y=\left(2^{\text {nd }} \text { random number }\right) \times \text { (width of reference grid }\right)
\end{aligned}
$$

The random numbers selected for this survey plan are $X=0.306383$ and $Y=0.637352$.

The number of sample points (static measurements denoted as variable $n$ ) required to be obtained in each Class 1 or Class 2 survey unit is survey unit specific. The approximate range is between 11 and 16 static measurements per survey unit. The number of survey points was calculated by using COMPASS computer software. The COMPASS results can be found in Appendix D.

With the number of sample points and survey unit area, the distance formula was used for determining the length between sample locations for a triangular sampling pattern. This distance is found by using the following equation:

$$
L=\sqrt{\frac{A}{0.866 n}}
$$

Where: $L$ is the distance between sampling locations

$A$ is the area of the survey unit

$n$ is the number of samples in a survey unit

With the values of $X, Y, A$, and $n$ known, $L$ was able to be determined for each survey unit. A summary of all Class 1 survey units and associated values of the variables aforementioned are found in Table 15. The number of sample points calculated by COMPASS for room 104 and survey unit "ledges - 1 " was calculated as 11 static measurements. However, the number of measurements was based off of the average sample values of similar rooms within the Class 1 areas. As a measure of conservatism, the number of static measurements was increased to 16 , which equals the highest number of static measurements of all class 1 areas.

Table 15. Survey Unit Specific Parameters

\begin{tabular}{||c|c|c|c|c|c||}
\hline & $\mathbf{X}(\mathbf{m})$ & $\begin{array}{c}\mathbf{Y} \\
\mathbf{( m )}\end{array}$ & $\begin{array}{c}\mathbf{A} \\
\left(\mathbf{m}^{\mathbf{2}}\right)\end{array}$ & $\mathbf{n}$ & $\mathbf{L} \mathbf{( m )}$ \\
\hline Room 102, Floor - Class 1 & & & & & \\
\hline Survey Unit 102-F-1 & 2.46 & 7.67 & 94.2 & 11 & 3.14 \\
\hline Survey Unit 102-F-2 & 2.46 & 7.27 & 89.4 & 11 & 3.06 \\
\hline Survey Unit 102-F-3 & 2.36 & 7.67 & 90.6 & 11 & 3.08 \\
\hline Survey Unit 102-F-4 & 0.76 & 3.54 & 13.4 & 11 & 1.19 \\
\hline
\end{tabular}


Table 15. Survey Unit Specific Parameters (continued)

\begin{tabular}{|c|c|c|c|c|c|}
\hline & $X(m)$ & $\begin{array}{c}\mathrm{Y} \\
(\mathrm{m})\end{array}$ & $\begin{array}{c}A \\
\left(\mathrm{~m}^{2}\right)\end{array}$ & $\mathbf{n}$ & $L(m)$ \\
\hline \multicolumn{6}{|l|}{ Room 102, Ceiling - Class 2} \\
\hline Survey Unit 102-C-1 & 8.03 & 8.85 & 335.3 & 11 & 5.93 \\
\hline \multicolumn{6}{|l|}{ Room 102, North Wall - Class 1} \\
\hline Survey Unit 102-NW-1 & 2.65 & 7.47 & 98.8 & 11 & 3.22 \\
\hline Survey Unit 102-NW-2 & 2.65 & 7.47 & 98.8 & 11 & 3.22 \\
\hline Survey Unit 102-NW-3 & 2.74 & 7.47 & 100 & 11 & 3.24 \\
\hline \multicolumn{6}{|l|}{ Room 102, East Wall - Class 1} \\
\hline Survey Unit 102-EW-1 & 2.55 & 6.88 & 75 & 11 & 2.81 \\
\hline \multicolumn{6}{|l|}{ Room 102 , South Wall - Class 1} \\
\hline Survey Unit 102-SW-1 & 2.65 & 7.47 & 89.5 & 11 & 3.07 \\
\hline Survey Unit 102-SW-2 & 2.65 & 7.47 & 93.6 & 11 & 3.13 \\
\hline Survey Unit 102-SW-3 & 2.74 & 7.47 & 94.4 & 11 & 3.15 \\
\hline \multicolumn{6}{|l|}{ Room 102, West Wall - Class 1} \\
\hline Survey Unit 102-WW-1 & 3.97 & 3.83 & 74.3 & 11 & 2.79 \\
\hline Survey Unit 102-WW-2 & 4.25 & 3.05 & 63.2 & 11 & 2.58 \\
\hline \multicolumn{6}{|l|}{$\begin{array}{c}\text { Room 102, Shield Doors and } \\
\text { Walls - Class } 1\end{array}$} \\
\hline Survey Unit 102-SDW-1 & 2.27 & 6.29 & 58 & 11 & 2.47 \\
\hline \multicolumn{6}{|l|}{ Room 104, Floor - Class 1} \\
\hline Survey Unit 104-F-1 & 2.83 & 5.11 & 60.6 & 16 & 2.09 \\
\hline \multicolumn{6}{|l|}{ Room 104, Ceiling - Class 2} \\
\hline Survey Unit 104-C-1 & 3.02 & 5.5 & 72.5 & 16 & 2.29 \\
\hline \multicolumn{6}{|l|}{ Room 104, North Wall - Class 1} \\
\hline Survey Unit 104-NW-1 & 2.83 & 6.88 & 83.5 & 16 & 2.45 \\
\hline \multicolumn{6}{|l|}{ Room 104, East Wall - Class 1} \\
\hline Survey Unit 104-EW-1 & 2.27 & 6.88 & 81 & 16 & 2.42 \\
\hline \multicolumn{6}{|l|}{ Room 104 , South Wall - Class 1} \\
\hline Survey Unit 104-SW-1 & 2.83 & 6.88 & 97.3 & 16 & 2.65 \\
\hline \multicolumn{6}{|l|}{ Room 104, West Wall - Class 1} \\
\hline Survey Unit 104-WW-1 & 2.83 & 6.88 & 67.2 & 16 & 2.2 \\
\hline \multicolumn{6}{|l|}{ Room 106, Floor - Class 1} \\
\hline Survey Unit 106-F-1 & 2.83 & 5.11 & 60.6 & 11 & 2.52 \\
\hline \multicolumn{6}{|l|}{ Room 106, Ceiling - Class 2} \\
\hline Survey Unit $106-C-1$ & 3.02 & 5.5 & 72.5 & 11 & 2.76 \\
\hline \multicolumn{6}{|l|}{ Room 106, North Wall - Class 1} \\
\hline Survey Unit 106-NW-1 & 2.83 & 6.88 & 83.5 & 11 & 2.76 \\
\hline
\end{tabular}


Table 15. Survey Unit Specific Parameters (continued)

\begin{tabular}{|c|c|c|c|c|c|}
\hline & $X(m)$ & $\begin{array}{c}\mathrm{Y} \\
(\mathrm{m})\end{array}$ & $\begin{array}{c}\text { A } \\
(\mathrm{m} 2)\end{array}$ & $\mathrm{n}$ & $L(m)$ \\
\hline \multicolumn{6}{|c|}{ Room 106, East Wall - Class 1} \\
\hline Survey Unit 106-EW-1 & 2.83 & 6.88 & 67.2 & 11 & 2.66 \\
\hline \multicolumn{6}{|c|}{ Room 106, South Wall - Class 1} \\
\hline Survey Unit 106-SW-1 & 2.83 & 6.88 & 97.3 & 11 & 3.2 \\
\hline \multicolumn{6}{|c|}{ Room 106, West Wall - Class 1} \\
\hline Survey Unit 106-WW-1 & 2.27 & 6.88 & 81 & 11 & 2.92 \\
\hline \multicolumn{6}{|l|}{ Room 107, Floor - Class 1} \\
\hline Survey Unit 107-F-1 & 2.36 & 8.45 & 84.5 & 16 & 2.47 \\
\hline \multicolumn{6}{|l|}{ Room 107, Ceiling - Class 2} \\
\hline Survey Unit $107-\mathrm{C}-1$ & 2.36 & 8.45 & 90.1 & 16 & 2.55 \\
\hline \multicolumn{6}{|c|}{ Room 107, North Wall - Class 1} \\
\hline Survey Unit 107-NW-1 & 3.69 & 3.14 & 65.4 & 16 & 2.17 \\
\hline Survey Unit 107-NW-2 & 3.59 & 3.73 & 66 & 16 & 2.18 \\
\hline \multicolumn{6}{|c|}{ Room 107, East Wall - Class 1} \\
\hline Survey Unit 107-EW-1 & 2.36 & 6.88 & 67.5 & 16 & 2.21 \\
\hline \multicolumn{6}{|c|}{ Room 107 , South Wall - Class 1} \\
\hline Survey Unit 107-SW-1 & 3.69 & 3.14 & 69.8 & 16 & 2.24 \\
\hline Survey Unit 107-SW-2 & 3.59 & 3.73 & 66 & 16 & 2.18 \\
\hline \multicolumn{6}{|c|}{ Room 107, West Wall - Class 1} \\
\hline Survey Unit 107-WW-1 & 2.36 & 6.88 & 67.5 & 16 & 2.21 \\
\hline \multicolumn{6}{|c|}{$\begin{array}{c}\text { Combined Ledges Rooms 102, } \\
104,106, \& 107 \text { - Class } 1\end{array}$} \\
\hline Survey Unit Ledges-1 & 7.28 & 1.57 & 99.6 & 16 & 2.68 \\
\hline
\end{tabular}

17.) Assessment Phase

As each survey unit is completed, the average $\mathrm{cpm}$ value and standard deviation will be determined. The $\mathrm{cpm}$ value will be corrected for background and converted to a value with units of $\mathrm{dpm} / 100 \mathrm{~cm} 2$. With a surface contamination value in the appropriate units, it can be compared to the surface contamination limits, or in this case the $D C G L_{w}$. The appropriate statistical test (sign test) will be performed and the results documented. If the survey unit is statistically determined to be below the $D C \mathrm{CL}_{w}$, then the survey unit passes and the null hypothesis (that the survey unit is above limits) is rejected. If the survey unit equals or is below the critical value in the sign test, the null hypothesis is not rejected. Further remediation will be required in that survey unit. 


\section{Contamination Surveys}

1) Scan surfaces for contamination (MARSSIM 6.4.2)

a) The primary purpose of the scan is to locate small areas of elevated contamination (i.e., hot spots). If hot spots are located, then the area around the hotspot are characterized by additional samples/measurements to determine that the contamination in below the $\mathrm{DCGL}_{\mathrm{emc}}$.

b) For alpha and beta scans, the probe is usually held around $1 \mathrm{~cm}$ above the surface. The scan rate for the NE Electra with DP8B probes is no greater than 1 foot per second. This scan rate will meet our required scan MDC.

2) Perform Static Measurements on Surfaces (MARSSIM 6.4.1, 7.5)

a) This is done to obtain accurate determinations of the contamination levels at a number of unbiased locations. This data will be assessed statistically to determine if the contamination levels in the survey unit are below the $\mathrm{DCGL}_{w}$ and used to determine that the survey unit was accurately classified.

b) Static measurements of alpha and beta concentrations are performed with the probe directly on, or just above, the surface. In the final status survey, the surfaces should be clean with little or no removable contamination. Measurements will be performed using 1 minute count times.

c) For the purposes of this survey plan, the static measurements will be recorded on the form FRM-0108B, "Radiological Survey Report - Data," in the units of cpm. These measurements will be directly transposed from the readout of the detector (i.e., no correction factors, efficiencies, or backgrounds are applied). The readout on the detector is simply the gross counts in cpm. This data set will later be adjusted by the reference area background and appropriately converted to $\mathrm{dpm} / 100 \mathrm{~cm}^{2}$ by the HPS or RE.

d) The number and location of static measurements in each survey unit were determined in step 16 of Section IV.

3) Removable Contamination Surveys

a) For the purposes of this survey plan, surveys for removable contamination are not required. Sufficient data has been obtained from the scoping and characterization surveys to adequately evaluate the potential for removable contamination.

4) Decontamination

a) If a trigger level is exceeded, the RCT shall physically mark the actual location and document the contamination levels and estimated size of the impacted area on the survey report. Allowances have been granted to perform remediation of such areas during the execution of the final status survey (See Appendix B). Decontamination will be performed to the extent necessary to be less than the $\mathrm{DCGL}_{\mathrm{w}}$. Decontamination of the impacted area may be performed utilizing a multitude of different methods depending on the physical characteristics of the location. As a general rule, 
decontamination methods should be performed using the least aggressive techniques such as using wet Kim Wipes, abrasive pads, and wire brushes. If these methods are ineffective more aggressive techniques may be used to sufficiently remediate the location such as needle guns, scabblers, grinders, and jack hammers. Decontamination activities that have the potential to generate removal contamination greater than Table 4-2 of the DOE/NV/25946--801 Revision 1, "Nevada Test Site Radiological Control Manual," shall be performed with the controls of a Radiological Work Permit (RWP).

b) Upon successful completion of decontamination, the entire impacted area remediated will be completely resurveyed and all immediately adjacent surfaces within a 1 meter radius of the impacted area.

5) Response to Abnormal Conditions

a) During this survey process if removable contamination is found that is above the suspension limits of the RWP, work shall be paused, personnel in the affected area or room notified of the condition and instructed to exit the area or room. RCTs will immediately notify the ER HPS and survey any personnel who were in the affected room or area. Based on the levels encountered, RCTs will also adjust radiological postings to be commensurate with the actual area conditions.

\section{Disposition}

Building 2201 Pluto Disassembly Facility structure is to be demolished and the structure size reduced. Much of the resulting building demolition debris will be disposed at an NTS onsite landfill. The remaining debris will be used to backfill the building's basement void space.

\section{References}

Abelquist, E. W., 2001. Decommissioning Health Physics - A Handbook for MARSSIM Users. New York, NY: Taylor \& Francis Group, LLC.

Argonne National Laboratory, 2001. RESRAD Documentation. [How Clean is Clean Fact Sheet] Available at: http://web.ead.anl.gov/resrad/documents/ [Accessed Novemeber 18, 2009]

National Security Technologies, LLC, 2008. Direct and Indirect Surveys. OI-0441.211. Las Vegas, NV.

National Security Technologies, LLC, 2009. Pluto Disassembly Facility - Building Radiological Characterization. Survey Plan 09-022. Las Vegas, NV.

National Security Technologies, LLC, 2009. Pluto Disassembly Facility - Radiological Characterization (Phase II) and Post Remediation Survey Requirements. Survey Plan 09-031. Las Vegas, NV. 
Ott, R. L., M. T. Longnecker, 2001. Statistical Methods and Data Analysis. $5^{\text {th }}$ ed. Duxbury: Wadsworth Group.

Stoller-Navarro Joint Venture, 2009. Closure Report for Corrective Action Unit 117: Area 26 Pluto Disassembly Facility, Nevada Test Site, Nevada. DOE/NV--1324. Las Vegas, NV.

U.S. Department of Energy Order 5400.5, "Radiation Protection of the Public and the Environment," 1990.

U.S. Nuclear Regulatory Commission, 1998. A Nonparametric Statistical Methodology for the Design and Analysis of Final Status Decommissioning Surveys. NUREG-1505 Rev. 1. June 1998. Washington, D.C.

U.S. Nuclear Regulatory Commission, 2001. Multi-Agency Radiation Survey and Site Investigation Manual (MARSSIM). NUREG-1575 Rev. 1. August 2000. Washington, D.C. 
Appendix A

Allowable Total Residual Surface Contamination Values in dpm/100 cm2 (Note 1)

\begin{tabular}{|l|c|c|c|}
\hline RADIONUCLIDE (See Note 2) & $\begin{array}{c}\text { REMOVABLE } \\
\text { (See Note 3) }\end{array}$ & $\begin{array}{c}\text { AVERAGE } \\
\text { (FIXED \& } \\
\text { REMOVABLE) } \\
\text { (See Note 4) }\end{array}$ & $\begin{array}{c}\text { MAXIMUM } \\
\text { ALLOWABLE } \\
\text { (FIXED \& } \\
\text { REMOVABLE) } \\
\text { (See Note 5) }\end{array}$ \\
\hline $\begin{array}{l}\text { Transuranics, 125I, 129I, 226Ra, } \\
\text { 227Ac, 228Ra, 228Th, 230Th, 231Pa, }\end{array}$ & 20 & 100 & 300 \\
\hline $\begin{array}{l}\text { Th-nat, 90Sr, 126I, 131I, 133I, 223Ra, } \\
\text { 224Ra, 232U, 232Th, }\end{array}$ & 200 & 1,000 & 3,000 \\
\hline $\begin{array}{l}\text { U-natural, 235U, 238U and } \\
\text { associated decay products, alpha } \\
\text { emitters }\end{array}$ & $1,000 \alpha$ & $5,000 \alpha$ & $15,000 \alpha$ \\
\hline $\begin{array}{l}\beta+\gamma \text { emitters (radionuclides with } \\
\text { decay modes other than } \alpha-e m i s s i o n \\
\text { or spontaneous fission) except 90Sr } \\
\text { and others noted above. (See Note 6) }\end{array}$ & $1,000 \beta+\gamma$ & $5,000 \beta+\gamma$ & $15,000 \beta+\gamma$ \\
\hline Tritium and Tritiated Compounds & 10,000 & N/A & N/A \\
\hline
\end{tabular}

Notes:

(from DOE O 5400.5)

1) Disintegrations per minute ( $\mathrm{dpm})$ means the rate of emission by radioactive material as determined by correcting the counts per minute measured by an appropriate detector for background, efficiency, and geometric factors associated with the instrumentation.

2) Where surface contamination by both alpha- and beta/gamma-emitting radionuclides exist, the limits established for alpha- and beta/gamma-emitting radionuclides apply independently.

3) The amount of removable material per $100 \mathrm{~cm} 2$ of surface area should be determined by wiping an area of that size with dry filter or soft absorbent paper, applying moderate pressure, and measuring the amount of radioactive material on the wiping with an appropriate instrument of known efficiency. When removable contamination on objects of surface area less than $100 \mathrm{~cm} 2$ is determined, the activity per unit area should be based on the actual area and the entire surface should be wiped. It is not necessary to use wiping techniques to measure removable contamination levels if direct scan surveys indicate that the total residual surface contamination levels are within the limits for removable contamination.

4) Measurements of average contamination should not be averaged over an area of more than $1 \mathrm{~m} 2$. For objects of less surface area, the average should be derived for each such object.

5) The maximum contamination level applies to an area of not more than $100 \mathrm{~cm} 2$.

6) This category of radionuclides includes mixed fission products, including the Sr-90, which is present in them. It does not apply to $\mathrm{Sr}-90$ that has been separated from the other fission products or mixtures where the Sr-90 has been enriched. 


\section{Appendix B \\ Approval of Parameters - via email string}

From: Boehlecke, Robert (NV) [mailto:BoehleckeR@nv.doe.gov]

Sent: Wednesday, December 09, 2009 9:34 AM

To: Thiele, Thomas

Subject: RE: Requested Information from NNSA for Pluto Final Status Survey

Tom,

Based on the information you provided at the meeting including that an independent verification of the FSS was not required for this work, my answers are below:

I concur with the approach identified in Doug's email below on the first three items and do not require an independent verification of the FSS. However, I am requesting that you provide the final survey plan to us for review.

Thanks

Rob

From: Thiele, Thomas [mailto:thieleta@nv.doe.gov]

Sent: Wednesday, December 09, 2009 9:01 AM

To: Boehlecke, Robert (NV)

Cc: Cabble, Kevin J. (NV); Lantow, Tiffany (NV); Primrose, Annette L (NSTec)

Subject: FW: Requested Information from NNSA for Pluto Final Status Survey

Rob:

Per our meeting last week about the MARSSIM work at Pluto, here's what we need from you to move forward. Please let me know if you have any questions or need additional information.

Thanks.

Tom

From: Traynor, James

To: Thiele, Thomas

Sent: Thu Dec 03 13:12:15 2009

Subject: FW: Requested Information from NNSA for Pluto Final Status Survey

Tom,

Here is the information we require from NNSA concerning the MARSSIM FSS for Pluto.

Thank you,

Jim 


\section{Appendix B \\ Approval of Parameters - via email string (continued)}

From: Frenette, Douglas $\mathrm{E}$

Sent: Thursday, December 03, 2009 12:50 PM

To: Traynor, James

Cc: Gwin, Jeremy S.

Subject: Requested Information from NNSA for Pluto Final Status Survey

Jim,

Below is the information that we would need concurrence/confirmation on from NNSA regarding the Pluto project and our Final Status Survey (FSS). I do apologize after a quick review with Jeremy today, there was one other item we needed from NNSA that we did not mention in the meeting yesterday (bullet \#3).

In order to complete some of the planning for the MARSSIM Final Status Survey (FSS) of the Pluto facility (Building 2201 or CAU 17), we need approval from NNSA to use some parameters. Here is what we propose to use for the FSS plan and request their concurrence:

We are requesting a $0.05(5 \%)$ Type I statistical error. (This is a typical value in most statistical tests.)

We are requesting permission to have the option of decontaminating a hot spot (i.e. radiologically impacted area) - if found during the FSS. The decontaminated area (unit), and all immediately adjacent areas (units) will then be surveyed. Provisions for this will be documented in the FSS plan.

We are requesting that we use a reference area (used for background measurements) inside the building, where offices were located. This reference area is inside a class 3 area (where little or no potential for contamination exists). It would be convenient to be able to run instrument backgrounds at Pluto, rather than performing backgrounds at a different site and then transporting them to Pluto.

We would also like to know if an independent verification of the FSS will be necessary before demolition of the building.

Douglas Frenette

Environmental Restoration

Health Physics Supervisor, NSTec

Desk: (702) 295-3784

Cell: (702) 241-4368

Fax: (702) 295-7918 
Survey Plan 10-015

Final Status Survey Plan for Corrective Action Unit 117 - Pluto Disassembly

Facility, Building 2201

Appendix C

1,000 Random Numbers between Zero and One

\begin{tabular}{|c|c|c|c|c|c|c|c|c|c|}
\hline 01 & 23 & 0.555548 & 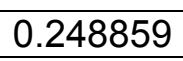 & 1 & 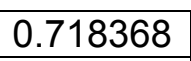 & -0 & 2 & 21 & 0.973160 \\
\hline & & & 132364 & & & & 0.896462 & & \\
\hline & & & & & & & & & \\
\hline & & & 26726 & & 993 & 7990 & 947022 & & 88081 \\
\hline & & & & & & & & & \\
\hline & & & & & & & & & \\
\hline & 34 & 92 & 161947 & 688925 & & 3577 & & & 66196 \\
\hline & & & & & & & & & \\
\hline & 72 & & & & & & & & \\
\hline & $\pi$ & 21 & .268003 & 27 & & & & & \\
\hline & & & & & & & & & \\
\hline & 3 & & & & & & & & \\
\hline & & & 37 & ת & & & & & \\
\hline & & & & & & & & & \\
\hline & 3 & & & & & & & & \\
\hline & & & & & & & & & \\
\hline & & & & & & & & & \\
\hline & & & 0.437 & & & & & & \\
\hline & & & & & & & & & \\
\hline & & & & & & & & & \\
\hline & & & & & & & & & \\
\hline & & & & & & & & & \\
\hline & & & & & & & & & \\
\hline & & & & & & & & & \\
\hline & & & & & & & & & \\
\hline & & & & & & & & & \\
\hline & & & & & & & & & \\
\hline & & & & & & & & & \\
\hline & & & & & & & & & \\
\hline & & & & & & & & & \\
\hline & & & & & & & & & \\
\hline & & & & & & & & & 169 \\
\hline & & & & & & & & & \\
\hline & & & & & & & & & \\
\hline & & & & & & & & & \\
\hline & & & & & & & & & \\
\hline & & & & & & & & & \\
\hline & & & & & & & & & \\
\hline & & & & & & & & & \\
\hline 66 & 0. & & 0.690208 & 0.74 & 009 & 0.6 & 931 & & 7899 \\
\hline & & & & & & & & & \\
\hline & & & & & & & & & \\
\hline 284 & & & 156 & 0.576129 & & & 748 & & 0.874416 \\
\hline & & & & & & & & & $00 n$ \\
\hline
\end{tabular}




\section{Appendix C 1,000 Random Numbers between Zero and One (continued)}

\begin{tabular}{|c|c|c|c|c|c|c|c|c|c|}
\hline 96 & 933631 & 4 & 3 & 77 & 1 & 3 & 0.066152 & 14 & 0.857606 \\
\hline & & & & & & & 0.228607 & & \\
\hline & & & & & & & & & \\
\hline & 540 & 775 & 0449 & 115 & & 14 & & & 28 \\
\hline & & & & & & & & & \\
\hline & & & & & & & & & \\
\hline & 061151 & 68 & & 52 & & & & & \\
\hline & & & & & & & & & \\
\hline & 29 & & & & & & & & \\
\hline & 926550 & 32 & & & & & & & \\
\hline & & & & & & & & & \\
\hline & & & & & & & & & \\
\hline & 237361 & 0 & & & & & & & \\
\hline & & & & & & & & & \\
\hline & 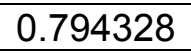 & & & & & & & & \\
\hline & & 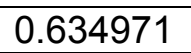 & & & & & & & \\
\hline & & & & & & & & & \\
\hline & $366 \mathrm{~s}$ & & & & & & & & \\
\hline & & & & & & & & & \\
\hline & & & & & & & & & \\
\hline & & & & & & & & & \\
\hline & & & & & & & & & \\
\hline & & & & & & & & & \\
\hline & & & & & & & & & \\
\hline & & & & & & & & & \\
\hline & & & & & & & & & 580 \\
\hline & & & & & & & & & \\
\hline & & & & & & & & & \\
\hline & & & & & & & & & \\
\hline & & & & & & & & & \\
\hline & & & & & & & & & \\
\hline & & & & & & & & & \\
\hline & & & & & & & & & \\
\hline & & & & & & & & & \\
\hline & & & & & & & & & \\
\hline & & & & & & & & & \\
\hline & & & & & & & & & \\
\hline & & & & & & & & & \\
\hline & & & & & & & & & \\
\hline 30 & 0.791992 & 75 & 52 & 561 & 34 & .447486 & 599 & 516 & 455018 \\
\hline & & & & & & & & & \\
\hline & & & & & & & & & \\
\hline & 0.800723 & & & & & & & & \\
\hline & 254 & & 22 & 02 & & & 36 & 69 & 0.377845 \\
\hline
\end{tabular}


Appendix C

\section{1,000 Random Numbers between Zero and One (continued)}

\begin{tabular}{|l|l|l|l|l|l|l|l|l|l|}
\hline 0.744807 & 0.960789 & 0.123099 & 0.163569 & 0.621969 & 0.571558 & 0.482449 & 0.346358 & 0.795845 & 0.207558 \\
\hline 0.642312 & 0.356643 & 0.797708 & 0.505570 & 0.418534 & 0.634642 & 0.033111 & 0.393330 & 0.105093 & 0.328848 \\
\hline 0.824625 & 0.855876 & 0.770743 & 0.678619 & 0.927298 & 0.204828 & 0.831460 & 0.979875 & 0.566627 & 0.056160 \\
\hline 0.755877 & 0.679791 & 0.442388 & 0.899944 & 0.563383 & 0.197074 & 0.679568 & 0.244433 & 0.786084 & 0.337991 \\
\hline 0.625370 & 0.967123 & 0.321605 & 0.697578 & 0.122418 & 0.475395 & 0.068207 & 0.070374 & 0.353248 & 0.461960 \\
\hline 0.124012 & 0.133851 & 0.761154 & 0.501578 & 0.204221 & 0.866481 & 0.925783 & 0.329001 & 0.327832 & 0.844681 \\
\hline 0.825392 & 0.382001 & 0.847909 & 0.520741 & 0.404959 & 0.308849 & 0.418976 & 0.972838 & 0.452438 & 0.600528 \\
\hline 0.999194 & 0.297058 & 0.617183 & 0.570478 & 0.875712 & 0.581618 & 0.284410 & 0.405575 & 0.362205 & 0.427077 \\
\hline 0.536855 & 0.667083 & 0.636883 & 0.043774 & 0.113509 & 0.980045 & 0.237797 & 0.618925 & 0.670767 & 0.814902 \\
\hline 0.361632 & 0.797162 & 0.136063 & 0.487575 & 0.682796 & 0.952708 & 0.759989 & 0.058556 & 0.292400 & 0.871674 \\
\hline 0.923253 & 0.479871 & 0.022855 & 0.673915 & 0.733795 & 0.811955 & 0.417970 & 0.095675 & 0.831670 & 0.043950 \\
\hline 0.845432 & 0.202336 & 0.348421 & 0.050704 & 0.171916 & 0.600557 & 0.284838 & 0.606715 & 0.758190 & 0.394811 \\
\hline
\end{tabular}




\section{Appendix D}

\section{COMPASS Results}

\section{Pages}




\section{BUILDING SURFACE SURVEY PLAN}

\section{Survey Plan Summary}

Site Name:

Pluto test

Planner(s):

Gwin

Survey Unit Name:

Room 102

Comments:

N/A

\section{Statistical Design Details}

\begin{tabular}{llll}
\hline Area $(\mathrm{m}):$ & 100 & Classification: & 1 \\
Selected Test: & Sign & Estimated Sigma (SOR): & 0.19 \\
DCGL (SOR): & 1 & Sample Size (N): & $\mathbf{1 1}$ \\
LBGR (SOR): & 0.36 & Estimated Conc. (SOR): & 0.40 \\
Alpha: & 0.050 & Estimated Power: & 1.0 \\
Beta: & 0.100 & Post-EMC Sample Size (N): & $\mathbf{1 1}$
\end{tabular}

NOTE: SOR = Sum-of-Ratios

\section{Prospective Power Curve}

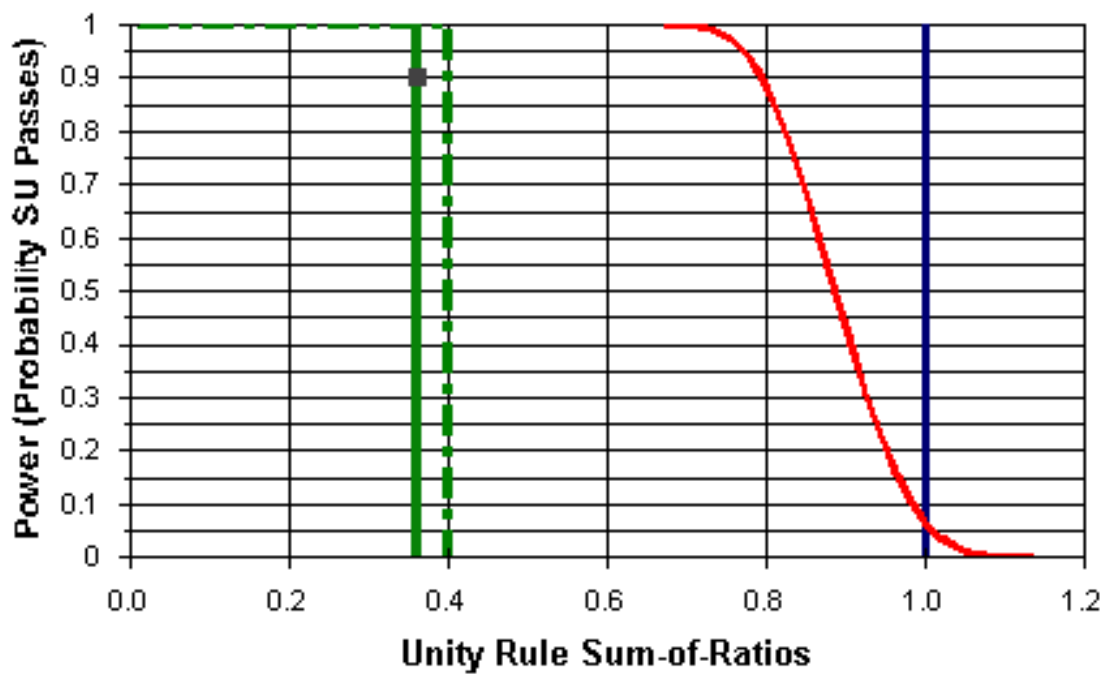

- Prospective Power $\quad$ DCGL
LBGR

- Estimated Power 


\section{Gross Alpha Efficiency Data}

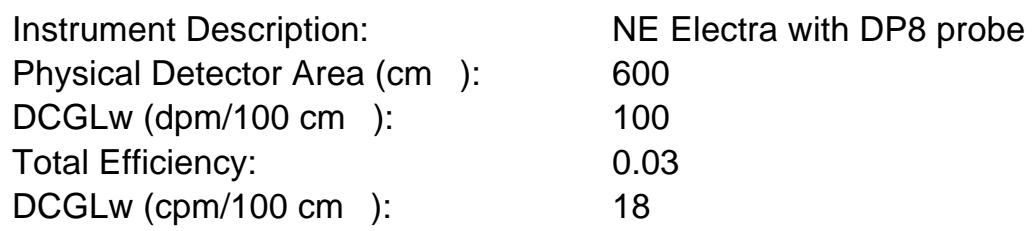

\begin{tabular}{|c|c|c|c|c|c|c|}
\hline Contaminant & HTDC $^{\mathbf{a}}$ & Energy $^{\mathbf{b}}$ & Fract $^{\mathbf{c}}$ & Inst. Eff. & Surf. Eff. & Total Eff. \\
\hline Pu-239 & No & N/A & 1.0 & 0.14 & 0.25 & 0.03 \\
\hline
\end{tabular}

a Hard-to-detect contaminant ${ }^{\mathrm{b}}$ Average beta energy (keV) [N/A indicates alpha emission] ${ }^{\mathrm{c}}$ Activity fraction

\section{Gross Beta Efficiency Data}

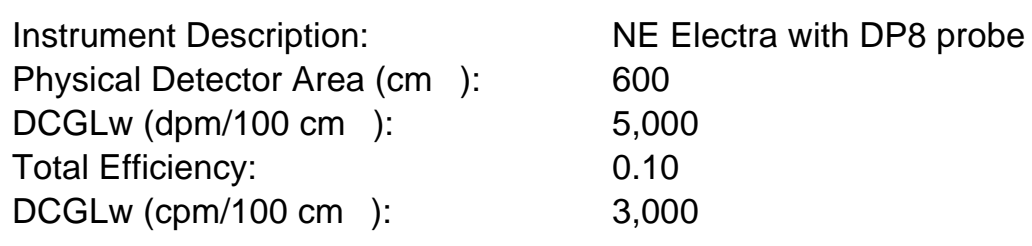

\begin{tabular}{|c|c|c|c|c|c|c|}
\hline Contaminant & HTDC $^{\mathbf{a}}$ & Energy $^{\mathbf{b}}$ & Fract $^{\mathbf{c}}$ & Inst. Eff. & Surf. Eff. & Total Eff. \\
\hline Cs-137 & No & 187.87 & 1.0 & 0.20 & 0.50 & 0.10 \\
\hline
\end{tabular}

${ }^{\mathrm{a}}$ Hard-to-detect contaminant ${ }^{\mathrm{b}}$ Average beta energy (keV) [N/A indicates alpha emission] ${ }^{\mathrm{c}}$ Activity fraction

\section{Gross Alpha Mean and Sigma Data}

$\begin{array}{ll}\text { Count Time }(\min ): & 1 \\ \text { Sign Test Sigma }(\mathrm{cpm} / 100 \mathrm{~cm}): & 2.8\end{array}$

\begin{tabular}{|l|c|c|c|}
\hline Data/Material & Mean (cpm/100 cm ) & Std. Dev. (cpm/100 cm ) & MDC (dpm/100 cm ) \\
\hline SU & 10 & 1.9 & 98 \\
\hline Concrete & 4.3 & 2.0 & 70 \\
\hline
\end{tabular}

\section{Gross Beta Mean and Sigma Data}

Count Time (min):
\begin{tabular}{l|c|c|c|} 
Sign Test Sigma (cpm/100 cm ): & 1 \\
\multicolumn{1}{|l|}{335} \\
Data/Material & Mean (cpm/100 cm ) & Std. Dev. (cpm/100 cm ) & MDC (dpm/100 cm ) \\
\hline SU & 1,276 & 325 & 282 \\
\hline Concrete & 1,041 & 83 & 255 \\
\hline
\end{tabular}

\section{Elevated Measurement Comparison (EMC) for Gross Alpha}

Scanning Instrumentation Description:

Scan MDC*:

Area Factor Table Interpolation Method:

\section{Statistical Design}

$\mathrm{N}$ :

Bounded Area (m ):

Area Factor:

DCGLw*:

Scan MDC Required*:

mhtml:file://C:\Documents and Settings \gwinjs \Desktop $\backslash E R \backslash P L U T O \backslash$ Compass Reports $\backslash$ Room 102.mht

\section{Hot Spot Design}

Actual Scan MDC*:

68

Area Factor:

Bounded Area (m ): N/A

Post-EMC N:

$\begin{array}{lll}9.1 & \text { Area Factor: } & \text { N/A } \\ 4.0 & \text { Bounded Area }(m): & 11 \\ 100 & \text { Post-EMC N: } & \end{array}$




\begin{tabular}{|c|c|c|c|}
\hline Contaminant & DCGLw $^{*}$ & Area Factor & Scan MDC Req'd $^{*}$ \\
\hline Pu-239 & 100 & 4.0 & 398 \\
\hline
\end{tabular}

* dpm/100 cm

\section{Elevated Measurement Comparison (EMC) for Gross Beta}

Scanning Instrumentation Description:

Background (cpm/100 cm ):

Total Scanning Efficiency:

True Positive Proportion:

False Positive Proportion:

Index of Sensitivity (d'):

Observation Interval (sec):

Surveyor Efficiency:

Area Factor Table Interpolation Method:
NE Electra with DP8 probe

1,041

0.10

0.9

0.05

2.92

10

0.50

Linear

\section{Statistical Design}

$\mathrm{N}$ :

Bounded Area (m ):

Area Factor:

DCGLw*:

Scan MDC Required*:
11

9.1

2.6

5,000

12,950
Hot Spot Design

Actual Scan MDC*:

Area Factor:

Bounded Area (m ): N/A

Post-EMC N:
3,264

N/A

11

\begin{tabular}{|c|c|c|c|c|c|}
\hline Contaminant & Energy $^{\mathbf{a}}$ & Fract $^{\mathbf{b}}$ & Inst. Eff. & Surf. Eff. & Total Eff. \\
\hline Cs-137 & 187.87 & 1.0 & 0.20 & 0.50 & 0.10 \\
\hline
\end{tabular}

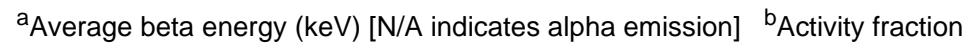

\begin{tabular}{|c|c|c|c|}
\hline Contaminant & DCGLw* $^{*}$ & Area Factor & Scan MDC Req'd $^{*}$ \\
\hline Cs-137 & 5,000 & 2.6 & 12,950 \\
\hline
\end{tabular}

* $\mathrm{dpm} / 100 \mathrm{~cm}$

Report Created 06/16/2010 0922 (COMPASS v1.1.0)

mhtml:file://C: $\backslash$ Documents and Settings $\backslash$ gwinjs $\backslash$ Desktop $\backslash E R \backslash P L U T O \backslash C o m p a s s$ Reports $\backslash$ Room 102.mht 


\section{BUILDING SURFACE SURVEY PLAN}

\section{Survey Plan Summary}

Site Name:

Planner(s):

Survey Unit Name:

Comments:
Pluto test

Gwin

test 102 ceiling

N/A

\section{Statistical Design Details}

\begin{tabular}{llll}
\hline Area $(\mathrm{m}):$ & 335 & Classification: & 2 \\
Selected Test: & Sign & Estimated Sigma (SOR): & 0.19 \\
DCGL (SOR): & 1 & Sample Size (N): & $\mathbf{1 1}$ \\
LBGR (SOR): & 0.36 & Estimated Conc. (SOR): & 0.40 \\
Alpha: & 0.050 & Estimated Power: & 1.0 \\
Beta: & 0.100 & &
\end{tabular}

NOTE: SOR = Sum-of-Ratios

\section{Prospective Power Curve}

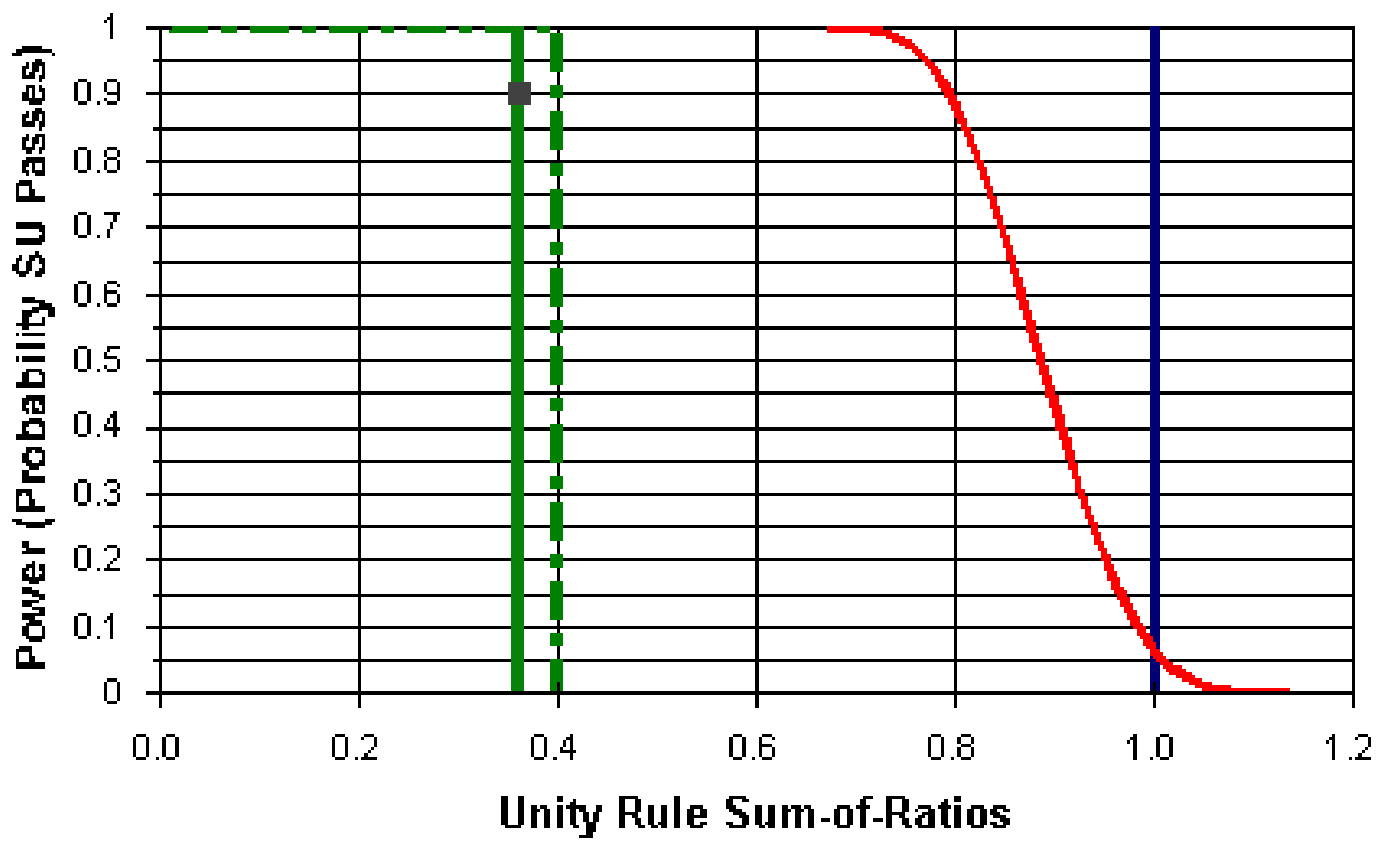

Prospective Power

DCGL

- Estimated Power

LBGR

- 1-beta 


\section{Gross Alpha Efficiency Data}

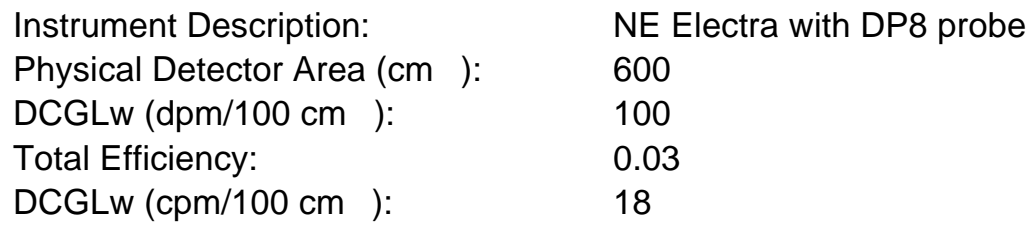

\begin{tabular}{|c|c|c|c|c|c|c|}
\hline Contaminant & HTDC $^{\mathbf{a}}$ & Energy $^{\mathbf{b}}$ & Fract $^{\mathbf{c}}$ & Inst. Eff. & Surf. Eff. & Total Eff. $^{\text {P }}$ \\
\hline Pu-239 & No & N/A & 1.0 & 0.14 & 0.25 & 0.03 \\
\hline
\end{tabular}

a Hard-to-detect contaminant ${ }^{\mathrm{b}}$ Average beta energy (keV) [N/A indicates alpha emission] ${ }^{\mathrm{c}}$ Activity fraction

\section{Gross Beta Efficiency Data}

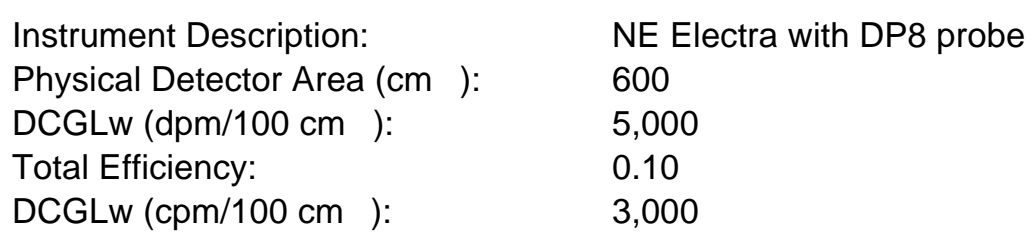

\begin{tabular}{|c|c|c|c|c|c|c|}
\hline Contaminant & HTDC $^{\mathbf{a}}$ & Energy $^{\mathbf{b}}$ & Fract $^{\mathbf{c}}$ & Inst. Eff. & Surf. Eff. & Total Eff. \\
\hline Cs-137 & No & 187.87 & 1.0 & 0.20 & 0.50 & 0.10 \\
\hline
\end{tabular}

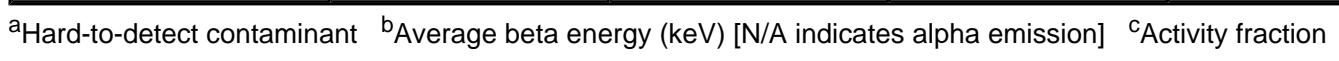

\section{Gross Alpha Mean and Sigma Data}

$\begin{array}{ll}\text { Count Time }(\mathrm{min}): & 1 \\ \text { Sign Test Sigma }(\mathrm{cpm} / 100 \mathrm{~cm}): & 2.8\end{array}$

\begin{tabular}{|l|c|c|c|}
\hline Data/Material & Mean (cpm/100 cm ) & Std. Dev. (cpm/100 cm ) & MDC (dpm/100 cm ) \\
\hline SU & 10 & 1.9 & 98 \\
\hline Concrete & 4.3 & 2.0 & 70 \\
\hline
\end{tabular}

\section{Gross Beta Mean and Sigma Data}

\begin{tabular}{|c|c|c|c|}
\hline $\begin{array}{l}\text { Count Time (min): } \\
\text { Sign Test Sigma }(\mathrm{cpm} / 100 \mathrm{~cm} \text { ): }\end{array}$ & $\begin{array}{l}1 \\
335\end{array}$ & & \\
\hline Data/Material & Mean $(\mathrm{cpm} / 100 \mathrm{~cm})$ & Std. Dev. (cpm/100 cm ) & MDC (dpm/100 cm ) \\
\hline SU & 1,276 & 325 & 282 \\
\hline Concrete & 1,041 & 83 & 255 \\
\hline
\end{tabular}




\section{BUILDING SURFACE SURVEY PLAN}

\section{Survey Plan Summary}

Site Name:

Pluto test

Planner(s):

Gwin

Survey Unit Name:

test room 104

Comments:

N/A

\section{Statistical Design Details}

\begin{tabular}{llll}
\hline Area $(\mathrm{m}):$ & 100 & Classification: & 1 \\
Selected Test: & Sign & Estimated Sigma (SOR): & 0.29 \\
DCGL (SOR): & 1 & Sample Size (N): & $\mathbf{1 1}$ \\
LBGR (SOR): & 0.26 & Estimated Conc. (SOR): & 0.33 \\
Alpha: & 0.050 & Estimated Power: & 1.0 \\
Beta: & 0.100 & Post-EMC Sample Size (N): & $\mathbf{1 1}$
\end{tabular}

NOTE: SOR = Sum-of-Ratios

\section{Prospective Power Curve}

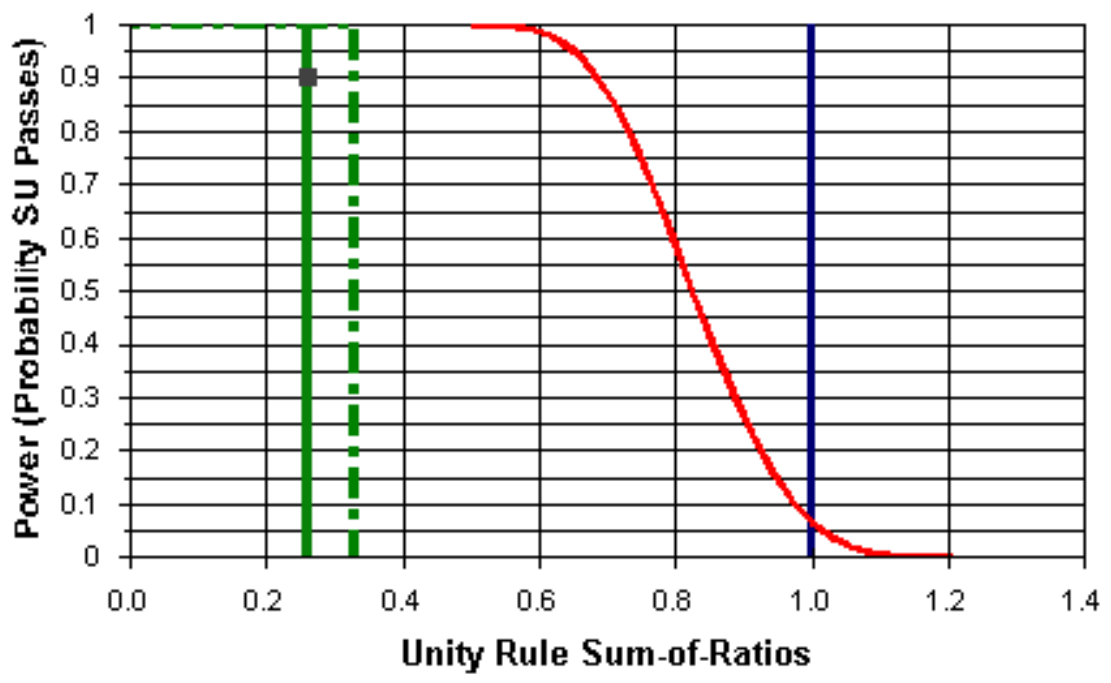

- Prospective Power $\quad$ DCGL
LBGR

- Estimated Power 


\section{Gross Alpha Efficiency Data}

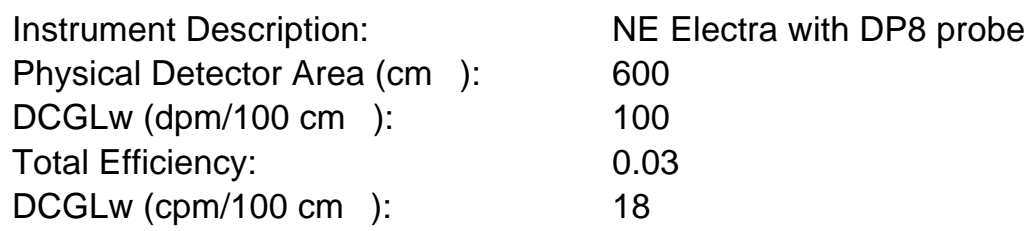

\begin{tabular}{|c|c|c|c|c|c|c|}
\hline Contaminant & HTDC $^{\mathbf{a}}$ & Energy $^{\mathbf{b}}$ & Fract $^{\mathbf{c}}$ & Inst. Eff. & Surf. Eff. & Total Eff. \\
\hline Pu-239 & No & N/A & 1.0 & 0.14 & 0.25 & 0.03 \\
\hline
\end{tabular}

a Hard-to-detect contaminant ${ }^{\mathrm{b}}$ Average beta energy (keV) [N/A indicates alpha emission] ${ }^{\mathrm{c}}$ Activity fraction

\section{Gross Beta Efficiency Data}

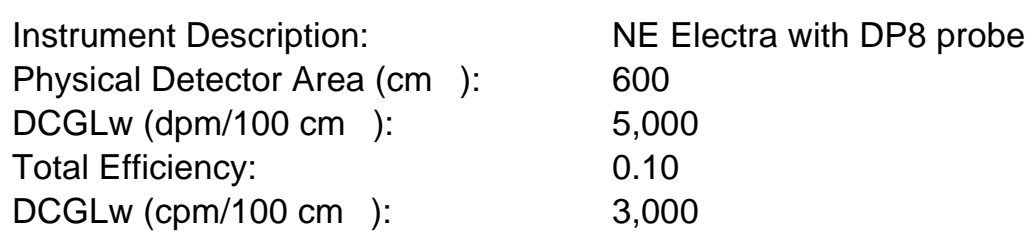

\begin{tabular}{|c|c|c|c|c|c|c|}
\hline Contaminant & HTDC $^{\mathbf{a}}$ & Energy $^{\mathbf{b}}$ & Fract $^{\mathbf{c}}$ & Inst. Eff. & Surf. Eff. & Total Eff. \\
\hline Cs-137 & No & 187.87 & 1.0 & 0.20 & 0.50 & 0.10 \\
\hline
\end{tabular}

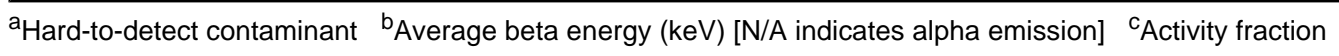

\section{Gross Alpha Mean and Sigma Data}

$\begin{array}{ll}\text { Count Time (min): } & 1 \\ \text { Sign Test Sigma }(\mathrm{cpm} / 100 \mathrm{~cm}): & 5.1\end{array}$

\begin{tabular}{|l|c|c|c|}
\hline Data/Material & Mean (cpm/100 cm ) & Std. Dev. (cpm/100 cm ) & MDC (dpm/100 cm ) \\
\hline SU & 10 & 4.7 & 98 \\
\hline Concrete & 4.3 & 2.0 & 70 \\
\hline
\end{tabular}

\section{Gross Beta Mean and Sigma Data}

\begin{tabular}{|c|c|c|c|}
\hline $\begin{array}{l}\text { Count Time (min): } \\
\text { Sign Test Sigma }(\mathrm{cpm} / 100 \mathrm{~cm}) \text { : }\end{array}$ & $\begin{array}{l}1 \\
196\end{array}$ & & \\
\hline Data/Material & Mean $(\mathrm{cpm} / 100 \mathrm{~cm}$ ) & Std. Dev. (cpm/100 cm ) & MDC (dpm/100 cm ) \\
\hline$\overline{\mathrm{SU}}$ & 1,069 & 178 & 258 \\
\hline Concrete & 1,041 & 83 & 255 \\
\hline
\end{tabular}

\section{Elevated Measurement Comparison (EMC) for Gross Alpha}

\begin{tabular}{ll}
\hline Scanning Instrumentation Description: & NE Electra with DP8 probe \\
Scan MDC*: & 67.63 \\
Area Factor Table Interpolation Method: & Linear
\end{tabular}

\section{Statistical Design}

$\mathrm{N}$ :

Bounded Area (m ):

Area Factor:

DCGLW*:

Scan MDC Required*:

\section{Hot Spot Design}

\begin{tabular}{|l|c|c|c|}
\hline act $^{\mathbf{c}}$ & Inst. Eff. & Surf. Eff. & Total Eff. \\
\hline 1.0 & 0.14 & 0.25 & 0.03 \\
\hline mission] & ${ }^{\mathrm{c}}$ Activity fraction & \\
\hline
\end{tabular}




\begin{tabular}{|c|c|c|c|}
\hline Contaminant & DCGLw $^{*}$ & Area Factor & Scan MDC Req'd $^{*}$ \\
\hline Pu-239 & 100 & 4.0 & 398 \\
\hline
\end{tabular}

* dpm/100 cm

\section{Elevated Measurement Comparison (EMC) for Gross Beta}

Scanning Instrumentation Description:

Background (cpm/100 cm ):

Total Scanning Efficiency:

True Positive Proportion:

False Positive Proportion:

Index of Sensitivity (d'):

Observation Interval (sec):

Surveyor Efficiency:

Area Factor Table Interpolation Method:
NE Electra with DP8 probe

1,041

0.10

0.9

0.05

2.92

10

0.50

Linear

\section{Statistical Design}

$\mathrm{N}$ :

Bounded Area (m ):

Area Factor:

DCGLw*:

Scan MDC Required*:

\section{1}

9.1

2.6

5,000

12,950
Hot Spot Design

Actual Scan MDC*:

Area Factor:

Bounded Area (m ): N/A

Post-EMC N:
3,255

N/A

11

\begin{tabular}{|c|c|c|c|c|c|}
\hline Contaminant & Energy $^{\mathbf{a}}$ & Fract $^{\mathbf{b}}$ & Inst. Eff. & Surf. Eff. & Total Eff. \\
\hline Cs-137 & 187.87 & 1.0 & 0.20 & 0.50 & 0.10 \\
\hline
\end{tabular}

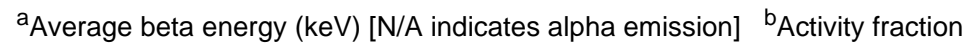

\begin{tabular}{|c|c|c|c|}
\hline Contaminant & DCGLw* $^{*}$ & Area Factor & Scan MDC Req'd $^{*}$ \\
\hline Cs-137 & 5,000 & 2.6 & 12,950 \\
\hline
\end{tabular}

* dpm/100 cm

Report Created 06/16/2010 0945 (COMPASS v1.1.0)

mhtml:file://C: $\backslash$ Documents and Settings $\backslash$ gwinjs $\backslash$ Desktop $\backslash E R \backslash P L U T O \backslash C o m p a s s$ Reports $\backslash$ Room 104.mht 


\section{BUILDING SURFACE SURVEY PLAN}

\section{Survey Plan Summary}

Site Name:

Planner(s):

Survey Unit Name:

Comments:
Pluto test

Gwin

test 104 ceiling

Average values used from 102,106, and 107

\section{Statistical Design Details}

\begin{tabular}{llll}
\hline Area $(\mathrm{m}):$ & 73 & Classification: & 2 \\
Selected Test: & Sign & Estimated Sigma (SOR): & 0.29 \\
DCGL (SOR): & 1 & Sample Size (N): & $\mathbf{1 1}$ \\
LBGR (SOR): & 0.26 & Estimated Conc. (SOR): & 0.33 \\
Alpha: & 0.050 & Estimated Power: & 1.0 \\
Beta: & 0.100 & &
\end{tabular}

NOTE: SOR = Sum-of-Ratios

\section{Prospective Power Curve}
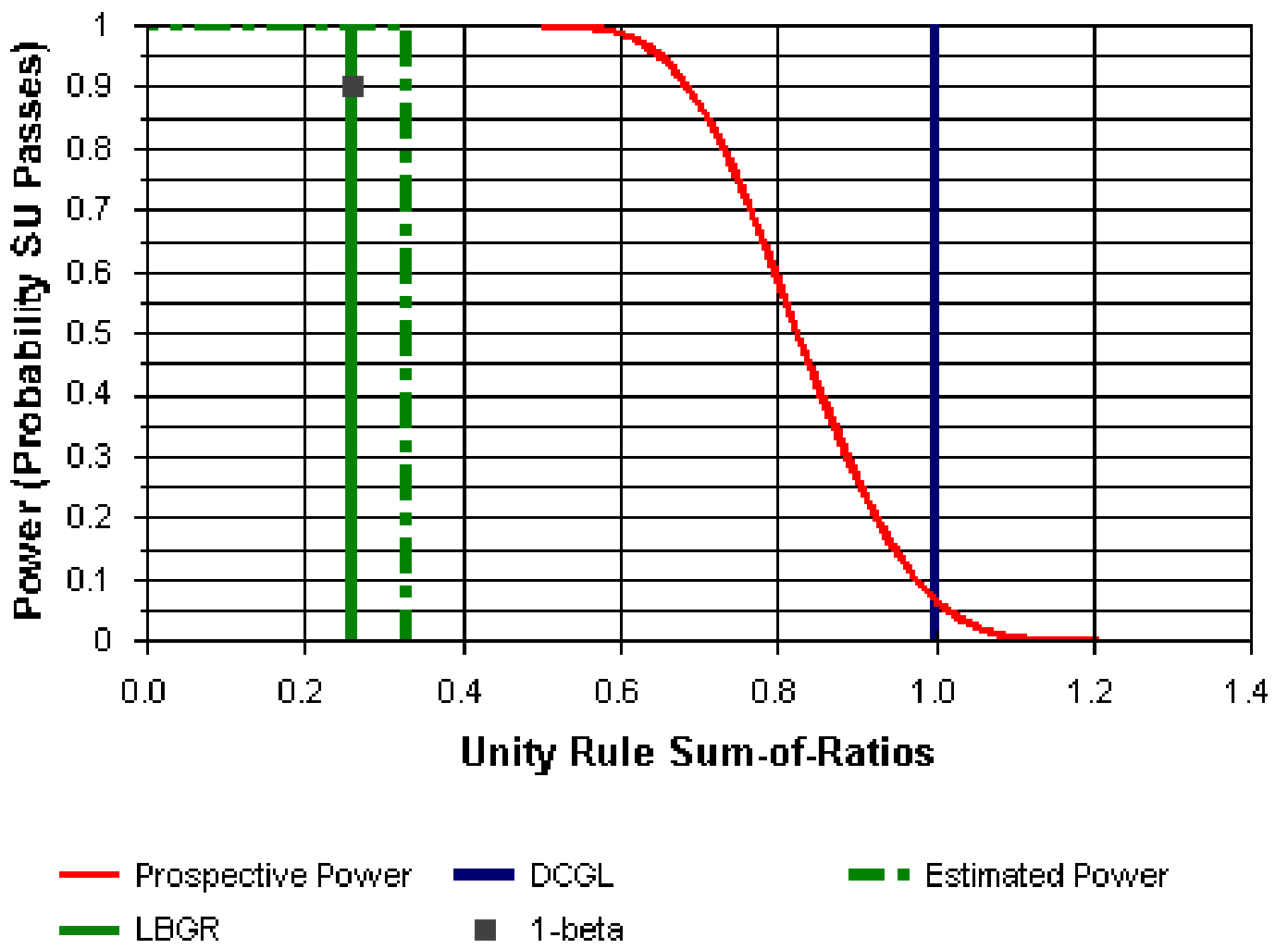


\section{Gross Alpha Efficiency Data}

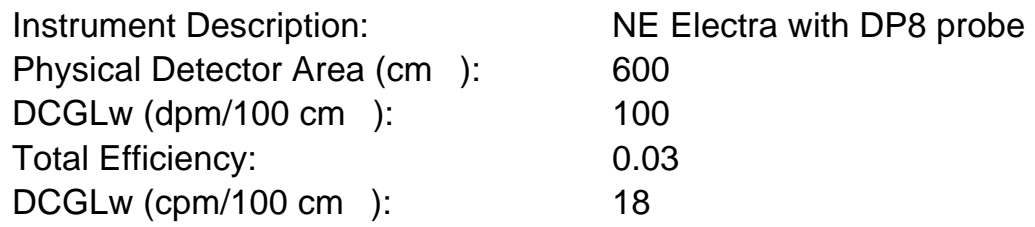

\begin{tabular}{|c|c|c|c|c|c|c|}
\hline Contaminant & HTDC $^{\mathbf{a}}$ & Energy $^{\mathbf{b}}$ & Fract $^{\mathbf{c}}$ & Inst. Eff. & Surf. Eff. & Total Eff. $^{\text {P }}$ \\
\hline Pu-239 & No & N/A & 1.0 & 0.14 & 0.25 & 0.03 \\
\hline
\end{tabular}

a Hard-to-detect contaminant ${ }^{\mathrm{b}}$ Average beta energy (keV) [N/A indicates alpha emission] ${ }^{\mathrm{c}}$ Activity fraction

\section{Gross Beta Efficiency Data}

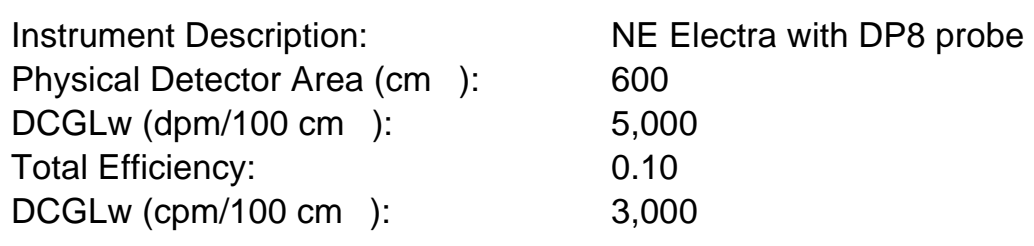

\begin{tabular}{|c|c|c|c|c|c|c|}
\hline Contaminant & HTDC $^{\mathbf{a}}$ & Energy $^{\mathbf{b}}$ & Fract $^{\mathbf{c}}$ & Inst. Eff. & Surf. Eff. & Total Eff. \\
\hline Cs-137 & No & 187.87 & 1.0 & 0.20 & 0.50 & 0.10 \\
\hline
\end{tabular}

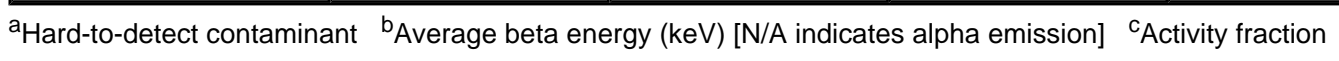

\section{Gross Alpha Mean and Sigma Data}

$\begin{array}{ll}\text { Count Time }(\mathrm{min}): & 1 \\ \text { Sign Test Sigma }(\mathrm{cpm} / 100 \mathrm{~cm}): & 5.1\end{array}$

\begin{tabular}{|l|c|c|c|}
\hline Data/Material & Mean (cpm/100 cm ) & Std. Dev. (cpm/100 cm ) & MDC (dpm/100 cm ) \\
\hline SU & 10 & 4.7 & 98 \\
\hline Concrete & 4.3 & 2.0 & 70 \\
\hline
\end{tabular}

\section{Gross Beta Mean and Sigma Data}

\begin{tabular}{|c|c|c|c|}
\hline $\begin{array}{l}\text { Count Time }(\mathrm{min}): \\
\text { Sign Test Sigma }(\mathrm{cpm} / 100 \mathrm{~cm}) \text { : }\end{array}$ & $\begin{array}{l}1 \\
197\end{array}$ & & \\
\hline Data/Material & Mean $(\mathrm{cpm} / 100 \mathrm{~cm})$ & Std. Dev. (cpm/100 cm ) & MDC (dpm/100 cm ) \\
\hline$S$ & 1,070 & 179 & 259 \\
\hline Concrete & 1,041 & 83 & 255 \\
\hline
\end{tabular}




\section{BUILDING SURFACE SURVEY PLAN}

\section{Survey Plan Summary}

Site Name:

Pluto test

Planner(s):

Gwin

Survey Unit Name:

test room 106

Comments:

N/A

\section{Statistical Design Details}

\begin{tabular}{llll}
\hline Area $(\mathrm{m}):$ & 100 & Classification: & 1 \\
Selected Test: & Sign & Estimated Sigma (SOR): & 0.24 \\
DCGL (SOR): & 1 & Sample Size (N): & $\mathbf{1 1}$ \\
LBGR (SOR): & 0.26 & Estimated Conc. (SOR): & 0.28 \\
Alpha: & 0.050 & Estimated Power: & 1.0 \\
Beta: & 0.100 & Post-EMC Sample Size (N): & $\mathbf{1 1}$
\end{tabular}

NOTE: SOR = Sum-of-Ratios

\section{Prospective Power Curve}

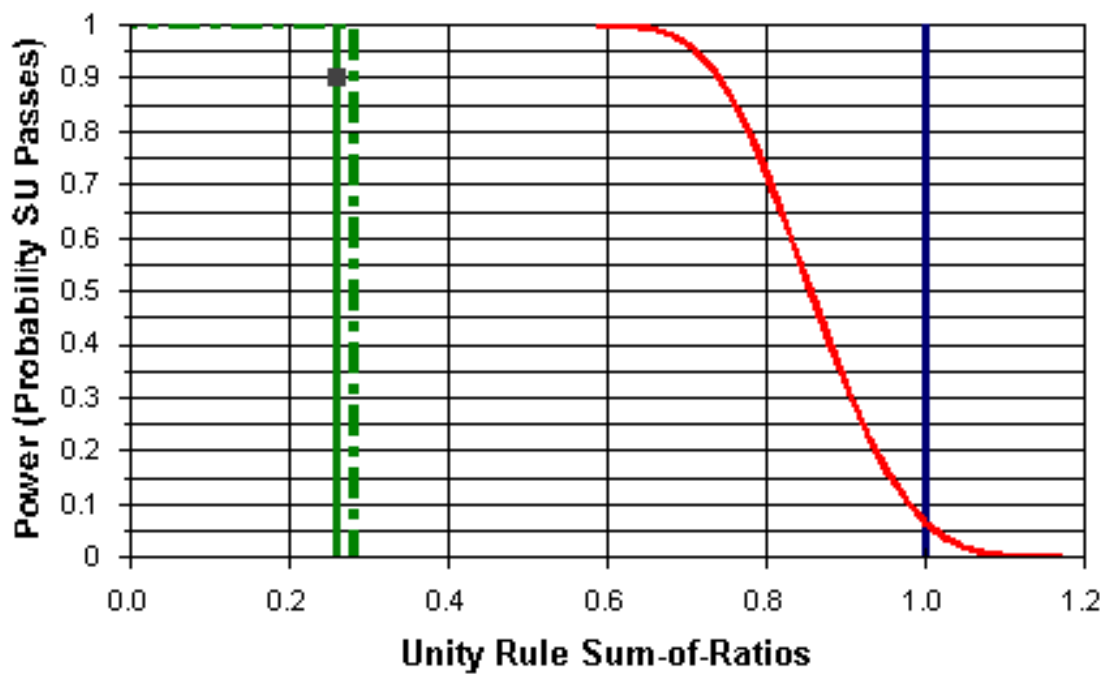

- Prospective Power $\quad$ DCGL
LBGR

- Estimated Power 


\section{Gross Alpha Efficiency Data}

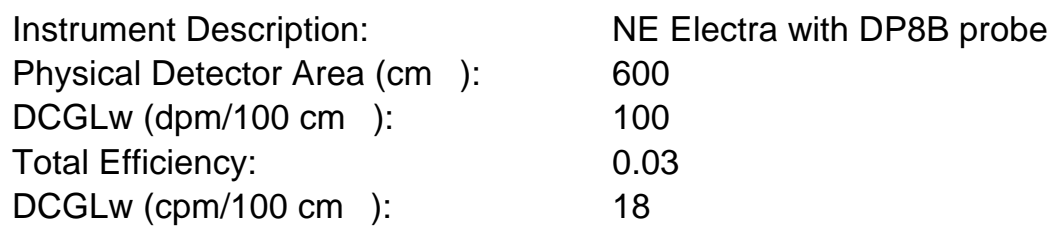

\begin{tabular}{|c|c|c|c|c|c|c|}
\hline Contaminant & HTDC $^{\mathbf{a}}$ & Energy $^{\mathbf{b}}$ & Fract $^{\mathbf{c}}$ & Inst. Eff. & Surf. Eff. & Total Eff. \\
\hline Pu-239 & No & N/A & 1.0 & 0.14 & 0.25 & 0.03 \\
\hline
\end{tabular}

a Hard-to-detect contaminant ${ }^{\mathrm{b}}$ Average beta energy (keV) [N/A indicates alpha emission] ${ }^{\mathrm{c}}$ Activity fraction

\section{Gross Beta Efficiency Data}

$\begin{array}{ll}\text { Instrument Description: } & \text { NE Electra with DP8B probe } \\ \text { Physical Detector Area (cm ): } & 600 \\ \text { DCGLw (dpm/100 cm ): } & 5,000 \\ \text { Total Efficiency: } & 0.10 \\ \text { DCGLw (cpm/100 cm ): } & 3,000\end{array}$

\begin{tabular}{|c|c|c|c|c|c|c|}
\hline Contaminant & HTDC $^{\mathbf{a}}$ & Energy $^{\mathbf{b}}$ & Fract $^{\mathbf{c}}$ & Inst. Eff. & Surf. Eff. & Total Eff. \\
\hline Cs-137 & No & 187.87 & 1.0 & 0.20 & 0.50 & 0.10 \\
\hline
\end{tabular}

${ }^{a}$ Hard-to-detect contaminant ${ }^{b}$ Average beta energy (keV) [N/A indicates alpha emission] ${ }^{\mathrm{c}}$ Activity fraction

\section{Gross Alpha Mean and Sigma Data}

Count Time (min):

Sign Test Sigma (cpm/100 cm ):
1

4.1

\begin{tabular}{|l|c|c|c|}
\hline Data/Material & Mean (cpm/100 cm ) & Std. Dev. (cpm/100 cm ) & MDC (dpm/100 cm ) \\
\hline SU & 9.6 & 3.6 & 97 \\
\hline Concrete & 4.3 & 2.0 & 70 \\
\hline
\end{tabular}

\section{Gross Beta Mean and Sigma Data}

Count Time $(\min ):$

Sign Test Sigma (cpm/100 cm ): 178

\begin{tabular}{|l|c|c|c|}
\hline Data/Material & Mean (cpm/100 cm ) & Std. Dev. (cpm/100 cm ) & MDC (dpm/100 cm ) \\
\hline SU & 991 & 158 & 249 \\
\hline Concrete & 1,041 & 83 & 255 \\
\hline
\end{tabular}

\section{Elevated Measurement Comparison (EMC) for Gross Alpha}

Scanning Instrumentation Description:

Scan MDC*:

Area Factor Table Interpolation Method:

\section{Statistical Design}

$\mathrm{N}$ :

Bounded Area (m ):

Area Factor:

DCGLw*:

Scan MDC Required*:

mhtml:file://C: Documents and Settings $\backslash$ gwinjs $\backslash$ Desktop $\backslash E R \backslash P L U T O \backslash C o m p a s s$ Reports $\backslash$ Room 106.mht

\section{Hot Spot Design}

Actual Scan MDC*:

71

N/A

N/A

11 


\begin{tabular}{|c|c|c|c|}
\hline Contaminant & DCGLw $^{*}$ & Area Factor & Scan MDC Req'd $^{*}$ \\
\hline Pu-239 & 100 & 4.0 & 398 \\
\hline
\end{tabular}

* dpm/100 cm

\section{Elevated Measurement Comparison (EMC) for Gross Beta}

Scanning Instrumentation Description:

Background (cpm/100 cm ):

Total Scanning Efficiency:

True Positive Proportion:

False Positive Proportion:

Index of Sensitivity (d'):

Observation Interval (sec):

Surveyor Efficiency:

Area Factor Table Interpolation Method:
NE Electra with DP8B probe

1,041

0.10

0.9

0.05

2.92

10

0.50

Linear

\section{Statistical Design}

$\mathrm{N}$ :

Bounded Area (m ):

Area Factor:

DCGLw*:

Scan MDC Required*:
11

9.1

2.6

5,000

12,950
Hot Spot Design

Actual Scan MDC*:

Area Factor:

Bounded Area (m ): N/A

Post-EMC N:
3,264

N/A

11

\begin{tabular}{|c|c|c|c|c|c|}
\hline Contaminant & Energy $^{\mathbf{a}}$ & Fract $^{\mathbf{b}}$ & Inst. Eff. & Surf. Eff. & Total Eff. \\
\hline Cs-137 & 187.87 & 1.0 & 0.20 & 0.50 & 0.10 \\
\hline
\end{tabular}

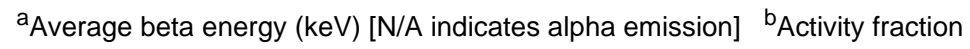

\begin{tabular}{|c|c|c|c|}
\hline Contaminant & DCGLw* $^{*}$ & Area Factor & Scan MDC Req'd $^{*}$ \\
\hline Cs-137 & 5,000 & 2.6 & 12,950 \\
\hline
\end{tabular}

* dpm/100 cm

Report Created 06/16/2010 0850 (COMPASS v1.1.0)

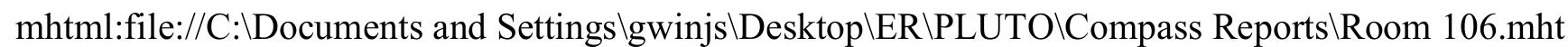




\section{BUILDING SURFACE SURVEY PLAN}

\section{Survey Plan Summary}

Site Name:

Planner(s):

Survey Unit Name:

Comments:
Pluto test

Gwin

test 106 ceiling

N/A

\section{Statistical Design Details}

\begin{tabular}{llll}
\hline Area $(\mathrm{m}):$ & 73 & Classification: & 2 \\
Selected Test: & Sign & Estimated Sigma (SOR): & 0.24 \\
DCGL (SOR): & 1 & Sample Size (N): & $\mathbf{1 1}$ \\
LBGR (SOR): & 0.26 & Estimated Conc. (SOR): & 0.28 \\
Alpha: & 0.050 & Estimated Power: & 1.0 \\
Beta: & 0.100 & &
\end{tabular}

NOTE: SOR = Sum-of-Ratios

\section{Prospective Power Curve}

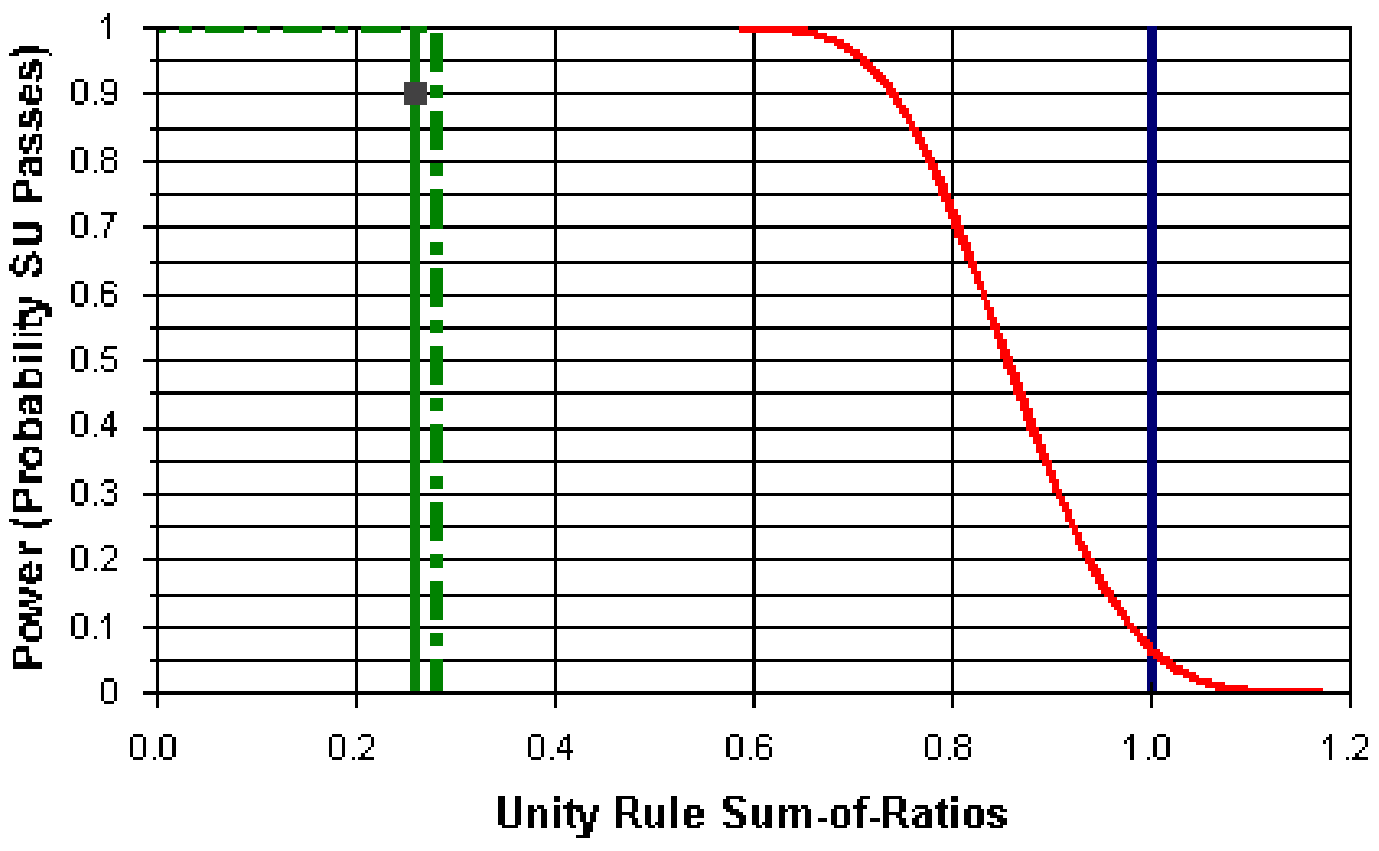

$\begin{array}{ll}\text { - Prospective Power } & \text { DCGL } \\ \text { LBGR } & \text { 1-beta }\end{array}$ 


\section{Gross Alpha Efficiency Data}

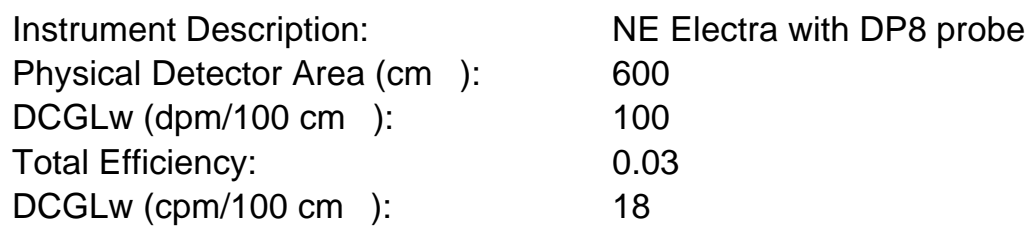

\begin{tabular}{|c|c|c|c|c|c|c|}
\hline Contaminant & HTDC $^{\mathbf{a}}$ & Energy $^{\mathbf{b}}$ & Fract $^{\mathbf{c}}$ & Inst. Eff. & Surf. Eff. & Total Eff. $^{\text {P }}$ \\
\hline Pu-239 & No & N/A & 1.0 & 0.14 & 0.25 & 0.03 \\
\hline
\end{tabular}

a Hard-to-detect contaminant ${ }^{\mathrm{b}}$ Average beta energy (keV) [N/A indicates alpha emission] ${ }^{\mathrm{c}}$ Activity fraction

\section{Gross Beta Efficiency Data}

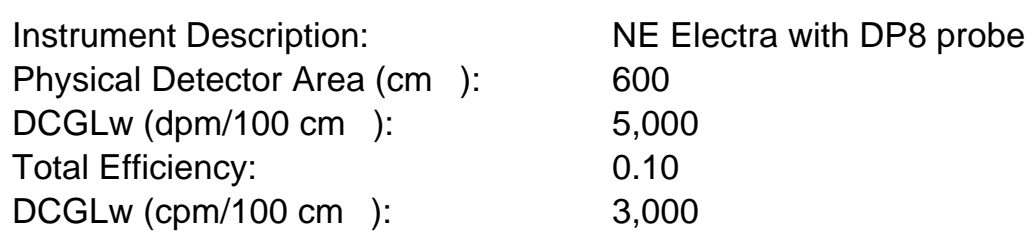

\begin{tabular}{|c|c|c|c|c|c|c|}
\hline Contaminant & HTDC $^{\mathbf{a}}$ & Energy $^{\mathbf{b}}$ & Fract $^{\mathbf{c}}$ & Inst. Eff. & Surf. Eff. & Total Eff. \\
\hline Cs-137 & No & 187.87 & 1.0 & 0.20 & 0.50 & 0.10 \\
\hline
\end{tabular}

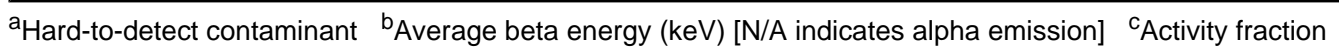

\section{Gross Alpha Mean and Sigma Data}

$\begin{array}{ll}\text { Count Time }(\mathrm{min}): & 1 \\ \text { Sign Test Sigma }(\mathrm{cpm} / 100 \mathrm{~cm}): & 4.1\end{array}$

\begin{tabular}{|l|c|c|c|}
\hline Data/Material & Mean (cpm/100 cm ) & Std. Dev. (cpm/100 cm ) & MDC (dpm/100 cm ) \\
\hline SU & 9.6 & 3.6 & 97 \\
\hline Concrete & 4.3 & 2.0 & 70 \\
\hline
\end{tabular}

\section{Gross Beta Mean and Sigma Data}

\begin{tabular}{|c|c|c|c|}
\hline $\begin{array}{l}\text { Count Time (min): } \\
\text { Sian Test Siama }(\mathrm{cnm} / 100 \mathrm{~cm} \text { ). }\end{array}$ & $\begin{array}{l}1 \\
178\end{array}$ & & \\
\hline Data/Material & Mean $(\mathrm{cpm} / 100 \mathrm{~cm})$ & Std. Dev. (cpm/100 cm ) & MDC (dpm/100 cm ) \\
\hline SU & 991 & 158 & 249 \\
\hline Concrete & 1,041 & 83 & 255 \\
\hline
\end{tabular}




\section{BUILDING SURFACE SURVEY PLAN}

\section{Survey Plan Summary}

Site Name:

Pluto test

Planner(s):

Gwin

Survey Unit Name:

test room 107

Comments:

N/A

\section{Statistical Design Details}

\begin{tabular}{llll}
\hline Area $(\mathrm{m}):$ & 100 & Classification: & 1 \\
Selected Test: & Sign & Estimated Sigma (SOR): & 0.46 \\
DCGL (SOR): & 1 & Sample Size (N): & $\mathbf{1 6}$ \\
LBGR (SOR): & 0.36 & Estimated Conc. (SOR): & 0.43 \\
Alpha: & 0.050 & Estimated Power: & 1.00 \\
Beta: & 0.100 & Post-EMC Sample Size (N): & $\mathbf{1 6}$
\end{tabular}

NOTE: SOR = Sum-of-Ratios

\section{Prospective Power Curve}

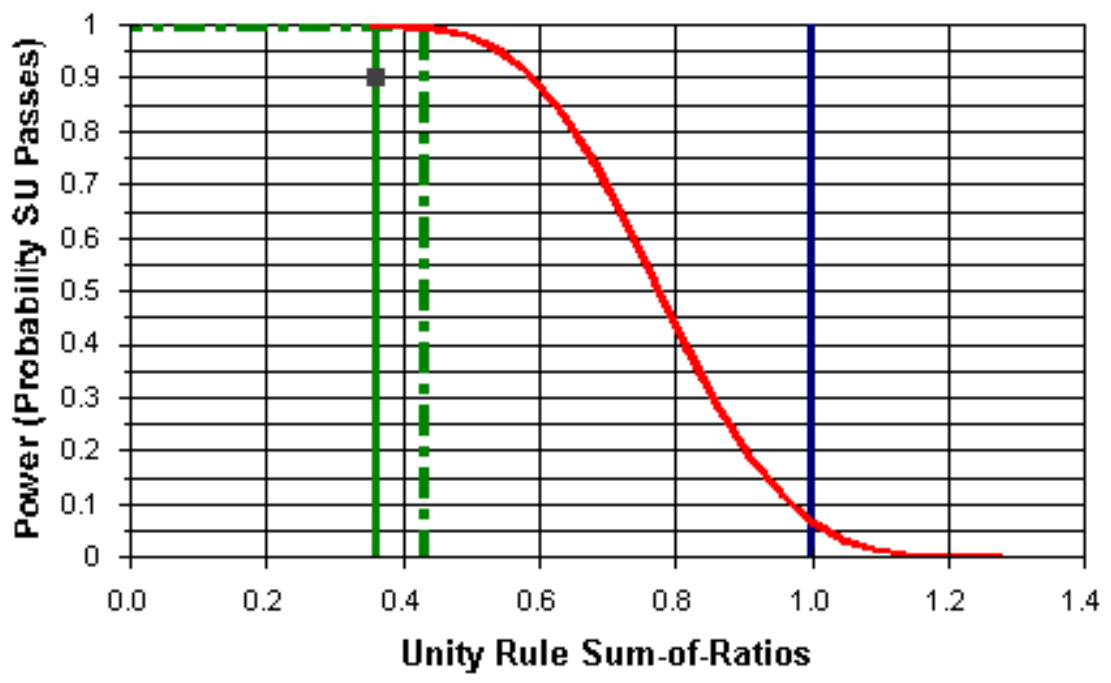

- Prospective Power $\quad$ DCGL
LBGR

- Estimated Power 


\section{Gross Alpha Efficiency Data}

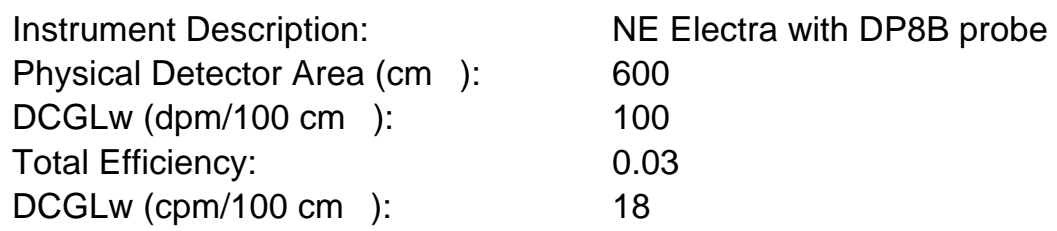

\begin{tabular}{|c|c|c|c|c|c|c|}
\hline Contaminant & HTDC $^{\mathbf{a}}$ & Energy $^{\mathbf{b}}$ & Fract $^{\mathbf{c}}$ & Inst. Eff. & Surf. Eff. & Total Eff. \\
\hline Pu-239 & No & N/A & 1.0 & 0.13 & 0.25 & 0.03 \\
\hline
\end{tabular}

a Hard-to-detect contaminant ${ }^{\mathrm{b}}$ Average beta energy (keV) [N/A indicates alpha emission] ${ }^{\mathrm{c}}$ Activity fraction

\section{Gross Beta Efficiency Data}

Instrument Description:

Physical Detector Area (cm ):

DCGLw (dpm/100 cm ):

Total Efficiency:

DCGLw (cpm/100 cm ):
NE Electra with DP8B probe

600

5,000

0.10

3,000

\begin{tabular}{|c|c|c|c|c|c|c|}
\hline Contaminant & HTDC $^{\mathbf{a}}$ & Energy $^{\mathbf{b}}$ & Fract $^{\mathbf{c}}$ & Inst. Eff. & Surf. Eff. $^{\text {Total Eff. }}$ \\
\hline Cs-137 & No & 187.87 & 1.0 & 0.20 & 0.50 & 0.10 \\
\hline
\end{tabular}

a Hard-to-detect contaminant ${ }^{b}$ Average beta energy (keV) [N/A indicates alpha emission] ${ }^{c}$ Activity fraction

\section{Gross Alpha Mean and Sigma Data}

Count Time (min):

Sign Test Sigma $(\mathrm{cpm} / 100 \mathrm{~cm})$ :
1

8.2

\begin{tabular}{|l|c|c|c|}
\hline Data/Material & Mean (cpm/100 cm ) & Std. Dev. (cpm/100 cm ) & MDC (dpm/100 cm ) \\
\hline SU & 12 & 7.9 & 106 \\
\hline Concrete & 4.3 & 2.0 & 70 \\
\hline
\end{tabular}

\section{Gross Beta Mean and Sigma Data}

Count Time (min): 1

Sign Test Sigma (cpm/100 cm ): 172

\begin{tabular}{|l|c|c|c|}
\hline Data/Material & Mean (cpm/100 cm ) & Std. Dev. (cpm/100 cm ) & MDC (dpm/100 cm ) \\
\hline SU & 1,045 & 151 & 256 \\
\hline Concrete & 1,041 & 83 & 255 \\
\hline
\end{tabular}

\section{Elevated Measurement Comparison (EMC) for Gross Alpha}

Scanning Instrumentation Description:

Scan MDC*:

Area Factor Table Interpolation Method:

Statistical Design

N:

Bounded Area (m ):

Area Factor:

DCGLw*:

Scan MDC Required*:

mhtml:file://C: Documents and Settings \gwinjs $\backslash$ Desktop $\backslash E R \backslash P L U T O \backslash$ Compass Reports $\backslash$ Room $107 . m h t$

\section{Hot Spot Design}

Actual Scan MDC*:

78

N/A

N/A

16 


\begin{tabular}{|c|c|c|c|}
\hline Contaminant & DCGLw $^{*}$ & Area Factor & Scan MDC Req'd ${ }^{*}$ \\
\hline Pu-239 & 100 & 6.7 & 670 \\
\hline
\end{tabular}

* dpm/100 cm

\section{Elevated Measurement Comparison (EMC) for Gross Beta}

Scanning Instrumentation Description:

Background (cpm/100 cm ):

Total Scanning Efficiency:

True Positive Proportion:

False Positive Proportion:

Index of Sensitivity (d'):

Observation Interval (sec):

Surveyor Efficiency:

Area Factor Table Interpolation Method:
NE Electra with DP8B probe

1,041

0.10

0.9

0.05

2.92

8.3

0.50

Linear

\section{Statistical Design}

$\mathrm{N}$ :

Bounded Area (m ):

Area Factor:

DCGLW*:

Scan MDC Required*:
16

6.3

4.3

5,000

21,350
Hot Spot Design

Actual Scan MDC*:

Area Factor:

Bounded Area (m ): N/A

Post-EMC N: $\quad 16$

\begin{tabular}{|c|c|c|c|c|c|}
\hline Contaminant & Energy $^{\mathbf{a}}$ & Fract $^{\mathbf{b}}$ & Inst. Eff. & Surf. Eff. & Total Eff. \\
\hline Cs-137 & 187.87 & 1.0 & 0.20 & 0.50 & 0.10 \\
\hline
\end{tabular}

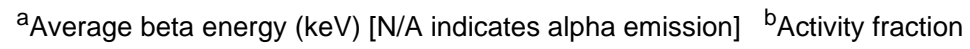

\begin{tabular}{|c|c|c|c|}
\hline Contaminant & DCGLw $^{*}$ & Area Factor & Scan MDC Req'd ${ }^{*}$ \\
\hline Cs-137 & 5,000 & 4.3 & 21,350 \\
\hline
\end{tabular}

${ }^{*} \mathrm{dpm} / 100 \mathrm{~cm}$

Report Created 06/16/2010 0807 (COMPASS v1.1.0)

mhtml:file://C: $\backslash$ Documents and Settings $\backslash$ gwinjs $\backslash$ Desktop $\backslash E R \backslash P L U T O \backslash C o m p a s s$ Reports $\backslash$ Room 107.mht 


\section{BUILDING SURFACE SURVEY PLAN}

\section{Survey Plan Summary}

Site Name:

Planner(s):

Survey Unit Name:

Comments:
Pluto test

Gwin

test 107 ceiling

N/A

\section{Statistical Design Details}

\begin{tabular}{llll}
\hline Area $(\mathrm{m}):$ & 81 & Classification: & 2 \\
Selected Test: & Sign & Estimated Sigma (SOR): & 0.46 \\
DCGL (SOR): & 1 & Sample Size (N): & $\mathbf{1 6}$ \\
LBGR (SOR): & 0.36 & Estimated Conc. (SOR): & 0.43 \\
Alpha: & 0.050 & Estimated Power: & 1.00 \\
Beta: & 0.100 & &
\end{tabular}

NOTE: SOR = Sum-of-Ratios

\section{Prospective Power Curve}

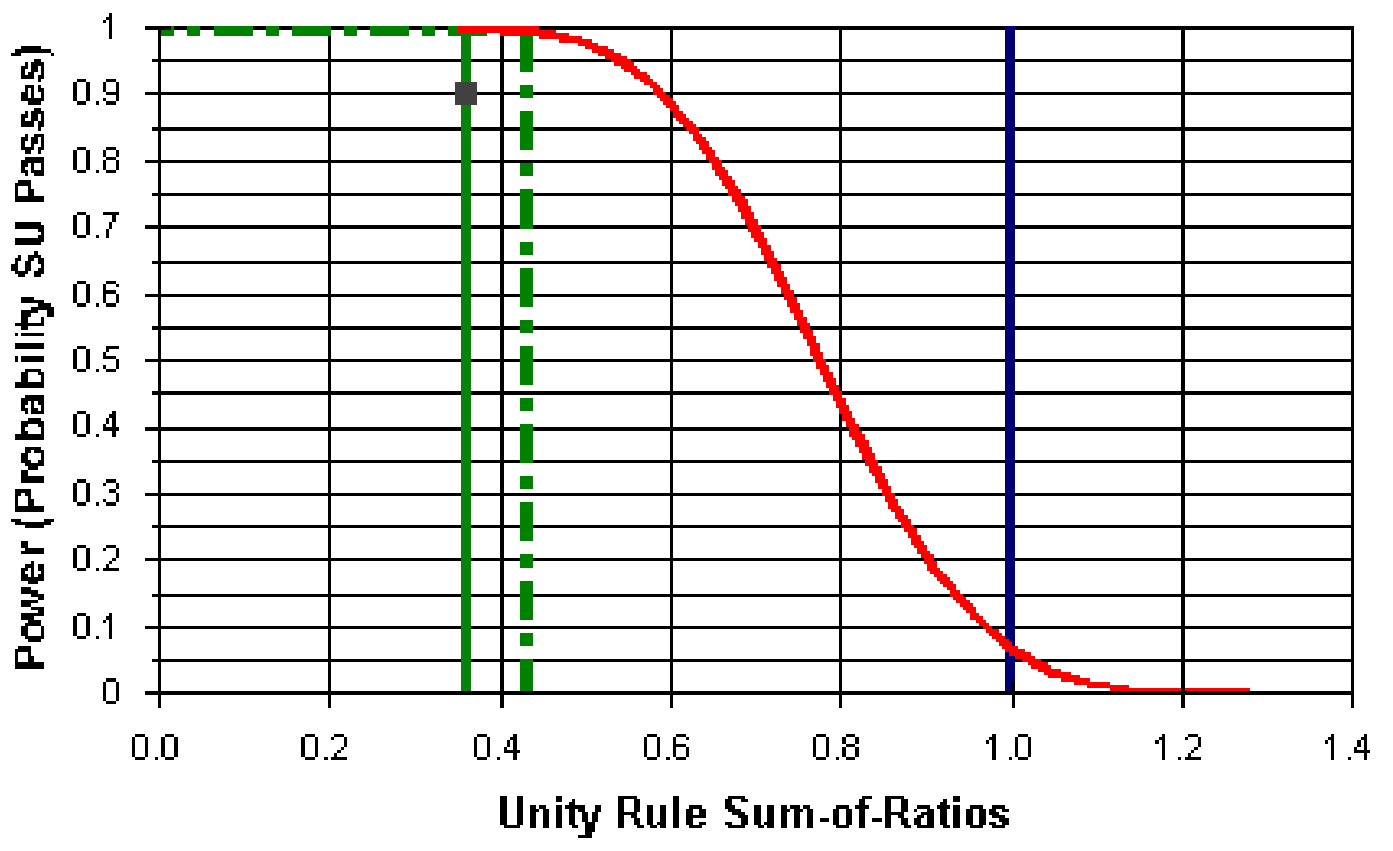

$\begin{array}{ll}\text { - Prospective Power } & \text { DCGL } \\ \text { LBGR } & \text { 1-beta }\end{array}$ 


\section{Gross Alpha Efficiency Data}

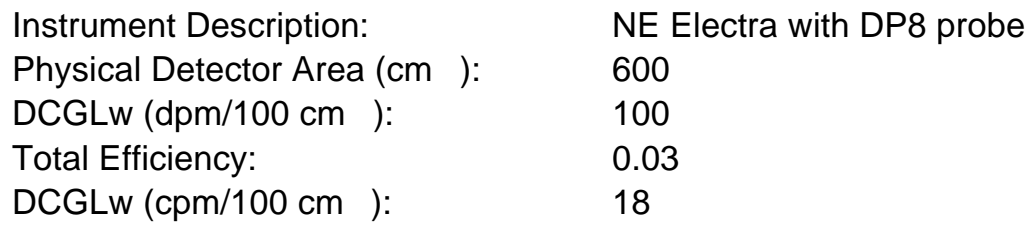

\begin{tabular}{|c|c|c|c|c|c|c|}
\hline Contaminant & HTDC $^{\mathbf{a}}$ & Energy $^{\mathbf{b}}$ & Fract $^{\mathbf{c}}$ & Inst. Eff. & Surf. Eff. & Total Eff. $^{\text {P }}$ \\
\hline Pu-239 & No & N/A & 1.0 & 0.14 & 0.25 & 0.03 \\
\hline
\end{tabular}

a Hard-to-detect contaminant ${ }^{\mathrm{b}}$ Average beta energy (keV) [N/A indicates alpha emission] ${ }^{\mathrm{c}}$ Activity fraction

\section{Gross Beta Efficiency Data}

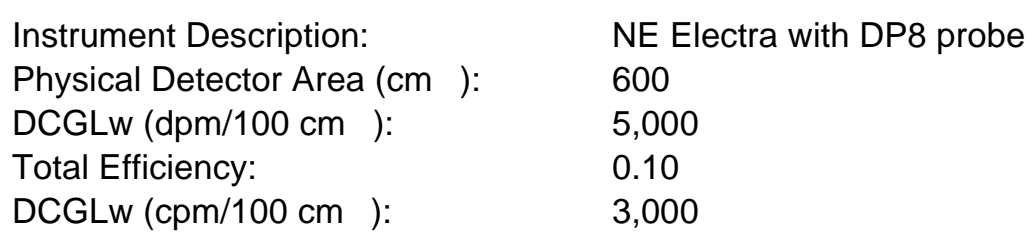

\begin{tabular}{|c|c|c|c|c|c|c|}
\hline Contaminant & HTDC $^{\mathbf{a}}$ & Energy $^{\mathbf{b}}$ & Fract $^{\mathbf{c}}$ & Inst. Eff. & Surf. Eff. & Total Eff. \\
\hline Cs-137 & No & 187.87 & 1.0 & 0.20 & 0.50 & 0.10 \\
\hline
\end{tabular}

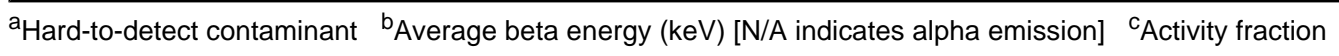

\section{Gross Alpha Mean and Sigma Data}

$\begin{array}{ll}\text { Count Time (min): } & 1 \\ \text { Sign Test Sigma (cpm/100 cm }): & 8.2\end{array}$

\begin{tabular}{|l|c|c|c|}
\hline Data/Material & Mean $(\mathbf{c p m} / \mathbf{1 0 0} \mathbf{~ c m ~})$ & Std. Dev. (cpm/100 cm $)$ & MDC (dpm/100 cm ) \\
\hline SU & 12 & 7.9 & 106 \\
\hline Concrete & 4.3 & 2.0 & 70 \\
\hline
\end{tabular}

\section{Gross Beta Mean and Sigma Data}

\begin{tabular}{|c|c|c|c|}
\hline $\begin{array}{l}\text { Count Time (min): } \\
\text { Sign Test Sigma }(\mathrm{cpm} / 100 \mathrm{~cm} \text { ): }\end{array}$ & $\begin{array}{l}1 \\
172\end{array}$ & & \\
\hline Data/Material & Mean (cpm/100 cm ) & Std. Dev. (cpm/100 cm ) & MDC (dpm/100 cm ) \\
\hline SU & 1,045 & 151 & 256 \\
\hline Concrete & 1,041 & 83 & 255 \\
\hline
\end{tabular}




\section{BUILDING SURFACE SURVEY PLAN}

\section{Survey Plan Summary}

Site Name:

Planner(s):

Survey Unit Name:

Comments:
Pluto test

Gwin

Room 102, 104, 106 ledges

average values used

\section{Statistical Design Details}

\begin{tabular}{llll}
\hline Area $(\mathrm{m}):$ & 100 & Classification: & 1 \\
Selected Test: & Sign & Estimated Sigma (SOR): & 0.29 \\
DCGL (SOR): & 1 & Sample Size (N): & $\mathbf{1 1}$ \\
LBGR (SOR): & 0.26 & Estimated Conc. (SOR): & 0.33 \\
Alpha: & 0.050 & Estimated Power: & 1.0 \\
Beta: & 0.100 & Post-EMC Sample Size (N): & $\mathbf{1 1}$
\end{tabular}

NOTE: SOR = Sum-of-Ratios

\section{Prospective Power Curve}

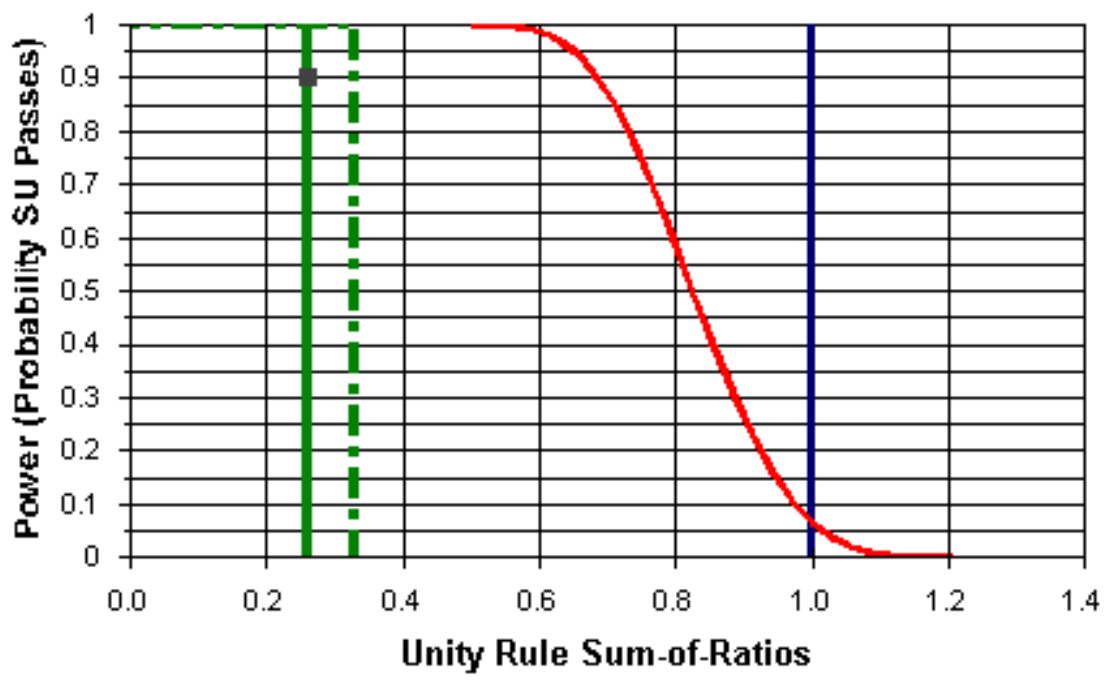

- Prospective Power $\quad$ DCGL
LBGR

- Estimated Power 


\section{Gross Alpha Efficiency Data}

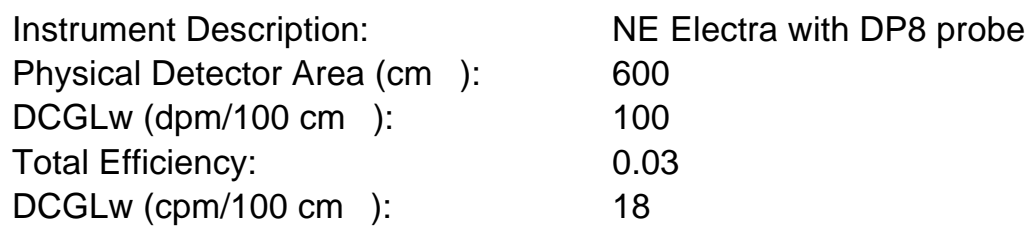

\begin{tabular}{|c|c|c|c|c|c|c|}
\hline Contaminant & HTDC $^{\mathbf{a}}$ & Energy $^{\mathbf{b}}$ & Fract $^{\mathbf{c}}$ & Inst. Eff. & Surf. Eff. & Total Eff. \\
\hline Pu-239 & No & N/A & 1.0 & 0.14 & 0.25 & 0.03 \\
\hline
\end{tabular}

a Hard-to-detect contaminant ${ }^{\mathrm{b}}$ Average beta energy (keV) [N/A indicates alpha emission] ${ }^{\mathrm{c}}$ Activity fraction

\section{Gross Beta Efficiency Data}

Instrument Description:

Physical Detector Area (cm ):

DCGLw (dpm/100 cm ):

Total Efficiency:

DCGLw (cpm/100 cm ):
NE Electra with DP8 probe

600

5,000

0.10

3,000

\begin{tabular}{|c|c|c|c|c|c|c|}
\hline Contaminant & HTDC $^{\mathbf{a}}$ & Energy $^{\mathbf{b}}$ & Fract $^{\mathbf{c}}$ & Inst. Eff. & Surf. Eff. $^{\text {Total Eff. }}$ \\
\hline Cs-137 & No & 187.87 & 1.0 & 0.20 & 0.50 & 0.10 \\
\hline
\end{tabular}

a Hard-to-detect contaminant ${ }^{b}$ Average beta energy (keV) [N/A indicates alpha emission] ${ }^{c}$ Activity fraction

\section{Gross Alpha Mean and Sigma Data}

Count Time (min):

Sign Test Sigma $(\mathrm{cpm} / 100 \mathrm{~cm})$ :
1

5.1

\begin{tabular}{|l|c|c|c|}
\hline Data/Material & Mean (cpm/100 cm ) & Std. Dev. (cpm/100 cm ) & MDC (dpm/100 cm ) \\
\hline SU & 10 & 4.7 & 98 \\
\hline Concrete & 4.3 & 2.0 & 70 \\
\hline
\end{tabular}

\section{Gross Beta Mean and Sigma Data}

Count Time (min): 1

Sign Test Sigma (cpm/100 cm ): 196

\begin{tabular}{|l|c|c|c|}
\hline Data/Material & Mean (cpm/100 cm ) & Std. Dev. (cpm/100 cm ) & MDC (dpm/100 cm ) \\
\hline SU & 1,069 & 178 & 258 \\
\hline Concrete & 1,041 & 83 & 255 \\
\hline
\end{tabular}

\section{Elevated Measurement Comparison (EMC) for Gross Alpha}

Scanning Instrumentation Description:

Scan MDC*:

Area Factor Table Interpolation Method:

Statistical Design

N:

Bounded Area (m ):

Area Factor:

DCGLW*:

Scan MDC Required*:

file://C:\Program Files $\backslash$ COMPASS $\backslash C O M P A S S$ Report.htm

67.63

Linear
NE Electra with DP8 probe

\section{Hot Spot Design}

\begin{tabular}{l|l|}
\hline Std. Dev. (cpm/100 cm ) & MDC (dpm/100 cm ) \\
\hline 178 & 258 \\
\hline 83 & 255 \\
\hline \multirow{2}{*}{ Alpha } \\
Hot Spot Design
\end{tabular}

Actual Scan MDC*: $\quad 68$

Area Factor: N/A

Bounded Area (m ): $\quad$ N/A

Post-EMC N: $\quad 11$ 


\begin{tabular}{|c|c|c|c|}
\hline Contaminant & DCGLw $^{*}$ & Area Factor & Scan MDC Req'd $^{*}$ \\
\hline Pu-239 & 100 & 4.0 & 398 \\
\hline
\end{tabular}

* dpm/100 cm

\section{Elevated Measurement Comparison (EMC) for Gross Beta}

Scanning Instrumentation Description:

Background (cpm/100 cm ):

Total Scanning Efficiency:

True Positive Proportion:

False Positive Proportion:

Index of Sensitivity (d'):

Observation Interval (sec):

Surveyor Efficiency:

Area Factor Table Interpolation Method:
NE Electra with DP8 probe

1,041

0.10

0.9

0.05

2.92

10

0.50

Linear

\section{Statistical Design}

$\mathrm{N}$ :

Bounded Area (m ):

Area Factor:

DCGLw*:

Scan MDC Required*:
11

9.1

2.6

5,000

12,950
Hot Spot Design

Actual Scan MDC*:

Area Factor:

Bounded Area (m ): N/A

Post-EMC N:
3,264

N/A

11

\begin{tabular}{|c|c|c|c|c|c|}
\hline Contaminant & Energy $^{\mathbf{a}}$ & Fract $^{\mathbf{b}}$ & Inst. Eff. & Surf. Eff. & Total Eff. \\
\hline Cs-137 & 187.87 & 1.0 & 0.20 & 0.50 & 0.10 \\
\hline
\end{tabular}

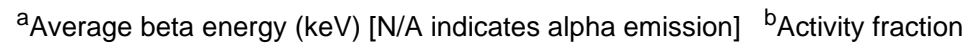

\begin{tabular}{|c|c|c|c|}
\hline Contaminant & DCGLw* $^{*}$ & Area Factor & Scan MDC Req'd $^{*}$ \\
\hline Cs-137 & 5,000 & 2.6 & 12,950 \\
\hline
\end{tabular}

* dpm/100 cm

Report Created 06/17/2010 0732 (COMPASS v1.1.0) 
Facility, Building 2201

\section{Appendix E}

Survey Maps

43 Pages 


\section{Survey \#}

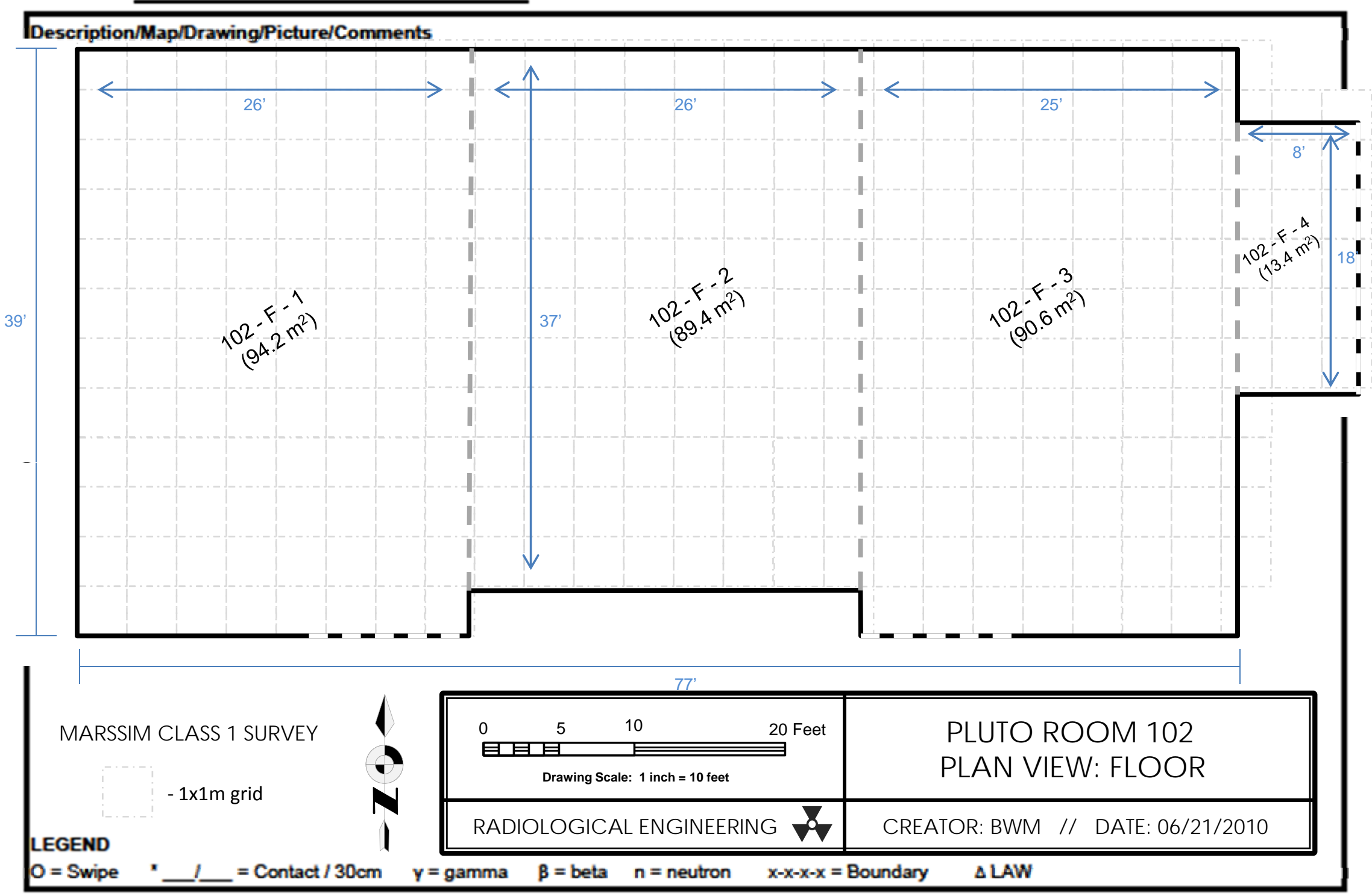


Survey \#

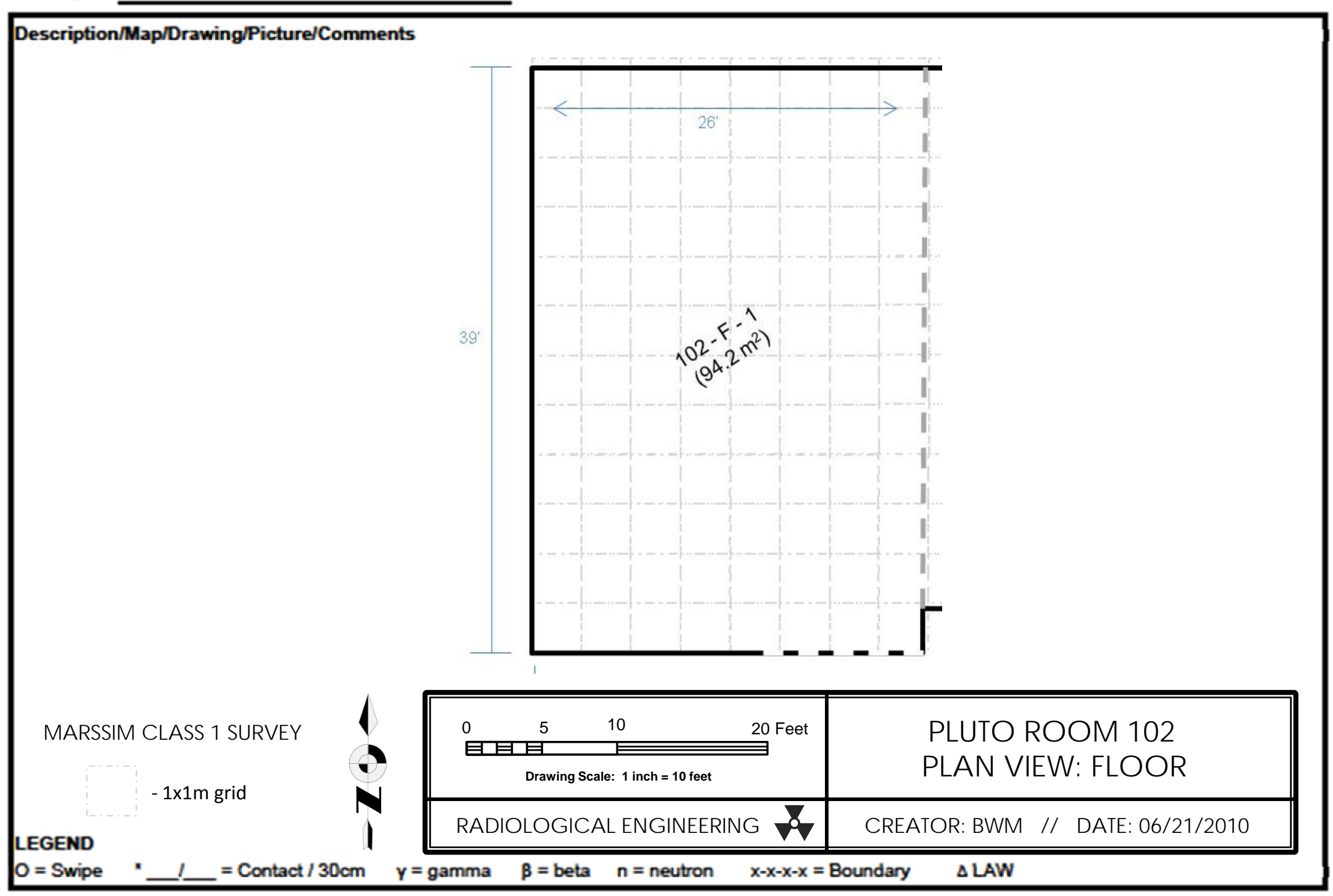


Survey \#

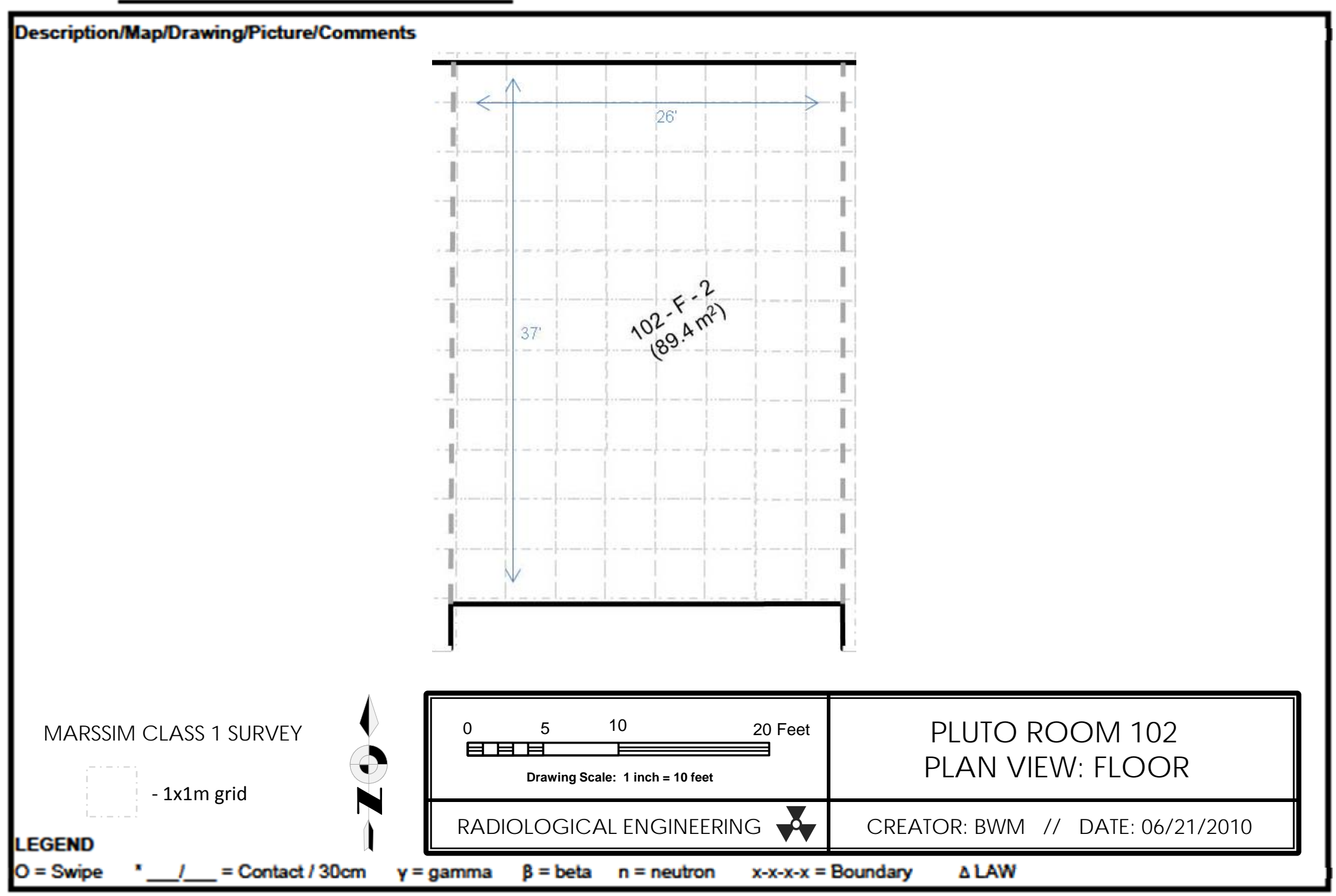


Survey \#

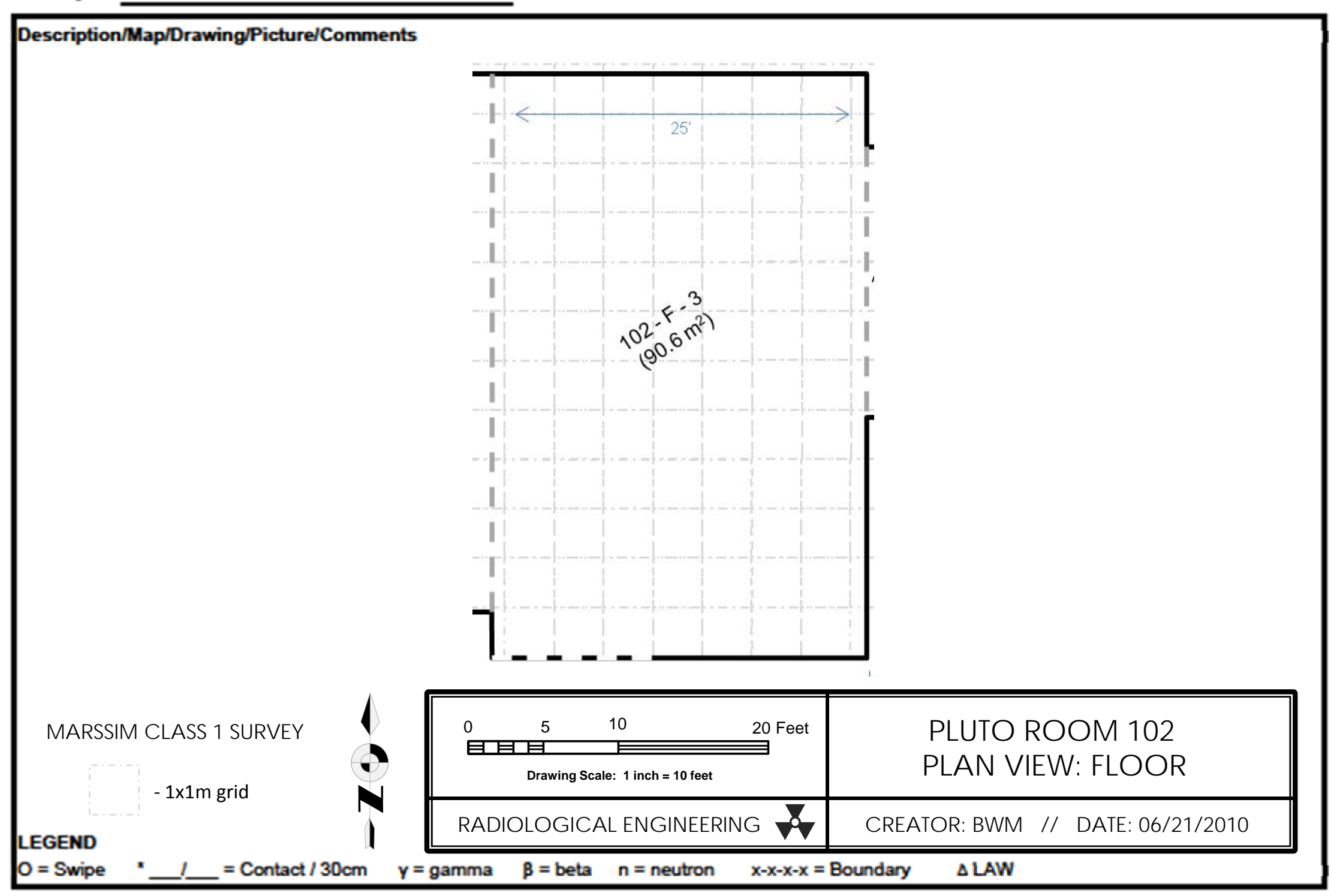


Survey \#

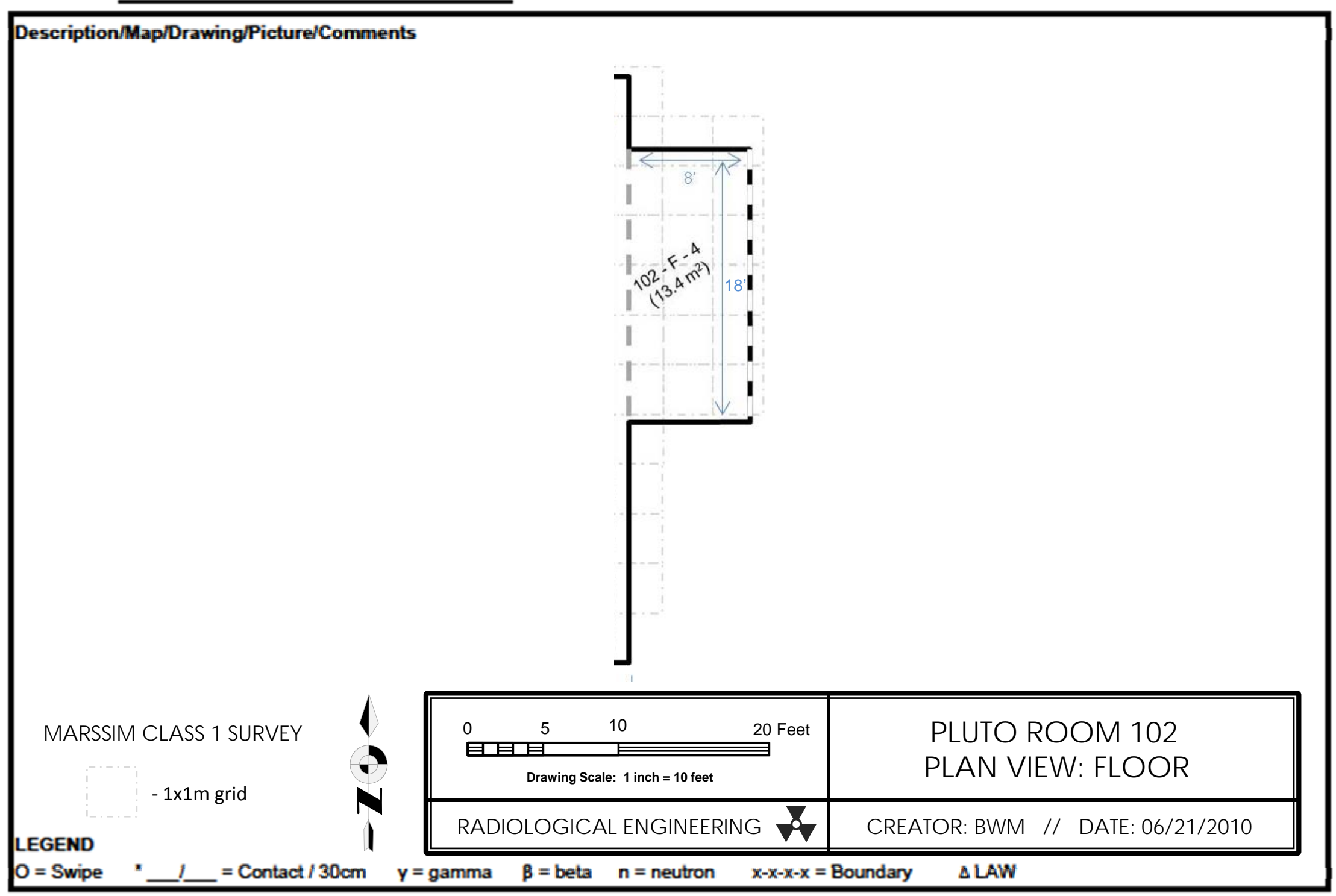


NSTec

Form

05/24/10

Rev. 01

FRM-0108C

RADIOLOGICAL SURVEY REPORT - FULL MAP

Page of

Survey \#

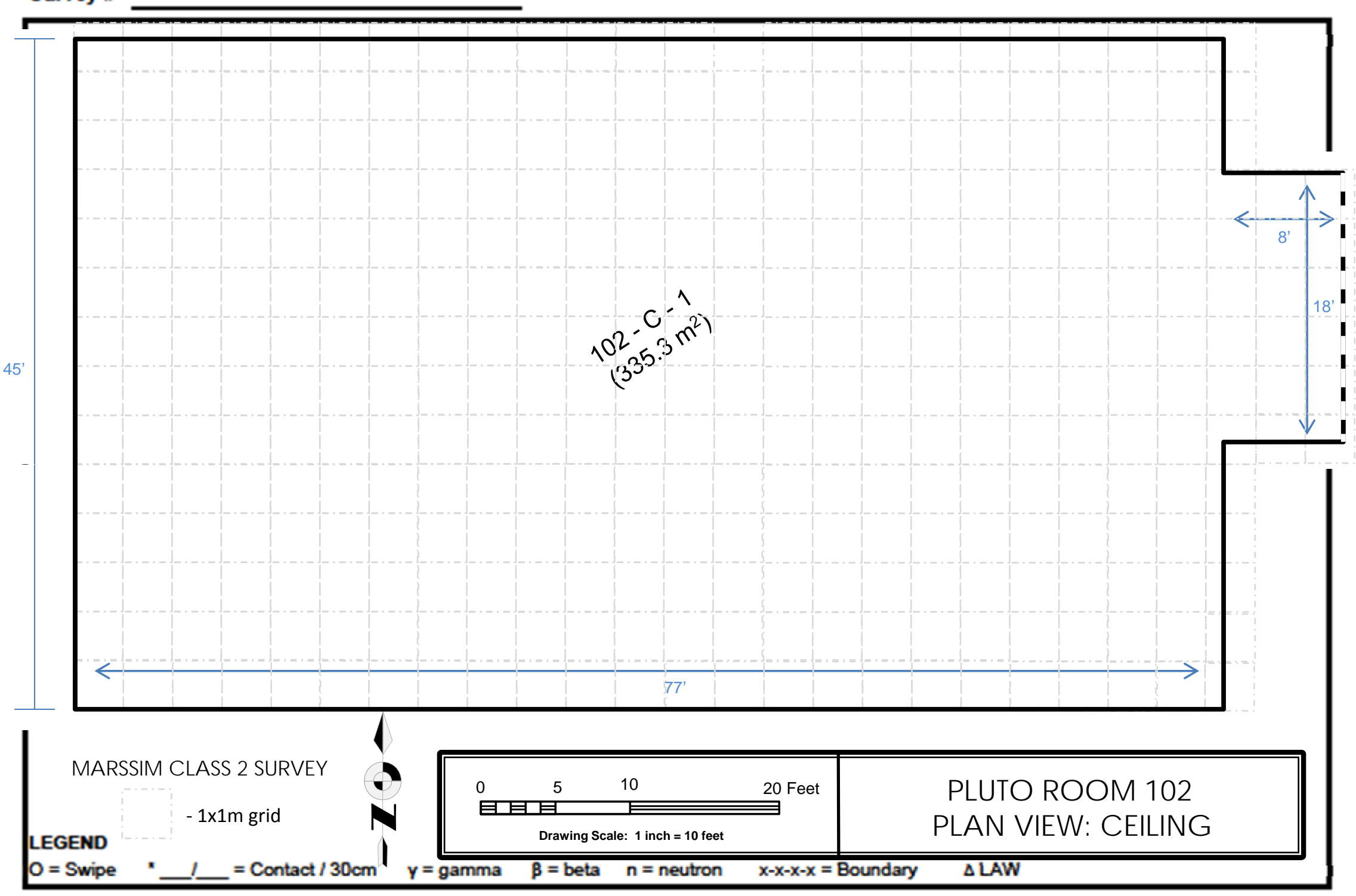


NSTec

Form

FRM-0108C

Horizontal surfaces,

RADIOLOGICAL SURVEY REPORT - FULL MAP

West-facing wall,

05/24/10

Rev. 01

\section{Survey \#}

Description/Map/Drawing/Pioture/Comments
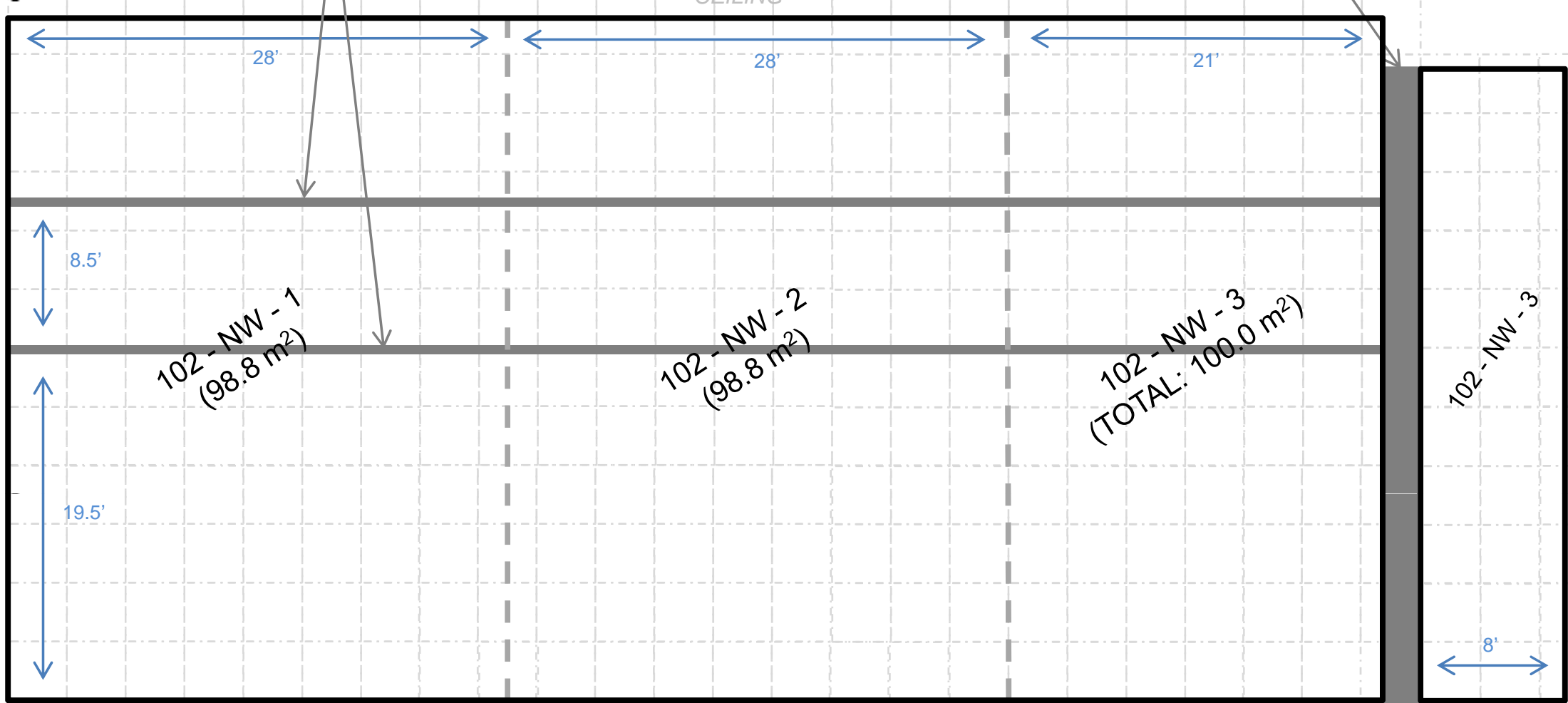

77

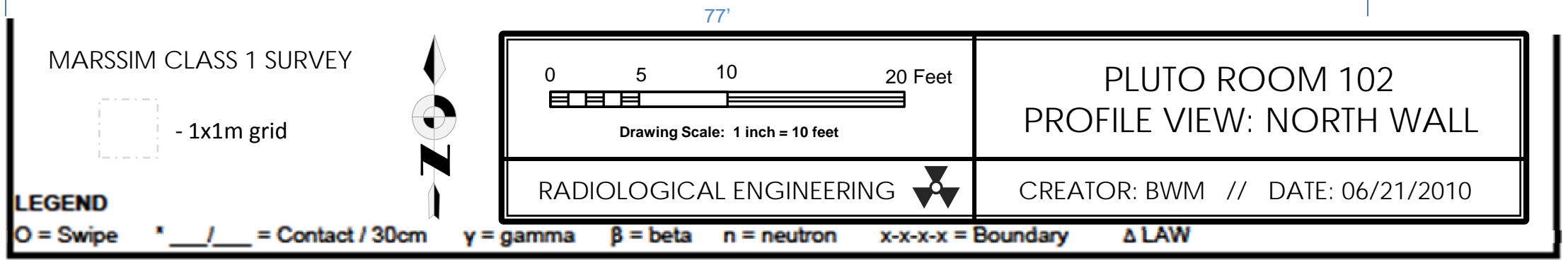


Survey \#

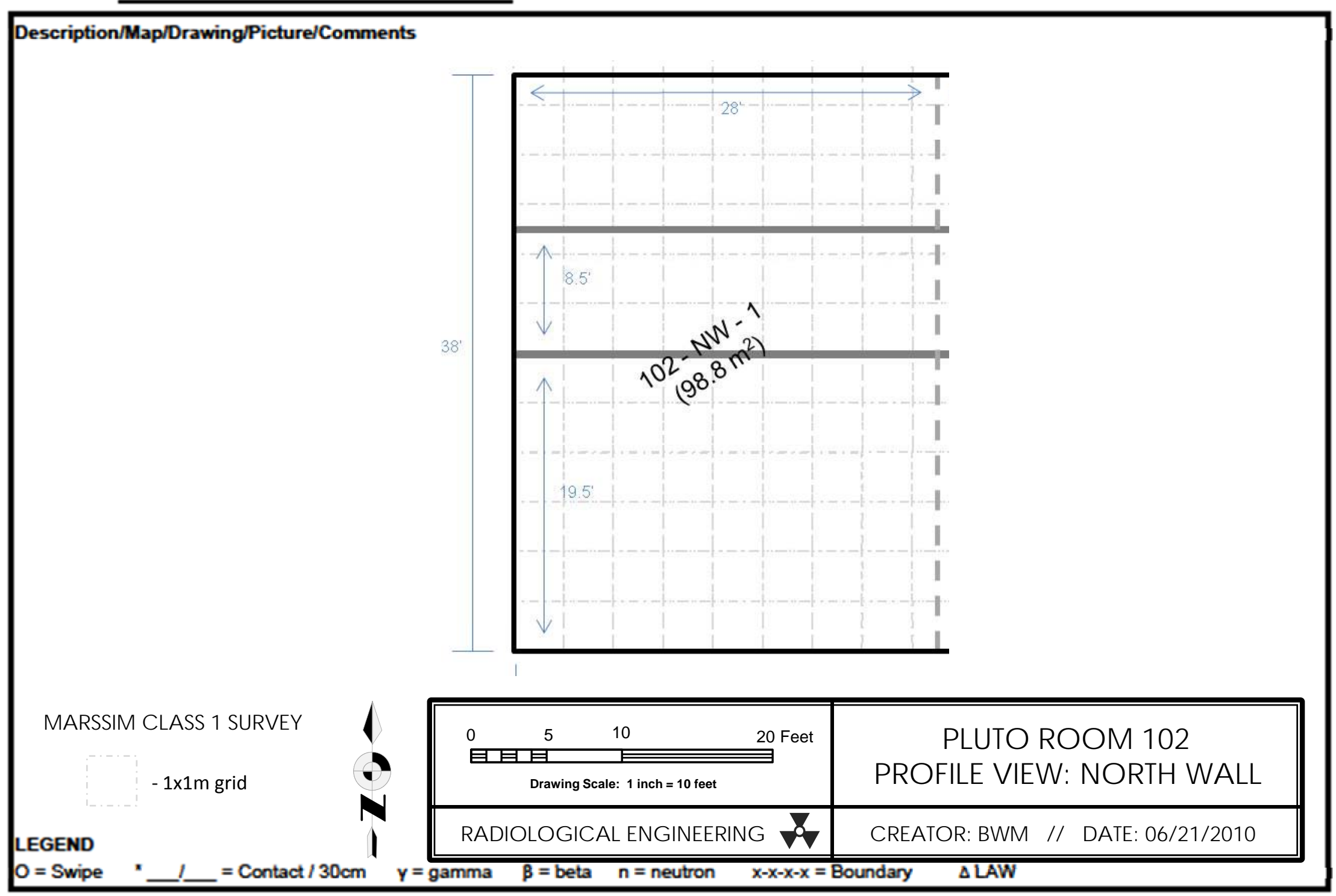


Survey \#

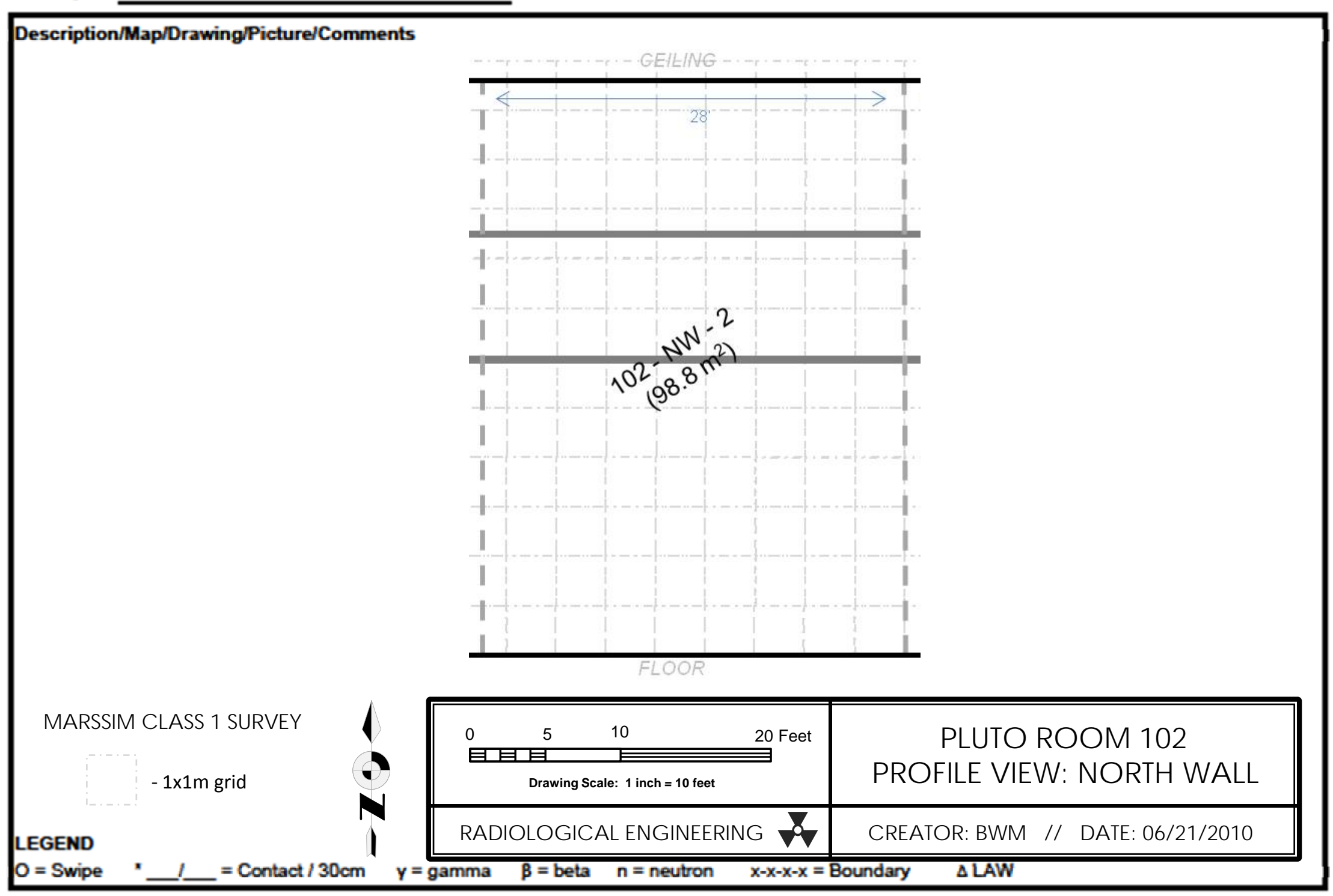


Survey \#

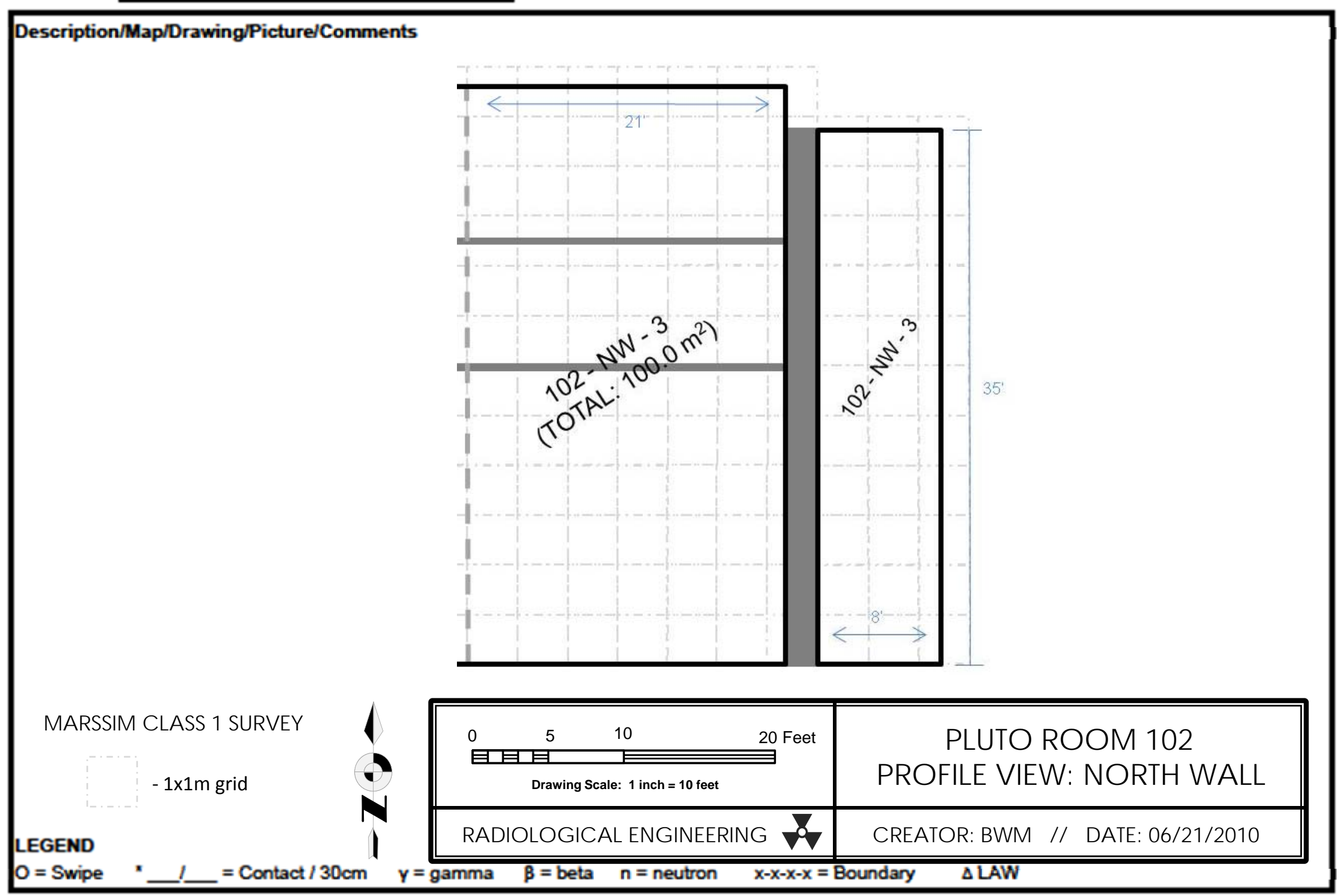


Survey \#

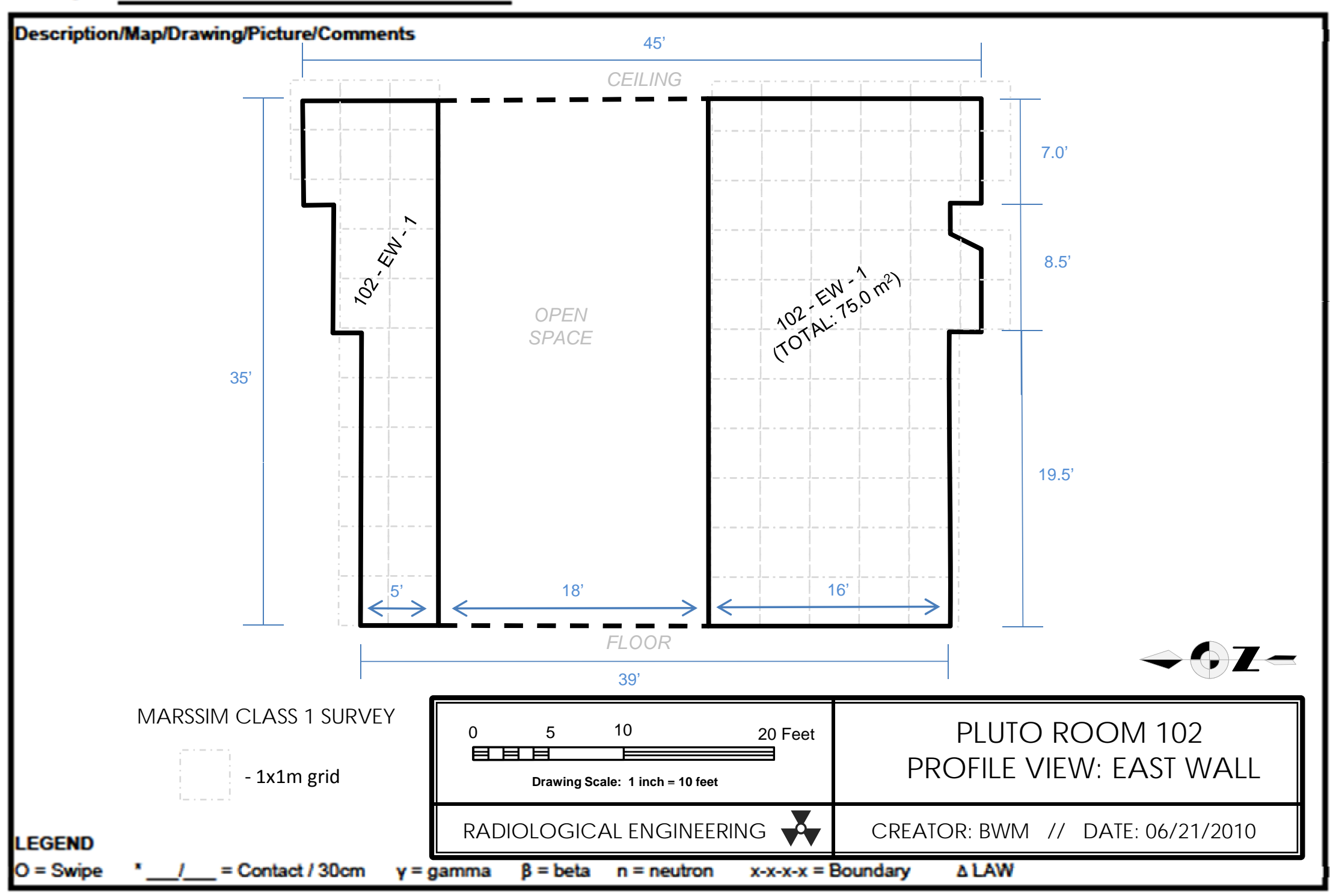


NSTec

East-facing wall,

Form

FRM-0108C

RADIOLOGICAL SURVEY REPORT - FULL MAP

Rev. 01

Survey \#

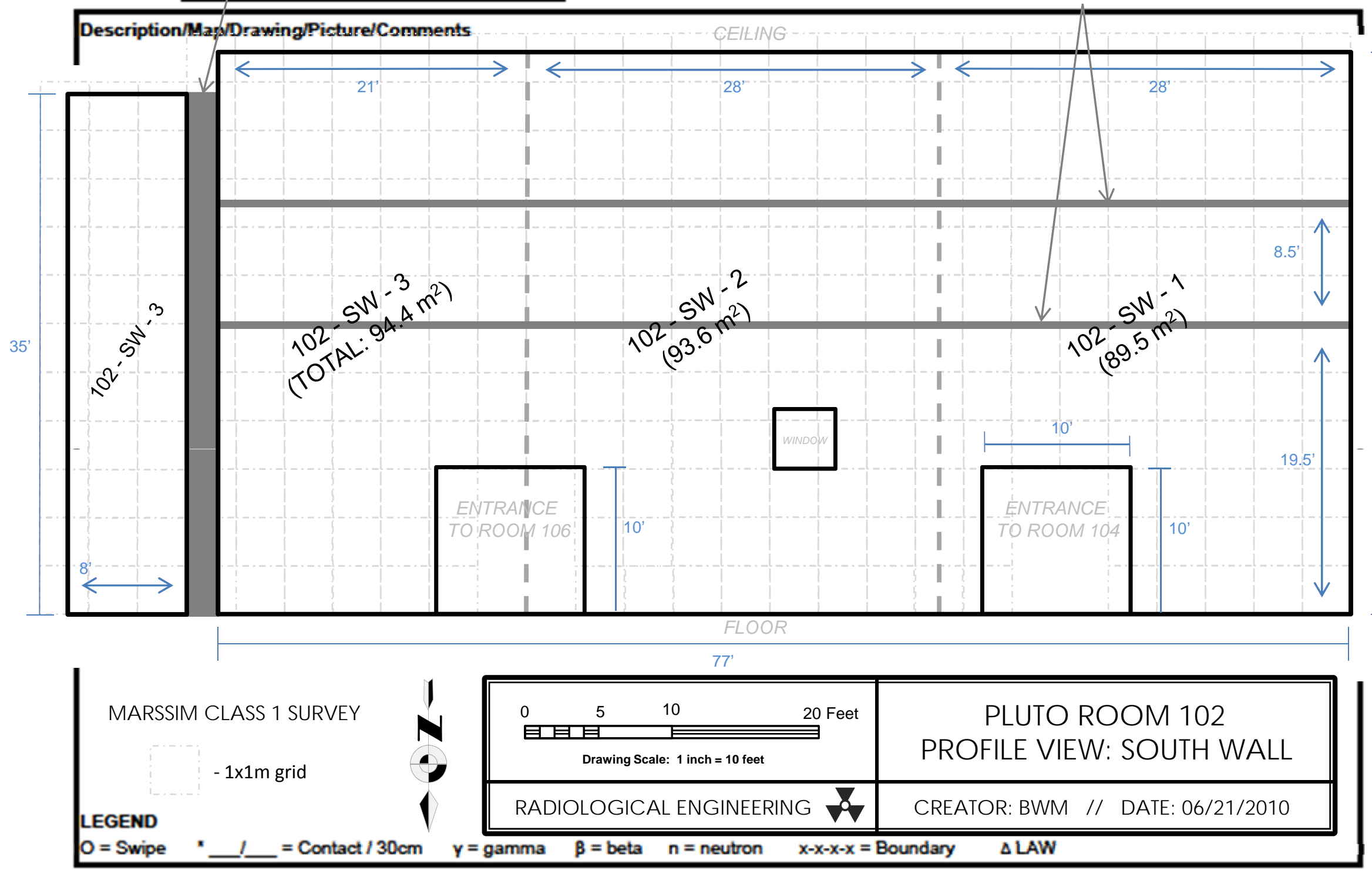


Survey \#

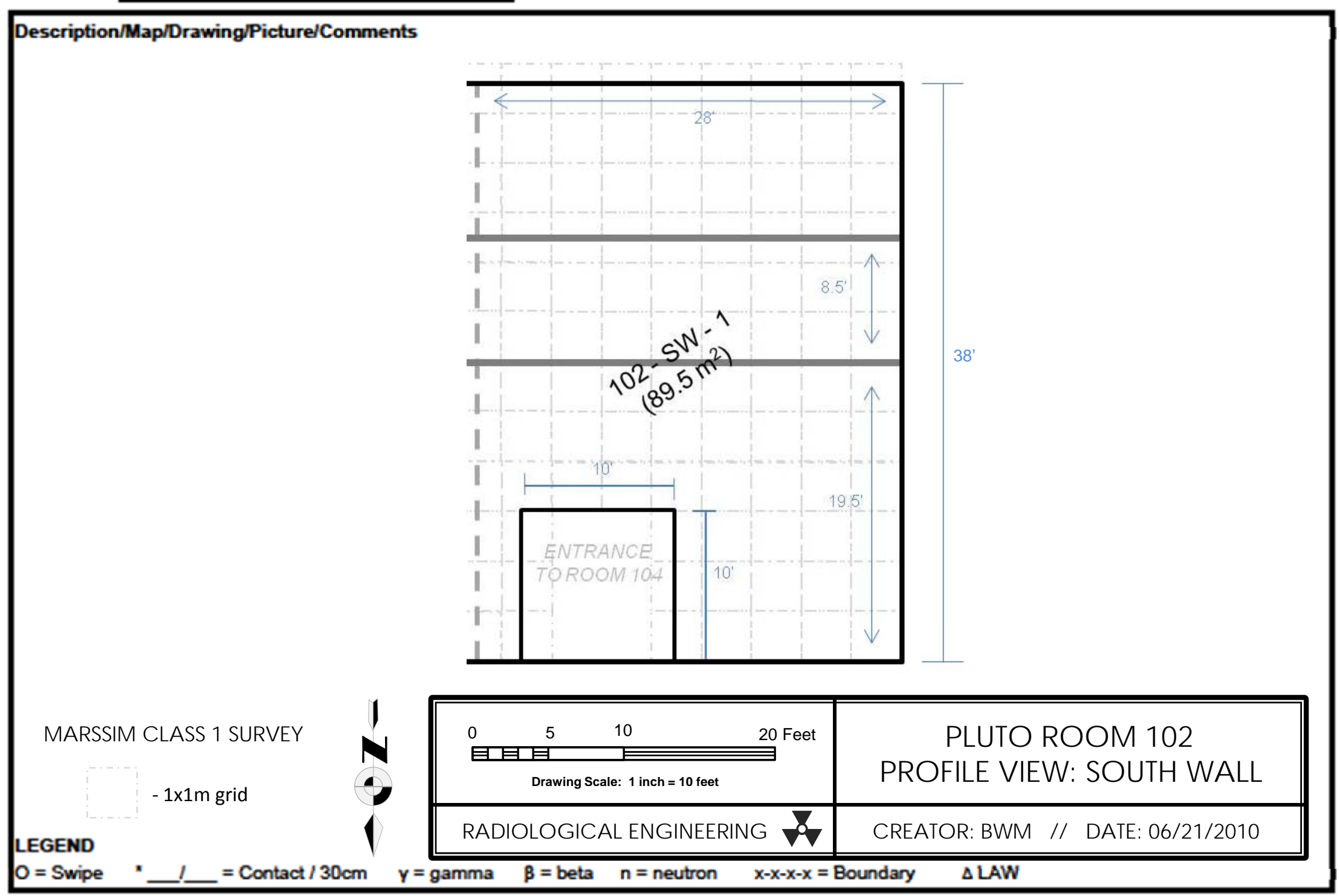


Survey \#

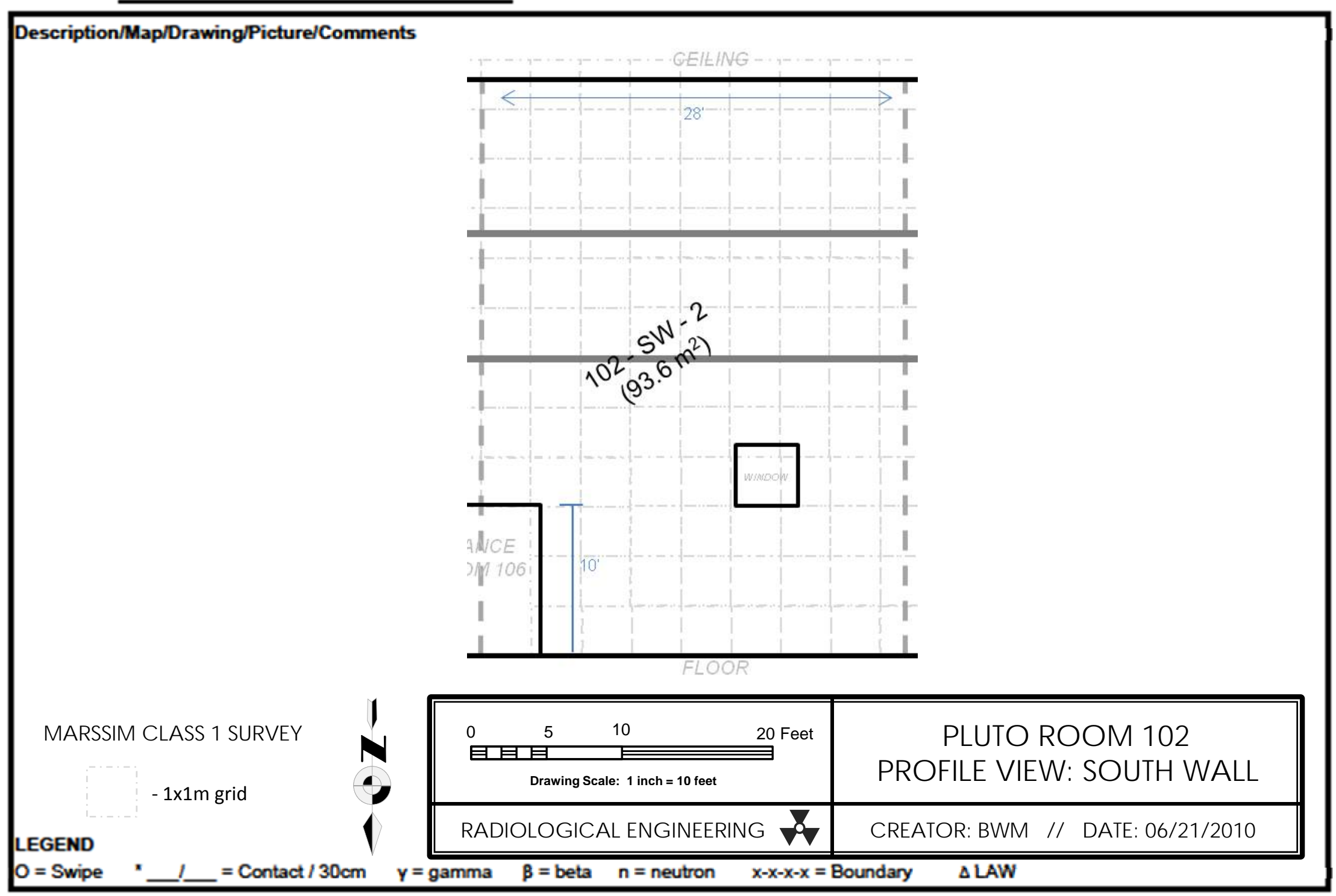


Survey \#

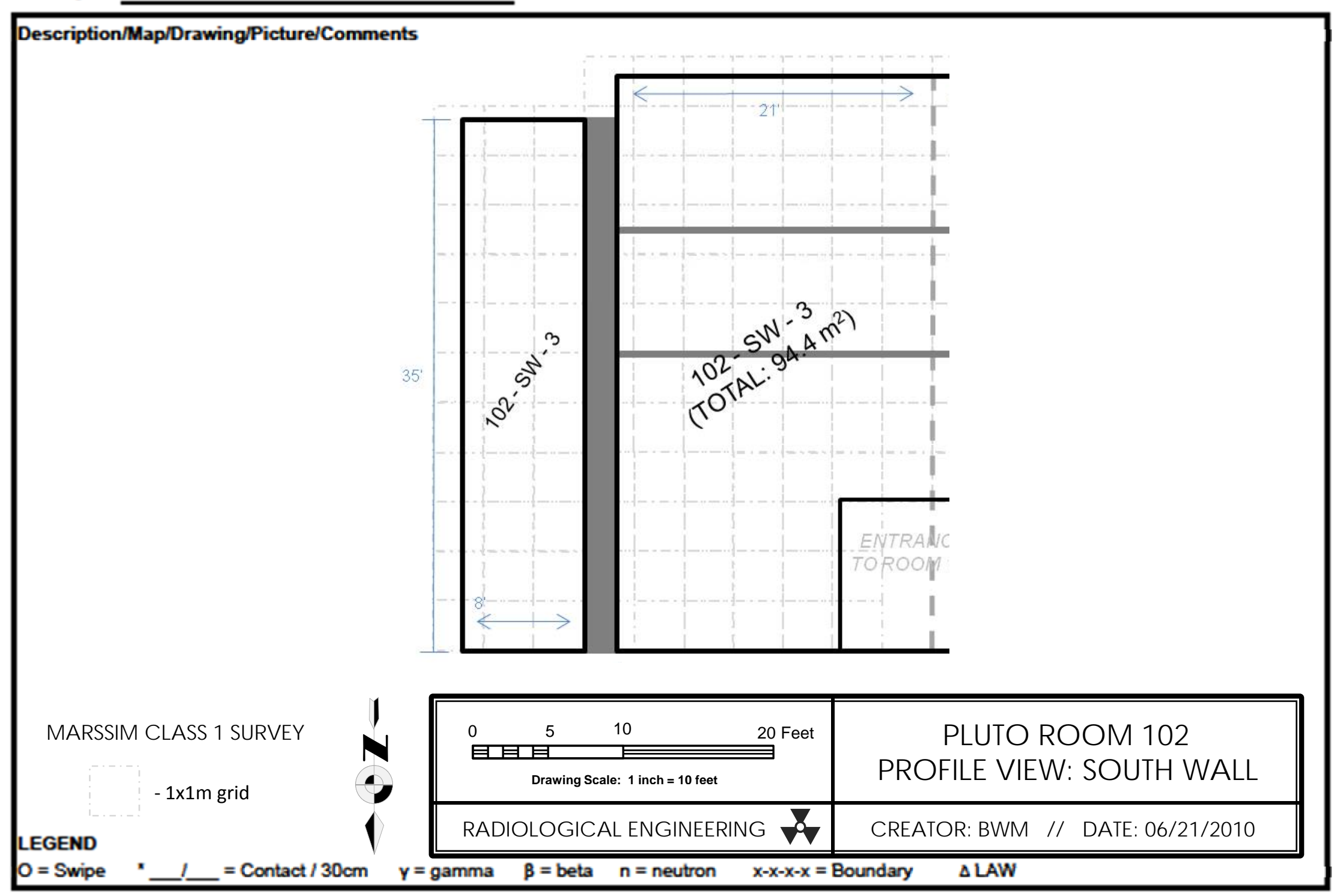


Survey \#

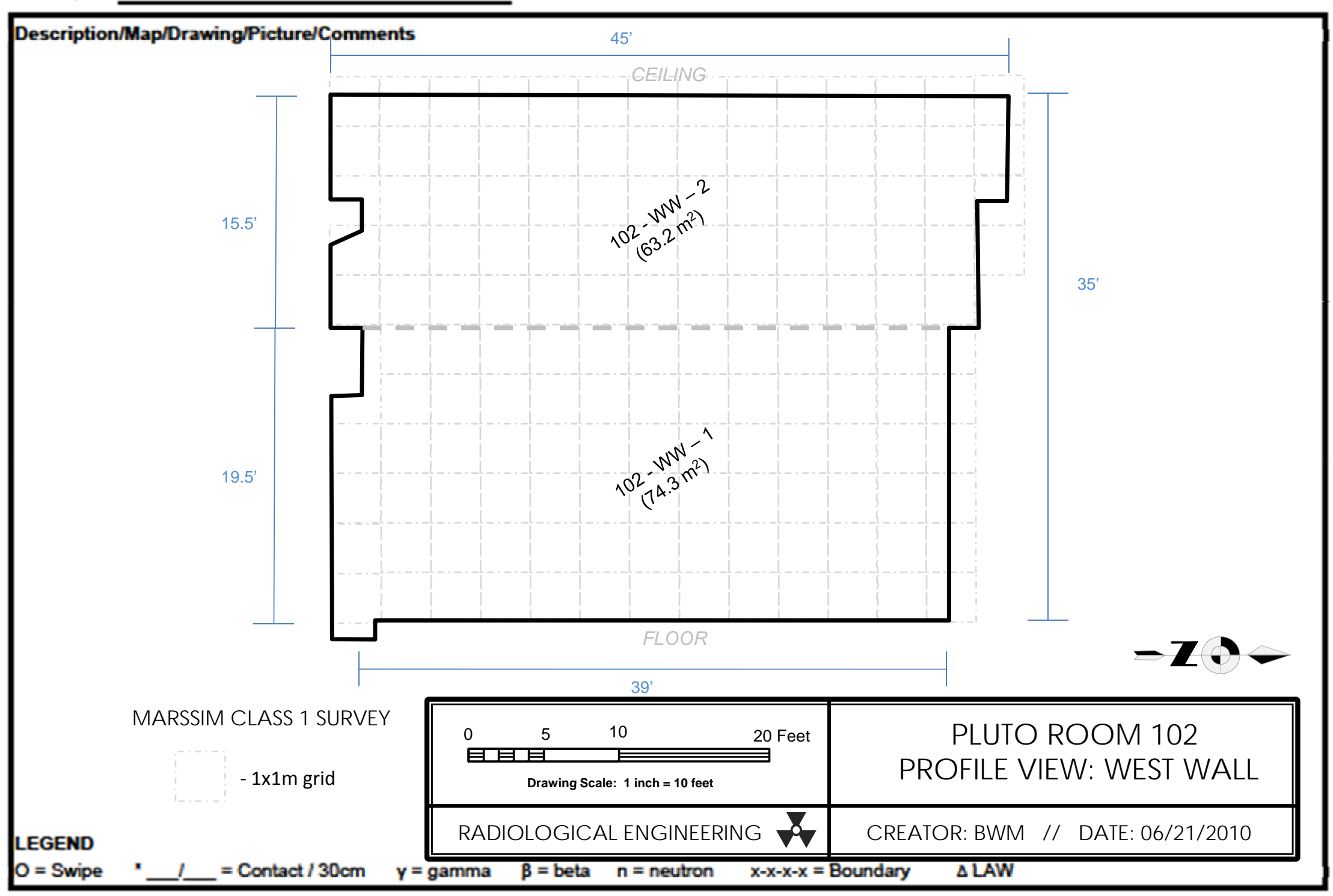


Survey \#

\section{Description/Map/Drawing/Picture/Comments}

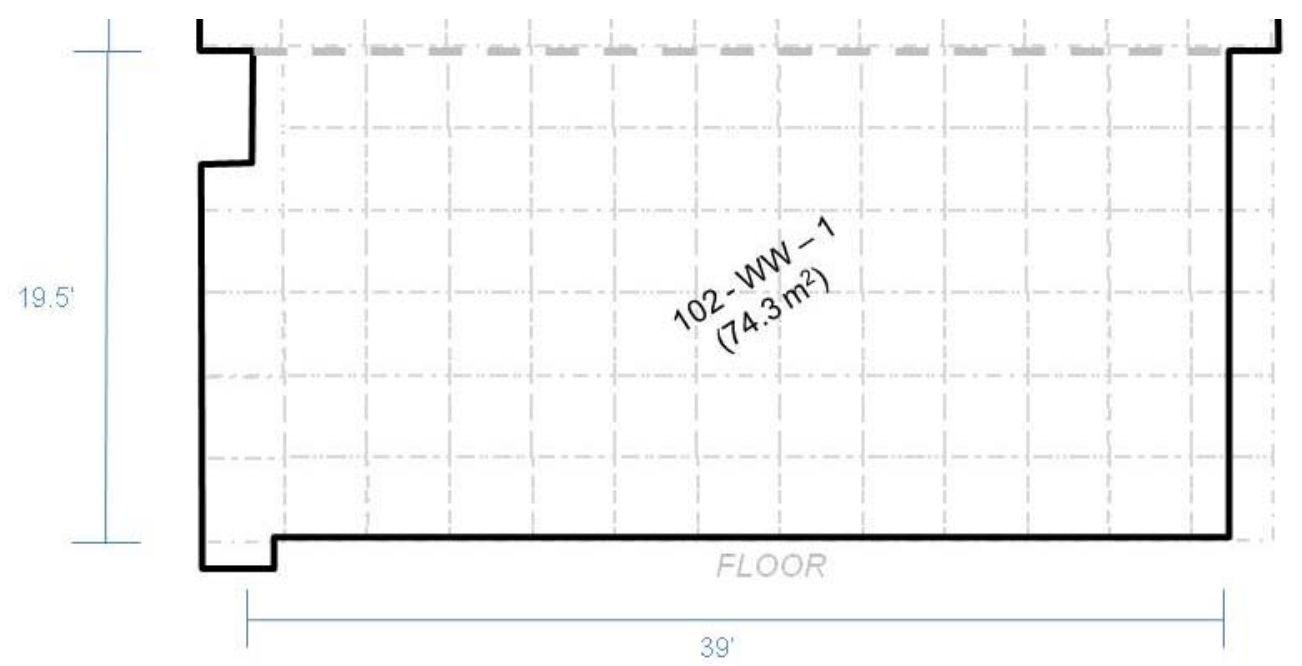

MARSSIM CLASS 1 SURVEY

$-1 \times 1 m$ grid

\begin{tabular}{|c|c|}
\hline 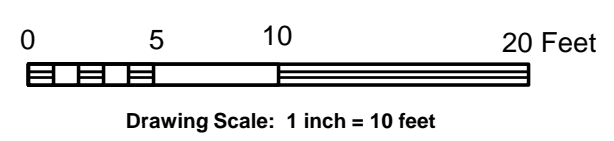 & $\begin{array}{c}\text { PLUTO ROOM } 102 \\
\text { PROFILE VIEW: WEST WAШ }\end{array}$ \\
\hline RADIOLOGICALENG INEERING & CREATOR: BWM // DATE: 06/21/2010 \\
\hline
\end{tabular}


Survey \#

Description/Map/Drawing/Picture/Comments

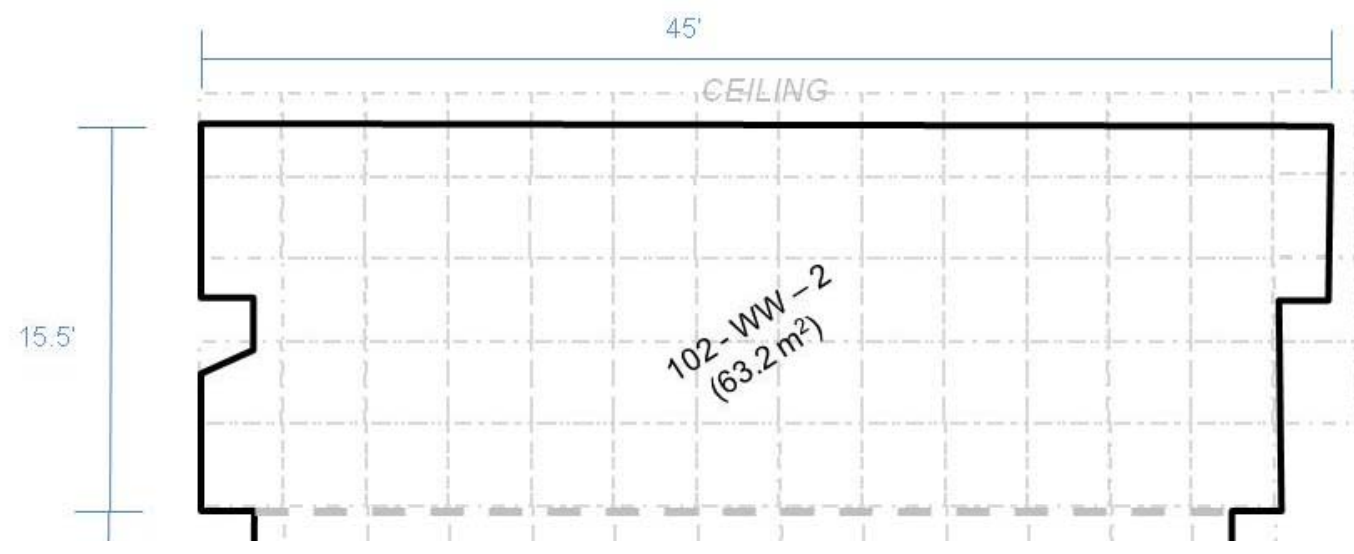

MARSSIM CLASS 1 SURVEY

$-1 \times 1 m$ grid

\begin{tabular}{|c|c|}
\hline$\underbrace{20}_{\substack{0 \\
\text { Drawing Scale: } 1 \text { inch }=10 \text { feet }}}$ Feet & $\begin{array}{c}\text { PLUTO ROOM } 102 \\
\text { PROFILE VIEW: WEST WAШ }\end{array}$ \\
\hline RADIOLOGICALENG INEERING & CREATOR: BWM // DATE: 06/21/2010 \\
\hline
\end{tabular}


Survey \#

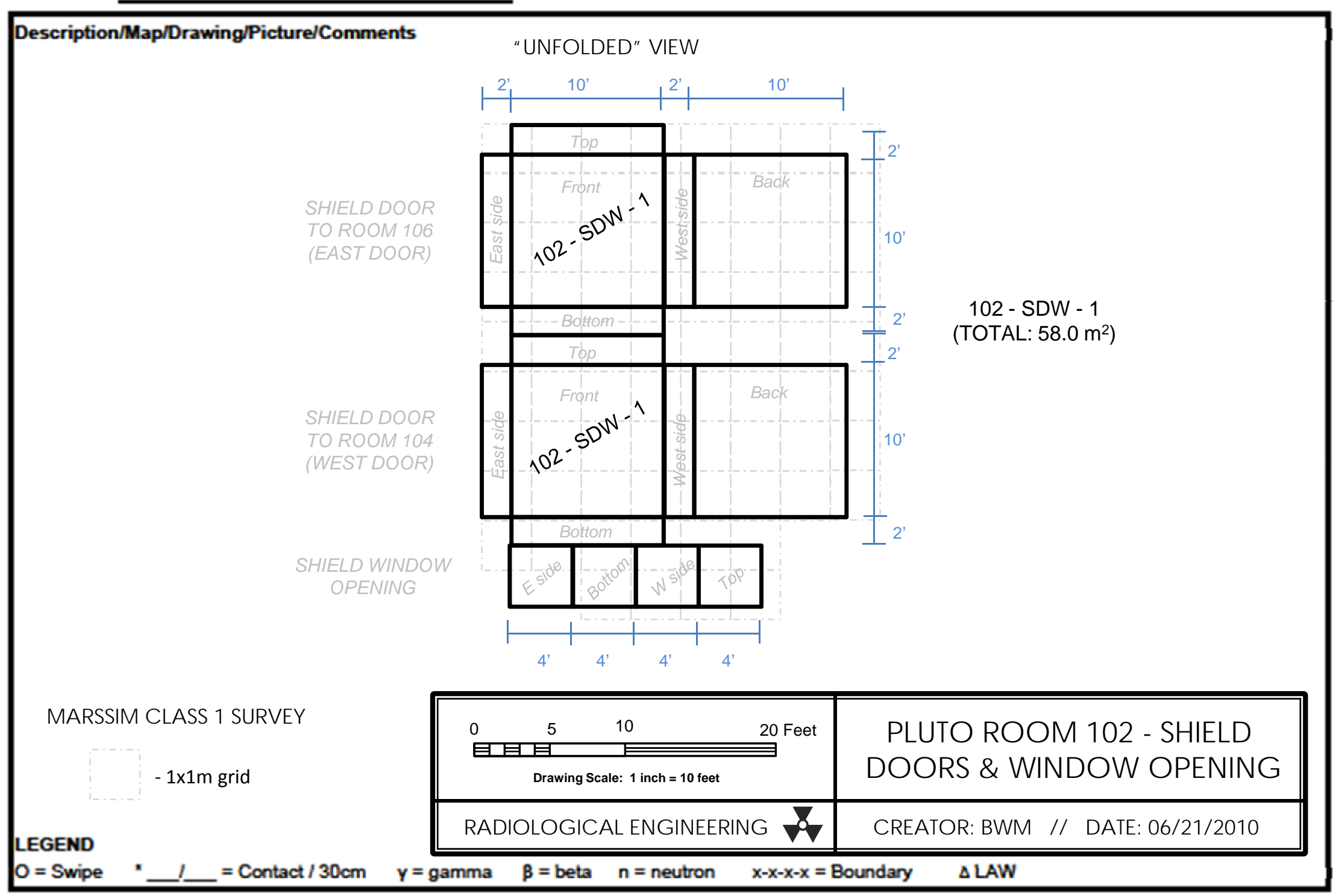


Survey \#

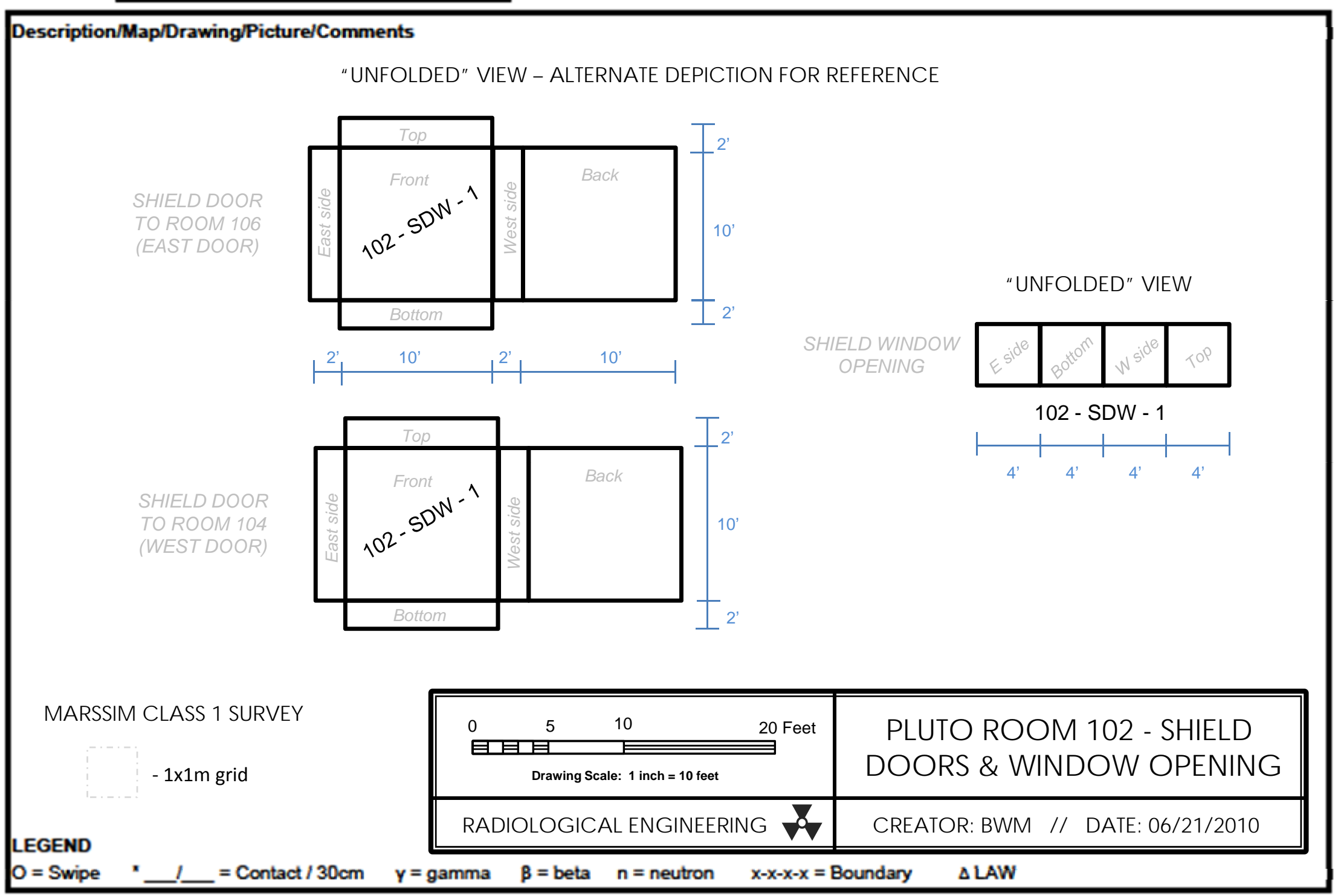


Survey \#

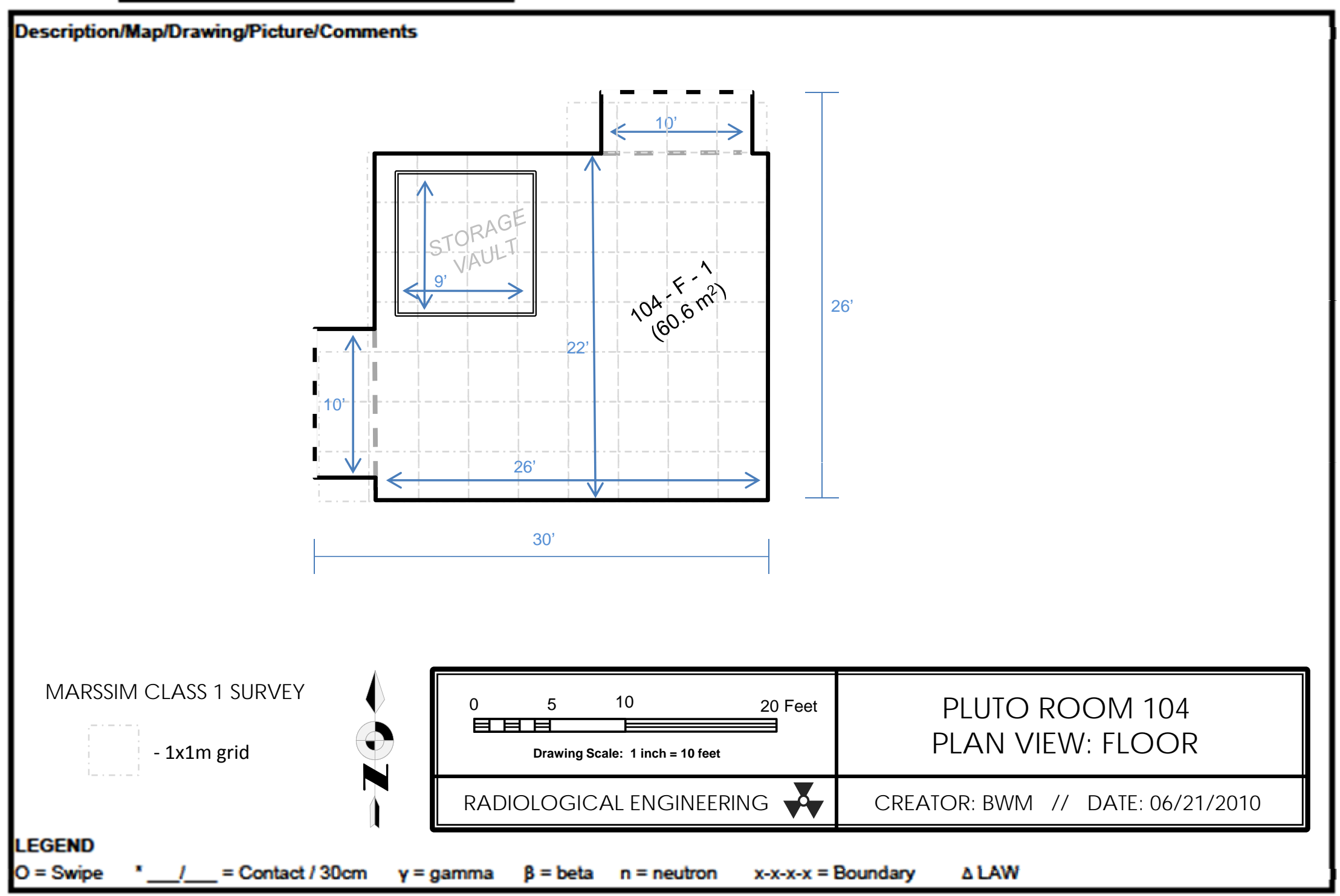


Survey \#

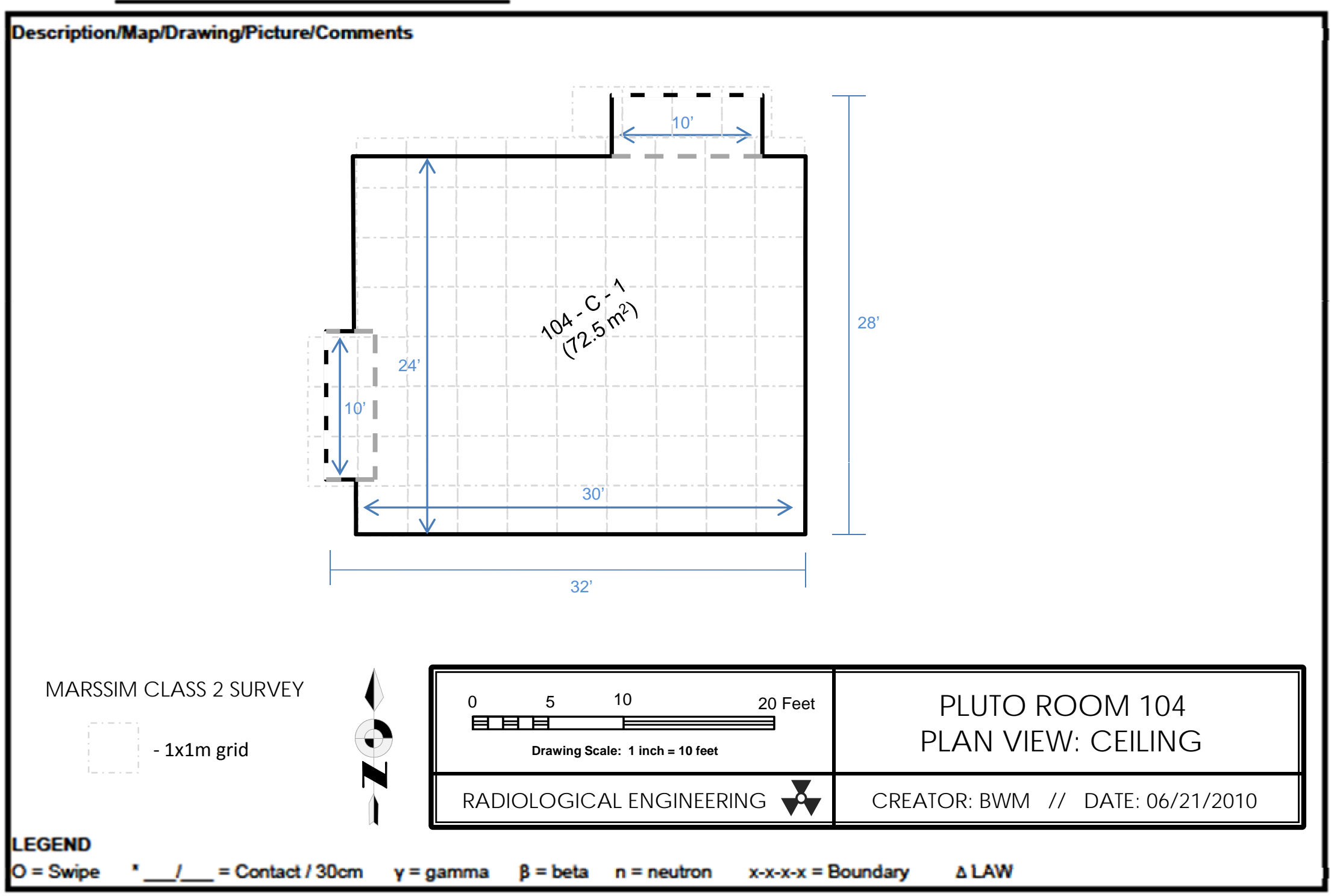


Survey \#

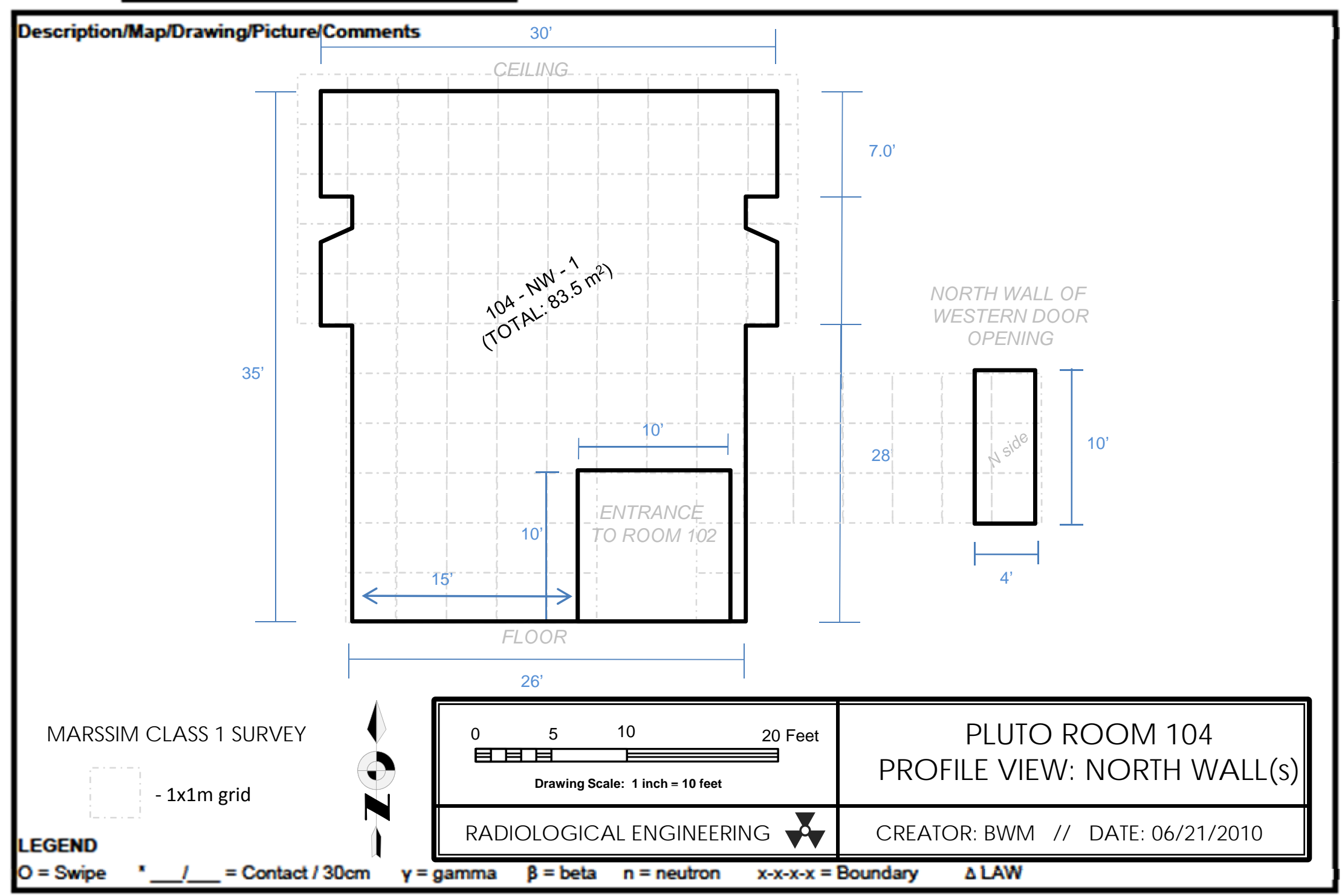


Survey \#

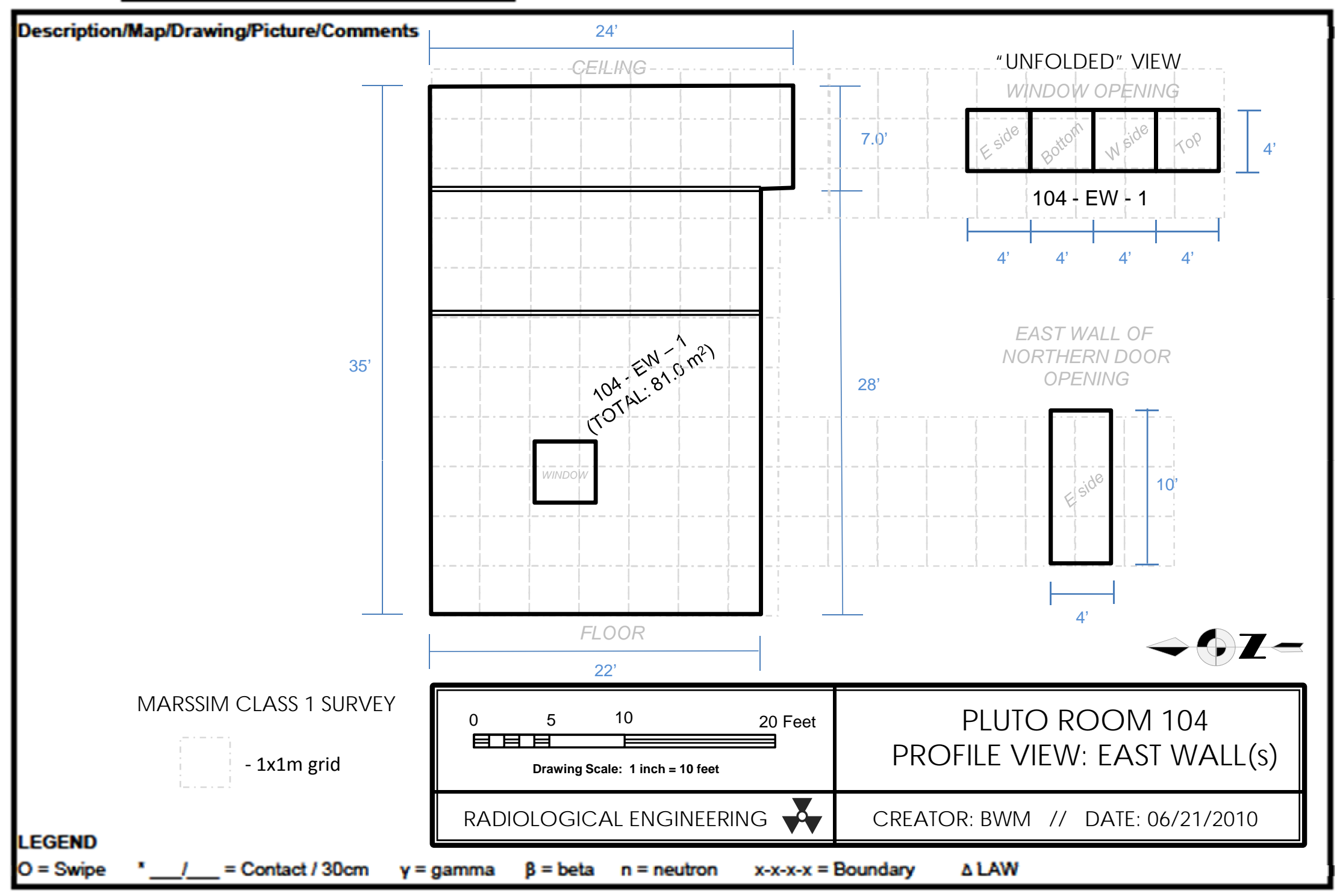


Survey \#

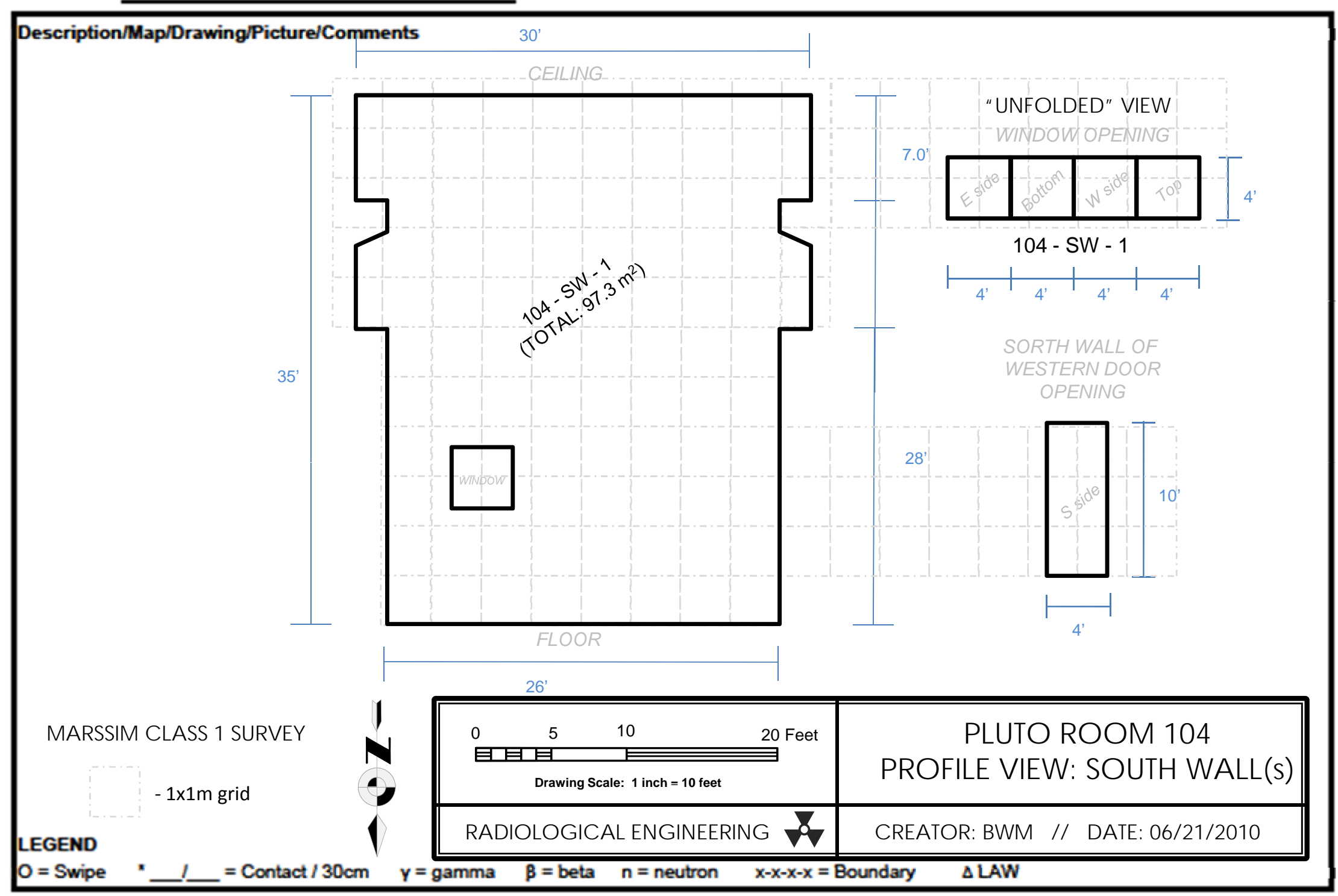


Survey \#

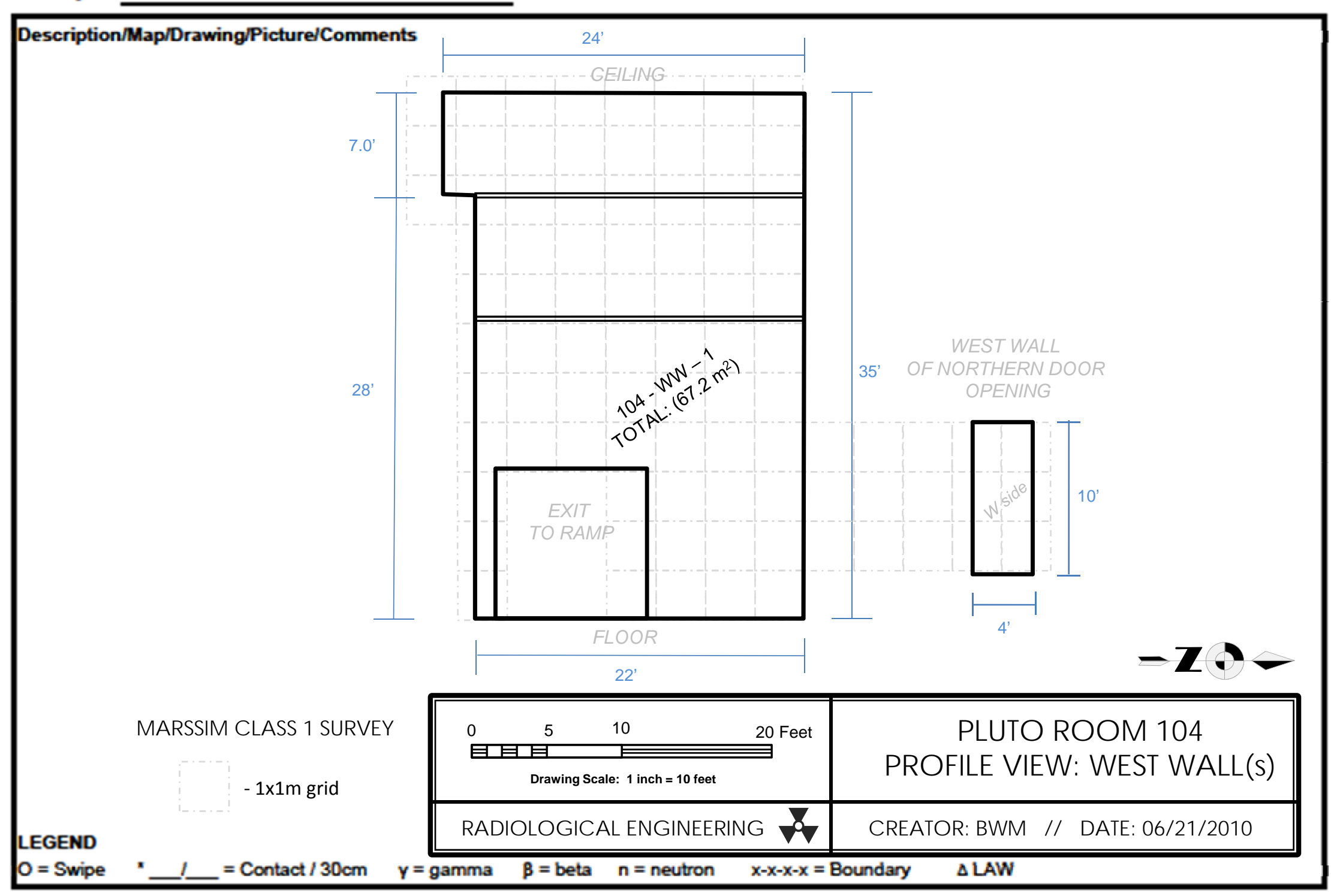


Survey \#

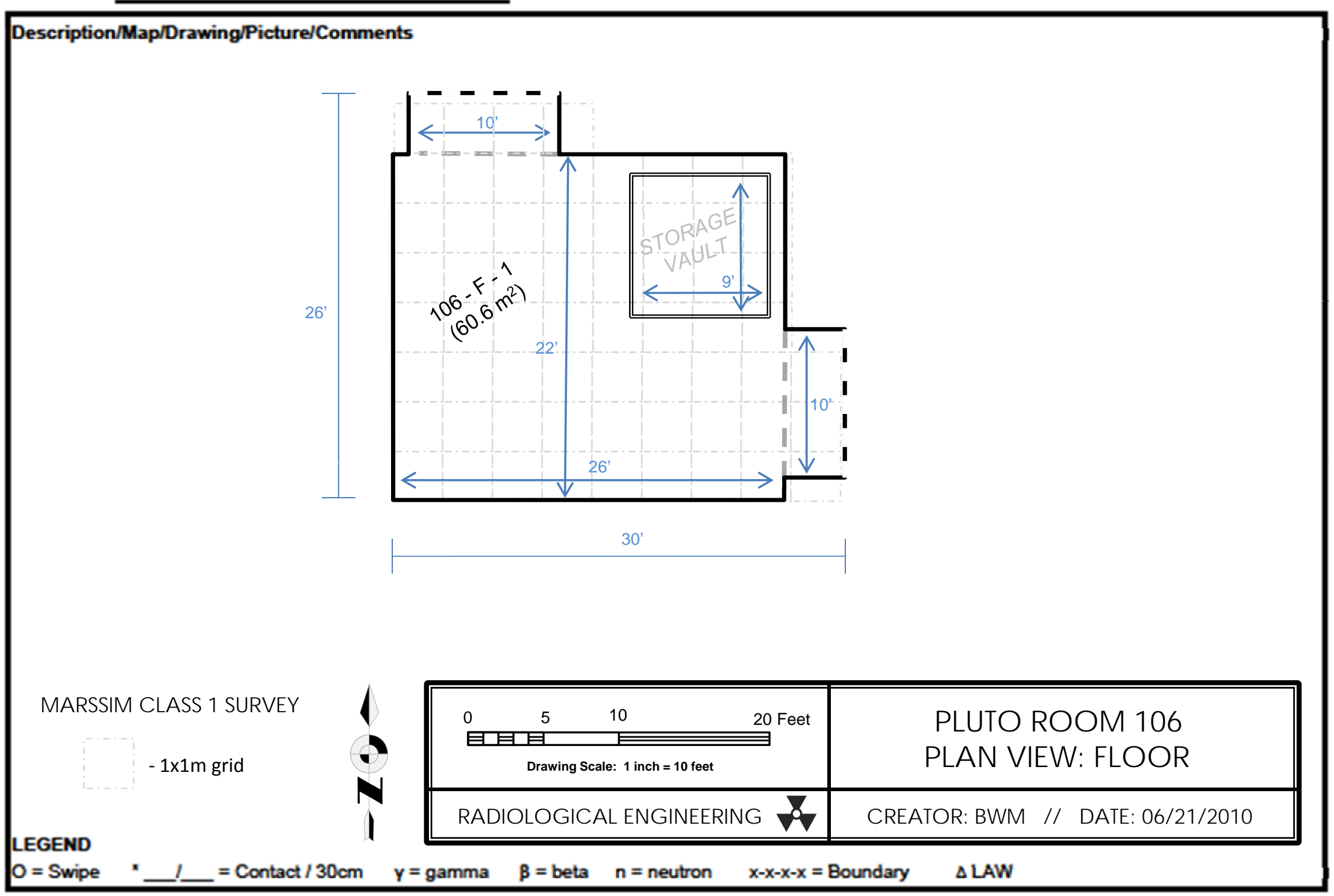


Survey \#

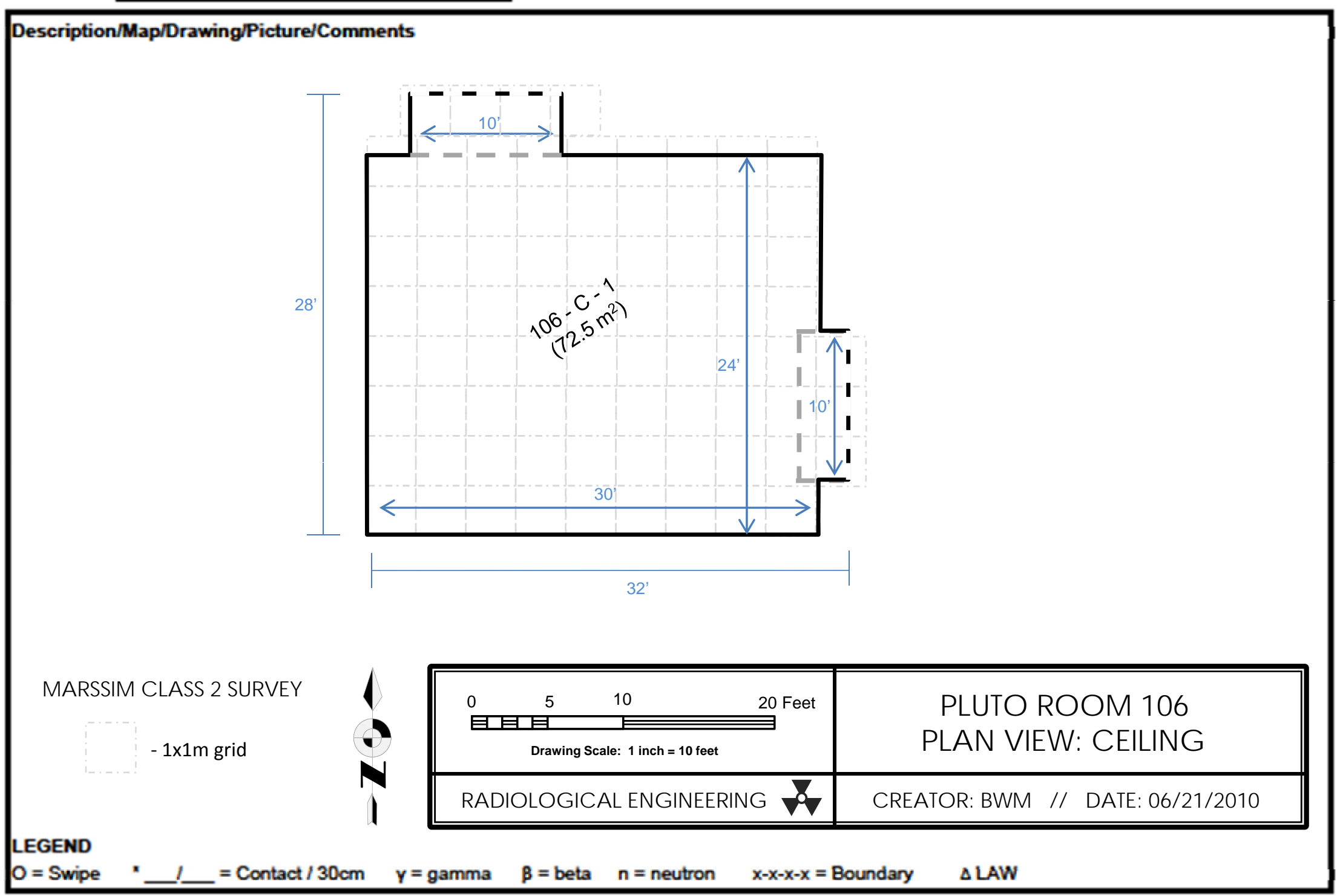


Survey \#

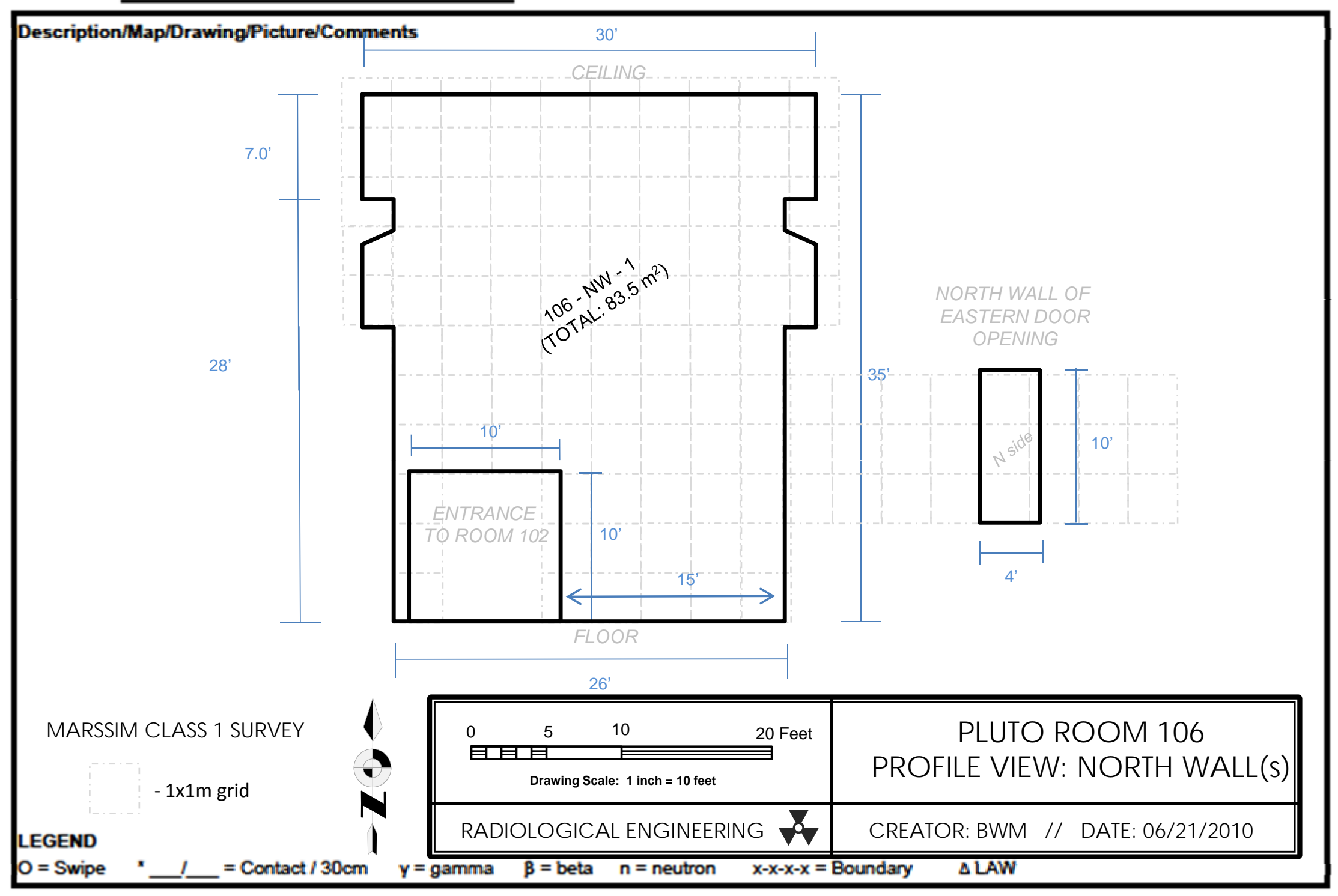


Survey \#

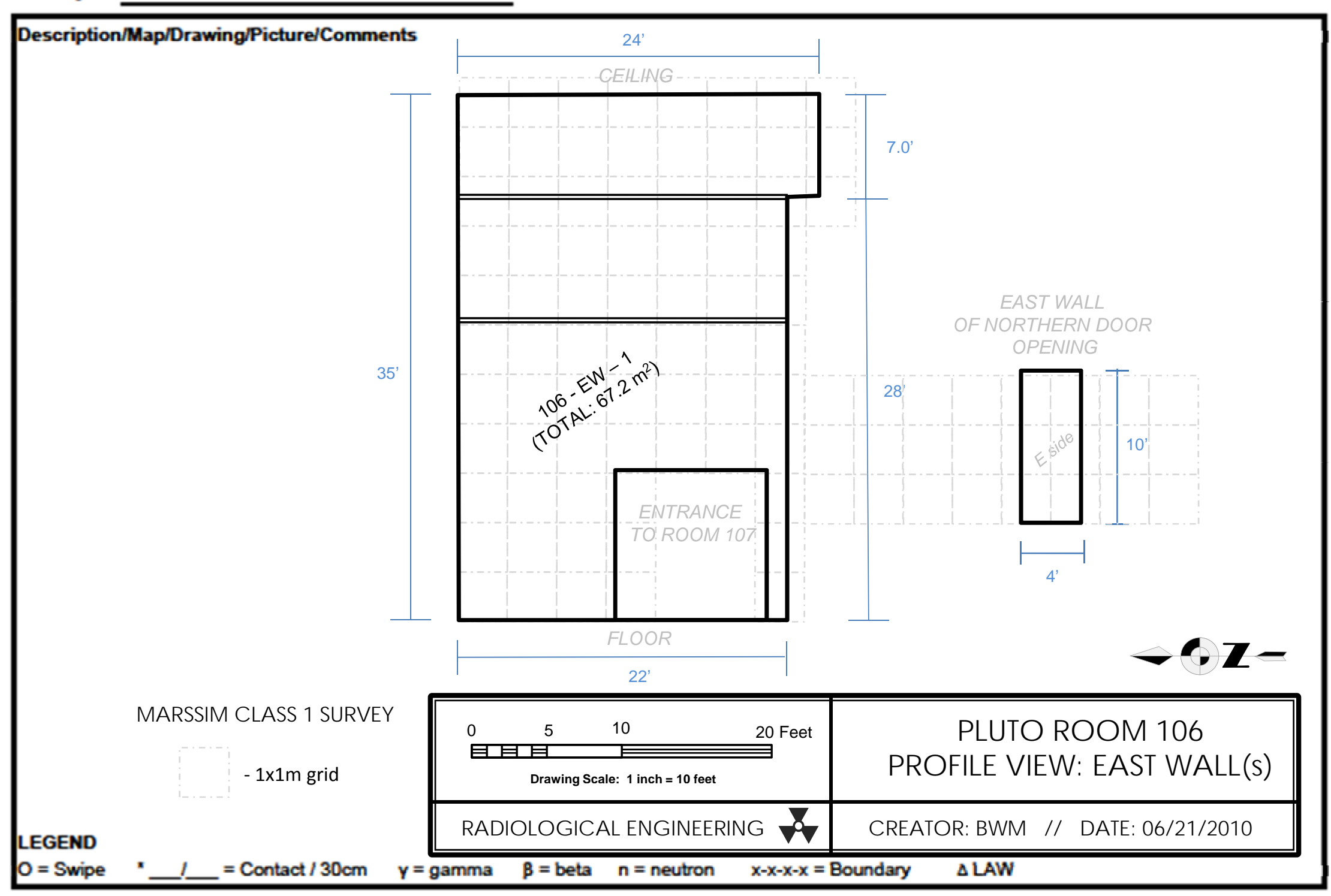


Survey \#

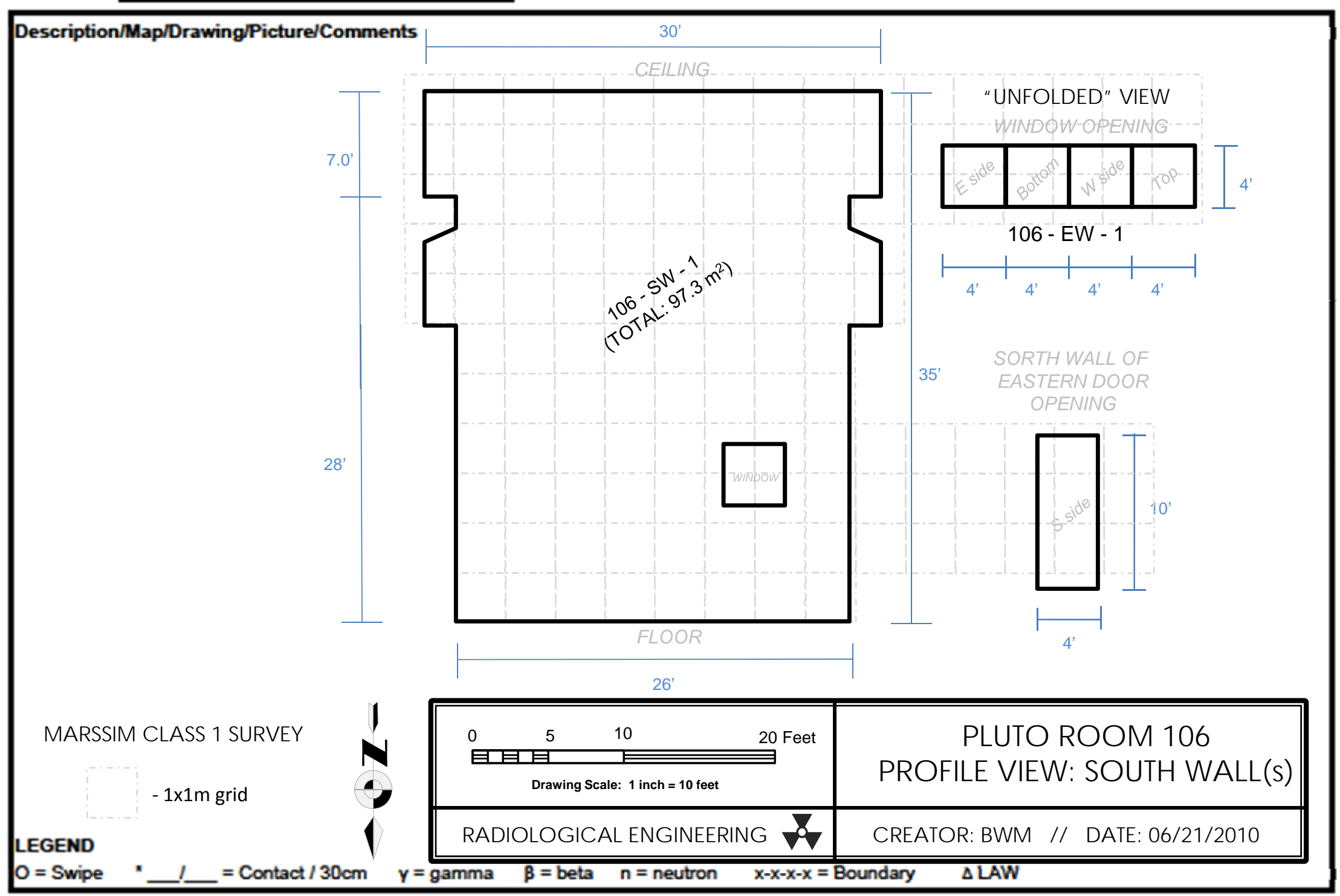


Survey \#

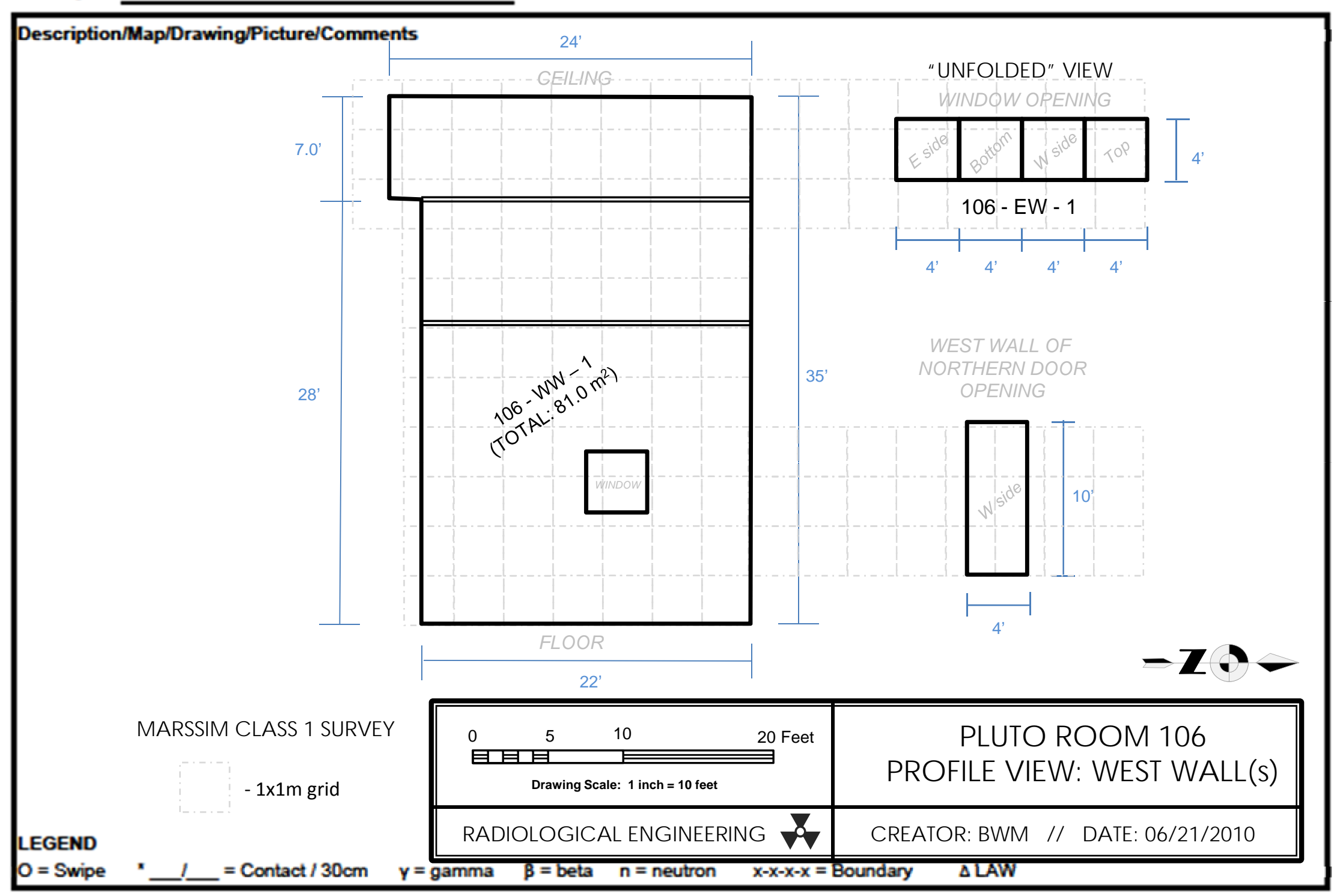


Survey \#

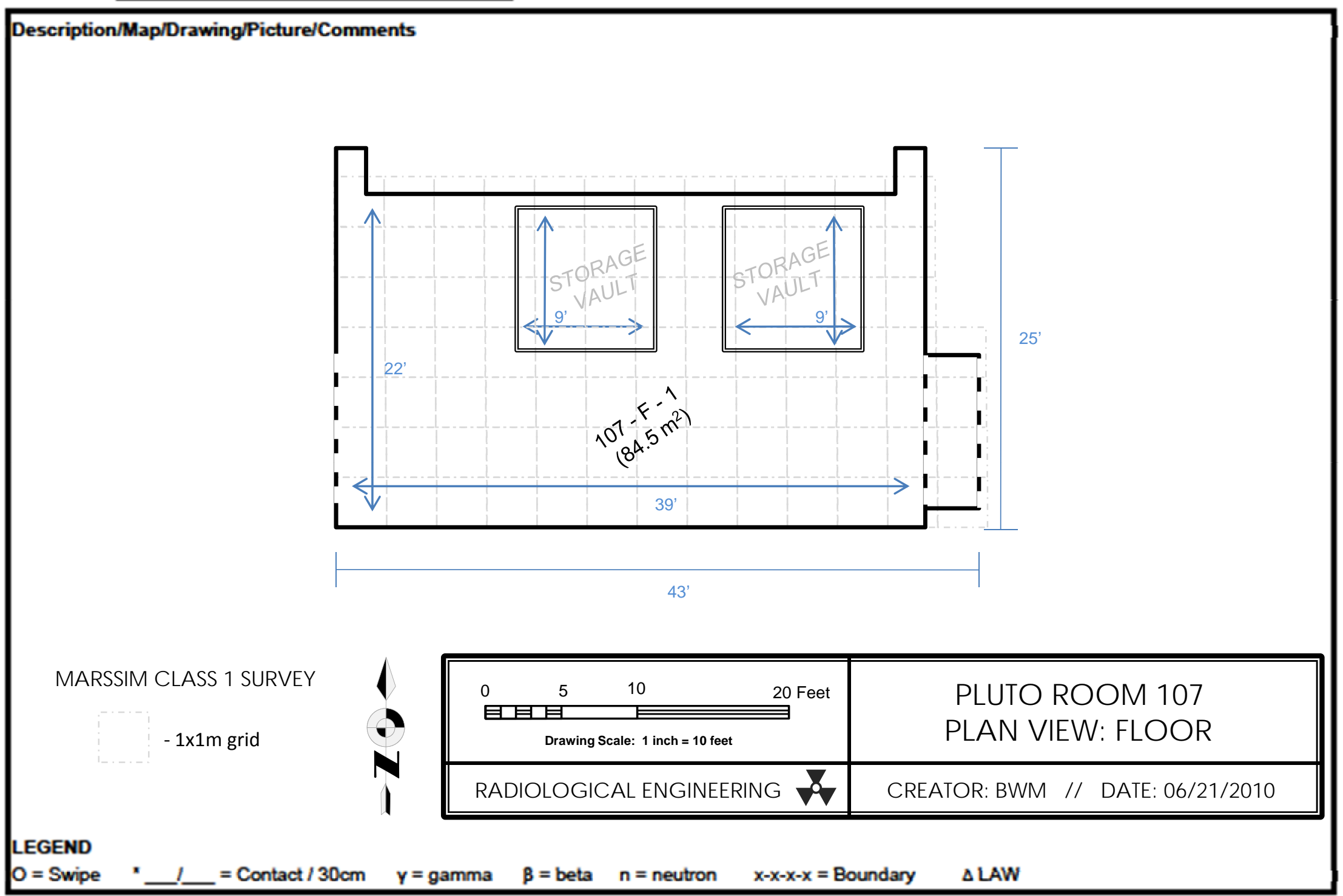


Survey \#

Description/Map/Drawing/Picture/Comments

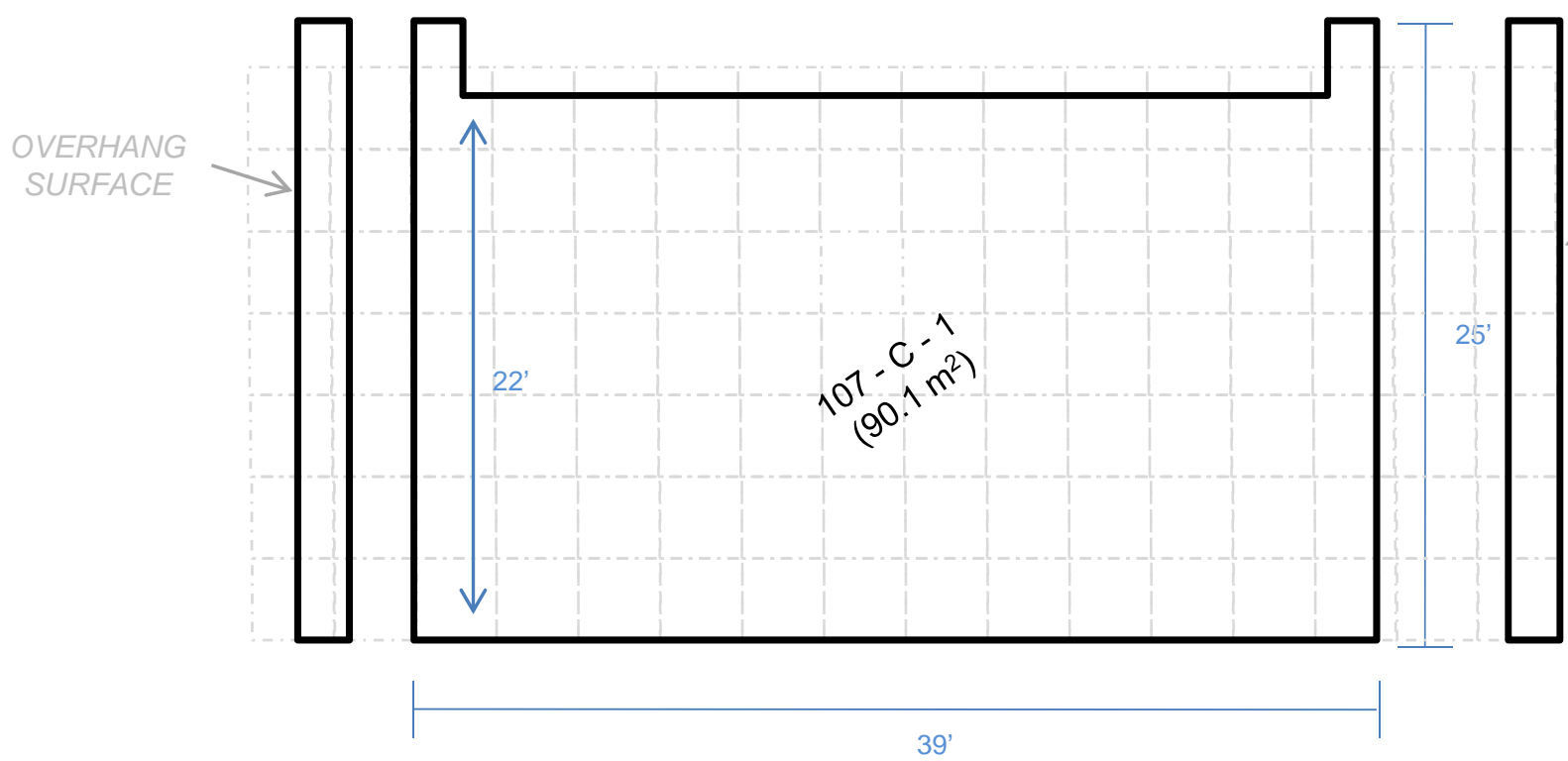

MARSSIM CLASS 2 SURVEY

$-1 \times 1 m$ grid

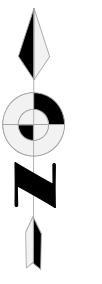

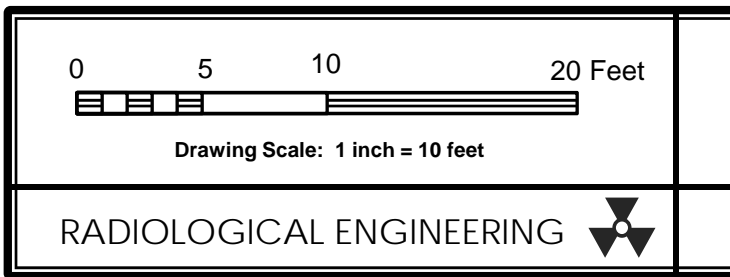

PLUTO ROOM 107

PLAN VIEW: CEIUNG

LEGEND

0 = Swipe $\quad x \quad 1 \quad=$ Contact $/ 30 \mathrm{~cm}$ 
Survey \#

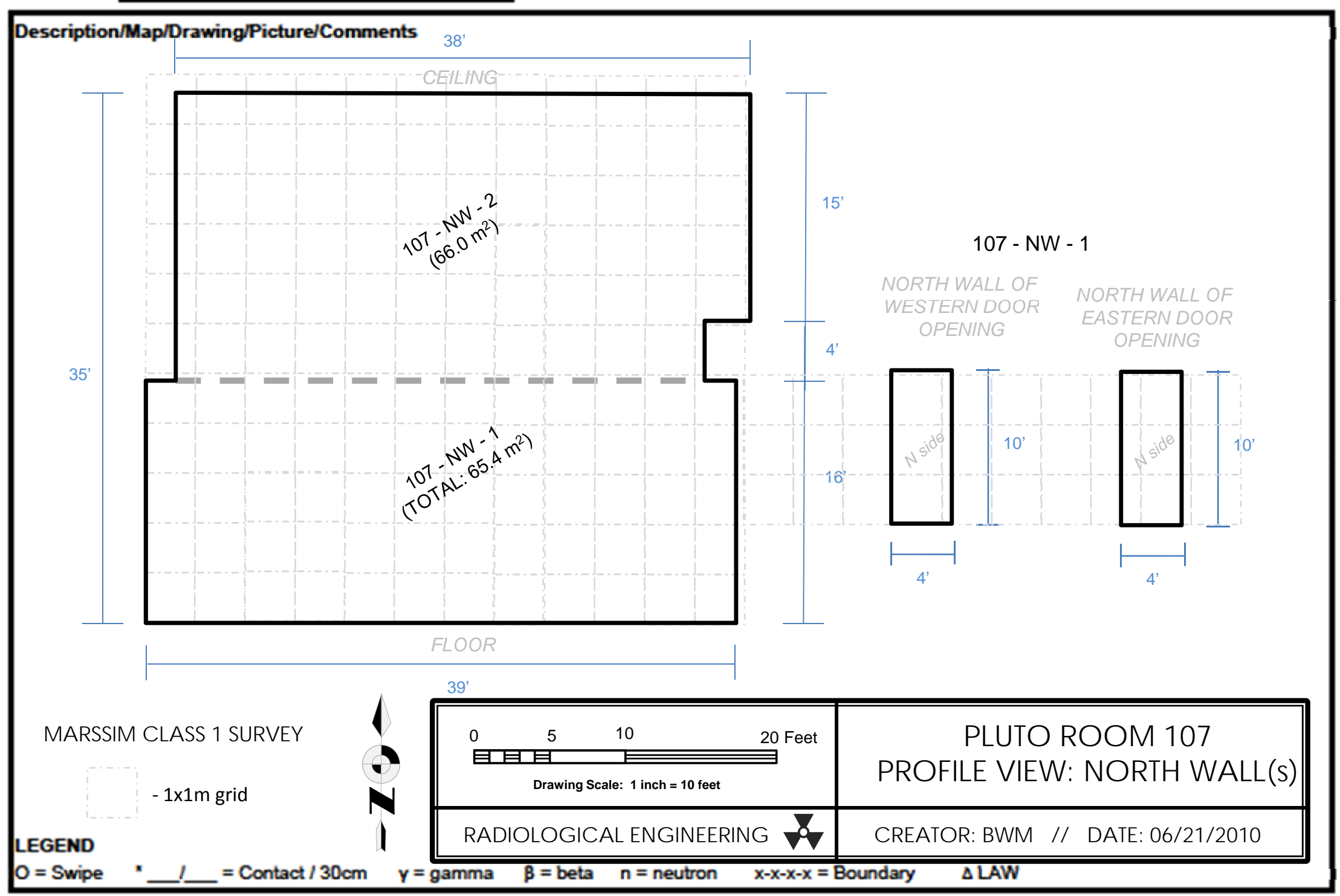


Survey \#

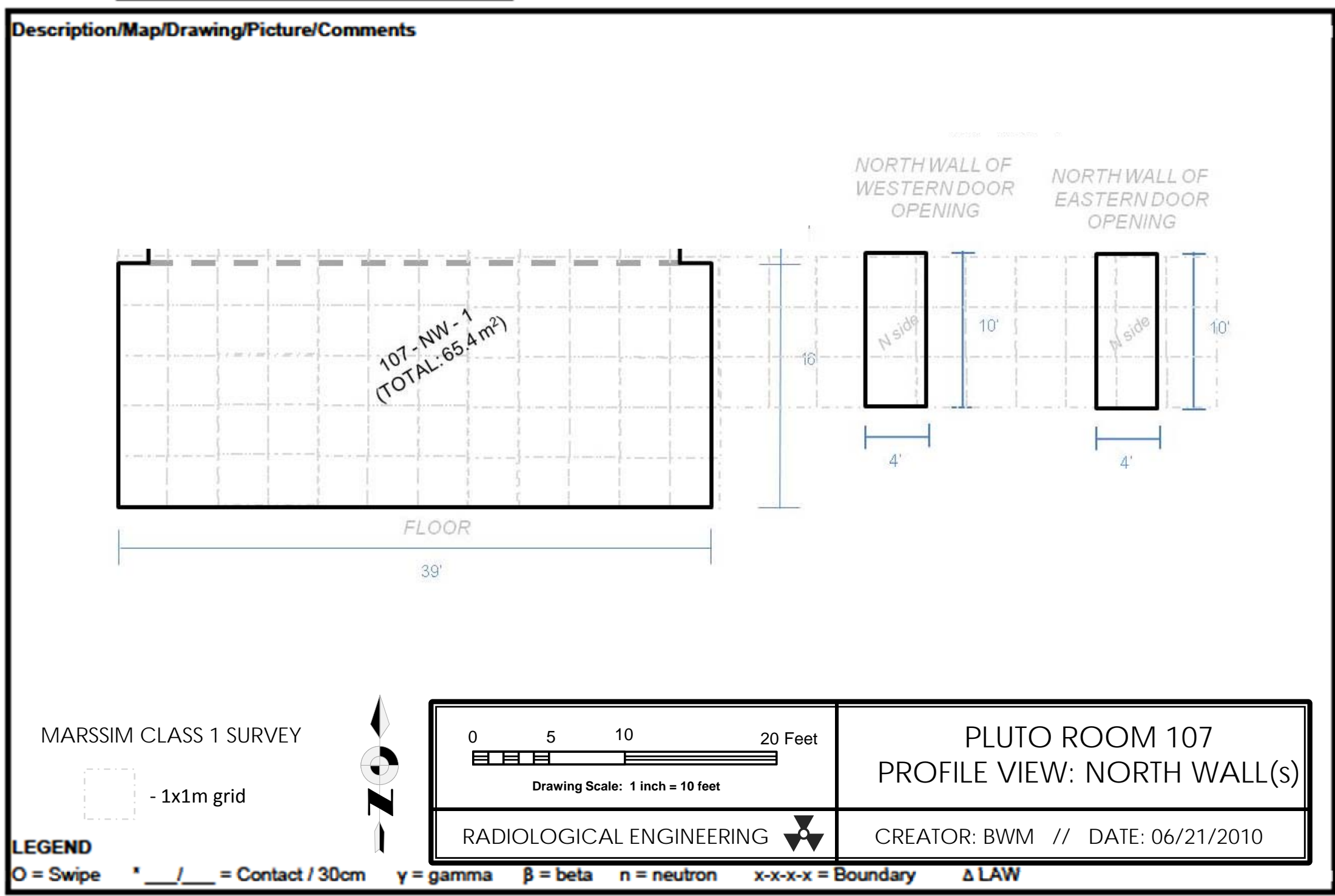


Survey \#

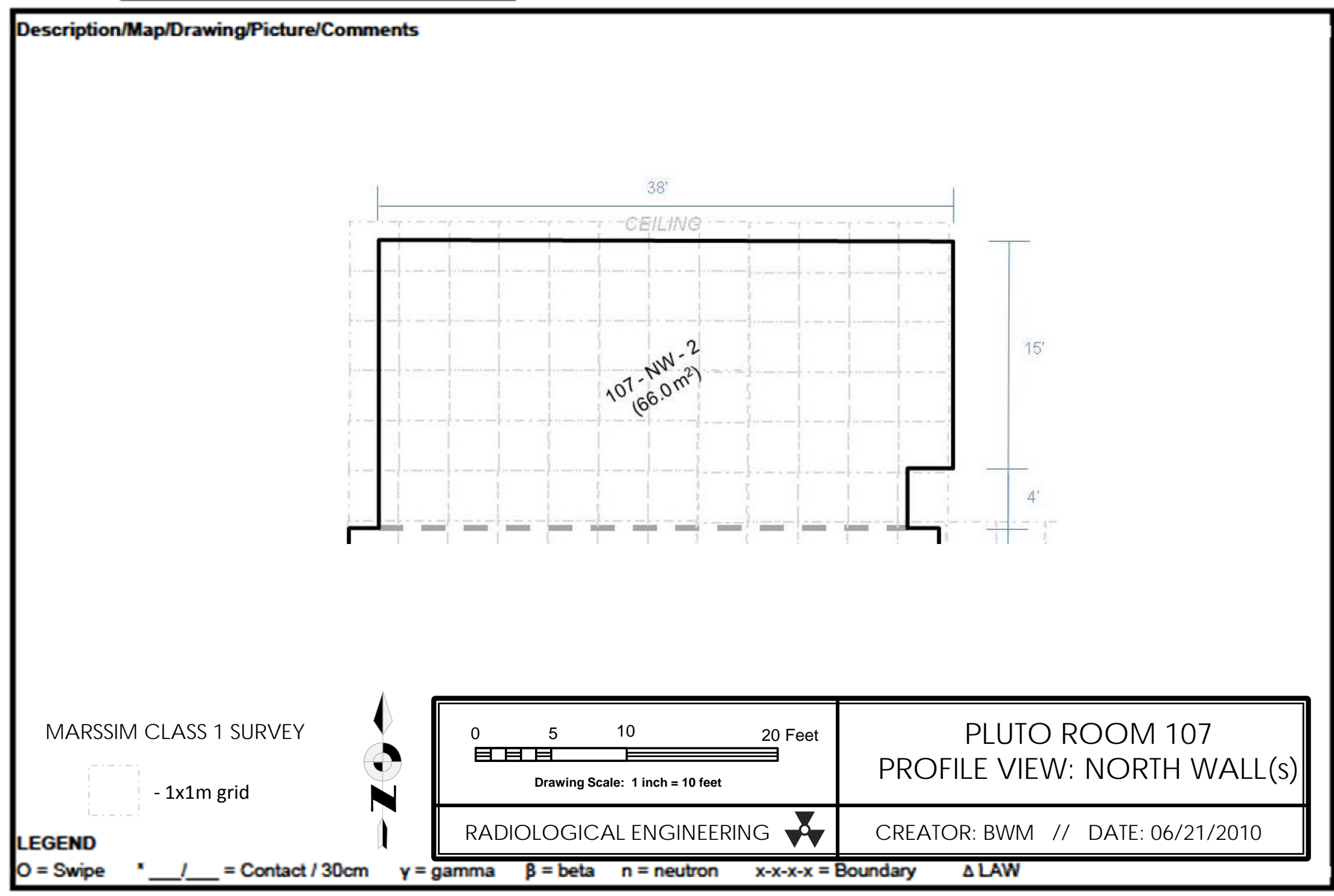


Survey \#

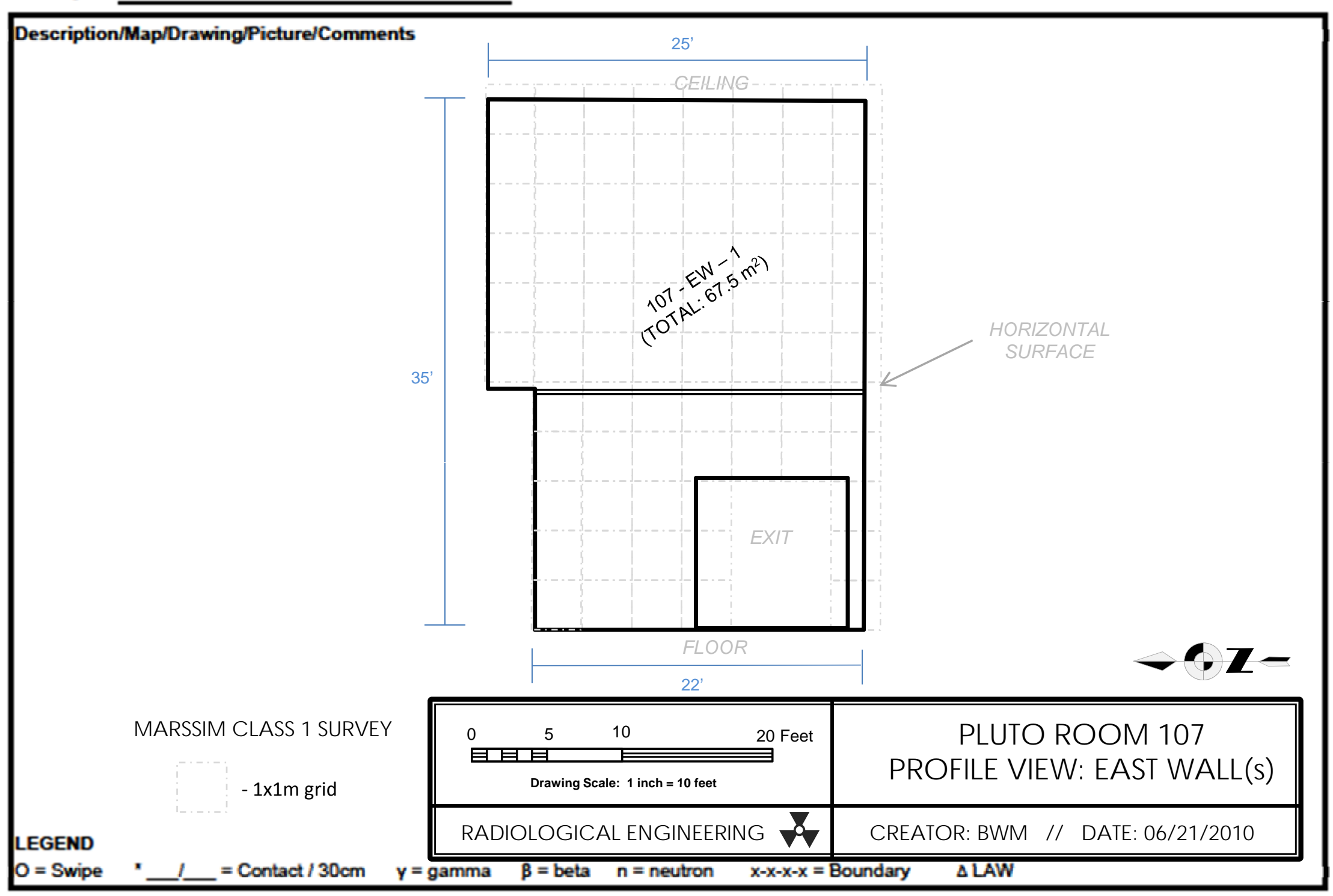


Survey \#

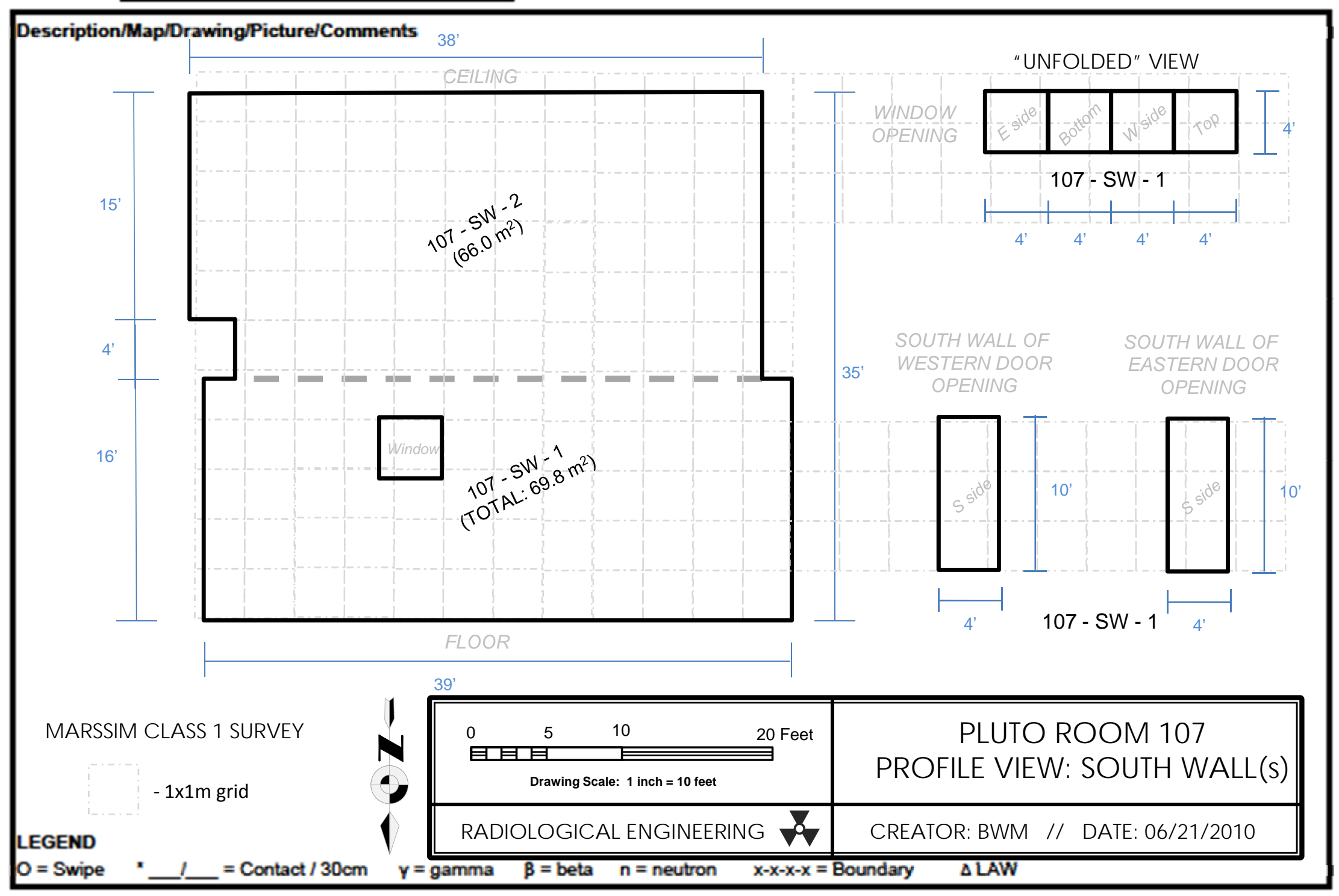


Survey \#

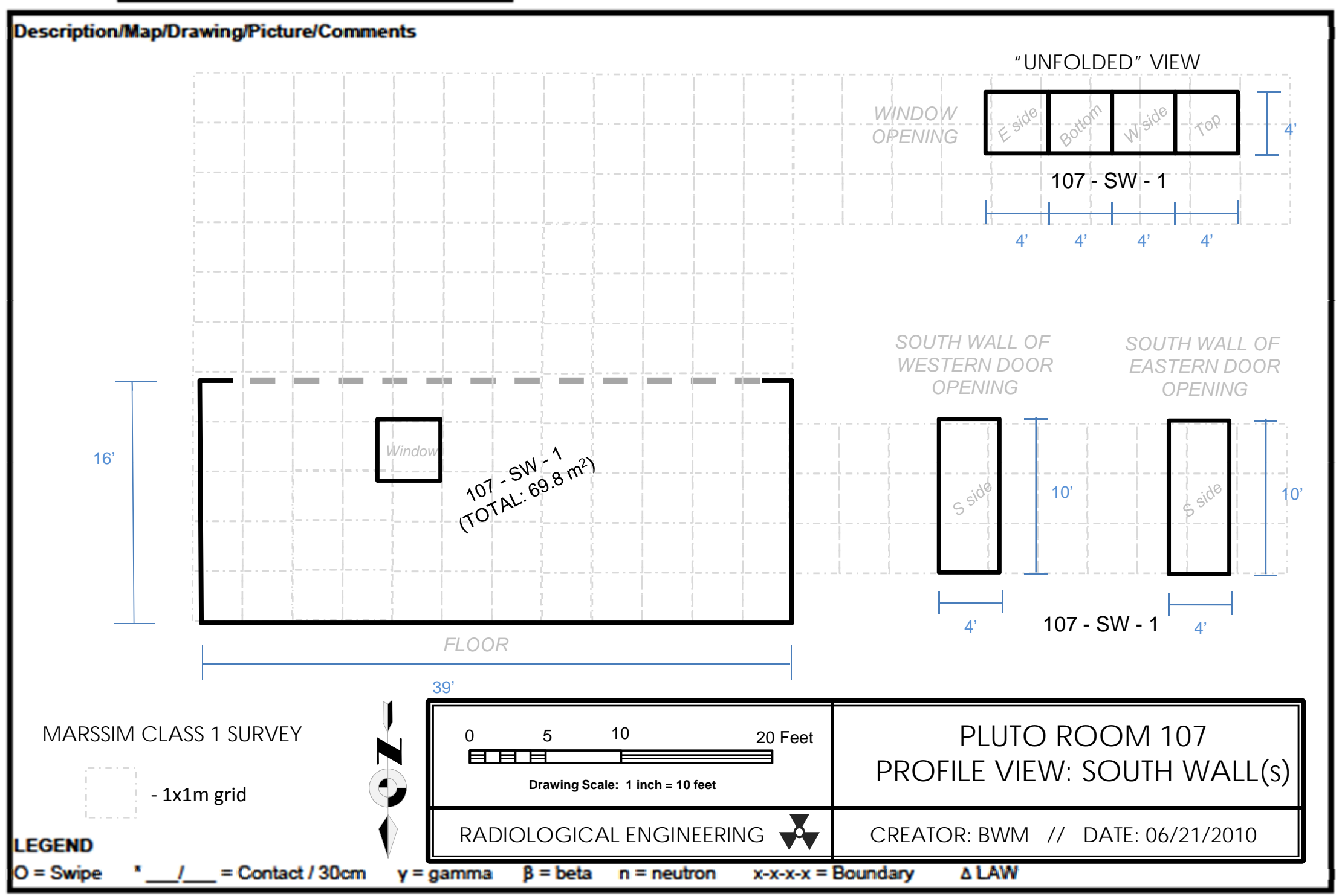


Survey \#

Description/Map/Drawing/Picture/Comments

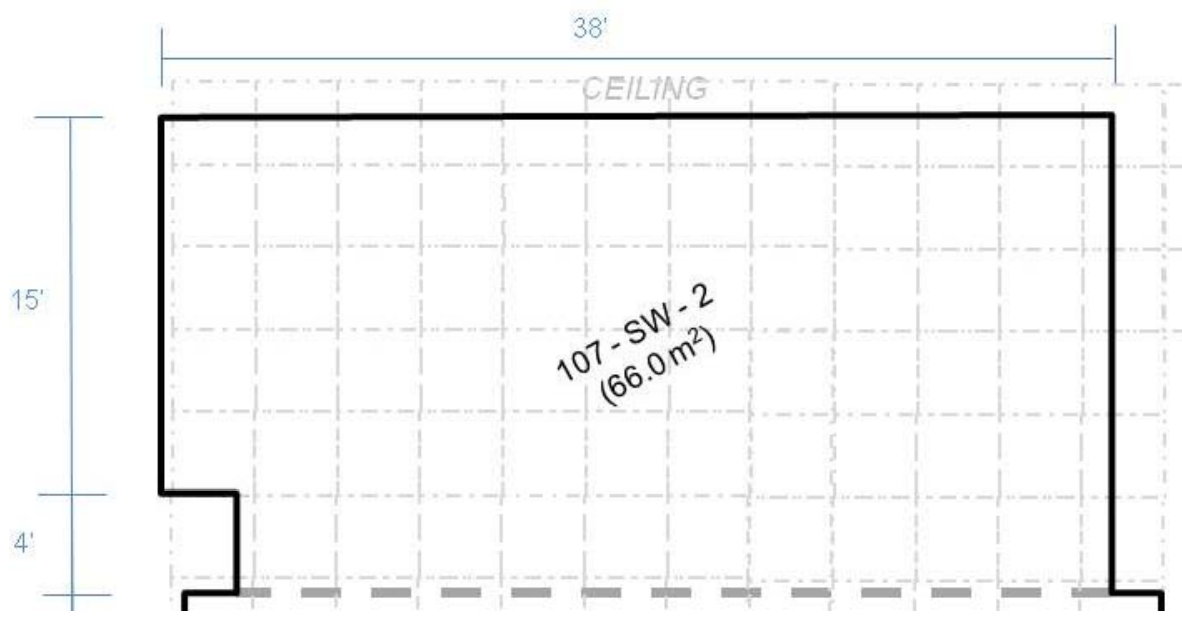

MARSSIM CLASS 1 SURVEY

$-1 \times 1 m$ grid

LEGEND

1

\begin{tabular}{|c|c|}
\hline 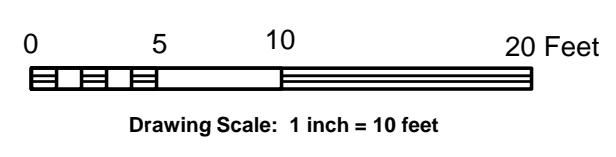 & $\begin{array}{c}\text { PLUTO ROOM } 107 \\
\text { PROFILE VIEW: SOUTH WAШ(s) }\end{array}$ \\
\hline 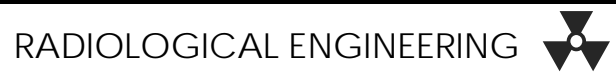 & CREATOR: BWM // DATE: 06/21/2010 \\
\hline
\end{tabular}

O = Swipe 
Survey \#

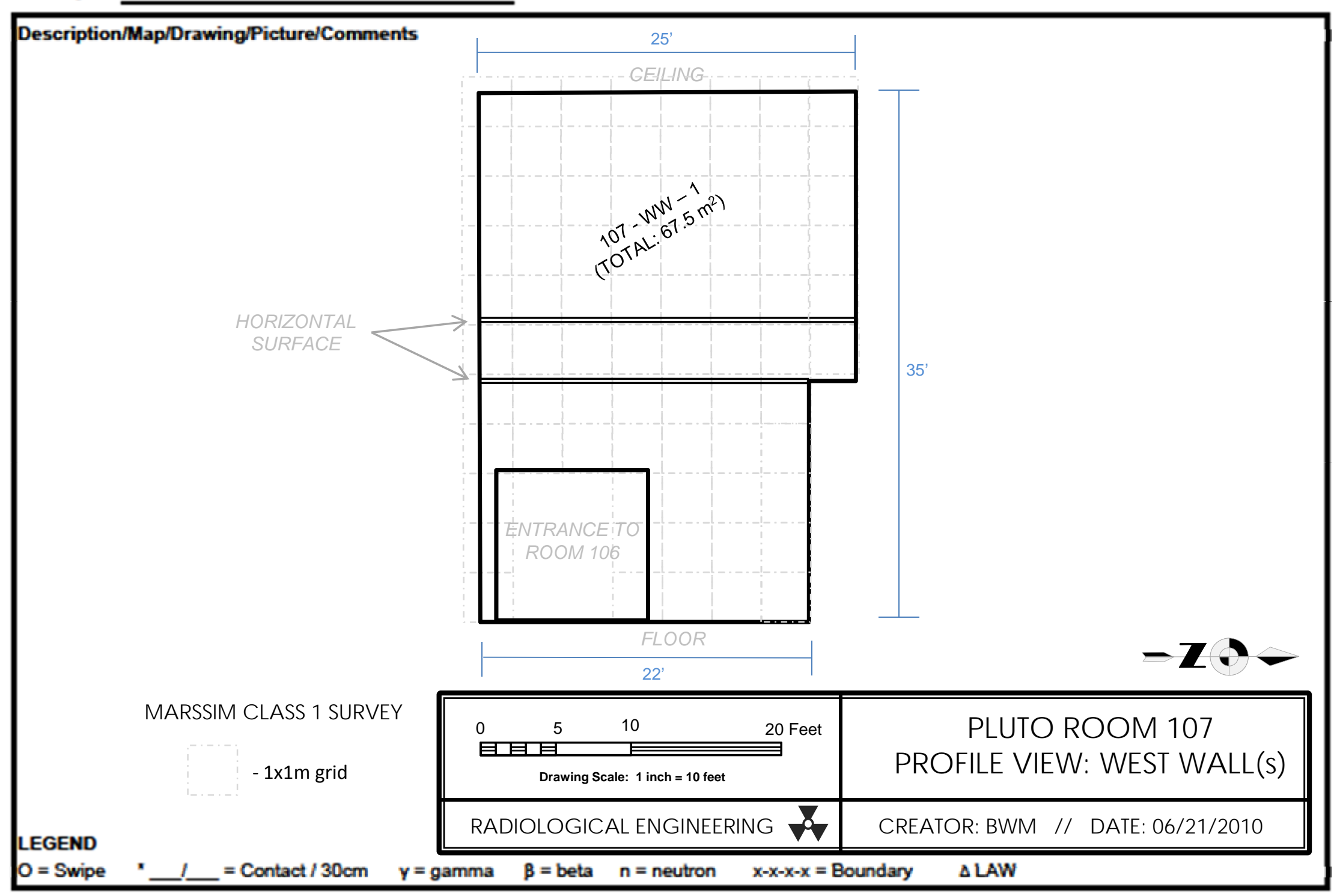


Survey \#

\section{Description/Map/Drawing/Picture/Comments}
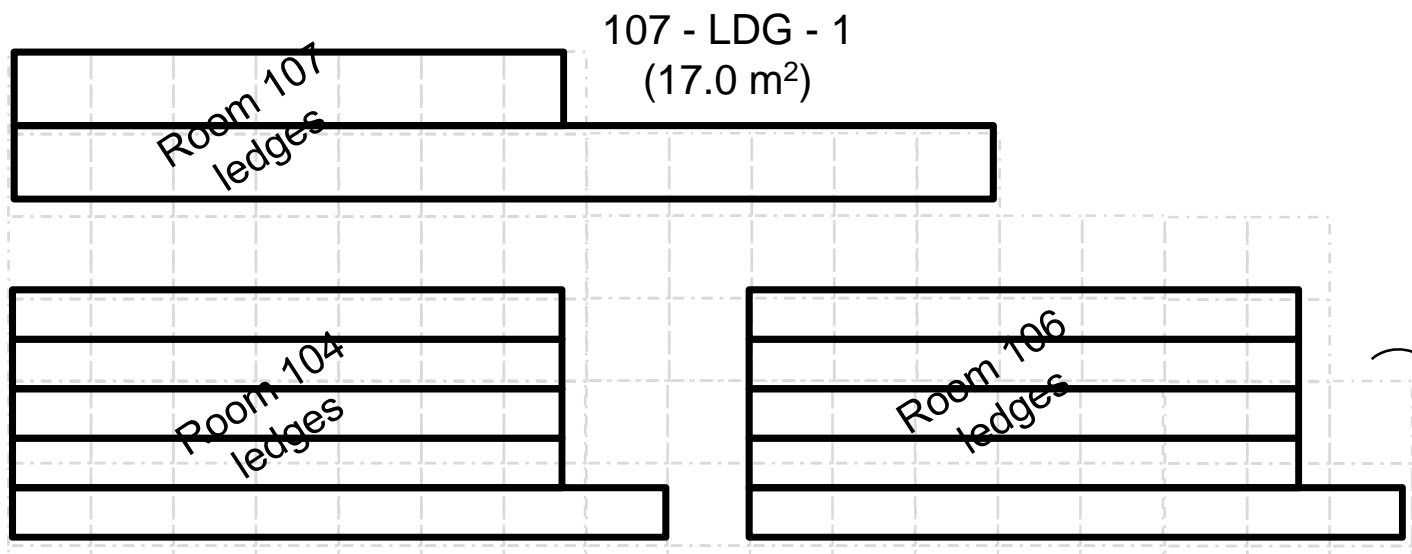

2201 - LDG - 1

[Inc. rooms 102, 104, 106] (TOTAL: $99.6 \mathrm{~m}^{2}$ )

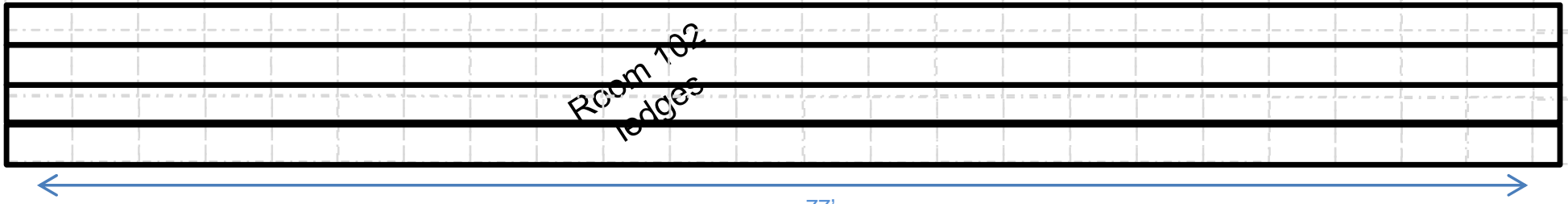

MARSSIM CLASS 1 SURVEY

$-1 \times 1 m$ grid

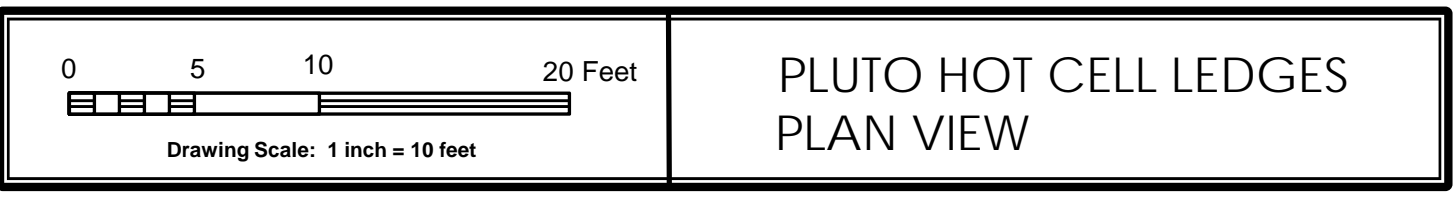

LEGEND

0 = Swipe $\quad x \quad 1 \quad=$ Contact $/ 30 \mathrm{~cm}$ 
Final Status Survey Report for

Corrective Action Unit 117-Pluto

Disassembly Facility, Building 2201

\section{ATTACHMENT 2}

Survey Plan 09-022, "Pluto Disassembly Facility - Building Radiological Characterization" 


\section{National Security Technologies LLc \\ Vision-Senvice - Partnership}

\section{Radiological Operations \\ Survey Plan}

Pluto Disassembly Facility - Building 2201

Radiological Characterization

09-022

July 22, 2009
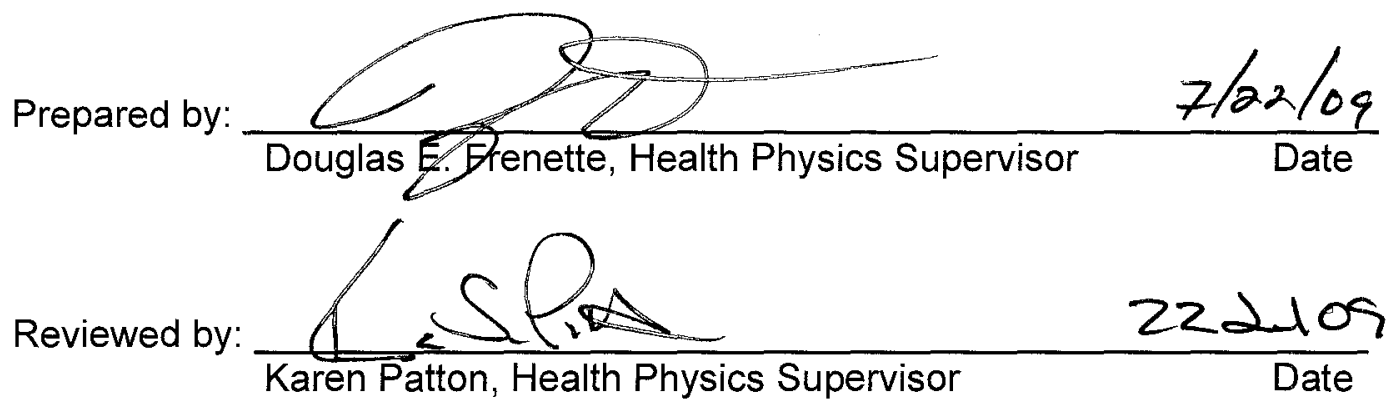

Approved by: 


\section{Pluto Disassembly Facility, Building 2201 - Radiological Characterization}

\section{Purpose}

This plan provides Radiological Control Technicians (RCTs) with the survey criteria required to characterize the building 2201, Pluto Disassembly Facility for radiological purposes.

\section{Scope}

This plan pertains to the entire building 2201 "Pluto Disassembly Facility" in area 26. The term "building" in reference to this survey plan applies to the physical structure (floors, ceilings, walls) and all equipment, components, systems, basement, stack and vaults contain on or within the structure. It does not pertain to the landmass, or exterior aboveground and below ground facility support systems (electrical, water, sewer, etc.).

Results of this survey may be used to;

- Fully determine the extent of contamination in rooms and equipment where radiological operations may have or were known to have occurred.

- Further validate the non-radiological status of rooms and equipment that are anticipated to be free of radiological material based off historical records.

- Provide data for risk/dose analysis and ALARA assessments, including potential Federal Facilities Agreement and Consent Order (FFACO) Use Restrictions.

- Evaluate future building disposition options.

- Determine future remedial action requirements, i.e. appropriate decontamination rigor, methods and costs based on proposed disposition.

Implementing the Multiagency Radiation Survey and Site Investigation Manual (MARSSIM) process.

As part of the MARSSIM approach to releasing building 2201 from radiological controls the standards require the following steps;

1. Historical Site Assessment (HSA) - This is the collection of data from historical documentation, observation or personnel interviews, also known as "process knowledge.” A brief synopsis of the facility and operations is included in later sections.

2. Scoping Survey - A scoping survey is a "big picture" type survey performed if the HSA indicates an area may be impacted. It should also determine the specific contaminant(s) of concern. In the case of the Pluto Disassembly Facility, it has already been established that various rooms and systems are or may be impacted. This step has been accomplished through pre-job and job coverage surveys performed during D\&D preparations last year including removal of hazardous materials, asbestos abatement, general equipment strip-out, stabilization of bi-parting doors, vault investigation and draining of all fluid systems. It is a culmination of several smaller surveys and analytical samples. 
Pluto Disassembly Facility, Building 2201 - Radiological Characterization

3. Characterization Survey - This is a more comprehensive survey and determines the nature and extent of the contamination, remedial alternatives, and provides data for the Final Status Survey. This is the intent of this survey plan.

4. Remedial Action Support Survey - This survey is more commonly referred to on the NTS as "in-process" or "job coverage” survey. Its purpose is to provide real time data that will help guide remedial action operations (i.e. decontamination and contaminated equipment removal.) This survey may or may not require a survey plan.

5. Final Status Survey - This survey is used to demonstrate that the potential dose from residual contamination is below release criteria. Release criteria depend on several factors but primarily on what the final disposition is (i.e. release to the public, release to an onsite landfill, below FFACO use restriction, etc.) This survey is the most comprehensive survey performed and has the most stringent requirements built into it for the type, quantity and quality of data acquired. This survey requires a detailed survey plan with a high level of approval including the regulatory agencies representatives.

6. Verification Survey - To determine if the site is acceptable for release, the regulatory agency might bring in an independent contractor to perform this survey. It is typically a survey with limited scope that might duplicate $10 \%$ of the final status survey measurements.

Note: Depending on the outcome of the characterization survey, and the subsequent cost analysis to decontaminate or remediate the Pluto Disassembly Facility, the Remedial Action Support survey, Final Status survey and Verification survey may not be warranted as the building may not be released from radiological controls and instead, disposed of as Low Level Waste.

\section{History}

Building 2201 was constructed between May 1959 and October 1960 in order to perform remote adjustment, component replacement and complete disassembly of Tory II reactors for Project Pluto.

Between the years 1961 - 1964, Lawrence Livermore National Laboratories (LLNL) conducted six nuclear ram-jet engine tests as part of Project Pluto. Four (4) tests of the Tory II-A Reactor and two (2) tests of the Tory II-C Reactor were conducted. Assembly and disassembly operations of the Tory II-A and Tory II-C took place in Building 2201. Each of these reactors contained highly enriched uranium dioxide $\left(\mathrm{UO}_{2}\right)$ contained in fuel rods that were remotely disassembled and inspected in the hot cell rooms after each test run. July 1, 1964 Project Pluto was cancelled.

Between the years 1971-1972, fuel repackaging operations took place in the hot cells of building 2201. During these activities, decontamination and radiological surveys were conducted revealing radiological contamination in the hot cell rooms. 


\section{Pluto Disassembly Facility, Building 2201 - Radiological Characterization}

In June of 1974, the Tory II-C Reactor was moved to another facility in Area 25 for final disassembly.

In the mid to late 1980's until 1992 Hydrogen Content Testing was performed in rooms 101 \& 102 utilizing radioactive sources containing isotopes such as Cs-137, Plutonium/Beryllium (PuBe), and Americium /Beryllium (AmBe).

In 1985, 1998 and 1999 walk-through surveys were performed inside building 2201, including the hot cell rooms. Varying levels of contamination were found during these surveys.

In 1998, Sandia National Laboratory intermittently used building 2201 in order to assemble and launch rocket test systems. Very little documentation is available during this time frame due to the classified nature of the project. This is the last known documented operation performed in the building.

Recent Activities - In the past few years building 2201 has been slated for D\&D and preparations are ongoing. These preparations include the removal of hazardous materials, asbestos abatement, general equipment strip-out, stabilization of bi-parting doors, vault investigation, grouting of drainage systems and draining of all fluid systems. The most recent radiological swipe surveys of the most potentially radiologically impacted rooms; Room $102-$ Main Disassembly Bay, Room 104 - Hot and Warm Cell, Room 106 - Kilo-Curie Hot Cell, Room 107 - Hot Storage and Packaging Room indicate past decontamination efforts were largely successful. Some areas and equipment in the aforementioned rooms; primarily located above 8 feet, indicate low levels of direct and removable, transuranic, alpha-emitting contamination; primarily (Plutonium-239 \& Americium-241), but also include mixed fission products and enriched uranium fuel. Removable levels have been found and documented above the limits specified in NV/YMP Radiological Control Manual, rev. 5, table 4-2 but are less than 100 times those values $\left(<2,000 \mathrm{dpm} / 100 \mathrm{~cm}^{2}\right.$ alpha.)

Ventilation System - The process ventilation system in the Pluto Disassembly Facility was also found to have gross contamination above NV/YMP Radiological Control Manual, rev. 5, table 4-2. A portion of the ventilation system is internal to the concrete reinforced walls and in other places the system is located below grade before recombining in Room 103 and exiting out the building through the building's stack.

The system supported operations in rooms 102, 104, 106, \& 107. Each of the rooms had a set, or "bank" of HEPA filters - these were the $1^{\text {st }}$ stage filters and intake locations for the system. The system then travels a short distance to either room 103 "Equipment room" or the facilities basement. At these locations air was forced through another bank of HEPA filters ( $2^{\text {nd }}$ stage) before it entered a common header with the other process rooms and was exhausted out through the building stack. All of the HEPA filters in all of the racks have been removed and disposed of. The only contamination found was at, or immediately downstream of the first stage HEPA filters. In all cases, the intake filter frames, or "racks" were removed and disposed of and the remaining framework embedded in the structure's concrete walls were encapsulated with a fixative. 


\title{
Pluto Disassembly Facility, Building 2201 - Radiological Characterization
}

Drains - The radioactive floor drains or "hot waste drainage system" is installed and was used in rooms 101, 102, 104, 106 \& 107. This system was connected to a radioactive leach field located approximately 1,600 feet southeast of the building. Other drains from the pits housing the pulley system for the bi-parting doors were also connected to this system. All drain points were surveyed between April 2008 and September 2008 and all readings were indistinguishable from background. The drains were all then sealed with grout to prevent any further infiltration of surface water.

Vaults - There are four known underground vaults in building 2201, these vaults were investigated between April 2008 and September 2008. During this time, each of the four vaults were core drilled, video monitored, sampled and surveyed. The vaults were then resealed. Each vault was described as being 5 feet square and 10 feet deep pits with a layered 3 foot thick cover. Each vault had a multi-level shelf system installed. Radiological control personnel noted all readings were indistinguishable from background and did not find evidence that any nuclear material remained.

\section{Instructions}

\author{
***Caution*** \\ In carrying out the instructions of this survey plan, RCTs may encounter areas meeting \\ the definition of a Confined Space (Permitted or Non-Permitted). RCTs are NOT to enter \\ these areas but should attempt to accomplish the goals of this survey plan through the use \\ of extension tools and remote reading instrumentation.
}

All surveys shall be performed by qualified personnel. Personnel must use their experience, knowledge of the area in which they are working, and workplace indicators to ensure adequate surveys are being performed, in addition to the information provided in this document.

Based on process knowledge, historical documentation and recent radiological surveys performed, the building will be divided into two categories to establish survey rigor - "Higher Risk," and "Lower Risk."

Any surveys performed above 8 feet by RCTs in rooms 102, 104, 106 \& 107 shall be done under the control of RWP 09-0023-06. These rooms are known to have removable contamination on equipment and surfaces above 8 feet above limits from the NV/YMP Radiological Control Manual, revision 5, table 4-2.

Since the intent of this survey plan is for characterization and not for release, release limits are not applicable. Instead, RCTs will operate under the suspension limits of the RWP and will record all data. 


\section{Pluto Disassembly Facility, Building 2201 - Radiological Characterization}

In order to determine the degree and rigor of future remediation, RCTs shall document with great accuracy any location found above the limits from the NV/YMP Radiological Control Manual, revision 5, table 4-2;

- $20 \mathrm{dpm} / 100 \mathrm{~cm}^{2}$ alpha (removable)

- $100 \mathrm{dpm} / 100 \mathrm{~cm}^{2}$ alpha (fixed \& removable)

- $1,000 \mathrm{dpm} / 100 \mathrm{~cm}^{2}$ beta (removable)

- $5,000 \mathrm{dpm} / 100 \mathrm{~cm}^{2}$ beta (fixed \& removable)

Physically gridding off the entire building is not necessary for this survey plan. However, in all rooms designated as "Higher Risk," gridding of the floors, ceiling and walls is mandatory. Grids in these rooms will be made as specified in Appendix A. For areas considered "Lower Risk," in lieu of gridding, RCTs must mark where survey data is obtained and in the unlikely event, unmistakably demarcate locations where contamination is found above the aforementioned levels; including the posting of approved radiological postings.

Background levels for direct surveys shall be less than $40 \mathrm{dpm} / 100 \mathrm{~cm}^{2}$ alpha and 4,000 $\mathrm{dpm} / 100 \mathrm{~cm}^{2}$ beta.

All surveys shall be performed and documented in accordance with OI-0441.211, "Direct and Indirect Surveys.” Survey results shall be recorded on a FRM-0108, "Radiological Survey Report,” and approved by the Environmental Restoration Health Physics Supervisor or designee.

During this survey process if contamination is found that is above the suspension limits of the RWP, work shall be paused, personnel in the affected area or room notified of the condition and instructed to exit the area or room. RCTs will immediately notify the ER Health Physics Supervisor and survey any personnel who were in the affected room or area. Based on the levels encountered, RCTs will also adjust radiological postings to be commensurate with the actual area conditions.

\section{Contamination Surveys}

Higher Risk - The following rooms in building 2201 are considered higher risk;

- Room 102 - Main Disassembly Bay

- Room 104 - Hot and Warm Cell

- Room 106 - Kilo-Curie Hot Cell

- Room 107 - Hot Storage and Packaging Room

Note: Included in this category are the ventilation system intakes for each room and the area immediately inside.

Each of these rooms shall be surveyed using the following guidelines;

Direct surveys $-100 \%$ of accessible surfaces on all walls, floors, ceilings and equipment shall be scanned with an NE Electra and DP6 Probe (or equivalent) followed with a direct static 


\section{Pluto Disassembly Facility, Building 2201 - Radiological Characterization}

measurement at the highest direct scanned reading in each grid location, or "survey unit" documented for both alpha and beta.

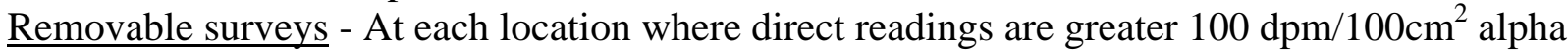
or $5,000 \mathrm{dpm} / 100 \mathrm{~cm}^{2}$ beta, a swipe shall be taken and documented. Otherwise, swipes are not required.

For equipment in these rooms (i.e. light fixtures, cranes, manipulators, piping, tanks, ventilation intakes, etc.) RCTs shall direct scan with an NE Electra and DP6 Probe (or equivalent) 100\% of accessible surfaces followed with a direct static measurement at the highest direct scanned reading. Swipes shall be performed whenever the direct static readings are greater than 100 $\mathrm{dpm} / 100 \mathrm{~cm}^{2}$ alpha or $5,000 \mathrm{dpm} / 100 \mathrm{~cm}^{2}$ beta. Equipment found to be contaminated shall be documented as such with the location and highest reading recorded.

Lower Risk - All other rooms, equipment and systems not listed in the previous section as "High Risk" will be considered Lower Risk, and surveyed under the following guidelines;

\section{Direct Surveys}

- Rooms - RCTs shall estimate 10 square meter sections of rooms and take one direct reading per section. The reading shall be documented for both alpha and beta results.

- Building Exterior - RCTs shall estimate 10 square meter sections of the building exterior surfaces (walls and roof) and take one direct reading per section. The reading shall be documented for both alpha and beta results.

- Stack and Ventilation System - RCTs shall open any and all readily accessible portions of the ventilation system and perform direct surveys of these areas to the greatest extent possible. The building's stack has access ports at the bottom of the stack and shall be accessed and direct survey readings obtained. All direct readings shall be documented for both alpha and beta results.

If survey results show no elevated readings, no further survey points need to be obtained due to considerations for personnel safety. Conversely, if survey results indicate the presence of radioactive materials at the bottom of the stack, further investigation will be required but only after, Field Operations and Health and Safety departments have evaluated and established a plan to provide RCTs required access to the internals of the stack.

Exception: The intake opening to the ventilation system in rooms 102, 104, 106 \& 107 are considered "Higher Risk," and therefore the survey requirements of that section are applicable.

- Vaults - Due to their current configuration and inaccessibility, surveys are not required to be performed in these vaults as part of this survey plan.

- Equipment - For any equipment RCTs shall direct scan $100 \%$ of accessible surfaces. RCTs should pay particular attention to those areas where staining and discoloration have occurred or areas where contamination would be likely to accumulate.

Note : These are the minimum required surveys and the RCT is encouraged to take more if an area looks suspicious or if direct readings indicate a potential for contamination exists. 
Pluto Disassembly Facility, Building 2201 - Radiological Characterization

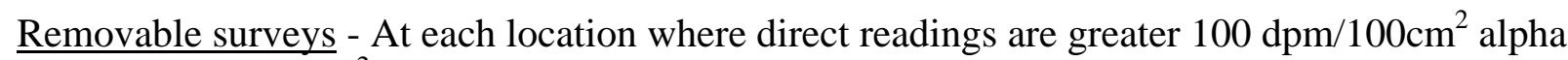
or $5,000 \mathrm{dpm} / 100 \mathrm{~cm}^{2}$ beta, a swipe shall be taken and documented. Otherwise, swipes are not required.

\section{Disposition}

There is no disposition of the building, equipment or system components scheduled at this time. 
Pluto Disassembly Facility, Building 2201 - Radiological Characterization

\section{Appendix A}

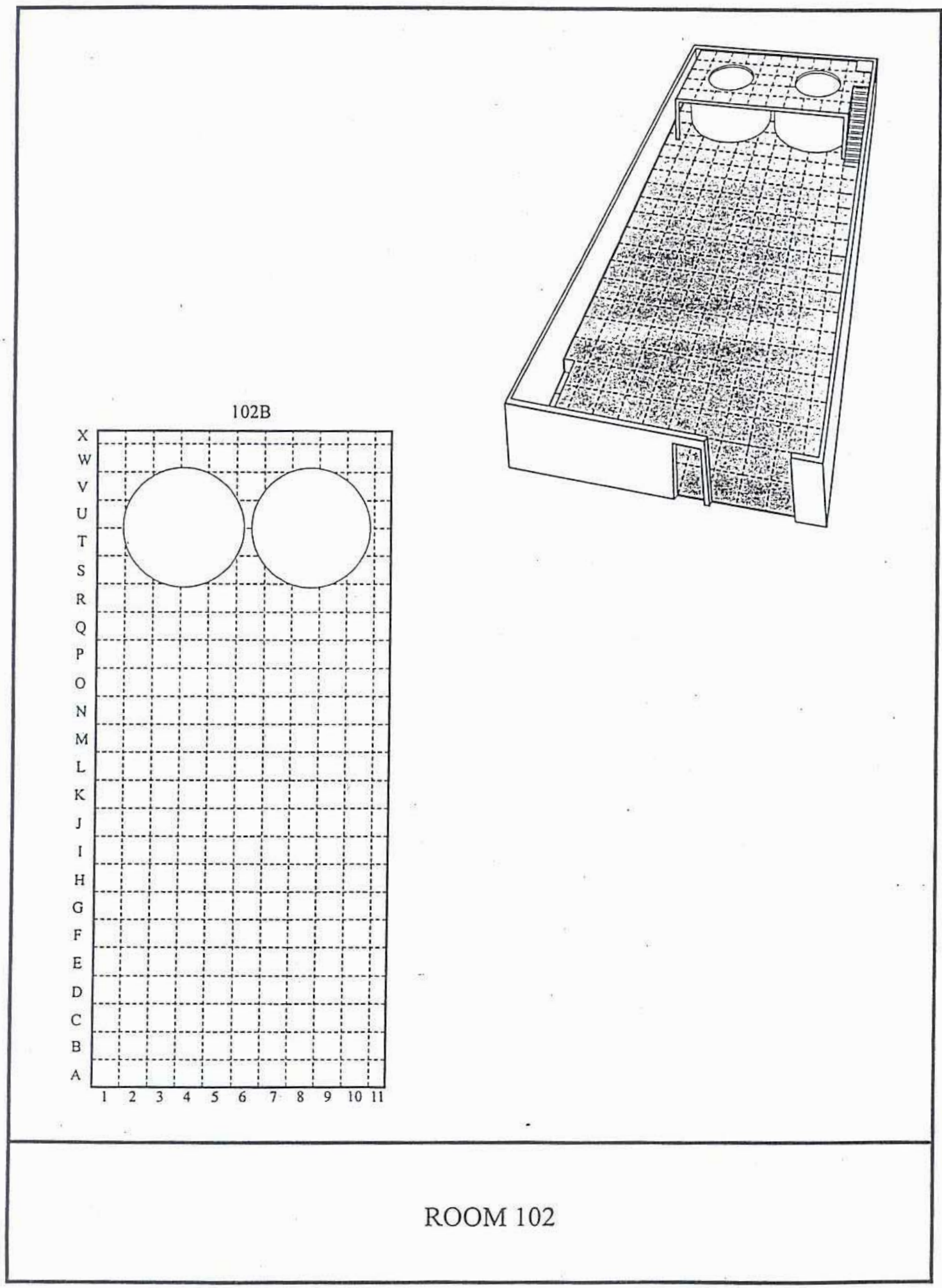


Pluto Disassembly Facility, Building 2201 - Radiological Characterization

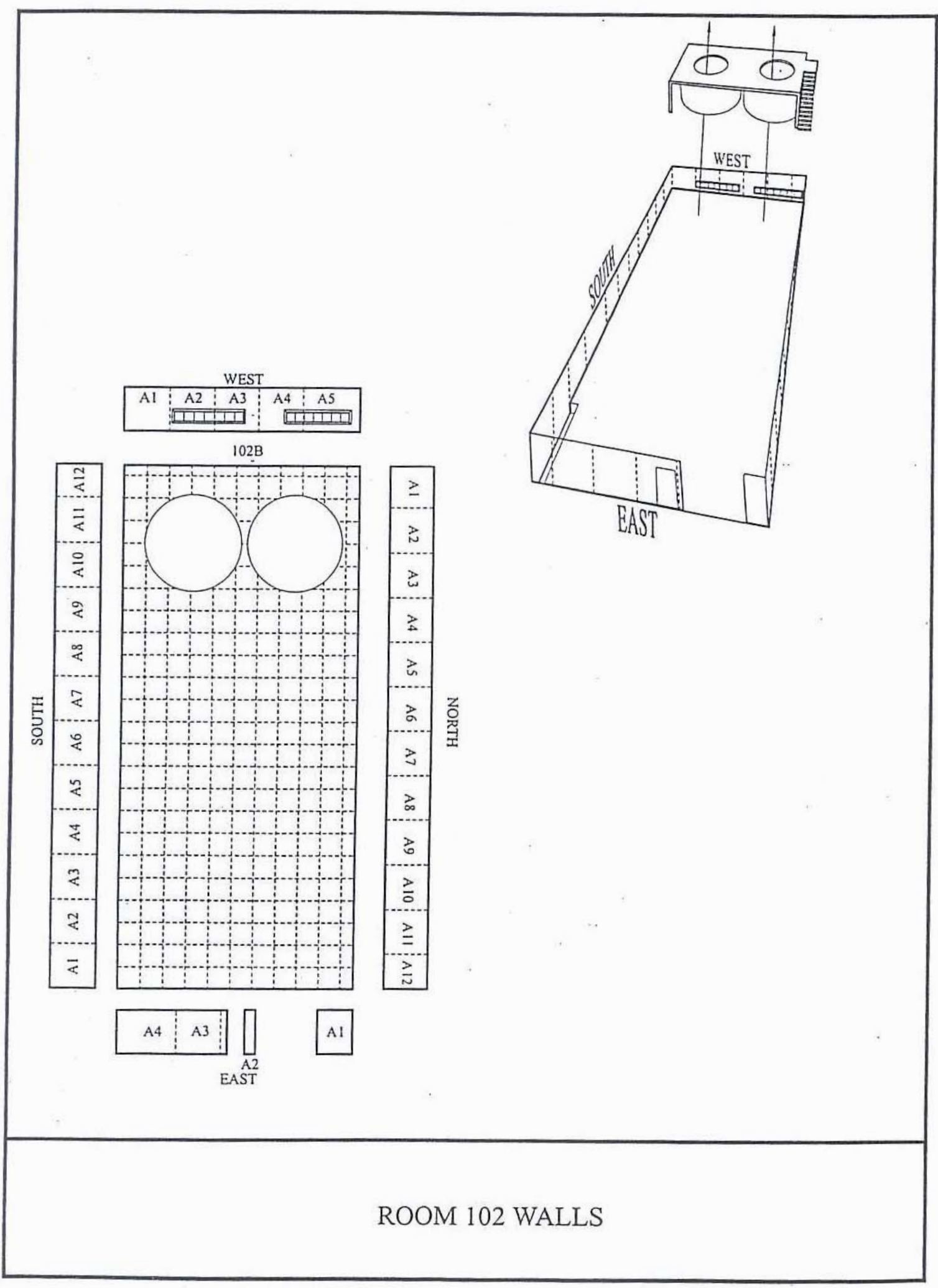


Pluto Disassembly Facility, Building 2201 - Radiological Characterization

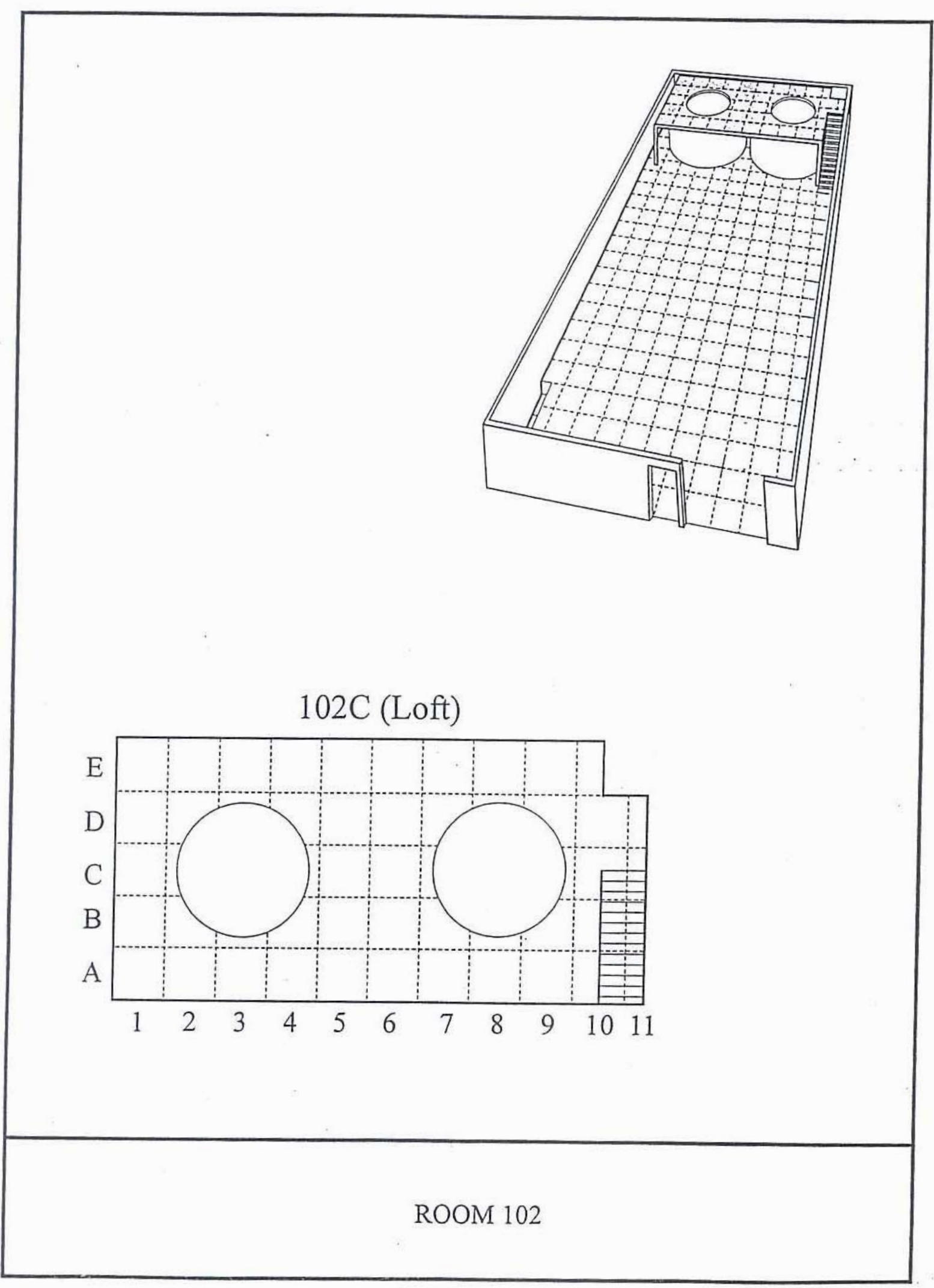


Pluto Disassembly Facility, Building 2201 - Radiological Characterization

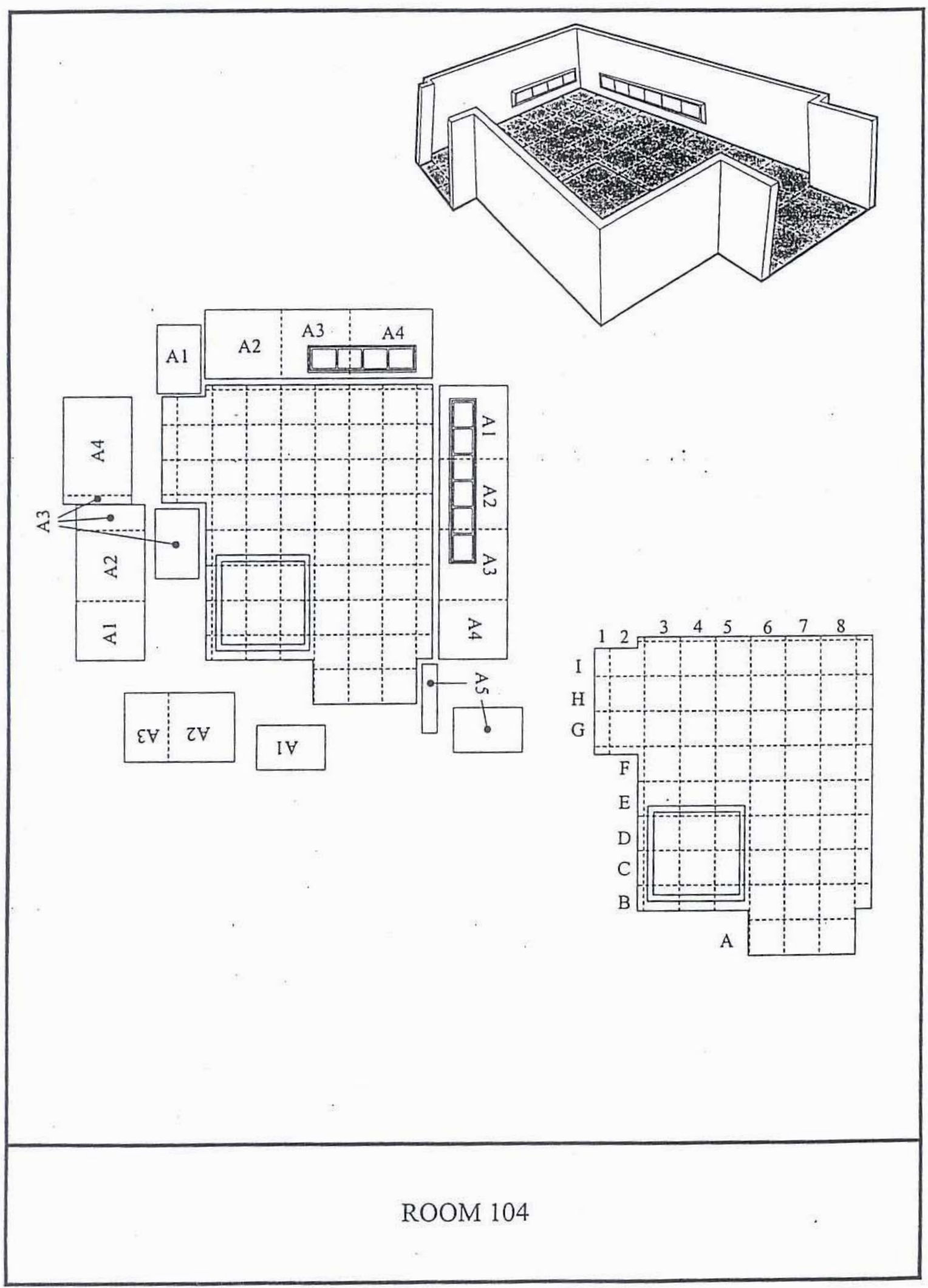


Pluto Disassembly Facility, Building 2201 - Radiological Characterization

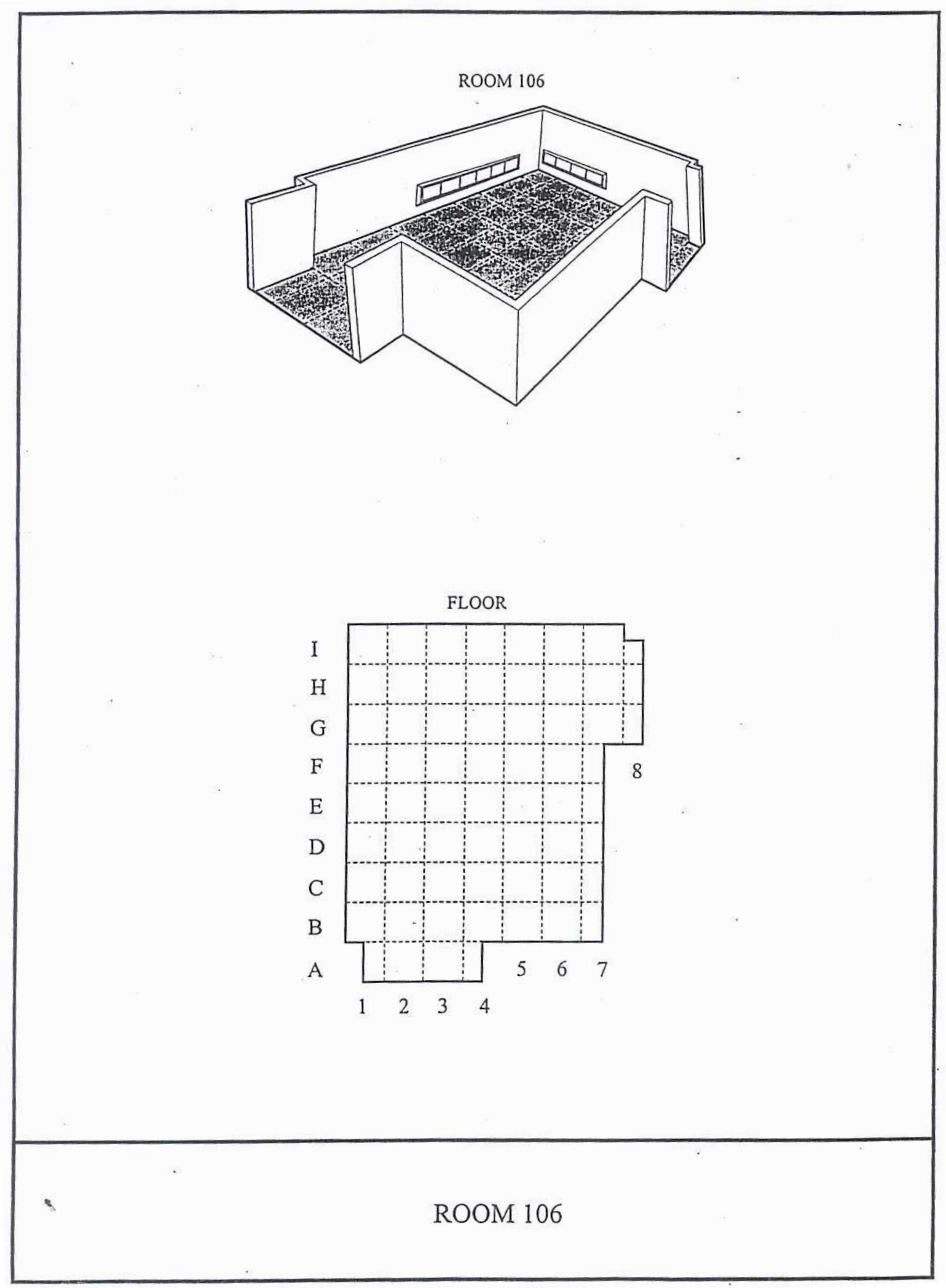


Pluto Disassembly Facility, Building 2201 - Radiological Characterization

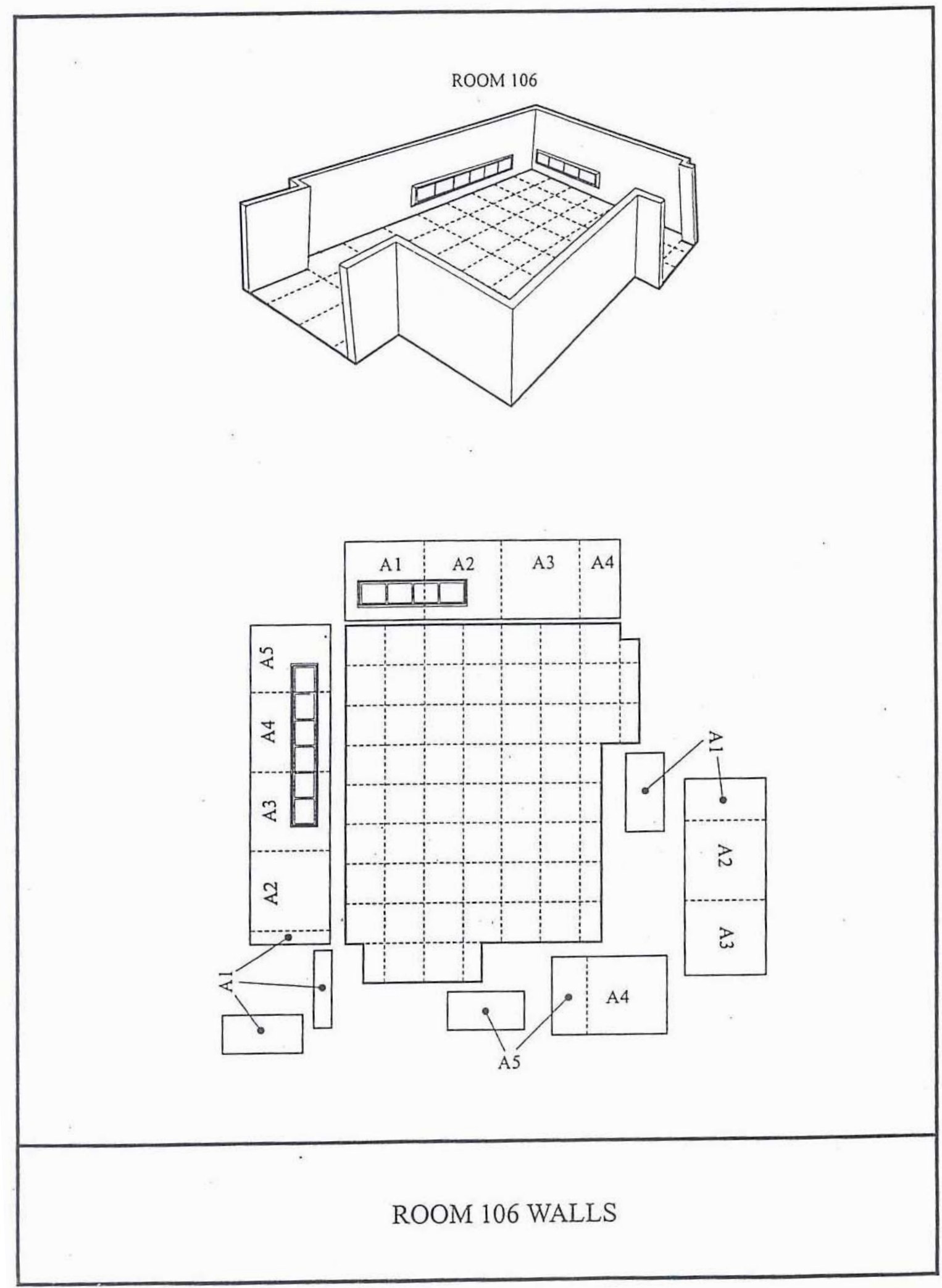


Pluto Disassembly Facility, Building 2201 - Radiological Characterization

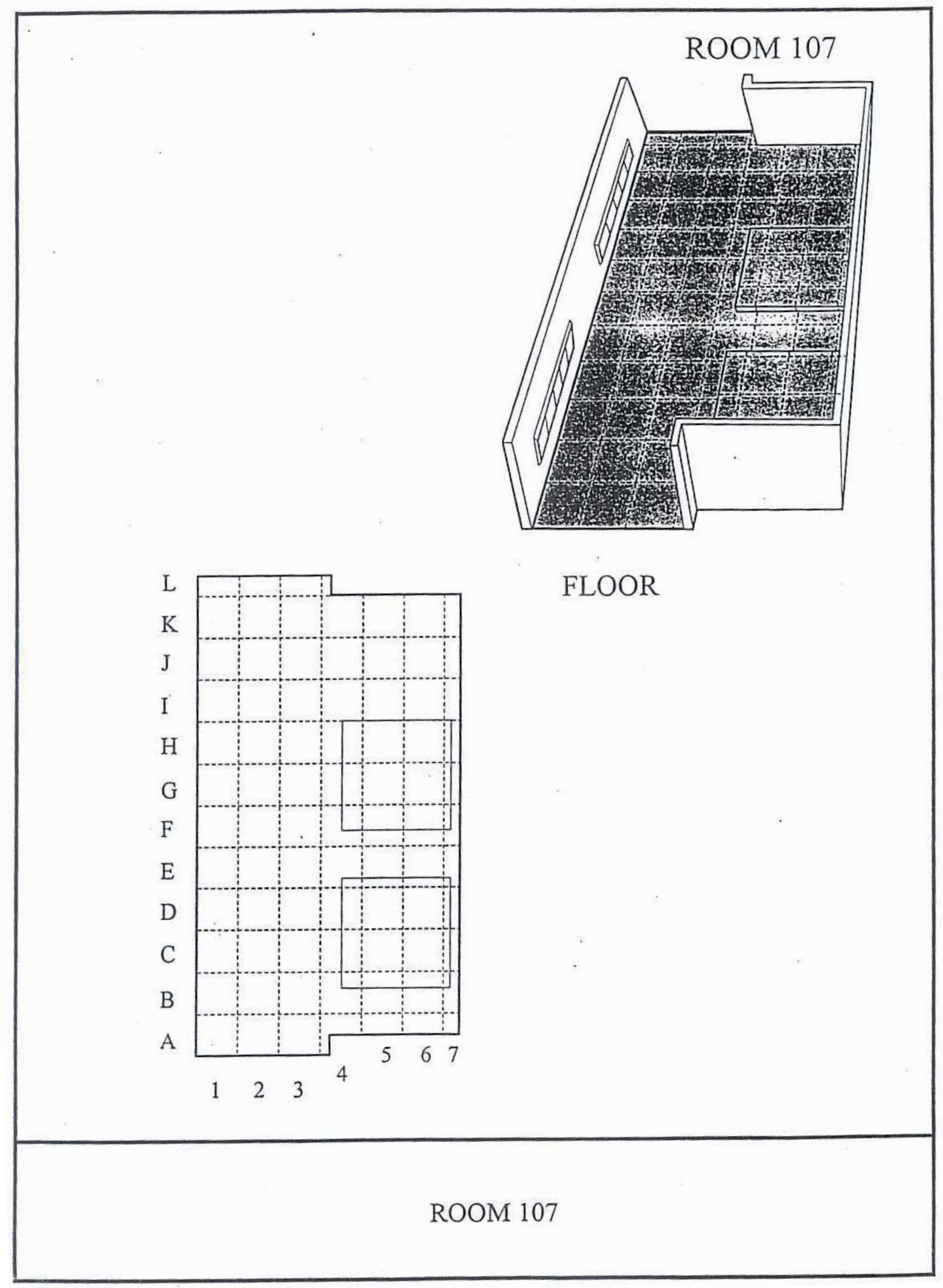


Pluto Disassembly Facility, Building 2201 - Radiological Characterization

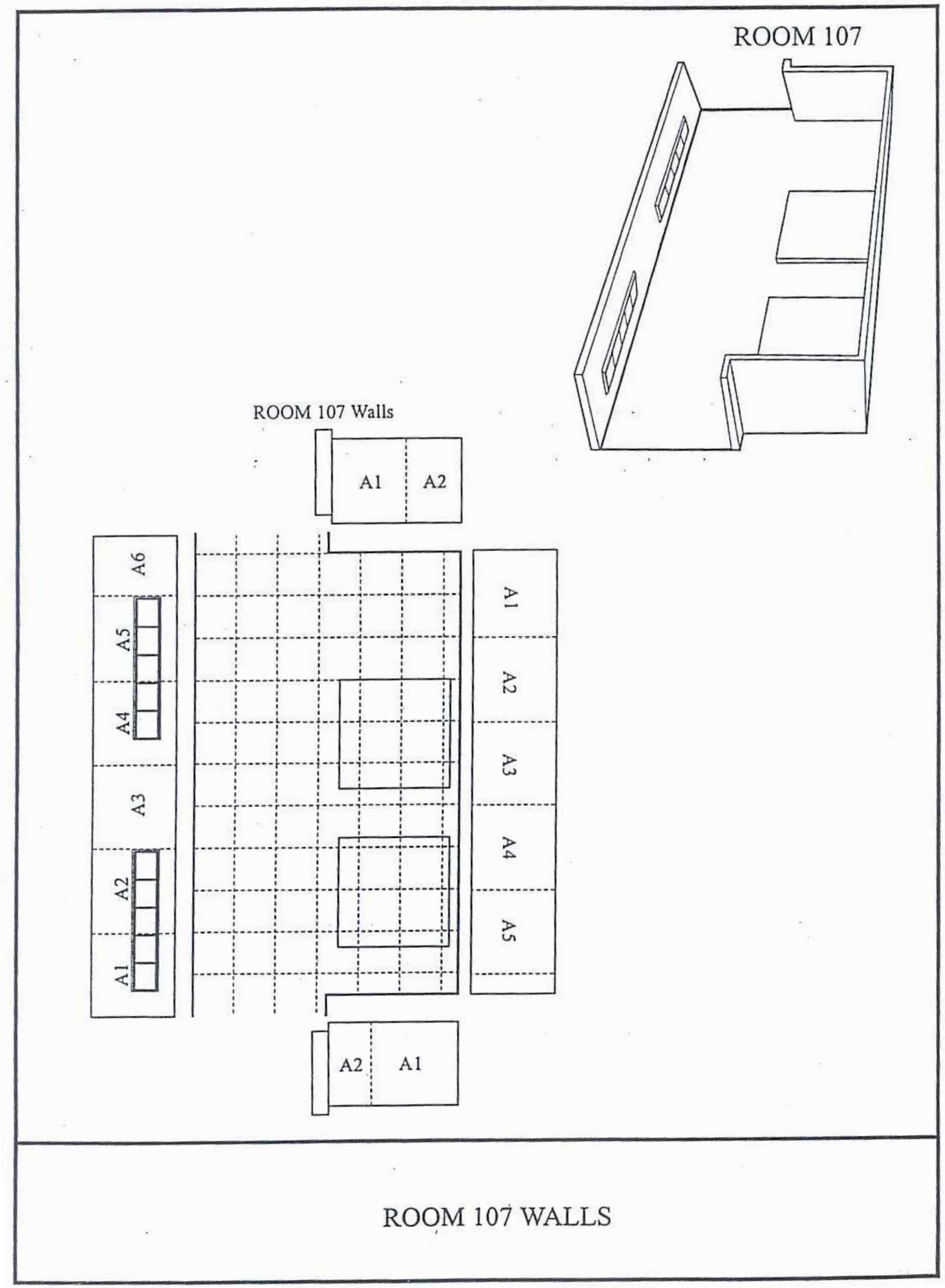


Final Status Survey Report for

Corrective Action Unit 117-Pluto

Disassembly Facility, Building 2201

\section{ATTACHMENT 3}

Survey Plan 09-031, "Pluto Disassembly Facility - Radiological Characterization (Phase II) and Post Remediation Survey Requirements" 


\section{National Security TechnologiesLLC}

Vision - Service - Partnership

Radiological Operations

Survey Plan

PLUTO DISASSEMBLY FACILITY - BUILDING 2201

RADIOLOGICAL CHARACTERIZATION (PHASE II) AND POST REMEDIATION SURVEY REQUIREMENTS

09-031

November 12, 2009
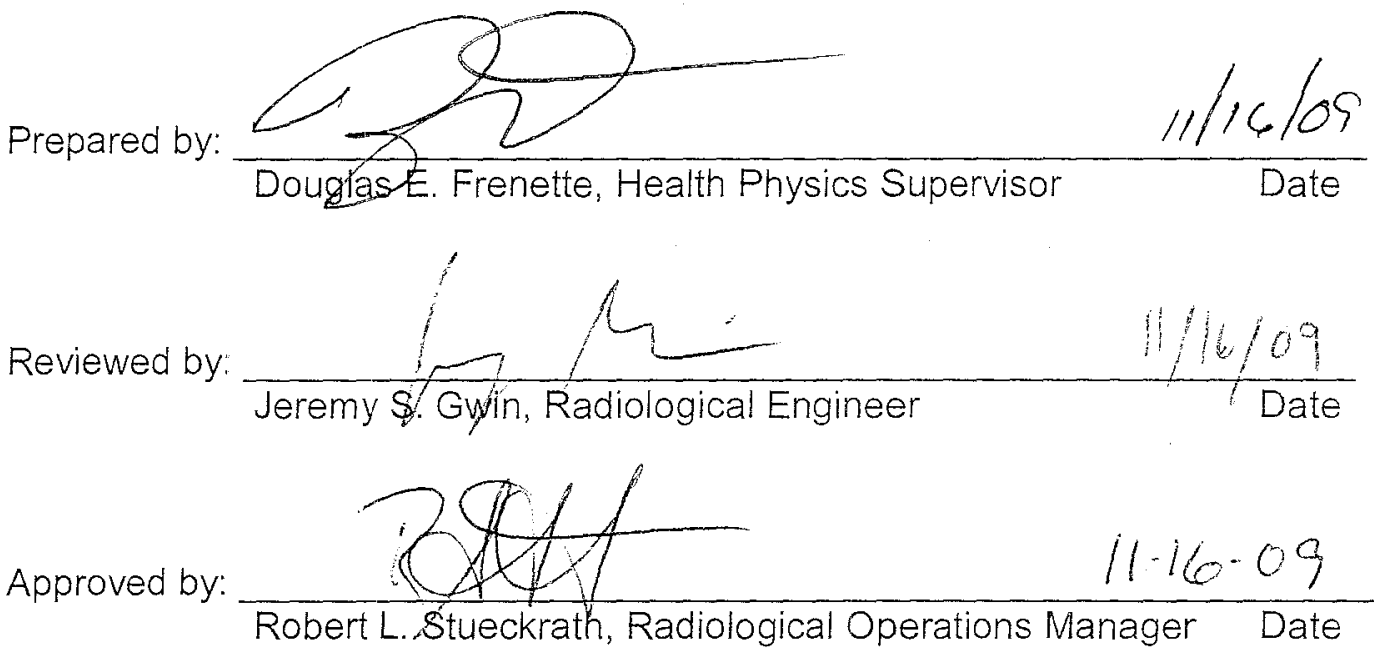


\section{Purpose}

This plan provides Radiological Control Technicians (RCTs) with the survey criteria required to complete the second phase of radiological characterization in Building 2201, Pluto Disassembly Facility.

This plan also provides RCTs with the survey criteria required after performing required remediation (i.e., decontamination) activities.

\section{Scope}

This plan pertains to specific locations inside Building 2201 in Area 26. The specific locations listed in Section V, "Contamination Surveys," were inaccessible during the first phase of characterization (Survey Plan 09-022) and will now be made accessible for RCT access and subsequent survey.

In addition, during the first phase of the characterization, multiple areas of fixed radioactive contamination were identified in rooms 102, 104, 106, and 107. Decontamination of these areas will be performed in accordance with the work package ENV-09-CAU117-0051 and Radiological Work Permit (RWP) 09-0026-01. Upon completion of decontamination activities at each identified location, RCTs will resurvey the area and surrounding space in accordance with the survey requirements of this survey plan in Section V.

Implementing the Multiagency Radiation Survey and Site Investigation Manual (MARSSIM) process

As part of the MARSSIM approach to releasing Building 2201 from radiological controls the standards recommend the following steps:

1. Historical Site Assessment (HSA) - This is the collection of data from historical documentation, observation or personnel interviews, also known as "process knowledge." A brief synopsis of the facility and operations is included in later sections.

2. Scoping Survey - A scoping survey is a "big picture" type survey performed if the HSA indicates an area may be impacted. It should also determine the specific contaminant(s) of concern. In the case of the Pluto Disassembly Facility, it has already been established that various rooms and systems are or may be impacted. This step has been accomplished through pre-job and job coverage surveys performed during Decontamination and Decommissioning (D\&D) preparations last year including removal of hazardous materials, asbestos abatement, equipment strip-out, stabilization of bi-parting doors, vault investigation, and draining of all fluid systems. It is a culmination of several smaller surveys and analytical samples.

3. Characterization Survey - This is a more comprehensive survey and determines the nature and extent of the contamination, remedial alternatives, and provides data for the Final Status Survey. This is the intent of this survey plan.

4. Remedial Action Support Survey - This survey is more commonly referred to on the Nevada Test Site (NTS) as "in-process" or "job coverage" survey. Its purpose is to provide real time data that will help guide remedial action operations (i.e., decontamination and contaminated equipment removal.) This is also the intent of this survey plan. 
5. Final Status Survey - This survey is used to demonstrate that the potential dose from residual contamination is below release criteria. Release criteria depend on several factors, but primarily on what the final disposition is (e.g., release to the public, release to an onsite landfill, below FFACO use restriction). This survey is the most comprehensive survey performed and has the most stringent requirements built into it for the type, quantity, and quality of data acquired. This survey requires a detailed survey plan with a high level of approval including the regulatory agencies representatives.

6. Verification Survey - To determine if the site is acceptable for release, the regulatory agency $\underline{\text { might }}$ bring in an independent contractor to perform this survey. It is typically a survey with limited scope that might duplicate $10 \%$ of the final status survey measurements.

Note: The intent of this survey plan covers both steps 3 and 4.

\section{History}

Building 2201 was constructed between May 1959 and October 1960 in order to perform remote adjustment, component replacement, and complete disassembly of Tory II reactors for Project Pluto.

Between the years 1961 - 1964, Lawrence Livermore National Laboratory (LLNL) conducted six nuclear ram-jet engine tests as part of Project Pluto. Four (4) tests of the Tory II-A Reactor and two (2) tests of the Tory II-C Reactor were conducted. Assembly and disassembly operations of the Tory II-A and Tory II-C took place in Building 2201. Each of these reactors contained highly enriched uranium dioxide $\left(\mathrm{UO}_{2}\right)$ contained in fuel rods that were remotely disassembled and inspected in the hot cell rooms after each test run. July 1, 1964, Project Pluto was cancelled.

Between the years 1971-1972, fuel repackaging operations took place in the hot cells of Building 2201. During these activities, decontamination and radiological surveys were conducted revealing radiological contamination in the hot cell rooms.

In June of 1974, the Tory II-C Reactor was moved to another facility in Area 25 for final disassembly.

In the mid to late 1980's until 1992, Hydrogen Content Testing was performed in rooms 101 \& 102 utilizing radioactive sources containing isotopes such as Cs-137, Plutonium/Beryllium (PuBe), and Americium /Beryllium (AmBe).

In 1985, 1998, and 1999 walk-through surveys were performed inside Building 2201, including the hot cell rooms. Varying levels of contamination were found during these surveys.

In 1998, Sandia National Laboratory intermittently used Building 2201 in order to assemble and launch rocket test systems. Very little documentation is available during this time frame due to the classified nature of the project. This is the last known documented operation performed in the building.

Building 2201 has been slated for D\&D and preparations are ongoing. Recent D\&D preparatory activities will aid in providing access to survey suspected radiological areas in the building. 
These activities include:

- Removal of a laminated wood floor covering in rooms 104, 106 and 107. To allow for access to the underlying floor foundation.

- Removal of the four underground vault lids and associated vault material racks residing in rooms 104, 106 and 107. To allow for access to the vault walls and floor.

- Performance of additional asbestos abatement in the basement's and Room 103's second stage filter plenums. To allow access to internal sections of the building ventilation system.

- Removal of the wooden ceiling in Room 101's west mezzanine. To allow access to the building's original ceiling surfaces in that location.

\section{Additional Historical and System Information}

Ventilation System - At one time, the process ventilation system in the Pluto Disassembly Facility was found to have gross contamination above DOE/NV/11718--079-REV 5, "NV/YMP Radiological Control Manual," Table 4-2. A portion of the ventilation system is internal to the concrete reinforced walls and in other places the system is located below grade before reconnecting in Room 103 and exiting out the building through the large exterior stack.

The system supported operations in rooms 102, 104, 106, \& 107. Each of the rooms had a set, or "bank" of HEPA filters; these were the first stage filters and intake locations for the system. The system then travels a short distance through internal passages in the walls to either Room 103 "Equipment room" or the facility basement. At these locations, air traversed through another bank of HEPA filters (second stage) before it entered a common header with the other process rooms and was exhausted out through the building stack. All of the HEPA filters in all of the racks have been removed and disposed of. The only contamination found was at, or immediately downstream of the first stage HEPA filters. In all cases, the intake filter frames or "racks" were removed and disposed of and the remaining framework embedded in the structure's concrete walls were encapsulated with a fixative and left in place.

Underground Vaults - There are four known underground vaults in Building 2201. These vaults were designed for the storage of nuclear and radioactive material. The vaults were investigated between April 2008 and September 2008. During this time, each of the four vaults were core drilled, video monitored, sampled, and surveyed. The vaults were then resealed. Each vault was described as being 5 feet square and 10 feet deep pits with a layered 3 foot thick cover. Each vault had a multi-level shelf or "rack" system installed. Radiological Control personnel noted all readings were indistinguishable from background and did not find evidence that any nuclear or radioactive material remained.

False Floors - Although the documentation is not conclusive in regards to the time frame, a "false floor" was installed in rooms 104, 106, and 107. This floor consisted of plywood and a laminated surface. Some consideration was given regarding the addition of the floor; it had been speculated that the floor had been installed to cover or contain underlying radiological contamination. However, interviews with craft personnel, who may have been on hand during the installation, reported that the flooring was to provide a smooth surface to move sensitive equipment over for operations occurring in those rooms during that time. In addition, during a previous characterization attempt, small sections (samples) were removed from the floor in various locations. Surveys were performed under the removed sections of flooring; no contamination was found at any of the locations. However, due to the inaccuracies that exist with the historical data on the floor, the determination was made to remove the entire false floor and survey the building's permanent concrete foundation underneath it. 


\title{
IV. Instructions
}

\author{
***Caution*** \\ In carrying out the instructions of this survey plan, RCTs may encounter areas \\ meeting the definition of a Confined Space (Permitted or Non-Permitted). RCTs are \\ NOT to enter these areas but should attempt to accomplish the goals of this survey \\ plan through the use of extension tools and remote reading instrumentation.
}

All surveys shall be performed by qualified personnel. Personnel must use their experience, knowledge of the area in which they are working, and workplace indicators to ensure adequate surveys are being performed, in addition to the information provided in this survey plan.

Based on process knowledge, historical documentation, and recent radiological surveys performed, the building will be divided into two categories to establish survey rigor - "Higher Risk," and "Lower Risk."

Since the intent of this survey plan is for both characterization and post remediation, (i.e., not for unrestricted release), release limits are not applicable at this time. Instead, RCTs will operate under the suspension limits of the RWP and will record all data.

In order to determine the degree and rigor of future remediation, RCTs shall document any location found above the limits from DOE/NV/25946--801, "Nevada Test Site Radiological Control Manual," (NTS RCM) Table 4-2:

- $20 \mathrm{dpm} / 100 \mathrm{~cm}^{2}$ alpha (removable)

- $100 \mathrm{dpm} / 100 \mathrm{~cm}^{2}$ alpha (fixed \& removable)

- $1,000 \mathrm{dpm} / 100 \mathrm{~cm}^{2}$ beta (removable)

- $5,000 \mathrm{dpm} / 100 \mathrm{~cm}^{2}$ beta (fixed \& removable)

During this survey process, if contamination is found that is above the suspension limits of the RWP, work shall be paused, personnel in the affected area or room notified of the condition, and instructed to exit the area or room. RCTs will immediately notify the Environmental Restoration Health Physics Supervisor (ER HPS) and survey any personnel who were in the affected room or area. Based on the levels encountered, RCTs will also adjust radiological postings to be commensurate with actual area conditions.

Physically gridding off the entire building is not necessary for this survey plan. However, in rooms 104, 106, and 107 after the false floor has been removed, gridding of the floors is mandatory. Grids for the floors in these rooms will be made as specified in Appendix A and will consist of 1 meter by 1 meter areas or "survey units." These grids shall be visually marked with paint, chalk lines, or any other method of demarcation. For areas considered "Lower Risk," in lieu of gridding, RCTs must mark where survey data is obtained and in the unlikely event, unmistakably demarcate locations where contamination is found above the aforementioned levels. RCTs shall post radiological areas according to actual area conditions.

Background levels for direct surveys shall be less than $40 \mathrm{dpm} / 100 \mathrm{~cm}^{2}$ alpha and $4,000 \mathrm{dpm} / 100 \mathrm{~cm}^{2}$ beta using the NE Electra. Surveys will consist of measurements for both Alpha and Beta contamination. All surveys shall be performed and documented in accordance with OI-0441.211, "Direct and Indirect Surveys." Survey results shall be recorded on a FRM-0108, "Radiological Survey Report," and approved by the ER HPS or designee. 


\section{Contamination Surveys}

\section{Second Phase Characterization}

Higher Risk - As part of the second phase of Pluto's radiological characterization, the following areas in Building 2201 are considered higher risk:

- Room 104 (under the false floor) - Hot and Warm Cell

- Room 106 (under the false floor) - Kilo-Curie Hot Cell

- Room 107 (under the false floor) - Hot Storage and Packaging Room

- Each of the four underground vaults (rooms 104, 106, and 107)

- Each of the four second stage filter plenums (Room 103 and facility's basement)

Each of these areas shall be surveyed using the following guidelines:

Direct surveys $-100 \%$ of accessible surfaces shall be scanned with an NE Electra and DP6 Probe (or equivalent) followed with a direct static measurement at the highest direct scanned reading in each grid location, or "survey unit" documented for both alpha and beta.

Note: After false floors are removed in rooms 104, 106, and 107, RCTs shall grid off the underlying floor surfaces into 1 meter by 1 meter grid sections.

Removable surveys - At each location where direct readings are greater than $100 \mathrm{dpm} / 100 \mathrm{~cm}^{2}$ alpha or $5,000 \mathrm{dpm} / 100 \mathrm{~cm}^{2}$ beta, a swipe shall be taken and documented. Count all swipes on a performance tested Tennelec, Protean, 3030 or 2929 scalers. Otherwise, swipes are not required for the purposes of this survey plan.

Lower Risk - As part of the second phase of Pluto's radiological characterization, the following areas will be considered Lower Risk, and surveyed under the following guidelines:

\section{Direct Surveys}

- Room 101 West mezzanine wood ceiling (two sections) - 100\% of newly exposed accessible surfaces shall be scanned with an NE Electra and DP6 Probe (or equivalent) followed with a direct static measurement at the highest direct scanned reading in both ceiling section and documented for both alpha and beta.

Removable surveys - At each location where direct readings are greater than $100 \mathrm{dpm} / 100 \mathrm{~cm}^{2}$ alpha or $5,000 \mathrm{dpm} / 100 \mathrm{~cm}^{2}$ beta, a swipe shall be taken and documented. Count all swipes on a performance tested Tennelec, Protean, 3030 or 2929 scalers. Otherwise, swipes are not required for the purposes of this survey plan.

\section{Post Remediation}

From the first phase of characterization, multiple areas were identified which require remediation in order to meet the requirements of the NTS RCM Table 4-2. These specific locations were physically marked and survey numbers are provided to give additional clarification. All areas noted were found only to have fixed contamination, no removable contamination above the NTS RCM Table 4-2 levels were discovered. 
Decontamination will be performed to the extent needed to meet the Table 4-2 requirements of the NTS RCM. Decontamination may be accomplished utilizing a multitude of different methods and may be as simple as wiping an area with wipes and a cleaning solution to much more aggressive methods such as needle guns, grinders, scabblers, or concrete shavers. In some cases, the most effective method may be to simply remove the affected area altogether such as an electrical panel door or in the case of the false floors, removal of the wooden flooring.

At the time of this survey plan, the known areas requiring remediation have been identified as follows:

\section{Room 102 (Survey \#09-ER-A26-029)}

- North Wall - $796 \mathrm{dpm} / 100 \mathrm{~cm}^{2}$ alpha

- North Wall (electrical panel) - 1,110 dpm/100 $\mathrm{cm}^{2}$ alpha

- South Wall - 1,200 dpm/100 $\mathrm{cm}^{2}$ beta

- Floor (multiple locations) - 5,210 to $19,500 \mathrm{dpm} / 100 \mathrm{~cm}^{2}$ beta

\section{Room 104 (Survey \#09-ER-A26-032)}

- South Wall (entire upper ledge) - 300 to $400 \mathrm{dpm} / 100 \mathrm{~cm}^{2}$ alpha

- South Wall (inside penetration) - $354 \mathrm{dpm} / 100 \mathrm{~cm}^{2}$ alpha

- East Wall (bottom, left side of filter rack) $-200 \mathrm{dpm} / 100 \mathrm{~cm}^{2}$ alpha

- East Wall (bottom of window ledge) $-12,000 \mathrm{dpm} / 100 \mathrm{~cm}^{2}$ beta

\section{Room 106 (Survey \#09-ER-A26-033)}

- South Wall (bottom, inside penetration) - $226 \mathrm{dpm} / 100 \mathrm{~cm}^{2}$ alpha

- South Wall (inside plenum) - 210 to $710 \mathrm{dpm} / 100 \mathrm{~cm}^{2}$ alpha

- South Wall (between grids A-3 and A-4) - 19,500 dpm/100 $\mathrm{cm}^{2}$ beta

- East Wall (3' high) - 2,640 dpm/100 $\mathrm{cm}^{2}$ beta

- East Wall (7' high) - 1,644 dpm/100 $\mathrm{cm}^{2}$ beta

\section{Room 107 (Survey \#09-ER-A26-034)}

- Floor (multiple locations) - 158 to $519 \mathrm{dpm} / 100 \mathrm{~cm}^{2}$ alpha

Additional Areas - Any additional areas found during the second phase of characterization that require remediation will be included in this survey plan as long as the level of potentially removable contamination does not exceed $2,000 \mathrm{dpm} / 100 \mathrm{~cm}^{2}$ alpha or $100,000 \mathrm{dpm} / 100 \mathrm{~cm}^{2}$ beta/gamma (i.e., does not have the potential to create a High Contamination Area).

Direct surveys $-100 \%$ of the remediated surface and each adjacent grid location shall be scanned with an NE Electra and DP6 Probe (or equivalent) followed with a direct static measurement at the highest direct scanned reading in each grid location and documented for both alpha and beta.

Note: After false floors are removed in rooms 104, 106, and 107, RCTs shall grid off the underlying floor surfaces into 1 meter by 1 meter grid sections (see Appendix A for gridding instructions).

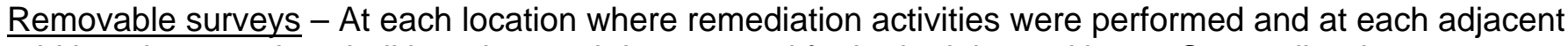
grid location, a swipe shall be taken and documented for both alpha and beta. Count all swipes on a performance tested Tennelec, Protean, 3030 or 2929 scalers. 


\section{Disposition}

There is no disposition of the building, equipment, or system components scheduled at this time. 


\section{Appendix A}

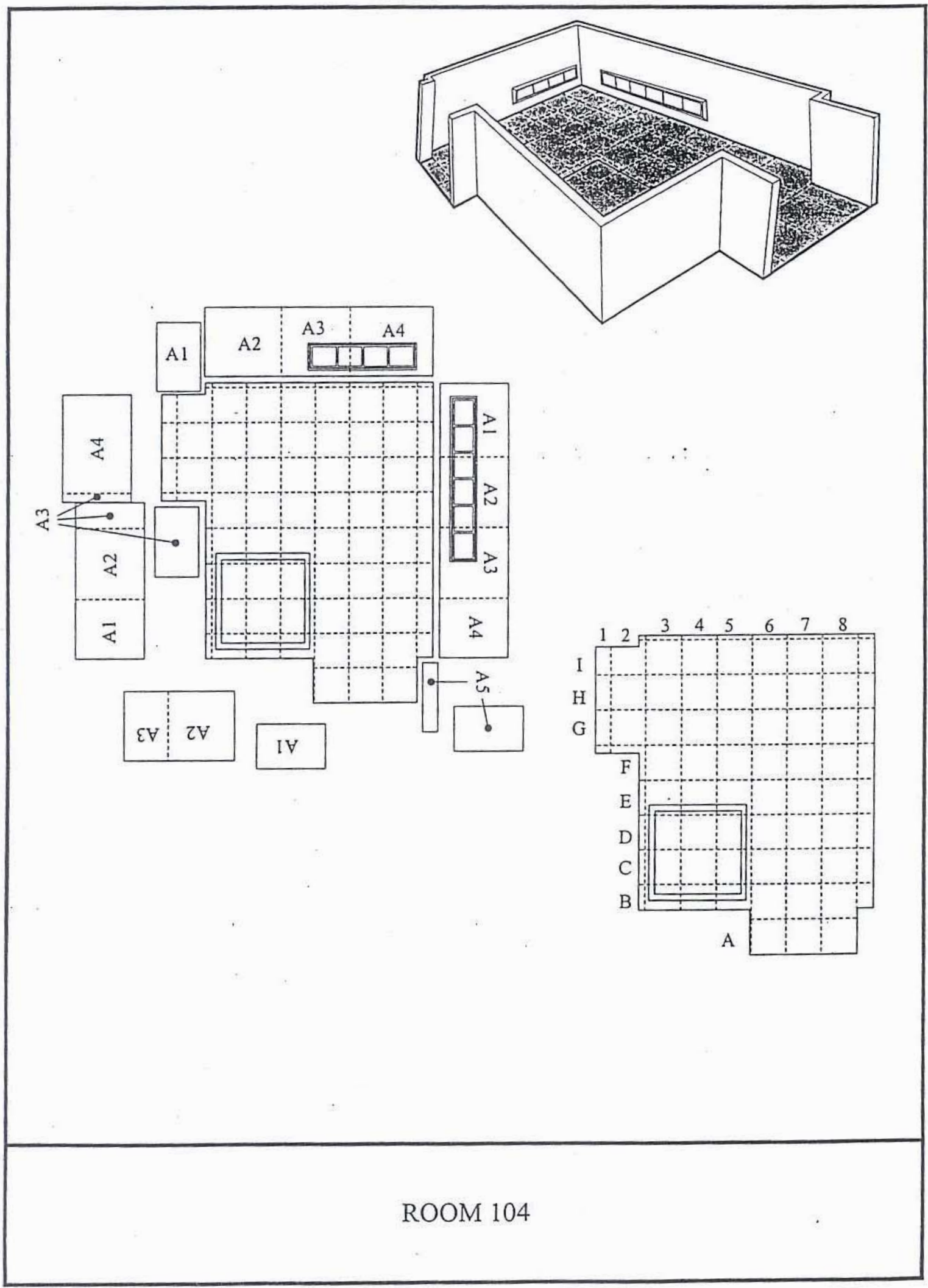




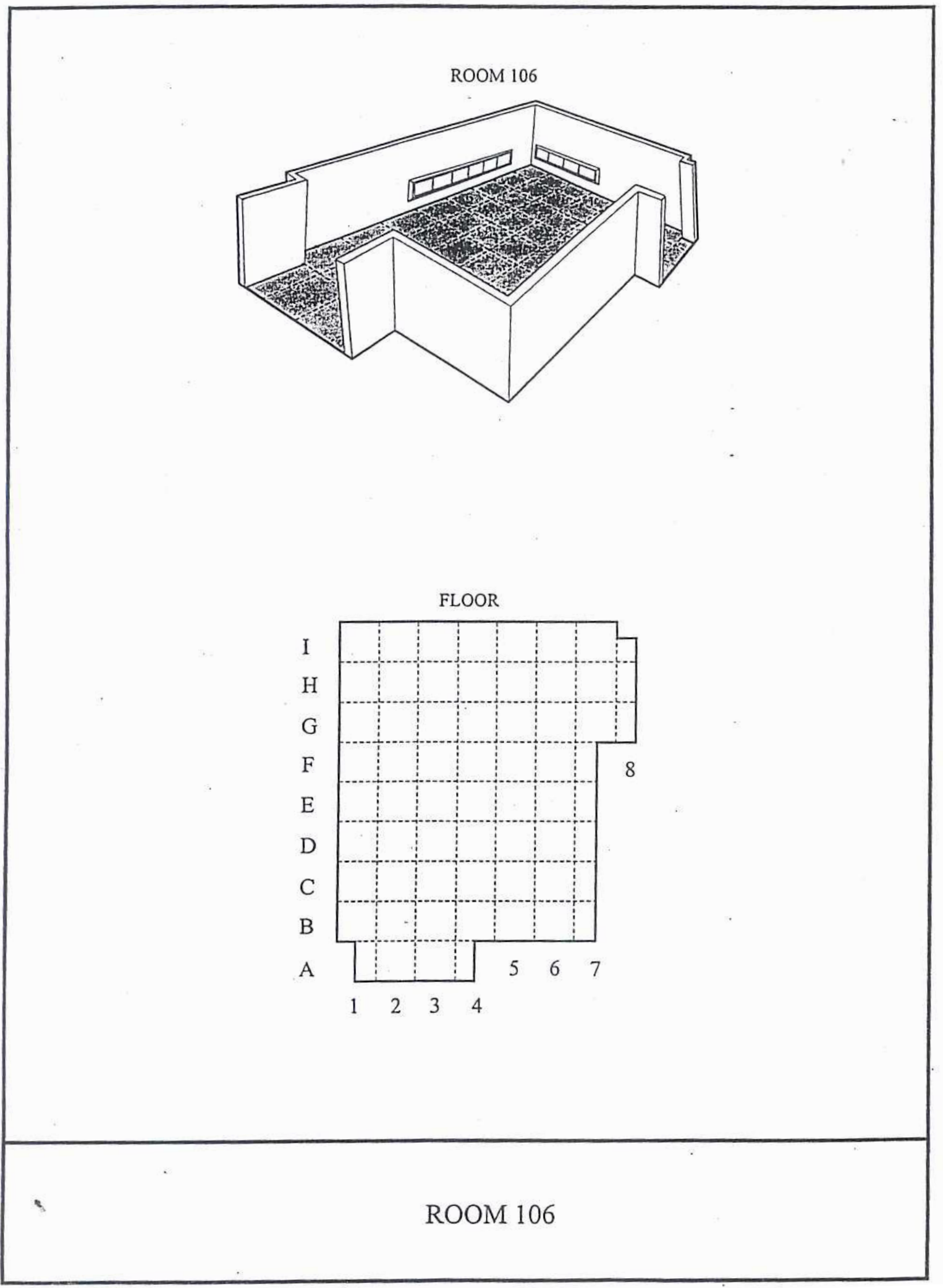



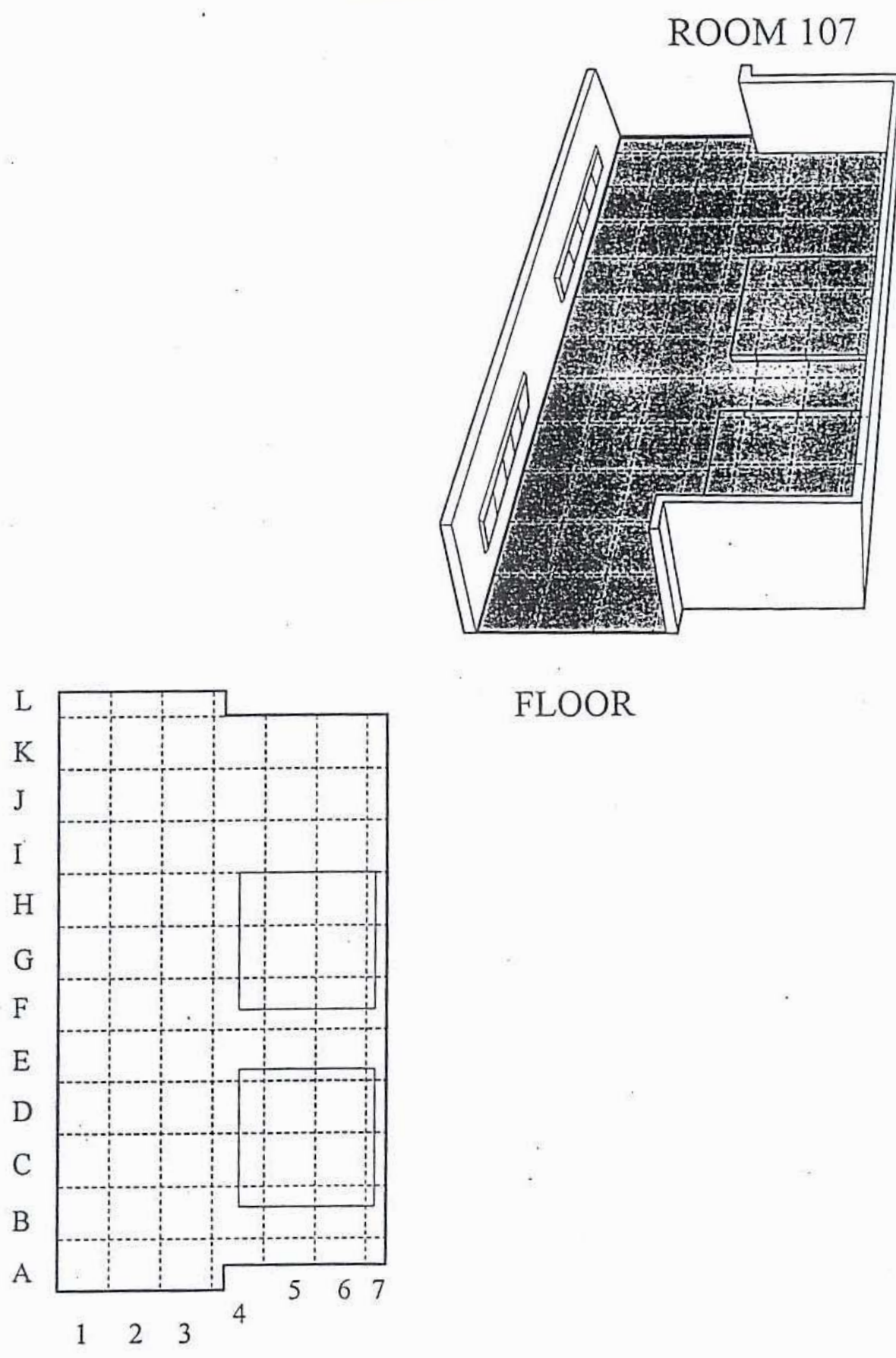

FLOOR

ROOM 107 